\title{
The Savannah River Site's Groundwater Monitoring Program First Quarter 1999 (January through March 1999)
}

by

J. B. Hutchison

Westinghouse Savannah River Company

Savannah River Site

Aiken, South Carolina 29808

This paper was prepared in connection with work done under the above contract number with the U. S. Department of Energy. By acceptance of this paper, the publisher and/or recipient acknowledges the U. S. Government's right to retain a nonexclusive, royalty-free license in and to any copyright covering this paper, along with the right to reproduce and to authorize others to reproduce all or part of the copyrighted paper. 


\title{
THE ENVIRONMENTAL PROTECTION DEPARTMENT ENVIRONMENTAL MONITORING SECTION
}

\author{
The Savannah River Site's \\ Groundwater Monitoring Program
}

\section{FIRST QUARTER 1999 (U) (January through March 1999)}

Environmental Protection Department

Westinghouse Savannah River Company

Aiken, SC

and

Exploration Resources, Inc.

Athens, GA

Reviewed and approved by

Jay Hutchison

EPD/EMS Groundwater Coordinator

Cover graphics supplied by R.A. Hiergesell

Savannah River Technology Center

Environmental Sciences Section

Publication Date: September 1999

Westinghouse Savannah River Company

Savannah River Site

Aiken, SC 29808

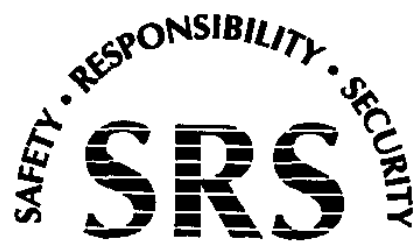

Prepared for the U.S. Department of Energy under Contract No. AB60294N 


\section{This Quarter at a Glance ...}

Executive Summary - table of all analytes detected at or above Flag 2 criteria

Flagging Criteria - standards for flagging results

Sample Scheduling-description of the sampling schedule

Field Notes-comments from the field-data books

Analytical Data Review-discrepancies in each laboratory's analytical data; laboratory-specific methods and estimated quantitation limits

Quality Control Samples-discussion of the quality of the analytical data in terms of precision, accuracy, representativeness, comparability, and completeness

Site Index-table of the well series and their site locations; also discusses the history of the sites Appendices:

A. Water-Level Data-tables listing field data obtained for hydrogeologic studies

B. Analytical Results - tables listing the quarter's analytical results and field data

C. Sampling Blanks Results-tables listing all analytical results for sampling blanks for the quarter

$$
\text { -.. }
$$

The following is a key to the numbered areas of the Savannah River Site.

\section{Site}

100 Areas - Reactors

200 Areas-Separations

300 Areas-Reactor Materials

400 Area-Heavy Water

600 Areas-General

700 Area-Administration

\section{Function}

To operate and support the reactors

To separate and purify the product from fuel and target assemblies; to process waste

To fabricate new fuel and target assemblies from raw materials

To produce steam and electrical power; to process heavy water

Other (general)

To nrovide administrative and support services

\section{DISCLAIMER}

This report was prepared as an account of work sponsored by an agency of the United States Government. Neither the United States Government nor any agency thereof, nor any of their for the accuracy, completenes, express or implied, or assumes any legal liability or responsibility fisclosed, or represents that its, or usefulness of any information, apparatus, product or process any specific commercial product, prould not infringe privately owned rights. Reference herein to otherwise does not necessarily constitute or service by trade name, trademark, manufacturer, or by the United States Government or any agency thereof. The recommendation, or favoring expressed herein do not necessarily state or reflect those of the views and opinions of authors agency thereof.

This report has been reproduced directly from the best available copy. Available for sale to the public, in paper, from: U.S. Department of Commerce, National Technical Information Service, 5285 Port Royal Road, Springfield, VA 22161, phone: (800) 553-6847 email: orders@ntis.fedworld.gov

7 online ordering: http://www.ntis.gov/ordering.htm

Available electronically at http://www.doe.gov/bridge

I Available for a processing fee to U.S. Department of U.S. Department of Energy, Office of Scientifict of Energy and its contractors, in paper, from: Ridge, TN 37831-0062, phone: (865) 576-8401 fax: (865) 576-5728

email: reports@adonis.osti.gov 


\section{Contents}

Page

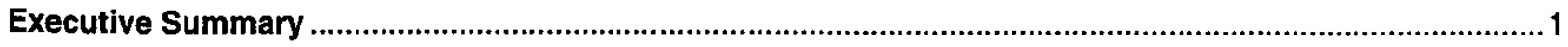

Introduction

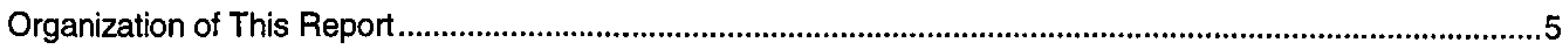

Flagging Criteria

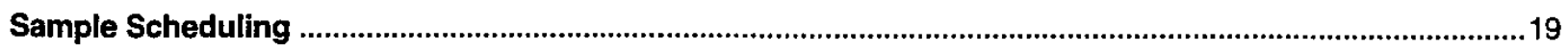

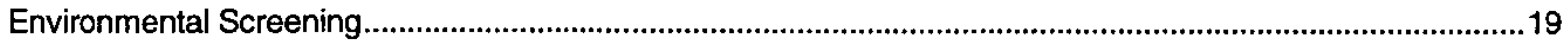

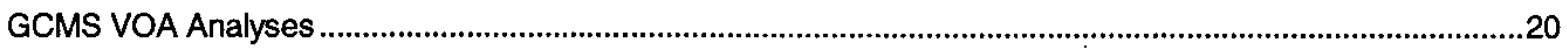

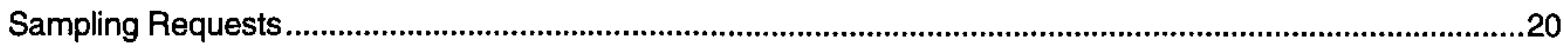

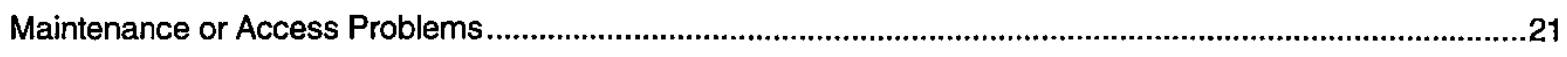

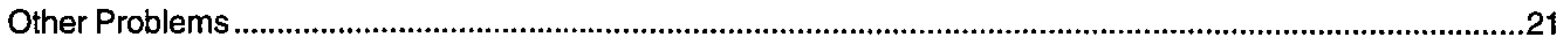

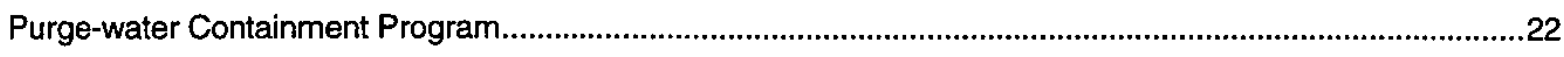

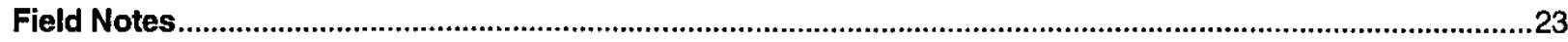

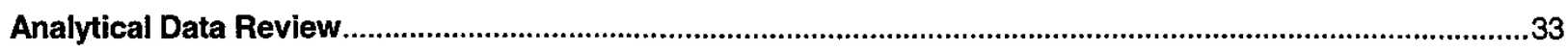

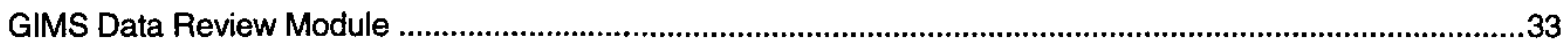

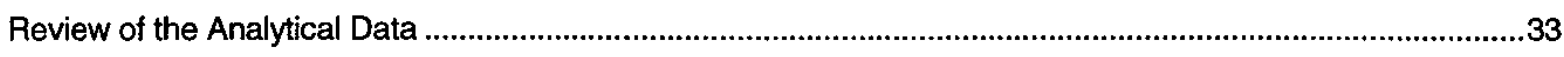

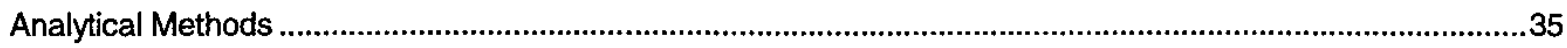

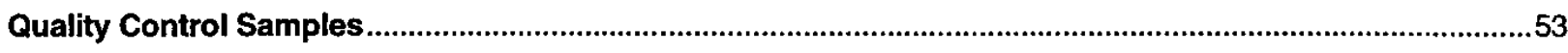

Precision

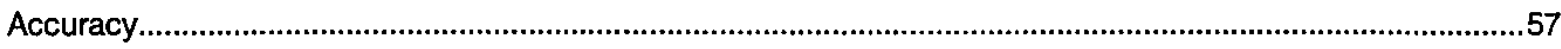

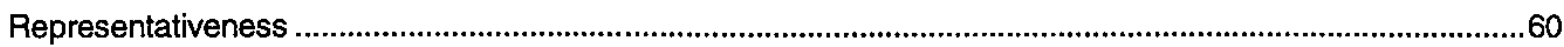

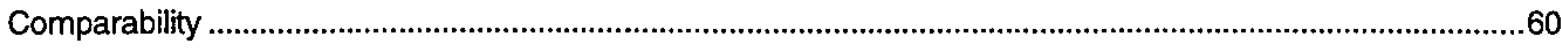

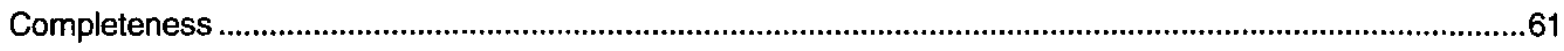

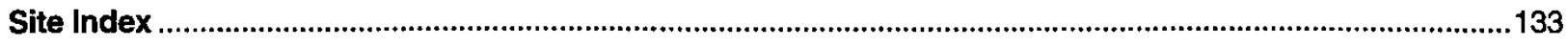

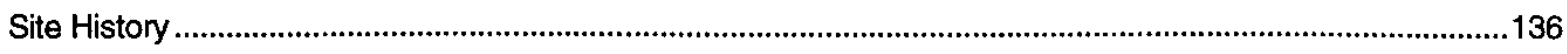

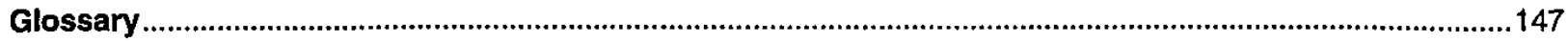


References

Appendix A. Water-Level Data .

A-1

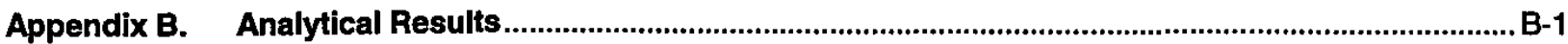

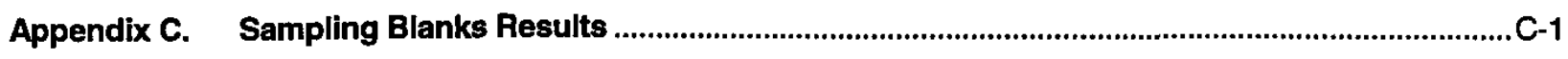

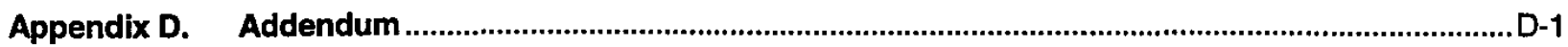




\section{LIST OF FIGURES}

Figure 1. Areas and Locations Monitored for Groundwater Quality ...................................................7

Figure 2. Separations and Waste Management Areas Monitored for Groundwater Quality ....................8

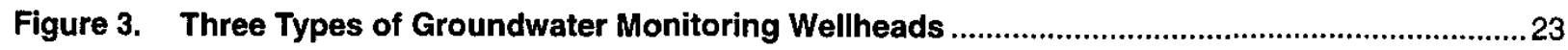

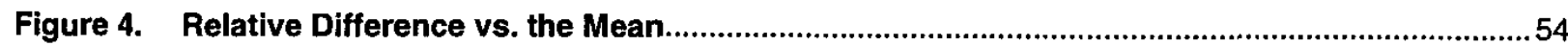

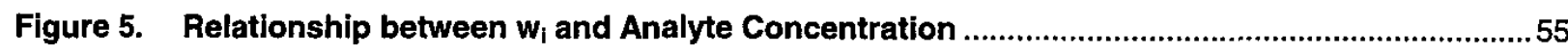

Figure 6. Effect of a Linear-Weight Function on the MRDadj.........................................................55 


\section{LIST OF TABLES}

Table 1. Analytes above Flag 2 Criteria

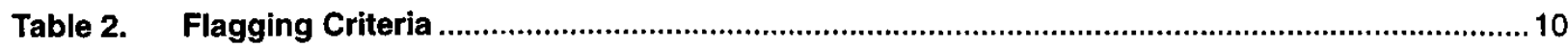

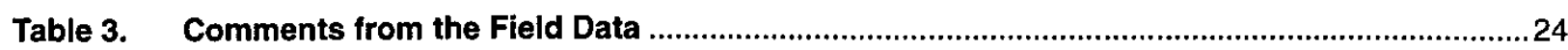

Table 4. EX Samples with High Analytical Results as Compared to Historical Data ............................35

Table 5. GE Samples with High Analytical Results as Compared to Historical Data ...........................36

Table 6. GE Samples with Low Analytical Results as Compared to Historical Data...........................36

Table 7. GP Samples with High Analytical Results as Compared to Historical Data ...........................37

Table 8. WA Samples with High Analytical Results as Compared to Historical Data ..........................37

Table 9. TM Samples with High Analytical Results as Compared to Historical Data ............................37

Table 10. Methods and Estimated Quantitation Limits Used by EX …..................................................37

Table 11. Methods and Estimated Quantitation Limits Used by GE ................................................... 41

Table 12. Methods and Estimated Quantitation Limits Used by WA ..................................................45

Table 13. Methods and Estimated Quantitation Limits Used by GP ...................................................50

Table 14. Methods and Estimated Quantitation Limits Used by TM ..................................................51

Table 15. Wells Providing Blind Replicate Samples and Associated Blanks .....................................61

Table 16. Analytes Not Showing Measurable Concentrations above Estimated Quantitation Limits in Any Replicated or Duplicated Samples for GE, WA, and EX ........................................62

Table 17. Analytes Not Showing Measurable Concentrations above Estimated Quantitation Limits in Any Replicated or Duplicated Samples for GP and TM ...................................................65

Table 18. Intralaboratory MRD Indices for EX

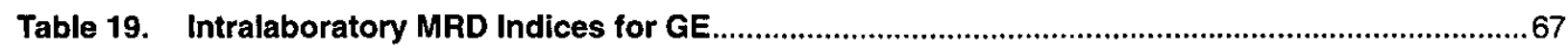

Table 20. Intralaboratory MRD Matrix Spike Indices for GE .........................................................68

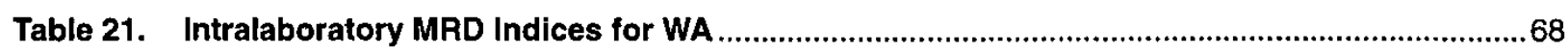

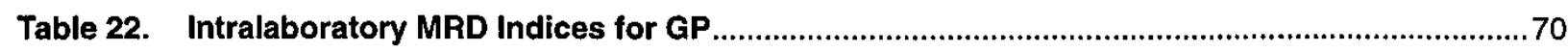

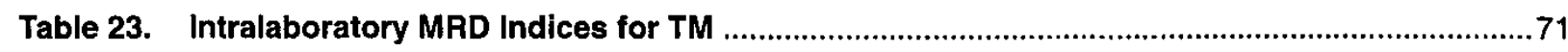

Table 24. Interlaboratory MRD and t-test Results for Analytes with at Least One Pair of Results

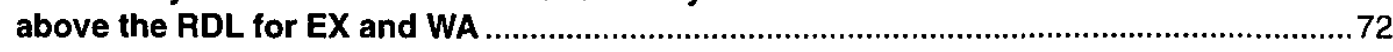


Table 25. Interlaboratory MRD and t-test Results for Analytes with at Least One Pair of Results above the RDL for GE and WA.

Table 26. Interlaboratory MRD and t-test Results for Analytes with at Least One Pair of Results above the RDL for GP and TM

Table 27. EX Samples and Blind Replicates Yielding Results Where One Is More Than Twice Another

Table 28. GE Samples and Blind Replicates Yielding Results Where One Is More Than Twice Another

Table 29. WA Samples and Blind Replicates Yielding Results Where One is More Than Twice Another

Table 30. WA Samples and Laboratory Duplicates Yielding Results Where One Is More Than Twice Another.

Table 31. GP Samples and Blind Replicates Yielding Results Where One Is More Than Twice Another

Table 32. GP Samples and Laboratory Duplicates Yielding Results Where One Is More Than Twice Another.

Table 33. TM Samples and Blind Replicates Yielding Results Where One Is More Than Twice Another.

Table 34. TM Samples and Laboratory Duplicates Yielding Results Where One Is More Than Twice Another.

Table 35. Analytes with One Laboratory's Result Greater Than Twice the Result from the Other Laboratory between EX and WA

Table 36. Analytes with One Laboratory's Result Greater Than Twice the Result from the Other Laboratory between GE and WA

Table 37. Analytes with One Laboratory's Result Greater Than Twice the Result from the Other Laboratory between GP and TM

Table 38. Quality Control Standards for Selected Analyses for EX .....................................................76

Table 39. Quality Control Standards for Selected Analyses for GE.......................................................79

Table 40. Quality Control Standards for Selected Analyses for WA .................................................... 82

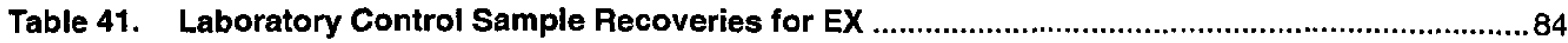

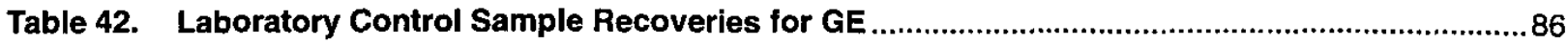

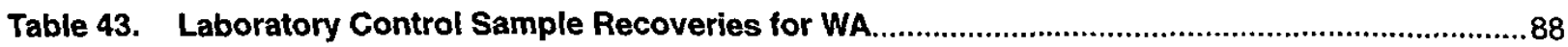

Table 44. Laboratory Control Sample Recoveries for GP ...................................................................

Table 45. Laboratory Control Sample Recoveries for TM ...................................................................92 
Table 46. Surrogate Recoveries for EX

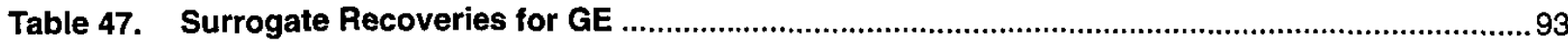

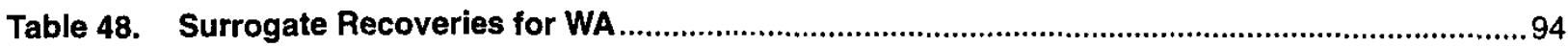

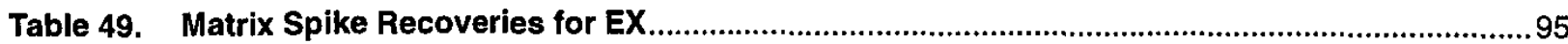

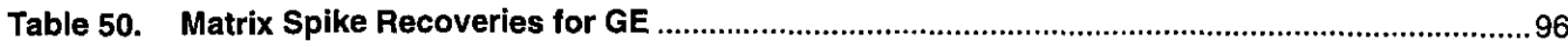

Table 51. Matrix Spike Recoveries for WA

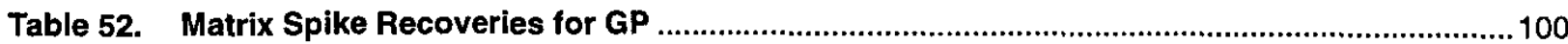

Table 53. Analytes Detected in Method Blanks for EX ..................................................................... 101

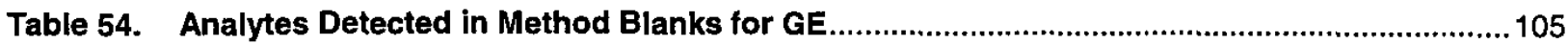

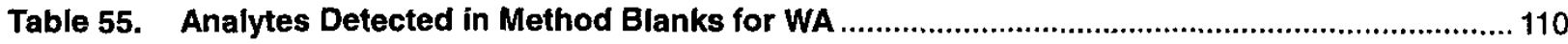

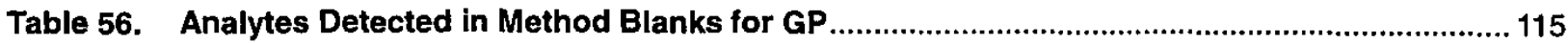

Table 57. Analytes Detected in Method Blanks for TM..................................................................... 117

Table 58. Analytes Detected in Field Blanks for EX

Table 59. Analytes Detected in Field Blanks for GE .......................................................................119

Table 60. Analytes Detected in Field Blanks for WA

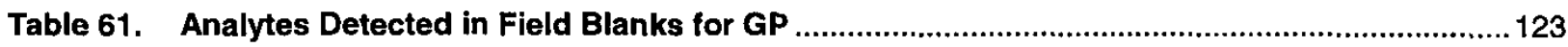

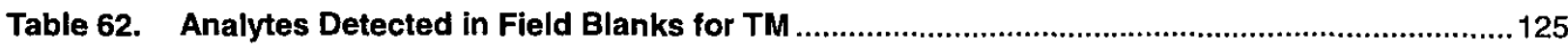

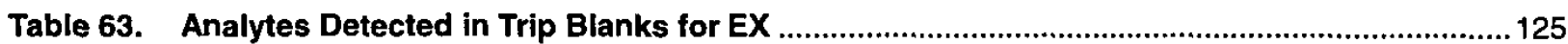

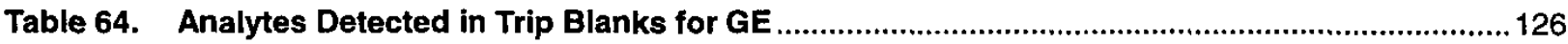

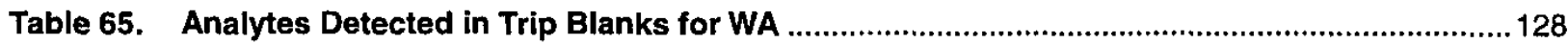

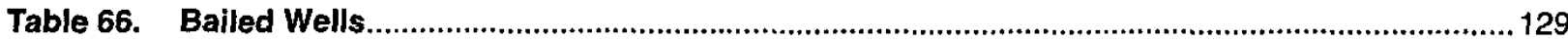

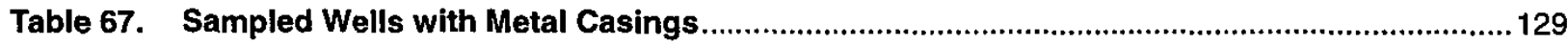

Table 68. Wells That Had Turbidity Greater Than 15 NTU............................................................... 130

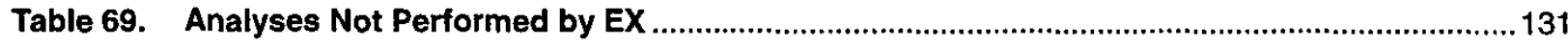

Table 70. Analyses Not Performed by GE

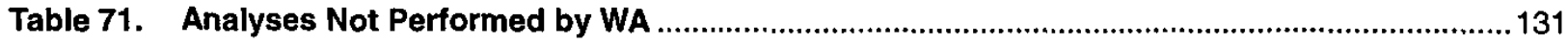

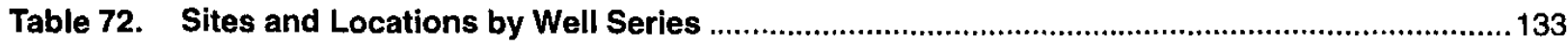




\section{Executive Summary}

The Environmental Protection Department/Environmental Monitoring Section (EPD/EMS) administers the Savannah River Site's (SRS) Groundwater Monitoring Program. During first quarter 1999, EPD/EMS conducted extensive sampling of monitoring wells.

EPD/EMS has established two sets of flagging criteria to assist in managing sample results. The flagging criteria do not define contamination levels; instead, they aid personnel in sample scheduling, data interpretation, and trend identification. Since 1991, the flagging criteria have been based on the U.S. Environmental Protection Agency (EPA) drinking water standards and on method detection limits. A detailed explanation of the flagging criteria is presented in the Flagging Criteria section of this document. Analytical results from first quarter 1999 are included in this report, which is distributed to all site custodians.

One or more analytes exceeded Flag 2 criteria during first quarter 1999 in 76 monitoring well series. Analytes exceeded the current Flag 2 criteria for the first time since 1984 in 11 of those 76 monitoring well series.

Table 1, organized alphabetically by well series, lists those well series with analytical results above Flag 2 criteria during first quarter 1999. Results from all laboratory analyses are used to generate this table. Specific conductance and $\mathrm{pH}$ data from field measurements also are included in this table.

\section{Table 1. Analytes above Flag 2 Criteria}

\begin{tabular}{|c|c|c|}
\hline Site & $\begin{array}{l}\text { Well } \\
\text { Series }\end{array}$ & Analytes above Flag 2 Criteria \\
\hline A-Area Metals Buming Pit & ABP & $\begin{array}{l}\text { Aluminum, dichloromethane, iron, lead, } \mathrm{pH}_{1} \text { specific } \\
\text { conductance, tetrachloroethylene, trichloroethylene }\end{array}$ \\
\hline $\begin{array}{l}\text { A-Area Cluster Perimeter Wells and } \mathrm{M} \text { - } \\
\text { Area Plume Definition Wells }\end{array}$ & $A C$ & Iron \\
\hline $\begin{array}{l}\text { A-Area Coal Pile Runoff Containment } \\
\text { Basin }\end{array}$ & ACB & Aluminum, gross alpha, iron, manganese, trichloroethylene \\
\hline Metallurgical Laboratory Seepage Basin & AMB & $\begin{array}{l}\text { Aluminum, iron, radium, total alpha-emitting, } \\
\text { tetrachloroethylene, total organic halogens, } \\
\text { trichloroethylene }\end{array}$ \\
\hline Motor Shop Oil Basin & $\mathrm{AOB}$ & Aluminum, iron, tetrachloroethylene, trichloroethylene \\
\hline A-Area Burning/Rubble Pits & ARP & Aluminum, iron, trichloroethylene \\
\hline $\begin{array}{l}\text { Savannah River Laboratory (SRL) } \\
\text { Seepage Basins }\end{array}$ & ASB & Aluminum, iron, $\mathrm{pH}$, tetrachloroethylene, trichloroethylene \\
\hline $\begin{array}{l}\text { Mixed Waste Management Facility (Site } \\
643-28 E \text { ) and Low-Level Radioactive } \\
\text { Waste Disposal Facility (643-7E) }\end{array}$ & $\mathrm{BGO}$ & $\begin{array}{l}\text { Aluminum, carbon tetrachloride, 1, } 1 \text {-dichloroethane, 1,1- } \\
\text { dichloroethylene, iron, } \mathrm{pH} \text {, specific conductance, } \\
\text { tetrachloroethylene, trichloroethylene, tritium }\end{array}$ \\
\hline E-Area Vaults & $B G X$ & Aluminum, manganese \\
\hline Road A Chemical Basin (Baxley Road) & BRD & Aluminum, iron \\
\hline Burma Road Rubble Pit & BRR & Aluminum, carbon tetrachloride \\
\hline Old Burial Ground (643-E) & BSE & Total organic halogens, trichloroethylene, tritium \\
\hline C-Area Disassembly Basin & $\mathrm{CDB}$ & Aluminum, iron, lead, specific conductance, tritium \\
\hline
\end{tabular}




\begin{tabular}{|c|c|c|}
\hline Site & $\begin{array}{l}\text { Well } \\
\text { Series }\end{array}$ & Analytes above Flag 2 Criteria \\
\hline Chemical, Metals, and Pesticides Pits & CMP & Aluminum, iron \\
\hline C-Area Burning/Rubble Pit & CRP & Aluminum, total organic halogens, trichloroethylene \\
\hline C-Area Reactor Seepage Basins & CSB & $\begin{array}{l}\text { Aluminum, iron, lead, manganese, total organic halogens, } \\
\text { trichloroethylene, tritium }\end{array}$ \\
\hline $\begin{array}{l}\text { N-Area (Central Shops) Diesel Spill } \\
\text { Characterization and Remediation Wells }\end{array}$ & CSD & Aluminum \\
\hline $\begin{array}{l}\text { N-Area (Central Shops) Burning/Rubble } \\
\text { Pits }\end{array}$ & CSR & Aluminum, manganese \\
\hline D-Area Burning/Rubble Pits & DBP & Aluminum, iron \\
\hline $\begin{array}{l}\text { D-Area Coal Pile Runoff Containment } \\
\text { Basin and Ash Basin }\end{array}$ & DCB & $\begin{array}{l}\text { Aluminum, gross alpha, iron, lithium, manganese, specific } \\
\text { conductance, sulfate, trichloroethylene, tritium }\end{array}$ \\
\hline $\begin{array}{l}\text { F-Area Coal Pile Runoff Containment } \\
\text { Basin }\end{array}$ & FCB & Aluminum, iron \\
\hline $\begin{array}{l}\text { F-Area Effluent Treatment Cooling Water } \\
\text { Basin }\end{array}$ & FET & Aluminum, iron \\
\hline $\begin{array}{l}\text { F-Area Seepage Basins Remediation } \\
\text { Extraction Well }\end{array}$ & FEX & Specific conductance \\
\hline $\begin{array}{l}\text { F-Area Seepage Basins Remediation } \\
\text { Injection Tank }\end{array}$ & FIN & $\begin{array}{l}\text { Aluminum, 1,2-dichloroethane, iodine-129, specific } \\
\text { conductance }\end{array}$ \\
\hline Old F-Area Seepage Basin & FNB & Aluminum, gross alpha, manganese, nonvolatile beta, tritium \\
\hline F-Area Retention Basin & FRB & Aluminum \\
\hline F-Area Seepage Basins & FSB & $\begin{array}{l}\text { Aluminum, americium-241, beryllium, cadmium, cesium-137, } \\
\text { cobalt, curium-243/244, dichloromethane, gross alpha, } \\
\text { iodine-129, iron, lead, mercury, nickel, nitrate-nitrite as } \\
\text { nitrogen, nonvolatile beta, pH, radium-226, radium-228, } \\
\text { specific conductance, strontium-90, thallium, } \\
\text { trichloroethylene, tritium, uranium-233/234, uranium-235, } \\
\text { uranium-238 }\end{array}$ \\
\hline F-Area Inactive Process Sewer Line & FSL & $\begin{array}{l}\text { Aluminum, cadmium, carbon tetrachloride, gross alpha, } \\
\text { iodine-129, iron, mercury, nitrate-nitrite as nitrogen, } \\
\text { nonvolatile beta, radium-226, strontium-90, tritium, } \\
\text { uranium-233/234, uranium-238 }\end{array}$ \\
\hline F-Area Tank Farm & FTF & Gross alpha, nonvolatile beta, $\mathrm{pH}$, specific conductance \\
\hline H-Area Tank Farm Operable Unit & HAA & $\begin{array}{l}\text { Aluminum, carbon tetrachloride, 1,1-dichloroethylene, iron, } \\
\text { lead, nonvolatile beta, } \mathrm{pH} \text {, specific conductance, 1,1,1- } \\
\text { trichloroethane, trichloroethylene, tritium }\end{array}$ \\
\hline $\begin{array}{l}\text { H-Area Coal Pile Runoff Containment } \\
\text { Basin }\end{array}$ & $\mathrm{HCB}$ & Aluminum, iron, manganese \\
\hline $\begin{array}{l}\text { H-Area Effluent Treatment Cooling Water } \\
\text { Basin }\end{array}$ & HET & Aluminum, iron \\
\hline $\mathrm{H}$-Area HP-52 Outfall & HHP & Aluminum, iron, manganese \\
\hline \multicolumn{3}{|c|}{ Executive Summary } \\
\hline ESH-EMS-990520 & & First Quarter 1999 \\
\hline
\end{tabular}




\begin{tabular}{lll}
\hline Site & $\begin{array}{l}\text { Well } \\
\text { Series }\end{array}$ & Analytes above Flag 2 Criteria \\
\hline
\end{tabular}

H-Area Seepage Basins Remediation HIN Injection Tank

Old H-Area Retention Basin

H-Area Seepage Basins

H-Area Inactive Process Sewer Line

H-Area Tank Farm

H-Area Wamer's Pond

Hazardous Waste Storage Facility

K-Area Ash Basin

K-Area Bingham Pump Outage Pits

K-Area Coal Pile Runoff Basin

K-Area Retention Basin

K-Area Burning/Rubble Pit

K-Area Tritium Sump Monitoring Well

L-Area Acid/Caustic Basin

L-Area Research Wells

L-Area Bingham Pump Outage Pit

L-Area Disassembly Basin

Interim Sanitary Landfill

Miscellaneous Chemical Basin

M-Area Hazardous Waste Management Facility (HWMF)
K-Area Disassembly Basin

K-Area Reactor Seepage Basin

lodine-129, radium-226, specific conductance

Aluminum, manganese, nitrate-nitrite as nitrogen, tritium

Aluminum, americium-241, curium-243/244, gross alpha, iodine-129, iron, lead, mercury, nickel-63, nitrate-nitrite as nitrogen, nonvolatile beta, $\mathrm{pH}$, radium-226, radium-228, specific conductance, strontium-90, tetrachloroethylene, tritium, uranium-233/234, uranium-238

HSL

HTF

HWP

HWS

$\mathrm{KAB}$

KBP

KCB

KDB

KRB

KRP

KSB

KSM

LAC

LAW

LBP

LDB

LFW

MCB

MSB

Executive Summary

ESH-EMS-990520
Aluminum, gross alpha, iodine-129, iron, nitrate-nitrite as nitrogen, nonvolatile beta, strontium-90, tritium

Aluminum, gross alpha, iron, $\mathrm{pH}$, specific conductance, trichloroethylene, tritium

Iron, manganese, nonvolatile beta, tritium

Aluminum, iron

Gross alpha, iron, manganese, specific conductance

Aluminum, manganese

Aluminum, iron, lead, manganese

Tritium

Tritium

Aluminum, manganese

Aluminum, iron, trichloroethylene, tritium

Tritium

Aluminum, iron

Tritium

Aluminum, iron, $\mathrm{pH}$, tritium

Tritium

Aluminum, benzene, benzoic acid, chloroethene, 1,4dichlorobenzene, dichlorodifluoromethane, 1,1dichloroethane, 1,2-dichloroethane, 1,1-dichloroethylene, dichloromethane, iron, mercury, tetrachloroethylene, trichloroethylene, trichlorofluoromethane, tritium

Aluminum, iron, $\mathrm{pH}$, specific conductance, tetrachloroethylene, total organic halogens, trichloroethylene

Aluminum, 1,1-dichloroethylene, dichloromethane, gross alpha, iron, lead, $\mathrm{pH}$, specific conductance, tetrachloroethylene, total organic halogens, trichloroethylene 


\begin{tabular}{|c|c|c|}
\hline Site & $\begin{array}{l}\text { Well } \\
\text { Series }\end{array}$ & Analytes above Flag 2 Criteria \\
\hline B-Area Microbiology Wells & $p$ & Iron, tritium \\
\hline P-Area Bingham Pump Outage Pit & PBP & Aluminum, iron \\
\hline $\begin{array}{l}\text { P-Area Coal Pile Runoff Containment } \\
\text { Basin }\end{array}$ & PCB & Aluminum, gross alpha, iron, manganese, sulfate \\
\hline P-Area Disassembly Basin & PDB & Aluminum, iron, tritium \\
\hline P.Area Reactor Seepage Basins & PSB & Aluminum, iron, strontium-90, tritium \\
\hline R-Area Bingham Pump Outage Pit & RBP & Aluminum \\
\hline R-Area Disassembly Basin & RDB & Aluminum, iron, manganese \\
\hline R-Area Burning/Rubble Pits & RRP & Aluminum, iron \\
\hline A/M-Area Recovery Well Network & RWM & Dichloromethane, tetrachloroethylene, trichloroethylene \\
\hline Silverton Road Waste Site & SRW & Aluminum, carbon tetrachloride, $\mathrm{pH}$, trichloroethylene \\
\hline TNX Burying Ground & TBG & $\begin{array}{l}\text { Aluminum, carbon tetrachloride, cis-1,2-dichloroethylene, } \\
\text { gross alpha, iron, manganese, mercury, nitrate as nitrogen, } \\
\text { tetrachloroethylene, trichloroethylene }\end{array}$ \\
\hline TNX-Area Operable Unit & TCM & $\begin{array}{l}\text { Aluminum, carbon tetrachloride, gross alpha, iron, } \\
\text { manganese, nitrate as nitrogen, trichloroethylene }\end{array}$ \\
\hline TNX-Area Operable Unit & TIR & $\begin{array}{l}\text { Aluminum, gross alpha, manganese, mercury, nitrate as } \\
\text { nitrogen, trichloroethylene }\end{array}$ \\
\hline TNX-Area Assessment Wells & TNX & $\begin{array}{l}\text { Aluminum, carbon tetrachloride, cis-1,2-dichloroethylene, iron } \\
\text { manganese, tetrachloroethylene, trichloroethylene }\end{array}$ \\
\hline TNX-Area Recovery Wells & TRW & $\begin{array}{l}\text { Aluminum, carbon tetrachloride, iron, manganese, } \\
\text { trichloroethylene }\end{array}$ \\
\hline New TNX Seepage Basin & xsB & Aluminum, iron, mercury, nitrate as nitrōg̀en, trichloroethylene \\
\hline Old TNX Seepage Basin & YSB & Aluminum, iron \\
\hline $\begin{array}{l}\text { Y-Area Waste Solidification and Disposal } \\
\text { Facility }\end{array}$ & YSC & Aluminum, iron \\
\hline Z-Area Low Point & ZDT & Aluminum, tritium \\
\hline
\end{tabular}

Note: The groundwater samples are unfiltered. Therefore, the results for metals are for total recoverable metals. Analytes in bold were detected at levels above the current Flag 2 criteria for the first time since 1984. 


\section{Introduction}

This report summarizes the Groundwater Monitoring Program conducted by SRS during first quarter 1999 . It includes the analytical data, field data, data review, quality control, and other documentation for this program; provides a record of the program's activities; and serves as an official record of the analytical results.

EPD/EMS is responsible for providing drilling, sampling, and analytical and data management support for the SRS Groundwater Monitoring Program at approximately 135 waste sites in 17 areas at SRS (see figures 1 and 2 at the end of this section). The majority of this monitoring is required by U.S. Department of Energy (DOE) orders and by federal and state regulations administered by the USEPA and the South Carolina Department of Health and Environmental Control (SCDHEC). The Groundwater Monitoring Program includes the following activities:

- installation, maintenance, and abandonment of monitoring wells

- environmental soil borings

- development of sampling and analytical schedules

- collection and analysis of groundwater samples

- review of analytical and other data

- maintenance of the databases containing groundwater monitoring data

- quality assurance (QA) evaluations of laboratory performance

- reports of results to waste-site facility custodians and the Environmental Protection Department

The custodian of each waste site is responsible for informing EPD/EMS of sampling and analytical requirements and special requests for the sampling schedule, assisting in review of the data, and making any decisions regarding groundwater monitoring at the waste site.

Each custodian receives a copy of this report. Each custodian also receives site-specific data on request, including the following:

- a computer printout of the analytical data for the current quarter and for the previous seven quarters, designed to assist in identifying trends

- a computer printout of analytical results at or above Flag 1 and Flag 2 criteria for the quarter, designed to assist in identifying elevated constituents

\section{ORGANIZATION OF THIS REPORT}

This report is divided into sections that focus on specific aspects of the SRS Groundwater Monitoring Program. The Executive Summary section presents a listing by waste site and well series of all analytes detected at or above Flag 2 criteria during the quarter. Analytes detected at or above Flag 2 criteria for the first time since 1984 are indicated in bold type.

The Flagging Criteria section lists flagging criteria for analytes and provides a short description of how the criteria were derived. The Sample Scheduling section discusses the preparation of the sampling schedule and the criteria for analyte selection. 
During sample collection, samplers write comments in the field logbooks that may be pertinent to the analysis of samples. Many of the comments concern wells that went dry during sampling or water that appeared colored, turbid, or aerated. These comments are included in the Field Notes section.

Samples are analyzed by the EPD/EMS (EM Lab or EM) Radiological Laboratory at SRS and by one or more offsite laboratories. During first quarter 1999, EMAX Laboratories, Inc. (EX), of Torrance, CA; General Engineering Laboratories (GE), of Charleston, SC; and Recra LabNet Philadelphia (WA), of Lionville, PA, were the primary offsite laboratories. Radionuclide analyses were conducted by Environmental Physics, Inc. (GP), a subcontractor for GE, and Thermo NUtech (TM), a subcontractor for WA. Analytical data from the EM Lab were not received in time to be included in the report for first quarter 1999. The Analytical Data Review section contains three subsections. The GIMS Data Review Module subsection discusses automated data management activities at EPD/EMS. The Review of the Analytical Data subsection includes a discussion of discrepancies in each laboratory's analytical data, including results that were considerably higher or lower than previous results. This subsection also includes information about the analytical narratives that were used as reference materials throughout the data validation process. The Analytical Methods subsection lists the methods the laboratories used for measuring concentrations of each analyte.

The Quality Control Samples section contains five subsections and discusses the analytical data in terms of the following indicators of data quality: precision, accuracy, representativeness, comparability, and completeness. The Precision subsection explains the replicate analysis program, gives the statistical methods used for comparison, and lists the results of the comparisons between the replicate and duplicate analyses. The Accuracy subsection examines the relationship between an observed value and an accepted reference value and/or the measure of the over- or underestimation of reported concentrations. The Representativeness subsection describes how groundwater samples can be affected to produce results that may be biased positively or negatively. The Comparability subsection discusses whether the laboratories use the same standardized procedures for sample preparation and analysis, whether the reporting units are the same, and whether similar quantitation limits were obtained. The Completeness section evaluates the amount of useable data that resulted from the data collection.

The Site Index section lists and gives a description of the sites associated with each well series, as well as historical information for the sites. A list of terms, abbreviations, and acronyms used in this report can be found in the Glossary section. References cited are included in the References section.

The Water-Level Data section (Appendix A) includes concurrent water elevations obtained in $A / M$ and other areas; these data are used by SRS personnel in hydrogeologic studies. The Analytical Results section (Appendix B) includes tables listing the analytical results from all laboratories and field data for all wells sampled during the quarter. The tables appear in alphabetical order by well name. The Sampling Blanks Results section (Appendix C) contains tables listing the analytical results of laboratory tests on sampling blanks. 


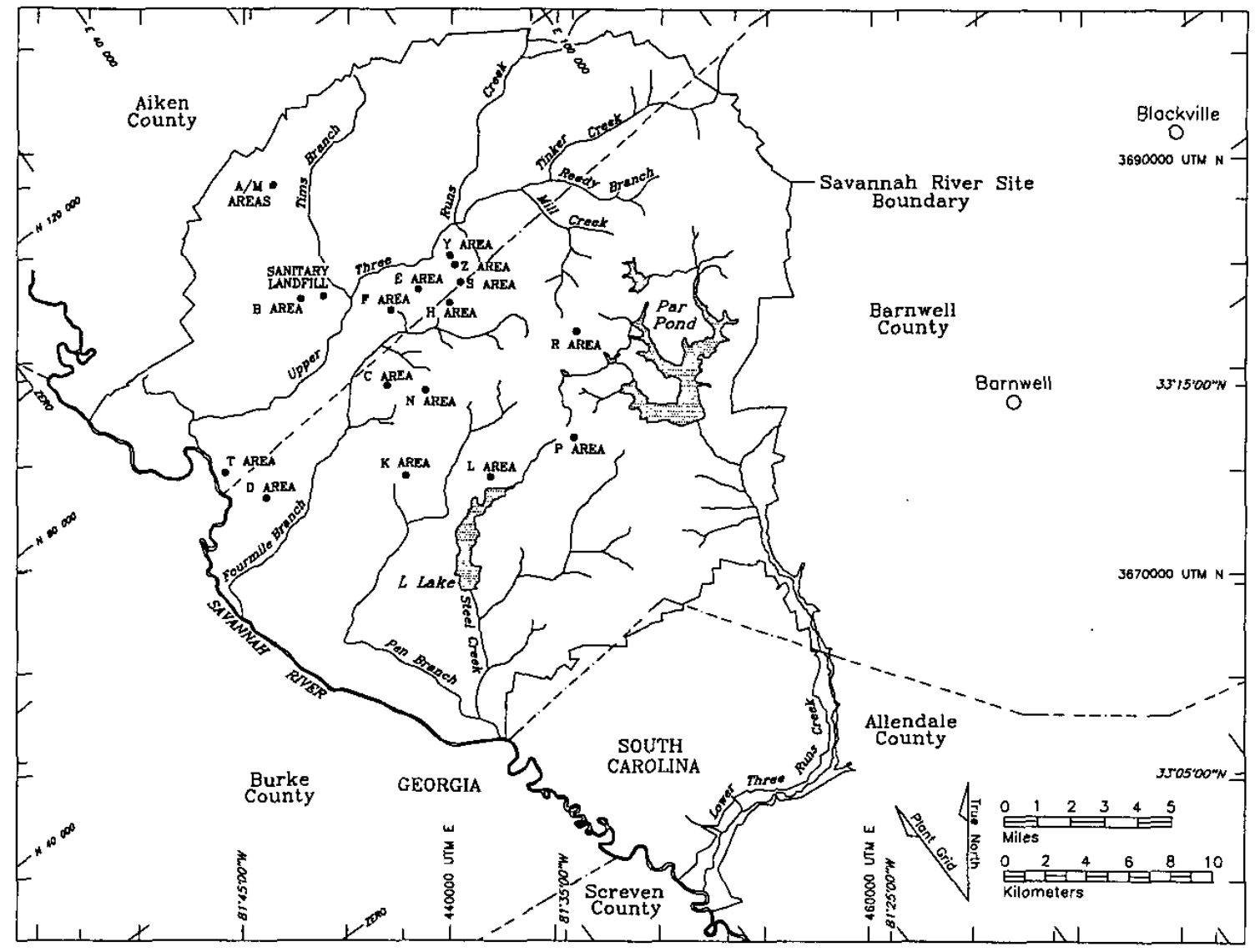

Figure 1. Areas and Locations Monitored for Groundwater Quality 


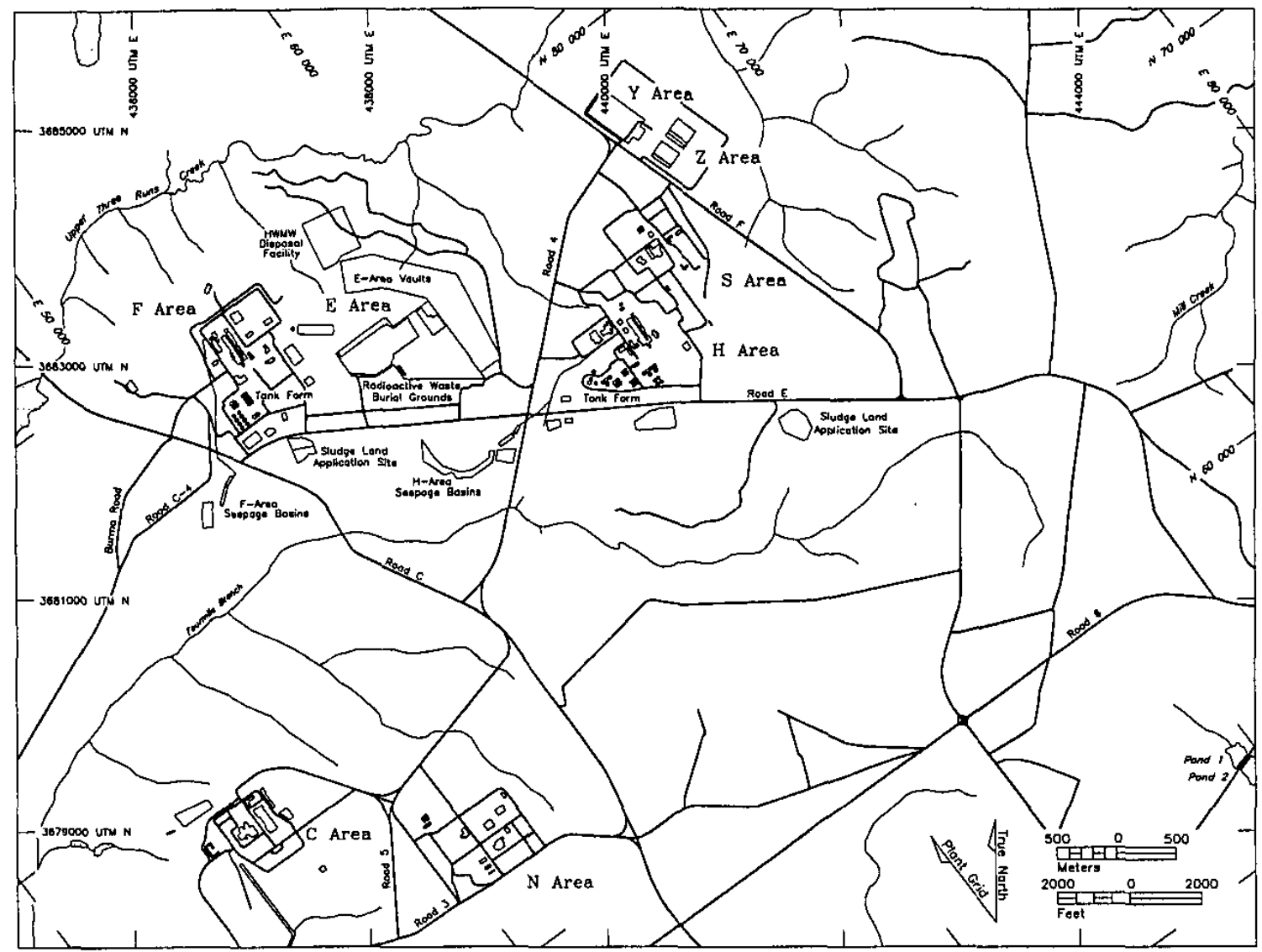

Figure 2. Separations and Waste Management Areas Monitored for Groundwater Quality 


\section{Flagging Criteria}

Analytes in the data tables are assigned flagging levels $(0,1$, or 2$)$ depending on their concentrations in a groundwater sample. The flagging levels dictate the scheduling and frequency of groundwater sampling. Beginning first quarter 1992, flagging criteria were established for all of the constituents currently being analyzed as part of the EPD/EMS Groundwater Monitoring Program, except for certain aesthetic constituents, indicator parameters, major cations, and common laboratory contaminants and cleaners, which can be analyzed by special request. The flagging criteria in table 2 were determined as follows:

Flag 0: Analytical results below Flag 1 and constituents having no flagging criteria were classified as Flag 0.

Flag 1: The Flag 1 criterion for a constituent was set as one-half of the EPA final primary drinking water standard, the EPA proposed primary drinking water standard, or the EPA secondary drinking water standard for that constituent. If a constituent did not have an EPA drinking water standard, the Flag 1 criterion was set as five times a recently published 90 th percentile detection limit obtained by one of the primary laboratories.

Flag 2: The Flag 2 criterion for a constituent was set as the EPA final primary drinking water standard, the EPA proposed primary drinking water standard, or the EPA secondary drinking water standard for that constituent. If a constituent did not have a drinking water standard, the Flag 2 criterion was set as 10 times a recently published 90th percentile detection limit obtained by one of the primary laboratories.

The following acronyms are used as abbreviated sources in the flagging criteria table. Complete information concerning documents cited can be found in the References section of this report.

APHA - American Public Health Association.

APHA Method - A specific analytical method for testing constituent levels in a sample as established by the APHA, American Water Works Association, and Water Pollution Control Federation. See American Public Health Association et al. in References.

EPA - U.S. Environmental Protection Agency.

EPA Method - A specific analytical method for testing constituent levels. Descriptions of these methods can be found in the EPA publications Methods for Chemical Analysis of Water and Wastes (1983) and Test Methods for Evaluating Solid Waste (1986b) and in the 1991 Code of Federal Regulations, Title 40, Part 136. See Environmental Protection Agency in References.

EPD/EMS - The Environmental Protection Department/Environmental Monitoring Section at the Savannah River Site.

PDWS — Primary Drinking Water Standards.

SCDHEC - South Carolina Department of Health and Environmental Control.

SDWS - Secondary Drinking Water Standards. 
Table 2. Flagging Criteria

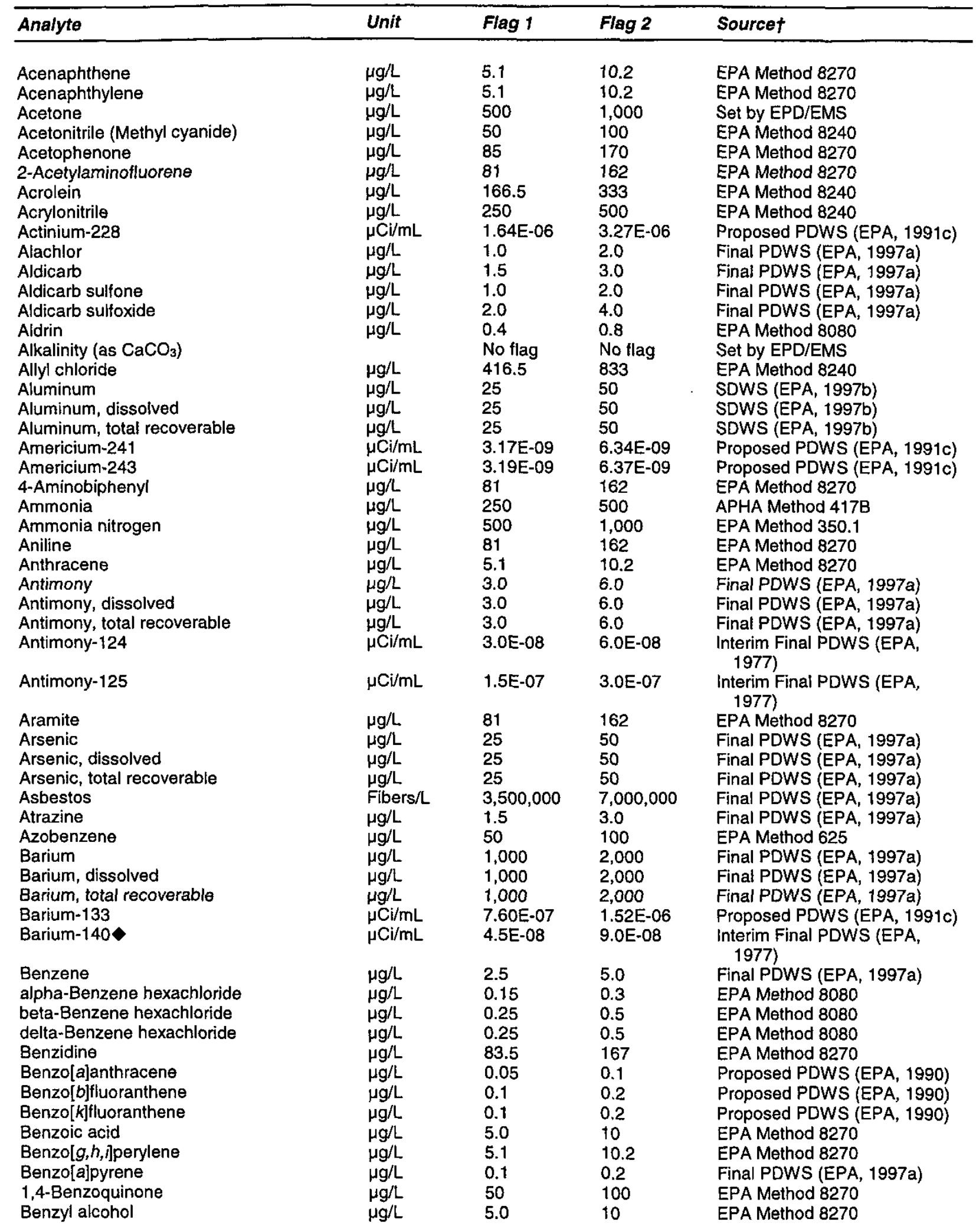




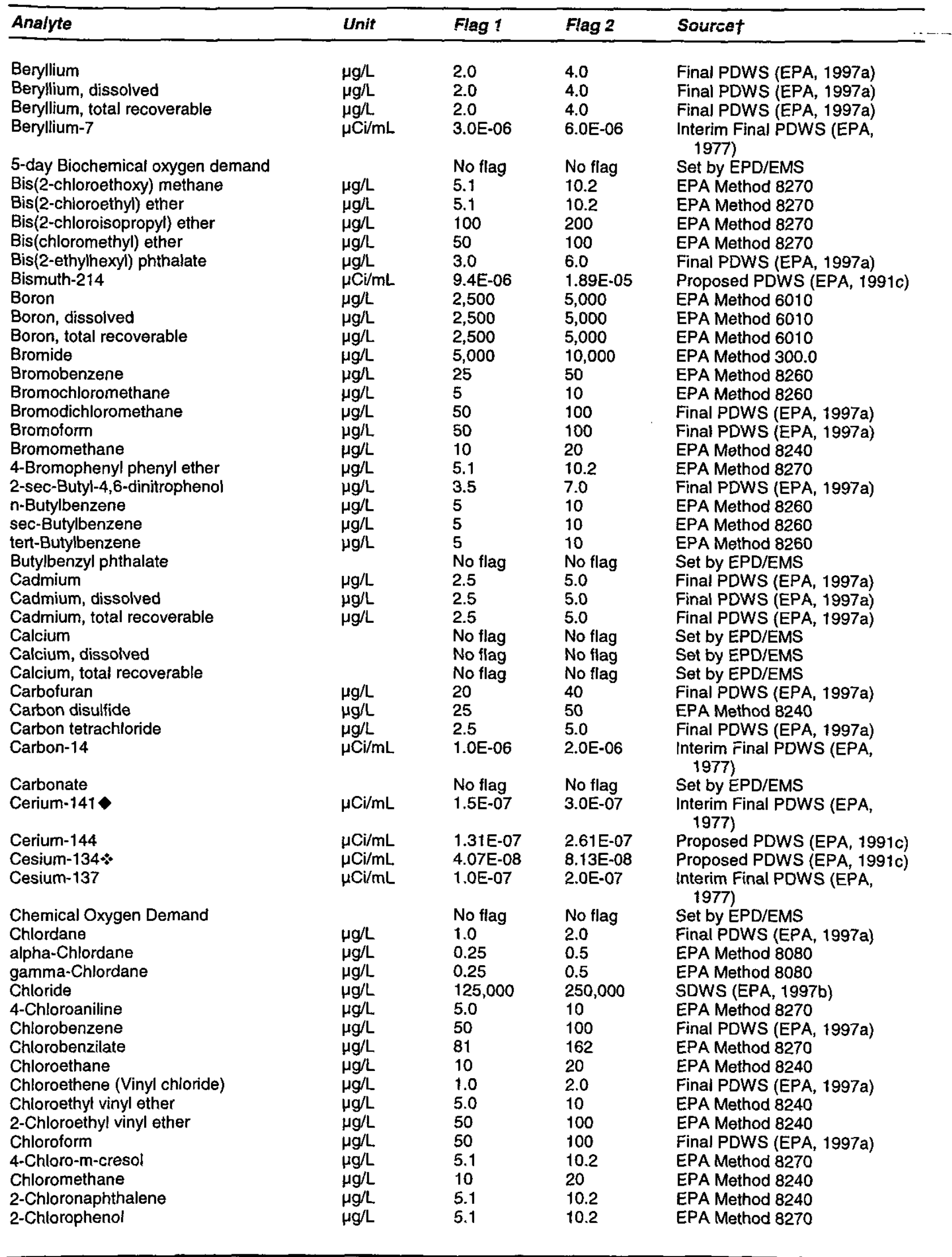




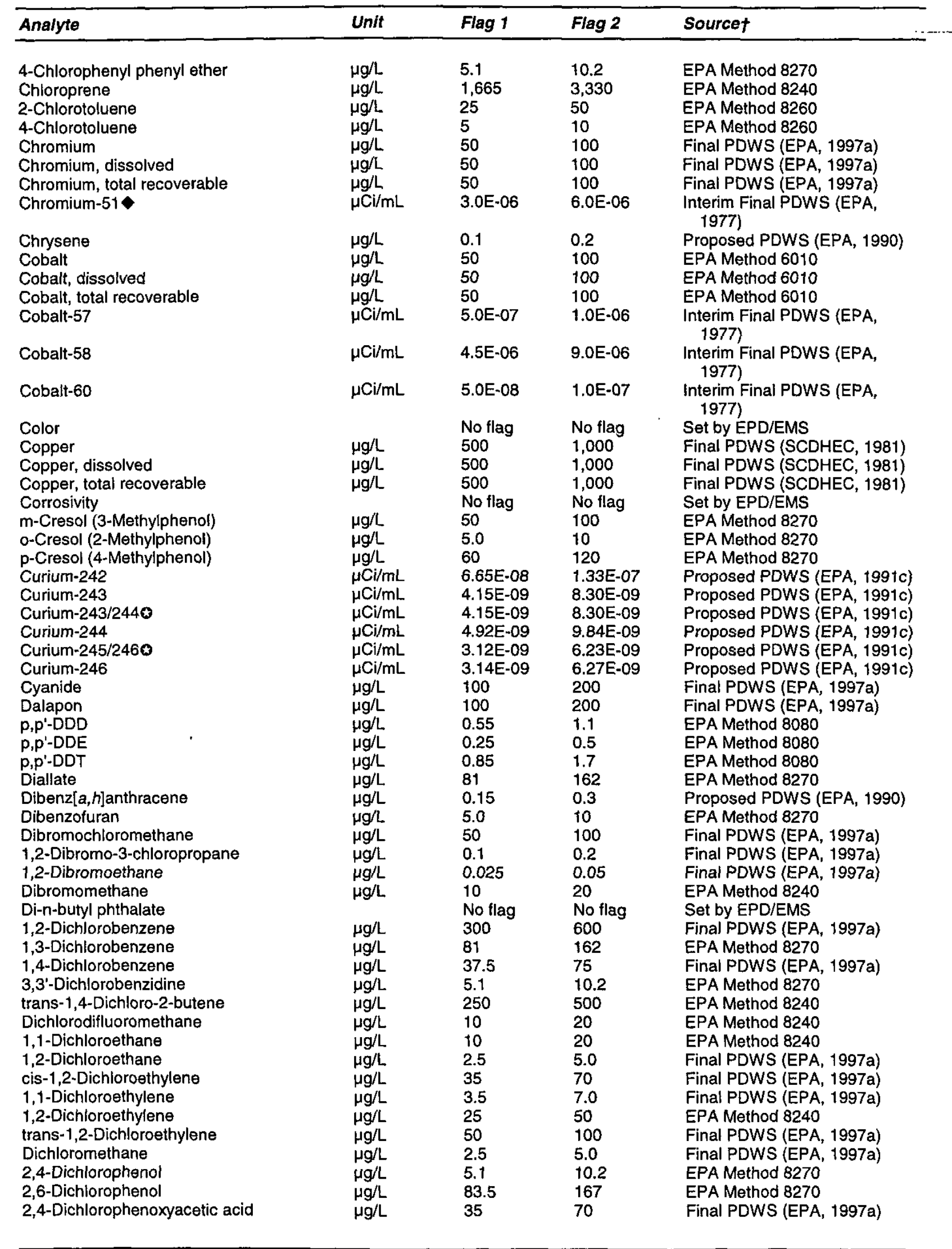




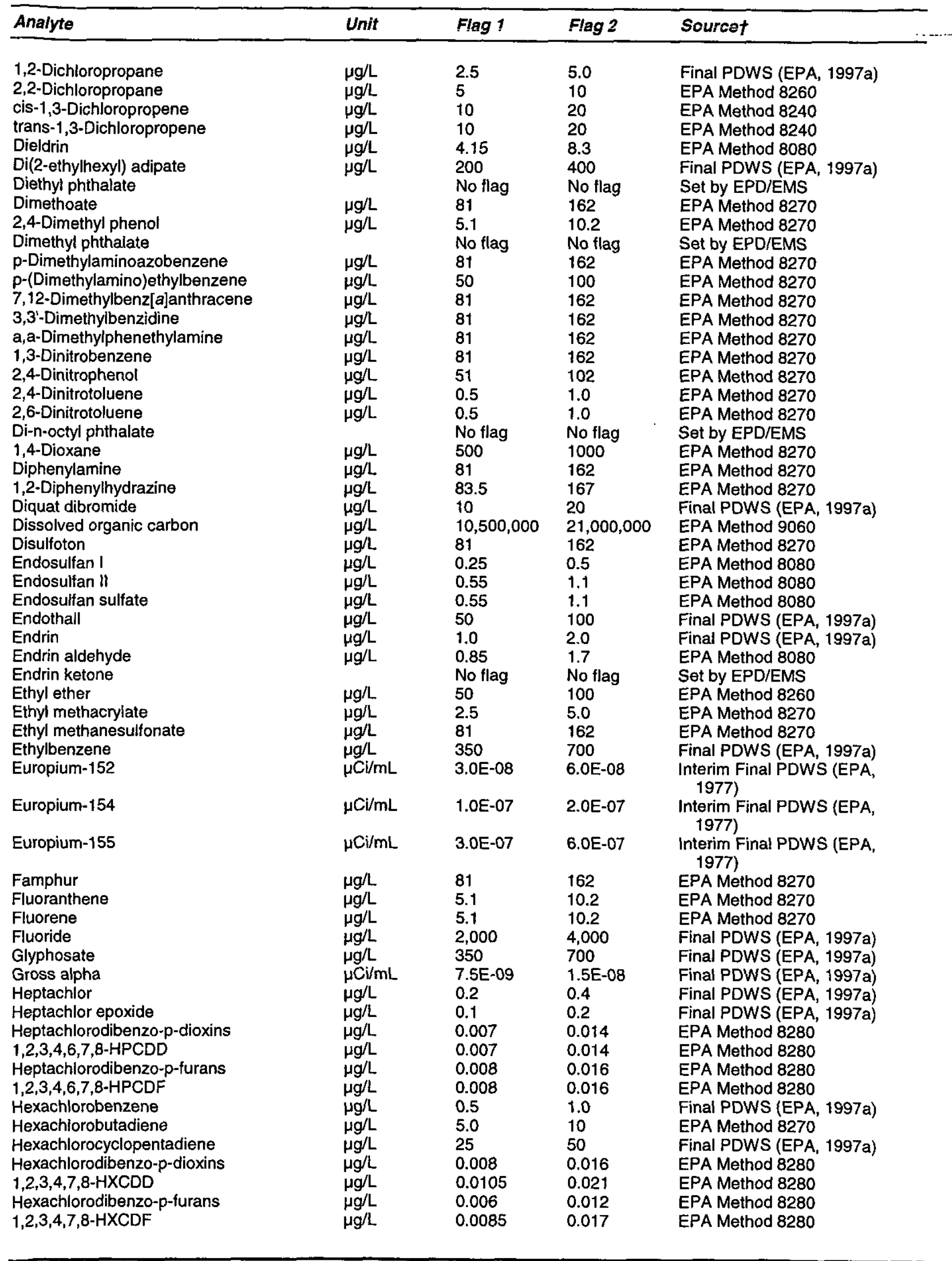




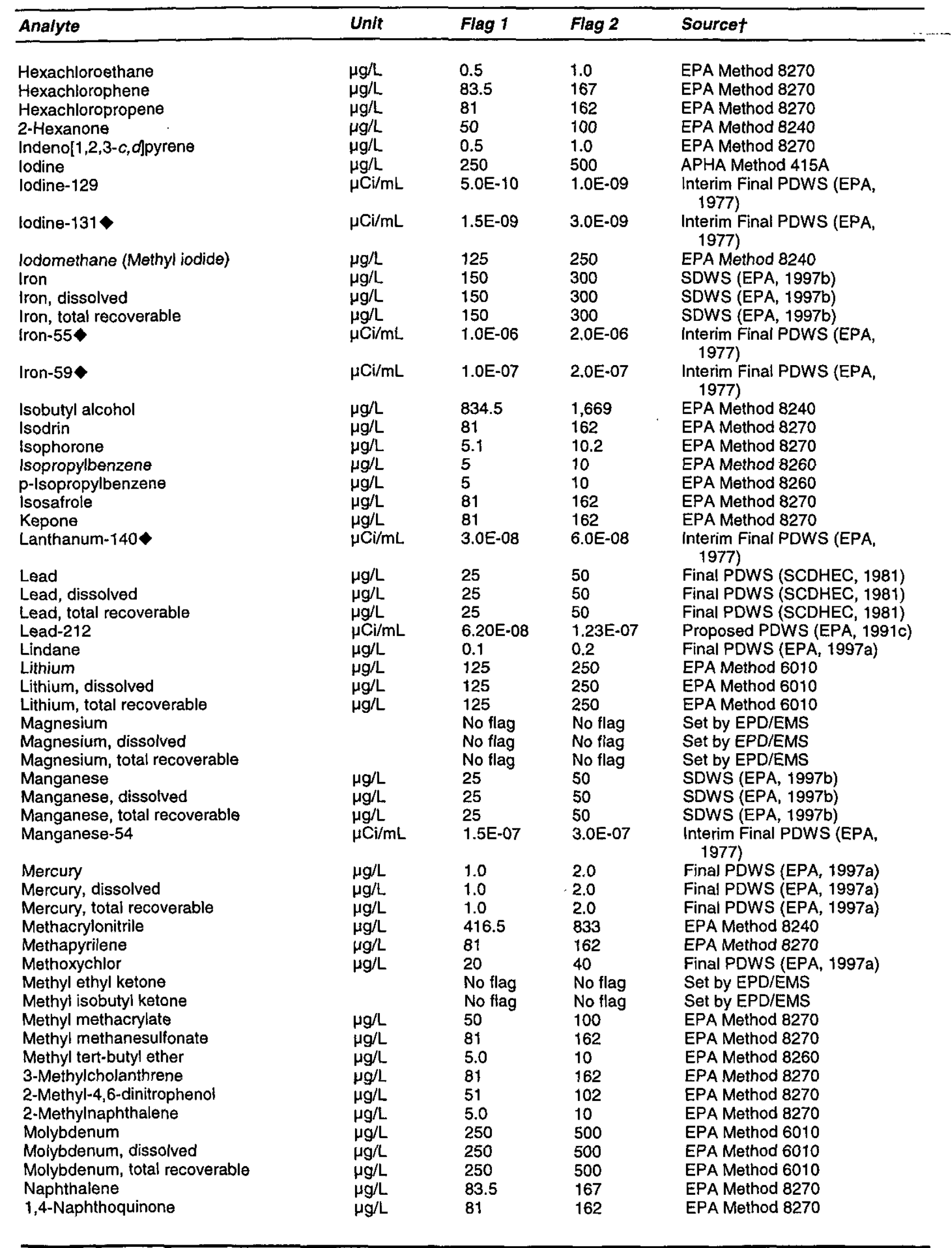




\begin{tabular}{|c|c|c|c|c|}
\hline Analyte & Unit & Flag 1 & Flag 2 & Sourcet \\
\hline 1-Naphthylamine & $\mu g / L$ & 81 & 162 & EPA Method 8270 \\
\hline 2-Naphthylamine & $\mu g / L$ & 81 & 162 & EPA Method 8270 \\
\hline Neptunium-237 & $\mu \mathrm{Ci} / \mathrm{mL}$ & 3.53E-09 & $7.06 \mathrm{E}-09$ & Proposed PDWS (EPA, 1991c) \\
\hline Neptunium-239 & $\mu \mathrm{Ci} / \mathrm{mL}$ & 8.40E-07 & 1.68E-06 & Proposed PDWS (EPA, 1991C) \\
\hline Nickel & $\mu g / L$ & 50 & 100 & Final PDWS (EPA, 1997a) \\
\hline Nickel, dissolved & $\mu g / L$ & 50 & 100 & Final PDWS (EPA, 1997a) \\
\hline Nickel, total recoverable & $\mu g / L$ & 50 & 100 & Final PDWS (EPA, 1997a) \\
\hline Nickel-59 & $\mu \mathrm{Ci} / \mathrm{mL}$ & $1.5 \mathrm{E}-07$ & 3.0E-07 & $\begin{array}{l}\text { Interim Final PDWS (EPA, } \\
1977 \text { ) }\end{array}$ \\
\hline Nickel-63 & $\mu \mathrm{Ci} / \mathrm{mL}$ & $2.5 E-08$ & 5.0E-08 & $\begin{array}{l}\text { Interim Final PDWS (EPA, } \\
1977 \text { ) }\end{array}$ \\
\hline Niobium-95 & $\mu \mathrm{Ci} / \mathrm{mL}$ & $1.5 E-07$ & 3.0E-07 & $\begin{array}{l}\text { Interim Final PDWS (EPA, } \\
1977 \text { ) }\end{array}$ \\
\hline Nitrate as nitrogen & $\mu g / L$ & 5,000 & 10,000 & Final PDWS (EPA, 1997a) \\
\hline Nitrate-nitrite as nitrogen & $\mu g / L$ & 5,000 & 10,000 & Final PDWS (EPA, 1997a) \\
\hline Nitrite as nitrogen & $\mu g / L$ & 500 & 1,000 & Final PDWS (EPA, 1997a) \\
\hline m-Nitroaniline & $\mu g / L$ & 5.0 & 10 & EPA Method 8270 \\
\hline o-Nitroaniline & $\mu g / L$ & 5.0 & 10 & EPA Method 8270 \\
\hline p-Nitroaniline & $\mu g / L$ & 5.0 & 10 & EPA Method 8270 \\
\hline Nitrobenzene & $\mu g / L$ & 5.1 & 10.2 & EPA Method 8270 \\
\hline Nitrogen by Kjeldahl method & $\mu g / L$ & 500 & 1,000 & EPA Method 351.2 \\
\hline 2-Nitrophenol & $\mu g / L$ & 5.1 & 10.2 & EPA Method 8270 \\
\hline 4-Nitrophenol & $\mu g / L$ & 5.1 & 10.2 & EPA Method 8270 \\
\hline 4-Nitroquinoline-1-oxide & $\mu g / L$ & 81 & 162 & EPA Method 8270 \\
\hline N-Nitrosodi-n-butylamine & $\mu g / L$ & 81 & 162 & EPA Method 8270 \\
\hline N-Nitrosodiethylamine & $\mu g / L$ & 81 & 162 & EPA Method 8270 \\
\hline N-Nitrosodimethylamine & $\mu g / L$ & 83.5 & 167 & EPA Method 8270 \\
\hline N-Nitrosodiphenylamine & $\mu g / L$ & 5.1 & 10.2 & EPA Method 8270 \\
\hline N-Nitrosodipropylamine & $\mu g / L$ & 5.1 & 10.2 & EPA Method 8270 \\
\hline N-Nitrosomethylethylamine & $\mu g / L$ & 81 & 162 & EPA Method 8270 \\
\hline N-Nitrosomorpholine & $\mu g / L$ & 81 & 162 & EPA Method 8270 \\
\hline N-Nitrosopiperidine & $\mu g / L$ & 81 & 162 & EPA Method 8270 \\
\hline $\mathrm{N}$-Nitrosopyrrolidine & $\mu g / L$ & 81 & 162 & EPA Method 8270 \\
\hline 5-Nitro-0-toluidine & $\mu g / L$ & 81 & 162 & EPA Method 8270 \\
\hline Nonvolatile beta & $\mu \mathrm{Ci} / \mathrm{mL}$ & 2.5E-08 & 5.0E-08 & $\begin{array}{l}\text { Interim Final PDWS (EPA, } \\
1977 \text { ) }\end{array}$ \\
\hline Octachlorodibenzo-p-dioxins & $\mu g / L$ & 0.0085 & 0.017 & EPA Method 8280 \\
\hline Octachlorodibenzo-p-furans & $\mu g / L$ & 0.0065 & 0.013 & EPA Method 8280 \\
\hline Odor & & No flag & No flag & Set by EPD/EMS \\
\hline Oil \& grease & $\mu g / L$ & 8,350 & 16,700 & EPA Method 413.1 \\
\hline Oxamyl & $\mu g / L$ & 100 & 200 & Final PDWS (EPA, 1997a) \\
\hline Parathion & $\mu g / L$ & 0.4 & 0.8 & EPA Method 8080 \\
\hline Parathion methyl & $\mu g / L$ & 0.4 & 0.8 & EPA Method 8080 \\
\hline PCB 1016 & $\mu g / L$ & 0.25 & 0.5 & Final PDWS (EPA, 1997a) \\
\hline PCB 1221 & $\mu \mathrm{g} / \mathrm{L}$ & 0.25 & 0.5 & Final PDWS (EPA, 1997a) \\
\hline PCB 1232 & $\mu g / L$ & 0.25 & 0.5 & Final PDWS (EPA, 1997a) \\
\hline PCB 1242 & $\mu g / L$ & 0.25 & 0.5 & Final PDWS (EPA, 1997a) \\
\hline PCB 1248 & $\mu g / L$ & 0.25 & 0.5 & Final PDWS (EPA, 1997a) \\
\hline PCB 1254 & $\mu g / L$ & 0.25 & 0.5 & Final PDWS (EPA, 1997a) \\
\hline PCB 1260 & $\mu g / L$ & 0.25 & 0.5 & Final PDWS (EPA, 1997a) \\
\hline PCB 1262 & $\mu g / L$ & 0.25 & 0.5 & Final PDWS (EPA, 1997a) \\
\hline Pentachlorobenzene & $\mu g / L$ & 81 & 162 & EPA Method 8270 \\
\hline Pentachlorodibenzo-p-dioxins & $\mu g / L$ & 0.008 & 0.016 & EPA Method 8280 \\
\hline $1,2,3,7,8-P C D D$ & $\mu g / L$ & 0.0075 & 0.015 & EPA Method 8280 \\
\hline Pentachlorodibenzo-p-furans & $\mu g / L$ & 0.0085 & 0.017 & EPA Method 8280 \\
\hline $1,2,3,7,8-P C D F$ & $\mu g / L$ & 0.0085 & 0.017 & EPA Method 8280 \\
\hline Pentachloroethane & $\mu g / L$ & 81 & 162 & EPA Method 8270 \\
\hline Pentachloronitrobenzene & $\mu g / L$ & 81 & 162 & EPA Method 8270 \\
\hline Pentachlorophenol & $\mu g / L$ & 0.5 & 1.0 & Final PDWS (EPA, 1997a) \\
\hline $\mathrm{pH}$ & $\mathrm{pH}$ & 8.0 & 10 & Set by EPD/EMS \\
\hline & $\mathrm{pH}$ & 4.0 & 3.0 & Set by EPD/EMS \\
\hline Phenacetin & $\mu g / L$ & 81 & 162 & EPA Method 8270 \\
\hline
\end{tabular}




\begin{tabular}{|c|c|c|c|c|}
\hline Analyte & Unit & Flag 1 & Flag 2 & Sourcet \\
\hline Phenanthrene & $\mu g / L$ & 5.1 & 10.2 & EPA Method 8270 \\
\hline Phenol & $\mu g / L$ & 83.5 & 167 & EPA Method 8270 \\
\hline Phenols & $\mu g / L$ & 50 & 100 & EPA Method 420.1 \\
\hline p-Phenylenediamine & $\mu g / L$ & 81 & 162 & EPA Method 8270 \\
\hline Phorate & $\mu g / L$ & 0.85 & 1.7 & EPA Method 8080 \\
\hline Picloram & $\mu g / L$ & 250 & 500 & Final PDWS (EPA, 1997a) \\
\hline 2-Picoline & $\mu g / L$ & 81 & 162 & EPA Method 8270 \\
\hline Plutonium-238 & $\mu \mathrm{Ci} / \mathrm{mL}$ & 3.51E-09 & 7.02E-09 & Proposed PDWS (EPA, 1991c) \\
\hline Plutonium-239 & $\mu \mathrm{Ci} / \mathrm{mL}$ & 3.11E-08 & $6.21 E-08$ & Proposed PDWS (EPA, 1991c) \\
\hline Plutonium-239/240@ & $\mu \mathrm{Ci} / \mathrm{mL}$ & 3.11E-08 & $6.21 E-08$ & Proposed PDWS (EPA, 1991C) \\
\hline Plutonium-240 & $\mu \mathrm{Ci} / \mathrm{mL}$ & $3.11 \mathrm{E}-08$ & $6.22 E-08$ & Proposed PDWS (EPA, 1991c) \\
\hline Plutonium-24t & $\mu \mathrm{Ci} / \mathrm{mL}$ & 3.13E-08 & $6.26 \mathrm{E}-08$ & Proposed PDWS (EPA, 1991c) \\
\hline Plutonium-242 & $\mu \mathrm{Ci} / \mathrm{mL}$ & 3.27E-08 & $6.54 \mathrm{E}-08$ & Proposed PDWS (EPA, 1991C) \\
\hline Potassium & & No flag & No flag & Set by EPD/EMS \\
\hline Potassium, dissolved & & No flag & No flag & Set by EPD/EMS \\
\hline Potassium, total recoverable & & No flag & No flag & Set by EPD/EMS \\
\hline Potassium-40 & $\mu \mathrm{Ci} / \mathrm{mL}$ & 1.5E-07 & $3.0 \mathrm{E}-07$ & Proposed PDWS (EPA, 1986a) \\
\hline Promethium-144 & $\mu \mathrm{Ci} / \mathrm{mL}$ & $5.0 E-08$ & $1.0 \mathrm{E}-07$ & EPA Method 901.1 \\
\hline Promethium-146 & $\mu \mathrm{Ci} / \mathrm{mL}$ & 5.0E-08 & $1.0 \mathrm{E}-07$ & EPA Method 901.1 \\
\hline Promethium-147 & $\mu \mathrm{Ci} / \mathrm{mL}$ & 2.62E-06 & $5.24 \mathrm{E}-06$ & Proposed PDWS (EPA, 1991c) \\
\hline Pronamid & $\mu g / L$ & 81 & 162 & EPA Method 8270 \\
\hline Propionitrile & $\mu g / L$ & 1,665 & 3,330 & EPA Method 8240 \\
\hline n-Propylbenzene & $\mu g / L$ & 5 & 10 & EPA Method 8260 \\
\hline Pyrene & $\mu g / L$ & 5.1 & 10.2 & EPA Method 8270 \\
\hline Pyridine & $\mu g / L$ & 81 & 162 & EPA Method 8270 \\
\hline Radium, total alpha-emitting & $\mu \mathrm{Ci} / \mathrm{mL}$ & 2.5E-09 & 5.0E-09 & $\begin{array}{l}\text { Interim Final PDWS (EPA, } \\
1977 \text { ) }\end{array}$ \\
\hline Radium-226 & $\mu \mathrm{Ci} / \mathrm{mL}$ & $2.5 \mathrm{E}-09$ & 5.0E-09 & $\begin{array}{l}\text { Interim Final PDWS (EPA, } \\
1977 \text { ) }\end{array}$ \\
\hline Radium-228 & $\mu \mathrm{Ci} / \mathrm{mL}$ & $2.5 E-09$ & 5.0E-09 & $\begin{array}{l}\text { Interim Final PDWS (EPA, } \\
\text { 1977) }\end{array}$ \\
\hline Radon-222 & $\mu \mathrm{Ci} / \mathrm{mL}$ & $1.5 \mathrm{E}-07$ & 3.0E-07 & Proposed PDWS (EPA, 1991c) \\
\hline Ruthenium-103 & $\mu \mathrm{Ci} / \mathrm{mL}$ & $1.0 E-07$ & 2.0E-07 & $\begin{array}{l}\text { Interim Final PDWS (EPA, } \\
1977 \text { ) }\end{array}$ \\
\hline Ruthenium-106 & $\mu \mathrm{Ci} / \mathrm{mL}$ & $1.5 \mathrm{E}-08$ & 3.0E-08 & $\begin{array}{l}\text { Interim Final PDWS (EPA, } \\
1977 \text { ) }\end{array}$ \\
\hline Safrole & $\mu g / L$ & 81 & 162 & EPA Method 8270 \\
\hline Selenium & $\mu g / L$ & 25 & 50 & Final PDWS (EPA, 1997a) \\
\hline Selenium, dissolved & $\mu g / L$ & 25 & 50 & Final PDWS (EPA, 1997a) \\
\hline Selenium, total recoverable & $\mu g / L$ & 25 & 50 & Final PDWS (EPA, 1997a) \\
\hline Silica & & No flag & No flag & Set by EPD/EMS \\
\hline Silica, dissolved & & No flag & No flag & Set by EPD/EMS \\
\hline Silica, total recoverable & & No flag & No flag & Set by EPD/EMS \\
\hline Silver & $\mu g / L$ & 50 & 100 & SDWS (EPA, 1997b) \\
\hline Silver, dissolved & $\mu g / L$ & 50 & 100 & SDWS (EPA, 1997b) \\
\hline Silver, total recoverable & $\mu g / L$ & 50 & 100 & SDWS (EPA, 1997b) \\
\hline Simazine & $\mu g / L$ & 2.0 & 4.0 & Final PDWS (EPA, 1997a) \\
\hline Sodium & & No flag & No flag & Set by EPD/EMS \\
\hline Sodium, dissolved & & No flag & No flag & Set by EPD/EMS \\
\hline Sodium, total recoverable & & No flag & No flag & Set by EPD/EMS \\
\hline $\begin{array}{l}\text { Sodium-22 } \\
\text { Specific conductance }\end{array}$ & $\begin{array}{l}\mu \mathrm{Ci} / \mathrm{mL} \\
\mu \mathrm{S} / \mathrm{cm}\end{array}$ & $\begin{array}{l}2.33 E-07 \\
250\end{array}$ & $\begin{array}{l}4.66 \mathrm{E}-07 \\
500\end{array}$ & $\begin{array}{l}\text { Proposed PDWS (EPA, 1991c) } \\
\text { Set by EPD/EMS }\end{array}$ \\
\hline Strontium-89 & $\mu \mathrm{Ci} / \mathrm{mL}$ & $1.0 E-08$ & $2.0 E-08$ & $\begin{array}{l}\text { Interim Final PDWS (EPA, } \\
\text { 1977) }\end{array}$ \\
\hline Strontium-89/900 & $\mu \mathrm{Ci} / \mathrm{mL}$ & 4.0E-09 & 8.0E-09 & Final PDWS (EPA, 1997a) \\
\hline Strontium-90 & $\mu \mathrm{Ci} / \mathrm{mL}$ & 4.0E-09 & 8.0E-09 & Final PDWS (EPA, 1997a) \\
\hline Styrene & $\mu g / L$ & 50 & 100 & Final PDWS (EPA, 1997a) \\
\hline Sulfate & $\mu g / L$. & 200,000 & 400,000 & Proposed PDWS (EPA, 1990) \\
\hline Sulfide & $\mu g / L$ & 8,350 & 16,700 & EPA Method $9030^{\circ}$ \\
\hline Sulfotepp & $\mu g / L$ & 81 & 162 & EPA Method 8270 \\
\hline Surfactants & & No flag & No flag & Set by EPD/EMS \\
\hline $2,3,7,8-T C D D$ & $\mu g / L$ & 0.007 & 0.014 & Final PDWS (EPA, 1997a) \\
\hline
\end{tabular}




\begin{tabular}{|c|c|c|c|c|}
\hline Analyte & Unit & Flag 1 & Flag 2 & Sourcet \\
\hline $\begin{array}{l}\text { 2,3,7,8-TCDF } \\
\text { Technetium-99 }\end{array}$ & $\begin{array}{l}\mu g / L \\
\mu \mathrm{Ci} / \mathrm{mL}\end{array}$ & $\begin{array}{l}0.00425 \\
4.5 E-07\end{array}$ & $\begin{array}{l}0.0085 \\
9.0 \mathrm{E}-07\end{array}$ & $\begin{array}{l}\text { EPA Method } 8280 \\
\text { Interim Final PDWS (EPA, } \\
1977 \text { ) }\end{array}$ \\
\hline $1,2,4,5$-Tetrachlorobenzene & $\mu g / L$ & 81 & 162 & EPA Method 8270 \\
\hline Tetrachlorodibenzo-p-dioxins & $\mu g / L$ & 0.007 & 0.014 & EPA Method 8280 \\
\hline Tetrachlorodibenzo-p-furans & $\mu g / L$ & 0.0055 & 0.011 & EPA Method 8280 \\
\hline 1,1,1,2-Tetrachloroethane & $\mu g / L$ & 10 & 20 & EPA Method 8240 \\
\hline $1,1,2,2$-Tetrachloroethane & $\mu \mathrm{g} / \mathrm{L}$ & 50 & 100 & EPA Method 8240 \\
\hline Tetrachloroethylene & $\mu g / L$ & 2.5 & 5.0 & Final PDWS (EPA, 1997a) \\
\hline 2,3,4,6-Tetrachlorophenol & $\mu g / L$ & 83.5 & 167 & EPA Method 8270 \\
\hline Thallium & $\mu g / L$ & 1.0 & 2.0 & Final PDWS (EPA, 1997a) \\
\hline Thallium, dissolved & $\mu g / L$ & 1.0 & 2.0 & Final PDWS (EPA, 1997a) \\
\hline Thallium, total recoverable & $\mu g / L$ & 1.0 & 2.0 & Final PDWS (EPA, 1997a) \\
\hline Thionazin & $\mu g / L$ & 81 & 162 & EPA Method 8270 \\
\hline Thorium-228 & $\mu \mathrm{Ci} / \mathrm{mL}$ & $6.25 E-08$ & $1.25 E-07$ & Proposed PDWS (EPA, 1991c) \\
\hline Thorium-230 & $\mu \mathrm{Ci} / \mathrm{mL}$ & $3.96 E-08$ & 7.92E-08 & Proposed PDWS (EPA, 1991C) \\
\hline Thorium-232 & $\mu \mathrm{Ci} / \mathrm{mL}$ & 4.4E-08 & $8.8 \mathrm{E}-08$ & Proposed PDWS (EPA, 1991c) \\
\hline Thorium-234 & $\mu \mathrm{Ci} / \mathrm{mL}$ & 2.0E-07 & $4.01 E-07$ & Proposed PDWS (EPA, 1991c) \\
\hline Tin & $\mu g / L$ & 250 & 500 & EPA Method 282.2 \\
\hline Tin, dissolved & $\mu g / L$ & 250 & 500 & EPA Method 282.2 \\
\hline Tin, total recoverable & $\mu g / L$ & 250 & 500 & EPA Method 282.2 \\
\hline $\operatorname{Tin}-113$ & $\mu \mathrm{Ci} / \mathrm{mL}$ & $1.5 \mathrm{E}-07$ & $3.0 E-07$ & $\begin{array}{l}\text { Interim Final POWS (EPA, } \\
1977 \text { ) }\end{array}$ \\
\hline Toluene & $\mu g / L$ & 500 & 1,000 & Final PDWS (EPA, 1997a) \\
\hline o-Toluidine & $\mu g / L$ & 81 & 162 & EPA Method 8270 \\
\hline Total carbon & $\mu g / L$ & 5,000 & 10,000 & EPA Method 9060 \\
\hline Total coliform & N/A & 0 & 0 & Final PDWS (EPA, 1997a) \\
\hline Total dissolved solids & & No flag & No flag & Set by EPD/EMS \\
\hline Total hydrocarbons & $\mu g / L$ & 5,000 & 10,000 & EPA Method 418.1 \\
\hline Total inorganic carbon & $\mu \mathrm{g} / \mathrm{L}$ & 8,350 & 16,700 & EPA Method 9060 \\
\hline Total organic carbon & $\mu g / L$ & 500,000 & $1,000,000$ & EPA Method 9060 \\
\hline Total organic halogens & $\mu \mathrm{g} / \mathrm{L}$ & 50 & 100 & EPA Method 9020 \\
\hline Total organic nitrogen & $\mu g / L$ & 500 & 1,000 & APHA Method 420 \\
\hline Total petroleum hydrocarbons & $\mu g / L$ & 8,350 & 16,700 & EPA Method 418.1 \\
\hline Total phosphates (as P) & & No flag & No flag & Set by EPD/EMS \\
\hline Total phosphorus & & No flag & No flag & Set by EPD/EMS \\
\hline Toxaphene & $\mu g / L$ & 1.5 & 3.0 & Final PDWS (EPA, 1997a) \\
\hline 2,4,5-TP (Silvex) & $\mu g / L$ & 25 & 50 & Final PDWS (EPA, 1997a) \\
\hline Tributyl phosphate & $\mu g / L$ & 86 & 172 & EPA Method 8270 \\
\hline 1,2,3-Trichlorobenzene & $\mu g / L$ & 5 & 10 & EPA Method 8260 \\
\hline 1,2,4-Trichlorobenzene & $\mu g / L$ & 35 & 70 & Final PDWS (EPA, 1997a) \\
\hline 1,1,1-Trichloroethane & $\mu g / L$ & 100 & 200 & Final PDWS (EPA, 1997a) \\
\hline 1,1,2-Trichloroethan $\theta$ & $\mu g / L$ & 2.5 & 5.0 & Final PDWS (EPA, 1997a) \\
\hline Trichloroethylene & $\mu g / L$ & 2.5 & 5.0 & Final PDWS (EPA, 1997a) \\
\hline Trichlorofluoromethane & $\mu g / L$ & 10 & 20 & EPA Method 8240 \\
\hline 2,4,5-Trichlorophenol & $\mu g / L$ & 5.0 & 10 & EPA Method 8270 \\
\hline 2,4,6-Trichlorophenol & $\mu \mathrm{g} / \mathrm{L}$ & 0.5 & 1.0 & EPA Method 8270 \\
\hline 2,4,5-Trichlorophenoxyacetic acid & $\mu g / L$ & 0.25 & 0.5 & EPA Method 8150 \\
\hline 1,2,3-Trichloropropane & $\mu g / L$ & 10 & 20 & EPA Method 8240 \\
\hline Trichlorotrifluoroethane & $\mu g / L$ & 50 & 100 & EPA Method 8260 \\
\hline $0,0,0$-Triethyl phosphorothioate & $\mu g / L$ & 81 & 162 & EPA Method 8270 \\
\hline $1,2,4$-Trimethylbenzene & $\mu g / L$ & 5 & 10 & EPA Method 8260 \\
\hline $1,3,5$ - Trimethylbenzene & $\mu \mathrm{g} / \mathrm{L}$ & 5 & 10 & EPA Method 8260 \\
\hline 1,3,5-Trinitrobenzene & $\mu g / L$ & 81 & 162 & EPA Method 8270 \\
\hline Tritium & $\mu \mathrm{Ci} / \mathrm{mL}$ & $1.0 \mathrm{E}-05$ & $2.0 \mathrm{E}-05$ & Final PDWS (EPA, 1997a) \\
\hline Turbidity & & No flag & No flag & Set by EPD/EMS \\
\hline Uranium & $\mu g / L$ & 10 & 20 & Proposed PDWS (EPA, 1991c) \\
\hline Uranium alpha activity & $\mu \mathrm{Ci} / \mathrm{mL}$ & $1.5 \mathrm{E}-08$ & 3.0E-08 & Proposed PDWS (EPA, 1991C) \\
\hline Uranium, dissolved & $\mu \mathrm{g} / \mathrm{L}$ & 10 & 20 & Proposed PDWS (EPA, 1991c) \\
\hline Uranium, total recoverable & $\mu g / L$ & 10 & 20 & Proposed PDWS (EPA, 1991c) \\
\hline Uranium-233/2340 & $\mu \mathrm{Ci} / \mathrm{mL}$ & $6.9 E-09$ & $1.38 \mathrm{E}-08$ & Proposed PDWS (EPA, 1991c) \\
\hline Uranium-234 & $\mu \mathrm{Ci} / \mathrm{mL}$ & $6.95 E-09$ & 1.39E-08 & Proposed PDWS (EPA, 1991c) \\
\hline
\end{tabular}




\begin{tabular}{|c|c|c|c|c|}
\hline Analyte & Unit & Flag 1 & Flag 2 & Sourcet \\
\hline Uranium-235 & $\mu \mathrm{Ci} / \mathrm{mL}$ & 7.25E-09 & $1.45 \mathrm{E}-08$ & Proposed PDWS (EPA, 1991c) \\
\hline Uranium-238 & $\mu \mathrm{Ci} / \mathrm{mL}$ & 7.3E-09 & $1.46 \mathrm{E}-08$ & Proposed PDWS (EPA, 1991c) \\
\hline Vanadium & $\mu g / L$ & 66.5 & 133 & EPA Method 6010 \\
\hline Vanadium, dissolved & $\mathrm{\mu g} / \mathrm{L}$ & 66.5 & 133 & EPA Method 6010 \\
\hline Vanadium, total recoverable & $\mu g / L$ & 66.5 & 133 & EPA Method 6010 \\
\hline Vinyl acetate & $\mu g / L$ & 50 & 100 & EPA Method 8240 \\
\hline $\mathrm{m} / \mathrm{p}$-Xylene & $\mu g / L$ & 81 & 162 & EPA Method 8260 \\
\hline $0-X y l e n e$ & $\mu g / L$ & 5 & 10 & EPA Method 8260 \\
\hline Xylenes & $\mu g / L$ & 5,000 & 10,000 & Final PDWS (EPA, 1997a) \\
\hline Yttrium-88 & $\mu \mathrm{Ci} / \mathrm{mL}$ & 5.0E-08 & 1.0E-07 & EPA Method 901.1 \\
\hline Zinc & $\mu g / L$ & 2,500 & 5,000 & SDWS (EPA, 1997b) \\
\hline Zinc, dissolved & $\mu g / L$ & 2,500 & 5,000 & SDWS (EPA, 1997b) \\
\hline Zinc, total recoverable & $\mu g / L$ & 2,500 & 5,000 & SDWS (EPA, 1997b) \\
\hline Zinc-65 & $\mu \mathrm{Ci} / \mathrm{mL}$ & $1.5 \mathrm{E}-07$ & $3.0 \mathrm{E}-07$ & $\begin{array}{l}\text { Interim Final PDWS (EPA, } \\
1977)\end{array}$ \\
\hline Zirconium-95 & $\mu \mathrm{Ci} / \mathrm{mL}$ & $1.0 \mathrm{E}-07$ & $2.0 E-07$ & $\begin{array}{l}\text { Interim Final PDWS (EPA, } \\
1977 \text { ) }\end{array}$ \\
\hline Zirconium/Niobium-95 & $\mu \mathrm{Ci} / \mathrm{mL}$ & $1.0 \mathrm{E}-07$ & $2.0 \mathrm{E}-07$ & $\begin{array}{l}\text { Interim Final PDWS (EPA, } \\
1977 \text { ) }\end{array}$ \\
\hline
\end{tabular}

† Analytical methods are discussed in the Analytical Data Review section of this document; references for dated sources are in the References section.

$\checkmark$ EMS discontinued monitoring this radionuclide because it is inappropriate for the SRS Groundwater Monitoring Program. * EPD/EMS set this flagging criterion using the 1991 proposed PDWS because the final PDWS in 1977 may have been in error.

When radionuclide analyses are combined, the lower DWS of the two isotopes is used for flagging.

The primary maximum contaminant level range for turbidity is $1-5$ NTU, which is inappropriate for the SRS Groundwater Monitoring Program.

Note: Beginning fourth quarter 1992, samples were no longer filtered at the wells. Therefore, the methods for analyzing metals now include a digestion step. Beginning fourth quarter 1993, the laboratories were required to report all metals as total recoverable metals. Flagging criteria remain unchanged. 


\section{Sample Scheduling}

Scheduling of sampling and analyses for the SRS Groundwater Monitoring Program conducted by EPD/EMS is based on several factors. Environmental screening is scheduled on a regular basis. Additional scheduling is based on previous flagging levels, regulatory requirements, and speciai requests that fall within the scope of the Groundwater Monitoring Program. This information is used to generate The Savannah River Site's Groundwater Monitoring Program 1999 Sampling Schedule.

A breakdown by laboratory of the total number of analyses performed during first quarter 1999 follows:

$\begin{array}{lr}\text { Laboratory } & \text { Number of Analyses } \\ \text { EMAX Laboratories, Inc. } & 19,222 \\ \text { Environmental Physics } & 14,498 \\ \text { General Engineering Laboratories } & 26,036 \\ \text { Recra LabNet Philadelphia } & 30,709 \\ \text { Thermo NUtech } & 3,054\end{array}$

\section{ENVIRONMENTAL SCREENING}

New wells designated as screening program wells are scheduled initially for four quarters of environmental screening. Environmental-screening constituents, which inciude indicator parameters, groundwater quality characteristics, and some drinking water characteristics, are listed below. After the initial four quarters of analyses for new wells, environmental screening is scheduled once every three years for wells identified as environmental-screening program wells. The wells are sampled only for the environmental-screening constituents that have not been analyzed within the past three years.

Beginning in 1996, EPD/EMS changed its policy concerning quarterly field measurements. Only wells scheduled by request or wells identified for environmental screening receive field measurements.

\section{Environmental-Screening Constituents}

\begin{tabular}{llll} 
Aluminum & $\mathrm{pH}$ & \multicolumn{1}{c}{ Well condition } & Mercury \\
Arsenic & Phenolphthalein alkalinity & Fluoride & Nitrate-nitrite as nitrogen \\
Barium & Program & Gross alpha & Nonvolatile beta \\
Boron & Sampling method & Iron & Selenium \\
Cadmium & Site code & Lead & Silver \\
Chloride & Specific conductance & Lithium & Sodium \\
Chromium & Stabilized (Yes or No) & Major ions & Sulfate \\
Field measurements & Time & Calcium & Total dissolved solids \\
Air temperature & Total alkalinity & Magnesium & Total organic carbon \\
Date & Turbidity & Potassium & Total organic halogens \\
Depth to water & Volume purged & Silica & Total phosphates (as P) \\
Flow rate & Water temperature & Manganese & Tritium
\end{tabular}

\section{Scheduling Based on Flagging Levels}

Only the flagging criteria for environmental screening and GC VOA (see Glossary) are used to trigger scheduling. Wells are grouped for scheduling by monitoring site or by the investigation for which they are sampled. Specific criteria for Flag 1 and Flag 2 designations are found in the Flagging Criteria section of this report.

Beginning in 1996, only wells in the environmental-screening program were scheduled by flagging criteria once a year. Constituents classified as Flag 0 in each well series are scheduled for analyses only by custodian 
request or as part of the triennial environmental-screening program. If an analytical result for an environmentalscreening or GC VOA analysis in any well exceeds Flag 2 or Flag 1, the environmental-screening wells in the same monitoring series are sampled and analyzed for that constituent once a year. If a constituent falls below Flag 2 for three consecutive sampling events, the individual well's flag is reduced from Flag 2 status to Flag 1 or Flag 0 status, depending on the results, and the well is scheduled according to the lower flag. If a constituent falls below Flag 1 for three consecutive sampling events, the individual well's flag is reduced from Flag 1 status to Flag 0 status, and the flagging-based sampling ceases.

If an environmental-screening or GC VOA constituent has ever been flagged in a well series, it automatically is flagged for all new wells of that series that are designated as environmental-screening wells. The rules previously referred to also apply to removal of a flag from a new well.

When one or more of the five constituents in the GC VOA suite are flagged, the entire suite is scheduled for analysis. The GC VOA suite includes the following: carbon tetrachloride, chloroform, tetrachloroethylene, 1,1,1-trichloroethane, and trichloroethylene.

The following constituents are exceptions to the flagging rules but still receive analyses by custodian request or during triennial environmental-screening analyses:

- Specific conductance and $\mathrm{pH}$, two indicator constituents, have flagging criteria but do not trigger the scheduling mechanism.

- No flags are set for the following indicator parameters and major cations: alkalinity, 5-day biochemical oxygen demand, calcium, carbonate, chemical oxygen demand, magnesium, potassium, silica, sodium, total dissolved solids, total phosphates (as P), and total phosphorus.

- Aesthetic analyses such as color, odor, corrosivity, Eh, turbidity, and surfactants will not be assigned flagging criteria but may be analyzed by special request.

- Cornmon laboratory contaminants and cleaners including phthalates, dichloromethane (methylene chloride), ketones, and toluene are not assigned flagging criteria unless they have primary drinking water standards. These constituents may be analyzed by special request.

\section{GCMS VOA ANALYSES}

All wells are reviewed for total organic halogens (TOH) results twice a year. GCMS VOA (see Glossary) is scheduled once for individual wells that are designated as environmental-screening wells, have had two results for $\mathrm{TOH}$ greater than $10 \mu \mathrm{g} / \mathrm{L}$ (excluding the first TOH analysis), and have never received GCMS VOA analysis.

\section{SAMPLING REOUESTS}

Many analyses are scheduled at the request of various SRS groups. The person or group requesting an analysis must submit a formal sampling request form to EPD/EMS. If the request is within the scope of the Groundwater Monitoring Program, and if provision for the analysis has been made in the current laboratory contract, the analysis is added to the sampling schedule. Likewise, if a sampling request should be deleted, the originator of the request must submit a deletion form.

\section{Regulatory Requirements}

All regulatory sampling requirements, such as those mandated by the Resource Conservation and Recovery Act (RCRA), are scheduled by request.

\section{Changes in Sampling}

For changes in sampling for first quarter 1999, please refer to The Savannah River Site's Groundwater Monitoring Program 1999 Sampling Schedule. 
The following RCRA Facility Investigation/Remedial Investigation (RFI/RI) and South Carolina Department of Health and Environmentai Control (SCDHEC) projects were in process during first quarter 1999:

- F- and H-Area Hazardous Waste Management Facilities

- $\quad F$ - and H-Area Injection Tanks

- M-Area Hazardous Waste Management Facility and Metallurgical Laboratory Hazardous Waste Management Facility

- Interim Saritary Landfill

- Old Burial Ground

- Sanitary Landfill

- TNX Area

- Z-Area Saltstone

\section{CERCLA Projects}

The following Comprehensive Environmental Response, Compensation, and Liability Act (CERCLA) projects were either in process or new during first quarter 1999:

- C-Area Reactor Seepage Basin

- D-Area Coal Pile Runoff Basin Containment Basin and Ash Basins

- H-Area Tank Farm

- P-Area Reactor Seepage Basin

\section{New Wells Scheduled for Sampling}

The following wells were scheduled for sampling for the first time during first quarter 1999: wells CSB 8D, and 9D; DCB 8C, 15R, 26A, 26AR, 26C, 27, 28, 29R, 30, 31, 32A, 33B, 33C, 33D, 34A, 34C, 35A, 35C, 36A, 36C, 37A, 37C, 8C, 39A, 39C, 40A, 41A, 41C, 43A, 43C, 44A, 44C, 45A, 45C, 46C, 47C, and 48D; HAA 5B, $5 \mathrm{D}, 7 \mathrm{~A}, 7 \mathrm{~B}, 7 \mathrm{C}, 7 \mathrm{D}, 8 \mathrm{~A}, 8 \mathrm{~B}, 8 \mathrm{C}, 8 \mathrm{D}, 9 \mathrm{~A}, 9 \mathrm{~B}, 9 \mathrm{C}, 9 \mathrm{D}, 10 \mathrm{~A}, 10 \mathrm{~B}, 10 \mathrm{C}, 10 \mathrm{D}, 11 \mathrm{~A}, 11 \mathrm{~B}, 11 \mathrm{C}, 11 \mathrm{D}, 12 \mathrm{~A}$,

12B, 12C, 12D, 13A, 13B, 13C, 13D, 14A, 14B, 14C, 14D, 15A, 15B, 15C, and 15D; and HTF 12D and 15D.

\section{MAINTENANCE OR ACCESS PROBLEMS}

Wells MSB 15C, 16C, 46C, and 48D were not sampled because of mechanical problems.

Recovery wells RWM 9 and 11 were not in operation for the February and March sampling events.

Wells FTF 3, 4, 5, and 7 were inaccessible due to construction. Well KDB 1 was inaccessible for the March sampling event because the road was flooded. Wells MSB 19A, 19B, and19C also were inaccessible and consequently not sampled.

\section{OTHER PROBLEMS}

Well FTF 1, 2, 6, 8, 11 and 14; LSB 4; MSB 9C and 87C; and ZBG 1A were dry during first quarter 1999. 
The following wells had flowmeter problems during first quarter 1999: AOB 2; BGX 10D; CSB 2A and 3A; FSB 79B and 108D; FSL 2D and 4D; HAA 11D; HSB101D, 107D, and 110C; HSL 6D and 8D, LDB 1; LFW 28; MSB 36B, 39D, 40B, and 42TA; P 26A, 26B, and 26D; and PCB 3A.

The following wells were sampled during first quarter 1999 using hand-held pumps: TBG 4 and TIR 1M (02/02/99), TCM 3 (02/08/99), and TNX 15D (02/09/99).

The following wells did not have water level measurements and did not have water in the standpipe: MCB 6 (01/06/99); HSB109D (01/15/99); TRW 3 (01/21/99); HSB152D (01/27/99 and 01/28/99); TRW 3 (02/03/99); RWM 1 (02/16/99); RWM 3 (02/17/99); TRW 3 (02/22/99); MSB 3B (02/24/99); MSB 26 (03/01/99); HWS 2 (03/03/99); RWM 2 and 3 (03/08/99); MSB 26 (03/09/99 and 03/10/99); RWM 1 (03/18/99); HSB152D (03/22/99); FSB 90D (03/23/99); TRW 3 (03/24/99); MSB 12D, 17C, and 35D (03/26/99); and MSB 3B, 9C, 10D, 11F, 14C, 15D, 23, 25, 26, and 45C; and SRW 7 and $10(03 / 29 / 99)$.

Wells FRB 2; FSB119D; HAA 7C and 9D; HSB115D; HSD 7D; HSL 5D; KDB 1, 3, 4, and 5; LDB 1 and 4; MSB 13D, 15D, and 78DR; and TNX 24D had purging problems during first quarter 1999.

Sampling scheduled for BTP and IDB wells was canceled by the project manager for the Accelerator for Production of Tritium project before sampling began.

\section{PURGE-WATER CONTAINMENT PROGRAM}

Beginning in 1991, a purge-water containment program was partially implemented to dispose properly of the water purged from certain wells before sampling. According to the Investigation-Derived Waste Management Plan (WSRC, 1995), additional wells were identified for purge-water treatment at the M-1 Air Stripper and Fand $\mathrm{H}$-Area Effluent Treatment Facility. The program has been implemented, and no well that was scheduled for analysis as part of the Groundwater Monitoring Program during first quarter 1999 was not sampled. 


\section{Field Notes}

A sampler may visit a well to collect field data, collect samples, and/or measure depth to water. A well may be visited multiple times during a quarter for any combination of these reasons. Field measurements generally include air temperature, depth to water prior to pumping, dissolved oxygen, Eh (REDOX potential), flow rate, $\mathrm{pH}$, phenolphthalein alkalinity, specific conductance, total alkalinity, turbidity, volume of water purged prior to sampling, and water temperature.

EPD/EMS personnel and RCS Corporation of Aiken, SC, performed well visitations during first quarter 1999. Each sampler maintained a field notebook. These notebooks are in the first quarter 1999 section of the EPD/EMS Groundwater Monitoring Library. All well visitations were routine during first quarter 1999, except as indicated in table 3. The table includes samplers' comments on conditions that may affect the samples or the data-collection process. The majority of wells sampled during first quarter 1999 were pumped. Bailed wells are listed in table 66 in the Quality Control Samples section.

If a well pumps or is bailed dry during purging and is revisited and sampled within 24 hours, this is considered one sampling event yielding a single set of field and analytical data. For such wells, table 3 lists the volume purged before the well went dry during the first visitation. The Analytical Results section gives the total amount of water purged from each well in one sampling event.

Comments about dry wells and continuously pumping wells are in the Analytical Results section.

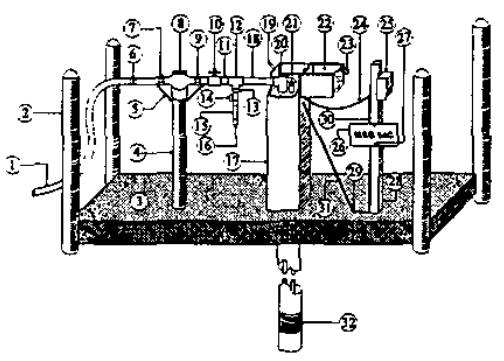

Water discharge hose

2. Protective or delineator post

3. Concrete pad

4. Flowmeter support post

5. Flowmeter tie down

6. Hose clamp

Flowmeter connector

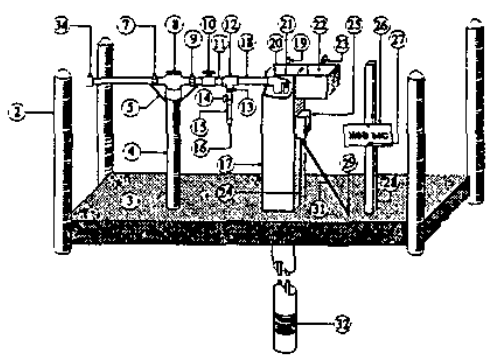

8. Flowmeter

9. Flowmeter connector

10. Gate or ball value

11. Nipple

12. Sampling tee

13. Bushing

14. Sample cock
15. Sample ripple

16. Sample nipple cap

17. Prolective casing

18. Nipple

19. Hasp

20. Riser pipe elbow

21. Liquid level pipe cap

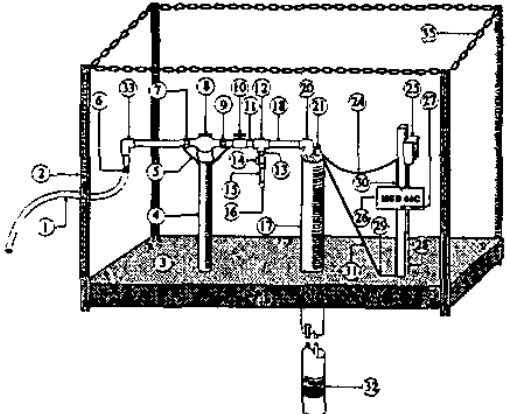

22. Protective casing lid

23. Lock

24. Electrical conduit

25. Electrical box

26. Well identification sign

27. Sign bolt

28. Mounting post
29. Grounding rod

30. Grounding wire

31. Security cable

32. Pump

33. Etbow

34. Quick disconnect

35. Yellow chain

Figure 3. Three Types of Groundwater Monitoring Wellheads 
Table 3. Comments from the Field Data

\begin{tabular}{lll}
\hline Well & Date & Comments \\
\hline
\end{tabular}

ABP Series

ABP $8 C$

$03 / 10 / 99$

$02 / 05 / 99$

$02 / 08 / 99$

$02 / 05 / 99$

$02 / 08 / 99$

$02 / 12 / 99$

$02 / 12 / 99$

$02 / 17 / 99$

$03 / 15 / 99$

$03 / 16 / 99$

$03 / 15 / 99$

AMB 15D

AOB Series

AOB 2

AOB 3

02/05/99

$01 / 06 / 99$

$03 / 10 / 99$

$02 / 11 / 99$

$02 / 11 / 99$

$02 / 22 / 99$

$02 / 22 / 99$

$01 / 14 / 99$

$02 / 18 / 99$

$03 / 15 / 99$

$01 / 12 / 99$

$02 / 17 / 99$

$01 / 13 / 99$

$02 / 17 / 99$

$03 / 11 / 99$

BGO 34D

$01 / 13 / 99$

$02 / 17 / 99$

$03 / 11 / 99$

$01 / 13 / 99$

$02 / 17 / 99$

$03 / 11 / 99$

BGO $36 D \quad 01 / 13 / 99$

$02 / 17 / 99$

$03 / 11 / 99$

BGO 370

BGO 38D

BGO 39D
$01 / 13 / 99$

$02 / 17 / 99$

$03 / 11 / 99$

$01 / 13 / 99$

02/17/99

$03 / 11 / 99$

$01 / 13 / 99$

$02 / 17 / 99$

$03 / 11 / 99$
Dry after $5 \mathrm{gal}$

Dry after $9 \mathrm{gal}$

Dry after $6 \mathrm{gal}$

Dry after $63 \mathrm{gal}$

Dry after $65 \mathrm{gal}$

Surges

Surges

Dry after $86 \mathrm{gal}$

Dry after $1 \mathrm{gal}$

Dry after $1 \mathrm{gal}$

Dry after $8 \mathrm{gal}$

Replace flowmeter

Dry after 12 gal

Undercurrent; not sampled

Dry after $11 \mathrm{gal}$

Dry after $36 \mathrm{gal}$

Well broken; not sampled

Dry after 12 gal

Dry after $4 \mathrm{gal}$

Dry after $5 \mathrm{gal}$

Dry after 2 gal

Dry after $12 \mathrm{gal}$

Dry after $6 \mathrm{gal}$

Dry after $9 \mathrm{gal}$

Dry after $8 \mathrm{gal}$

Dry after $7 \mathrm{gal}$

Dry after $11 \mathrm{gal}$

Dry after $10 \mathrm{gal}$

Dry after $10 \mathrm{gal}$

Dry after $8 \mathrm{gal}$

Dry after $9 \mathrm{gal}$

Dry after $7 \mathrm{gal}$

Dry after $8 \mathrm{gal}$

Dry after $7 \mathrm{gal}$

Dry after $7 \mathrm{gal}$

Dry after $7 \mathrm{gal}$

Dry after $7 \mathrm{gal}$

Dry after $4 \mathrm{gal}$

Dry after $9 \mathrm{gal}$

Dry after $8 \mathrm{gal}$

Dry after $8 \mathrm{gal}$

Dry after $4 \mathrm{gal}$

Dry after $3 \mathrm{gal}$

Dry after $4 \mathrm{gal}$ 


\begin{tabular}{lll}
\hline Well & Date & Comments \\
\hline \multirow{2}{*}{ BGO 49D } & $01 / 13 / 99$ & Dry after 4 gal \\
& $02 / 17 / 99$ & Dry after 4 gal \\
BGO 51D & $03 / 11 / 99$ & Dry after 4 gal \\
& $02 / 17 / 99$ & Dry after 3 gal \\
BGO 52D & $03 / 12 / 99$ & Dry after 2 gal \\
& $01 / 13 / 99$ & Dry after 2 gal \\
& $03 / 15 / 99$ & Dry after 3 gal
\end{tabular}

BGX Series

$B G X 1 D$

BGX 3D

$\mathrm{BG} \times 10 \mathrm{D}$

$02 / 25 / 99$

$02 / 22 / 99$

$02 / 25 / 99$

BSE Series

BSE 1D4

$01 / 05 / 99$

CBR Series

CBR 1D

$01 / 25 / 99$

\section{CDB Series}

CDB 1

CDB 2

$03 / 01 / 99$

$03 / 01 / 99$

CMP Series

CMP 11D

03/23/99

CRP Series

CAP 3D

CsB Series

CSB $2 A$

CSB 3A

$03 / 10 / 99$

$03 / 10 / 99$

DCB Series

DCB 13

$03 / 12 / 99$

$03 / 24 / 99$

FCB Series

FCB 5

$01 / 26 / 99$

FET Series

FET 1D

$01 / 29 / 99$

FRB Series

FRB 2

03/04/99

FSB Series

FSB 78C

FSB 79B

FSB 87D

FSB 88D

FSB $90 \mathrm{C}$

FSB 90D
$01 / 13 / 99$

$01 / 06 / 99$

$01 / 07 / 99$

$01 / 14 / 99$

$01 / 06 / 99$

01/04/99
Dry after $5 \mathrm{gal}$

Dry after $13 \mathrm{gal}$

Dry after $3 \mathrm{gal}$; flowmeter broken, estimated volume purged; purged through sample port; high turbidity

Resample

Dry after 12 gal

Dry after 26 gal

Dry after $20 \mathrm{gal}$

Dry after $8 \mathrm{gal}$

Dry after 2 gai

Flowmeter broken; estimated volume purged

Flowmeter broken; estimated volume purged

Dry after $18 \mathrm{gal}$

Dry after 9 gal

Dry after $8 \mathrm{gal}$

Dry after $9 \mathrm{gal}$

17 gal purged through sample port

Dry after 43 gal

Flowmeter broken

Hard to start

Dry after $7 \mathrm{gal}$

Dry after $34 \mathrm{gal}$

Dry after $9 \mathrm{gal}$ 


\begin{tabular}{|c|c|c|}
\hline Well & Date & Comments \\
\hline $\begin{array}{l}\text { FSB 91C } \\
\text { FSB 92C } \\
\text { FSB 93D } \\
\text { FSB 94C } \\
\text { FSB 97D } \\
\text { FSB 98D } \\
\text { FSB106D }\end{array}$ & $\begin{array}{l}01 / 14 / 99 \\
01 / 14 / 99 \\
01 / 13 / 99 \\
01 / 13 / 99 \\
01 / 11 / 99 \\
01 / 28 / 99 \\
01 / 08 / 99 \\
01 / 12 / 99\end{array}$ & $\begin{array}{l}\text { Dry after } 45 \mathrm{gal} \\
\text { Dry after } 33 \mathrm{gal} \\
\text { Dry after } 6 \mathrm{gal} \\
\text { Dry after } 26 \mathrm{gal} \\
\text { Dry after } 10 \mathrm{gal} \\
\text { Dry after } 12 \mathrm{gal} \\
\text { Dry after } 0.5 \mathrm{gal} \\
\text { Dry after } 0.25 \mathrm{gal} \text {; highly aerated }\end{array}$ \\
\hline FSB108D & $01 / 11 / 99$ & $\begin{array}{l}\text { After } 1 \text { volume, slowed to } 1 \mathrm{gal} / \mathrm{min} \text {; meter not turning at } \\
\text { low volume }\end{array}$ \\
\hline $\begin{array}{l}\text { FSB111D } \\
\text { FSB113A } \\
\text { FSB113C } \\
\text { FSB115D } \\
\text { FSB116D } \\
\text { FSB119D }\end{array}$ & $\begin{array}{l}01 / 07 / 99 \\
01 / 07 / 99 \\
01 / 07 / 99 \\
01 / 11 / 99 \\
01 / 11 / 99 \\
01 / 12 / 99 \\
01 / 13 / 99\end{array}$ & $\begin{array}{l}\text { Dry after } 4 \mathrm{gal} \\
\text { Dry after } 40 \mathrm{gal} \\
\text { Dry after } 26 \mathrm{gal} \\
\text { Turbidity }>15 \mathrm{NTU} \\
\text { Dry after } 2 \mathrm{gal} \\
\text { Dry after } 8 \mathrm{gal} \\
\text { Purged } 9 \text { gal through sample port to lower turbidity }\end{array}$ \\
\hline $\begin{array}{l}\text { FSB120A } \\
\text { FSB120D } \\
\text { FSB121DR }\end{array}$ & $\begin{array}{l}01 / 08 / 99 \\
01 / 08 / 99 \\
01 / 08 / 99\end{array}$ & $\begin{array}{l}\text { Dry after } 42 \mathrm{gal} \\
\text { Dry after } 11 \mathrm{gal} \\
\text { Dry after } 6 \mathrm{gal}\end{array}$ \\
\hline \multicolumn{3}{|l|}{ FSL Series } \\
\hline $\begin{array}{l}\text { FSL 1D } \\
\text { FSL 2D } \\
\text { FSL 3D } \\
\text { FSL 4D } \\
\text { FSL 6D } \\
\text { FSL 7D }\end{array}$ & $\begin{array}{l}01 / 26 / 99 \\
01 / 13 / 99 \\
01 / 12 / 99 \\
01 / 12 / 99 \\
01 / 12 / 99 \\
01 / 12 / 99\end{array}$ & $\begin{array}{l}\text { Dry after } 1 \mathrm{gal} \\
\text { Volume too low for meter } \\
\text { Slowed to about } 200 \mathrm{~mL} / \mathrm{min} \text { after purging } \\
\text { Volume too low for meter } \\
\text { Turbidity in excess of limits first three readings } \\
\text { Dry after } 4 \text { gal }\end{array}$ \\
\hline \multicolumn{3}{|l|}{ FTF Series } \\
\hline $\begin{array}{l}\text { FTF } 22 \\
\text { FTF } 23 \\
\text { FTF 24A } \\
\text { FTF 25A } \\
\text { FTF 26 } \\
\text { FTF } 27\end{array}$ & $\begin{array}{l}01 / 21 / 99 \\
01 / 21 / 99 \\
01 / 26 / 99 \\
01 / 26 / 99 \\
01 / 26 / 99 \\
01 / 26 / 99\end{array}$ & $\begin{array}{l}\text { Muddy } \\
\text { No sign } \\
\text { Pumped dry } \\
\text { Pumped dry } \\
\text { Pumped dry } \\
\text { Pumped dry }\end{array}$ \\
\hline \multicolumn{3}{|l|}{ HAA series } \\
\hline HAA 3D & $\begin{array}{l}01 / 04 / 99 \\
01 / 05 / 99\end{array}$ & $\begin{array}{l}\text { Dry after } 3 \text { gal } \\
\text { Cloudy }\end{array}$ \\
\hline $\begin{array}{l}\text { HAA 4D } \\
\text { HAA 5A }\end{array}$ & $\begin{array}{l}01 / 10 / 99 \\
01 / 10 / 99\end{array}$ & $\begin{array}{l}\text { Dry after } 1 \mathrm{gal} \\
\text { Ball valve split }\end{array}$ \\
\hline $\begin{array}{l}\text { HAA 5BR } \\
\text { HAA 5D }\end{array}$ & $\begin{array}{l}01 / 10 / 99 \\
02 / 24 / 99\end{array}$ & $\begin{array}{l}\text { Flowmeter broken and leaks } \\
\text { Dry after } 9 \mathrm{gal}\end{array}$ \\
\hline HAA 6D & $01 / 04 / 99$ & $\begin{array}{l}\text { Dry after } 2 \mathrm{gal} \\
\text { Cloudy }\end{array}$ \\
\hline HAA 7C & $01 / 04 / 99$ & $\begin{array}{l}\text { Dry after } 10 \mathrm{gal} \text {; tried to lower turbidity by purging through } \\
\text { sample port }\end{array}$ \\
\hline $\begin{array}{l}\text { HAA 9D } \\
\text { HAA } 110\end{array}$ & $\begin{array}{l}01 / 05 / 99 \\
02 / 16 / 99 \\
02 / 18 / 99\end{array}$ & $\begin{array}{l}\text { Dry after } 25 \text { gal; purged slowly through sample port } \\
\text { Gate valve broken; purged through sample port } \\
\text { Flowmeter broken; estimated volume purged in } 5 \mathrm{gal} \\
\text { bucket }\end{array}$ \\
\hline HAA 13A & $01 / 06 / 99$ & Dry after $3 \mathrm{gal}$ \\
\hline HAA 13D & $\begin{array}{l}01 / 05 / 99 \\
01 / 06 / 99 \\
02 / 16 / 99 \\
02 / 17 / 99 \\
02 / 18 / 99 \\
02 / 24 / 99 \\
\end{array}$ & $\begin{array}{l}\text { Dry after } 2 \mathrm{gal} \\
\text { Dry after } 2 \mathrm{gal} \\
\text { Dry after } 2 \mathrm{gal} \\
\text { Dry after } 2 \mathrm{gal} \\
\text { Pumped dry } \\
\text { Dry after } 2 \mathrm{gal} \\
\end{array}$ \\
\hline
\end{tabular}




\begin{tabular}{lll}
\hline Well & Date & Comments \\
\hline HAA 14D & $02 / 02 / 99$ & $\begin{array}{l}\text { Dry after 2 gal; high turbidity } \\
\text { Muddy }\end{array}$ \\
& $02 / 18 / 99$ &
\end{tabular}

\section{HET Serles}

HET 1D

HET 2D

HET 3D

HET 4D

HSB Series

HSB 68B

HSB $68 \mathrm{C}$

HSB 70C

HSB 71C

HSB 84B

HSB $84 C$

HSB 85B

HSB 98D

HSB101C

HSB101D

HSB102D

HSB107D

HSB108D

HSB109D

HSB110C

HSB112E

HSB115D

HSB1 16D

HSB119A

HSB123A

HSB126D

HSB139C

HSB141A

HSB141D

HSB $145 \mathrm{C}$

HSB147D

HSB $148 \mathrm{C}$

HSB148D

HSB150D

HSB152D

HSL Series

HSL 3D

HSL 5D

HSL 60

HSL 7D

HSL 8D
01/29/99

$01 / 29 / 99$

$01 / 29 / 99$

$01 / 29 / 99$

$01 / 08 / 99$

$01 / 08 / 99$

$01 / 12 / 99$

$01 / 12 / 99$

$01 / 11 / 99$

01/13/99

$01 / 13 / 99$

$01 / 28 / 99$

$01 / 21 / 99$

$01 / 14 / 99$

$01 / 27 / 99$

$01 / 28 / 99$

$01 / 15 / 99$

$01 / 28 / 99$

$01 / 15 / 99$

$01 / 15 / 99$

$01 / 15 / 99$

$01 / 15 / 99$

$01 / 27 / 99$

$01 / 15 / 99$

$01 / 18 / 99$

$01 / 18 / 99$

$01 / 18 / 99$

$01 / 21 / 99$

$01 / 19 / 99$

$01 / 19 / 99$

$01 / 20 / 99$

$01 / 21 / 99$

$01 / 20 / 99$

$01 / 21 / 99$

$01 / 21 / 99$

$01 / 21 / 99$

$01 / 27 / 99$

$01 / 21 / 99$

$01 / 27 / 99$

$01 / 28 / 99$

$01 / 21 / 99$

$01 / 22 / 99$

$01 / 25 / 99$

$01 / 22 / 99$

$01 / 22 / 99$

$01 / 25 / 99$
Dry after $15 \mathrm{gal}$

Dry after 9 gal

Dry after $11 \mathrm{gal}$

Dry after $16 \mathrm{gal}$

Dry after $50 \mathrm{gal}$

Dry after $19 \mathrm{gal}$

Dry after $24 \mathrm{gal}$

Dry after $19 \mathrm{gal}$

Dry after 43 gal

Dry after $13 \mathrm{gal}$

Dry after $45 \mathrm{gal}$

Dry after $12 \mathrm{gal}$

Dry after $10 \mathrm{gal}$

Flowmeter broken; volume purged was approximated

Dry after 6 gal

Dry after $6 \mathrm{gal}$

Meter not working

Flowmeter broken; estimated volume purged

Dry after $24 \mathrm{gal}$

Gate valve broken; not sampled; no water in standpipe

Meter not working

Dry after $5 \mathrm{gal}$

Dry after 2 gal; purged slowly through sample port to lower turbidity

Dry after $8 \mathrm{gal}$

Dry after $79 \mathrm{gal}$

Dry after 33 gal

Dry after $11 \mathrm{gal}$

Dry after 28 gal

Dry after $79 \mathrm{gal}$

Dry after $12 \mathrm{gal}$

Dry after $2 \mathrm{gal}$

Turbidity went up during sampling

Well pumping coarse sand

Dry after 8 gal

Dry after $23 \mathrm{gal}$

Dry after $4 \mathrm{gal}$

Dry after $3 \mathrm{gal}$

Dry after $9 \mathrm{gal}$

Ory after $5 \mathrm{gal}$, no water in standpipe

Dry after $1 \mathrm{gal}$, no water in standpipe; turbidity increased while fitling bottles

High turbidity

Dry after $4 \mathrm{gal}$; very turbid

Bucket volume

Flow too slow for meter; turbidity stable, high

Bucket volume; water turned very turbid after filling $1 / 3$ of the bottles

Bucket volume 


\section{Well}

HTF 6

HTF 7

HTF 10

HTF 12

HTF 12D

HTF 13

HTF 15

HTF 15D

HTF 34

HWS Series

HWS 2

KBP Series

KBP 10

$03 / 08 / 99$

KCB Series

KCB 7

$01 / 26 / 99$

KDB Series

KDB 1

$01 / 14 / 99$

02/24/99

KDB 3

KDB 4

KDB 5

01/14/99

$01 / 14 / 99$

02/24/99

03/22/99

$01 / 14 / 99$

$02 / 24 / 99$

$03 / 22 / 99$

KRB Series

KRB 19D

$03 / 22 / 99$

KRP Series

KRP 3

$01 / 28 / 99$

KSB Series

KSB 5D

LBP Series

LBP 1D

$01 / 28 / 99$

$03 / 01 / 99$

03/08/99

LDB Series

LDB 1

$01 / 15 / 99$

$02 / 25 / 99$

03/22/99
Comments

Muddy

Muddy

No sign

No sign

No sign, no lock; flowmeter needs to have tie downs

No sign

No sign

No sign, no lock; muddy

No sign; muddy

No water in standpipe

Dry after 5 gal

Dry after $4 \mathrm{gal}$

Dry after $17 \mathrm{gal}$

Dry after $16 \mathrm{gal}$

Dry after $18 \mathrm{gal}$

Dry after $17 \mathrm{gal}$; purged $1 \mathrm{gal}$ through sample port

Dry after 8 gal

Dry after 8 gal; gate valve was broken, gallons purged measured using a 2.5 gal bucket

Purged 3 gal through sample port

Dry after $8 \mathrm{gal}$

Dry after 8 gal; purged $5 \mathrm{gal}$ through sample port

Dry after $7 \mathrm{gal}$

Broken pump; not sampled

Inaccessible, road flooded; not sampled

Dry after $3 \mathrm{gal}$

Dry after $2 \mathrm{gal}$

Dry after $2 \mathrm{gal}$

Dry after $1 \mathrm{gal}$

Dry after $16 \mathrm{gal}$; flowmeter broken

Dry after $15 \mathrm{gal}$; flowmeter broken; purged $6 \mathrm{gal}$ through sample port to lower turbidity

Dry after 26 gal; purged through sample port 


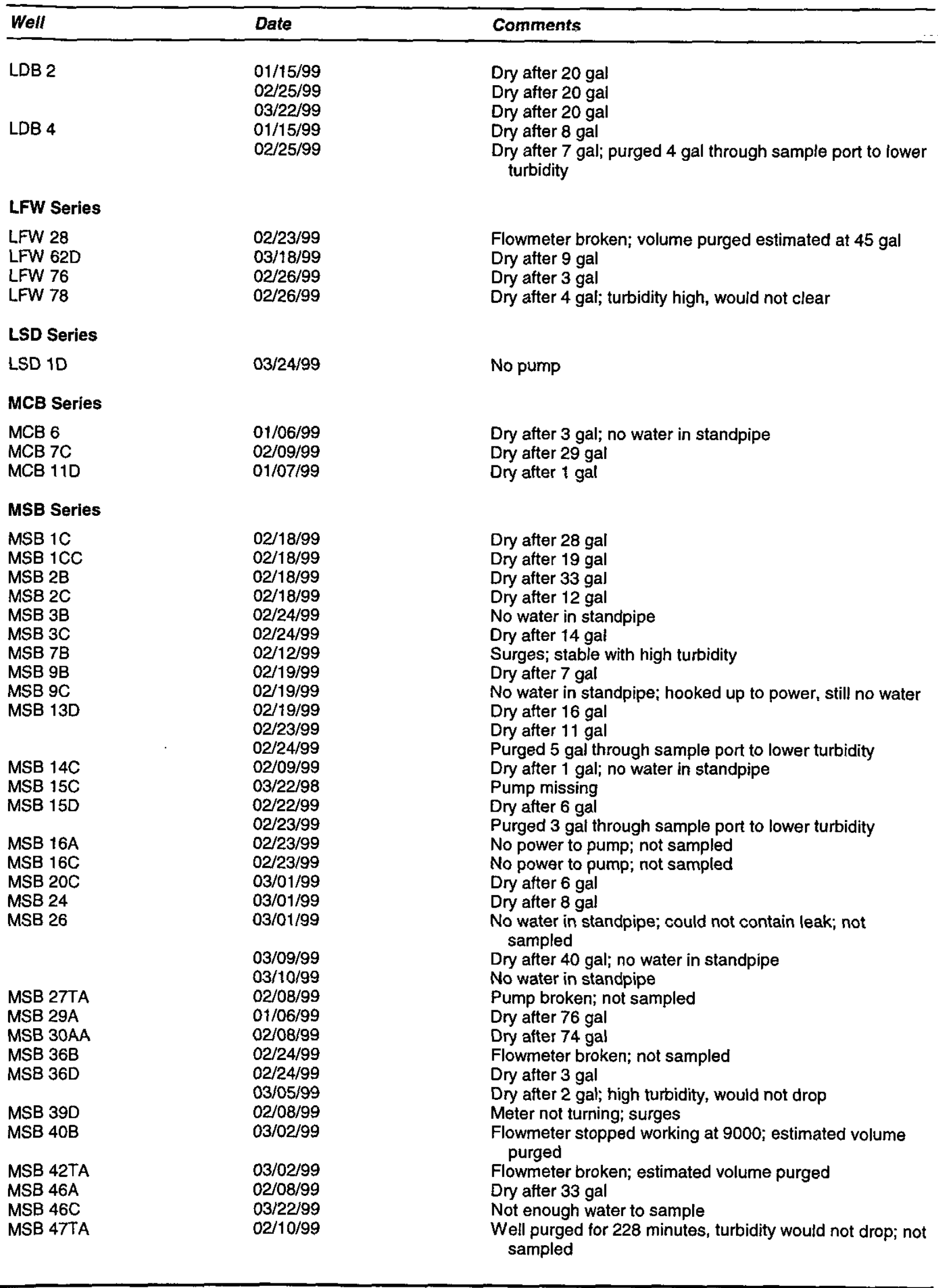




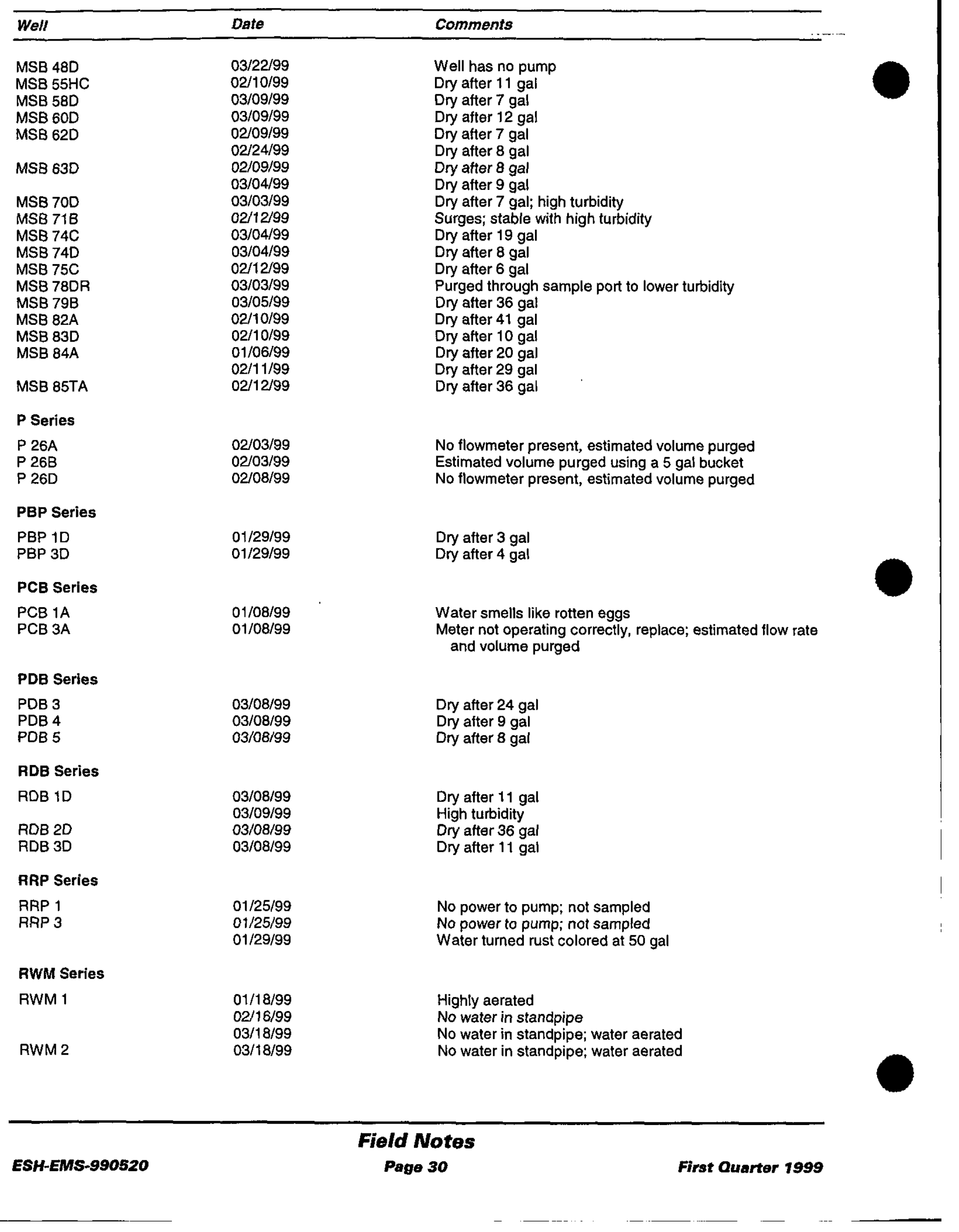




\begin{tabular}{|c|c|c|}
\hline Well & Date & Comments \\
\hline RWM 3 & $\begin{array}{l}02 / 17 / 99 \\
03 / 18 / 99\end{array}$ & $\begin{array}{l}\text { No water in standpipe } \\
\text { No water in standpipe }\end{array}$ \\
\hline \multicolumn{3}{|c|}{ SRW Series } \\
\hline SRW $16 \mathrm{~A}$ & $02 / 09 / 99$ & Dry after $42 \mathrm{gal}$ \\
\hline \multicolumn{3}{|c|}{ TBG Series } \\
\hline $\begin{array}{l}\text { TBG } 3 \\
\text { TBG } 4 \\
\text { TBG } 6\end{array}$ & $\begin{array}{l}02 / 08 / 99 \\
02 / 08 / 99 \\
02 / 08 / 99\end{array}$ & $\begin{array}{l}\text { Dry after } 7 \text { gal } \\
\text { Hand-held pump will not reach water; not sampled } \\
\text { Dry after } 18 \mathrm{gal}\end{array}$ \\
\hline \multicolumn{3}{|c|}{ TCM Series } \\
\hline $\begin{array}{l}\text { TCM } 2 \\
\text { TCM } 3\end{array}$ & $\begin{array}{l}02 / 02 / 99 \\
02 / 08 / 99\end{array}$ & $\begin{array}{l}\text { Used hand-held submersible pump } \\
\text { Used hand-held submersible pump }\end{array}$ \\
\hline \multicolumn{3}{|l|}{ TIR Series } \\
\hline TIR $1 \mathrm{M}$ & $02 / 02 / 99$ & Used hand-held submersible pump \\
\hline \multicolumn{3}{|c|}{ TNX Series } \\
\hline TNX 2D & $\begin{array}{l}02 / 02 / 99 \\
02 / 03 / 99\end{array}$ & $\begin{array}{l}\text { Dry after } 8 \text { gal; needs discharge hose } \\
\text { Aerated }\end{array}$ \\
\hline TNX 3D & $02 / 08 / 99$ & Dry after $4 \mathrm{gal}$ \\
\hline TNX 4D & 02/02/99 & Dry after $6 \mathrm{gal}$ \\
\hline TNX 5D & 02/02/99 & Dry after $5 \mathrm{gal}$ \\
\hline TNX 6D & $02 / 02 / 99$ & Dry after $7 \mathrm{gal}$ \\
\hline TNX 15D & 02/02/99 & Used hand-held submersible pump \\
\hline TNX 18D & 02/03/99 & $\begin{array}{l}\text { Continues flowing well; could not determine depth to } \\
\text { water }\end{array}$ \\
\hline $\begin{array}{l}\text { TNX } 210 \\
\text { TNX 24D } \\
\text { TNX 26D }\end{array}$ & $\begin{array}{l}02 / 03 / 99 \\
02 / 03 / 99 \\
02 / 04 / 99\end{array}$ & $\begin{array}{l}\text { Turbidity stabilized at } 27.2 \text { NTU; all samples collected } \\
5 \text { gal purged through sample port } \\
\text { Dry after } 2 \text { gal }\end{array}$ \\
\hline \multicolumn{3}{|c|}{ TRW Serles } \\
\hline TRW 3 & $\begin{array}{l}01 / 21 / 99 \\
02 / 03 / 99 \\
02 / 22 / 99 \\
03 / 24 / 99\end{array}$ & $\begin{array}{l}\text { No water in standpipe } \\
\text { No water in standpipe } \\
\text { No water in standpipe } \\
\text { No water in standpipe }\end{array}$ \\
\hline \multicolumn{3}{|c|}{ XSB Series } \\
\hline XSB $3 A$ & $02 / 03 / 99$ & No hose \\
\hline \multicolumn{3}{|c|}{ YSB Series } \\
\hline YSB 3A & $02 / 03 / 99$ & Very turbid at start \\
\hline \multicolumn{3}{|c|}{ YSC Series } \\
\hline YSC 2D & 03/09/99 & Dry after $12 \mathrm{gal}$ \\
\hline \multicolumn{3}{|c|}{ ZBG Series } \\
\hline$Z B G \uparrow A$ & $01 / 25 / 99$ & Used hand-held submersible pump \\
\hline
\end{tabular}




\section{NOTES}




\section{Analytical Data Review}

The SRS Groundwater Monitoring Program evaluates all data systematically to provide high-quality data for reporting on the environmental monitoring and cleanup efforts at SRS. Data verification and validation are continuous, interactive processes, usually completed within 60 days after the last data are received for a quarter.

$E X, G E$, and WA, the primary contracting laboratories for sample analyses, performed all analyses with the following exceptions:

- The EM Lab at SRS conducted total-activity analyses of samples for shipping clearance. The EM Lab also conducted tritium analyses of samples from specified well series. Data from the EM Lab for first quarter 1999 were not available in time for publication in this report.

- GE subcontracted radionuclide analyses to GP, and WA subcontracted radionuclide analyses to TM. GP and TM conducted gross alpha, nonvolatile beta, tritium, and selected radionuclide analyses.

\section{GIMS DATA REVIEW MODULE}

The Geochemical Information Management System (GIMS) is a combination of hardware, software, data, and procedures that supports EPD/EMS' data management activities. The GIMS Data Review Module provides automated data loading, validation and verification functions, data editing, determination of data review status, report generation, and data review QA. The data editing program allows users to correct errors in loaded analytical, field, and shipping data. When the review process is complete, data are loaded into the permanent production database tables in GIMS and are available sitewide.

\section{REVIEW OF THE ANALYTICAL DATA}

EPD/EMS reviews analytical data from the laboratories for errors and unusual results before releasing the data for use. The laboratories are asked to review and comment on suspect data.

Typical errors identified during data loading into GIMS include incorrect sample dates, run dates, and sample identifications; incorrectly entered analytical units, methods, and corresponding detection limits; and incorrect dilution factor calculations.

Analytical results that appear different from historical data collected since 1991 are brought to the attention of the appropriate laboratory. Thus, the laboratory is able to identify problems with some of the analyses, including incorrect dilution factor calculations and data entry errors. EPD/EMS corrects data files after receiving written notification from the laboratory. Specific details concerning the corrections are entered in the EMS Groundwater Monitoring Program Changes to the Database Logbook.

Samples that exceeded holding times are indicated by an EPA STORET code $Q$ in the analytical results tables (see Appendix B for further information). The EPA STORET code $V$ is used to indicate sample results associated with method laboratory blanks at the preparation step that are elevated above the instrument detection limit. Samples that were preserved incorrectly are marked with a YEPA STORET code in the analytical results tables (see Appendix B). Usually, the $Y$ indicates that the sample coolers were not cold enough. An EMS code $/$ indicates that a sample's matrix spike recovery was not within control limits.

To determine if a new analytical result for a sampling site is similar to or relatively higher or lower than historical results, new results for each well are compared to its historical results using the following procedure:

- GIMS calculates the mean of the historical results and the mean of the historical results above detection for all analytes in the wells being compared. The historical results that are below their detection limit value are considered at their detection limits for the purpose of the calculation. The process eliminates any false high values due to diluted samples. 
- GIMS factors in trends in the data calculated from the previous eight sampling events. If no previous data are available for a particular well/analyte combination, the program includes previous results from other wells in the same vicinity.

- Results greater than 10 times the calculated mean of the previous results are marked as "high." Results (or their detection limits if the results are below detection) less than 10 percent of the calculated mean of the previous results are marked as "low."

GIMS flags the potentially anomalous results for review. The data reviewer examines the results and takes into account individual historical values, variations of certain values, general trends in the data, and data in the prep batch associated with the current result. The data reviewer eliminates results if anomalous historical results have skewed the calculated mean. Another data reviewer inspects and confirms that the results marked as anomalous are properly identified. Anomalous results are presented to the lab for review and comment. Results significantly high or low compared with historical data are rerun by the lab.

\section{Review of the Analytical Narratives}

EPD/EMS reviews the analytical narratives received from the laboratories, which are used as reference materials throughout the data validation process. Any discrepancies between the narratives and the analytical or chain-of-custody $(\mathrm{COC})$ data must be resolved by the laboratories. The narratives include the following types of problems: QA samples that do not meet the criteria specified by the analytical method, problems with matrix interference, sample-specific adjustments to the method caused by high concentrations of some analytes, problems with sample preservation and holding time, instrument calibration problems, and contaminated blanks. The narratives also include additional information about $\mathrm{COC}$ and analytical data.

The three primary laboratories (EX, GE, and WA) differ in their analytical suite assignments for certain constituents. Thus, some analytes may not be analyzed by all laboratories. See the Sample Scheduling, Field Notes, Quality Control Samples, and Analytical Results sections of this report for more information on wells scheduled but not sampled this quarter.

\section{Review of EX's Analytical Data}

A technical review of the quarter's analytical data identified at least one reported result for each of the analyses in table 4 as high compared with historical data. A review of the laboratory records did not reveal any problems with the analyses.

A technical review of the quarter's analytical data identified no reported results as low compared with historical data.

\section{Review of GE's Analytical Data}

A technical review of the quarter's analytical data identified at least one reported result for each of the analyses in table 5 as high compared with historical data. A review of the laboratory records did not reveal any problems with the analyses.

A technical review of the quarter's analytical data identified at least one reported result for each of the analyses in table 6 as low as compared with historical data. A review of the laboratory records did not reveal any problems.

\section{Review of GP's Analytical Data}

A technical review of the quarter's analytical data identified at least one reported result for each of the analyses in table 7 as high compared with historical data. A review of the laboratory records did not reveal any problems other than those listed below.

A technical review of the quarter's analytical data identified no reported results as low compared with historical data. 


\section{Review of WA's Analytical Data}

A technical review of the quarter's analytical data identified at least one reported result for each of the analyses in table 8 as high as compared with historical data. A review of the laboratory records did not reveal any problems.

A technical review of the quarter's analytical data identified no reported results as low compared with historical data.

\section{Review of TM's Analytical Data}

A technical review of the quarter's analytical data identified at least one reported result for each of the analyses in table 9 as high as compared with historical data. A review of the laboratory records did not reveal any problems.

A technical review of the quarter's analytical data identified no reported results as low compared with historical data.

\section{ANALYTICAL METHODS}

Sample analyses performed for EPD/EMS during first quarter 1999 were conducted using EPA and other methods as noted in tables 10-14 at the end of this section. EX, GE, and WA performed most of the analyses conducted during the quarter. Their methods and estimated quantitation limits (EQLs) are listed in table 10 for EX, table 11 for GE, and table 12 for WA.

GP and TM performed the radionuclide analyses during first quarter 1999. Radionuclide methods generally are modified by the laboratories performing the analyses. Their methods and EQLs are listed in table 13 for GP and table 14 for TM.

The EM Lab conducted selected radionuclide analyses of samples required by the Groundwater Monitoring Program. The total activity method used by the EM Lab is an in-house method based on applicable EPA, DOE, or other procedures. Methods used by EPD/EMS for testing other radioisotopes also are in-house analytical methods. The EM Lab radioactivity determinations are typically reported as the absolute concentrations calculated from the analytical tests. Analytical data from the EM Lab for first quarter 1999 were not available in time for publication of this report.

If the laboratories used more than one analytical method for an analyte, the methods are listed in the tables in descending order according to frequency of use. Generally, the method listed first was used for at least half of the analyses.

Table 4. EX Samples with High Analytical Results as Compared to Historical Data

\begin{tabular}{ll}
\hline Analyte & Well(s) \\
\hline Aluminum & MSB 70Dt \\
Barium & MSB 70D \\
Chloroethane (Vinyl chloride) & LFW 21 \\
Dichloromethane (Methylene chloride) & MSB 26B, MSB 70C \\
Fluoride & MSB 29D \\
Iron & MSB 7A, MSB 70D†, TRW 2 \\
Lead & MSB 6C \\
Nickel & MSB 6C
\end{tabular}




\begin{tabular}{|c|c|}
\hline Analyte & Well(s) \\
\hline $\begin{array}{l}\text { Nitrate as nitrogen } \\
\text { Tetrachloroethylene } \\
\text { Total organic carbon }\end{array}$ & $\begin{array}{l}\text { P 26A, XSB 5A } \\
\text { MSB 10A } \\
\text { AMB 7B }\end{array}$ \\
\hline \multirow{2}{*}{\multicolumn{2}{|c|}{$\begin{array}{l}\text { The questioned value was at least } 10 \text { times higher than historical data. Because holding times had not been e } \\
\text { the laboratory was asked to reanalyze the sample. } \\
\text { Table 5. GE Samples with High Analytical Results as Compared to Historical Data }\end{array}$}} \\
\hline & \\
\hline Analyte & Well(s) \\
\hline $\begin{array}{l}\text { Aluminum } \\
\text { Barium } \\
\text { Chromium } \\
\text { Copper } \\
\text { Dichloromethane (Methylene chloride) } \\
\text { Iron } \\
\text { Mercury } \\
\text { Nitrate-nitrite as nitrogen } \\
\text { Trichloroethylene } \\
\text { Zinc } \\
\end{array}$ & 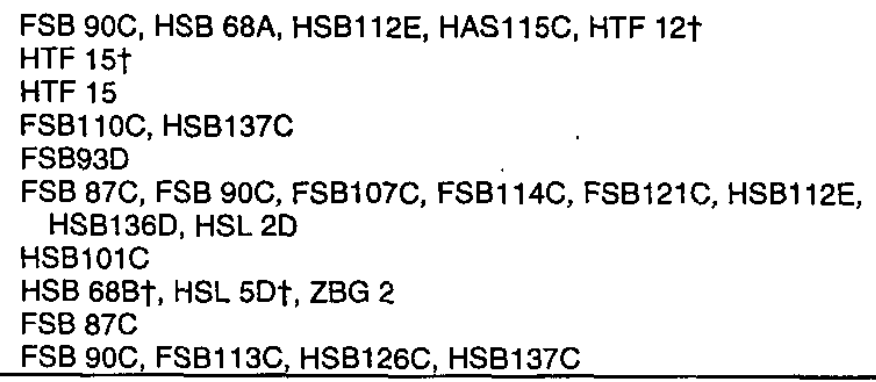 \\
\hline
\end{tabular}

† The questioned value was at least 10 times higher than historical data. Because insufficient sample was left, the laboratory was not asked to reanalyze the sample.

Table 6. GE Samples with Low Analytical Results as Compared to Historical Data

\begin{tabular}{ll}
\hline Analyte & Well(s) \\
\hline Aluminum & HSB117C \\
Nitrate-nitrite as N & FSB102C \\
Specific conductance & HSB 68B \\
\hline
\end{tabular}


Table 7. GP Samples with High Analytical Results as Compared to Historical Data

\begin{tabular}{|c|c|}
\hline Analyte & Well(s) \\
\hline $\begin{array}{l}\text { Americum-241 } \\
\text { Carbon- } 14 \\
\text { Curium } 243 / 244 \\
\text { lodine-129 } \\
\text { Nonvolatile beta } \\
\text { Radium-226 } \\
\text { Strontium-90 } \\
\text { Technetium-99 } \\
\text { Tritium } \\
\text { Uranium-233/234 } \\
\text { Uranium-235 }\end{array}$ & 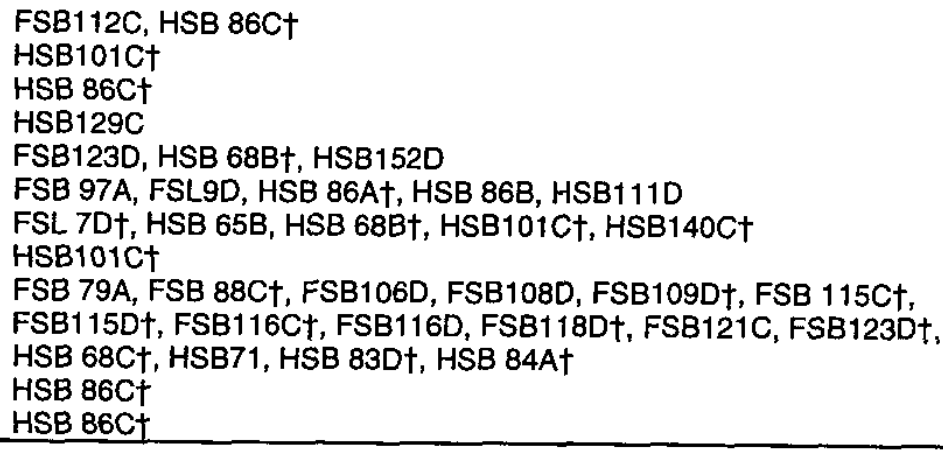 \\
\hline
\end{tabular}

$\dagger$ The questioned value was at least 10 times higher than historical data. Because insufficient sample was left, the laboratory was not asked to reanalyze the sample.

Table 8. WA Samples with High Analytical Results as Compared to Historical Data

\begin{tabular}{ll}
\hline Analyte & Well(s) \\
\hline Aluminum & LAC 2, PSB 7At \\
Barium & ASB 8C \\
Cyanide & ASB 1A \\
Iron & DCB 4A, DCB 8, FET 1D, HWS 1A, LAC 2, PCB 1A \\
Mercury & LFW 34 \\
Total Dissolved Solids & ACB 2A \\
\hline
\end{tabular}

$\dagger$ The questioned value was at least 10 times higher than historical data. Because holding times had been exceeded, the laboratory was asked to reanalyze the sample.

Table 9. TM Samples with High Analytical Results as Compared to Historical Data

\begin{tabular}{ll}
\hline Analyte & Well(s) \\
\hline & \\
Gross Aipha & P 26Dt \\
Radium-228 & HSB100D \\
Tritium & KDB 1, LDB 4, P 29Dt \\
\hline
\end{tabular}

$\dagger$ The questioned value was at least 10 times higher than historical data. Because hoiding times had not been exceeded, the laboratory was asked to reanalyze the sample.

Table 10. Methods and Estimated Quantitation Limits Used by EX

\begin{tabular}{llll}
\hline Analyte & Unit & Method & Minimum/Maximum EQLs \\
\hline & & & \\
Acenaphthene & $\mu g / L$ & EPA8270C & 10.0 \\
Acenaphthylene & $\mu g / L$ & EPA8270C & 10.0 \\
Acetone & $\mu g / L$ & EPA8260B & $10.0 / 50.0$
\end{tabular}




\begin{tabular}{|c|c|c|c|}
\hline Analyte & Unit & Method & Minimum/Maximum EQLs \\
\hline 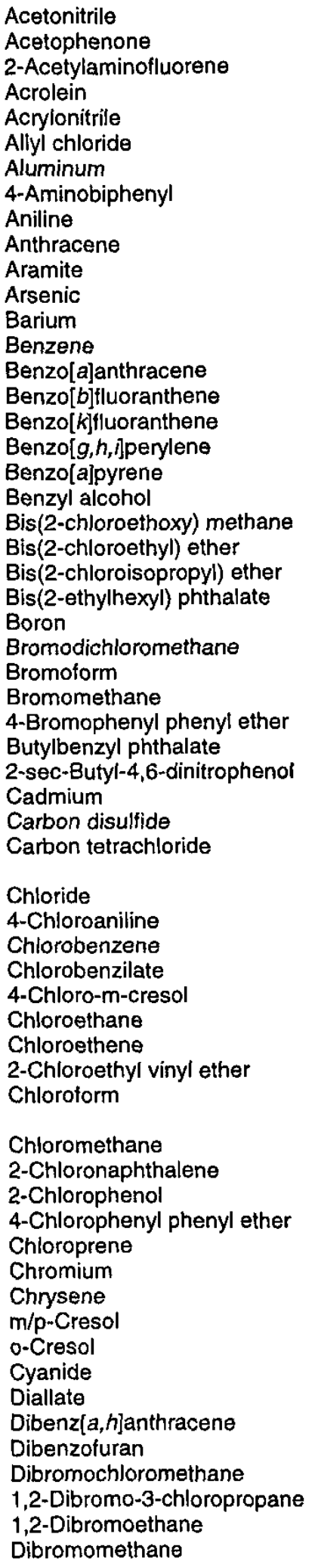 & $\begin{array}{l}\mu g / L \\
\mu g / L \\
\mu g / L \\
\mu g / L \\
\mu g / L \\
\mu g / L \\
\mu g / L \\
\mu g / L \\
\mu g / L \\
\mu g / L \\
\mu g / L \\
\mu g / L \\
\mu g / L \\
\mu g / L \\
\mu g / L \\
\mu g / L \\
\mu g / L \\
\mu g / L \\
\mu g / L \\
\mu g / L \\
\mu g / L \\
\mu g / L \\
\mu g / L \\
\mu g / L \\
\mu g / L \\
\mu g / L \\
\mu g / L \\
\mu g / L \\
\mu g / L \\
\mu g / L \\
\mu g / L \\
\mu g / L \\
\mu g / L \\
\mu g / L \\
\mu g / L \\
\mu g / L \\
\mu g / L \\
\mu g / L \\
\mu g / L \\
\mu g / L \\
\mu g / L \\
\mu g / L \\
\mu g / L \\
\mu g / L \\
\mu g / L \\
\mu g / L \\
\mu g / L \\
\mu g / L \\
\mu g / L \\
\mu g / L \\
\mu g / L \\
\mu g / L \\
\mu g / L \\
\mu g / L \\
\mu g / L \\
\mu g / L \\
\mu g / L \\
\mu g / L \\
\mu g / L \\
\mu g / L \\
\mu g / L \\
\end{array}$ & $\begin{array}{l}\text { EPA8260B } \\
\text { EPA8270C } \\
\text { EPA8270C } \\
\text { EPA8260B } \\
\text { EPA8260B } \\
\text { EPA8260B } \\
\text { EPA6010B } \\
\text { EPA8270C } \\
\text { EPA8270C } \\
\text { EPA8270C } \\
\text { EPA8270C } \\
\text { EPA6010B } \\
\text { EPA6010B } \\
\text { EPA8260B } \\
\text { EPA8270C } \\
\text { EPA8270C } \\
\text { EPA8270C } \\
\text { EPA8270C } \\
\text { EPA8270C } \\
\text { EPA8270C } \\
\text { EPA8270C } \\
\text { EPA8270C } \\
\text { EPA8270C } \\
\text { EPA8270C } \\
\text { EPA6010B } \\
\text { EPA8260B } \\
\text { EPA8260B } \\
\text { EPA8260B } \\
\text { EPA8270C } \\
\text { EPA8270C } \\
\text { EPA8270C } \\
\text { EPA6010B } \\
\text { EPA8260B } \\
\text { EPA8260B } \\
\text { EPA8021B } \\
\text { EPA300.0 } \\
\text { EPA8270C } \\
\text { EPA8260B } \\
\text { EPA8270C } \\
\text { EPA8270C } \\
\text { EPA88270 } \\
\text { EPA8260B } \\
\text { EPA8260B } \\
\text { EPA8260B } \\
\text { EPA8260B } \\
\text { EPA8270C } \\
\text { EPA98014 } \\
\text { EPA8270C } \\
\text { EPA8260B } \\
\text { EPA8021B } \\
\text { EPA8260B } \\
\text { EPA8270C } \\
\text { EPA270C } \\
\text { EPA260B }\end{array}$ & 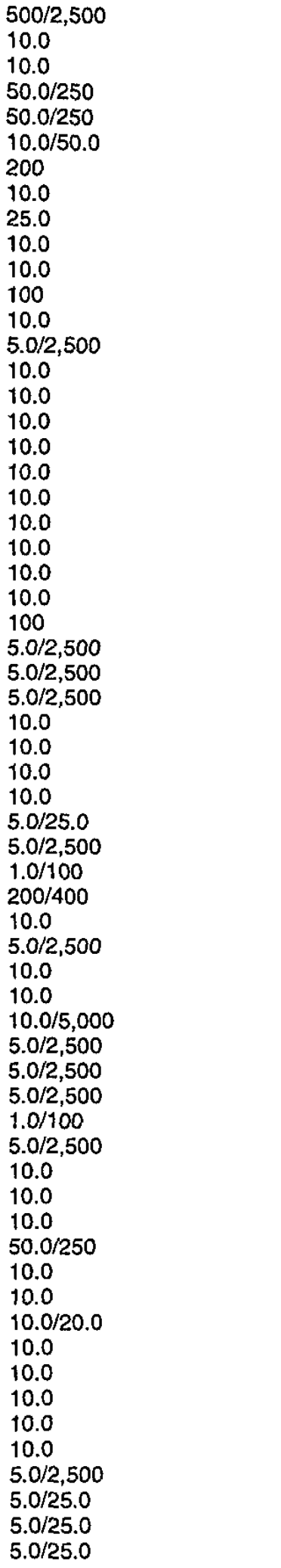 \\
\hline
\end{tabular}




\begin{tabular}{|c|c|c|c|}
\hline Analyte & Unit & Method & MInimum/Maximum EQLs \\
\hline 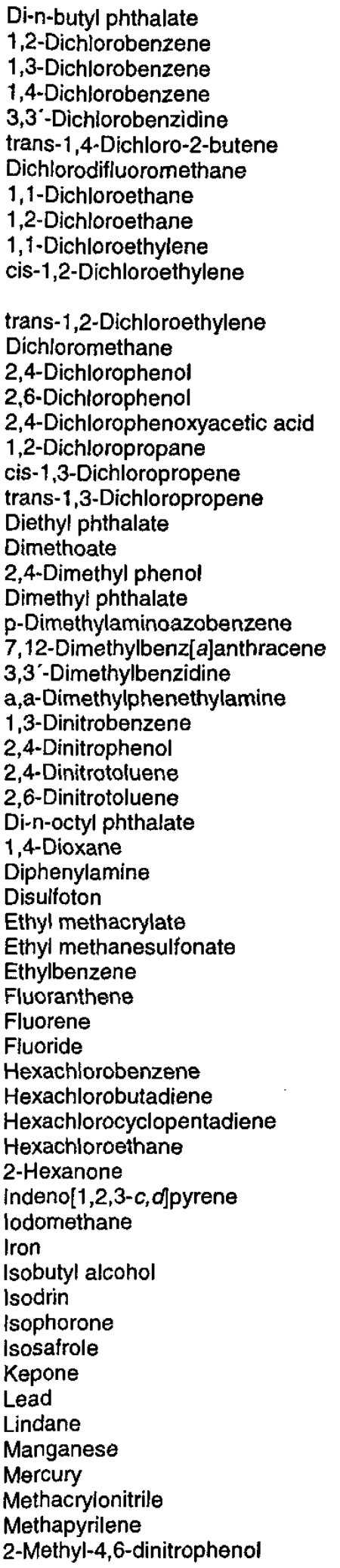 & $\begin{array}{l}\mu g / L \\
\mu g / L \\
\mu g / L \\
\mu g / L \\
\mu g / L \\
\mu g / L \\
\mu g / L \\
\mu g / L \\
\mu g / L \\
\mu g / L \\
\mu g / L \\
\mu g / L \\
\mu g / L \\
\mu g / L \\
\mu g / L \\
\mu g / L \\
\mu g / L \\
\mu g / L \\
\mu g / L \\
\mu g / L \\
\mu g / L \\
\mu g / L \\
\mu g / L \\
\mu g / L \\
\mu g / L \\
\mu g / L \\
\mu g / L \\
\mu g / L \\
\mu g / L \\
\mu g / L \\
\mu g / L \\
\mu g / L \\
\mu g / L \\
\mu g / L \\
\mu g / L \\
\mu g / L \\
\mu g / L \\
\mu g / L \\
\mu g / L \\
\mu g / L \\
\mu g / L \\
\mu g / L \\
\mu g / L \\
\mu g / L \\
\mu g / L \\
\mu g / L \\
\mu g / L \\
\mu g / L \\
\mu g / L \\
\mu g / L \\
\mu g / L \\
\mu g / L \\
\mu g / L \\
\mu g / L \\
\mu g / L \\
\mu g / L \\
\mu g / L \\
\mu g / L \\
\mu g / L \\
\mu g / L \\
\mu g / L\end{array}$ & 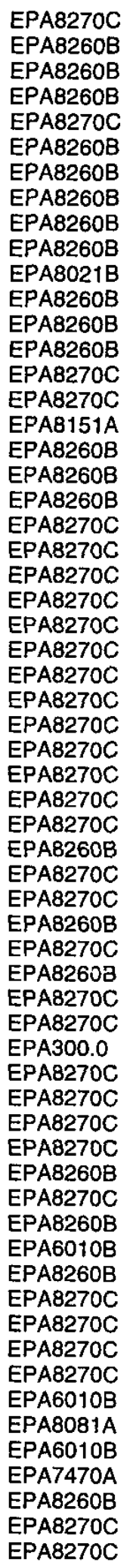 & 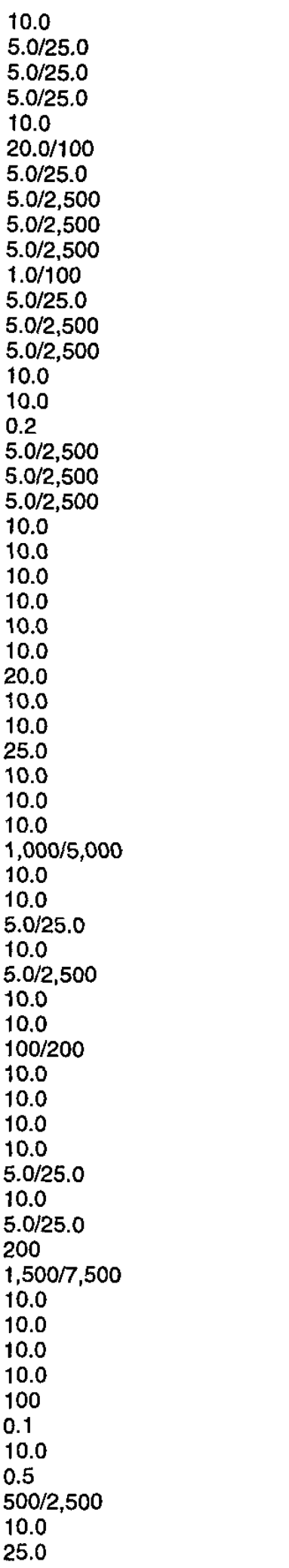 \\
\hline
\end{tabular}




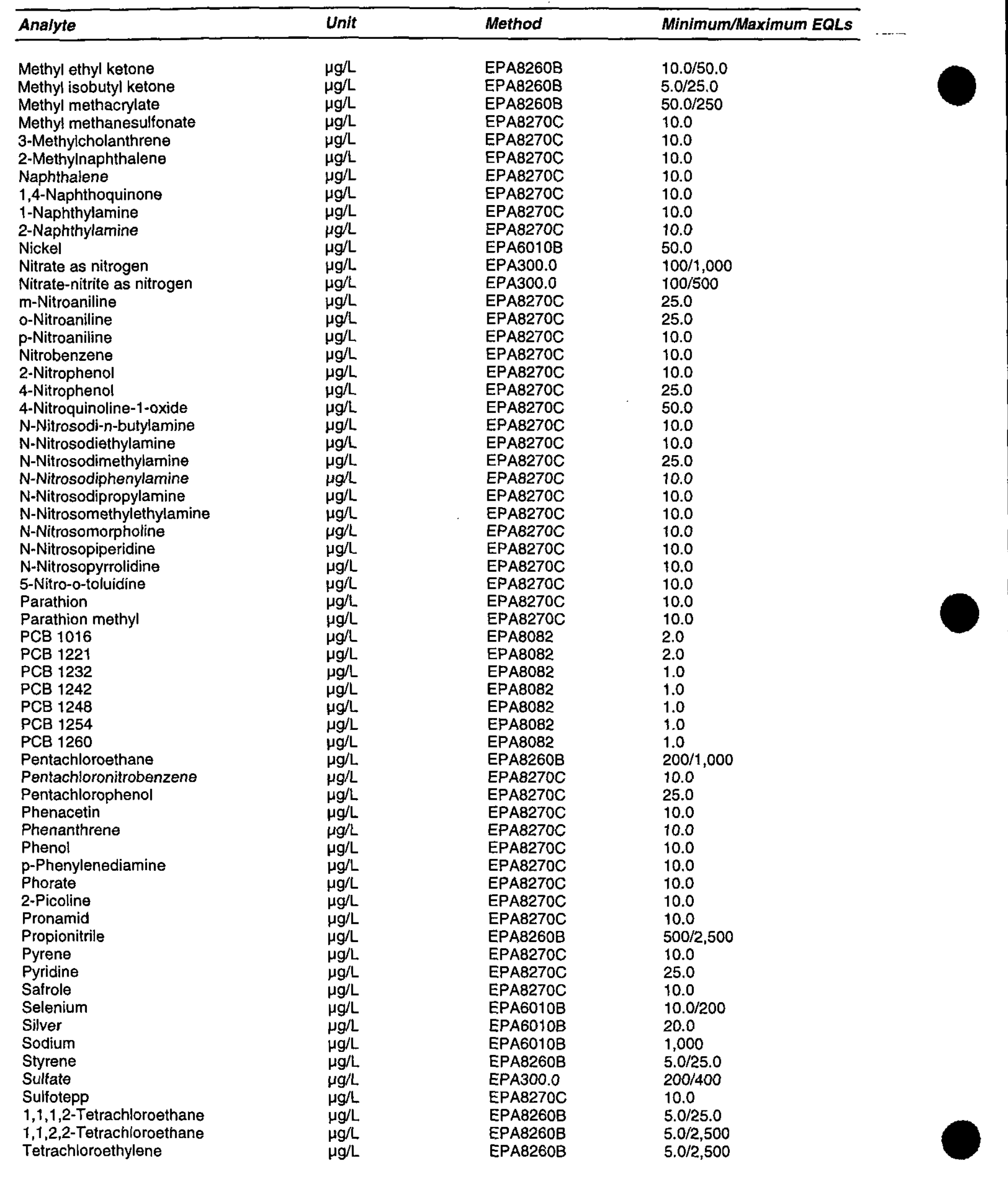




\begin{tabular}{|c|c|c|c|}
\hline Analyte & Unit & Method & Minimum/Maximum EQLs \\
\hline $\begin{array}{l}\text { 2,3,4,6-Tetrachlorophenol } \\
\text { Thallium } \\
\text { Thionazin } \\
\text { Toluene } \\
\text { o-Toluidine } \\
\text { Total organic carbon } \\
\text { Total phosphates (as P) } \\
\text { 1,2,4-Trichlorobenzene } \\
\text { 1,1,1-Trichloroethane } \\
\text { 1,1,2-Trichloroethane } \\
\text { Trichloroethylene } \\
\text { Trichlorofluoromethane } \\
\text { 2,4,5-Trichlorophenol } \\
\text { 2,4,6-Trichlorophenol } \\
\text { 1,2,3-Trichloropropane } \\
\text { O,O,O-Triethyl phosphorothioate } \\
1,3,5-\text { Trinitrobenzene } \\
\text { Vanadium } \\
\text { Vinyl acetate } \\
\text { Xylenes }\end{array}$ & $\begin{array}{l}\mu g / L \\
\mu g / L \\
\mu g / L \\
\mu g / L \\
\mu g / L \\
\mu g / L \\
\mu g / L \\
\mu g / L \\
\mu g / L \\
\mu g / L \\
\mu g / L \\
\mu g / L \\
\mu g / L \\
\mu g / L \\
\mu g / L \\
\mu g / L \\
\mu g / L \\
\mu g / L \\
\mu g / L \\
\mu g / L \\
\mu g / L \\
\mu g / L \\
\mu g / L \\
\mu g / L\end{array}$ & $\begin{array}{l}\text { EPA8021B } \\
\text { EPA8270C } \\
\text { EPA7841 } \\
\text { EPA8270C } \\
\text { EPA8260B } \\
\text { EPA8270C } \\
\text { AP-3086 } \\
\text { EPA9060 } \\
\text { EPA300.0 } \\
\text { EPA8270C } \\
\text { EPA8260B } \\
\text { EPA8021B } \\
\text { EPA8260B } \\
\text { EPA8260B } \\
\text { EPA8021B } \\
\text { EPA8260B } \\
\text { EPA8270C } \\
\text { EPA8270C } \\
\text { EPA8260B } \\
\text { EPA8270C } \\
\text { EPA8270C } \\
\text { EPA6010B } \\
\text { EPA8260B } \\
\text { EPA8260B }\end{array}$ & $\begin{array}{l}1.0 / 100 \\
10.0 \\
2.0 \\
10.0 \\
5.0 / 2,500 \\
10.0 \\
5,000 \\
5,000 \\
500 / 2,500 \\
10.0 \\
5.0 / 2,500 \\
1.0 / 100 \\
5.0 / 2,500 \\
5.0 / 2,500 \\
1.0 / 100 \\
5.0 / 2,500 \\
10.0 \\
25.0 \\
5.0 / 25.0 \\
10.0 \\
10.0 \\
10.0 \\
20.0 / 100 \\
10.0 / 50.0 \\
\end{array}$ \\
\hline
\end{tabular}

Table 11. Methods and Estimated Quantitation Limits Used by GE

\begin{tabular}{|c|c|c|c|}
\hline Analyte & Unit & Method & Minimum/Maximum EQLs \\
\hline $\begin{array}{l}\text { Acenaphthene } \\
\text { Acenaphthylene } \\
\text { Acetone } \\
\text { Acetonitrile } \\
\text { Acrolein } \\
\text { Acrylonitrile } \\
\text { Aldrin } \\
\text { Allyl chloride } \\
\text { Aluminum } \\
\text { Anthracene } \\
\text { Antimony } \\
\text { Arsenic } \\
\text { Barium } \\
\text { Benzene } \\
\text { alpha-Benzene hexachloride } \\
\text { beta-Benzene hexachloride } \\
\text { deita-Benzene hexachloride } \\
\text { Benzidine } \\
\text { Benzo[a]anthracene } \\
\text { Benzo[b]fluoranthene } \\
\text { Benzo[ } k \text { ]fluoranthene } \\
\text { Benzoic acid } \\
\text { Benzo[g, } h \text {,i]perylene } \\
\text { Benzo[a]pyrene } \\
\text { Benzyl alcohol }\end{array}$ & $\begin{array}{l}\mu g / L \\
\mu g / L \\
\mu g / L \\
\mu g / L \\
\mu g / L \\
\mu g / L \\
\mu g / L \\
\mu g / L \\
\mu g / L \\
\mu g / L \\
\mu g / L \\
\mu g / L \\
\mu g / L \\
\mu g / L \\
\mu g / L \\
\mu g / L \\
\mu g / L \\
\mu g / L \\
\mu g / L \\
\mu g / L \\
\mu g / L \\
\mu g / L \\
\mu g / L \\
\mu g / L \\
\mu g / L \\
\mu g / L \\
\mu g / L \\
\mu g / L \\
\mu g / L\end{array}$ & $\begin{array}{l}\text { EPA8270C } \\
\text { EPA8270C } \\
\text { EPA8260B } \\
\text { EPA8260B } \\
\text { EPA8260B } \\
\text { EPA8260B } \\
\text { EPA8081A } \\
\text { EPA8260B } \\
\text { EPA6020 } \\
\text { EPA6010B } \\
\text { EPA8270C } \\
\text { EPA6020 } \\
\text { EPA6010B } \\
\text { EPA6020 } \\
\text { EPA6010B } \\
\text { EPA6020 } \\
\text { EPA6010B } \\
\text { EPA8260B } \\
\text { EPA8081A } \\
\text { EPA8081A } \\
\text { EPA8081A } \\
\text { EPA8270C } \\
\text { EPA8270C } \\
\text { EPA8270C } \\
\text { EPA8270C } \\
\text { EPA8270C } \\
\text { EPA8270C } \\
\text { EPA8270C } \\
\text { EPA8270C }\end{array}$ & $\begin{array}{l}10.0 \\
10.0 \\
5.0 \\
25.0 \\
10.0 \\
10.0 \\
0.0198 / 0.02 \\
5.0 \\
10.0 / 300 \\
50.0 \\
10.0 \\
0.2 / 2.0 \\
10.0 \\
3.0 / 60.0 \\
5.0 \\
2.0 \\
5.0 \\
1.0 / 25.0 \\
0.0198 / 0.02 \\
0.0198 / 0.02 \\
0.0198 / 0.02 \\
50.0 \\
10.0 \\
10.0 \\
10.0 \\
20.0 \\
10.0 \\
10.0 \\
10.0\end{array}$ \\
\hline
\end{tabular}




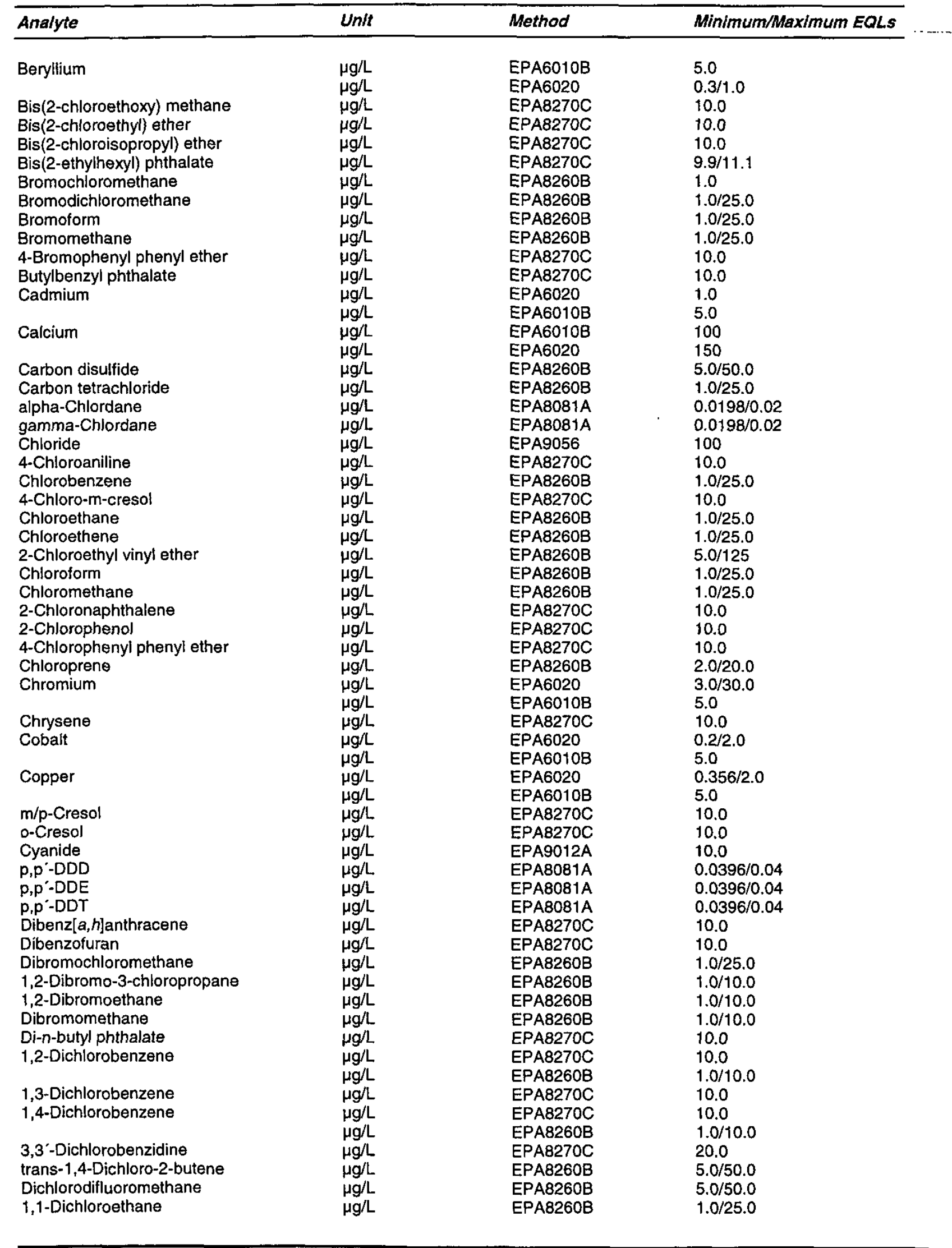




\begin{tabular}{|c|c|c|c|}
\hline Analyte & Unit & Method & Minimum/Maximum EQLs \\
\hline 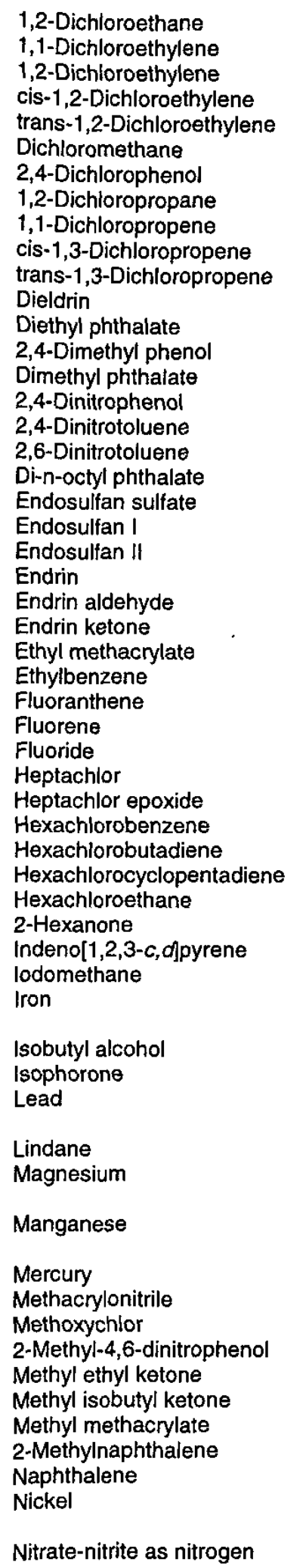 & $\begin{array}{l}\mu g / L \\
\mu g / L \\
\mu g / L \\
\mu g / L \\
\mu g / L \\
\mu g / L \\
\mu g / L \\
\mu g / L \\
\mu g / L \\
\mu g / L \\
\mu g / L \\
\mu g / L \\
\mu g / L \\
\mu g / L \\
\mu g / L \\
\mu g / L \\
\mu g / L \\
\mu g / L \\
\mu g / L \\
\mu g / L \\
\mu g / L \\
\mu g / L \\
\mu g / L \\
\mu g / L \\
\mu g / L \\
\mu g / L \\
\mu g / L \\
\mu g / L \\
\mu g / L \\
\mu g / L \\
\mu g / L \\
\mu g / L \\
\mu g / L \\
\mu g / L \\
\mu g / L \\
\mu g / L \\
\mu g / L \\
\mu g / L \\
\mu g / L \\
\mu g / L \\
\mu g / L \\
\mu g / L \\
\mu g / L \\
\mu g / L \\
\mu g / L \\
\mu g / L \\
\mu g / L \\
\mu g / L \\
\mu g / L \\
\mu g / L \\
\mu g / L \\
\mu g / L \\
\mu g / L \\
\mu g / L \\
\mu g / L \\
\mu g / L \\
\mu g / L \\
\mu g / L \\
\mu g / L \\
\end{array}$ & $\begin{array}{l}\text { EPA8260B } \\
\text { EPA8260B } \\
\text { EPA8260B } \\
\text { EPA8260B } \\
\text { EPA8260B } \\
\text { EPA8260B } \\
\text { EPA8270C } \\
\text { EPA8260B } \\
\text { EPA8260B } \\
\text { EPA8260B } \\
\text { EPA8260B } \\
\text { EPA8081A } \\
\text { EPA8270C } \\
\text { EPA8270C } \\
\text { EPA8270C } \\
\text { EPA8270C } \\
\text { EPA8270C } \\
\text { EPA8270C } \\
\text { EPA8270C } \\
\text { EPA8081A } \\
\text { EPA8081A } \\
\text { EPA8081A } \\
\text { EPA8081A } \\
\text { EPA8081A } \\
\text { EPA80B1A } \\
\text { EPA8260B } \\
\text { EPA8260B } \\
\text { EPA8270C } \\
\text { EPA8270C } \\
\text { EPA9056 } \\
\text { EPA8081A } \\
\text { EPA8081A } \\
\text { EPA8270C } \\
\text { EPA8270C } \\
\text { EPA8270C } \\
\text { EPA8270C } \\
\text { EPA8260B } \\
\text { EPA8270C } \\
\text { EPA8260B } \\
\text { EPA6020 } \\
\text { EPA8270C } \\
\text { EPA6020 } \\
\text { EPA6010B } \\
\text { EPA353.1 }\end{array}$ & $\begin{array}{l}1.0 / 25.0 \\
1.0 / 100 \\
1.0 \\
1.17 / 11.7 \\
1.0 / 25.0 \\
5.0 / 50.0 \\
10.0 \\
1.0 / 25.0 \\
1.0 \\
1.0 / 25.0 \\
1.0 / 25.0 \\
0.0396 / 0.04 \\
10.0 \\
10.0 \\
10.0 \\
20.0 \\
10.0 \\
10.0 \\
10.0 \\
0.0396 / 0.04 \\
0.0198 / 0.02 \\
0.0396 / 0.04 \\
0.0396 / 0.04 \\
0.0396 / 0.04 \\
0.0396 / 0.04 \\
5.0 / 50.0 \\
1.0 / 25.0 \\
10.0 \\
10.0 \\
50.0 \\
0.0198 / 0.02 \\
0.0198 / 0.02 \\
10.0 \\
10.0 \\
10.0 \\
10.0 \\
5.0 / 50.0 \\
10.0 \\
10.0 / 100 \\
15.0 / 25.0 \\
50.0 \\
50.0 / 500 \\
10.0 \\
2.0 / 40.0 \\
5.0 \\
0.0198 / 0.02 \\
10.0 \\
3.0 \\
10.0 \\
0.2 \\
0.2 / 0.8 \\
5.0 / 50.0 \\
0.198 / 0.2 \\
10.0 \\
10.0 / 100 \\
5.0 / 50.0 \\
5.0 / 50.0 \\
10.0 \\
10.0 \\
2.0 / 20.0 \\
5.0 \\
50.0 / 10,000\end{array}$ \\
\hline
\end{tabular}




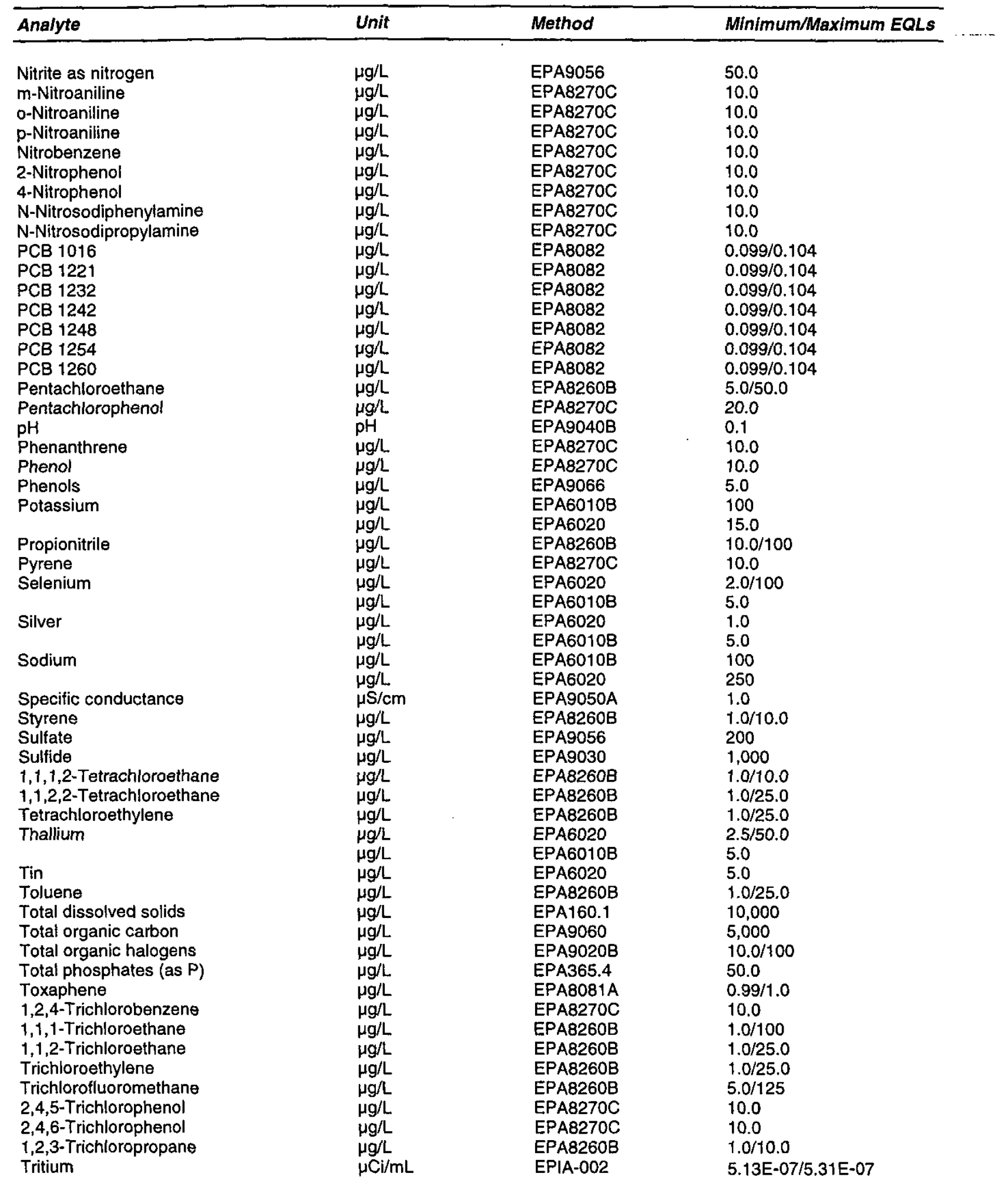




\begin{tabular}{llll}
\hline Analyte & Unit & Method & Minimum/Maximum EQLs \\
\hline \multirow{2}{*}{ Vanadium } & & & \\
& $\mu \mathrm{g} / \mathrm{L}$ & EPA6020 & $4.08 / 100$ \\
Vinyl acetate & $\mu \mathrm{g} / \mathrm{L}$ & EPA6010B & 5.0 \\
Xylenes & $\mu \mathrm{g} / \mathrm{L}$ & EPA8260B & $5.0 / 50.0$ \\
Zinc & $\mu \mathrm{g} / \mathrm{L}$ & EPA8260B & $2.0 / 20.0$ \\
& $\mu \mathrm{g} / \mathrm{L}$ & EPA6020 & 10.0 \\
\hline
\end{tabular}

Note: The groundwater samples are unfiltered; thus, the methods for metals are for total recoverable metals. Method 6010 is an inductively coupled plasma atomic emission spectroscopy method for metals determination and is published for RCRA determinations.

Tablo 12. Methods and Estimated Quantitation Limits Used by WA

\begin{tabular}{|c|c|c|c|}
\hline Analyte & Unit & Method & Minimum/Maximum EQLs \\
\hline 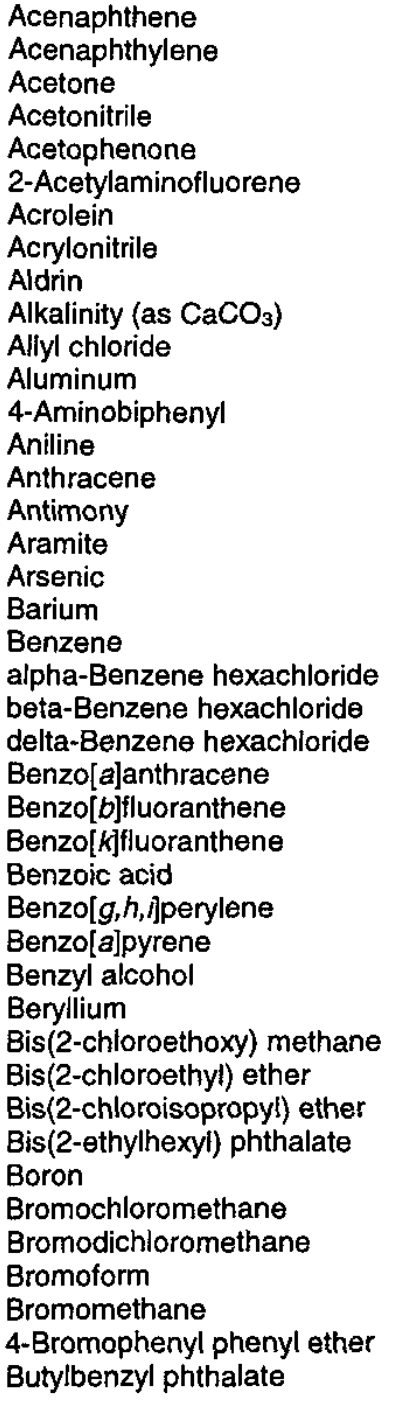 & $\begin{array}{l}\mu g / L \\
\mu g / L \\
\mu g / L \\
\mu g / L \\
\mu g / L \\
\mu g / L \\
\mu g / L \\
\mu g / L \\
\mu g / L \\
\mathrm{meq} / L \\
\mu g / L \\
\mu g / L \\
\mu g / L \\
\mu g / L \\
\mu g / L \\
\mu g / L \\
\mu g / L \\
\mu g / L \\
\mu g / L \\
\mu g / L \\
\mu g / L \\
\mu g / L \\
\mu g / L \\
\mu g / L \\
\mu g / L \\
\mu g / L \\
\mu g / L \\
\mu g / L \\
\mu g / L \\
\mu g / L \\
\mu g / L \\
\mu g / L \\
\mu g / L \\
\mu g / L \\
\mu g / L \\
\mu g / L \\
\mu g / L \\
\mu g / L \\
\mu g / L \\
\mu g / L \\
\mu g / L \\
\mu g / L\end{array}$ & $\begin{array}{l}\text { EPA8270C } \\
\text { EPA8270C } \\
\text { EPA8260B } \\
\text { EPA8260B } \\
\text { EPA8270C } \\
\text { EPA8270C } \\
\text { EPA8260B } \\
\text { EPA8260B } \\
\text { EPA8081A } \\
\text { EPA310.1 } \\
\text { EPA8260B } \\
\text { EPA6010B } \\
\text { EPA8270C } \\
\text { EPA8270C } \\
\text { EPA8270C } \\
\text { EPA6010B } \\
\text { EPA8270C } \\
\text { EPA6010B } \\
\text { EPA6010B } \\
\text { EPA8260B } \\
\text { EPA8081A } \\
\text { EPA8081A } \\
\text { EPA8081A } \\
\text { EPA8270C } \\
\text { EPA8270C } \\
\text { EPA8270C } \\
\text { EPA8270C } \\
\text { EPA8270C } \\
\text { EPA8270C } \\
\text { EPA8270C } \\
\text { EPA6010B } \\
\text { EPA8270C } \\
\text { EPA8270C } \\
\text { EPA8270C } \\
\text { EPA8270C } \\
\text { EPA6010B } \\
\text { EPA8260B } \\
\text { EPA8260B } \\
\text { EPA8260B } \\
\text { EPA8260B } \\
\text { EPA8270C } \\
\text { EPA8270C }\end{array}$ & $\begin{array}{l}10.0 / 22.0 \\
10.0 / 22.0 \\
10.0 / 50.0 \\
20.0 / 100 \\
10.0 / 22.0 \\
10.0 / 22.0 \\
20.0 / 100 \\
5.0 / 25.0 \\
0.05 / 0.1 \\
6,700 / 13,400 \\
10.0 / 50.0 \\
146 \\
10.0 / 22.0 \\
10.0 / 22.0 \\
10.0 / 22.0 \\
27.0 \\
20.0 / 22.0 \\
40.0 \\
1.8 \\
5.0 / 1,000 \\
0.05 / 0.106 \\
0.05 / 0.116 \\
0.05 / 0.106 \\
10.0 / 22.0 \\
10.0 / 22.0 \\
10.0 / 22.0 \\
25.0 / 55.0 \\
10.0 / 22.0 \\
10.0 / 22.0 \\
10.0 / 22.0 \\
1.6 \\
10.0 / 22.0 \\
10.0 / 22.0 \\
10.0 / 22.0 \\
10.0 / 22.0 \\
266 \\
5.0 \\
5.0 / 1,000 \\
5.0 / 1,000 \\
10.0 / 2,000 \\
10.0 / 22.0 \\
10.0 / 22.0 \\
10 \\
10 \\
10 \\
10\end{array}$ \\
\hline
\end{tabular}




\begin{tabular}{|c|c|c|c|}
\hline Analyte & Unit & Method & Minimum/Maximum EQLs \\
\hline 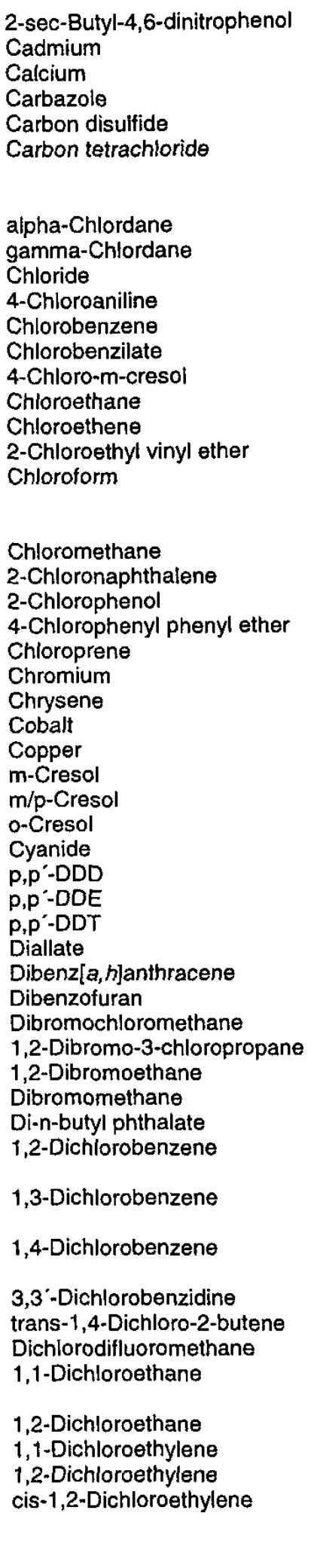 & $\begin{array}{l}\mu g / L \\
\mu g / L \\
\mu g / L \\
\mu g / L \\
\mu g / L \\
\mu g / L \\
\mu g / L \\
\mu g / L \\
\mu g / L \\
\mu g / L \\
\mu g / L \\
\mu g / L \\
\mu g / L \\
\mu g / L \\
\mu g / L \\
\mu g / L \\
\mu g / L \\
\mu g / L \\
\mu g / L \\
\mu g / L \\
\mu g / L \\
\mu g / L \\
\mu g / L \\
\mu g / L \\
\mu g / L \\
\mu g / L \\
\mu g / L \\
\mu g / L \\
\mu g / L \\
\mu g / L \\
\mu g / L \\
\mu g / L \\
\mu g / L \\
\mu g / L \\
\mu g / L \\
\mu g / L \\
\mu g / L \\
\mu g / L \\
\mu g / L \\
\mu g / L \\
\mu g / L \\
\mu g / L \\
\mu g / L \\
\mu g / L \\
\mu g / L \\
\mu g / L \\
\mu g / L \\
\mu g / L \\
\mu g / L \\
\mu g / L \\
\mu g / L \\
\mu g / L \\
\mu g / L \\
\mu g / L \\
\mu g / L \\
\mu g / L \\
\mu g / L \\
\mu g / L \\
\mu g / L \\
\mu g / L \\
\mu g / L\end{array}$ & $\begin{array}{l}\text { EPA8270C } \\
\text { EPA6010B } \\
\text { EPA6010B } \\
\text { EPA8270C } \\
\text { EPA8260B } \\
\text { EPA8260B } \\
\text { EPA8010 } \\
\text { EPA8021B } \\
\text { EPA8081A } \\
\text { EPA8081A } \\
\text { EPA9056 } \\
\text { EPA8270C } \\
\text { EPA8260B } \\
\text { EPA8270C } \\
\text { EPA8270C } \\
\text { EPA8260B } \\
\text { EPA8260B } \\
\text { EPA8260B } \\
\text { EPA8260B } \\
\text { EPA8010 } \\
\text { EPA8021B } \\
\text { EPA8260B } \\
\text { EPA8270C } \\
\text { EPA8270C } \\
\text { EPA8270C } \\
\text { EPA8260B } \\
\text { EPA6010B } \\
\text { EPA8270C } \\
\text { EPA6010B } \\
\text { EPA6010B } \\
\text { EPA8270C } \\
\text { EPA8270C } \\
\text { EPA8270C } \\
\text { EPA9014 } \\
\text { EPA8081A } \\
\text { EPA8081A } \\
\text { EPA8081A } \\
\text { EPA888260B } \\
\text { EPA8 } \\
\text { EPA8260B } \\
\text { EPA8260B } \\
\text { EPA8010 } \\
\text { EPA8270C } \\
\text { EPA8270C } \\
\text { EPA8270 } \\
\text { EPA8270C } \\
\text { EPA8260B } \\
\text { EPA8260B } \\
\text { EPA8260B } \\
\text { EPA8260B } \\
\text { EPA8270C } \\
\text { EPA }\end{array}$ & 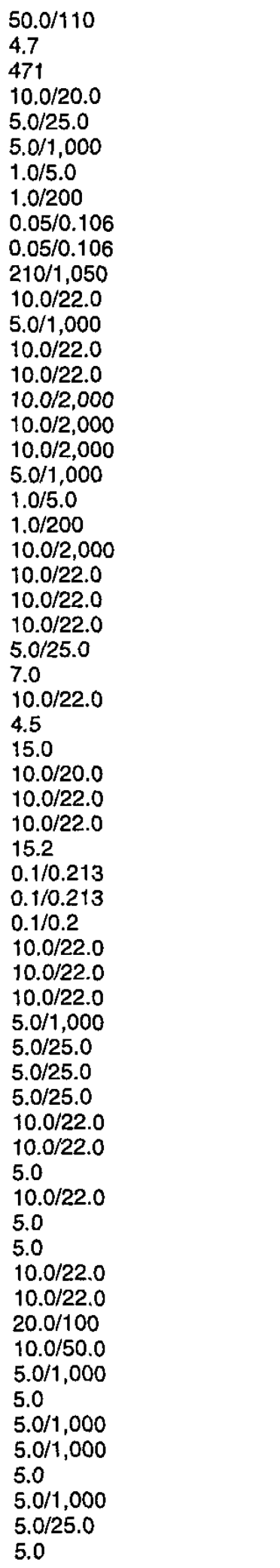 \\
\hline
\end{tabular}




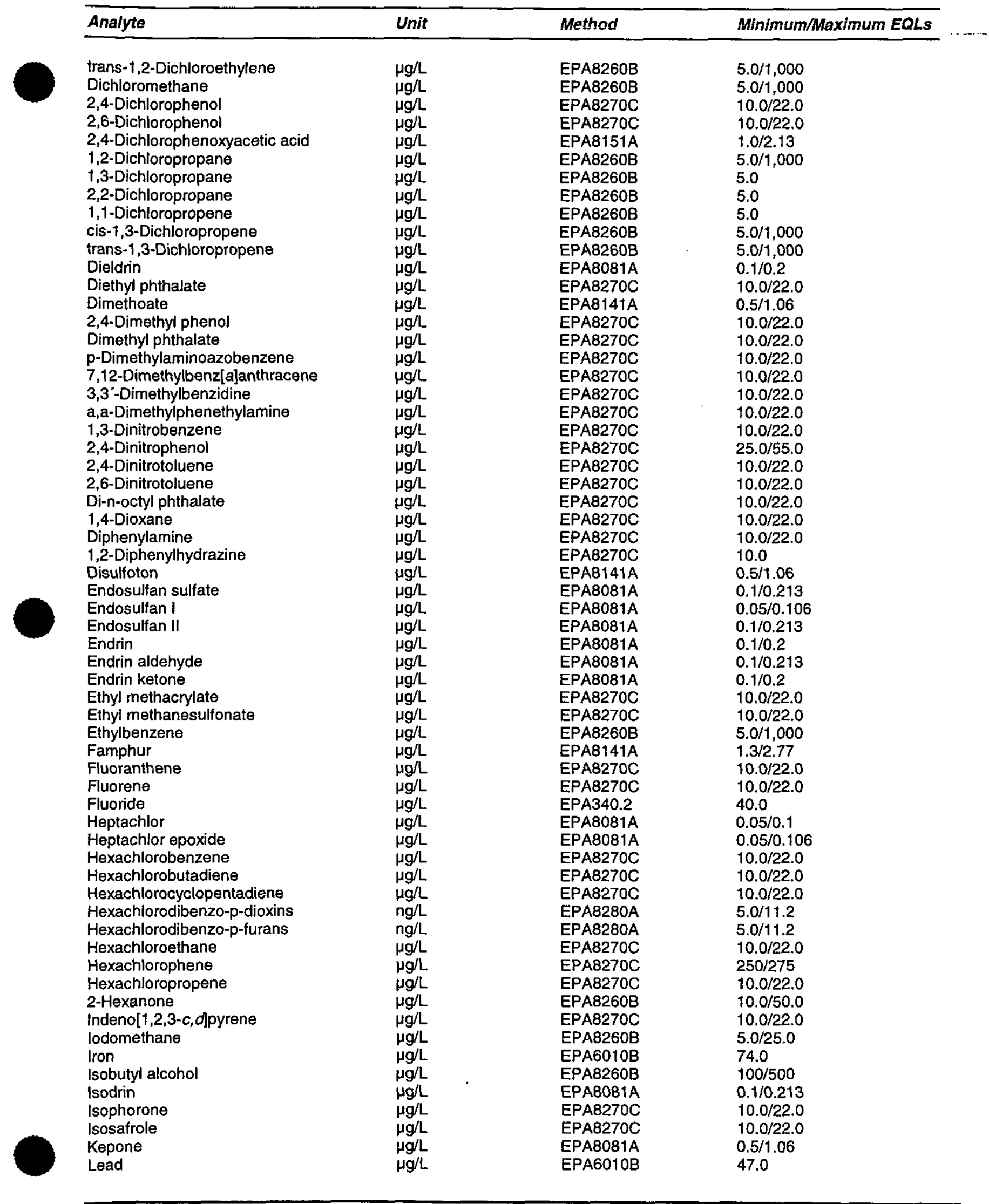




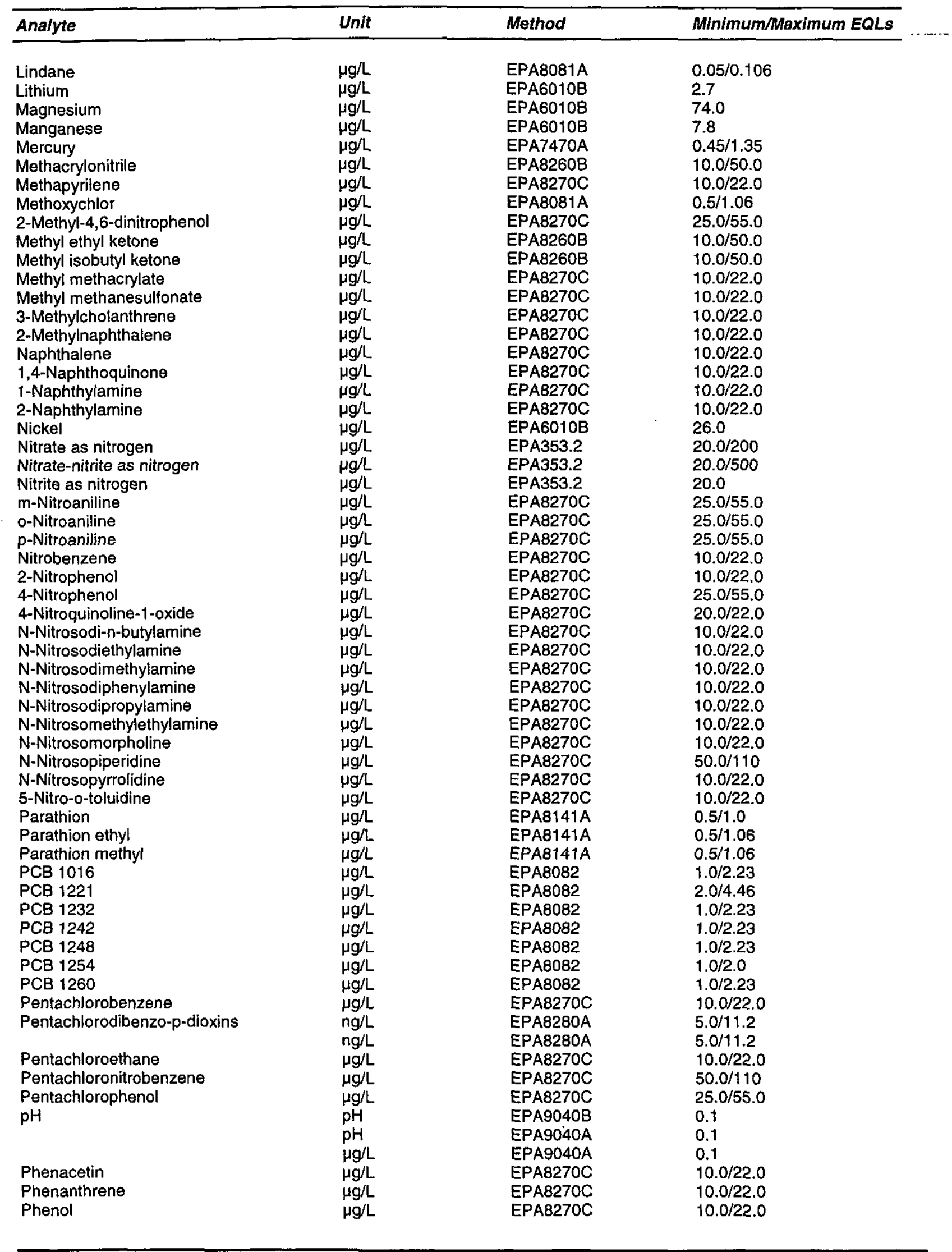




\begin{tabular}{|c|c|c|c|}
\hline Analyte & Unit & Method & Minimum/Maximum EQLs \\
\hline 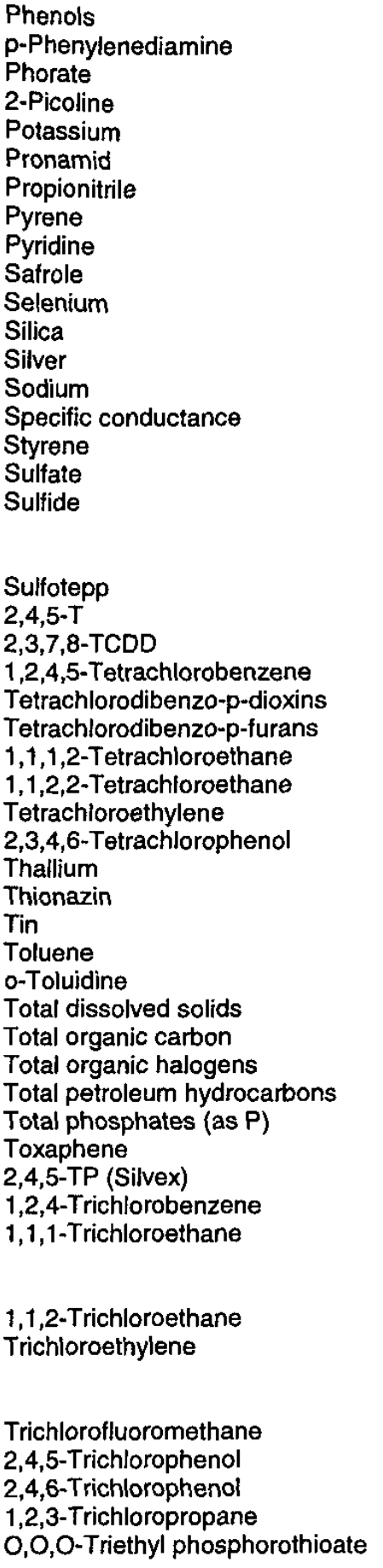 & $\begin{array}{l}\mu g / L \\
\mu g / L \\
\mu g / L \\
\mu g / L \\
\mu g / L \\
\mu g / L \\
\mu g / L \\
\mu g / L \\
\mu g / L \\
\mu g / L \\
\mu g / L \\
\mu g / L \\
\mu g / L \\
\mu g / L \\
\mu S / C m \\
\mu g / L \\
\mu g / L \\
\mu g / L \\
\mu g / L \\
\mu g / L \\
\mu g / L \\
\mu g / L \\
n g / L \\
\mu g / L \\
n g / L \\
n g / L \\
\mu g / L \\
\mu g / L \\
\mu g / L \\
\mu g / L \\
\mu g / L \\
\mu g / L \\
\mu g / L \\
\mu g / L \\
\mu g / L \\
\mu g / L \\
\mu g / L \\
\mu g / L \\
\mu g / L \\
\mu g / L \\
\mu g / L \\
\mu g / L \\
\mu g / L \\
\mu g / L \\
\mu g / L \\
\mu g / L \\
\mu g / L \\
\mu g / L \\
\mu g / L\end{array}$ & $\begin{array}{l}\text { EPA9066 } \\
\text { EPA8270C } \\
\text { EPA8141A } \\
\text { EPA8270C } \\
\text { EPA6010B } \\
\text { EPA8270C } \\
\text { EPA8260B } \\
\text { EPA8270C } \\
\text { EPA8270C } \\
\text { EPA8270C } \\
\text { EPA6010B } \\
\text { EPA6010B } \\
\text { EPA6010B } \\
\text { EPA6010B } \\
\text { EPA9050A } \\
\text { EPA8260B } \\
\text { EPA9056 } \\
\text { EPA9030B } \\
\text { EPA9034 } \\
\text { EPA376.2 } \\
\text { EPA8141A } \\
\text { EPA8151A } \\
\text { EPA8280A } \\
\text { EPA8270C } \\
\text { EPA8280A } \\
\text { EPA8280A } \\
\text { EPA8260B } \\
\text { EPA8260B } \\
\text { EPA8260B } \\
\text { EPA8270C } \\
\text { EPA6010B } \\
\text { EPA8141A } \\
\text { EPA6010B } \\
\text { EPA8260B } \\
\text { EPA8270C } \\
\text { EPA160.1 } \\
\text { EPA9060 } \\
\text { EPA8270C } \\
\text { EPA8260B } \\
\text { EPA8141A } \\
\text { EPA418.1 } \\
\text { EPA365.2 } \\
\text { EPA8081A } \\
\text { EPA8151A } \\
\text { EPA8270C } \\
\text { EPA8260B } \\
\text { EPA8010 } \\
\text { EPA8021B } \\
\text { EPA8260B } \\
\text { EPA8260B }\end{array}$ & $\begin{array}{l}37.0 \\
10.0 / 22.0 \\
1.0 / 2.13 \\
10.0 / 22.0 \\
187 \\
10.0 / 22.0 \\
50.0 / 250 \\
10.0 / 22.0 \\
10.0 / 22.0 \\
10.0 / 22.0 \\
66.0 \\
1,350 / 1,370 \\
5.0 \\
285 \\
8.9 / 10.0 \\
5.0 / 25.0 \\
340 / 68,000 \\
10,000 \\
10,000 \\
10,000 \\
1.0 / 2.13 \\
0.5 / 1.06 \\
5.0 / 11.2 \\
10.0 / 22.0 \\
5.0 / 11.2 \\
5.0 / 11.2 \\
5.0 / 25.0 \\
5.0 / 1,000 \\
5.0 / 1,000 \\
10.0 / 22.0 \\
55.0 \\
0.5 / 1.06 \\
70.0 \\
5.0 / 1,000 \\
10.0 / 22.0 \\
47,000 / 50,000 \\
1,000 \\
120 / 1,200 \\
10,000 \\
67.0 \\
5.0 / 10.6 \\
0.5 / 1.06 \\
10.0 / 22.0 \\
5.0 / 1,000 \\
1.0 / 5.0 \\
1.0 / 200 \\
5.0 / 1,000 \\
5.0 / 1,000 \\
1.0 / 5.0 \\
1.0 / 200 \\
5.0 / 1,000 \\
25.0 / 55.0 \\
10.0 / 22.0 \\
5.0 / 25.0 \\
0.5 / 1.06 \\
\\
\\
\end{array}$ \\
\hline
\end{tabular}




\begin{tabular}{llll}
\hline Analyte & Unit & Method & Minimum/Maximum EQLs \\
\hline & & & \\
1,3,5-Trinitrobenzene & $\mu \mathrm{g} / \mathrm{L}$ & EPA8270C & $10.0 / 22.0$ \\
Vanadium & $\mu \mathrm{g} / \mathrm{L}$ & EPA6010B & 6.9 \\
Vinyl acetate & $\mu \mathrm{g} / \mathrm{L}$ & EPA8260B & $10.0 / 50.0$ \\
Xylenes & $\mu \mathrm{g} / \mathrm{L}$ & EPA8260B & $5.0 / 1,000$ \\
Zinc & $\mu \mathrm{g} / \mathrm{L}$ & EPA6010B & 53.0 \\
\hline
\end{tabular}

Note: The groundwater samples are unfiltered; thus, the methods for metals are for total recoverable metals. Method 200.7 is an inductively coupled plasma atomic emission spectroscopy method for metals determination and is published for Safe Drinking Water Act investigations.

Table 13. Methods and Estimated Quantitation Limits Used by GP

\begin{tabular}{|c|c|c|c|}
\hline Analyte & Unit & Method & Minimum/Maximum EQLs \\
\hline Actinium-228 & $\mu \mathrm{Ci} / \mathrm{mL}$ & EPIA-013 & $9.88 \mathrm{E}-09 / 2.4 \mathrm{E}-08$ \\
\hline Americium-241 & $\mu \mathrm{Ci} / \mathrm{mL}$ & EPIA-011 & $1.43 \mathrm{E}-11 / 4.22 \mathrm{E}-09$ \\
\hline Antimony-125 & $\mu \mathrm{Ci} / \mathrm{mL}$ & EPIA-013 & $6.5 \mathrm{E}-09 / 1.56 \mathrm{E}-08$ \\
\hline Carbon-14 & $\mu \mathrm{Ci} / \mathrm{mL}$ & EPIA-003 & $5.16 \mathrm{E}-09 / 1.84 \mathrm{E}-08$ \\
\hline Cerium-144 & $\mu \mathrm{Cl} / \mathrm{mL}$ & EPIA-013 & $1.5 \mathrm{E}-08 / 4.1 \mathrm{E}-08$ \\
\hline Cesium-134 & $\mu \mathrm{Ci} / \mathrm{mL}$ & EPIA-013 & 2.19E-09/4.99E-09 \\
\hline Cesium-137 & $\mu \mathrm{Ci} / \mathrm{mL}$ & EPIA-013 & 2.3E-09/1.01E-07 \\
\hline Cobalt-57 & $\mu \mathrm{Ci} / \mathrm{mL}$ & EPIA-013 & $1.92 \mathrm{E}-09 / 5.29 \mathrm{E}-09$ \\
\hline Cobalt-60 & $\mu \mathrm{Ci} / \mathrm{mL}$ & EPIA-013 & 2.04E-09/6.16E-09 \\
\hline Curium-242 & $\mu \mathrm{Ci} / \mathrm{mL}$ & EPIA-011 & $1.5 \mathrm{E}-11 / 3.29 \mathrm{E}-09$ \\
\hline Curium-243/244 & $\mu \mathrm{Ci} / \mathrm{mL}$ & EPIA-011 & 1.31E-11/3.11E-09 \\
\hline Curium-245/246 & $\mu \mathrm{Ci} / \mathrm{mL}$ & EPIA-011 & $1.26 \mathrm{E}-11 / 1.55 \mathrm{E}-09$ \\
\hline Europium-152 & $\mu \mathrm{Ci} / \mathrm{mL}$ & EPIA-013 & $6.89 E-09 / 1.69 E-08$ \\
\hline Europium-154 & $\mu \mathrm{Ci} / \mathrm{mL}$ & EPIA-013 & $5.78 \mathrm{E}-09 / 1.66 \mathrm{E}-08$ \\
\hline Europium-155 & $\mu \mathrm{Ci} / \mathrm{mL}$ & EPIA-013 & 7.96E-09/2.19E-08 \\
\hline Gross alpha & $\mu \mathrm{Ci} / \mathrm{mL}$ & EPIA-001 & $2.54 \mathrm{E}-10 / 4.27 \mathrm{E}-07$ \\
\hline lodine-129 & $\mu \mathrm{Ci} / \mathrm{mL}$ & EPIA-006 & $1.1 \mathrm{E}-10 / 5.06 \mathrm{E}-09$ \\
\hline Lead-212 & $\mu \mathrm{Ci} / \mathrm{mL}$ & EPIA-013 & 4.01E-09/1.1E-08 \\
\hline Manganese-54 & $\mu \mathrm{Ci} / \mathrm{mL}$ & EPIA-013 & $1.98 E-09 / 4.88 E-09$ \\
\hline Nickel-63 & $\mu \mathrm{Ci} / \mathrm{mL}$ & EPIA-022 & $1.29 \mathrm{E}-08 / 1.51 \mathrm{E}-07$ \\
\hline Nonvolatile beta & $\mu \mathrm{Ci} / \mathrm{mL}$ & EPIA-001 & $5.39 E-10 / 6.7 E-07$ \\
\hline Plutonium-238 & $\mu \mathrm{Ci} / \mathrm{mL}$ & EPIA-011 & $9.09 \mathrm{E}-12 / 2.67 \mathrm{E}-09$ \\
\hline Plutonium-239/240 & $\mu \mathrm{Ci} / \mathrm{mL}$ & EPIA-011 & $9.25 \mathrm{E}-12 / 1.96 \mathrm{E}-09$ \\
\hline Potassium-40 & $\mu \mathrm{Ci} / \mathrm{mL}$ & EPIA-013 & $1.9 E-08 / 6.81 E-08$ \\
\hline Promethium-144 & $\mu \mathrm{Ci} / \mathrm{mL}$ & EPIA-013 & 2.17E-09/4.6E-09 \\
\hline Promethium-146 & $\mu \mathrm{Ci} / \mathrm{mL}$ & EPIA-013 & 3.05E-09/7.09E-09 \\
\hline Radium, total alpha-emitting & $\mu \mathrm{Ci} / \mathrm{mL}$ & EPIA-010 & $6.21 E-10 / 8.74 E-10$ \\
\hline Radium-226 & $\mu \mathrm{Ci} / \mathrm{mL}$ & EPIA-008 & $1.11 \mathrm{E}-10 / 1.04 \mathrm{E}-09$ \\
\hline Radium-228 & $\mu \mathrm{Ci} / \mathrm{mL}$ & EPIA-009 & 4.02E-10/3.22E-09 \\
\hline Ruthenium-106 & $\mu \mathrm{Ci} / \mathrm{mL}$ & EPIA-013 & 2.07E-08/4.69E-08 \\
\hline Sodium-22 & $\mu \mathrm{Ci} / \mathrm{mL}$ & EPIA-013 & 2.06E-09/5.92E-09 \\
\hline Strontium-89/90 & $\mu \mathrm{Ci} / \mathrm{mL}$ & EPIA-004 & $9.58 E-10 / 6.14 E-09$ \\
\hline Strontium-90 & $\mu \mathrm{Ci} / \mathrm{mL}$ & EPIA-004 & $6.32 \mathrm{E}-10 / 1.46 \mathrm{E}-08$ \\
\hline Technetium-99 & $\mu \mathrm{Ci} / \mathrm{mL}$ & EPIA-005 & 3.76E-09/4.89E-08 \\
\hline Thorium-228 & $\mu \mathrm{Ci} / \mathrm{mL}$ & EPIA-012 & $4.13 E-11 / 6.2 E-09$ \\
\hline Thorium-230 & $\mu \mathrm{Ci} / \mathrm{mL}$ & EPIA-012 & $9.86 E-12 / 3.67 E-09$ \\
\hline Thorium-232 & $\mu \mathrm{Ci} / \mathrm{mL}$ & EPIA-012 & 8.76E-12/3.22E-09 \\
\hline Tritium & $\mu \mathrm{Ci} / \mathrm{mL}$ & EPIA-002 & $5.2 E-07 / 1.09 E-05$ \\
\hline Uranium-233/234 & $\mu \mathrm{Ci} / \mathrm{mL}$ & EPIA-011 & $9.8 E-12 / 3.97 E-09$ \\
\hline
\end{tabular}




\begin{tabular}{llll}
\hline Analyte & Unit & Method & Minimum/Maximum EQLs \\
\hline Uranium-235 & $\mu \mathrm{Ci} / \mathrm{mL}$ & EPIA-011 & $8.87 \mathrm{E}-12 / 3.38 \mathrm{E}-09$ \\
Uranium-238 & $\mu \mathrm{Ci} / \mathrm{mL}$ & EPIA-011 & $1.9 \mathrm{E}-11 / 2.94 \mathrm{E}-09$ \\
Yttrium-88 & $\mu \mathrm{Ci} / \mathrm{mL}$ & EPIA-013 & $2.25 \mathrm{E}-09 / 6.15 \mathrm{E}-09$ \\
Zinc-65 & $\mu \mathrm{Ci} / \mathrm{mL}$ & EPIA-013 & $7.52 \mathrm{E}-10 / 1.45 \mathrm{E}-08$ \\
\hline
\end{tabular}

Table 14. Methods and Estimated Quantitation Limits Used by TM

\begin{tabular}{|c|c|c|c|}
\hline Analyte & Unit & Method & MInimum/Maximum EQLs \\
\hline Actinium-228 & $\mu \mathrm{Ci} / \mathrm{mL}$ & EPA901.1MOD & $1.571 \mathrm{E}-08 / 2.874 \mathrm{E}-08$ \\
\hline Americium-241/Curium-246 & $\mu \mathrm{Ci} / \mathrm{mL}$ & EMLAM01MOD & $1.2 \mathrm{E}-10 / 7.9 \mathrm{E}-10$ \\
\hline Antimony-124 & $\mu \mathrm{Ci} / \mathrm{mL}$ & EPA901.1MOD & $4.48 \mathrm{E}-09 / 6.76 \mathrm{E}-09$ \\
\hline Antimony-125 & $\mu \mathrm{Ci} / \mathrm{mL}$ & EPA901.1MOD & $9.22 \mathrm{E}-09 / 1.751 \mathrm{E}-08$ \\
\hline Barium-133 & $\mu \mathrm{Ci} / \mathrm{mL}$ & EPA901.1MOD & 4.56E-09/7.57E-09 \\
\hline Carbon-14 & $\mu \mathrm{Ci} / \mathrm{mL}$ & ENICMOD & $1.6994 \mathrm{E}-07 / 1.8243 \mathrm{E}-07$ \\
\hline Cerium-144 & $\mu \mathrm{Ci} / \mathrm{mL}$ & EPA901.1MOD & 2.283E-08/2.864E-08 \\
\hline Cesium-134 & $\mu \mathrm{Ci} / \mathrm{mL}$ & EPA901.1MOD & 4.03E-09/6.44E-09 \\
\hline Cesium-137 & $\mu \mathrm{Ci} / \mathrm{mL}$ & EPA901.1MOD & 4.38E-09/7.28E-09 \\
\hline Cobalt-57 & $\mu \mathrm{Ci} / \mathrm{mL}$ & EPA901.1MOD & 2.74E-09/3.67E-09 \\
\hline Cobalt-58 & $\mu \mathrm{Ci} / \mathrm{mL}$ & EPA901.1MOD & $4.22 \mathrm{E}-09 / 7.41 \mathrm{E}-09$ \\
\hline Cobalt-60 & $\mu \mathrm{Ci} / \mathrm{mL}$ & EPA901.1MOD & 4.33E-09/8.28E-09 \\
\hline Curium-242 & $\mu \mathrm{Ci} / \mathrm{mL}$ & EMLAM01MOD & 1.5E-10/6.8E-10 \\
\hline Curium-243/244 & $\mu \mathrm{Ci} / \mathrm{mL}$ & EMLAM01MOD & 2.2E-10/1.19E-09 \\
\hline Europium-152 & $\mu \mathrm{Ci} / \mathrm{mL}$ & EPA901.1MOD & 2.994E-08/5.317E-08 \\
\hline Europium-154 & $\mu \mathrm{Ci} / \mathrm{mL}$ & EPA901.1MOD & $1.087 \mathrm{E}-08 / 2.188 \mathrm{E}-08$ \\
\hline Europium-155 & $\mu \mathrm{Ci} / \mathrm{mL}$ & EPA901.1MOD & $8.0 \mathrm{E}-09 / 1.389 \mathrm{E}-08$ \\
\hline Gross alpha & $\mu \mathrm{Ci} / \mathrm{mL}$ & EPA900.0MOD & $2.0 \mathrm{E}-11 / 3.44 \mathrm{E}-09$ \\
\hline Jodine-129 & $\mu \mathrm{Ci} / \mathrm{mL}$ & EPA902.0MOD & $1.34 \mathrm{E}-09 / 1.356 \mathrm{E}-08$ \\
\hline Lead-212 & $\mu \mathrm{Ci} / \mathrm{mL}$ & EPA901.1MOD & $5.92 \mathrm{E}-09 / 1.058 \mathrm{E}-08$ \\
\hline Manganese-54 & $\mu \mathrm{Ci} / \mathrm{mL}$ & EPA901.1MOD & 4.24E-09/7.73E-09 \\
\hline Neptunium-239 & $\mu \mathrm{Ci} / \mathrm{mL}$ & EPA901.1MOD & 3.856E-08/7.313E-07 \\
\hline Nickel-63 & $\mu \mathrm{Ci} / \mathrm{mL}$ & 3500 NIEMOD & 2.22E-09/2.4E-08 \\
\hline Nonvolatile beta & $\mu \mathrm{Ci} / \mathrm{mL}$ & EPA900.0MOD & 1.0E-10/6.75E-09 \\
\hline Plutonium-238 & $\mu \mathrm{Ci} / \mathrm{mL}$ & EMLPUO2MOD & $1.5 \mathrm{E}-10 / 2.12 \mathrm{E}-09$ \\
\hline Plutonium-239/240 & $\mu \mathrm{Ci} / \mathrm{mL}$ & EMLPU02MOD & $6.0 \mathrm{E}-11 / 1.48 \mathrm{E}-09$ \\
\hline Potassium-40 & $\mu \mathrm{Ci} / \mathrm{mL}$ & EPA901.1MOD & $3.825 E-08 / 8.659 E-08$ \\
\hline Promethium-144 & $\mu \mathrm{Ci} / \mathrm{mL}$ & EPA901.1MOD & 3.99E-09/7.24E-09 \\
\hline Promethium-146 & $\mu \mathrm{Ci} / \mathrm{mL}$ & EPA901.1MOD & 7.04E-09/1.172E-08 \\
\hline \multirow[t]{2}{*}{ Radium, total alpha-emitting } & $\mu \mathrm{Ci} / \mathrm{mL}$ & EPA903.0MOD & 2.2E-10/1.73E-09 \\
\hline & $\mu \mathrm{Ci} / \mathrm{mL}$ & EPA900.0MOD & $5.1 E-10$ \\
\hline Radium-226 & $\mu \mathrm{Ci} / \mathrm{mL}$ & EPA903.0MOD & $7.0 \mathrm{E}-11 / 4.2 \mathrm{E}-10$ \\
\hline Radium-228 & $\mu \mathrm{Ci} / \mathrm{mL}$ & EPA904.0MOD & 9.6E-10/2.64E-09 \\
\hline Ruthenium-106 & $\mu \mathrm{Ci} / \mathrm{mL}$ & EPA901.1MOD & 3.656E-08/6.143E-08 \\
\hline Sodium-22 & $\mu \mathrm{Ci} / \mathrm{mL}$ & EPA901.1MOD & 3.87E-09/7.8E-09 \\
\hline Strontium-90 & $\mu \mathrm{Ci} / \mathrm{mL}$ & EMLSR02MOD & $6.6 \mathrm{E}-10 / 1.87 \mathrm{E}-09$ \\
\hline Technetium-99 & $\mu \mathrm{Ci} / \mathrm{mL}$ & EICHROMTC1MOD & 1.26E-09/1.686E-08 \\
\hline Thorium-228 & $\mu \mathrm{Ci} / \mathrm{mL}$ & EMLTH01MOD & 1.5E-10/6.3E-10 \\
\hline Thorium-230 & $\mu \mathrm{Ci} / \mathrm{mL}$ & EMLTH01MOD & $1.1 \mathrm{E}-10 / 4.8 \mathrm{E}-10$ \\
\hline Thorium-232 & $\mu \mathrm{Ci} / \mathrm{mL}$ & EMLTH01MOD & $1.1 E-10 / 5.1 E-10$ \\
\hline Tin-113 & $\mu \mathrm{Ci} / \mathrm{mL}$ & EPA901.1MOD & 4.38E-09/7.65E-09 \\
\hline Tritium & $\mu \mathrm{Ci} / \mathrm{mL}$ & EPA906.0MOD & 4.8E-07/6.3195E-04 \\
\hline Uranium-234 & $\mu \mathrm{Ci} / \mathrm{mL}$ & EMLU02MOD & $1.0 \mathrm{E}-10 / 3.8 \mathrm{E}-10$ \\
\hline Uranium-235 & $\mu \mathrm{Ci} / \mathrm{mL}$ & EMLU02MOD & $1.0 \mathrm{E}-10 / 3.4 \mathrm{E}-10$ \\
\hline
\end{tabular}




\begin{tabular}{llll}
\hline Analyte & Unit & Method & Minimum/Maximum EQLs \\
\hline Uranium-238 & & & \\
Yttrium-88 & $\mu \mathrm{Ci} / \mathrm{mL}$ & EMLU02MOD & $1.0 \mathrm{E}-10 / 3.1 \mathrm{E}-10$ \\
Zinc-65 & $\mu \mathrm{Ci} / \mathrm{mL}$ & EPA901.1MOD & $3.54 \mathrm{E}-09 / 9.41 \mathrm{E}-09$ \\
Zirconium-95 & $\mu \mathrm{Ci} / \mathrm{mL}$ & EPA901.1MOD & $8.65 \mathrm{E}-09 / 1.601 \mathrm{E}-08$ \\
\hline
\end{tabular}




\section{Quality Control Samples}

This section discusses the analytical data in terms of the following indicators of data quality: precision, accuracy, representativeness, comparability, and completeness. Precision is determined from the field and laboratory duplicate or replicate analyses and indicates the consistency of field and laboratory techniques. Accuracy is determined from the quality control standards, laboratory control samples or biank spikes, surrogates, matrix spikes, and the results of method, field, and trip blanks and indicates the ability of the laboratory to generate correct results. (Equipment blanks are used to evaluate the effectiveness of the cleaning procedures used in the field.) Representativeness is the determination of how well the sample reflects the site's characteristics. Comparability expresses the confidence with which data from different laboratories are considered to be equivalent. Completeness measures the amount of useable data resulting from the data collection activity.

\section{PRECISION}

Precision is a measure of the repeatability of a measurement and is evaluated from the results of duplicate samples and splits. Blind replicates, or field replicates, measure the repeatability of the sampling and analytical techniques, and laboratory duplicates measure the ability of the laboratory to reproduce a result. Split samples measure whether two laboratories using comparable procedures obtain equivalent results. Low precision can be caused by poor instrument performance, poor operator technique, inconsistent application of method protocols, laboratory environment, time between analyses, or by a difficult, heterogeneous sample matrix.

\section{Replicate and Duplicate Analyses of Samples}

Blind replicate and duplicate samples are analyzed to establish the precision of scheduled analyses. The replicate and duplicate analytical results are used to generate Mean Relative Difference (MRD) indices, which are used to evaluate the laboratories' performances.

The primary laboratories EX, GE, and WA, performed all analyses with the following exception: GP and TM performed radionuclide analyses for EX, GE, and WA.

For intralaboratory comparisons, generally $10 \%$ of the samples are analyzed in duplicate. In addition, EPD/EMS sends blind replicates of approximately $5 \%$ of the total samples to the laboratories for analysis. The results of the blind replicate analyses are used for both intralaboratory and interlaboratory comparisons.

All these results are included in the Analytical Results section (Appendix B) of this report. Results from duplicate samples are included in the main table for a given well and sample date. Results from analyses of replicate samples and duplicate analyses of the replicates are reported in a second table for the same well and sample date.

Table 15 lists the well names, sample dates, and associated blanks for wells used as blind replicates for EX, $\mathrm{GE}$, and WA.

Certain analytes were not present in concentrations above estimated quantitation limits in any well samples having replicates or duplicates. These analytes are not considered in further evaluation of replicate and duplicate analyses and are listed in tables 16 and 17. See tables 10-14 for the estimated quantitation limits that are applicable this quarter.

\section{Intralaboratory Comparisons}

Intralaboratory comparisons are of two types: in-house duplicates and blind replicates. The MRD was developed by R.C. Tuckfield of the Applied Statistics Group at the Savannah River Technology Center, in conjunction with M.M. Khalil of EPD/EMS, to assess the reproducibility of identical chemical analyses. For both intralaboratory comparisons, the MRD is defined as the average absolute difference between an original sample and its duplicate or blind replicate, expressed as a percentage of the mean of those two values. It is calculated as 


$$
\begin{aligned}
& \mathrm{MRD}=\left\{\frac{\sum_{i=1}^{n}\left(\left|x_{i}-y_{i}\right| /\left[\left(x_{i}+y_{i}\right) / 2\right]\right)}{n}\right\} \times 100 \\
& \text { where } \\
& x_{i}=\text { an analyte's mean concentration } \\
& \text { in a water sample for the } i^{\text {th }} \text { well, } \\
& y_{i}=\text { the analyte's mean concentration } \\
& \text { in the replicate or duplicate, and } \\
& n=\text { the number of pairs of observations. }
\end{aligned}
$$

For the in-house duplicate comparisons, the quantities $x_{i}$ and $y_{i}$ represent the results for the original sample and the in-house duplicate, respectively. For the blind replicate comparisons, $x_{i}$ and $y_{i}$ represent the results for the known sample and the EPD blind replicate, respectively. Generally, the closer the original results and their replicate or duplicate results are to each other, the lower the MRD.

\section{An Adjusted Mean Relative Difference}

A drawback to the MRD statistic occurs when $x_{i}$ and $y_{i}$ are close to zero. This drawback can be illustrated by determining the relative difference (RD) for the $i^{\text {th }}$ well or sample as follows:

$$
\begin{gathered}
\mathrm{RD}_{i}=\frac{\left|x_{i}-y_{i}\right|}{z_{i}} \\
\text { where } z_{i}=\left(\frac{x_{i}+y_{i}}{2}\right)
\end{gathered}
$$

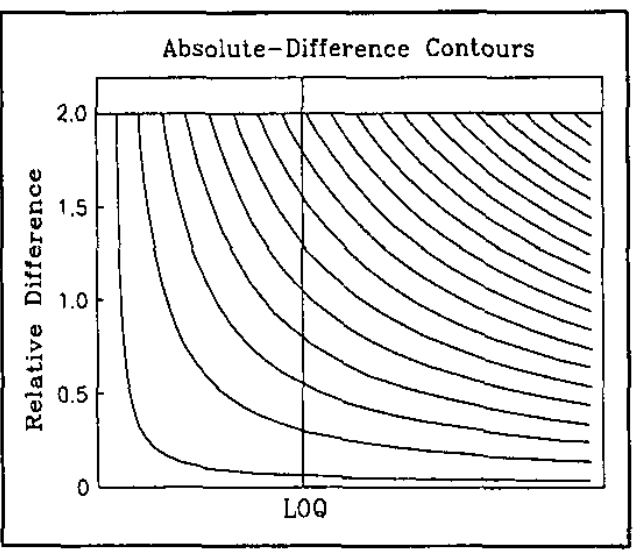

Figure 4. Relative Difference vs. the Mean

The $\mathrm{RD}_{i}$ is an individual term in the MRD calculation for the $i^{\text {th }}$ replicated sample. For example, if $x_{i}=99$ and $y_{i}$ $=101$, then $\mathrm{RD}_{i}=2 \%$. However, if $x_{i}=3$ and $y_{i}=1$, then $\mathrm{RD}_{i}=100 \%$. Both situations have the same absolute difference, but the latter situation has a much larger relative difference. The effect can be shown by graphing the relative difference vs. the mean $\left(z_{i}\right)$ and marking contours for constant levels of absolute difference 
(figure 4). The first contour, in the lower left corner of the figure, represents the smallest absolute difference. The last contour, in the upper right corner of the figure, represents the largest absolute difference.

The inordinate inflation of the MRD when $x_{i}$ and $y_{i}$ are near zero is of particular concern when the results are below the limit of quantitation (LOQ). Briefly, the LOQ is defined by L.H. Keith (1991) as 10 times the instrument signal standard deviation $(\sigma)$ for blank samples. For perspective, the limit of detection is defined as $3 \sigma$.

The reproducibility of analytical results less than the LOQ is considered by environmental chemists to be questionable. In this situation, the $\mathrm{RD}_{i}$ may reflect variation more in the measuring device itself than in the measuring process. However, the MRD can be a useful statistic if adjusted so that results below the LOQ have less influence than more reproducible results above the LOQ.

The simplest adjustment to the MRD to reduce the influence of analyte concentrations near zero is to weight

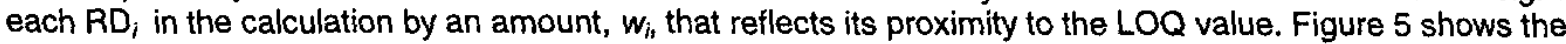
relationship between $w_{i}$ and analyte concentration. This relationship is a linear-weight function.

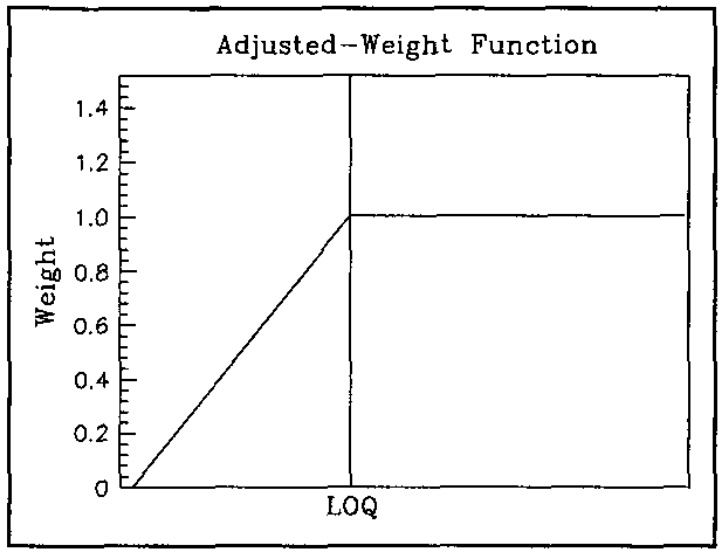

Figure 5. Relationship betweon $\mathrm{w}_{1}$ and Analyte Concentration

Figure 6 shows the computer simulation results for the effect of a linear-weight function on the now-adjusted MRD (MRDadj), developed by Tuckfield and Khalil, again by determining constant contours of absolute difference. Below the LOQ, all samples with the same absolute difference are given the same adjusted RD value. Above the LOQ, the unadjusted RD is preserved because the weight function is unity when $z_{i}$ is greater than the LOQ.

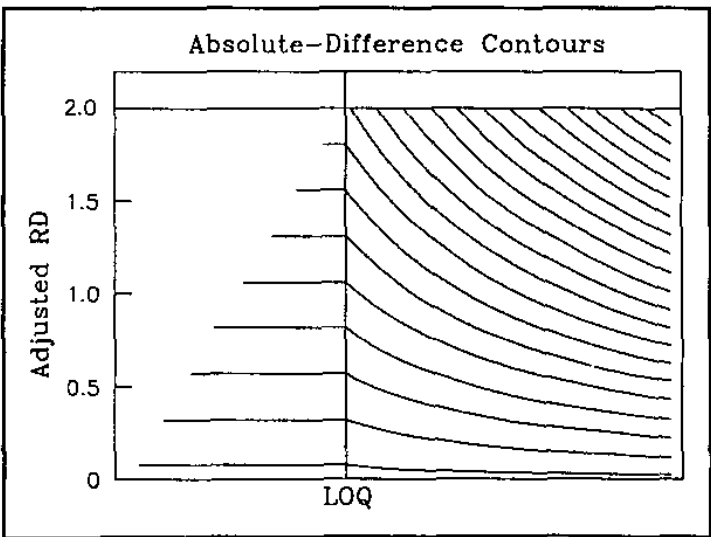

Figure 6. Effect of a Linear-Woight Function on the MRDadj 
The MRDadj, then, has the following form:

$$
\begin{gathered}
\text { MRDadj }=\frac{\sum_{i=1}^{n} \mathrm{w}_{i} \mathrm{RD}_{i}}{n}, \\
\text { where } \mathrm{w}_{i}=\left\{\begin{array}{cl}
\frac{z_{i}}{\mathrm{LOQ}} & ; \text { if } z_{i}<\mathrm{LOQ} \\
1 & ; \text { otherwise. }
\end{array}\right.
\end{gathered}
$$

This adjustment has several advantages. For example, the weight function reflects the chemist's view of the reliability of the measurement. If analyses are conducted on different equipment (i.e., with different LOQs), the precision of the equipment is included automatically in the MRD. Data from more precise equipment are given more influence. Also, no data are removed from the computation completely, so the sample size $(n)$ is not affected.

\section{Normalizing Date to the Reference Detection Limit}

Because some detection limits may be anomalously high (because of dilution or other effects, for example), it is necessary to use a reference detection limit (RDL) in the MRD calculations. This is set as the 90th percentile value of the detection limits of the not-detected samples. All the results less than the RDL are adjusted up to that value. Results that are detection limit values above the RDL are eliminated from the MRD index calculations. By definition, fewer than $10 \%$ of the detection limit values are above the RDL. The intralaboratory MRD indices are listed in tables 18-23.

\section{Interlaboratory Comparisons}

For interlaboratory comparisons, the MRD is calculated as the average absolute difference between the laboratories for the $i^{\text {th }}$ well expressed as a percentage of the mean of both laboratories. For these comparisons, $x_{i}$ and $y_{i}$ represent the mean analyte concentrations for the $i^{\text {th }}$ well; $x_{i}$ represents the mean from one laboratory, and $y_{i}$ represents the mean from the other. The means are calculated from the known sample results and the EPD blind replicate results.

\section{Choosing an RDL}

For interlaboratory comparisons, a new RDL must be established for calculation of the MRD. The interlaboratory $\mathrm{RDL}$ is chosen as the 90 th percentile value from the combined array of non-detected sample results from both laboratories.

\section{Normalizing Date to the RDL}

All results less than the RDL are adjusted to the new $R D L$ value. Detection limit values above the $R D L$ are eliminated from the MRD index comparison and from the $t$-tests. By definition, fewer than $10 \%$ of the detection limit values are above the RDL. In addition to the interlaboratory MRD calculations, paired $t$-tests are performed to see if the difference between the mean concentrations of an analyte from the same well reported by each laboratory is significant. The t-test tests the null hypothesis that there is no significant difference in the concentrations reported by the two laboratories. The MRD and the $t$-test resuits for analytes with at least one pair of results above the interlaboratory $R D L$ are listed in tables 24-26.

Analytes with significance-of-probability values less than .050 (tables 24-26) indicate a probability of less than $5 \%$ that the results for that analyte are the same from both laboratories.

\section{Presentation of the Replicate and Duplicate Analyses}

In tables 24-26, high MRDs (greater than or equal to 20) appear in bold type. Low MRDs (less than or equal to .050) appear in bold italic type. 
Table 27 lists analytes and wells for which samples and blind replicates analyzed by EX yielded results where one was more than twice another.

Table 28 lists analytes and wells for which samples and blind replicates analyzed by GE yielded results where one was more than twice another.

Table 29 lists analytes and wells for which samples and blind replicates analyzed by WA yielded results where one was more than twice another.

Table 30 lists analytes and wells for which samples and laboratory duplicates analyzed by WA yielded results where one was more than twice another.

Table 31 lists analytes and wells for which samples and blind replicates analyzed by GP yielded results where one was more than twice another.

Table 32 lists analytes and wells for which samples and laboratory duplicates analyzed by GP yielded results where one was more than twice another.

Table 33 lists analytes and wells for which samples and blind replicates analyzed by TM yielded results where one was more than twice another.

Table 34 lists analytes and wells for which samples and laboratory duplicates analyzed by TM yielded results where one was more than twice another.

Tables 35-37 list analytes and wells where a result from one laboratory was more than twice the corresponding result from the other laboratory.

See the Analytical Methods subsection of the Analytical Data Review section of this report for more information.

\section{ACCURACY}

Accuracy is defined as the closeness of agreement between an observed value and an accepted reference value or as a measure of the over- or underestimation of reported concentrations. Accuracy is especially important when the concentration of concern approaches the detection limit and/or the action limit. When the concentration is underestimated near the detection limit, the analyte may be present but reported as not detected; near the action limit, the analyte may be at a concentration that would require remediation, but the remediation would not be performed. When the concentration is overestimated near the detection limit, the analyte may not be present but reported as detected; near the action limit, the analyte may not be at a concentration that would require remediation, but the remediation would be performed. Quality control standards, performance evaluation studies, laboratory control samples, surrogate and matrix spikes, and method blanks are used to evaluate accuracy.

\section{Quality Control Standards}

During first quarter 1999, EPD/EMS conducted quality assessments of EX, GE, and WA laboratories. Each laboratory received a set of certified environmental quality control standards from Environmental Resource Associates (ERA) of Arvada, CO (lot numbers 439, 586, 3227, 3427, 8922, and 9987). Each laboratory's results were compared with the ERA-certified values and performance acceptance limits (PALs). The PALs are listed as guidelines for acceptable analytical results given the limitations of the EPA methods used to determine these parameters. The PALs closely approximate the $95 \%$ confidence interval. EX, GE, and WA all returned results for first quarter 1999 quality control assessments. The laboratories' results and the certified values and limits are listed in tables 38-40.

EX, GE, and WA analyzed total petroleum hydrocarbons by the infrared method. WSRC cancelled the analysis of a performance evaluation sample for toxaphene for two of the laboratories: GE, due to a broken vial; WA, because the toxaphene ampule was received empty by the laboratory. 
Of 103 analyses reported by EX, 98 , or $95.1 \%$, were within the PALs. Of the 103 analyses reported by GE, 102 , or $99.0 \%$, were within the PALs. Of 103 analyses reported by WA, 90 , or $87.4 \%$, were within the PALs.

\section{Laboratory Control Samples}

Laboratory control samples are used to monitor the performance of all steps in the analysis process, including sample preparation, and are used to identify problems with the analytical procedure. Laboratory control samples are deionized water that is spiked with the target analyte, digested, and analyzed with the regular samples for inorganic parameters. Blank spikes are organic-free water that is spiked with selected target analytes, extracted, and analyzed with the regular samples for organic parameters. The spiking solutions for laboratory control samples are obtained from the EPA or a third-party supplier, or they are prepared in the laboratory with chemicals from a different source than the calibration standards. All laboratory control standards are validated to EPA standards, as detailed in the EGG Operating Handbook, section 1.800, Analytical Data Qualification.

The percent recovery $(\% \mathrm{R})$ for laboratory control samples or blank spikes is calculated as

Tables 41-45 list the statistical information for the percent recovery for laboratory control samples by analyte for EX, GE, WA, GP, and TM. The Qualified Out of Range column provides the number of laboratory control samples or blank spikes that had percent recoveries outside the acceptance limits compared to the total number analyzed; the other columns provide the mean recovery, standard deviation, and the minimum and maximum recoveries.

\section{Surrogates}

Surrogates are analytes not normaliy found in environmental samples that are used to spike all samples, QC samples, and calibration standards for organic analyses. Surrogates are added prior to analysis for VOAs (volatile organic analyses) and prior to extraction for semivolatiles, pesticides, and herbicides. Low surrogate recovery is a measure of the effect of the sample matrix, high analyte concentration, or laboratory error. High surrogate recovery usually indicates instrument or sample preparation errors. All surrogates are validated to EPA standards, as detailed in the WSRC EGG Operating Handbook, section 1.800, Analytical Data Qualification.

Tables 46-48 list the statistical information for the percent recovery for the surrogates by analyte for EX, GE, and WA. The Qualified Out of Range column gives the number of surrogates that had percent recoveries outside the acceptance limits compared to the total number analyzed; the other columns provide the mean recovery, standard deviation, and the minimum and maximum recoveries.

\section{Matrix Spikes}

Matrix spikes are used to evaluate the effect of the sample matrix on the analytical procedure. Matrix spikes are prepared by adding a known quantity of the target analyte to at least $5 \%$ of the samples prior to sample preparation. For the inorganic analyses, all target analytes are spiked. For the organic analyses, selected target analytes are used in the spiking solution. Results from the matrix spike are used to evaluate the extent of matrix interference and to determine the bias of the procedure for the sample matrix. Matrix spikes have the same recovery limits as laboratory control samples. 
The percent recovery for matrix spikes is calculated as

$$
\begin{aligned}
& \text { where } \\
& \begin{array}{l}
\%=\text { percent recovery } \\
S S R=\text { spiked sample result } \\
S R=\text { sample result, and } \\
S A=\text { spike added. }
\end{array}
\end{aligned}
$$

Percent bias in tables $49-52$ is the difference between $100 \%$ and the mean recovery; a negative value indicates that the mean recovery was below $100 \%$. If the bias is consistently positive, the laboratory may be overestimating the concentration of the analyte, and if the bias is consistently negative, the laboratory may be underestimating the concentration of the analyte. Results close to the quantitation and action limits should be closely examined, and their use in decision-making should be carefully considered.

Matrix spikes are rejected if the concentration of the analyte in the sample is more than four times the amount of the spike. Results for matrix spikes are provided in tables $49-52$ for EX, GE, WA, and GP. The Qualified Out of Range column provides the number of matrix spikes that had percent recoveries outside the acceptance limits compared to the total number analyzed; the other columns provide the mean recovery, standard deviation, percent bias, and the minimum and maximum recoveries.

\section{Method Blanks}

Method blanks, or laboratory blanks, are used to determine the existence and magnitude of contamination problems resulting from the analytical process. Method blanks are deionized water to which all reagents are added in the same proportions used in sample processing. When method blanks have detectable concentrations of the analytes, the laboratory must determine the cause and take corrective action to eliminate the contamination.

Tables 53-57 list the statistical information for analytes detected in method blanks for EX, GE, WA, GP, and TM. The Frequency of Detection column provides the number of method blanks analyzed for each analyte during the quarter that had detectable concentrations compared to the total number that were analyzed. The other columns list the mean result, standard deviation, and minimum and maximum results.

\section{Field Blanks}

Field blanks (called QA blanks in the tables) are used to identify possible sources of contamination from the processing and shipping of samples. Field blanks are sample bottles filled with deionized water prior to well sampling; the bottles are not opened at the sampling site. The field blanks are sent along with, and analyzed in the same manner as, the samples. Positive results from field blanks can result from analytical bias, contaminated sample bottles, contaminated deionized water, or contamination during shipping or analysis. The results from all samples in the sample delivery group are evaluated by the laboratory and data validators to determine the cause of the contamination and the corrective action to be taken.

Tables 58-62 list the statistical information for the field blanks by analyte for EX, GE, WA, GP, and TM. The Frequency of Detection column gives the number of field blanks analyzed for each analyte during the quarter that had detectable concentrations compared to the total number analyzed. The other columns list the mean result, standard deviation, and minimum and maximum results. 


\section{Trip Blanks}

Trip blanks are vials of deionized water sent to the laboratory for volatiles analysis with each shipping cooler containing volatiles samples. Trip blanks are used to check for contamination resulting from shipping, primarily due to the breaking of the vial's seal because of depressurization during air transport. Trip blanks are used also to test the laboratories' reliability. The blanks are prepared by adding preservative to a $40 \mathrm{~mL}$ vial, filling it completely with deionized water, and sealing the top with a teflon-lined septum cap. The results from all samples in the sample delivery group are evaluated by the laboratory and data validators to determine the cause of the contamination and the corrective action to be taken.

Tables 63-65 list the statistical information for the analytes detected in trip blanks by EX, GE, and WA. The Frequency of Detection column gives the number of trip blanks analyzed for each analyte during the quarter that had detectable concentrations compared to the total number analyzed. The other columns list the mean result, standard deviation, and minimum and maximum results.

\section{Equipment Blanks or Rinsates}

Equipment blanks (called EPT blanks in the tables) or rinsates are used to determine if sampling equipment that has been cleaned in the field is contaminated. Prior to sampling, deionized water is poured over or pumped through portions of the sampling equipment that come in contact with the sample. If the equipment blank is contaminated, the field cleaning procedure must be evaluated to determine the cause of the contamination. Results for all samples collected with equipment cleaned in the field must be evaluated to determine whether the contamination is isolated or generalized.

No information about equipment blanks was provided for first quarter 1999.

\section{Blanks Results}

The blanks results tables in Appendix $\mathrm{C}$ list the dates, field measurements, and analytical results for the sampling blanks. See Appendix B for a key to the abbreviations used in the tables.

\section{REPRESENTATIVENESS}

A representative sample is a sample that can be expected to exhibit the average properties of the population being sampled. Representativeness for groundwater samples can be affected by using a bailer to collect the sample from the well, metal casings in the well, and turbidity (suspended particulates) in the sample. The results may be biased positively or negatively.

If a well is bailed, VOAs are biased negatively due to aeration of the sample in the sampling process. Table 66 lists wells that were bailed during first quarter 1999.

For metal casings, the bias for metals can be positive or negative depending on whether the casing is releasing or absorbing metals. Table 67 lists the wells with metal casings that were sampled during first quarter 1999.

If turbidity is greater than 15 NTU, the metals can be biased positively or negatively, and the radionuclidesparticularly those that are determined by gamma spectroscopy-can be masked due to self-absorption. Table 68 lists the wells that had turbidity results greater than 15 NTU during first quarter 1999.

\section{COMPARABILITY}

Comparability is evaluated by confirming that the laboratories used the same standardized procedures for sample preparation and analysis, that the reporting units are the same, and that similar quantitation limits were obtained. The analytical methods, reporting units, and EQLs reported by each laboratory are given in tables 1014 in the Analytical Data Review section. Tables 35-37 list the analytes and wells where a result from one laboratory was more than twice the corresponding result from the other laboratory. 


\section{COMPLETENESS}

Completeness is evaluated by comparing the wells scheduled for sampling with the wells sampled and comparing the requested analyses with the analytical data received. The number of wells sampled and the requested analyses are determined from the chains of custody. Tables 69-71 list the reasons the laboratories did not perform certain analyses on samples from wells that could be sampled. See the Sample Scheduling, Field Notes, and Analytical Results sections of this report for more information on wells scheduled but not sampled this quarter.

Table 15. Wells Providing Blind Replicate Samples and Associated Blanks

\begin{tabular}{|c|c|c|c|}
\hline Well & $\begin{array}{l}\text { Sample } \\
\text { Date }\end{array}$ & Replicate & $\begin{array}{l}\text { Associated } \\
\text { Blank }\end{array}$ \\
\hline $\begin{array}{l}\text { ABP 2A } \\
\text { AMB 7B } \\
\text { AMB 11B } \\
\text { ASB 8B } \\
\text { ASB 8TA } \\
\text { BGO 35C } \\
\text { BGO 37C } \\
\text { BGO 49C } \\
\text { CSB 2C } \\
\text { CSB 8D } \\
\text { FSB 76C } \\
\text { FSB 79A } \\
\text { FSB 89C } \\
\text { FSB121C } \\
\text { FSB123C } \\
\text { HAA 4D } \\
\text { HAA 5A } \\
\text { HAA 5C } \\
\text { HAA 11C } \\
\text { HAA 15C } \\
\text { HSB 68B } \\
\text { HSB 83C } \\
\text { HSB105C } \\
\text { HSB112C } \\
\text { HSB122A } \\
\text { HSB135C } \\
\text { HSB144A } \\
\text { LFW 43B } \\
\text { LFW 58D } \\
\text { LFW 67C } \\
\text { MSB 12A } \\
\text { MSB 17BB } \\
\text { MSB 29TA } \\
\text { MSB 31C } \\
\text { MSB 37B } \\
\text { MSB 42B } \\
\text { MSB 54B } \\
\text { MSB 62B } \\
\text { MSB 83TA } \\
\text { MSB 86C } \\
\text { P 26A } \\
\text { PSB 3A } \\
\text { TBG 5B } \\
\text { TRW 2 } \\
\text { ZBG 1 }\end{array}$ & $\begin{array}{l}02 / 12 / 99 \\
03 / 11 / 99 \\
02 / 23 / 99 \\
02 / 11 / 99 \\
02 / 22 / 99 \\
01 / 19 / 99 \\
02 / 25 / 99 \\
03 / 11 / 99 \\
03 / 19 / 99 \\
03 / 19 / 99 \\
01 / 06 / 99 \\
01 / 11 / 99 \\
01 / 20 / 99 \\
01 / 11 / 99 \\
01 / 20 / 99 \\
01 / 10 / 99 \\
01 / 10 / 99 \\
03 / 02 / 99 \\
01 / 05 / 99 \\
02 / 18 / 99 \\
01 / 20 / 99 \\
01 / 20 / 99 \\
01 / 20 / 99 \\
01 / 15 / 99 \\
01 / 27 / 99 \\
01 / 19 / 99 \\
01 / 27 / 99 \\
02 / 25 / 99 \\
03 / 18 / 99 \\
03 / 16 / 99 \\
02 / 22 / 99 \\
03 / 03 / 99 \\
02 / 02 / 99 \\
03 / 02 / 99 \\
03 / 10 / 99 \\
03 / 02 / 99 \\
02 / 23 / 99 \\
03 / 08 / 99 \\
02 / 22 / 99 \\
02 / 22 / 99 \\
02 / 03 / 99 \\
01 / 26 / 99 \\
02 / 02 / 99 \\
02 / 03 / 99 \text { and } 02 / 22 / 99 \\
01 / 20 / 99\end{array}$ & $\begin{array}{l}\text { QA 25A } \\
\text { QA 27A } \\
\text { QA 29A } \\
\text { QA 31A } \\
\text { QA 33A } \\
\text { QA 55A } \\
\text { QA 57A } \\
\text { QA 59A } \\
\text { QA 97A } \\
\text { QA 99A } \\
\text { QA 1A } \\
\text { QA 3A } \\
\text { QA 5A } \\
\text { QA 7A } \\
\text { QA 9AD } \\
\text { QA 61A } \\
\text { QA 63A } \\
\text { QA 65A } \\
\text { QA 67A } \\
\text { QA 69A } \\
\text { QA 11A } \\
\text { QA 13A } \\
\text { QA 15A } \\
\text { QA 17A } \\
\text { QA 19A } \\
\text { QA 21A } \\
\text { QA 23A } \\
\text { QA 77A } \\
\text { QA 79A } \\
\text { QA 81A } \\
\text { QA 35A } \\
\text { QA 37A } \\
\text { QA 39A } \\
\text { QA 41A } \\
\text { QA 43A } \\
\text { QA 45A } \\
\text { QA 47A } \\
\text { QA 49A } \\
\text { QA 51A } \\
\text { QA 53A } \\
\text { QA 85A } \\
\text { QA 75A } \\
\text { QA 87A } \\
\text { QA 83A } \\
\text { QA 73A }\end{array}$ & $\begin{array}{l}\text { QA 26A } \\
\text { QA 28A } \\
\text { QA 30A } \\
\text { QA 32A } \\
\text { QA 34A } \\
\text { QA 56A } \\
\text { QA 58A } \\
\text { QA 60A } \\
\text { QA 98A } \\
\text { QA 100A } \\
\text { QA 2A } \\
\text { QA 4A } \\
\text { QA 6A } \\
\text { QA 8A } \\
\text { QA 10A } \\
\text { QA 62A } \\
\text { QA 64A } \\
\text { QA 66A } \\
\text { QA 68A } \\
\text { QA 70A } \\
\text { QA 12A } \\
\text { QA 14A } \\
\text { QA 16A } \\
\text { QA 28A } \\
\text { QA 20A } \\
\text { QA 22A } \\
\text { QA 24A } \\
\text { QA 78A } \\
\text { QA 80A } \\
\text { QA 82A } \\
\text { QA 36A } \\
\text { QA 38A } \\
\text { QA 40A } \\
\text { QA 42A } \\
\text { QA 44A } \\
\text { QA 46A } \\
\text { QA 48A } \\
\text { QA 50A } \\
\text { QA 52A } \\
\text { QA 54 } \\
\text { QA 86A } \\
\text { Not applicable } \\
\text { QA 88A } \\
\text { QA 84A } \\
\text { QA 74A }\end{array}$ \\
\hline
\end{tabular}


Table 16. Analytes Not Showing Measurable Concentrations above Estimated Quantitation Limits in Any Replicated or Duplicated Samples for GE, WA, and EX

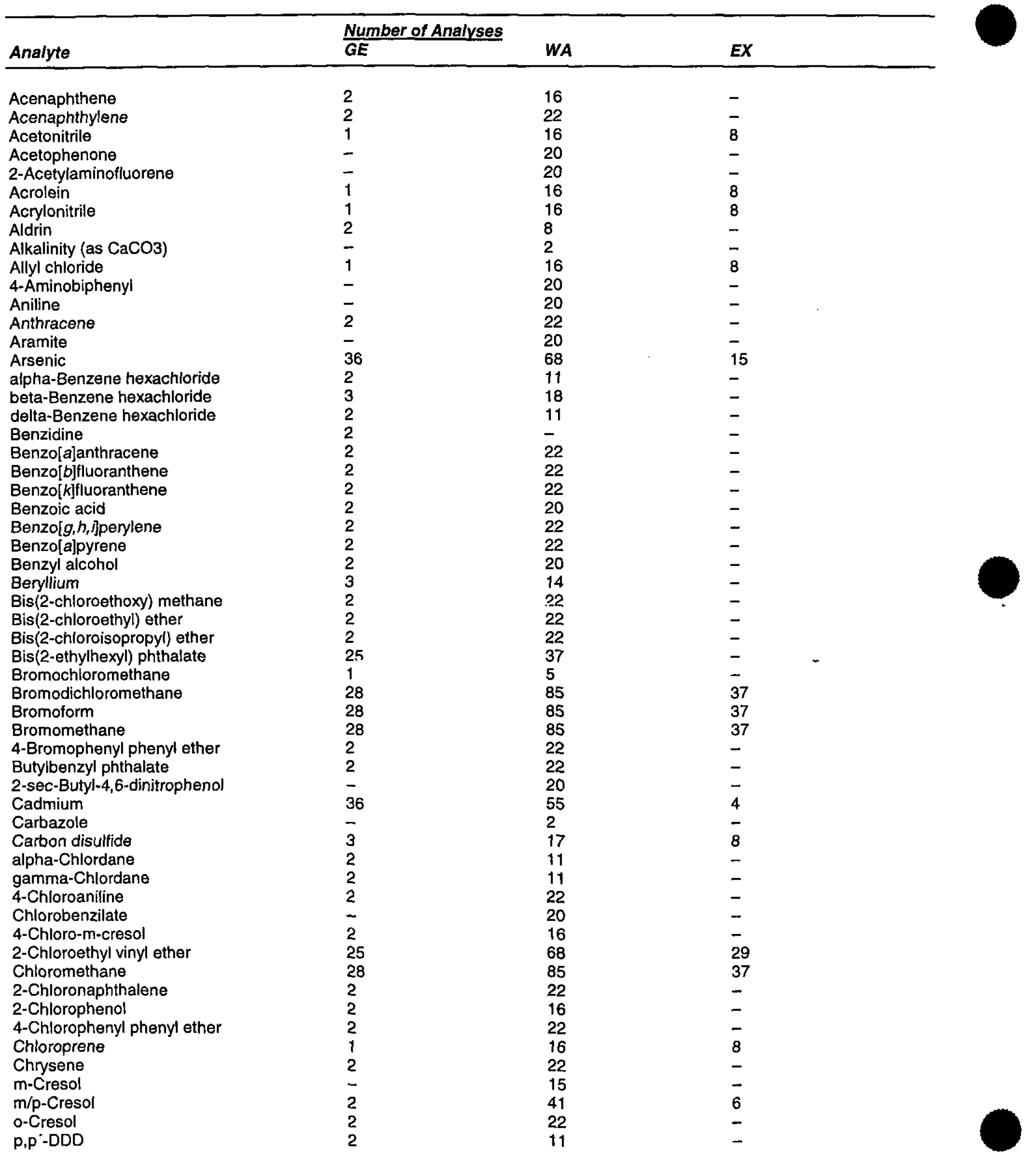




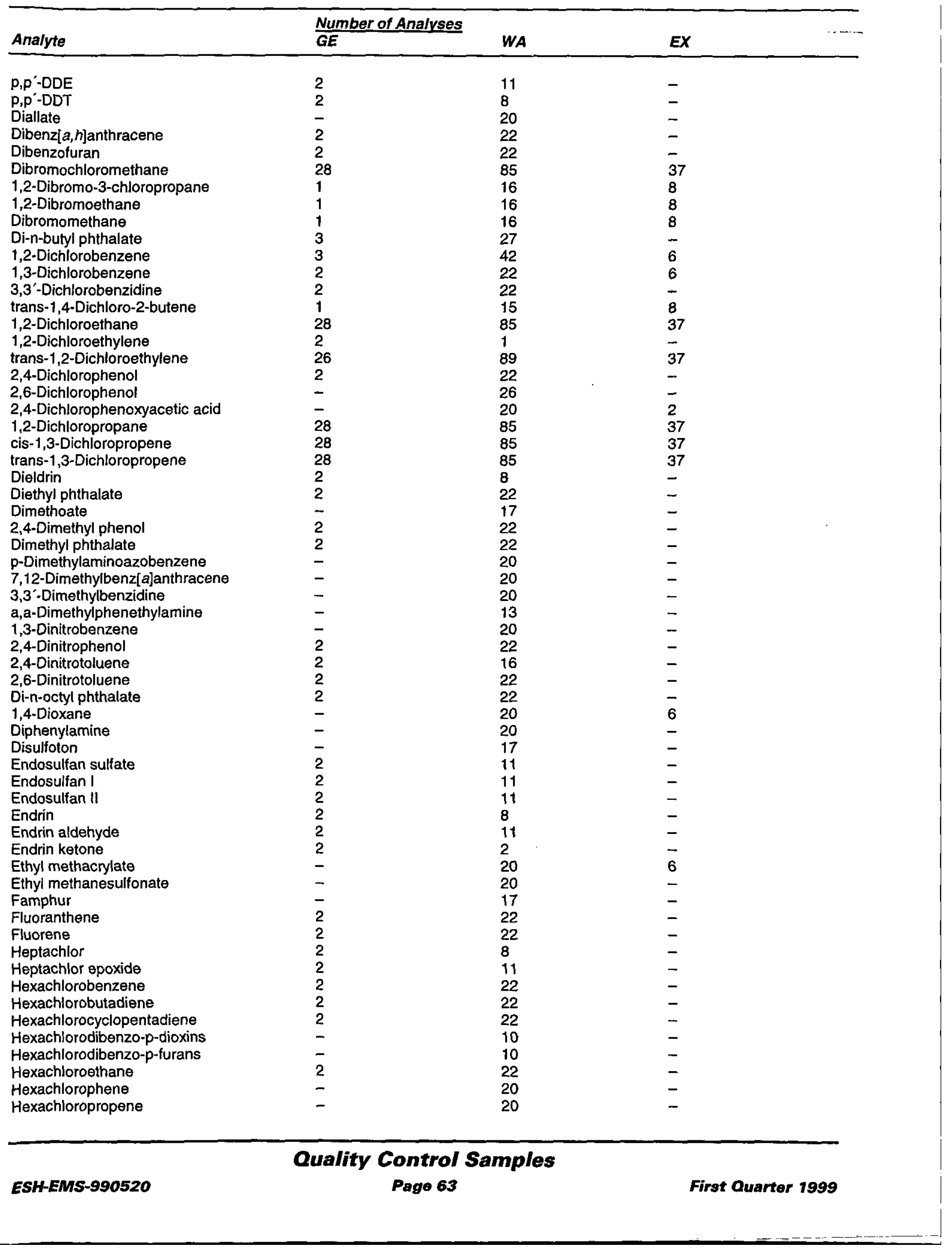




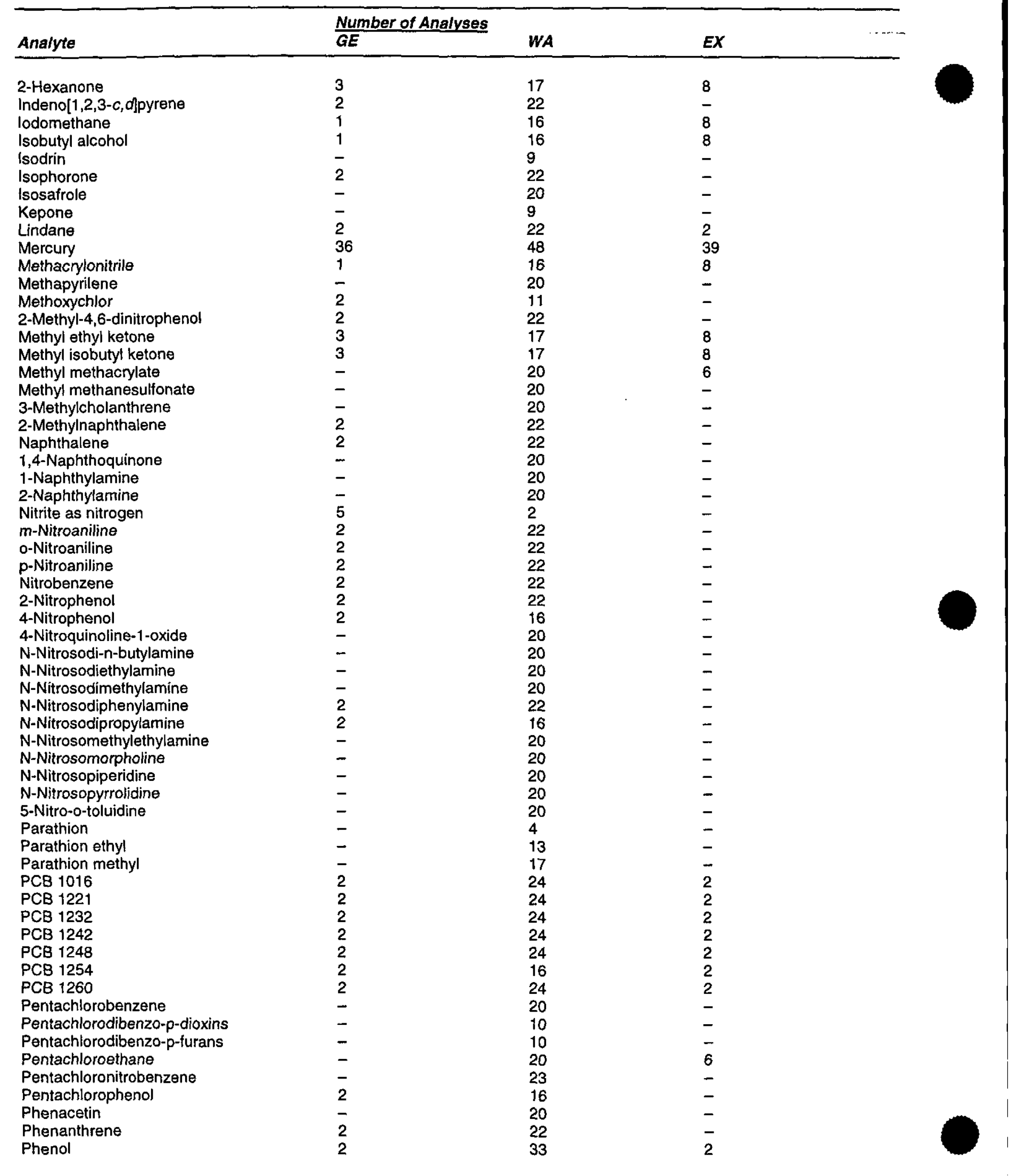




\begin{tabular}{|c|c|c|c|}
\hline Analyte & $\frac{\text { Num }}{G E}$ & WA & $E X$ \\
\hline Phenols & 37 & 7 & - \\
\hline p-Phenylenediamine & - & 20 & - \\
\hline Phorate & - & 17 & - \\
\hline 2-Picoline & - & 20 & - \\
\hline Pronamid & - & 20 & - \\
\hline Propionitrile & 1 & 16 & 8 \\
\hline Pyrene & 2 & 16 & - \\
\hline Pyridine & - & 20 & - \\
\hline Safrole & - & 20 & - \\
\hline Selenium & 36 & 82 & 84 \\
\hline Styrene & 3 & 17 & 8 \\
\hline Sulfotepp & - & 17 & - \\
\hline $2,4,5-T$ & - & 8 & - \\
\hline $2,3,7,8-T C D D$ & - & 10 & - \\
\hline $1,2,4,5$-Tetrachlorobenzene & - & 20 & - \\
\hline Tetrachlorodibenzo-p-dioxins & - & 10 & - \\
\hline Tetrachlorodibenzo-p-furans & - & 10 & - \\
\hline 1,1,1,2-Tetrachloroethane & 1 & 16 & 8 \\
\hline 1,1,2,2-Tetrachloroethane & 28 & 85 & 37 \\
\hline 2,3,4,6-Tetrachiorophenol & - & 20 & - \\
\hline Thallium & 13 & 21 & - \\
\hline Thionazin & - & 17 & - \\
\hline o-Toluidine & - & 20 & - \\
\hline Total organic halogens & 4 & 40 & - \\
\hline Toxaphene & 2 & 11 & - \\
\hline 2,4,5-TP (Silvex) & - & $B$ & - \\
\hline 1,2,4-Trichlorobenzene & 2 & 16 & - \\
\hline 1,1,1-Trichloroethane & 36 & 114 & 71 \\
\hline 1,1,2-Trichloroethane & 28 & 85 & 37 \\
\hline 2,4,5-Trichlorophenol & 2 & 22 & - \\
\hline 2,4,6-Trichlorophenol & 2 & 22 & - \\
\hline 1,2,3-Trichloropropane & 1 & 16 & 8 \\
\hline $0,0,0$-Triethyl phosphorothioate & - & 17 & - \\
\hline 1,3,5-Trinitrobenzene & - & 20 & - \\
\hline Vanadium & 26 & 30 & - \\
\hline Vinyl acetate & 3 & 17 & 8 \\
\hline
\end{tabular}

- No replicate or duplicate analyses were performed.

Table 17. Analytes Not Showing Measurable Concentrations above Estimated Quantitation Limits in Any Replicated or Duplicated Samples for GP and TM

\begin{tabular}{lll}
\hline & Number of Analyses & $T M$ \\
\hline Analyte & GP & \\
Antimony-124 & & 14 \\
Antimony-125 & - & 22 \\
Barium-133 & 75 & 27 \\
Cerium-144 & - & 22 \\
Cesium-134 & 75 & 27 \\
Cobalt-57 & 75 & 27 \\
Cobalt-58 & 75 & 27 \\
Curium-242 & - & 22 \\
Europium-154 & 79 & 19 \\
Manganese-54 & 75 & 27 \\
Neptunium-239 & 75 & 27 \\
& - & 22 \\
\hline
\end{tabular}




\begin{tabular}{lll}
\hline Analyte & Number of Analvses & TM \\
\hline Promethium-146 & GP & 27 \\
Sodium-22 & 75 & 27 \\
Tin-113 & 75 & 22 \\
Uranium-234 & - & 14 \\
Yttrium-88 & - & 27 \\
Zirconium-95 & 75 & 22 \\
\hline
\end{tabular}

- No replicate or duplicate analyses were performed.

Table 18. Intralaboratory MRD Indices for EX

\begin{tabular}{|c|c|c|c|c|c|c|c|}
\hline Analyte & $R D L$ & $\begin{array}{l}\text { In-house D } \\
\text { Number of } \\
\text { Dup. Pairs }\end{array}$ & MRD & MRDadj & $\begin{array}{l}\text { Blind Replic } \\
\text { Number of } \\
\text { Dup. Pairs }\end{array}$ & $M R D$ & MRDadj \\
\hline Acetone & $5.0 \mathrm{E}+01 \mu \mathrm{g} / \mathrm{L}$ & 0 & $\sim$ & - & 2 & 0.00 & 0.00 \\
\hline Aluminum & $2.0 \mathrm{E}+02 \mu \mathrm{g} / \mathrm{L}$ & 0 & - & - & 14 & 0.00 & 0.00 \\
\hline Barium & $1.0 \mathrm{E}+01 \mu \mathrm{g} / \mathrm{L}$ & 0 & - & - & 13 & 6.73 & 4.28 \\
\hline Benzene & $2.5 E+02 \mu g / L$ & 0 & - & - & 11 & 0.00 & 0.00 \\
\hline Boron & $1.0 \mathrm{E}+02 \mu \mathrm{g} / \mathrm{L}$ & 0 & - & - & 2 & 0.00 & 0.00 \\
\hline Carbon tetrachloride & $1.25 \mathrm{E}+02 \mu \mathrm{g} / \mathrm{L}$ & & - & - & 13 & 0.00 & 0.00 \\
\hline Chloride & $t$ & 2 & 1.17 & 1.17 & 0 & - & $\cdot$ \\
\hline Chlorobenzene & $2.5 \mathrm{E}+02 \mu \mathrm{g} / \mathrm{L}$ & 0 & - & - & 11 & 0.00 & 0.00 \\
\hline Chloroethane & $5.0 \mathrm{E}+02 \mu \mathrm{g} / \mathrm{L}$ & 0 & - & - & 11 & 0.00 & 0.00 \\
\hline Chloroethene & $2.5 \mathrm{E}+02 \mu \mathrm{g} / \mathrm{L}$ & 0 & - & - & 11 & 0.00 & 0.00 \\
\hline Chloroform & $1.25 \mathrm{E}+02 \mu \mathrm{g} / \mathrm{L}$ & & - & - & 13 & 0.00 & 0.00 \\
\hline Chromium & $1.0 \mathrm{E}+01 \mu \mathrm{g} / \mathrm{L}$ & 0 & - & - & 2 & 0.00 & 0.00 \\
\hline Cyanide & $1.0 \mathrm{E}+01 \mu \mathrm{g} / \mathrm{L}$ & 0 & - & - & 10 & 0.00 & 0.00 \\
\hline 1,4-Dichlorobenzene & $2.5 E+01 \mu g / L$ & 0 & - & - & 2 & 3.36 & 2.99 \\
\hline Dichlorodifluoromethane & $2.5 E+01 \mu g / L$ & 0 & - & - & 2 & 0.00 & 0.00 \\
\hline 1,1-Dichloroethane & $2.5 \mathrm{E}+02 \mu \mathrm{g} / \mathrm{L}$ & 0 & - & - & 11 & 0.00 & 0.00 \\
\hline 1,1-Dichloroethylene & $2.5 E+02 \mu g / L$ & 0 & - & - & 11 & 0.00 & 0.00 \\
\hline cis-1,2-Dichloroethylene & $5.0 \mathrm{E}+01 \mu \mathrm{g} / \mathrm{L}$ & 0 & - & - & 4 & 1.87 & 0.63 \\
\hline Dichloromethane & $2.5 \mathrm{E}+02 \mu \mathrm{g} / \mathrm{L}$ & 0 & - & - & 11 & 0.00 & 0.00 \\
\hline Ethylbenzene & $2.5 E+02 \mu g / L$ & 0 & - & - & 11 & 0.00 & 0.00 \\
\hline Fluoride & $2.0 \mathrm{E}+02 \mu \mathrm{g} / \mathrm{L}$ & 2 & 0.40 & 0.40 & 0 & - & - \\
\hline Iron & $2.0 \mathrm{E}+02 \mu \mathrm{g} / \mathrm{L}$ & 0 & - & - & 14 & 0.81 & 0.81 \\
\hline Lead & $1.0 \mathrm{E}+02 \mu \mathrm{g} / \mathrm{L}$ & 0 & - & - & 14 & 0.00 & 0.00 \\
\hline Manganese & $1.0 \mathrm{E}+01 \mu \mathrm{g} / \mathrm{L}$ & 0 & - & - & 3 & 3.81 & 2.20 \\
\hline Nickel & $5.0 \mathrm{E}+01 \mu \mathrm{g} / \mathrm{L}$ & 0 & - & - & 10 & 0.00 & 0.00 \\
\hline Nitrate as nitrogen & $2.0 \mathrm{E}+02 \mu \mathrm{g} / \mathrm{L}$ & 10 & 0.43 & 0.43 & 2 & 1.50 & 1.50 \\
\hline Nitrate-nitrite as nitrogen & $5.0 \mathrm{E}+02 \mu \mathrm{g} / \mathrm{L}$ & 1 & 2.16 & 2.16 & 0 & - & - \\
\hline Silver & $2.0 \mathrm{E}+01 \mu \mathrm{g} / \mathrm{L}$ & 0 & - & - & 2 & 0.00 & 0.00 \\
\hline Sodium & $1.0 \mathrm{E}+03 \mu \mathrm{g} / \mathrm{L}$ & 0 & - & - & 0 & - & - \\
\hline Sulfate & $4.0 \mathrm{E}+02 \mu \mathrm{g} / \mathrm{L}$ & 2 & 0.00 & 0.00 & 0 & - & - \\
\hline Tetrachloroethylene & $5.0 \mathrm{E}+01 \mu \mathrm{g} / \mathrm{L}$ & 0 & - & - & 13 & 0.81 & 0.81 \\
\hline Toluene & $2.5 \mathrm{E}+02 \mu \mathrm{g} / \mathrm{L}$ & 0 & - & - & 11 & 0.00 & 0.00 \\
\hline Total organic carbon & $5.0 E+03 \mu \mathrm{g} / \mathrm{L}$ & 0 & - & - & 0 & - & $\cdot$ \\
\hline
\end{tabular}




\begin{tabular}{|c|c|c|c|c|c|c|c|}
\hline \multirow[b]{2}{*}{ Analyte } & \multirow[b]{2}{*}{$R D L$} & \multicolumn{3}{|c|}{ In-house Duplicates } & \multicolumn{3}{|c|}{ Blind Replicates } \\
\hline & & $\begin{array}{l}\text { Number of } \\
\text { Dup. Pairs }\end{array}$ & MRD & MRDadj & $\begin{array}{l}\text { Number of } \\
\text { Dup. Pairs }\end{array}$ & MRD & MRDadJ \\
\hline Total phosphates (as P) & $2.5 \mathrm{E}+03 \mu \mathrm{g} / \mathrm{L}$ & 1 & 0.00 & 0.00 & 0 & - & - \\
\hline Trichloroethylene & $5.0 \mathrm{E}+00 \mu \mathrm{g} / \mathrm{L}$ & 0 & - & - & 14 & 3.37 & 2.75 \\
\hline Trichlorofluoromethane & $2.5 \mathrm{E}+02 \mu \mathrm{g} / \mathrm{L}$ & 0 & - & - & 11 & 0.00 & 0.00 \\
\hline Xylenes & $5.0 \mathrm{E}+01 \mu \mathrm{g} / \mathrm{L}$ & 0 & - & - & 2 & 0.31 & 0.12 \\
\hline
\end{tabular}

† No detection limit, or no replicate or duplicate results below detection limit.

- No replicate or duplicate analyses could be calculated.

Note: An MRD of 0.00 indicates no difference between any of the pairs of results used in calculating the MRD.

Table 19. Intralaboratory MRD Indices for GE

\begin{tabular}{|c|c|c|c|c|c|c|c|}
\hline Analyte & $R D L$ & $\begin{array}{l}\text { In-house Du } \\
\text { Number of } \\
\text { Dup. Pairs }\end{array}$ & $\frac{\text { eates }}{\text { MRD }}$ & MRDadj & $\begin{array}{l}\text { Blind Repli } \\
\text { Number of } \\
\text { Dup. Pairs }\end{array}$ & $M R D$ & MRDadj \\
\hline Acetone & $5.0 \mu \mathrm{g} / \mathrm{L}$ & 0 & - & . & 1 & 0.00 & 0.00 \\
\hline Aluminum & $50.0 \mu \mathrm{g} / \mathrm{L}$ & 0 & - & - & 17 & 4.79 & 3.89 \\
\hline Antimony & $10.0 \mu \mathrm{g} / \mathrm{L}$ & 0 & - & - & 13 & 0.00 & 0.00 \\
\hline Barium & $15.5 \mu g / L$ & 0 & - & - & 17 & 12.24 & 11.31 \\
\hline Benzene & $1.0 \mu \mathrm{g} / \mathrm{L}$ & 0 & - & - & 17 & 0.00 & 0.00 \\
\hline Calcium & $t$ & 0 & - & - & 1 & 3.86 & 3.86 \\
\hline Carbon tetrachloride & $1.0 \mu \mathrm{g} / \mathrm{L}$ & 0 & - & - & 17 & 0.00 & 0.00 \\
\hline Chlorobenzene & $1.0 \mu \mathrm{g} / \mathrm{L}$ & 0 & - & - & 17 & 0.00 & 0.00 \\
\hline Chloroethane & $1.0 \mathrm{\mu g} / \mathrm{L}$ & 0 & - & - & 13 & 0.23 & 0.07 \\
\hline Chloroethene & $1.0 \mu \mathrm{g} / \mathrm{L}$ & 0 & - & - & 13 & 0.00 & 0.00 \\
\hline Chloroform & $1.0 \mu \mathrm{g} / \mathrm{L}$ & 0 & - & - & 16 & 0.00 & 0.00 \\
\hline Chromium & $5.0 \mu g / L$ & 0 & - & - & 17 & 1.93 & 0.70 \\
\hline Cobalt & $5.0 \mu \mathrm{g} / \mathrm{L}$ & 0 & - & - & 12 & 0.00 & 0.00 \\
\hline Copper & $5.0 \mu \mathrm{g} / \mathrm{L}$ & 0 & - & - & 12 & 7.93 & 4.64 \\
\hline $\begin{array}{l}\text { Cyanide } \\
\text { 1,4-Dichlorobenzene }\end{array}$ & $\begin{array}{l}10.0 \mu \mathrm{g} / \mathrm{L} \\
10.0 \mu \mathrm{g} / \mathrm{L}\end{array}$ & $\begin{array}{l}32 \\
0\end{array}$ & 0.00 & 0.00 & $\begin{array}{l}12 \\
1\end{array}$ & $\begin{array}{l}0.00 \\
0.00\end{array}$ & $\begin{array}{l}0.00 \\
0.00\end{array}$ \\
\hline Dichlorodifluoromethane & $5.0 \mu \mathrm{g} / \mathrm{L}$ & 0 & - & - & 0 & - & - \\
\hline 1,1-Dichloroethane & $1.0 \mu \mathrm{g} / \mathrm{L}$ & 0 & - & - & 13 & 0.00 & 0.00 \\
\hline 1,1-Dichloroethylene & $1.0 \mu \mathrm{g} / \mathrm{L}$ & 0 & - & - & 17 & 0.00 & 0.00 \\
\hline cis-1,2-Dichloroethylene & $1.17 \mu \mathrm{g} / \mathrm{L}$ & 0 & - & - & 0 & - & - \\
\hline Dichloromethane & $9.44 \mu \mathrm{g} / \mathrm{L}$ & 0 & - & - & 11 & 0.00 & 0.00 \\
\hline Ethylbenzene & $1.0 \mu \mathrm{g} / \mathrm{L}$ & 0 & - & - & 13 & 0.00 & 0.00 \\
\hline Iron & $53.9 \mu \mathrm{g} / \mathrm{L}$ & 0 & - & - & 17 & 30.85 & 25.65 \\
\hline Lead & $5.0 \mu \mathrm{g} / \mathrm{L}$ & 0 & - & - & 16 & 4.04 & 1.40 \\
\hline Magnesium & $\dagger$ & 0 & - & - & 1 & 10.57 & 10.57 \\
\hline Manganese & $10.0 \mu g / L$ & 0 & - & - & 1 & 0.00 & 0.00 \\
\hline Nickel & $5.0 \mu \mathrm{g} / \mathrm{L}$ & 0 & - & - & 12 & 0.00 & 0.00 \\
\hline Nitrate-nitrite as nitrogen & $50.0 \mu \mathrm{g} / \mathrm{L}$ & 29 & 1.91 & 1.78 & 11 & 30.29 & 30.29 \\
\hline $\mathrm{pH}$ & $\dagger$ & 34 & 0.23 & 0.23 & 12 & 3.26 & 3.26 \\
\hline Potassium & $t$ & 0 & - & - & 1 & 9.50 & 9.50 \\
\hline Silver & $5.0 \mu \mathrm{g} / \mathrm{L}$ & 0 & - & - & 17 & 0.00 & 0.00 \\
\hline Sodium & $t$ & 0 & - & - & 1 & 2.88 & 2.88 \\
\hline Specific conductance & $t$ & 29 & 2.16 & 2.16 & 11 & 4.64 & 4.64 \\
\hline Sulfide & $t$ & 1 & 11.87 & 11.87 & 0 & - & - \\
\hline Tetrachloroethylene & $1.0 \mu \mathrm{g} / \mathrm{L}$ & 0 & - & - & 17 & 1.09 & 0.46 \\
\hline Tin & $5.0 \mu \mathrm{g} / \mathrm{L}$ & 0 & - & - & 6 & 0.00 & 0.00 \\
\hline Toluene & $1.0 \mu \mathrm{g} / \mathrm{L}$ & 0 & - & - & 17 & 0.00 & 0.00 \\
\hline
\end{tabular}




\begin{tabular}{|c|c|c|c|c|c|c|c|}
\hline Analyte & $A D L$ & $\begin{array}{l}\text { In-house } D \\
\text { Number of } \\
\text { Dup. Pairs }\end{array}$ & Mates & MRDadj & $\begin{array}{l}\text { Blind Repll } \\
\text { Number of } \\
\text { Dup. Pairs }\end{array}$ & $M R D$ & MRDad \\
\hline Trichloroethylene & $1.0 \mu \mathrm{g} / \mathrm{L}$ & 0 & - & - & 17 & 0.69 & 0.34 \\
\hline Trichlorofluoromethane & $5.0 \mu \mathrm{g} / \mathrm{L}$ & 0 & - & - & 12 & 0.00 & 0.00 \\
\hline Xylenes & $2.0 \mu \mathrm{g} / \mathrm{L}$ & 0 & - & - & 1 & 0.00 & 0.00 \\
\hline Zinc & $10.0 \mu \mathrm{g} / \mathrm{L}$ & 0 & - & - & 11 & 11.92 & 7.04 \\
\hline
\end{tabular}

† No detection limit, or no replicate or duplicate results below detection limit.

- No replicate or duplicate analyses could be calculated.

Note: An MRD of 0.00 indicates no difference between any of the pairs of results used in calculating the MRD. MRD results greater than or equal to 20 appear in bold.

Table 20. Intralaboratory MRD Matrix Spike Indices for GE

\begin{tabular}{|c|c|c|c|c|}
\hline Analyte & $R D L$ & $\begin{array}{l}\text { In-house D } \\
\text { Number of } \\
\text { Dup. Pairs }\end{array}$ & $M R D$ & MRDadj \\
\hline Acenaphthene & $\uparrow$ & 2 & 10.34 & 10.34 \\
\hline Aldrin & $t$ & 2 & 4.65 & 4.65 \\
\hline Benzene & $t$ & 60 & 3.43 & 3.43 \\
\hline Chlorobenzene & $t$ & 60 & 3.27 & 3.27 \\
\hline 4-Chloro-m-cresol & $t$ & 2 & 13.23 & 13.23 \\
\hline 2-Chlorophenol & $t$ & 2 & 6.57 & 6.57 \\
\hline $\mathrm{p}, \mathrm{p}^{\prime}-\mathrm{DDT}$ & $t$ & 2 & 0.63 & 0.63 \\
\hline 1,4-Dichlorobenzene & $t$ & 2 & 6.51 & 6.51 \\
\hline 1,1-Dichloroethylene & $t$ & 60 & 4.49 & 4.49 \\
\hline Dieldrin & $t$ & 2 & 2.63 & 2.63 \\
\hline 2,4-Dinitrotoluene & $\dagger$ & 2 & 12.43 & 12.43 \\
\hline Endrin & $t$ & 2 & 1.18 & 1.18 \\
\hline Heptachlor & $t$ & 2 & 1.98 & 1.98 \\
\hline Lindane & $t$ & 2 & 1.96 & 1.96 \\
\hline 4-Nitrophenol & $t$ & 2 & 8.30 & 8.30 \\
\hline N-Nitrosodipropylamine & $t$ & 2 & 8.57 & 8.57 \\
\hline PCB 1260 & $t$ & 2 & 21.07 & 21.07 \\
\hline Pentachtorophenol & $t$ & 2 & 11.61 & 11.61 \\
\hline Phenol & $t$ & 2 & 6.47 & 6.47 \\
\hline Pyrene & $t$ & 2 & 4.95 & 4.95 \\
\hline Toluene & $\dagger$ & 60 & 4.50 & 4.50 \\
\hline 1,2,4-Trichlorobenzene & $t$ & 2 & 7.47 & 7.47 \\
\hline Trichloroethylene & \pm & 60 & 3.91 & 3.91 \\
\hline
\end{tabular}

† No detection limit, or no replicate or duplicate results below detection limit.

Note: An MRD of 0.00 indicates no difference between any of the pairs of results used in calculating the MRD. MRD results greater than or equal to 20 appear in bold.

Table 21. Intralaboratory MRD Indices for WA

\begin{tabular}{|c|c|c|c|c|c|c|c|}
\hline Analyte & $R D L$ & $\begin{array}{l}\text { In-house DL } \\
\text { Number of } \\
\text { Dup. Pairs }\end{array}$ & $\begin{array}{l}\text { cates } \\
\text { MRD }\end{array}$ & MRDadJ & $\begin{array}{l}\text { Blind Repli } \\
\text { Number of } \\
\text { Dup. Pairs }\end{array}$ & $M R D$ & MRDad \\
\hline $\begin{array}{l}\text { Acetone } \\
\text { Aluminum }\end{array}$ & $\begin{array}{l}10.0 \mu \mathrm{g} / \mathrm{L} \\
146 \mu \mathrm{g} / \mathrm{L}\end{array}$ & $\begin{array}{l}3 \\
26\end{array}$ & $\begin{array}{l}0.00 \\
1.89\end{array}$ & $\begin{array}{l}0.00 \\
0.86\end{array}$ & $\begin{array}{l}3 \\
6\end{array}$ & $\begin{array}{l}0.00 \\
0.78\end{array}$ & $\begin{array}{l}0.00 \\
0.24\end{array}$ \\
\hline
\end{tabular}




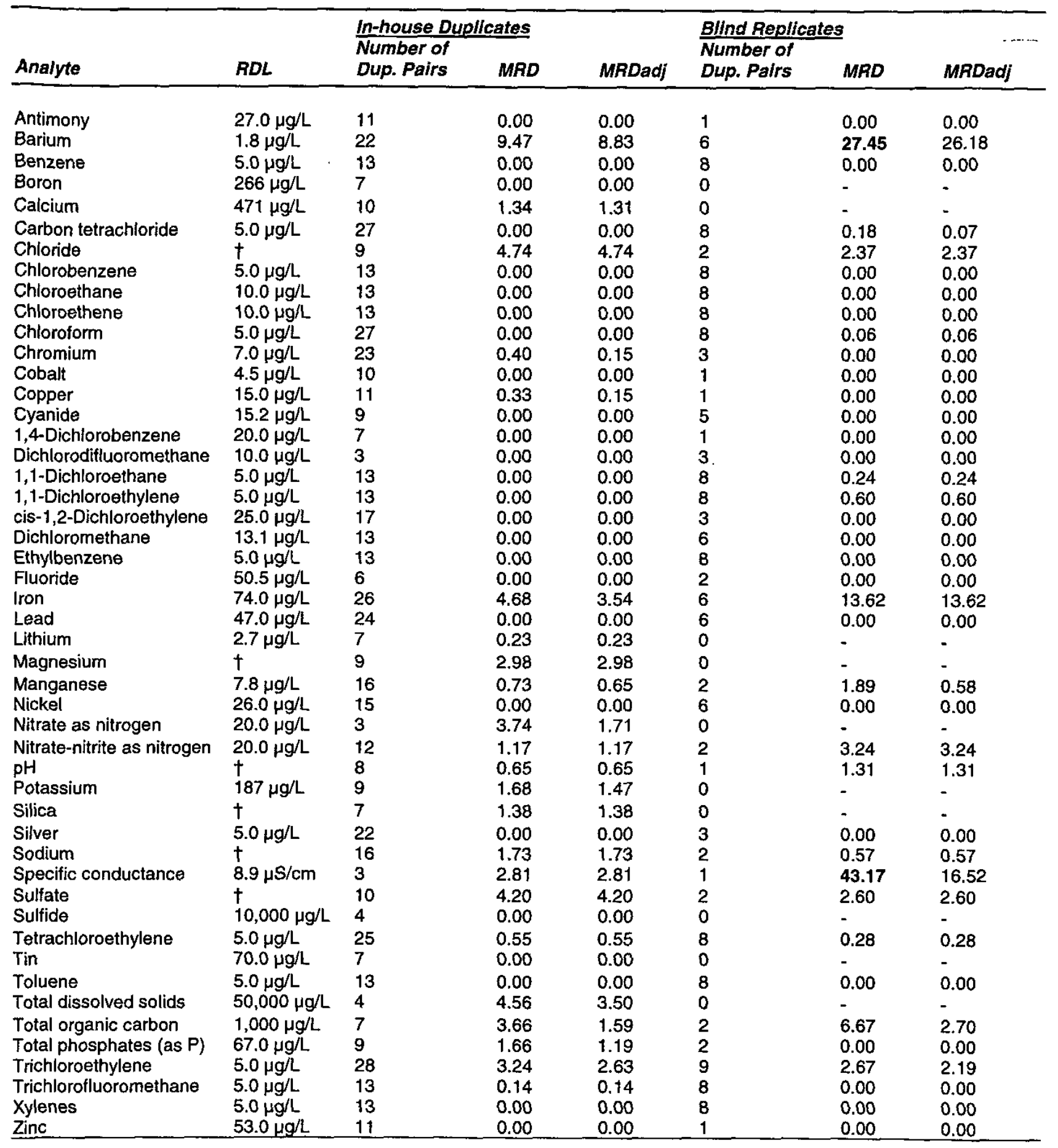

† No detection limit, or no replicate or duplicate results below detection limit.

- No replicate or duplicate analyses could be calculated.

Note: An MRD of 0.00 indicates no difference between any of the pairs of results used in calculating the MRD. MRD results greater than or equal to 20 appear in bold. 
Table 22. Intralaboratory MRD Indices for GP

\begin{tabular}{|c|c|c|c|c|c|c|c|}
\hline Analyte & $R D L$ & $\begin{array}{l}\text { In-house Du } \\
\text { Number of } \\
\text { Dup. Pairs }\end{array}$ & $\begin{array}{l}\text { gates } \\
\text { MRD }\end{array}$ & MRDadj & $\begin{array}{l}\text { Blind Replic } \\
\text { Number of } \\
\text { Dup. Pairs }\end{array}$ & MRD & MRDadj \\
\hline Actinium-228 & $1.74 \mathrm{E}-08 \mu \mathrm{Ci} / \mathrm{mL}$ & 23 & 1.73 & 1.09 & 10 & 0.00 & 0.00 \\
\hline Americium-241 & $9.91 \mathrm{E}-10 \mu \mathrm{Ci} / \mathrm{mL}$ & 24 & 0.87 & 0.87 & 11 & 0.00 & 0.00 \\
\hline Carbon-14 & 1.33E-08 $\mu \mathrm{Ci} / \mathrm{mL}$ & 19 & 15.24 & 9.40 & 12 & 6.19 & 3.06 \\
\hline Cesium-137 & 4.05E-09 $\mu \mathrm{Ci} / \mathrm{mL}$ & 23 & 1.46 & 0.70 & 10 & 21.43 & 13.85 \\
\hline Cobalt-60 & 4.38E-09 $\mu \mathrm{Ci} / \mathrm{mL}$ & 20 & 0.46 & 0.27 & 11 & 0.00 & 0.00 \\
\hline Curium-243/244 & 7.73E-10 $\mu \mathrm{Ci} / \mathrm{mL}$ & 26 & 3.73 & 1.98 & 10 & 0.00 & 0.00 \\
\hline Curium-245/246 & $3.6\} \mathrm{E}-10 \mu \mathrm{Ci} / \mathrm{mL}$ & 24 & 3.97 & 1.84 & 10 & 0.00 & 0.00 \\
\hline Europium-152 & 1.11E-08 $\mu \mathrm{Ci} / \mathrm{mL}$ & 23 & 0.91 & 0.31 & 11 & 0.00 & 0.00 \\
\hline Europium-155 & $1.43 \mathrm{E}-08 \mu \mathrm{Ci} / \mathrm{mL}$ & 22 & 0.00 & 0.00 & 12 & 0.00 & 0.00 \\
\hline Gross alpha & 1.03E-09 $\mu \mathrm{Ci} / \mathrm{mL}$ & 32 & 10.55 & 7.98 & 16 & 6.11 & 2.36 \\
\hline lodine-129 & 1.31E-09 $\mu \mathrm{Ci} / \mathrm{mL}$ & 19 & 6.09 & 4.54 & 11 & 1.05 & 0.33 \\
\hline Lead-212 & 7.57E-09 $\mu \mathrm{Ci} / \mathrm{mL}$ & 23 & 9.02 & 3.98 & 10 & 5.76 & 2.32 \\
\hline Nickel-63 & 1.47E-07 $\mu \mathrm{Ci} / \mathrm{mL}$ & 11 & 10.69 & 6.90 & 6 & 10.27 & 4.46 \\
\hline Nonvolatile beta & 1.39E-09 $\mu \mathrm{Ci} / \mathrm{mL}$ & 32 & 12.77 & 10.46 & 16 & 14.22 & 8.84 \\
\hline Plutonium-238 & $7.72 \mathrm{E}-10 \mu \mathrm{Ci} / \mathrm{mL}$ & 23 & 0.00 . & 0.00 & 11 & 0.00 & 0.00 \\
\hline Plutonium-239/240 & $4.51 \mathrm{E}-10 \mu \mathrm{Ci} / \mathrm{mL}$ & 22 & 2.42 & 0.86 & 10 & 0.00 & 0.00 \\
\hline Potassium-40 & 5.26E-08 $\mu \mathrm{Ci} / \mathrm{mL}$ & 20 & 0.00 & 0.00 & 12 & 4.13 & 1.65 \\
\hline Promethium-144 & 3.76E-09 $\mu \mathrm{Ci} / \mathrm{mL}$ & 22 & 0.00 & 0.00 & 9 & 0.00 & 0.00 \\
\hline $\begin{array}{l}\text { Radium, total alpha- } \\
\text { emitting }\end{array}$ & 8.74E-10 $\mu \mathrm{Ci} / \mathrm{mL}$ & 4 & 23.20 & 12.46 & 1 & 2.93 & 0.89 \\
\hline Radium-226 & $7.73 \mathrm{E}-10 \mu \mathrm{Ci} / \mathrm{mL}$ & 25 & 7.80 & 6.26 & 10 & 17.78 & 10.93 \\
\hline Radium-228 & $1.59 \mathrm{E}-09 \mu \mathrm{Ci} / \mathrm{mL}$ & 23 & 6.41 & 3.64 & 11 & 0.00 & 0.00 \\
\hline Ruthenium-106 & 3.42E-08 $\mu \mathrm{Ci} / \mathrm{mL}$ & 22 & 0.84 & 0.28 & 9 & 0.00 & 0.00 \\
\hline Strontium-89/90 & 6.14E-09 $\mu \mathrm{Ci} / \mathrm{mL}$ & 5 & 0.00 & 0.00 & 0 & - & - \\
\hline Strontium-90 & 2.07E-09 $\mu \mathrm{Ci} / \mathrm{mL}$ & 19 & 6.41 & 6.03 & 12 & 1.50 & 0.50 \\
\hline Technetium-99 & 2.4E-08 $\mu \mathrm{Ci} / \mathrm{mL}$ & 20 & 3.36 & 1.86 & 11 & 0.48 & 0.15 \\
\hline Thorium-228 & 7.27E-10 $\mu \mathrm{Ci} / \mathrm{mL}$ & 23 & 3.74 & 1.33 & 11 & 0.00 & 0.00 \\
\hline Thorium-230 & 4.13E-10 $\mu \mathrm{Ci} / \mathrm{mL}$ & 23 & 0.00 & 0.00 & 11 & 0.00 & 0.00 \\
\hline Thorium-232 & 3.37E-10 $\mu \mathrm{Ci} / \mathrm{mL}$ & 22 & 0.00 & 0.00 & 11 & 0.00 & 0.00 \\
\hline Tritium & 7.14E-07 $\mu \mathrm{Ci} / \mathrm{mL}$ & 23 & 5.73 & 5.06 & 17 & 13.44 & 12.17 \\
\hline Uranium-233/234 & $6.18 \mathrm{E}-10 \mu \mathrm{Ci} / \mathrm{mL}$ & 26 & 6.41 & 4.68 & 11 & 0.00 & 0.00 \\
\hline Uranium-235 & $5.24 \mathrm{E}-10 \mu \mathrm{Ci} / \mathrm{mL}$ & 23 & 1.17 & 1.17 & 11 & 0.00 & 0.00 \\
\hline Uranium-238 & 3.17E-10 $\mu \mathrm{Ci} / \mathrm{mL}$ & 25 & 7.09 & 6.27 & 11 & 0.00 & 0.00 \\
\hline Zinc-65 & 8.56E-09 $\mu \mathrm{Ci} / \mathrm{mL}$ & 22 & 2.34 & 0.95 & 11 & 0.00 & 0.00 \\
\hline
\end{tabular}

- No replicate or duplicate analyses could be calculated.

Note: An MRD of 0.00 indicates no difference between any of the pairs of results used in calculating the MRD. MRD results greater than or equal to 20 appear in bold. 
Table 23. Intralaboratory MRD Indices for TM

\begin{tabular}{|c|c|c|c|c|c|c|c|}
\hline Analyte & $R D L$ & $\begin{array}{l}\text { In-house Du } \\
\text { Number of } \\
\text { Dup. Pairs }\end{array}$ & Mates & MRDad J & $\begin{array}{l}\text { Blind Replic } \\
\text { Number of } \\
\text { Dup. Pairs }\end{array}$ & $M R D$ & MRDadJ \\
\hline Actinium-228 & $2.742 \mathrm{E}-08 \mu \mathrm{Ci} / \mathrm{mL}$ & 9 & 0.00 & 0.00 & 0 & - & - \\
\hline Carbon-14 & $1.8131 \mathrm{E}-07 \mu \mathrm{Ci} / \mathrm{mL}$ & 7 & 4.55 & 2.32 & 0 & - & - \\
\hline Cesium-137 & $6.99 \mathrm{E}-09 \mu \mathrm{Ci} / \mathrm{mL}$ & 10 & 0.00 & 0.00 & 0 & - & - \\
\hline Cobalt-60 & 8.05E-09 $\mu \mathrm{Ci} / \mathrm{mL}$ & 9 & 0.00 & 0.00 & 0 & - & - \\
\hline Curium-243/244 & 8.2E-10 $\mu \mathrm{Ci} / \mathrm{mL}$ & 6 & 0.00 & 0.00 & 0 & - & - \\
\hline Europium-152 & 5.036E- $08 \mu \mathrm{Ci} / \mathrm{mL}$ & 10 & 0.34 & 0.10 & 0 & - & - \\
\hline Europium-155 & 1.366E-08 $\mu \mathrm{Ci} / \mathrm{mL}$ & 10 & 0.00 & 0.00 & 0 & - & - \\
\hline Gross alpha & $1.51 \mathrm{E}-09 \mu \mathrm{Ci} / \mathrm{mL}$ & 84 & 11.99 & 8.75 & 8 & 16.73 & 9.91 \\
\hline lodine-129 & $1.206 \mathrm{E}-08 \mu \mathrm{Ci} / \mathrm{mL}$ & 7 & 2.25 & 0.73 & 0 & - & - \\
\hline Lead-212 & $8.48 \mathrm{E}-09 \mu \mathrm{Ci} / \mathrm{mL}$ & 10 & 0.00 & 0.00 & 0 & - & - \\
\hline Nickel-63 & 2.3E-08 $\mu \mathrm{Ci} / \mathrm{mL}$ & 4 & 0.00 & 0.00 & 0 & - & - \\
\hline Nonvolatile beta & $3.56 \mathrm{E}-09 \mu \mathrm{Ci} / \mathrm{mL}$ & 65 & 3.66 & 2.51 & 6 . & 13.33 & 5.00 \\
\hline Plutonium-238 & $5.2 \mathrm{E}-10 \mu \mathrm{Ci} / \mathrm{mL}$ & 7 & 0.00 & 0.00 & 0 & - & - \\
\hline Plutonium-239/240 & $5.1 \mathrm{E}-10 \mu \mathrm{Ci} / \mathrm{mL}$ & 7 & 0.00 & 0.00 & 0 & - & - \\
\hline Potassium-40 & 8.293E- $08 \mu \mathrm{Ci} / \mathrm{mL}$ & 9 & 0.00 & 0.00 & 0 & - & - \\
\hline Promethium-144 & $6.48 \mathrm{E}-09 \mu \mathrm{Ci} / \mathrm{mL}$ & 10 & 0.00 & 0.00 & 0 & - & - \\
\hline $\begin{array}{l}\text { Radium, total aipha- } \\
\text { emitting }\end{array}$ & $1.52 \mathrm{E}-09 \mu \mathrm{Ci} / \mathrm{mL}$ & 16 & 6.33 & 3.47 & 2 & 0.00 & 0.00 \\
\hline Radium-226 & $3.9 \mathrm{E}-10 \mu \mathrm{Ci} / \mathrm{mL}$ & 7 & 0.00 & 0.00 & 0 & - & - \\
\hline Radium-228 & 2.59E-09 $\mu \mathrm{Ci} / \mathrm{mL}$ & 7 & 0.00 & 0.00 & 0 & - & - \\
\hline Ruthenium-106 & $5.706 \mathrm{E}-08 \mu \mathrm{Ci} / \mathrm{mL}$ & 10 & 0.00 & 0.00 & 0 & - & . \\
\hline Strontium-90 & $1.7 \mathrm{E}-09 \mu \mathrm{Ci} / \mathrm{mL}$ & 7 & 0.00 & 0.00 & 0 & - & - \\
\hline Technetium-99 & $1.502 \mathrm{E}-08 \mu \mathrm{Ci} / \mathrm{mL}$ & 7 & 4.24 & 1.76 & 0 & - & - \\
\hline Thorium-228 & $5.7 \mathrm{E}-10 \mu \mathrm{Ci} / \mathrm{mL}$ & 6 & 0.00 & 0.00 & 0 & - & - \\
\hline Thorium-230 & $4.8 \mathrm{E}-10 \mu \mathrm{Ci} / \mathrm{mL}$ & 7 & 2.45 & 0.80 & 0 & - & - \\
\hline Thorium-232 & $5.0 \mathrm{E}-10 \mu \mathrm{Ci} / \mathrm{mL}$ & 7 & 0.00 & 0.00 & 0 & - & - \\
\hline Tritium & $7.8 \mathrm{E}-07 \mu \mathrm{Ci} / \mathrm{mL}$ & 89 & 12.03 & 10.32 & 6 & 11.30 & 9.06 \\
\hline Uranium-235 & $3.4 \mathrm{E}-10 \mu \mathrm{Ci} / \mathrm{mL}$ & 7 & 0.00 & 0.00 & 0 & - & - \\
\hline Uranium-238 & 3.0E-10 $\mu \mathrm{Ci} / \mathrm{mL}$ & 6 & 0.00 & 0.00 & 0 & - & - \\
\hline Zinc-65 & $1.551 \mathrm{E}-08 \mu \mathrm{Ci} / \mathrm{mL}$ & 10 & 0.00 & 0.00 & 0 & - & - \\
\hline
\end{tabular}

- No replicate or duplicate analyses could be calculated.

Note: An MRD of 0.00 indicates no difference between any of the pairs of results used in calculating the MRD. 
Table 24. Interlaboratory MRD and t-test Results for Analytes with at Least One Pair of Results above the RDL for EX and WA

\begin{tabular}{|c|c|c|c|c|}
\hline Analyte & $R D L$ & Unit & $M R D$ & $\begin{array}{l}\text { t-test } \\
\text { Probability }\end{array}$ \\
\hline Acetone & 10.0 & $\mu g / L$ & 18.75 & .391 \\
\hline Aluminum & 200 & $\mu g / L$ & 6.11 & .389 \\
\hline Barium & 10.0 & $\mu g / L$ & 20.60 & .164 \\
\hline Benzene & 125 & $\mu g / L$ & 0.00 & - \\
\hline Boron & 266 & $\mu g / L$ & 0.00 & - \\
\hline Carbon tetrachioride & 50.0 & $\mu g / L$ & 0.00 & - \\
\hline Chlorobenzene & 100 & $\mu g / L$ & 0.00 & - \\
\hline Chloroethene & 125 & $\mu g / L$ & 1.34 & .334 \\
\hline Chloroform & 50.0 & $\mu g / L$ & 0.00 & - \\
\hline Chromium & 10.0 & $\mu g / L$ & 0.00 & - \\
\hline Cyanide & 15.2 & $\mu g / L$ & 1.75 & .334 \\
\hline 1,4-Dichlorobenzene & 20.0 & $\mu g / L$ & 4.69 & .058 \\
\hline Dichlorodifluoromethane & 10.0 & $\mu g / L$ & 23.63 & .250 \\
\hline 1,1-Dichtoroethane & 125 & $\mu g / L$ & 0.00 & - \\
\hline 1,1-Dichloroethylene & 100 & $\mu \mathrm{g} / \mathrm{L}$ & 0.00 & - \\
\hline cis-1,2-Dichloroethylene & 25.0 & $\mu g / L$ & 0.00 & - \\
\hline Dichloromethane & 125 & $\mu g / L$ & 0.71 & .333 \\
\hline Ethylbenzene & 125 & $\mu g / L$ & 0.00 & - \\
\hline Fluoride & 200 & $\mu g / L$ & 0.00 & - \\
\hline Iron & 200 & . $\mu g / L$ & 4.72 & .279 \\
\hline Lead & 100 & $\mu g / L$ & 0.00 & - \\
\hline Manganese & 10.0 & $\mu g / L$ & 11.90 & .508 \\
\hline Nickel & 50.0 & $\mu \mathrm{g} / \mathrm{L}$ & 0.00 & - \\
\hline Nitrate as nitrogen & 200 & $\mu g / L$ & 3.85 & .423 \\
\hline Nitrate-nitrite as nitrogen & 500 & $\mu g / \mathrm{L}$ & 11.33 & .500 \\
\hline Sodium & 1,000 & $\mu g / L$ & 15.04 & .193 \\
\hline Sulfate & 400 & $\mu g / L$ & 25.73 & .100 \\
\hline Tetrachloroethylene & 20.0 & $\mu g / L$ & 3.38 & .300 \\
\hline Toluene & 100 & $\mu g / L$ & 0.00 & - \\
\hline Total organic carbon & 5,000 & $\mu g / L$ & 9.91 & .500 \\
\hline Total phosphates (as P) & 2,500 & $\mu g / L$ & 0.00 & - \\
\hline Trichloroethylene & 5.0 & $\mu g / L$ & 13.74 & .119 \\
\hline Trichlorofluoromethane & 125 & $\mu g / L$ & 0.00 & - \\
\hline Xylenes & 10.0 & $\mathrm{\mu g} / \mathrm{L}$ & 0.71 & .391 \\
\hline
\end{tabular}

- Could not calculate because there are no differences between pairs.

Note: Values less than .050 indicate a probability of less than 1 in 20 that the results for that analyte are the same from both laboratories. MRD results greater than or equal to 20 appear in bold; less than or equal to .050 , in bold italic. 
Table 25. Interlaboratory MRD and t-test Results for Analytes with at Least One Pair of Results above the RDL for GE and WA

\begin{tabular}{|c|c|c|c|c|}
\hline Analyte & $R D L$ & Unit & MRD & $\begin{array}{l}\text { t-test } \\
\text { Probability }\end{array}$ \\
\hline $\begin{array}{l}\text { Aluminum } \\
\text { Antimony }\end{array}$ & $\begin{array}{l}146 \\
27.0\end{array}$ & $\begin{array}{l}\mu g / L \\
\mu g / L\end{array}$ & $\begin{array}{l}3.67 \\
0.00\end{array}$ & $\begin{array}{l}.450 \\
-\end{array}$ \\
\hline $\begin{array}{l}\text { Barium } \\
\text { Calcium }\end{array}$ & $\begin{array}{l}15.5 \\
471\end{array}$ & $\begin{array}{l}\mu g / L \\
\mu g / L\end{array}$ & $\begin{array}{l}9.83 \\
6.48\end{array}$ & .220 \\
\hline Carbon tetrachloride & 5.0 & $\mu g / L$ & 0.00 & - \\
\hline Chloroethane & 10.0 & $\mu g / L$ & 0.00 & - \\
\hline Chloroform & 5.0 & $\mu g / L$ & 0.00 & - \\
\hline $\begin{array}{l}\text { Chromium } \\
\text { Cobalt }\end{array}$ & $\begin{array}{l}7.0 \\
4.5\end{array}$ & $\mu g / L$ & $\begin{array}{l}1.31 \\
0.00\end{array}$ & .406 \\
\hline $\begin{array}{l}\text { Copper } \\
\text { 1,1-Dichloroethane }\end{array}$ & $\begin{array}{l}15.0 \\
5.0\end{array}$ & $\begin{array}{l}\mu g / L \\
\mu g / L\end{array}$ & $\begin{array}{l}1.77 \\
0.00\end{array}$ & .295 \\
\hline $1, t$-Dichloroethylene & 5.0 & $\mu g / L$ & 0.00 & - \\
\hline Dichloromethane & 10.7 & $\mu g / L$ & 0.00 & - \\
\hline Iron & 74.0 & $\mu g / L$ & 25.25 & .068 \\
\hline Lead & 47.0 & $\mu g / L$ & 0.00 & - \\
\hline Nickel & 26.0 & $\mu g / L$ & 0.00 & - \\
\hline Nitrate-nitrite as nitrogen & 50.0 & $\mu g / L$ & 22.14 & .482 \\
\hline Potassium & 187 & $\mu g / L$ & 3.55 & - \\
\hline Silver & 5.0 & $\mu g / L$ & 3.78 & .331 \\
\hline Specific conductance & 8.9 & $\mu \mathrm{S} / \mathrm{cm}$ & 21.66 & .000 \\
\hline Tetrachloroethylene & 5.0 & $\mu g / L$ & 0.00 & - \\
\hline Tin & 70.0 & $\mu g / L$ & 0.00 & - \\
\hline Trichloroethylene & 5.0 & $\mu g / L$ & 0.00 & - \\
\hline Trichlorofluoromethane & 5.0 & $\mu g / L$ & 0.00 & - \\
\hline Zinc & 53.0 & $\mu g / L$ & 0.51 & .336 \\
\hline
\end{tabular}

- Could not calculate because there are no differences between pairs.

Note: Values less than .050 indicate a probability of less than 1 in 20 that the results for that analyte are the same from both laboratories. MRD results greater than or equal to 20 appear in bold; less than or equal to .050 , in bold italic..

Table 26. Interlaboratory MRD and t-test Results for Analytes with at Least One Pair of Results above the RDL for GP and TM

\begin{tabular}{|c|c|c|c|c|}
\hline Analyte & $R D L$ & Unit & $M R D$ & $\begin{array}{l}\text { t-test } \\
\text { Probability }\end{array}$ \\
\hline Actinium-228 & $2.419 E-08$ & $\mu \mathrm{Ci} / \mathrm{mL}$ & 0.00 & - \\
\hline Carbon-14 & $1.789 E-07$ & $\mu \mathrm{Ci} / \mathrm{mL}$ & 6.26 & .302 \\
\hline Cesium-137 & $6.2 \mathrm{E}-09$ & $\mu \mathrm{Ci} / \mathrm{mL}$ & 5.97 & .177 \\
\hline Cobalt-60 & $6.93 \mathrm{E}-09$ & $\mu \mathrm{Ci} / \mathrm{mL}$ & 0.00 & - \\
\hline Curium-243/244 & $8.0 \mathrm{E}-10$ & $\mu \mathrm{Ci} / \mathrm{mL}$ & 0.00 & - \\
\hline Europium-152 & 4.352E-08 & $\mu \mathrm{Ci} / \mathrm{mL}$ & 0.85 & .341 \\
\hline Europium-155 & 1.41E-08 & $\mu \mathrm{Ci} / \mathrm{mL}$ & 0.00 & - \\
\hline Gross alpha & $1.51 E-09$ & $\mu \mathrm{Ci} / \mathrm{mL}$ & 19.22 & .067 \\
\hline lodine-129 & $1.109 E-08$ & $\mu \mathrm{Ci} / \mathrm{mL}$ & 1.42 & .347 \\
\hline Lead-212 & 8.17E-09 & $\mu \mathrm{Ci} / \mathrm{mL}$ & 5.69 & .527 \\
\hline Nickel-63 & $7.87 \mathrm{E}-08$ & $\mu \mathrm{Ci} / \mathrm{mL}$ & 18.62 & .363 \\
\hline
\end{tabular}




\begin{tabular}{lllll}
\hline Analyte & RDL & Unit & MRD & $\begin{array}{l}\text { t-test } \\
\text { Probability }\end{array}$ \\
\hline Nonvolatile beta & & & & .348 \\
Plutonium-238 & $3.55 \mathrm{E}-09$ & $\mu \mathrm{Ci} / \mathrm{mL}$ & 21.96 & - \\
Plutonium-239/240 & $7.64 \mathrm{E}-10$ & $\mu \mathrm{Ci} / \mathrm{mL}$ & 0.00 & - \\
Potassium-40 & $5.1 \mathrm{E}-10$ & $\mu \mathrm{Ci} / \mathrm{mL}$ & 0.00 & - \\
Promethium-144 & $6.604 \mathrm{E}-08$ & $\mu \mathrm{Ci} / \mathrm{mL}$ & 0.00 & - \\
Radium, total alpha-emitting & $5.8 \mathrm{E}-09$ & $\mu \mathrm{Ci} / \mathrm{mL}$ & 0.00 & - \\
Radium-226 & $1.52 \mathrm{E}-09$ & $\mu \mathrm{Ci} / \mathrm{mL}$ & 0.00 & .084 \\
Radium-228 & $7.38 \mathrm{E}-10$ & $\mu \mathrm{Ci} / \mathrm{mL}$ & 13.72 & .341 \\
Ruthenium-106 & $2.35 \mathrm{E}-09$ & $\mu \mathrm{Ci} / \mathrm{mL}$ & 16.70 & - \\
Strontium-90 & $5.183 \mathrm{E}-08$ & $\mu \mathrm{Ci} / \mathrm{mL}$ & 0.00 & .304 \\
Technetium-99 & $1.92 \mathrm{E}-09$ & $\mu \mathrm{Ci} / \mathrm{mL}$ & 1.65 & .336 \\
Thorium-228 & $2.34 \mathrm{E}-08$ & $\mu \mathrm{Ci} / \mathrm{mL}$ & 0.21 & - \\
Thorium-230 & $6.3 \mathrm{E}-10$ & $\mu \mathrm{Ci} / \mathrm{mL}$ & 0.00 & .225 \\
Thorium-232 & $4.3 \mathrm{E}-10$ & $\mu \mathrm{Ci} / \mathrm{mL}$ & 5.07 & .341 \\
Tritium & $3.7 \mathrm{E}-10$ & $\mu \mathrm{Ci} / \mathrm{mL}$ & 2.54 & .773 \\
Uranium-235 & $7.17 \mathrm{E}-07$ & $\mu \mathrm{Ci} / \mathrm{mL}$ & 13.19 & - \\
Uranium-238 & $4.54 \mathrm{E}-10$ & $\mu \mathrm{Ci} / \mathrm{mL}$ & 0.00 & - \\
Zinc-65 & $3.0 \mathrm{E}-10$ & $\mu \mathrm{Ci} / \mathrm{mL}$ & 0.00 & - \\
\hline
\end{tabular}

- Could not calculate because there are no differences between pairs.

Note: Values less than .050 indicate a probability of less than 1 in 20 that the results for that analyte are the same from both laboratories. MRD results greater than or equal to 20 appear in bold; less than or equal to .050 , in bold italic.

Table 27. EX Samples and Blind Replicates Yielding Results Where One Is More Than Twice Another

\begin{tabular}{ll}
\hline Analyte & Wells \\
\hline Barium & LFW 67C \\
\hline
\end{tabular}

Table 28. GE Samples and Blind Replicates Yielding Results Where One Is More Than Twice Another

\begin{tabular}{ll}
\hline Analyte & Wells \\
\hline & \\
Barium & FSB121C \\
Copper & CSB 2C \\
Iron & CSB 2C, FSB 121C, HAA 5C \\
Nitrate-nitrite as nitrogen & FSB 79A, FSB 89C \\
\hline
\end{tabular}

Table 29. WA Samples and Blind Replicates Yielding Results Where One Is More Than Twice Another

\begin{tabular}{ll}
\hline Analyte & Wells \\
\hline Barium & AMB 11B
\end{tabular}

\section{Quality Control Samples}




\begin{tabular}{ll}
\hline Analyte & Wells \\
\hline Iron & ASB 8TA \\
\hline
\end{tabular}

Table 30. WA Samples and Laboratory Duplicates Yielding Results Where One Is More Than Twice Another

\begin{tabular}{ll}
\hline Analyte & Wells \\
\hline Barium & AMB 11B \\
\hline
\end{tabular}

Table 31. GP Samples and Blind Replicates Yielding Results Where One Is More Than Twice Another

\begin{tabular}{ll}
\hline Analyte & Wells \\
\hline & \\
Cesium-137 & FSB121C, ZBG 1 \\
Radium-226 & FSB 76C, FSB121C \\
\hline
\end{tabular}

Table 32. GP Samples and Laboratory Duplicates Yielding Results Where One Is More Than Twice Another

\begin{tabular}{ll}
\hline Analyte & Wells \\
\hline Carbon-14 & QA 98A \\
Nickel-63 & HSB152D \\
Nonvolatile beta & HIN600TK \\
Radium, total alpha-emitting & ZBG 2 \\
Radium-228 & HSB114D \\
\hline
\end{tabular}

Note: Results for blind blanks are given in Appendix C.

Table 33. TM Samples and Blind Replicates Vielding Results Where One Is More Than Twice Another

\begin{tabular}{ll}
\hline Analyte & Wells \\
\hline Gross alpha & $P 26 \mathrm{~A}$ \\
\hline
\end{tabular}

Table 34. TM Samples and Laboratory Duplicates Vielding Results Where One Is More Than Twice Another

\begin{tabular}{ll}
\hline Analyte Wells & W___n \\
\hline
\end{tabular}




\begin{tabular}{ll}
\hline Analyte & Wells \\
\hline Tritium & $\mathrm{P} 29 \mathrm{D}$ \\
\hline
\end{tabular}

Table 35. Analytes with One Laboratory's Result Greater Than Twice the Result from the Other Laboratory between EX and WA

\begin{tabular}{ll}
\hline Analyte & Wells \\
\hline & \\
Acetone & LFW 67C \\
Aluminum & TNX 26D \\
Barium & ABP 2A, MSB 42B \\
Trichloroethylene & MSB 12A \\
\hline
\end{tabular}

Table 36. Analytes with One Laboratory's Result Greater Than Twice the Result from the Other Laboratory between GE and WA

\begin{tabular}{ll}
\hline Analyte & Wells \\
\hline & \\
Barium & FSB121C \\
Iron & FSB121C, HSB122A \\
Nitrate-nitrite as nitrogen & FSB 89C, HSB144A \\
Silver & HSB 83C \\
\hline
\end{tabular}

Table 37. Analytes with One Laboratory's Result Greater Than Twice the Result from the Other Laboratory between GP and TM

\begin{tabular}{ll}
\hline Analyte & Wells \\
\hline & \\
Gross alpha & HAA 5A, HAA 15C, P 26A \\
Nickel-63 & HSB144A \\
Nonvolatile beta & FSB121C, HSB100D, HSB112C, TBG 5B \\
Radium-226 & FSB 76C \\
Radium-228 & HSB100D \\
\hline
\end{tabular}

Table 38. Quality Control Standards for Selected Analyses for EX

\begin{tabular}{lllll}
\hline & Certified & Performance & EX & Functional \\
Analyte & Value & Acceptance Limits & Result & Guideline Code \\
\hline
\end{tabular}

Acids (Lot 586)

2,6-Dichlorophenol $(\mu g / L)$

2-Methylphenol $(\mu g / L)$

4-Methylphenol $(\mu \mathrm{g} / \mathrm{L})$

Pentachlorophenol $(\mu \mathrm{g} / \mathrm{L})$

2,4,5-Trichlorophenol $(\mu \mathrm{g} / \mathrm{L})$

2,4,6-Trichlorophenol $(\mu \mathrm{g} / \mathrm{L})$

132
59.9
82.8
129
60.5
55.7

$72.9-153$

19.0-69.3

25.4-95.9

$40.2-162$

23.8-69.9

$24.0-63.8$

$\dagger$

37.7

53.0

96.4

43.2

38.5 


\begin{tabular}{lllll}
\hline Analyte & Certified & Performance & EX & Functional \\
Value & Acceptance Limits & Result & Guideline Code \\
\hline
\end{tabular}

Base/Neutrals (Lot 586)

Anthracene $(\mu g / L)$

Benzo $[g, h, i]$ perylene $(\mu g / L)$

Bis(2-ethylhexyl) phthalate $(\mu \mathrm{g} / \mathrm{L})$

4-Bromophenyl-phenylether ( $\mu g / L)$

Chrysene ( $\mu \mathrm{g} / \mathrm{L})$

185
28.7

Dibenz[a, $h]$ anthracene $(\mu g / L)$

Dibenzofuran $(\mu \mathrm{g} / \mathrm{L})$

1,2-Dichlorobenzene $(\mu \mathrm{g} / \mathrm{L})$

Diethylphthlate $(\mu \mathrm{g} / \mathrm{L})$

Dimethylphthlate $(\mu g / L)$

2,4-Dinitrotoluene $(\mu \mathrm{g} / \mathrm{L})$

Naphthalene $(\mu g / L)$

N-Nitroso-di-n-propylamine ( $\mu \mathrm{g} / \mathrm{L})$

Phenanthrene $(\mu g / L)$

Pyrene $(\mu g / L)$

1,2,4-Trichlorobenzene $(\mu g / L)$

40.7-101

7.16-24.3

31.0-98.1

91.4-221

13.3-35.2

5.26-19.0

56.1-131

26.4-129

21.8-117

59.4-160

43.5-120

31.9-102

26.7-77.6

29.9-63.5

46.6-121

29.7-116

101,000

74,900

Calcium $(\mu g / L)$

Magnesium $(\mu g / L)$

Potassium $(\mu g / L)$

Sodium $(\mu g / L)$

90,300

94,900

Cyanide and Phenol (Lot 9987)

Cyanide, total $(\mu \mathrm{g} / \mathrm{L})$

Phenol $(\mu g / L)$

82.6

106

50.6

151,000
56,800
4,730
3,260
9.23
18,800
144,000
676
79,500
607,000

3,560

5,420

4,060

Total phosphates (as P) ( $\mu g / L)$

PCBs (Lot 586)

PCB $1254(\mu g / L)$

Pesticides (Lot 586)

Aldrin ( $\mu g / L)$

beta-Benzene hexachloride $(\mu \mathrm{g} / \mathrm{L})$

delta-Benzene hexachloride $(\mu g / L)$

gamma-Benzene hexachloride (Lindane)
5.71

1.60

7.12

5.74

1.04
$90,900-111,000$

$66,700-83,100$

$82,200-98,400$

$84,500-106,000$

60.3-105

80.6-131

$30.4-63.3$

$141,000-170,000$

$50,500-64,100$

$4,260-5,200$

$2,930-3,590$

9.03-9.43

$16,100-21,900$

$130,000-159,000$

$566-769$

$68,200-89,900$

$497,000-680,000$

$2,990-4,130$

$4,820-6,020$

$3,450-4,670$

$3.42-7.17$

$0.830-1.97$

3.80-9.00

2.53-7.37

$0.585-1.35$
43.2

4.50

1.00

5.50

73.4

14.0

70.1

128

26.2

10.8

79.3

58.1

11.7

4.03

74.4

49.6

30.1

45.7

86.5

53.4

103,000

72,100

81,700

87,700

77.1

109

166,000

58,800

3,930

3,160

9.18

18,700

142,000

685

80,600

600,000

2,910

5,250

4,060

5.20

0.64 


\begin{tabular}{|c|c|c|c|c|}
\hline Analyte & $\begin{array}{l}\text { Certified } \\
\text { Value }\end{array}$ & $\begin{array}{l}\text { Performance } \\
\text { Acceptance Limits }\end{array}$ & $\begin{array}{l}\text { EX } \\
\text { Result }\end{array}$ & $\begin{array}{l}\text { Functional } \\
\text { Guideline Code }\end{array}$ \\
\hline $\begin{array}{l}\quad(\mu g / L) \\
\text { gamma-Chlordane }(\mu g / L) \\
4,4^{\prime}-D D D(\mu g / L) \\
4,4^{\prime}-D D E(\mu g / L) \\
4,4^{\prime}-D D T(\mu g / L) \\
\text { Dieldrin }(\mu g / L) \\
\text { Endrin }(\mu g / L) \\
\text { Heptachlor }(\mu g / L) \\
\text { Heptachlor epoxide }(\mu g / L)\end{array}$ & $\begin{array}{l}8.56 \\
6.50 \\
0.877 \\
1.19 \\
3.65 \\
1.43 \\
5.83 \\
0.915\end{array}$ & $\begin{array}{l}5.96-10.2 \\
3.99-8.44 \\
0.511-1.10 \\
0.692-1.49 \\
2.26-4.72 \\
0.896-1.85 \\
2.64-7.22 \\
0.566-1.12\end{array}$ & $\begin{array}{l}7.60 \\
6.90 \\
0.91 \\
1.30 \\
3.50 \\
1.40 \\
3.50 \\
0.77\end{array}$ & \\
\hline \multicolumn{5}{|l|}{ Pesticides/Herbicides (Lot 3227) } \\
\hline $\begin{array}{l}\text { 2-sec-Butyl-4,6-dinitrophenol }(\mu g / L) \\
\text { 2,4-Dichlorophenoxyacetic acid }(\mu g / L) \\
\text { 2,4,5-TP (Silvex) }(\mu g / L)\end{array}$ & $\begin{array}{l}4.24 \\
5.92 \\
4.90\end{array}$ & $\begin{array}{l}1.39-5.48 \\
2.96-8.88 \\
2.45-7.35\end{array}$ & $\begin{array}{l}0.48 \\
3.50 \\
3.72\end{array}$ & \\
\hline \multicolumn{5}{|l|}{ Total Petroleum Hydrocarbons (Lot 8922) } \\
\hline $\begin{array}{l}\text { Total petroleum hydrocarbons, infrared } \\
\text { (mg/bottle) }\end{array}$ & 54.6 & $34,0-70.8$ & 59.9 & \\
\hline \multicolumn{5}{|l|}{ Toxaphene (Lot 3227) } \\
\hline Toxaphene $(\mu g / L)$ & 3.02 & $1.66-4.38$ & 3.60 & \\
\hline \multicolumn{5}{|l|}{ Trace Metals (Lot 9987) } \\
\hline $\begin{array}{l}\text { Aluminum }(\mu g / L) \\
\text { Antimony }(\mu g / L) \\
\text { Arsenic }(\mu g / L) \\
\text { Barium }(\mu g / L) \\
\text { Beryllium }(\mu g / L) \\
\text { Boron }(\mu g / L) \\
\text { Cadmium }(\mu g / L) \\
\text { Chromium }(\mu g / L) \\
\text { Cobalt }(\mu g / L) \\
\text { Copper }(\mu g / L) \\
\text { Iron }(\mu g / L) \\
\text { Lead }(\mu g / L) \\
\text { Manganese }(\mu g / L) \\
\text { Mercury }(\mu g / L) \\
\text { Molybdenum }(\mu g / L) \\
\text { Nickel }(\mu g / L) \\
\text { Selenium }(\mu g / L) \\
\text { Silver }(\mu g / L) \\
\text { Strontium }(\mu g / L) \\
\text { Thallium }(\mu g / L) \\
\text { Vanadium }(\mu g / L) \\
\text { Zinc }(\mu g / L)\end{array}$ & $\begin{array}{l}233 \\
192 \\
117 \\
617 \\
105 \\
641 \\
175 \\
242 \\
517 \\
275 \\
467 \\
433 \\
558 \\
6.67 \\
142 \\
342 \\
139 \\
183 \\
533 \\
108 \\
458 \\
117\end{array}$ & $\begin{array}{l}191-275 \\
144-240 \\
87.8-138 \\
506-728 \\
86.1-124 \\
526-801 \\
144-207 \\
198-285 \\
424-610 \\
226-325 \\
383-551 \\
355-511 \\
458-658 \\
5.00-8.34 \\
116-168 \\
280-404 \\
104-164 \\
150-216 \\
437-629 \\
81.0-135 \\
376-540 \\
95.9-138\end{array}$ & $\begin{array}{l}249 \\
220 \\
118 \\
636 \\
106 \\
709 \\
162 \\
249 \\
547 \\
265 \\
494 \\
430 \\
572 \\
5.92 \\
155 \\
338 \\
139 \\
173 \\
539 \\
112 \\
451 \\
109\end{array}$ & \\
\hline \multicolumn{5}{|l|}{ Turbidity (Lot 3427) } \\
\hline Turbidity (NTU) & 3.36 & $2.86-3.93$ & 3.35 & \\
\hline \multicolumn{5}{|l|}{ Volatiles (Lot 586) } \\
\hline $\begin{array}{l}\text { Benzene }(\mu g / L) \\
\text { Bromodichloromethane }(\mu g / L) \\
\text { Bromoform }(\mu g / L) \\
\text { Carbon tetrachloride }(\mu g / L) \\
\text { Chlorobenzene }(\mu g / L) \\
\text { Chloroform }(\mu g / L) \\
\text { Dibromochloromethane }(\mu g / L) \\
\text { 1,2-Dichlorobenzene }(\mu g / L)\end{array}$ & $\begin{array}{l}110 \\
61.3 \\
86.8 \\
175 \\
78.1 \\
96.1 \\
15.9 \\
62.6\end{array}$ & $\begin{array}{l}85.4-137 \\
47.1-76.3 \\
63.5-112 \\
129-218 \\
61.1-93.7 \\
73.7-117 \\
12.4-19.6 \\
47.5-76.9\end{array}$ & $\begin{array}{l}120 \\
72.0 \\
110 \\
190 \\
81.0 \\
100 \\
16.0 \\
66.0\end{array}$ & \\
\hline
\end{tabular}




\begin{tabular}{|c|c|c|c|c|}
\hline Analyte & $\begin{array}{l}\text { Certified } \\
\text { Value }\end{array}$ & $\begin{array}{l}\text { Performance } \\
\text { Acceptance Limits }\end{array}$ & $\begin{array}{l}\text { EX } \\
\text { Result }\end{array}$ & $\begin{array}{l}\text { Functional } \\
\text { Guideline Code }\end{array}$ \\
\hline $\begin{array}{l}\text { 1,3-Dichlorobenzene }(\mu g / L) \\
\text { 1,4-Dichlorobenzene }(\mu g / L) \\
\text { 1,2-Dichloroethane }(\mu g / L) \\
\text { Dichloromethane (methylene chloride) }(\mu g / L) \\
\text { Ethylbenzene }(\mu g / L) \\
\text { 4-Methyl-2-pentanone }(\mathrm{M} / \mathrm{BK})(\mu \mathrm{g} / \mathrm{L}) \\
\text { 1,1,2,2-Tetrachloroethane }(\mu g / L) \\
\text { Tetrachloroethylene }(\mu g / L) \\
\text { Toluene }(\mu g / L) \\
1,1,1-\text { Trichloroethane }(\mu g / L) \\
1,1,2-\text { Trichloroethane }(\mu g / L) \\
\text { Trichloroethylene }(\mu g / L) \\
\text { m/p-Xylene }(\mu g / L) \\
\end{array}$ & $\begin{array}{l}127 \\
57.6 \\
60.1 \\
60.8 \\
49.3 \\
63.5 \\
81.0 \\
64.3 \\
84.4 \\
59.0 \\
36.4 \\
37.6 \\
41.2 \\
\end{array}$ & $\begin{array}{l}97.0-153 \\
43.3-70.2 \\
46.9-75.7 \\
43.0-79.2 \\
36.9-57.6 \\
36.7-86.7 \\
59.0-102 \\
47.4-77.6 \\
65.1-102 \\
42.6-70.4 \\
27.7-45.7 \\
27.9-45.5 \\
26.6-51.8\end{array}$ & $\begin{array}{l}120 \\
53.0 \\
67.0 \\
62.0 \\
54.0 \\
62.0 \\
80.0 \\
60.0 \\
88.0 \\
60.0 \\
37.0 \\
39.0 \\
42.0\end{array}$ & \\
\hline
\end{tabular}

$\dagger$ Result was not reported by laboratory.

- Result is out of range.

$\checkmark$ The analytical result is an estimated quantity.

Table 39. Quality Control Standards for Selected Analyses for GE

\begin{tabular}{|c|c|c|c|c|}
\hline Analyte & $\begin{array}{l}\text { Certified } \\
\text { Value }\end{array}$ & $\begin{array}{l}\text { Performance } \\
\text { Acceptance Limits }\end{array}$ & $\begin{array}{l}\text { GE } \\
\text { Result }\end{array}$ & $\begin{array}{l}\text { Functional } \\
\text { Guideline Code }\end{array}$ \\
\hline
\end{tabular}

Acids (Lot 586)

2,6-Dichlorophenol $(\mu \mathrm{g} / \mathrm{L})$

2-Methylphenol $(\mu g / L)$

4-Methylphenol $(\mu \mathrm{g} / \mathrm{L})$

Pentachlorophenol $(\mu \mathrm{g} / \mathrm{L})$

2,4,5-Trichlorophenol $(\mu g / L)$

2,4,6-Trichlorophenol $(\mu g / L)$

Base/Neutrals (Lot 586)

Anthracene $(\mu \mathrm{g} / \mathrm{L})$

Benzo[ $g, h, i]$ perylene $(\mu g / L)$

Bis(2-ethylhexyl) phthalate $(\mu g / L)$

4-Bromophenyl-phenylether $(\mu \mathrm{g} / \mathrm{L})$

Chrysene $(\mu \mathrm{g} / \mathrm{L})$

Dibenz $[a, h]$ anthracene $(\mu g / L)$

Dibenzofuran $(\mu g / L)$

1,2-Dichlorobenzene ( $\mu g / L)$

Diethylphthlate $(\mu \mathrm{g} / \mathrm{L})$

Dimethylphthlate $(\mu g / L)$

2,4-Dinitrotoluene $(\mu \mathrm{g} / \mathrm{L})$

Naphthalene $(\mu \mathrm{g} / \mathrm{L})$

N-Nitroso-di-n-propylamine ( $\mu g / L)$

Phenanthrene $(\mu \mathrm{g} / \mathrm{L})$

Pyrene $(\mu \mathrm{g} / \mathrm{L})$

1,2,4-Trichlorobenzene $(\mu g / L)$

Cations (Lot 439)

Calcium $(\mu g / L)$

Magnesium $(\mu \mathrm{g} / \mathrm{L})$

Potassium $(\mu g / L)$

Sodium $(\mu g / L)$
132

59.9

82.8

129

60.5

55.7

86.3

17.3

76.9

185

28.7

14.7

118

113

90.9

138

104

89.9

63.1

55.2

99.6

102

101,000

74,900

90,300

94,900

$\begin{array}{ll}72.9-153 & 97.2 \\ 19.0-69.3 & 40.5 \\ 25.4-95.9 & 47.6 \\ 40.2-162 & 110 \\ 23.8-69.9 & 39.9 \\ 24.0-63.8 & 35.5\end{array}$

40.7-101

73.2

7.16-24.3 12.9

$31.0-98.1 \quad 71.9$

91.4-221 $\quad 144$

13.3-35.2 25.1

5.26-19.0 10.4

56.1-131 91.4

26.4-129 64.3

21.8-117 $\quad 58.1$

$59.4-160$

43.5-120 75.4

31.9-102 64.3

26.7-77.6 50.3

29.9-63.5 44.8

46.6-121 84.6

29.7-116 $\quad 58.7$

$90,900-111,000 \quad 104,000$

$66,700-83,100 \quad 75,400$

$82,200-98,400 \quad 82,500$

$84,500-106,000 \quad 98,300$ 


\begin{tabular}{lllll}
\hline & Certified & Performance & GE & Functional \\
Analyte & Value & Acceptance Limits & Result & Guldeline Code \\
\hline
\end{tabular}

Cyanide and Phenol (Lot 9987)

Cyanide, total $(\mu g / L)$

Phenol $(\mu g / L)$

$\begin{array}{lll}82.6 & 60.3-105 & 82.6\end{array}$

Grease and Oil (Lot 9987)

Grease and oil (gravimetric) (mg/bottle)

50.6

80.6-131

89.7

Inorganics (Lot 3427)

Alkalinity (as $\left.\mathrm{CaCO}_{3}\right)(\mu \mathrm{g} / \mathrm{L})$

Chloride $(\mu \mathrm{g} / \mathrm{L})$

Fluoride $(\mu \mathrm{g} / \mathrm{L})$

Nitrate as nitrogen $(\mu g / L)$

$\mathrm{pH}$ (pH units)

Potassium $(\mu \mathrm{g} / \mathrm{L})$

Sodium $(\mu g / L)$

Specific conductance $(\mu \mathrm{S} / \mathrm{cm})$

Sulfate $(\mu g / L)$

Total dissolved solids $(\mu g / L)$

Nutrients (Lot 9987)

Ammonia as nitrogen $(\mu \mathrm{g} / \mathrm{L})$

Nitrate-nitrite as nitrogen $(\mu g / L)$

Total phosphates (as P) ( $\mu \mathrm{g} / \mathrm{L})$

PCBs (Lot 586)

PCB $1254(\mu g / L)$

5.71

$30.4-63.3$

41.4

151,000
56,800
4,730
3,260
9.23
18,800
144,000
676
79,500
607,000

$141,000-170,000$

145,000

$50,500-64,100 \quad 54,300$

$4,260-5,200 \quad 4,530$

$2,930-3,590 \quad 3,010$

$9.03-9.43 \quad 9.07$

$16,100-21,900 \quad 19,100$

$130,000-159,000 \quad 151,000$

566-769 718

$68,200-89,900 \quad \cdot 75,700$

$497,000-680,000 \quad 580,000$

3,560

5,420

$2,990-4,130$

3,350

$4,820-6,020$

5,450

4,060

$3,450-4,670$

3,630

Pesticides (Lot 586)

Aldrin ( $\mu g / L)$

beta-Benzene hexachloride $(\mu g / L)$

delta-Benzene hexachloride $(\mu \mathrm{g} / \mathrm{L})$

gamma-Benzene hexachloride (Lindane) ( $\mu \mathrm{g} / \mathrm{L})$

gamma-Chlordane $(\mu \mathrm{g} / \mathrm{L})$

4,4'-DDD $(\mu g / L)$

4,4'-DDE $(\mu g / L)$

4,4'-DDT $(\mu g / L)$

Dieldrin $(\mu g / L)$

Endrin $(\mu g / L)$

Heptachlor $(\mu \mathrm{g} / \mathrm{L})$

Heptachlor epoxide $(\mu g / L)$

$3.42-7.17$

5.30

J

1.60

7.12

5.74

1.04

8.56

6.50

0.877

1.19

3.65

1.43

5.83

0.915

$0.830-1.97$

3.80-9.00

$2.53-7.37$

0.585-1.35

1.51

6.69

5.78

0.87

5.96-10.2

3.99-8.44

$0.511-1.10$

$0.692-1.49$

$2.26-4.72$

$0.896-1.85$

2.64-7.22

$0.566-1.12$

8.0

5.52

0.76

1.15

3.22

1.36

5.20

0.79

Pesticides/Herbicides (Lot 3227)

2-sec-Butyl-4,6-dinitrophenol ( $\mu \mathrm{g} / \mathrm{L})$

2,4-Dichlorophenoxyacetic acid $(\mu \mathrm{g} / \mathrm{L})$

4.24

5.92

$1.39-5.48$

$2.96-8.88$

$2.45-7.35$

3.92

5.66

2,4,5-TP (Silvex) $(\mu g / L)$

4.90

Total Petroleum Hydrocarbons (Lot 8922)

Total petroleum hydrocarbons, infrared (mg/bottle)

54.6

$34.0-70.8$

49.5

Toxaphene (Lot 3227)

Toxaphene $(\mu g / L)$

3.02

$1.66-4.38$

$\dagger$

\section{Quality Control Samples}




\begin{tabular}{lllll}
\hline & Certified & Performance & GE & Functional \\
Analyte & Value & Acceptance Limits & Result & Guideline Code \\
\hline
\end{tabular}

Trace Metals (Lot 9987)

Aluminum $(\mu g / L)$

Antimony $(\mu g / L)$

Arsenic $(\mu \mathrm{g} / \mathrm{L}) \quad 117$

Barium $(\mu g / L) \quad 617$

Beryllium $(\mu \mathrm{g} / \mathrm{L})$

Boron $(\mu \mathrm{g} / \mathrm{L})$

Cadmium $(\mu g / L)$

Chromium $(\mu \mathrm{g} / \mathrm{L})$

Cobalt $(\mu g / L) \quad 517$

Copper $(\mu g / L)$

Iron $(\mu g / L) \quad 467$

Lead $(\mu g / L)$

Manganese $(\mu g / L)$

Mercury $(\mu g / L) \quad 6.67$

Molybdenum $(\mu \mathrm{g} / \mathrm{L})$

Nickel $(\mu g / L)$

Selenium $(\mu g / L)$

Silver $(\mu \mathrm{g} / \mathrm{L})$

Strontium ( $\mu g / L)$

Thallium $(\mu g / L) \quad 108$

Vanadium $(\mu \mathrm{g} / \mathrm{L})$

Zinc $(\mu g / L)$

191-275 253

$144-240$

87.8-138 118

$506-728 \quad 604$

86.1-124 103

526-801 706

144-207 176

198-285 240

424-610 528

226-325 267

383-551 461

355-511 414

458-658 556

$5.00-8.34 \quad 6.08$

116-168 132

280-404 352

104-164 140

150-216 175

$437-629 \quad 521$

81.0-135 110

$376-540 \quad 448$

95.9-138 118

Turbidity (Lot 3427)

Turbidity (NTU)

3.36

$2.86-3.93$

3.22

\section{Volatiles (Lot 586)}

Benzene $(\mu g / L)$

Bromodichloromethane $(\mu \mathrm{g} / \mathrm{L})$

Bromoform $(\mu g / L)$

Carbon tetrachloride $(\mu g / L)$

Chlorobenzene $(\mu g / L)$

Chlorotorm $(\mu g / L)$

Dibromochloromethane $(\mu \mathrm{g} / \mathrm{L})$

1,2-Dichlorobenzene $(\mu \mathrm{g} / \mathrm{L})$

1,3-Dichlorobenzene $(\mu \mathrm{g} / \mathrm{L})$

1,4-Dichlorobenzene $(\mu g / L)$

1,2-Dichloroethane $(\mu g /$ L)

Dichloromethane (methylene chloride) $(\mu g / L) 60.8$

Ethylbenzene $(\mu g / L)$

4-Methyl-2-pentanone (MIBK) ( $\mu \mathrm{g} / \mathrm{L}$ )

$1,1,2,2-$ Tetrachloroethane $(\mu g / L) \quad 81.0$

Tetrachloroethylene $(\mu \mathrm{g} / \mathrm{L})$

Toluene $(\mu \mathrm{g} / \mathrm{L}) \quad 84.4$

$1,1,1-$ Trichloroethane $(\mu g / L) \quad 59.0$

1,1,2-Trichloroethane $(\mu g / L) \quad 36.4$

Trichloroethylene $(\mu \mathrm{g} / \mathrm{L})$

$\mathrm{m} / \mathrm{p}$-Xylene $(\mu \mathrm{g} / \mathrm{L})$

85.4-137 104

47.1-76.3 $\quad 59.0$

63.5-112 93.2

$129-218 \quad 188$

$\begin{array}{ll}61.1-93.7 & 79.2\end{array}$

$73.7-117 \quad 95.2$

$12.4-19.6 \quad 16.3$

$47.5-76.9 \quad 63.3$

97.0-153 134

43.3-70.2 65.4

46.9-75.7 61.9

43.0-79.2 61.8

$36.9-57.6 \quad 49.5$

36.7-86.7 60.0

$\begin{array}{ll}59.0-102 & 72.4\end{array}$

47.4-77.6 71.7

65.1-102 $\quad 80.7$

42.6-70.4 62.5

$27.7-45.7 \quad 34.7$

$27.9-45.5 \quad 38.4$

$26.6-51.8 \quad 43.3$

$\dagger$ Result was not reported by laboratory.

- Result is out of range.

$\mathrm{J}$ The analytical result is an estimated quantity. 
Table 40. Quality Control Standards for Selected Analyses for WA

\begin{tabular}{lllll}
\hline & Certified & Performance & WA & Functional \\
Analyte & Value & Acceptance Limits & Result & Guideline Code \\
\hline
\end{tabular}

\section{Acids (Lot 586)}

2,6-Dichlorophenol $(\mu g / \mathrm{L})$

2-Methylphenol $(\mu g / L)$

4-Methyiphenol $(\mu g / L)$

Pentachlorophenol $(\mu \mathrm{g} / \mathrm{L})$

2,4,5-Trichlorophenol $(\mu \mathrm{g} / \mathrm{L})$

2,4,6-Trichlorophenol $(\mu g / L)$

Base/Neutrals (Lot 586)

Anthracene $(\mu g / L)$

Benzo[g,h,]perylene $(\mu g / L)$

Bis(2-ethylhexyl) phthalate $(\mu \mathrm{g} / \mathrm{L})$

4-Bromophenyl-phenylether $(\mu g / L)$

Chrysene $(\mu \mathrm{g} / \mathrm{L})$

Dibenz[a, h]anthracene $(\mu g / L)$

Dibenzofuran $(\mu \mathrm{g} / \mathrm{L})$

1,2-Dichlorobenzene $(\mu \mathrm{g} / \mathrm{L})$

Diethylphthlate $(\mu g / L)$

Dimethylphthlate $(\mu g / \mathrm{L})$

2,4-Dinitrotoluene $(\mu g / L)$

Naphthalene $(\mu g / L)$

N-Nitroso-di-n-propylamine $(\mu \mathrm{g} / \mathrm{L})$

Phenanthrene $(\mu g / L)$

Pyrene $(\mu g / L)$

1,2,4-Trichlorobenzene ( $\mu \mathrm{g} / \mathrm{L}$ )

\section{Cations (Lot 439)}

Calcium $(\mu g / L)$

Magnesium $(\mu \mathrm{g} / \mathrm{L})$

Potassium $(\mu g / L)$

Sodium $(\mu g / L)$

Cyanide and Phenol (Lot 9987)

Cyanide, total ( $\mu \mathrm{g} / \mathrm{L})$

Phenol $(\mu g / L)$

\section{Grease and Oil (Lot 9987)}

Grease and oil (gravimetric) (mg/bottle)

Inorganics (Lot 3427)

Alkalinity (as $\left.\mathrm{CaCO}_{3}\right)(\mu \mathrm{g} / \mathrm{L})$

Chloride ( $\mu \mathrm{g} / \mathrm{L})$

Fluoride $(\mu \mathrm{g} / \mathrm{L})$

Nitrate as nitrogen $(\mu g / L)$

$\mathrm{pH}$ (pH units)

Potassium ( $\mu g / L)$

Sodium ( $\mu g / L$ )

Specific conductance $(\mu \mathrm{S} / \mathrm{cm})$

Sulfate $(\mu \mathrm{g} / \mathrm{L})$

Total dissolved solids $(\mu g / L)$

\section{Nutrients (Lot 9987)}

Ammonia as nitrogen $(\mu \mathrm{g} / \mathrm{L})$

Nitrate-nitrite as nitrogen $(\mu \mathrm{g} / \mathrm{L})$
50.6

132

59.9

82.8

129

60.5

55.7

86.3

17.3

76.9

185

28.7

14.7

118

113

90.9

138

104

89.9

63.1

55.2

99.6

102

101,000

74,900

90,300

94,900

82.6

106

151,000

56,800

4,730

3,260

9.23

18,800

144,000

676

79,500

607,000

3,560

5,420
72.9-153

$19.0-69.3$

25.4-95.9

40.2-162

23.8-69.9

$24.0-63.8$

106

42.7

59.8

108

52.2

49.0

40.7-101

64.1

$7.16-24.3$

$31.0-98.1$

91.4-221

13.3-35.2

5.26-19.0

$56.1-131$

26.4-129

21.8-117

59.4-160

43.5-120

31.9-102

26.7-77.6

$29.9-63.5$

46.6-121

29.7-116

17.3

61.4

165

24.4

16.0

106

85.0

68.0

123

85.1

62.3

50.2

48.4

74.9

68.8

$90,900-111,000$

$66,700-83,100$

102,000

$82,200-98,400$

$84,500-106,000$

71,100

90,800

91,100

60.3-105

80.6-131

83.1

102

$30.4-63.3$

36.1

J

$141,000-170,000$

151,000

$50,500-64,100$

63,000

$4,260-5,200$

$2,930-3,590$

9.03-9.43

4,740

3,230

$16,100-21,900$

9.17

19,200

$130,000-159,000$

134,000

$566-769$

559

81,600

$497,000-680,000 \quad 581,000$

$2,990-4,130$

3,500

$4,820-6,020$

5,410 


\begin{tabular}{|c|c|c|c|c|}
\hline Analyte & $\begin{array}{l}\text { Certified } \\
\text { Value }\end{array}$ & $\begin{array}{l}\text { Performance } \\
\text { Acceptance Limits }\end{array}$ & $\begin{array}{l}\text { WA } \\
\text { Result }\end{array}$ & $\begin{array}{l}\text { Functional } \\
\text { Guideline Code }\end{array}$ \\
\hline Total phosphates (as P) $(\mu g / L)$ & 4,060 & $3,450-4,670$ & 4,080 & \\
\hline \multicolumn{5}{|l|}{ PCBs (Lot 586) } \\
\hline PCB $1254(\mu g / L)$ & 5.71 & $3.42-7.17$ & 5.28 & \\
\hline \multicolumn{5}{|l|}{ Pesticides (Lot 586) } \\
\hline $\begin{array}{l}\text { Aldrin }(\mu g / L) \\
\text { beta-Benzene hexachloride }(\mu g / L) \\
\text { delta-Benzene hexachloride }(\mu g / L) \\
\text { gamma-Benzene hexachloride (Lindane) } \\
\quad(\mu g / L) \\
\text { gamma-Chlordane }(\mu g / L) \\
4,4^{\prime}-D D D(\mu g / L) \\
4,4^{\prime}-D D E(\mu g / L) \\
4,4^{\prime}-D D T(\mu g / L) \\
\text { Dieldrin }(\mu g / L) \\
\text { Endrin }(\mu g / L) \\
\text { Heptachlor }(\mu g / L) \\
\text { Heptachlor epoxide }(\mu g / L)\end{array}$ & $\begin{array}{l}1.60 \\
7.12 \\
5.74 \\
1.04 \\
\\
8.56 \\
6.50 \\
0.877 \\
1.19 \\
3.65 \\
1.43 \\
5.83 \\
0.915\end{array}$ & $\begin{array}{l}0.830-1.97 \\
3.80-9.00 \\
2.53-7.37 \\
0.585-1.35 \\
\\
5.96-10.2 \\
3.99-8.44 \\
0.511-1.10 \\
0.692-1.49 \\
2.26-4.72 \\
0.896-1.85 \\
2.64-7.22 \\
0.566-1.12\end{array}$ & $\begin{array}{l}1.82 \\
8.01 \\
5.92 \\
1.03 \\
\\
8.48 \\
5.93 \\
0.76 \\
1.23 \\
3.72 \\
1.69 \\
6.01 \\
0.84\end{array}$ & \\
\hline \multicolumn{5}{|l|}{ Pesticides/Herbicides (Lot 3227) } \\
\hline $\begin{array}{l}\text { 2-sec-Butyl-4,6-dinitrophenol }(\mu g / L) \\
\text { 2,4-Dichlorophenoxyacetic acid }(\mu g / L) \\
\text { 2,4,5-TP (Silvex) }(\mu g / L)\end{array}$ & $\begin{array}{l}4.24 \\
5.92 \\
4.90\end{array}$ & $\begin{array}{l}1.39-5.48 \\
2.96-8.88 \\
2.45-7.35\end{array}$ & $\begin{array}{l}4.19 \\
6.24 \\
4.56\end{array}$ & \\
\hline \multicolumn{5}{|l|}{ Total Petroleum Hydrocarbons (Lot 8922) } \\
\hline $\begin{array}{l}\text { Total petroleum hydrocarbons, infrared } \\
\text { (mg/bottle) }\end{array}$ & 54.6 & $34.0-70.8$ & $<1,000 *$ & \\
\hline \multicolumn{5}{|l|}{ Toxaphene (Lot 3227) } \\
\hline Toxaphene $(\mu g / L)$ & 3.02 & $1.66-4.38$ & $\dagger$ & \\
\hline \multicolumn{5}{|l|}{ Trace Metals (Lot 9987) } \\
\hline $\begin{array}{l}\text { Aluminum }(\mu g / L) \\
\text { Antimony }(\mu g / L) \\
\text { Arsenic }(\mu g / L) \\
\text { Barium }(\mu g / L) \\
\text { Beryllium }(\mu g / L) \\
\text { Boron }(\mu g / L) \\
\text { Cadmium }(\mu g / L) \\
\text { Chromium }(\mu g / L) \\
\text { Cobalt }(\mu g / L) \\
\text { Copper }(\mu g / L) \\
\text { Iron }(\mu g / L) \\
\text { Lead }(\mu g / L) \\
\text { Manganese }(\mu g / L) \\
\text { Mercury }(\mu g / L) \\
\text { Molybdenum }(\mu g / L) \\
\text { Nickel }(\mu g / L) \\
\text { Selenium }(\mu g / L) \\
\text { Silver }(\mu g / L) \\
\text { Strontium }(\mu g / L) \\
\text { Thallium }(\mu g / L) \\
\text { Vanadium }(\mu g / L) \\
\text { Zinc }(\mu g / L)\end{array}$ & $\begin{array}{l}233 \\
192 \\
117 \\
617 \\
105 \\
641 \\
175 \\
242 \\
517 \\
275 \\
467 \\
433 \\
558 \\
6.67 \\
142 \\
342 \\
139 \\
183 \\
533 \\
108 \\
458 \\
117\end{array}$ & $\begin{array}{l}191-275 \\
144-240 \\
87.8-138 \\
506-728 \\
86.1-124 \\
526-801 \\
144-207 \\
198-285 \\
424-610 \\
226-325 \\
383-551 \\
355-511 \\
458-658 \\
5.00-8.34 \\
116-168 \\
280-404 \\
104-164 \\
150-216 \\
437-629 \\
81.0-135 \\
376-540 \\
95.9-138\end{array}$ & $\begin{array}{l}308 \\
200 \\
118 \\
623 \\
101 \\
660 \\
176 \\
241 \\
521 \\
269 \\
461 \\
441 \\
576 \\
6.58 \\
144 \\
350 \\
148 \\
183 \\
531 \\
110 \\
454 \\
119\end{array}$ & \\
\hline
\end{tabular}




\begin{tabular}{|c|c|c|c|c|}
\hline Analyte & $\begin{array}{l}\text { Certified } \\
\text { Value }\end{array}$ & $\begin{array}{l}\text { Performance } \\
\text { Acceptance Limits }\end{array}$ & $\begin{array}{l}\text { WA } \\
\text { Result }\end{array}$ & $\begin{array}{l}\text { Functional } \\
\text { Guideline Code }\end{array}$ \\
\hline \multicolumn{5}{|l|}{ Turbidity (Lot 3427) } \\
\hline Turbidity (NTU) & 3.36 & $2.86-3.93$ & 3.30 & \\
\hline \multicolumn{5}{|l|}{ Volatiles (Lot 586) } \\
\hline $\begin{array}{l}\text { Benzene }(\mu g / L) \\
\text { Bromodichloromethane }(\mu g / L) \\
\text { Bromoform }(\mu g / L) \\
\text { Carbon tetrachloride }(\mu g / L) \\
\text { Chlorobenzene }(\mu g / L) \\
\text { Chloroform }(\mu g / L) \\
\text { Dibromochloromethane }(\mu g / L) \\
\text { 1,2-Dichlorobenzene }(\mu g / L) \\
\text { 1,3-Dichlorobenzene }(\mu g / L) \\
\text { 1,4-Dichlorobenzene }(\mu g / L) \\
\text { 1,2-Dichloroethane }(\mu g / L) \\
\text { Dichloromethane }(\text { methylene chloride) }(\mu g / L) \\
\text { Ethylbenzene }(\mu g / L) \\
\text { 4-Methyl-2-pentanone }(\mathrm{MIBK})(\mu g / L) \\
\text { 1,1,2,2-Tetrachloroethane }(\mu g / L) \\
\text { Tetrachloroethylene }(\mu g / L) \\
\text { Toluene }(\mu g / L) \\
\text { 1,1,1-Trichloroethane }(\mu g / L) \\
1,1,2-\text { Trichloroethane }(\mu g / L) \\
\text { Trichloroethylene }(\mu g / L) \\
\text { m/p-Xylene }(\mu g / L) \\
\end{array}$ & $\begin{array}{l}110 \\
61.3 \\
86.8 \\
175 \\
78.1 \\
96.1 \\
15.9 \\
62.6 \\
127 \\
57.6 \\
60.1 \\
60.8 \\
49.3 \\
63.5 \\
81.0 \\
64.3 \\
84.4 \\
59.0 \\
36.4 \\
37.6 \\
41.2 \\
\end{array}$ & $\begin{array}{l}85.4-137 \\
47.1-76.3 \\
63.5-112 \\
129-218 \\
61.1-93.7 \\
73.7-117 \\
12.4-19.6 \\
47.5-76.9 \\
97.0-153 \\
43.3-70.2 \\
46.9-75.7 \\
43.0-79.2 \\
36.9-57.6 \\
36.7-86.7 \\
59.0-102 \\
47.4-77.6 \\
65.1-102 \\
42.6-70.4 \\
27.7-45.7 \\
27.9-45.5 \\
26.6-51.8 \\
\end{array}$ & 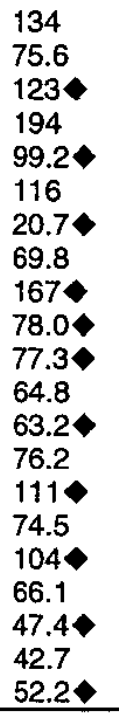 & \\
\hline
\end{tabular}

$\dagger$ Result was not reported by laboratory.

- Result is out of range.

* Not enough information to determine if result is within performance acceptance limits.

$\mathrm{J}$ The analytical result is an estimated quantity.

Table 41. Laboratory Control Sample Recoveries for EX

\begin{tabular}{|c|c|c|c|c|c|}
\hline Analyte & $\begin{array}{l}\text { Qualified } \\
\text { Out of Ranget }\end{array}$ & $\begin{array}{l}\text { Mean } \\
\text { Recovery (\%) }\end{array}$ & $\begin{array}{l}\text { Standard } \\
\text { Deviation }\end{array}$ & $\begin{array}{l}\text { Minimum } \\
\text { Recovery (\%) }\end{array}$ & $\begin{array}{l}\text { Maximum } \\
\text { Recovery (\%) }\end{array}$ \\
\hline \multicolumn{6}{|l|}{ AP-3086 } \\
\hline Total organic carbon & $0 / 2$ & 97.0 & 0.0 & 97.0 & 97.0 \\
\hline \multicolumn{6}{|l|}{ EPA300.0 } \\
\hline Chloride & $0 / 8$ & 104 & 5.90 & 97.0 & 114 \\
\hline Fluoride & $0 / 8$ & 103 & 3.55 & 96.0 & 105 \\
\hline Nitrate as nitrogen & $0 / 22$ & 101 & 2.73 & 98.0 & 108 \\
\hline Nitrate-nitrite as nitrogen & $0 / 12$ & 103 & 4.64 & 96.0 & 109 \\
\hline Sulfate & $0 / 8$ & 98.1 & 4.88 & 91.0 & 105 \\
\hline Total phosphates (as P) & $0 / 8$ & 99.6 & 5.85 & 91.0 & 107 \\
\hline \multicolumn{6}{|l|}{ EPA6010B } \\
\hline Aluminum & $0 / 56$ & 104 & 4.30 & 94.0 & 113 \\
\hline Arsenic & $0 / 12$ & 103 & 5.23 & 96.0 & 112 \\
\hline Barium & $0 / 40$ & 103 & 3.90 & 94.0 & 112 \\
\hline Boron & $0 / 20$ & 104 & 6.14 & 94.0 & 114 \\
\hline Cadmium & $0 / 6$ & 106 & 3.49 & 102 & 111 \\
\hline Chromium & $0 / 12$ & 105 & 4.47 & 99.0 & 112 \\
\hline Iron & $0 / 56$ & 105 & 4.48 & 95.0 & 114 \\
\hline Lead & $0 / 56$ & 105 & 5.37 & 95.0 & 117 \\
\hline
\end{tabular}




\begin{tabular}{|c|c|c|c|c|c|}
\hline Analyte & $\begin{array}{l}\text { Qualified } \\
\text { Out of Ranget }\end{array}$ & $\begin{array}{l}\text { Mean } \\
\text { Recovery (\%) }\end{array}$ & $\begin{array}{l}\text { Standard } \\
\text { Deviation }\end{array}$ & $\begin{array}{l}\text { Minimum } \\
\text { Recovery (\%) }\end{array}$ & $\begin{array}{l}\text { Maximum } \\
\text { Recovery (\%) }\end{array}$ \\
\hline $\begin{array}{l}\text { Manganese } \\
\text { Nickel } \\
\text { Selenium } \\
\text { Silver } \\
\text { Sodium }\end{array}$ & $\begin{array}{l}0 / 22 \\
0 / 38 \\
0 / 39 \\
0 / 12 \\
0 / 6\end{array}$ & $\begin{array}{l}103 \\
104 \\
100 \\
106 \\
106\end{array}$ & $\begin{array}{l}5.21 \\
4.24 \\
8.13 \\
4.75 \\
4.75\end{array}$ & $\begin{array}{l}94.0 \\
95.0 \\
87.0 \\
100 \\
101\end{array}$ & $\begin{array}{l}113 \\
112 \\
114 \\
114 \\
111\end{array}$ \\
\hline $\begin{array}{l}\text { EPA7470A } \\
\text { Mercury }\end{array}$ & $1 / 32$ & 98.4 & 5.56 & 79.0 & 107 \\
\hline $\begin{array}{l}\text { EPA7841 } \\
\text { Thallium }\end{array}$ & $0 / 2$ & 103 & 0.0 & 103 & 103 \\
\hline $\begin{array}{l}\text { EPA8021B } \\
\text { Carbon tetrachioride } \\
\text { Chloroform } \\
\text { cis-1,2-Dichloroethylene } \\
\text { Tetrachloroethylene } \\
\text { 1,1,1-Trichloroethane } \\
\text { Trichloroethylene }\end{array}$ & $\begin{array}{l}0 / 20 \\
0 / 20 \\
0 / 20 \\
0 / 20 \\
0 / 20 \\
4 / 20\end{array}$ & $\begin{array}{l}110 \\
113 \\
113 \\
116 \\
111 \\
110\end{array}$ & $\begin{array}{l}9.27 \\
9.62 \\
9.51 \\
13.8 \\
11.2 \\
10.4\end{array}$ & $\begin{array}{l}93.0 \\
96.0 \\
95.0 \\
94.0 \\
94.0 \\
91.0\end{array}$ & $\begin{array}{l}127 \\
129 \\
133 \\
141 \\
130 \\
130\end{array}$ \\
\hline $\begin{array}{l}\text { EPA8081A } \\
\text { Lindane }\end{array}$ & $0 / 5$ & 72.6 & 9.50 & 62.0 & 86.0 \\
\hline $\begin{array}{l}\text { EPA8082 } \\
\text { PCB } 1260\end{array}$ & $0 / 11$ & 102 & 6.59 & 96.0 & 117 \\
\hline $\begin{array}{l}\text { EPA8151A } \\
\text { 2,4-Dichlorophenoxyacetic } \\
\text { acid }\end{array}$ & $0 / 6$ & 103 & 22.1 & 80.0 & 127 \\
\hline $\begin{array}{l}\text { EPA8260B } \\
\text { Benzene } \\
\text { Chlorobenzene } \\
\text { 1,1-Dichloroethylene } \\
\text { Toluene } \\
\text { Trichloroethylene }\end{array}$ & $\begin{array}{l}0 / 66 \\
0 / 66 \\
0 / 66 \\
0 / 66 \\
0 / 66\end{array}$ & $\begin{array}{l}101 \\
100 \\
99.2 \\
99.8 \\
101\end{array}$ & $\begin{array}{l}4.89 \\
5.34 \\
7.88 \\
5.14 \\
6.13\end{array}$ & $\begin{array}{l}91.0 \\
90.0 \\
83.0 \\
87.0 \\
86.0\end{array}$ & $\begin{array}{l}113 \\
113 \\
116 \\
110 \\
115\end{array}$ \\
\hline $\begin{array}{l}\text { EPA8270C } \\
\text { Acenaphthene } \\
\text { 4-Chloro-m-cresol } \\
\text { 2-Chlorophenol } \\
\text { 2,4-Dinitrotoluene } \\
\text { 4-Nitrophenol } \\
\text { N-Nitrosodipropylamine } \\
\text { Pentachlorophenol } \\
\text { Phenol } \\
\text { Pyrene } \\
\text { 1,2,4-Trichlorobenzene }\end{array}$ & $\begin{array}{l}2 / 4 \\
2 / 4 \\
1 / 4 \\
2 / 4 \\
2 / 4 \\
2 / 4 \\
4 / 4 \\
0 / 10 \\
0 / 4 \\
3 / 4\end{array}$ & $\begin{array}{l}51.8 \\
76.0 \\
52.5 \\
85.0 \\
76.5 \\
32.5 \\
212 \\
60.5 \\
67.3 \\
26.8\end{array}$ & $\begin{array}{l}22.8 \\
34.7 \\
31.2 \\
22.7 \\
19.7 \\
15.6 \\
57.2 \\
28.6 \\
4.79 \\
16.6\end{array}$ & $\begin{array}{l}31.0 \\
44.0 \\
23.0 \\
64.0 \\
59.0 \\
18.0 \\
162 \\
17.0 \\
63.0 \\
11.0\end{array}$ & $\begin{array}{l}72.0 \\
106 \\
90.0 \\
111 \\
99.0 \\
46.0 \\
271 \\
96.0 \\
74.0 \\
46.0\end{array}$ \\
\hline $\begin{array}{l}\text { EPA9014 } \\
\text { Cyanide }\end{array}$ & $0 / 30$ & 95.5 & 1.48 & 93.0 & 99.0 \\
\hline $\begin{array}{l}\text { EPA9060 } \\
\text { Total organic carbon }\end{array}$ & $0 / 3$ & 96.3 & 2.08 & 94.0 & 98.0 \\
\hline
\end{tabular}

† Number of batches qualified that exhibit poor laboratory control sample and blank spike recovery due to interference compared to the total number of batches containing laboratory control samples and blank spikes.

Note: A value of 0 is reported as 0.0 . 
Table 42. Laboratory Control Sample Recoveries for GE

\begin{tabular}{|c|c|c|c|c|c|}
\hline Analyte & $\begin{array}{l}\text { Quallfied } \\
\text { Out of Ranget }\end{array}$ & $\begin{array}{l}\text { Mean } \\
\text { Recovery (\%) }\end{array}$ & $\begin{array}{l}\text { Standard } \\
\text { Deviation }\end{array}$ & $\begin{array}{l}\text { Minimum } \\
\text { Recovery (\%) }\end{array}$ & $\begin{array}{l}\text { Maximum } \\
\text { Recovery (\%) }\end{array}$ \\
\hline \multicolumn{6}{|l|}{ EPA160.1 } \\
\hline Total dissolved solids & $0 / 1$ & 98.3 & - & 98.3 & 98.3 \\
\hline \multicolumn{6}{|l|}{ EPA353.1 } \\
\hline Nitrate-nitrite as nitrogen & $0 / 17$ & 103 & 5.71 & 94.0 & 118 \\
\hline \multicolumn{6}{|l|}{ EPA365.4 } \\
\hline Total phosphates (as P) & $0 / 1$ & 106 & - & 106 & 106 \\
\hline \multicolumn{6}{|l|}{ EPA6010B } \\
\hline Aluminum & $0 / 18$ & 101 & 3.47 & 93.4 & 106 \\
\hline Antimony & $0 / 5$ & 101 & 3.58 & 95.5 & 105 \\
\hline Arsenic & $0 / 17$ & 102 & 3.64 & 93.4 & 108 \\
\hline Barium & $0 / 17$ & 99.3 & 4.02 & 92.0 & 104 \\
\hline Beryllium & $0 / 2$ & 99.1 & 6.93 & 94.2 & 104 \\
\hline Cadmium & $0 / 17$ & 101 & 4.55 & 93.1 & 109 \\
\hline Calcium & $0 / 1$ & 95.9 & $\rightarrow$ & 95.9 & 95.9 \\
\hline Chromium & $0 / 17$ & 101 & 3.80 & 93.0 & 107 \\
\hline Cobalt & $0 / 2$ & 99.5 & 7.85 & 93.9 & 105 \\
\hline Copper & $0 / 2$ & 98.7 & 8.98 & 92.3 & 105 \\
\hline Iron & $0 / 19$ & 101 & 4.84 & 89.5 & 108 \\
\hline Lead & $0 / 17$ & 103 & 4.31 & 93.0 & 111 \\
\hline Magnesium & $0 / 1$ & 95.2 & - & 95.2 & 95.2 \\
\hline Manganese & $0 / 2$ & 95.1 & 1.77 & 93.8 & 96.3 \\
\hline Nickel & $0 / 2$ & 100 & 8.49 & 94.0 & 106 \\
\hline Potassium & $0 / 1$ & 91.5 & - & 91.5 & 91.5 \\
\hline Selenium & $0 / 17$ & 99.9 & 3.78 & 92.8 & 106 \\
\hline Silver & $1 / 17$ & 98.3 & 24.4 & 4.57 & 109 \\
\hline Sodium & $0 / 1$ & 92.6 & 一 & 92.6 & 92.6 \\
\hline Thallium & $0 / 2$ & 100 & 11.4 & 91.9 & 108 \\
\hline Vanadium & $0 / 2$ & 98.7 & 7.57 & 93.3 & 104 \\
\hline Zinc & $0 / 2$ & 101 & 8.98 & 94.3 & 107 \\
\hline \multicolumn{6}{|l|}{ EPA6020 } \\
\hline Aluminum & $1 / 25$ & 105 & 8.61 & 85.1 & 122 \\
\hline Antimony & $0 / 21$ & 94.8 & 9.22 & 84.9 & 112 \\
\hline Arsenic & $0 / 21$ & 103 & 5.61 & 86.9 & 110 \\
\hline Barium & $0 / 21$ & 91.5 & 7.74 & 80.8 & 105 \\
\hline Beryllium & $0 / 2$ & 107 & 4.95 & 103 & 110 \\
\hline Cadmium & $0 / 22$ & 101 & 3.75 & 87.2 & 105 \\
\hline Calcium & $0 / 2$ & 98.6 & 0.07 & 98.5 & 98.6 \\
\hline Chromium & $0 / 22$ & 91.9 & 7.74 & 83.0 & 105 \\
\hline Cobalt & $0 / 21$ & 100 & 4.40 & 85.0 & 105 \\
\hline Copper & $0 / 21$ & 104 & 5.33 & 85.2 & 110 \\
\hline Iron & $2 / 25$ & 97.3 & 14.2 & 70.7 & 133 \\
\hline Lead & $0 / 22$ & 103 & 5.09 & 89.2 & 112 \\
\hline Magnesium & $0 / 2$ & 88.4 & 2.40 & 86.7 & 90.1 \\
\hline Manganese & $0 / 2$ & 102 & 0.0 & 102 & 102 \\
\hline Nickel & $0 / 22$ & 101 & 5.46 & 81.8 & 109 \\
\hline Potassium & $0 / 2$ & 82.6 & 0.71 & 82.1 & 83.1 \\
\hline Selenium & $0 / 21$ & 104 & 7.28 & 83.4 & 112 \\
\hline Silver & $4 / 26$ & 96.4 & 27.3 & 15.4 & 114 \\
\hline Sodium & $0 / 2$ & 101 & 1.70 & 99.6 & 102 \\
\hline Thallium & $0 / 16$ & 98.6 & 6.13 & 88.6 & 105 \\
\hline Tin & $2 / 17$ & 93.9 & 31.9 & 16.4 & 190 \\
\hline Vanadium & $1 / 22$ & 99.8 & 14.8 & 40.0 & 113 \\
\hline Zinc & $0 / 21$ & 98.5 & 7.25 & 86.8 & 110 \\
\hline
\end{tabular}




\begin{tabular}{|c|c|c|c|c|c|}
\hline Analyte & $\begin{array}{l}\text { Qualified } \\
\text { Out of Ranget }\end{array}$ & $\begin{array}{l}\text { Mean } \\
\text { Recovery (\%) }\end{array}$ & $\begin{array}{l}\text { Standard } \\
\text { Deviation }\end{array}$ & $\begin{array}{l}\text { Minimum } \\
\text { Recovery (\%) }\end{array}$ & $\begin{array}{l}\text { Maximum } \\
\text { Recovery (\%) }\end{array}$ \\
\hline \multicolumn{6}{|l|}{ EPA7470A } \\
\hline Mercury & $0 / 36$ & 99.3 & 6.95 & 88.1 & 115 \\
\hline \multicolumn{6}{|l|}{ EPA8081A } \\
\hline Aldrin & $0 / 1$ & 120 & - & 120 & 120 \\
\hline$p, p^{\prime}-D D T$ & $0 / 1$ & 127 & - & 127 & 127 \\
\hline Dieldrin & $0 / 1$ & 112 & 一 & 112 & 112 \\
\hline Endrin & $1 / 1$ & 136 & 一 & 136 & 136 \\
\hline Heptachlor & $0 / 1$ & 124 & - & 124 & 124 \\
\hline Lindane & $0 / 1$ & 115 & 一 & 115 & 115 \\
\hline \multicolumn{6}{|l|}{ EPA8082 } \\
\hline PCB 1260 & $0 / 2$ & 88.0 & 4.24 & 85.0 & 91.0 \\
\hline \multicolumn{6}{|l|}{ EPA8260B } \\
\hline Benzene & $0 / 82$ & 102 & 6.46 & 86.8 & 116 \\
\hline Chlorobenzene & $1 / 82$ & 96.4 & 12.2 & 0.0 & 108 \\
\hline 1,1-Dichloroethylene & $1 / 82$ & 106 & 14.4 & 0.0 & 124 \\
\hline Toluene & $0 / 82$ & 96.2 & 7.47 & 81.2 & 109 \\
\hline Trichloroethylene & $1 / 82$ & 96.9 & 13.1 & 0.0 & 119 \\
\hline \multicolumn{6}{|l|}{ EPA8270C } \\
\hline Acenaphthene & $0 / 1$ & 67.2 & - & 67.2 & 67.2 \\
\hline 4-Chloro-m-cresol & $0 / 1$ & 78.3 & 一 & 78.3 & 78.3 \\
\hline 2-Chlorophenol & $0 / 1$ & 53.3 & 一 & 53.3 & 53.3 \\
\hline 1,4-Dichlorobenzene & $0 / 1$ & 47.0 & 一 & 47.0 & 47.0 \\
\hline 2,4-Dinitrotoluene & $0 / 1$ & 75.5 & - & 75.5 & 75.5 \\
\hline 4-Nitrophenol & $0 / 1$ & 27.7 & - & 27.7 & 27.7 \\
\hline N-Nitrosodipropylamine & $0 / 1$ & 76.4 & - & 76.4 & 76.4 \\
\hline Pentachlorophenol & $0 / 1$ & 93.4 & - & 93.4 & 93.4 \\
\hline Phenol & $0 / 1$ & 28.9 & - & 28.9 & 28.9 \\
\hline Pyrene & $0 / 1$ & 76.2 & - & 76.2 & 76.2 \\
\hline 1,2,4-Trichlorobenzene & $0 / 1$ & 49.4 & - & 49.4 & 49.4 \\
\hline \multicolumn{6}{|l|}{ EPA9012A } \\
\hline Cyanide & $0 / 23$ & 93.7 & 8.39 & 80.7 & 112 \\
\hline \multicolumn{6}{|l|}{ EPA9030 } \\
\hline Sulfide & $0 / 1$ & 113 & - & 113 & 113 \\
\hline \multicolumn{6}{|l|}{ EPA9040B } \\
\hline $\mathrm{pH}$ & $0 / 25$ & 99.7 & 0.41 & 98.8 & 101 \\
\hline \multicolumn{6}{|l|}{ EPA9050A } \\
\hline Specific conductance & $0 / 19$ & 102 & 3.60 & 92.8 & 105 \\
\hline \multicolumn{6}{|l|}{ EPA9056 } \\
\hline Chloride & $0 / 1$ & 96.7 & - & 96.7 & 96.7 \\
\hline Fluoride & $0 / 1$ & 97.9 & - & 97.9 & 97.9 \\
\hline Nitrite as nitrogen & $0 / 2$ & 99.6 & 7.64 & 94.2 & 105 \\
\hline Sulfate & $0 / 1$ & 98.4 & - & 98.4 & 98.4 \\
\hline
\end{tabular}




\begin{tabular}{|c|c|c|c|c|c|}
\hline Analyte & $\begin{array}{l}\text { Qualified } \\
\text { Out of Ranget }\end{array}$ & $\begin{array}{l}\text { Mean } \\
\text { Recovery (\%) }\end{array}$ & $\begin{array}{l}\text { Standard } \\
\text { Deviatlon }\end{array}$ & $\begin{array}{l}\text { Minimum } \\
\text { Recovery (\%) }\end{array}$ & $\begin{array}{l}\text { Maximum } \\
\text { Recovery (\%) }\end{array}$ \\
\hline $\begin{array}{l}\text { EPA9060 } \\
\text { Total organic carbon }\end{array}$ & $0 / 1$ & 99.3 & - & 99.3 & 99.3 \\
\hline $\begin{array}{l}\text { EPA9066 } \\
\text { Phenols }\end{array}$ & $0 / 9$ & 101 & 4.49 & 96.7 & 111 \\
\hline
\end{tabular}

$\uparrow$ Number of batches qualified that exhibit poor laboratory control sample and blank spike recovery due to interference compared to the total number of batches containing laboratory control samples and blank spikes.

- Standard deviation cannot be determined.

Note: $A$ value of 0 is reported as 0.0 .

Tablo 43. Laboratory Control Samplo Recoveries for WA

\begin{tabular}{|c|c|c|c|c|c|}
\hline Analyte & $\begin{array}{l}\text { Qualified } \\
\text { Out of Ranget }\end{array}$ & $\begin{array}{l}\text { Mean } \\
\text { Recovery (\%) }\end{array}$ & $\begin{array}{l}\text { Standard } \\
\text { Deviation }\end{array}$ & $\begin{array}{l}\text { Minimum } \\
\text { Recovery (\%) }\end{array}$ & $\begin{array}{l}\text { Maximum } \\
\text { Recovery (\%) }\end{array}$ \\
\hline \multicolumn{6}{|l|}{ EPA160.1 } \\
\hline Total dissolved solids & $0 / 34$ & 100 & 3.43 & 94.0 & 107 \\
\hline \multicolumn{6}{|l|}{ EPA310.1 } \\
\hline Alkalinity (as $\mathrm{CaCO}_{3}$ ) & $0 / 2$ & 99.5 & 0.71 & 99.0 & 100 \\
\hline \multicolumn{6}{|l|}{ EPA340.2 } \\
\hline Fluoride & $0 / 28$ & 97.7 & 4.35 & 88.8 & 102 \\
\hline \multicolumn{6}{|l|}{ EPA353.2 } \\
\hline Nitrate as nitrogen & $0 / 6$ & 99.7 & 4.34 & 96.0 & 108 \\
\hline Nitrate-nitrite as nitrogen & $0 / 52$ & 99.1 & 1.61 & 96.0 & 103 \\
\hline Nitrite as nitrogen & $0 / 2$ & 102 & 0.71 & 101 & 102 \\
\hline \multicolumn{6}{|l|}{ EPA365.2 } \\
\hline Total phosphates (as P) & $0 / 30$ & 100 & 2.04 & 98.2 & 107 \\
\hline \multicolumn{6}{|l|}{ EPA376.2 } \\
\hline Sulfide & $0 / 2$ & 101 & 0.0 & 101 & 101 \\
\hline \multicolumn{6}{|l|}{ EPA6010B } \\
\hline Aluminum & $0 / 51$ & 102 & 1.50 & 99.5 & 106 \\
\hline Antimony & $0 / 16$ & 99.4 & 1.06 & 97.0 & 101 \\
\hline Arsenic & $0 / 41$ & 99.6 & 1.42 & 97.8 & 104 \\
\hline Barium & $0 / 39$ & 98.6 & 1.59 & 96.4 & 105 \\
\hline Beryllium & $0 / 11$ & 98.8 & 1.53 & 97.0 & 101 \\
\hline Boron & $0 / 15$ & 96.7 & 1.42 & 95.1 & 99.4 \\
\hline Cadmium & $0 / 39$ & 98.7 & 1.17 & 97.2 & 102 \\
\hline Calcium & $0 / 17$ & 101 & 1.75 & 98.6 & 104 \\
\hline Chromium & $0 / 38$ & 99.5 & 1.38 & 97.5 & 104 \\
\hline Cobalt & $0 / 13$ & 98.4 & 1.08 & 96.6 & 100 \\
\hline Copper & $0 / 15$ & 99.2 & 1.35 & 96.2 & 101 \\
\hline Iron & $0 / 50$ & 98.9 & 1.33 & 97.4 & 103 \\
\hline Lead & $0 / 44$ & 99.5 & 1.46 & 96.5 & 103 \\
\hline Lithium & $0 / 15$ & 104 & 2.58 & 99.9 & 108 \\
\hline Magnesium & $0 / 17$ & 101 & 1.45 & 98.7 & 104 \\
\hline Manganese & $0 / 32$ & 101 & 2.07 & 98.3 & 107 \\
\hline Nickel & $0 / 24$ & 97.9 & 1.56 & 95.8 & 103 \\
\hline Potassium & $0 / 17$ & 103 & 3.03 & 100 & 108 \\
\hline Selenium & $0 / 43$ & 101 & 1.62 & 99.0 & 106 \\
\hline
\end{tabular}




\begin{tabular}{|c|c|c|c|c|c|}
\hline Analyte & $\begin{array}{l}\text { Quallfied } \\
\text { Out of Ranget }\end{array}$ & $\begin{array}{l}\text { Mean } \\
\text { Recovery (\%) }\end{array}$ & $\begin{array}{l}\text { Standard } \\
\text { Deviation }\end{array}$ & $\begin{array}{l}\text { Minimum } \\
\text { Recovery (\%) }\end{array}$ & $\begin{array}{l}\text { Maximum } \\
\text { Recovery (\%) }\end{array}$ \\
\hline $\begin{array}{l}\text { Silica } \\
\text { Silver } \\
\text { Sodium } \\
\text { Thallium } \\
\text { Tin } \\
\text { Vanadium } \\
\text { Zinc }\end{array}$ & $\begin{array}{l}0 / 17 \\
0 / 36 \\
0 / 22 \\
0 / 12 \\
0 / 10 \\
0 / 13 \\
0 / 15\end{array}$ & $\begin{array}{l}98.7 \\
99.9 \\
99.2 \\
100 \\
98.9 \\
101 \\
98.7\end{array}$ & $\begin{array}{l}3.16 \\
1.25 \\
1.55 \\
0.93 \\
1.29 \\
1.18 \\
1.28\end{array}$ & $\begin{array}{l}95.1 \\
98.0 \\
97.1 \\
98.6 \\
97.4 \\
99.2 \\
97.5\end{array}$ & $\begin{array}{l}107 \\
105 \\
102 \\
101 \\
101 \\
103 \\
102\end{array}$ \\
\hline $\begin{array}{l}\text { EPA7470A } \\
\text { Mercury }\end{array}$ & $0 / 35$ & 103 & 2.27 & 99.5 & 107 \\
\hline $\begin{array}{l}\text { EPA8010 } \\
\text { Carbon tetrachloride } \\
\text { Chloroform } \\
\text { cis-1,2-Dichloroethylene } \\
\text { t,1,1-Trichloroethane } \\
\text { Trichloroethylene }\end{array}$ & $\begin{array}{l}0 / 7 \\
0 / 7 \\
0 / 9 \\
0 / 7 \\
0 / 7\end{array}$ & $\begin{array}{l}93.7 \\
94.6 \\
93.6 \\
96.9 \\
98.8\end{array}$ & $\begin{array}{l}8.34 \\
7.79 \\
7.27 \\
8.60 \\
9.68\end{array}$ & $\begin{array}{l}84.7 \\
84.3 \\
86.0 \\
87.9 \\
86.3\end{array}$ & $\begin{array}{l}110 \\
109 \\
107 \\
114 \\
117\end{array}$ \\
\hline $\begin{array}{l}\text { EPA8021B } \\
\text { Carbon tetrachloride } \\
\text { Chloroform } \\
\text { cis-1,2-Dichloroethylene } \\
\text { 1,1,1-Trichloroethane } \\
\text { Trichloroethylene }\end{array}$ & $\begin{array}{l}0 / 15 \\
0 / 15 \\
0 / 2 \\
0 / 15 \\
0 / 15\end{array}$ & $\begin{array}{l}100 \\
99.2 \\
92.2 \\
98.5 \\
96.7\end{array}$ & $\begin{array}{l}8.43 \\
8.08 \\
7.78 \\
7.57 \\
5.47\end{array}$ & $\begin{array}{l}88.7 \\
86.8 \\
86.7 \\
88.3 \\
89.2\end{array}$ & $\begin{array}{l}112 \\
110 \\
97.7 \\
109 \\
106\end{array}$ \\
\hline $\begin{array}{l}\text { EPA8081A } \\
\text { Aldrin } \\
\text { p,p'-DDT } \\
\text { Dieldrin } \\
\text { Endrin } \\
\text { Heptachlor } \\
\text { Lindane }\end{array}$ & $\begin{array}{l}0 / 9 \\
0 / 9 \\
1 / 9 \\
2 / 9 \\
0 / 9 \\
3 / 15\end{array}$ & $\begin{array}{l}86.1 \\
107 \\
108 \\
117 \\
95.0 \\
95.0\end{array}$ & $\begin{array}{l}12.2 \\
12.6 \\
18.2 \\
17.7 \\
14.4 \\
27.8\end{array}$ & $\begin{array}{l}75.0 \\
94.0 \\
94.0 \\
86.0 \\
80.0 \\
20.0\end{array}$ & $\begin{array}{l}110 \\
130 \\
150 \\
152 \\
125 \\
140\end{array}$ \\
\hline $\begin{array}{l}\text { EPA8082 } \\
\text { PCB } 1254\end{array}$ & $0 / 10$ & 82.9 & 5.49 & 77.9 & 92.8 \\
\hline $\begin{array}{l}\text { EPA8141A } \\
\text { Dimethoate } \\
\text { Disulfoton } \\
\text { Famphur } \\
\text { Parathion } \\
\text { Parathion ethyl } \\
\text { Parathion methyl } \\
\text { Phorate } \\
\text { Sulfotepp } \\
\text { Thionazin } \\
\text { O,O,O-Triethyl } \\
\text { phosphorothioate }\end{array}$ & $\begin{array}{l}0 / 10 \\
0 / 10 \\
0 / 10 \\
0 / 2 \\
0 / 8 \\
0 / 10 \\
0 / 10 \\
0 / 10 \\
0 / 10 \\
0 / 10\end{array}$ & $\begin{array}{l}68.7 \\
85.9 \\
105 \\
86.8 \\
90.8 \\
91.5 \\
88.5 \\
80.0 \\
92.3 \\
120\end{array}$ & $\begin{array}{l}22.4 \\
11.5 \\
12.0 \\
11.7 \\
5.62 \\
10.9 \\
9.41 \\
9.19 \\
11.9 \\
20.8\end{array}$ & $\begin{array}{l}48.5 \\
68.5 \\
92.0 \\
78.5 \\
86.5 \\
81.0 \\
80.5 \\
72.0 \\
83.0 \\
90.0\end{array}$ & $\begin{array}{l}112 \\
112 \\
136 \\
95.0 \\
104 \\
119 \\
112 \\
102 \\
120 \\
154\end{array}$ \\
\hline $\begin{array}{l}\text { EPAB151A } \\
\text { 2,4-Dichlorophenoxyacetic } \\
\text { acid } \\
2,4,5-\mathrm{T} \\
2,4,5-\mathrm{TP} \text { (Silvex) }\end{array}$ & $\begin{array}{l}0 / 10 \\
0 / 8 \\
0 / 8\end{array}$ & $\begin{array}{l}85.1 \\
99.1 \\
82.2\end{array}$ & $\begin{array}{l}19.8 \\
20.9 \\
14.9\end{array}$ & $\begin{array}{l}49.2 \\
66.0 \\
60.8\end{array}$ & $\begin{array}{l}106 \\
123 \\
98.8\end{array}$ \\
\hline $\begin{array}{l}\text { EPA8260B } \\
\text { Benzene } \\
\text { Chlorobenzene } \\
\text { 1,1-Dichloroethylene } \\
\text { 1,2-Dichloroethylene }\end{array}$ & $\begin{array}{l}0 / 31 \\
0 / 31 \\
0 / 32 \\
0 / 1\end{array}$ & $\begin{array}{l}96.6 \\
95.8 \\
91.0 \\
105\end{array}$ & $\begin{array}{l}5.94 \\
5.38 \\
9.08 \\
-\end{array}$ & $\begin{array}{l}85.2 \\
84.6 \\
75.1 \\
105\end{array}$ & $\begin{array}{l}111 \\
110 \\
116 \\
105\end{array}$ \\
\hline
\end{tabular}




\begin{tabular}{|c|c|c|c|c|c|}
\hline Analyte & $\begin{array}{l}\text { Qualified } \\
\text { Out of Ranget }\end{array}$ & $\begin{array}{l}\text { Mean } \\
\text { Recovery (\%) }\end{array}$ & $\begin{array}{l}\text { Standard } \\
\text { Deviation }\end{array}$ & $\begin{array}{l}\text { Minimum } \\
\text { Recovery (\%) }\end{array}$ & $\begin{array}{l}\text { Maximum } \\
\text { Recovery (\%) }\end{array}$ \\
\hline $\begin{array}{l}\text { cis-1,2-Dichloroethylene } \\
\text { Tetrachloroethylene } \\
\text { Toluene } \\
\text { Trichloroethylene }\end{array}$ & $\begin{array}{l}0 / 15 \\
0 / 22 \\
0 / 31 \\
0 / 31\end{array}$ & $\begin{array}{l}98.7 \\
99.2 \\
98.0 \\
92.4\end{array}$ & $\begin{array}{l}8.16 \\
7.24 \\
5.42 \\
5.19\end{array}$ & $\begin{array}{l}86.7 \\
86.5 \\
87.6 \\
78.1\end{array}$ & $\begin{array}{l}111 \\
114 \\
112 \\
102\end{array}$ \\
\hline $\begin{array}{l}\text { EPA8270C } \\
\text { Acenaphthene } \\
\text { Bis(2-ethylhexyl) phthalate } \\
\text { 4-Chloro-m-cresol } \\
\text { 2-Chlorophenol } \\
\text { 1,4-Dichlorobenzene } \\
\text { 2,4-Dinitrotoluene } \\
\text { 4-Nitrophenol } \\
\text { N-Nitrosodipropylamine } \\
\text { Pentachlorophenol } \\
\text { Phenol } \\
\text { Pyrene } \\
\text { 1,2,4-Trichlorobenzene }\end{array}$ & $\begin{array}{l}1 / 11 \\
0 / 4 \\
1 / 11 \\
0 / 11 \\
2 / 10 \\
0 / 11 \\
1 / 11 \\
1 / 11 \\
1 / 11 \\
0 / 18 \\
0 / 11 \\
2 / 11\end{array}$ & $\begin{array}{l}56.9 \\
72.8 \\
52.2 \\
55.8 \\
40.9 \\
65.3 \\
51.8 \\
62.8 \\
55.2 \\
59.5 \\
68.3 \\
44.5\end{array}$ & $\begin{array}{l}9.47 \\
18.1 \\
12.5 \\
9.58 \\
6.87 \\
17.6 \\
19.5 \\
14.5 \\
22.3 \\
13.6 \\
17.4 \\
9.18\end{array}$ & $\begin{array}{l}36.1 \\
46.3 \\
21.6 \\
34.5 \\
31.6 \\
27.2 \\
17.9 \\
38.3 \\
0.0 \\
26.3 \\
34.6 \\
23.4\end{array}$ & $\begin{array}{l}71.0 \\
87.4 \\
65.5 \\
71.0 \\
52.6 \\
85.4 \\
87.2 \\
85.1 \\
83.8 \\
75.8 \\
88.5 \\
58.2\end{array}$ \\
\hline $\begin{array}{l}\text { EPA8280A } \\
\text { Hexachlorodibenzo-p-dioxins } \\
\text { Hexachlorodibenzo-p-furans } \\
\text { Pentachlorodibenzo-p-furans } \\
2,3,7,8-T C D D \\
\text { Tetrachlorodibenzo-p-dioxins } \\
\text { Tetrachlorodibenzo-p-furans }\end{array}$ & $\begin{array}{l}0 / 5 \\
0 / 5 \\
0 / 10 \\
0 / 6 \\
0 / 5 \\
0 / 5\end{array}$ & $\begin{array}{l}99.8 \\
96.4 \\
104 \\
107 \\
107 \\
92.0\end{array}$ & $\begin{array}{l}11.5 \\
6.07 \\
6.41 \\
2.32 \\
2.59 \\
1.58\end{array}$ & $\begin{array}{l}91.0 \\
92.0 \\
92.0 \\
104 \\
104 \\
90.0\end{array}$ & $\begin{array}{l}120 \\
107 \\
110 \\
111 \\
111 \\
94.0\end{array}$ \\
\hline $\begin{array}{l}\text { EPA9014 } \\
\text { Cyanide }\end{array}$ & $0 / 62$ & 94.1 & 6.67 & 82.5 & 109 \\
\hline $\begin{array}{l}\text { EPA9020B } \\
\text { Total organic halogens }\end{array}$ & $0 / 106$ & 103 & 3.36 & 90.6 & 108 \\
\hline $\begin{array}{l}\text { EPA9030B } \\
\text { Sulfide }\end{array}$ & $0 / 10$ & 99.7 & 0.42 & 99.0 & 100 \\
\hline $\begin{array}{l}\text { EPA9034 } \\
\text { Sulfide }\end{array}$ & $0 / 4$ & 99.3 & 0.29 & 99.0 & 99.5 \\
\hline $\begin{array}{l}\text { EPA9050A } \\
\text { Specific conductance }\end{array}$ & $0 / 8$ & 96.5 & 2.16 & 93.5 & 98.7 \\
\hline $\begin{array}{l}\text { EPA9056 } \\
\text { Chloride } \\
\text { Sulfate }\end{array}$ & $\begin{array}{l}0 / 27 \\
0 / 28\end{array}$ & $\begin{array}{l}95.9 \\
96.9\end{array}$ & $\begin{array}{l}4.16 \\
1.68\end{array}$ & $\begin{array}{l}91.9 \\
95.2\end{array}$ & $\begin{array}{l}104 \\
102\end{array}$ \\
\hline $\begin{array}{l}\text { EPA9060 } \\
\text { Total organic carbon }\end{array}$ & $0 / 34$ & 106 & 3.49 & 96.4 & 110 \\
\hline $\begin{array}{l}\text { EPA9066 } \\
\text { Phenols }\end{array}$ & $0 / 2$ & 96.5 & 0.64 & 96.0 & 96.9 \\
\hline
\end{tabular}

$†$ Number of batches qualified that exhibit poor laboratory control sample and blank spike recovery due to interference compared to the total number of batches containing laboratory control samples and blank spikes.

- Standard deviation cannot be determined.

Note: A value of $O$ is reported as 0.0 
Table 44. Laboratory Control Sample Recoveries for GP

\begin{tabular}{|c|c|c|c|c|c|}
\hline Analyte & $\begin{array}{l}\text { Qualified } \\
\text { Out of Ranget }\end{array}$ & $\begin{array}{l}\text { Mean } \\
\text { Recovery (\%) }\end{array}$ & $\begin{array}{l}\text { Standard } \\
\text { Deviation }\end{array}$ & $\begin{array}{l}\text { Minimum } \\
\text { Recovery (\%) }\end{array}$ & $\begin{array}{l}\text { Maximum } \\
\text { Recovery (\%) }\end{array}$ \\
\hline \multicolumn{6}{|l|}{ EPIA-001 } \\
\hline Gross alpha & $1 / 33$ & 99.6 & 7.46 & 84.3 & 122 \\
\hline Nonvolatile beta & $1 / 31$ & 107 & 8.03 & 83.7 & 123 \\
\hline \multicolumn{6}{|l|}{ EPIA-002 } \\
\hline Tritium & $1 / 27$ & 269 & 874 & 88.9 & 4,640 \\
\hline \multicolumn{6}{|l|}{ EPIA-003 } \\
\hline Carbon-14 & $1 / 21$ & 94.1 & 6.57 & 78.5 & 108 \\
\hline \multicolumn{6}{|l|}{ EPIA-004 } \\
\hline Strontium-89/90 & $0 / 5$ & 88.9 & 12.2 & 80.1 & 109 \\
\hline Strontium-90 & $3 / 23$ & 97.7 & 14.3 & 80.4 & 125 \\
\hline \multicolumn{6}{|l|}{ EPIA-005 } \\
\hline Technetium-99 & $0 / 24$ & 106 & 5.55 & 96.2 & 116 \\
\hline \multicolumn{6}{|l|}{ EPIA-006 } \\
\hline lodine-129 & $1 / 23$ & 104 & 7.92 & 91.4 & 123 \\
\hline \multicolumn{6}{|l|}{ EPIA-008 } \\
\hline Radium-226 & $0 / 29$ & 102 & 8.39 & 87.4 & 114 \\
\hline \multicolumn{6}{|l|}{ EPIA-009 } \\
\hline Radium-228 & $2 / 25$ & 98.0 & 12.1 & 71.3 & 116 \\
\hline \multicolumn{6}{|l|}{ EPIA-010 } \\
\hline Radium, total alpha-emitting & $1 / 4$ & 85.5 & 5.22 & 79.2 & 90.6 \\
\hline \multicolumn{6}{|l|}{ EPIA-011 } \\
\hline Americium-241 & $2 / 29$ & 100 & 11.6 & 78.6 & 125 \\
\hline Curium-243/244 & $2 / 29$ & 98.5 & 11.5 & 74.6 & 121 \\
\hline Plutonium-238 & $3 / 3$ & 0.0 & 0.0 & 0.0 & 0.0 \\
\hline Plutonium-239/240 & $1 / 25$ & 94.0 & 9.62 & 77.9 & 116 \\
\hline Uranium-238 & $1 / 29$ & 94.2 & 7.43 & 79.8 & 110 \\
\hline \multicolumn{6}{|l|}{ EPIA-012 } \\
\hline Thorium-232 & $2 / 26$ & 90.9 & 7.56 & 75.8 & 106 \\
\hline \multicolumn{6}{|l|}{ EPIA-013 } \\
\hline Cesium-134 & $1 / 19$ & 90.6 & 6.06 & 79.0 & 104 \\
\hline Cesium-137 & $2 / 9$ & 79.4 & 45.0 & 0.0 & 106 \\
\hline \multicolumn{6}{|l|}{ EPIA-022 } \\
\hline
\end{tabular}

† Number of batches qualified that exhibit poor laboratory control sample and blank spike recovery due to interference compared to the total number of batches containing laboratory control samples and blank spikes.

Note: $A$ value of 0 is reported as 0.0 . 
Table 45. Laboratory Control Sample Recoveries for TM

\begin{tabular}{|c|c|c|c|c|c|}
\hline Analyte & $\begin{array}{l}\text { Qualified } \\
\text { Out of Ranget }\end{array}$ & $\begin{array}{l}\text { Mean } \\
\text { Recovery (\%) }\end{array}$ & $\begin{array}{l}\text { Standard } \\
\text { Deviation }\end{array}$ & $\begin{array}{l}\text { Minimum } \\
\text { Recovery (\%) }\end{array}$ & $\begin{array}{l}\text { Maximum } \\
\text { Recovery (\%) }\end{array}$ \\
\hline \multicolumn{6}{|l|}{ 3500NIEMOD } \\
\hline Nicke!-63 & $1 / 4$ & 103 & 24.3 & 80.3 & 127 \\
\hline \multicolumn{6}{|l|}{ EICHROMTC1MOD } \\
\hline Technetium-99 & $0 / 9$ & 104 & 4.83 & 97.6 & 113 \\
\hline \multicolumn{6}{|l|}{ EMLAM01MOD } \\
\hline Americium-241/Curium-246 & $0 / 7$ & 98.2 & 6.86 & 88.9 & 108 \\
\hline Curium-243/244 & $0 / 7$ & 93.0 & 4.03 & 89.3 & 101 \\
\hline \multicolumn{6}{|l|}{ EMLPU02MOD } \\
\hline Plutonium-238 & $0 / 7$ & 94.7 & 2.85 & 90.3 & 99.0 \\
\hline Plutonium-239/240 & $0 / 7$ & 105 & 6.60 & 98.1 & 113 \\
\hline \multicolumn{6}{|l|}{ EMLSR02MOD } \\
\hline Strontium-90 & $0 / 9$ & 98.6 & 7.38 & 80.7 & 105 \\
\hline \multicolumn{6}{|l|}{ EMLTH01MOD } \\
\hline Thorium-228 & $0 / 7$ & 104 & 10.1 & 93.7 & 120 \\
\hline Thorium-230 & $0 / 7$ & 98.0 & 6.98 & 88.1 & 107 \\
\hline Thorium-232 & $0 / 7$ & 105 & 7.69 & 94.9 & 117 \\
\hline \multicolumn{6}{|l|}{ EMLU02MOD } \\
\hline Uranium-234 & $0 / 8$ & 94.2 & 5.92 & 89.5 & 106 \\
\hline Uranium-235 & $6 / 8$ & 68.7 & 34.9 & 25.0 & 125 \\
\hline Uranium-238 & $0 / 8$ & 96.8 & 5.18 & 90.9 & 106 \\
\hline \multicolumn{6}{|l|}{ ENICMOD } \\
\hline Carbon-14 & $0 / 8$ & 102 & 1.40 & 99.5 & 104 \\
\hline \multicolumn{6}{|l|}{ EPA900.0MOD } \\
\hline Gross alpha & $17 / 91$ & 112 & 8.20 & 96.2 & 134 \\
\hline Nonvolatile beta & $0 / 72$ & 104 & 3.82 & 91.9 & 114 \\
\hline \multicolumn{6}{|l|}{ EPA901.1MOD } \\
\hline Cesium-137 & $0 / 11$ & 97.9 & 2.57 & 91.6 & 101 \\
\hline Cobalt-60 & $0 / 11$ & 99.6 & 3.0 & 94.0 & 103 \\
\hline \multicolumn{6}{|l|}{ EPA902.0MOD } \\
\hline lodine-129 & $0 / 8$ & 103 & 10.4 & 87.3 & 118 \\
\hline \multicolumn{6}{|l|}{ EPA903.0MOD } \\
\hline Radium, total alpha-emitting & $0 / 17$ & 102 & 5.37 & 90.1 & 117 \\
\hline Radium-226 & $0 / 9$ & 94.0 & 5.83 & 85.5 & 105 \\
\hline \multicolumn{6}{|l|}{ EPA904.0MOD } \\
\hline Radium-228 & $3 / 9$ & 96.1 & 18.9 & 71.0 & 129 \\
\hline \multicolumn{6}{|l|}{ EPA906.0MOD } \\
\hline Tritium & $8 / 97$ & 100 & 11.3 & 74.9 & 132 \\
\hline
\end{tabular}

† Number of batches qualified that exhibit poor laboratory control sample and blank spike recovery due to interference compared to the total number of batches containing laboratory control samples and blank spikes.

Note: $A$ value of 0 is reported as 0.0 . 
Table 46. Surrogate Recoveries for EX

\begin{tabular}{|c|c|c|c|c|c|}
\hline Analyte & $\begin{array}{l}\text { Qualified } \\
\text { Out of Ranget }\end{array}$ & $\begin{array}{l}\text { Mean } \\
\text { Recovery (\%) }\end{array}$ & $\begin{array}{l}\text { Standard } \\
\text { Deviation }\end{array}$ & $\begin{array}{l}\text { Minimum } \\
\text { Recovery (\%) }\end{array}$ & $\begin{array}{l}\text { Maximum } \\
\text { Recovery (\%) }\end{array}$ \\
\hline $\begin{array}{l}\text { EPA8021B } \\
\text { p-Bromofluorobenzene }\end{array}$ & $40 / 143$ & 107 & 11.3 & 78.0 & 138 \\
\hline $\begin{array}{l}\text { EPAB081A } \\
\text { Decachlorobiphenyl } \\
\text { Tetrachloro-m-xylene }\end{array}$ & $\begin{array}{l}0 / 16 \\
0 / 16\end{array}$ & $\begin{array}{l}103 \\
60.4\end{array}$ & $\begin{array}{l}10.3 \\
14.0\end{array}$ & $\begin{array}{l}84.0 \\
49.0\end{array}$ & $\begin{array}{l}129 \\
96.0\end{array}$ \\
\hline $\begin{array}{l}\text { EPA8082 } \\
\text { Decachlorobiphenyl } \\
\text { Tetrachloro-m-xylene }\end{array}$ & $\begin{array}{l}0 / 33 \\
0 / 33\end{array}$ & $\begin{array}{l}110 \\
67.8\end{array}$ & $\begin{array}{l}10.4 \\
12.7\end{array}$ & $\begin{array}{l}95.0 \\
46.0\end{array}$ & $\begin{array}{l}125 \\
96.0\end{array}$ \\
\hline $\begin{array}{l}\text { EPA8151A } \\
\text { 2,4-Dichlorophenylacetic acid }\end{array}$ & $0 / 19$ & 87.0 & 16.8 & 60.0 & 107 \\
\hline $\begin{array}{l}\text { EPA8260B } \\
\text { p-Bromofluorobenzene } \\
\text { 1,2-Dichloroethane-d4 } \\
\text { Toluene-d8 }\end{array}$ & $\begin{array}{l}0 / 434 \\
0 / 433 \\
0 / 434\end{array}$ & $\begin{array}{l}101 \\
103 \\
101\end{array}$ & $\begin{array}{l}5.79 \\
10.4 \\
4.76\end{array}$ & $\begin{array}{l}84.0 \\
78.0 \\
88.0\end{array}$ & $\begin{array}{l}125 \\
133 \\
120\end{array}$ \\
\hline $\begin{array}{l}\text { EPA8270C } \\
\text { 2-Fluorobiphenyl } \\
\text { 2-Fluorophenol } \\
\text { Nitrobenzene-d5 } \\
\text { Phenol-d5 } \\
\text { p-Terphenyl-d14 } \\
\text { 2,4,6-Tribromophenol (surr) }\end{array}$ & $\begin{array}{l}5 / 26 \\
4 / 26 \\
5 / 26 \\
1 / 26 \\
0 / 26 \\
0 / 26 \\
\end{array}$ & $\begin{array}{l}54.6 \\
46.6 \\
55.0 \\
52.0 \\
78.9 \\
70.0 \\
\end{array}$ & $\begin{array}{l}21.0 \\
22.2 \\
24.4 \\
22.0 \\
16.2 \\
34.9 \\
\end{array}$ & $\begin{array}{l}14.0 \\
10.0 \\
11.0 \\
12.0 \\
54.0 \\
20.0 \\
\end{array}$ & $\begin{array}{l}101 \\
103 \\
109 \\
104 \\
117 \\
161 \\
\end{array}$ \\
\hline
\end{tabular}

$\dagger$ Number of batches qualified that exhibit poor surrogate recovery due to interference compared to the total number of batches containing surrogates.

Table 47. Surrogate Recoveries for GE

\begin{tabular}{|c|c|c|c|c|c|}
\hline Analyte & $\begin{array}{l}\text { Qualified } \\
\text { Out of Ranget }\end{array}$ & $\begin{array}{l}\text { Mean } \\
\text { Recovery (\%) }\end{array}$ & $\begin{array}{l}\text { Standard } \\
\text { Deviation }\end{array}$ & $\begin{array}{l}\text { Minimum } \\
\text { Recovery (\%) }\end{array}$ & $\begin{array}{l}\text { Maximum } \\
\text { Recovery (\%) }\end{array}$ \\
\hline \multicolumn{6}{|l|}{ EPA8081A } \\
\hline $\begin{array}{l}\text { Decachlorobiphenyl } \\
\text { Tetrachloro-m-xylene }\end{array}$ & $\begin{array}{l}0 / 12 \\
0 / 12\end{array}$ & $\begin{array}{l}99.0 \\
92.9\end{array}$ & $\begin{array}{l}8.34 \\
7.56\end{array}$ & $\begin{array}{l}82.4 \\
79.4\end{array}$ & $\begin{array}{l}110 \\
108\end{array}$ \\
\hline \multicolumn{6}{|l|}{ EPA8082 } \\
\hline $\begin{array}{l}\text { Decachlorobiphenyl } \\
\text { Tetrachloro-m-xylene }\end{array}$ & $\begin{array}{l}0 / 13 \\
0 / 13\end{array}$ & $\begin{array}{l}100 \\
80.2\end{array}$ & $\begin{array}{l}10.6 \\
6.93\end{array}$ & $\begin{array}{l}80.5 \\
68.5\end{array}$ & $\begin{array}{l}114 \\
96.0\end{array}$ \\
\hline \multicolumn{6}{|l|}{ EPA8260B } \\
\hline $\begin{array}{l}\text { p-Bromofluorobenzene } \\
\text { Dibromofluoromethane } \\
\text { Toluene-d8 }\end{array}$ & $\begin{array}{l}341 / 622 \\
182 / 622 \\
419 / 622\end{array}$ & $\begin{array}{l}96.4 \\
93.2 \\
94.3\end{array}$ & $\begin{array}{l}32.6 \\
27.8 \\
32.5\end{array}$ & $\begin{array}{l}63.6 \\
71.4 \\
69.1\end{array}$ & $\begin{array}{l}776 \\
750 \\
810\end{array}$ \\
\hline \multicolumn{6}{|l|}{ EPA8270C } \\
\hline $\begin{array}{l}\text { 2-Fluorobiphenyl } \\
\text { 2-Fluorophenol }\end{array}$ & $\begin{array}{l}7 / 391 \\
0 / 12\end{array}$ & $\begin{array}{l}65.2 \\
32.8\end{array}$ & $\begin{array}{l}10.4 \\
6.73\end{array}$ & $\begin{array}{l}7.12 \\
15.5\end{array}$ & $\begin{array}{l}89.4 \\
39.8\end{array}$ \\
\hline
\end{tabular}




\begin{tabular}{llllll}
\hline Analyte & $\begin{array}{l}\text { Qualified } \\
\text { Out of Ranget }\end{array}$ & $\begin{array}{l}\text { Mean } \\
\text { Recovery (\%) }\end{array}$ & $\begin{array}{l}\text { Standard } \\
\text { Deviation }\end{array}$ & $\begin{array}{l}\text { Minimum } \\
\text { Recovery (\%) }\end{array}$ & $\begin{array}{l}\text { Maximum } \\
\text { Recovery (\%) }\end{array}$ \\
\hline & & & & & \\
Nitrobenzene-d5 & $3 / 391$ & 59.8 & 10.2 & 6.64 & 81.8 \\
Phenol-d6 & $0 / 12$ & 25.0 & 4.55 & 17.4 & 33.0 \\
p-Terphenyl-d14 & $6 / 391$ & 61.0 & 16.9 & 8.40 & 108 \\
2,4,6-Tribromophenol (surr) & $0 / 12$ & 55.8 & 11.0 & 31.0 & 78.1 \\
\hline
\end{tabular}

$\dagger$ Number of batches qualified that exhibit poor surrogate recovery due to interference compared to the total number of batches containing surrogates.

Table 48. Surrogate Recoveries for WA

\begin{tabular}{|c|c|c|c|c|c|}
\hline Analyte & $\begin{array}{l}\text { Qualified } \\
\text { Out of Ranget }\end{array}$ & $\begin{array}{l}\text { Mean } \\
\text { Recovery (\%) }\end{array}$ & $\begin{array}{l}\text { Standard } \\
\text { Deviation }\end{array}$ & $\begin{array}{l}\text { Minimum } \\
\text { Recovery (\%) }\end{array}$ & $\begin{array}{l}\text { Maximum } \\
\text { Recovery (\%) }\end{array}$ \\
\hline $\begin{array}{l}\text { EPA8010 } \\
\text { Bromochloromethane }\end{array}$ & $3 / 79$ & 95.1 & 15.4 & 4.62 & 135 \\
\hline $\begin{array}{l}\text { EPA8021B } \\
\text { Bromochloromethane }\end{array}$ & $0 / 83$ & 103 & 8.84 & 75.3 & 116 \\
\hline $\begin{array}{l}\text { EPA8081A } \\
\text { Decachlorobipheny! } \\
\text { Tetrachloro-m-xylene }\end{array}$ & $\begin{array}{l}0 / 102 \\
0 / 102\end{array}$ & $\begin{array}{l}104 \\
77.5\end{array}$ & $\begin{array}{l}28.4 \\
25.2\end{array}$ & $\begin{array}{l}29.0 \\
12.5\end{array}$ & $\begin{array}{l}260 \\
185\end{array}$ \\
\hline $\begin{array}{l}\text { EPA8082 } \\
\text { Decachlorobiphenyl } \\
\text { Tetrachloro-m-xylene }\end{array}$ & $\begin{array}{l}0 / 58 \\
0 / 56\end{array}$ & $\begin{array}{l}99.8 \\
75.5\end{array}$ & $\begin{array}{l}32.9 \\
33.7\end{array}$ & $\begin{array}{l}27.0 \\
10.0\end{array}$ & $\begin{array}{l}293 \\
282\end{array}$ \\
\hline $\begin{array}{l}\text { EPA8141A } \\
\text { TPP } \\
\text { Tributyl phosphate }\end{array}$ & $\begin{array}{l}0 / 50 \\
0 / 50\end{array}$ & $\begin{array}{l}77.7 \\
88.8\end{array}$ & $\begin{array}{l}37.3 \\
39.0\end{array}$ & $\begin{array}{l}7.0 \\
11.0\end{array}$ & $\begin{array}{l}162 \\
202\end{array}$ \\
\hline $\begin{array}{l}\text { EPA8151A } \\
\text { 2,4-Dichlorophenylacetic acid }\end{array}$ & $7 / 73$ & 91.7 & 16.6 & 46.3 & 124 \\
\hline $\begin{array}{l}\text { EPA8260B } \\
\text { p-Bromofluorobenzene } \\
\text { 1,2-Dichloroethane-d4 } \\
\text { Toluene-d8 }\end{array}$ & $\begin{array}{l}1 / 399 \\
1 / 399 \\
2 / 399\end{array}$ & $\begin{array}{l}97.2 \\
97.4 \\
99.8\end{array}$ & $\begin{array}{l}5.28 \\
8.37 \\
3.75\end{array}$ & $\begin{array}{l}87.0 \\
76.0 \\
88.0\end{array}$ & $\begin{array}{l}116 \\
115 \\
112\end{array}$ \\
\hline $\begin{array}{l}\text { EPAB270C } \\
\text { 2-Fluorobiphenyl } \\
\text { 2-Fluorophenol } \\
\text { Nitrobenzene-d5 } \\
\text { Phenol-d5 } \\
\text { p-Terphenyl-d14 } \\
\text { 2,4,6-Tribromophenol (surr) }\end{array}$ & $\begin{array}{l}4 / 93 \\
0 / 105 \\
2 / 93 \\
0 / 105 \\
1 / 93 \\
0 / 105\end{array}$ & $\begin{array}{l}58.4 \\
57.4 \\
62.1 \\
50.3 \\
69.5 \\
65.2\end{array}$ & $\begin{array}{l}13.6 \\
14.0 \\
14.6 \\
17.5 \\
18.7 \\
18.3\end{array}$ & $\begin{array}{l}9.36 \\
4.73 \\
6.67 \\
5.19 \\
12.2 \\
6.44\end{array}$ & $\begin{array}{l}83.6 \\
78.8 \\
84.1 \\
86.6 \\
102 \\
103\end{array}$ \\
\hline $\begin{array}{l}\text { EPA8280A } \\
\text { Carbon 13-labeled 1,2,3,6,7,8- } \\
\text { HXCDD }\end{array}$ & $.0 / 26$ & 86.5 & 14.4 & 66.0 & 114 \\
\hline $\begin{array}{l}\text { Carbon 13-labeled 2,3,7,8- } \\
\text { TCDD }\end{array}$ & $0 / 28$ & 79.1 & 6.36 & 67.0 & 89.0 \\
\hline $\begin{array}{l}\text { Carbon 13-labeled 2,3,7,8- } \\
\text { TCDF }\end{array}$ & $0 / 26$ & 76.0 & 5.91 & 62.0 & 85.0 \\
\hline Carbon 13-labeled HPCDF & $0 / 26$ & 81.8 & 14.1 & 50.0 & 107 \\
\hline
\end{tabular}

$\dagger$ Number of batches qualified that exhibit poor surrogate recovery due to interference compared to the total number of batches containing surrogates. 
Table 49. Matrix Spike Recoveries for EX

\begin{tabular}{|c|c|c|c|c|c|c|}
\hline Analyte & $\begin{array}{l}\text { Qualified } \\
\text { Out of Ranget }\end{array}$ & $\begin{array}{l}\text { Mean } \\
\text { Recovery (\%) }\end{array}$ & $\begin{array}{l}\text { Standard } \\
\text { Deviation }\end{array}$ & Bias (\%) & $\begin{array}{l}\text { Minimum } \\
\text { Recovery (\%) }\end{array}$ & $\begin{array}{l}\text { Maximum } \\
\text { Recovery (\%) }\end{array}$ \\
\hline \multicolumn{7}{|l|}{ EPA300.0 } \\
\hline Chloride & $0 / 2$ & 95.0 & 4.24 & -5.0 & 92.0 & 98.0 \\
\hline Fluoride & $0 / 2$ & 103 & 16.3 & 3.0 & 91.0 & 114 \\
\hline Nitrate as nitrogen & $0 / 16$ & 96.0 & 12.3 & -4.0 & 54.0 & 108 \\
\hline Nitrate-nitrite as nitrogen & $0 / 3$ & 105 & 8.08 & 5.0 & 100 & 114 \\
\hline Sulfate & $0 / 2$ & 92.5 & 3.54 & -7.50 & 90.0 & 95.0 \\
\hline Total phosphates (as P) & $0 / 1$ & 100 & - & 0.0 & 100 & 100 \\
\hline \multicolumn{7}{|l|}{ EPA6010B } \\
\hline Aluminum & $0 / 62$ & 104 & 5.05 & 4.0 & 96.0 & 114 \\
\hline Arsenic & $0 / 6$ & 103 & 3.25 & 3.0 & 100 & 108 \\
\hline Barium & $0 / 44$ & 104 & 4.70 & 4.0 & 96.0 & 113 \\
\hline Boron & $0 / 20$ & 106 & 8.27 & 6.0 & 94.0 & 124 \\
\hline Cadmium & $0 / 6$ & 106 & 4.68 & 6.0 & 100 & 113 \\
\hline Chromium & $0 / 6$ & 105 & 4.22 & 5.0 & 101 & 111 \\
\hline Iron & $0 / 62$ & 105 & 5.67 & 5.0 & 97.0 & 119 \\
\hline Lead & $0 / 60$ & 106 & 6.24 & 6.0 & 96.0 & 119 \\
\hline Manganese & $0 / 22$ & 104 & 7.28 & 4.0 & 94.0 & 115 \\
\hline Nickel & $0 / 36$ & 104 & 5.44 & 4.0 & 95.0 & 116 \\
\hline Selenium & $0 / 36$ & 101 & 9.92 & 1.0 & 80.0 & 116 \\
\hline Silver & $0 / 6$ & 106 & 4.67 & 6.0 & 100 & 113 \\
\hline \multicolumn{7}{|l|}{ EPA7470A } \\
\hline Mercury & $0 / 14$ & 97.1 & 7.10 & -2.90 & 82.0 & 108 \\
\hline \multicolumn{7}{|l|}{ EPA8021B } \\
\hline Carbon tetrachloride & $0 / 24$ & 110 & 10.4 & 10.0 & 82.0 & 125 \\
\hline Chloroform & $0 / 24$ & 113 & 10.1 & 13.0 & 87.0 & 124 \\
\hline cis-1,2-Dichloroethylene & $0 / 24$ & 107 & 12.1 & 7.0 & 76.0 & 122 \\
\hline Tetrachloroethylene & $0 / 24$ & 114 & 13.8 & 14.0 & 88.0 & 141 \\
\hline 1,1,1-Trichloroethane & $0 / 24$ & 110 & 9.38 & 10.0 & 90.0 & 123 \\
\hline Trichioroethylene & $0 / 24$ & 95.7 & 17.1 & -4.30 & 71.0 & 125 \\
\hline \multicolumn{7}{|l|}{ EPA8082 } \\
\hline PCB 1260 & $0 / 4$ & 108 & 10.4 & 8.0 & 95.0 & 117 \\
\hline \multicolumn{7}{|l|}{ EPAB151A } \\
\hline $\begin{array}{l}\text { 2,4-Dichlorophenoxyacetic } \\
\text { acid }\end{array}$ & $0 / 2$ & 99.5 & 0.71 & -0.50 & 99.0 & 100 \\
\hline \multicolumn{7}{|l|}{ EPA8260B } \\
\hline Benzene & $0 / 46$ & 101 & 6.51 & 1.0 & 92.0 & 117 \\
\hline Chlorobenzene & $0 / 46$ & 98.8 & 6.92 & -1.20 & 83.0 & 109 \\
\hline 1,1-Dichloroethylene & $0 / 46$ & 98.9 & 8.42 & -1.10 & 78.0 & 118 \\
\hline Toluene & $0 / 46$ & 99.1 & 6.43 & -0.90 & 86.0 & 112 \\
\hline Trichloroethylene & $0 / 46$ & 96.7 & 9.10 & -3.30 & 69.0 & 116 \\
\hline \multicolumn{7}{|l|}{ EPA9014 } \\
\hline Cyanide & $0 / 46$ & 89.1 & 23.5 & -10.9 & 1.0 & 103 \\
\hline
\end{tabular}

$\dagger$ Number of batches qualified that exhibit poor spike recovery due to interference compared to the total number of batches containing spikes.

- Standard deviation cannot be determined.

Note: A value of 0 is reported as 0.0 . 
Table 50. Matrix Spike Recoveries for GE

\begin{tabular}{|c|c|c|c|c|c|c|}
\hline Analyte & $\begin{array}{l}\text { Qualified } \\
\text { Out of Ranget }\end{array}$ & $\begin{array}{l}\text { Mean } \\
\text { Recovery (\%) }\end{array}$ & $\begin{array}{l}\text { Standard } \\
\text { Deviation }\end{array}$ & Bias (\%) & $\begin{array}{l}\text { Minimum } \\
\text { Recovery (\%) }\end{array}$ & $\begin{array}{l}\text { Maximum } \\
\text { Recovery (\%) }\end{array}$ \\
\hline \multicolumn{7}{|l|}{ EPA353.1 } \\
\hline Nitrate-nitrite as nitrogen & $0 / 31$ & 101 & 7.93 & 1.0 & 72.0 & 114 \\
\hline \multicolumn{7}{|l|}{ EPA6010B } \\
\hline Aluminum & $0 / 28$ & 64.2 & 83.8 & -35.8 & -163 & 106 \\
\hline Antimony & $0 / 6$ & 101 & 5.48 & 1.0 & 92.5 & 105 \\
\hline Arsenic & $0 / 28$ & 102 & 4.61 & 2.0 & 90.8 & 110 \\
\hline Barium & $0 / 28$ & 98.2 & 4.31 & -1.80 & 90.3 & 104 \\
\hline Beryllium & $0 / 2$ & 92.6 & 1.13 & -7.40 & 91.8 & 93.4 \\
\hline Cadmium & $0 / 28$ & 101 & 5.47 & 1.0 & 90.6 & 110 \\
\hline Calcium & $0 / 2$ & 94.7 & 1.63 & -5.30 & 93.5 & 95.8 \\
\hline Chromium & $0 / 28$ & 101 & 4.78 & 1.0 & 90.1 & 108 \\
\hline Cobalt & $0 / 2$ & 92.3 & 1.06 & -7.70 & 91.5 & 93.0 \\
\hline Copper & $0 / 2$ & 90.9 & 1.20 & -9.10 & 90.0 & 91.7 \\
\hline Iron & $0 / 30$ & 97.3 & 29.8 & -2.70 & -43.0 & 144 \\
\hline Lead & $0 / 28$ & 104 & 5.99 & 4.0 & 90.9 & 115 \\
\hline Magnesium & $0 / 2$ & 93.6 & 1.48 & -6.40 & 92.5 & 94.6 \\
\hline Manganese & $0 / 4$ & 94.0 & 2.39 & -6.0 & 91.4 & 96.9 \\
\hline Nickel & $0 / 2$ & 92.0 & 1.13 & -8.0 & 91.2 & 92.8 \\
\hline Potassium & $0 / 2$ & 90.2 & 1.41 & -9.80 & 89.2 & 91.2 \\
\hline Selenium & $0 / 28$ & 100 & 4.36 & 0.0 & 90.4 & 106 \\
\hline Silver & $0 / 28$ & 97.0 & 26.6 & -3.0 & -3.51 & 110 \\
\hline Sodium & $0 / 2$ & 91.5 & 0.64 & -8.50 & 91.0 & 91.9 \\
\hline Thallium & $0 / 2$ & 90.1 & 0.85 & -9.90 & 89.5 & 90.7 \\
\hline Vanadium & $0 / 2$ & 92.1 & 1.13 & -7.90 & 91.3 & 92.9 \\
\hline Zinc & $0 / 2$ & 92.7 & 1.77 & -7.30 & 91.4 & 93.9 \\
\hline \multicolumn{7}{|l|}{ EPA6020 } \\
\hline Aluminum & $0 / 36$ & 102 & 172 & 2.0 & -354 & 680 \\
\hline Antimony & $0 / 36$ & 94.2 & 8.79 & -5.80 & 83.4 & $\$ 11$ \\
\hline Arsenic & $0 / 36$ & 99.7 & 8.50 & -0.30 & 69.9 & 109 \\
\hline Barium & $0 / 36$ & 91.7 & 9.15 & -8.30 & 80.3 & 116 \\
\hline Beryilium & $0 / 2$ & 101 & 1.63 & 1.0 & 99.7 & 102 \\
\hline Cadmium & $0 / 36$ & 99.5 & 4.55 & -0.50 & 82.0 & 105 \\
\hline Calcium & $0 / 2$ & 109 & 0.71 & 9.0 & 108 & 109 \\
\hline Chromium & $0 / 36$ & 90.1 & 7.79 & -9.90 & 78.4 & 109 \\
\hline Cobalt & $0 / 36$ & 97.6 & 6.22 & -2.40 & 79.4 & 107 \\
\hline Copper & $0 / 36$ & 99.2 & 7.77 & -0.80 & 78.9 & 109 \\
\hline Iron & $0 / 36$ & 79.6 & 27.0 & -20.4 & -1.22 & 119 \\
\hline Lead & $0 / 36$ & 102 & 6.22 & 2.0 & 84.4 & 112 \\
\hline Magnesium & $0 / 2$ & 80.7 & 1.34 & -19.3 & 79.7 & 81.6 \\
\hline Manganese & $0 / 2$ & 100 & 0.0 & 0.0 & 100 & 100 \\
\hline Nickel & $0 / 36$ & 97.9 & 7.02 & -2.10 & 78.8 & 111 \\
\hline Potassium & $0 / 2$ & 89.3 & 1.48 & -10.7 & 88.2 & 90.3 \\
\hline Selenium & $0 / 36$ & 101 & 9.11 & 1.0 & 71.5 & 114 \\
\hline Silver & $0 / 44$ & 92.5 & 27.8 & -7.50 & 14.9 & 116 \\
\hline Sodium & $0 / 2$ & 108 & 2.83 & 8.0 & 106 & 110 \\
\hline Thallium & $0 / 14$ & 95.4 & 6.79 & -4.60 & 84.0 & 105 \\
\hline Tin & $0 / 24$ & 82.1 & 26.9 & -17.9 & 0.0 & 108 \\
\hline Vanadium & $0 / 38$ & 103 & 8.83 & 3.0 & 79.6 & 124 \\
\hline Zinc & $0 / 36$ & 93.3 & 8.97 & -6.70 & 80.2 & 113 \\
\hline \multicolumn{7}{|l|}{ EPA7470A } \\
\hline Mercury & $0 / 60$ & 101 & 10.1 & 1.0 & 86.1 & 130 \\
\hline \multicolumn{7}{|l|}{ EPA8081A } \\
\hline Aldrin & $0 / 2$ & 108 & 3.54 & 8.0 & 105 & 110 \\
\hline p,p'-DDT & $0 / 2$ & 128 & 0.71 & 28.0 & 127 & 128 \\
\hline
\end{tabular}




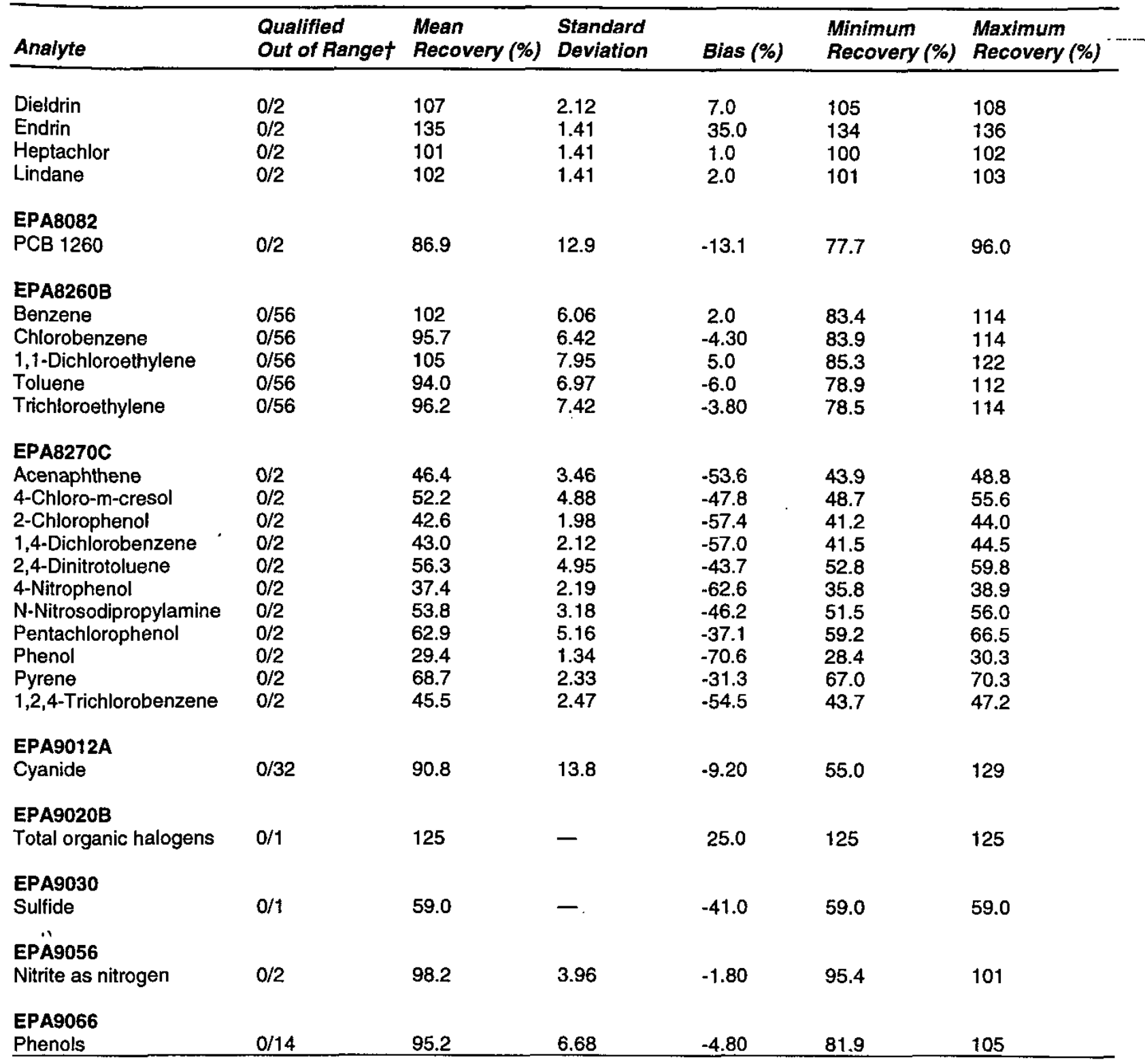

$\dagger$ Number of batches qualified that exhibit poor spike recovery due to interference compared to the total number of batches containing spikes.

- Standard deviation cannot be determined.

Note: $A$ value of 0 is reported as 0.0 . 
Table 51. Matrix Spike Recoveries for WA

\begin{tabular}{|c|c|c|c|c|c|c|}
\hline Analyte & $\begin{array}{l}\text { Qualified } \\
\text { Out of Ranget }\end{array}$ & $\begin{array}{l}\text { Mean } \\
\text { Recovery (\%) }\end{array}$ & $\begin{array}{l}\text { Standard } \\
\text { Deviation }\end{array}$ & Bias (\%) & $\begin{array}{l}\text { Minimum } \\
\text { Recovery (\%) }\end{array}$ & $\begin{array}{l}\text { Maximum } \\
\text { Recovery (\%) }\end{array}$ \\
\hline \multicolumn{7}{|l|}{ EPA310.1 } \\
\hline Alkalinity (as $\mathrm{CaCO}_{3}$ ) & $0 / 1$ & 101 & - & 1.0 & 101 & 101 \\
\hline \multicolumn{7}{|l|}{ EPA340.2 } \\
\hline Fluoride & $0 / 9$ & 91.6 & 11.5 & -8.40 & 72.2 & 109 \\
\hline \multicolumn{7}{|l|}{ EPA353.2 } \\
\hline Nitrate as nitrogen & $0 / 3$ & 105 & 4.16 & 5.0 & 100 & 108 \\
\hline Nitrate-nitrite as nitrogen & $0 / 12$ & 101 & 3.85 & 1.0 & 92.4 & 107 \\
\hline Nitrite as nitrogen & $0 / 1$ & 105 & - & 5.0 & 105 & 105 \\
\hline \multicolumn{7}{|l|}{ EPA365.2 } \\
\hline Total phosphates (as P) & $0 / 8$ & 105 & 4.71 & 5.0 & 97.6 & 111 \\
\hline \multicolumn{7}{|l|}{ EPA6010B } \\
\hline Aluminum & $0 / 50$ & 104 & 4.37 & 4.0 & 98.8 & 126 \\
\hline Antimony & $0 / 22$ & 101 & 2.30 & 1.0 & 97.2 & 104 \\
\hline Arsenic & $0 / 48$ & 102 & 2.44 & 2.0 & 94.5 & 108 \\
\hline Barium & $0 / 44$ & 99.3 & 2.88 & -0.70 & 92.5 & 108 \\
\hline Beryllium & $0 / 12$ & 101 & 2.29 & 1.0 & 96.8 & 103 \\
\hline Boron & $0 / 16$ & 97.5 & 3.05 & -2.50 & 89.2 & 101 \\
\hline Cadmium & $0 / 39$ & 99.8 & 2.49 & -0.20 & 94.4 & 104 \\
\hline Calcium & $0 / 21$ & 101 & 2.79 & 1.0 & 95.2 & 105 \\
\hline Chromium & $0 / 46$ & 101 & 2.44 & 1.0 & 95.2 & 107 \\
\hline Cobalt & $0 / 20$ & 99.7 & 1.85 & -0.30 & 96.2 & 103 \\
\hline Copper & $0 / 22$ & 99.7 & 1.95 & -0.30 & 96.3 & 103 \\
\hline Iron & $0 / 52$ & 102 & 6.05 & 2.0 & 92.0 & 129 \\
\hline Lead & $0 / 49$ & 101 & 2.78 & 1.0 & 93.6 & 107 \\
\hline Lithium & $0 / 16$ & 107 & 3.54 & 7.0 & 100 & 113 \\
\hline Magnesium & $0 / 19$ & 102 & 2.79 & 2.0 & 95.6 & 106 \\
\hline Manganese & $0 / 31$ & 102 & 2.83 & 2.0 & 95.9 & 107 \\
\hline Nickel & $0 / 29$ & 100 & 2.84 & 0.0 & 93.5 & 105 \\
\hline Potassium & $0 / 19$ & 104 & 3.46 & 4.0 & 97.5 & 110 \\
\hline Selenium & $0 / 46$ & 103 & 3.03 & 3.0 & 95.6 & 112 \\
\hline Silica & $0 / 16$ & 121 & 31.2 & 21.0 & 97.3 & 213 \\
\hline Silver & $0 / 44$ & 99.6 & 3.36 & -0.40 & 91.2 & 110 \\
\hline Sodium & $0 / 32$ & 96.3 & 3.81 & -3.70 & 81.6 & 100 \\
\hline Thallium & $0 / 16$ & 102 & 1.48 & 2.0 & 100 & 105 \\
\hline Tin & $0 / 14$ & 99.2 & 1.88 & -0.80 & 96.0 & 102 \\
\hline Vanadium & $0 / 20$ & 102 & 2.72 & 2.0 & 98.8 & 108 \\
\hline Zinc & $0 / 22$ & 99.2 & 1.26 & -0.80 & 97.0 & 102 \\
\hline \multicolumn{7}{|l|}{ EPA7470A } \\
\hline Mercury & $0 / 24$ & 92.2 & 13.3 & -7.80 & 51.4 & 104 \\
\hline \multicolumn{7}{|l|}{ EPA8010 } \\
\hline Carbon tetrachloride & $0 / 7$ & 102 & 10.6 & 2.0 & 87.6 & 121 \\
\hline Chloroform & $0 / 7$ & 103 & 9.62 & 3.0 & 91.8 & 122 \\
\hline cis-1,2-Dichloroethylene & $0 / 7$ & 100 & 14.2 & 0.0 & 80.9 & 128 \\
\hline $1,1,1$-Trichloroethane & $0 / 7$ & 104 & 11.1 & 4.0 & 88.8 & 125 \\
\hline Trichloroethylene & $0 / 7$ & 99.8 & 15.9 & -0.20 & 75.0 & 127 \\
\hline \multicolumn{7}{|l|}{ EPA8021B } \\
\hline Carbon tetrachloride & $0 / 9$ & 97.1 & 7.54 & -2.90 & 81.4 & 105 \\
\hline Chloroform & $0 / 9$ & 97.8 & 6.31 & -2.20 & 83.4 & 105 \\
\hline cis-1,2-Dichloroethylene & $0 / 1$ & 91.1 & - & -8.90 & 91.1 & 91.1 \\
\hline 1,1,1-Trichloroethane & $0 / 9$ & 95.4 & 6.64 & -4.60 & 80.6 & 103 \\
\hline Trichloroethylene & $0 / 9$ & 88.1 & 16.1 & -11.9 & 51.7 & 102 \\
\hline
\end{tabular}




\begin{tabular}{|c|c|c|c|c|c|c|}
\hline Analyte & $\begin{array}{l}\text { Qualified } \\
\text { Out of Ranget }\end{array}$ & $\begin{array}{l}\text { Mean } \\
\text { Recovery (\%) }\end{array}$ & $\begin{array}{l}\text { Standard } \\
\text { Deviation }\end{array}$ & Bias (\%) & $\begin{array}{l}\text { Minimum } \\
\text { Recovery (\%) }\end{array}$ & $\begin{array}{l}\text { Maximum } \\
\text { Recovery (\%) }\end{array}$ \\
\hline
\end{tabular}

\section{EPA8081A}

Aldrin

p,p'-DDT

Dieldrin

Endrin

Heptachlor

Lindane

EPA8082

PCB 1016

PCB 1221

PCB 1232

PCB 1242

PCB 1248

PCB 1254

PCB 1260

\section{EPA8141A}

Dimethoate

Disulfoton

Famphur

Parathion

Parathion ethyl

Parathion methyl

Phorate

Sulfotepp

Thionazin

$0,0,0$-Triethyl

phosphorothioate

EPA8151A

2,4-Dichlorophenoxyacetic $0 / 8$ acid

2,4,5-T

2,4,5-TP (Silvex)

$0 / 4$

$0 / 4$

\section{EPA8260B}

Benzene

Chlorobenzene

1,1-Dichloroethylene

1,2-Dichloroethylene

cis-1,2-Dichloroethylene

Tetrachloroethylene

Toluene

Trichloroethylene

$$
0 / 15
$$

$0 / 15$

$0 / 15$

$0 / 1$

$0 / 7$

$0 / 16$

$0 / 15$

$0 / 15$

EPA8270C

Acenaphthene

4-Chloro-mil) phthalate $0 / 5$

2-Chlorophenol $\quad 0 / 9$

1,4-Dichlorobenzene $\quad 0 / 9$

2,4-Dinitrotoluene $\quad 0 / 9$

4-Nitrophenol

N-Nitrosodipropylamine

Pentachlorophenol

Phenol

Pyrene

1,2,4-Trichlorobenzene

$0 / 9$

$0 / 9$
78.8

81.0

88.4

108

90.0

89.9

97.3

91.0

82.8

95.1

125

87.5

109

87.2

98.3

96.8

93.0

103

97.6

98.6

98.8

90.0

56.0

74.5

52.6

56.0

44.5

66.0

47.8

63.5

59.7

59.9

61.4

49.9

78.8
92.0
91.0
105
85.0
78.8

10.3

11.8

8.87

11.5

9.17

23.9

-
-
-
$\overline{7} .61$

8.09

11.1

10.8

9.19

9.28

11.3

12.2

11.4

11.7

26.4

25.0

13.5

11.5

6.86

6.97

6.32

$-$

7.46

10.0

7.41

9.15

16.5

12.9

16.0

15.6

14.3

21.3

16.4

17.7

22.0

13.7

18.0

17.1

$\begin{array}{cc}-21.2 & 70.0 \\ -8.0 & 80.0 \\ -9.0 & 80.0 \\ 5.0 & 98.0 \\ -15.0 & 75.0 \\ -21.2 & 19.2\end{array}$

90.0

104

100

122

95.1

100

$-16.2$

67.2

92.5

$-19.0$

$-11.6$

8.0

$-10.0$

$-10.1$

$-2.70$

$-9.0$

$-17.2$

$-4.90$

25.0

74.5

74.5

97.0

83.5

79.0

82.0

78.0

70.5

80.5

86.5

96.0

106

128

96.5

99.0

114

111

104

113

150
$-12.5$

9.0

$-12.8$

$-1.70$

$-3.20$

$-7.0$

3.0

$-2.40$

$-1.40$

$-1.20$

$-10.0$

$-44.0$

$-25.5$

$-47.4$

$-44.0$

$-55.5$

$-34.0$

$-52.2$

$-36.5$

$-40.3$

$-40.1$

$-38.6$

$-50.1$
42.8

121

122

96.4

90.8

88.1

87.1

80.9

103

83.2

75.4

85.3

65.0

110

110

103

103

105

124

111

102

22.0

53.4

14.8

22.0

19.4

15.3

11.8

24.3

8.74

17.8

21.3

13.2
80.2

88.2

72.2

77.6

66.5

86.2

68.2

84.1

90.4

78.0

85.3

72.8 


\begin{tabular}{|c|c|c|c|c|c|c|}
\hline Analyte & $\begin{array}{l}\text { Qualified } \\
\text { Out of Ranget }\end{array}$ & $\begin{array}{l}\text { Mean } \\
\text { Recovery (\%) }\end{array}$ & $\begin{array}{l}\text { Standard } \\
\text { Deviation }\end{array}$ & Bias (\%) & $\begin{array}{l}\text { Minimum } \\
\text { Recovery (\%) }\end{array}$ & $\begin{array}{l}\text { Maximum } \\
\text { Recovery (\%) }\end{array}$ \\
\hline \multicolumn{7}{|l|}{ EPA8280A } \\
\hline $\begin{array}{l}\text { Hexachlorodibenzo-p- } \\
\text { dioxins }\end{array}$ & $0 / 5$ & 96.0 & 6.16 & -4.0 & 91.0 & 106 \\
\hline $\begin{array}{l}\text { Hexachlorodibenzo-p- } \\
\text { furans }\end{array}$ & $0 / 5$ & 95.2 & 5.85 & -4.80 & 85.0 & 99.0 \\
\hline $\begin{array}{l}\text { Pentachlorodibenzo-p- } \\
\text { furans }\end{array}$ & $0 / 10$ & 103 & 8.52 & 3.0 & 93.0 & 115 \\
\hline $2,3,7,8-T C D D$ & $0 / 5$ & 104 & 4.30 & 4.0 & 100 & 111 \\
\hline $\begin{array}{l}\text { Tetrachlorodibenzo-p- } \\
\text { dioxins }\end{array}$ & $0 / 5$ & 104 & 4.30 & 4.0 & 100 & 111 \\
\hline $\begin{array}{l}\text { Tetrachlorodibenzo-p- } \\
\text { furans }\end{array}$ & $0 / 5$ & 94.6 & 1.67 & -5.40 & 92.0 & 96.0 \\
\hline \multicolumn{7}{|l|}{ EPA9014 } \\
\hline Cyanide & $0 / 10$ & 94.1 & 5.85 & -5.90 & 83.3 & 103 \\
\hline \multicolumn{7}{|l|}{ EPA9020B } \\
\hline Total organic halogens & $0 / 12$ & 105 & 6.29 & 5.0 & 89.5 & 115 \\
\hline \multicolumn{7}{|l|}{ EPA9030B } \\
\hline Sulfide & $0 / 4$ & 99.2 & 0.76 & -0.80 & 98.2 & 100 \\
\hline \multicolumn{7}{|l|}{ EPA9056 } \\
\hline Chloride & $0 / 9$ & 98.5 & 7.72 & -1.50 & 82.4 & 109 \\
\hline Sulfate & $0 / 10$ & 97.1 & 9.08 & -2.90 & 77.0 & 109 \\
\hline \multicolumn{7}{|l|}{ EPA9060 } \\
\hline Total organic carbon & $0 / 7$ & 109 & 7.04 & 9.0 & 99.6 & 118 \\
\hline \multicolumn{7}{|l|}{ EPA9066 } \\
\hline Phenols & $0 / 2$ & 98.0 & 0.71 & -2.0 & 97.5 & 98.5 \\
\hline \multicolumn{7}{|c|}{$\begin{array}{l}\text { Number of batches qualified that exhibit poor spike recovery due to interference compared to the total number of } \\
\text { batches containing spikes. } \\
\text { - Standard deviation cannot be determined. }\end{array}$} \\
\hline \multicolumn{7}{|c|}{ Note: $A$ value of 0 is reported as 0.0} \\
\hline \multicolumn{7}{|c|}{ Table 52. Matrix Spike Recoveries for GP } \\
\hline Analyte & $\begin{array}{l}\text { Qualified } \\
\text { Out of Ranget }\end{array}$ & $\begin{array}{l}\text { Mean } \\
\text { Recovery (\%) }\end{array}$ & $\begin{array}{l}\text { Standard } \\
\text { Deviation }\end{array}$ & Blas (\%) & $\begin{array}{l}\text { Minimum } \\
\text { Recovery (\%) }\end{array}$ & $\begin{array}{l}\text { Maximum } \\
\text { Recovery (\%) }\end{array}$ \\
\hline \multicolumn{7}{|l|}{ EPIA-001 } \\
\hline Gross alpha & $0 / 34$ & 94.2 & 10.1 & -5.80 & 79.4 & 120 \\
\hline Nonvolatile beta & $0 / 32$ & 92.0 & 65.0 & -8.0 & -214 & 229 \\
\hline \multicolumn{7}{|l|}{ EPIA-002 } \\
\hline Tritium & $0 / 27$ & 258 & 867 & 158 & -308 & 4,560 \\
\hline \multicolumn{7}{|l|}{ EPIA-003 } \\
\hline Carbon-14 & $0 / 21$ & 93.9 & 7.25 & -6.10 & 75.3 & 103 \\
\hline \multicolumn{7}{|l|}{ EPIA-004 } \\
\hline $\begin{array}{l}\text { Strontium-89/90 } \\
\text { Strontium-90 }\end{array}$ & $\begin{array}{l}0 / 5 \\
0 / 21\end{array}$ & $\begin{array}{l}96.8 \\
108\end{array}$ & $\begin{array}{l}15.5 \\
33.8\end{array}$ & $\begin{array}{l}-3.20 \\
8.0\end{array}$ & $\begin{array}{l}79.1 \\
84.7\end{array}$ & $\begin{array}{l}118 \\
246\end{array}$ \\
\hline EPIA-005 & & & & & & \\
\hline
\end{tabular}




\begin{tabular}{|c|c|c|c|c|c|c|}
\hline Analyte & $\begin{array}{l}\text { Qualified } \\
\text { Out of Ranget }\end{array}$ & $\begin{array}{l}\text { Mean } \\
\text { Recovery (\%) }\end{array}$ & $\begin{array}{l}\text { Standard } \\
\text { Deviation }\end{array}$ & Bias (\%) & $\begin{array}{l}\text { Minimum } \\
\text { Recovery (\%) }\end{array}$ & $\begin{array}{l}\text { Maximum } \\
\text { Recovery (\%) }\end{array}$ \\
\hline Technetium-99 & $0 / 23$ & 108 & 6.22 & 8.0 & 96.2 & 119 \\
\hline $\begin{array}{l}\text { EPIA-006 } \\
\text { lodine-129 }\end{array}$ & $0 / 20$ & 107 & 8.51 & 7.0 & 85.2 & 119 \\
\hline $\begin{array}{l}\text { EPIA-008 } \\
\text { Radium-226 }\end{array}$ & $0 / 28$ & 104 & 9.38 & 4.0 & 79.7 & 119 \\
\hline $\begin{array}{l}\text { EPIA-009 } \\
\text { Radium-228 }\end{array}$ & $0 / 25$ & 98.5 & 13.8 & -1.50 & 75.6 & 120 \\
\hline $\begin{array}{l}\text { EPIA-010 } \\
\text { Radium, total alpha- } \\
\text { emitting }\end{array}$ & $0 / 4$ & 83.4 & 8.83 & -16.6 & 75.7 & 94.0 \\
\hline $\begin{array}{l}\text { EPIA-011 } \\
\text { Americium-241 } \\
\text { Curium-243/244 } \\
\text { Plutonium-238 } \\
\text { Plutonium-239/240 } \\
\text { Uranium-238 }\end{array}$ & $\begin{array}{l}0 / 30 \\
0 / 30 \\
0 / 3 \\
0 / 27 \\
0 / 30\end{array}$ & $\begin{array}{l}96.2 \\
98.7 \\
0.0 \\
92.8 \\
85.3\end{array}$ & $\begin{array}{l}10.2 \\
10.9 \\
0.0 \\
8.73 \\
41.7\end{array}$ & $\begin{array}{l}-3.80 \\
-1.30 \\
-100 \\
-7.20 \\
-14.7\end{array}$ & $\begin{array}{l}75.7 \\
75.1 \\
0.0 \\
78.6 \\
-69.1\end{array}$ & $\begin{array}{l}114 \\
118 \\
0.0 \\
109 \\
114\end{array}$ \\
\hline $\begin{array}{l}\text { EPIA-012 } \\
\text { Thorium-232 }\end{array}$ & $0 / 26$ & 93.9 & 11.5 & -6.10 & 75.9 & 118 \\
\hline $\begin{array}{l}\text { EPIA-013 } \\
\text { Cesium-134 } \\
\text { Cesium-137 }\end{array}$ & $\begin{array}{l}0 / 19 \\
0 / 8\end{array}$ & $\begin{array}{l}87.6 \\
77.6\end{array}$ & $\begin{array}{l}8.11 \\
48.2\end{array}$ & $\begin{array}{l}-12.4 \\
-22.4\end{array}$ & $\begin{array}{l}75.6 \\
0.0\end{array}$ & $\begin{array}{l}106 \\
112\end{array}$ \\
\hline $\begin{array}{l}\text { EPIA-022 } \\
\text { Nickel-63 } \\
\end{array}$ & $0 / 11$ & 99.7 & 7.02 & -0.30 & 86.8 & 110 \\
\hline
\end{tabular}

$\dagger$ Number of batches qualified that exhibit poor spike recovery due to interference compared to the total number of batches containing spikes.

Note: $A$ value of 0 is reported as 0.0 .

Tablo 53. Analytes Detected in Method Blanks for EX

\begin{tabular}{|c|c|c|c|c|}
\hline Analyte & $\begin{array}{l}\text { Frequency } \\
\text { of Detectiont }\end{array}$ & Mean Result & $\begin{array}{l}\text { Standard } \\
\text { Deviation }\end{array}$ & Minimum/Maximum Results \\
\hline \multicolumn{5}{|l|}{ AP-3086 } \\
\hline Total organic carbon & $0 / 1$ & 5,000 & - & $5,000 / 5,000 \mu g / L$ \\
\hline \multicolumn{5}{|l|}{ EPA300.0 } \\
\hline Chloride & $0 / 5$ & 200 & 0.0 & $200 / 200 \mu g / L$ \\
\hline Fluoride & $0 / 5$ & 100 & 0.0 & $100 / 100 \mu \mathrm{g} / \mathrm{L}$ \\
\hline Nitrate as nitrogen & $0 / 11$ & 100 & 0.0 & $100 / 100 \mu \mathrm{g} / \mathrm{L}$ \\
\hline Nitrate-nitrite as nitrogen & $0 / 6$ & 100 & 0.0 & $100 / 100 \mu g / L$ \\
\hline Sulfate & $0 / 5$ & 200 & 0.0 & $200 / 200 \mu g / L$ \\
\hline Total phosphates (as P) & $0 / 5$ & 500 & 0.0 & $500 / 500 \mu g / L$ \\
\hline \multicolumn{5}{|l|}{ EPA6010B } \\
\hline Aluminum & $0 / 33$ & 200 & 0.0 & $200 / 200 \mu g / L$ \\
\hline Arsenic & $0 / 7$ & 100 & 0.0 & $100 / 100 \mu \mathrm{g} / \mathrm{L}$ \\
\hline Barium & $0 / 24$ & 10.0 & 0.0 & $10.0 / 10.0 \mu g / L$ \\
\hline Boron & $1 / 11$ & 91.9 & 26.8 & $11.0 / 100 \mu g / L$ \\
\hline
\end{tabular}




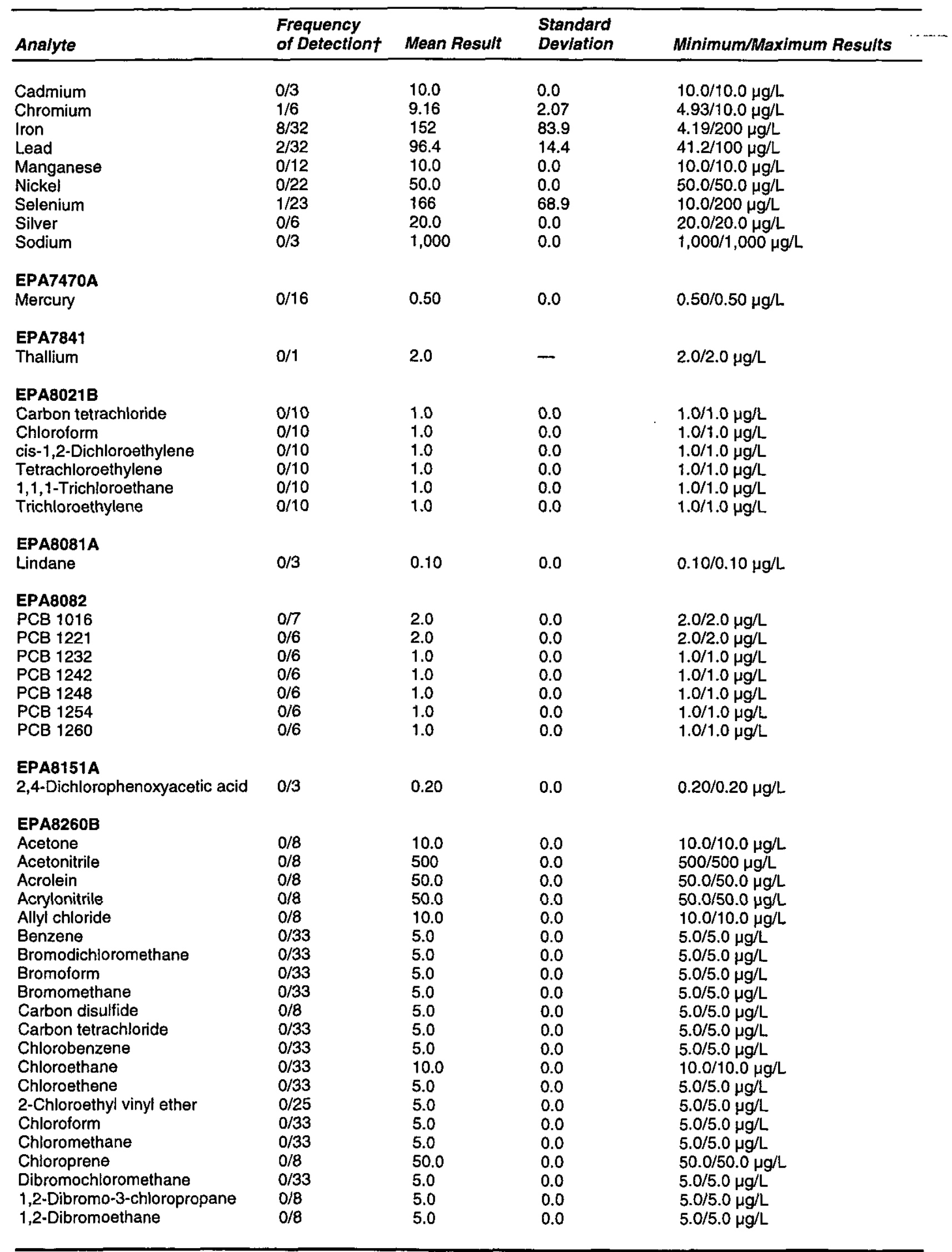




\begin{tabular}{|c|c|c|c|c|}
\hline Analyte & $\begin{array}{l}\text { Frequency } \\
\text { of Detectiont }\end{array}$ & Mean Result & $\begin{array}{l}\text { Standard } \\
\text { Deviation }\end{array}$ & Minimum/Maximum Results \\
\hline 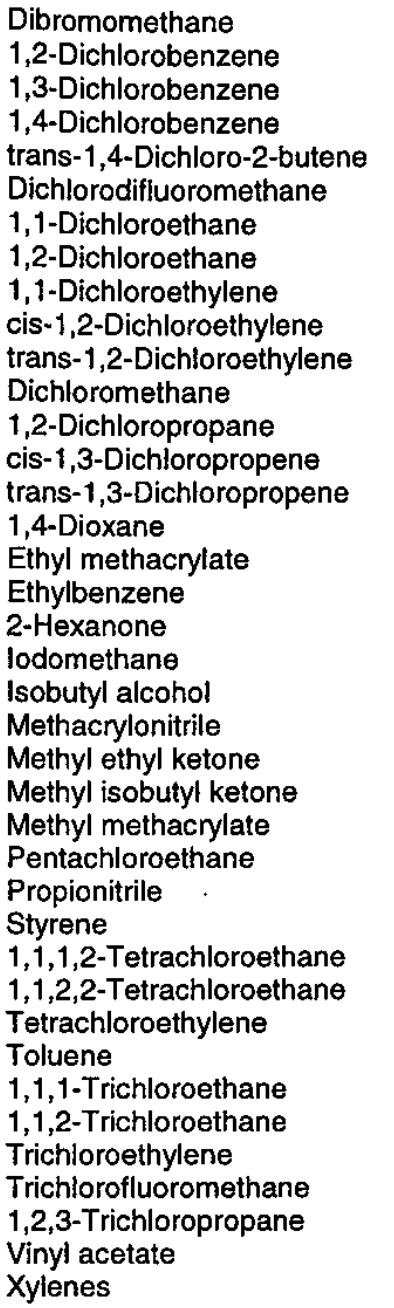 & $\begin{array}{l}0 / 8 \\
0 / 8 \\
0 / 8 \\
0 / 8 \\
0 / 8 \\
0 / 8 \\
0 / 33 \\
0 / 33 \\
0 / 33 \\
0 / 8 \\
0 / 33 \\
2 / 33 \\
0 / 33 \\
0 / 33 \\
0 / 33 \\
0 / 8 \\
0 / 8 \\
0 / 33 \\
0 / 8 \\
0 / 8 \\
0 / 8 \\
0 / 8 \\
0 / 8 \\
0 / 8 \\
0 / 8 \\
0 / 8 \\
0 / 8 \\
0 / 8 \\
0 / 10 \\
0 / 33 \\
0 / 33 \\
0 / 33 \\
0 / 38 \\
0 / 33 \\
0 / 33 \\
0 / 33 \\
0 / 8 \\
0 / 8 \\
0 / 8\end{array}$ & $\begin{array}{l}5.0 \\
5.0 \\
5.0 \\
5.0 \\
20.0 \\
5.0 \\
5.0 \\
5.0 \\
5.0 \\
5.0 \\
5.0 \\
5.03 \\
5.0 \\
5.0 \\
5.0 \\
1,000 \\
5.0 \\
5.0 \\
5.0 \\
5.0 \\
1,500 \\
500 \\
10.0 \\
5.0 \\
50.0 \\
200 \\
500 \\
5.0 \\
5.0 \\
5.0 \\
5.0 \\
5.0 \\
5.0 \\
5.0 \\
5.0 \\
5.0 \\
5.0 \\
20.0 \\
10.0\end{array}$ & $\begin{array}{l}0.0 \\
0.0 \\
0.0 \\
0.0 \\
0.0 \\
0.0 \\
0.0 \\
0.0 \\
0.0 \\
0.0 \\
0.0 \\
0.18 \\
0.0 \\
0.0 \\
0.0 \\
0.0 \\
0.0 \\
0.0 \\
0.0 \\
0.0 \\
0.0 \\
0.0 \\
0.0 \\
0.0 \\
0.0 \\
0.0 \\
0.0 \\
0.0 \\
0.0 \\
0.0 \\
0.0 \\
0.0 \\
0.0 \\
0.0 \\
0.0 \\
0.0 \\
0.0 \\
0.0 \\
0.0\end{array}$ & $\begin{array}{l}5.0 / 5.0 \mu g / \mathrm{L} \\
5.0 / 5.0 \mu \mathrm{g} / \mathrm{L} \\
5.0 / 5.0 \mu \mathrm{g} / \mathrm{L} \\
5.0 / 5.0 \mu \mathrm{g} / \mathrm{L} \\
20.0 / 20.0 \mu \mathrm{g} / \mathrm{L} \\
5.0 / 5.0 \mu \mathrm{g} / \mathrm{L} \\
5.0 / 5.0 \mu \mathrm{g} / \mathrm{L} \\
5.0 / 5.0 \mu \mathrm{g} / \mathrm{L} \\
5.0 / 5.0 \mu \mathrm{g} / \mathrm{L} \\
5.0 / 5.0 \mu \mathrm{g} / \mathrm{L} \\
5.0 / 5.0 \mu \mathrm{g} / \mathrm{L} \\
5.0 / 6.01 \mu \mathrm{g} / \mathrm{L} \\
5.0 / 5.0 \mu \mathrm{g} / \mathrm{L} \\
5.0 / 5.0 \mu \mathrm{g} / \mathrm{L} \\
5.0 / 5.0 \mu \mathrm{g} / \mathrm{L} \\
1,000 / 1,000 \mu \mathrm{g} / \mathrm{L} \\
5.0 / 5.0 \mu \mathrm{g} / \mathrm{L} \\
5.0 / 5.0 \mu \mathrm{g} / \mathrm{L} \\
5.0 / 5.0 \mu \mathrm{g} / \mathrm{L} \\
5.0 / 5.0 \mu \mathrm{g} / \mathrm{L} \\
1,500 / 1,500 \mu \mathrm{g} / \mathrm{L} \\
500 / 500 \mu \mathrm{g} / \mathrm{L} \\
10.0 / 10.0 \mu \mathrm{g} / \mathrm{L} \\
5.0 / 5.0 \mu \mathrm{g} / \mathrm{L} \\
50.0 / 50.0 \mu \mathrm{g} / \mathrm{L} \\
200 / 200 \mu \mathrm{g} / \mathrm{L} \\
500 / 500 \mu \mathrm{g} / \mathrm{L} \\
5.0 / 5.0 \mu \mathrm{g} / \mathrm{L} \\
5.0 / 5.0 \mu \mathrm{g} / \mathrm{L} \\
5.0 / 5.0 \mu \mathrm{g} / \mathrm{L} \\
5.0 / 5.0 \mu \mathrm{g} / \mathrm{L} \\
5.0 / 5.0 \mu \mathrm{g} / \mathrm{L} \\
5.0 / 5.0 \mu \mathrm{g} / \mathrm{L} \\
5.0 / 5.0 \mu \mathrm{g} / \mathrm{L} \\
5.0 / 5.0 \mu \mathrm{g} / \mathrm{L} \\
5.0 / 5.0 \mu \mathrm{g} / \mathrm{L} \\
5.0 / 5.0 \mu \mathrm{g} / \mathrm{L} \\
20.0 / 20.0 \mu \mathrm{g} / \mathrm{L} \\
10.0 / 10.0 \mu \mathrm{g} / \mathrm{L}\end{array}$ \\
\hline $\begin{array}{l}\text { EPA8270C } \\
\text { Acenaphthene } \\
\text { Acenaphthylene } \\
\text { Acetophenone } \\
\text { 2-Acetylaminofluorene } \\
\text { 4-Aminobiphenyl } \\
\text { Aniline } \\
\text { Anthracene } \\
\text { Aramite } \\
\text { Benzo[a]anthracene } \\
\text { Benzo[b]fluoranthene } \\
\text { Benzo[k]fluoranthene } \\
\text { Benzo[g,h,i]perylene } \\
\text { Benzo[a]pyrene } \\
\text { Benzyl alcohol } \\
\text { Bis(2-chloroethoxy) methane } \\
\text { Bis(2-chloroethyl) ether } \\
\text { Bis(2-chloroisopropyl) ether } \\
\text { Bis(2-ethylhexyl) phthalate } \\
\text { 4-Bromophenyl phenyl ether } \\
\text { Butylbenzyl phthalate }\end{array}$ & $\begin{array}{l}0 / 2 \\
0 / 2 \\
0 / 2 \\
0 / 2 \\
0 / 2 \\
0 / 2 \\
0 / 2 \\
0 / 2 \\
0 / 2 \\
0 / 2 \\
0 / 2 \\
0 / 2 \\
0 / 2 \\
0 / 2 \\
0 / 2 \\
0 / 2 \\
0 / 2 \\
1 / 2 \\
0 / 2 \\
0 / 2\end{array}$ & $\begin{array}{l}10.0 \\
10.0 \\
10.0 \\
10.0 \\
10.0 \\
25.0 \\
10.0 \\
10.0 \\
10.0 \\
10.0 \\
10.0 \\
10.0 \\
10.0 \\
10.0 \\
10.0 \\
10.0 \\
10.0 \\
11.2 \\
10.0 \\
10.0\end{array}$ & $\begin{array}{l}0.0 \\
0.0 \\
0.0 \\
0.0 \\
0.0 \\
0.0 \\
0.0 \\
0.0 \\
0.0 \\
0.0 \\
0.0 \\
0.0 \\
0.0 \\
0.0 \\
0.0 \\
0.0 \\
0.0 \\
1.63 \\
0.0 \\
0.0\end{array}$ & $\begin{array}{l}10.0 / 10.0 \mu \mathrm{g} / \mathrm{L} \\
10.0 / 10.0 \mu \mathrm{g} / \mathrm{L} \\
10.0 / 10.0 \mu \mathrm{g} / \mathrm{L} \\
10.0 / 10.0 \mu \mathrm{g} / \mathrm{L} \\
10.0 / 10.0 \mu \mathrm{g} / \mathrm{L} \\
25.0 / 25.0 \mu \mathrm{L} / \mathrm{L} \\
10.0 / 10.0 \mu \mathrm{g} / \mathrm{L} \\
10.0 / 10.0 \mu \mathrm{g} / \mathrm{L} \\
10.0 / 10.0 \mu \mathrm{g} / \mathrm{L} \\
10.0 / 10.0 \mu \mathrm{L} / \mathrm{L} \\
10.0 / 10.0 \mu \mathrm{L} / \mathrm{L} \\
10.0 / 10.0 \mu \mathrm{g} / \mathrm{L} \\
10.0 / 10.0 \mu \mathrm{g} / \mathrm{L} \\
10.0 / 10.0 \mu \mathrm{g} / \mathrm{L} \\
10.0 / 10.0 \mu \mathrm{g} / \mathrm{L} \\
10.0 / 10.0 \mu \mathrm{g} / \mathrm{L} \\
10.0 / 10.0 \mu \mathrm{g} / \mathrm{L} \\
10.0 / 12.3 \mu \mathrm{g} / \mathrm{L} \\
10.0 / 10.0 \mu \mathrm{L} / \mathrm{L} \\
10.0 / 10.0 \mu \mathrm{g} / \mathrm{L}\end{array}$ \\
\hline
\end{tabular}




\begin{tabular}{|c|c|c|c|c|}
\hline Analyte & $\begin{array}{l}\text { Frequency } \\
\text { of Detectiont }\end{array}$ & Mean Result & $\begin{array}{l}\text { Standard } \\
\text { Deviation }\end{array}$ & Minimum/Maximum Results \\
\hline 2-sec-Butyl-4,6-dinitrophenol & $0 / 2$ & 10.0 & 0.0 & $10.0 / 10.0 \mu \mathrm{g} / \mathrm{L}$ \\
\hline 4-Chloroaniline & $0 / 2$ & 10.0 & 0.0 & $10.0 / 10.0 \mu \mathrm{g} / \mathrm{L}$ \\
\hline Chlorobenzilate & $0 / 2$ & 10.0 & 0.0 & $10.0 / 10.0 \mu \mathrm{g} / \mathrm{L}$ \\
\hline 4-Chloro-m-cresol & $0 / 2$ & 10.0 & 0.0 & $10.0 / 10.0 \mu \mathrm{g} / \mathrm{L}$ \\
\hline 2-Chloronaphthalene & $0 / 2$ & 10.0 & 0.0 & $10.0 / 10.0 \mu \mathrm{g} / \mathrm{L}$ \\
\hline 2-Chlorophenol & $0 / 2$ & 10.0 & 0.0 & $10.0 / 10.0 \mu g / L$ \\
\hline 4-Chlorophenyl phenyl ether & $0 / 2$ & 10.0 & 0.0 & $10.0 / 10.0 \mu \mathrm{g} / \mathrm{L}$ \\
\hline Chrysene & $0 / 2$ & 10.0 & 0.0 & $10.0 / 10.0 \mu \mathrm{g} / \mathrm{L}$ \\
\hline $\mathrm{m} / \mathrm{p}$-Cresol & $0 / 4$ & 15.0 & 5.77 & $10.0 / 20.0 \mu \mathrm{g} / \mathrm{L}$ \\
\hline o-Cresol & $0 / 2$ & 10.0 & 0.0 & $10.0 / 10.0 \mu \mathrm{g} / \mathrm{L}$ \\
\hline Diallate & $0 / 2$ & 10.0 & 0.0 & $10.0 / 10.0 \mu g / L$ \\
\hline Dibenz $[a, h]$ anthracene & $0 / 2$ & 10.0 & 0.0 & $10.0 / 10.0 \mu g / L$ \\
\hline Dibenzofuran & $0 / 2$ & 10.0 & 0.0 & $10.0 / 10.0 \mu \mathrm{g} / \mathrm{L}$ \\
\hline Di-n-butyl phthalate & $0 / 2$ & 10.0 & 0.0 & $10.0 / 10.0 \mu \mathrm{g} / \mathrm{L}$ \\
\hline 3,3'-Dichlorobenzidine & $0 / 2$ & 10.0 & 0.0 & $10.0 / 10.0 \mu \mathrm{g} / \mathrm{L}$ \\
\hline 2,4-Dichlorophenol & $0 / 2$ & 10.0 & 0.0 & $10.0 / 10.0 \mu \mathrm{g} / \mathrm{L}$ \\
\hline 2,6-Dichlorophenol & $0 / 2$ & 10.0 & 0.0 & $10.0 / 10.0 \mu \mathrm{g} / \mathrm{L}$ \\
\hline Diethyl phthalate & $0 / 2$ & 10.0 & 0.0 & $10.0 / 10.0 \mu \mathrm{g} / \mathrm{L}$ \\
\hline Dimethoate & $0 / 2$ & 10.0 & 0.0 & $10.0 / 10.0 \mu \mathrm{g} / \mathrm{L}$ \\
\hline 2,4-Dimethyl phenol & $0 / 2$ & 10.0 & 0.0 & $10.0 / 10.0 \mu \mathrm{g} / \mathrm{L}$ \\
\hline Dimethyl phthalate & $0 / 2$ & 10.0 & 0.0 & $10.0 / 10.0 \mu \mathrm{g} / \mathrm{L}$ \\
\hline p-Dimethylaminoazobenzene & $0 / 2$ & 10.0 & 0.0 & $10.0 / 10.0 \mu g / L$ \\
\hline 7,12-Dimethylbenz[a]anthracene & $0 / 2$ & 10.0 & 0.0 & $10.0 / 10.0 \mu \mathrm{g} / \mathrm{L}$ \\
\hline 3,3'-Dimethylbenzidine & $0 / 2$ & 20.0 & 0.0 & $20.0 / 20.0 \mu \mathrm{g} / \mathrm{L}$ \\
\hline a,a-Dimethylphenethylamine & $0 / 2$ & 10.0 & 0.0 & $10.0 / 10.0 \mu \mathrm{g} / \mathrm{L}$ \\
\hline 1,3-Dinitrobenzene & $0 / 2$ & 10.0 & 0.0 & $10.0 / 10.0 \mu \mathrm{g} / \mathrm{L}$ \\
\hline 2,4-Dinitrophenol & $0 / 2$ & 25.0 & 0.0 & $25.0 / 25.0 \mu \mathrm{g} / \mathrm{L}$ \\
\hline 2,4-Dinitrotoluene & $0 / 2$ & 10.0 & 0.0 & $10.0 / 10.0 \mu \mathrm{g} / \mathrm{L}$ \\
\hline 2,6-Dinitrotoluene & $0 / 2$ & 10.0 & 0.0 & $10.0 / 10.0 \mu \mathrm{g} / \mathrm{L}$ \\
\hline Di-n-octyl phthalate & $0 / 2$ & 10.0 & 0.0 & $10.0 / 10.0 \mu \mathrm{g} / \mathrm{L}$ \\
\hline Diphenylamine & $0 / 2$ & 10.0 & 0.0 & $10.0 / 10.0 \mu \mathrm{g} / \mathrm{L}$ \\
\hline Disulfoton & $0 / 2$ & 10.0 & 0.0 & $10.0 / 10.0 \mu \mathrm{g} / \mathrm{L}$ \\
\hline Ethyl methanesulfonate & $0 / 2$ & 10.0 & 0.0 & $10.0 / 10.0 \mu \mathrm{g} / \mathrm{L}$ \\
\hline Fluoranthene & $0 / 2$ & 10.0 & 0.0 & $10.0 / 10.0 \mu g / L$ \\
\hline Fluorene & $0 / 2$ & 10.0 & 0.0 & $10.0 / 10.0 \mu \mathrm{g} / \mathrm{L}$ \\
\hline Hexachlorobenzene & $0 / 2$ & 10.0 & 0.0 & $10.0 / 10.0 \mu \mathrm{g} / \mathrm{L}$ \\
\hline Hexachlorobutadiene & $0 / 2$ & 10.0 & 0.0 & $10.0 / 10.0 \mu \mathrm{g} / \mathrm{L}$ \\
\hline Hexachlorocyclopentadiene & $0 / 2$ & 10.0 & 0.0 & $10.0 / 10.0 \mu \mathrm{g} / \mathrm{L}$ \\
\hline Hexachloroethane & $0 / 2$ & 10.0 & 0.0 & $10.0 / 10.0 \mu \mathrm{g} / \mathrm{L}$ \\
\hline Indeno[1,2,3-c, d]pyrene & $0 / 2$ & 10.0 & 0.0 & $10.0 / 10.0 \mu \mathrm{g} / \mathrm{L}$ \\
\hline Isodrin & $0 / 2$ & 10.0 & 0.0 & $10.0 / 10.0 \mu \mathrm{g} / \mathrm{L}$ \\
\hline Isophorone & $0 / 2$ & 10.0 & 0.0 & $10.0 / 10.0 \mu \mathrm{g} / \mathrm{L}$ \\
\hline Isosafrole & $0 / 2$ & 10.0 & 0.0 & $10.0 / 10.0 \mu \mathrm{g} / \mathrm{L}$ \\
\hline Kepone & $0 / 2$ & 10.0 & 0.0 & $10.0 / 10.0 \mu \mathrm{g} / \mathrm{L}$ \\
\hline Methapyrilene & $0 / 2$ & 10.0 & 0.0 & $10.0 / 10.0 \mu \mathrm{g} / \mathrm{L}$ \\
\hline 2-Methyl-4,6-dinitrophenol & $0 / 2$ & 25.0 & 0.0 & $25.0 / 25.0 \mu \mathrm{g} / \mathrm{L}$ \\
\hline Methyl methanesulfonate & $0 / 2$ & 10.0 & 0.0 & $10.0 / 10.0 \mu \mathrm{g} / \mathrm{L}$ \\
\hline 3-Methylcholanthrene & $0 / 2$ & 10.0 & 0.0 & $10.0 / 10.0 \mu \mathrm{g} / \mathrm{L}$ \\
\hline 2-Methylnaphthalene & $0 / 2$ & 10.0 & 0.0 & $10.0 / 10.0 \mu \mathrm{g} / \mathrm{L}$ \\
\hline Naphthalene & $0 / 2$ & 10.0 & 0.0 & $10.0 / 10.0 \mu \mathrm{g} / \mathrm{L}$ \\
\hline 1,4-Naphthoquinone & $0 / 2$ & 10.0 & 0.0 & $10.0 / 10.0 \mu \mathrm{g} / \mathrm{L}$ \\
\hline 1-Naphthylamine & $0 / 2$ & 10.0 & 0.0 & $10.0 / 10.0 \mu \mathrm{g} / \mathrm{L}$ \\
\hline 2-Naphthylamine & $0 / 2$ & 10.0 & 0.0 & $10.0 / 10.0 \mu \mathrm{g} / \mathrm{L}$ \\
\hline m-Nitroaniline & $0 / 2$ & 25.0 & 0.0 & $25.0 / 25.0 \mu \mathrm{g} / \mathrm{L}$ \\
\hline o-Nitroaniline & $0 / 2$ & 25.0 & 0.0 & $25.0 / 25.0 \mu \mathrm{g} / \mathrm{L}$ \\
\hline p-Nitroaniline & $0 / 2$ & 10.0 & 0.0 & $10.0 / 10.0 \mu \mathrm{g} / \mathrm{L}$ \\
\hline Nitrobenzene & $0 / 2$ & 10.0 & 0.0 & $10.0 / 10.0 \mu g / t$ \\
\hline 2-Nitrophenol & $0 / 2$ & 10.0 & 0.0 & $10.0 / 10.0 \mu \mathrm{g} / \mathrm{L}$ \\
\hline 4-Nitrophenol & $0 / 2$ & 25.0 & 0.0 & $25.0 / 25.0 \mu \mathrm{g} / \mathrm{L}$ \\
\hline 4-Nitroquinoline-1-oxide & $0 / 2$ & 50.0 & 0.0 & $50.0 / 50.0 \mu \mathrm{g} / \mathrm{L}$ \\
\hline N-Nitrosodi-n-butylamine & $0 / 2$ & 10.0 & 0.0 & $10.0 / 10.0 \mu \mathrm{g} / \mathrm{L}$ \\
\hline
\end{tabular}




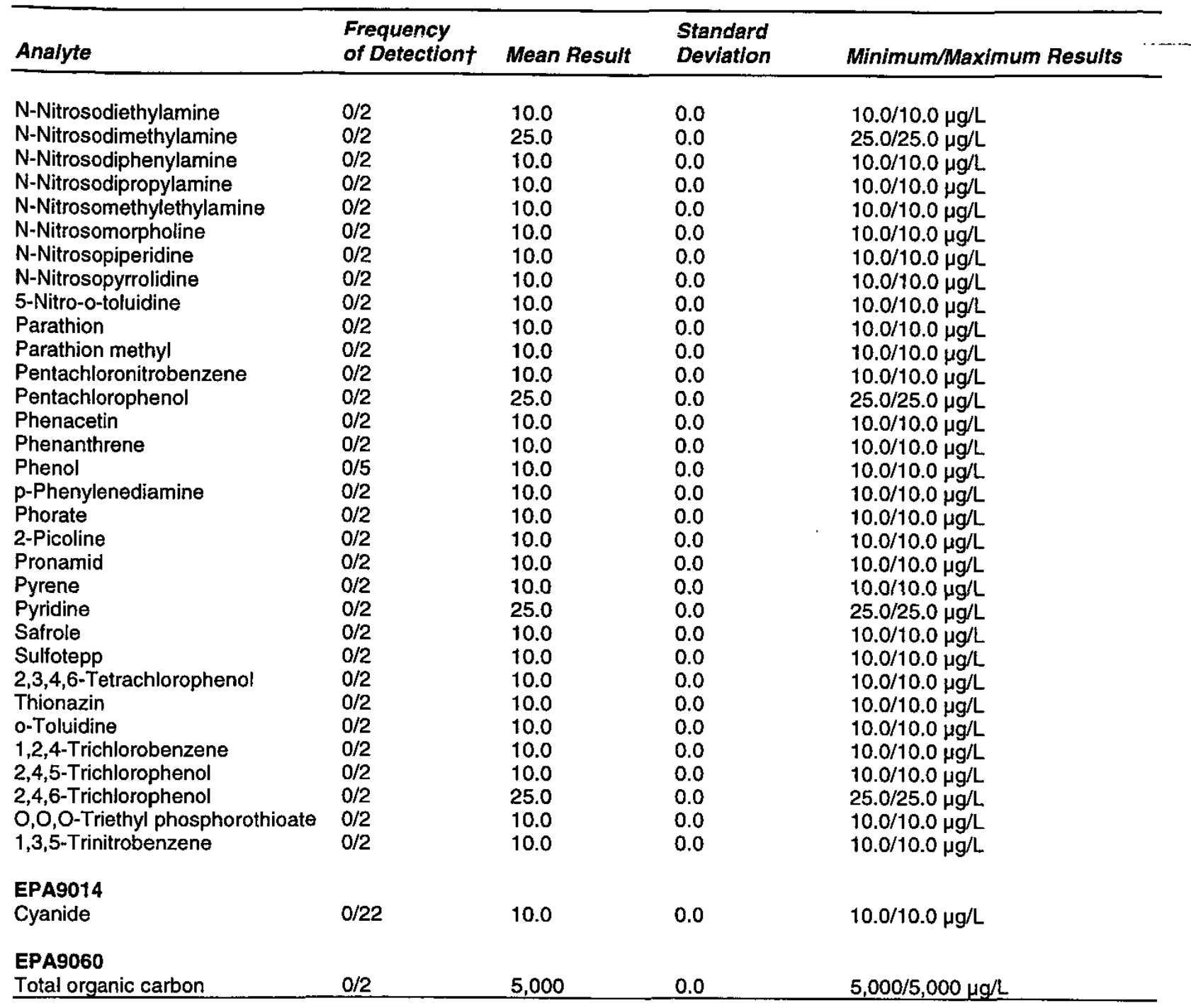

$\uparrow$ Number of times analyte was detected compared to the total number of method blanks for the analyte.

- Standard deviation cannot be determined.

Note: If the analyte was not detected in the method blank(s), detection limit information appears in the Mean Result and Minimum/Maximum Results columns.

Table 54. Analytes Detected in Mothod Blanks for GE

\begin{tabular}{lllll}
\hline Analyte & $\begin{array}{l}\text { Frequency } \\
\text { of Detectiont }\end{array}$ & Mean Result & $\begin{array}{l}\text { Standard } \\
\text { Deviation }\end{array}$ & Minimum/Maximum Results \\
\hline $\begin{array}{l}\text { EPA160.1 } \\
\text { Total dissolved solids }\end{array}$ & $1 / 1$ & 10,000 & - & $10,000 / 10,000 \mu \mathrm{g} / \mathrm{L}$ \\
$\begin{array}{l}\text { EPA353.1 } \\
\text { Nitrate-nitrite as nitrogen }\end{array}$ & $6 / 17$ & 38.2 & 18.8 & $10.0 / 50.0 \mu \mathrm{g} / \mathrm{L}$ \\
$\begin{array}{l}\text { EPA365.4 } \\
\text { Total phosphates (as P) }\end{array}$ & $1 / 1$ & 50.0 & - & $50.0 / 50.0 \mu \mathrm{g} / \mathrm{L}$ \\
\hline
\end{tabular}




\begin{tabular}{|c|c|c|c|c|}
\hline Analyte & $\begin{array}{l}\text { Frequency } \\
\text { of Detectiont }\end{array}$ & Mean Result & $\begin{array}{l}\text { Standard } \\
\text { Deviation }\end{array}$ & Minimum/Maximum Results \\
\hline \multicolumn{5}{|l|}{ EPA60t0B } \\
\hline Aluminum & $6 / 16$ & 37.0 & 17.4 & $12.8 / 50.0 \mu \mathrm{g} / \mathrm{L}$ \\
\hline Antimony & $0 / 4$ & 10.0 & 0.0 & $10.0 / 10.0 \mu \mathrm{g} / \mathrm{L}$ \\
\hline Arsenic & $0 / 15$ & 5.0 & 0.0 & $5.0 / 5.0 \mu \mathrm{g} / \mathrm{L}$ \\
\hline Barium & $1 / 15$ & 4.71 & 1.12 & $0.67 / 5.0 \mu \mathrm{g} / \mathrm{L}$ \\
\hline Beryllium & $0 / 2$ & 5.0 & 0.0 & $5.0 / 5.0 \mu \mathrm{g} / \mathrm{L}$ \\
\hline Cadmium & $0 / 15$ & 5.0 & 0.0 & $5.0 / 5.0 \mu \mathrm{g} / \mathrm{L}$ \\
\hline Calcium & $0 / 1$ & 100 & - & $100 / 100 \mu \mathrm{g} / \mathrm{L}$ \\
\hline Chromium & $1 / 15$ & 4.71 & 1.11 & $0.70 / 5.0 \mu \mathrm{g} / \mathrm{L}$ \\
\hline Cobalt & $0 / 2$ & 5.0 & 0.0 & $5.0 / 5.0 \mu \mathrm{g} / \mathrm{L}$ \\
\hline Copper & $0 / 2$ & 5.0 & 0.0 & $5.0 / 5.0 \mu \mathrm{g} / \mathrm{L}$ \\
\hline Iron & $1 / 17$ & 51.5 & 6.23 & $50.0 / 75.7 \mu g / L$ \\
\hline Lead & $0 / 15$ & 5.0 & 0.0 & $5.0 / 5.0 \mu \mathrm{g} / \mathrm{L}$ \\
\hline Magnesium & $0 / 1$ & 10.0 & - & $10.0 / 10.0 \mu \mathrm{g} / \mathrm{L}$ \\
\hline Manganese & $1 / 2$ & 10.2 & 0.28 & $10.0 / 10.4 \mu \mathrm{g} / \mathrm{L}$ \\
\hline Nickel & $0 / 2$ & 5.0 & 0.0 & $5.0 / 5.0 \mu \mathrm{g} / \mathrm{L}$ \\
\hline Potassium & $0 / 1$ & 100 & - & $100 / 100 \mu \mathrm{g} / \mathrm{L}$ \\
\hline Selenium & $1 / 15$ & 4.85 & 0.58 & $2.74 / 5.0 \mu \mathrm{g} / \mathrm{L}$ \\
\hline Silver & $5 / 15$ & 3.90 & 1.68 & $0.85 / 5.0 \mu \mathrm{g} / \mathrm{L}$ \\
\hline Sodium & $0 / 1$ & 100 & - & $100 / 100 \mu \mathrm{g} / \mathrm{L}$ \\
\hline Thallium & $0 / 2$ & 5.0 & 0.0 & $5.0 / 5.0 \mu \mathrm{g} / \mathrm{L}$ \\
\hline Vanadium & $0 / 2$ & 5.0 & 0.0 & $5.0 / 5.0 \mu \mathrm{g} / \mathrm{L}$ \\
\hline Zinc & $1 / 2$ & 4.46 & 0.76 & $3.92 / 5.0 \mu \mathrm{g} / \mathrm{L}$ \\
\hline \multicolumn{5}{|l|}{ EPA6020 } \\
\hline Aluminum & $1 / 23$ & 14.8 & 1.04 & $10.0 / 15.0 \mu \mathrm{g} / \mathrm{L}$ \\
\hline Antimony & $4 / 20$ & 0.30 & 0.41 & $0.15 / 2.0 \mu \mathrm{g} / \mathrm{L}$ \\
\hline Arsenic & $0 / 20$ & 3.0 & 0.0 & $3.0 / 3.0 \mu \mathrm{g} / \mathrm{L}$ \\
\hline Barium & $0 / 20$ & 2.0 & 0.0 & $2.0 / 2.0 \mu \mathrm{g} / \mathrm{L}$ \\
\hline Beryllium & $0 / 3$ & 0.77 & 0.40 & $0.30 / 1.0 \mu \mathrm{g} / \mathrm{L}$ \\
\hline Cadmium & $2 / 21$ & 0.97 & 0.16 & $0.26 / 1.0 \mu \mathrm{g} / \mathrm{L}$ \\
\hline Calcium & $1 / 2$ & 150 & 0.0 & $150 / 150 \mu \mathrm{g} / \mathrm{L}$ \\
\hline Chromium & $1 / 21$ & 3.0 & 0.0 & $3.0 / 3.0 \mu \mathrm{g} / \mathrm{L}$ \\
\hline Cobalt & $3 / 20$ & 0.22 & 0.20 & $0.03 / 1.0 \mu \mathrm{g} / \mathrm{L}$ \\
\hline Copper & $3 / 20$ & 0.98 & 0.30 & $0.33 / 2.0 \mu \mathrm{g} / \mathrm{L}$ \\
\hline Iron & $2 / 23$ & 15.6 & 2.15 & $15.0 / 25.0 \mu \mathrm{g} / \mathrm{L}$ \\
\hline Lead & $1 / 21$ & 2.0 & 0.0 & $2.0 / 2.0 \mu \mathrm{g} / \mathrm{L}$ \\
\hline Magnesium & $1 / 2$ & 3.0 & 0.0 & $3.0 / 3.0 \mu \mathrm{g} / \mathrm{L}$ \\
\hline Manganese & $1 / 2$ & 0.20 & 0.0 & $0.20 / 0.20 \mu \mathrm{g} / \mathrm{L}$ \\
\hline Nickel & $1 / 21$ & 2.04 & 0.50 & $0.94 / 4.0 \mu \mathrm{g} / \mathrm{L}$ \\
\hline Potassium & $1 / 2$ & 15.0 & 0.0 & $15.0 / 15.0 \mu \mathrm{g} / \mathrm{L}$ \\
\hline Selenium & $1 / 20$ & 4.74 & 0.81 & $2.0 / 5.0 \mu \mathrm{g} / \mathrm{L}$ \\
\hline Silver & $0 / 23$ & 1.0 & 0.0 & $1.0 / 1.0 \mu g / L$ \\
\hline Sodium & $1 / 2$ & 250 & 0.0 & $250 / 250 \mu \mathrm{g} / \mathrm{L}$ \\
\hline Thallium & $8 / 16$ & 1.30 & 1.24 & $0.03 / 2.50 \mu \mathrm{g} / \mathrm{L}$ \\
\hline Tin & $0 / 16$ & 5.0 & 0.0 & $5.0 / 5.0 \mu \mathrm{g} / \mathrm{L}$ \\
\hline Vanadium & $1 / 21$ & 9.69 & 1.43 & $3.46 / 10.0 \mu \mathrm{g} / \mathrm{L}$ \\
\hline Zinc & $0 / 20$ & 10.0 & 0.0 & $10.0 / 10.0 \mu \mathrm{g} / \mathrm{L}$ \\
\hline \multicolumn{5}{|l|}{ EPA7470A } \\
\hline Mercury & $3 / 31$ & 0.19 & 0.03 & $0.05 / 0.20 \mu g / L$ \\
\hline \multicolumn{5}{|l|}{ EPAB081A } \\
\hline Aldrin & $0 / 1$ & 0.02 & - & $0.02 / 0.02 \mu \mathrm{g} / \mathrm{L}$ \\
\hline alpha-Benzene hexachloride & $0 / 1$ & 0.02 & - & $0.02 / 0.02 \mu \mathrm{g} / \mathrm{L}$ \\
\hline beta-Benzene hexachloride & $0 / 2$ & 0.02 & 0.0 & $0.02 / 0.02 \mu \mathrm{g} / \mathrm{L}$ \\
\hline delta-Benzene hexachloride & $0 / 1$ & 0.02 & - & $0.02 / 0.02 \mu \mathrm{g} / \mathrm{L}$ \\
\hline alpha-Chlordane & $0 / 1$ & 0.02 & - & $0.02 / 0.02 \mu \mathrm{g} / \mathrm{L}$ \\
\hline gamma-Chlordane & $0 / 1$ & 0.02 & - & $0.02 / 0.02 \mu \mathrm{g} / \mathrm{L}$ \\
\hline$p, p^{\prime}-D D D$ & $0 / 1$ & 0.04 & $\rightarrow$ & $0.04 / 0.04 \mu g / L$ \\
\hline
\end{tabular}




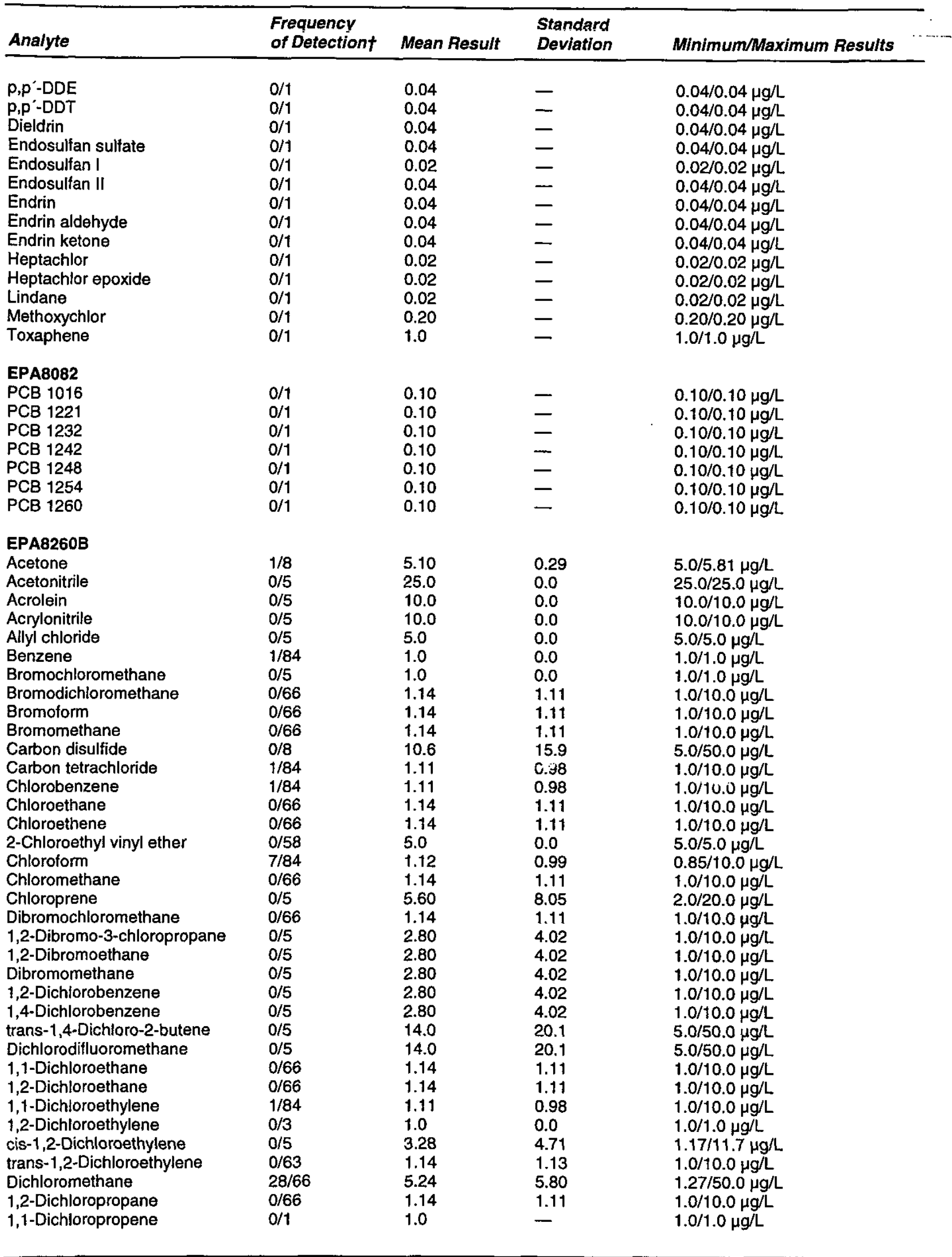

\section{Quality Control Samples}




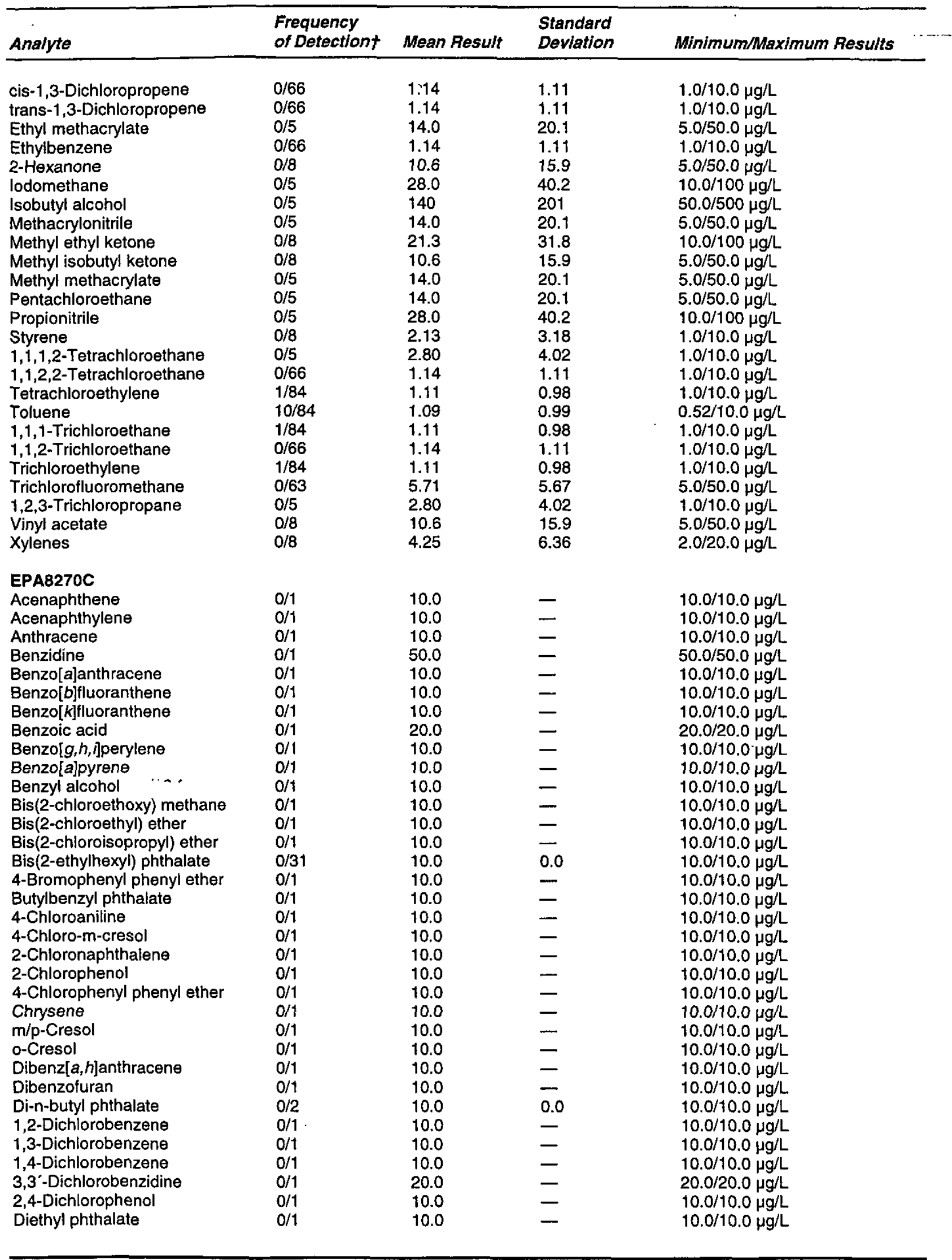




\begin{tabular}{|c|c|c|c|c|}
\hline Analyte & $\begin{array}{l}\text { Frequency } \\
\text { of Detectiont }\end{array}$ & Mean Result & $\begin{array}{l}\text { Standard } \\
\text { Deviation }\end{array}$ & Minimum/Maximum Results \\
\hline 2,4-Dimethyl phenol & $0 / 1$ & 10.0 & - & $10.0 / 10.0 \mu \mathrm{q} / \mathrm{L}$ \\
\hline Dimethyl phthalate & $0 / 1$ & 10.0 & - & $10.0 / 10.0 \mu \mathrm{g} / \mathrm{L}$ \\
\hline 2,4-Dinitrophenol & $0 / 1$ & 20.0 & - & $20.0 / 20.0 \mu \mathrm{g} / \mathrm{L}$ \\
\hline 2,4-Dinitrotoluene & $0 / 1$ & 10.0 & - & $10.0 / 10.0 \mu \mathrm{g} / \mathrm{L}$ \\
\hline 2,6-Dinitrotoluene & $0 / 1$ & 10.0 & - & $10.0 / 10.0 \mu \mathrm{g} / \mathrm{L}$ \\
\hline Di-n-octyl phthalate & $0 / 1$ & 10.0 & - & $10.0 / 10.0 \mu g / L$ \\
\hline Fluoranthene & $0 / 1$ & 10.0 & - & $10.0 / 10.0 \mu \mathrm{g} / \mathrm{L}$ \\
\hline Fluorene & $0 / 1$ & 10.0 & - & $10.0 / 10.0 \mu g / L$ \\
\hline Hexachlorobenzene & $0 / 1$ & 10.0 & - & $10.0 / 10.0 \mu \mathrm{g} / \mathrm{L}$ \\
\hline Hexachlorobutadiene & $0 / 1$ & 10.0 & - & $10.0 / 10.0 \mu g / \mathrm{L}$ \\
\hline Hexachlorocyclopentadiene & $0 / 1$ & 10.0 & - & $10.0 / 10.0 \mu \mathrm{g} / \mathrm{L}$ \\
\hline Hexachloroethane & $0 / 1$ & 10.0 & - & $10.0 / 10.0 \mu g / L$ \\
\hline Indeno[1,2,3-c,d]pyrene & $0 / 1$ & 10.0 & - & $10.0 / 10.0 \mu \mathrm{g} / \mathrm{L}$ \\
\hline Isophorone & $0 / 1$ & 10.0 & - & $10.0 / 10.0 \mu \mathrm{g} / \mathrm{L}$ \\
\hline 2-Methyl-4,6-dinitrophenol & $0 / 1$ & 10.0 & - & $10.0 / 10.0 \mu \mathrm{g} / \mathrm{L}$ \\
\hline 2-Methylnaphthalene & $0 / 1$ & 10.0 & - & $10.0 / 10.0 \mu \mathrm{g} / \mathrm{L}$ \\
\hline Naphthalene & $0 / 1$ & 10.0 & - & $10.0 / 10.0 \mu \mathrm{g} / \mathrm{L}$ \\
\hline m-Nitroaniline & $0 / 1$ & 10.0 & - & $10.0 / 10.0 \mu g / L$ \\
\hline o-Nitroaniline & $0 / 1$ & 10.0 & - & $10.0 / 10.0 \mu \mathrm{g} / \mathrm{L}$ \\
\hline p-Nitroaniline & $0 / 1$ & 10.0 & - & $10.0 / 10.0 \mu \mathrm{g} / \mathrm{L}$ \\
\hline Nitrobenzene & $0 / 1$ & 10.0 & - & $10.0 / 10.0 \mu g / L$ \\
\hline 2-Nitrophenol & $0 / 1$ & 10.0 & - & $10.0 / 10.0 \mu \mathrm{g} / \mathrm{L}$ \\
\hline 4-Nitrophenol & $0 / 1$ & 10.0 & 一 & $10.0 / 10.0 \mu \mathrm{g} / \mathrm{L}$ \\
\hline N-Nitrosodiphenylamine & $0 / 1$ & 10.0 & - & $10.0 / 10.0 \mu g / L$ \\
\hline N-Nitrosodipropylamine & $0 / 1$ & 10.0 & - & $10.0 / 10.0 \mu \mathrm{g} / \mathrm{L}$ \\
\hline Pentachlorophenol & $0 / 1$ & 20.0 & - & $20.0 / 20.0 \mu \mathrm{g} / \mathrm{L}$ \\
\hline $\begin{array}{l}\text { Phenanthrene } \\
\text { P }\end{array}$ & $0 / 1$ & 10.0 & - & $10.0 / 10.0 \mu \mathrm{g} / \mathrm{L}$ \\
\hline Phenol & $0 / 1$ & 10.0 & - & $10.0 / 10.0 \mu \mathrm{g} / \mathrm{L}$ \\
\hline Pyrene & $0 / 1$ & 10.0 & 一 & $10.0 / 10.0 \mu \mathrm{g} / \mathrm{L}$ \\
\hline 1,2,4-Trichlorobenzene & $0 / 1$ & 10.0 & - & $10.0 / 10.0 \mu \mathrm{g} / \mathrm{L}$ \\
\hline $2,4,5$-Trichlorophenol & $0 / 1$ & 10.0 & - & $10.0 / 10.0 \mu \mathrm{g} / \mathrm{h}$ \\
\hline 2,4,6-Trichlorophenol & $0 / 1$ & 10.0 & - & $10.0 / 10.0 \mu \mathrm{g} / \mathrm{L}$ \\
\hline \multicolumn{5}{|l|}{ EPA9012A } \\
\hline Cyanide & $0 / 21$ & 10.0 & 0.0 & $10.0 / 10.0 \mu \mathrm{g} / \mathrm{L}$ \\
\hline \multicolumn{5}{|l|}{ EPA9030 } \\
\hline Sulfide & $0 / 1$ & 1,000 & - & $1,000 / 1,000 \mu \mathrm{g} / \mathrm{L}$ \\
\hline \multicolumn{5}{|l|}{ EPA9056 } \\
\hline Chloride & $1 / 1$ & 100 & - & $100 / 100 \mu \mathrm{g} / \mathrm{L}$ \\
\hline Fluoride & $1 / 1$ & 50.0 & - & $50.0 / 50.0 \mu \mathrm{gg} / \mathrm{L}$ \\
\hline Nitrite as nitrogen & $0 / 2$ & 50.0 & 0.0 & $50.0 / 50.0 \mu g / L$ \\
\hline Sulfate & $1 / 1$ & 200 & - & $200 / 200 \mu g / L$ \\
\hline \multicolumn{5}{|l|}{ EPA9060 } \\
\hline Total organic carbon & $1 / 1$ & 200 & - & $200 / 200 \mu g / L$ \\
\hline \multicolumn{5}{|l|}{ EPA9066 } \\
\hline
\end{tabular}

$\dagger$ Number of times analyte was detected compared to the total number of method blanks for the analyte.

- Standard deviation cannot be determined.

Note: If the analyte was not detected in the method blank(s), detection limit information appears in the Mean Result and Minimum/Maximum Results columns. 
Table 55. Analytes Detected in Mothod Blanks for WA

\begin{tabular}{|c|c|c|c|c|}
\hline Analyte & $\begin{array}{l}\text { Frequency } \\
\text { of Detectiont }\end{array}$ & Mean Result & $\begin{array}{l}\text { Standard } \\
\text { Deviation }\end{array}$ & Minimum/Maximum Results \\
\hline $\begin{array}{l}\text { EPA160.1 } \\
\text { Total dissolved solids }\end{array}$ & $1 / 17$ & 48,800 & 1,520 & $47,000 / 50,000 \mu \mathrm{g} / \mathrm{L}$ \\
\hline $\begin{array}{l}\text { EPA310.1 } \\
\text { Alkalinity (as } \mathrm{CaCO}_{3} \text { ) }\end{array}$ & $0 / 1$ & 6.70 & 一 & $6.70 / 6.70 \mathrm{mq} / \mathrm{L}$ \\
\hline $\begin{array}{l}\text { EPA340.2 } \\
\text { Fluoride }\end{array}$ & $15 / 15$ & 22.2 & 3.52 & $18.6 / 30.9 \mu \mathrm{g} / \mathrm{L}$ \\
\hline $\begin{array}{l}\text { EPA353.2 } \\
\text { Nitrate as nitrogen } \\
\text { Nitrate-nitrite as nitrogen } \\
\text { Nitrite as nitrogen }\end{array}$ & $\begin{array}{l}0 / 3 \\
2 / 26 \\
0 / 1\end{array}$ & $\begin{array}{l}20.0 \\
18.7 \\
20.0\end{array}$ & $\begin{array}{l}0.0 \\
4.62 \\
-\end{array}$ & $\begin{array}{l}20.0 / 20.0 \mu \mathrm{g} / \mathrm{L} \\
3.0 / 20.0 \mu \mathrm{g} / \mathrm{L} \\
20.0 / 20.0 \mu \mathrm{g} / \mathrm{L}\end{array}$ \\
\hline $\begin{array}{l}\text { EPA365.2 } \\
\text { Total phosphates (as P) }\end{array}$ & $0 / 15$ & 67.0 & 0.0 & $67.0 / 67.0 \mu \mathrm{g} / \mathrm{L}$ \\
\hline $\begin{array}{l}\text { EPA376.2 } \\
\text { Sulfide }\end{array}$ & $0 / 1$ & 10,000 & 一 & $10,000 / 10,000 \mu \mathrm{g} / \mathrm{L}$ \\
\hline $\begin{array}{l}\text { EPA6010B } \\
\text { Aluminum } \\
\text { Antimony } \\
\text { Arsenic } \\
\text { Barium } \\
\text { Beryllium } \\
\text { Boron } \\
\text { Cadmium } \\
\text { Calcium } \\
\text { Chromium } \\
\text { Cobalt } \\
\text { Copper } \\
\text { Iron } \\
\text { Lead } \\
\text { Lithium } \\
\text { Magnesium } \\
\text { Manganese } \\
\text { Nickel } \\
\text { Potassium } \\
\text { Selenium } \\
\text { Silica } \\
\text { Silver } \\
\text { Sodium } \\
\text { Thallium } \\
\text { Tin } \\
\text { Vanadium } \\
\text { Zinc }\end{array}$ & $\begin{array}{l}6 / 54 \\
2 / 16 \\
0 / 42 \\
26 / 40 \\
0 / 11 \\
0 / 15 \\
0 / 40 \\
0 / 17 \\
2 / 38 \\
0 / 13 \\
8 / 15 \\
22 / 58 \\
0 / 45 \\
6 / 15 \\
6 / 18 \\
0 / 32 \\
0 / 24 \\
0 / 17 \\
0 / 45 \\
0 / 17 \\
6 / 37 \\
4 / 22 \\
0 / 12 \\
0 / 10 \\
0 / 13 \\
0 / 15\end{array}$ & $\begin{array}{l}132 \\
24.2 \\
40.0 \\
0.89 \\
1.60 \\
266 \\
4.70 \\
471 \\
6.68 \\
4.50 \\
8.03 \\
50.9 \\
47.0 \\
1.80 \\
53.2 \\
7.80 \\
26.0 \\
187 \\
66.0 \\
1,350 \\
4.37 \\
244 \\
55.0 \\
70.0 \\
6.90 \\
53.0\end{array}$ & $\begin{array}{l}39.2 \\
7.75 \\
0.0 \\
0.70 \\
0.0 \\
0.0 \\
0.0 \\
0.0 \\
1.37 \\
0.0 \\
6.76 \\
30.2 \\
0.0 \\
1.14 \\
30.4 \\
0.0 \\
0.0 \\
0.0 \\
0.0 \\
6.64 \\
1.44 \\
89.0 \\
0.0 \\
0.0 \\
0.0 \\
0.0\end{array}$ & $\begin{array}{l}16.1 / 146 \mu \mathrm{g} / \mathrm{L} \\
4.30 / 27.0 \mu \mathrm{g} / \mathrm{L} \\
40.0 / 40.0 \mu \mathrm{g} / \mathrm{L} \\
0.19 / 1.80 \mu \mathrm{g} / \mathrm{L} \\
1.60 / 1.60 \mu \mathrm{g} / \mathrm{L} \\
266 / 266 \mu \mathrm{g} / \mathrm{L} \\
4.70 / 4.70 \mu \mathrm{g} / \mathrm{L} \\
471 / 471 \mu \mathrm{g} / \mathrm{L} \\
0.95 / 7.0 \mu \mathrm{g} / \mathrm{L} \\
4.50 / 4.50 \mu \mathrm{g} / \mathrm{L} \\
1.50 / 15.0 \mu \mathrm{g} / \mathrm{L} \\
7.80 / 74.0 \mu \mathrm{g} / \mathrm{L} \\
47.0 / 47.0 \mu \mathrm{g} / \mathrm{L} \\
0.33 / 2.70 \mu \mathrm{g} / \mathrm{L} \\
7.40 / 74.0 \mu \mathrm{g} / \mathrm{L} \\
7.80 / 7.80 \mu \mathrm{g} / \mathrm{L} \\
26.0 / 26.0 \mu \mathrm{g} / \mathrm{L} \\
187 / 187 \mu \mathrm{g} / \mathrm{L} \\
66.0 / 66.0 \mu \mathrm{g} / \mathrm{L} \\
1,350 / 1,370 \mu \mathrm{g} / \mathrm{L} \\
0.90 / 5.0 \mu \mathrm{g} / \mathrm{L} \\
46.0 / 285 \mu \mathrm{L} / \mathrm{L} \\
55.0 / 55.0 \mu \mathrm{L} / \mathrm{L} \\
70.0 / 70.0 \mu \mathrm{g} / \mathrm{L} \\
6.90 / 6.90 \mu \mathrm{g} / \mathrm{L} \\
53.0 / 53.0 \mu \mathrm{L} / \mathrm{L}\end{array}$ \\
\hline $\begin{array}{l}\text { EPA7470A } \\
\text { Mercury }\end{array}$ & $0 / 34$ & 0.45 & 0.0 & $0.45 / 0.45 \mu \mathrm{g} / \mathrm{L}$ \\
\hline $\begin{array}{l}\text { EPA8010 } \\
\text { Carbon tetrachloride } \\
\text { Chloroform } \\
\text { cis-1,2-Dichloroethylene } \\
\text { 1,1,1-Trichloroethane } \\
\text { Trichloroethylene }\end{array}$ & $\begin{array}{l}0 / 8 \\
0 / 7 \\
0 / 10 \\
0 / 7 \\
0 / 7\end{array}$ & $\begin{array}{l}1.0 \\
1.0 \\
5.0 \\
1.0 \\
1.0\end{array}$ & $\begin{array}{l}0.0 \\
0.0 \\
0.0 \\
0.0 \\
0.0\end{array}$ & $\begin{array}{l}1.0 / 1.0 \mu \mathrm{g} / \mathrm{L} \\
1.0 / 1.0 \mu \mathrm{g} / \mathrm{L} \\
5.0 / 5.0 \mu \mathrm{g} / \mathrm{L} \\
1.0 / 1.0 \mu \mathrm{g} / \mathrm{L} \\
1.0 / 1.0 \mu \mathrm{g} / \mathrm{L}\end{array}$ \\
\hline
\end{tabular}




\begin{tabular}{|c|c|c|c|c|}
\hline Analyte & $\begin{array}{l}\text { Frequency } \\
\text { of Detectiont }\end{array}$ & Mean Result & $\begin{array}{l}\text { Standard } \\
\text { Deviation }\end{array}$ & Minimum/Maximum Results \\
\hline \multicolumn{5}{|l|}{ EPA8021B } \\
\hline Carbon tetrachloride & $0 / 15$ & 1.0 & 0.0 & $1.0 / 1.0 \mu \mathrm{g} / \mathrm{L}$ \\
\hline Chlorotorm & $0 / 15$ & 1.0 & 0.0 & $1.0 / 1.0 \mu \mathrm{g} / \mathrm{L}$ \\
\hline cis-1,2-Dichloroethylene & $0 / 2$ & 5.0 & 0.0 & $5.0 / 5.0 \mu \mathrm{g} / \mathrm{L}$ \\
\hline $1,1,1-$ Trichloroethane & $0 / 15$ & 1.0 & 0.0 & $1.0 / 1.0 \mu \mathrm{g} / \mathrm{L}$ \\
\hline Trichloroethylene & $0 / 15$ & 1.0 & 0.0 & $1.0 / 1.0 \mu \mathrm{g} / \mathrm{L}$ \\
\hline \multicolumn{5}{|l|}{ EPA8081A } \\
\hline Aldrin & $0 / 10$ & 0.05 & 0.0 & $0.05 / 0.05 \mu \mathrm{g} / \mathrm{L}$ \\
\hline alpha-Benzene hexachloride & $0 / 10$ & 0.05 & 0.0 & $0.05 / 0.05 \mu \mathrm{g} / \mathrm{L}$ \\
\hline beta-Benzene hexachloride & $0 / 13$ & 0.05 & 0.0 & $0.05 / 0.05 \mu \mathrm{g} / \mathrm{L}$ \\
\hline delta-Benzene hexachioride & $0 / 10$ & 0.05 & 0.0 & $0.05 / 0.05 \mu \mathrm{g} / \mathrm{L}$ \\
\hline alpha-Chlordane & $0 / 10$ & 0.05 & 0.0 & $0.05 / 0.05 \mu \mathrm{g} / \mathrm{L}$ \\
\hline gamma-Chlordane & $0 / 10$ & 0.05 & 0.0 & $0.05 / 0.05 \mu \mathrm{g} / \mathrm{L}$ \\
\hline$p, p^{\prime}-D D D$ & $0 / 10$ & 0.10 & 0.0 & $0.10 / 0.10 \mu \mathrm{g} / \mathrm{L}$ \\
\hline$p, p^{\prime}-D D E$ & $0 / 10$ & 0.10 & 0.0 & $0.10 / 0.10 \mu \mathrm{g} / \mathrm{L}$ \\
\hline$p, p^{\prime}-D D T$ & $0 / 10$ & 0.10 & 0.0 & $0.10 / 0.10 \mu \mathrm{g} / \mathrm{L}$ \\
\hline Dieldrin & $0 / 10$ & 0.10 & 0.0 & $0.10 / 0.10 \mu \mathrm{g} / \mathrm{L}$ \\
\hline Endosulfan sulfate & $0 / 10$ & 0.10 & 0.0 & $0.10 / 0.10 \mu \mathrm{g} / \mathrm{L}$ \\
\hline Endosulfan I & $0 / 10$ & 0.05 & 0.0 & $0.05 / 0.05 \mu \mathrm{g} / \mathrm{L}$ \\
\hline Endosulfan II & $0 / 10$ & 0.10 & 0.0 & $0.10 / 0.10 \mu \mathrm{g} / \mathrm{L}$ \\
\hline Endrin & $0 / 10$ & 0.10 & 0.0 & $0.10 / 0.10 \mu \mathrm{g} / \mathrm{L}$ \\
\hline Endrin aldehyde & $0 / 10$ & 0.10 & 0.0 & $0.10 / 0.10 \mu \mathrm{g} / \mathrm{L}$ \\
\hline Endrin ketone & $0 / 1$ & 0.10 & - & $0.10 / 0.10 \mu \mathrm{g} / \mathrm{L}$ \\
\hline Heptachlor & $0 / 10$ & 0.05 & 0.0 & $0.05 / 0.05 \mu \mathrm{g} / \mathrm{L}$ \\
\hline Heptachlor epoxide & $0 / 10$ & 0.05 & 0.0 & $0.05 / 0.05 \mu \mathrm{g} / \mathrm{L}$ \\
\hline Isodrin & $0 / 8$ & 0.10 & 0.0 & $0.10 / 0.10 \mu \mathrm{g} / \mathrm{L}$ \\
\hline Kepone & $0 / 8$ & 0.50 & 0.0 & $0.50 / 0.50 \mu \mathrm{g} / \mathrm{L}$ \\
\hline Lindane & $0 / 14$ & 0.05 & 0.0 & $0.05 / 0.05 \mu \mathrm{g} / \mathrm{L}$ \\
\hline Methoxychlor & $0 / 10$ & 0.50 & 0.0 & $0.50 / 0.50 \mu \mathrm{g} / \mathrm{L}$ \\
\hline Toxaphene & $0 / 10$ & 5.0 & 0.0 & $5.0 / 5.0 \mu \mathrm{g} / \mathrm{L}$ \\
\hline \multicolumn{5}{|l|}{ EPA8082 } \\
\hline PCB 1016 & $0 / 12$ & 1.0 & 0.0 & $1.0 / 1.0 \mu \mathrm{g} / \mathrm{L}$ \\
\hline PCB 1221 & $0 / 12$ & 2.0 & 0.0 & $2.0 / 2.0 \mu \mathrm{g} / \mathrm{L}$ \\
\hline PCB 1232 & $0 / 12$ & 1.0 & 0.0 & $1.0 / 1.0 \mu \mathrm{g} / \mathrm{L}$ \\
\hline PCB 1242 & $0 / 12$ & 1.0 & 0.0 & $1.0 / 1.0 \mu \mathrm{g} / \mathrm{L}$ \\
\hline PCB 1248 & $0 / 12$ & 1.0 & 0.0 & $1.0 / 1.0 \mu \mathrm{g} / \mathrm{L}$ \\
\hline PCB 1254 & $0 / 12$ & 1.0 & 0.0 & $1.0 / 1.0 \mu \mathrm{g} / \mathrm{L}$ \\
\hline PCB 1260 & $0 / 12$ & 1.0 & 0.0 & $1.0 / 1.0 \mu \mathrm{g} / \mathrm{L}$ \\
\hline \multicolumn{5}{|l|}{ EPA8141A } \\
\hline Dimethoate & $0 / 10$ & 0.50 & 0.0 & $0.50 / 0.50 \mu \mathrm{g} / \mathrm{L}$ \\
\hline Disulfoton & $0 / 10$ & 0.50 & 0.0 & $0.50 / 0.50 \mu \mathrm{g} / \mathrm{L}$ \\
\hline Famphur & $0 / 10$ & 1.30 & 0.0 & $1.30 / 1.30 \mathrm{\mu g} / \mathrm{L}$ \\
\hline Parathion & $0 / 2$ & 0.50 & 0.0 & $0.50 / 0.50 \mu \mathrm{g} / \mathrm{L}$ \\
\hline Parathion ethyl & $0 / 8$ & 0.50 & 0.0 & $0.50 / 0.50 \mu g / L$ \\
\hline Parathion methyl & $0 / 10$ & 0.50 & 0.0 & $0.50 / 0.50 \mu \mathrm{g} / \mathrm{L}$ \\
\hline Phorate & $0 / 10$ & 1.0 & 0.0 & $1.0 / 1.0 \mu \mathrm{g} / \mathrm{L}$ \\
\hline Sulfotepp & $0 / 10$ & 1.0 & 0.0 & $1.0 / 1.0 \mu \mathrm{g} / \mathrm{L}$ \\
\hline Thionazin & $0 / 10$ & 0.50 & 0.0 & $0.50 / 0.50 \mu \mathrm{g} / \mathrm{L}$ \\
\hline $0,0,0-$ Triethyl phosphorothioate & $0 / 10$ & 0.50 & 0.0 & $0.50 / 0.50 \mu \mathrm{g} / \mathrm{L}$ \\
\hline \multicolumn{5}{|l|}{ EPAB151A } \\
\hline 2,4-Dichlorophenoxyacetic acid & $0 / 11$ & 1.0 & 0.0 & $1.0 / 1.0 \mu \mathrm{g} / \mathrm{L}$ \\
\hline $2,4,5-\mathrm{T}$ & $0 / 8$ & 0.50 & 0.0 & $0.50 / 0.50 \mu \mathrm{g} / \mathrm{L}$ \\
\hline $2,4,5-\mathrm{TP}$ (Silvex) & $0 / 8$ & 0.50 & 0.0 & $0.50 / 0.50 \mu \mathrm{g} / \mathrm{L}$ \\
\hline \multicolumn{5}{|l|}{ EPA8260B } \\
\hline Acetone & $2 / 21$ & 10.1 & 0.87 & $8.42 / 13.6 \mu \mathrm{g} / \mathrm{L}$ \\
\hline Acetonitrile & $0 / 20$ & 20.0 & 0.0 & $20.0 / 20.0 \mu g h$ \\
\hline
\end{tabular}




\begin{tabular}{|c|c|c|c|c|}
\hline Analyte & $\begin{array}{l}\text { Frequency } \\
\text { of Detectiont }\end{array}$ & Mean Result & $\begin{array}{l}\text { Standard } \\
\text { Deviation }\end{array}$ & Minimum/Maximum Results \\
\hline Acrolein & $0 / 21$ & 20.0 & 0.0 & $20.0 / 20.0 \mu \mathrm{g} / \mathrm{L}$ \\
\hline Acrylonitrile & $0 / 21$ & 5.24 & 1.09 & $5.0 / 10.0 \mu \mathrm{g} / \mathrm{L}$ \\
\hline Aliyl chloride & $0 / 20$ & 10.0 & 0.0 & $10.0 / 10.0 \mu \mathrm{g} / \mathrm{L}$ \\
\hline Benzene & $0 / 68$ & 5.0 & 0.0 & $5.0 / 5.0 \mu \mathrm{g} / \mathrm{L}$ \\
\hline Bromochloromethane & $0 / 7$ & 5.0 & 0.0 & $5.0 / 5.0 \mu \mathrm{g} / \mathrm{L}$ \\
\hline Bromodichloromethane & $0 / 69$ & 5.0 & 0.0 & $5.0 / 5.0 \mu \mathrm{g} / \mathrm{L}$ \\
\hline Bromoform & $0 / 69$ & 5.0 & 0.0 & $5.0 / 5.0 \mu \mathrm{g} / \mathrm{L}$ \\
\hline Bromomethane & $4 / 69$ & 9.60 & 1.66 & $1.90 / 10.0 \mu \mathrm{g} / \mathrm{L}$ \\
\hline Carbon disulfide & $0 / 21$ & 5.0 & 0.0 & $5.0 / 5.0 \mu \mathrm{g} / \mathrm{L}$ \\
\hline Carbon tetrachloride & $0 / 69$ & 5.0 & 0.0 & $5.0 / 5.0 \mu \mathrm{g} / \mathrm{L}$ \\
\hline Chlorobenzene & $0 / 68$ & 5.0 & 0.0 & $5.0 / 5.0 \mu \mathrm{g} / \mathrm{L}$ \\
\hline Chloroethane & $0 / 69$ & 10.0 & 0.0 & $10.0 / 10.0 \mu \mathrm{g} / \mathrm{L}$ \\
\hline Chloroethene & $0 / 69$ & 10.0 & 0.0 & $10.0 / 10.0 \mu \mathrm{g} / \mathrm{L}$ \\
\hline 2-Chloroethyl vinyl ether & $0 / 48$ & 10.0 & 0.0 & $10.0 / 10.0 \mu \mathrm{g} / \mathrm{L}$ \\
\hline Chloroform & $0 / 69$ & 5.0 & 0.0 & $5.0 / 5.0 \mu \mathrm{g} / \mathrm{L}$ \\
\hline Chloromethane & $2 / 69$ & 9.77 & 1.33 & $1.58 / 10.0 \mu \mathrm{g} / \mathrm{L}$ \\
\hline Chloroprene & $0 / 20$ & 5.0 & 0.0 & $5.0 / 5.0 \mu g / L$ \\
\hline Dibromochloromethane & $0 / 69$ & 5.0 & 0.0 & $5.0 / 5.0 \mu \mathrm{g} / \mathrm{L}$ \\
\hline 1,2-Dibromo-3-chloropropane & $0 / 20$ & 5.0 & 0.0 & $5.0 / 5.0 \mu \mathrm{g} / \mathrm{L}$ \\
\hline 1,2-Dibromoethane & $0 / 20$ & 5.0 & 0.0 & $5.0 / 5.0 \mu \mathrm{g} / \mathrm{L}$ \\
\hline Dibromomethane & $0 / 20$ & 5.0 & 0.0 & $5.0 / 5.0 \mu \mathrm{g} / \mathrm{L}$ \\
\hline 1,2-Dichlorobenzene & $0 / 8$ & 5.0 & 0.0 & $5.0 / 5.0 \mu \mathrm{g} / \mathrm{L}$ \\
\hline 1,3-Dichlorobenzene & $0 / 1$ & 5.0 & - & $5.0 / 5.0 \mu \mathrm{g} / \mathrm{L}$ \\
\hline 1,4-Dichlorobenzene & $0 / 10$ & 5.0 & 0.0 & $5.0 / 5.0 \mu \mathrm{g} / \mathrm{L}$ \\
\hline trans-1,4-Dichloro-2-butene & $0 / 19$ & 20.0 & 0.0 & $20.0 / 20.0 \mu \mathrm{g} / \mathrm{L}$ \\
\hline Dichlorodifluoromethane & $0 / 20$ & 10.0 & 0.0 & $10.0 / 10.0 \mu \mathrm{g} / \mathrm{L}$ \\
\hline 1,1-Dichloroethane & $0 / 75$ & 5.0 & 0.0 & $5.0 / 5.0 \mu \mathrm{g} / \mathrm{h}$ \\
\hline 1,2-Dichloroethane & $0 / 69$ & 5.0 & 0.0 & $5.0 / 5.0 \mu \mathrm{g} / \mathrm{L}$ \\
\hline 1,1-Dichloroethylene & $0 / 68$ & 5.0 & 0.0 & $5.0 / 5.0 \mu \mathrm{g} / \mathrm{L}$ \\
\hline 1,2-Dichloroethylene & $0 / 2$ & 5.0 & 0.0 & $5.0 / 5.0 \mu \mathrm{g} / \mathrm{L}$ \\
\hline cis-1,2-Dichloroethylene & $0 / 35$ & 6.14 & 4.71 & $5.0 / 25.0 \mu \mathrm{g} / \mathrm{L}$ \\
\hline trans-1,2-Dichloroethylene & $0 / 69$ & 5.0 & 0.0 & $5.0 / 5.0 \mu \mathrm{g} / \mathrm{L}$ \\
\hline Dichloromethane & $26 / 70$ & 6.61 & 3.03 & $4.11 / 17.6 \mu \mathrm{g} / \mathrm{L}$ \\
\hline 1,2-Dichloropropane & $0 / 69$ & 5.0 & 0.0 & $5.0 / 5.0 \mu \mathrm{g} / \mathrm{L}$ \\
\hline 1,3-Dichloropropane & $0 / 1$ & 5.0 & - & $5.0 / 5.0 \mu \mathrm{g} / \mathrm{L}$ \\
\hline 2,2-Dichloropropane & $0 / 1$ & 5.0 & - & $5.0 / 5.0 \mu \mathrm{g} / \mathrm{L}$ \\
\hline 1,1-Dichloropropene & $0 / 1$ & 5.0 & - & $5.0 / 5.0 \mu \mathrm{g} / \mathrm{L}$ \\
\hline cis-1,3-Dichloropropene & $0 / 69$ & 5.0 & 0.0 & $5.0 / 5.0 \mu \mathrm{g} / \mathrm{L}$ \\
\hline trans-1,3-Dichloropropene & $0 / 69$ & 5.0 & 0.0 & $5.0 / 5.0 \mu g / L$ \\
\hline Ethylbenzene & $0 / 69$ & 5.0 & 0.0 & $5.0 / 5.0 \mu \mathrm{g} / \mathrm{L}$ \\
\hline 2-Hexanone & $0 / 21$ & 10.0 & 0.0 & $10.0 / 10.0 \mu \mathrm{g} / \mathrm{L}$ \\
\hline lodomethane & $0 / 20$ & 5.0 & 0.0 & $5.0 / 5.0 \mu \mathrm{g} / \mathrm{L}$ \\
\hline Isobutyl alcohol & $0 / 20$ & 100 & 0.0 & $100 / 100 \mu g / L$ \\
\hline Methacrylonitrile & $0 / 20$ & 10.0 & 0.0 & $10.0 / 10.0 \mu \mathrm{g} / \mathrm{L}$ \\
\hline Methyl ethyl ketone & $0 / 21$ & 10.0 & 0.0 & $10.0 / 10.0 \mu \mathrm{g} / \mathrm{L}$ \\
\hline Methyl isobutyl ketone & $0 / 21$ & 10.0 & 0.0 & $10.0 / 10.0 \mu \mathrm{g} / \mathrm{L}$ \\
\hline Propionitrile & $0 / 20$ & 50.0 & 0.0 & $50.0 / 50.0 \mu \mathrm{g} / \mathrm{L}$ \\
\hline Styrene & $0 / 21$ & 5.0 & 0.0 & $5.0 / 5.0 \mu \mathrm{g} / \mathrm{L}$ \\
\hline $1,1,1,2-$ Tetrachloroethane & $0 / 20$ & 5.0 & 0.0 & $5.0 / 5.0 \mu \mathrm{g} / \mathrm{L}$ \\
\hline $1,1,2,2-$ Tetrachloroethane & $0 / 69$ & 5.0 & 0.0 & $5.0 / 5.0 \mu \mathrm{g} / \mathrm{L}$ \\
\hline Tetrachloroethylene & $0 / 91$ & 5.0 & 0.0 & $5.0 / 5.0 \mu \mathrm{g} / \mathrm{L}$ \\
\hline Toluene & $0 / 68$ & 5.0 & 0.0 & $5.0 / 5.0 \mu g / L$ \\
\hline 1,1,1-Trichloroethane & $0 / 69$ & 5.0 & 0.0 & $5.0 / 5.0 \mu \mathrm{g} / \mathrm{L}$ \\
\hline $1,1,2-$ Trichloroethane & $0 / 69$ & 5.0 & 0.0 & $5.0 / 5.0 \mu \mathrm{g} / \mathrm{L}$ \\
\hline Trichloroethylene & $0 / 68$ & 5.0 & 0.0 & $5.0 / 5.0 \mu \mathrm{g} / \mathrm{L}$ \\
\hline Trichlorofluoromethane & $0 / 67$ & 5.0 & 0.0 & $5.0 / 5.0 \mu \mathrm{g} / \mathrm{L}$ \\
\hline 1,2,3-Trichloropropane & $0 / 20$ & 5.0 & 0.0 & $5.0 / 5.0 \mu \mathrm{g} / \mathrm{L}$ \\
\hline Vinyl acetate & $0 / 20$ & 10.0 & 0.0 & $10.0 / 10.0 \mu \mathrm{g} / \mathrm{L}$ \\
\hline Xylenes & $0 / 68$ & 5.0 & 0.0 & $5.0 / 5.0 \mu \mathrm{g} / \mathrm{L}$ \\
\hline
\end{tabular}




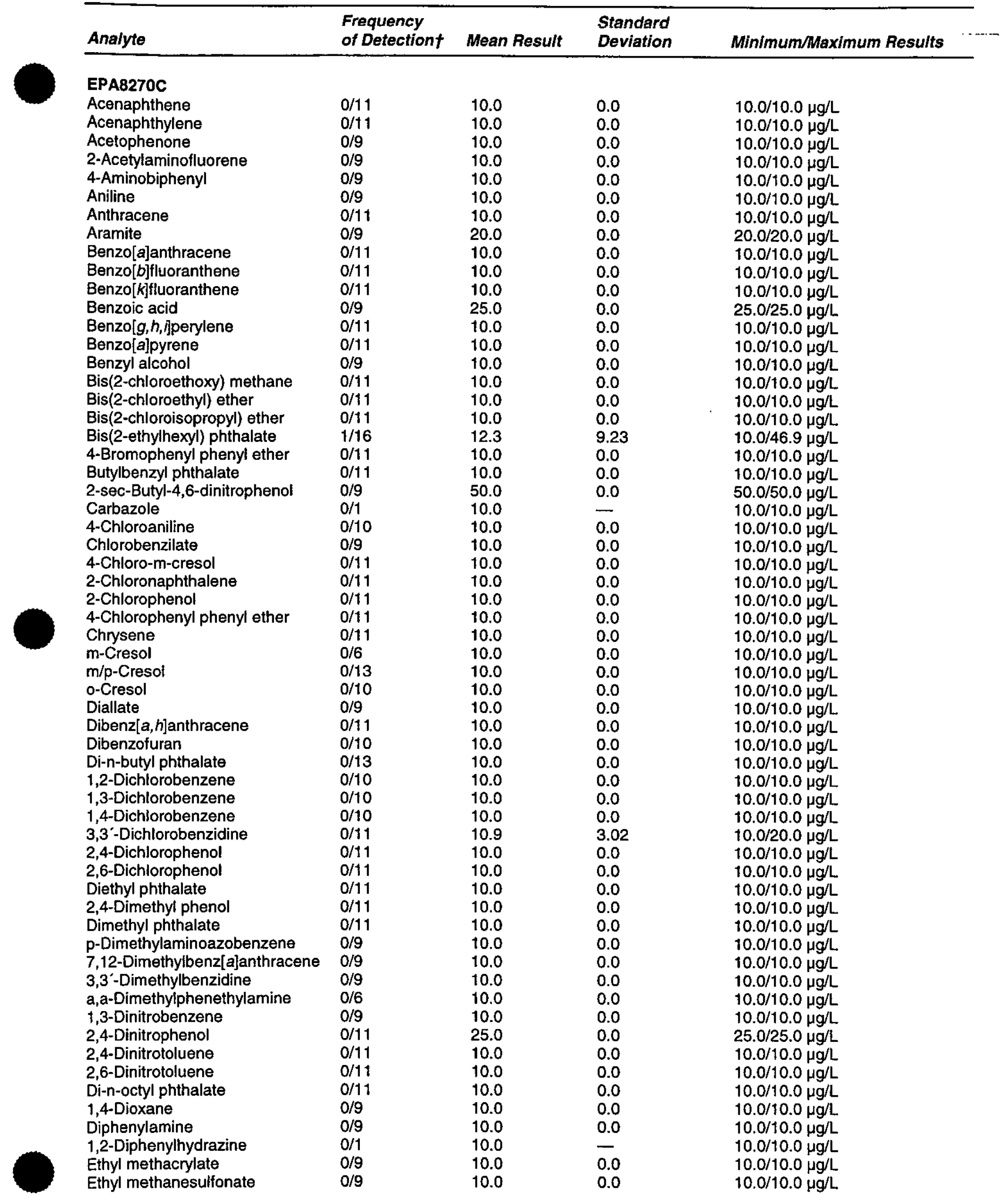




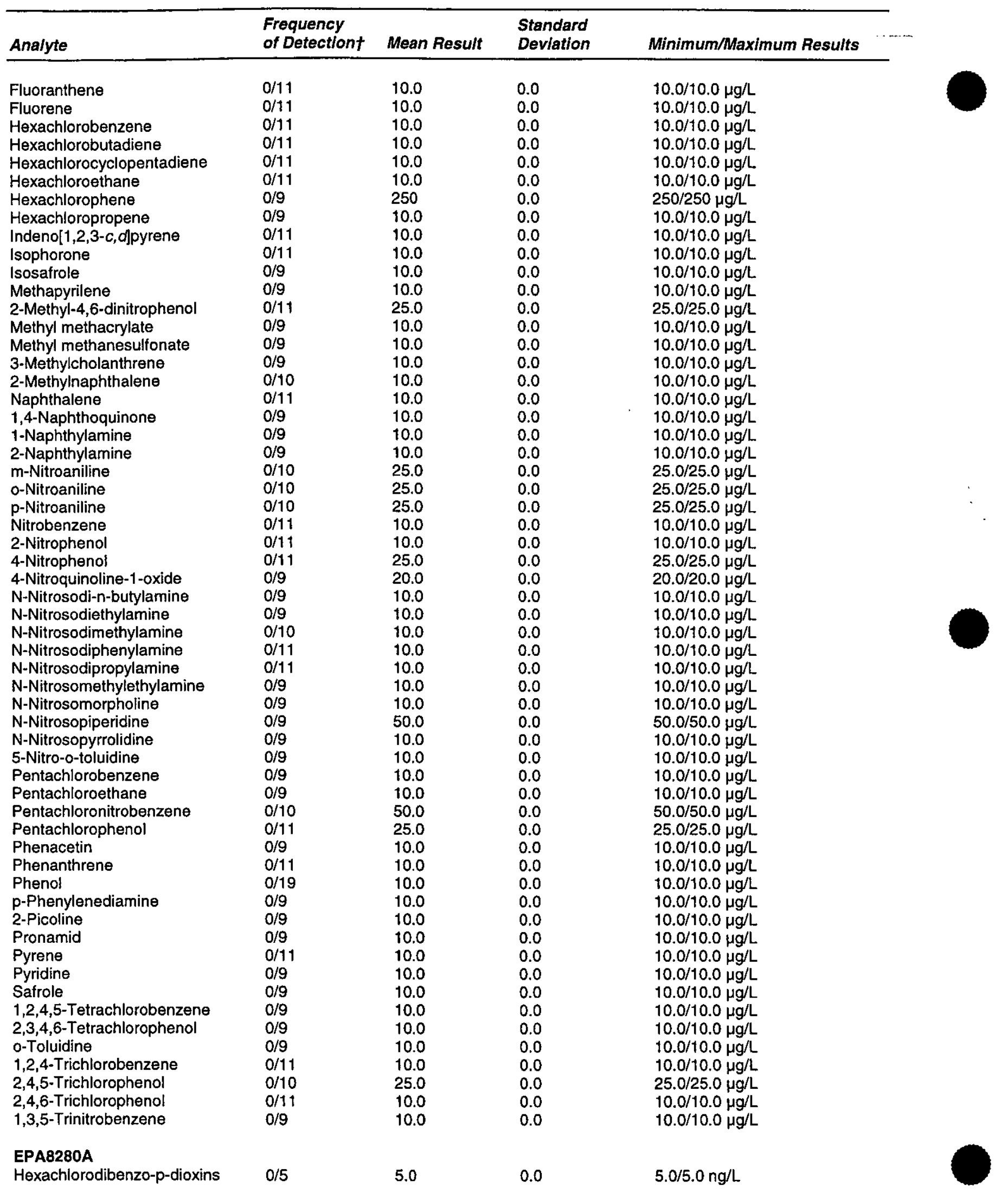




\begin{tabular}{|c|c|c|c|c|}
\hline Analyte & $\begin{array}{l}\text { Frequency } \\
\text { of Detectiont }\end{array}$ & Mean Result & $\begin{array}{l}\text { Standard } \\
\text { Deviation }\end{array}$ & Minimum/Maximum Results \\
\hline $\begin{array}{l}\text { Hexachlorodibenzo-p-furans } \\
\text { Pentachlorodibenzo-p-furans } \\
2,3,7,8-T C D D \\
\text { Tetrachlorodibenzo-p-dioxins } \\
\text { Tetrachlorodibenzo-p-furans }\end{array}$ & $\begin{array}{l}0 / 5 \\
0 / 10 \\
0 / 6 \\
0 / 5 \\
0 / 5\end{array}$ & $\begin{array}{l}5.0 \\
5.0 \\
5.0 \\
5.0 \\
5.0\end{array}$ & $\begin{array}{l}0.0 \\
0.0 \\
0.0 \\
0.0 \\
0.0\end{array}$ & $\begin{array}{l}5.0 / 5.0 \mathrm{ng} / \mathrm{L} \\
5.0 / 5.0 \mathrm{ng} / \mathrm{L} \\
5.0 / 5.0 \mathrm{ng} / \mathrm{L} \\
5.0 / 5.0 \mathrm{ng} / \mathrm{L} \\
5.0 / 5.0 \mathrm{ng} / \mathrm{L}\end{array}$ \\
\hline $\begin{array}{l}\text { EPA9014 } \\
\text { Cyanide }\end{array}$ & $0 / 31$ & 15.2 & 0.0 & $15.2 / 15.2 \mu \mathrm{g} / \mathrm{L}$ \\
\hline $\begin{array}{l}\text { EPA9020B } \\
\text { Total organic halogens }\end{array}$ & $0 / 56$ & 120 & 0.0 & $120 / 120 \mu g / L$ \\
\hline $\begin{array}{l}\text { EPA9030B } \\
\text { Sulfide }\end{array}$ & $0 / 5$ & 10,000 & 0.0 & $10,000 / 10,000 \mu g / L$ \\
\hline $\begin{array}{l}\text { EPA9034 } \\
\text { Sulfide }\end{array}$ & $0 / 2$ & 10,000 & 0.0 & $10,000 / 10,000 \mu g / L$ \\
\hline $\begin{array}{l}\text { EPA9050A } \\
\text { Specific conductance }\end{array}$ & $0 / 5$ & 8.90 & 0.0 & $8.90 / 8.90 \mu \mathrm{S} / \mathrm{cm}$ \\
\hline $\begin{array}{l}\text { EPA9056 } \\
\text { Chloride } \\
\text { Sulfate }\end{array}$ & $\begin{array}{l}4 / 24 \\
0 / 25\end{array}$ & $\begin{array}{l}204 \\
340\end{array}$ & $\begin{array}{l}26.8 \\
0.0\end{array}$ & $\begin{array}{l}111 / 229 \mu g / L \\
340 / 340 \mu g / L\end{array}$ \\
\hline $\begin{array}{l}\text { EPA9060 } \\
\text { Total organic carbon }\end{array}$ & $0 / 18$ & 1,000 & 0.0 & $1,000 / 1,000 \mu \mathrm{g} / \mathrm{L}$ \\
\hline $\begin{array}{l}\text { EPA9066 } \\
\text { Phenols }\end{array}$ & $0 / 1$ & 37.0 & - & $37.0 / 37.0 \mu \mathrm{g} / \mathrm{L}$ \\
\hline
\end{tabular}

$\uparrow$ Number of times analyte was detected compared to the total number of method blanks for the analyte.

- Standard deviation cannot be determined.

Note: If the analyte was not detected in the method blank(s), detection limit information appears in the Mean Result and Minimum/Maximum Results columns.

Table 56. Analytes Dotected in Mothod Blanks for GP

\begin{tabular}{|c|c|c|c|c|}
\hline Analyte & $\begin{array}{l}\text { Frequency } \\
\text { of Detectiont }\end{array}$ & Mean Result & $\begin{array}{l}\text { Standard } \\
\text { Deviation }\end{array}$ & Minimum/Maximum Results \\
\hline \multicolumn{5}{|l|}{ EPIA-001 } \\
\hline Gross alpha & $1 / 33$ & $8.25 E-11$ & $1.65 E-10$ & $-2.70 \mathrm{E}-10 / 4.90 \mathrm{E}-10 \mu \mathrm{Ci} / \mathrm{mL}$ \\
\hline Nonvolatile beta & $1 / 31$ & $2.95 E-10$ & $4.31 \mathrm{E}-10$ & $-4.20 \mathrm{E}-10 / 1.31 \mathrm{E}-09 \mu \mathrm{Ci} / \mathrm{mL}$ \\
\hline \multicolumn{5}{|l|}{ EPIA-002 } \\
\hline Tritium & $1 / 27$ & $-3.17 \mathrm{E}-08$ & $1.68 \mathrm{E}-07$ & $-4.27 \mathrm{E}-07 / 2.58 \mathrm{E}-07 \mu \mathrm{Ci} / \mathrm{mL}$ \\
\hline \multicolumn{5}{|l|}{ EPIA-003 } \\
\hline Carbon-14 & $0 / 21$ & $-8.24 \mathrm{E}-10$ & $2.85 E-09$ & $-4.81 \mathrm{E}-09 / 3.72 \mathrm{E}-09 \mu \mathrm{Ci} / \mathrm{mL}$ \\
\hline \multicolumn{5}{|l|}{ EPIA-004 } \\
\hline Strontium-89/90 & $0 / 5$ & $-4.31 E-10$ & $4.02 E-10$ & $-9.07 \mathrm{E}-10 / 1.13 \mathrm{E}-10 \mu \mathrm{Ci} / \mathrm{mL}$ \\
\hline Strontium-90 & $0 / 23$ & $6.00 \mathrm{E}-11$ & $5.08 \mathrm{E}-10$ & $-6.96 \mathrm{E}-10 / 1.26 \mathrm{E}-09 \mu \mathrm{Ci} / \mathrm{mL}$ \\
\hline
\end{tabular}




\begin{tabular}{|c|c|c|c|c|}
\hline Analyte & $\begin{array}{l}\text { Frequency } \\
\text { of Detectiont }\end{array}$ & Mean Result & $\begin{array}{l}\text { Standard } \\
\text { Deviation }\end{array}$ & Minimum/Maximum Results \\
\hline \multicolumn{5}{|l|}{ EPIA-005 } \\
\hline Technetium-99 & $0 / 24$ & $-1.11 \mathrm{E}-09$ & $6.55 \mathrm{E}-09$ & $-1.51 \mathrm{E}-08 / 1.31 \mathrm{E}-08 \mu \mathrm{Ci} / \mathrm{mL}$ \\
\hline \multicolumn{5}{|l|}{ EPIA-006 } \\
\hline lodine-129 & $2 / 23$ & $2.95 E-10$ & $4.24 \mathrm{E}-10$ & $-7.88 \mathrm{E}-10 / 1.46 \mathrm{E}-09 \mu \mathrm{Ci} / \mathrm{mL}$ \\
\hline \multicolumn{5}{|l|}{ EPIA-008 } \\
\hline Radium-226 & $0 / 29$ & $2.17 E-10$ & $1.30 \mathrm{E}-10$ & $0.0 / 6.08 \mathrm{E}-10 \mu \mathrm{Ci} / \mathrm{mL}$ \\
\hline \multicolumn{5}{|l|}{ EPIA-009 } \\
\hline Radium-228 & $4 / 25$ & $6.84 E-11$ & $7.52 \mathrm{E}-10$ & -1.77E-09/9.99E-10 $\mu \mathrm{Ci} / \mathrm{mL}$ \\
\hline \multicolumn{5}{|l|}{ EPIA-010 } \\
\hline Radium, total alpha-emitting & $0 / 4$ & 2.25E-10 & $1.89 \mathrm{E}-10$ & $1.00 \mathrm{E}-10 / 5.00 \mathrm{E}-10 \mu \mathrm{Ci} / \mathrm{mL}$ \\
\hline \multicolumn{5}{|l|}{ EPIA-011 } \\
\hline Americium-241 & $3 / 28$ & $1.16 \mathrm{E}-11$ & $7.24 \mathrm{E}-11$ & $-1.07 \mathrm{E}-10 / 2.52 \mathrm{E}-10 \mu \mathrm{Ci} / \mathrm{mL}$ \\
\hline Curium-242 & $0 / 28$ & $-9.84 \mathrm{E}-12$ & $2.82 \mathrm{E}-11$ & $-9.31 \mathrm{E}-11 / 3.94 \mathrm{E}-11 \mu \mathrm{Ci} / \mathrm{mL}$ \\
\hline Curium-243/244 & $1 / 28$ & $2.75 \mathrm{E}-11$ & $5.46 \mathrm{E}-11$ & $-2.37 \mathrm{E}-11 / 1.88 \mathrm{E}-10 \mu \mathrm{Ci} / \mathrm{mL}$ \\
\hline Curium-245/246 & $1 / 28$ & $1.45 E-11$ & $3.23 E-11$ & $-8.00 \mathrm{E}-12 / 1.61 \mathrm{E}-10 \mu \mathrm{Ci} / \mathrm{mL}$ \\
\hline Plutonium-238 & $1 / 25$ & $2.92 \mathrm{E}-11$ & $6.48 E-11$ & -3.79E-11/2.77E-10 $\mu \mathrm{Ci} / \mathrm{mL}$ \\
\hline Plutonium-239/240 & $1 / 25$ & $1.41 \mathrm{E}-11$ & $2.07 E-11$ & $-4.38 \mathrm{E}-12 / 9.06 \mathrm{E}-11 \mu \mathrm{Ci} / \mathrm{mL}$ \\
\hline Uranium-233/234 & $3 / 29$ & 4.19E-11 & $1.11 E-10$ & $-1.18 \mathrm{E}-11 / 5.66 \mathrm{E}-10 \mu \mathrm{Ci} / \mathrm{mL}$ \\
\hline Uranium-235 & $0 / 29$ & $9.91 \mathrm{E}-12$ & $2.78 \mathrm{E}-11$ & $-9.42 \mathrm{E}-12 / 1.38 \mathrm{E}-10 \mu \mathrm{Ci} / \mathrm{mL}$ \\
\hline Uranium-238 & $1 / 29$ & $2.36 E-11$ & $5.35 E-11$ & $-6.52 \mathrm{E}-12 / 2.56 \mathrm{E}-10 \mu \mathrm{Ci} / \mathrm{mL}$ \\
\hline \multicolumn{5}{|l|}{ EPIA-012 } \\
\hline Thorium-228 & $0 / 26$ & $2.44 \mathrm{E}-11$ & $3.63 E-11$ & $-2.14 \mathrm{E}-11 / 1.08 \mathrm{E}-10 \mu \mathrm{Ci} / \mathrm{mL}$ \\
\hline Thorium-230 & $1 / 26$ & $1.39 E-11$ & $9.89 E-12$ & $-1.87 \mathrm{E}-12 / 4.23 \mathrm{E}-11 \mu \mathrm{Ci} / \mathrm{mL}$ \\
\hline Thorium-232 & $0 / 26$ & $2.19 \mathrm{E}-12$ & $4.84 \mathrm{E}-12$ & $-2.95 \mathrm{E}-12 / 1.45 \mathrm{E}-11 \mu \mathrm{Ci} / \mathrm{mL}$ \\
\hline \multicolumn{5}{|l|}{ EPIA-013 } \\
\hline Actinium-228 & $0 / 26$ & $6.28 E-09$ & 4.17E-09 & $-6.51 \mathrm{E}-10 / 1.61 \mathrm{E}-08 \mu \mathrm{Ci} / \mathrm{mL}$ \\
\hline Antimony-125 & $0 / 26$ & $7.61 \mathrm{E}-10$ & 4.11E-09 & $-4.72 \mathrm{E}-09 / 1.54 \mathrm{E}-08 \mu \mathrm{Ci} / \mathrm{mL}$ \\
\hline Cerium-144 & $0 / 26$ & $2.57 E-09$ & $6.60 \mathrm{E}-09$ & $-1.02 \mathrm{E}-08 / 1.76 \mathrm{E}-08 \mu \mathrm{Ci} / \mathrm{mL}$ \\
\hline Cesium-134 & $0 / 26$ & $-1.28 \mathrm{E}-10$ & $1.45 E-09$ & $-6.07 \mathrm{E}-09 / 1.68 \mathrm{E}-09 \mu \mathrm{Ci} / \mathrm{mL}$ \\
\hline Cesium-137 & $0 / 26$ & $1.51 \mathrm{E}-09$ & $3.11 E-09$ & $-1.62 \mathrm{E}-09 / 1.23 \mathrm{E}-08 \mu \mathrm{Ci} / \mathrm{mL}$ \\
\hline Cobalt-57 & $0 / 26$ & 1.79E-10 & $6.61 E-10$ & $-1.03 \mathrm{E}-09 / 1.87 \mathrm{E}-09 \mu \mathrm{Ci} / \mathrm{mL}$ \\
\hline Cobalt-60 & $0 / 26$ & $3.05 E-10$ & $1.02 \mathrm{E}-09$ & $-1.80 \mathrm{E}-09 / 2.49 \mathrm{E}-09 \mu \mathrm{Ci} / \mathrm{mL}$ \\
\hline Europium-152 & $0 / 26$ & $1.46 \mathrm{E}-09$ & $2.29 E-09$ & $-3.09 \mathrm{E}-09 / 5.64 \mathrm{E}-09 \mu \mathrm{Ci} / \mathrm{mL}$ \\
\hline Europium-154 & $0 / 26$ & $6.00 E-10$ & $3.24 E-09$ & -3.23E-09/8.91E-09 $\mu \mathrm{Ci} / \mathrm{mL}$ \\
\hline Europium-155 & $0 / 26$ & $-9.01 E-10$ & $3.21 \mathrm{E}-09$ & $-7.18 \mathrm{E}-09 / 4.74 \mathrm{E}-09 \mu \mathrm{Ci} / \mathrm{mL}$ \\
\hline Lead-212 & $0 / 26$ & $3.16 \mathrm{E}-09$ & $1.72 \mathrm{E}-09$ & 4.59E-10/8.26E-09 $\mu \mathrm{Ci} / \mathrm{mL}$ \\
\hline Manganese-54 & $0 / 26$ & 2.71E-10 & 1.34E-09 & $-2.73 \mathrm{E}-09 / 3.81 \mathrm{E}-09 \mu \mathrm{Ci} / \mathrm{mL}$ \\
\hline Potassium-40 & $1 / 26$ & 1.95E-08 & $1.59 E-08$ & $-7.98 \mathrm{E}-09 / 5.27 \mathrm{E}-08 \mu \mathrm{Ci} / \mathrm{mL}$ \\
\hline Promethium-144 & $0 / 26$ & $2.27 \mathrm{E}-10$ & $8.81 E-10$ & $-1.26 \mathrm{E}-09 / 2.48 \mathrm{E}-09 \mu \mathrm{Ci} / \mathrm{mL}$ \\
\hline Promethium- 146 & $0 / 26$ & $1.87 E-10$ & $1.86 E-09$ & $-3.04 \mathrm{E}-09 / 5.48 \mathrm{E}-09 \mu \mathrm{Ci} / \mathrm{mL}$ \\
\hline Ruthenium-106 & $0 / 26$ & $1.84 E-09$ & 8.31E-09 & $-1.52 \mathrm{E}-08 / 1.58 \mathrm{E}-08 \mu \mathrm{Ci} / \mathrm{mL}$ \\
\hline Sodium-22 & $0 / 26$ & $2.40 E-10$ & 1.13E-09 & $-1.14 \mathrm{E}-09 / 3.17 \mathrm{E}-09 \mu \mathrm{Ci} / \mathrm{mL}$ \\
\hline Yttrium-88 & $0 / 26$ & $-8.71 E-11$ & $1.55 E-09$ & $-3.86 \mathrm{E}-09 / 3.25 \mathrm{E}-09 \mu \mathrm{Ci} / \mathrm{mL}$ \\
\hline Zinc-65 & $0 / 26$ & $-1.09 \mathrm{E}-09$ & 2.47E-09 & $-5.97 \mathrm{E}-09 / 3.83 \mathrm{E}-09 \mu \mathrm{Ci} / \mathrm{mL}$ \\
\hline
\end{tabular}




\begin{tabular}{|c|c|c|c|c|}
\hline Analyte & $\begin{array}{l}\text { Frequency } \\
\text { of Detectiont }\end{array}$ & Mean Result & $\begin{array}{l}\text { Standard } \\
\text { Deviation }\end{array}$ & Minimum/Maximum Results \\
\hline \multicolumn{5}{|l|}{ EPIA-022 } \\
\hline Nickel-63 & $0 / 11$ & 9.00E-09 & 1.77E-08 & $-2.04 \mathrm{E}-08 / 4.85 \mathrm{E}-08 \mu \mathrm{Ci} / \mathrm{mL}$ \\
\hline
\end{tabular}

$\dagger$ Number of times analyte was detected compared to the total number of method blanks for the analyte.

Note: If the analyte was not detected in the method blank(s), detection limit information appears in the Mean Result and Minimum/Maximum Results columns.

Table 57. Analytes Detected in Method Blanks for TM

\begin{tabular}{|c|c|c|c|c|}
\hline Analyte & $\begin{array}{l}\text { Frequency } \\
\text { of Detectiont }\end{array}$ & Mean Result & $\begin{array}{l}\text { Standard } \\
\text { Deviation }\end{array}$ & Minimum/Maximum Results \\
\hline \multicolumn{5}{|l|}{ 3500NIEMOD } \\
\hline Nickel-63 & $0 / 4$ & $-1.13 E-08$ & $1.51 E-08$ & -3.39E-08/-2.75E-09 $\mu \mathrm{Ci} / \mathrm{mL}$ \\
\hline \multicolumn{5}{|l|}{ EICHROMTC1MOD } \\
\hline Technetium-99 & $0 / 9$ & $2.66 \mathrm{E}-10$ & $2.55 E-10$ & $-3.00 \mathrm{E}-10 / 5.20 \mathrm{E}-10 \mu \mathrm{Ci} / \mathrm{mL}$ \\
\hline \multicolumn{5}{|l|}{ EMLAM01MOD } \\
\hline Americium-241/Curium-246 & $4 / 7$ & $1.74 E-10$ & $9.74 \mathrm{E}-11$ & 2.00E-11/2.90E-10 $\mu \mathrm{Ci} / \mathrm{mL}$ \\
\hline Curium-242 & $0 / 7$ & $2.86 \mathrm{E}-12$ & $2.21 E-11$ & $-2.00 \mathrm{E}-11 / 4.00 \mathrm{E}-11 \mu \mathrm{Ci} / \mathrm{mL}$ \\
\hline Curium-243/244 & $0 / 7$ & $2.00 \mathrm{E}-11$ & 4.16E-11 & $-3.00 \mathrm{E}-11 / 9.00 \mathrm{E}-11 \mu \mathrm{Ci} / \mathrm{mL}$ \\
\hline \multicolumn{5}{|l|}{ EMLPU02MOD } \\
\hline Plutonium-238 & $0 / 7$ & $2.71 E-11$ & 2.93E-11 & $0.0 / 9.00 \mathrm{E}-11 \mu \mathrm{Ci} / \mathrm{mL}$ \\
\hline Plutonium-239/240 & $0 / 7$ & $2.57 \mathrm{E}-11$ & $3.05 E-11$ & $0.0 / 9.00 \mathrm{E}-11 \mu \mathrm{Ci} / \mathrm{mL}$ \\
\hline \multicolumn{5}{|l|}{ EMLSR02MOD } \\
\hline Strontium-90 & $2 / 9$ & $4.29 \mathrm{E}-10$ & 8.95E-10 & $-4.50 \mathrm{E}-10 / 2.20 \mathrm{E}-09 \mu \mathrm{Ci} / \mathrm{mL}$ \\
\hline \multicolumn{5}{|l|}{ EML.TH01MOD } \\
\hline Thorium-228 & $0 / 7$ & $6.43 \mathrm{E}-11$ & $3.15 \mathrm{E}-11$ & $2.00 \mathrm{E}-11 / 1.20 \mathrm{E}-10 \mu \mathrm{Ci} / \mathrm{mL}$ \\
\hline Thorium-230 & $4 / 7$ & $1.73 \mathrm{E}-10$ & $6.73 E-11$ & 1.10E-10/2.70E-10 $\mu \mathrm{Ci} / \mathrm{mL}$ \\
\hline Thorium-232 & $0 / 7$ & $6.86 \mathrm{E}-11$ & $4.14 \mathrm{E}-11$ & 2.00E-11/1.30E-10 $\mu \mathrm{Ci} / \mathrm{mL}$ \\
\hline \multicolumn{5}{|l|}{ EMLU02MOD } \\
\hline Uranium-234 & $0 / 8$ & $7.50 \mathrm{E}-11$ & $6.14 \mathrm{E}-11$ & $3.00 \mathrm{E}-11 / 2.20 \mathrm{E}-10 \mu \mathrm{Ci} / \mathrm{mL}$ \\
\hline Uranium-235 & $0 / 8$ & $1.88 \mathrm{E}-11$ & $9.91 \mathrm{E}-12$ & $0.0 / 3.00 \mathrm{E}-11 \mu \mathrm{Ci} / \mathrm{mL}$ \\
\hline Uranium-238 & $0 / 8$ & 3.63E- 11 & $2.88 \mathrm{E}-11$ & $0.0 / 8.00 \mathrm{E}-11 \mu \mathrm{Ci} / \mathrm{mL}$ \\
\hline \multicolumn{5}{|l|}{ ENICMOD } \\
\hline Carbon-14 & $0 / 8$ & 3.52E-09 & $6.18 \mathrm{E}-08$ & $-8.08 \mathrm{E}-08 / 9.65 \mathrm{E}-08 \mu \mathrm{Ci} / \mathrm{mL}$ \\
\hline \multicolumn{5}{|l|}{ EPA900.0MOD } \\
\hline Gross alpha & $7 / 91$ & $4.29 E-12$ & $1.69 E-10$ & $-2.70 \mathrm{E}-10 / 9.40 \mathrm{E}-10 \mu \mathrm{Ci} / \mathrm{mL}$ \\
\hline Nonvolatile beta & $2 / 72$ & $-2.26 \mathrm{E}-10$ & $4.21 \mathrm{E}-10$ & $-1.11 \mathrm{E}-09 / 1.43 \mathrm{E}-09 \mu \mathrm{Ci} / \mathrm{mL}$ \\
\hline \multicolumn{5}{|l|}{ EPA901.1MOD } \\
\hline Cesium-137 & $0 / 11$ & $7.98 \mathrm{E}-10$ & $1.20 E-09$ & $-1.19 \mathrm{E}-09 / 2.38 \mathrm{E}-09 \mu \mathrm{Ci} / \mathrm{mL}$ \\
\hline Cobalt- 60 & $0 / 11$ & $3.46 \mathrm{E}-10$ & 1.16E-09 & $-1.46 \mathrm{E}-09 / 1.65 \mathrm{E}-09 \mu \mathrm{Ci} / \mathrm{mL}$ \\
\hline \multicolumn{5}{|l|}{ EPA902.0MOD } \\
\hline lodine-129 & $0 / 8$ & $3.86 \mathrm{E}-10$ & $5.80 \mathrm{E}-10$ & $-2.50 \mathrm{E}-10 / 1.18 \mathrm{E}-09 \mu \mathrm{Ci} / \mathrm{mL}$ \\
\hline
\end{tabular}

\section{Quality Control Samples}




\begin{tabular}{|c|c|c|c|c|}
\hline Analyte & $\begin{array}{l}\text { Frequency } \\
\text { of Detectiont }\end{array}$ & Mean Result & $\begin{array}{l}\text { Standard } \\
\text { Deviation }\end{array}$ & Minimum/Maximum Results \\
\hline \multicolumn{5}{|l|}{ EPA903.OMOD } \\
\hline Radium, total alpha-emitting & $2 / 17$ & $1.15 \mathrm{E}-10$ & $1.30 \mathrm{E}-10$ & $-9.00 \mathrm{E}-11 / 3.60 \mathrm{E}-10 \mu \mathrm{Ci} / \mathrm{mL}$ \\
\hline Radium-226 & $1 / 9$ & $7.22 \mathrm{E}-11$ & $3.96 \mathrm{E}-11$ & $0.0 / 1.30 \mathrm{E}-10 \mu \mathrm{Ci} / \mathrm{mL}$ \\
\hline \multicolumn{5}{|l|}{ EPA904.0MOD } \\
\hline Radium-228 & $1 / 9$ & $-2.89 E-11$ & $5.69 \mathrm{E}-10$ & $-6.70 \mathrm{E}-10 / 1.16 \mathrm{E}-09 \mu \mathrm{Ci} / \mathrm{mL}$ \\
\hline \multicolumn{5}{|l|}{ EPA906.0MOD } \\
\hline Tritium & $14 / 97$ & $2.74 \mathrm{E}-07$ & $1.06 \mathrm{E}-06$ & $-2.10 \mathrm{E}-06 / 6.20 \mathrm{E}-06 \mu \mathrm{Ci} / \mathrm{mL}$ \\
\hline
\end{tabular}

+ Number of times analyte was detected compared to the total number of method blanks for the analyte.

Table 58. Analytes Detected in Field Blanks for EX

\begin{tabular}{|c|c|c|c|c|}
\hline Analyte & $\begin{array}{l}\text { Frequency } \\
\text { of Detectiont }\end{array}$ & Mean Result & $\begin{array}{l}\text { Standard } \\
\text { Deviation }\end{array}$ & Minimum/Maximum Results \\
\hline \multicolumn{5}{|l|}{ EPA300.0 } \\
\hline Nitrate as nitrogen & $0 / 3$ & 133 & 57.7 & $100 / 200 \mu g / L$ \\
\hline \multicolumn{5}{|l|}{ EPA6010B } \\
\hline Aluminum & $0 / 18$ & 200 & 0.0 & $200 / 200 \mu \mathrm{g} / \mathrm{L}$ \\
\hline Arsenic & $0 / 2$ & 100 & 0.0 & $100 / 100 \mu g / L$ \\
\hline Barium & $0 / 14$ & 10.0 & 0.0 & $10.0 / 10.0 \mu \mathrm{g} / \mathrm{L}$ \\
\hline Boron & $0 / 5$ & 100 & 0.0 & $100 / 100 \mu \mathrm{g} / \mathrm{L}$ \\
\hline Cadmium & $0 / 2$ & 10.0 & 0.0 & $10.0 / 10.0 \mu g / \mathrm{L}$ \\
\hline Chromium & $0 / 2$ & 10.0 & 0.0 & $10.0 / 10.0 \mu \mathrm{g} / \mathrm{L}$ \\
\hline Iron & $2 / 18$ & 148 & 85.6 & $6.58 / 200 \mu \mathrm{g} / \mathrm{L}$ \\
\hline Lead & $0 / 17$ & 96.8 & 13.0 & $46.3 / 100 \mu g / L$ \\
\hline Manganese & $0 / 6$ & 10.0 & 0.0 & $10.0 / 10.0 \mu \mathrm{g} / \mathrm{L}$ \\
\hline Nickel & $0 / 11$ & 42.5 & 16.7 & $7.80 / 50.0 \mu \mathrm{g} / \mathrm{L}$ \\
\hline Selenium & $0 / 13$ & 156 & 83.3 & $10.0 / 200 \mu \mathrm{g} / \mathrm{L}$ \\
\hline Silver & $0 / 2$ & 20.0 & 0.0 & $20.0 / 20.0 \mu \mathrm{g} / \mathrm{L}$ \\
\hline \multicolumn{5}{|l|}{ EPA7470A } \\
\hline Mercury & $1 / 5$ & 0.44 & 0.14 & $0.19 / 0.50 \mu \mathrm{g} / \mathrm{L}$ \\
\hline \multicolumn{5}{|l|}{ EPA8082 } \\
\hline PCB 1016 & $0 / 1$ & 2.0 & - & $2.0 / 2.0 \mu \mathrm{g} / \mathrm{L}$ \\
\hline PCB 1221 & $0 / 1$ & 2.0 & - & $2.0 / 2.0 \mu \mathrm{g} / \mathrm{L}$ \\
\hline PCB 1232 & $0 / 1$ & 1.0 & - & $1.0 / 1.0 \mu g / L$ \\
\hline PCB 1242 & $0 / 1$ & 1.0 & - & $1.0 / 1.0 \mu \mathrm{g} / \mathrm{L}$ \\
\hline PCB 1248 & $0 / 1$ & 1.0 & - & $1.0 / 1.0 \mu \mathrm{g} / \mathrm{L}$ \\
\hline PCB 1254 & $0 / 1$ & 1.0 & - & $1.0 / 1.0 \mu \mathrm{g} / \mathrm{L}$ \\
\hline PCB 1260 & $0 / 1$ & 1.0 & - & $1.0 / 1.0 \mu \mathrm{g} / \mathrm{L}$ \\
\hline \multicolumn{5}{|l|}{ EPA9014 } \\
\hline Cyanide & $0 / 10$ & 10.0 & 0.0 & $10.0 / 10.0 \mu \mathrm{g} / \mathrm{L}$ \\
\hline
\end{tabular}

t Number of times analyte was detected compared to the total number of field blanks for the analyte.

- Standard deviation cannot be determined.

Note: If the analyte was not detected in the field blank(s), detection limit information appears in the Mean Result and Minimum/Maximum Results columns. 
Table 59. Analytes Detected in Field Blanks for GE

\begin{tabular}{|c|c|c|c|c|}
\hline Analyte & $\begin{array}{l}\text { Frequency } \\
\text { of Detectiont }\end{array}$ & Mean Result & $\begin{array}{l}\text { Standard } \\
\text { Deviation }\end{array}$ & Minimum/Maximum Results \\
\hline \multicolumn{5}{|l|}{ EPA353.1 } \\
\hline Nitrate-nitrite as nitrogen & $3 / 13$ & 46.9 & 27.2 & $10.0 / 120 \mu \mathrm{g} / \mathrm{L}$ \\
\hline \multicolumn{5}{|l|}{ EPA6010B } \\
\hline Aluminum & $1 / 6$ & 113 & 159 & $22.4 / 435 \mu g / L$ \\
\hline Antimony & $0 / 2$ & 10.0 & 0.0 & $10.0 / 10.0 \mu \mathrm{g} / \mathrm{L}$ \\
\hline Arsenic & $0 / 6$ & 5.0 & 0.0 & $5.0 / 5.0 \mu \mathrm{g} / \mathrm{L}$ \\
\hline Barium & $1 / 6$ & 7.27 & 5.55 & $5.0 / 18.6 \mu \mathrm{g} / \mathrm{L}$ \\
\hline Beryllium & $0 / 1$ & 5.0 & - & $5.0 / 5.0 \mu \mathrm{g} / \mathrm{L}$ \\
\hline Cadmium & $0 / 6$ & 5.0 & 0.0 & $5.0 / 5.0 \mu \mathrm{g} / \mathrm{h}$ \\
\hline Calcium & $0 / 1$ & 100 & - & $100 / 100 \mu \mathrm{g} / \mathrm{L}$ \\
\hline Chromium & $2 / 6$ & 3.56 & 2.23 & $0.58 / 5.0 \mu \mathrm{g} / \mathrm{L}$ \\
\hline Cobalt & $0 / 1$ & 5.0 & - & $5.0 / 5.0 \mu \mathrm{g} / \mathrm{L}$ \\
\hline Copper & $0 / 1$ & 5.0 & - & $5.0 / 5.0 \mu \mathrm{gg} / \mathrm{L}$ \\
\hline Iron & $1 / 6$ & 51.8 & 4.33 & $50.0 / 60.6 \mu \mathrm{g} / \mathrm{L}$ \\
\hline Lead & $2 / 6$ & 7.92 & 7.44 & 4.44/23.1 $\mathrm{\mu g} / \mathrm{L}$ \\
\hline Magnesium & $0 / 1$ & 10.0 & - & $10.0 / 10.0 \mu \mathrm{g} / \mathrm{L}$ \\
\hline Manganese & $0 / 1$ & 10.0 & - & $10.0 / 10.0 \mu \mathrm{g} / \mathrm{L}$ \\
\hline Nickel & $0 / 1$ & 5.0 & - & $5.0 / 5.0 \mu \mathrm{g} / \mathrm{L}$ \\
\hline Potassium & $0 / 1$ & 100 & - & $100 / 100 \mu \mathrm{g} / \mathrm{L}$ \\
\hline Selenium & $0 / 6$ & 5.0 & 0.0 & $5.0 / 5.0 \mu \mathrm{g} / \mathrm{L}$ \\
\hline Silver & $1 / 6$ & 2.70 & 1.89 & $0.80 / 5.0 \mu \mathrm{g} / \mathrm{L}$ \\
\hline Sodium & $0 / 1$ & 100 & - & $100 / 100 \mu \mathrm{g} / \mathrm{L}$ \\
\hline Thallium & $0 / 1$ & 5.0 & - & $5.0 / 5.0 \mu \mathrm{g} / \mathrm{h}$ \\
\hline Vanadium & $0 / 1$ & 5.0 & - & $5.0 / 5.0 \mu \mathrm{g} / \mathrm{L}$ \\
\hline Zinc & $0 / 1$ & 2.94 & - & $2.94 / 2.94 \mu g / L$ \\
\hline \multicolumn{5}{|l|}{ EPA6020 } \\
\hline Aluminum & $0 / 12$ & 15.0 & 0.0 & $15.0 / 15.0 \mu \mathrm{g} / \mathrm{L}$ \\
\hline Antimony & $0 / 12$ & 0.19 & 0.02 & $0.13 / 0.20 \mu \mathrm{g} / \mathrm{L}$ \\
\hline Arsenic & $0 / 12$ & 3.0 & 0.0 & $3.0 / 3.0 \mu \mathrm{g} / \mathrm{L}$ \\
\hline Barium & $2 / 12$ & 1.81 & 0.45 & $0.76 / 2.0 \mu \mathrm{g} / \mathrm{L}$ \\
\hline Cadmium & $0 / 12$ & 0.87 & 0.30 & $0.23 / 1.0 \mu \mathrm{g} / \mathrm{L}$ \\
\hline Chromium & $2 / 12$ & 2.53 & 0.91 & $0.64 / 3.0 \mu \mathrm{g} / \mathrm{L}$ \\
\hline Cobalt & $0 / 12$ & 0.19 & 0.04 & $0.08 / 0.20 \mu \mathrm{g} / \mathrm{L}$ \\
\hline Copper & $5 / 12$ & 0.75 & 0.38 & $0.28 / 1.22 \mu \mathrm{g} / \mathrm{L}$ \\
\hline Iron & $1 / 12$ & 21.0 & 20.8 & $15.0 / 87.1 \mu \mathrm{g} / \mathrm{L}$ \\
\hline Lead & $1 / 12$ & 1.87 & 0.46 & $0.40 / 2.0 \mu \mathrm{g} / \mathrm{L}$ \\
\hline Nickel & $1 / 12$ & 1.90 & 0.35 & $0.80 / 2.0 \mu \mathrm{g} / \mathrm{L}$ \\
\hline Selenium & $0 / 12$ & 5.0 & 0.0 & $5.0 / 5.0 \mu \mathrm{g} / \mathrm{L}$ \\
\hline Silver & $0 / 12$ & 1.0 & 0.0 & $1.0 / 1.0 \mu \mathrm{g} / \mathrm{L}$ \\
\hline Thallium & $1 / 5$ & 1.64 & 1.19 & $0.12 / 2.50 \mu \mathrm{g} / \mathrm{L}$ \\
\hline Tin & $0 / 7$ & 5.0 & 0.0 & $5.0 / 5.0 \mu \mathrm{g} / \mathrm{L}$ \\
\hline Vanadium & $0 / 12$ & 6.69 & 3.46 & $3.15 / 10.0 \mu \mathrm{g} / \mathrm{L}$ \\
\hline Zinc & $0 / 12$ & 10.0 & 0.0 & $10.0 / 10.0 \mu \mathrm{g} / \mathrm{L}$ \\
\hline \multicolumn{5}{|l|}{ EPA7470A } \\
\hline Mercury & $1 / 18$ & 0.19 & 0.03 & $0.05 / 0.20 \mu \mathrm{g} / \mathrm{L}$ \\
\hline \multicolumn{5}{|l|}{ EPA8081A } \\
\hline Aldrin & $0 / 1$ & 0.02 & 一 & $0.02 / 0.02 \mu \mathrm{g} / \mathrm{L}$ \\
\hline alpha-Benzene hexachloride & $0 / 1$ & 0.02 & - & $0.02 / 0.02 \mu \mathrm{g} / \mathrm{L}$ \\
\hline beta-Benzene hexachloride & $0 / 1$ & 0.02 & - & $0.02 / 0.02 \mu \mathrm{g} / \mathrm{L}$ \\
\hline delta-Benzene hexachloride & $0 / 1$ & 0.02 & 一 & $0.02 / 0.02 \mu \mathrm{g} / \mathrm{L}$ \\
\hline alpha-Chlordane & $0 / 1$ & 0.02 & - & $0.02 / 0.02 \mu \mathrm{g} / \mathrm{L}$ \\
\hline gamma-Chlordane & $0 / 1$ & 0.02 & 一 & $0.02 / 0.02 \mu \mathrm{g} / \mathrm{L}$ \\
\hline$p, p^{\prime}-D D D$ & $0 / 1$ & 0.04 & - & $0.04 / 0.04 \mu \mathrm{g} / \mathrm{L}$ \\
\hline p,p-DDE & $0 / 1$ & 0.04 & - & $0.04 / 0.04 \mu \mathrm{g} / \mathrm{L}$ \\
\hline
\end{tabular}




\begin{tabular}{|c|c|c|c|c|}
\hline Analyte & $\begin{array}{l}\text { Frequency } \\
\text { of Detectiont }\end{array}$ & Mean Result & $\begin{array}{l}\text { Standard } \\
\text { Deviation }\end{array}$ & Minimum/Maximum Results \\
\hline $\begin{array}{l}\text { p,p'-DDT } \\
\text { Dieldrin } \\
\text { Endosulfan sulfate } \\
\text { Endosulfan I } \\
\text { Endosulfan II } \\
\text { Endrin } \\
\text { Endrin aldehyde } \\
\text { Endrin ketone } \\
\text { Heptachlor } \\
\text { Heptachlor epoxide } \\
\text { Lindane } \\
\text { Methoxychlor } \\
\text { Toxaphene }\end{array}$ & $\begin{array}{l}0 / 1 \\
0 / 1 \\
0 / 1 \\
0 / 1 \\
0 / 1 \\
0 / 1 \\
0 / 1 \\
0 / 1 \\
0 / 1 \\
0 / 1 \\
0 / 1 \\
0 / 1 \\
0 / 1\end{array}$ & $\begin{array}{l}0.04 \\
0.04 \\
0.04 \\
0.02 \\
0.04 \\
0.04 \\
0.04 \\
0.04 \\
0.02 \\
0.02 \\
0.02 \\
0.20 \\
1.0\end{array}$ & $\begin{array}{l}- \\
- \\
- \\
- \\
- \\
- \\
- \\
-\end{array}$ & $\begin{array}{l}0.04 / 0.04 \mu \mathrm{g} / \mathrm{L} \\
0.04 / 0.04 \mu \mathrm{g} / \mathrm{L} \\
0.04 / 0.04 \mu \mathrm{g} / \mathrm{L} \\
0.02 / 0.02 \mu \mathrm{g} / \mathrm{L} \\
0.04 / 0.04 \mu \mathrm{g} / \mathrm{L} \\
0.04 / 0.04 \mu \mathrm{g} / \mathrm{L} \\
0.04 / 0.04 \mu \mathrm{g} / \mathrm{L} \\
0.04 / 0.04 \mu \mathrm{g} / \mathrm{L} \\
0.02 / 0.02 \mu \mathrm{g} / \mathrm{L} \\
0.02 / 0.02 \mu \mathrm{g} / \mathrm{L} \\
0.02 / 0.02 \mu \mathrm{g} / \mathrm{L} \\
0.20 / 0.20 \mu \mathrm{g} / \mathrm{L} \\
1.0 / 1.0 \mu \mathrm{g} / \mathrm{L}\end{array}$ \\
\hline $\begin{array}{l}\text { EPA8082 } \\
\text { PCB } 1016 \\
\text { PCB } 1221 \\
\text { PCB } 1232 \\
\text { PCB } 1242 \\
\text { PCB } 1248 \\
\text { PCB } 1254 \\
\text { PCB } 1260\end{array}$ & $\begin{array}{l}0 / 1 \\
0 / 1 \\
0 / 1 \\
0 / 1 \\
0 / 1 \\
0 / 1 \\
0 / 1\end{array}$ & $\begin{array}{l}0.10 \\
0.10 \\
0.10 \\
0.10 \\
0.10 \\
0.10 \\
0.10\end{array}$ & $\begin{array}{l}- \\
- \\
- \\
-\end{array}$ & $\begin{array}{l}0.10 / 0.10 \mu \mathrm{g} / \mathrm{L} \\
0.10 / 0.10 \mu \mathrm{g} / \mathrm{L} \\
0.10 / 0.10 \mu \mathrm{g} / \mathrm{L} \\
0.10 / 0.10 \mu \mathrm{g} / \mathrm{L} \\
0.10 / 0.10 \mu \mathrm{g} / \mathrm{L} \\
0.10 / 0.10 \mu \mathrm{g} / \mathrm{L} \\
0.10 / 0.10 \mu \mathrm{g} / \mathrm{L}\end{array}$ \\
\hline 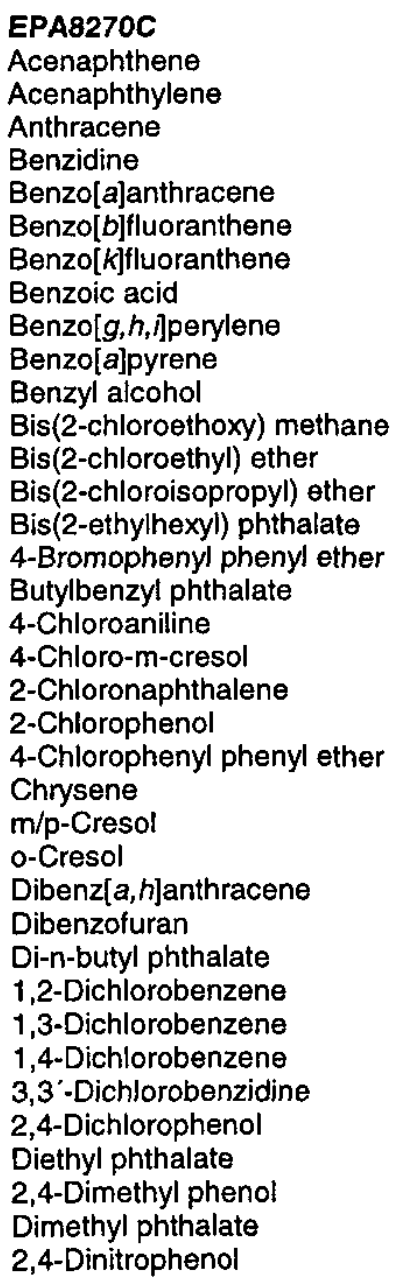 & $\begin{array}{l}0 / 1 \\
0 / 1 \\
0 / 1 \\
0 / 1 \\
0 / 1 \\
0 / 1 \\
0 / 1 \\
0 / 1 \\
0 / 1 \\
0 / 1 \\
0 / 1 \\
0 / 1 \\
0 / 1 \\
0 / 1 \\
8 / 13 \\
0 / 1 \\
0 / 1 \\
0 / 1 \\
0 / 1 \\
0 / 1 \\
0 / 1 \\
0 / 1 \\
0 / 1 \\
0 / 1 \\
0 / 1 \\
0 / 1 \\
0 / 1 \\
0 / 1 \\
0 / 1 \\
0 / 1 \\
0 / 1 \\
0 / 1 \\
0 / 1 \\
0 / 1 \\
0 / 1 \\
0 / 1 \\
0 / 1\end{array}$ & $\begin{array}{l}10.0 \\
10.0 \\
10.0 \\
50.0 \\
10.0 \\
10.0 \\
10.0 \\
20.0 \\
10.0 \\
10.0 \\
10.0 \\
10.0 \\
10.0 \\
10.0 \\
12.6 \\
10.0 \\
10.0 \\
10.0 \\
10.0 \\
10.0 \\
10.0 \\
10.0 \\
10.0 \\
10.0 \\
10.0 \\
10.0 \\
10.0 \\
10.0 \\
10.0 \\
10.0 \\
10.0 \\
20.0 \\
10.0 \\
10.0 \\
10.0 \\
10.0 \\
20.0\end{array}$ & $\begin{array}{l}- \\
- \\
- \\
- \\
- \\
- \\
- \\
- \\
- \\
- \\
5 \\
- \\
- \\
- \\
- \\
- \\
- \\
- \\
- \\
- \\
- \\
- \\
- \\
- \\
- \\
- \\
- \\
- \\
- \\
- \\
- \\
- \\
- \\
- \\
- \\
- \\
- \\
- \\
- \\
- \\
- \\
- \\
- \\
-\end{array}$ & $\begin{array}{l}10.0 / 10.0 \mu \mathrm{g} / \mathrm{L} \\
10.0 / 10.0 \mu \mathrm{g} / \mathrm{L} \\
10.0 / 10.0 \mu \mathrm{g} / \mathrm{L} \\
50.0 / 50.0 \mu \mathrm{g} / \mathrm{L} \\
10.0 / 10.0 \mu \mathrm{g} / \mathrm{L} \\
10.0 / 10.0 \mu \mathrm{g} / \mathrm{L} \\
10.0 / 10.0 \mu \mathrm{g} / \mathrm{L} \\
20.0 / 20.0 \mu \mathrm{g} / \mathrm{L} \\
10.0 / 10.0 \mu \mathrm{g} / \mathrm{L} \\
10.0 / 10.0 \mu \mathrm{g} / \mathrm{L} \\
10.0 / 10.0 \mu \mathrm{g} / \mathrm{L} \\
10.0 / 10.0 \mu \mathrm{g} / \mathrm{L} \\
10.0 / 10.0 \mu \mathrm{g} / \mathrm{L} \\
10.0 / 10.0 \mu \mathrm{g} / \mathrm{L} \\
9.27 / 27.9 \mu \mathrm{L} / \mathrm{L} \\
10.0 / 10.0 \mu \mathrm{g} / \mathrm{L} \\
10.0 / 10.0 \mu \mathrm{g} / \mathrm{L} \\
10.0 / 10.0 \mu \mathrm{g} / \mathrm{L} \\
10.0 / 10.0 \mu \mathrm{g} / \mathrm{L} \\
10.0 / 10.0 \mu \mathrm{L} / \mathrm{L} \\
10.0 / 10.0 \mu \mathrm{g} / \mathrm{L} \\
10.0 / 10.0 \mu \mathrm{L} / \mathrm{L} \\
10.0 / 10.0 \mu \mathrm{g} / \mathrm{L} \\
10.0 / 10.0 \mu \mathrm{L} / \mathrm{L} \\
10.0 / 10.0 \mu \mathrm{g} / \mathrm{L} \\
10.0 / 10.0 \mu \mathrm{g} / \mathrm{L} \\
10.0 / 10.0 \mu \mathrm{g} / \mathrm{L} \\
10.0 / 10.0 \mu \mathrm{g} / \mathrm{L} \\
10.0 / 10.0 \mu \mathrm{g} / \mathrm{L} \\
10.0 / 10.0 \mu \mathrm{g} / \mathrm{L} \\
10.0 / 10.0 \mu \mathrm{g} / \mathrm{L} \\
20.0 / 20.0 \mu \mathrm{g} / \mathrm{L} \\
10.0 / 10.0 \mu \mathrm{g} / \mathrm{L} \\
10.0 / 10.0 \mu \mathrm{g} / \mathrm{L} \\
10.0 / 10.0 \mu \mathrm{g} / \mathrm{L} \\
10.0 / 10.0 \mu \mathrm{g} / \mathrm{L} \\
20.0 / 20.0 \mu \mathrm{g} / \mathrm{L}\end{array}$ \\
\hline
\end{tabular}




\begin{tabular}{|c|c|c|c|c|}
\hline Analyte & $\begin{array}{l}\text { Frequency } \\
\text { of Detectiont }\end{array}$ & Mean Result & $\begin{array}{l}\text { Standard } \\
\text { Deviation }\end{array}$ & Minimum/Maximum Results \\
\hline $\begin{array}{l}\text { 2,4-Dinitrotoluene } \\
\text { 2,6-Dinitrotoluene } \\
\text { Di-n-octyl phthalate } \\
\text { Fluoranthene } \\
\text { Fluorene } \\
\text { Hexachlorobenzene } \\
\text { Hexachlorobutadiene } \\
\text { Hexachlorocyclopentadiene } \\
\text { Hexachloroethane } \\
\text { Indeno[1,2,3-c, d]pyrene } \\
\text { Isophorone } \\
\text { 2-Methyl-4,6-dinitrophenol } \\
\text { 2-Methylnaphthalene } \\
\text { Naphthalene } \\
\text { m-Nitroaniline } \\
\text { o-Nitroaniline } \\
\text { p-Nitroaniline } \\
\text { Nitrobenzene } \\
\text { 2-Nitrophenol } \\
\text { 4-Nitrophenol } \\
\text { N-Nitrosodiphenylamine } \\
\text { N-Nitrosodipropylamine } \\
\text { Pentachlorophenol } \\
\text { Phenanthrene } \\
\text { Phenol } \\
\text { Pyrene } \\
\text { 1,2,4-Trichlorobenzene } \\
\text { 2,4,5-Trichlorophenol } \\
\text { 2,4,6-Trichlorophenol }\end{array}$ & $\begin{array}{l}0 / 1 \\
0 / 1 \\
0 / 1 \\
0 / 1 \\
0 / 1 \\
0 / 1 \\
0 / 1 \\
0 / 1 \\
0 / 1 \\
0 / 1 \\
0 / 1 \\
0 / 1 \\
0 / 1 \\
0 / 1 \\
0 / 1 \\
0 / 1 \\
0 / 1 \\
0 / 1 \\
0 / 1 \\
0 / 1 \\
0 / 1 \\
0 / 1 \\
0 / 1 \\
0 / 1 \\
0 / 1 \\
0 / 1 \\
0 / 1 \\
0 / 1 \\
0 / 1\end{array}$ & $\begin{array}{l}10.0 \\
10.0 \\
10.0 \\
10.0 \\
10.0 \\
10.0 \\
10.0 \\
10.0 \\
10.0 \\
10.0 \\
10.0 \\
10.0 \\
10.0 \\
10.0 \\
10.0 \\
10.0 \\
10.0 \\
10.0 \\
10.0 \\
10.0 \\
10.0 \\
10.0 \\
20.0 \\
10.0 \\
10.0 \\
10.0 \\
10.0 \\
10.0 \\
10.0\end{array}$ & $\begin{array}{l}- \\
- \\
- \\
- \\
- \\
- \\
- \\
- \\
- \\
- \\
- \\
- \\
- \\
- \\
- \\
- \\
-\end{array}$ & $\begin{array}{l}10.0 / 10.0 \mu \mathrm{g} / \mathrm{L} \\
10.0 / 10.0 \mu \mathrm{g} / \mathrm{L} \\
10.0 / 10.0 \mu \mathrm{g} / \mathrm{L} \\
10.0 / 10.0 \mu \mathrm{g} / \mathrm{L} \\
10.0 / 10.0 \mu \mathrm{g} / \mathrm{L} \\
10.0 / 10.0 \mu \mathrm{g} / \mathrm{L} \\
10.0 / 10.0 \mu \mathrm{g} / \mathrm{L} \\
10.0 / 10.0 \mu \mathrm{h} / \mathrm{L} \\
10.0 / 10.0 \mu \mathrm{g} / \mathrm{L} \\
10.0 / 10.0 \mu \mathrm{g} / \mathrm{L} \\
10.0 / 10.0 \mu \mathrm{g} / \mathrm{L} \\
10.0 / 10.0 \mu \mathrm{g} / \mathrm{L} \\
10.0 / 10.0 \mu \mathrm{g} / \mathrm{L} \\
10.0 / 10.0 \mu \mathrm{g} / \mathrm{L} \\
10.0 / 10.0 \mu \mathrm{g} / \mathrm{L} \\
10.0 / 10.0 \mu \mathrm{g} / \mathrm{L} \\
10.0 / 10.0 \mu \mathrm{g} / \mathrm{L} \\
10.0 / 10.0 \mu \mathrm{g} / \mathrm{L} \\
10.0 / 10.0 \mu \mathrm{g} / \mathrm{L} \\
10.0 / 10.0 \mu \mathrm{g} / \mathrm{L} \\
10.0 / 10.0 \mu \mathrm{g} / \mathrm{L} \\
10.0 / 10.0 \mu \mathrm{g} / \mathrm{L} \\
20.0 / 20.0 \mu \mathrm{g} / \mathrm{L} \\
10.0 / 10.0 \mu \mathrm{g} / \mathrm{L} \\
10.0 / 10.0 \mu \mathrm{g} / \mathrm{L} \\
10.0 / 10.0 \mu \mathrm{g} / \mathrm{L} \\
10.0 / 10.0 \mu \mathrm{g} / \mathrm{L} \\
10.0 / 10.0 \mu \mathrm{g} / \mathrm{L} \\
10.0 / 10.0 \mu \mathrm{L} / \mathrm{L}\end{array}$ \\
\hline $\begin{array}{l}\text { EPA9012A } \\
\text { Cyanide }\end{array}$ & $0 / 15$ & 10.0 & 0.0 & 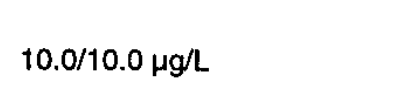 \\
\hline $\begin{array}{l}\text { EPA9020B } \\
\text { Total organic halogens }\end{array}$ & $1 / 1$ & 13.8 & - & $13.8 / 13.8 \mu \mathrm{g} / \mathrm{L}$ \\
\hline $\begin{array}{l}\text { EPA9040B } \\
\mathrm{pH}\end{array}$ & $16 / 16$ & 5.67 & 0.27 & $5.26 / 6.18 \mathrm{pH}$ \\
\hline $\begin{array}{l}\text { EPA9050A } \\
\text { Specific conductance }\end{array}$ & $13 / 13$ & 1.74 & 0.53 & $1.19 / 3.30 \mu \mathrm{S} / \mathrm{cm}$ \\
\hline $\begin{array}{l}\text { EPA9056 } \\
\text { Nitrite as nitrogen }\end{array}$ & $0 / 1$ & 50.0 & 一 & $50.0 / 50.0 \mu \mathrm{g} / \mathrm{L}$ \\
\hline $\begin{array}{l}\text { EPA9066 } \\
\text { Phenols }\end{array}$ & $0 / 7$ & 5.0 & 0.0 & $5.0 / 5.0 \mu \mathrm{g} / \mathrm{L}$ \\
\hline $\begin{array}{l}\text { EPIA-002 } \\
\text { Tritium }\end{array}$ & $0 / 1$ & $-8.29 E-09$ & 一 & $-8.29 \mathrm{E}-09 /-8.29 \mathrm{E}-09 \mu \mathrm{Ci} / \mathrm{mL}$ \\
\hline
\end{tabular}

$t$ Number of times analyte was detected compared to the total number of field blanks for the analyte.

- Standard deviation cannot be determined.

Note: If the analyte was not detected in the field blank(s), detection limit information appears in the Mean Result and Minimum/Maximum Results columns. 
Table 60. Analytes Detected in Field Blanks for WA

\begin{tabular}{|c|c|c|c|c|}
\hline Analyte & $\begin{array}{l}\text { Frequency } \\
\text { of Detectiont }\end{array}$ & Mean Result & $\begin{array}{l}\text { Standard } \\
\text { Deviation }\end{array}$ & Minimum/Maximum Resu/ts \\
\hline $\begin{array}{l}\text { EPA340.2 } \\
\text { Fluoride }\end{array}$ & $0 / 3$ & 16.0 & 2.40 & $14.1 / 18.7 \mu g / L$ \\
\hline $\begin{array}{l}\text { EPA353.2 } \\
\text { Nitrate-nitrite as nitrogen }\end{array}$ & $1 / 2$ & 11.0 & 12.7 & $2.0 / 20.0 \mu \mathrm{g} / \mathrm{L}$ \\
\hline $\begin{array}{l}\text { EPA365.2 } \\
\text { Total phosphates (as P) }\end{array}$ & $1 / 2$ & 38.2 & 40.8 & $9.34 / 67.0 \mu \mathrm{g} / \mathrm{L}$ \\
\hline $\begin{array}{l}\text { EPA6010B } \\
\text { Aluminum } \\
\text { Antimony } \\
\text { Arsenic } \\
\text { Barium } \\
\text { Beryllium } \\
\text { Cadmium } \\
\text { Chromium } \\
\text { Cobalt } \\
\text { Copper } \\
\text { Iron } \\
\text { Lead } \\
\text { Manganese } \\
\text { Nickel } \\
\text { Selenium } \\
\text { Silver } \\
\text { Sodium } \\
\text { Thallium } \\
\text { Vanadium } \\
\text { Zinc }\end{array}$ & $\begin{array}{l}1 / 6 \\
0 / 1 \\
0 / 3 \\
0 / 6 \\
0 / 1 \\
0 / 1 \\
0 / 3 \\
0 / 1 \\
0 / 1 \\
1 / 6 \\
0 / 6 \\
0 / 2 \\
0 / 6 \\
0 / 6 \\
0 / 3 \\
1 / 2 \\
0 / 1 \\
0 / 1 \\
0 / 1\end{array}$ & $\begin{array}{l}109 \\
27.0 \\
40.0 \\
1.21 \\
1.60 \\
4.70 \\
7.0 \\
4.50 \\
15.0 \\
45.4 \\
47.0 \\
7.80 \\
26.0 \\
66.0 \\
5.0 \\
37.7 \\
55.0 \\
6.90 \\
53.0\end{array}$ & $\begin{array}{l}57.7 \\
\overline{0.0} \\
0.72 \\
- \\
\overline{0.0} \\
- \\
\overline{32.1} \\
0.0 \\
0.0 \\
0.0 \\
0.0 \\
0.0 \\
11.6 \\
- \\
- \\
-\end{array}$ & $\begin{array}{l}26.7 / 146 \mu \mathrm{g} / \mathrm{L} \\
27.0 / 27.0 \mu \mathrm{L} / \mathrm{L} \\
40.0 / 40.0 \mu \mathrm{g} / \mathrm{L} \\
0.31 / 1.80 \mu \mathrm{g} / \mathrm{L} \\
1.60 / 1.60 \mu \mathrm{g} / \mathrm{L} \\
4.70 / 4.70 \mu \mathrm{g} / \mathrm{L} \\
7.0 / 7.0 \mu \mathrm{g} / \mathrm{L} \\
4.50 / 4.50 \mu \mathrm{g} / \mathrm{L} \\
15.0 / 15.0 \mu \mathrm{g} / \mathrm{L} \\
8.50 / 74.0 \mu \mathrm{g} / \mathrm{L} \\
47.0 / 47.0 \mu \mathrm{L} / \mathrm{L} \\
7.80 / 7.80 \mu \mathrm{g} / \mathrm{L} \\
26.0 / 26.0 \mu \mathrm{h} / \mathrm{L} \\
66.0 / 66.0 \mu \mathrm{g} / \mathrm{L} \\
5.0 / 5.0 \mu \mathrm{g} / \mathrm{L} \\
29.5 / 45.9 \mu \mathrm{g} / \mathrm{L} \\
55.0 / 55.0 \mu \mathrm{g} / \mathrm{L} \\
6.90 / 6.90 \mu \mathrm{g} / \mathrm{L} \\
53.0 / 53.0 \mu \mathrm{g} / \mathrm{L}\end{array}$ \\
\hline $\begin{array}{l}\text { EPA7470A } \\
\text { Mercury }\end{array}$ & $0 / 3$ & 0.45 & 0.0 & $0.45 / 0.45 \mu \mathrm{g} / \mathrm{L}$ \\
\hline $\begin{array}{l}\text { EPA8081A } \\
\text { beta-Benzene hexachloride } \\
\text { Lindane }\end{array}$ & $\begin{array}{l}0 / 1 \\
0 / 2\end{array}$ & $\begin{array}{l}0.05 \\
0.05\end{array}$ & $\overline{0.0}$ & $\begin{array}{l}0.05 / 0.05 \mu g / L \\
0.05 / 0.05 \mu g / L\end{array}$ \\
\hline $\begin{array}{l}\text { EPAB151A } \\
\text { 2,4-Dichlorophenoxyacetic acid }\end{array}$ & $0 / 2$ & 1.05 & 0.01 & $1.04 / 1.05 \mu \mathrm{g} / \mathrm{L}$ \\
\hline $\begin{array}{l}\text { EPA8270C } \\
\text { Di-n-butyl phthalate } \\
\text { Phenol }\end{array}$ & $\begin{array}{l}0 / 1 \\
0 / 2\end{array}$ & $\begin{array}{l}10.2 \\
10.2\end{array}$ & $\overline{0.0}$ & $\begin{array}{l}10.2 / 10.2 \mu \mathrm{g} / \mathrm{L} \\
10.2 / 10.2 \mu \mathrm{g} / \mathrm{L}\end{array}$ \\
\hline $\begin{array}{l}\text { EPA9014 } \\
\text { Cyanide }\end{array}$ & $1 / 5$ & 13.2 & 4.40 & $5.37 / 15.2 \mu \mathrm{g} / \mathrm{L}$ \\
\hline $\begin{array}{l}\text { EPA9020B } \\
\text { Total organic halogens }\end{array}$ & $0 / 2$ & 120 & 0.0 & $120 / 120 \mu g / L$ \\
\hline $\begin{array}{l}\text { EPA9040B } \\
\mathrm{pH}\end{array}$ & $1 / 1$ & 5.77 & - & $5.77 / 5.77 \mathrm{pH}$ \\
\hline $\begin{array}{l}\text { EPA9050A } \\
\text { Specific conductance }\end{array}$ & $0 / 1$ & 8.90 & - & $8.90 / 8.90 \mu \mathrm{S} / \mathrm{cm}$ \\
\hline
\end{tabular}




\begin{tabular}{|c|c|c|c|c|}
\hline Analyte & $\begin{array}{l}\text { Frequency } \\
\text { of Detectiont }\end{array}$ & Mean Resu/t & $\begin{array}{l}\text { Standard } \\
\text { Deviation }\end{array}$ & Minimum/Maximum Results \\
\hline EPA9056 & & & & \\
\hline $\begin{array}{l}\text { Chloride } \\
\text { Sulfate }\end{array}$ & $\begin{array}{l}1 / 2 \\
0 / 2\end{array}$ & $\begin{array}{l}219 \\
340\end{array}$ & $\begin{array}{l}12.0 \\
0.0\end{array}$ & $\begin{array}{l}210 / 227 \mu g / L \\
340 / 340 \mu g / L\end{array}$ \\
\hline $\begin{array}{l}\text { EPA9060 } \\
\text { Total organic carbon }\end{array}$ & $2 / 2$ & 1,090 & 305 & $878 / 1,310 \mu \mathrm{g} / \mathrm{L}$ \\
\hline
\end{tabular}

$\uparrow$ Number of times analyte was detected compared to the total number of field blanks for the analyte.

- Standard deviation cannot be determined.

Note: If the analyte was not detected in the field blank(s), detection limit information appears in the Mean Result and Minimum/Maximum Results columns.

Table 61. Analytes Detected in Field Blanks for GP

\begin{tabular}{|c|c|c|c|c|}
\hline Analyte & $\begin{array}{l}\text { Frequency } \\
\text { of Detectiont }\end{array}$ & Mean Result & $\begin{array}{l}\text { Standard } \\
\text { Deviation }\end{array}$ & Minimum/Maximum Results \\
\hline \multicolumn{5}{|l|}{ EPIA-001 } \\
\hline Gross alpha & $1 / 21$ & $1.48 \mathrm{E}-10$ & $3.01 E-10$ & $-2.43 \mathrm{E}-10 / 1.22 \mathrm{E}-09 \mu \mathrm{Ci} / \mathrm{mL}$ \\
\hline Nonvolatile beta & $2 / 20$ & $1.13 \mathrm{E}-09$ & $3.06 \mathrm{E}-09$ & $-1.84 \mathrm{E}-10 / 1.39 \mathrm{E}-08 \mu \mathrm{Ci} / \mathrm{mL}$ \\
\hline \multicolumn{5}{|l|}{ EPIA-002 } \\
\hline Tritium & $1 / 20$ & $1.43 \mathrm{E}-06$ & $6.91 \mathrm{E}-06$ & $-3.54 \mathrm{E}-07 / 3.08 \mathrm{E}-05 \mu \mathrm{Ci} / \mathrm{mL}$ \\
\hline \multicolumn{5}{|l|}{ EPIA-003 } \\
\hline Carbon-14 & $1 / 14$ & 3.73E-09 & 7.77E-09 & $-3.56 \mathrm{E}-09 / 2.85 \mathrm{E}-08 \mu \mathrm{Ci} / \mathrm{mL}$ \\
\hline \multicolumn{5}{|l|}{ EPIA-004 } \\
\hline Strontium-90 & $1 / 15$ & $2.78 \mathrm{E}-09$ & $1.08 \mathrm{E}-08$ & $-1.57 \mathrm{E}-09 / 4.17 \mathrm{E}-08 \mu \mathrm{Ci} / \mathrm{mL}$ \\
\hline \multicolumn{5}{|l|}{ EPIA-005 } \\
\hline Technetium-99 & $0 / 16$ & $-1.24 \mathrm{E}-08$ & $1.60 \mathrm{E}-08$ & $-3.71 \mathrm{E}-08 / 6.42 \mathrm{E}-09 \mu \mathrm{Ci} / \mathrm{mL}$ \\
\hline \multicolumn{5}{|l|}{ EPIA-006 } \\
\hline lodine-129 & $1 / 17$ & $3.59 \mathrm{E}-10$ & $3.61 \mathrm{E}-10$ & $-5.02 \mathrm{E}-10 / 1.02 \mathrm{E}-09 \mu \mathrm{Ci} / \mathrm{mL}$ \\
\hline \multicolumn{5}{|l|}{ EPIA-008 } \\
\hline Radium-226 & $3 / 16$ & $4.22 \mathrm{E}-10$ & $2.36 E-10$ & 1.07E-10/9.15E-10 $\mu \mathrm{Ci} / \mathrm{mL}$ \\
\hline \multicolumn{5}{|l|}{ EPIA-009 } \\
\hline Radium-228 & $2 / 17$ & 7.54E-10 & $8.54 E-10$ & $-3.59 \mathrm{E}-12 / 3.80 \mathrm{E}-09 \mu \mathrm{Ci} / \mathrm{mL}$ \\
\hline \multicolumn{5}{|l|}{ EPIA-010 } \\
\hline Radium, total alpha-emitting & $0 / 1$ & $-2.00 E-10$ & - & $-2.00 \mathrm{E}-10 /-2.00 \mathrm{E}-10 \mu \mathrm{Ci} / \mathrm{mL}$ \\
\hline \multicolumn{5}{|l|}{ EPIA-011 } \\
\hline Americium-241 & $0 / 15$ & $3.26 \mathrm{E}-11$ & $5.55 \mathrm{E}-11$ & $-5.93 \mathrm{E}-11 / 1.43 \mathrm{E}-10 \mu \mathrm{Ci} / \mathrm{mL}$ \\
\hline Curium-242 & $0 / 15$ & $2.54 \mathrm{E}-12$ & $1.67 \mathrm{E}-11$ & $-2.79 \mathrm{E}-11 / 4.02 \mathrm{E}-11 \mu \mathrm{Ci} / \mathrm{mL}$ \\
\hline Curium-243/244 & $0 / 15$ & $9.86 \mathrm{E}-12$ & $1.73 E-11$ & $-1.49 \mathrm{E}-11 / 4.17 \mathrm{E}-11 \mu \mathrm{Ci} / \mathrm{mL}$ \\
\hline Curium-245/246 & $1 / 15$ & $7.64 \mathrm{E}-11$ & $1.61 \mathrm{E}-10$ & $0.0 / 6.28 \mathrm{E}-10 \mu \mathrm{Ci} / \mathrm{mL}$ \\
\hline Plutonium-238 & $1 / 15$ & $6.29 \mathrm{E}-11$ & $1.34 \mathrm{E}-10$ & $-9.07 \mathrm{E}-11 / 4.69 \mathrm{E}-10 \mu \mathrm{Ci} / \mathrm{mL}$ \\
\hline
\end{tabular}




\begin{tabular}{|c|c|c|c|c|}
\hline Analyte & $\begin{array}{l}\text { Frequency } \\
\text { of Detectiont }\end{array}$ & Mean Result & $\begin{array}{l}\text { Standard } \\
\text { Deviation }\end{array}$ & Minimum/Maximum Results \\
\hline Plutonium-239/240 & $0 / 15$ & $3.97 E-11$ & $6.08 \mathrm{E}-11$ & $-4.87 \mathrm{E}-11 / 1.97 \mathrm{E}-10 \mu \mathrm{Ci} / \mathrm{mL}$ \\
\hline Uranium-233/234 & $2 / 15$ & $1.05 E-10$ & $9.05 E-11$ & $-8.16 \mathrm{E}-12 / 2.66 \mathrm{E}-10 \mu \mathrm{Ci} / \mathrm{mL}$ \\
\hline Uranium-235 & $1 / 15$ & $2.64 \mathrm{E}-11$ & $4.57 E-11$ & $-4.27 \mathrm{E}-11 / 1.49 \mathrm{E}-10 \mu \mathrm{Ci} / \mathrm{mL}$ \\
\hline Uranium-238 & $0 / 15$ & 2. $16 \mathrm{E}-11$ & $3.29 E-11$ & $-1.44 \mathrm{E}-11 / 1.10 \mathrm{E}-10 \mu \mathrm{Ci} / \mathrm{mL}$ \\
\hline \multicolumn{5}{|l|}{ EPIA-012 } \\
\hline Thorium-228 & $0 / 15$ & $2.59 E-11$ & $6.37 \mathrm{E}-11$ & $-8.84 \mathrm{E}-11 / 1.28 \mathrm{E}-10 \mu \mathrm{Ci} / \mathrm{mL}$ \\
\hline Thorium-230 & $1 / 15$ & $7.33 E-11$ & $7.95 E-11$ & 1.19E-11/3.14E-10 $\mu \mathrm{Ci} / \mathrm{mL}$ \\
\hline Thorium-232 & $0 / 15$ & $2.03 \mathrm{E}-12$ & 2.81E-11 & $-3.58 \mathrm{E}-11 / 8.87 \mathrm{E}-11 \mu \mathrm{Ci} / \mathrm{mL}$ \\
\hline \multicolumn{5}{|l|}{ EPIA-013 } \\
\hline Actinium-228 & $0 / 19$ & 5.32E-09 & 3.13E-09 & 1.17E-09/1.24E-08 $\mu \mathrm{Ci} / \mathrm{mL}$ \\
\hline Antimony-125 & $0 / 19$ & $9.55 \mathrm{E}-10$ & $2.63 \mathrm{E}-09$ & $-2.65 \mathrm{E}-09 / 5.39 \mathrm{E}-09 \mu \mathrm{Ci} / \mathrm{mL}$ \\
\hline Cerium-144 & $0 / 19$ & $-6.30 E-10$ & $5.76 \mathrm{E}-09$ & $-9.15 \mathrm{E}-09 / 1.25 \mathrm{E}-08 \mu \mathrm{Ci} / \mathrm{mL}$ \\
\hline Cesium-134 & $0 / 19$ & $3.39 E-11$ & $1.02 E-09$ & $-1.80 E-09 / 2.02 E-09 \mu \mathrm{Ci} / \mathrm{mL}$ \\
\hline Cesium-137 & $2 / 19$ & $1.02 E-09$ & 2.43E-09 & $-1.26 \mathrm{E}-09 / 8.91 \mathrm{E}-09 \mu \mathrm{Ci} / \mathrm{mL}$ \\
\hline Cobalt-57 & $1 / 19$ & $-2.93 E-12$ & $9.28 \mathrm{E}-10$ & $-1.43 \mathrm{E}-09 / 2.12 \mathrm{E}-09 \mu \mathrm{Ci} / \mathrm{mL}$ \\
\hline Cobalt-60 & $0 / 19$ & 4.15E-10 & $1.14 \mathrm{E}-09$ & -1.93E-09/2.47E-09 $\mu \mathrm{Ci} / \mathrm{mL}$ \\
\hline Europium-152 & $0 / 19$ & $-3.72 E-10$ & 2.16E-09 & $-4.33 \mathrm{E}-09 / 4.68 \mathrm{E}-09 \mu \mathrm{Ci} / \mathrm{mL}$ \\
\hline Europium-154 & $0 / 19$ & $6.15 E-10$ & $2.96 \mathrm{E}-09$ & $-6.14 \mathrm{E}-09 / 4.97 \mathrm{E}-09 \mu \mathrm{Ci} / \mathrm{mL}$ \\
\hline Europium-155 & $0 / 19$ & $1.37 \mathrm{E}-10$ & $3.82 \mathrm{E}-09$ & $-7.15 \mathrm{E}-09 / 7.62 \mathrm{E}-09 \mu \mathrm{Ci} / \mathrm{mL}$ \\
\hline Lead-212 & $1 / 19$ & $3.50 E-09$ & $3.00 E-09$ & 3.74E-10/1.27E-08 $\mu \mathrm{Ci} / \mathrm{mL}$ \\
\hline Manganese-54 & $0 / 19$ & $-1.44 \mathrm{E}-10$ & $7.38 \mathrm{E}-10$ & $-1.68 \mathrm{E}-09 / 8.22 \mathrm{E}-10 \mu \mathrm{Ci} / \mathrm{mL}$ \\
\hline Potassium-40 & $2 / 19$ & $1.94 \mathrm{E}-08$ & $1.63 E-08$ & $-8.71 \mathrm{E}-10 / 5.37 \mathrm{E}-08 \mu \mathrm{Ci} / \mathrm{mL}$ \\
\hline Promethium-144 & $0 / 19$ & $2.08 \mathrm{E}-10$ & $8.20 E-10$ & $-1.14 \mathrm{E}-09 / 1.62 \mathrm{E}-09 \mu \mathrm{Ci} / \mathrm{mL}$ \\
\hline Promethium-146 & $0 / 19$ & $4.26 E-11$ & $1.36 \mathrm{E}-09$ & $-2.88 \mathrm{E}-09 / 2.22 \mathrm{E}-09 \mu \mathrm{Ci} / \mathrm{mL}$ \\
\hline Ruthenium-106 & $0 / 19$ & $1.27 \mathrm{E}-10$ & $7.54 E-09$ & $-1.15 \mathrm{E}-08 / 1.28 \mathrm{E}-08 \mu \mathrm{Ci} / \mathrm{mL}$ \\
\hline Sodium-22 & $0 / 19$ & $2.34 \mathrm{E}-10$ & $1.07 E-09$ & $-2.21 \mathrm{E}-09 / 1.78 \mathrm{E}-09 \mu \mathrm{Ci} / \mathrm{mL}$ \\
\hline Yttrium-88 & $0 / 19$ & $1.09 E-11$ & $1.51 \mathrm{E}-09$ & $-2.17 \mathrm{E}-09 / 3.73 \mathrm{E}-09 \mu \mathrm{Ci} / \mathrm{mL}$ \\
\hline Zinc-65 & $0 / 19$ & $-1.10 \mathrm{E}-09$ & $2.53 \mathrm{E}-09$ & $-4.98 \mathrm{E}-09 / 2.55 \mathrm{E}-09 \mu \mathrm{Ci} / \mathrm{mL}$ \\
\hline \multicolumn{5}{|l|}{ EPIA-022 } \\
\hline Nickel-63 & $0 / 7$ & $-4.57 E-09$ & $2.10 \mathrm{E}-08$ & $-2.63 \mathrm{E}-08 / 2.52 \mathrm{E}-08 \mu \mathrm{Ci} / \mathrm{mL}$ \\
\hline
\end{tabular}

$\dagger$ Number of times analyte was detected compared to the total number of field blanks for the analyte.

- Standard deviation cannot be determined.

Note: If the analyte was not detected in the field blank(s), detection limit information appears in the Mean Result and Minimum/Maximum Results columns. 
Table 62. Analytes Detected in Field Blanks for TM

\begin{tabular}{lllll}
\hline Analyte & $\begin{array}{l}\text { Frequency } \\
\text { of Detectiont }\end{array}$ & Mean Result & $\begin{array}{l}\text { Standard } \\
\text { Deviation }\end{array}$ & Minimum/Maximum Results \\
\hline $\begin{array}{l}\text { EPA900.0MOD } \\
\text { Gross alpha }\end{array}$ & $2 / 12$ & $1.02 \mathrm{E}-10$ & $3.45 \mathrm{E}-10$ & $-4.90 \mathrm{E}-10 / 6.70 \mathrm{E}-10 \mu \mathrm{Ci} / \mathrm{mL}$ \\
Nonvolatile beta & $1 / 9$ & $-1.31 \mathrm{E}-10$ & $1.05 \mathrm{E}-09$ & $-1.47 \mathrm{E}-09 / 2.15 \mathrm{E}-09 \mu \mathrm{Ci} / \mathrm{mL}$ \\
$\begin{array}{l}\text { EPA903.0MOD } \\
\text { Radium, total alpha-emitting }\end{array}$ & $0 / 2$ & $-3.00 \mathrm{E}-11$ & $1.84 \mathrm{E}-10$ & $-1.60 \mathrm{E}-10 / 1.00 \mathrm{E}-10 \mu \mathrm{Ci} / \mathrm{mL}$ \\
$\begin{array}{l}\text { EPA906.0MOD } \\
\text { Tritium }\end{array}$ & $1 / 7$ & & \\
\hline
\end{tabular}

$\uparrow$ Number of times analyte was detected compared to the total number of field blanks for the analyte.

Note: If the analyte was not detected in the field blank(s), detection limit information appears in the Mean Result and Minimum/Maximum Results columns.

Table 63. Analytes Detected in Trip Blanks for EX

\begin{tabular}{|c|c|c|c|c|}
\hline Analyte & $\begin{array}{l}\text { Frequency } \\
\text { of Detectiont }\end{array}$ & Mean Result & $\begin{array}{l}\text { Standard } \\
\text { Deviation }\end{array}$ & Minimum/Maximum Results \\
\hline \multicolumn{5}{|l|}{ EPA8021B } \\
\hline Carbon tetrachloride & $0 / 7$ & 1.0 & 0.0 & $1.0 / 1.0 \mu \mathrm{g} / \mathrm{L}$ \\
\hline Chloroform & $0 / 7$ & 1.0 & 0.0 & $1.0 / 1.0 \mu \mathrm{g} / \mathrm{L}$ \\
\hline cis-1,2-Dichloroethylene & $0 / 7$ & 1.0 & 0.0 & $1.0 / 1.0 \mu \mathrm{g} / \mathrm{L}$ \\
\hline Tetrachioroethylene & $0 / 7$ & 1.0 & 0.0 & $1.0 / 1.0 \mu \mathrm{g} / \mathrm{L}$ \\
\hline $1,1,1$-Trichloroethane & $0 / 7$ & 1.0 & 0.0 & $1.0 / 1.0 \mu \mathrm{g} / \mathrm{L}$ \\
\hline Trichloroethylene & $0 / 7$ & 1.0 & 0.0 & $1.0 / 1.0 \mu g / L$ \\
\hline \multicolumn{5}{|l|}{ EPA8260B } \\
\hline Acetone & $0 / 7$ & 10.0 & 0.0 & $10.0 / 10.0 \mu g / L$ \\
\hline Acetonitrile & $0 / 7$ & 500 & 0.0 & $500 / 500 \mu g / L$ \\
\hline Acrolein & $0 / 7$ & 50.0 & 0.0 & $50.0 / 50.0 \mu \mathrm{g} / \mathrm{L}$ \\
\hline Acrylonitrile & $0 / 7$ & 50.0 & 0.0 & $50.0 / 50.0 \mu \mathrm{g} / \mathrm{L}$ \\
\hline Allyl chloride & $0 / 7$ & 10.0 & 0.0 & $10.0 / 10.0 \mu \mathrm{g} / \mathrm{L}$ \\
\hline Benzene & $0 / 29$ & 5.0 & 0.0 & $5.0 / 5.0 \mu \mathrm{g} / \mathrm{L}$ \\
\hline Bromodichloromethane & $0 / 29$ & 5.0 & 0.0 & $5.0 / 5.0 \mu \mathrm{g} / \mathrm{L}$ \\
\hline Bromoform & $0 / 29$ & 5.0 & 0.0 & $5.0 / 5.0 \mu \mathrm{g} / \mathrm{L}$ \\
\hline Bromomethane & $0 / 29$ & 5.0 & 0.0 & $5.0 / 5.0 \mu \mathrm{g} / \mathrm{L}$ \\
\hline Carbon disulfide & $0 / 7$ & 5.0 & 0.0 & $5.0 / 5.0 \mu \mathrm{g} / \mathrm{L}$ \\
\hline Carbon tetrachloride & $0 / 29$ & 5.0 & 0.0 & $5.0 / 5.0 \mu \mathrm{g} / \mathrm{L}$ \\
\hline Chlorobenzene & $0 / 29$ & 5.0 & 0.0 & $5.0 / 5.0 \mu g / \mathrm{L}$ \\
\hline Chloroethane & $0 / 29$ & 10.0 & 0.0 & $10.0 / 10.0 \mu g / L$ \\
\hline Chloroethene & $0 / 29$ & 5.0 & 0.0 & $5.0 / 5.0 \mu \mathrm{g} / \mathrm{h}$ \\
\hline 2-Chloroethyl vinyl ether & $0 / 22$ & 5.0 & 0.0 & $5.0 / 5.0 \mu \mathrm{g} / \mathrm{L}$ \\
\hline Chloroform & $0 / 29$ & 5.0 & 0.0 & $5.0 / 5.0 \mu \mathrm{g} / \mathrm{L}$ \\
\hline Chloromethane & $0 / 29$ & 5.0 & 0.0 & $5.0 / 5.0 \mu \mathrm{g} / \mathrm{L}$ \\
\hline Chloroprene & $0 / 7$ & 50.0 & 0.0 & $50.0 / 50.0 \mu \mathrm{g} / \mathrm{L}$ \\
\hline Dibromochloromethane & $0 / 29$ & 5.0 & 0.0 & $5.0 / 5.0 \mu \mathrm{g} / \mathrm{L}$ \\
\hline 1,2-Dibromo-3-chloropropane & $0 / 7$ & 5.0 & 0.0 & $5.0 / 5.0 \mu \mathrm{g} / \mathrm{L}$ \\
\hline 1,2-Dibromoethane & $0 / 7$ & 5.0 & 0.0 & $5.0 / 5.0 \mu \mathrm{g} / \mathrm{L}$ \\
\hline Dibromomethane & $0 / 7$ & 5.0 & 0.0 & $5.0 / 5.0 \mu \mathrm{g} / \mathrm{L}$ \\
\hline 1,2-Dichlorobenzene & $0 / 7$ & 5.0 & 0.0 & $5.0 / 5.0 \mu \mathrm{g} / \mathrm{L}$ \\
\hline 1,3-Dichlorobenzene & $0 / 7$ & 5.0 & 0.0 & $5.0 / 5.0 \mu \mathrm{g} / \mathrm{L}$ \\
\hline 1,4-Dichlorobenzene & $0 / 7$ & 5.0 & 0.0 & $5.0 / 5.0 \mu \mathrm{g} / \mathrm{L}$ \\
\hline trans-1,4-Dichloro-2-butene & $0 / 7$ & 20.0 & 0.0 & $20.0 / 20.0 \mu \mathrm{g} / \mathrm{L}$ \\
\hline
\end{tabular}




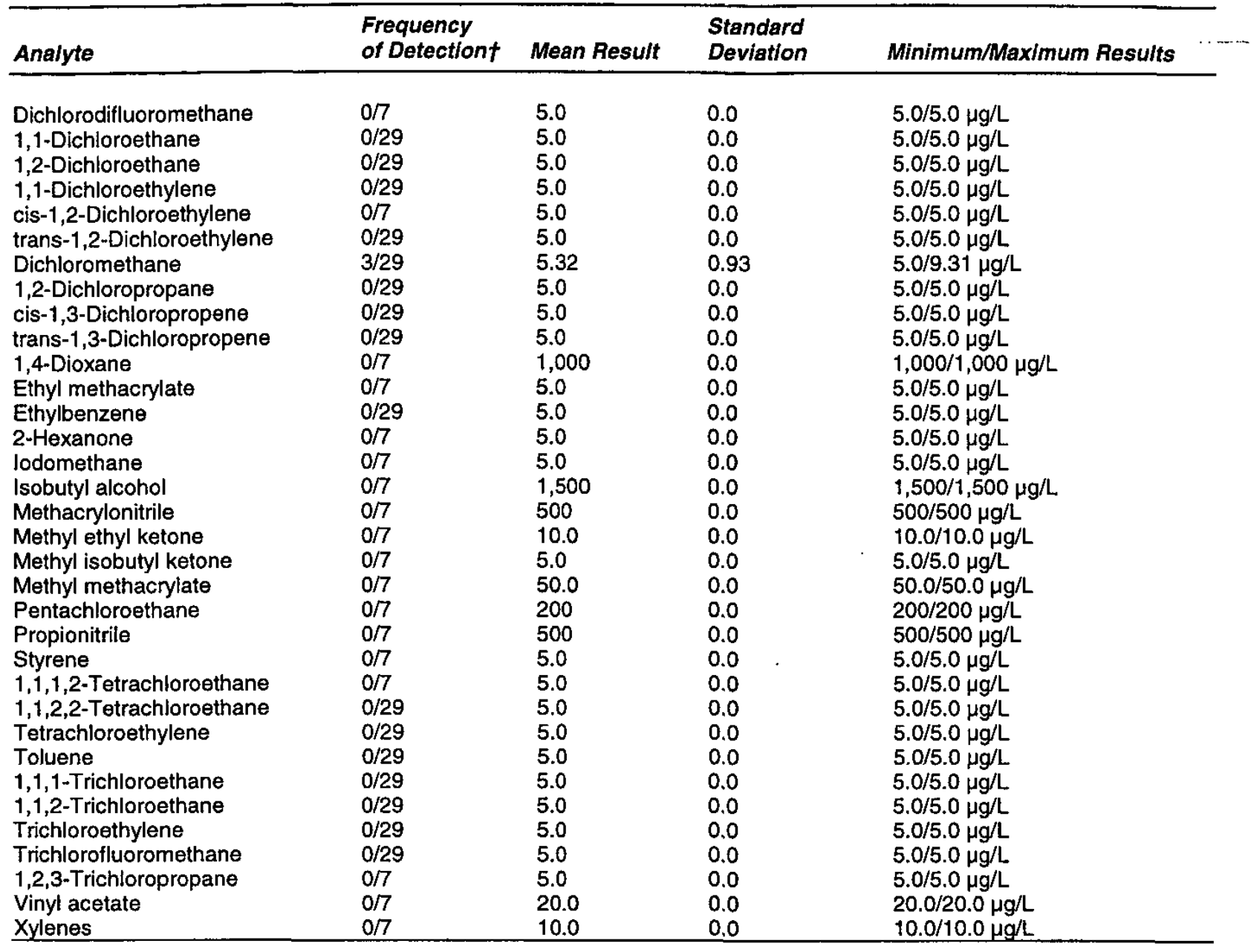

† Number of times analyte was detected compared to the total number of field blanks for the analyte.

Note: If the analyte was not detected in the trip blank(s), detection limit information appears in the Mean Result and Minimum/Maximum Results columns.

Table 64. Analytes Detected in Trip Blanks for GE

\begin{tabular}{lllll}
\hline Analyte & $\begin{array}{l}\text { Frequency } \\
\text { of Detectiont }\end{array}$ & Mean Result & $\begin{array}{l}\text { Standard } \\
\text { Deviation }\end{array}$ & Minimum/Maximum Results \\
\hline & & & & \\
EPA8260B & $0 / 2$ & 5.0 & 0.0 & $5.0 / 5.0 \mu \mathrm{g} / \mathrm{L}$ \\
Acetone & $0 / 1$ & 25.0 & - & $25.0 / 25.0 \mu \mathrm{g} / \mathrm{L}$ \\
Acetonitrile & $0 / 1$ & 10.0 & - & $10.0 / 10.0 \mu \mathrm{g} / \mathrm{L}$ \\
Acrolein & $0 / 1$ & 10.0 & - & $10.0 / 10.0 \mu \mathrm{g} / \mathrm{L}$ \\
Acrylonitrile & $0 / 1$ & 5.0 & 0.0 & $5.0 / 5.0 \mu \mathrm{g} / \mathrm{L}$ \\
Allyl chloride & $1 / 46$ & 1.0 & $-0 / 1.0 \mu \mathrm{g} / \mathrm{L}$ \\
Benzene & $0 / 1$ & 1.0 & 0.0 & $1.0 / 1.0 \mu \mathrm{g} / \mathrm{L}$ \\
Bromochloromethane & $0 / 33$ & 1.0 & 0.0 & $1.0 / 1.0 \mu \mathrm{g} / \mathrm{L}$ \\
Bromodichloromethane & $0 / 33$ & 1.0 & 0.0 & $1.0 / 1.0 \mu \mathrm{g} / \mathrm{L}$ \\
Bromoform & $0 / 33$ & 1.0 & 0.0 & $1.0 / 1.0 \mu \mathrm{g} / \mathrm{L}$ \\
Bromomethane & $0 / 2$ & 5.0 & 0.0 & $1.0 / 1.0 \mu \mathrm{gg} / \mathrm{L}$ \\
Carbon disulfide & $1 / 46$ & 1.0 & & \\
Carbon tetrachloride & & & & \\
& & & &
\end{tabular}




\begin{tabular}{|c|c|c|c|c|}
\hline Analyte & $\begin{array}{l}\text { Frequency } \\
\text { of Detectiont }\end{array}$ & Mean Result & $\begin{array}{l}\text { Standard } \\
\text { Deviation }\end{array}$ & Minimum/Maximum Results \\
\hline Chlorobenzene & $1 / 46$ & 1.0 & 0.0 & $1.0 / 1.0 \mu \mathrm{g} / \mathrm{L}$ \\
\hline Chloroethane & $0 / 33$ & 1.0 & 0.0 & $1.0 / 1.0 \mu \mathrm{g} / \mathrm{L}$ \\
\hline Chloroethene & $0 / 33$ & 1.0 & 0.0 & $1.0 / 1.0 \mu \mathrm{g} / \mathrm{L}$ \\
\hline 2-Chloroethyl vinyl ether & $0 / 31$ & 5.0 & 0.0 & $5.0 / 5.0 \mu \mathrm{g} / \mathrm{L}$ \\
\hline Chloroform & $1 / 46$ & 1.01 & 0.11 & $0.93 / 1.76 \mu \mathrm{g} / \mathrm{L}$ \\
\hline Chloromethane & $0 / 33$ & 1.0 & 0.0 & $1.0 / 1.0 \mu \mathrm{g} / \mathrm{L}$ \\
\hline Chloroprene & $0 / 1$ & 2.0 & - & $2.0 / 2.0 \mu \mathrm{g} / \mathrm{L}$ \\
\hline Dibromochloromethane & $0 / 33$ & 1.0 & 0.0 & $1.0 / 1.0 \mu \mathrm{g} / \mathrm{L}$ \\
\hline 1,2-Dibromo-3-chloropropane & $0 / 1$ & 1.0 & - & $1.0 / 1.0 \mu \mathrm{g} / \mathrm{L}$ \\
\hline 1,2-Dibromoethane & $0 / 1$ & 1.0 & - & $1.0 / 1.0 \mu \mathrm{g} / \mathrm{L}$ \\
\hline Dibromomethane & $0 / 1$ & 1.0 & - & $1.0 / 1.0 \mu \mathrm{g} / \mathrm{L}$ \\
\hline 1,2-Dichlorobenzene & $0 / 1$ & 1.0 & - & $1.0 / 1.0 \mu \mathrm{g} / \mathrm{L}$ \\
\hline 1,4-Dichlorobenzene & $0 / 1$ & 1.0 & - & $1.0 / 1.0 \mu g / L$ \\
\hline trans-1,4-Dichloro-2-butene & $0 / 1$ & 5.0 & - & $5.0 / 5.0 \mu \mathrm{g} / \mathrm{L}$ \\
\hline Dichlorodifluoromethane & $0 / 1$ & 5.0 & - & $5.0 / 5.0 \mu g / L$ \\
\hline 1,1-Dichloroethane & $0 / 33$ & 1.0 & 0.0 & $1.0 / 1.0 \mu \mathrm{g} / \mathrm{L}$ \\
\hline 1,2-Dichloroethane & $0 / 33$ & 1.0 & 0.0 & $1.0 / 1.0 \mu \mathrm{g} / \mathrm{L}$ \\
\hline 1,1-Dichloroethylene & $1 / 46$ & 1.0 & 0.0 & $1.0 / 1.0 \mu \mathrm{g} / \mathrm{L}$ \\
\hline 1,2-Dichloroethylene & $0 / 1$ & 1.0 & - & $1.0 / 1.0 \mu \mathrm{g} / \mathrm{L}$ \\
\hline cis-1,2-Dichloroethylene & $0 / 1$ & 1.17 & - & $1.17 / 1.17 \mu \mathrm{g} / \mathrm{L}$ \\
\hline trans-1,2-Dichloroethylene & $0 / 32$ & 1.0 & 0.0 & $1.0 / 1.0 \mu g / L$ \\
\hline Dichloromethane & $3 / 33$ & 4.28 & 1.22 & $1.56 / 6.61 \mu \mathrm{g} / \mathrm{L}$ \\
\hline 1,2-Dichloropropane & $0 / 33$ & 1.0 & 0.0 & $1.0 / 1.0 \mu \mathrm{g} / \mathrm{L}$ \\
\hline cis-1,3-Dichloropropene & $0 / 33$ & 1.0 & 0.0 & $1.0 / 1.0 \mu \mathrm{g} / \mathrm{L}$ \\
\hline trans-1,3-Dichloropropene & $0 / 33$ & 1.0 & 0.0 & $1.0 / 1.0 \mu \mathrm{g} / \mathrm{L}$ \\
\hline Ethyl methacrylate & $0 / 1$ & 5.0 & - & $5.0 / 5.0 \mu \mathrm{g} / \mathrm{L}$ \\
\hline Ethylbenzene & $0 / 33$ & 1.0 & 0.0 & $1.0 / 1.0 \mu \mathrm{g} / \mathrm{L}$ \\
\hline 2-Hexanone & $0 / 2$ & 5.0 & 0.0 & $5.0 / 5.0 \mu \mathrm{g} / \mathrm{L}$ \\
\hline lodomethane & $0 / 1$ & 10.0 & 一 & $10.0 / 10.0 \mu \mathrm{g} / \mathrm{L}$ \\
\hline Isobutyl alcohol & $0 / 1$ & 50.0 & 一 & $50.0 / 50.0 \mu \mathrm{g} / \mathrm{L}$ \\
\hline Methacrylonitrile & $0 / 1$ & 5.0 & - & $5.0 / 5.0 \mu g / L$ \\
\hline Methyl ethyl ketone & $0 / 2$ & 10.0 & 0.0 & $10.0 / 10.0 \mu \mathrm{g} / \mathrm{L}$ \\
\hline Methyl isobutyl ketone & $0 / 2$ & 5.0 & 0.0 & $5.0 / 5.0 \mu \mathrm{g} / \mathrm{L}$ \\
\hline Methyl methacrylate & $0 / 1$ & 5.0 & - & $5.0 / 5.0 \mu \mathrm{g} / \mathrm{L}$ \\
\hline Pentachloroethane & $0 / 1$ & 5.0 & - & $5.0 / 5.0 \mu \mathrm{g} / \mathrm{L}$ \\
\hline Propionitrile & $0 / 1$ & 10.0 & - & $10.0 / 10.0 \mu \mathrm{g} / \mathrm{L}$ \\
\hline Styrene & $0 / 2$ & 1.0 & 0.0 & $1.0 / 1.0 \mu \mathrm{g} / \mathrm{L}$ \\
\hline $1,1,1,2-$ Tetrachloroethane & $0 / 1$ & 1.0 & - & $1.0 / 1.0 \mu \mathrm{g} / \mathrm{L}$ \\
\hline 1,1,2,2-Tetrachloroethane & $0 / 33$ & 1.0 & 0.0 & $1.0 / 1.0 \mu \mathrm{g} / \mathrm{L}$ \\
\hline Tetrachloroethylene & $1 / 46$ & 1.0 & 0.0 & $1.0 / 1.0 \mu \mathrm{g} / \mathrm{L}$ \\
\hline Toluene & $3 / 46$ & 0.98 & 0.11 & $0.53 / 1.24 \mu \mathrm{g} / \mathrm{L}$ \\
\hline $1,1,1$-Trichloroethane & $1 / 46$ & 1.0 & 0.0 & $1.0 / 1.0 \mu \mathrm{g} / \mathrm{L}$ \\
\hline 1,1,2-Trichloroethane & $0 / 33$ & 1.0 & 0.0 & $1.0 / 1.0 \mu g / L$ \\
\hline Trichloroethylene & $1 / 46$ & 1.0 & 0.0 & $1.0 / 1.0 \mu \mathrm{g} / \mathrm{L}$ \\
\hline Trichlorofluoromethane & $0 / 32$ & 5.0 & 0.0 & $5.0 / 5.0 \mu \mathrm{g} / \mathrm{L}$ \\
\hline 1,2,3-Trichloropropane & $0 / 1$ & 1.0 & 一 & $1.0 / 1.0 \mu \mathrm{g} / \mathrm{L}$ \\
\hline Vinyl acetate & $0 / 2$ & 5.0 & 0.0 & $5.0 / 5.0 \mu \mathrm{g} / \mathrm{L}$ \\
\hline Xylenes & $0 / 2$ & 2.0 & 0.0 & $2.0 / 2.0 \mu \mathrm{g} / \mathrm{L}$ \\
\hline
\end{tabular}

$\dagger$ Number of times analyte was detected compared to the total number of trip blanks for the analyte.

- Standard deviation cannot be determined.

Note: If the analyte was not detected in the trip blank(s), detection limit information appears in the Mean Result and Minimum/Maximum Results columns. 
Table 65. Analytes Detected in Trip Blanks for WA

\begin{tabular}{|c|c|c|c|c|}
\hline Analyte & $\begin{array}{l}\text { Frequency } \\
\text { of Detectiont }\end{array}$ & Mean Result & $\begin{array}{l}\text { Standard } \\
\text { Deviation }\end{array}$ & Minimum/Maximum Resu/ts \\
\hline \multicolumn{5}{|l|}{ EPA8010 } \\
\hline Carbon tetrachloride & $0 / 11$ & 1.0 & 0.0 & $1.0 / 1.0 \mu \mathrm{g} / \mathrm{L}$ \\
\hline Chloroform & $0 / 11$ & 1.0 & 0.0 & $1.0 / 1.0 \mu \mathrm{g} / \mathrm{L}$ \\
\hline cis-1,2-Dichloroethylene & $0 / 11$ & 5.0 & 0.0 & $5.0 / 5.0 \mu \mathrm{g} / \mathrm{L}$ \\
\hline 1,1,1-Trichloroethane & $0 / 11$ & 1.0 & 0.0 & $1.0 / 1.0 \mu \mathrm{g} / \mathrm{L}$ \\
\hline Trichloroethylene & $0 / 11$ & 1.0 & 0.0 & $1.0 / 1.0 \mu \mathrm{g} / \mathrm{L}$ \\
\hline \multicolumn{5}{|l|}{ EPA8021B } \\
\hline Carbon tetrachloride & $0 / 8$ & 1.0 & 0.0 & $1.0 / 1.0 \mu \mathrm{g} / \mathrm{L}$ \\
\hline Chloroform & $0 / 8$ & 1.0 & 0.0 & $1.0 / 1.0 \mu \mathrm{g} / \mathrm{L}$ \\
\hline $1,1,1$-Trichloroethane & $0 / 8$ & 1.0 & 0.0 & $1.0 / 1.0 \mu \mathrm{g} / \mathrm{L}$ \\
\hline Trichioroethylene & $1 / 8$ & 1.01 & 0.03 & $1.0 / 1.09 \mu \mathrm{g} / \mathrm{L}$ \\
\hline \multicolumn{5}{|l|}{ EPA8260B } \\
\hline Acetone & $0 / 12$ & 9.34 & 2.27 & $2.12 / 10.0 \mu \mathrm{g} / \mathrm{L}$ \\
\hline Acetonitrile & $0 / 11$ & 20.0 & 0.0 & $20.0 / 20.0 \mu \mathrm{g} / \mathrm{L}$ \\
\hline Acrolein & $0 / 11$ & 20.0 & 0.0 & $20.0 / 20.0 \mu \mathrm{g} / \mathrm{L}$ \\
\hline Acrylonitrile & $0 / 11$ & 5.0 & 0.0 & $5.0 / 5.0 \mu \mathrm{g} / \mathrm{L}$ \\
\hline Allyl chloride & $0 / 11$ & 10.0 & 0.0 & $10.0 / 10.0 \mu \mathrm{g} / \mathrm{L}$ \\
\hline Benzene & $0 / 36$ & 5.0 & 0.0 & $5.0 / 5.0 \mu \mathrm{g} / \mathrm{L}$ \\
\hline Bromochloromethane & $0 / 3$ & 5.0 & 0.0 & $5.0 / 5.0 \mu \mathrm{g} / \mathrm{L}$ \\
\hline Bromodichloromethane & $0 / 36$ & 5.0 & 0.0 & $5.0 / 5.0 \mu \mathrm{g} / \mathrm{L}$ \\
\hline Bromoform & $0 / 36$ & 5.0 & 0.0 & $5.0 / 5.0 \mu \mathrm{g} / \mathrm{L}$ \\
\hline Bromomethane & $0 / 36$ & 10.0 & 0.0 & $10.0 / 10.0 \mu \mathrm{g} / \mathrm{L}$ \\
\hline Carbon disulfide & $0 / 12$ & 5.0 & 0.0 & $5.0 / 5.0 \mu \mathrm{g} / \mathrm{L}$ \\
\hline Carbon tetrachloride & $0 / 36$ & 5.0 & 0.0 & $5.0 / 5.0 \mu \mathrm{g} / \mathrm{L}$ \\
\hline Chlorobenzene & $0 / 36$ & 5.0 & 0.0 & $5.0 / 5.0 \mu \mathrm{g} / \mathrm{L}$ \\
\hline Chloroethane & $0 / 36$ & 10.0 & 0.0 & $10.0 / 10.0 \mu g / L$ \\
\hline Chloroethene & $0 / 36$ & 10.0 & 0.0 & $10.0 / 10.0 \mu g / L$ \\
\hline 2-Chloroethyl vinyl ether & $0 / 24$ & 10.0 & 0.0 & $10.0 / 10.0 \mu \mathrm{g} / \mathrm{L}$ \\
\hline Chloroform & $0 / 36$ & 5.0 & 0.0 & $5.0 / 5.0 \mu \mathrm{g} / \mathrm{L}$ \\
\hline Chloromethane & $0 / 36$ & 10.0 & 0.0 & $10.0 / 10.0 \mu \mathrm{g} / \mathrm{L}$ \\
\hline Chloroprene & $0 / 11$ & 5.0 & 0.0 & $5.0 / 5.0 \mu \mathrm{g} / \mathrm{L}$ \\
\hline Dibromochloromethane & $0 / 36$ & 5.0 & 0.0 & $5.0 / 5.0 \mu \mathrm{g} / \mathrm{L}$ \\
\hline 1,2-Dibromo-3-chloropropane & $0 / 11$ & 5.0 & 0.0 & $5.0 / 5.0 \mu \mathrm{g} / \mathrm{L}$ \\
\hline 1,2-Dibromoethane & $0 / 11$ & 5.0 & 0.0 & $5.0 / 5.0 \mu \mathrm{g} / \mathrm{L}$ \\
\hline Dibromomethane & $0 / 11$ & 5.0 & 0.0 & $5.0 / 5.0 \mu \mathrm{g} / \mathrm{L}$ \\
\hline 1,2-Dichlorobenzene & $0 / 3$ & 5.0 & 0.0 & $5.0 / 5.0 \mu \mathrm{g} / \mathrm{L}$ \\
\hline 1,4-Dichlorobenzene & $1 / 5$ & 4.22 & 1.75 & $1.08 / 5.0 \mu \mathrm{g} / \mathrm{L}$ \\
\hline trans-1,4-Dichloro-2-butene & $0 / 10$ & 20.0 & 0.0 & $20.0 / 20.0 \mu \mathrm{g} / \mathrm{L}$ \\
\hline Dichlorodifluoromethane & $0 / 11$ & 10.0 & 0.0 & $10.0 / 10.0 \mu \mathrm{g} / \mathrm{L}$ \\
\hline 1,1-Dichloroethane & $0 / 37$ & 5.0 & 0.0 & $5.0 / 5.0 \mu \mathrm{g} / \mathrm{L}$ \\
\hline 1,2-Dichloroethane & $0 / 36$ & 5.0 & 0.0 & $5.0 / 5.0 \mu \mathrm{g} / \mathrm{L}$ \\
\hline 1,1-Dichloroethylene & $0 / 36$ & 5.0 & 0.0 & $5.0 / 5.0 \mu \mathrm{g} / \mathrm{L}$ \\
\hline 1,2-Dichloroethylene & $0 / 1$ & 5.0 & - & $5.0 / 5.0 \mu \mathrm{g} / \mathrm{L}$ \\
\hline cis-1,2-Dichloroethylene & $0 / 16$ & 7.50 & 6.83 & $5.0 / 25.0 \mu \mathrm{g} / \mathrm{L}$ \\
\hline trans-1,2-Dichloroethylene & $0 / 36$ & 5.0 & 0.0 & $5.0 / 5.0 \mu \mathrm{g} / \mathrm{L}$ \\
\hline Dichloromethane & $0 / 36$ & 5.56 & 2.29 & $3.20 / 14.1 \mu \mathrm{g} / \mathrm{L}$ \\
\hline 1,2-Dichloropropane & $0 / 36$ & 5.0 & 0.0 & $5.0 / 5.0 \mu \mathrm{g} / \mathrm{L}$ \\
\hline 1,3-Dichloropropane & $0 / 3$ & 5.0 & 0.0 & $5.0 / 5.0 \mu \mathrm{g} / \mathrm{L}$ \\
\hline 2,2-Dichloropropane & $0 / 3$ & 5.0 & 0.0 & $5.0 / 5.0 \mu \mathrm{g} / \mathrm{L}$ \\
\hline 1,1-Dichloropropene & $0 / 3$ & 5.0 & 0.0 & $5.0 / 5.0 \mu g / L$ \\
\hline cis-1,3-Dichloropropene & $0 / 36$ & 5.0 & 0.0 & $5.0 / 5.0 \mu \mathrm{g} / \mathrm{L}$ \\
\hline trans-1,3-Dichloropropene & $0 / 36$ & 5.0 & 0.0 & $5.0 / 5.0 \mu \mathrm{g} / \mathrm{L}$ \\
\hline Ethylbenzene & $0 / 36$ & 5.0 & 0.0 & $5.0 / 5.0 \mu \mathrm{g} / \mathrm{L}$ \\
\hline 2-Hexanone & $0 / 12$ & 10.0 & 0.0 & $10.0 / 10.0 \mu \mathrm{g} / \mathrm{L}$ \\
\hline lodomethane & $0 / 11$ & 5.0 & 0.0 & $5.0 / 5.0 \mu \mathrm{g} / \mathrm{L}$ \\
\hline Isobutyl alcohol & $0 / 11$ & 100 & 0.0 & $100 / 100 \mu \mathrm{g} / \mathrm{L}$ \\
\hline
\end{tabular}




\begin{tabular}{lllll}
\hline Analyte & $\begin{array}{l}\text { Frequency } \\
\text { of Detectiont }\end{array}$ & Mean Result & $\begin{array}{l}\text { Standard } \\
\text { Deviation }\end{array}$ & Minimum/Maximum Resu/ts \\
\hline Methacrylonitrile & & & & \\
Methyl ethyl ketone & $0 / 11$ & 10.0 & 0.0 & $10.0 / 10.0 \mu \mathrm{g} / \mathrm{L}$ \\
Methyl isobutyl ketone & $0 / 12$ & 10.0 & 0.0 & $10.0 / 10.0 \mu \mathrm{g} / \mathrm{L}$ \\
Propionitrile & $0 / 12$ & 10.0 & 0.0 & $10.0 / 10.0 \mu \mathrm{g} / \mathrm{L}$ \\
Styrene & $0 / 11$ & 50.0 & 0.0 & $50.0 / 50.0 \mu \mathrm{g} / \mathrm{L}$ \\
$1,1,1,2-$ Tetrachloroethane & $0 / 12$ & 5.0 & 0.0 & $5.0 / 5.0 \mu \mathrm{g} / \mathrm{L}$ \\
$1,1,2,2-$ Tetrachloroethane & $0 / 11$ & 5.0 & 0.0 & $5.0 / 5.0 \mu \mathrm{g} / \mathrm{L}$ \\
Tetrachloroethylene & $0 / 36$ & 5.0 & 0.0 & $5.0 / 5.0 \mu \mathrm{g} / \mathrm{L}$ \\
Toluene & $0 / 55$ & 5.0 & 0.0 & $5.0 / 5.0 \mu \mathrm{h} / \mathrm{L}$ \\
$1,1,1-$ Trichloroethane & $0 / 36$ & 5.0 & 0.0 & $5.0 / 5.0 \mu \mathrm{g} / \mathrm{L}$ \\
$1,1,2-$ Trichloroethane & $0 / 36$ & 5.0 & 0.0 & $5.0 / 5.0 \mu \mathrm{g} / \mathrm{L}$ \\
Trichloroethylene & $0 / 36$ & 5.0 & 0.0 & $5.0 / 5.0 \mu \mathrm{g} / \mathrm{L}$ \\
Trichlorofluoromethane & $0 / 36$ & 5.0 & 0.0 & $5.0 / 5.0 \mu \mathrm{g} / \mathrm{L}$ \\
1,2,3-Trichloropropane & $0 / 35$ & 5.0 & 0.0 & $5.0 / 5.0 \mu \mathrm{g} / \mathrm{L}$ \\
Vinyl acetate & $0 / 11$ & 5.0 & 0.0 & $5.0 / 5.0 \mu \mathrm{g} / \mathrm{L}$ \\
Xylenes & $0 / 12$ & 10.0 & 0.0 & $10.0 / 10.0 \mu \mathrm{g} / \mathrm{L}$ \\
\hline
\end{tabular}

$\uparrow$ Number of times analyte was detected compared to the total number of trip blanks for the analyte.

- Standard deviation cannot be determined.

Note: If the analyte was not detected in the trip blank(s), detection limit information appears in the Mean Result and Minimum/Maximum Results columns.

Tablo 66. Bailod Wolls

\begin{tabular}{llll}
\hline Well & Date & Well & Date \\
\hline FTF 9 & $01 / 21 / 99$ & FTF 22 & $01 / 21 / 99$ \\
FTF 10 & $01 / 21 / 99$ & FTF 23 & $01 / 21 / 99$ \\
FTF 12 & $01 / 21 / 99$ & HTF 2 & $01 / 09 / 99$ \\
FTF 13 & $01 / 21 / 99$ & HTF 3 & $01 / 09 / 99$ \\
FTF 15 & $01 / 21 / 99$ & HTF 4 & $01 / 10 / 99$ \\
FTF 16 & $01 / 21 / 99$ & HTF 5 & $01 / 10 / 99$ \\
FTF 17 & $01 / 21 / 99$ & HTF 6 & $01 / 10 / 99$ \\
FTF 18 & $01 / 21 / 99$ & HTF 7 & $01 / 11 / 99$ \\
FTF 19 & $01 / 21 / 99$ & HTF 8 & $01 / 10 / 99$ \\
FTF 20 & $01 / 21 / 99$ & HTF 34 & $01 / 07 / 99$ \\
FTF 21 & $01 / 21 / 99$ & & \\
\hline
\end{tabular}

Table 67. Sampled Wolls with Motal Casings

\begin{tabular}{llll}
\hline Well & Casing & Well & Casing \\
\hline AC 1A & Steel & MSB 54TA & Carbon steel \\
AC 1B & Steel & MSB 55TA & Carbon steel \\
ASB 8TA & Carbon steel & MSB 82TA & Carbon steel \\
FTF 12 & Steel & RWM 1 & Carbon steel \\
FTF 13 & Steel & RWM 3 & Carbon steel \\
HTF 1 & Steel & RWM 4 & Carbon steel \\
HTF 2 & Steel & RWM 5 & Carbon steel \\
HTF 3 & Steel & RWM 7 & Carbon steel \\
HTF 4 & Steel & RWM 8 & Carbon steel \\
HTF 5 & Steel & RWM 9 & Carbon steel \\
HTF 6 & Steel & RWM 10 & Carbon steel \\
HTF 7 & Steel & & Carbon steel
\end{tabular}




\begin{tabular}{llll}
\hline Well & Casing & Well & Casing \\
\hline HTF 8 & Steel & RWM 11 & Carbon steel \\
HTF 9 & Steel & RWM 13B & Carbon steel \\
HTF 10 & Steel & RWM 13C & Carbon steel \\
HTF 11 & Steel & RWM 14B & Carbon steel \\
HTF 13 & Steel & RWM 14C & Carbon steel \\
MSB 12TA & Steel & RWM 15B & Carbon steel \\
MSB 23TA & Steel & TNX 13D & Stainless steel \\
MSB 27TA & Carbon steel & TNX 14D & Stainless steel \\
MSB 29TA & Carbon steel & TNX 15D & Stainless steel \\
MSB 34TA & Steel & TNX 16D & Stainless steel \\
MSB 35TA & Carbon steel & TNX 17D & Stainless steel \\
MSB 36TA & Carbon steel & TNX 18D & Stainless steel \\
MSB 39TA & Carbon steel & TNX 19D & Stainless steel \\
MSB 41TA & Carbon steel & TNX 20D & Stainless steel \\
MSB 42TA & Carbon steel & TNX 21D & Stainless steel \\
MSB 43TA & Carbon steel & TNX 22D & Stainless steel \\
MSB 47TA & Carbon steel & TNX 26D & Stainless steel \\
MSB 48TA & Carbon steel & & \\
\hline
\end{tabular}

Table 68. Wells That Had Turbidity Greater Than 15 NTU

\begin{tabular}{|c|c|c|}
\hline$W e l$ & Date & Results (in NTU) \\
\hline $\begin{array}{l}\text { ABP 10D } \\
\text { FSB115D } \\
\text { FTF } 9 \\
\text { FTF 10 } \\
\text { FTF 12 } \\
\text { FTF 13 } \\
\text { FTF 15 } \\
\text { FTF 16 } \\
\text { FTF 17 } \\
\text { FTF 18 } \\
\text { FTF 19 } \\
\text { FTF 20 } \\
\text { FTF 21 } \\
\text { FTF 22 } \\
\text { FTF 23 } \\
\text { HAA 4D } \\
\text { HAA 5A } \\
\text { HAA 5C } \\
\text { HAA 5D } \\
\text { HAA 10AR } \\
\text { HAA 13A } \\
\text { HAA 13D } \\
\text { HAA 14D } \\
\text { HAA 15C } \\
\text { HAA 15D } \\
\text { HSL 3D } \\
\text { HSL 6D } \\
\text { HSL 7D } \\
\text { HTF } 2 \\
\text { HTF 3 } \\
\text { HTF 4 } \\
\text { HTF 5 } \\
\text { HTF 6 } \\
\text { HTF 7 } \\
\text { HTF 8 }\end{array}$ & $\begin{array}{l}01 / 07 / 99 \\
01 / 11 / 99 \\
01 / 21 / 99 \\
01 / 21 / 99 \\
01 / 21 / 99 \\
01 / 21 / 99 \\
01 / 21 / 99 \\
01 / 21 / 99 \\
01 / 21 / 99 \\
01 / 21 / 99 \\
01 / 21 / 99 \\
01 / 21 / 99 \\
01 / 21 / 99 \\
01 / 21 / 99 \\
01 / 21 / 99 \\
01 / 10 / 99 \\
01 / 10 / 99 \\
01 / 10 / 99 \\
02 / 25 / 99 \\
011 / 06 / 99 \\
01 / 07 / 99 \\
01 / 06 / 99 \\
02 / 18 / 99 \\
01 / 11 / 99 \\
01 / 19 / 99 \\
01 / 21 / 99 \\
01 / 22 / 99 \\
01 / 22 / 99 \\
01 / 09 / 99 \\
01 / 09 / 99 \\
01 / 10 / 99 \\
01 / 10 / 99 \\
01 / 10 / 99 \\
01 / 11 / 99 \\
01 / 10 / 99\end{array}$ & 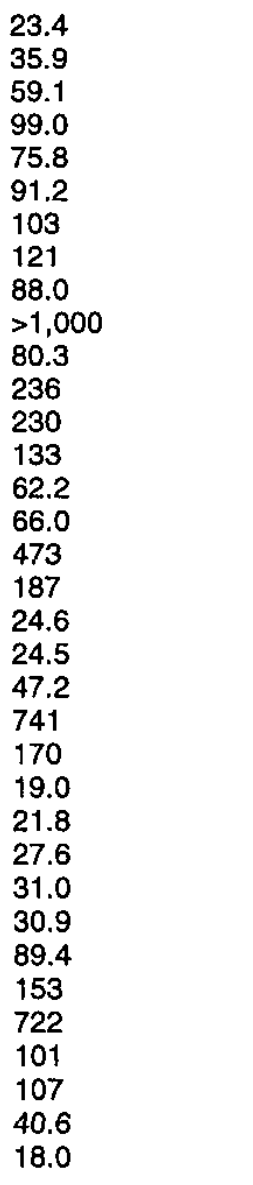 \\
\hline
\end{tabular}

\section{Quality Control Samplos}




\begin{tabular}{lll}
\hline WeI & Date & Results (in NTU) \\
\hline HTF 9 & $01 / 10 / 99$ & \\
HTF 10 & $01 / 08 / 99$ & 114 \\
HTF 11 & $01 / 08 / 99$ & 341 \\
HTF 13 & $01 / 08 / 99$ & 190 \\
HTF 15D & $03 / 03 / 99$ & 710 \\
HTF 18 & $01 / 08 / 99$ & 21,000 \\
HTF 19 & $01 / 08 / 99$ & 531 \\
HTF 20 & $01 / 08 / 99$ & 16.8 \\
HTF 21 & $01 / 08 / 99$ & 284 \\
HTF 34 & $01 / 07 / 99$ & 1,000 \\
LBP 1D & $01 / 29 / 99$ & 18.1 \\
MSB 71B & $02 / 12 / 99$ & 94.1 \\
RDB 1D & $03 / 09 / 99$ & 32.8 \\
TCM 3 & $02 / 08 / 99$ & 18.0 \\
TNX 4D & $02 / 03 / 99$ & 53.8 \\
TNX 21D & $02 / 03 / 99$ & 27.2 \\
\hline
\end{tabular}

Table 69. Analyses Not Performed by EX

\begin{tabular}{lll}
\hline Well & Analyte & Reason \\
\hline $\mathrm{P} 260$ & Nitrate-nitrite as nitrogen & Canceled \\
\hline
\end{tabular}

Table 70. Analyses Not Performed by GE

\begin{tabular}{lll}
\hline Well & Analyte & Reason \\
\hline HSB 67 & Bis-2-ethylhexylphthlate & Canceled \\
\hline
\end{tabular}

Table 71. Analyses Not Performed by WA

\begin{tabular}{lll}
\hline Well & Analyte & Reason \\
\hline AMB 18C & Tin & Not logged for analysis \\
HAA 5A & GC VOA & Not sampled \\
\hline
\end{tabular}


NOTES
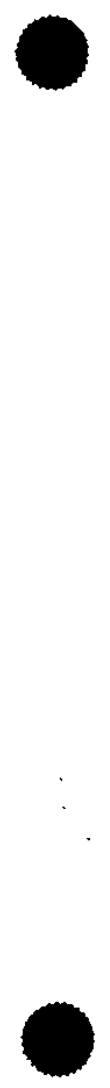


\section{Site Index}

Table 72 provides information about sites, locations, and well series. Some site names and locations were not available.

Table 72. Sites and Locations by Well Series

\begin{tabular}{|c|c|c|}
\hline Well Series & Site & Location \\
\hline $\begin{array}{l}A B P \\
A B W \\
A C\end{array}$ & $\begin{array}{l}\text { A-Area Metals Burning Pit } \\
\text { A Area near Firing Range } \\
\text { A-Area Cluster Perimeter Wells and M-Area Plume Definition } \\
\text { Wells }\end{array}$ & $\begin{array}{l}\text { South of the burning/rubble pits } \\
\text { North of Road D-1 and east of Road 1-7 }\end{array}$ \\
\hline $\begin{array}{l}\text { ACB } \\
\text { AMB } \\
\text { AMP }\end{array}$ & $\begin{array}{l}\text { A-Area Coal Pile Runoff Containment Basin } \\
\text { Metallurgical Laboratory Seepage Basin } \\
\text { A-Area Rubble Pile }\end{array}$ & $\begin{array}{l}\text { Southeast of A Area } \\
\text { At the eastern edge of A Area }\end{array}$ \\
\hline AOB & Motor Shop Oil Basin & $\begin{array}{l}\text { At the south edge of A Area near NPDES } \\
\text { Outfall A-14 }\end{array}$ \\
\hline $\begin{array}{l}\text { ARP } \\
\text { ASB }\end{array}$ & $\begin{array}{l}\text { A-Area Burning/Rubble Pits and A-Area Ash Pile } \\
\text { Savannah River Laboratory Seepage Basins }\end{array}$ & $\begin{array}{l}\text { West of Road D, south of A Area } \\
\text { Across the road from the Savannah River } \\
\text { Technology Center (formerly the Savannah } \\
\text { River Laboratory) }\end{array}$ \\
\hline $\begin{array}{l}\text { BGO } \\
\text { BGX } \\
\text { BRD } \\
\text { BRR } \\
\text { BSE } \\
\text { BTP }\end{array}$ & $\begin{array}{l}\text { Burial Grounds Perimeter } \\
\text { E-Area Vaults/Burial Ground Expansion } \\
\text { Road A Chemical Basin (Baxley Road) } \\
\text { Burma Road Rubble Pit } \\
\text { Old Burial Ground } \\
\text { Characterization Piezometers for the Proposed Sanitary Landfill }\end{array}$ & $\begin{array}{l}\text { Southem E Area } \\
\text { Northem E Area } \\
\text { East of D Area } \\
\text { Southwest of F Area } \\
\text { Southeast edge of the Old Burial Ground } \\
\text { Site B, off Road E-2 }\end{array}$ \\
\hline CBR & $\begin{array}{l}\text { N-Area (Central Shops) Burning/Rubble Pit south of the Ford } \\
\text { Building Seepage Basin }\end{array}$ & Southeast of N Area \\
\hline $\begin{array}{l}\text { CCB } \\
\text { CDB } \\
\text { CDS } \\
\text { CMP }\end{array}$ & $\begin{array}{l}\text { C-Area Coal Pile Runoff Containment Basin } \\
\text { C-Area Disassembly Basin } \\
\text { 108-3C Bioremediation Facility } \\
\text { Chemicals, Metals, and Pesticides Pits }\end{array}$ & $\begin{array}{l}\text { Southeast of C Area } \\
\text { Near the C-Area reactor building } \\
\text { Near the C-Area reactor building } \\
\text { West of Road C, approximately two miles } \\
\text { southeast of N Area }\end{array}$ \\
\hline $\begin{array}{l}\text { CRP } \\
\text { CSA } \\
\text { CSB } \\
\text { CSR } \\
\text { CSO }\end{array}$ & $\begin{array}{l}\text { C-Area Burning/Rubble Pit } \\
\text { Hydrofluoric Acid Spill Area } \\
\text { C-Area Reactor Seepage Basins } \\
\text { N-Area (Central Shops) Burning/Rubble Pits } \\
\text { Fire Department Training Facility }\end{array}$ & $\begin{array}{l}\text { Southeast of } N \text { Area } \\
\text { South of Road } 3 \text { in } N \text { Area } \\
\text { Southem } C \text { Area, west of the reactor building } \\
\text { North of N Area } \\
\text { Southeast portion of N Area }\end{array}$ \\
\hline $\begin{array}{l}\text { DBP } \\
\text { DCB }\end{array}$ & $\begin{array}{l}\text { D-Area Burning/Rubble Pits } \\
\text { D-Area Coal Pile Runoff Containment Basin and Ash Basins }\end{array}$ & $\begin{array}{l}\text { Westem portion of D Area } \\
\text { South (containment basin) and southwest } \\
\text { (ash basins) of D Area }\end{array}$ \\
\hline $\begin{array}{l}\text { DOB } \\
\text { DOL }\end{array}$ & $\begin{array}{l}\text { D-Area Oil Seepage Basin } \\
\text { D-Area Oil Seepage Basin }\end{array}$ & $\begin{array}{l}\text { North of D Area } \\
\text { North of D Area }\end{array}$ \\
\hline FAB & F-Area Ash Basin 288-1 Groundwater Quality Assessment & $\begin{array}{l}\text { East of F Area and south of the F-Area } \\
\text { acid/caustic basin }\end{array}$ \\
\hline $\begin{array}{l}\text { FAL } \\
\text { FBP } \\
\text { FCA } \\
\text { FCB } \\
\text { FET } \\
\text { FEX } \\
\text { FIN } \\
\text { FIW } \\
\text { FNB } \\
\text { FOB }\end{array}$ & $\begin{array}{l}\text { F-Area A Line } \\
\text { F-Area Burning/Rubble Pits } \\
\text { F-Area Canyon Buitding } \\
\text { F-Area Coal Pile Runoff Containment Basin } \\
\text { F-Area Effluent Treatment Cooling Water Basin } \\
\text { F-Area Seepage Basins Pemediation Extraction Wells } \\
\text { F-Area Seepage Basins Remediation Injection Tanks } \\
\text { F-Area Seepage Basins } \\
\text { Old F-Area Seepage Basin } \\
\text { F-Area Seepage Basins }\end{array}$ & $\begin{array}{l}\text { Adjacent to the F-Area canyon building } \\
\text { North of Road C and west of F Area } \\
\text { Central F Area } \\
\text { Southeast of F Area } \\
\text { South of F Area }\end{array}$ \\
\hline
\end{tabular}




\begin{tabular}{|c|c|}
\hline Well Series & Site \\
\hline $\begin{array}{l}\text { FRB } \\
\text { FSB } \\
\text { FSL } \\
\text { FSS } \\
\text { FST }\end{array}$ & $\begin{array}{l}\text { F-Area Retention Basin } \\
\text { F-Area Seepage Basins } \\
\text { F-Area Inactive Process Sewer Line } \\
\text { F-Area Sludge Land Application Site } \\
\text { Savannah River Ecology Laboratory Flowing Springs Site }\end{array}$ \\
\hline FTF & F-Area Tank Farm \\
\hline GBW & Background Well near Hawthorne Fire Tower \\
\hline $\begin{array}{l}\text { HAA } \\
\text { HAP }\end{array}$ & $\begin{array}{l}\text { H-Area Tank Farm Groundwater Operable Unit } \\
\text { H-Area Auxiliary Pump Pit }\end{array}$ \\
\hline $\begin{array}{l}\text { HCA } \\
\text { HCB } \\
\text { HET } \\
\text { HEX } \\
\text { HHP } \\
\text { HIN } \\
\text { HIW } \\
\text { HMD } \\
\text { HR3 }\end{array}$ & $\begin{array}{l}\text { H-Area Canyon Building } \\
\text { H-Area Coal Pile Runoff Containment Basin } \\
\text { H-Area Effluent Treatment Cooling Water Basin } \\
\text { H-Area Seepage Basins Remediation Extraction Wells } \\
\text { HP-52 Outfall Area and Warners Pond } \\
\text { H-Area Injection Tank } \\
\text { H-Area Injection Wells } \\
\text { Hazardous Waste/Mixed Waste Disposal Facility } \\
\text { Old H-Area Retention Basin }\end{array}$ \\
\hline HR8 & $\mathrm{H}$-Area Retention Basin \\
\hline HSB & H-Area Seepage Basins \\
\hline HSL & $\mathrm{H}$-Area Inactive Process Sewer Line \\
\hline $\begin{array}{l}\text { HSS } \\
\text { HTF } \\
\text { HWP } \\
\text { HWS } \\
\text { HXB }\end{array}$ & $\begin{array}{l}\text { H-Area Sludge Land Application Site } \\
\text { H-Area Tank Farm } \\
\text { Warner's Pond and HP-52 Outfall } \\
\text { Hazardous Waste Storage Facility } \\
\text { Ford Building Seepage Basin }\end{array}$ \\
\hline $\begin{array}{l}\text { IDB } \\
\text { IDP } \\
\text { IDQ }\end{array}$ & $\begin{array}{l}\text { Interim Waste Technology Site Characterization Wells, Site B } \\
\text { Interim Waste Technology Site Characterization Wells, Site P } \\
\text { Interim Waste Technology Site Characterization Wells, Site Q }\end{array}$ \\
\hline $\begin{array}{l}\mathrm{KAB} \\
\mathrm{KBP} \\
\mathrm{KCB}\end{array}$ & $\begin{array}{l}\text { K-Area Ash Basin } \\
\text { K-Area Bingham Pump Outage Pii } \\
\text { K-Area Coal Pile Runoff Containment Basin }\end{array}$ \\
\hline $\begin{array}{l}\mathrm{KDB} \\
\mathrm{KDT}\end{array}$ & $\begin{array}{l}\text { K-Area Disassembly Basin } \\
\text { K-Area Diesel Tank }\end{array}$ \\
\hline $\begin{array}{l}\text { KRB } \\
\text { KRP } \\
\text { KSB } \\
\text { KSM } \\
\text { KSS }\end{array}$ & $\begin{array}{l}\text { K-Area Retention Basin } \\
\text { K-Area Burning/Rubble Pit } \\
\text { K-Area Reactor Seepage Basin } \\
\text { K-Area Tritium Sump } \\
\text { K-Area Sludge Land Application Site }\end{array}$ \\
\hline $\begin{array}{l}\text { LAC } \\
\text { LAW } \\
\text { LBP } \\
\text { LCO } \\
\text { LDB } \\
\text { LDS } \\
\text { LFW } \\
\text { LRP } \\
\text { LSB }\end{array}$ & $\begin{array}{l}\text { L-Area Acid/Caustic Basin } \\
\text { L-Area Research Wells } \\
\text { L-Area Bingham Pump Outage Pit } \\
\text { L-Area Oil and Chemical Basin } \\
\text { L-Area Disassembly Basin } \\
\text { 108-3L Bioremediation Facility } \\
\text { Sanitary Landfill } \\
\text { L-Area Burning/Rubble Pit } \\
\text { L-Area Reactor Seepage Basin }\end{array}$ \\
\hline
\end{tabular}

Location

South of Road C; east of Road C-4 South of Road C; east of Road C.4

Adjacent to Aquatic Ecology Laboratory (Road C)

West of Road 2-1.1F

At the east end of $\mathrm{H}$ Area near the coal pile runoff containment basin

East of $\mathrm{H}$ Area

Southwest of H Area

East of Road 4

South of Road E

Near the H-Area seepage basins

Northwest of the burial ground expansion

Southeast of the intersection of Roads 4 and $\mathrm{E}$

Southeast of the intersection of Roads 4 and $E$

Southwest of $\mathrm{H}$ Area and the intersection of Roads 4 and $E$

Extends from the southwest portion of $\mathrm{H}$ Area to north of the H-Area seepage basins

Southeast of $\mathrm{H}$ Area

At the south end of $\mathrm{H}$ Area

Northwest of N Area

in the southeast portion of $\mathrm{N}$ Area

Two miles northeast of $\mathrm{H}$ Area

South of B Area and north of Highway 125

Adjacent to Site P, South or B Area and north of Highway 125

Southwest of $\mathrm{K}$ Area

West of K Area, between the K-Area ash basin and reactor seepage basin

Central K Area, north of the disassembly basin

Northwest of K Area

West of K Area

Near the K-Area process water storage tank Southeast of K Area

North of Road B and east of Road B-2.13

South of L Area

South of Road C

Northwest of $L$ Area

Southeast of $L$ Area, adjacent to the L-Area oil and chemical basin 


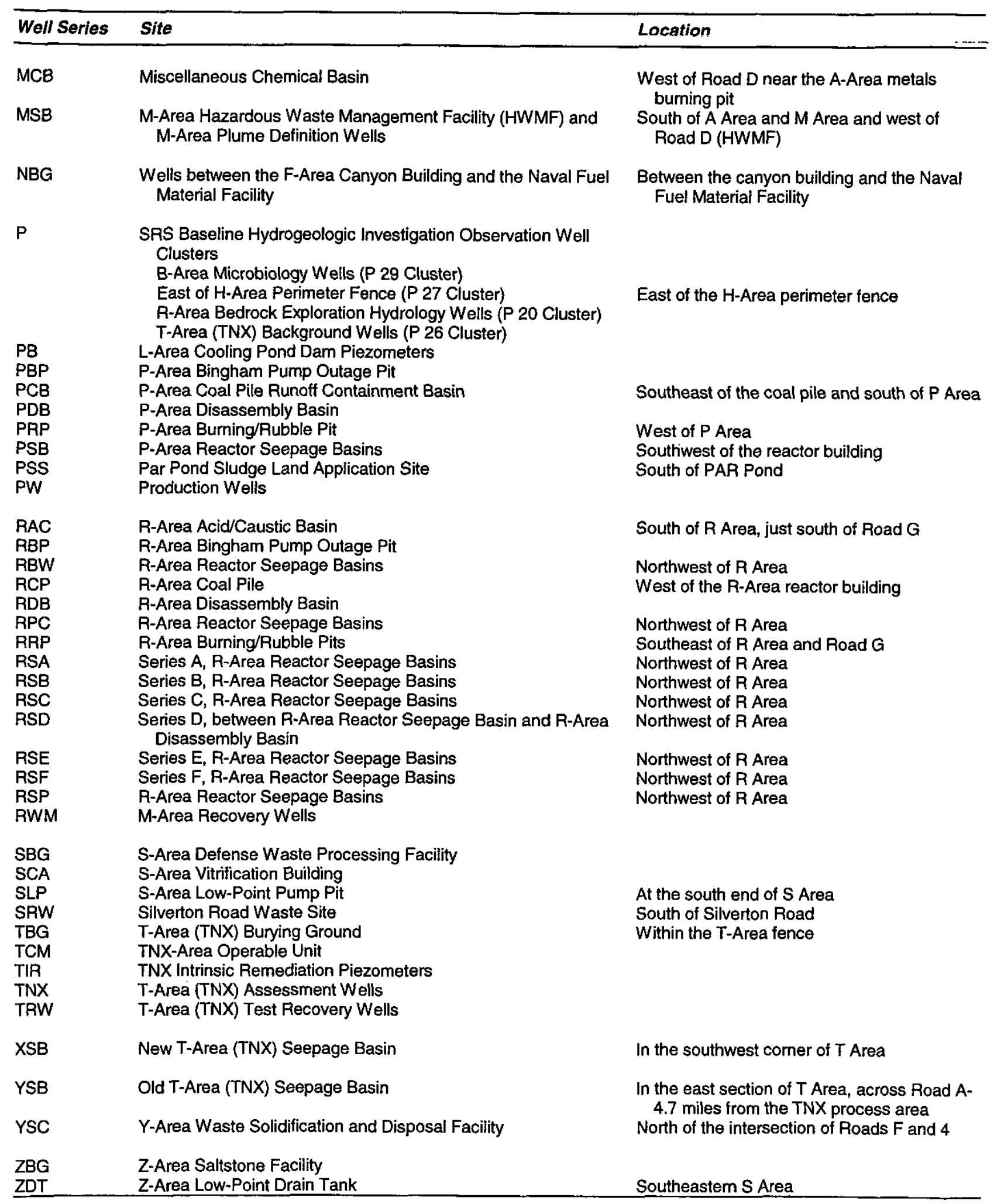




\section{SITE HISTORY}

Geographical descriptions in the text are based on true north rather than SRS grid coordinates.

The following sections describe facilities at approximately 100 locations within designated areas at SRS. The sections are arranged in the following order:

- $\quad$ acid/caustic basins

- burning/rubble, rubble, and metals burning pits

- coal pile runoff containment basins, ash basins, and coal piles

- disassembly basins

- seepage and retention basins

- operating buildings and facilities

- plume monitoring

- radioactive waste storage and disposal facilities

- sanitary landfill and interim sanitary landfill

- sludge application sites

- other sites

\section{Acid/Caustic Basins}

The acid/caustic basins in F Area, $H$ Area, $K$ Area, L Area, P Area, and $R$ Area are unlined earthen pits (approximately 50 by 50 by 7 feet deep). These pits received dilute sulfuric acid and sodium hydroxide solutions used to regenerate ion-exchange units in power plant water purification processes at the reactor and separations areas in the center of SRS. The basins allowed mixing and neutralization of the dilute solutions before their discharge to nearby streams.

The basins were constructed between 1952 and 1955. They are uncovered, and most are dry except during periods of prolonged precipitation. The R-Area and L-Area basins were abandoned in 1964 and 1968 , respectively. The other basins remained in service until 1982, when the water purification systems either were shut down or modernized. However, the $\mathrm{H}$-Area basin continued to receive steam condensate from a hose box and drainage from a chemical pad until the basin was abandoned in 1985. During July through September 1993, the F-, H-, K-, and P-Area basins were dewatered, vegetation was removed and disposed of, the basins were filled with compacted soil from the Burma Road clay pit, a grass cover was established, and the fences were reinstalled.

\section{Burning/Rubble, Rubble, and Metals Burning Pits}

From 1951 to 1973, burnable wastes-such as paper, wood, plastics, rubber, oil, degreasers, and drummed solvents - were received and burned monthly in one or more of the burning/rubble pits in the following areas: A, C, D, F, K, L, N, P, and R. In 1973, waste no longer was burned at the pits, which were covered with a layer of soil. Rubble wastes-including paper, wood, cans, concrete, and empty galvanized-steel barrels and drumsthen were disposed of in the pits until they reached capacity and were covered with soil. All burning/rubble pits were inactive by 1981, and all are covered except the R-Area pit, which has not been backfilled. Lithiumaluminum alloy, aluminum pieces, metal drums, other metal scraps, and plastic pipe were deposited and burned periodically in the A-Area metals burning pit, beginning about 1952. In 1974, the solid materials remaining on the site were covered with soil, and the pit was regraded. The site is inactive. 
The Burma Road rubble pit consists of two excavated earthen pits that may contain paint cans, fluorescent light fixtures, metal, concrete, lumber, poles, and glass. Unknown quantities of refuse were deposited here from approximately 1973 through 1983 . The pit is inactive and has been covered with soil.

\section{Coal Pile Runoff Containment Basins, Ash Basins, and Coal Piles}

Electricity and steam at SRS are generated by burning coal. Coal piles originally existed in the following areas: $A, C, D, F, H, K, L, P$, and $R$. The facilities generally contained a 90 -day reserve of coal that was not rotated. During long-term exposure to the environment, chemical and biological oxidation of sulfur compounds in coal resulted in the formation of sulfuric acid.

The R-Area coal pile was removed in 1964, and the L-Area coal pile was removed in 1968. To achieve compliance with the National Pollutant Discharge Elimination System (NPDES) permit issued in 1977, coal pile runoff containment basins in A Area and D Area were completed in October 1978, and basins in C Area, F Area, $\mathrm{H}$ Area, $\mathrm{K}$ Area, and $\mathrm{P}$ Area were completed in March 1981. The coal piles in C Area and $F$ Area were removed in 1985. In 1991, the K-Area coal pile was reduced to a 2-inch base, and 75 percent of the P-Area coal pile was removed.

Currently, rainwater runoff from the remaining coal piles in $A, D, H, K$, and $P$ Areas flows into the coal pile runoff containment basins via gravity flow ditches and sewers. The basins allow mixing of the runoff and its seepage into the subsurface, thus preventing the entry of large surges of low-pH runoff into surface streams. The basins in $\mathrm{C}$ and $\mathrm{F}$ Areas also still collect runoff, although no coal remains at either location. Ash sluice water from the D-Area and K-Area powerhouses has been discharged to the D-Area ash basins and the K-Area ash basin, respectively, since 1951.

\section{F-Area Ash Basin}

The F-Area ash basin was monitored for the first time during second quarter 1994.

\section{R-Area Coal Pile}

Two wells were installed in 1990 inside the boundaries of the former coal storage area, originally for groundwater assessment in relation to the R-Area coal pile.

\section{Disassembly Basins}

The disassembly basins, also called fuel and target storage basins, are concrete-lined, open tanks of water next to the reactor rooms inside the reactor buildings in C, K, L, P, and R Areas. Irradiated assemblies (reactor fuel and target rods) were rinsed and stored in the basins prior to their shipment to the separations areas. Some radioactivity was transferred to the basin water from leaks in porous components and as a liquid or oxide corrosion film on the irradiated components.

Sand filters were used to remove radioactive particulates from the disassembly basin water. The filtered water was circulated through deionizers to remove additional constituents and was purged periodically through regenerated deionizers to the reactor seepage basins.

\section{Seepage and Retention Basins}

Seepage, retention, and settling basins have been used at SRS to store or dispose of wastewater from various operations. Seepage and retention basins in the following areas are monitored: $A, C, F, H, K, L, M, N, P, R, T$, and the Savannah River Laboratory.

\section{C-Area Reactor Seepage Basins}

These basins have received low-level radioactive purge water from the disassembly basin since 1957. 


\section{F-Area Seepage Basins and Inactive Process Sewer Line}

Beginning in 1955, the F-Area seepage basins received F-Area wastewater containing low-level radioactivity and chemicals, including chromium, mercury, nitric acid, and sodium hydroxide. Clay caps were completed in 1991 when the basins were closed.

\section{Ford Building Seepage Basin}

The Ford Building seepage basin received low-level radioactive wastewater from Ford Building operations (repairing heat exchangers) from 1964 to January 1984.

\section{H-Area Retention Basins}

A small, unlined earthen retention basin (the old $\mathrm{H}$-Area retention basin) was used from 1955 to 1973 to provide temporary emergency storage for cooling water from the chemical separations process that contained radionuclides and possible trace quantities of chemicals.

A larger, rubber-lined retention basin replaced the original basin in 1973 and still is in use for receipt of diverted cooling water or tank farm stormwater runoff.

\section{H-Area Seepage Basins and Inactive Process Sewer Line}

Starting in 1955, the $\mathrm{H}$-Area seepage basins received wastewater from $\mathrm{H}$ Area containing low-level radioactivity and chemicals, including nitric acid, mercury, and sodium hydroxide. Basin 3 has been inactive since 1962.

Basins 1, 2, and 4 operated from 1980 until they were taken out of service in the fourth quarter of 1988. Clay caps were completed early in 1991 when the basins were closed.

\section{K-Area Reactor Seopage Basin}

This basin has received low-level radioactive purge water from the disassembly basin since 1957.

\section{L-Area Reactor Seepage Basin}

This basin has received low-level radioactive purge water from the disassembiy basin since 1957.

\section{M-Area Hazardous Waste Management Facility}

The unlined M-Area settling basin, in operation from 1958 until 1985, received wastewater containing metalcleaning solvents, depleted uranium, and other chemicals and metals from fuel fabrication processes in $M$ Area. Because surface water flowed from this basin, it is classified as a settling basin rather than a seepage basin. Water from the basin flowed through an overflow ditch to Lost Lake, a shallow upland depression. A seepage area formed adjacent to the ditch and Lost Lake. The M-Area hazardous waste management facility comprises the settling basin, overflow ditch, seepage area, and Lost Lake. A closure cap was completed on the basin during 1989/1990.

Since the beginning of a full-scale recovery system for groundwater remediation in April 1985, groundwater flow has changed markedly near this facility, and changes over time in concentrations of analytes are difficult to interpret. See the Plume Monitoring section of this chapter for more information on remediation.

\section{Metallurgical Laboratory Seepage Basin}

The Metallurgical Laboratory seepage basin received wastewater effluent from the Metallurgical Laboratory building from 1956 until 1985. Wastewater released to the basin consisted of small quantities (5 to 10 gallons per day) of laboratory wastes-mostly rinse water-from metallographic sample preparation (degreasing, cleaning, etching) and corrosion testing of stainless steel and nickel-based alloys. Noncontact cooling water (approximately 900 gallons per day) also was discharged. The basin has been dewatered, backfilled, and capped with low-permeability clay.

\section{New T-Area (TNX) Seepage Basin}

The new TNX seepage basin replaced the old TNX seepage basin and operated from 1980 to 1988 . 


\section{Old F-Area Seepage Basin}

The old F-Area seepage basin, the first seepage basin constructed in F Area, was used for disposal of wastewater from the canyon building from November 1954 until May 1955, when it was abandoned. During operation, the seepage basin received a variety of wastewaters, including evaporator overheads, laundry wastewater, and an unknown amount of chemicals. For three months in 1969, spent nitric acid solutions used to etch depleted uranium were discharged to the basin. In 1984, low-level contaminated water was released to the basin.

\section{Old T-Area (TNX) Seepage Basin}

The old TNX seepage basin received waste from pilot-scale tests conducted at TNX from 1958 to 1980 . In 1981, the basin wall was breached and the impounded water was drained into the adjacent wetlands. The basin then was backfilled with a sand and clay mixture, and the top was capped with clay.

\section{P-Area Reactor Seepage Basins}

These basins have received low-level radioactive purge water from the P-Area disassembly basin since 1957.

\section{R-Area Reactor Seepage Basins}

On November 8,1957 , an experimental fuel element failed during a calorimeter test in the emergency section of the R-Area disassembly basin. Following this incident, the original seepage basin received approximately $2,700 \mathrm{Ci}$ of nonvolatile beta activity, including strontium- 90 and cesium-137, each of which has a half-life of about 30 years. Much of the released radioactivity was contained in that basin, which was backfilled in December 1957. Five more basins were put into operation in 1957 and 1958 to assist in containing the radioactivity.

In 1960, Basins 2 through 5 were closed and backfilled. The ground surface above Basins 1 through 5 was treated with herbicide and covered with asphalt. In addition, a kaolinite cap and dike were constructed over and around Basin 1 and the northwest end of Basin 3 to minimize lateral movement of the radioactive contamination. Basin 6, which received water directly from the disassembly basin from 1960 until 1964, was backfilled in 1977.

\section{Savannoh River Laboratory Seepage Basins}

The Savannah River Laboratory seepage basins received low-level radioactive laboratory wastewater through underground drains until they were taken out of service in October 1982. Two basins were put into operation in 1954; one more was added in 1958 and another in 1960 to provide additional holding capacity.

An exception to the practice of discharging only low-level alpha or beta-gamma wastewater was made in 1971, when $0.68 \mathrm{Ci}$ of curium from a leaking separator pit in the Savannah River Laboratory radioactive waste tanks was disposed of in the basins. Approximately 34 million gallons of wastewater were discharged to the basins during their operating life.

\section{Operating Buildings and Facilities}

\section{Defense Waste Processing Facility (S-Area Vitrification Building)}

The DWPF, also known as the S-Area vitrification building or S-Area canyon, contains the process and auxiliary equipment to incorporate high-level radioactive waste into leach-resistant glass. The facility began radioactive operations in 1996.

\section{F-Area Canyon Building and A-Line Uranium Recovery Facility}

At the canyon building, irradiated product from the reactors is dissolved using nitric acid, and the desired radionuclides are separated from fission products. At the A-Line uranium recovery facility, adjacent to the canyon building, uranium oxide is produced from uranyl nitrate. 


\section{F-Area Effluent Treatment Cooling Water Basin}

The F-Area effluent treatment cooling water basin receives diverted cooling water from the separations processes. The cooling water is sent from the basin to the F-Area and H-Area effluent treatment facility (ETF) if contaminated or to a permitted outfall if uncontaminated. The ETF, on the south side of $\mathrm{H}$ Area, was placed in service in 1988 to treat wastewater formerly sent to the F-Area and $\mathrm{H}$-Area seepage basins. In addition to cooling water, it also receives separations area stormwater runoff and condensed overheads from the evaporators in the tank farms. The treatment facility removes hazardous and radioactive contaminants from these low-level liquid wastes and concentrates them for immobilization as saltstone.

\section{H-Area Auxiliary Pump Pit}

The $\mathrm{H}$-Area auxiliary pump pit facility will pump high-level radioactive sludge and precipitate from the $\mathrm{H}$-Area tank farm to the S-Area low-point pump pit en route to the vitrification facility. When the pumps are shut down, this facility will collect the solution in a temporary holding tank via gravity flow lines.

\section{H-Area Canyon Building}

As in F Area, materials from the reactors are dissolved at the canyon building, and the desired radionuclides are separated from waste products.

\section{H-Area Effluent Treatment Cooling Water Basin}

For more information, see the F-Area Effluent Treatment Cooling Water Basin section.

\section{K-Area Tritium Sump}

A single well, installed in 1992, monitors the water table just west of the K-Area reactor. The well was placed near the K-Area process water storage tank, which stores water collected in sumps within the K-Area reactor building. Tritium activity in this sump water has been reported at greater than $5 \mathrm{Ci} / \mathrm{mL}$.

\section{N-Area Hazardous Waste Storage Facility}

Building 645- $\mathrm{N}$ of the hazardous waste storage facility has been in service since 1983, 645-2N since 1987, and $645-4 \mathrm{~N}$ since 1984 . Buildings $645-\mathrm{N}$ and $645-4 \mathrm{~N}$ contain hazardous waste, and building 645-2N contains mixed waste (a mixture of low-level radioactive waste and hazardous waste). Wastes are stored inside the buildings in drums placed on diked concrete floors designed to contain liquid spills.

\section{Naval Fuel Material Facility}

The Naval Fuel Material Facility was used to produce HEU (highly enriched uranium) for naval reactors until shutdown in 1989. Monitoring wells in the NBG series are located between the canyon building and the Naval Fuel Material Facility.

\section{S-Area Facilities}

S-Area contains several facilities for processing high-level radioactive waste from the F-Area and $\mathrm{H}$-Area tank farms into borosilicate glass solidified within stainless steel canisters. The glass is stored temporarily in specially designed storage buildings within S Area. Eventual permanent disposal is expected to be in an offsite federal geologic repository.

\section{S-Area Low-Point Pump Pit}

The S-Area low-point pump pit receives high-level radioactive sludge and precipitate from the H-Area tank farm and pumps it to the defense waste processing facility (DWPF) vitrification building; it also receives waste being recycled from the vitrification building back to the tank farm. As at the $\mathrm{H}$-Area auxiliary pump pit, when the pumps are shut down, the sludge and precipitate remaining in the line drain back into a temporary holding tank via gravity flow lines. 


\section{Z-Area Low-Point Drain Tank}

The Z-Area low-point drain tank facility receives low-level radioactive salt solution from the H-Area tank farm and pumps it to the Z-Area salt solution hoiding tank. When the H-Area pump is shut down, the low-point drain tank can collect the solution remaining in the lines via gravity flow.

\section{Z-Area Saltstone Manufacturing and Disposal Facility}

The Z-Area saltstone manufacturing and disposal facility processes and permanently disposes of low-level radioactive salt solution supernatant from the underground storage tanks at $F$ Area and $H$ Area and from ETF concentrate.

The facility began radioactive operations in June 1990. In November 1992, a tank in the Z-Area saltstone manufacturing and disposal facility overflowed, and a portion of the liquid leaked from the building into a storm drain. Approximately 2 gallons of solution reached a drainage pipe that flows into a series of sedimentation basins and eventually into McQueen Branch. Sediment samples showed small amounts of cesium-137 exceeding those amounts observed in the Savannah River, but within the activity ranges in site streams.

\section{Plume Monitoring}

\section{A Area and M Area}

In addition to the groundwater monitoring conducted at specific locations in A Area and $M$ Area, numerous plume definition weils also monitor a 5-square-mile area to assess the extent of volatile organic contamination. The first plume definition wells were installed soon after discovery of the contamination in June 1981.

The plume definition well network extends from the region north of SRTC, between Road 1 and the SRS boundary, south to wells near the miscellaneous chemical basin and the metals burning pit, and from Tims Branch in the east toward the Silverton Road waste site in the west. The plume encompasses approximately three square miles and consists primarily of trichloroethylene, tetrachloroethylene, and 1,1,1-trichloroethane.

\section{Separations and Waste Management Areas}

A number of wells were installed in the separations areas in 1951 and 1952. These wells, which range from approximately 15 to 90 feet in depth, are used to measure water table elevations and monitor for radioactive constituents (gross alpha, nonvolatile beta, and tritium) in the groundwater in and around F Area and $\mathrm{H}$ Area. They have steel casings that could affect the metal concentrations in the water.

\section{Radioactive Waste Storage and Disposal Facilities}

\section{Burial Grounds}

The burial grounds have been used for storage and disposal of radioactive solid waste produced at SRS or shipped from other facilities since 1952. The original area, known as the old burial ground, contains low-level alpha and beta-gamma trenches, intermediate-level beta-gamma trenches, and alpha waste trenches. As the trenches were filled, they were covered with soil. When the old burial ground was filled in 1974, operations moved to the adjacent low-level radioactive waste disposal facility (LLRWDF).

The sections of the LLRWDF currently being operated, known as the Solid Waste Disposal Facility (SWDF), contain trenches for only radioactive waste. Concrete vaults, known as the E-Area vaults, have been constructed east and north of the LLRWDF for disposal of solid radioactive waste. The first waste was placed there in September 1994.

Mixed waste storage building 643/29E, within the boundaries of the LLRWDF, has been in use since March 1987. The adjacent mixed waste storage building, $643 / 43 \mathrm{E}$, was completed in July 1995 , and the facility began receiving waste later that same month.

Until 1965, transuranic (TRU) waste was placed in plastic bags and cardboard boxes and buried in earthen trenches. Between 1965 and 1974, lower level TRU waste was buried unencapsulated in trenches, and higher level TRU waste was buried in retrievable concrete containers or encapsulated in concrete. Since 1974, TRU 
wastes contaminated with greater than $0.01 \mathrm{Ci} / \mathrm{g}$ have been stored in watertight containers on concrete pads with monitoring sumps. TRU waste storage pads 1-19 are on the FFA's list of RCRA-regulated units.

Since mid-1984, newly generated low-level beta-gamma waste has been placed in metal boxes or metal drums. Currently, it is disposed of in engineered trenches and covered with at least 4 feet of soil. Some wastes that do not have forms that are easily placed in containers are disposed of in shallow land-burial slit trenches.

Mixed wastes stored or disposed of within the old burial ground and portions of the LLRWDF include cadmium, lead, mercury, and tritiated pump oil. Some of the waste is contained in welded stainless steel containers or metal drums and stored within concrete cylinders. Degraded radioactive organic solvents and tritiated pump oil have been stored in 22 underground storage tanks in the old burial ground. In addition, two areas of the old burial ground were used for incineration of solvents.

The burial ground complex, comprising the old burial ground, solvent storage tanks S01-S22, and portions of the LLRWDF, is monitored by the following:

Burial Ground Expansion (E-Area Vaults)-This site is located in the northern section of E Area and is monitored by the BGX well series.

Hazardous Waste/Mixed Waste Disposal Facility-This site is northwest of the burial ground expansion and is monitored by the HMD well series.

Old Burial Ground-The old burial ground is in the southern portion of E Area and is monitored by wells in the $B G$ and $B G O$ well series.

Radioactive Waste Burial Ground-The LLRWDF, which includes the mixed waste management facility (MWMF), is monitored by wells in the BGO well series.

\section{Tank Farms}

Liquid radioactive wastes are stored and processed at the tank farms, which comprise subsurface tanks containing high-level aqueous radioactive wastes in the form of sludges, supernatant liquid of varying salt concentrations, and saltcake. Approximately 129 million liters of waste are stored in the tanks.

The high-level liquid waste volume is reduced in the tank farm evaporators. Certain tanks are used for pretreatment of the wastes before they are processed at the DWPF into saltstone (low-level waste) or a glass form (high-level waste). As described earlier, saltstone manufacturing and disposal is ongoing; vitrification was tested during 1995, and the DWPF began production operations in 1996. Pretreatment processes at the tank farms include in-tank precipitation and extended sludge processing.

More information about the function of the tank farms may be found in previous sections of this chapter, including the discussions of the F-Area effluent treatment cooling water basin, the H-Area auxiliary pump pit, $S$ Area, the S-Area low-point pump pit, the DWPF, the Z-Area low-point drain tank, and the Z-Area saltstone manufacturing and disposal facility.

Because of restrictions on the disposal of purge water, monitoring wells at the tank farms are bailed and not purged.

F-Area Tank Farm-The F-Area tank farm comprises 22 subsurface tanks. In 1961, Tank 8 was overfilled, causing soil and possible groundwater contamination.

H-Area Tank Farm-The H-Area tank farm comprises 29 subsurface tanks. In 1960, Tank 16 leaked an unknown quantity (a few tens of gallons to a few hundred gallons) of waste into the soil. The tank's remaining waste was removed by 1972 .

Several other releases of waste from $\mathrm{H}$-Area tanks have occurred, including a spill of approximately 100 gallons at Tank 13 in 1983. In 1989, approximately 500 pounds of volume-reduced waste leaked from a transfer line at 
Tank 37. The leak sites have been cleaned up or stabilized to prevent the spread of contamination. Both the FArea and $\mathrm{H}$-Area sites are being monitored for gross alpha, nonvolatile beta, and tritium.

\section{Sanitary Landfill and Interim Sanitary Landfill}

The sanitary landfill began receiving waste from office, cafeteria, and industrial activities during 1974. Materials such as paper, plastics, rubber, wood, cardboard, rags, metal debris, pesticide bags, empty cans, carcasses, asbestos in bags, and sludge from the site's wastewater treatment plant are placed in unlined trenches and covered daily with soil or a fabric substitute. The original section of the landfill and its southern expansion, with a total area of approximately 54 acres, have been filled. Operations at the portion of approximately 16 acres known as the northern expansion, or the interim sanitary landfill, were discontinued in November 1994.

Sanitary landfills are intended to receive only nonradioactive, nonhazardous waste. However, until October 1992, some hazardous wastes (specifically, solvent-laden rags and wipes used for cleaning, decontamination, and instrument calibration) were buried in portions of the original 32-acre landfill and its southern expansion.

\section{Sludge Application Sites}

These sites originally were the subject of a research program using domestic sewage sludge to reclaim borrow pits and to enhance forest productivity at SRS. In 1980, sludge was applied to the following application sites: K Area, Kato Road, Lower Kato Road, Orangeburg, PAR Pond, Road F, Sandy (Lucy), Second PAR Pond Borrow Pit, and 40-Acre Hardwood. After sludge was applied to the sites, hardwoods and pines were planted to quantify the effectiveness of the sludge as a fertilizer and soil conditioner.

Sludge from Aiken and Augusta municipal wastewater treatment plants was applied to the following sites: F Area, H Area, Kato Road, Lower Kato Road, Orangeburg, Road F, Sandy (Lucy), Second PAR Pond Borrow Pit, and 40-Acre Hardwood. Wastewater sludge was applied to the K Area and PAR Pond sites in 1981 and 1988. Revegetating of the sites is continuing.

In November 1993, groundwater monitoring was discontinued at the Kato Road, Lower Kato Road, Orangeburg, Road F, Sandy (Lucy), and 40-Acre Hardwood sites because they have not received applications of sewage sludge since 1981, and historical monitoring results show no impact from sludge applications. Monitoring was canceled after first quarter 1994.

\section{H-Area Sanitary Sludge Land Application Site}

Sewage sludge from SRS sanitary wastewater treatment plants was disposed of at this 13-acre site southeast of H Area from November 1990 to May 1992.

\section{K-Area and PAR Pond Sludge Land Application Sites (Formerly K-Area Borrow Pit and PAR Pond Borrow Pit Sites)}

In 1981, sludge from Aiken and Augusta municipal wastewater treatment plants was applied to the K-Area and PAR Pond borrow pits. In 1988, the N-Area sanitary sewage sludge lagoon was closed, and the lagoon sludge was applied to the K Area and PAR Pond borrow pits. In 1989, the K-Area location (now called the K-Area sludge land application site) was declared a RCRAVCERCLA unit because of the presence of chlordane in the lagoon sludge applied to the site.

\section{Other Sites}

\section{B-Area Gas Station}

Elevated benzene, which could be the result of old underground gasoline or diesel storage tanks, has been detected near B Area. EMS has inspected the area and believes there may be two underground storage tanks southeast of B Area. The first suspected tank appears to be at an abandoned gas station between Kato Road and Road C-2. The second appears to be an old diesel tank in front of a storage and laboratory facility. 


\section{Baseline Hydrogeologic Investigation Observation Well Clusters}

Wells in the $\mathrm{P}$ series that provide baseline hydrogeologic investigation data are located in numerous locations across SRS.

\section{Chemicals, Metals, and Pesticides Pits}

The chemicals, metals, and pesticides pits were used from 1971 to 1979 to dispose of oil in drums, organic solvents, and small amounts of pesticides and metals. In 1984, the pits were excavated to form two trenches, backfilled, and capped. During excavation, most of the contaminated material (liquid in original drums, free liquid placed in drums during excavation, and contaminated soil) was moved to the hazardous waste storage facility.

\section{D-Area Oil Disposal Basin}

The D-Area oil disposal basin was constructed in 1952 and received waste oil products from D Area that were unacceptable for incineration in the powerhouse boilers. These waste oils may have contained hydrogen sulfide, chlorinated organics, or other chemicals. In 1975, the oil basin was removed from service and backfilled with soil.

\section{Interim Waste Technology Site Characterization Wells}

Characterization wells monitor interim waste technology sites $B, L, Q$, and $P$.

\section{K-Area Diesel Tank Spill}

Following the discovery in 1989 of a leaking buried diesel supply line, most of the diesel-contaminated soil was removed from this area except where continued excavation would have jeopardized the structural integrity of an underground storage tank.

\section{L-Area Acid/Caustic Basin and L-Area Oil and Chemical Basin}

From 1961 to 1979 , the L-Area oil and chemical basin received small quantities of radioactive oil and chemical waste that could not be discharged to effluent streams, regular seepage basins, or the 200 Areas' waste management systems. The waste came from throughout SRS, primarily from the reactor areas and the contaminated-equipment workshop through a pipeline known to have leaked. The basin has been inactive since 1979.

\section{M-Area Recovery Welis}

The RWM well series identifies the M-Area recovery wells. The first wells were installed in 1982 and 1983, with pumps added in 1985. Additional wells were installed in 1985, 1990, 1993, and 1996. The RWM wells pump contaminated groundwater to air strippers, which remove volatile organic compounds from the water before it is returned to the ground.

\section{Miscellaneous Chemical Basin}

The miscellaneous chemical basin, in operation by 1956 , was closed and graded in 1974. No records document the materials disposed of at this location. However, soil gas investigations revealed volatile organics in the nearsurface soils at the basin. It is assumed that the site was used for disposal of waste solvents, liquid chemical wastes, and possibly waste oil. The basin is inactive.

\section{Motor Shop Oil Basin}

This unlined basin was placed in service in 1977 to receive liquid effluent from the Motor Shop, including trace quantities of engine oil, grease, kerosene, ethylene glycol, and soap. All waste passed through an oil skimmer prior to discharge into the basin. All discharges to the basin were terminated in August 1983. The basin is inactive but collects rainwater during periods of heavy precipitation.

\section{N-Area Diesel Spill Hazardous Waste Storage Facility}

The tanks have been filled with inert material, and the pipelines have been disconnected at this site. 


\section{N-Area Fire Department Training Facility}

The fire department training facility, also known as the $\mathrm{N}$-Area burnable-oil basin, is a shallow pit surrounded by an 18-inch-high asphalt dike. It was used from 1979 to 1982 by the SRS Fire Department to train personnel in the use of firefighting equipment. After this time, the area was excavated and backfilled.

\section{N-Area Hydrofluoric Acid Spill}

It is uncertain whether a spill occurred at the hydrofluoric acid spill area or if contaminated soil or containers were buried there. The spill or burial occurred prior to 1970 , and an identification sign is the only evidence that material was released.

\section{Production Wells}

The PW series wells are production wells scattered across SRS.

\section{Road A (Baxley Road) Chemical Basin}

The Road A chemical basin is reported to have received miscellaneous radioactive and chemical aqueous waste, but no records of the materials disposed of at the basin are available. The basin was closed and backfilled in 1973. The BRD well series is being monitored for environmental-screening constituents only.

\section{Silverton Road Waste Site}

The Silverton Road waste site, south of Silverton Road, was used for disposal of metal shavings, construction debris, tires, drums, tanks, and miscellaneous other items. The startup date is unknown, and no records of waste disposal activities were kept. Operations at this location ended in 1974, and the waste material is covered with soil and vegetation.

\section{TNX Burying Ground}

The TNX burying ground was created to dispose of debris from an experimental evaporator that exploded at T Area in 1953. The buried material included contaminated conduit, tin, drums, structural steel, and depleted uranium. Although most of this material was excavated and sent to the LLRWDF between 1980 and 1984, an estimated $27 \mathrm{~kg}$ of uranyl nitrate remains buried at this location. See the New TNX Seepage Basin section for more information on the unit. 


\section{Glossary}

Also see p. B-1 for abbreviations and qualifiers used in the results tables in Appendix B.

2,4-D. 2,4-Dichlorophenoxyacetic acid.

absolute difference. The unsigned result of the subtraction of any two numbers.

accuracy. The degree of agreement between an observed value and an accepted reference value or a measure of the over- or underestimation of reported concentrations.

advisory range. A range of acceptable analytical results established by the provider of known samples.

aerated sample. Groundwater sample supplied or charged with air. Aeration can occur naturally or during well pumping.

aliquot. A portion of a sample being used for analysis.

analyte. Analyzed constituent.

analytical detection limit. The lowest reasonably accurate concentration of an analyte that can be detected. This value varies depending on the method, instrument, and dilution used.

APHA. American Public Health Association.

Appendix IX. A list of constituents specified by Appendix IX in the Code of Federal Regulations, Title 40, Part 264 (EPA, 1991d). Analysis for Appendix IX constituents is required by the Resource Conservation and Recovery Act (RCRA) under specified conditions.

associated samples. Samples analyzed by a laboratory in the same batch with field or laboratory blanks.

ASTM. American Society for Testing and Materials.

bail. To remove water from a well by lowering a container into the water, allowing it to fill with water, and removing it from the well.

blank. Aliquot of deionized water generated by laboratory or sampling personnel and analyzed like a groundwater sample. See equipment blank, field blank, laboratory blank, and trip blank.

blank spike. An organic-free water sample spiked with target analytes, extracted, and analyzed with the regular samples for organic parameters to monitor the performance of all steps in the analysis process.

blind replicate. A second sample taken from a well at the same time as the primary sample and sent to the laboratory for analysis as an unknown.

BNA. Base/neutral and acid extractables. Groups of organic compounds analyzed as part of the Appendix IX and Priority Pollutants suites; also, a group of compounds that can be analyzed by EPA Method 8270.

Bq/L. Becquerels per liter. A measurement of radioactivity.

cation. Positively charged ion.

CERCLA. Comprehensive Environmental Response, Compensation, and Liability Act, commonly known as Superfund. 
certified value. The known concentration of an analyte in a referenced sample.

CFR. Code of Federal Regulations. Sections of this annual document contain EPA standards and regulations for environmental monitoring.

chain-of-custody record. A form that documents the collection, transport, analysis, and disposal of well samples.

common analyses. Common parameters tested for, and generally found, in drinking water.

comparability. An evaluation made by confirming that the laboratories used the same standardized procedures for sample preparation and analysis, that the reporting units are the same, and that similar detection and quantitation limits were obtained.

completeness. An evaluation based on a comparison of the wells scheduled for sampling to the wells sampled, atso a comparison of the requested analyses to the analytical data received.

deionized water. Water from which all charged species or ionizable organic and inorganic salts have been removed.

detection limit. See analytical detection limit.

dilution factor. The mathematical factor by which a sample is diluted to bring the concentration of an analyte in the sample within the analytical range of an instrument (e.g., $1 \mathrm{~mL}$ sample $+9 \mathrm{~mL}$ solvent $=1: 10$ dilution, or a dilution factor of 10 ).

DL. See analytical detection limit.

DNAPL. Dense nonaqueous phase liquid.

DOE. U.S. Department of Energy.

drinking water standards. Federal primary and secondary drinking water standards, as set forth by the EPA.

duplicate. Duplicate sample; an aliquot of a primary sample.

duplicate result. A result obtained from identical analyses performed on more than one aliquot of a primary sample.

DWS. See drinking water standards.

E. A code letter used in the analytical data tables that signifies exponential notation (e.g., $3.4 E+03=3.4 \times 10^{3}=$ $3,400)$.

EM. EPD/EMS Laboratory at SRS.

EMAX Laboratories. EMAX Laboratories, Inc., of Torrance, CA.

EMS. The Environmental Monitoring Section of the Environmental Protection Department at SRS.

EMS code. See qualifier.

Environmental Physics. Environmental Physics, Inc., of Charleston, SC (subcontractor for General Engineering).

environmental-screening analyses. A group of analyses that forms the core of the EPD/EMS Groundwater Monitoring Program each quarter. See the Sample Scheduling section of this report for a complete list of constituents. 
EPA. U.S. Environmental Protection Agency.

EPD. Environmental Protection Department at SRS.

EPD/EMS. Environmental Protection Department's Environmental Monitoring Section at SRS.

\section{EQL. See estimated quantitation limit.}

equipment blank. A sample of deionized water that is opened at the sampling location and poured or pumped through the sampling device. Equipment blanks are used to identify possible contaminants in the sampling equipment.

\section{ES. See QST Environmental.}

estimated quantitation limit (EQL). The lowest concentration reliably achieved within specified limits of precision and accuracy during routine laboratory operating conditions. The EQL is generally $5 \times$ to $10 \times$ the method detection limit (MDL); however, it may be nominally chosen within these guidelines to simplify data reporting. For many analytes, the EQL analyte concentration is selected as the lowest nonzero standard in the calibration curve.

\section{EX. See EMAX Laboratories.}

Fibers/L. Fibers per liter. A unit of measurement for asbestos.

field blank. A sample container of deionized water sent to a laboratory under an alias as a quality control check.

field qualifier. See sample interference field qualifier. Due to space limitations, sample interference field qualifiers are referred to as field qualifiers in the analytical results tables in Appendix B.

flagging criteria. Criteria established to help determine the relative concentration and testing frequency for analytes. See the Flagging Criteria section of this report for further information.

functional guideline code. See qualifier.

gamma PHA. A group of analyses performed to determine activities of gamma-emitting radionuclides.

GC. See Gulf Coast.

GC VOA. Gas chromatographic volatile organics analyses. Also used to refer to a group of volatile organic compounds that can be analyzed by gas chromatography.

GCMS VOA. Gas chromatograph/mass spectrometer volatile organics analyses. Also used to refer to a group of volatile organic compounds analyzed by gas chromatography and mass spectrometry methods.

\section{GE. See General Engineering.}

General Engineering. General Engineering Laboratories of Charleston, SC.

General Engineering Laboratories Mobile Laboratory. The Mobile Laboratory, associated with General Engineering Laboratories of Charleston, SC.

\section{GP. See Environmental Physics.}

Gulf Coast. Gulf Coast of Chicago, IL. (owned by Recra).

halogen. Any of the elements of the halogen family, which consists of fluorine, chlorine, bromine, iodine, and astatine. 
herbicides/pesticides. A suite of analyses. See the Sample Scheduling section of this report for further information.

holding time. The length of time during which an analysis of a sample can be reliably performed. Holding times vary depending on which constituents are being analyzed.

interlaboratory comparisons. Comparisons conducted between two or more laboratories.

intralaboratory comparisons. Comparisons conducted within a single laboratory.

ion. An isolated electron or positron or an atom or molecule that has acquired a net electric charge by the loss or gain of one or more electrons.

laboratory blank. Deionized water or solvent sample generated by the laboratory. One blank is analyzed with each batch of samples as an in-house check of analytical procedures and equipment.

laboratory control sample. A deionized water sample that is spiked with the target analyte, digested, and analyzed with the regular samples for inorganic parameters to monitor the performance of all steps in the analysis process.

\section{MA. See Microanalytical Laboratories.}

major ions. A group of analyses performed in the EPD/EMS Groundwater Monitoring Program to determine the concentrations of calcium, magnesium, potassium, and silica ions and the alkalinity of a sample.

matrix spike. A known quantity of a target analyte added to at least $5 \%$ of the samples prior to sample preparation to evaluate the effect of the sample matrix on the analytical procedure.

\section{See method detection limit.}

mean. The arithmetic mean; a single number that typifies a set of numbers.

method detection limit (MDL). A reproducible analyte- and method-specific detection limit: the minimum concentration of a substance that can be measured and reported with $99 \%$ confidence that the analyte concentration is greater than zero.

mg/L. Milligrams per liter.

$\mu \mathrm{Ci}$. Microcurie; unit of radioactivity equivalent to $3.7 \times 10^{4}$ disintegrations per second.

$\boldsymbol{\mu C} \mathbf{i} / \mathbf{m L}$. Microcuries per milliliter.

$\mu \mathrm{g} / \mathrm{L}$. Micrograms per liter.

$\boldsymbol{\mu S} / \mathrm{cm}$. Microsiemens per centimeter, equivalent to micromhos per centimeter. The unit of conductance across two points, used as the measure of specific conductance in analytical data tables.

Microanalytical Laboratories. Microanalytical Laboratories, Inc., of Gainesville, FL (subcontractor for QST Environmental).

\section{See General Engineering Laboratories Mobile Laboratory.}

modifier. See qualifier.

MRD. Mean relative difference. See the Quality Control Samples section of this report for further information. 
msl. Mean sea level.

NTU. Nephelometric turbidity units. The standard unit of turbidity measurement.

null hypothesis. A statement, which can be tested statistically, of no difference in a characteristic of a population or distribution.

organic. A chemical compound based on carbon chains or rings and containing hydrogen with or without oxygen, nitrogen, or other elements.

PCB. Polychlorinated biphenyl.

pCi. Picocurie; a unit of radioactivity equivalent to $3.7 \times 10^{-2}$ disintegrations per second.

pCi/L. Picocuries per liter.

pCi/mL. Picocuries per milliliter.

piezometer. An instrument used to measure the potentiometric surface of groundwater. Also, a well designed for this purpose.

plume. A volume of contaminated air or water originating at a point-source emission (e.g., a smokestack) or a waste source (e.g., a hazardous-waste disposal site).

potentiometric surface. The surface to which water in an aquifer would rise by hydrostatic pressure if unconfined.

precision. A measure of the repeatability of a measurement, evaluated from the results of duplicate samples and splits.

primary laboratory. A laboratory having a contract with EPD/EMS to perform a specific set of analyses; a primary laboratory may subcontract this work to other laboratories.

purge. To remove water from a well prior to sampling, generally by pumping or bailing. Under the EPD/EMS Groundwater Monitoring Program, two well volumes generally are purged before sampling.

QA. Quality assurance.

QC. Quality control.

QST Environmental. QST Environmental, of Gainesville, FL.

qualifier. A code used to convey additional information about an analytical result. Also called a modifier. Specific types include functional guideline codes, STORET codes, and EMS codes. See Appendix B for additional information.

radioisotopes. Radioactive isotopes.

radionuclide. A nuclide at an unstable, high-energy level that seeks a more stable, low-energy level by emitting particles of energy. Through these emissions, the nuclear configuration decays to simpler nuclides.

RCRA. See Resource Conservation and Recovery Act.

RCRA site. Solid-waste management unit under RCRA regulation.

RDL. See reference detection limit. 
Recra LabNet Philadelphia. Recra LabNet Philadelphia, of Lionville, PA.

reference detection limit (RDL). The detection limit chosen to allow comparison of several analyses with different detection limits. For the purposes of this report, the individual detection limits of at least $90 \%$ of the analyses are less than the reference detection limit. See the Quality Control Samples section of this report for further information.

relative percent difference (RPD). A commonly used estimate of precision when only two samples are available. Precision is the agreement among a set of replicate measurements without assumption of the true value. Precision is estimated by means of duplicate analyses.

replicate. Replicate sample. Used in this report to mean only those duplicate samples sent to the laboratory as unknowns. See blind replicate.

representativeness. The quality of exhibiting the average properties of the population being sampled.

Resource Conservation and Recovery Act (RCRA). Federal legislation that regulates the transport, treatment, and disposal of solid and hazardous wastes.

RFI Program. RCRA Facility Investigation Program. EPA-regulated investigation of a solid-waste management unit with regard to its potential impact on the environment.

RFVRI Program. RCRA Facility Investigation/Remedial Investigation Program. At SRS, an expansion of the RFI Program that includes CERCLA and hazardous-substance regulations.

\section{RPD. See relative percent difference.}

run date. The calendar date denoting when an analysis is performed.

sample interference field qualifier. See also field qualifier. This describes interferences encountered during sample collection that could affect analytical results. It is used to qualify analytical data based on field condition.

sample quantitation limit (SQL). The sample-specific EQL, which is the EQL multiplied by factors of concentration, dilution, aliquot size, and percent solids.

sample-specific EQL (sSEQL). The EQL multiplied by factors of concentration, dilution, aliquot size, and percent solids. Also called the SQL.

sample-specific MDL (ssMDL). The MDL multiplied by factors of concentration, dilution, aliquot size, and percent solids. For radiological analyses it is known as the sample-specific minimum detectable concentration.

sampling device. Anything used in sampling, especially portable (nondedicated) pumps and bailers. Possible source of sample contamination if not cleaned thoroughly between uses.

SCDHEC. South Carolina Department of Health and Environmental Control.

seepage basin. An excavation that receives wastewater. Designed to prevent overflow or surface runoff.

settling basin. A temporary holding basin (excavation) that receives wastewater.

significance of probability. The probability of observing a statistical value as significant as, or more significant than, the value actually observed.

site custodian. WSRC employee responsible for a site being monitored.

SQL. See sample quantitation limit. 
SRL. Savannah River Laboratory at SRS; now Savannah River Technology Center (SRTC).

SRP. Savannah River Plant; now Savannah River Site (SRS).

SRS. Savannah River Site.

SRTC. Savannah River Technology Center.

STORET. EPA national database for storage and retrieval of water quality information and monitoring data; some of the qualifiers listed in the Analytical Results section of this report (Appendix B) are based on STORET codes.

STORET code. See qualifier.

surrogate. An organic compound similar in composition and test performance to one of the analytes of interest; known quantities are used in an analysis as a quality assurance measure.

tank farm. An installation of interconnected underground tanks used for storage of high-level radioactive liquid wastes.

Thermo NUtech. Thermo NUtech, of Oak Ridge, TM (subcontractor for Recra LabNet Philadelphia and QST Environmental).

\section{TL. See Triangle Laboratories.}

TM. See Thermo NUtech.

TOC. Top of casing. The elevation of the casing at the top of a well; used as a reference for water-level measurements.

Triangle Laboratories. Triangle Laboratories, Inc., of Durham, NC (subcontractor for Environmental Science \& Engineering).

trip blank. A sample container of deionized water that is transported to the well sample location, treated as a well sample, and sent to the laboratory for analysis; trip blanks are used to check for contamination resulting from transport, shipping, and site conditions.

t-test. Statistical method used to determine if the means of groups of observations are equal.

turbidity. A measure of the concentration of sediment or suspended particles in solution.

U. Unclassified.

USDWS. U.S. Public Health Service drinking water standard.

volatile organic compounds. A broad range of organic compounds, commonly halogenated, that vaporize at ambient, or relatively low, temperatures (e.g., acetone, benzene, chloroform, and methyl alcohol).

\section{WA. See Recra LabNet Philadelphia.}

well volume. The volume of water between the well water surface and the bottom of the screen; the volume of water standing inside the well casing.

wellhead. The top of a well.

WSRC. Westinghouse Savannah River Company. 


\section{References}

American Public Health Association, American Water Works Association, and Water Pollution Control Federation. 1985. Standard Methods for the Examination of Water and Wastewater, 16th edition. Washington, DC.

American Society for Testing and Materials. 1992. Annual Book of ASTM Standards, Volume 11.02. Philadelphia, PA.

Environmental Protection Agency. 1977. National Interim Primary Drinking Water Regulations, EPA-570/9-76-003. Washington, DC.

Environmental Protection Agency. 1980. Prescribed Procedures for Measurement of Radioactivity in Drinking Water, EPA-600/4-80-032 (method 901.1). Cincinnati, OH.

Environmental Protection Agency. 1982. Methods for Organic Chemical Analysis of Municipal and Industrial Wastewater, PB83-201798 (method 625). Cincinnati, OH.

Environmental Protection Agency. 1983. Methods for Chemical Analysis of Water and Wastes, PB84-128677 (methods 200.7, 282.2, 310.1, 350.1, 351.2, 413.1, 418.1, and 420.1). Cincinnati, $\mathrm{OH}$.

Environmental Protection Agency. 1986a. RCRA Ground-Water Monitoring Technical Enforcement Guidance Document, OSWER-9950.1. Washington, DC: Office of Waste Programs Enforcement.

Environmental Protection Agency. 1986b. Test Methods for Evaluating Solid Waste, Volumes 1A, 1B, and 1C, third edition, SW-846 (methods 6010, 8080, 8150, 8240, 8270, 8280, 9020, 9030, and 9060). Washington, DC.

Environmental Protection Agency. 1988a. Laboratory Data Validation Functional Guidelines for Evaluating Organics Analyses. Washington, DC: Office of Solid Waste and Emergency Response.

Environmental Protection Agency. 1988b. Laboratory Data Validation Functional Guidelines for Inorganics Analyses, Draft. Washington, DC: Office of Solid Waste and Emergency Response.

Environmental Protection Agency. 1990. "National Primary and Secondary Drinking Water Regulations; Synthetic Organic Chemicals and Inorganic Chemicals; Proposed Rule" in Federal Register, July 25, 1990, pp.

30369-30448. Washington, DC.

Environmental Protection Agency. 1991a. "Guidelines Establishing Test Procedures for the Analysis of Pollutants" in Code of Federal Regulations, Title 40, Part 136, pp. 293-575. Washington, DC.

Environmental Protection Agency. 1991b. National Functional Guidelines for Organic Data Review, Draft. Washington, DC: Office of Solid Waste and Emergency Response.

Environmental Protection Agency. 1991c. "National Primary Drinking Water Regulations; Radionuclides; Proposed Rule" in Federal Register, July 18, 1991, pp. 33052-33127. Washington, DC.

Environmental Protection Agency. 1991d. "Standards for Owners and Operators of Hazardous Waste Treatment, Storage, and Disposal Facilities" in Code of Federal Regulations, Title 40, Part 264, App. IX, pp. 310-316. Washington, DC.

Environmental Protection Agency. 1991e. Test Method: The Determination of Inorganic Anions in Water by lon Chromatography Method 300.0, Revised August 1991. Cincinnati, OH.

Environmental Protection Agency. 1991f. USEPA Contract Laboratory Program, Statement of Work (CLP SOW) for Organics Analysis, Multi-Media, Multi-Concentration, ILM03.0. Washington, DC. 
Environmental Protection Agency. 1997a. "National Primary Drinking Water Regulations" in Code of Federal Regulations, pp. 288-432. Washington, DC.

Environmental Protection Agency. 1997b. "National Secondary Drinking Water Regulations" in Code of Federal Regulations, pp. 473-474. Washington, DC.

Keith, L.H. 1991. Environmental Sampling and Analysis: A Practical Guide. Chelsea, Ml: Lewis Publishers.

South Carolina Department of Health and Environmental Control. 1981. State Primary Drinking Water Regulations, R.61-58.5. Columbia, SC.

U.S. Department of Energy. 1986. Savannah River Plant Environmental Report for 1985, Volumes I and II, DPSPU-86-30-1. Aiken, SC.

U.S. Department of Energy. 1987. Savannah River Plant Environmental Report for 1986, Volumes I and II, DPSPU-87-30-1. Aiken, SC.

U.S. Department of Energy. 1988a. Geohydrology Program Report. Aiken, SC: Environmental Division, Savannah River Operations Office.

U.S. Department of Energy. 1988b. Savannah River Plant Environmental Report for 1987, Volumes I and II, DPSPU-88-30-1. Aiken, SC.

U.S. Department of Energy. 1989a. Geoscience Implementation Plan. Aiken, SC: Environmental Division, Savannah River Operations Office.

U.S. Department of Energy. 1989b. Savannah River Site Environmental Report for 1988, Volumes I and II, WSRCRP-89-59-1. Aiken, SC.

Westinghouse Savannah River Company. Environmental Geochemistry Group Operating Handbook, Section 1.800, "Analytical Data Qualification," October 19, 1998. ESH-EMS-970437. Aiken, SC. 


\section{Appendix A. Water-Level Data}

During first quarter 1999, water-level measurements were obtained for hydrogeologic projects. Most of the data presented on the following pages were obtained as concurrent data for hydrogeologic interpretation in the A/M and $\mathrm{F} / \mathrm{H}$ areas. Only water levels were measured for this project; no field tests of water characteristics were conducted. RCS Corporation of Aiken, SC, collected the data. 


\section{NOTES}
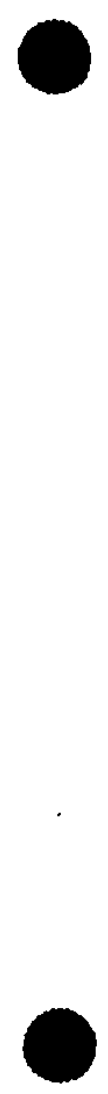


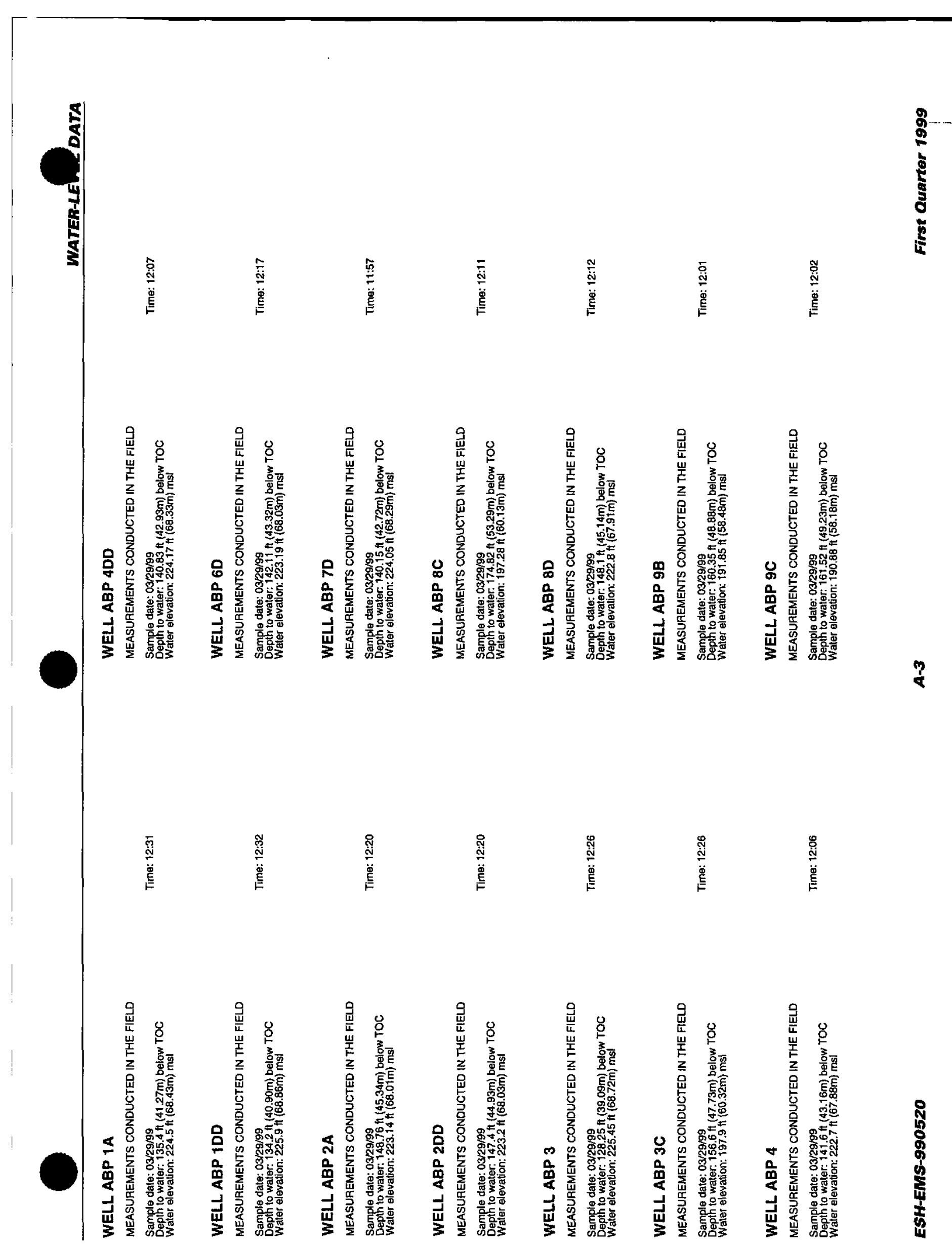




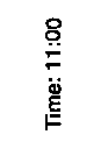

$\stackrel{8}{\stackrel{8}{+}}$

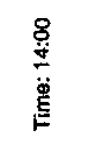

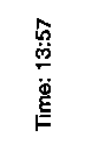

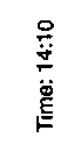

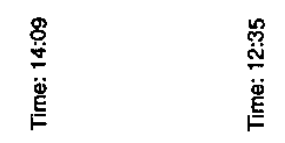

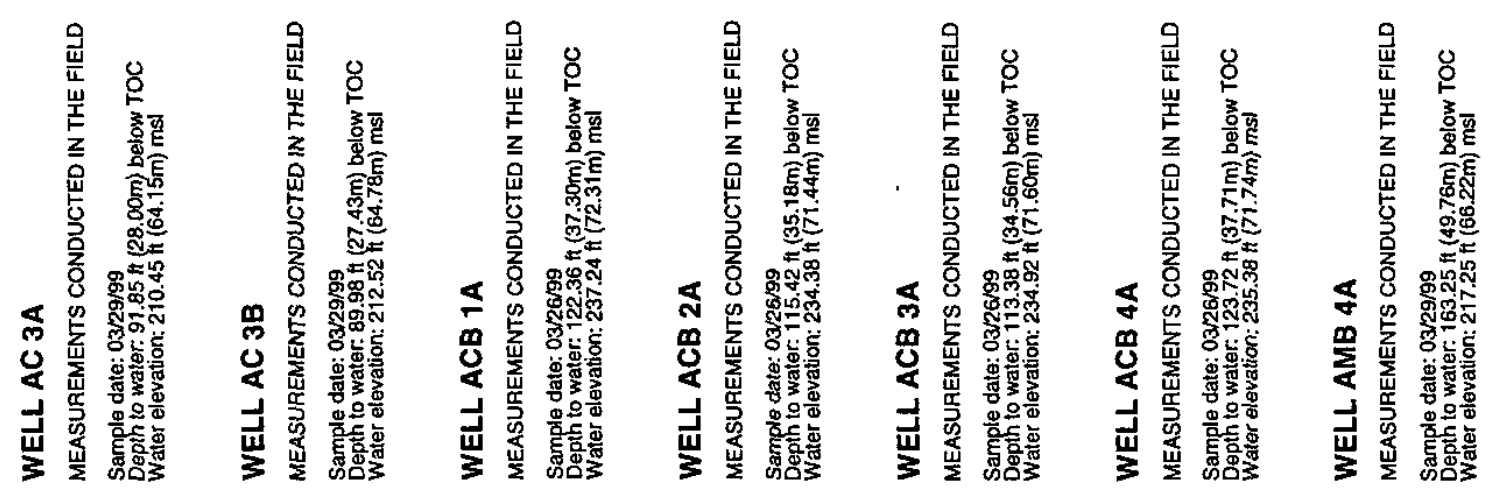

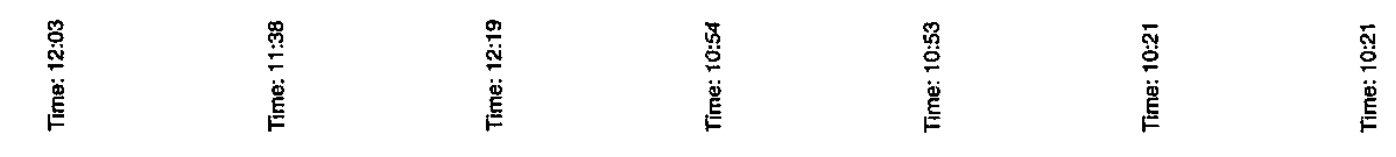

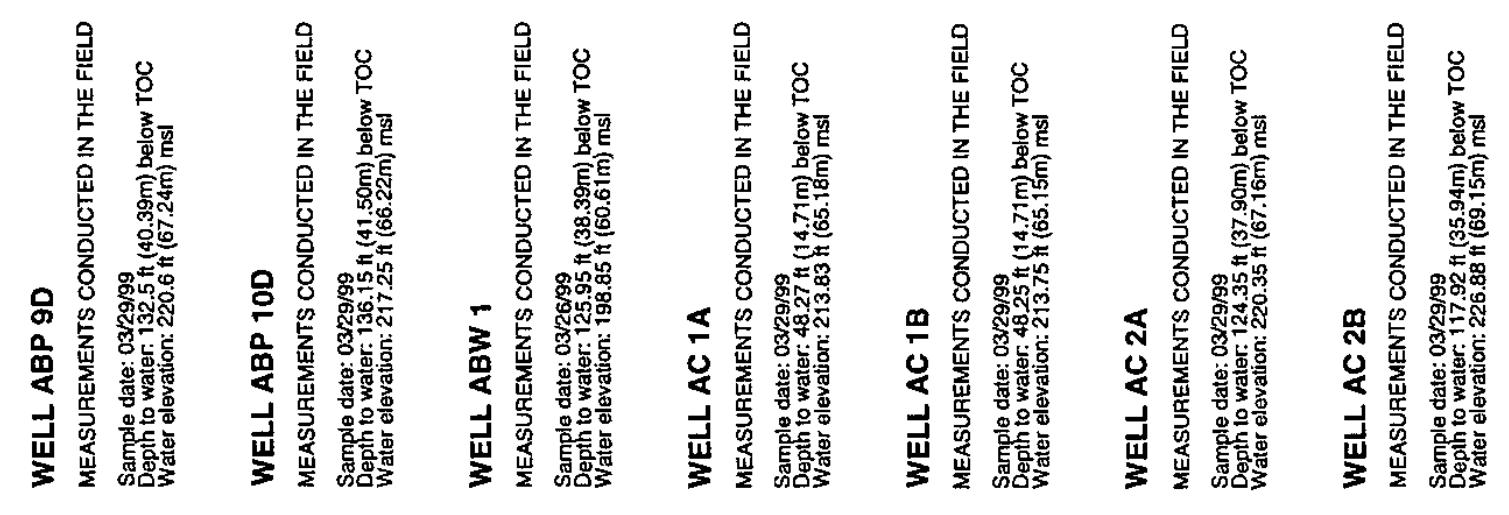

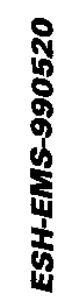




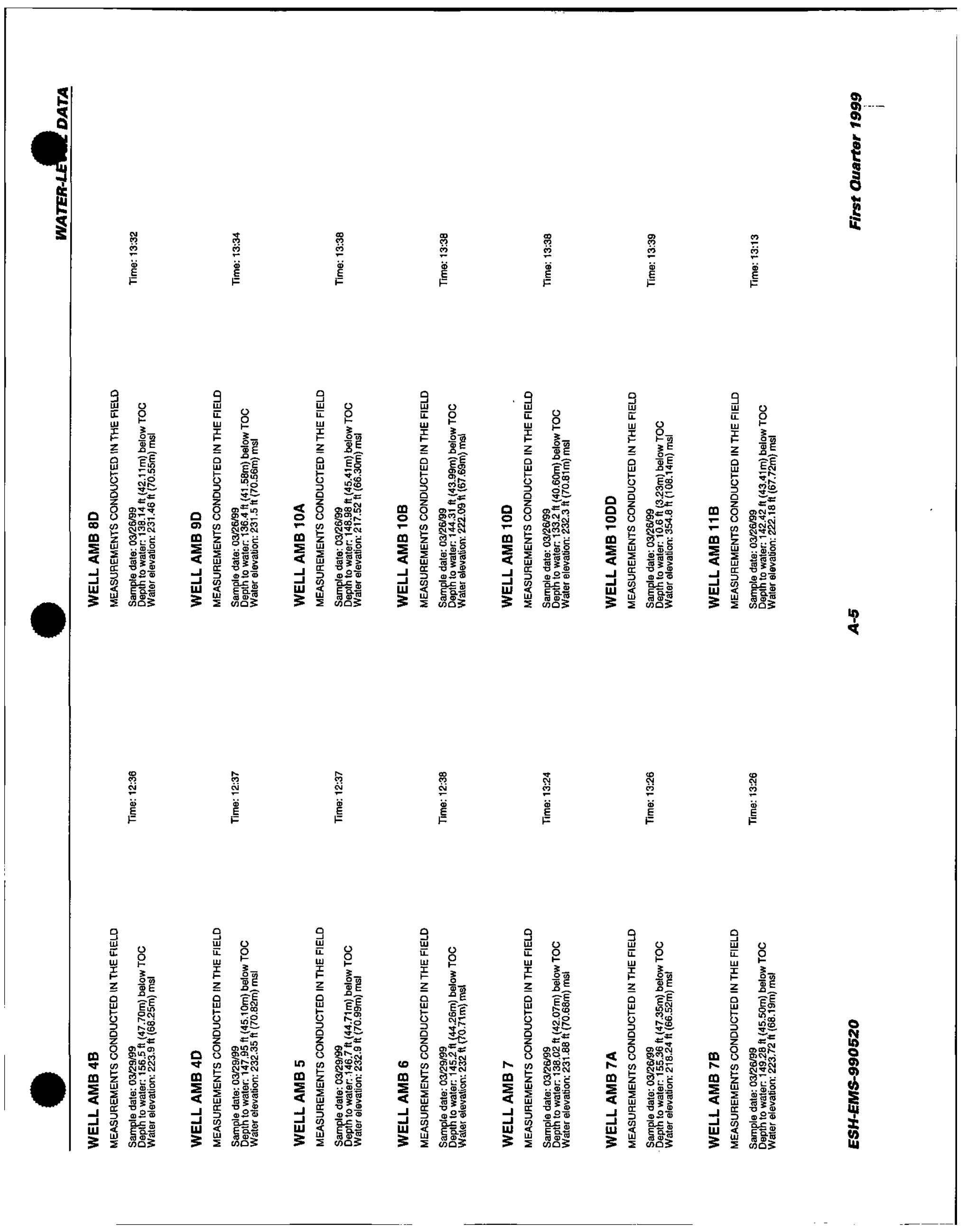


\begin{tabular}{l}
$\mathbf{8}$ \\
$\mathbf{5}$ \\
$\mathbf{1}$ \\
$\mathbf{4}$ \\
\hdashline \\
$\mathbf{4}$ \\
$\mathbf{4}$ \\
$\mathbf{5}$
\end{tabular}

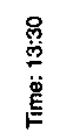

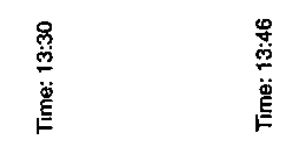

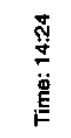

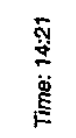

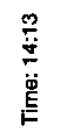

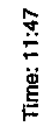

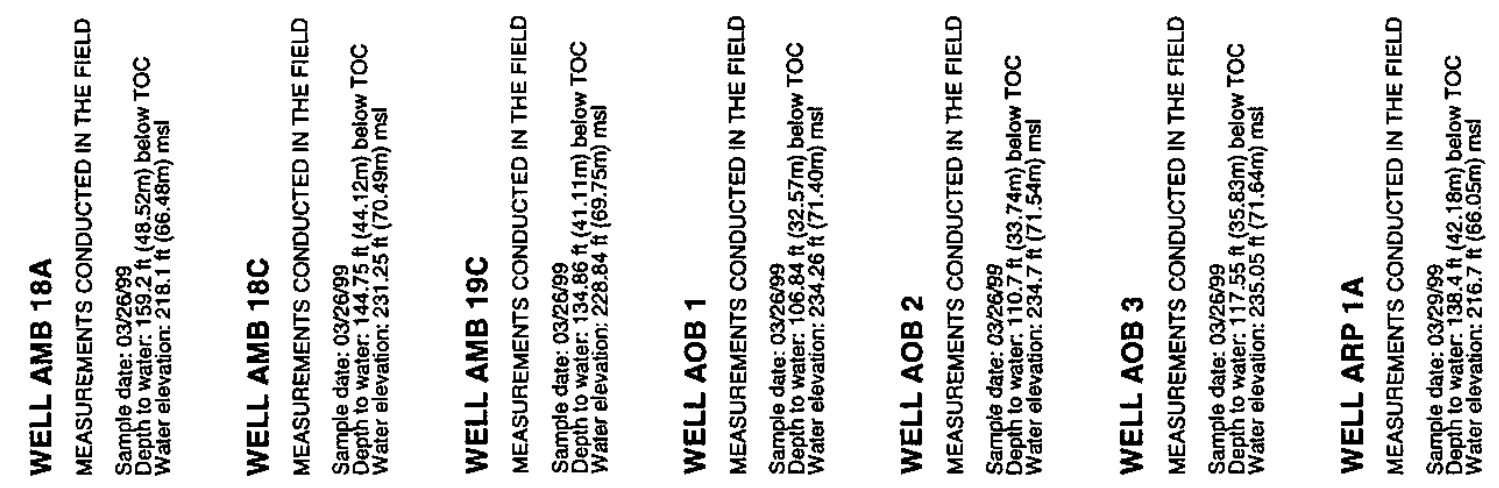

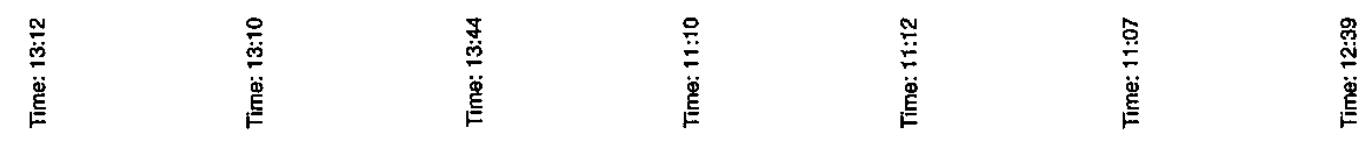

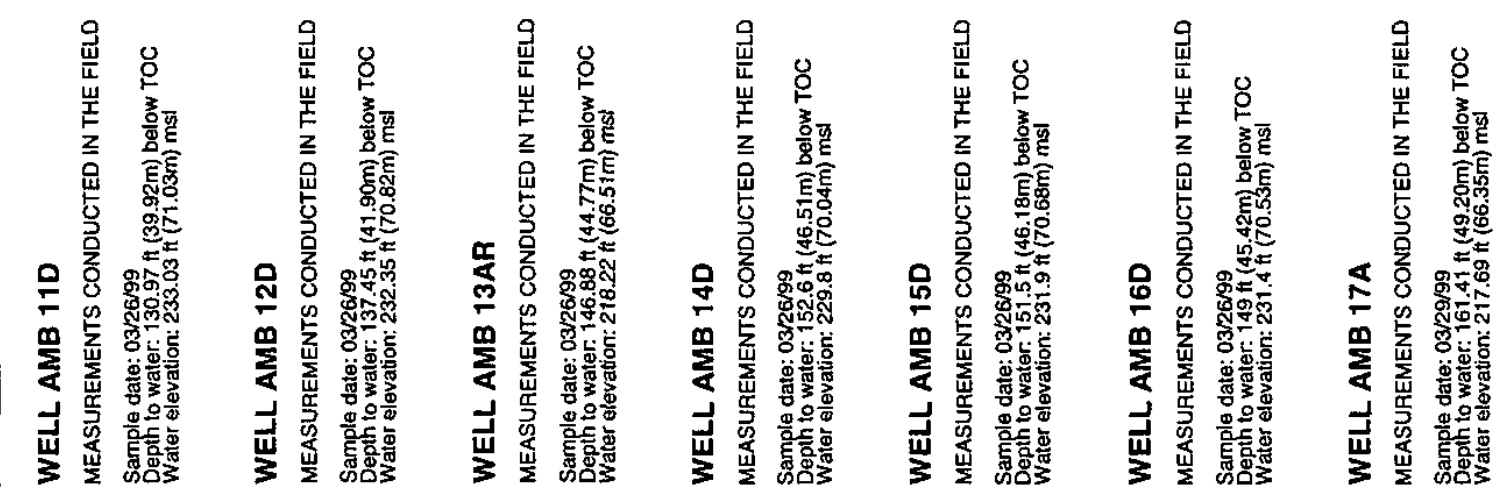

$q$
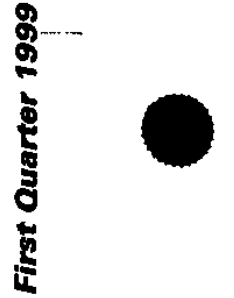


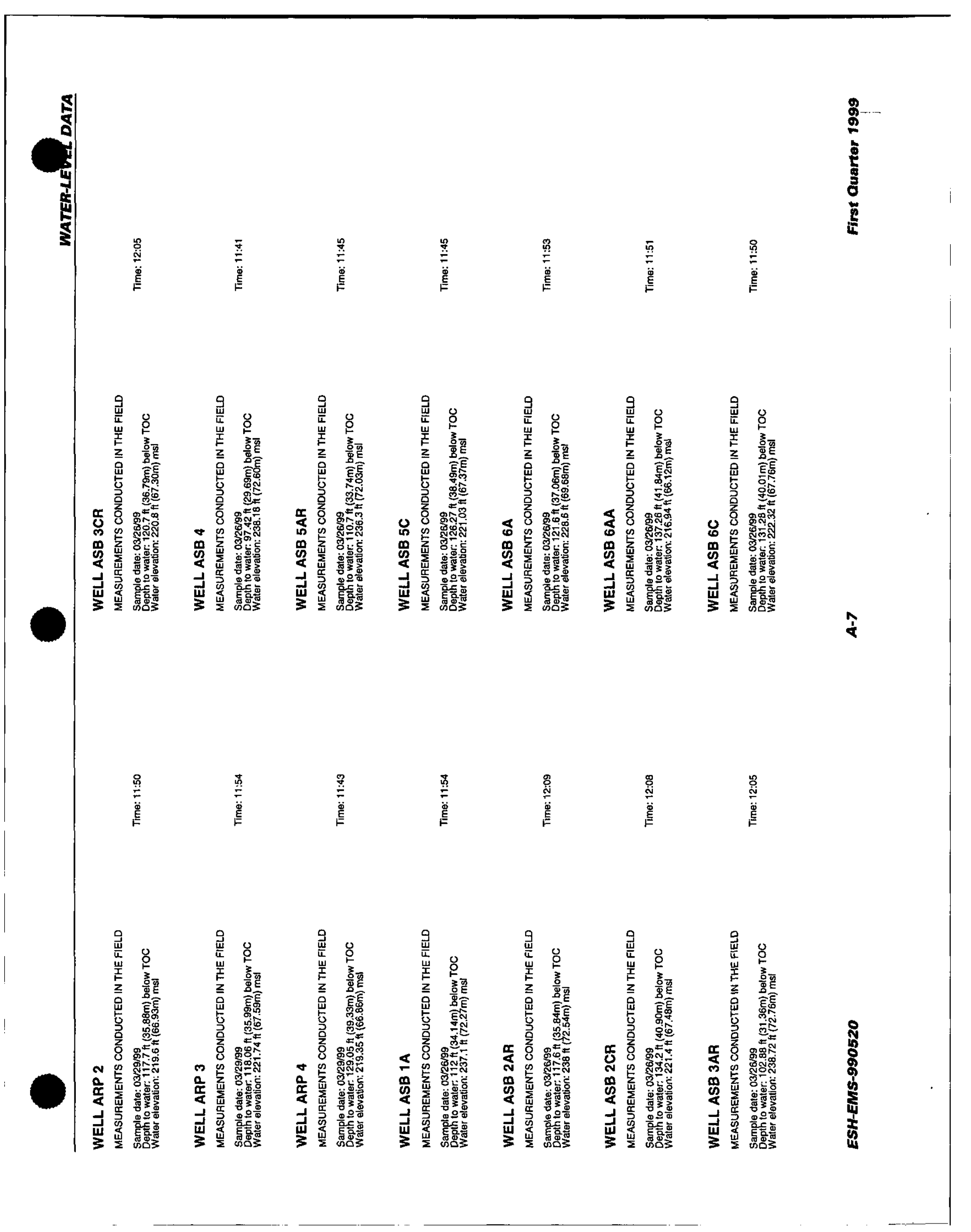




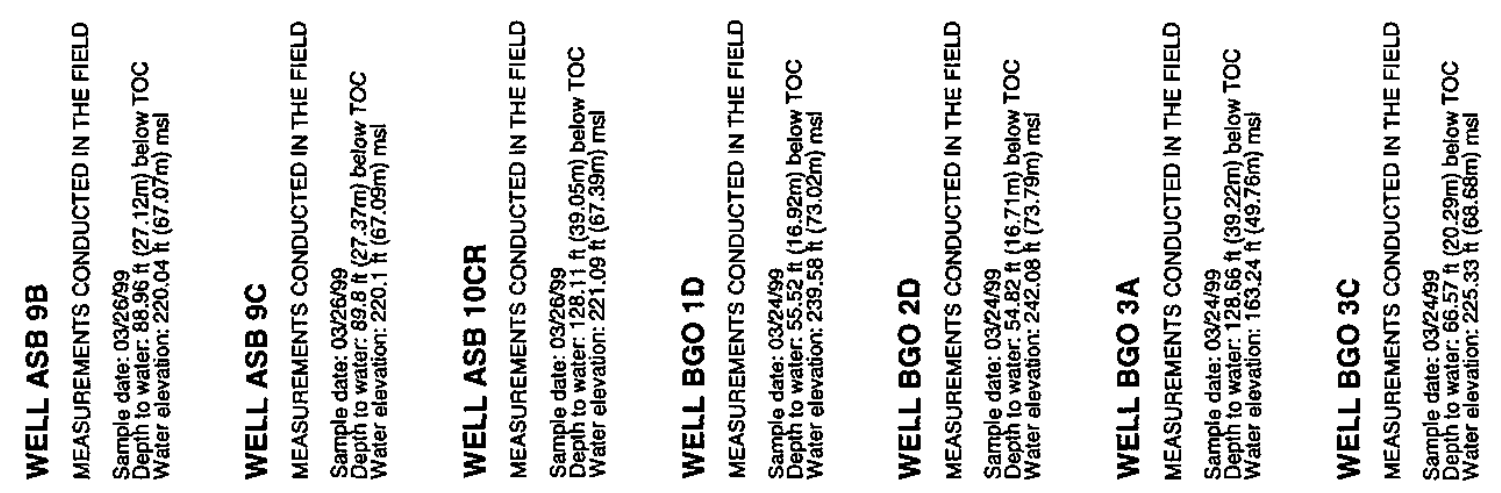

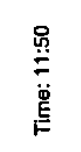
岕
畩

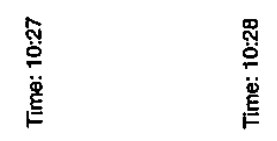

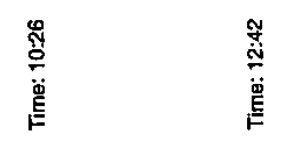

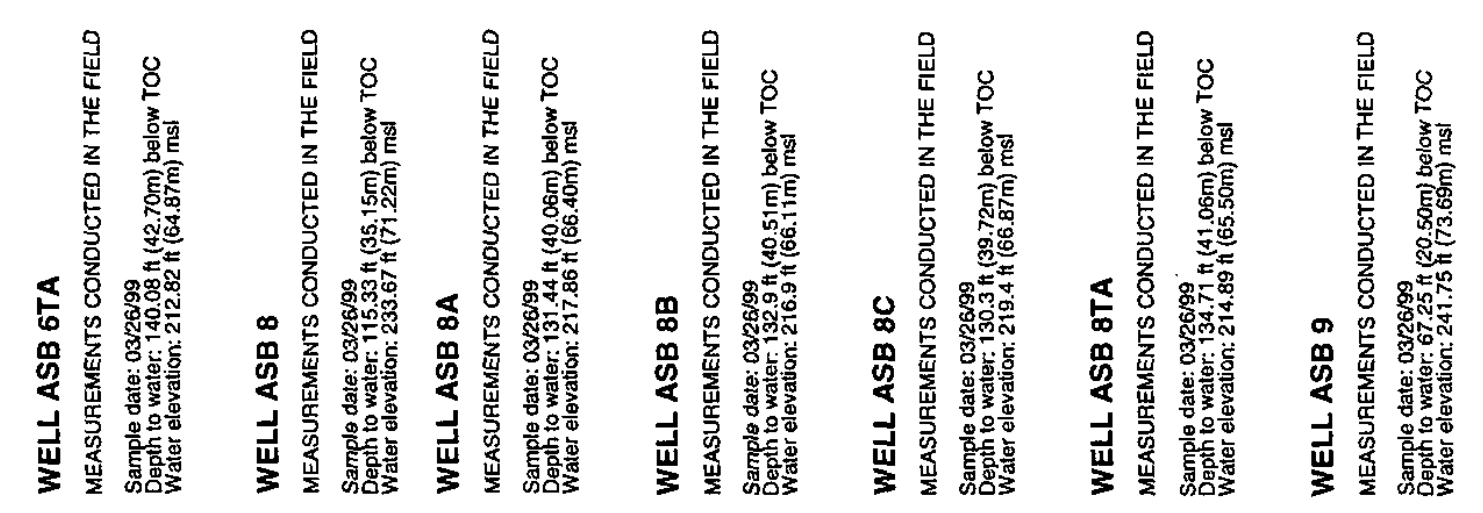




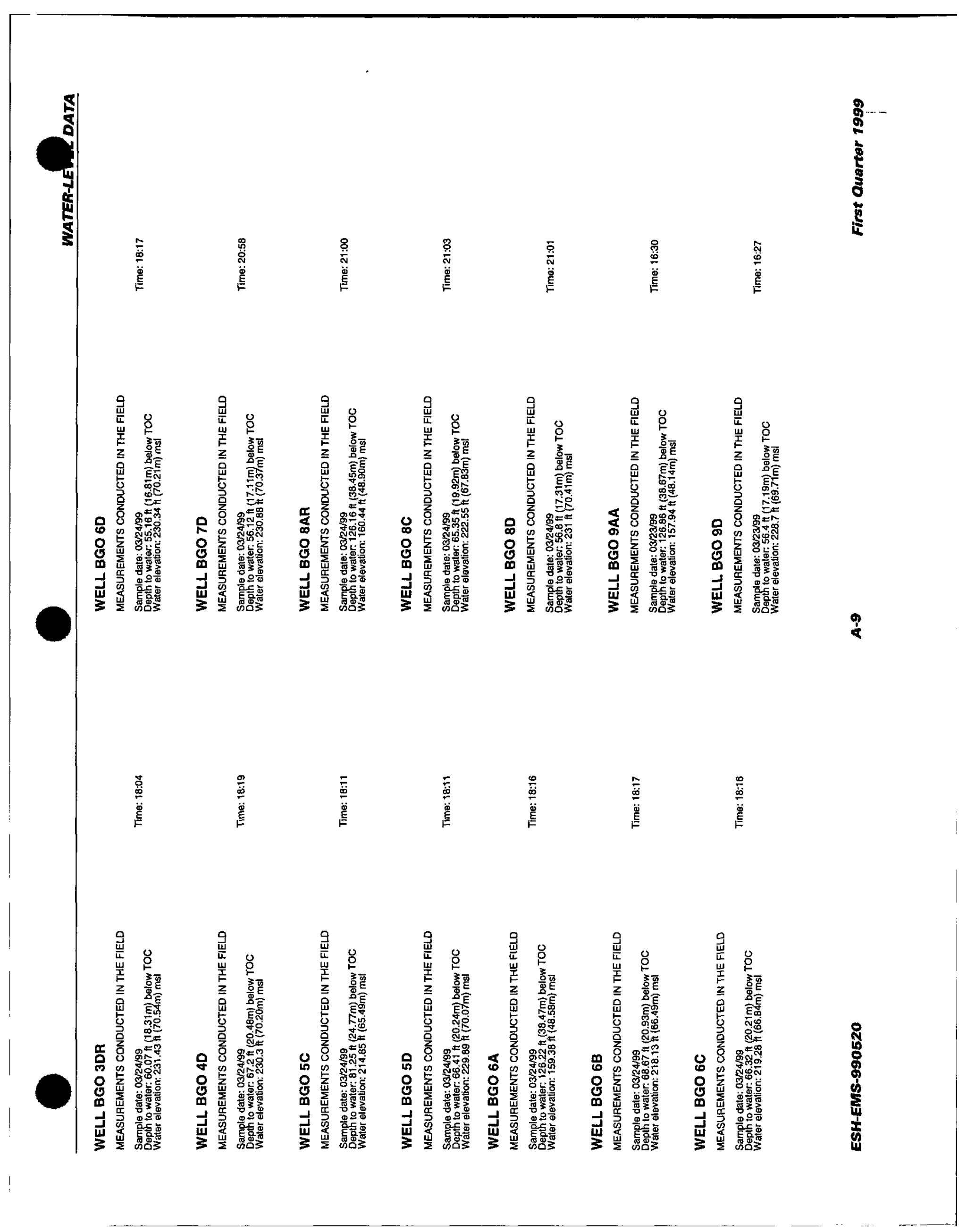


$\mathbf{5}$
$\mathbf{5}$
$\mathbf{w}$
$\mathbf{4}$
$\mathbf{5}$
$\mathbf{4}$
$\mathbf{5}$

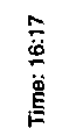

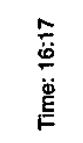

$\overline{\dot{\omega}}$
$\ddot{\Phi}$
$\underline{E}$

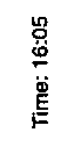

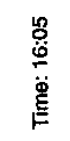

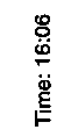

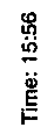

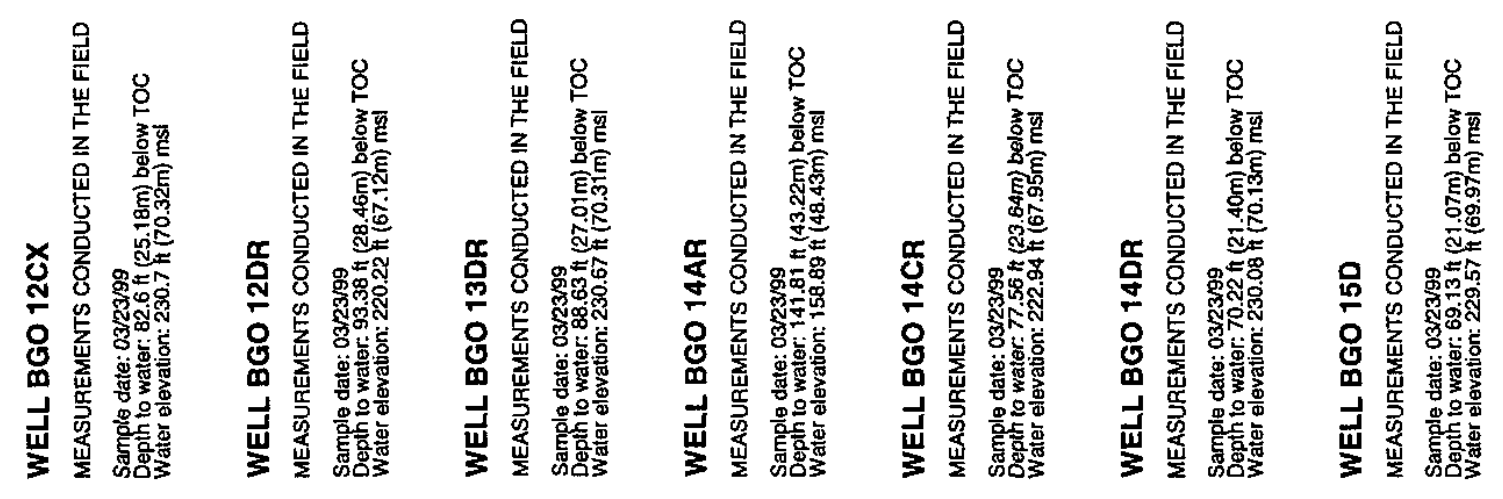

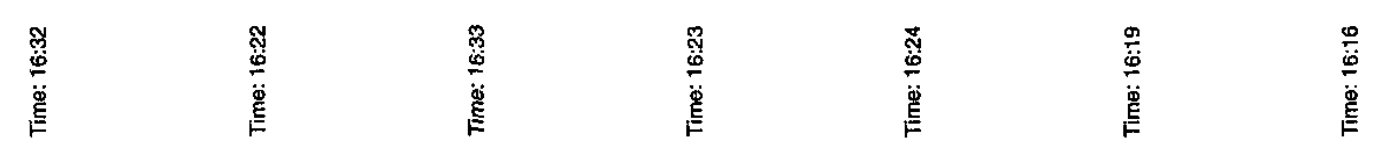

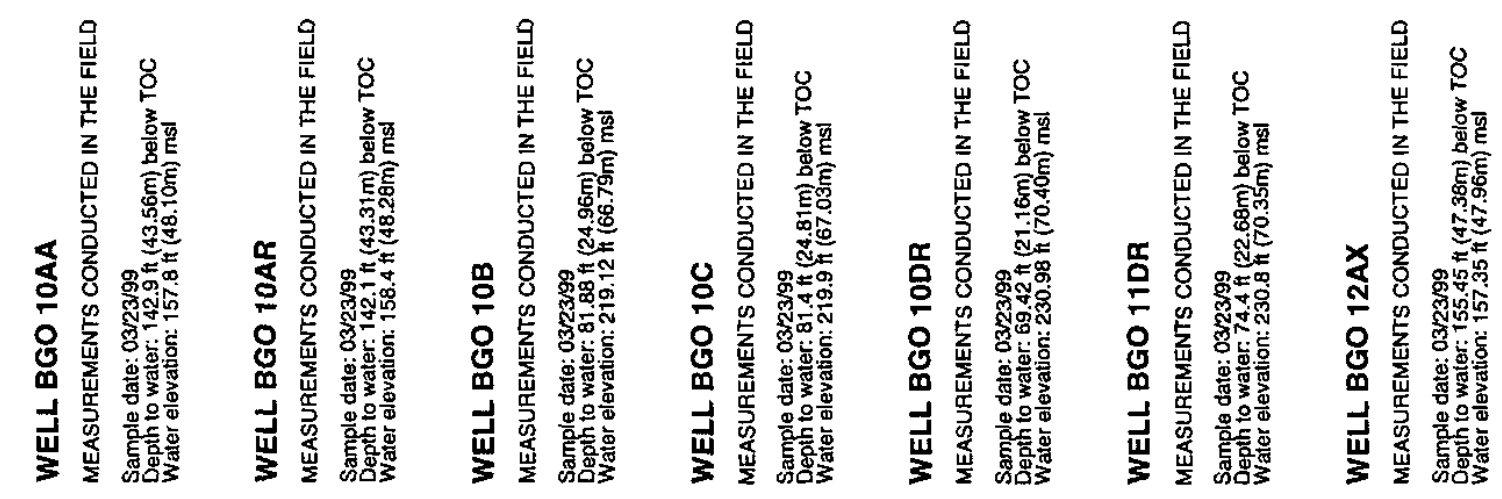

$\frac{1}{4}$
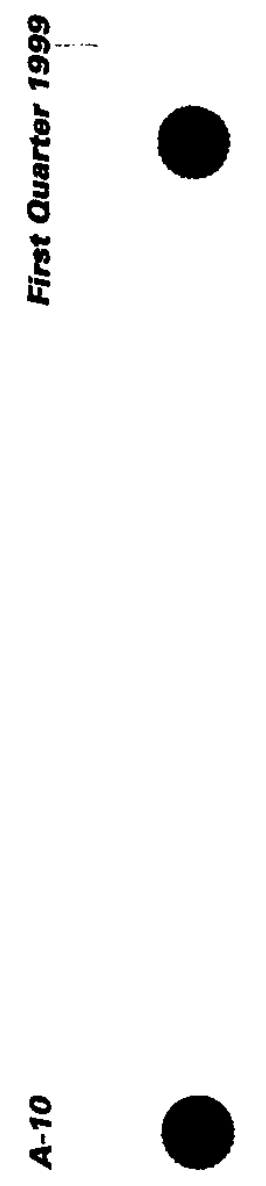


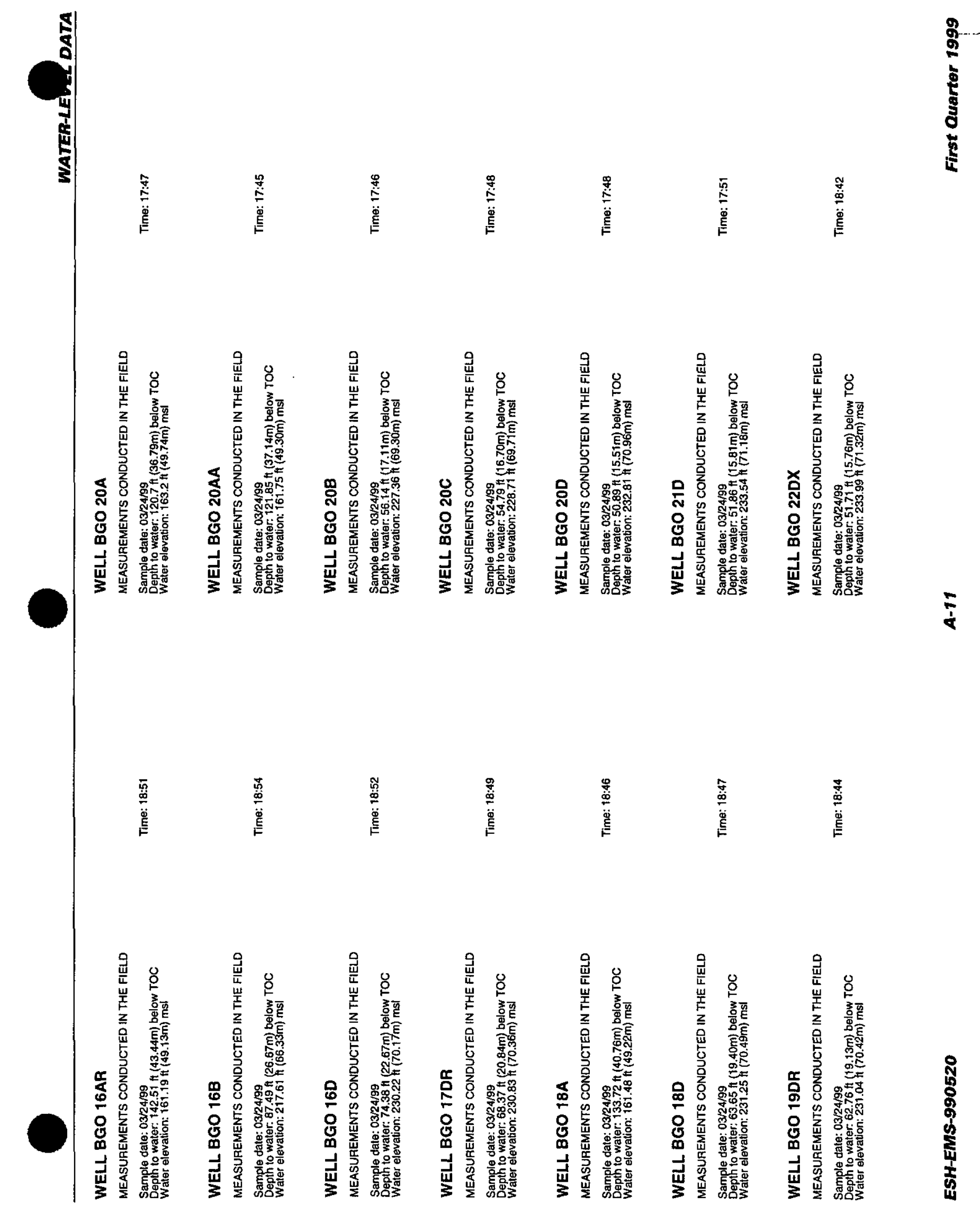




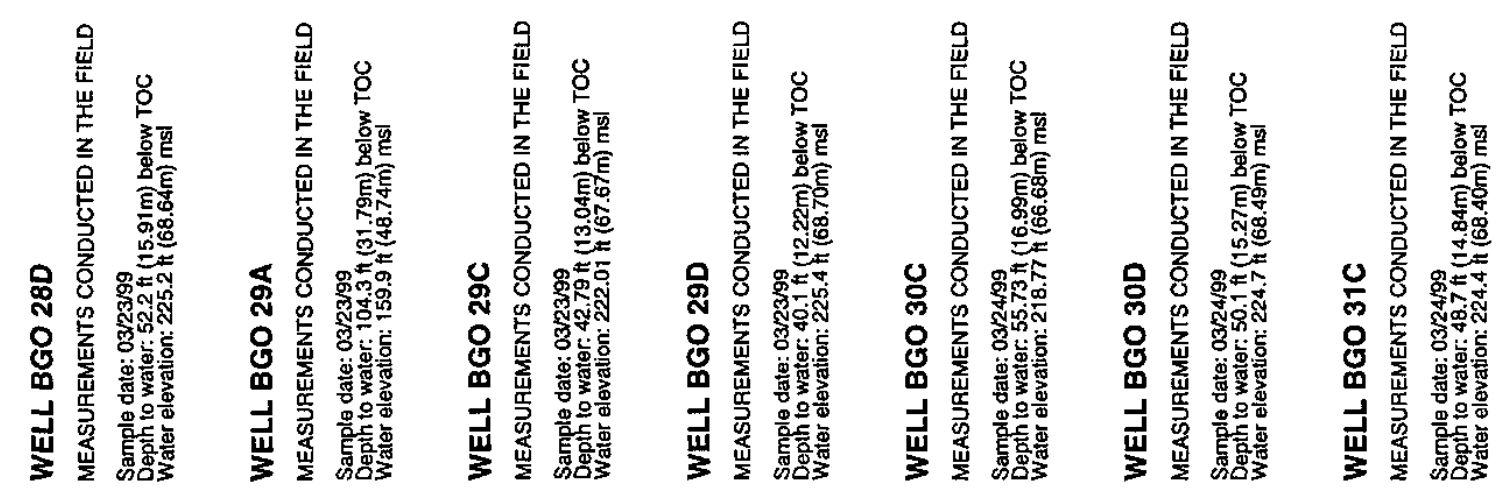

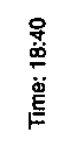
预

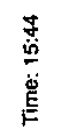

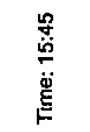

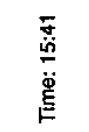

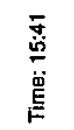

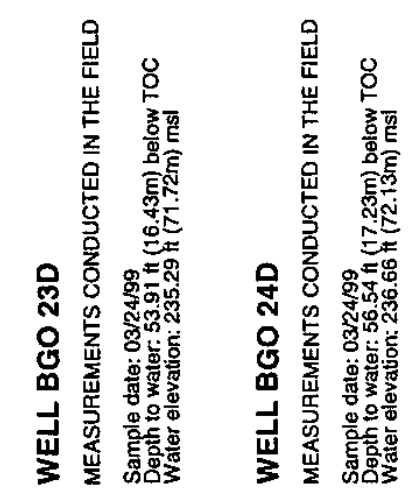
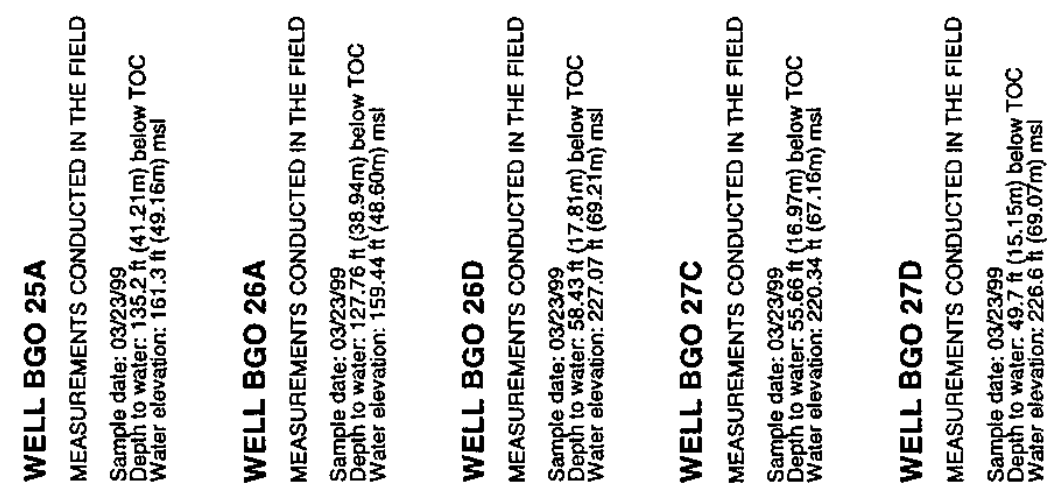

$\frac{N}{4}$ 


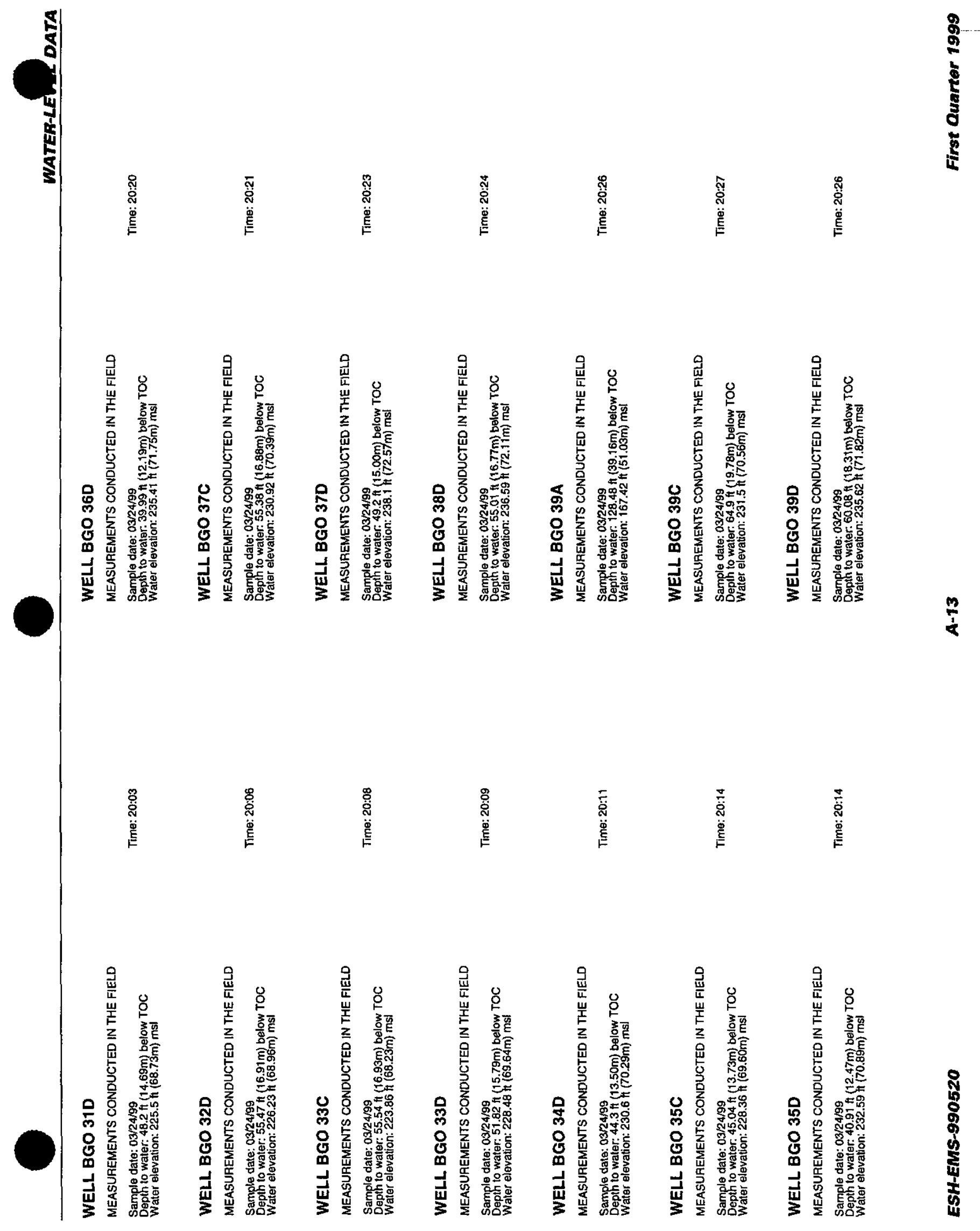


$\underset{\substack{\stackrel{\infty}{*} \\ \stackrel{\oplus}{\oplus}}}{\stackrel{\oplus}{*}}$

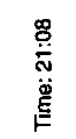

$\underset{\substack{\stackrel{N}{N} \\ \stackrel{q}{q}}}{\stackrel{F}{F}}$

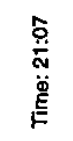

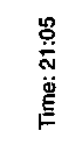

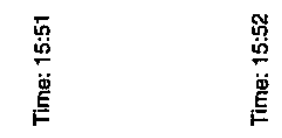

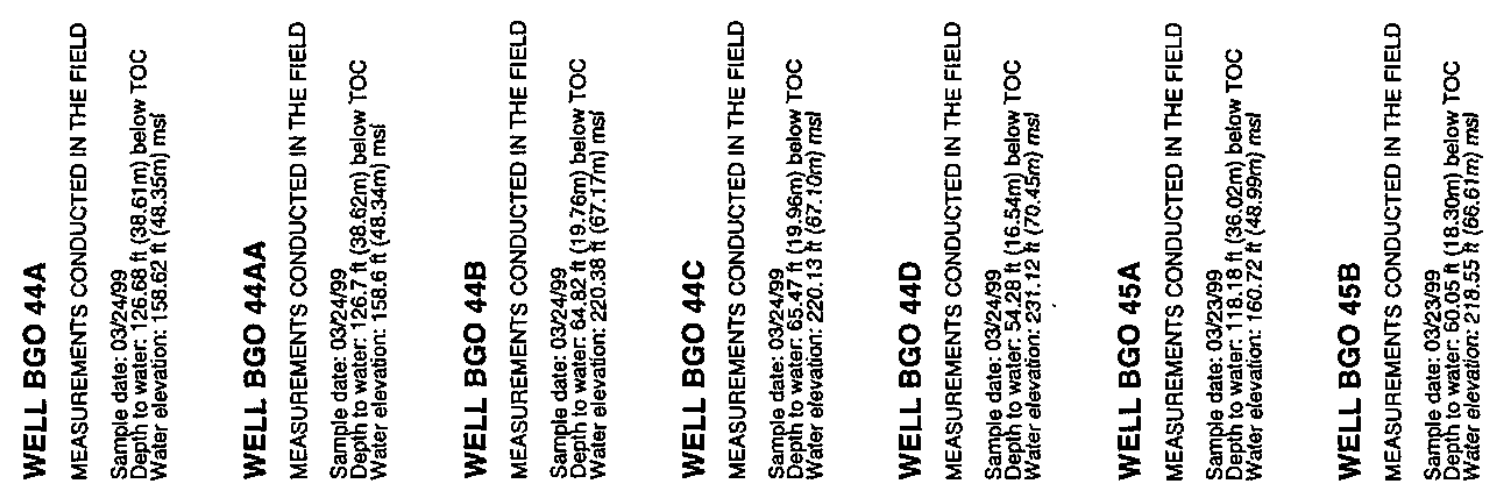

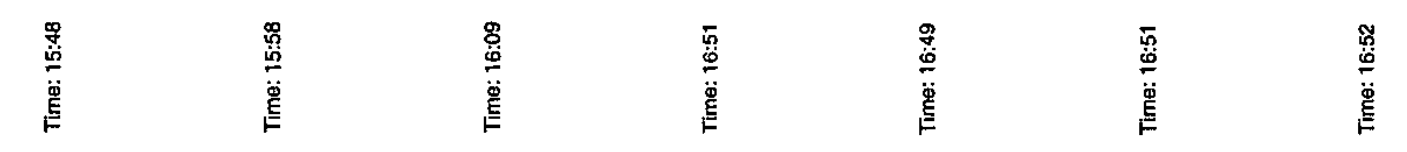

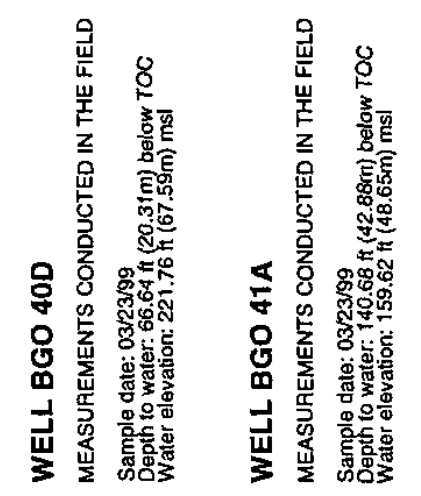
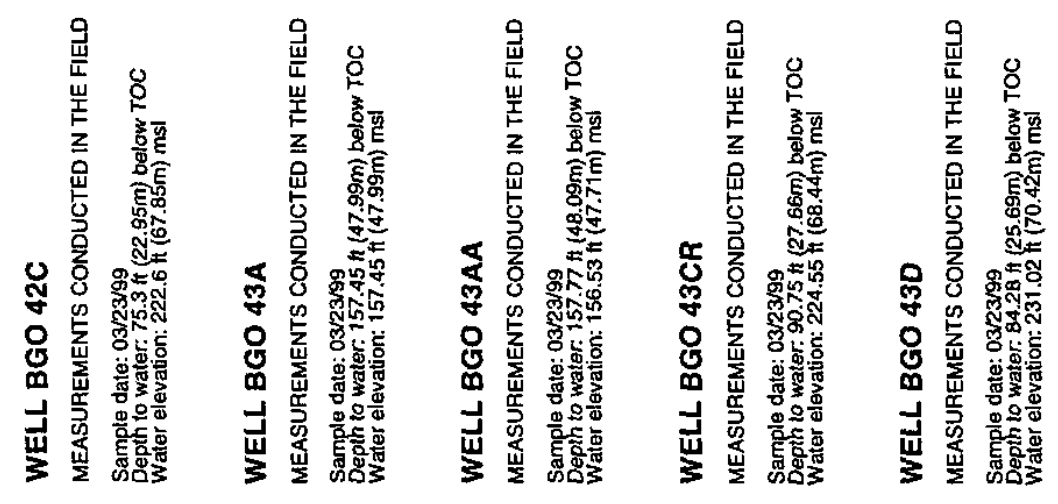

E

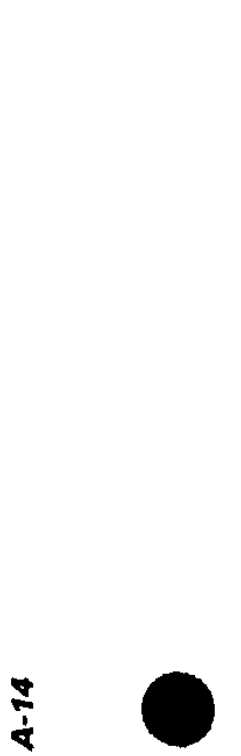



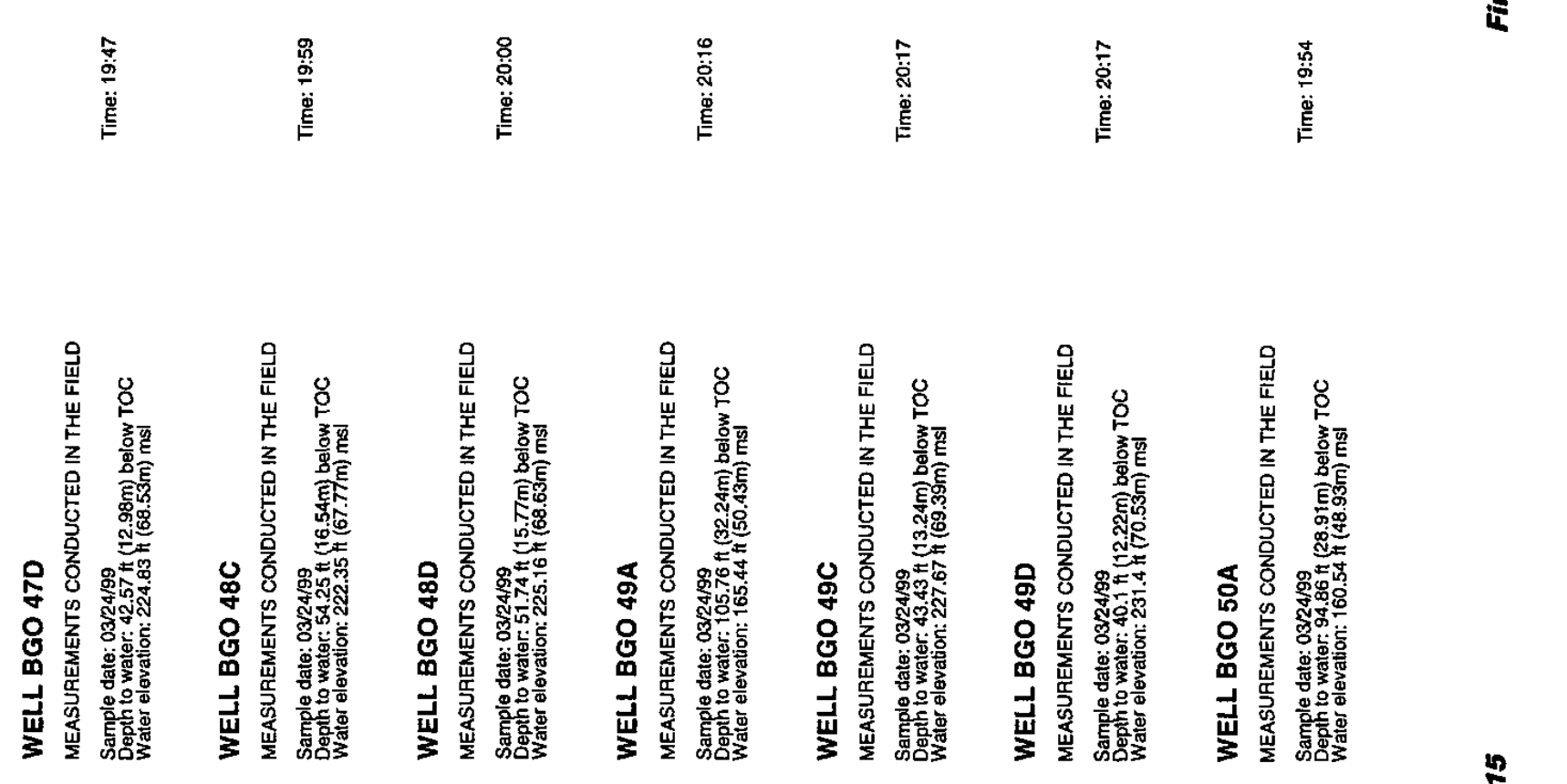

$\stackrel{p}{\dot{q}}$
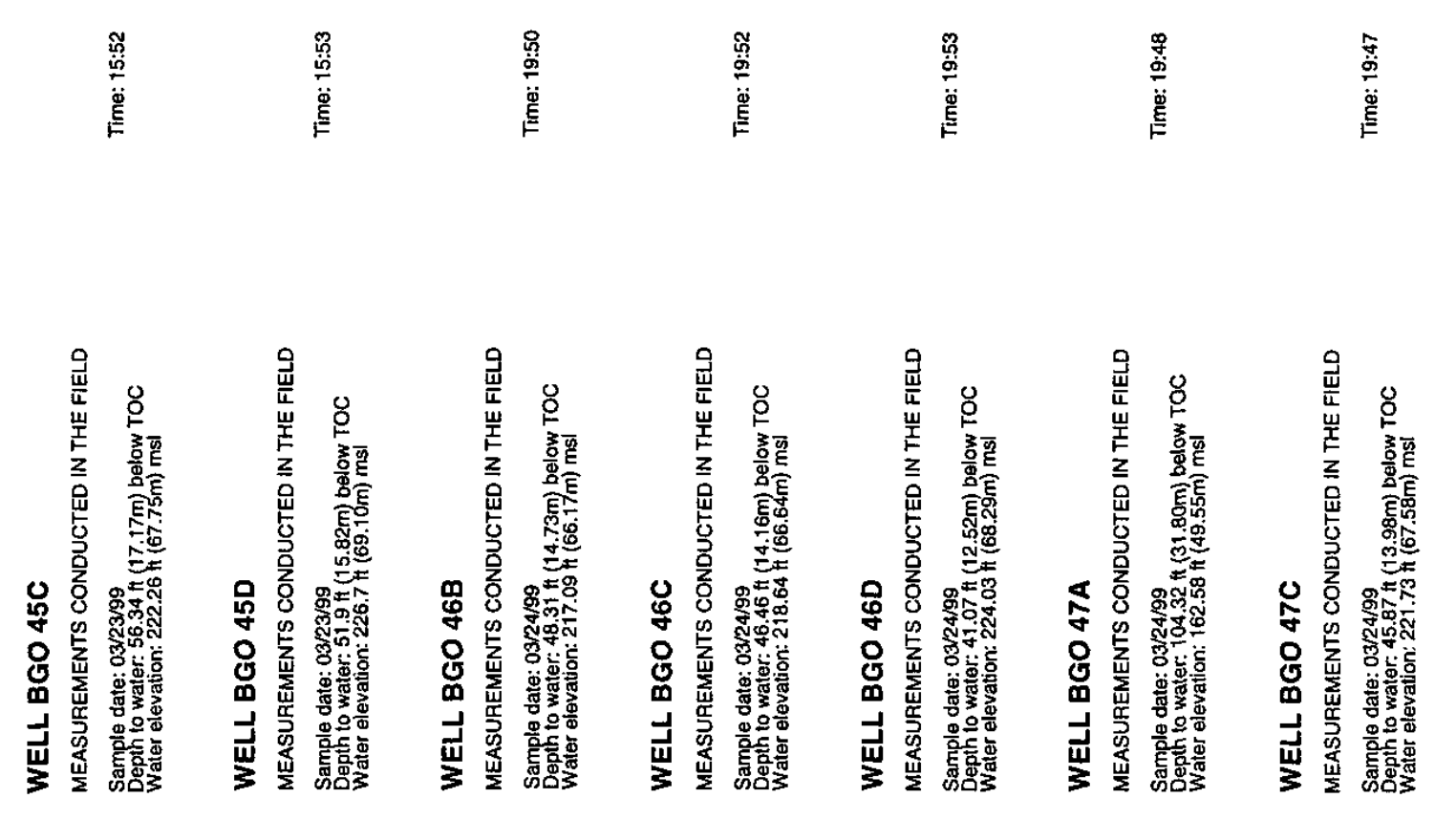


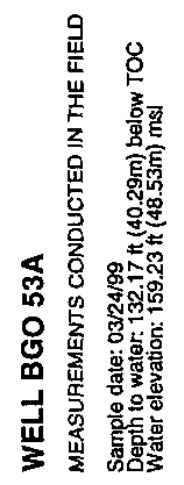

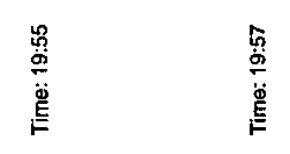

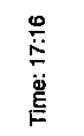

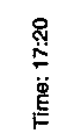

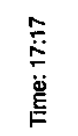

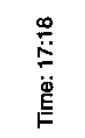

$\underset{\stackrel{9}{ }}{\stackrel{\circ}{i \oplus}}$

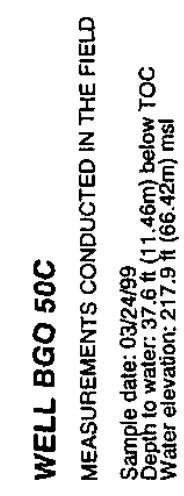
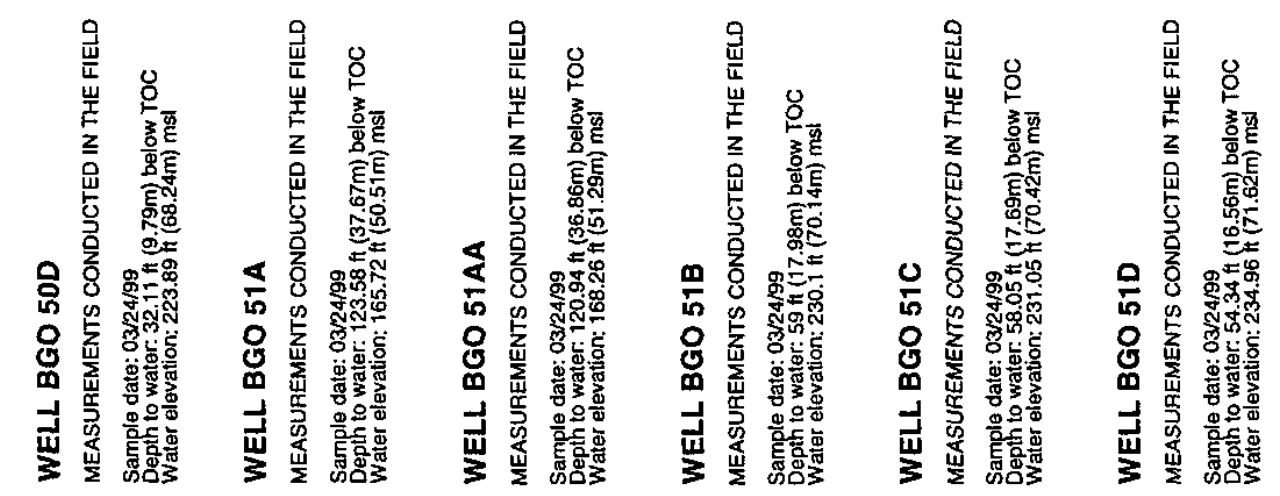

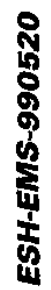




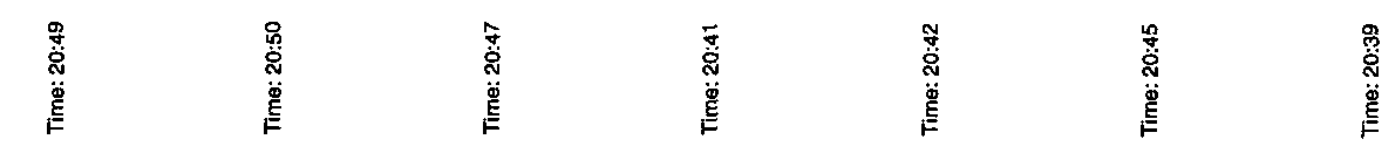

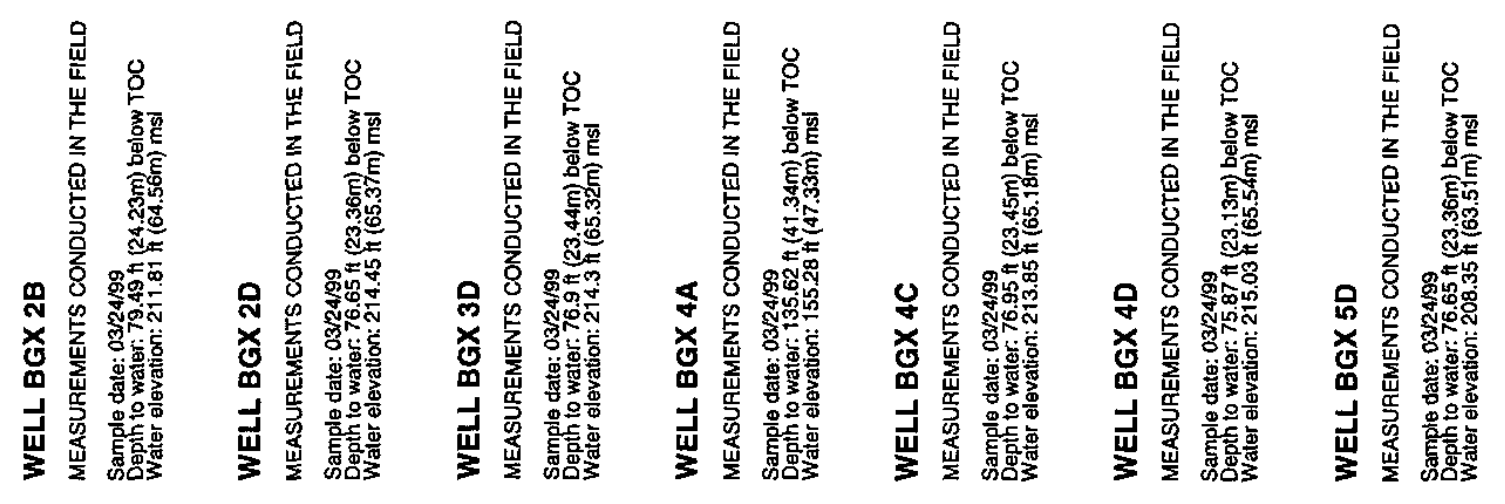

$\hat{\xi}$

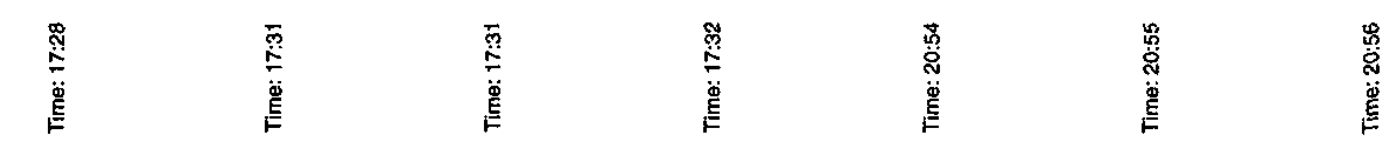

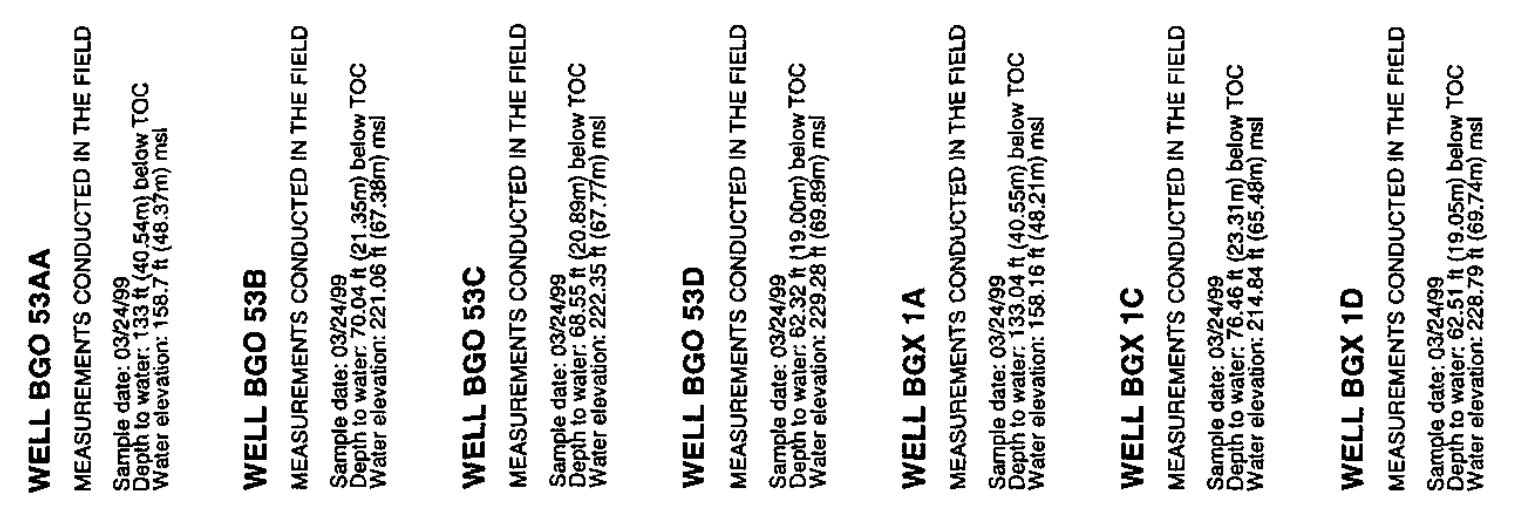




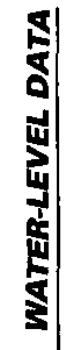

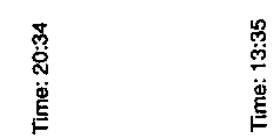

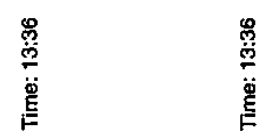

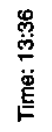

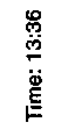

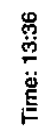

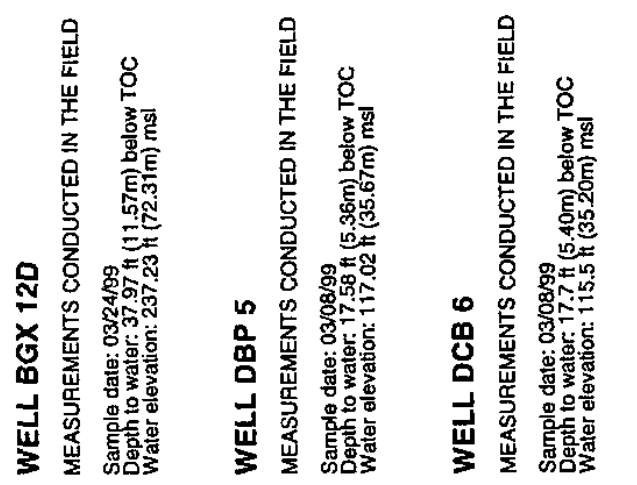
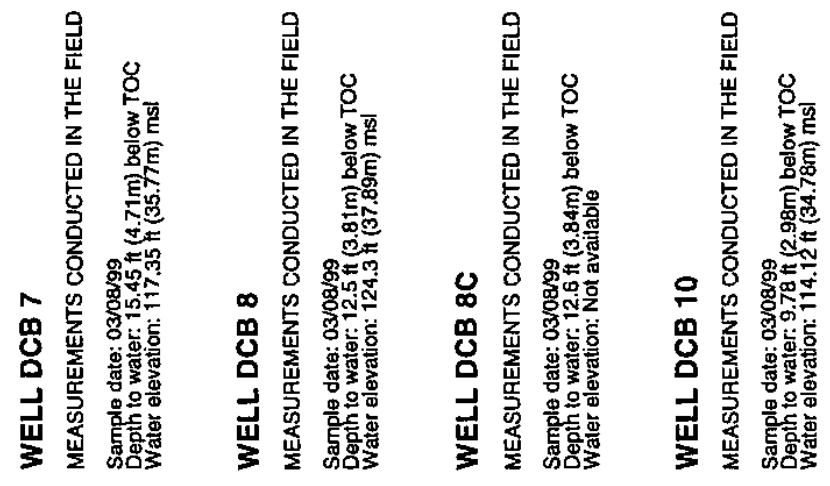

$\frac{\infty}{\dot{\alpha}}$

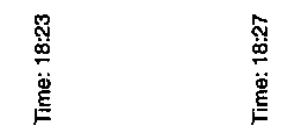

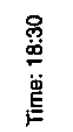

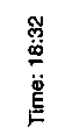

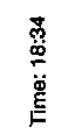

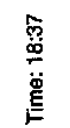

o
芦
$\stackrel{\Phi}{\Phi}$
点

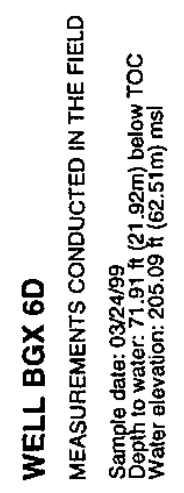
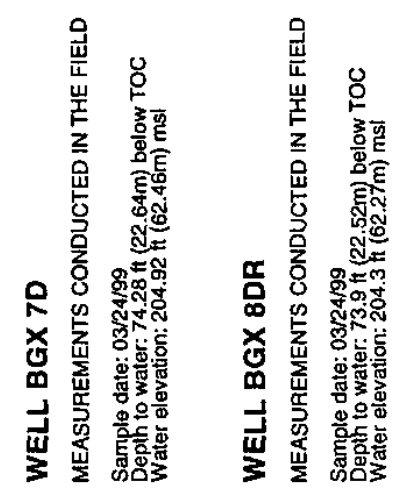

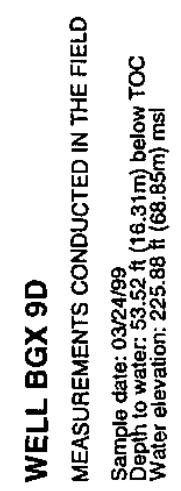
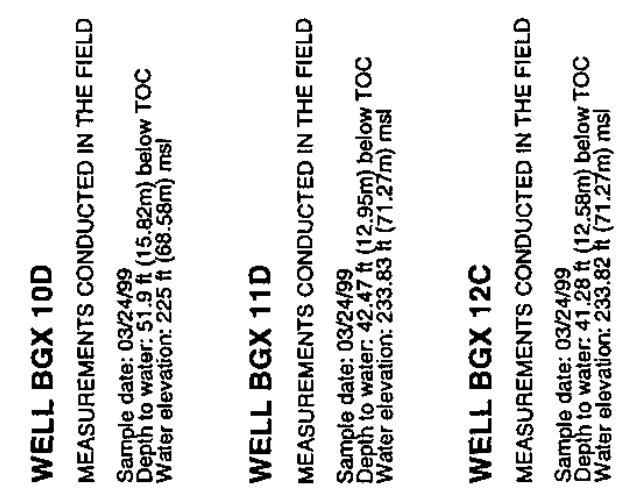

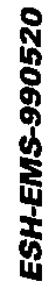




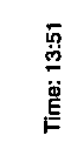

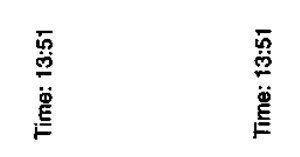

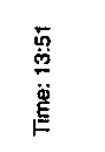

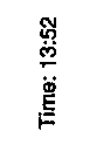

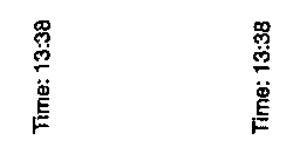

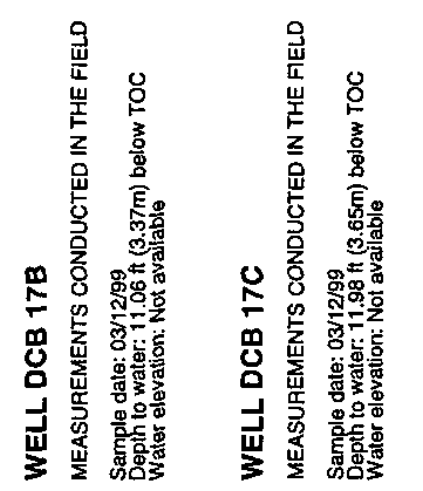
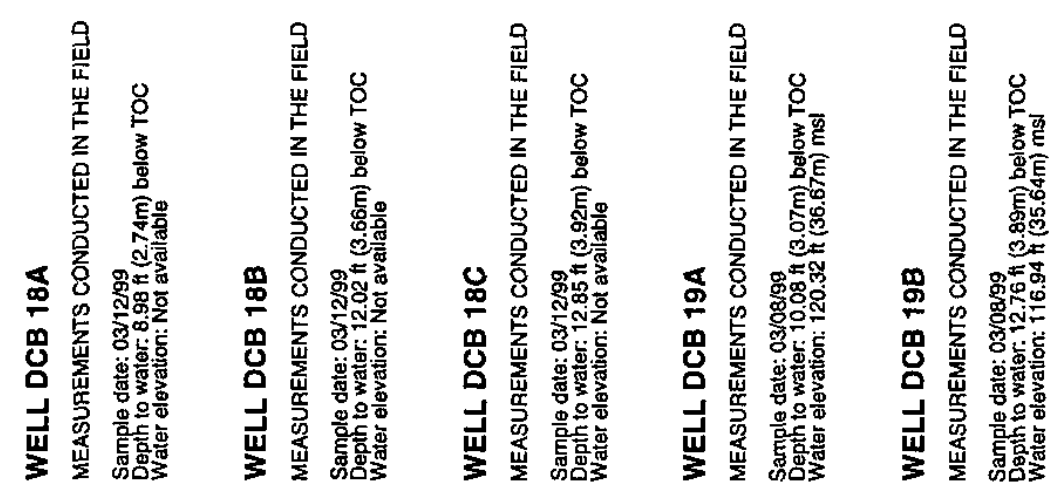

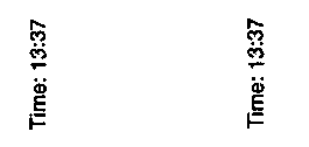
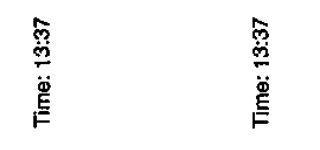

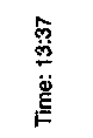

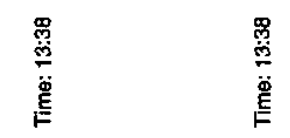
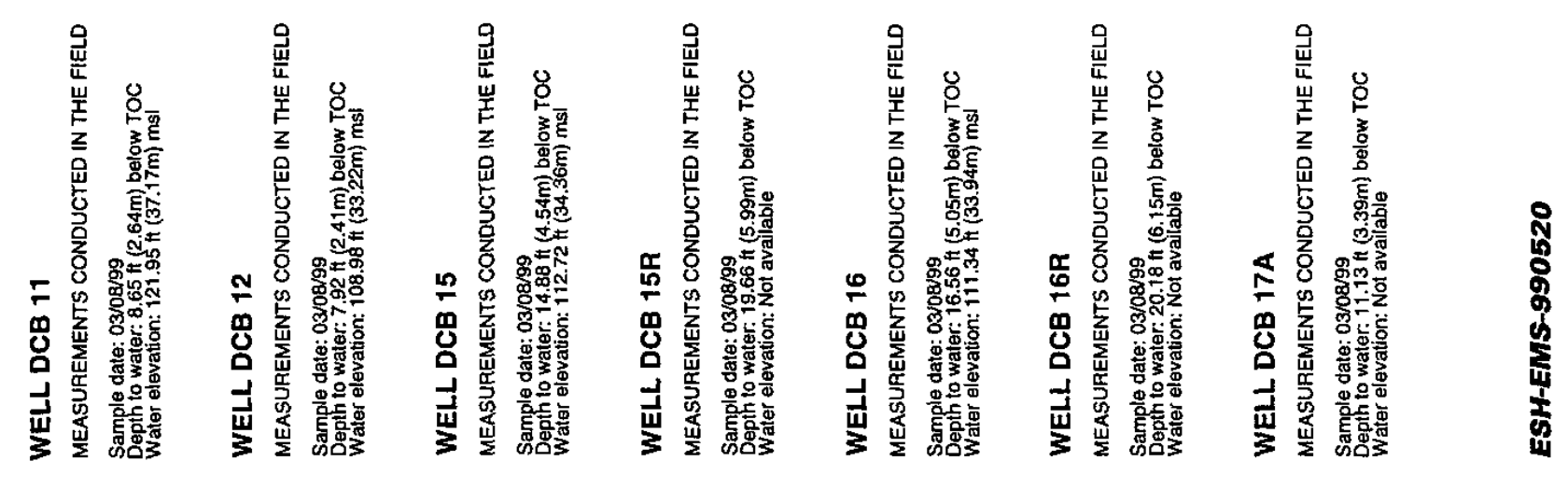

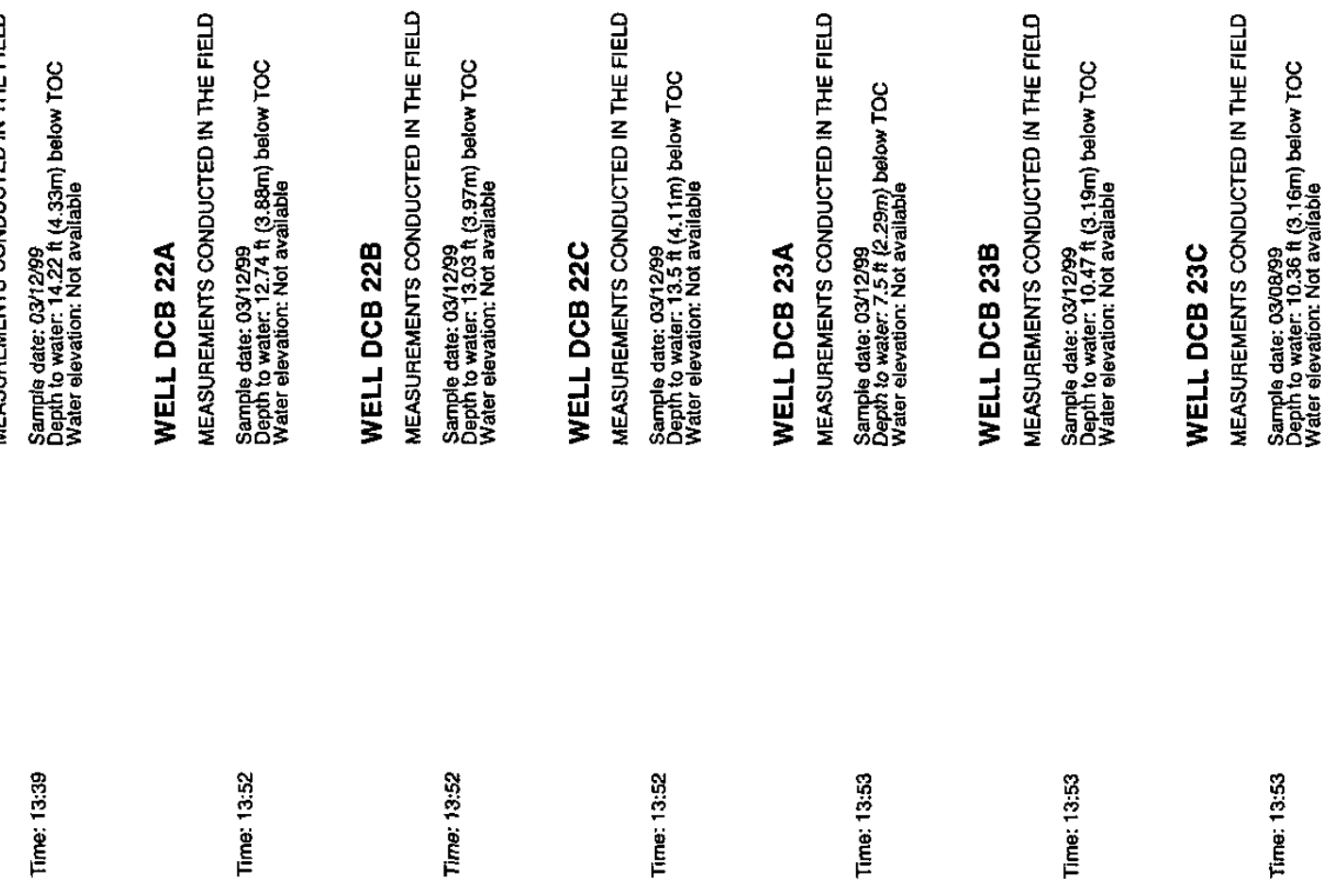

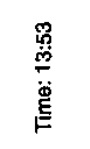

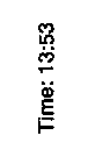

总
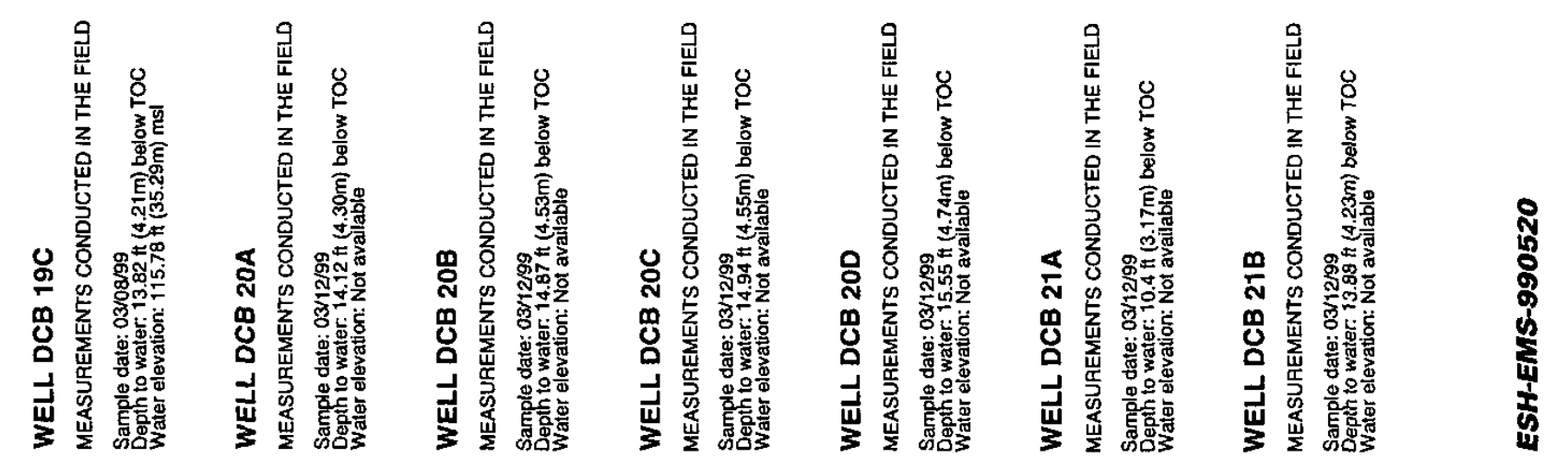


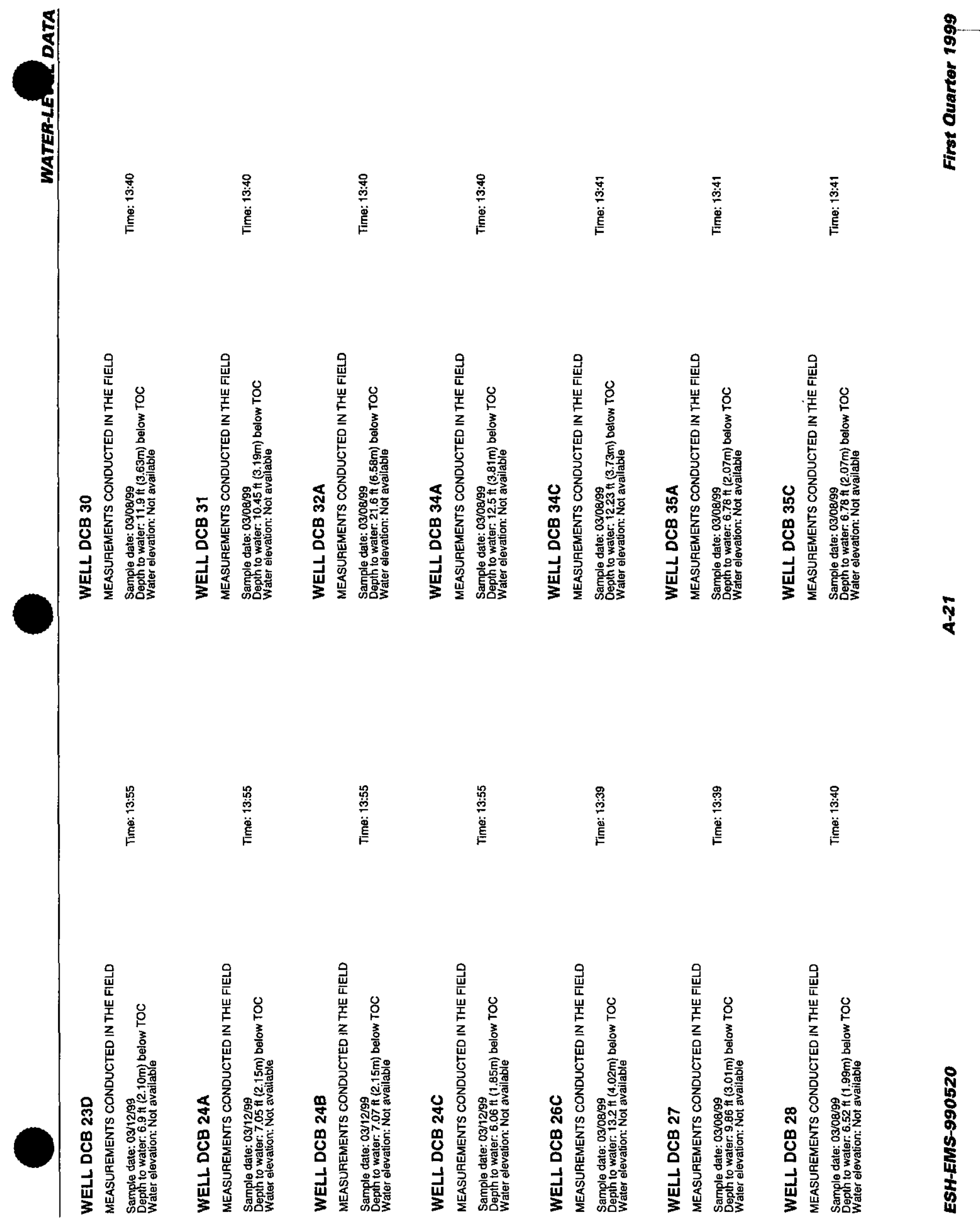




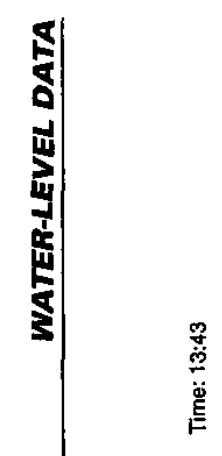

$\frac{8}{1 !}$

I

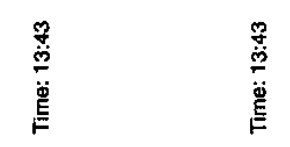

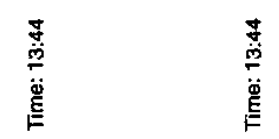

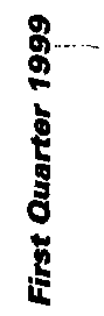

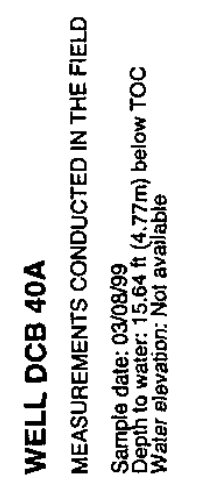
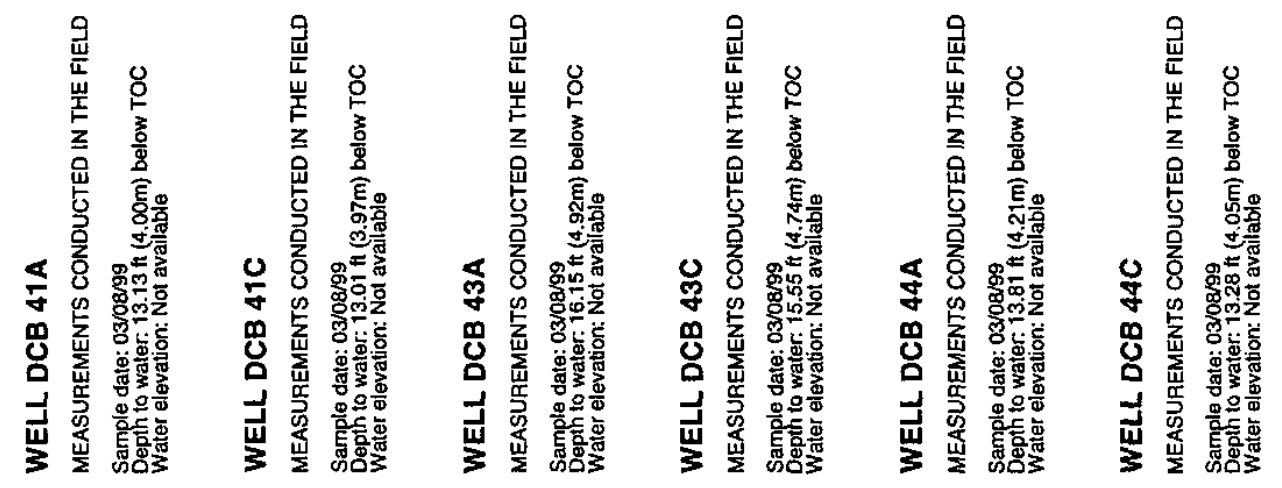

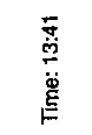

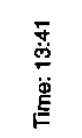

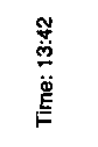

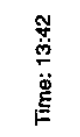

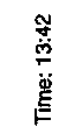

誉

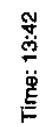
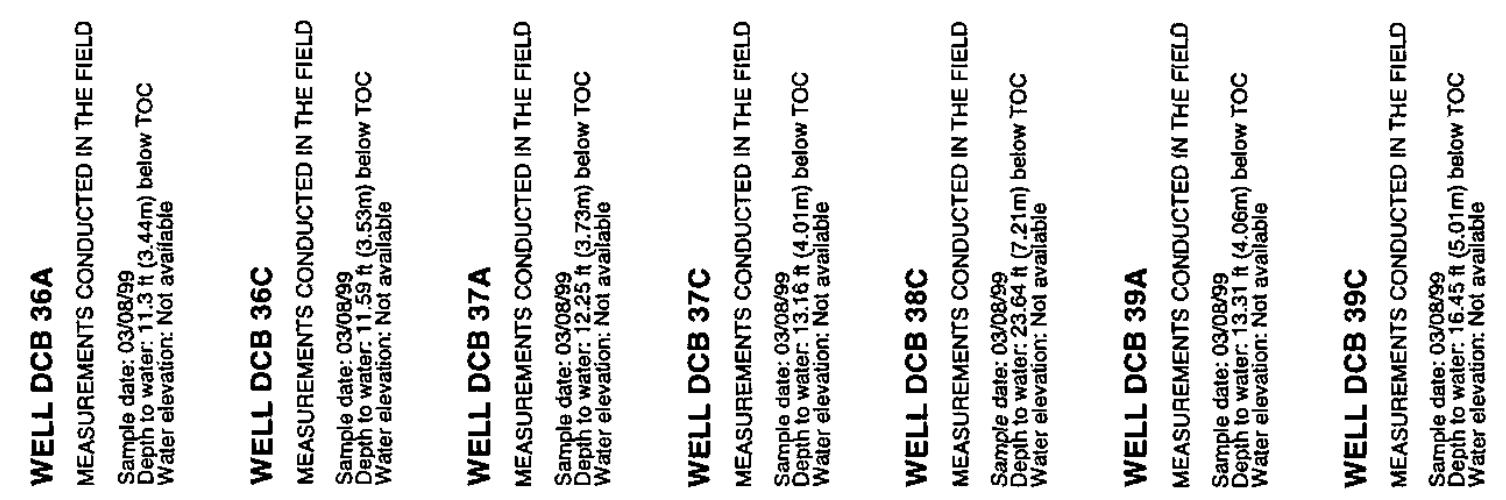

รู

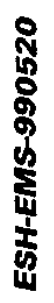




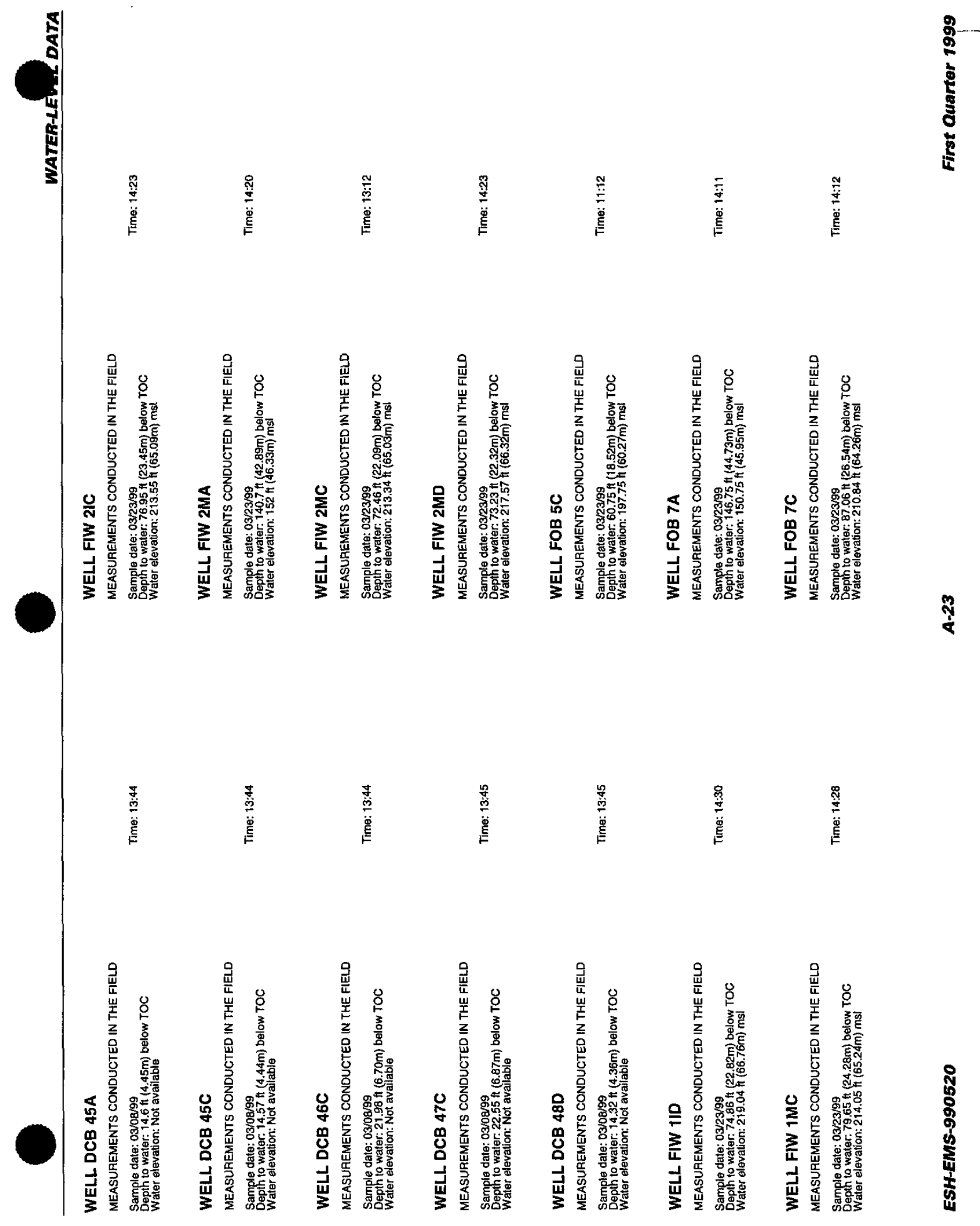



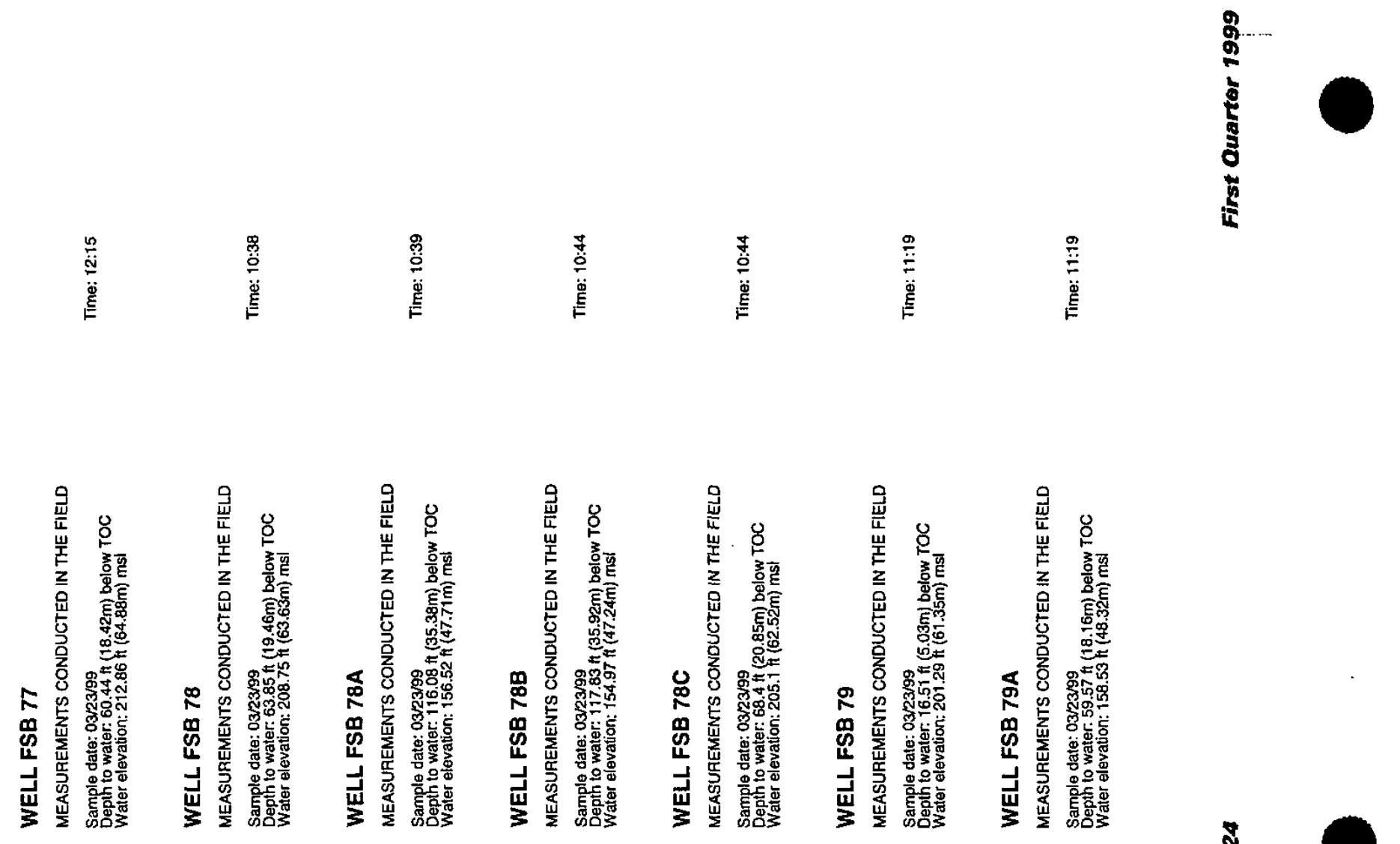

1
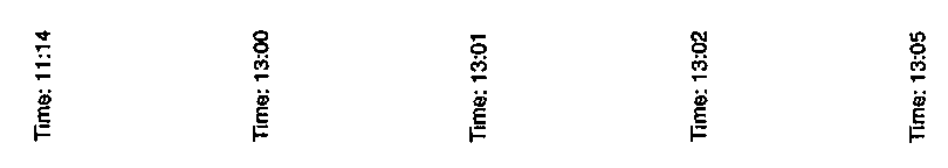


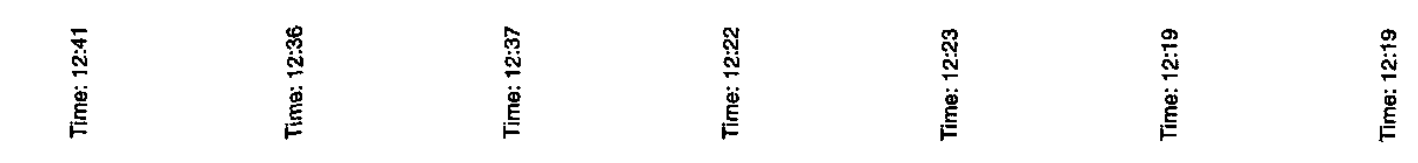

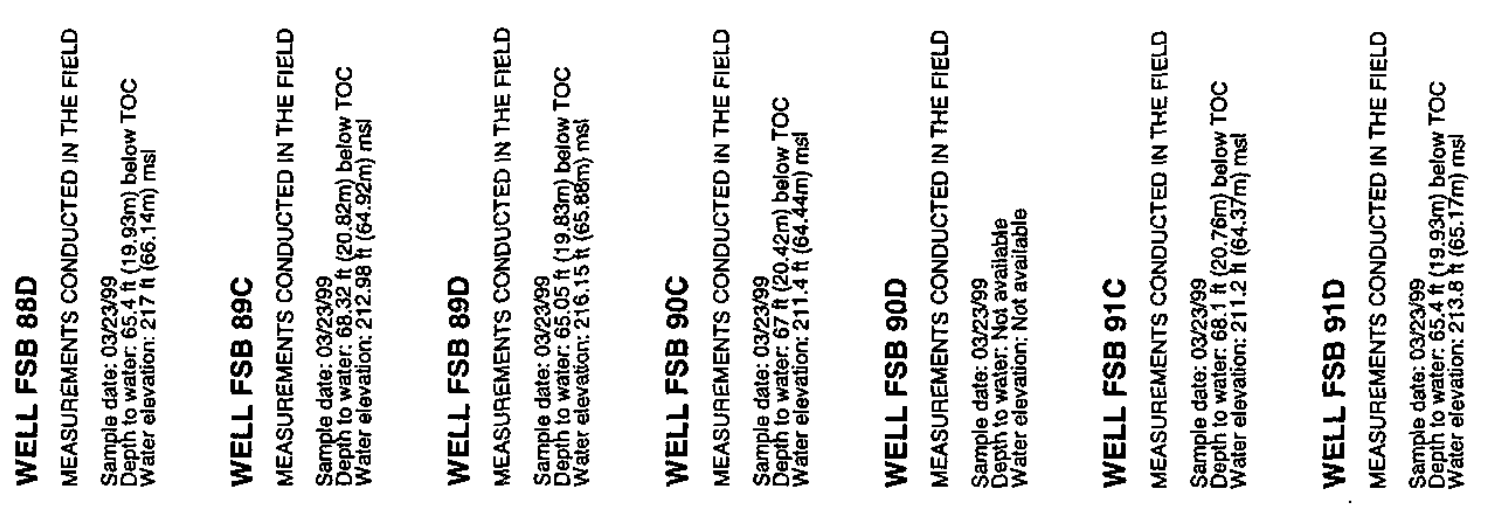

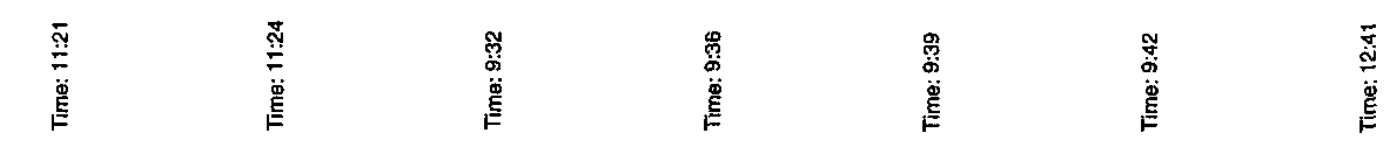

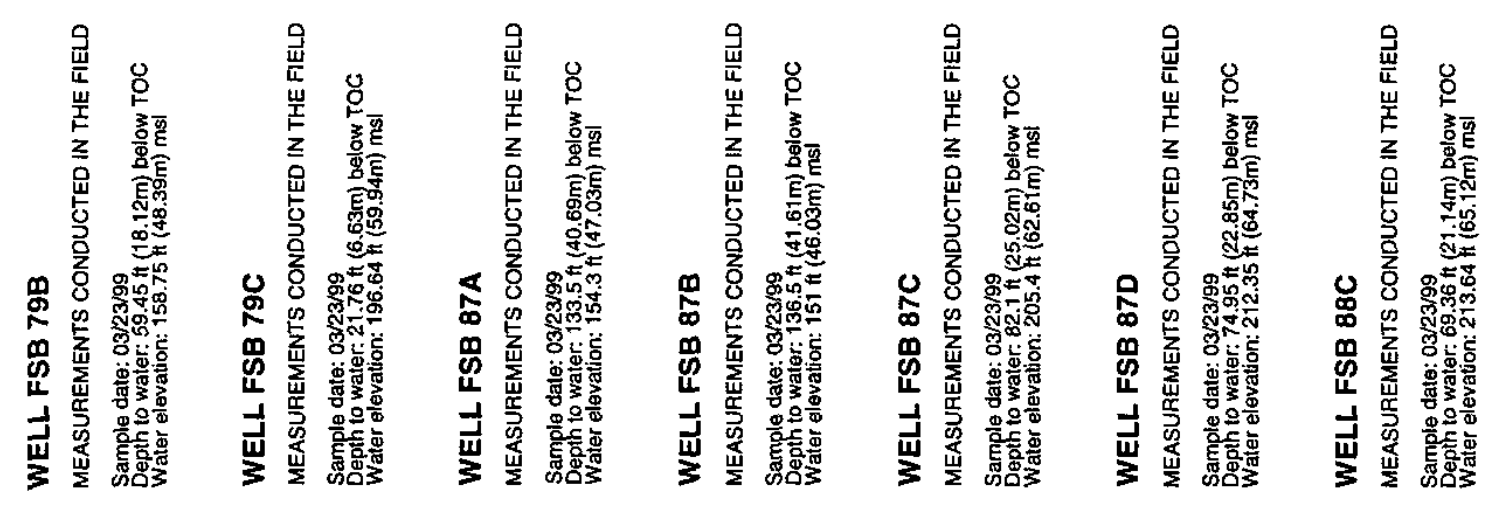

㩊 

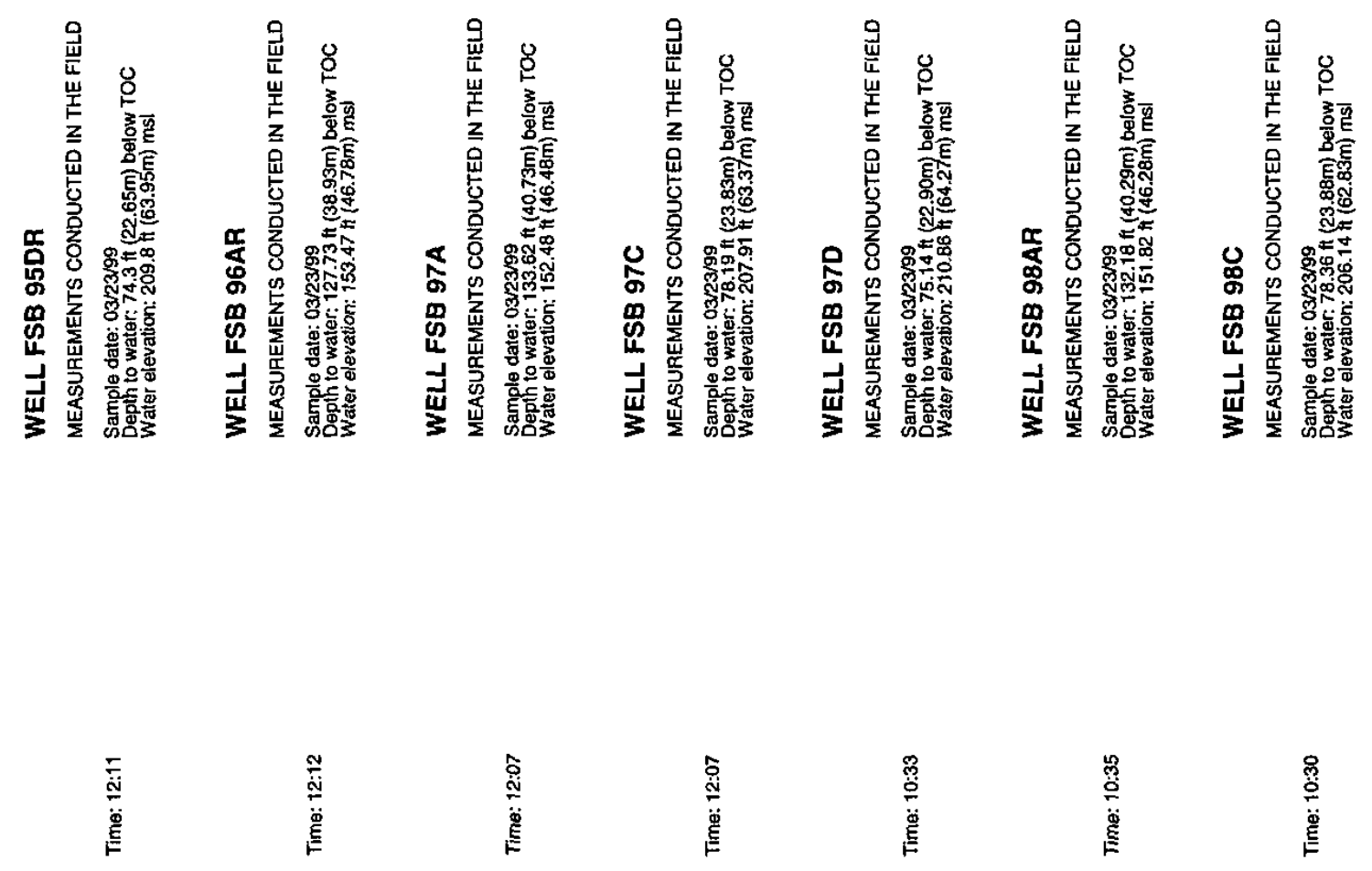

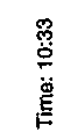

兯

尽
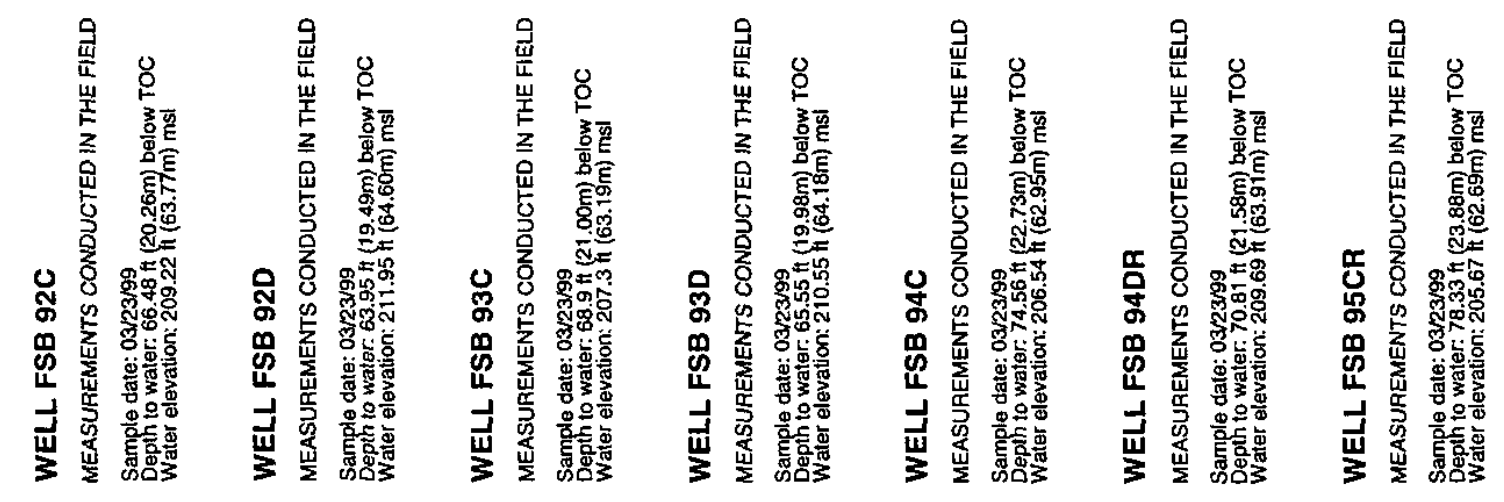

\& 


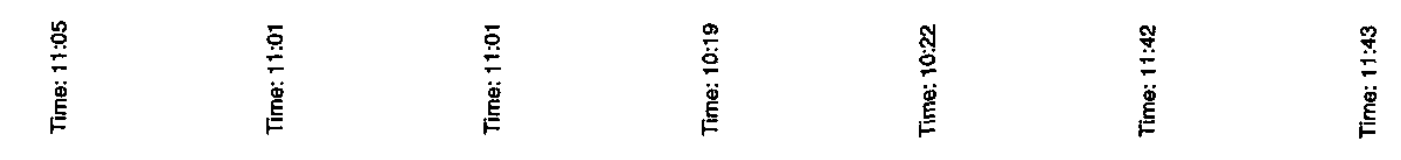

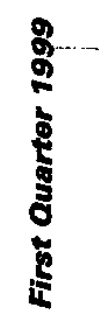

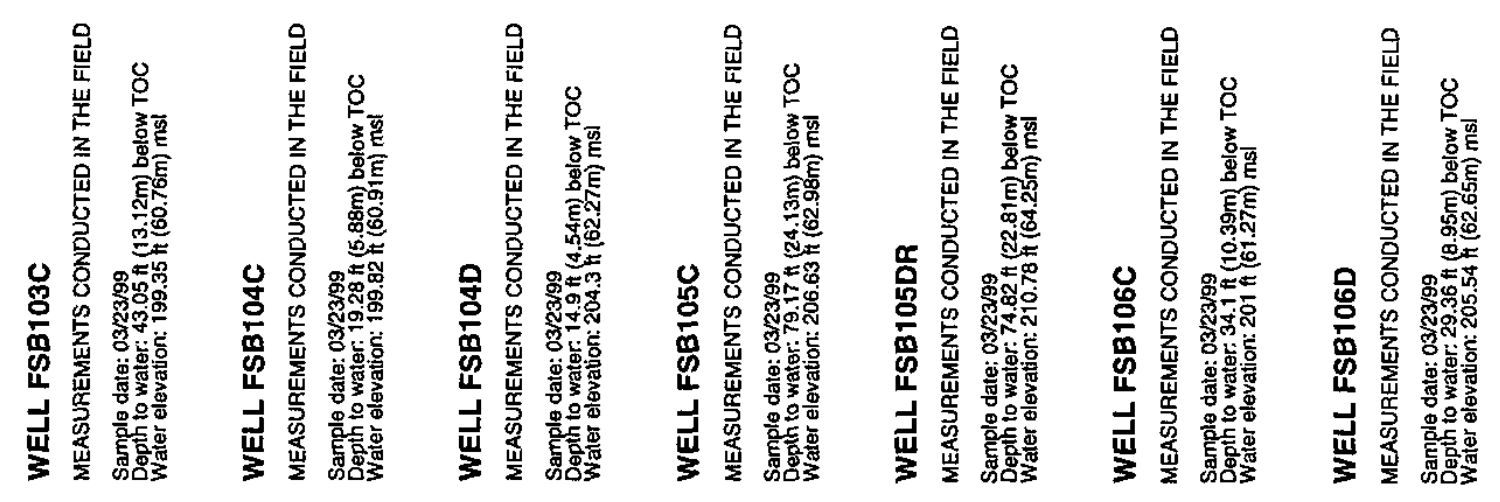

q

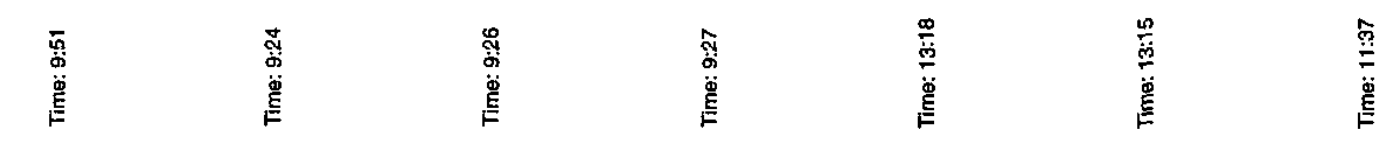

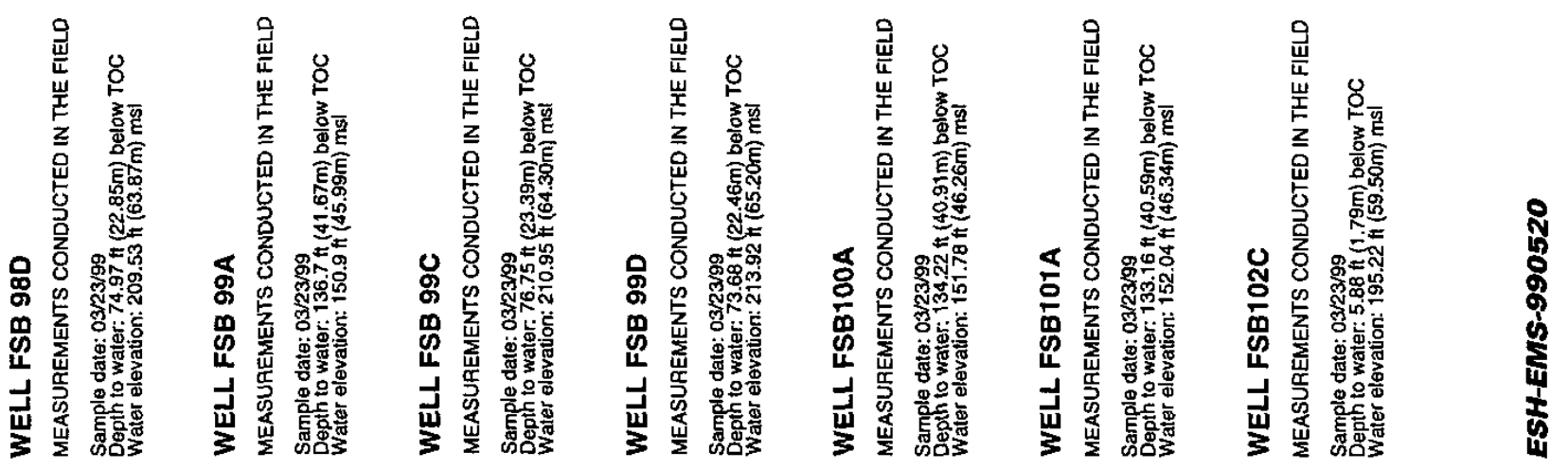



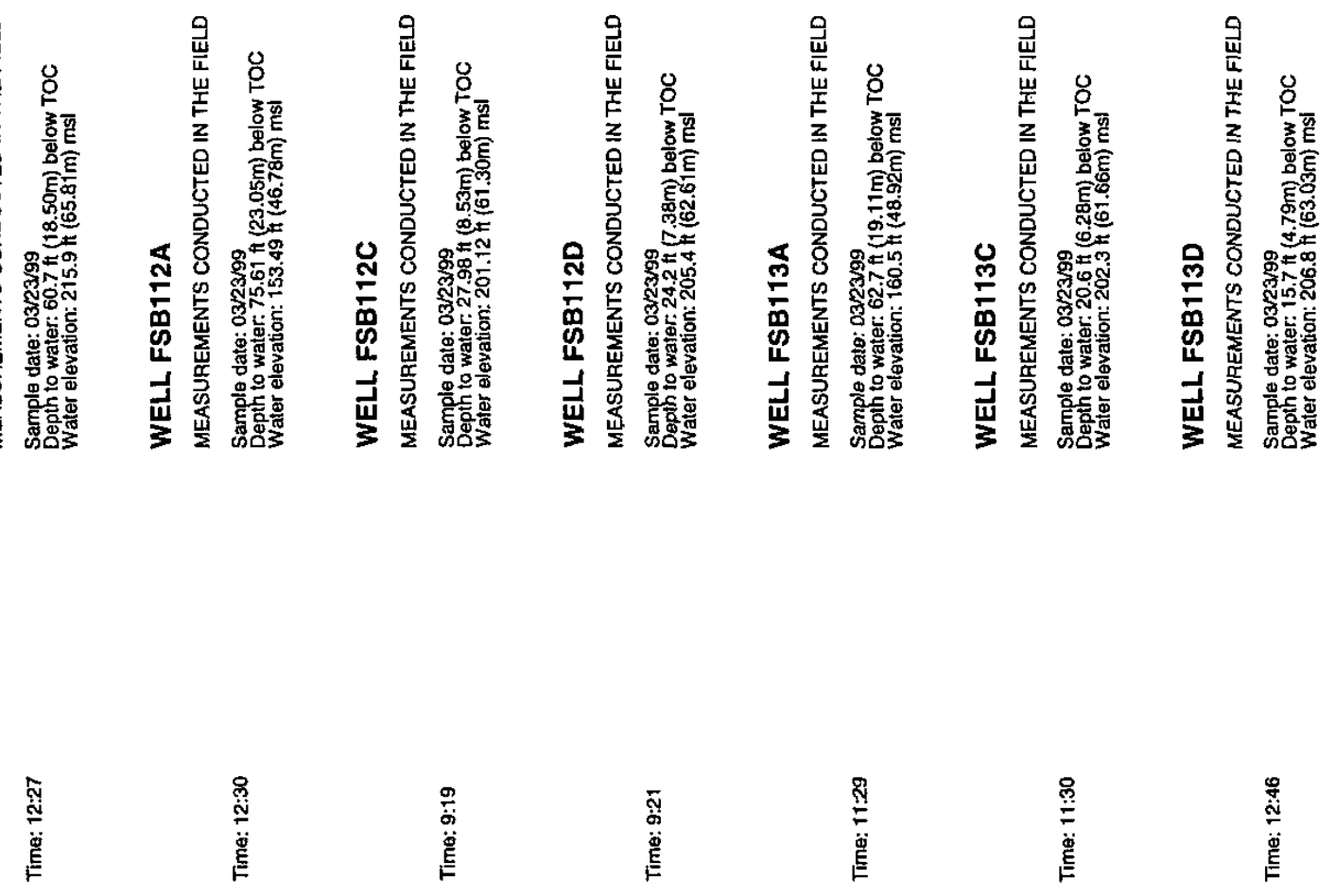

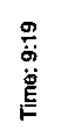

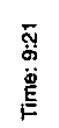

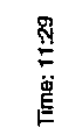

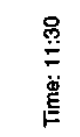

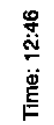

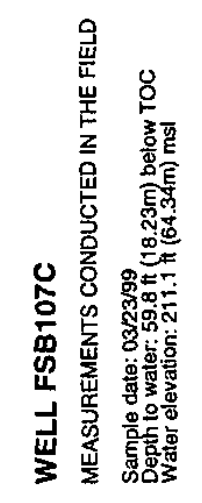
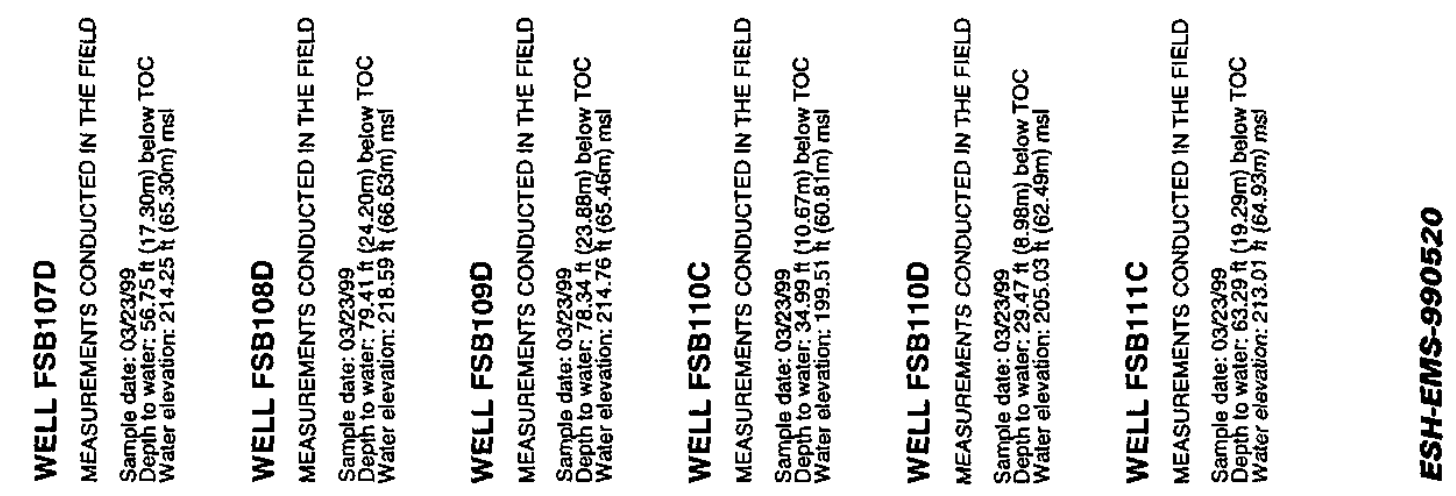


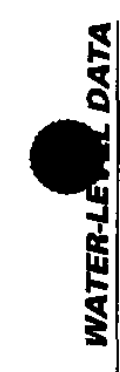

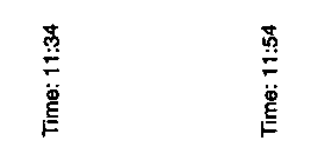

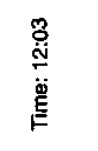

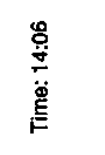

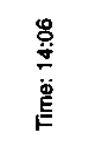

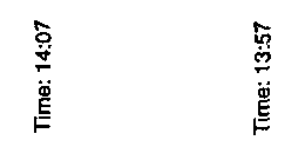

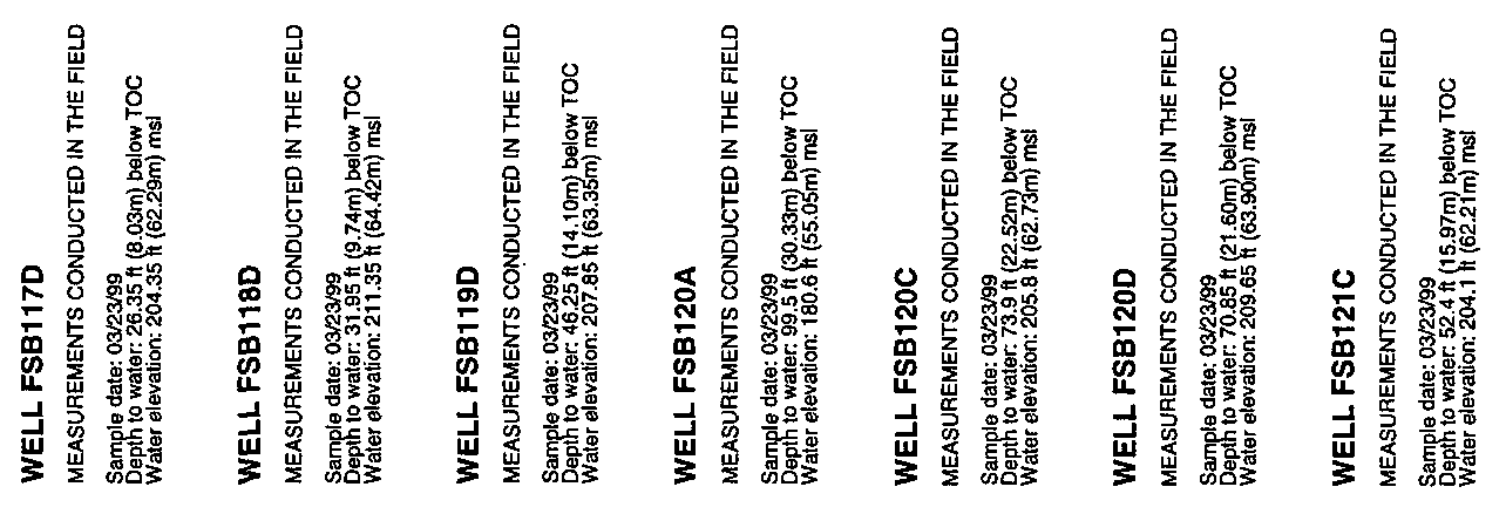

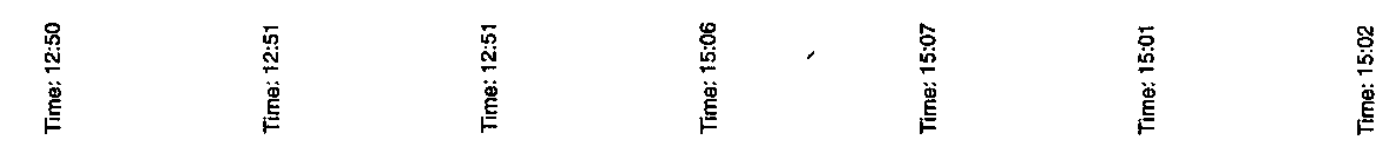

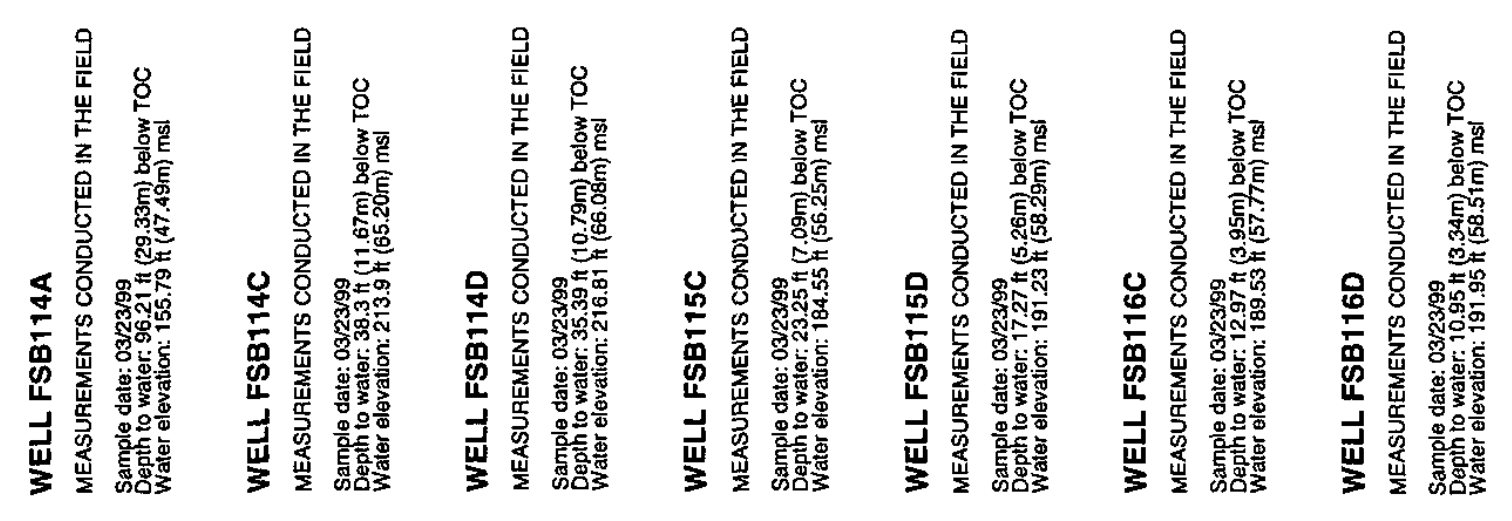

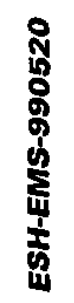




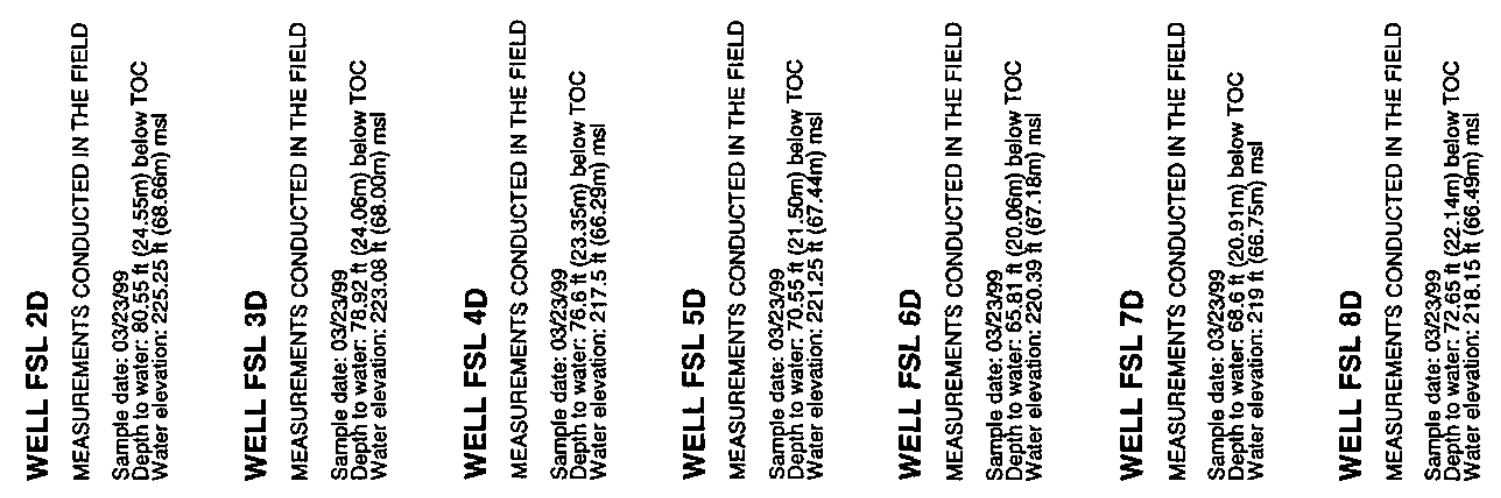

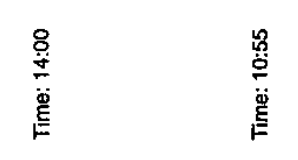

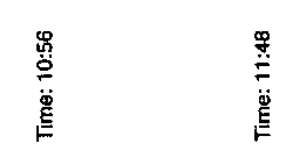

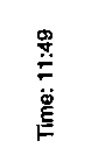

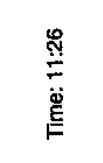

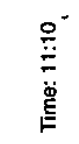




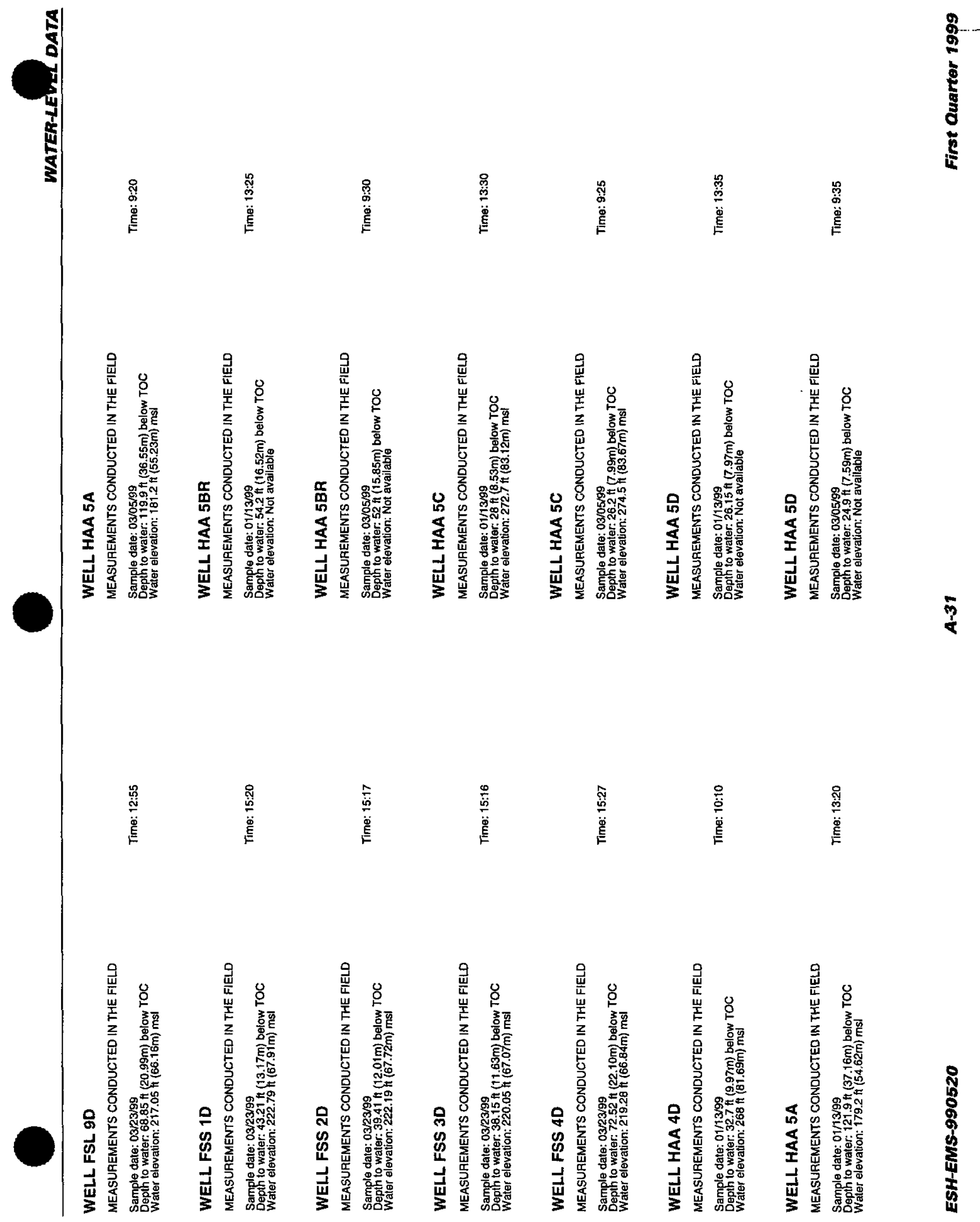



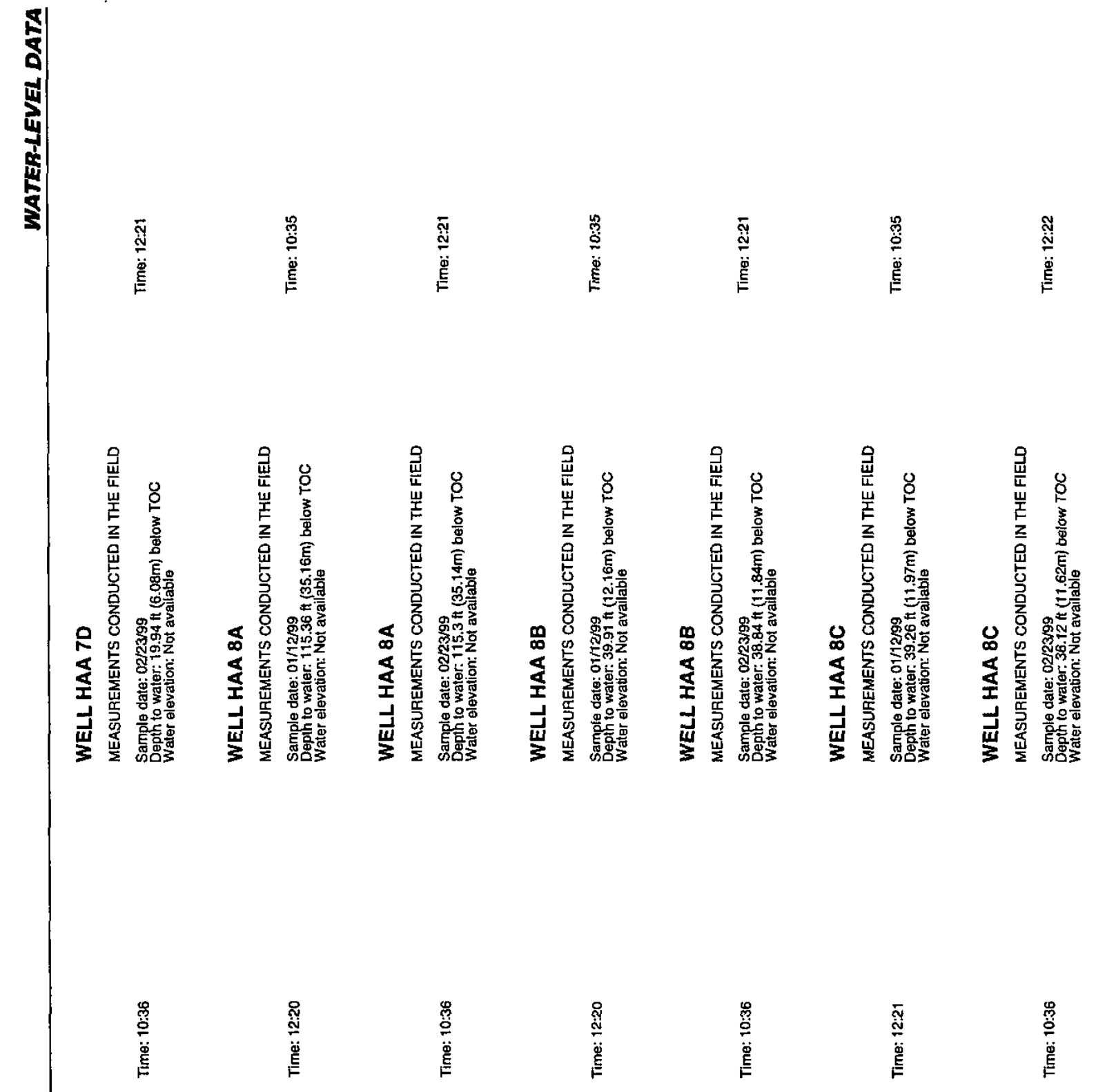

导
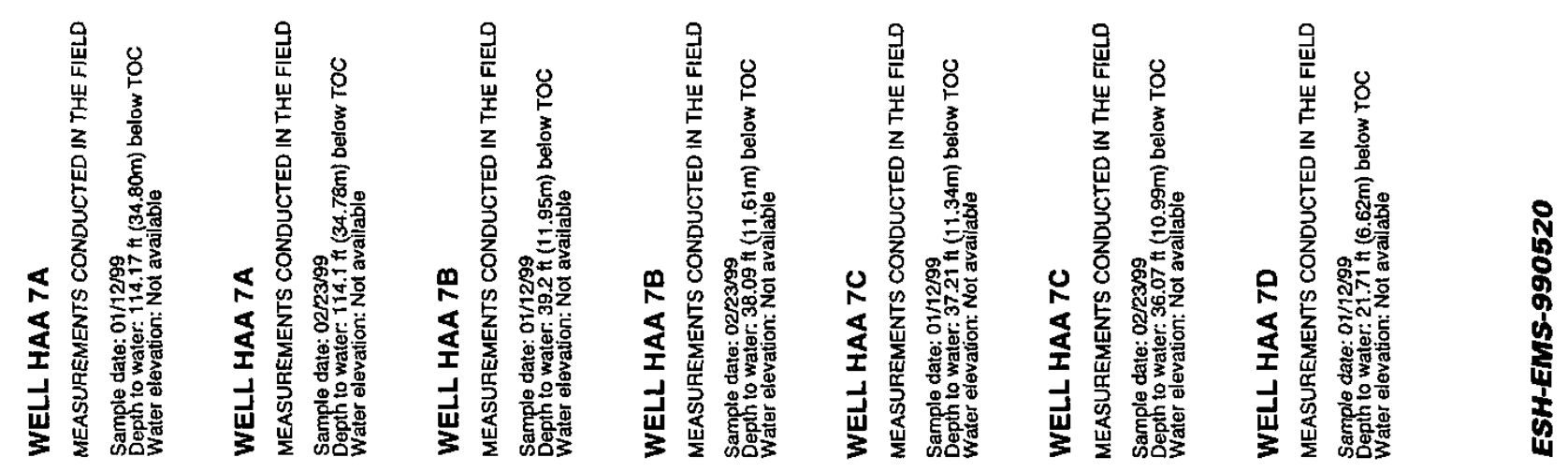


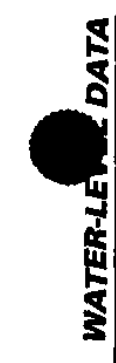

1

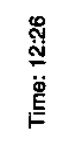

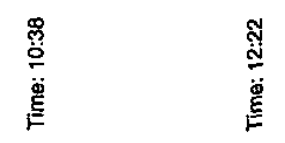

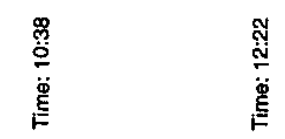

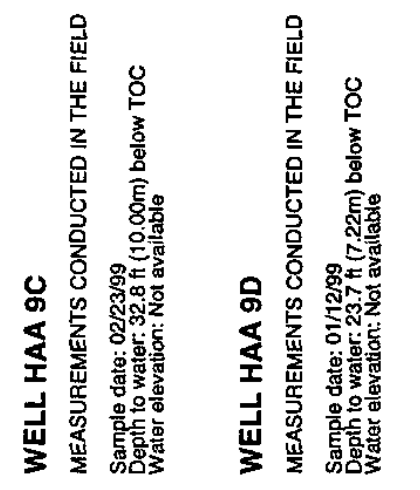
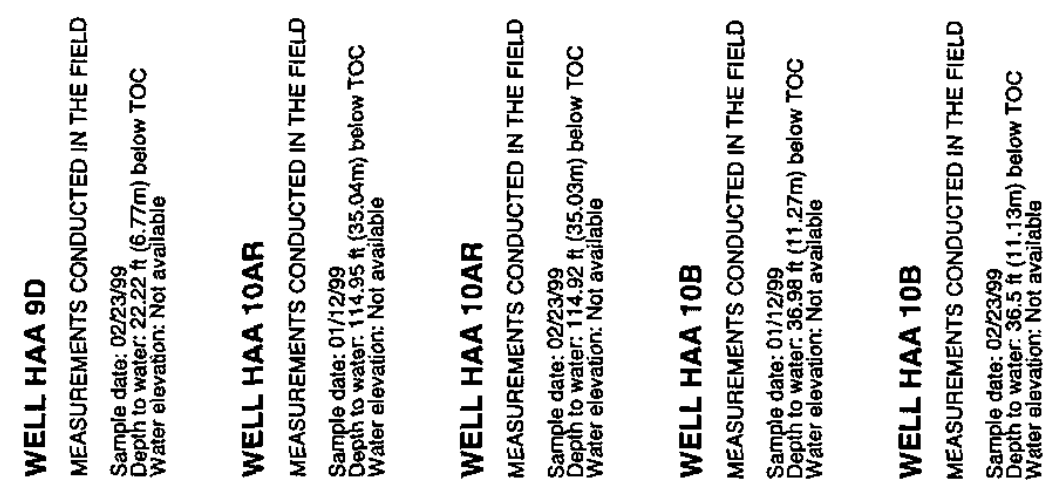

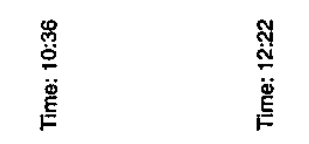

矛

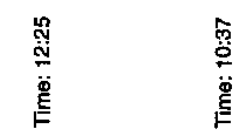

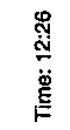

商

\&

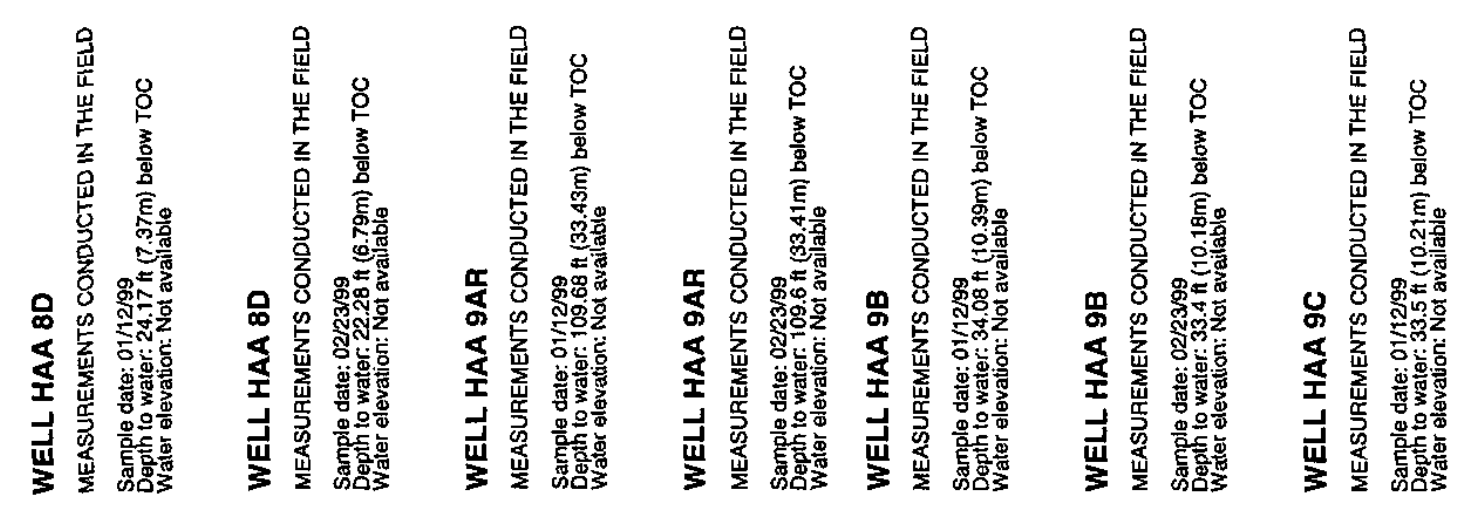

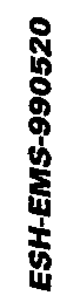




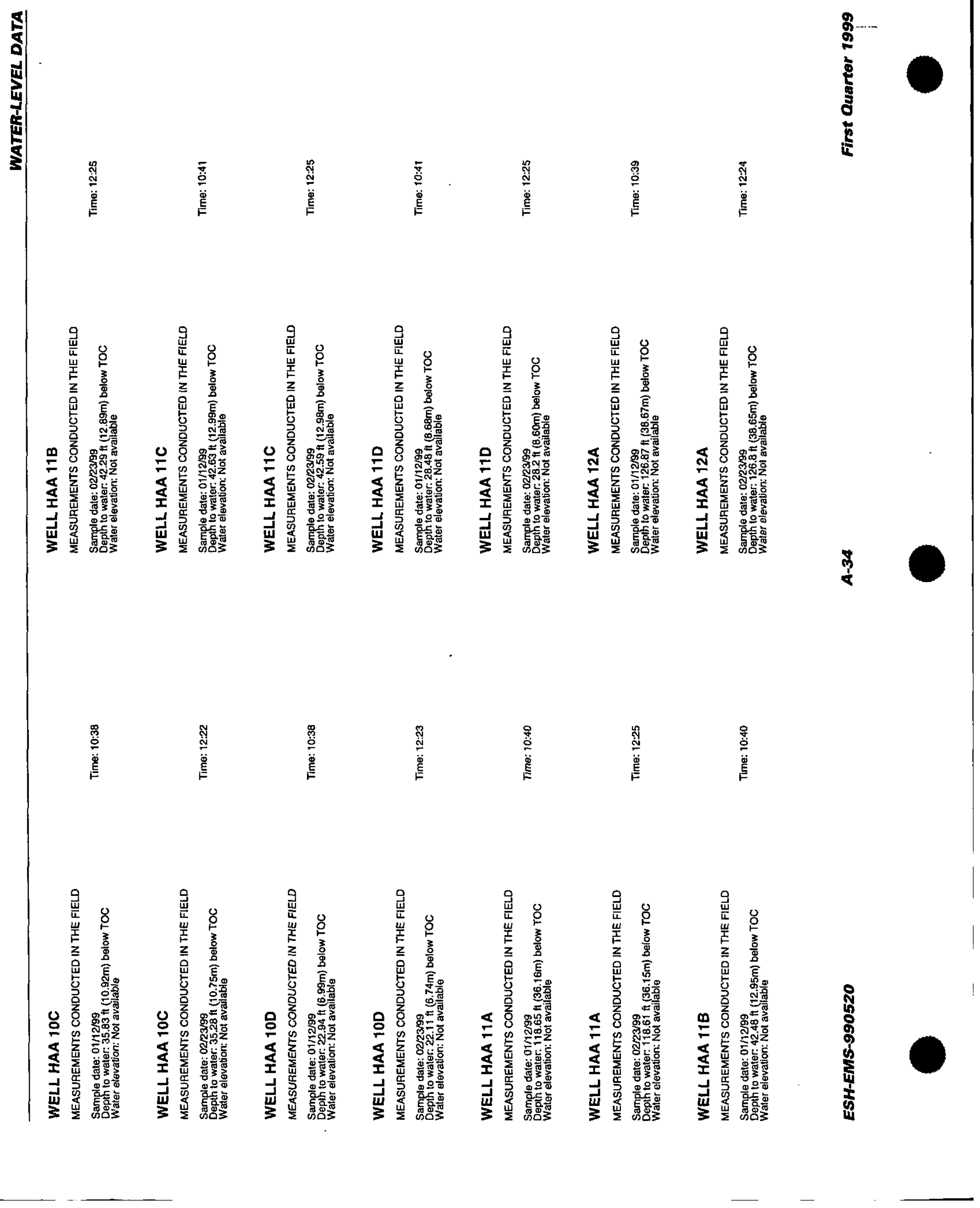




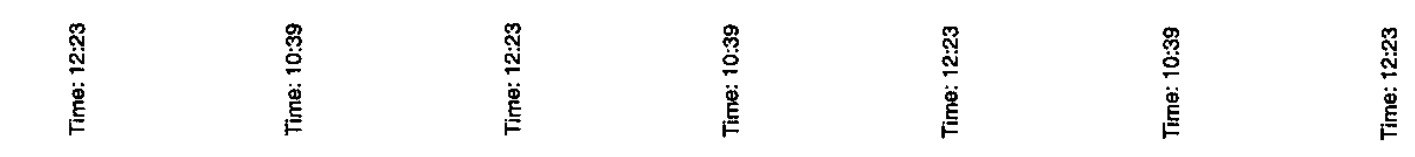

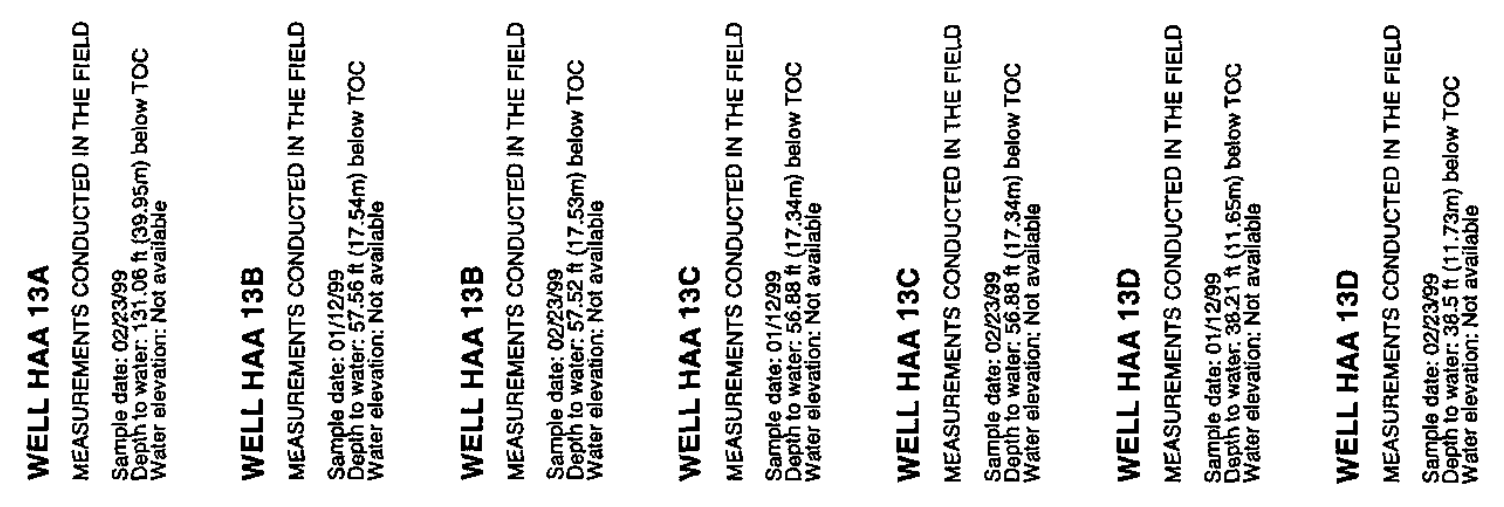

是

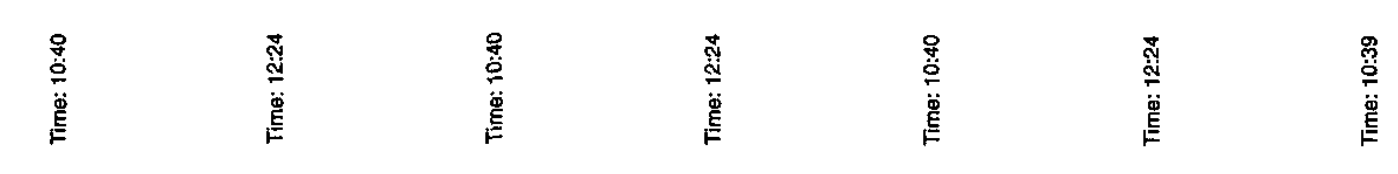

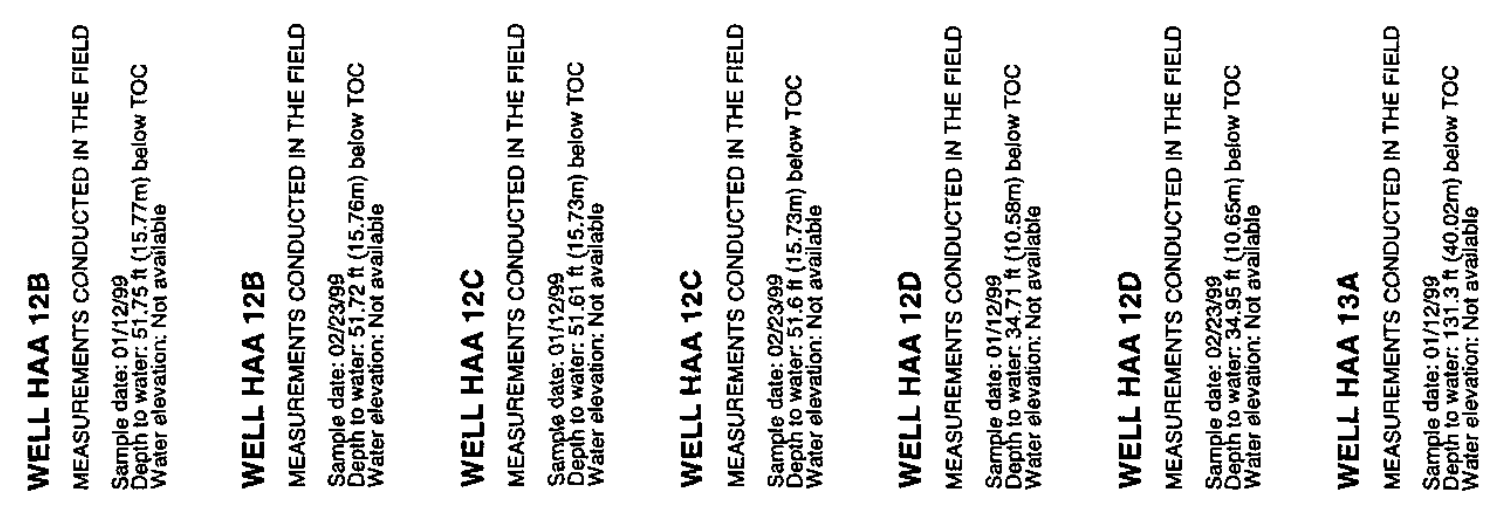

棠 


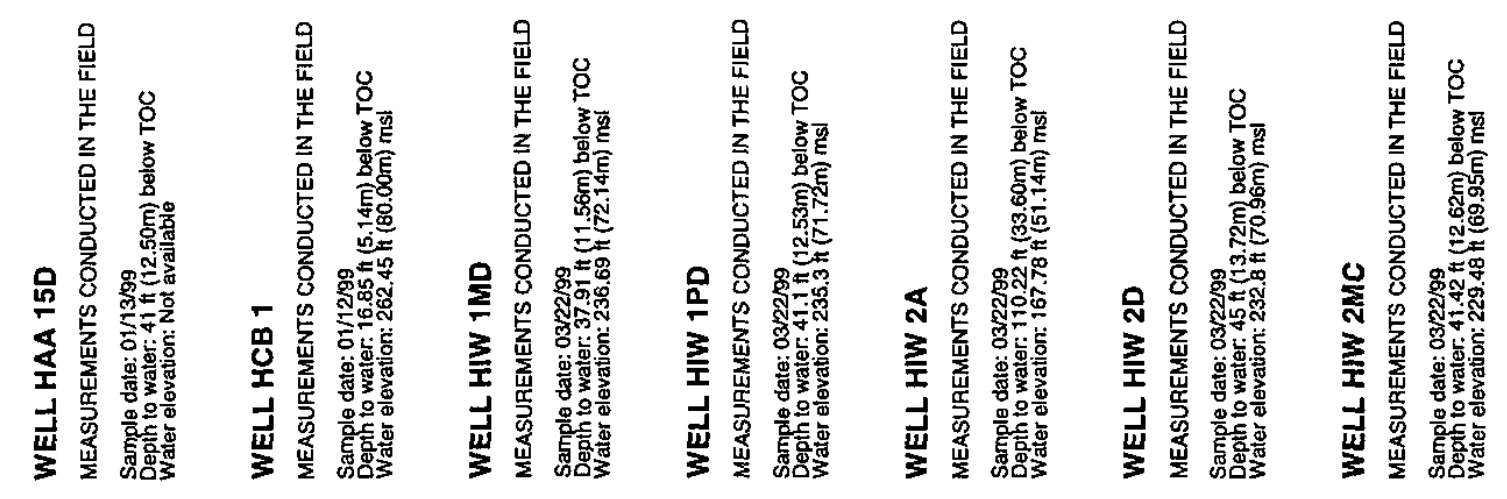

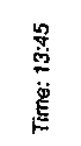

惫

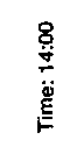

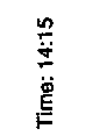

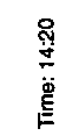
蚿

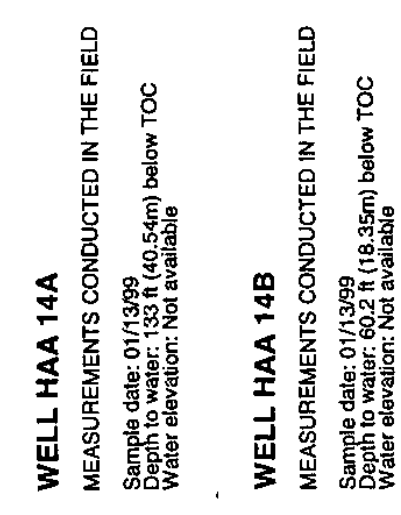

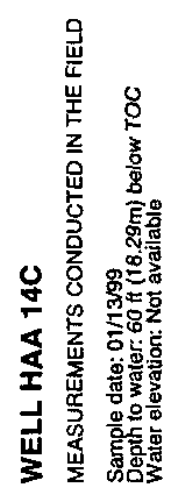

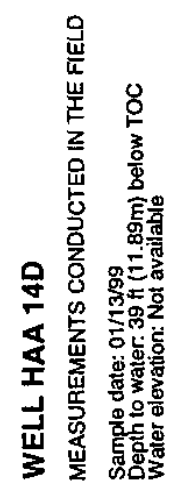

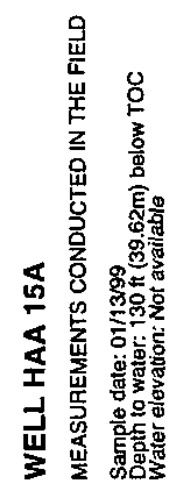
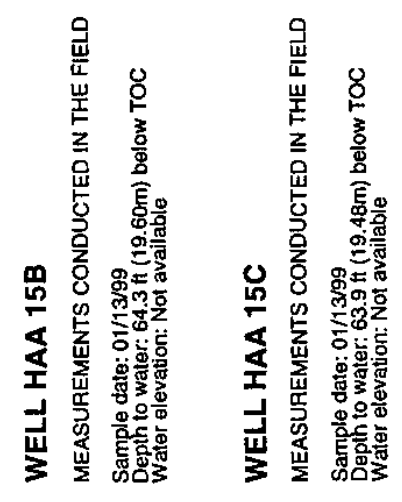

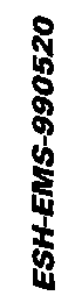




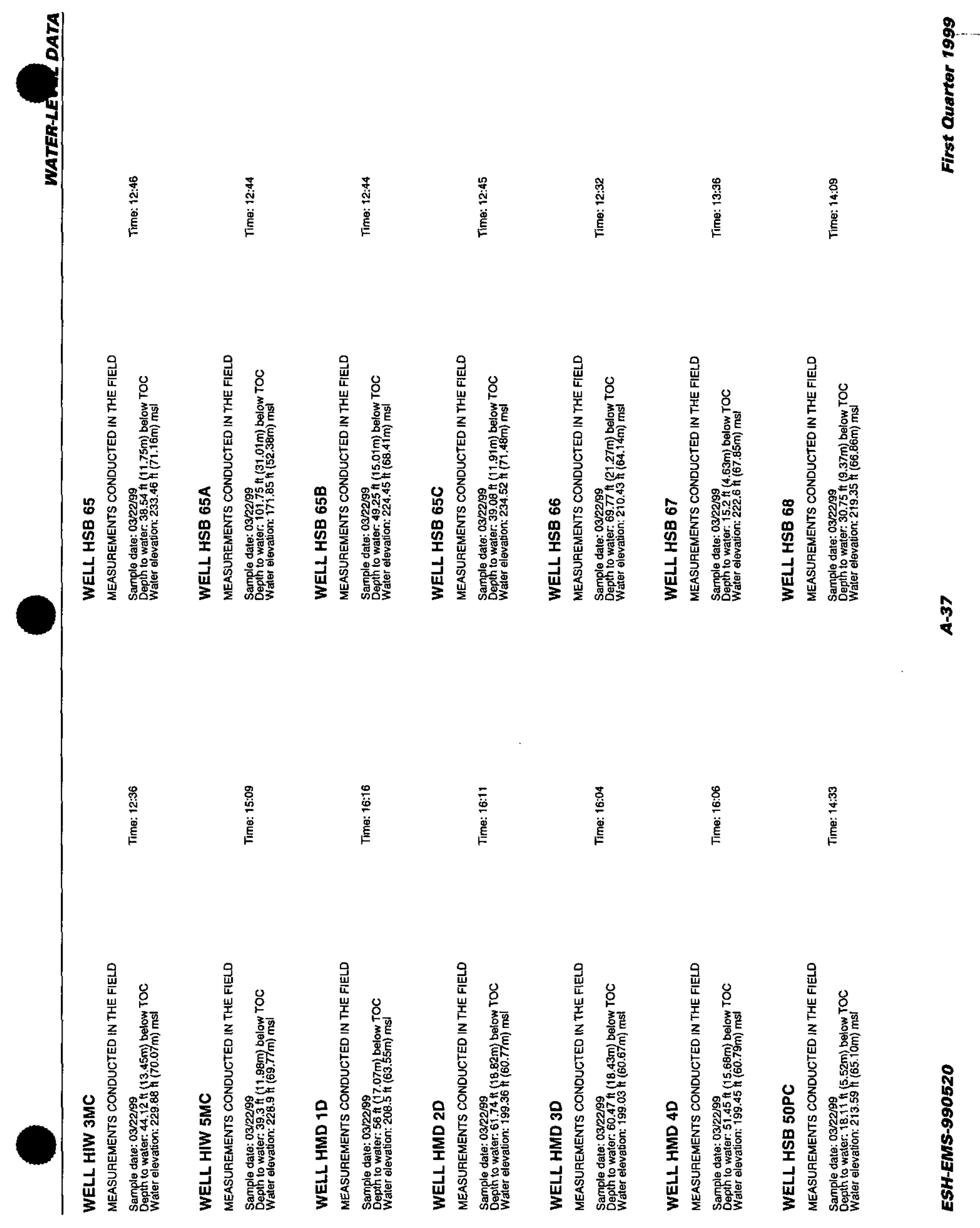




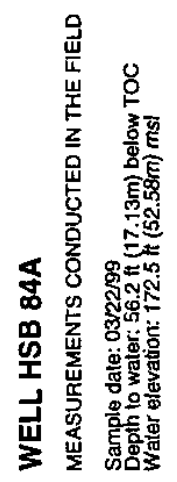

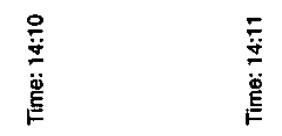

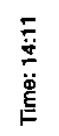

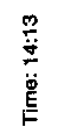

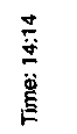

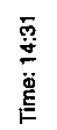

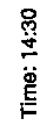

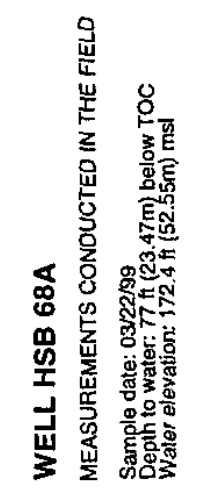

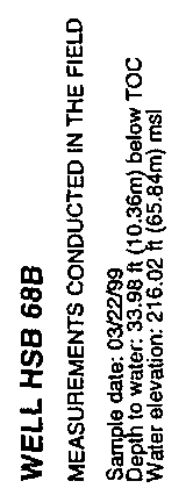
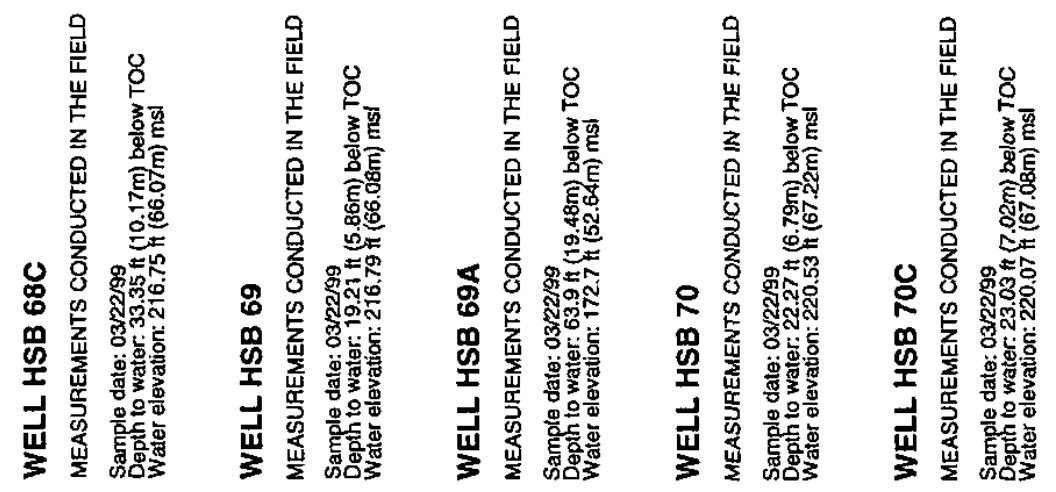


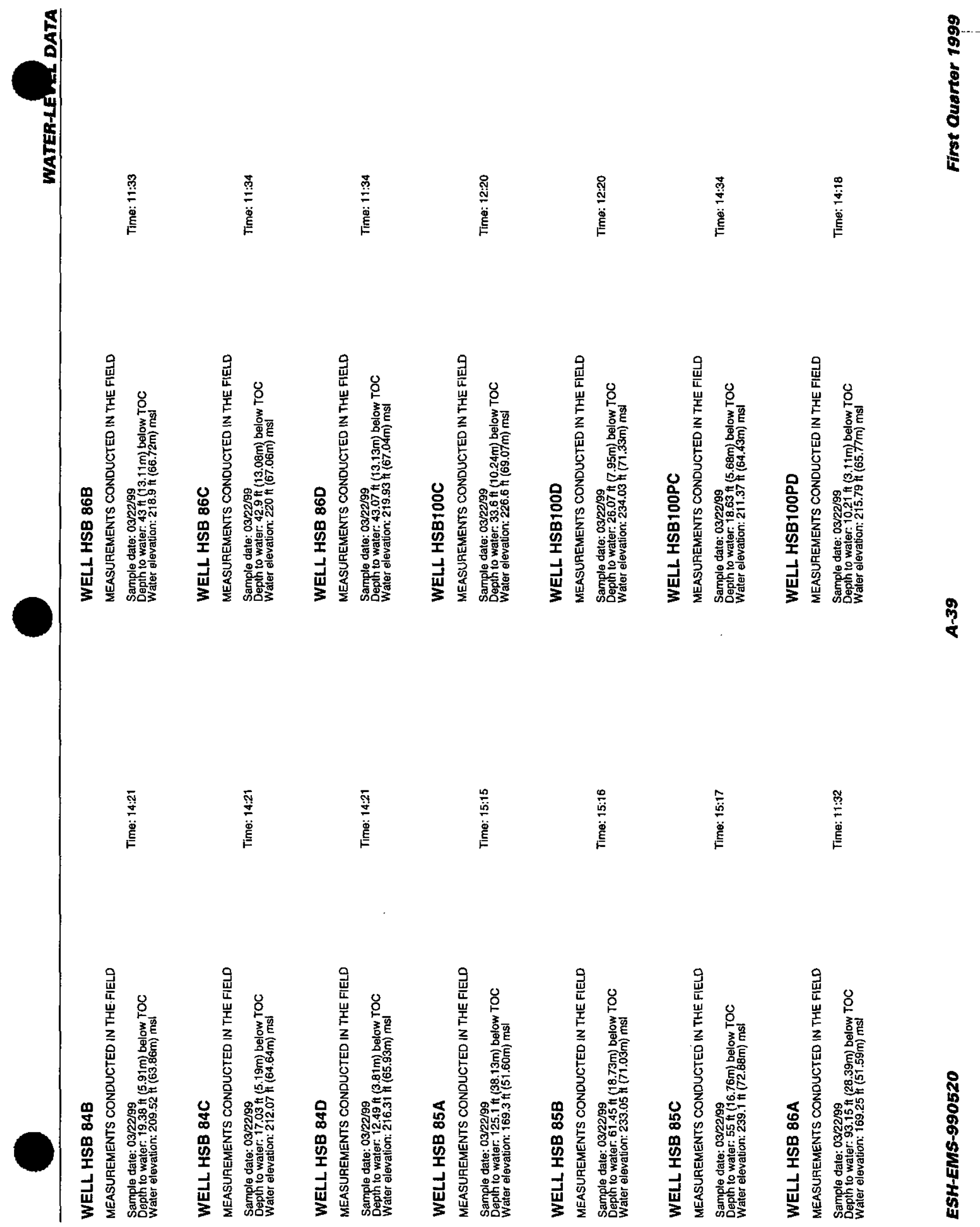



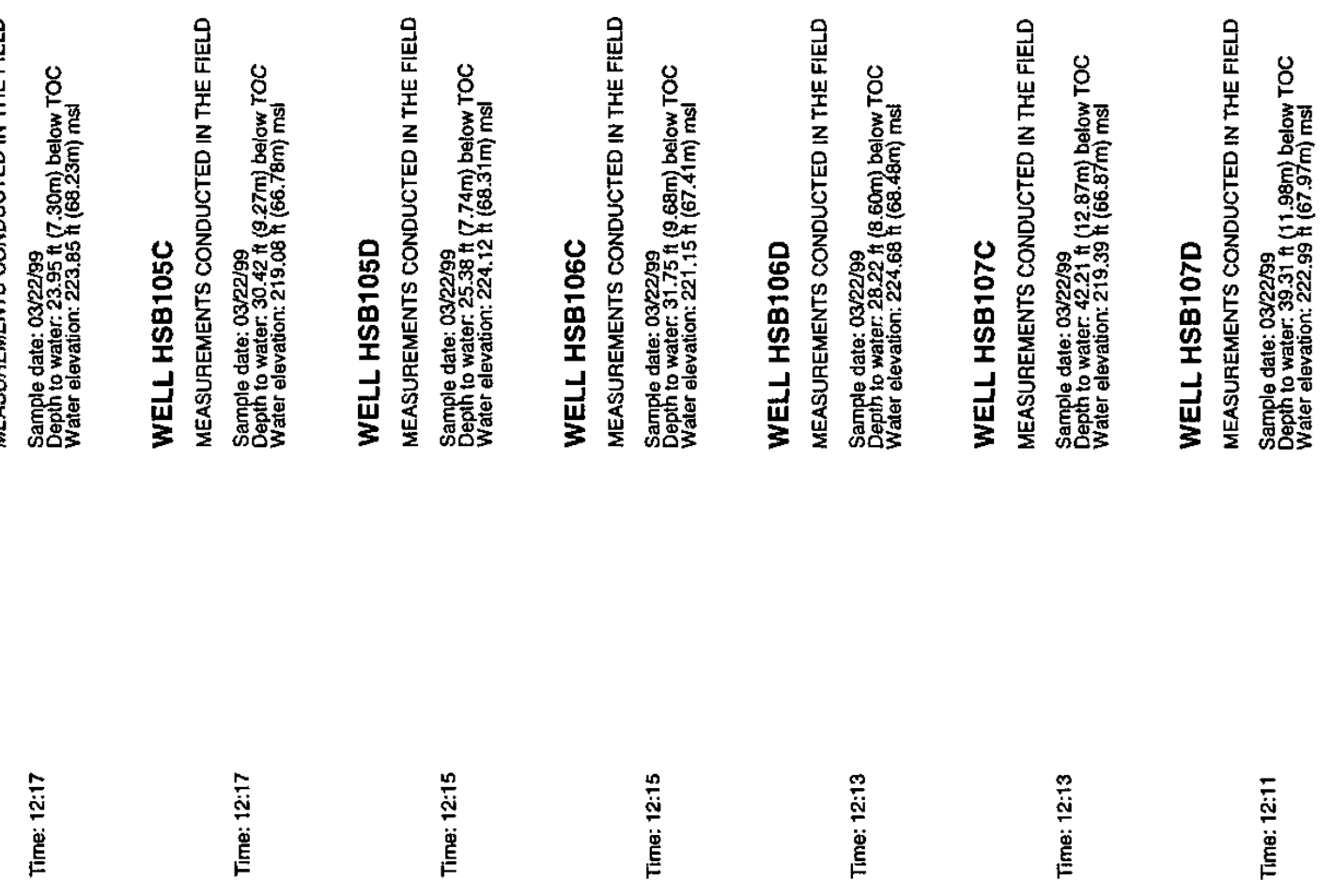

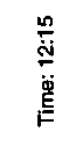

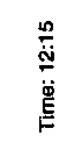

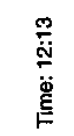

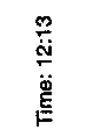

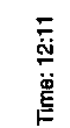




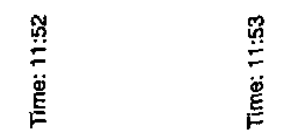

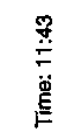

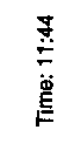

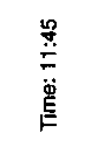

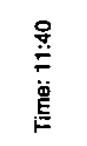

$\underset{⿱ 亠 幺}{\stackrel{\Xi}{*}}$

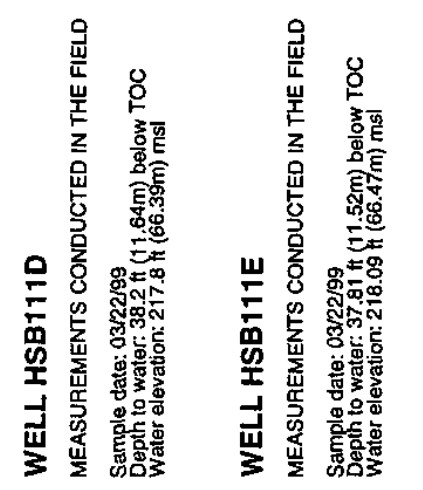
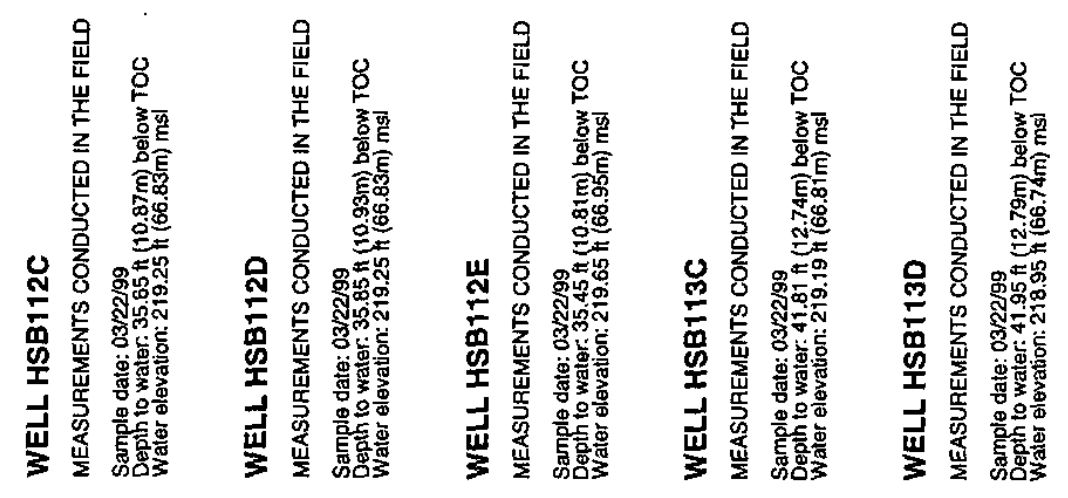

\$

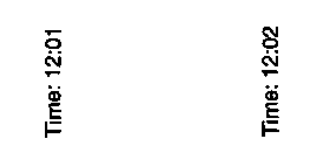

兽



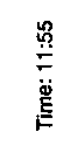

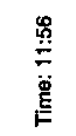

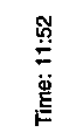
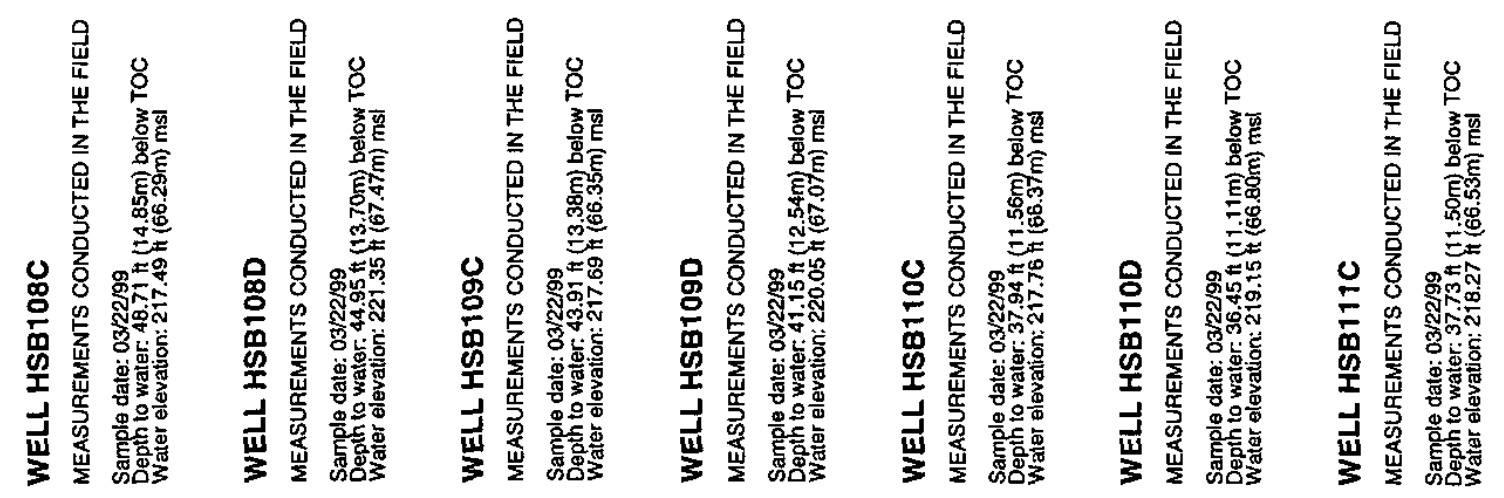

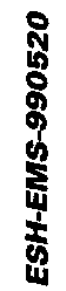




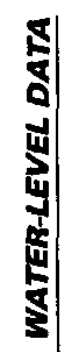

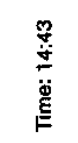

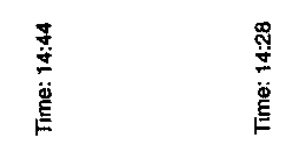

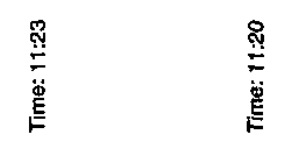

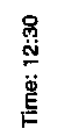

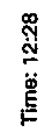
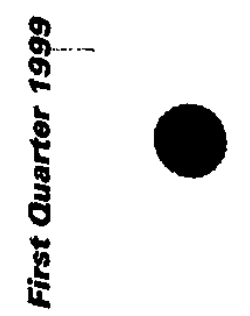

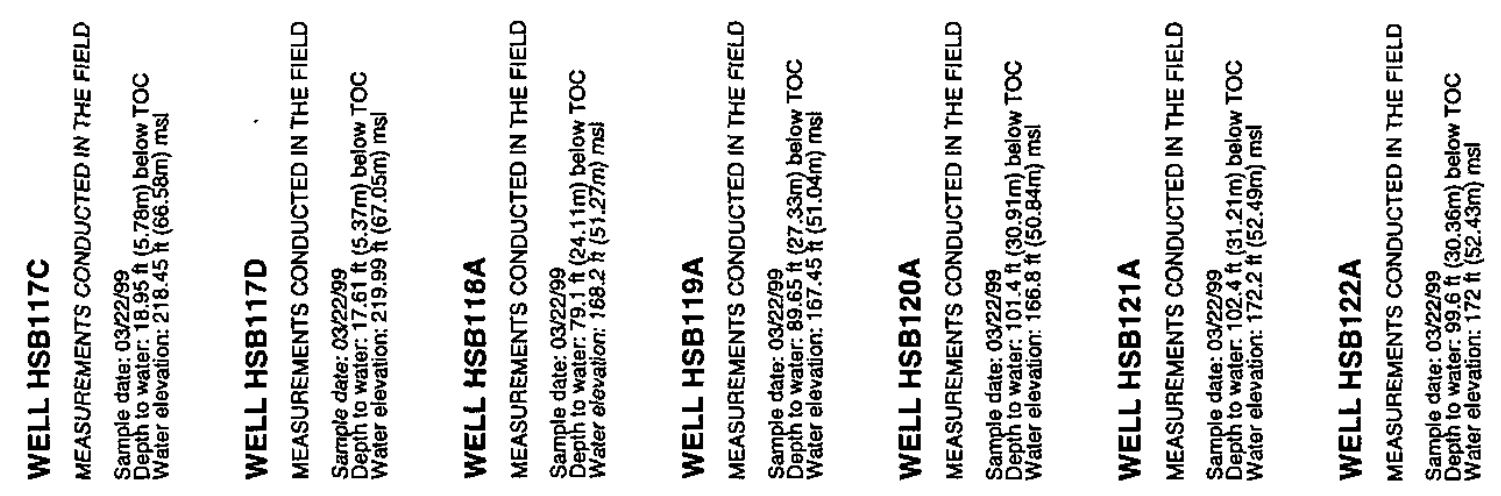

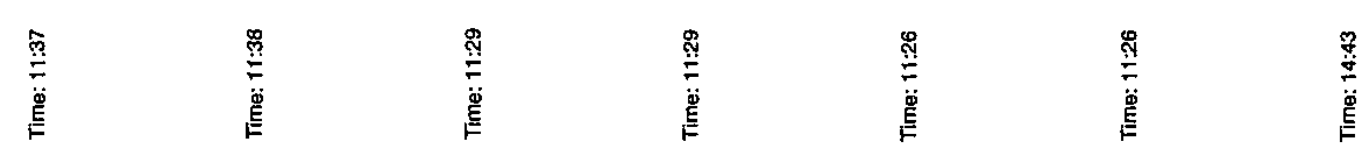

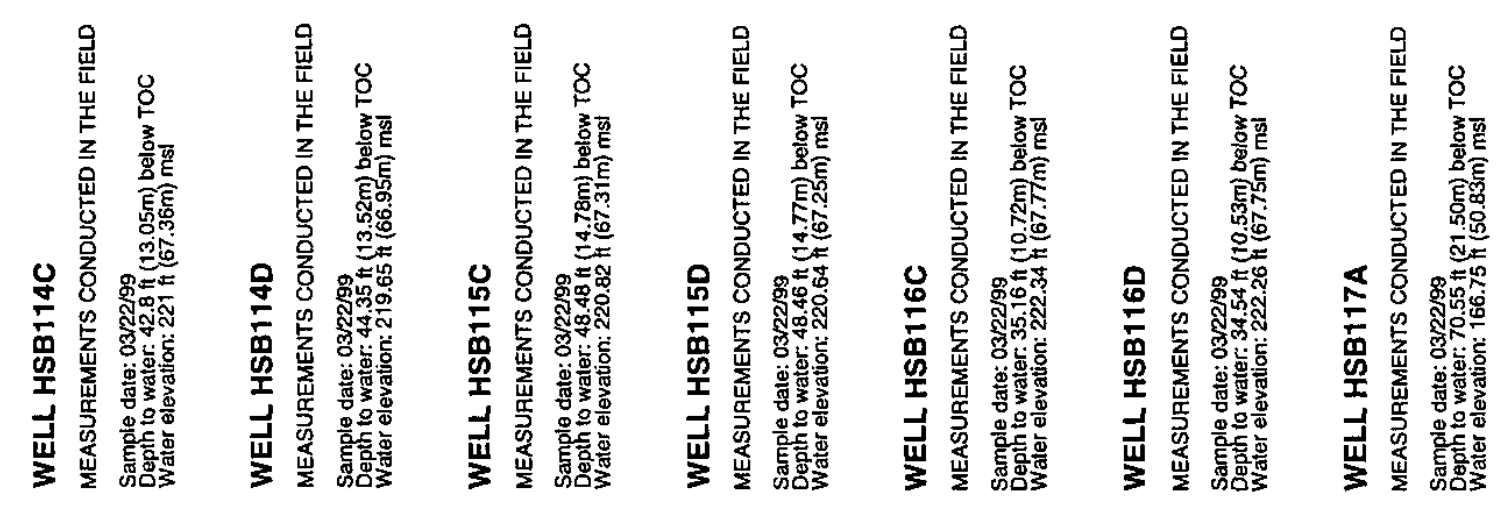

亭

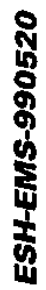




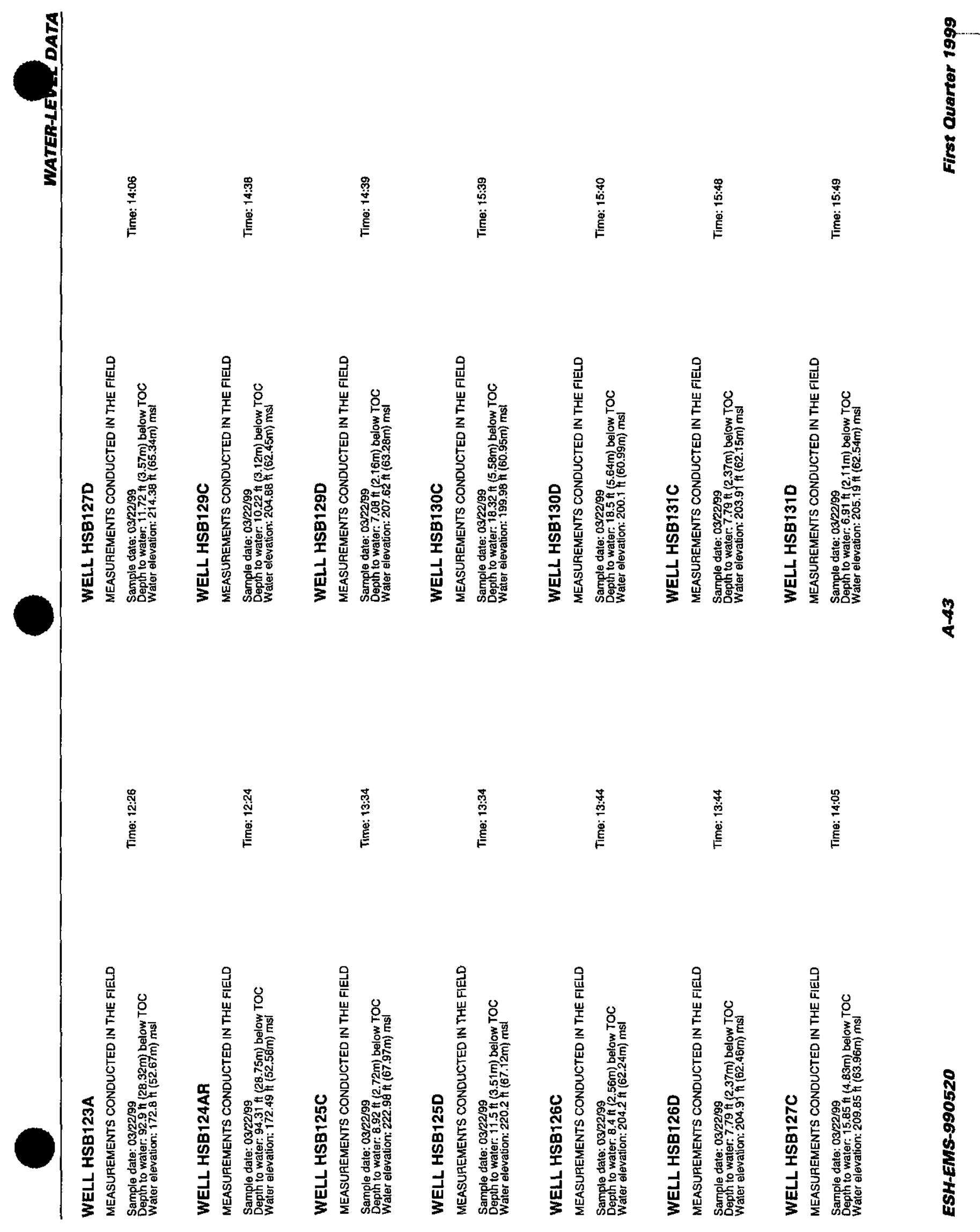




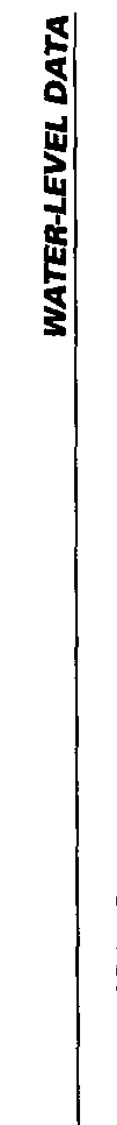

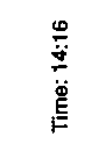

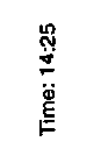

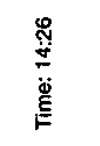

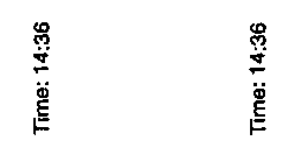

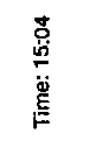

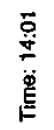

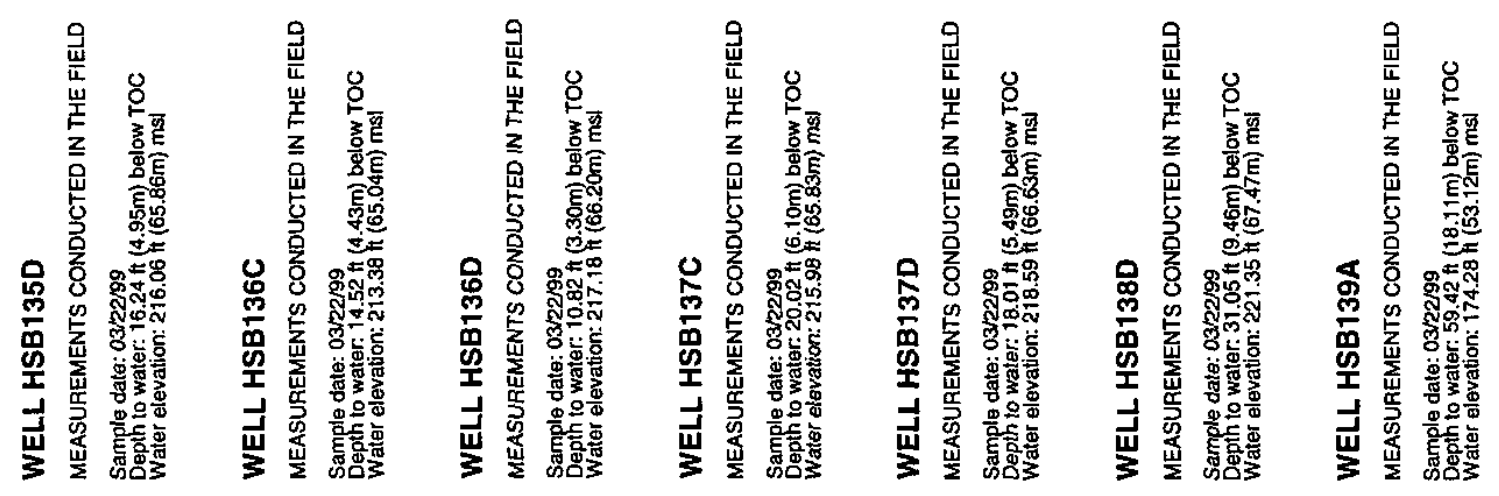

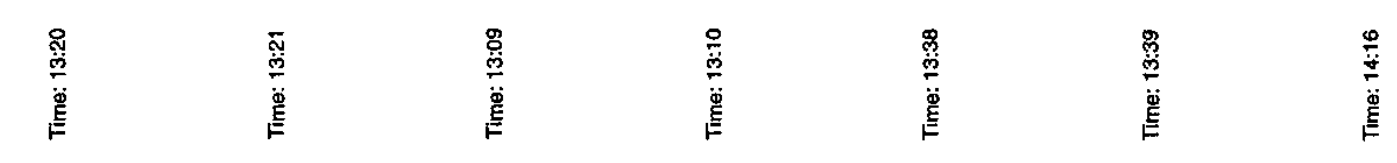

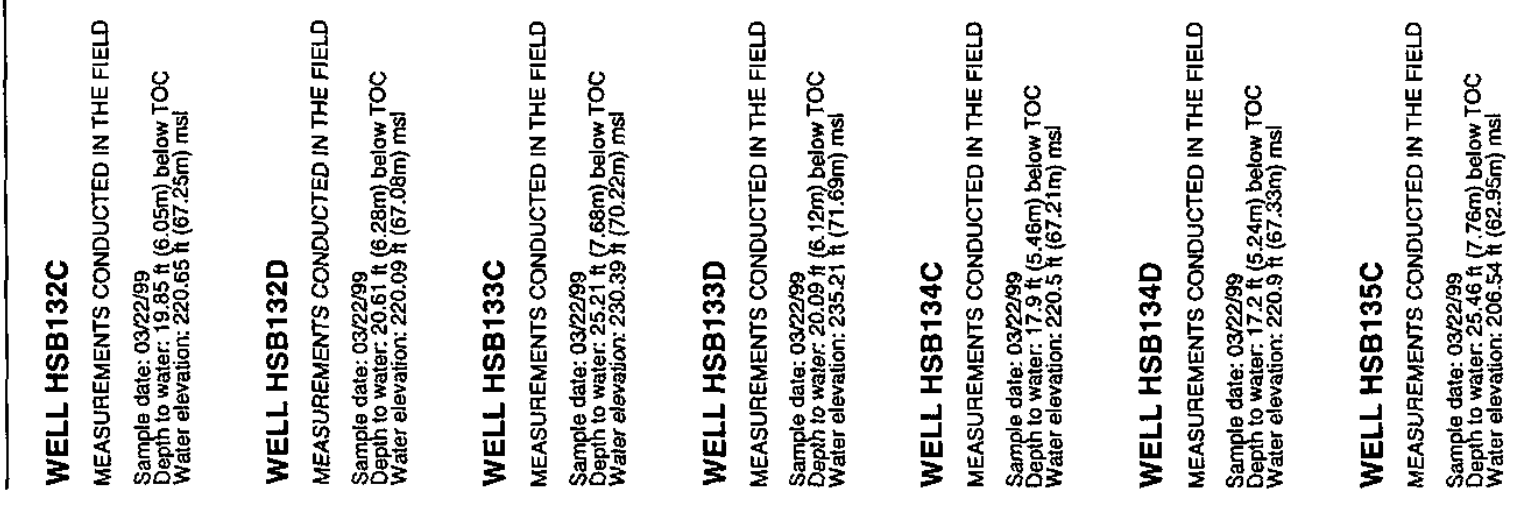

\$

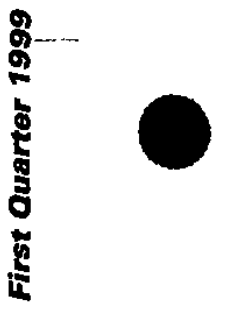




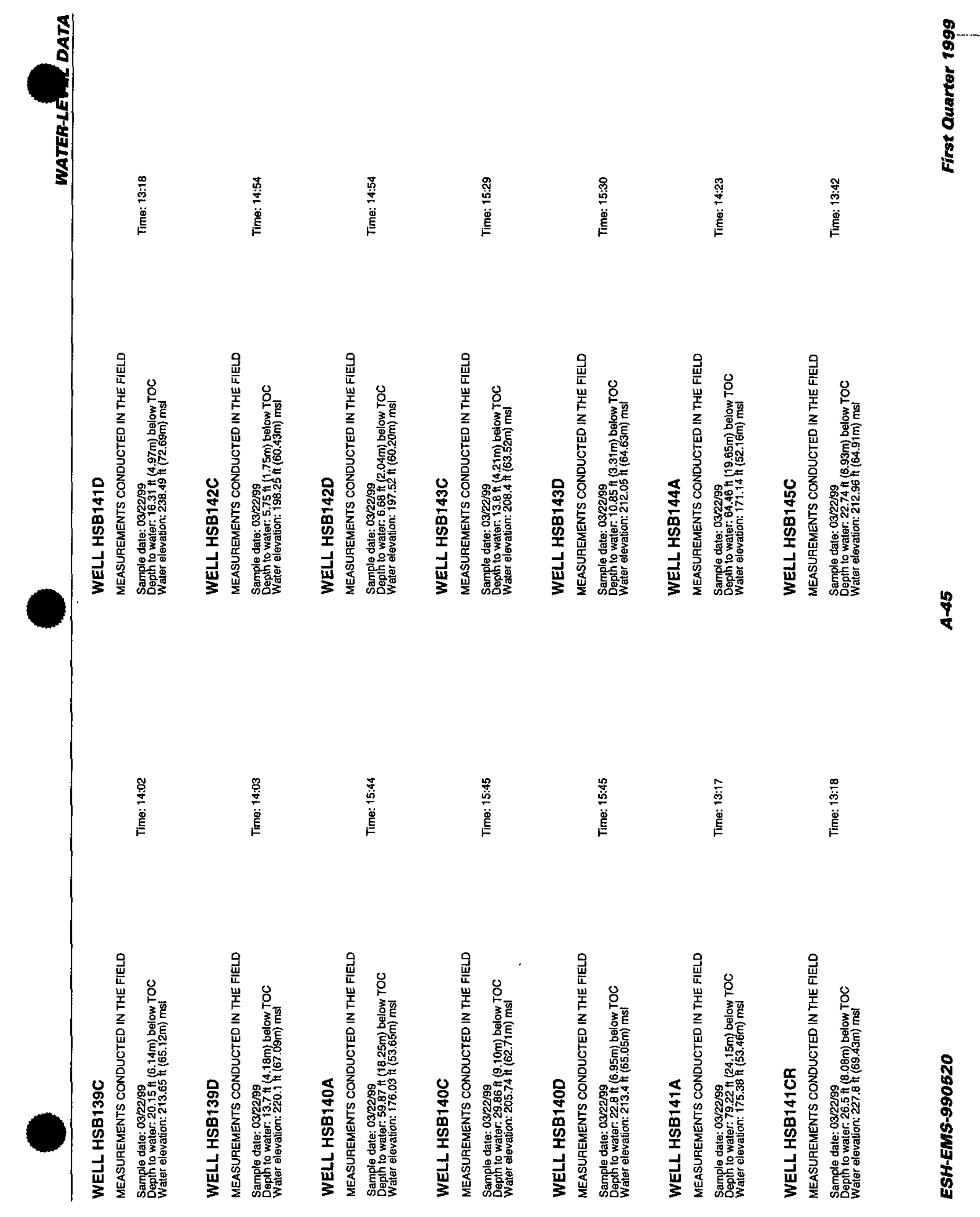


$\mathbf{x}$
$\mathbf{5}$
$\mathbf{1}$
$\mathbf{4}$
$\mathbf{7}$
$\mathbf{4}$
$\mathbf{4}$
$\mathbf{5}$

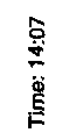

产

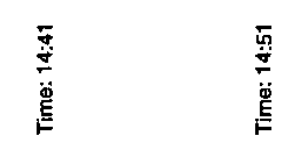

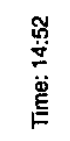

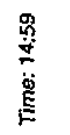

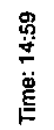

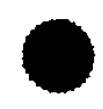

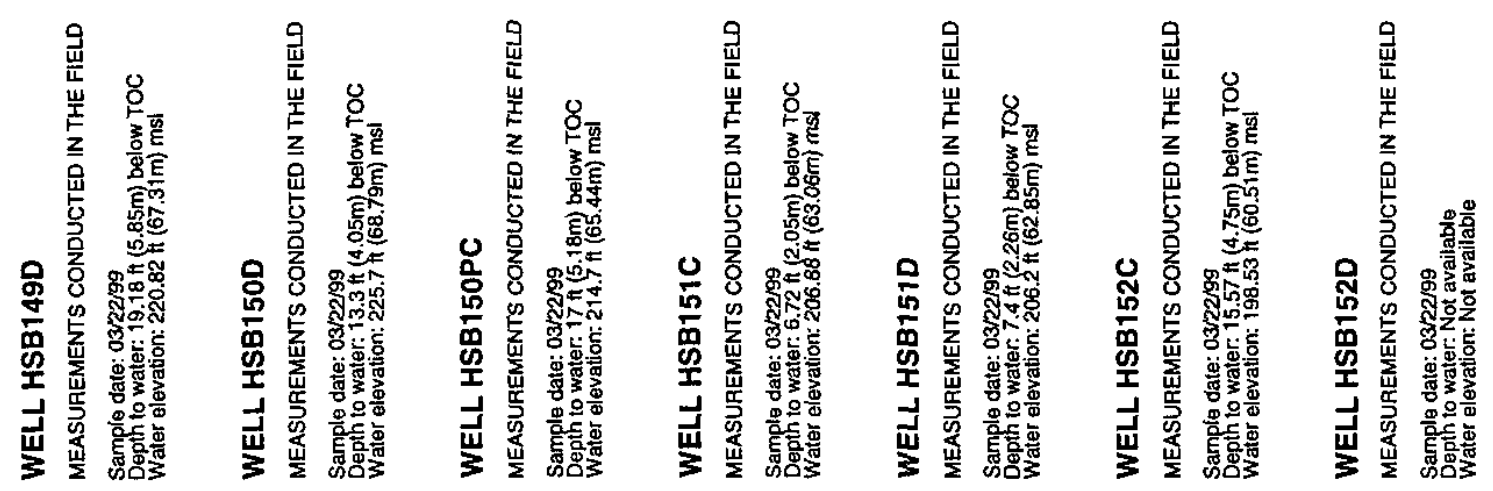

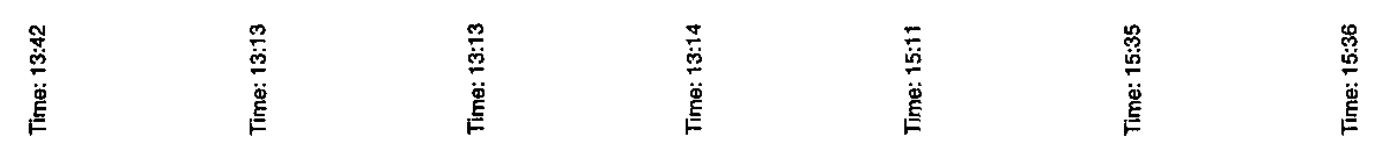

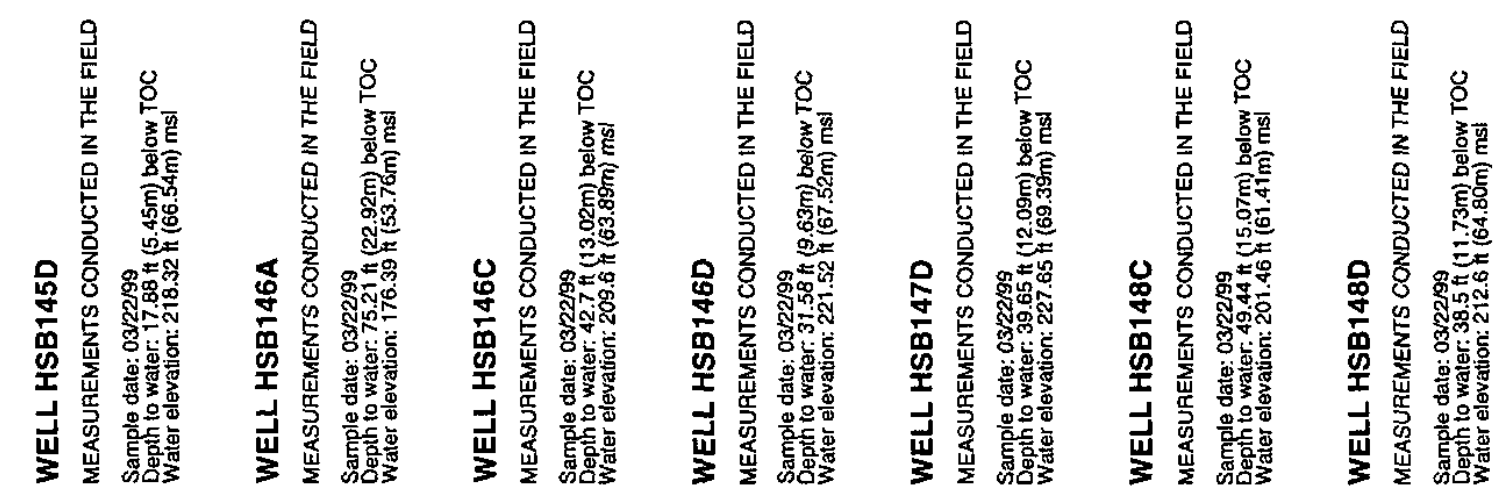

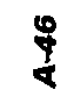




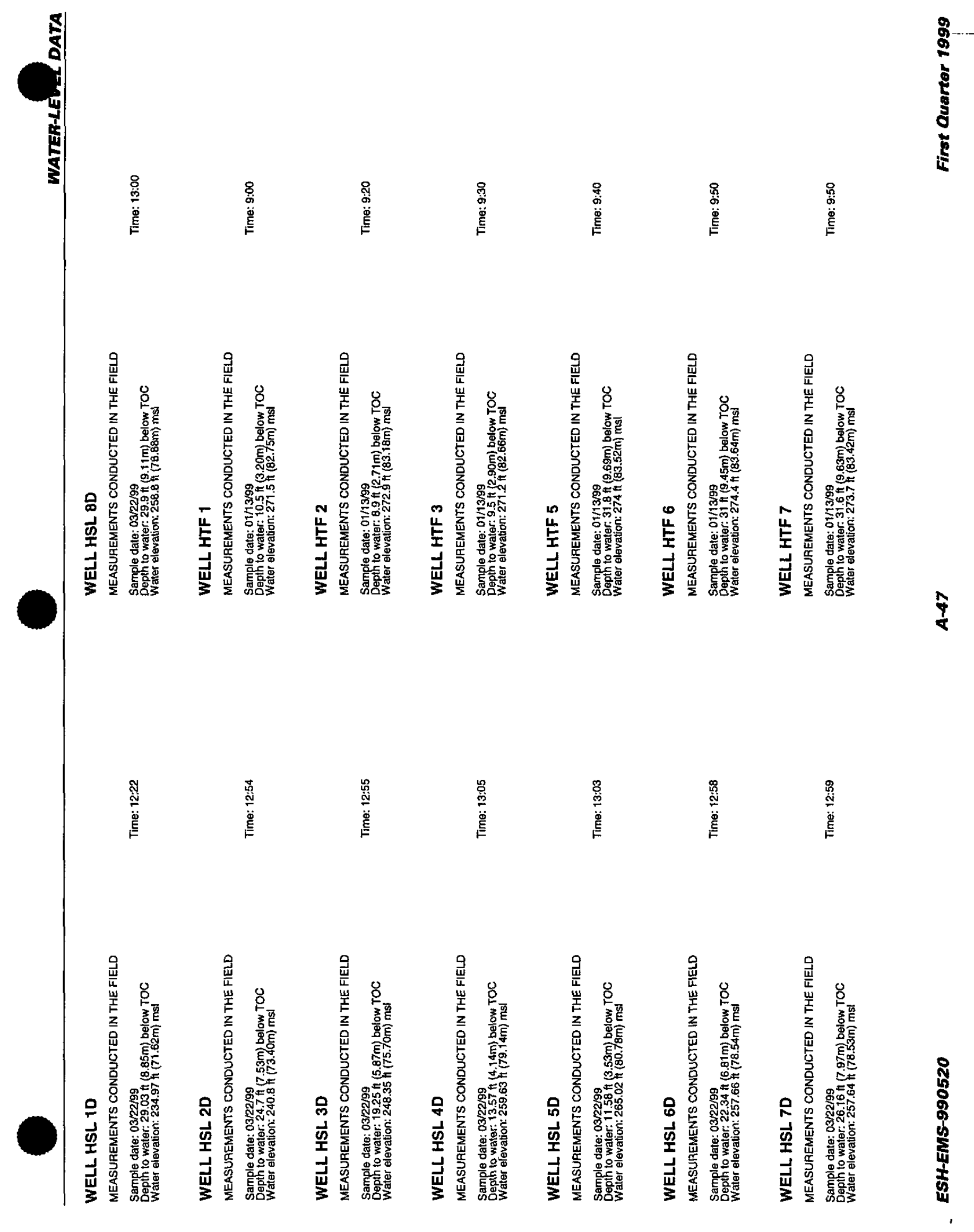




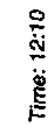

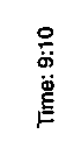

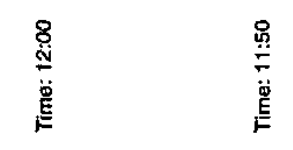

$\stackrel{?}{\stackrel{P}{*}}$

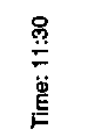

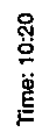

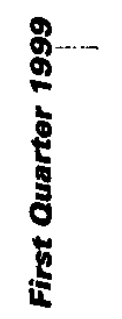

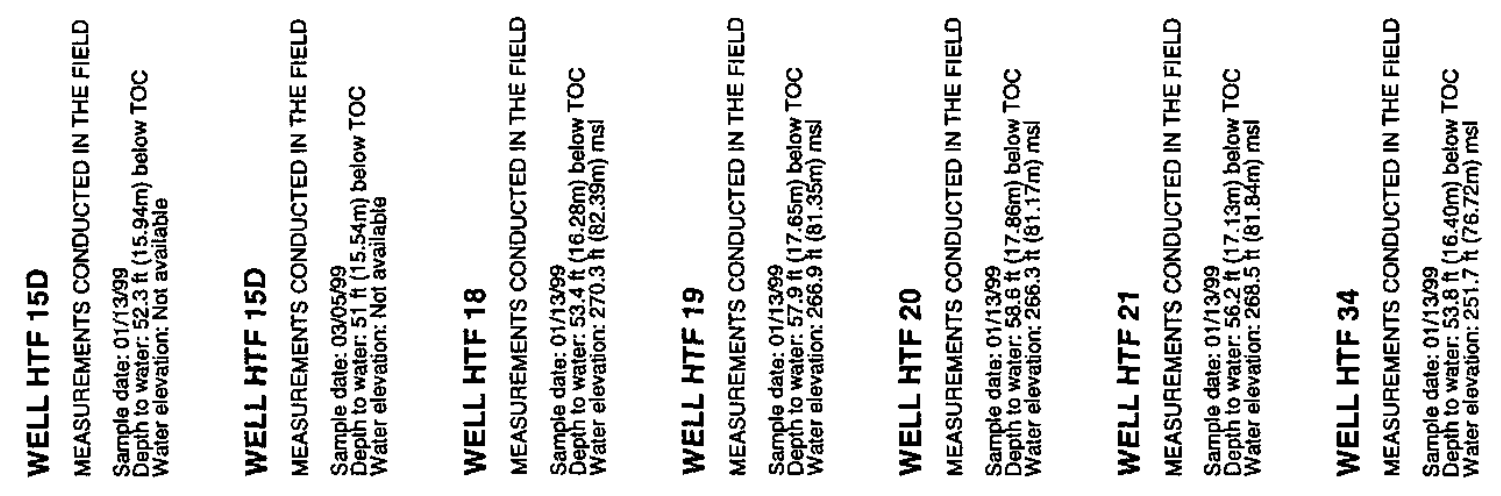

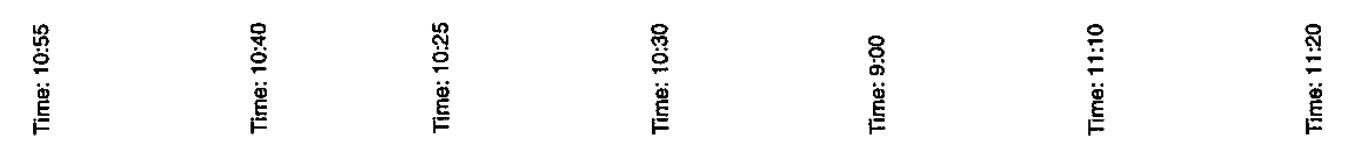

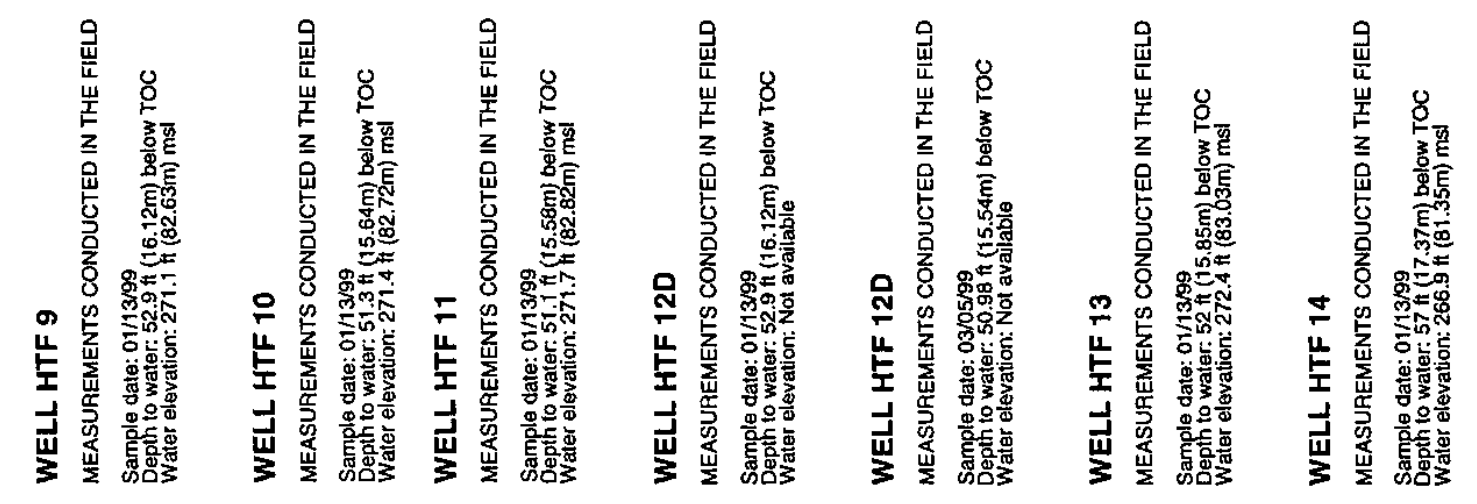

疋 


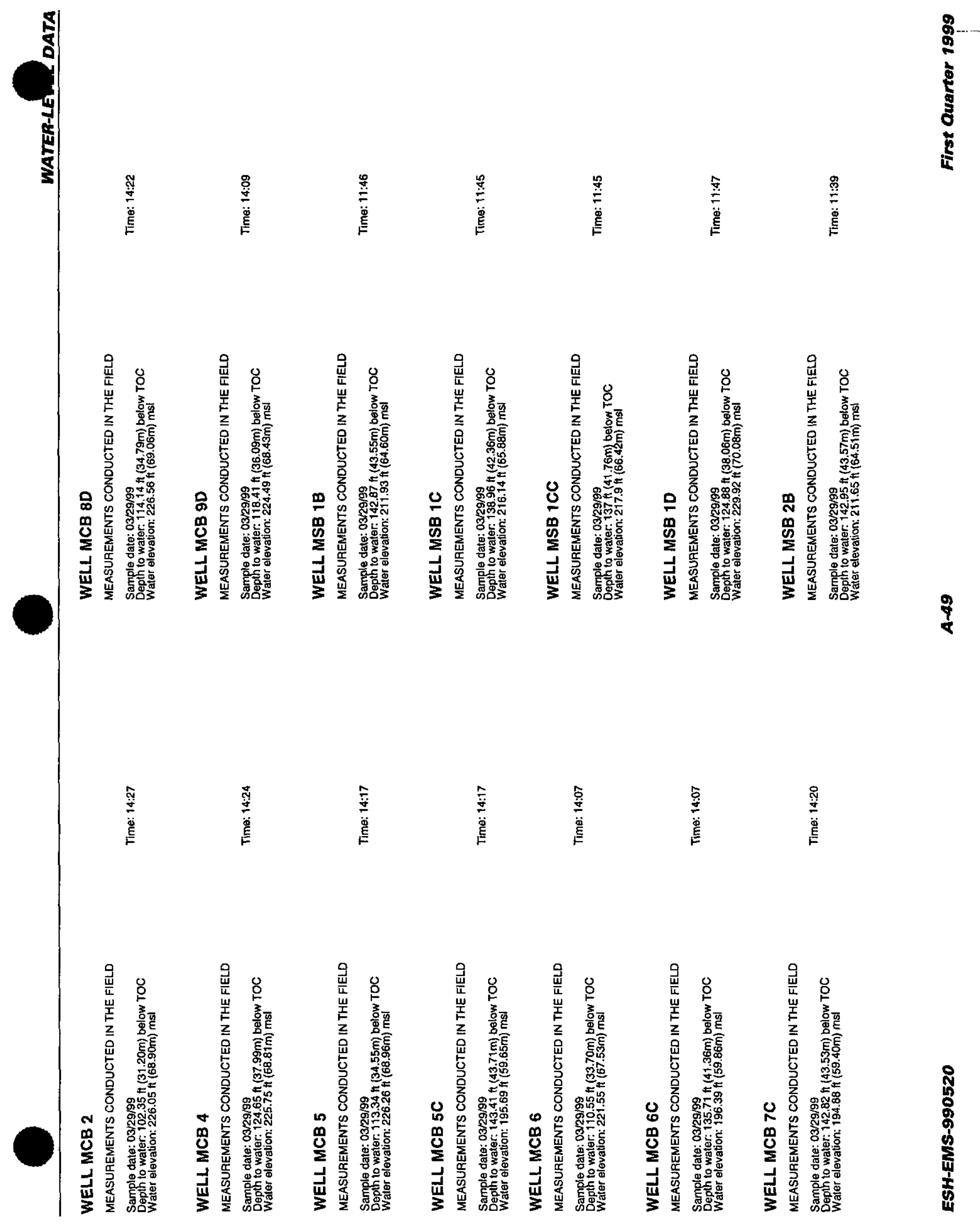




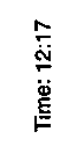

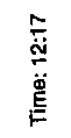

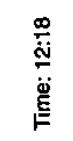

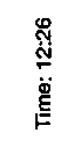



胥

总
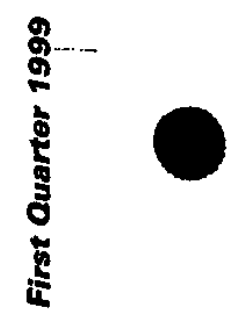

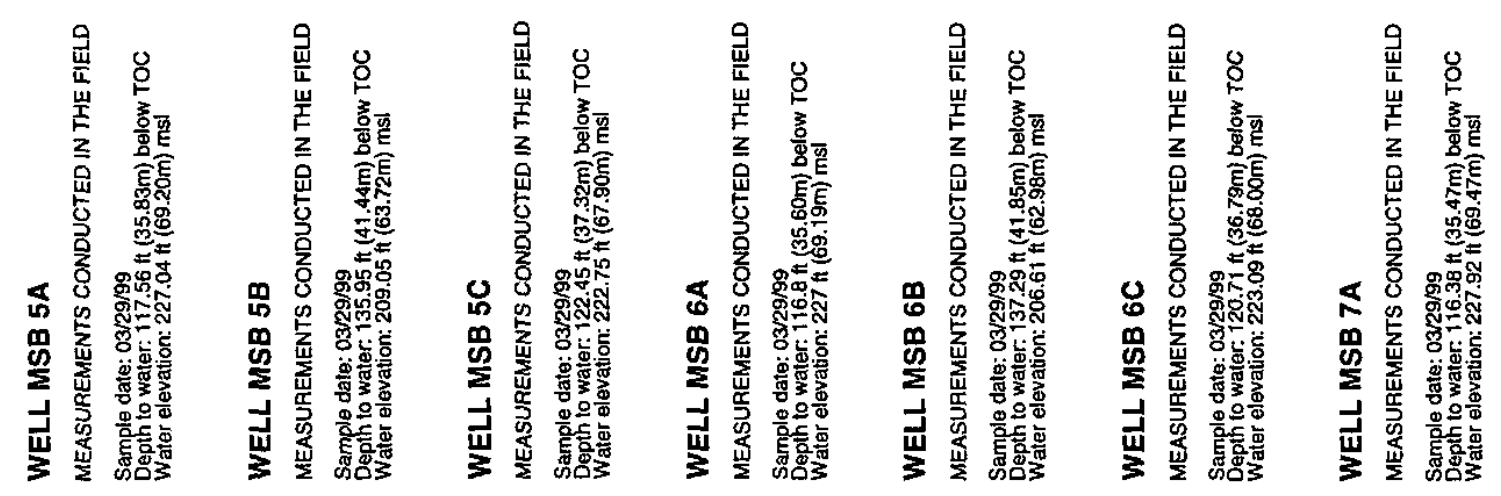

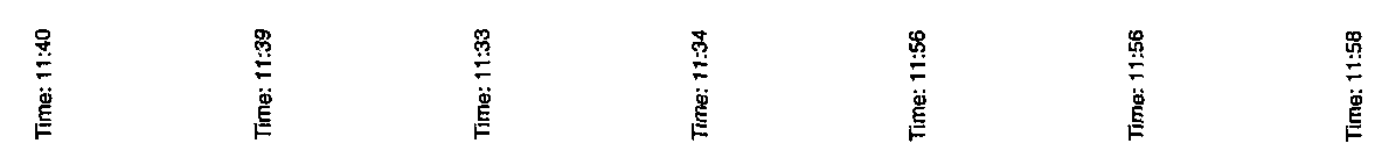

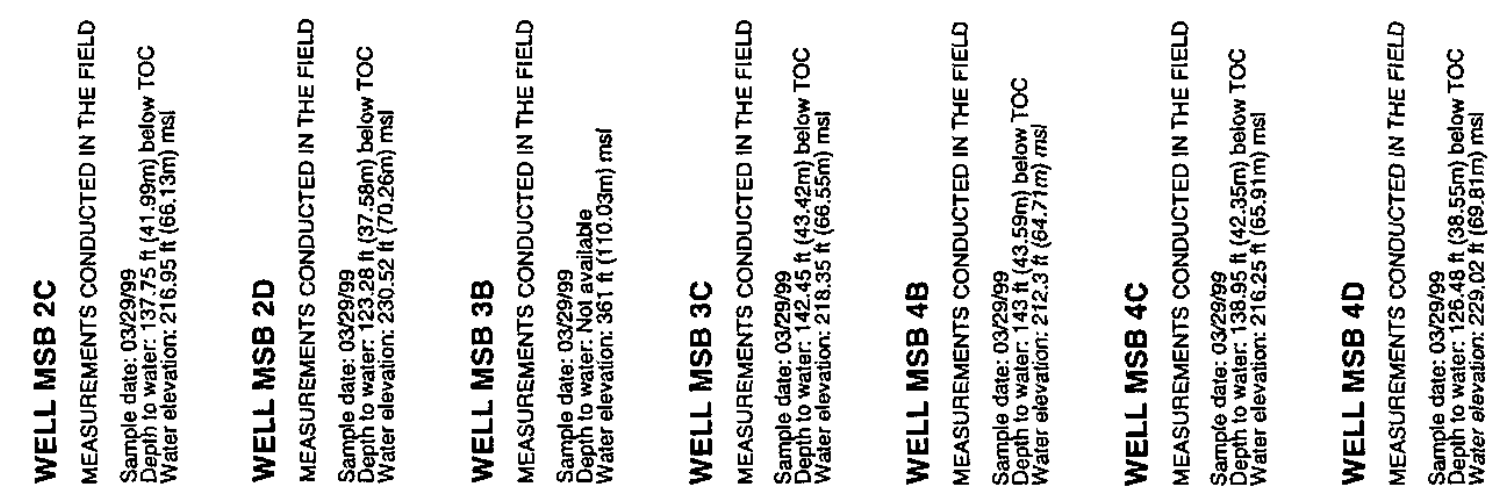

4

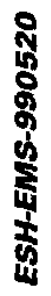




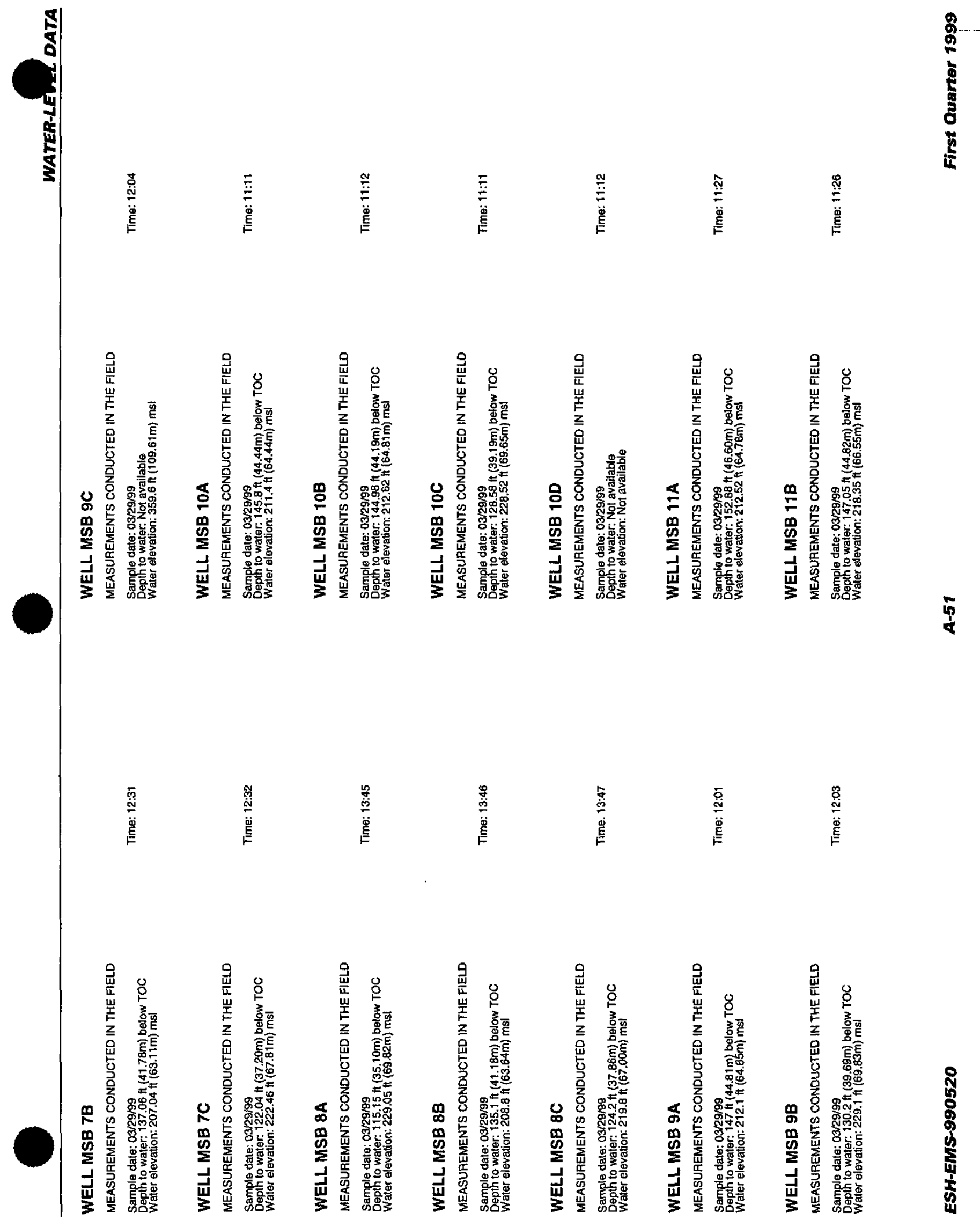




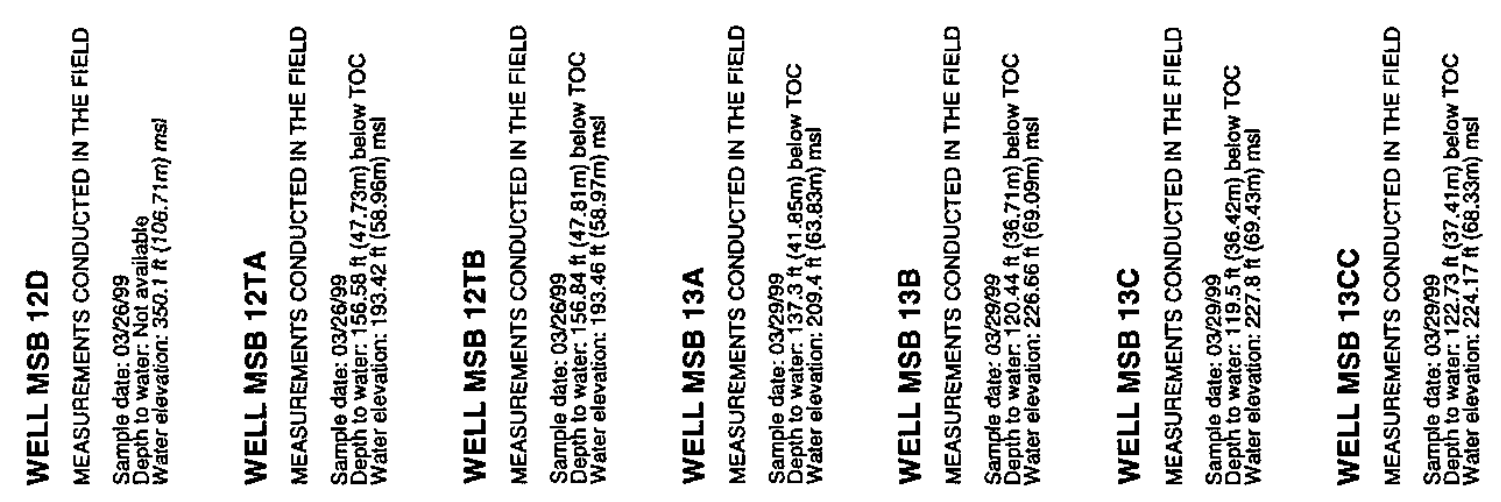


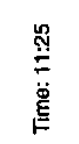

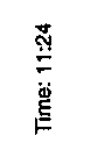

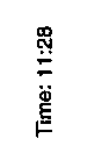

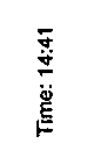

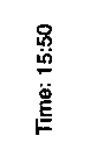

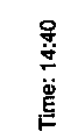
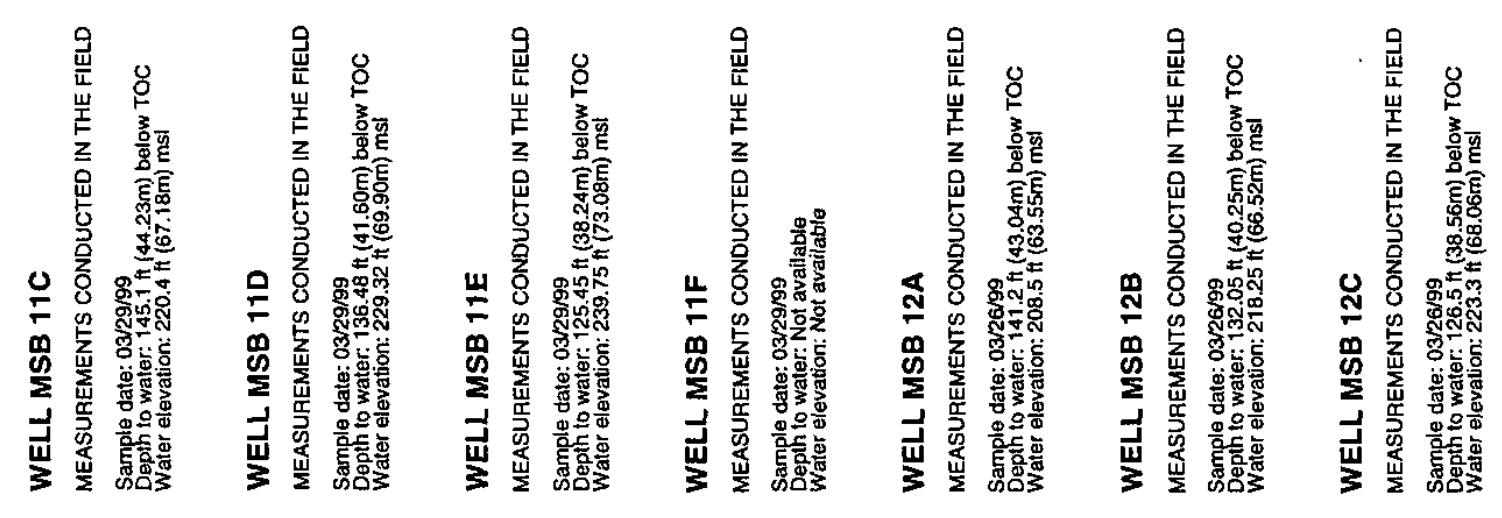

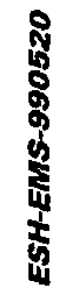



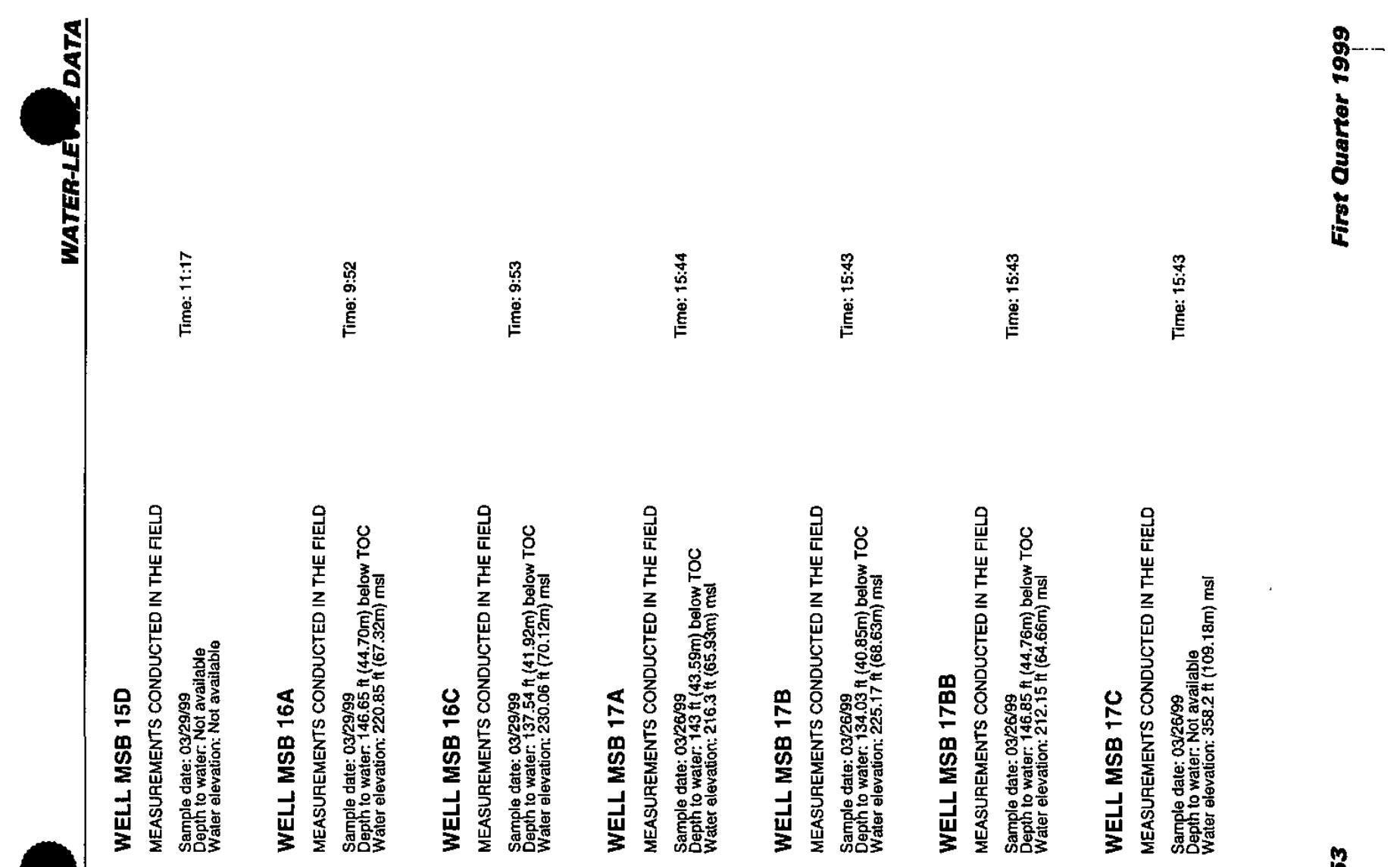

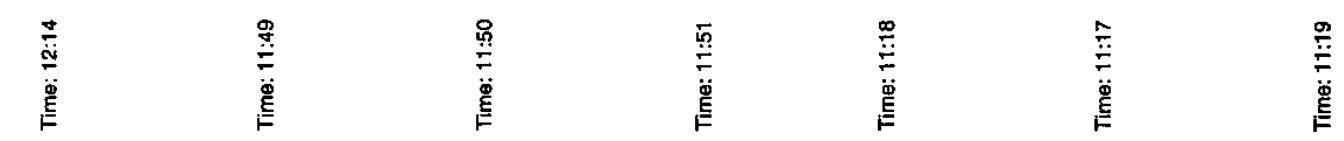

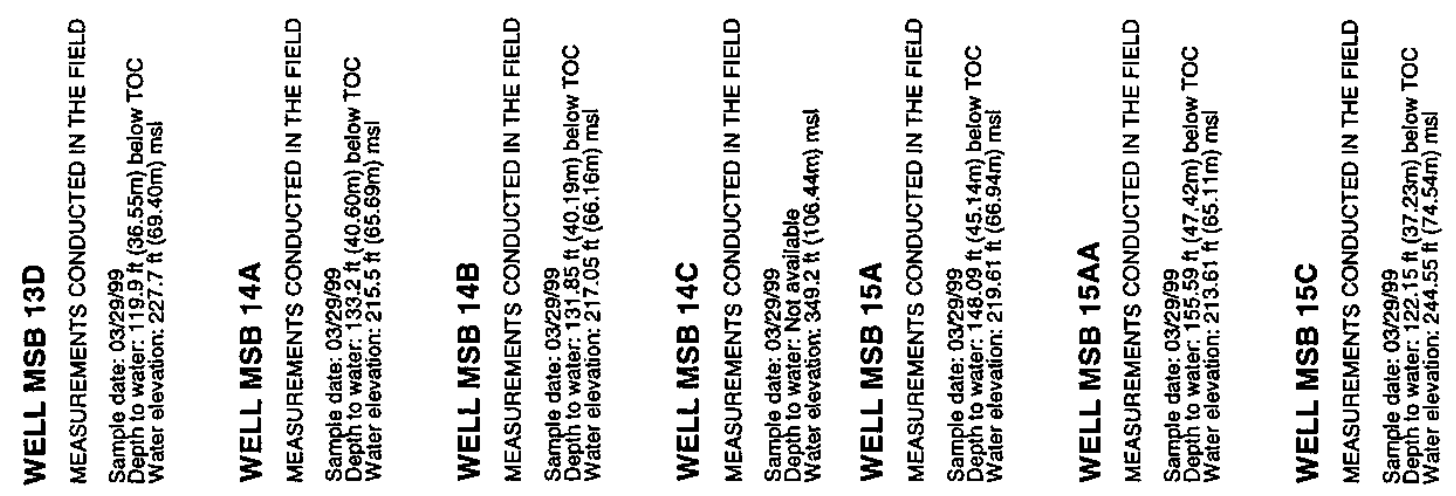

㝵 

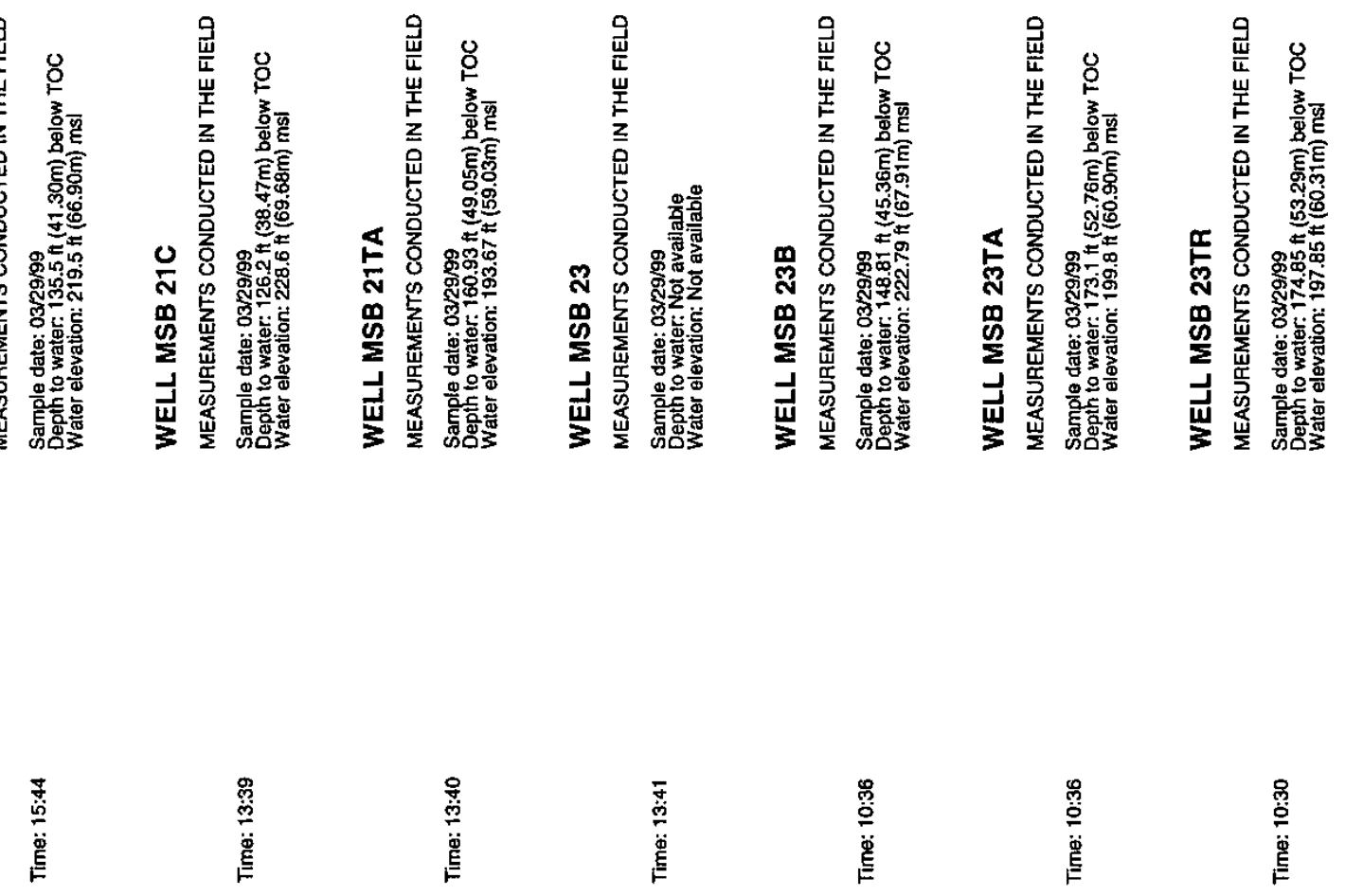

导

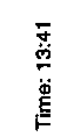

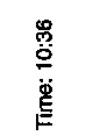

邑

兽
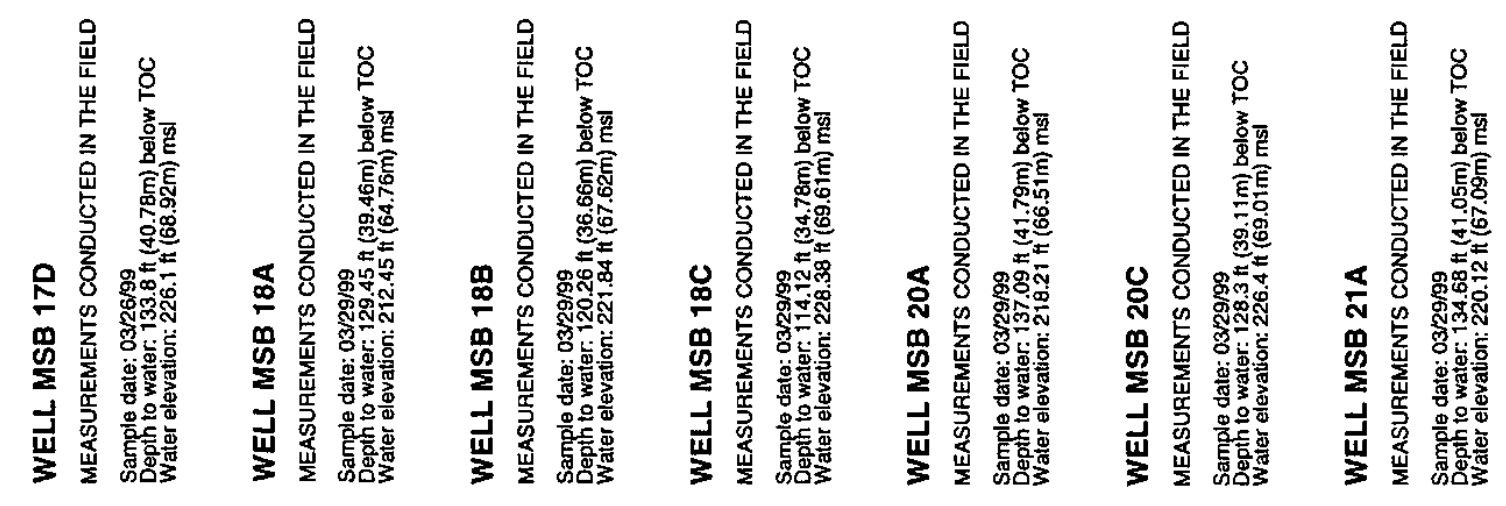

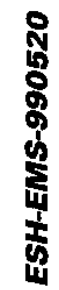



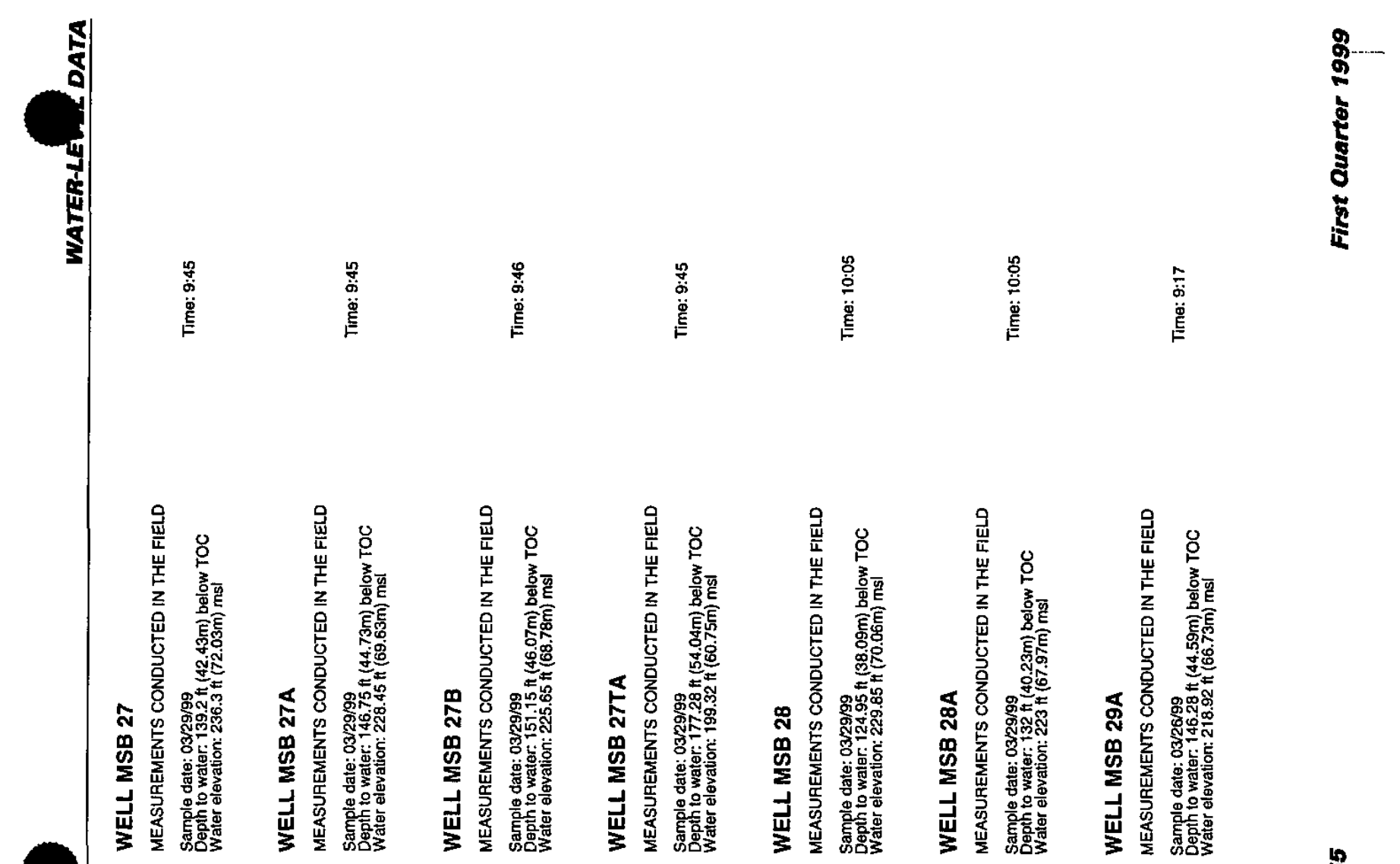

果
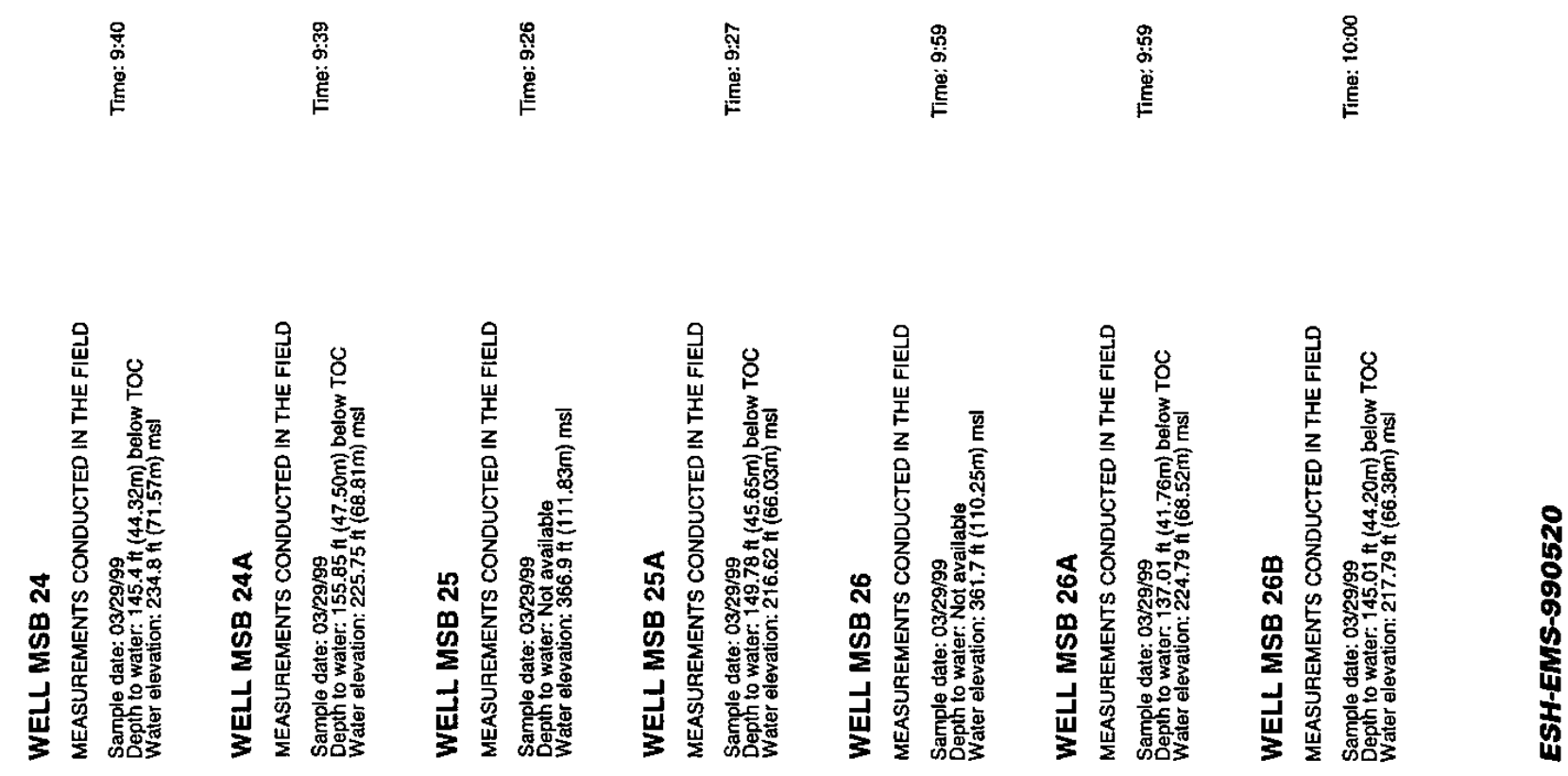


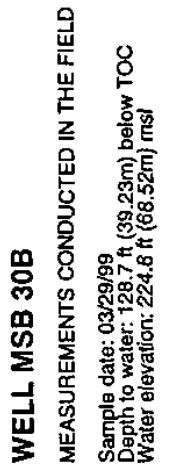

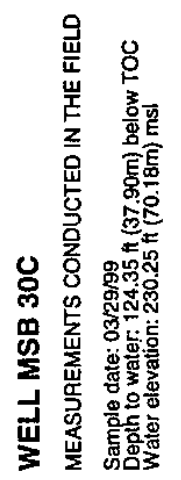

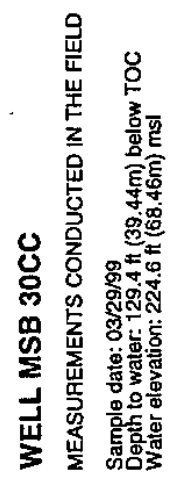

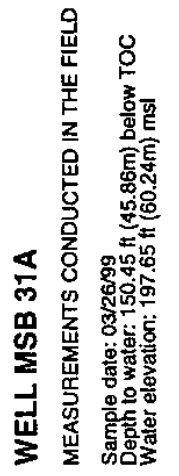

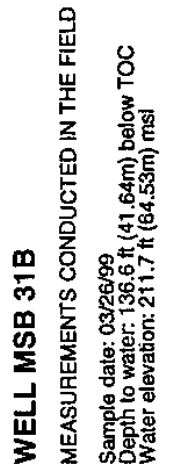
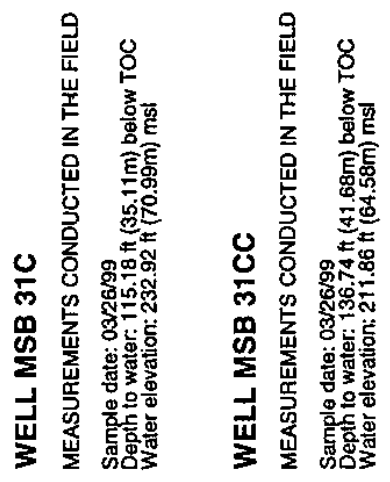

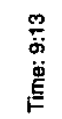

高

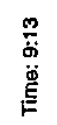

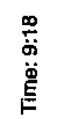

$\stackrel{\frac{0}{0}}{\stackrel{0}{\dot{\phi}}}$

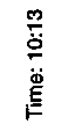

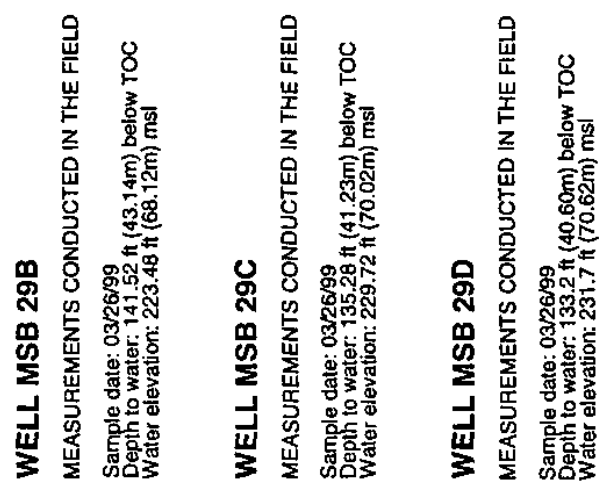
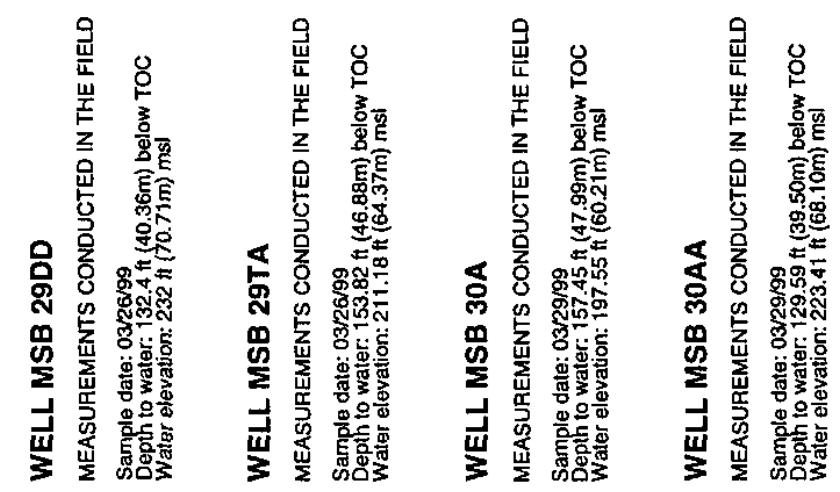

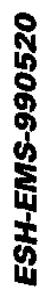




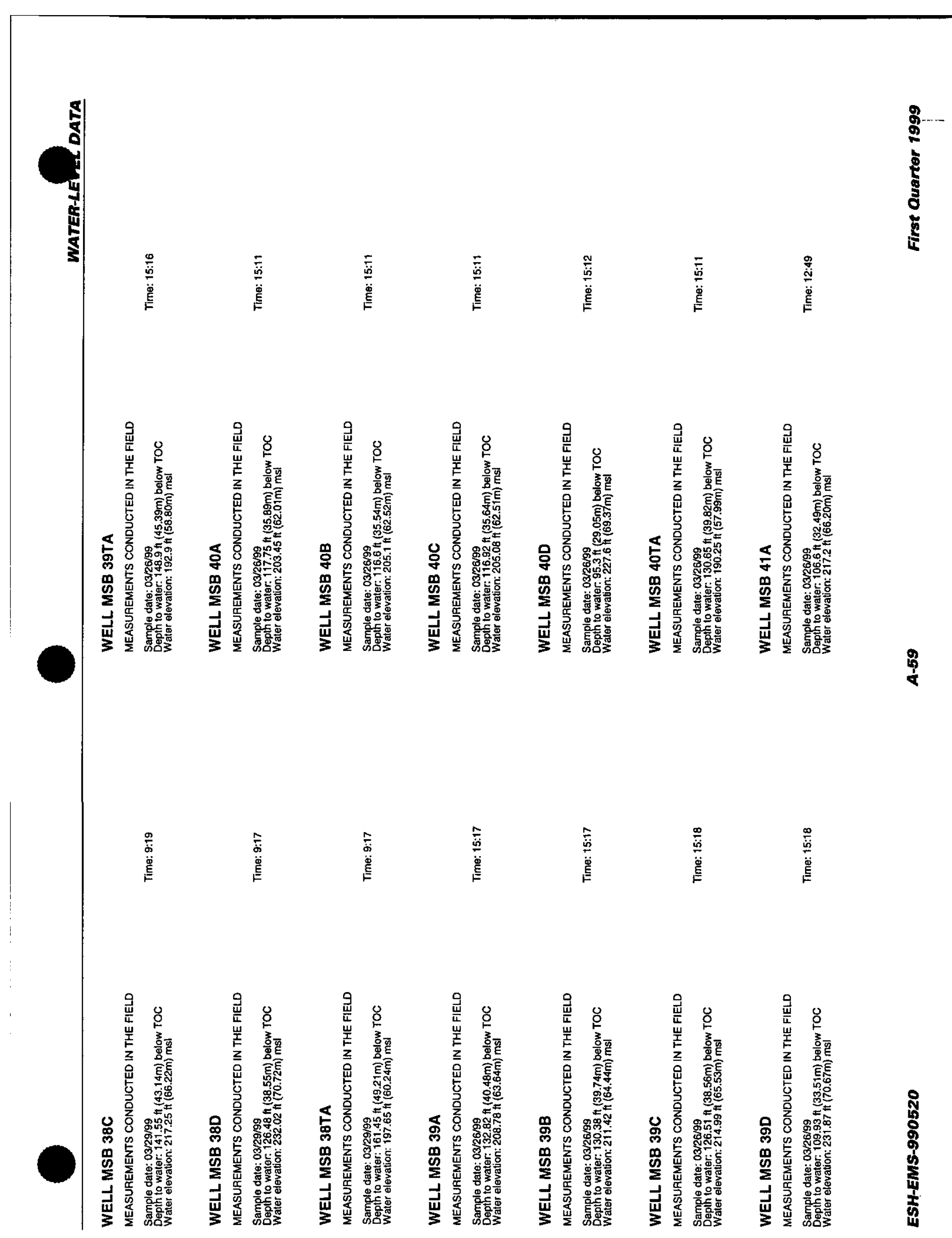




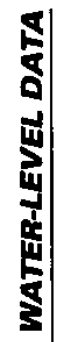

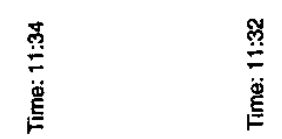

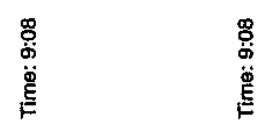

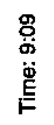

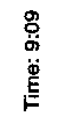

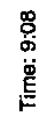

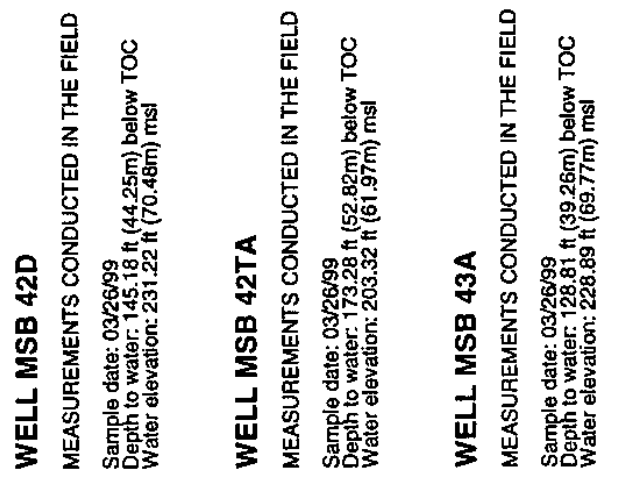
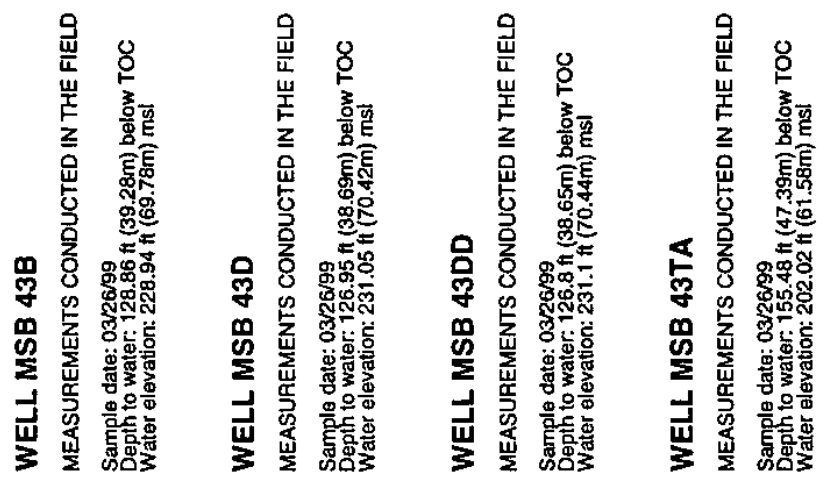

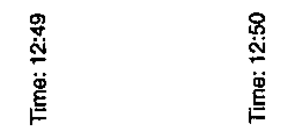

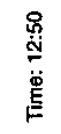

高

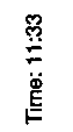

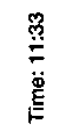

$\frac{\stackrel{p}{\%}}{=}$

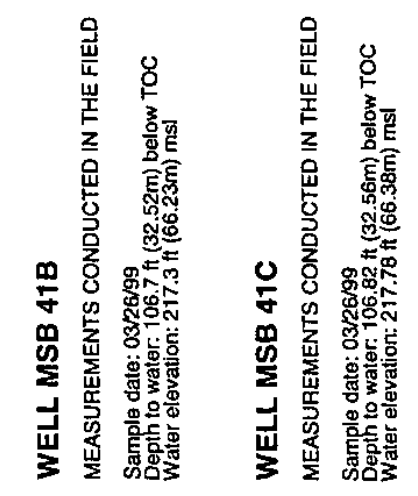

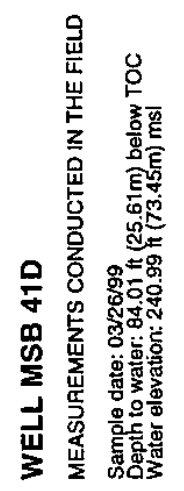
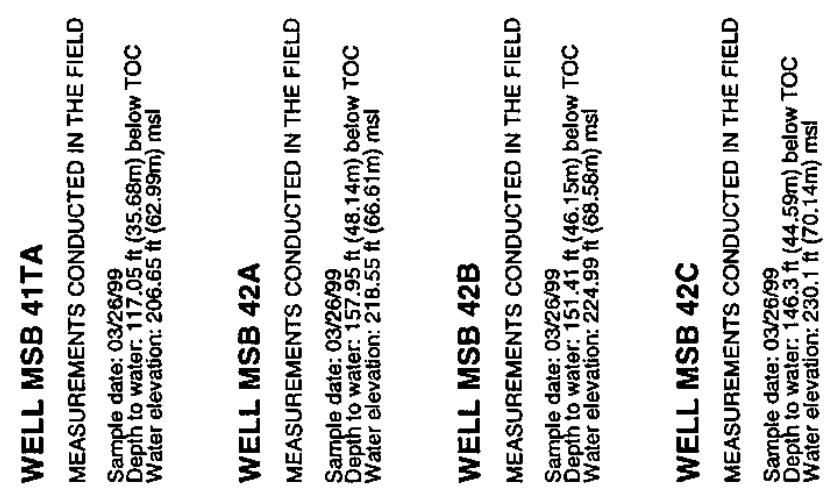

\$
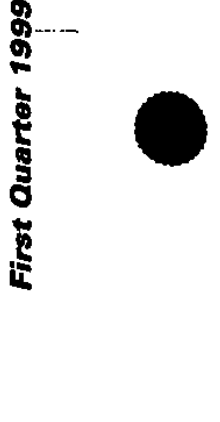


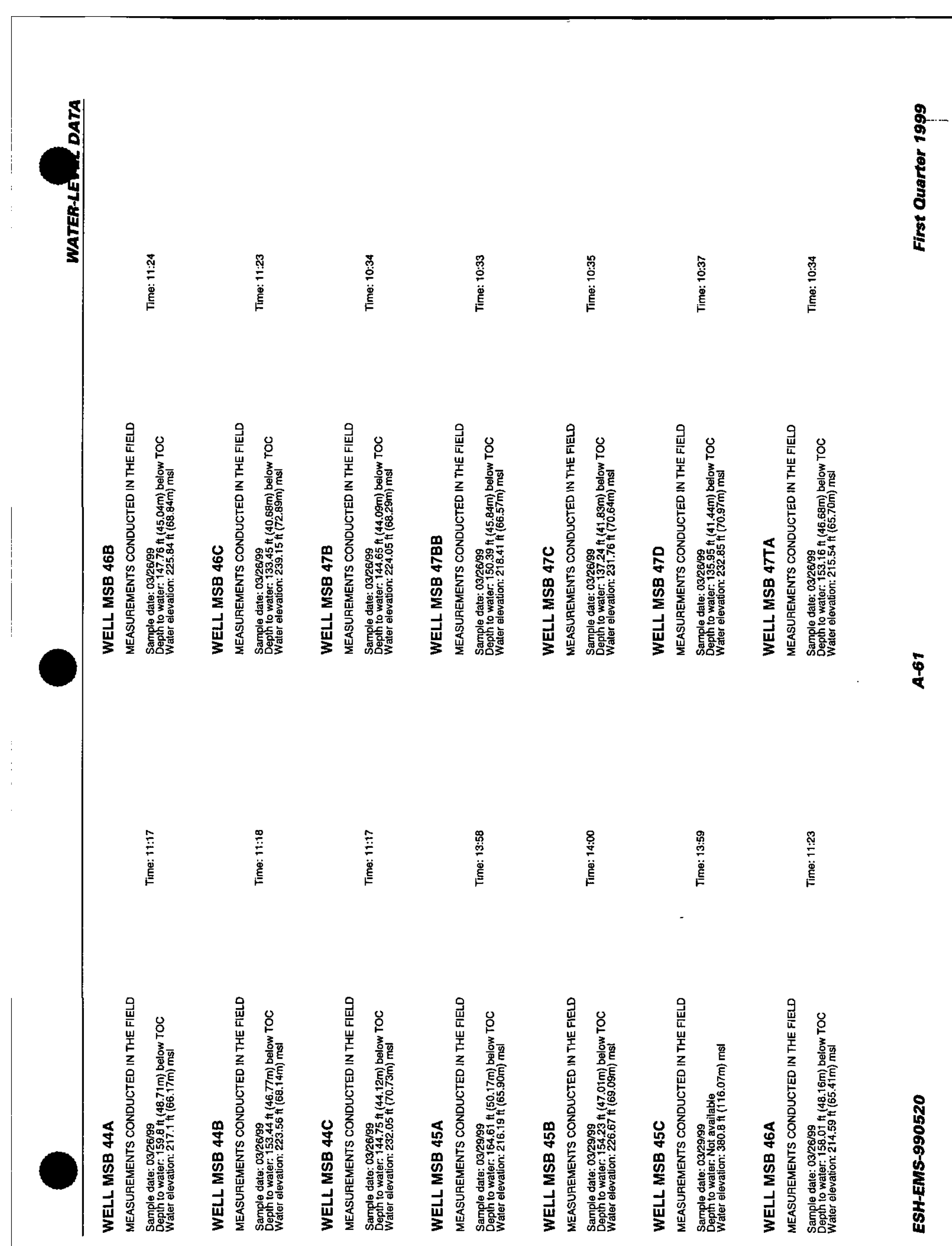



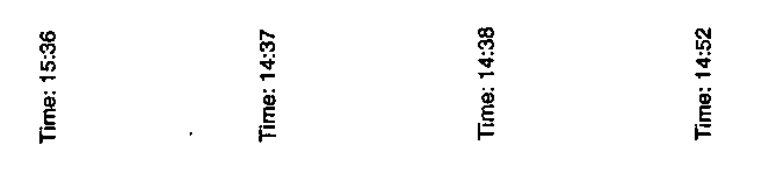

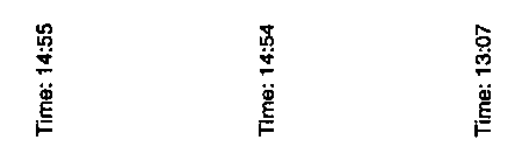

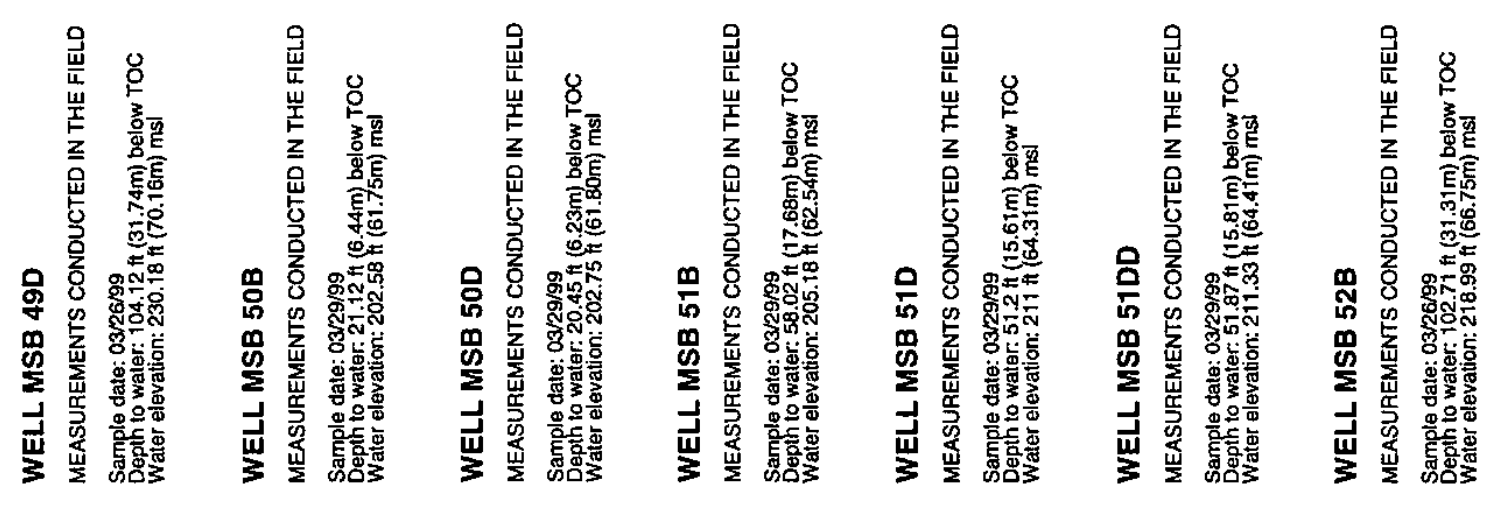

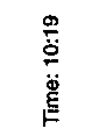

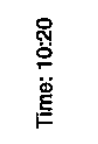

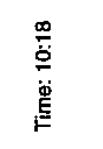

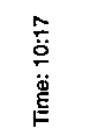
$\underset{\stackrel{\infty}{\ddot{0}}}{\stackrel{\infty}{\underline{*}}}$

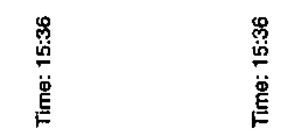
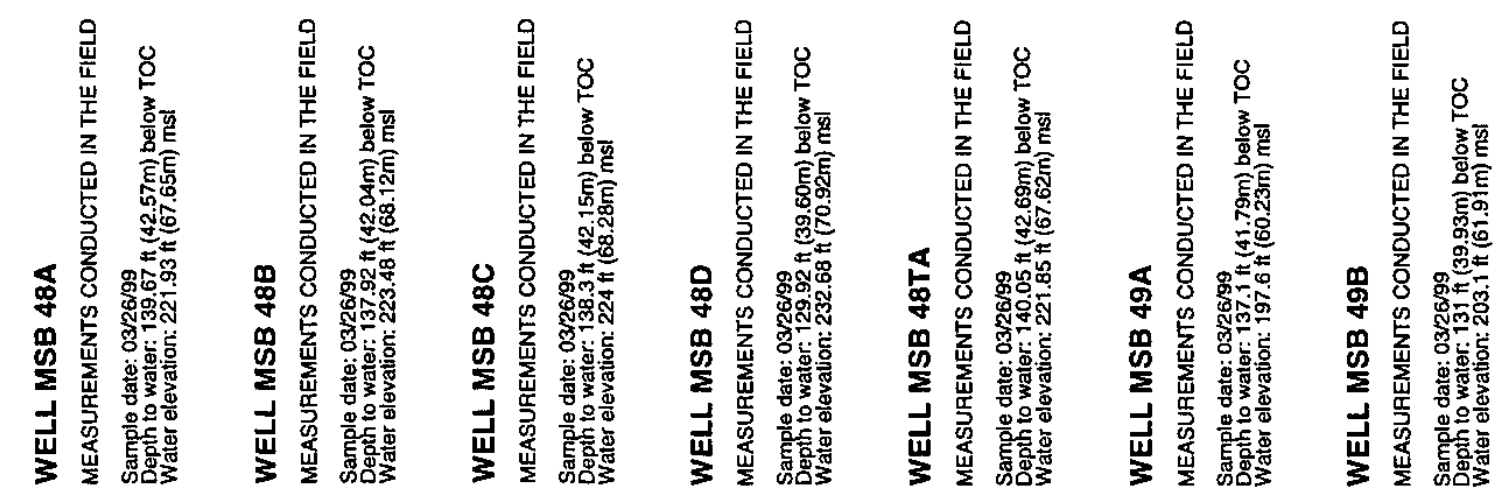

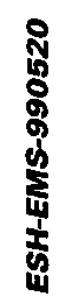




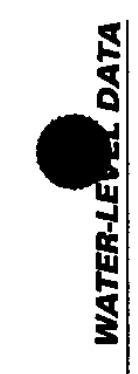

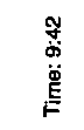

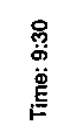

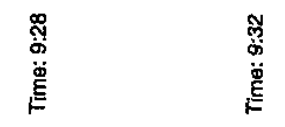

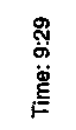

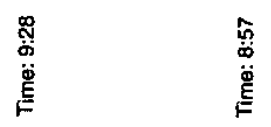

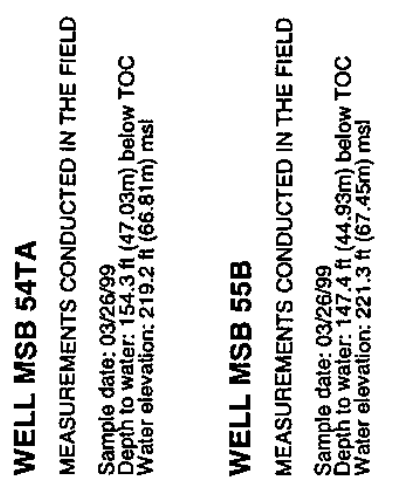
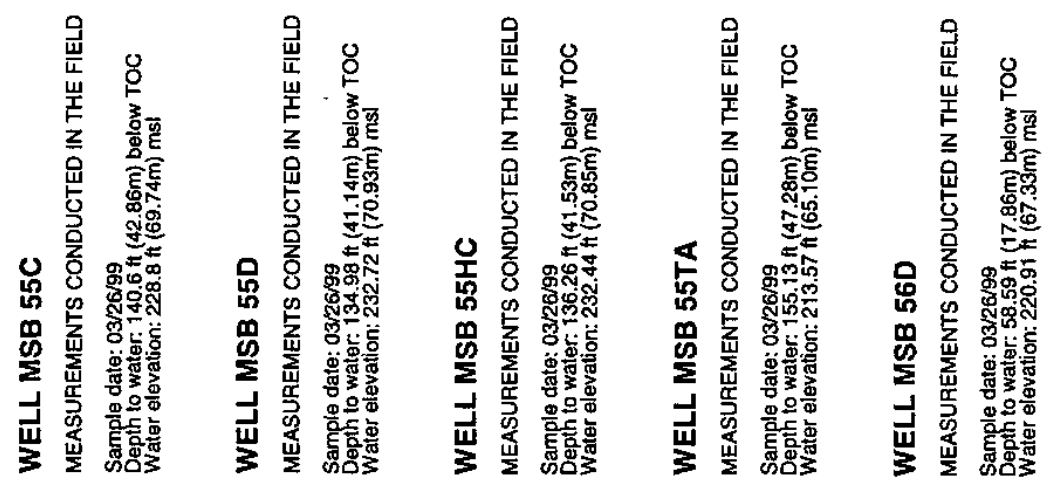

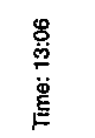

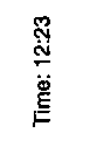

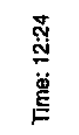

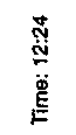

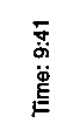

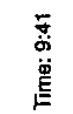

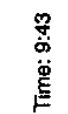
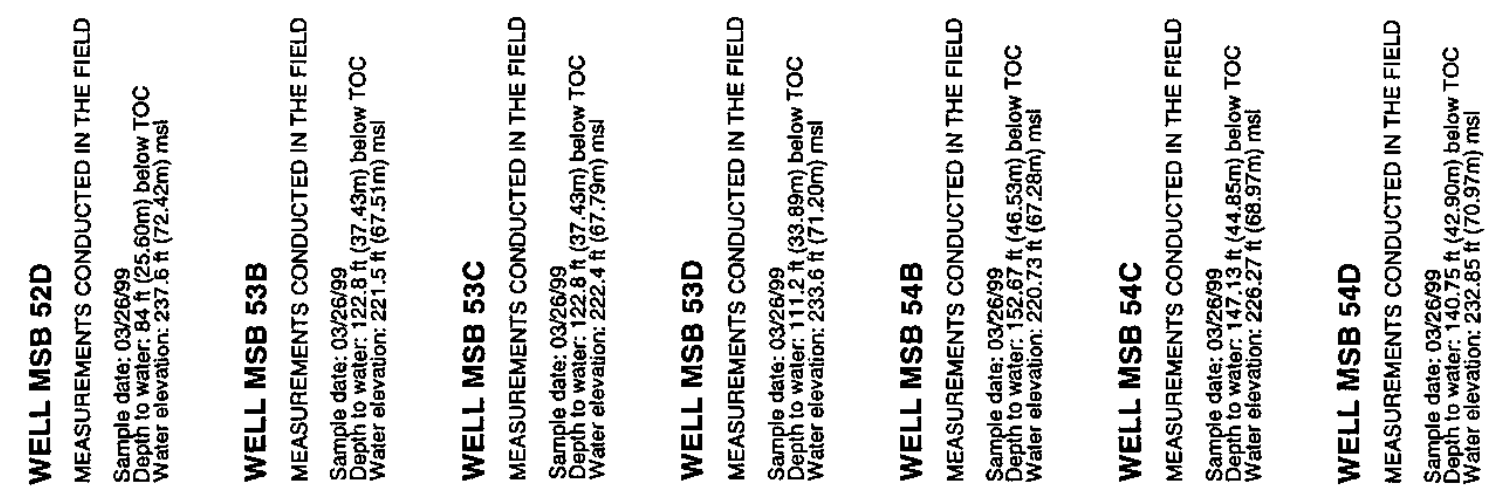


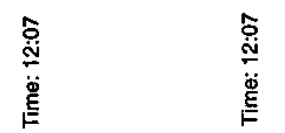



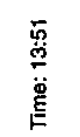

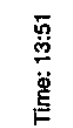

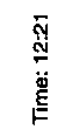

苂

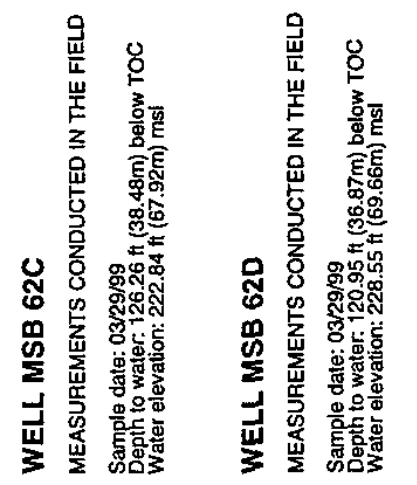
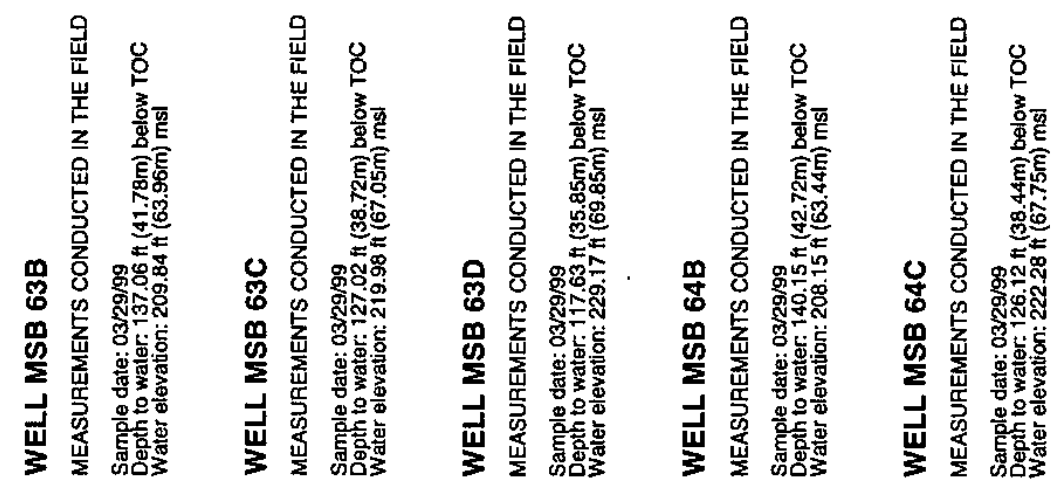

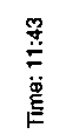

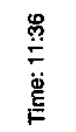

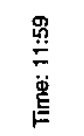

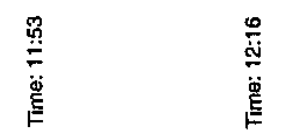

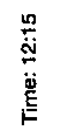

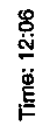

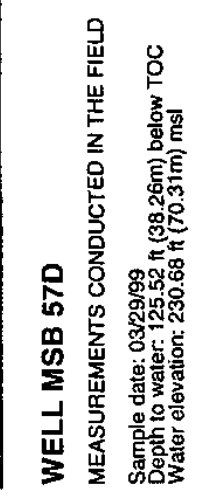

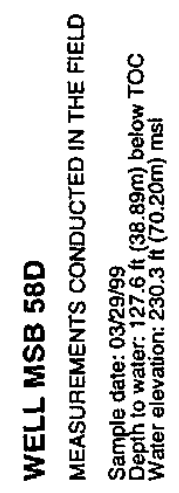

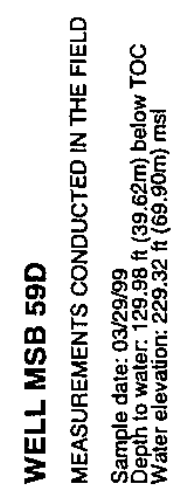

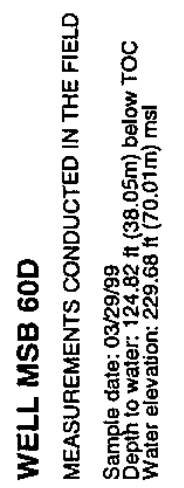

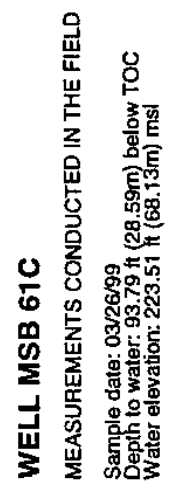
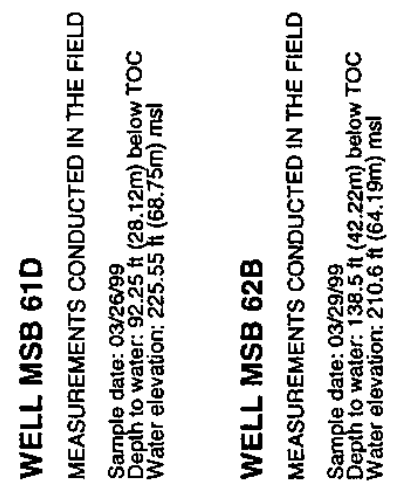

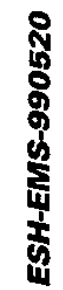



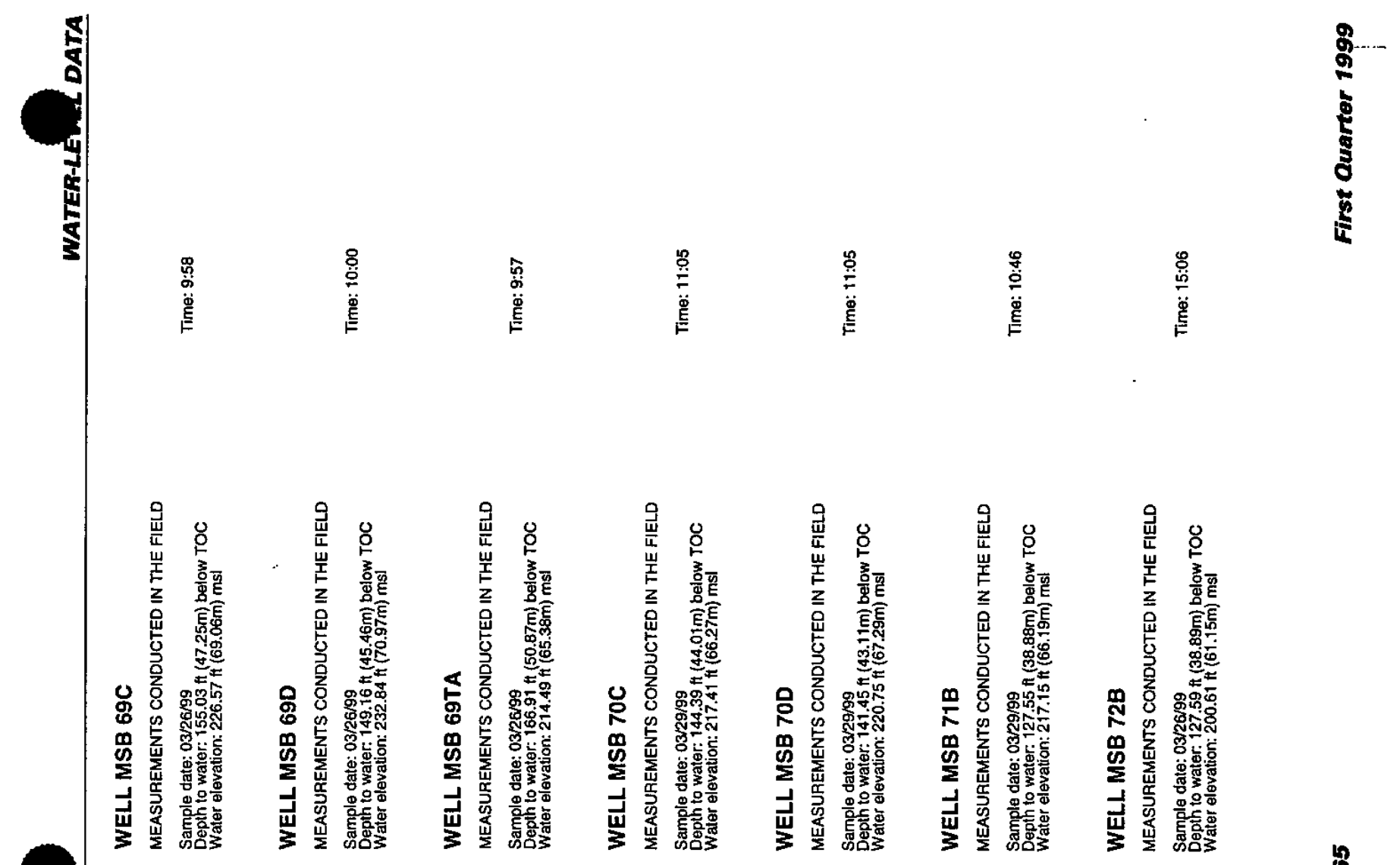

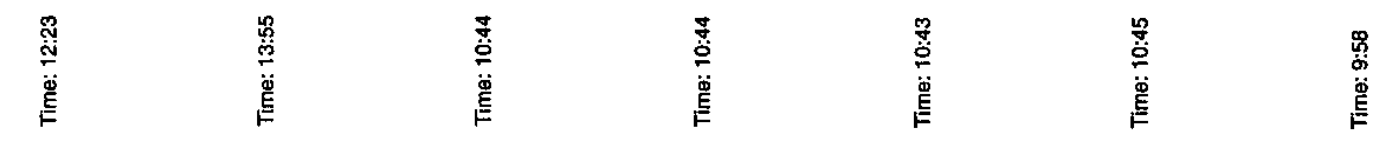
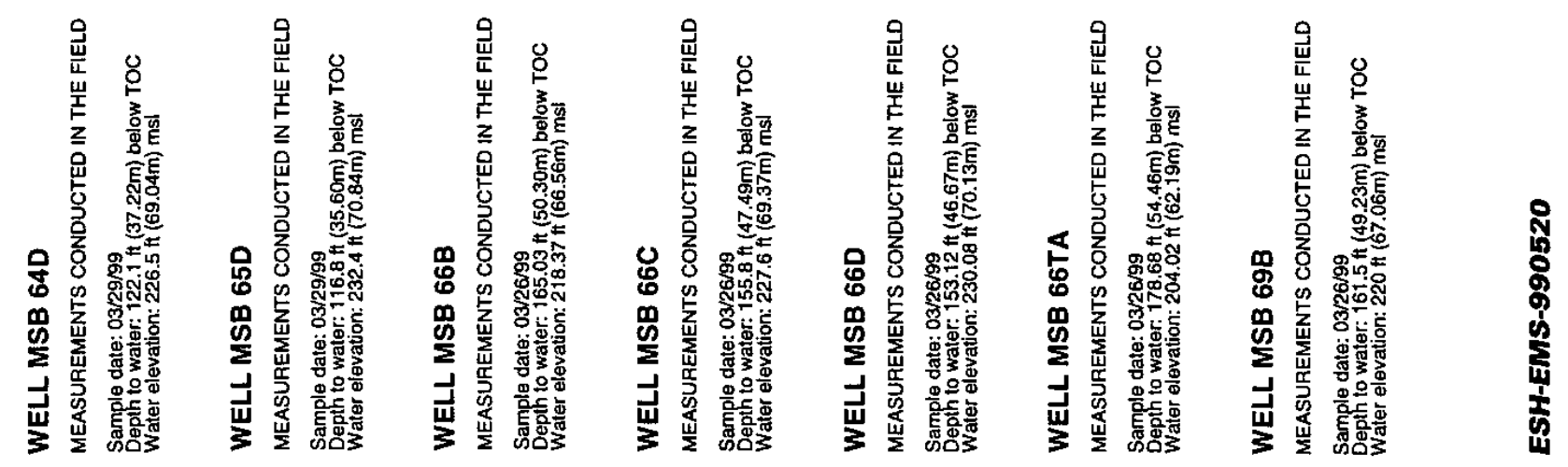


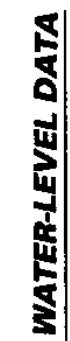

䎹

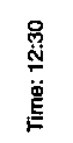

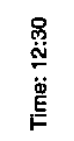

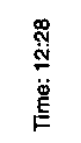

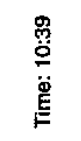

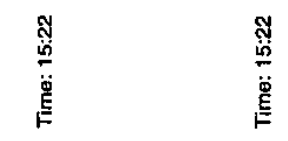

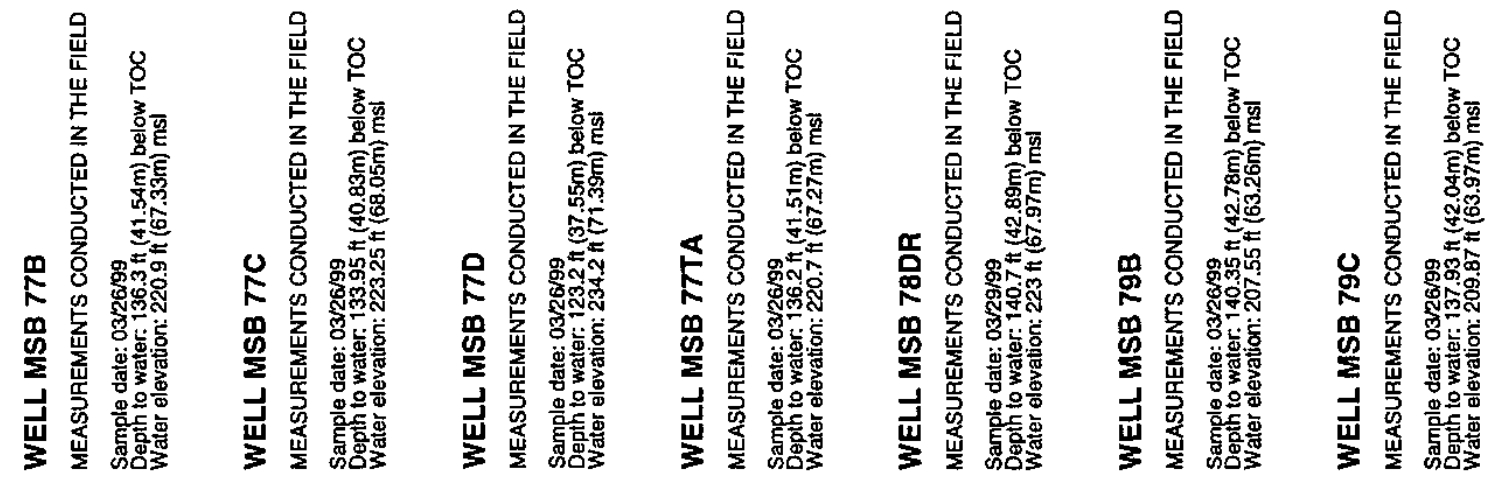

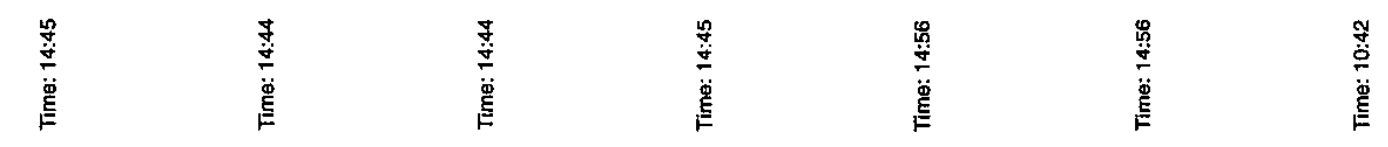

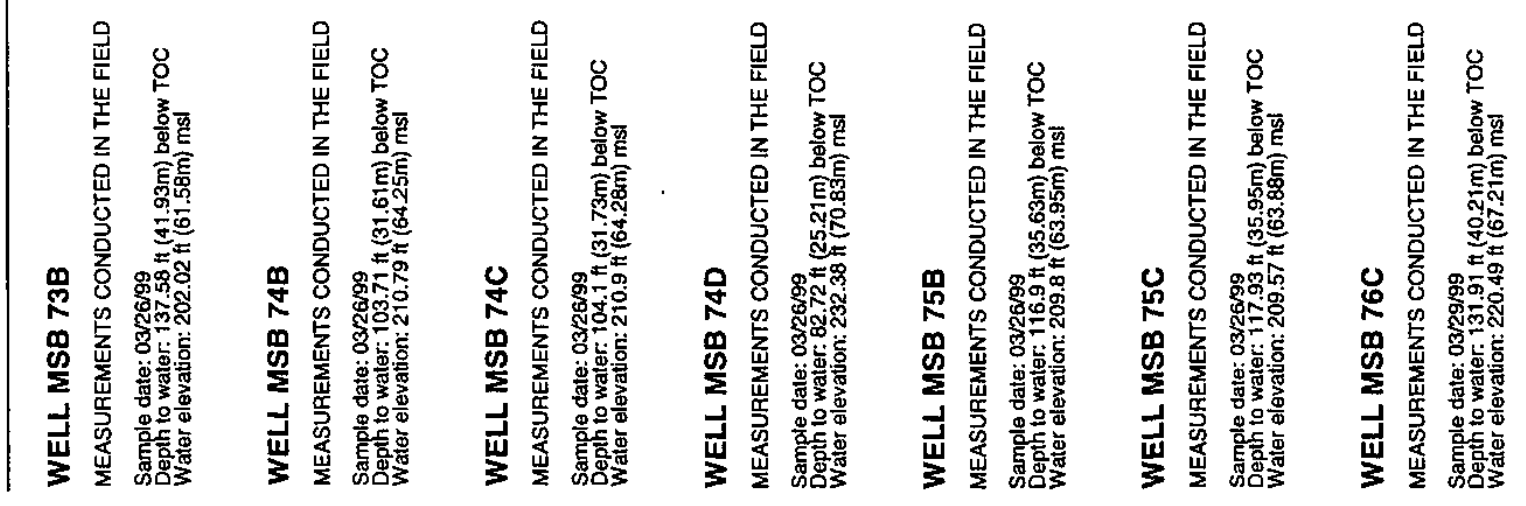

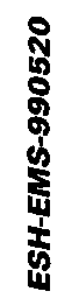




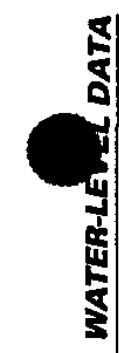

ণ্ণু

$$
\begin{aligned}
& \stackrel{p}{0} \\
& \stackrel{0}{0} \\
& \stackrel{\Phi}{E}
\end{aligned}
$$

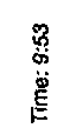

$$
\text { 怘 }
$$

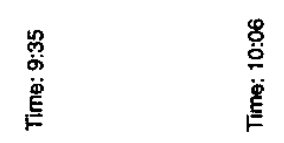

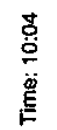
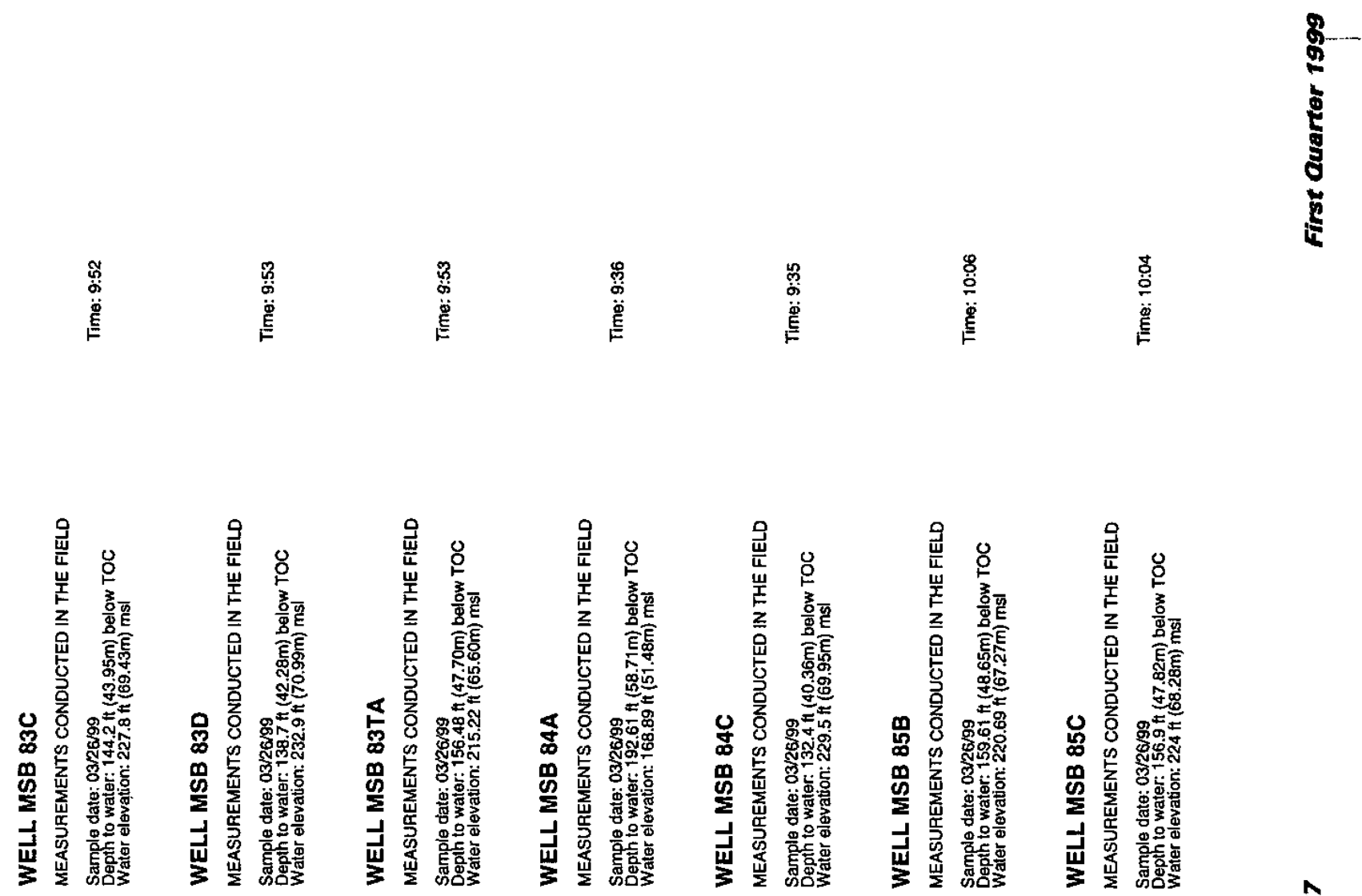

$\frac{8}{8}$

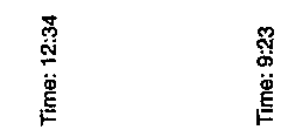<smiles>C=[18O]</smiles><smiles>C=[18O]</smiles><smiles></smiles>

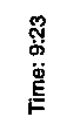

宊
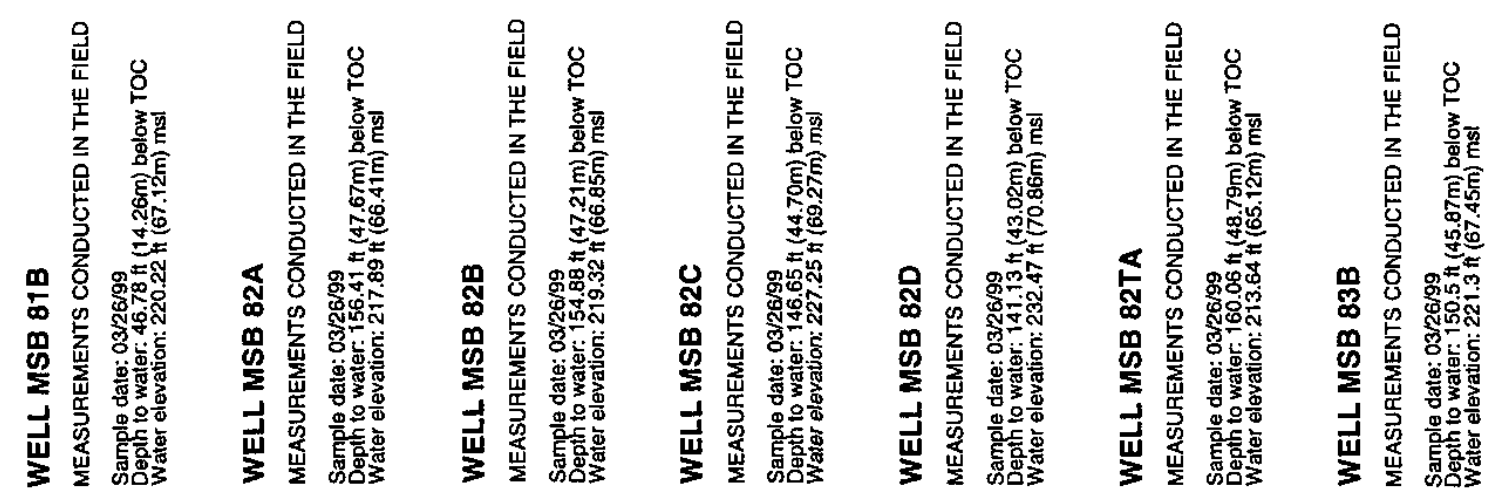


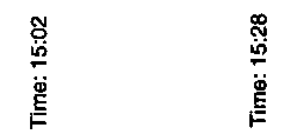

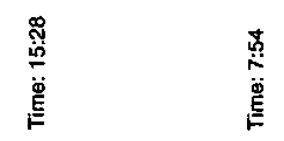

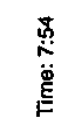

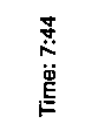

志

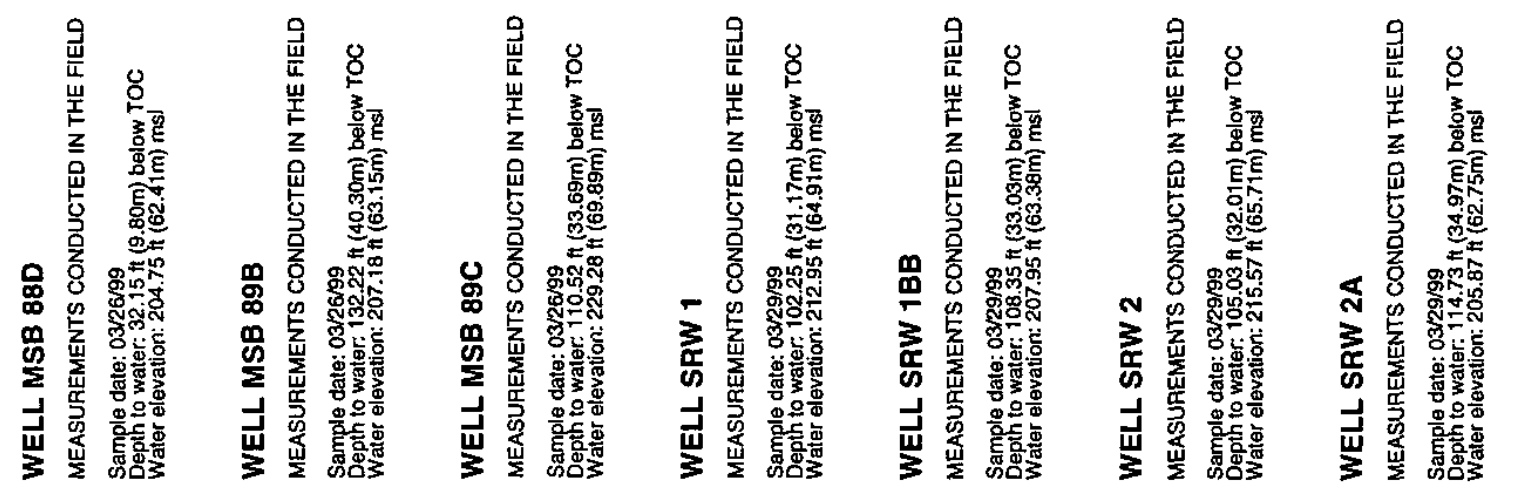

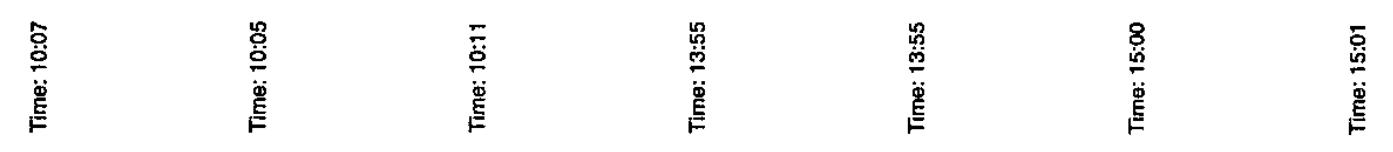

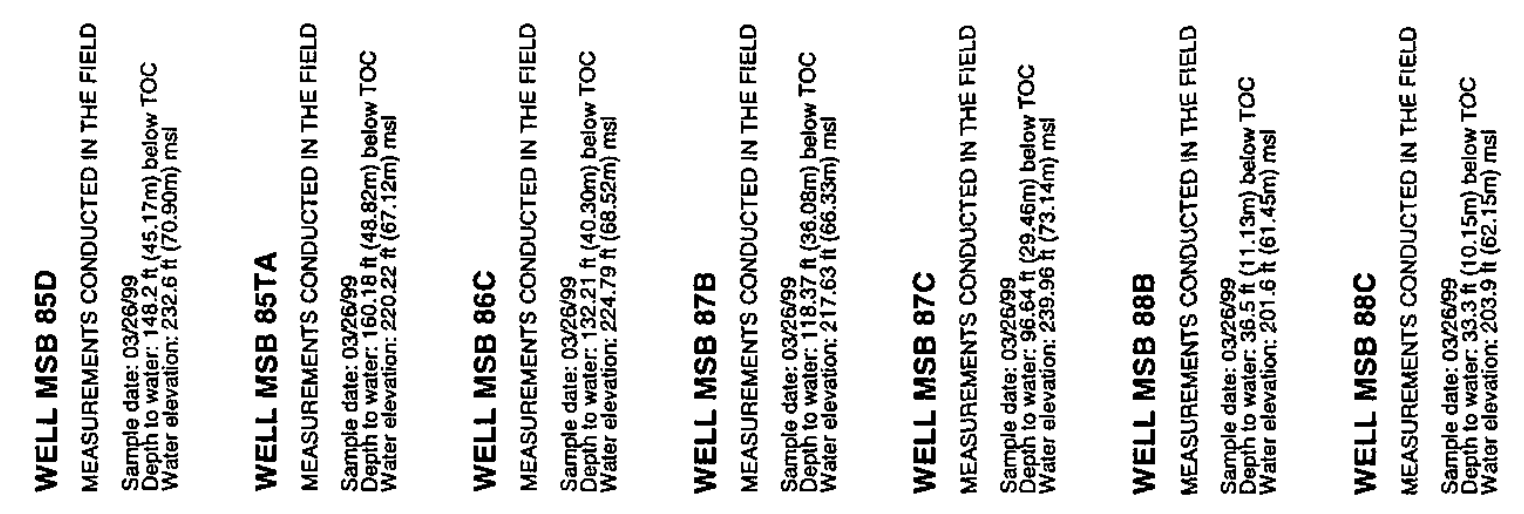

\$

8
5
8
8
0
5
$\frac{1}{4}$
$\frac{5}{4}$
4 


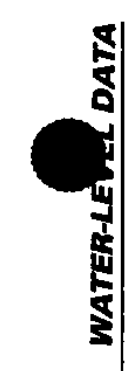

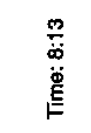

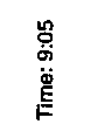

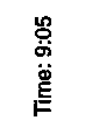

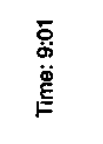

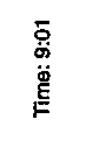

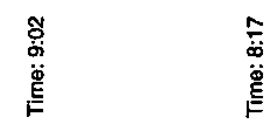

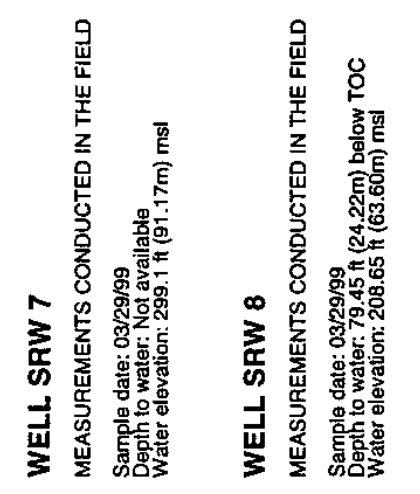
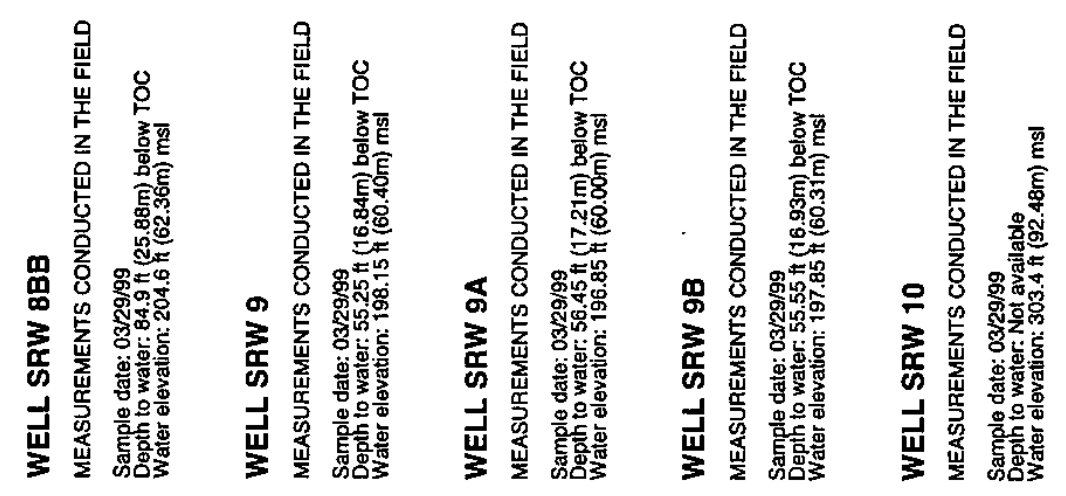

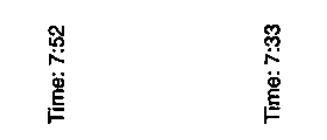

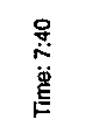

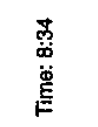

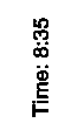

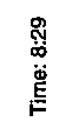

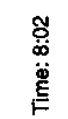
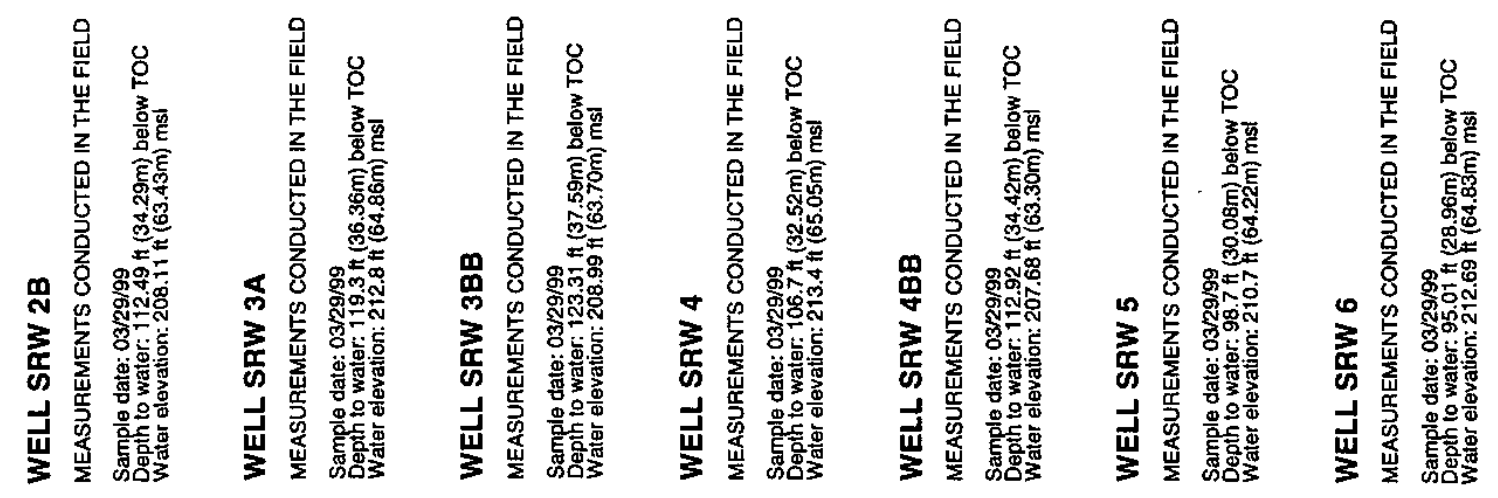

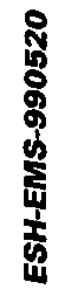



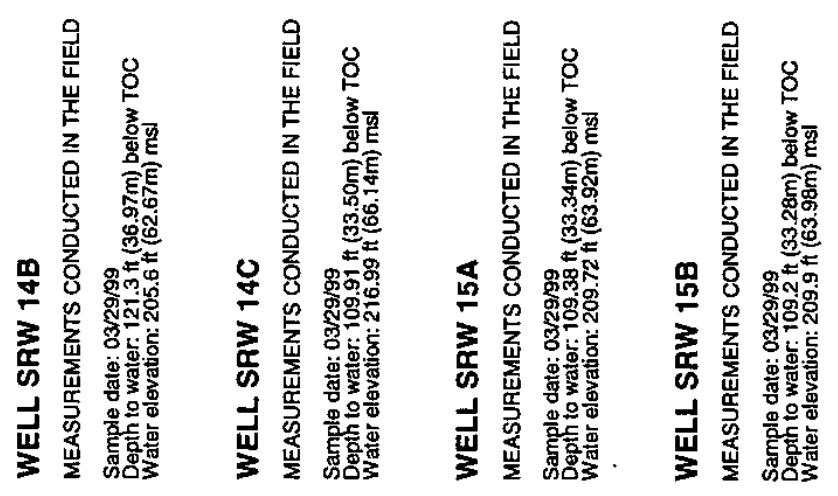

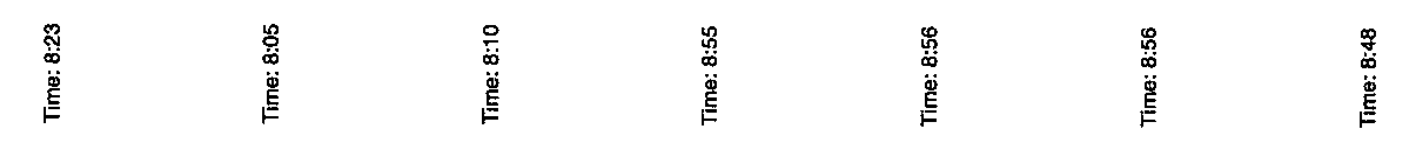
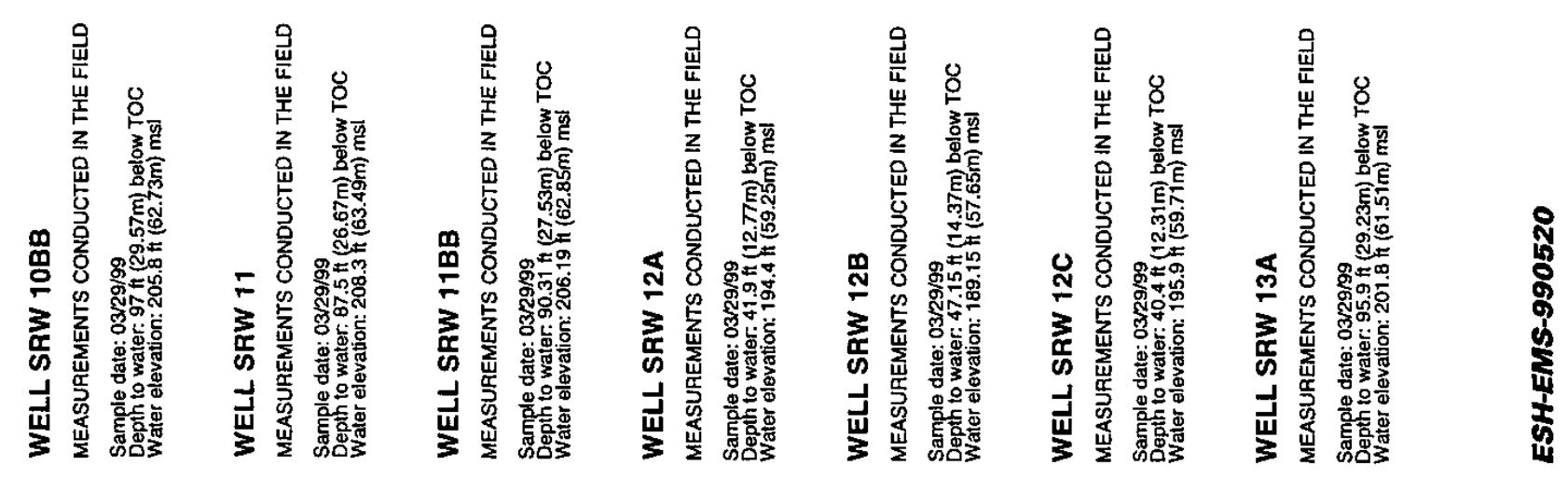


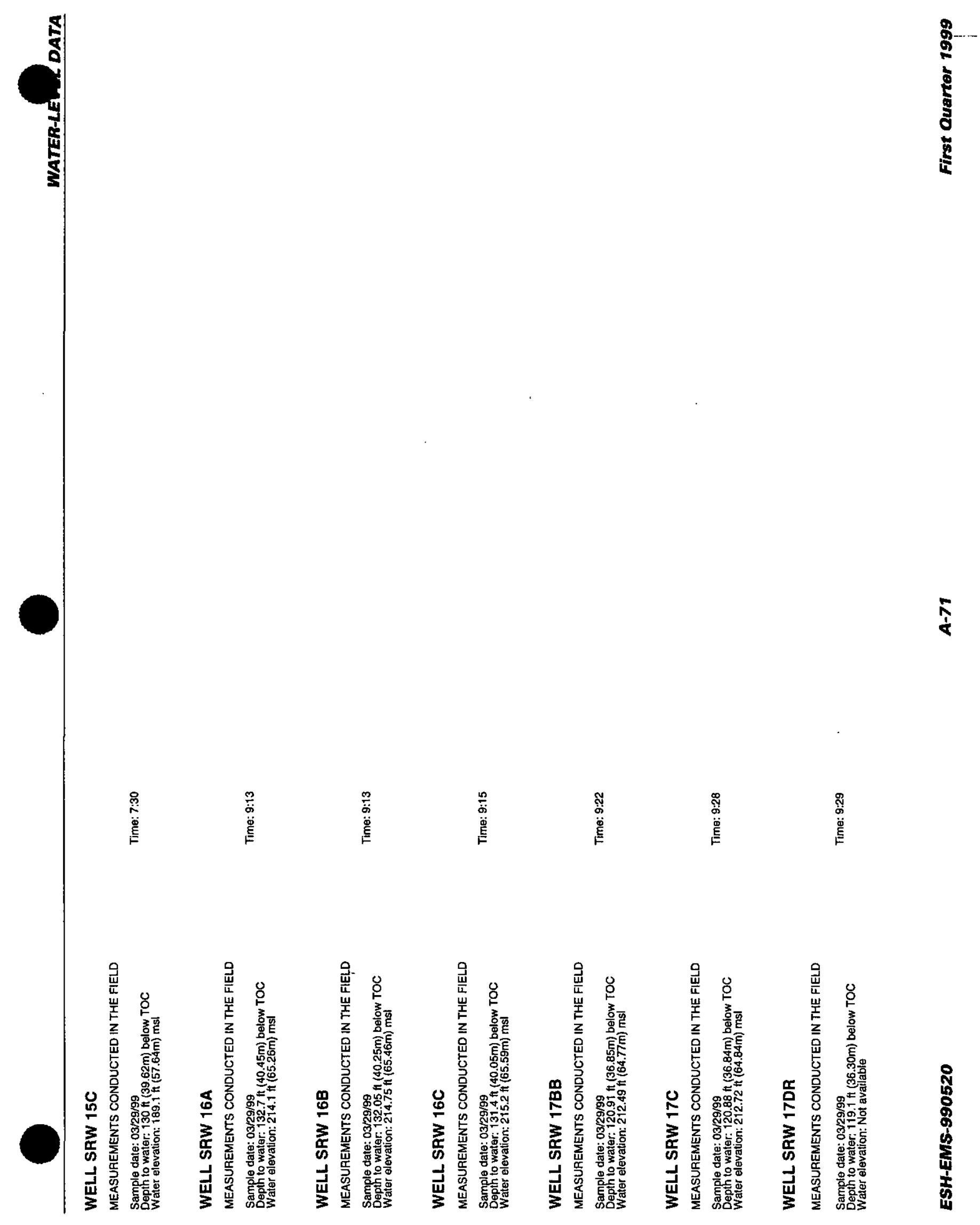




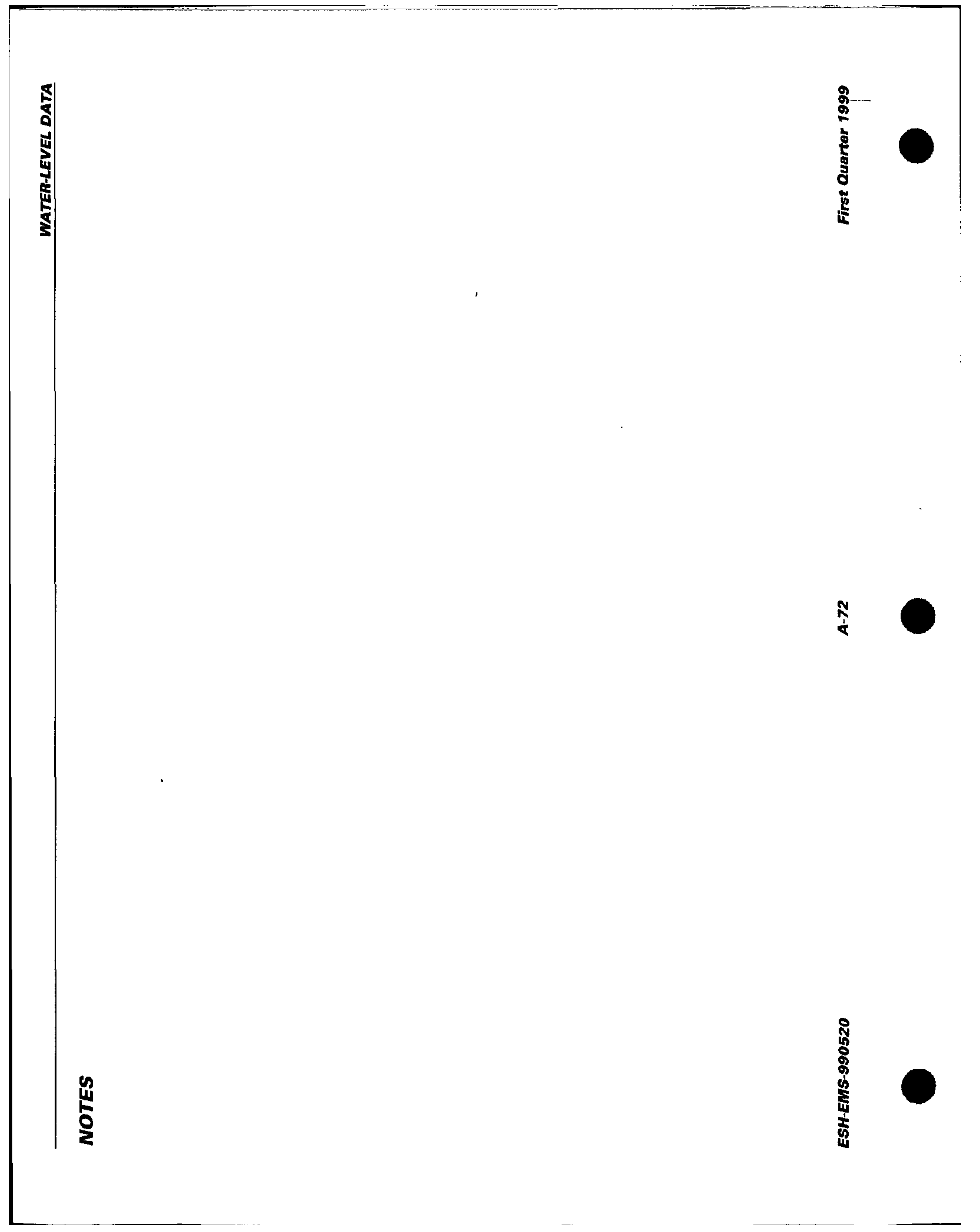




\section{Appendix B. Analytical Results}

This section presents the field and analytical results for samples collected during first quarter 1999. The results tables are presented in alphabetical order by well series and in numerical order within each series. The Site Index section of this report contains the area name(s) for each series.

The tabular data contain all field and analytical results for well samples collected during this quarter. Results of laboratory analyses on sampling blanks are in Appendix $\mathbf{C}$ of this report.

Due to space limitations, the following abbreviations are used in the analytical and sampling blanks results tables.

\begin{tabular}{ll}
\hline Method or Analyte & Abbreviation \\
\hline EICHROMTC1M & EICHROM \\
MMES16009MOD & MMES16009 \\
ASTMD888-92B & ASTMD888 \\
EICHROMSRW01M & EICHROMS \\
5-day biochemical oxygen demand & 5-day biochem oxygen demand \\
ESESOPM008 & ESOPM008 \\
ESESOPM017 & ESOPM017 \\
ESESOPM020 & ESOPM020 \\
ESESOPM022 & ESOPM022 \\
ESESOPM029 & ESOPM029 \\
ESESOPM030 & ESOPM030 \\
ESESOPM031 & ESOPM031 \\
ESESOPM032 & ESOPM032 \\
\hline
\end{tabular}

The Field Notes section of this report contains information about the inability to collect samples, unusual conditions during sample collection, and samplers' observations.

Properly defined and used modifiers or qualifiers can be a key component in assessing data usability. Modifiers designated by EPD/EMS and provided to the primary laboratories are defined below.

Key to the Tables

$\begin{array}{cl}E & \left.\text { exponential notation (e.g., 1.1E-09=1.1 } \times 10^{-9}=0.0000000011\right) \\ \text { EMS } & \text { EMS codes } \\ F & \text { Flag } \\ F G & \text { EPA functional guideline codes }\end{array}$

Fibers $/$ fibers per liter 


\begin{tabular}{cl}
\hline Key to the Tables & \\
\hline$\mu \mathrm{Ci} / \mathrm{mL}$ & microcuries per milliliter \\
$\mu \mathrm{g} / \mathrm{L}$ & micrograms per liter \\
$\mu \mathrm{S} / \mathrm{cm}$ & Microsiemens per centimeter \\
$\mathrm{mg} / \mathrm{L}$ & milligrams per liter \\
$\mathrm{mSl}$ & mean sea level \\
$\mathrm{NTU}$ & Nephelometric turbidity units \\
$\mathrm{S}$ & EPA STORET codes \\
$\mathrm{SQL}$ & sample quantitation limit \\
$\mathrm{TOC}$ & top of casing \\
\hline
\end{tabular}

\section{EPA Functional Definition}

Guideline Codes

(Blank) Data not remarked. The analytical result is acceptable for use as reported.

$\mathrm{J} \quad$ The analyte was positively identified; the associated numerical value is an estimated concentration of the analyte in the sample.

$\mathrm{N} \quad$ The analysis indicated the presence of an analyte for which there is presumptive evidence to make a tentative identification. Use for all TIC results.

R The sample results are rejected due to serious deficiencies in the ability to analyze the sample and meet quality control criteria. The presence or absence of the analyte cannot be verified. Assignment of $R$ requires approval by the appropriate WSRC data validation coordinator.

$U \quad$ The analyte was analyzed for but was not detected above the reported sample quantitation limit.

NJ The analysis indicates the presence of an analyte that has been tentatively identified and the associated numerical value represents its approximate concentration.

UJ

The analyte was not detected above the reported sample quantitation limit. The reported quantitation limit is approximate, and may not represent the actual limit of quantitation necessary to accurately and precisely measure the analyte in the sample.

\section{EPA STORET Codes Definition}

(Blank) Data not remarked.

A The result is the mean of two or more results.

Bf The result is based on colony counts outside the acceptance range.

C The result is calculated. 
D

E†

F

G

H

1

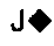

K

L

M

$\mathrm{N} \bullet$

0

$\mathrm{P} \dagger$

Q

$\mathrm{R}+$

$\mathrm{s}$

$\mathrm{Tt}$

u

v

Wt

$x+$

$Y$

$\mathrm{Z \dagger}$

Field measurement.

Extra samples were taken at composite stations.

Indicates female of the species.

The result reported is the maximum of two or more results.

The result is from a field kit determination and may not be accurate.

The result is less than the sSEQL, but equal to or greater than the MDL.

The result is estimated.

The actual concentration is known to be less than the reported result.

The actual concentration is known to be greater than the reported result.

Indicates male of the species.

There is presumptive evidence of the presence of the analyte.

The sample was received by the laboratory, but the analysis was lost or not found.

Too numerous to count.

The sample was held beyond the normal holding time prior to analysis.

There was significant rain in the past 48 hours.

Laboratory test.

The result is less than the criteria of detection.

Undetermined sex of the species.

The analyte was detected in both the method blank and the sample.

The result is less than the lowest reportable under TSTORET code.

The value is from a quasi-vertically integrated sample.

The result is from an unpreserved or incorrectly preserved sample; the data may not be accurate.

There were too many colonies present to count (TNTC); the numeric value represents the filtration volume.

$\dagger$ The code is not currently used for EMS/EGG programs, but may be used in the future or in some other SRS programs. - Indicated STORET code or secondary code definition is redundant to a Functional Guideline code and will not be used at this time.

EMS Codes

(Blank)

A

\section{Definition}

Data not remarked. The analytical result is acceptable for use as reported.

Compound identification criteria were not met.

\section{Appendix B. Analytical Results}

ESH-EMS-990520
B-3

First Quarter 1999 


\begin{tabular}{|c|c|}
\hline EMS Codes & Definition \\
\hline B & Not currently used. \\
\hline $\mathrm{C}$ & LCS or BS criteria were not met. \\
\hline $\mathrm{D}$ & ICP serial dilution criteria were not met. \\
\hline$E$ & Not currently used. \\
\hline $\mathrm{F}$ & Not currently used. \\
\hline G & Not currently used. \\
\hline $\mathrm{H}$ & Internal standard criteria were not met when the IS was used for quantitation. \\
\hline 1 & Matrix spike recovery was not within the control limits. \\
\hline $\mathrm{K}$ & A tentatively identified compound is a suspected aldol-condensation product. \\
\hline$L$ & Initial or continuing calibration criteria were not met. \\
\hline$M$ & Not currently used. \\
\hline$N$ & Not currently used. \\
\hline O & Surrogate or tracer spike recovery is out of specification. \\
\hline$P$ & $\begin{array}{l}\text { Graphite furnace atomic absorption QC } \\
\text { a. Duplication injection criteria were not met. } \\
\text { b. Post-digestion spike recovery was not within control limits and the sample absorbance is } \\
>50 \% \text { of the post-digestion spike absorbance. }\end{array}$ \\
\hline$Q$ & Not currently used. \\
\hline $\mathrm{R}$ & Not currently used. \\
\hline $\mathrm{s}$ & The sample was analyzed by the method of standard additions. \\
\hline $\mathrm{T}$ & Not currently used. \\
\hline $\mathrm{U}$ & Not currently used. \\
\hline $\mathrm{V}$ & Not currently used. \\
\hline W & $\begin{array}{l}\text { Graphite furnace atomic absorption QC: the post-digestion spike recovery is not within control limits } \\
\text { and the sample absorbance is }<50 \% \text { of the post-digestion spike absorbance. }\end{array}$ \\
\hline $\mathrm{x}$ & The laboratory duplicate RPD or MS/MSD RPD was not within control limits. \\
\hline Y & Not currently used. \\
\hline Z & Not currently used. \\
\hline 4 & Matrix interference is present. \\
\hline 6 & The analyte was detected in both the sample and associated field blank. \\
\hline 7 & The analyte was detected in both the sample and associated rinsate. \\
\hline 8 & The analyte was detected in both the sample and associated trip blank. \\
\hline 9 & The field duplicate RPD was not within control limits. \\
\hline
\end{tabular}




\section{Field Qualifiers}

Sample interference field qualifiers were added to the field data in the analytical results tables beginning fourth quarter 1996. The qualifiers describe sampling interferences encountered during sample collection that could affect analytical results. They are used to qualify analytical data based on field conditions. Due to space limitations, the sample interference field qualifiers are referred to as field qualifiers in the following table and in the field data section of the analytical results tables.

$\begin{array}{cl}\text { Field Qualifiers } & \text { Definition } \\ \text { A } & \begin{array}{l}\text { The pump was surging excessively. Aeration could cause oxidation reactions and loss of } \\ \text { volatiles (low results). Analytical results may be of poor precision (high variability) due to } \\ \text { sampling bias. The sample qualifier shall include an } A \text { if the site code is an } A .\end{array}\end{array}$

If the method code for a sample is $B$, then the sample qualifier shall include a $B$. This indicates that an open bucket bailer was used to collect the sample, which typically agitates the sample, increasing aeration and suspended solids. All analytical results may be of poor precision, volatile organic results may be biased low, and some metal and radionuclide results may be biased high.

Analytical results may be unrepresentative of true values due to reactions with metal well casing. This value will be automatically filled in if the casing type in the well inventory table is $A l, C S$, Iron, SS, or Steel. Analytical results for some metals and radionuclides may be higher or lower than actual groundwater concentrations.

If the method code for a sample is $G$, then the sample qualifier shall include a $G$. This indicates that an open bucket bailer was used to collect the sample without purging the well to attain stabilized field parameters. The grab sample method collects water that has undergone chemical reactions with the atmosphere and typically agitates the sample, increasing aeration and suspended solids. All analytical results may be of poor precision, volatile organic results may be biased low, and some metal and radionuclide results may be biased high. Analytical results may differ significantly for actual groundwater concentrations.

Analytical results may be unrepresentative of actual groundwater concentrations due to an elevated pH, possibly due to well installation materials (drilling mud, grout). Results for some inorganic constituents (i.e., sodium, metals, radionuclides) may be affected. If the $\mathrm{pH}$ for a sample is greater than eight, then the sample qualifier shall include an $\mathrm{H}$.

Analytical results may be unrepresentative of actual groundwater concentrations due to well installation or formation interferences causing elevated turbidity. Results for particle reactive constituents (i.e., metals, radionuclides) may be elevated. If the turbidity for a sample is greater than $15 \mathrm{NTU}$, then the sample qualifier shall include an $N$.

If the sample method is an $S$, then the sample qualifier shall include an $S$. Single-speed centrifugal submersible pump flow rates vary from 1 to $15 \mathrm{gpm}$, and agitation of the sample may occur at higher flow rates, causing poor precision, low volatile organic results, or elevated metal or radionuclide results.

One or more of the field parameters (i.e., pH, conductivity, turbidity) did not stabilize prior to sample collection. The results may be of poor precision (high variability) due to sampling bias. The sample qualifier shall be a $U$ if the stabilized field is $N$ or the method code is $G$.

If the method code is a $V$, then the sample qualifier shall include a $V$. Sample collection with variable-speed pumps indicates that flow rates were less than one liter per minute. Sample collection at low flow rates provides the best estimates of actual groundwater concentrations due to reduced sampling bias.

If the site code is an $X$, then the sample qualifier shall include an $X$. Analytical results may be of poor precision for many constituents, and volatile organic results may be biased low because the well went dry during purging. 


\section{Calculation of Alkalinity Relationships}

The results obtained from the phenolphthalein and total alkalinity determinations offer a means for stoichiometric classification of the three principal forms of alkalinity present in many waters. The classification ascribes the entire alkalinity to bicarbonate, carbonate, and hydroxide, and assumes the absence of other (weak) inorganic or organic acids, such as silicic, phosphoric, and boric acids. It further presupposes the incompatibility of hydroxide and bicarbonate alkalinities. Because the calculations are made on a stoichiometric basis, ion concentrations in the strictest sense are not represented in the results, which may differ significantly from actual concentrations, especially at $\mathrm{pH}>10$. According to this scheme:

(1) Carbonate $\left(\mathrm{CO}_{3}{ }^{2}\right)$ alkalinity is present when phenolphthalein alkalinity is not zero but is less than total alkalinity.

(2) Hydroxide $\left(\mathrm{OH}^{-}\right)$alkalinity is present if phenolphthalein alkalinity is more than half the total alkalinity.

(3) Bicarbonate $\left(\mathrm{HCO}_{3}\right)$ alkalinity is present if phenolphthalein alkalinity is less than half the total alkalinity. These relationships may be calculated by the following scheme, where $P$ is phenolphthalein alkalinity and $T$ is total alkalinity:

Select the smaller value of $P$ or $(T-P)$. Then, carbonate alkalinity equals twice the smaller value. When the smaller value is $P$, the balance $(T-2 P)$ is bicarbonate. When the smaller value is $(T-P)$, the balance $(2 P-T)$ is hydroxide. All results are expressed as $\mathrm{CaCO}_{3}$.

\begin{tabular}{llll}
\hline $\begin{array}{l}\text { If Phenolphthalein } \\
\text { Alkalinity Result }=\end{array}$ & $\begin{array}{l}\text { then Hydroxide } \\
\text { Alkalinity }=\end{array}$ & $\begin{array}{l}\text { then Carbonate } \\
\text { Alkallnity }=\end{array}$ & $\begin{array}{l}\text { then Bicarbonate } \\
\text { Alkalinity }=\end{array}$ \\
\hline 0 & 0 & 0 & Total Alk \\
$<1 / 2$ Total Alk & 0 & 2 (Phen Alk) & Total Alk - 2(Phen Alk) \\
$=1 / 2$ Total Alk & 0 & 2 (Phen Alk) & 0 \\
$>1 / 2$ Total Alk & 2(Phen Alk) - Total Alk & 2(Total Alk - Phen Alk) & 0 \\
Phen Alk = Total Alk & Total Alk & 0 & 0 \\
\hline
\end{tabular}




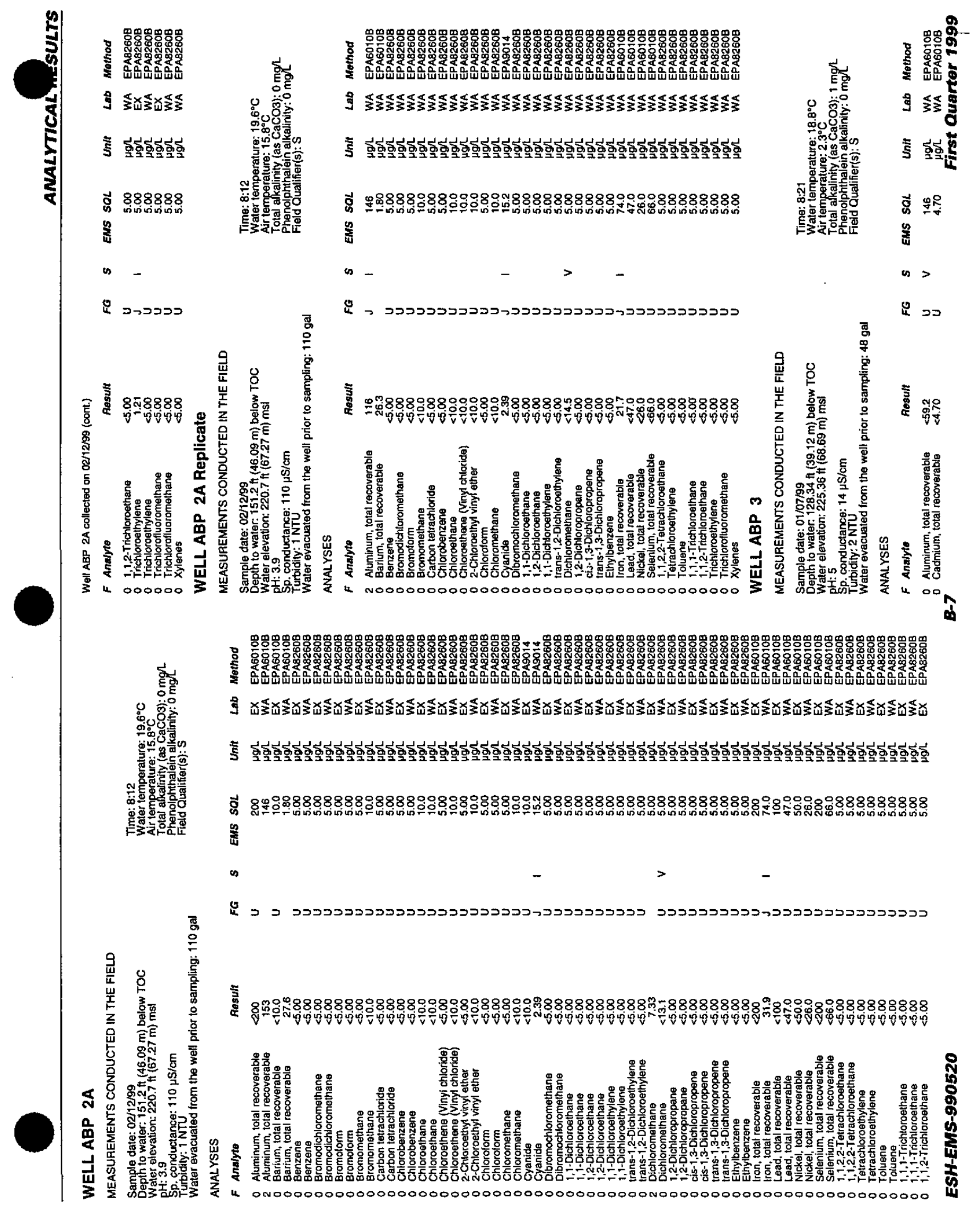




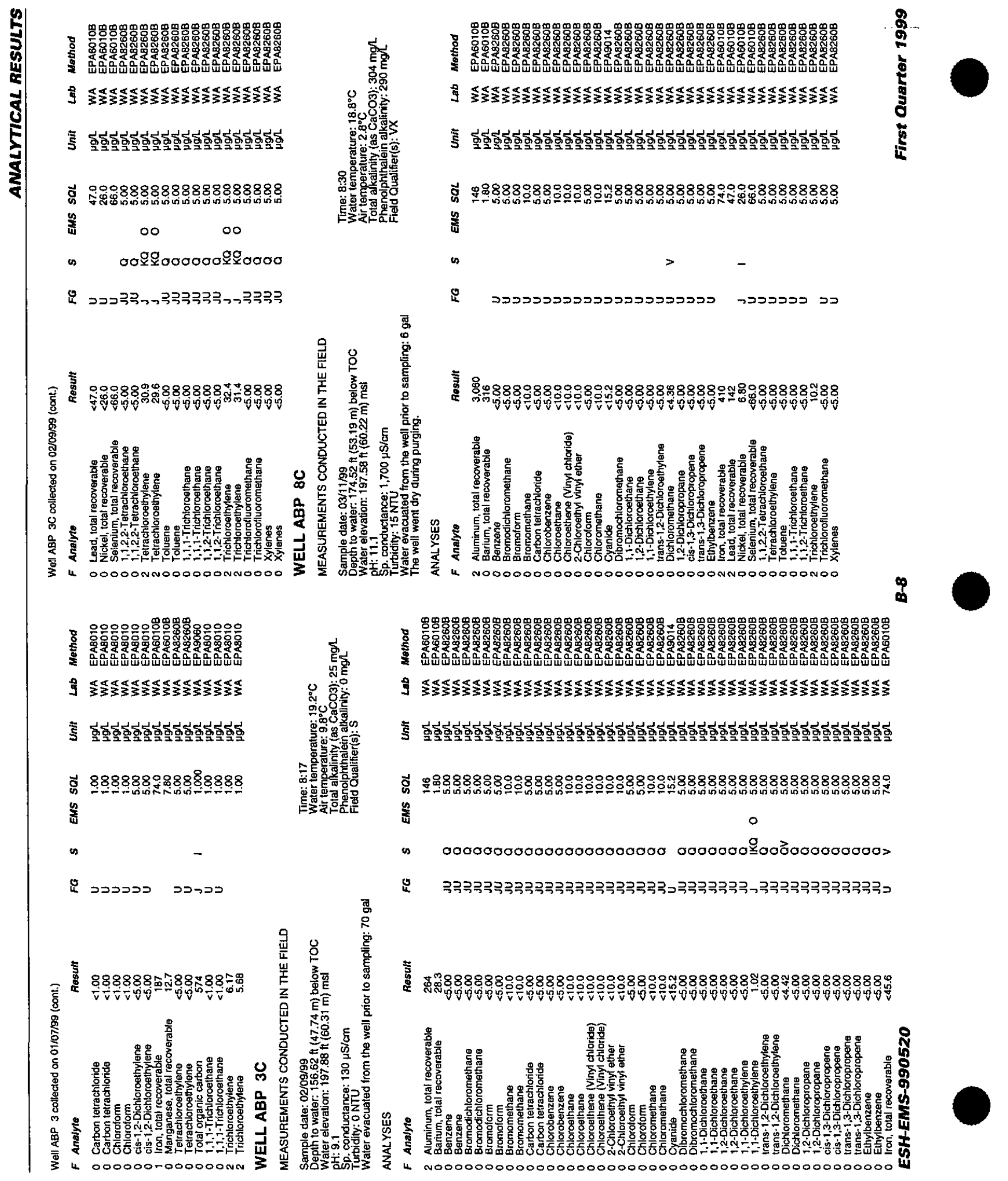




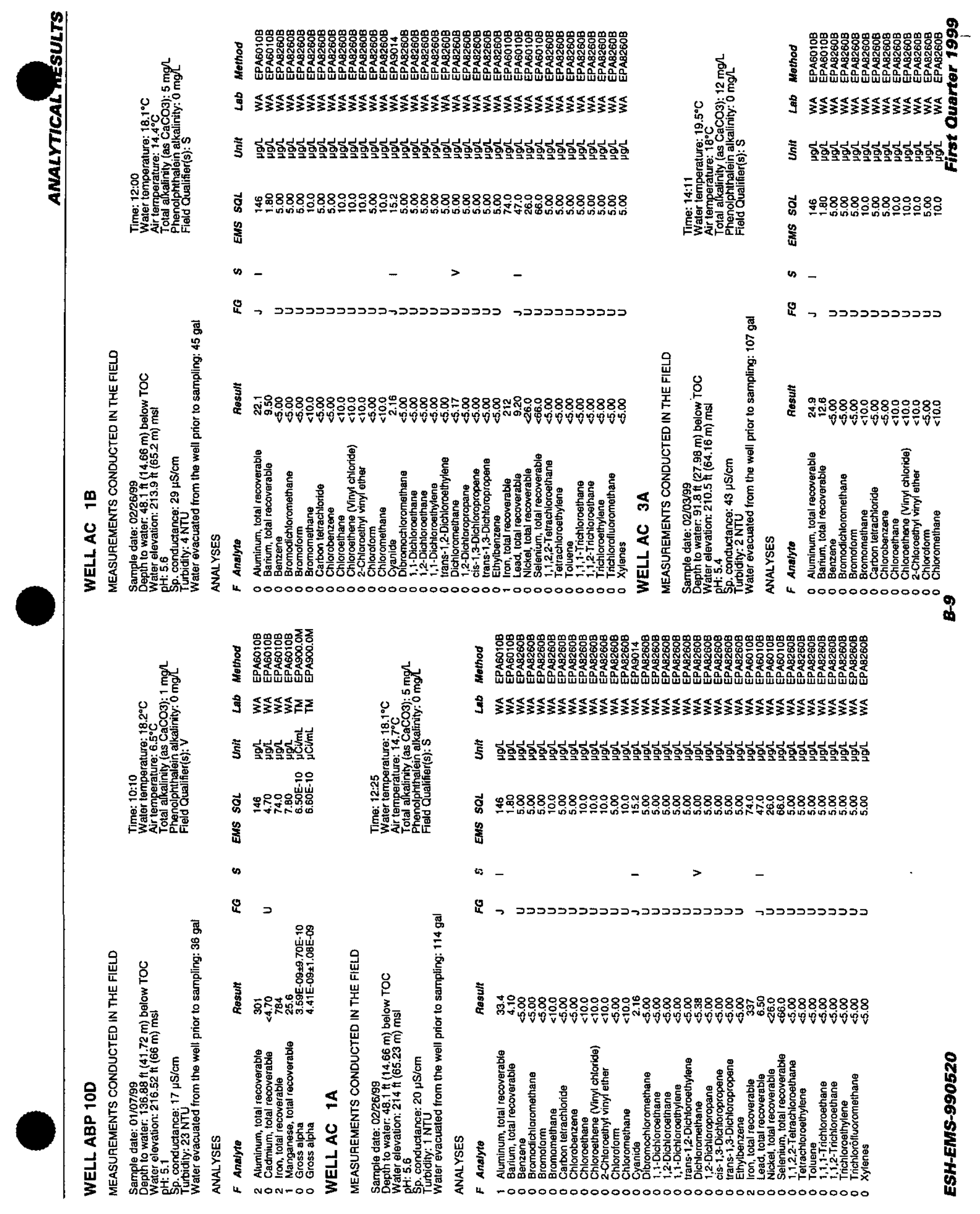




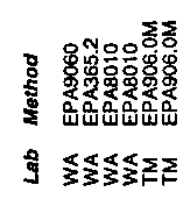

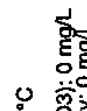

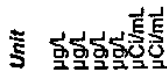

2.

尊

$2-$

בכנית

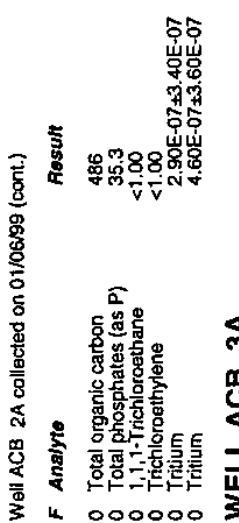

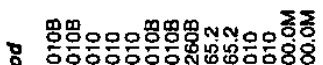

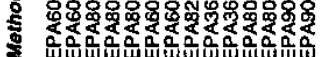

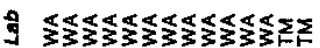

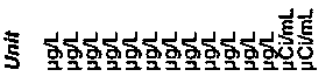

ธิ

总

בכירכ בכבי

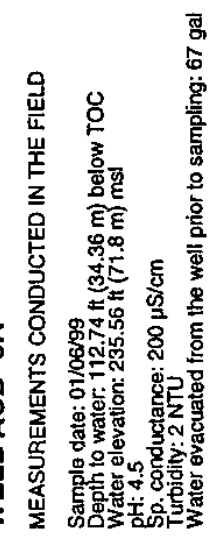

\section{g.}

מבירכ בכנד

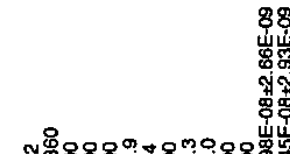

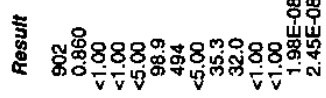

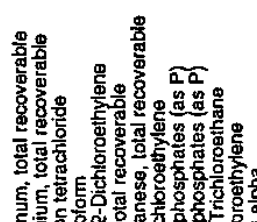

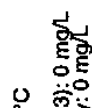

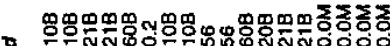

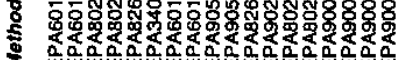

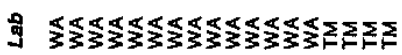

(1)

昰产

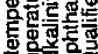

年

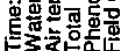

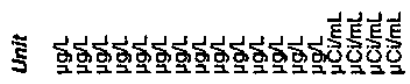

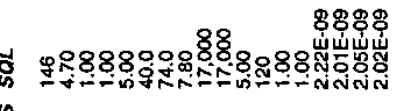

急

בכב 20

8 要

8888

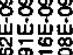

F⿻ำ

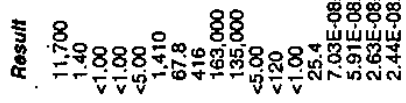

8
$\frac{8}{8}$
8
8
8

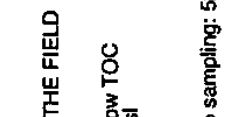

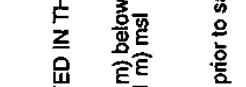

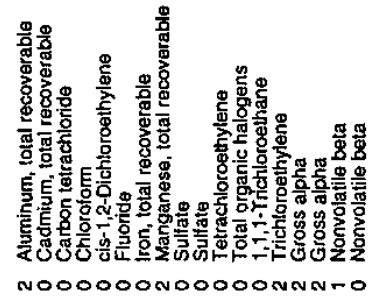

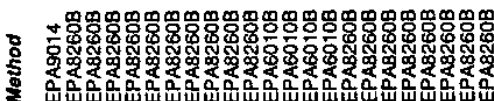

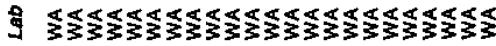

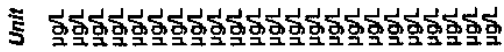

ప

罂

$\sqrt{162}$

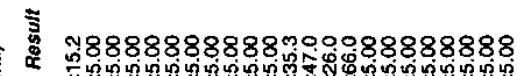

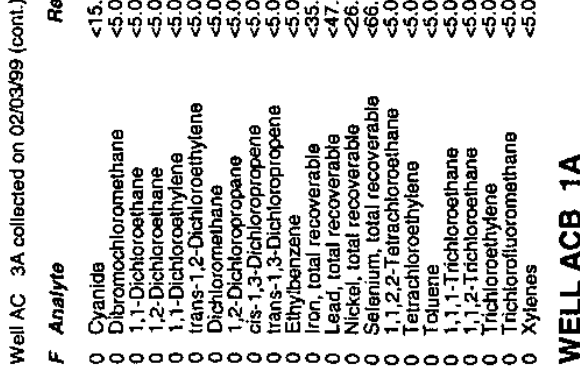

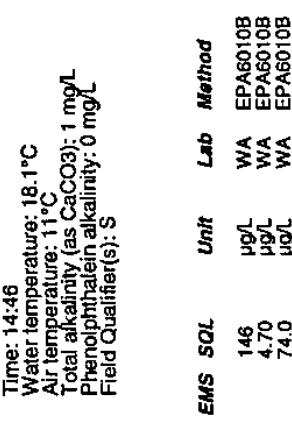

$\infty$

U.

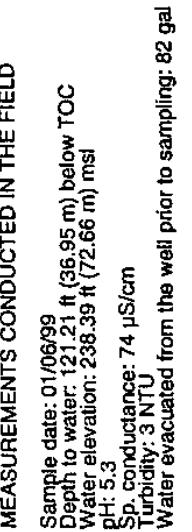

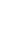

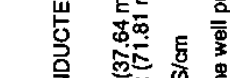

\%

展

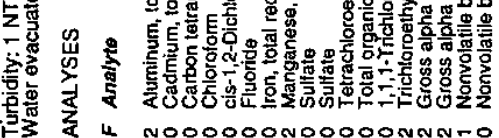

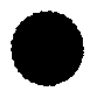

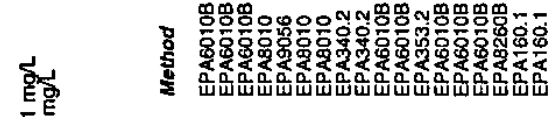

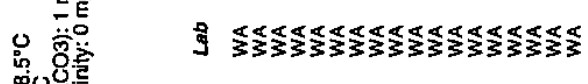

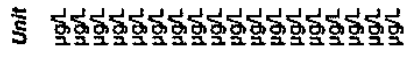

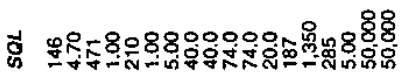

紊

$\infty \quad>>-\infty$

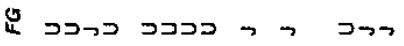

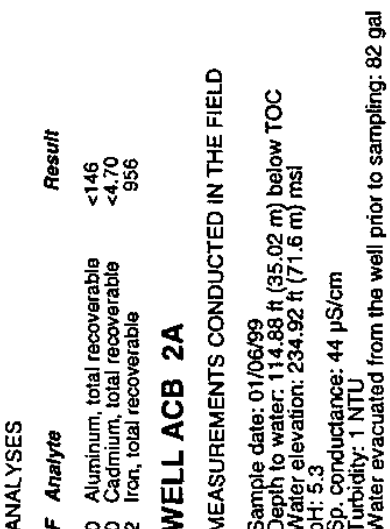

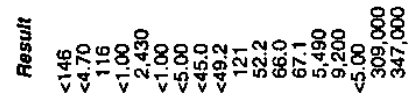

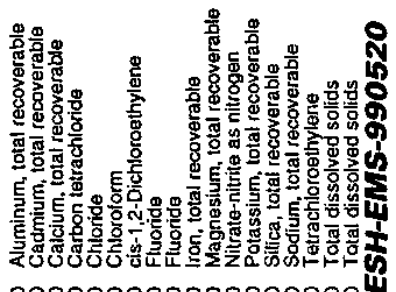




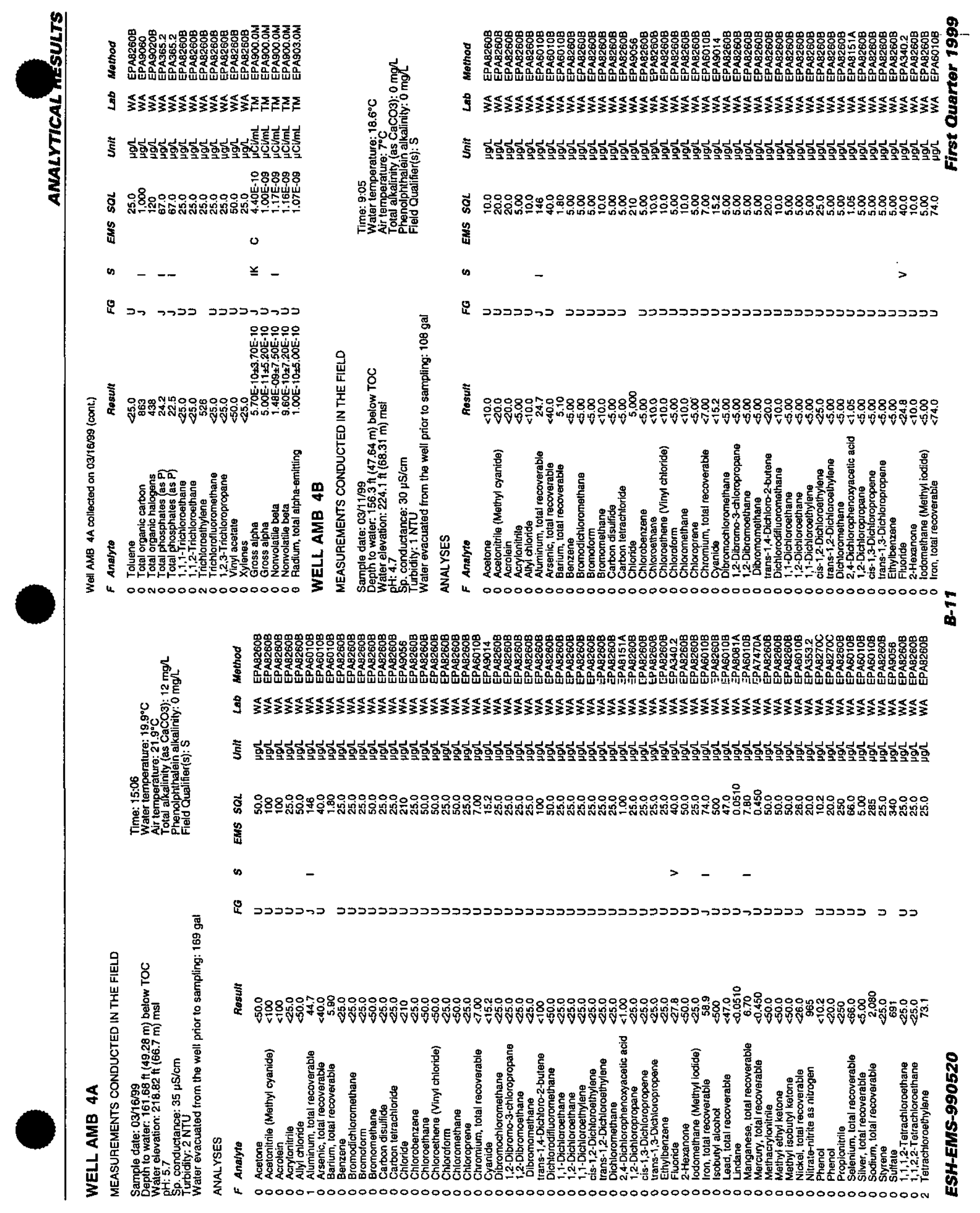




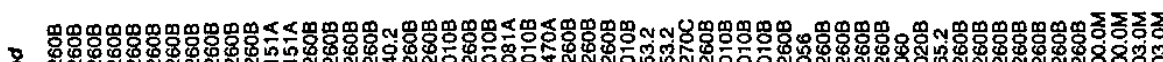

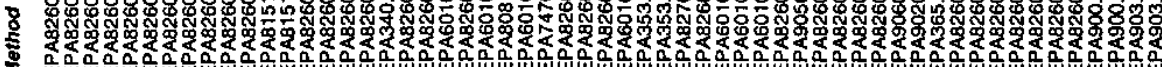

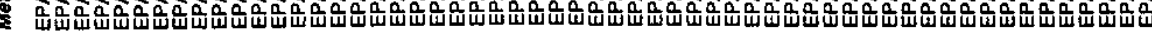

9.

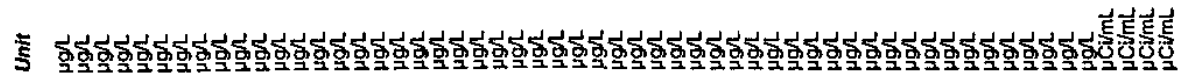

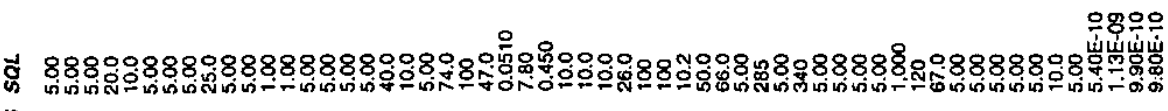

密

0

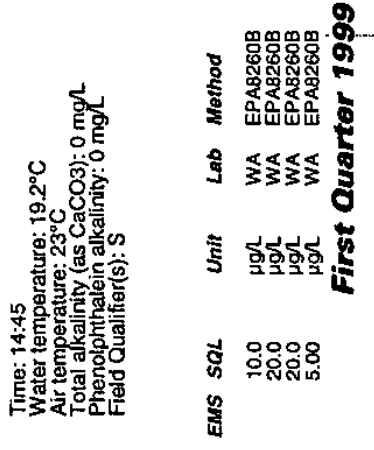

n

\&

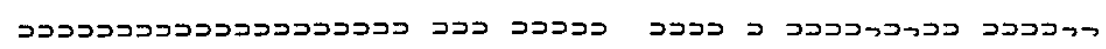

8 우88

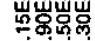

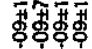

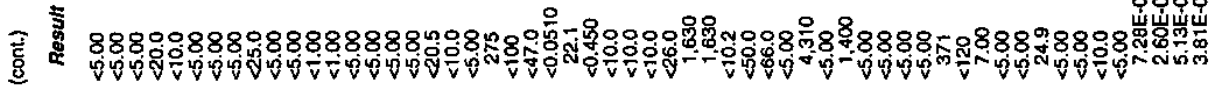

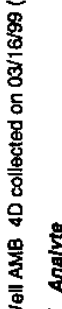

꽁뮴

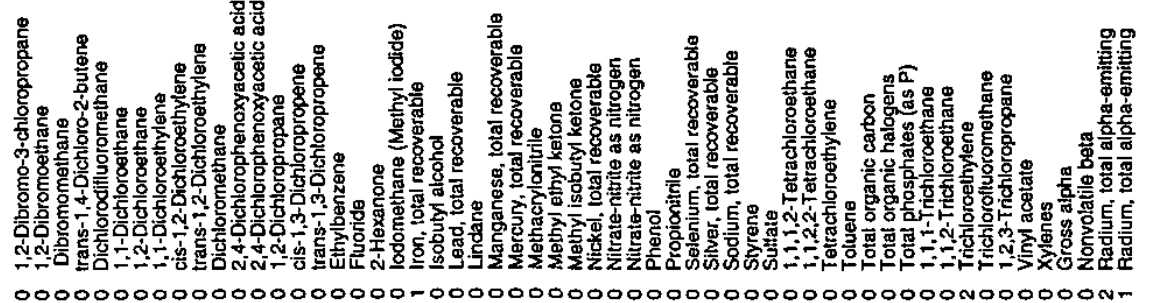

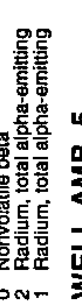

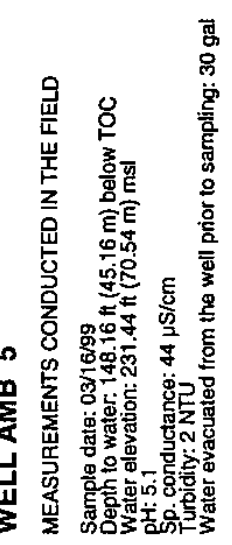

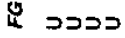

要

is

产 웅요

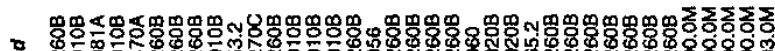

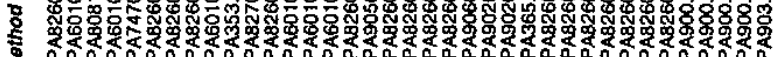

- -

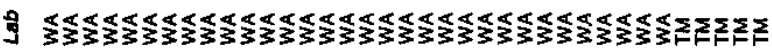

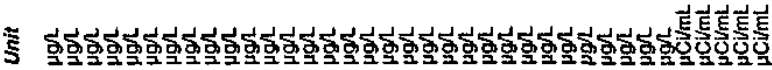

$8888 \%$

현 운

善

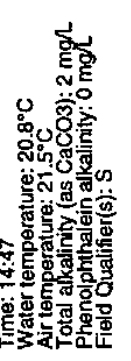

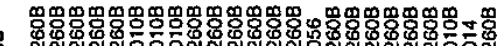
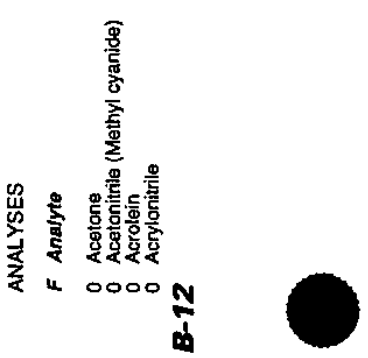

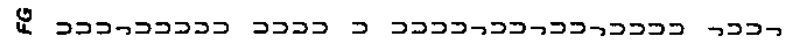

$888 \%$

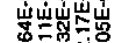

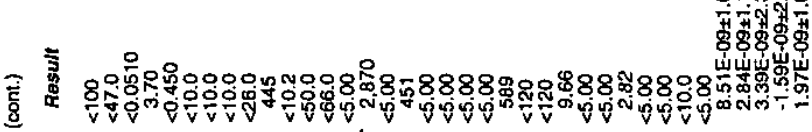

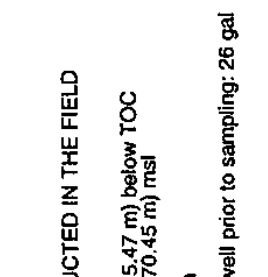

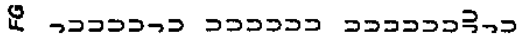

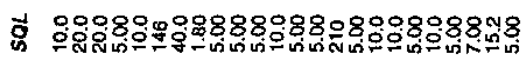

紊

$\infty$

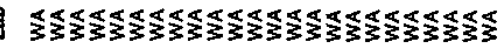

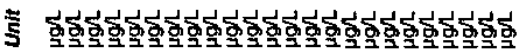

(

$80080000088088 \%$

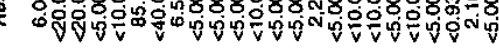
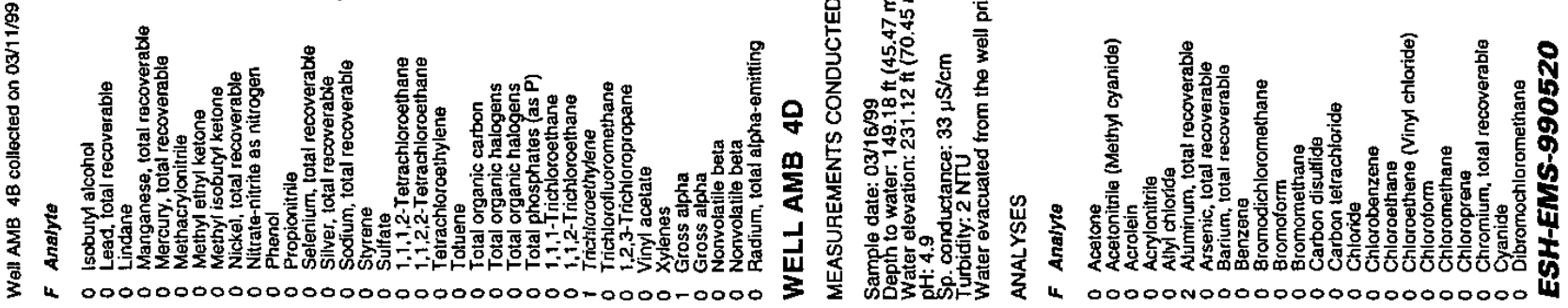


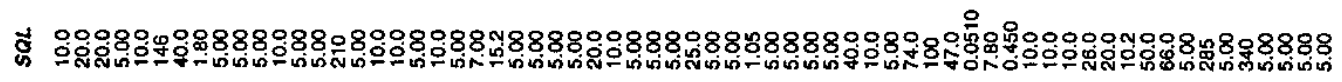
孳

a

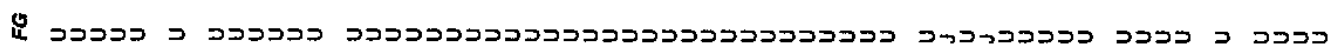

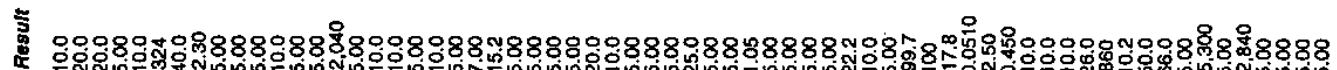

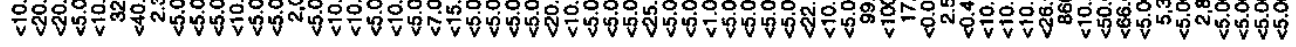

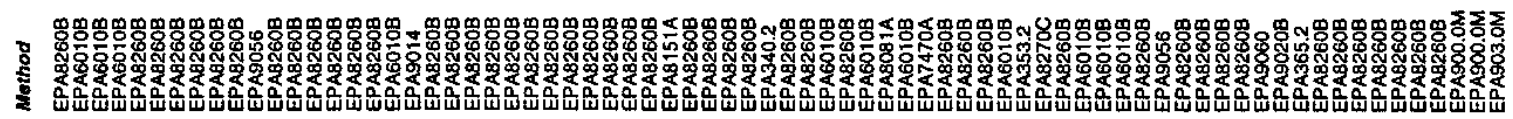

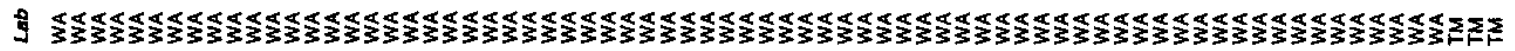

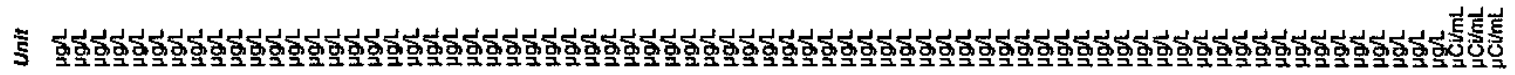

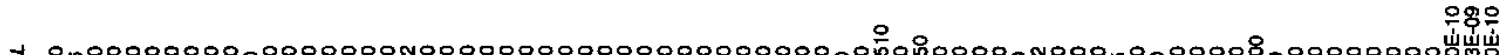

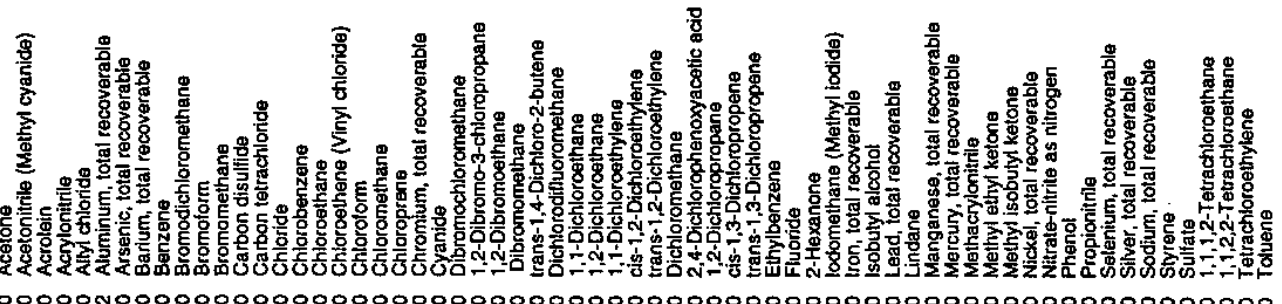

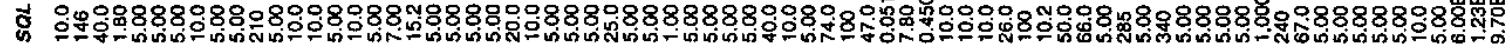
恙

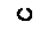

0

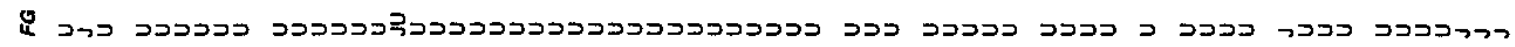

우요용

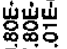

क्षेत्रें

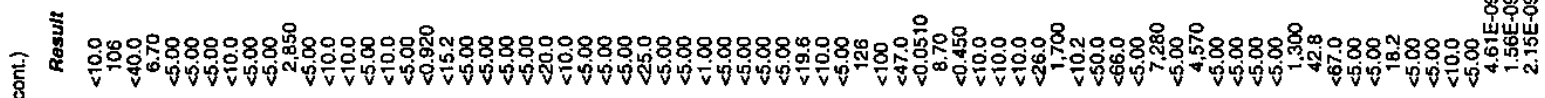




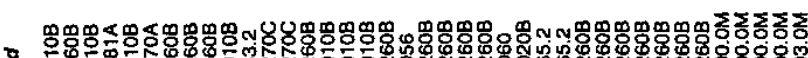

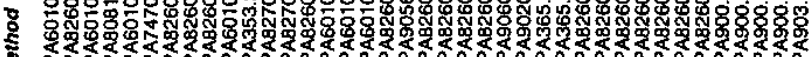

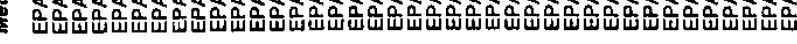

行

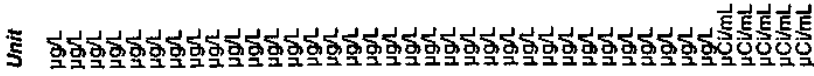

으우우유으

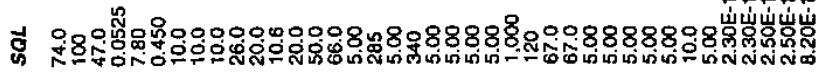

辤

as $0_{-}-000_{-} \quad 0>000000_{-}-0000000>>$

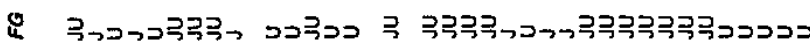

우웅ㅇㅇㅇ

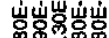

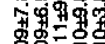

言

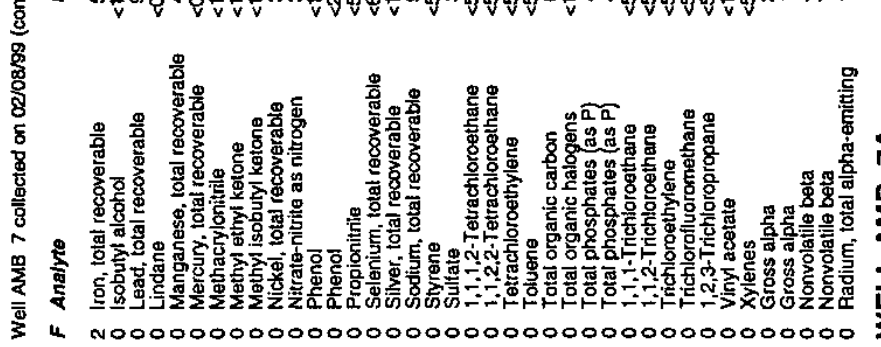

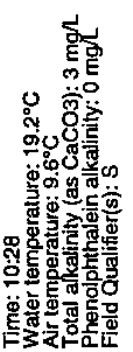

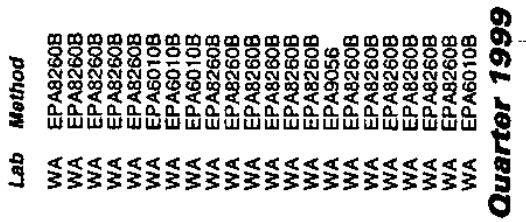

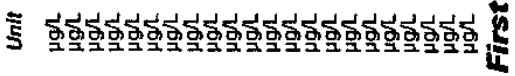

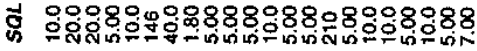

紊

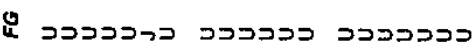

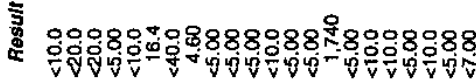

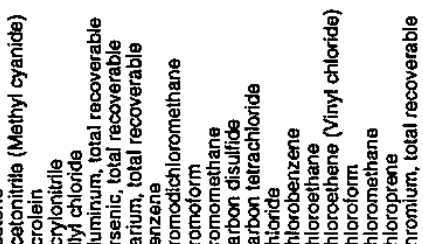

\%

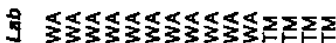

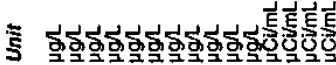

우유융ㅇ

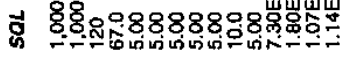

章

的

כככדבכבככבכדני

으용ㅇㅇ

㖆岁岲岁

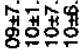

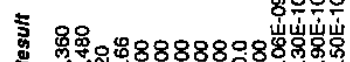

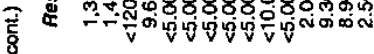

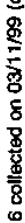

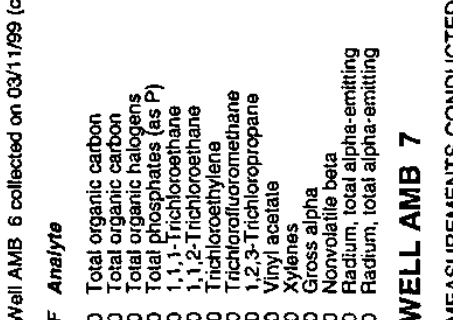

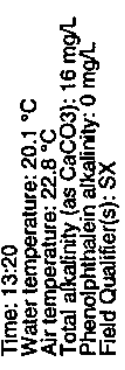

క 蛋

is $00000000000000000>--0000000000000000>00$

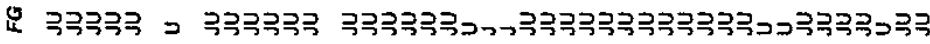

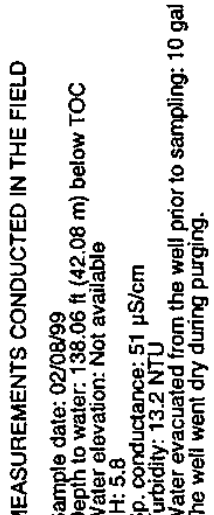

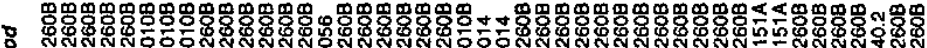

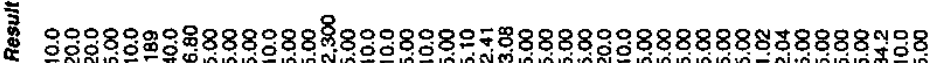

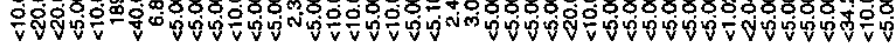

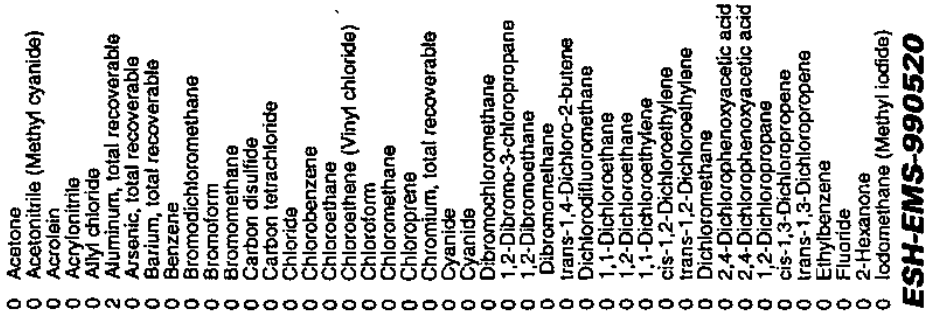




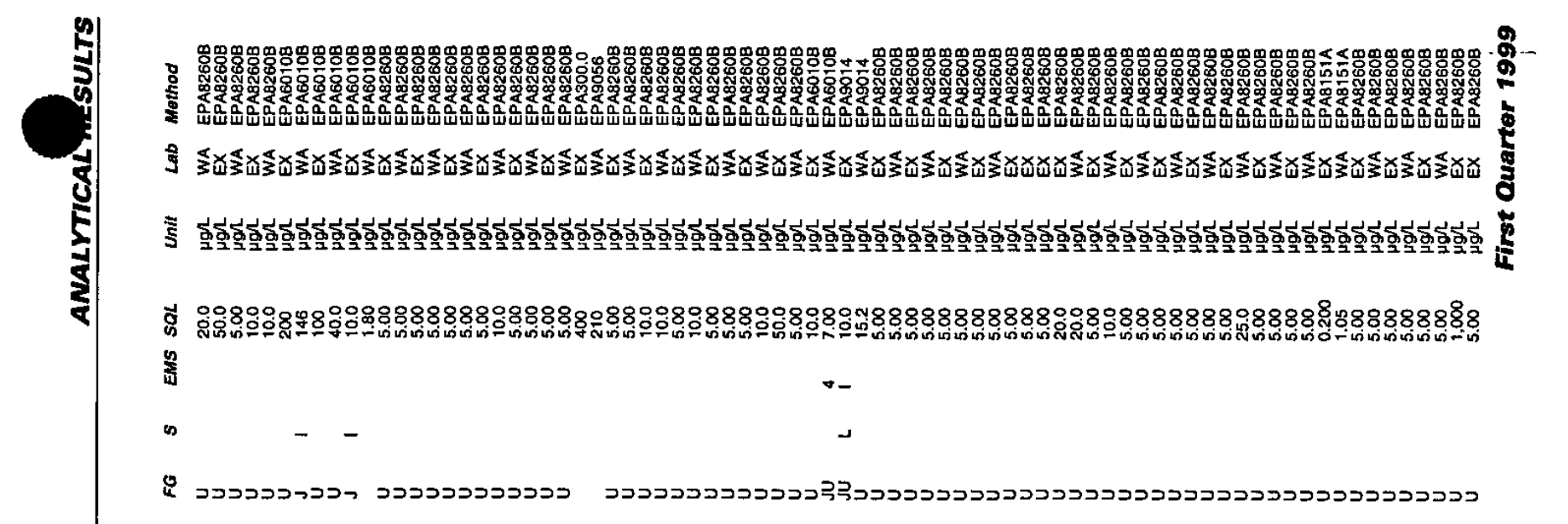

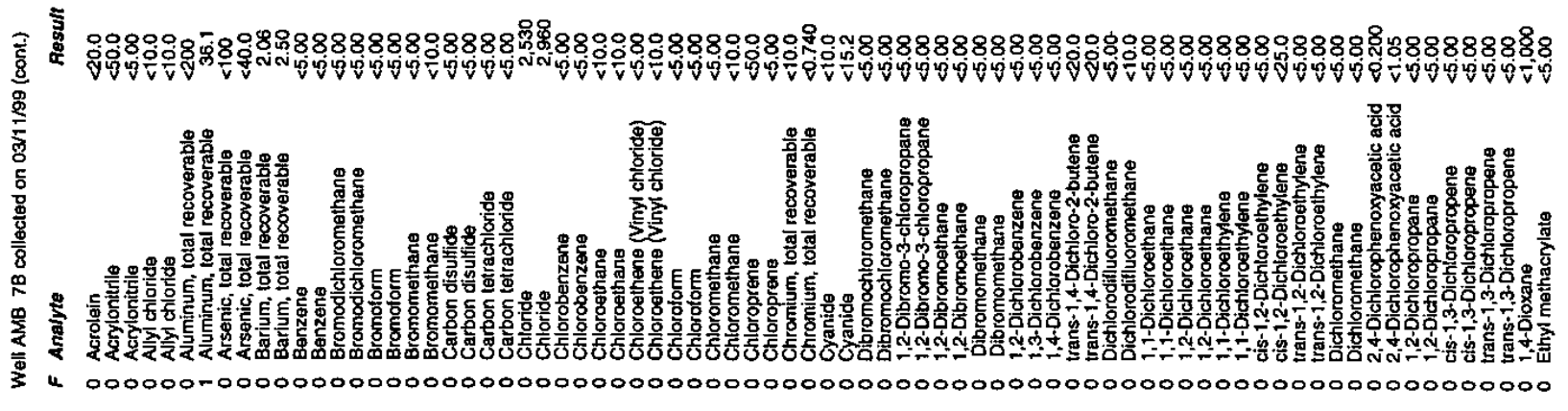

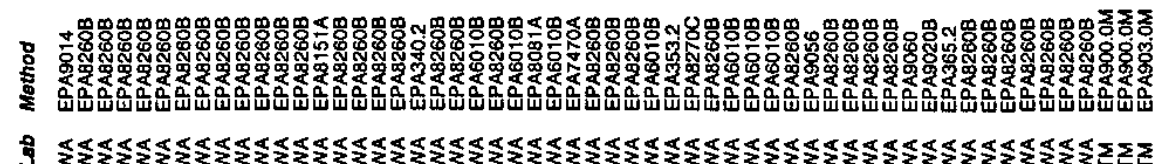

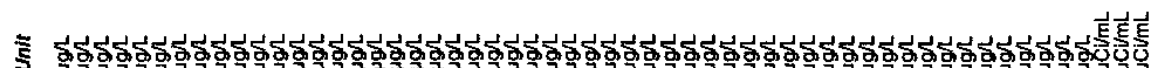

1

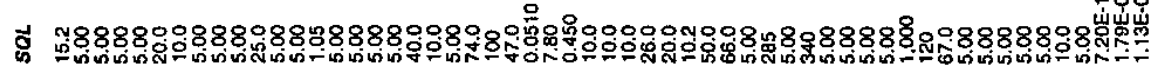
量 (1)

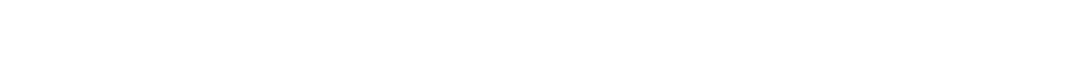

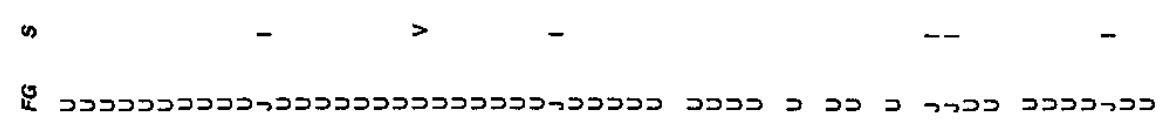

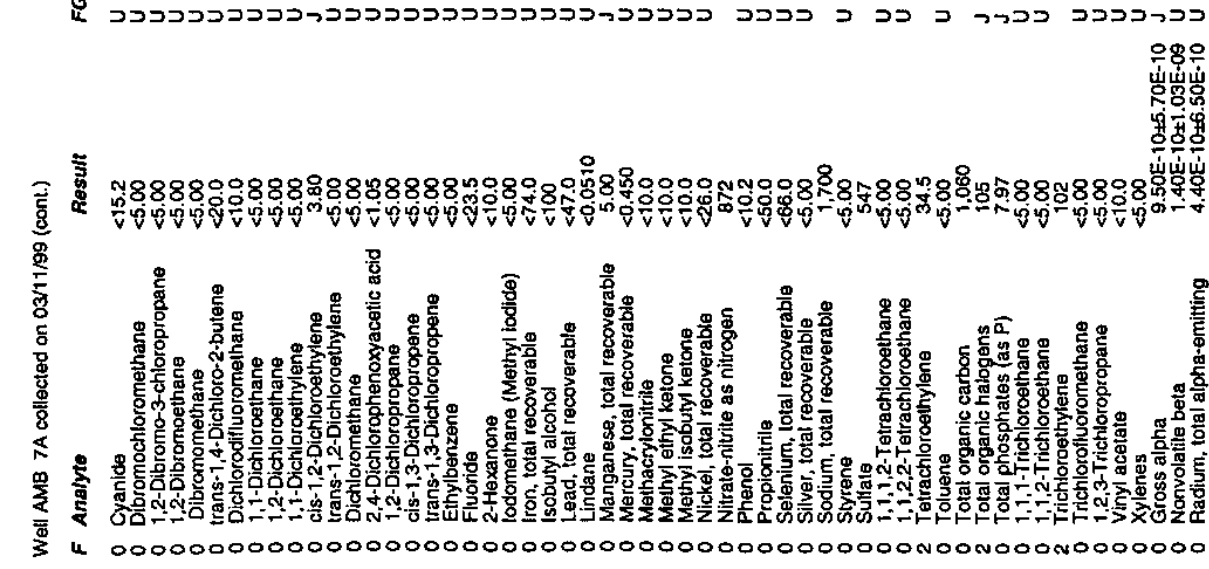

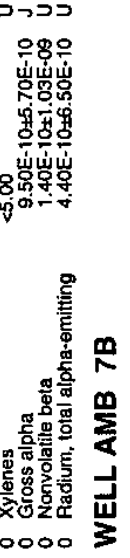

응

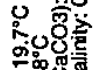

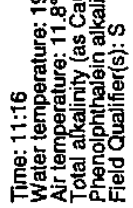<smiles>C1CCCC1</smiles>

8

क्ष

ง

รั 히ㅎㅐㅗ호호

호 웅우웄옹ㅇํ

嵒

क

ככככב

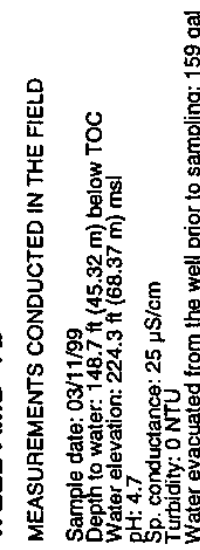

ฮू

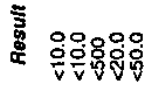

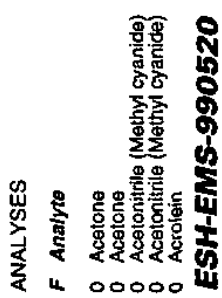




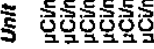

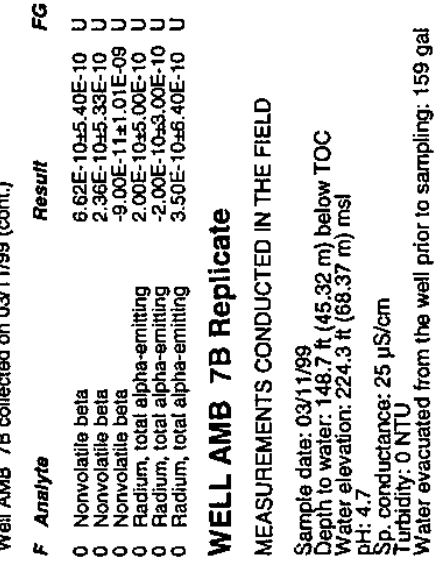

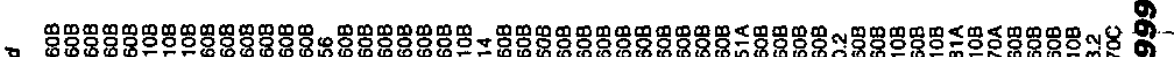
5 1

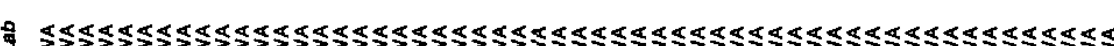

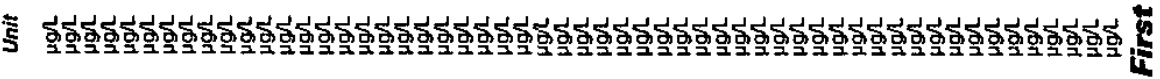

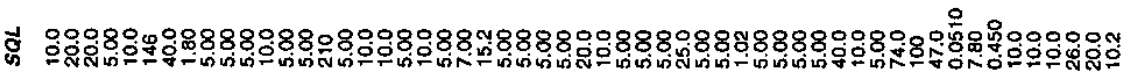
姜

as

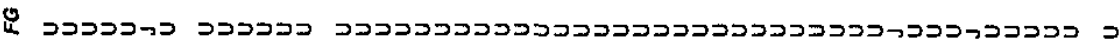

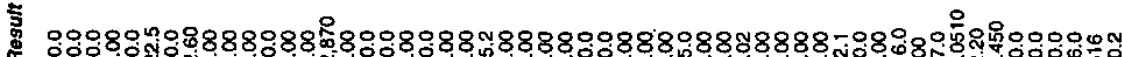

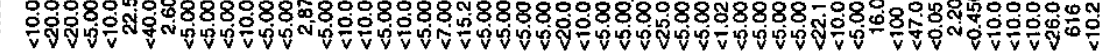

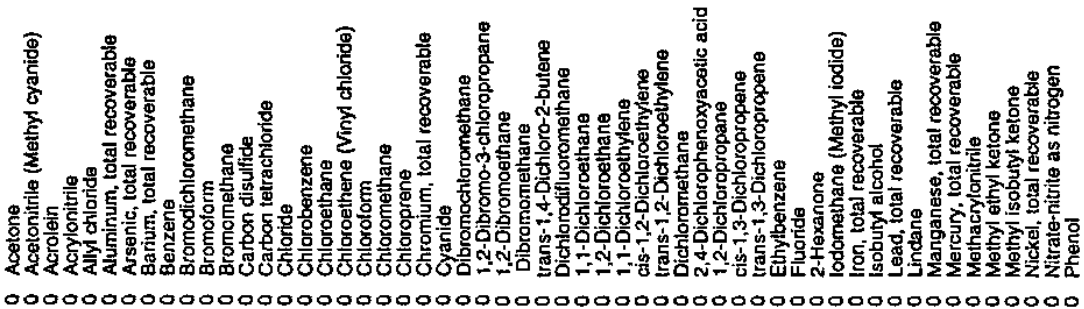

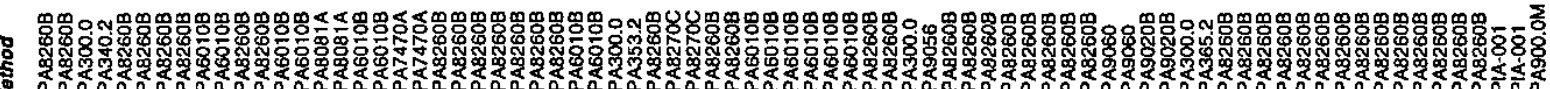
o of

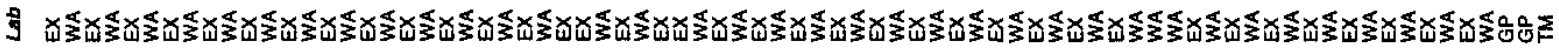

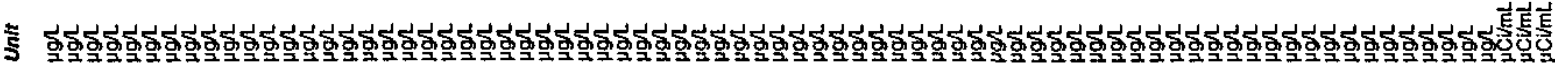

으으눈

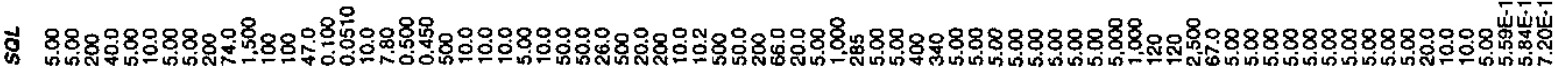
影

$>\quad--\quad-$

a.

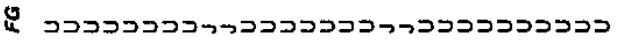

כככככככנ

$د$

ככככבכבכ

כככつכフ

רכבככבכבכנב

우으우

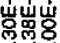

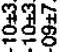

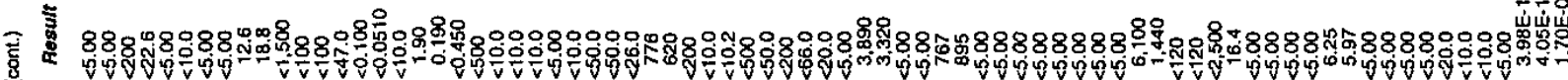

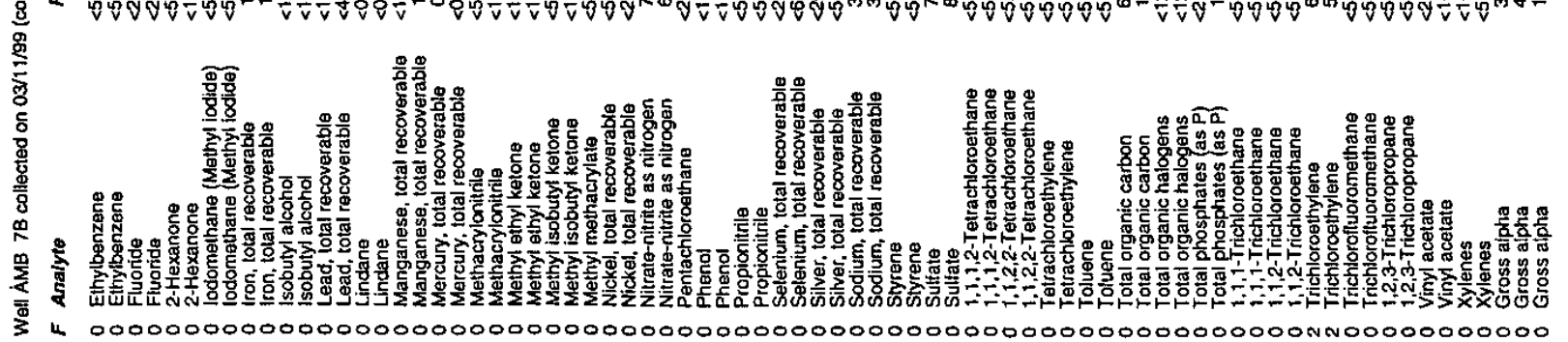




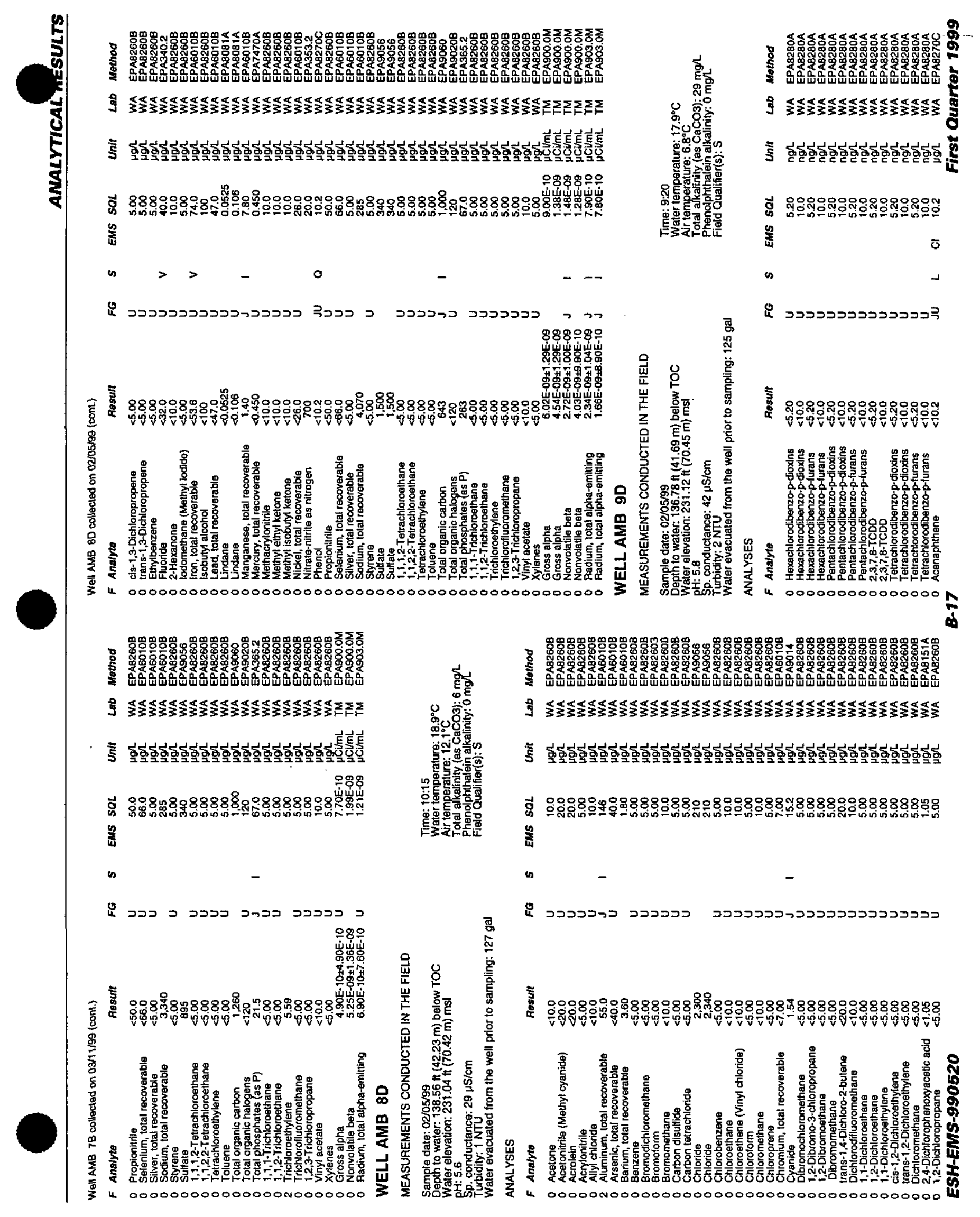


:

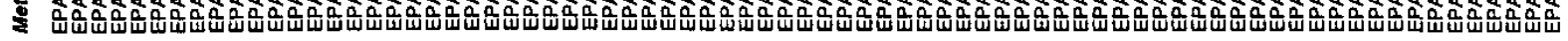

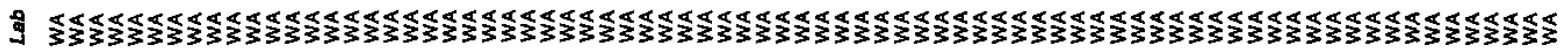

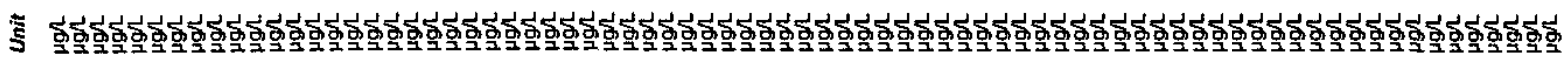

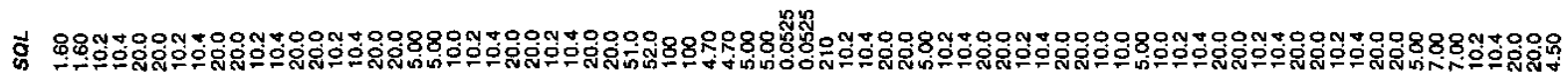

量

$\overline{0} \overline{0}$

$-$

0000000000000000

○

$0 \quad 0 \mu \sigma\lrcorner \sigma \quad 0 \quad 0>0 \quad 0$

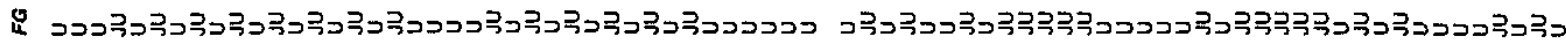

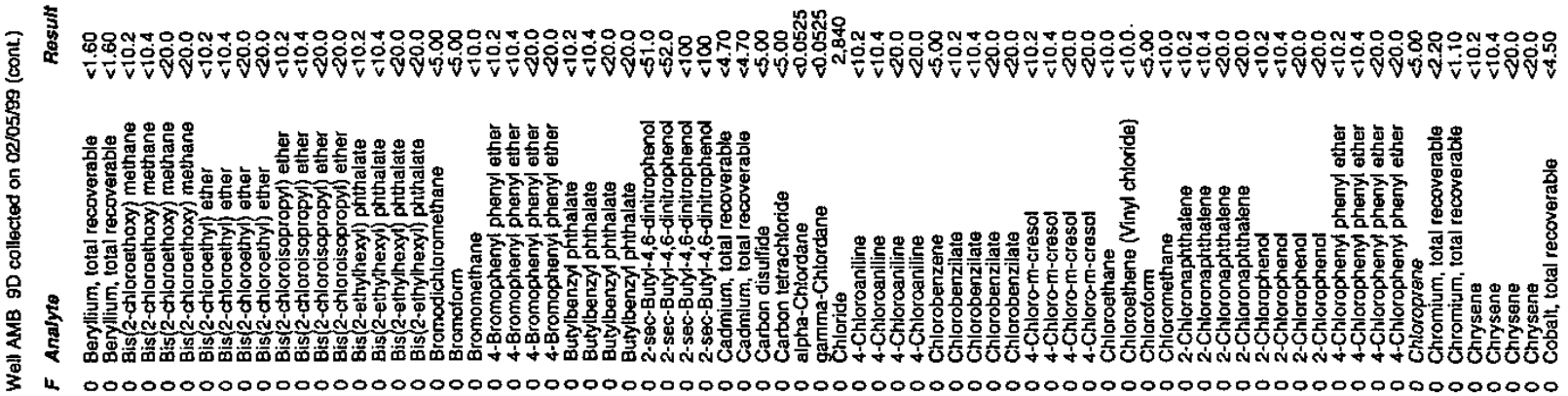

$888880 \%$

o

:

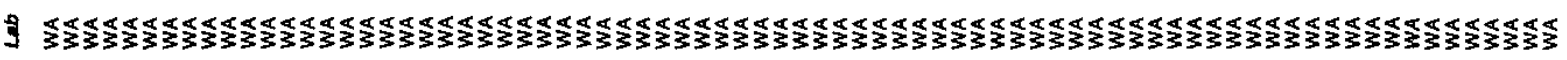

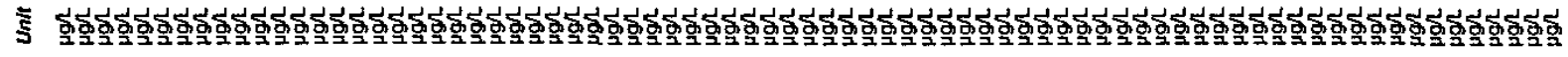

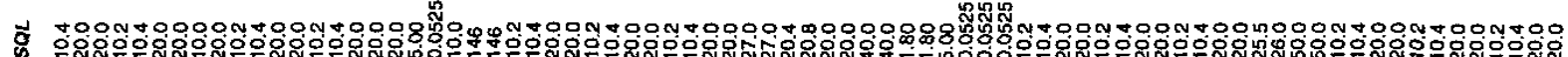
密

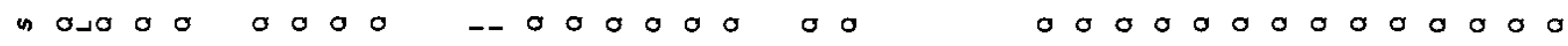

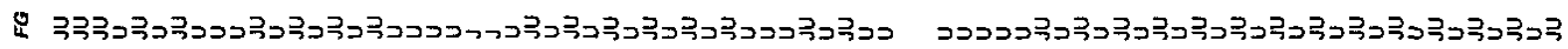

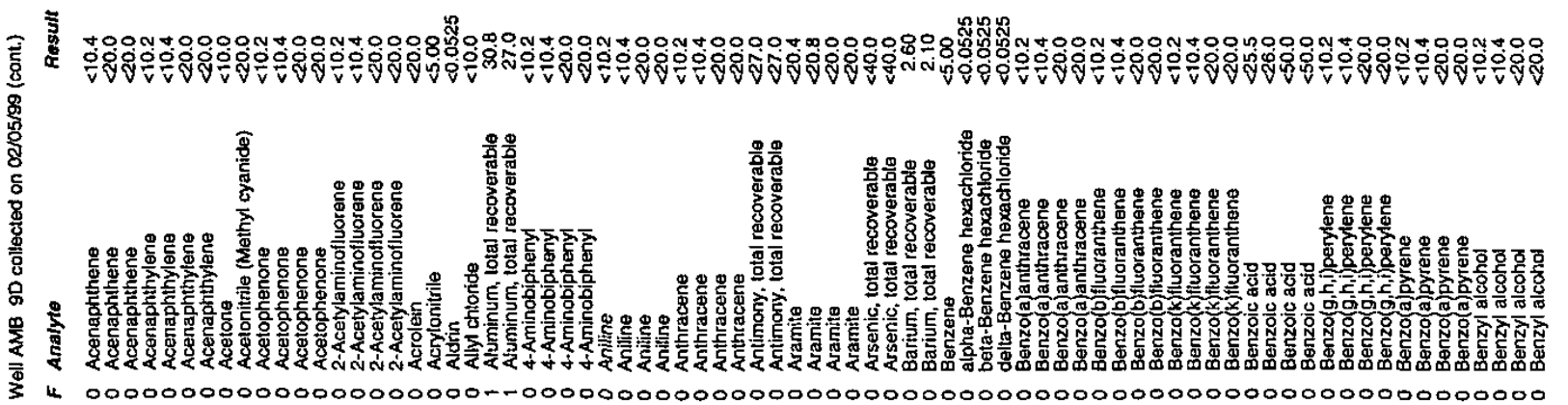


!



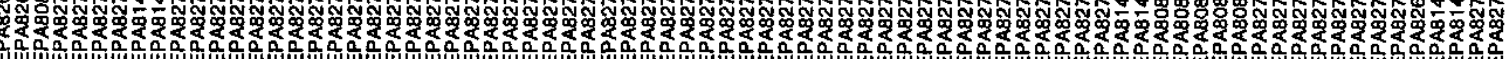

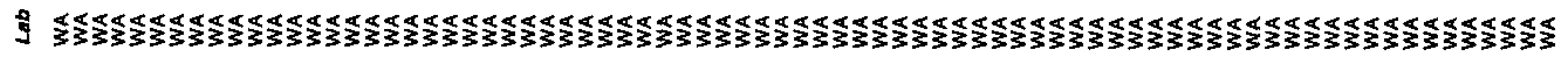

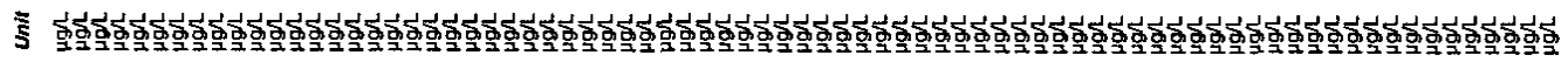

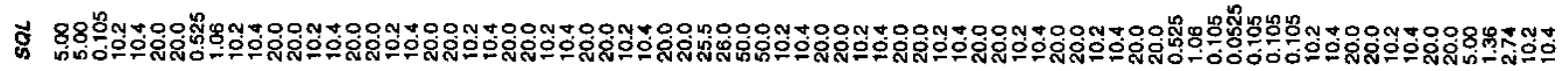
蛋

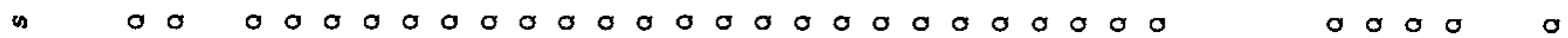

4

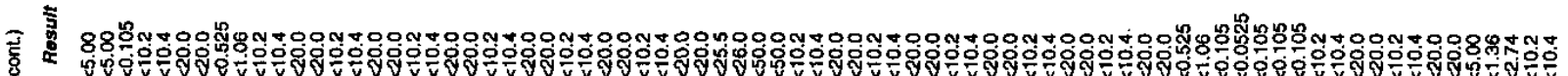

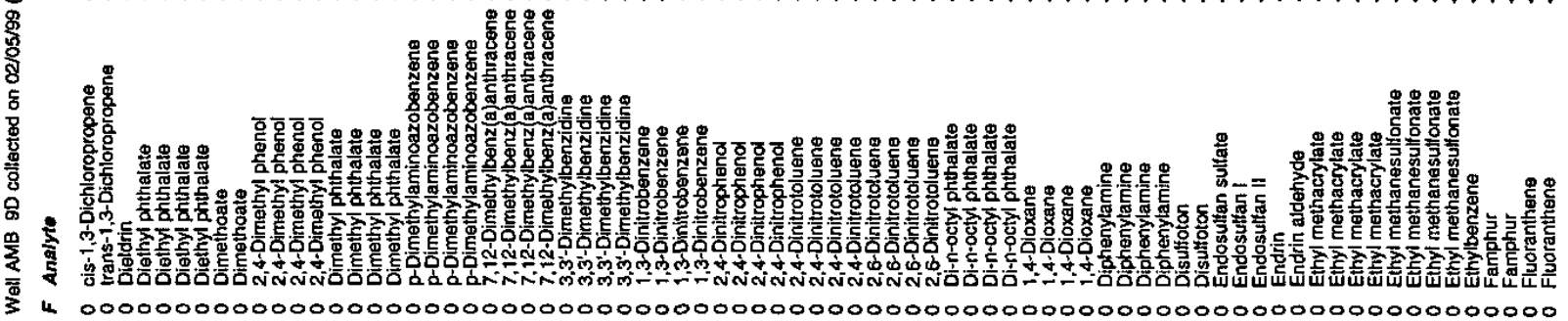

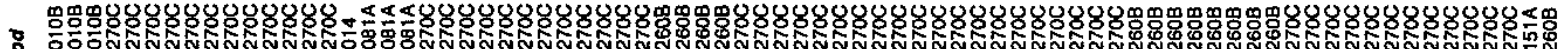

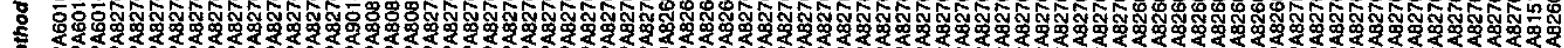

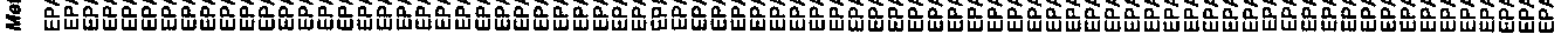

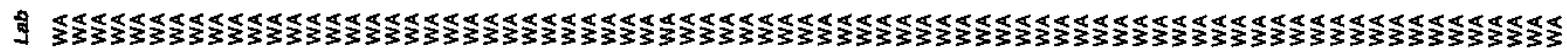

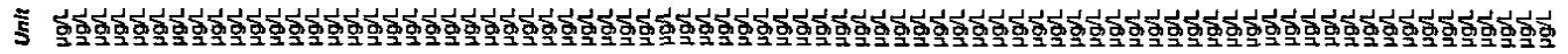

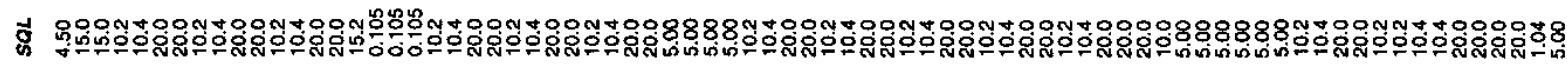
墨

$\overline{0} \overline{0}$

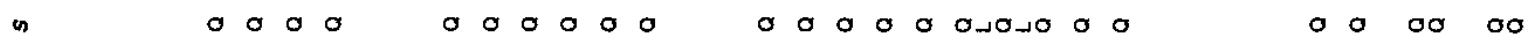

ע ע

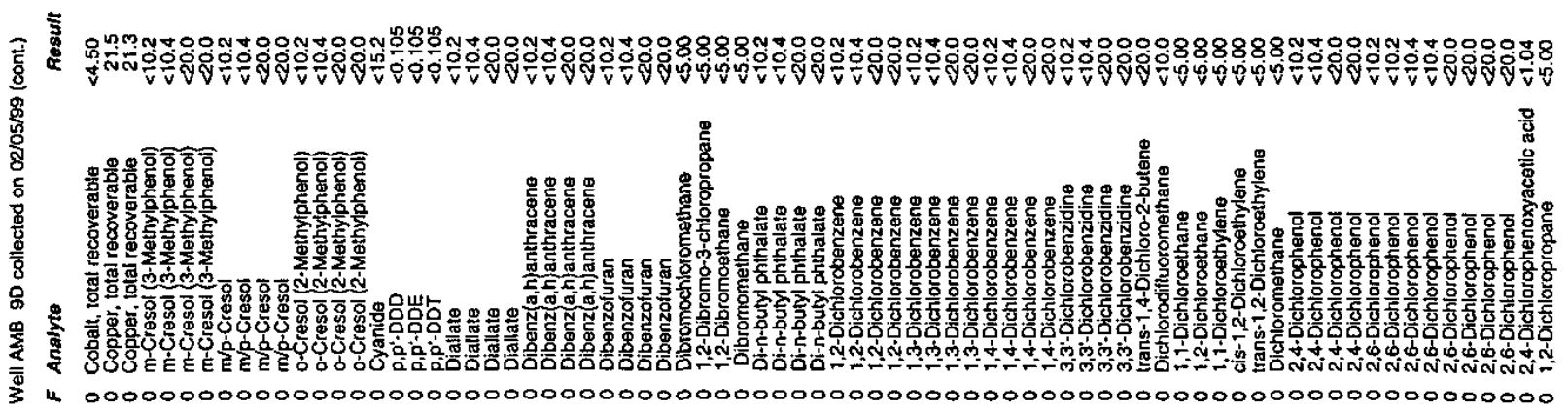




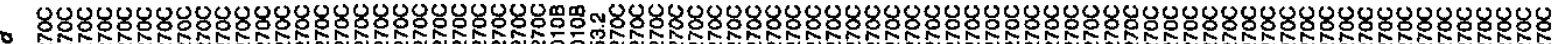
号

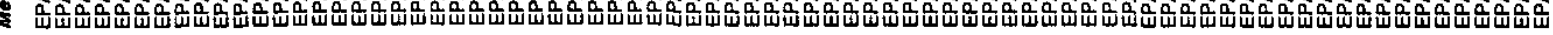

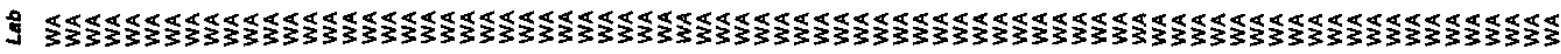

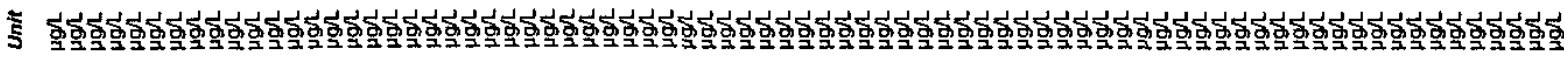

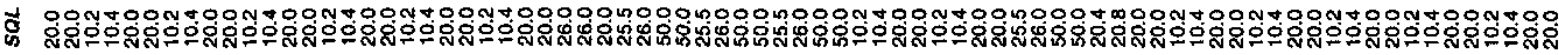
紊

\& 00000000000000

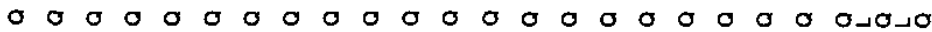

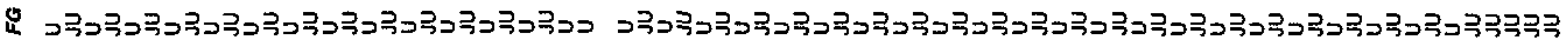

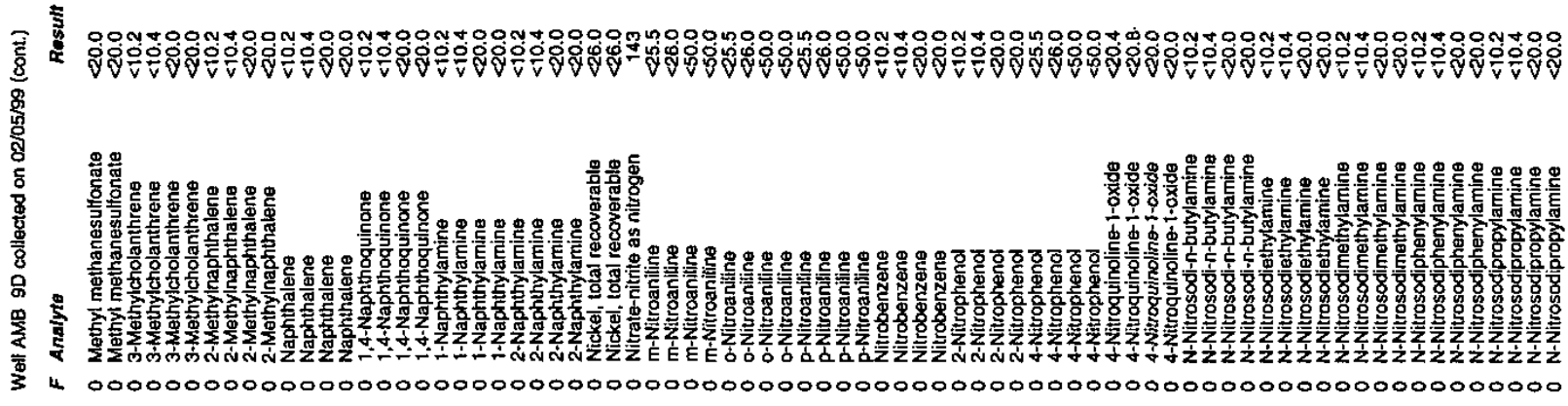

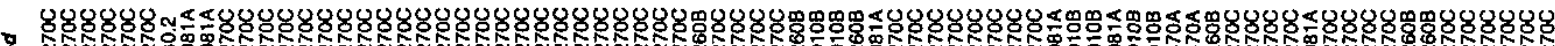

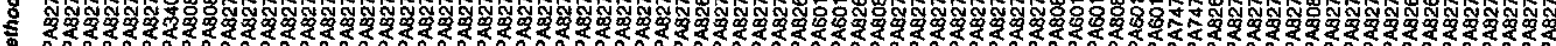

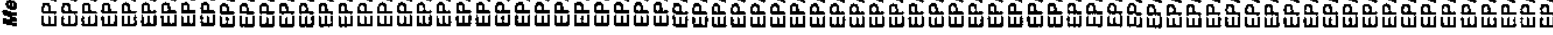

包

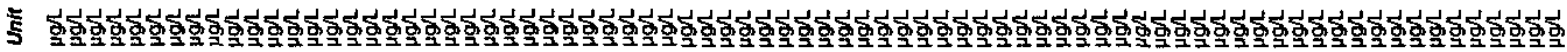

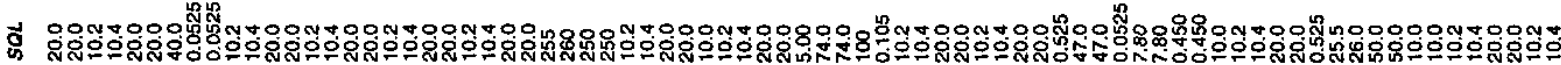
查

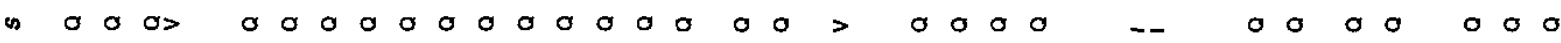

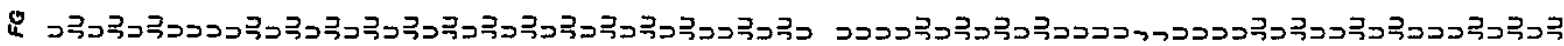

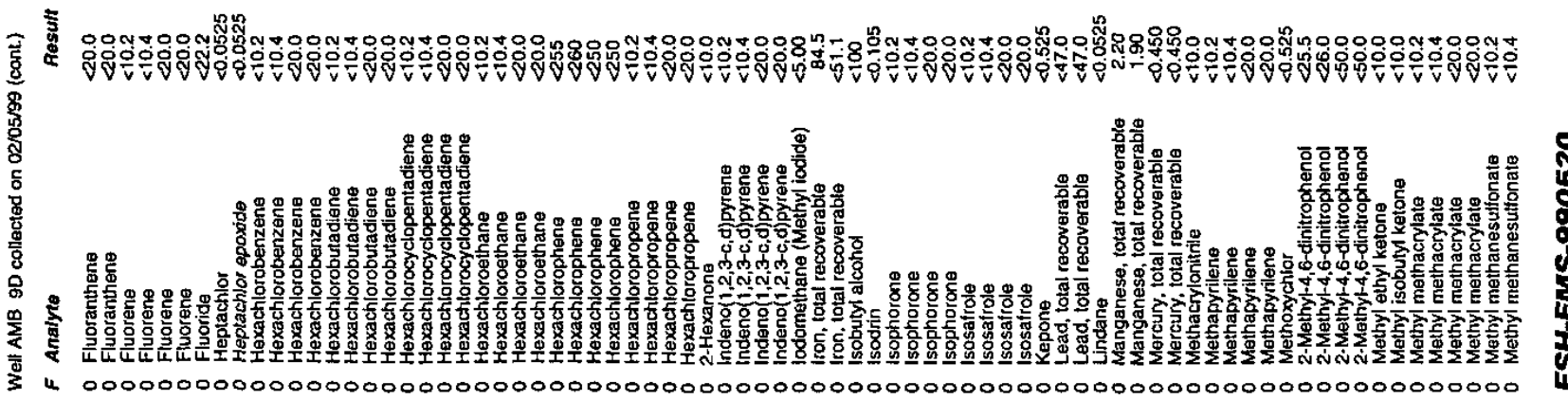




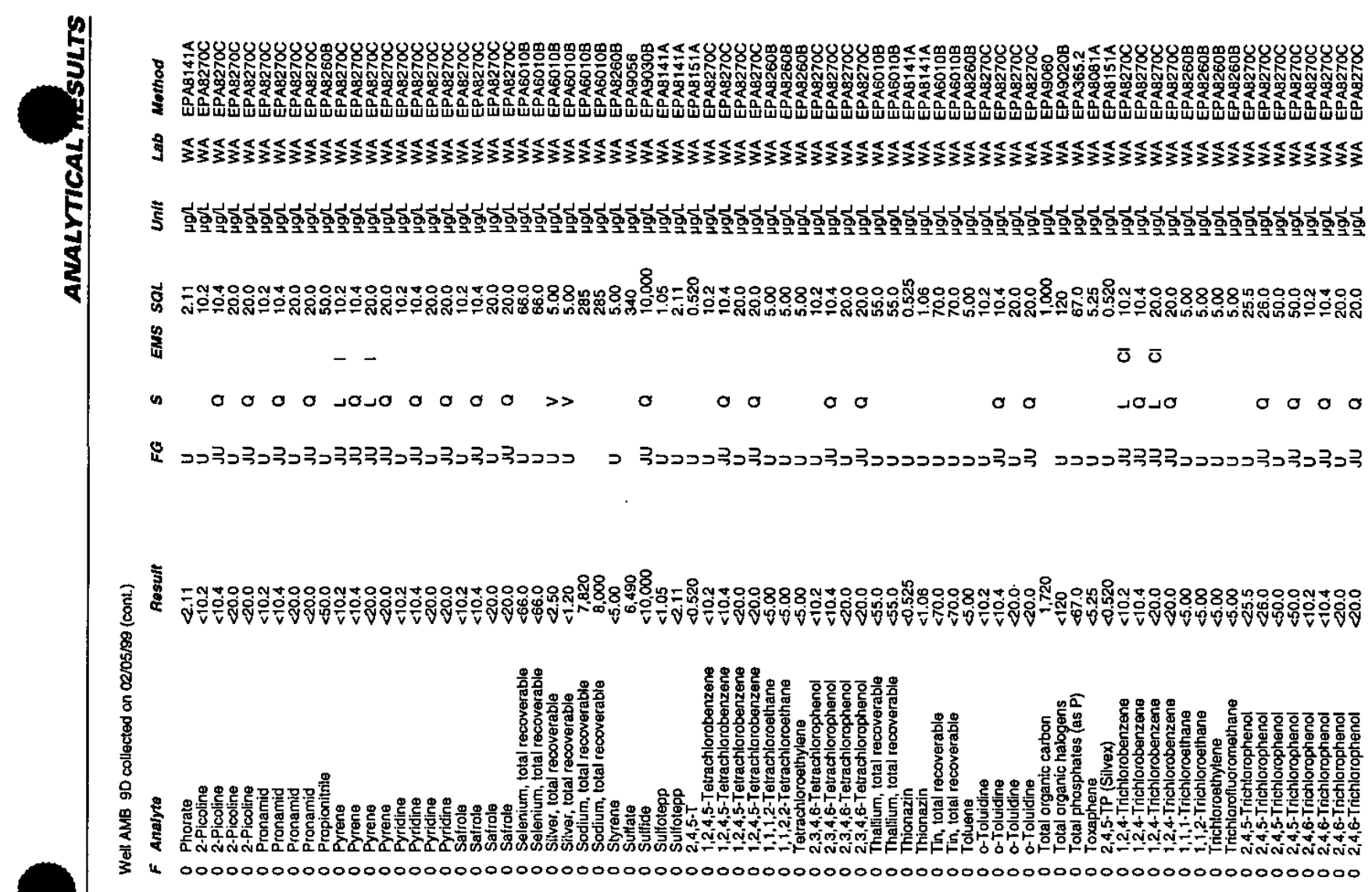

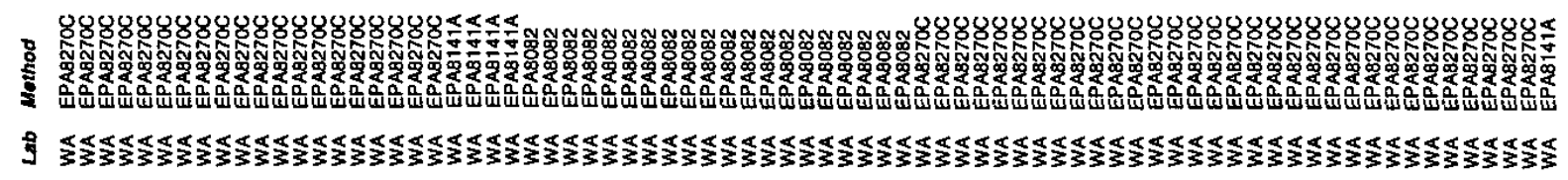

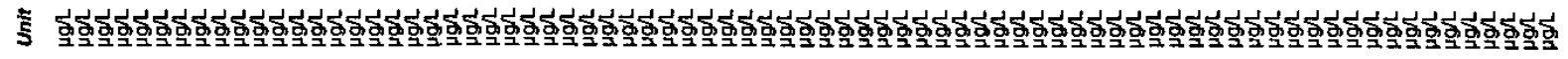

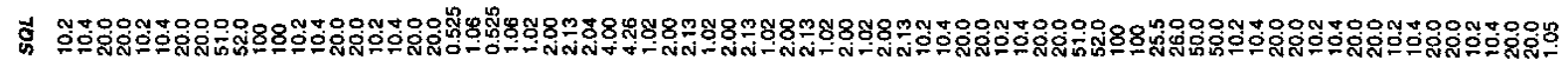
密

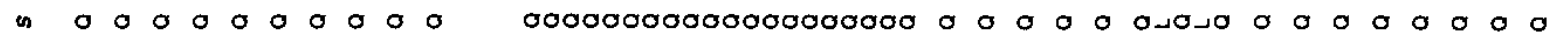

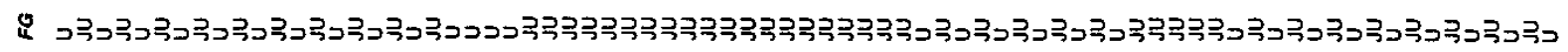

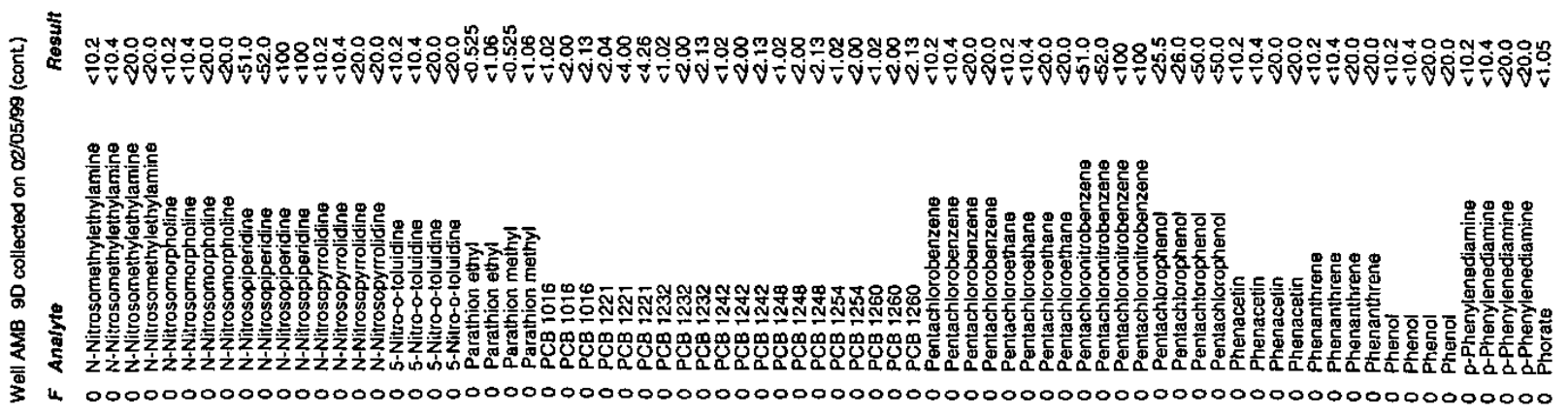


จ

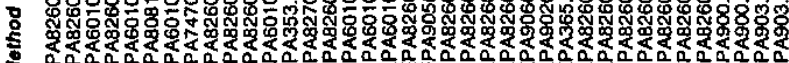

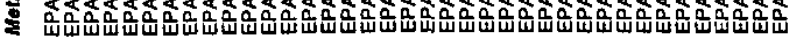

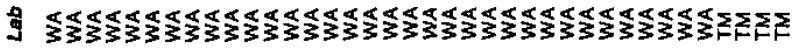

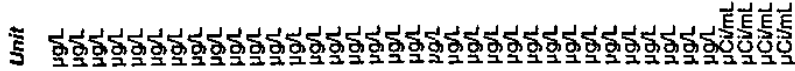

우우우으

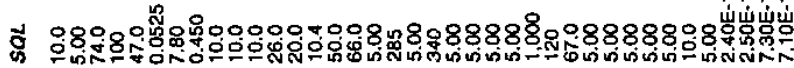

紊

os $00>0$ - $000 \quad 0>00000-0000000>-$

क्रू

:유류.

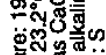

氙通产

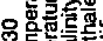

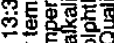

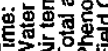

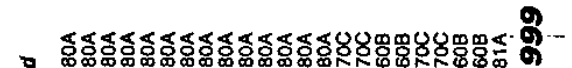
空

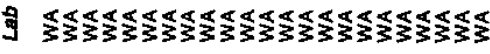

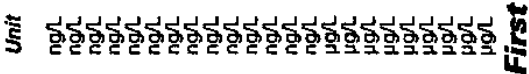

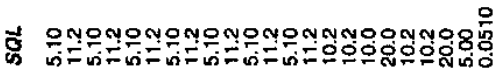

密

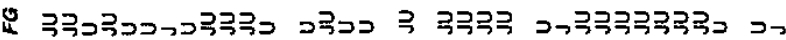

요용ㅇㅇ

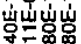

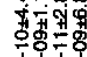

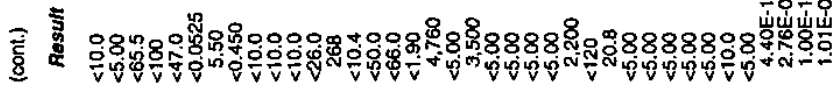

旁
옹
음

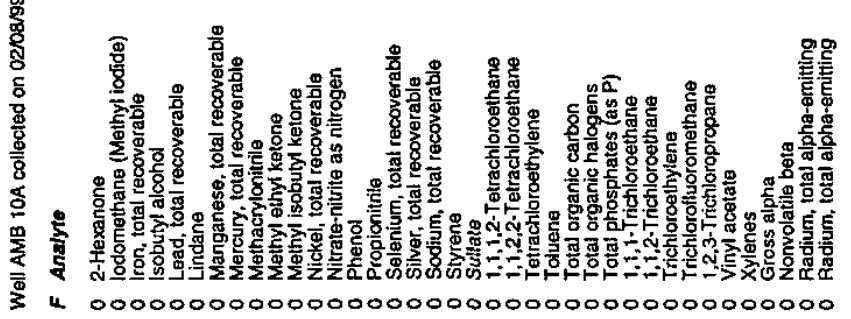

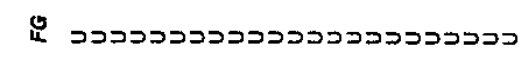

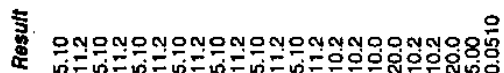

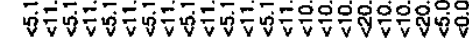

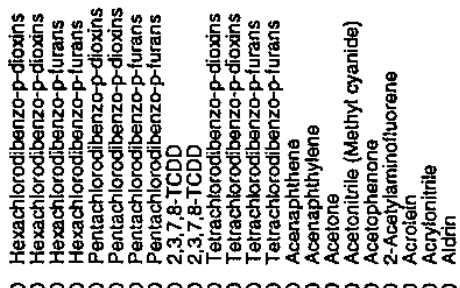

哭过

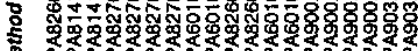

\%

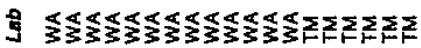

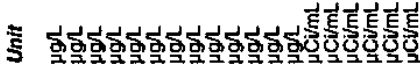

영용ㅇㅇㅇㅇㅇㅇ

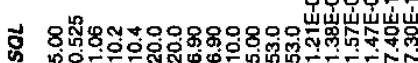

辤

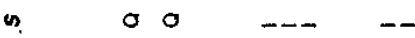

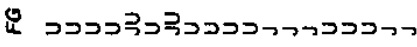

으응ㅇ으으으

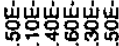

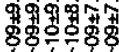

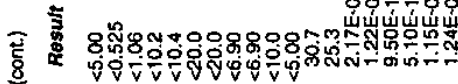

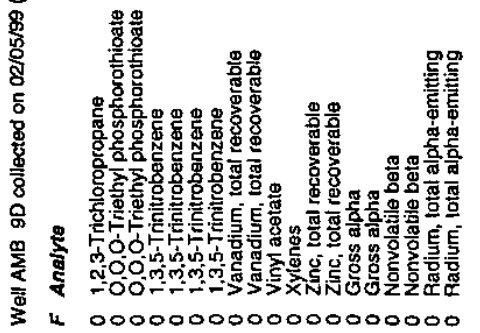

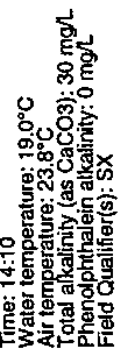

5ू.

తి

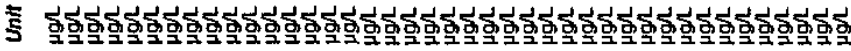

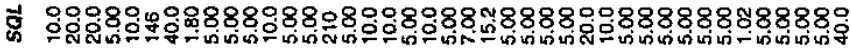

蛋

is 00000 - $000000000000>-0000000000000000>$

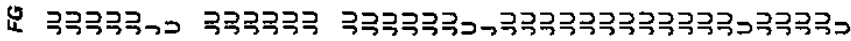

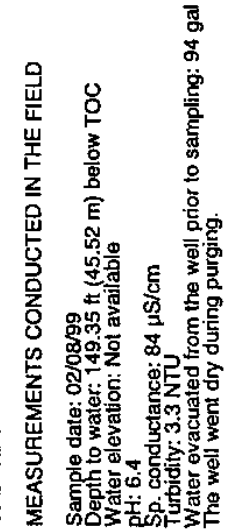

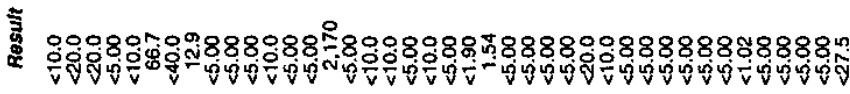

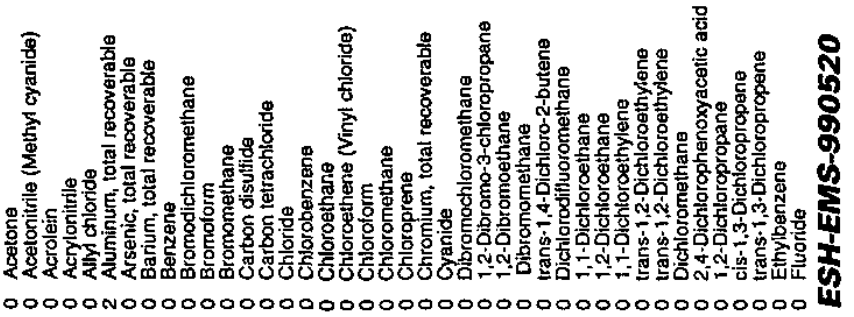




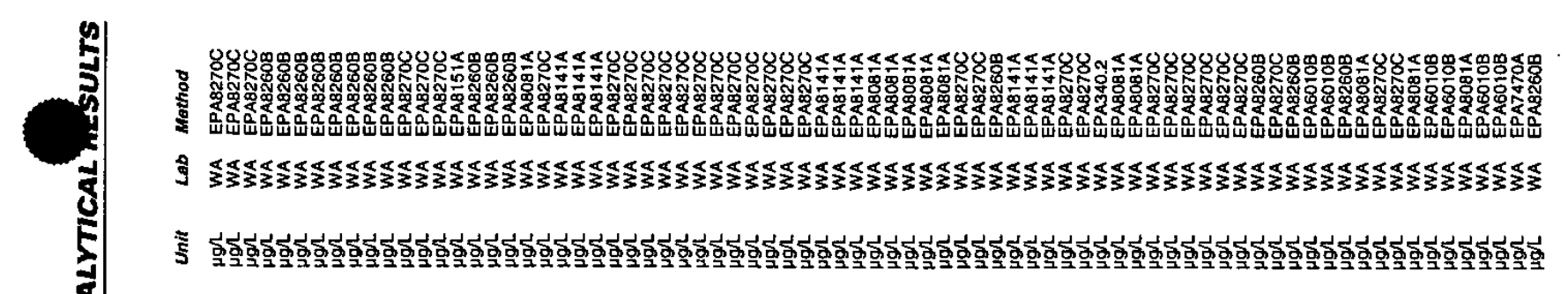

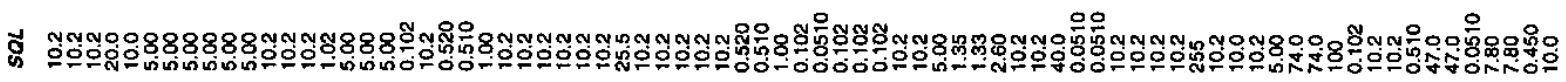
:

is

$\circ$

is

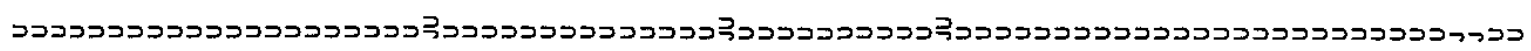

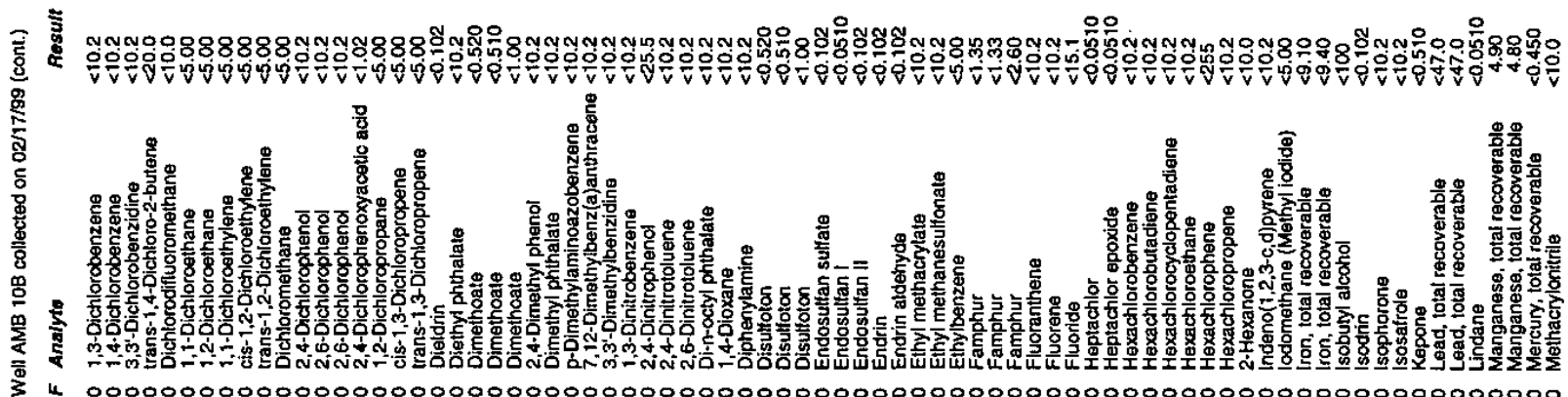

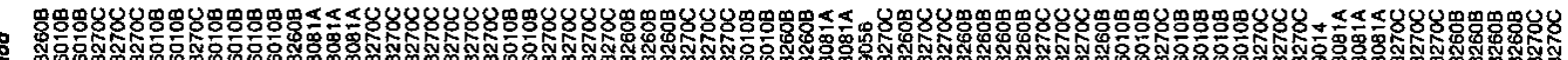

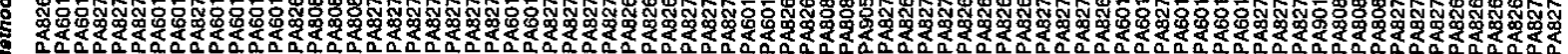

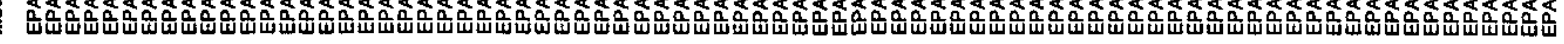

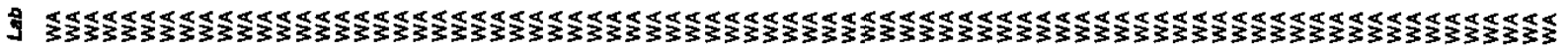

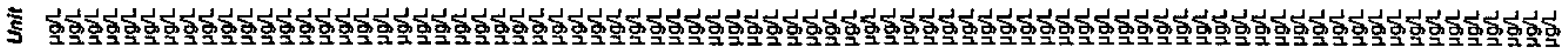

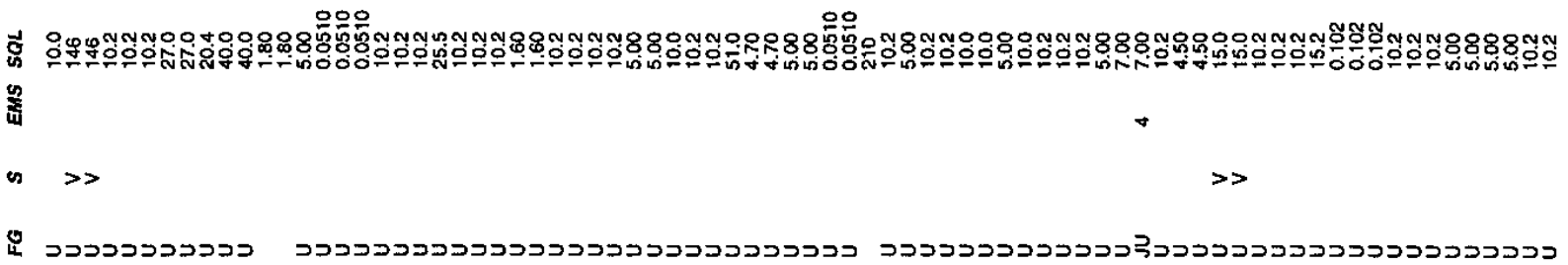

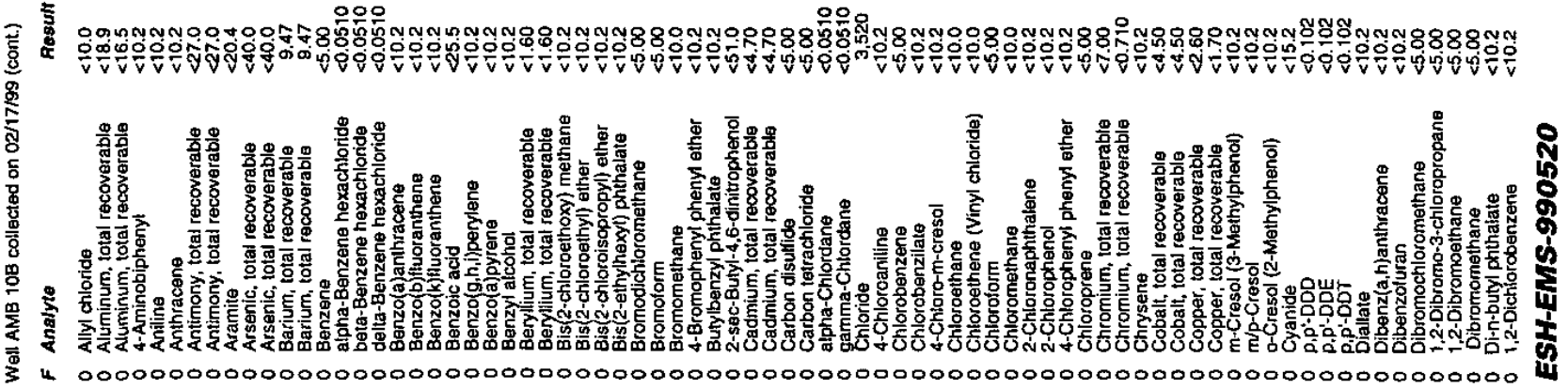




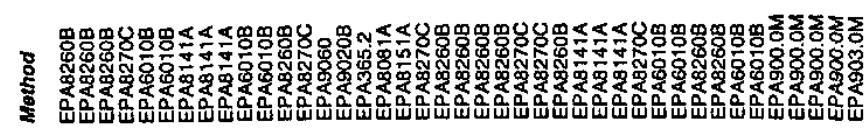

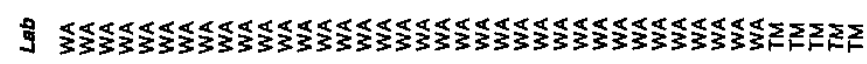

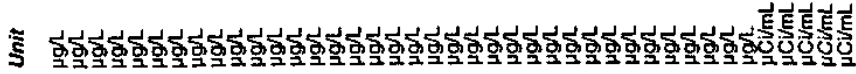

으으양으

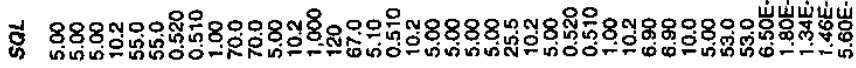

离

$\infty$

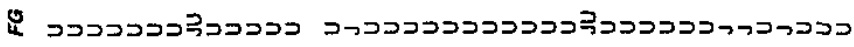
우으으으으

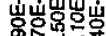

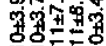

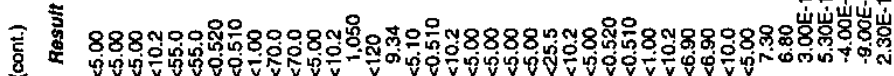

श्र

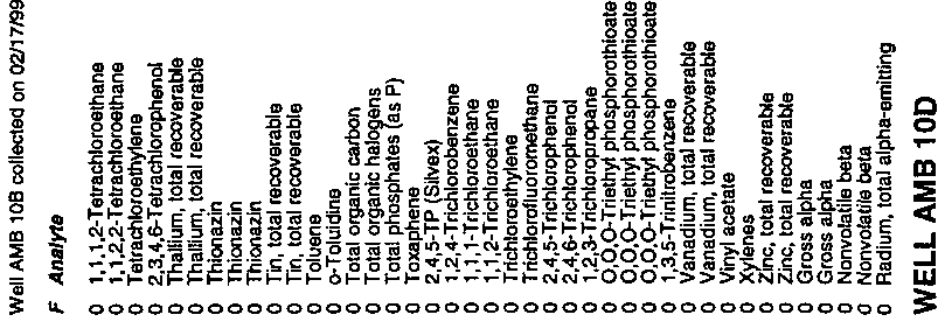

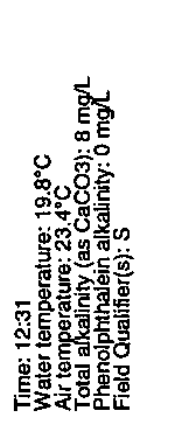

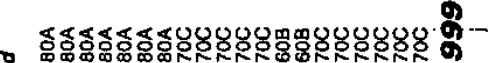

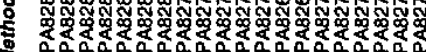

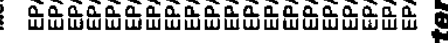

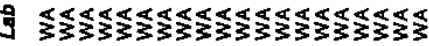

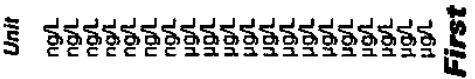

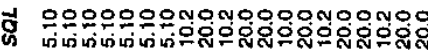

善

in

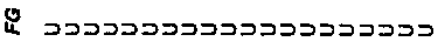

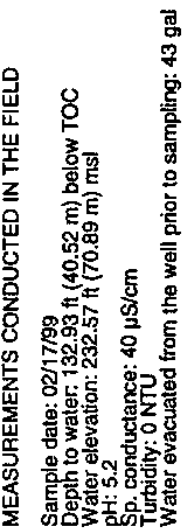

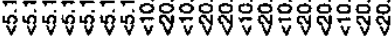

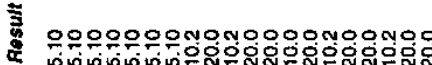

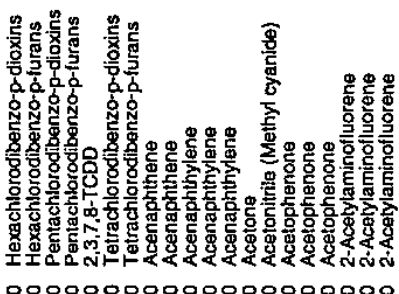

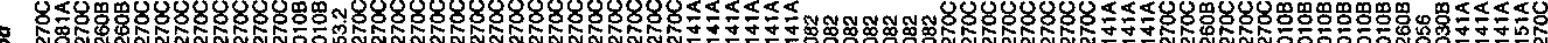

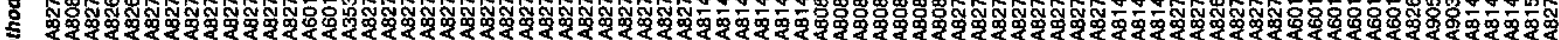

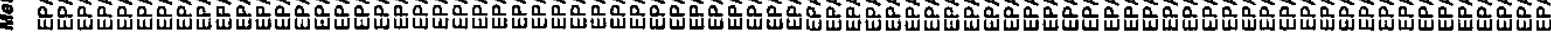

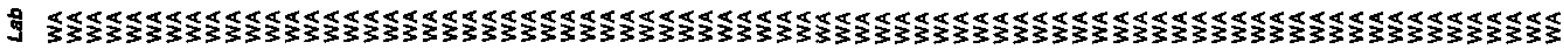

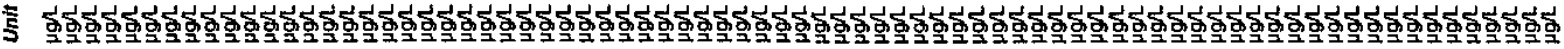

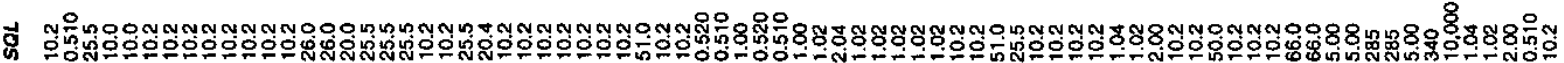
密

o

$\circ$

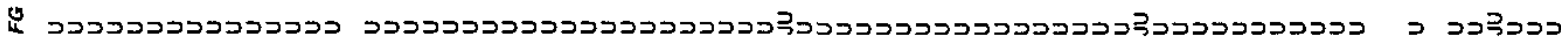

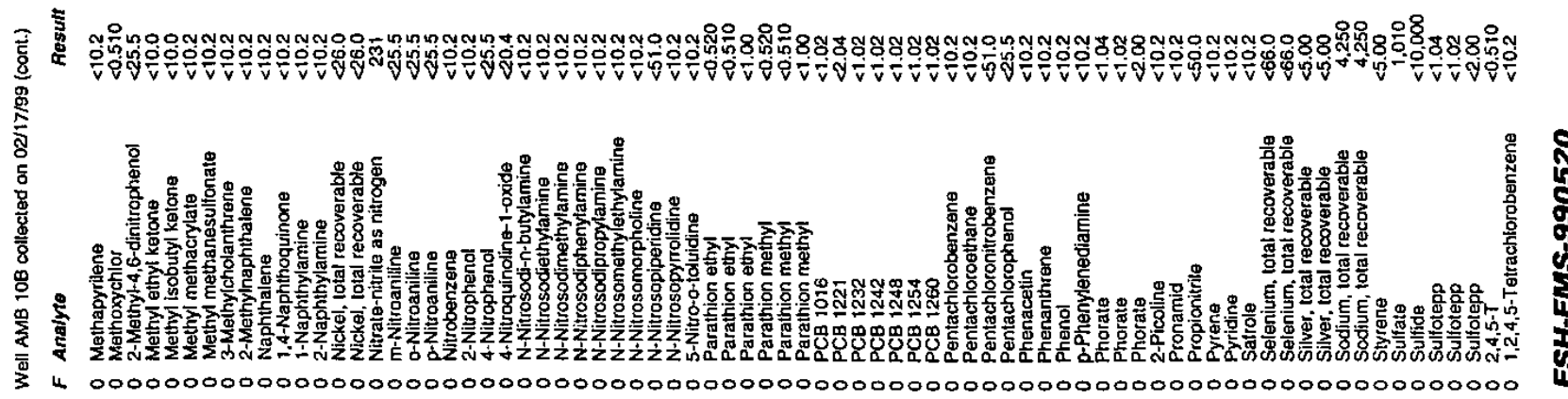


害

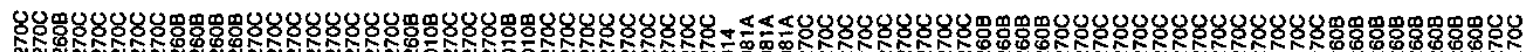
罗 W. W.

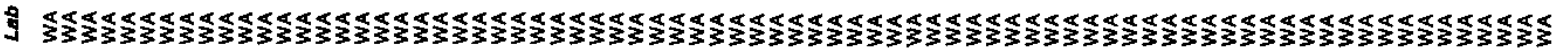

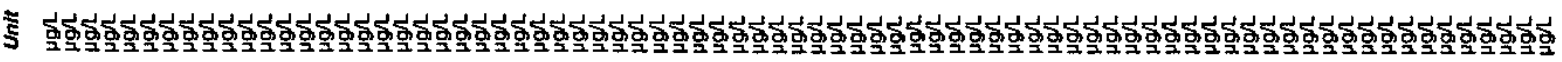

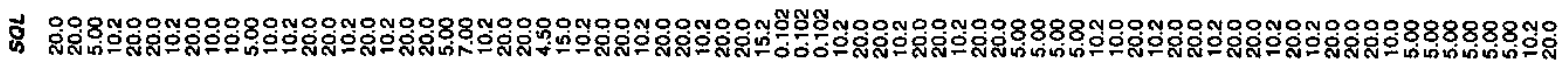
蛋

2

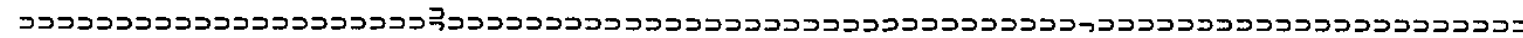

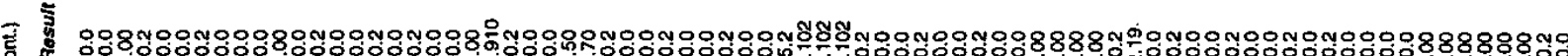

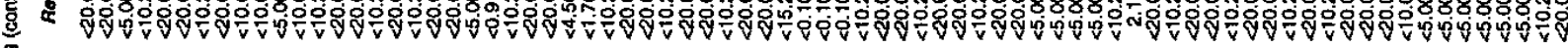

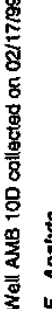

4 L 00000000000000000000000000000000000000000000000000000000000000000000000000000

है:

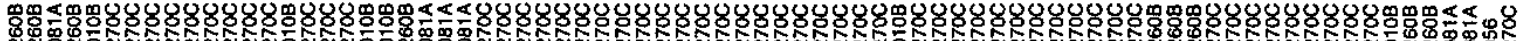

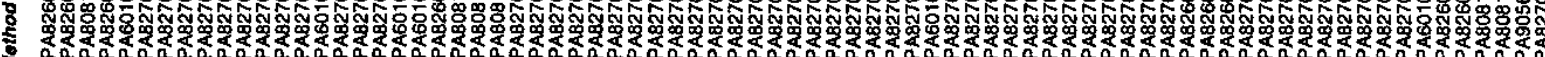

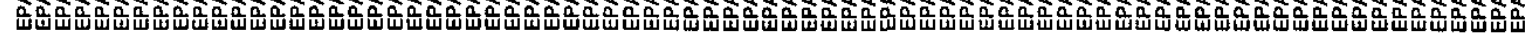

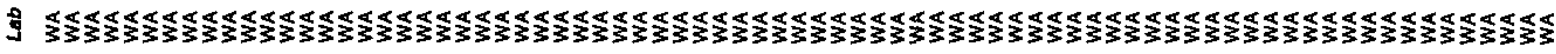

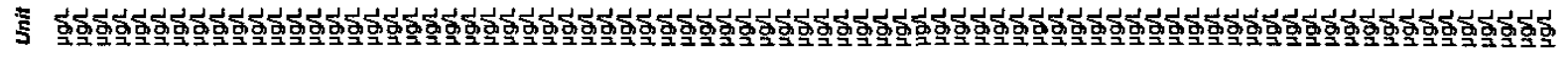

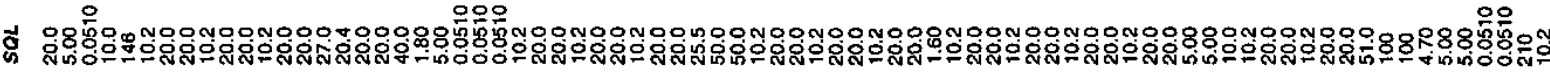
密

is $>$

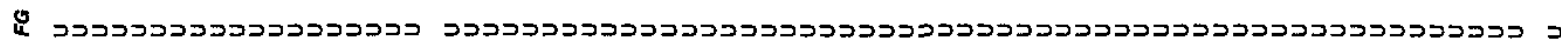

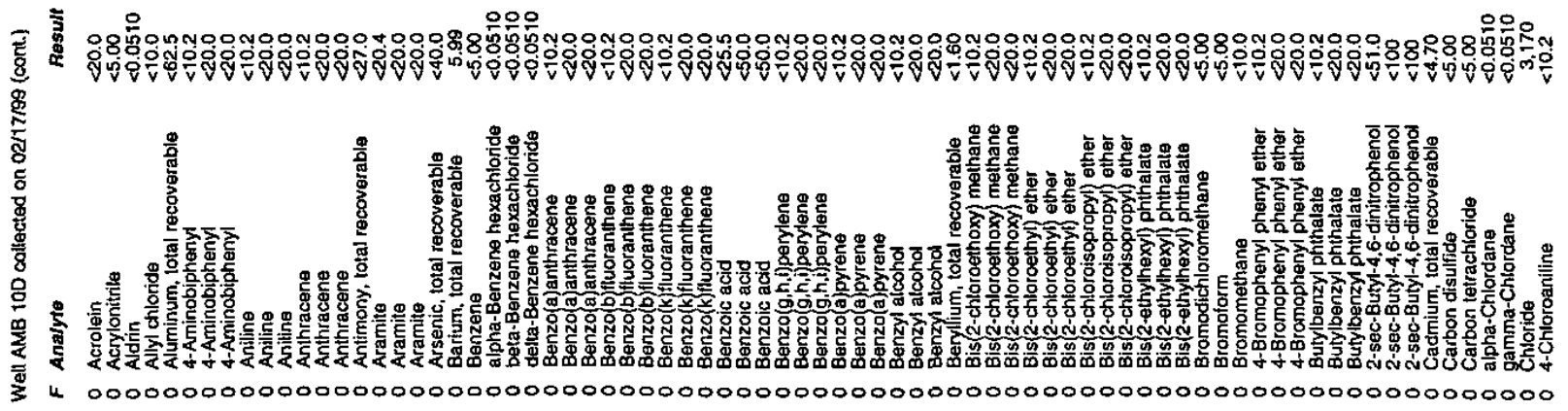


- 808 × 荡

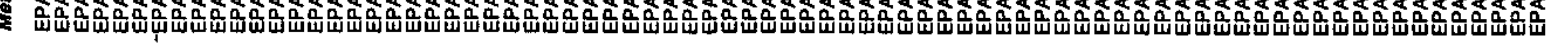

す

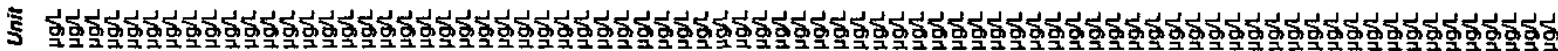

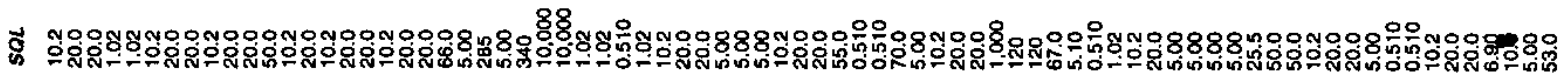
离

is 0

$-$

o

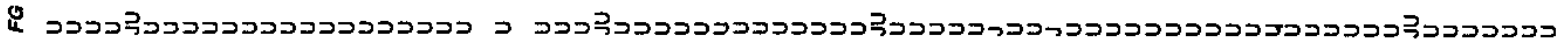

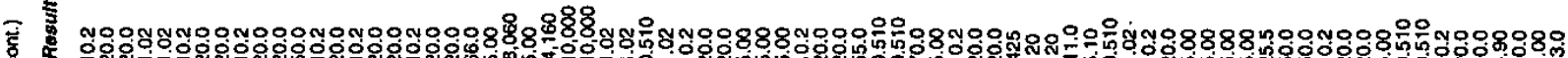

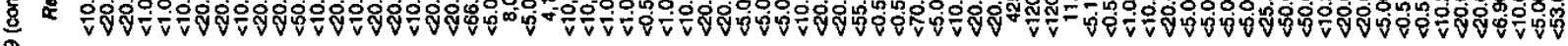

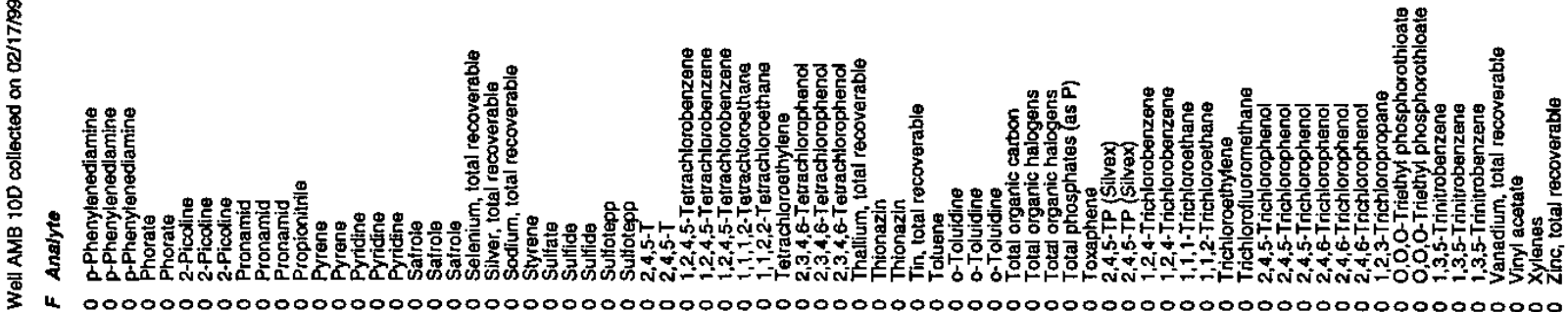

u 00000000000000000000000000000000000000000000000000000000000000000000000000000

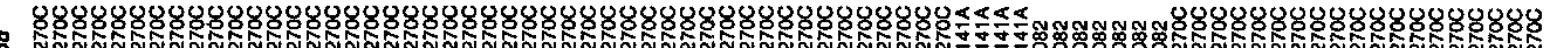
芦

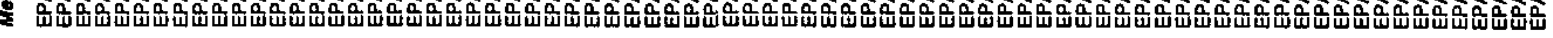

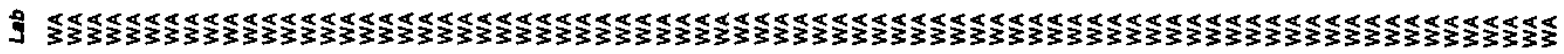

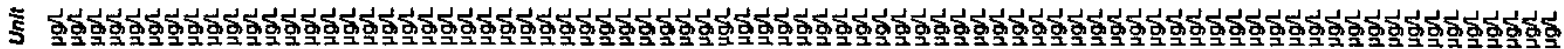

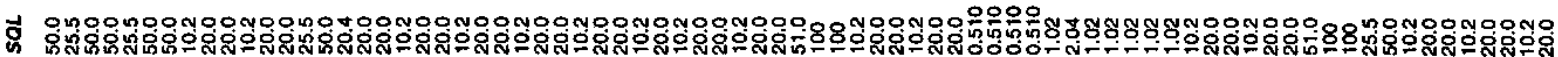
蛋

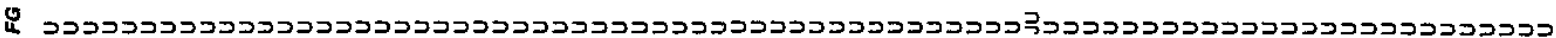

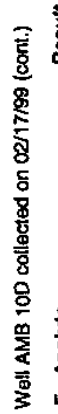

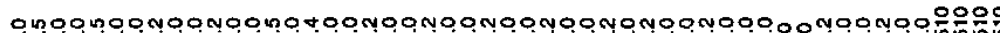

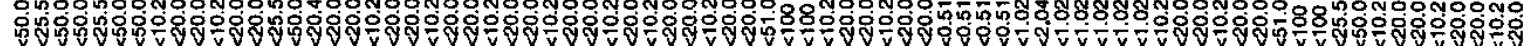

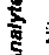

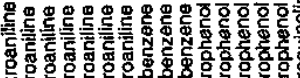

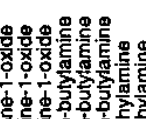

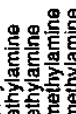

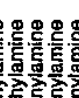
. 


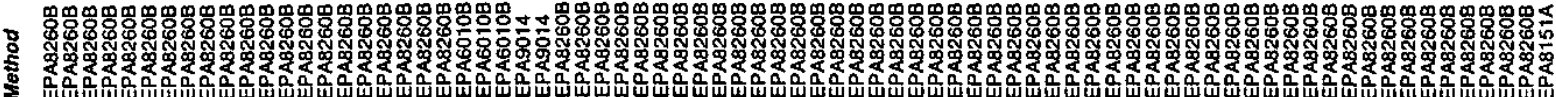

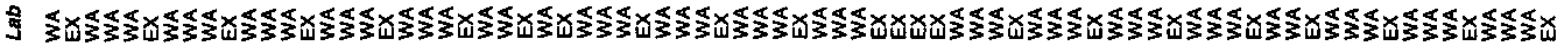

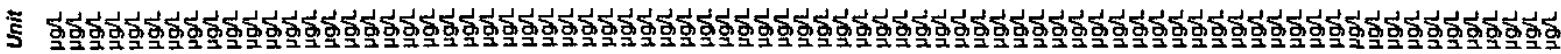

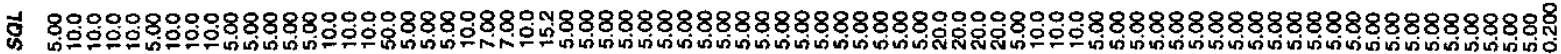

妾

us

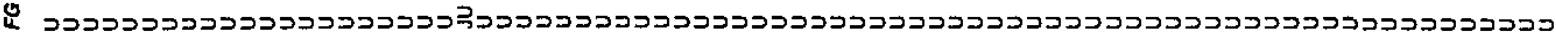

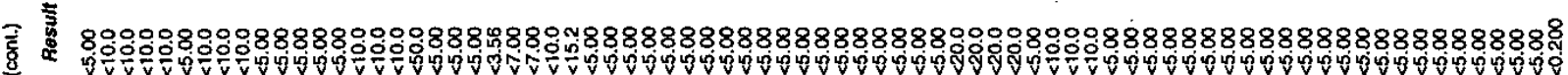

:

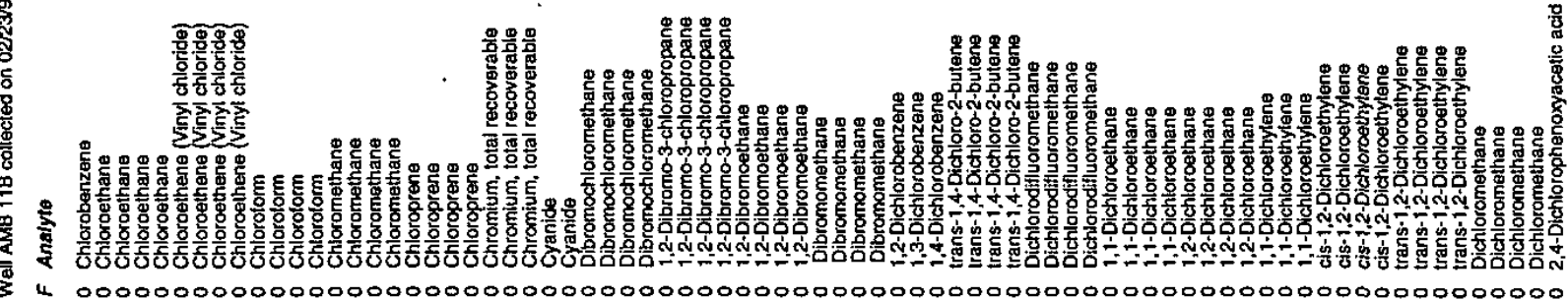

\section{공홍종중}

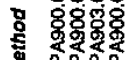

눈믄ㅁㄴ

ఏ $\sum \sum \sum \sum$

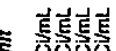

5 ond

우웅웅

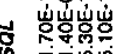

着

is - >>

\&

오웅ㅇ

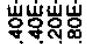

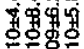

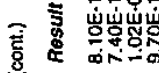

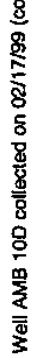

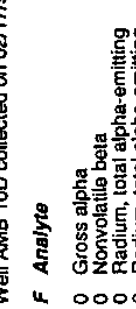

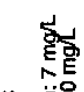

落范

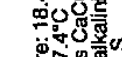

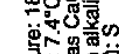

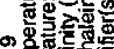

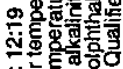

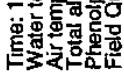

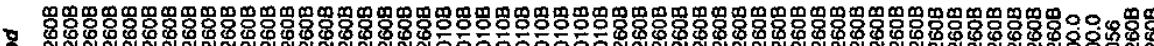
5

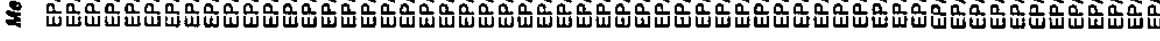

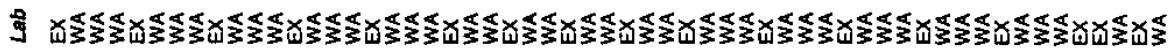

. 혹 허허

If 盖

is $>>$

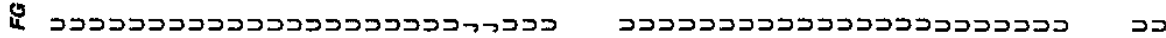
需

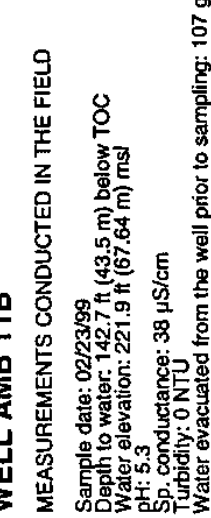

产

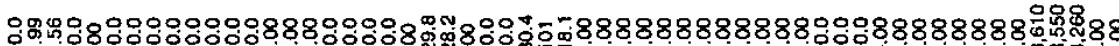
งท

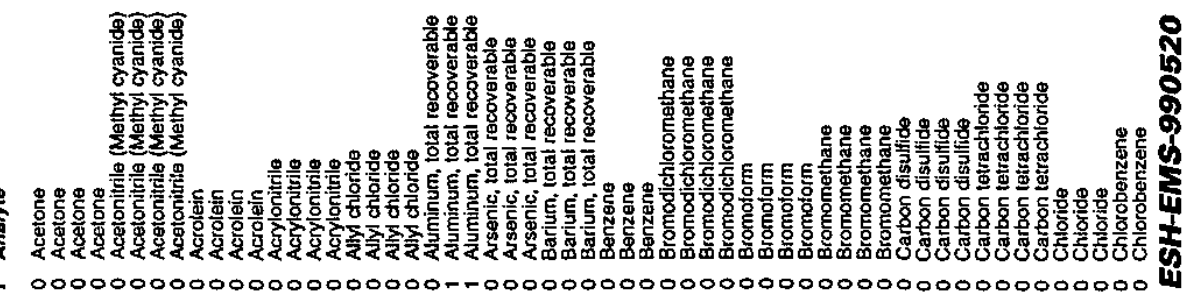




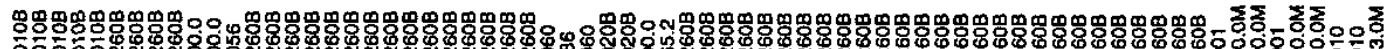

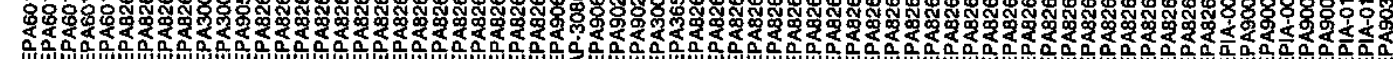

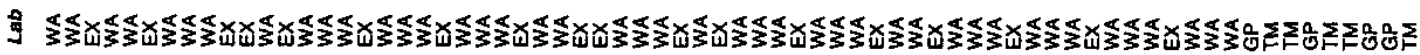

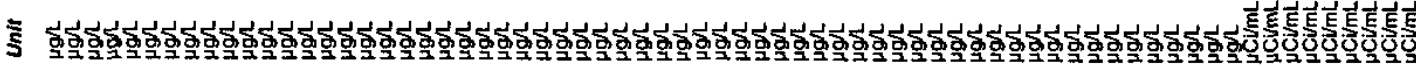

우우으용용ㅇㅇ우요

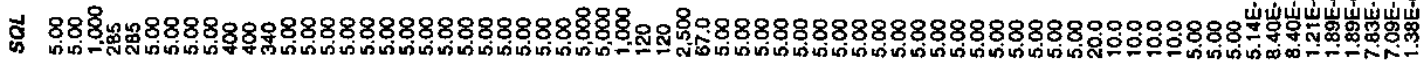

旁

a

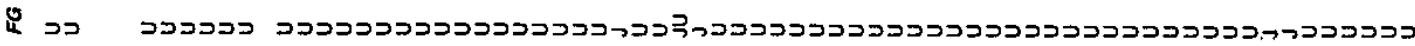
으우유으웅웅ㅇㅇㅇㅇ

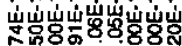

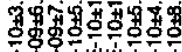

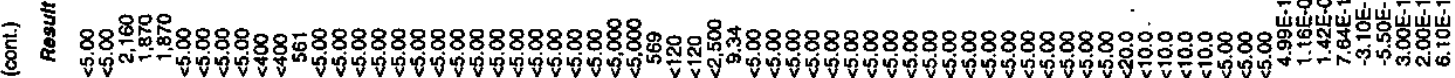

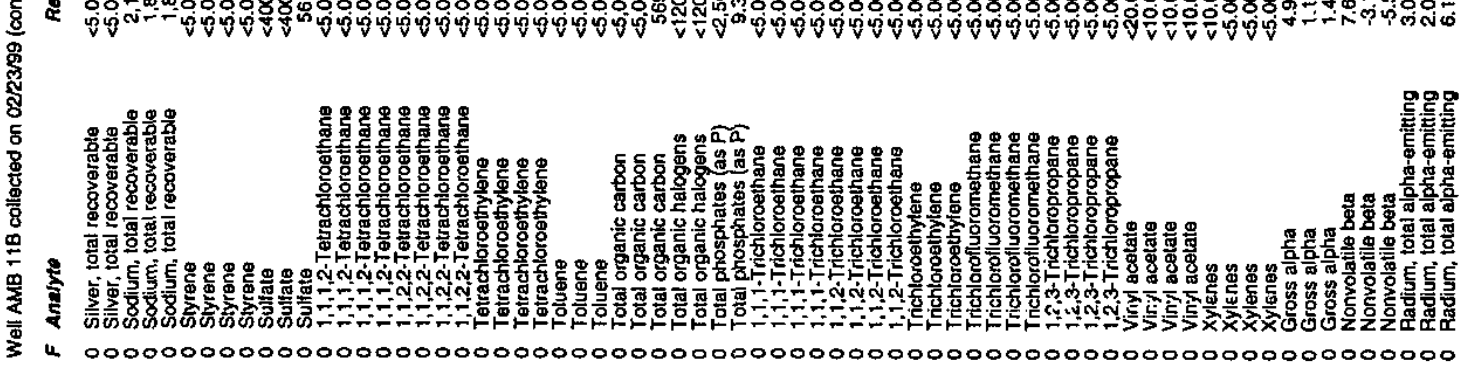

누유. \$

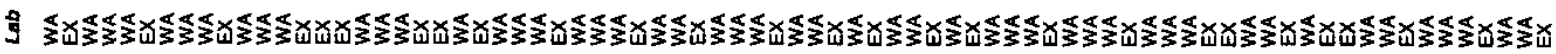

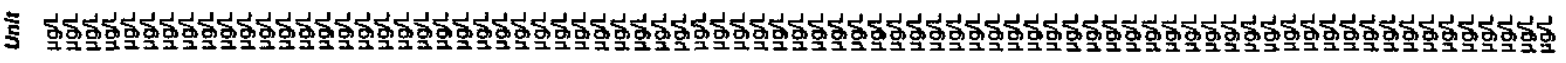

흔 竞

is

\&

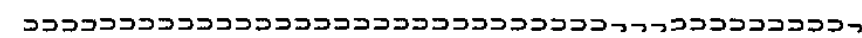

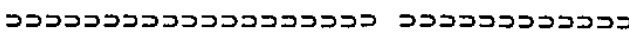

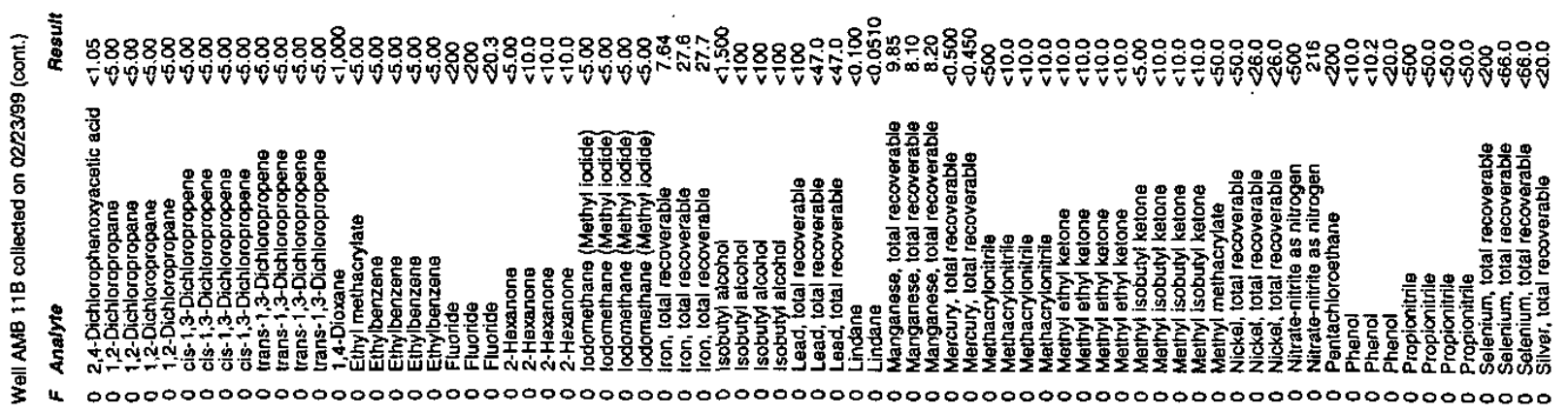




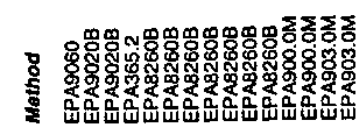

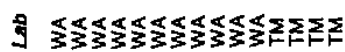

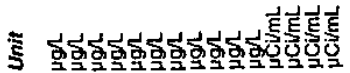
으용으으

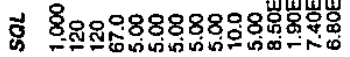
蛋

n - -

政

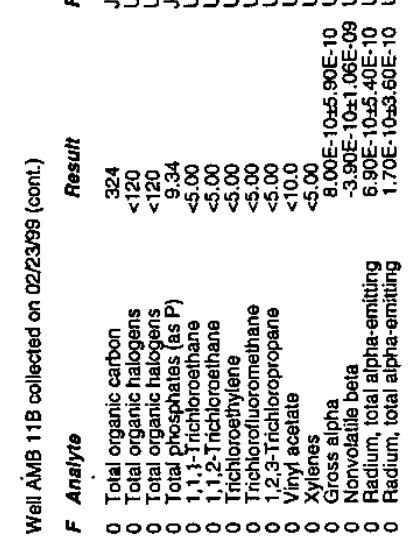

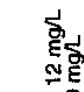

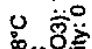

:.0

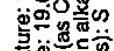

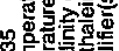

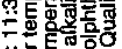

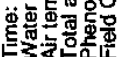

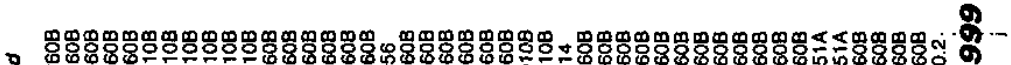
落

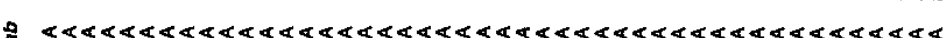

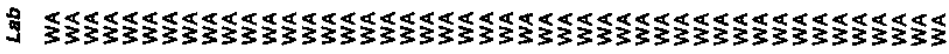

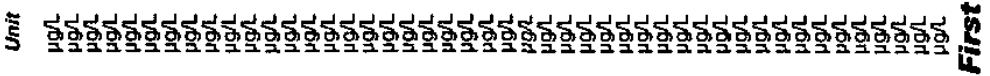

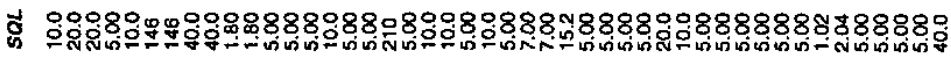

竞

$\circ$

n

ㄴำ

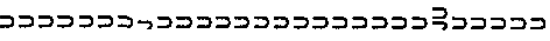

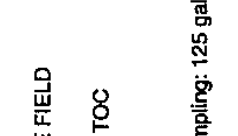

害

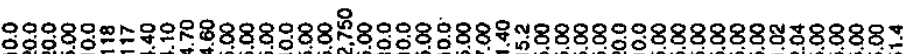

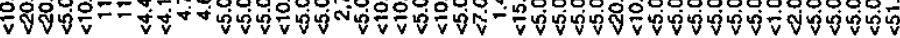

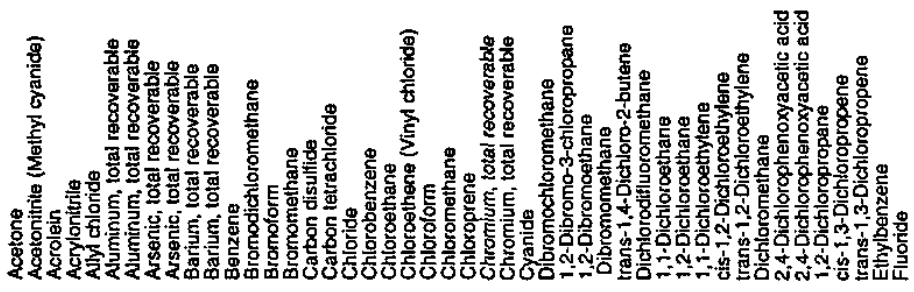

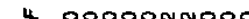

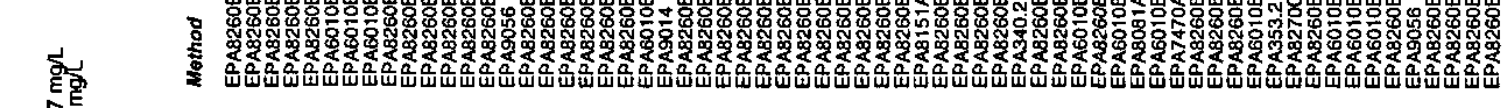

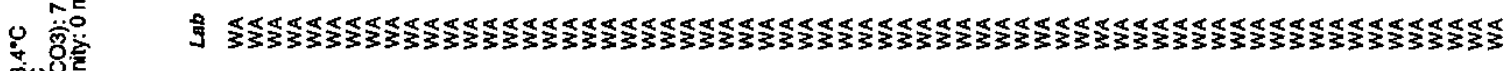

我记

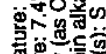

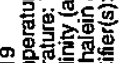

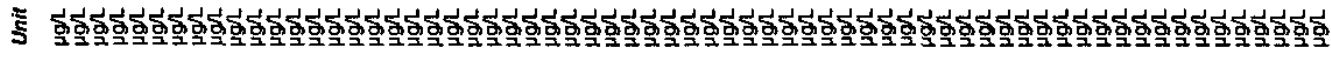

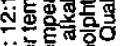

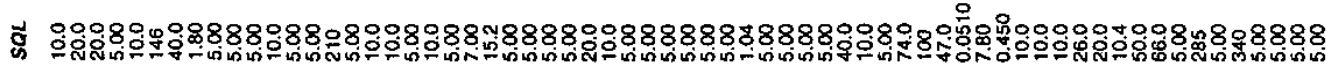

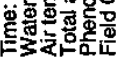
㮍

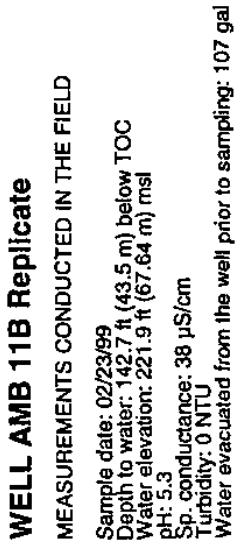

0

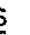

$\infty>$

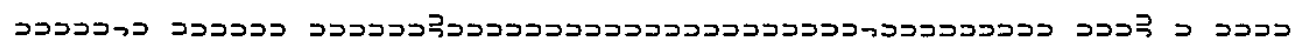

$$
\text { 产 }
$$

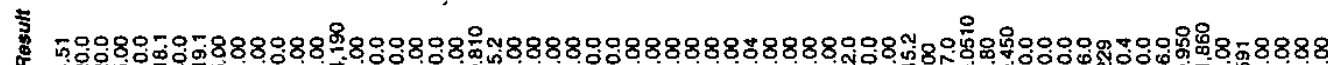
*

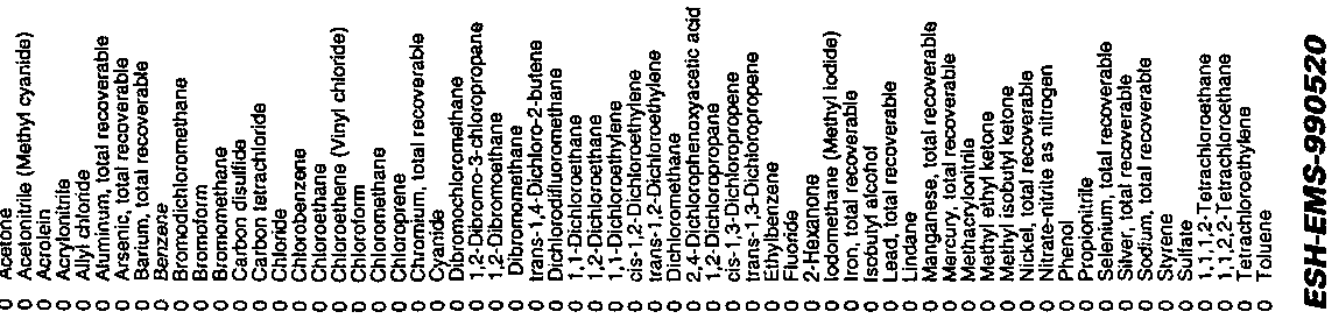


\&

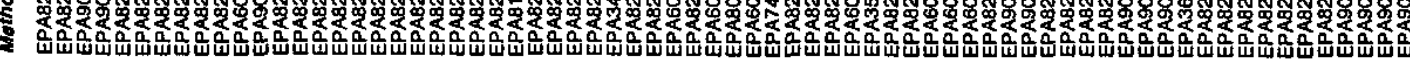

ป

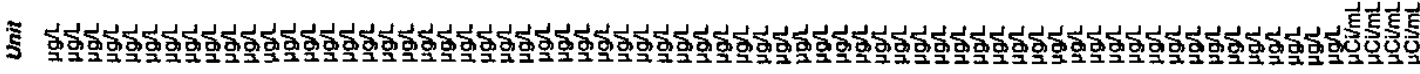

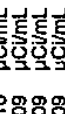

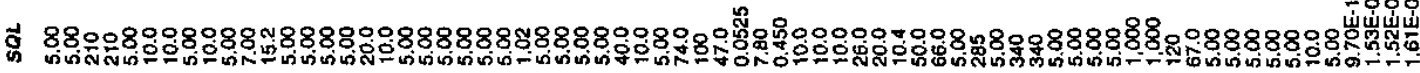

咅

$\rightarrow$

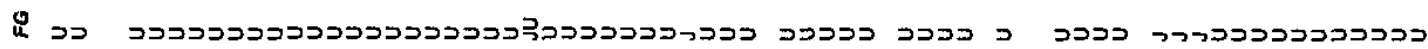
우웅으

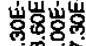

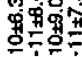

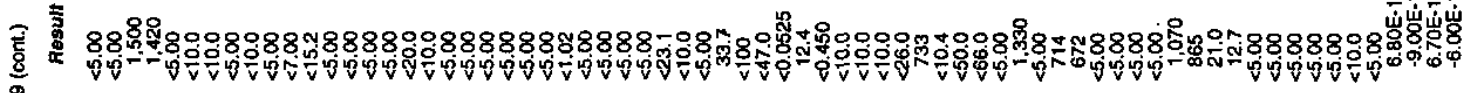

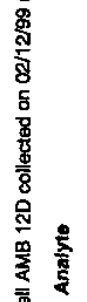

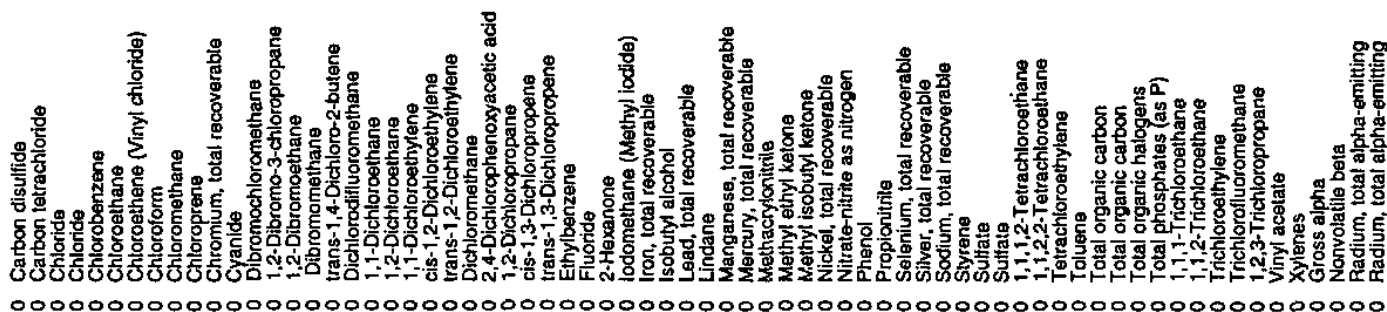

40000000000000000000000000000000000000000000000000000000000000000000000

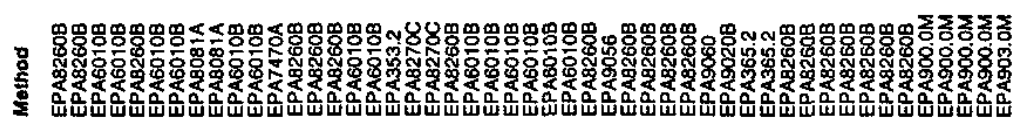

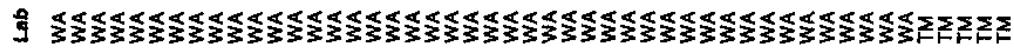

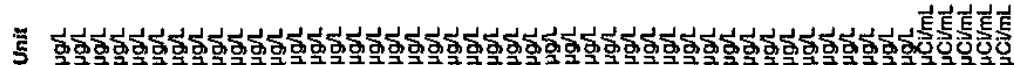

$8 \% 888$

t. 悉

$\infty$

i

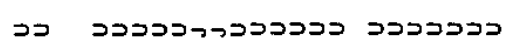

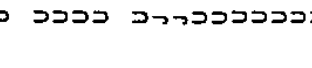

웅으응으으

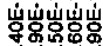

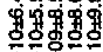

言

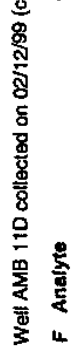

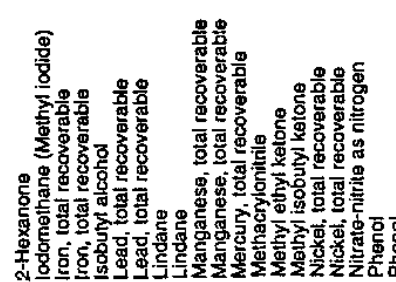

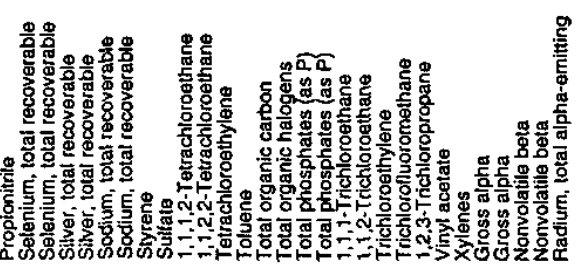

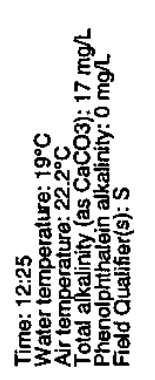

:

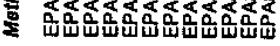

మ

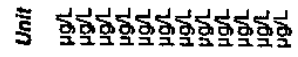

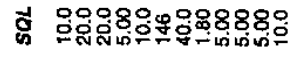

诏

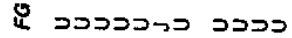

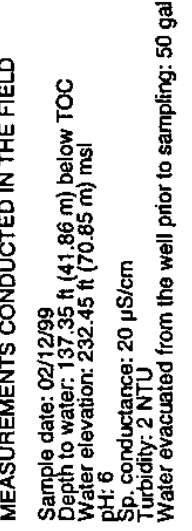

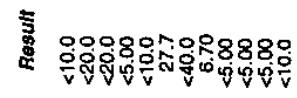

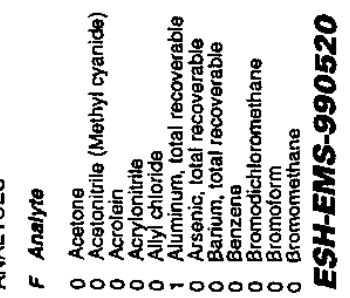




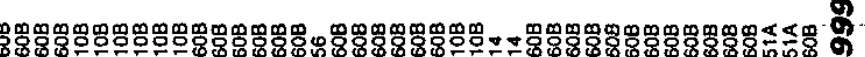

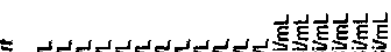

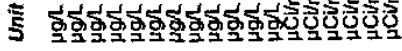

密

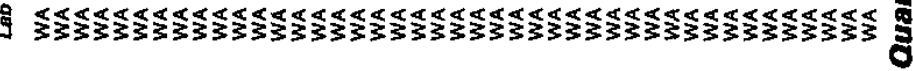

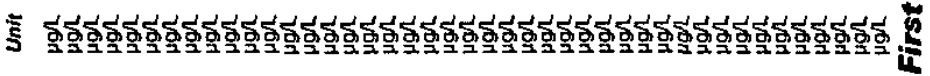

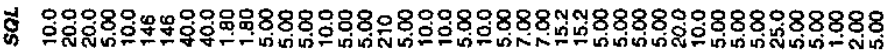
紊

$\infty>$

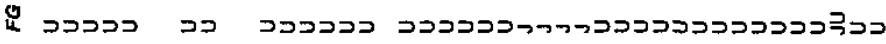

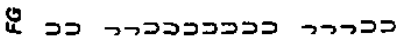

으용ㅇㅇ은

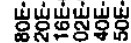

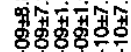

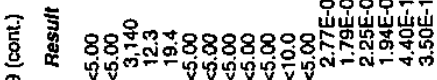

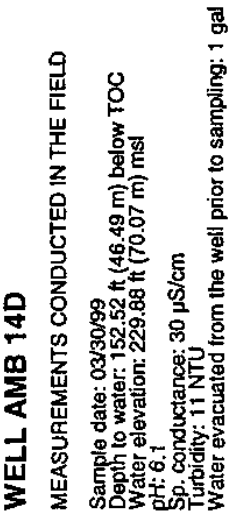

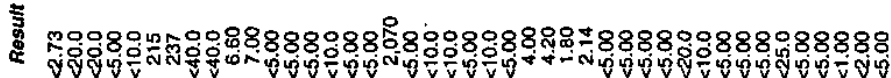
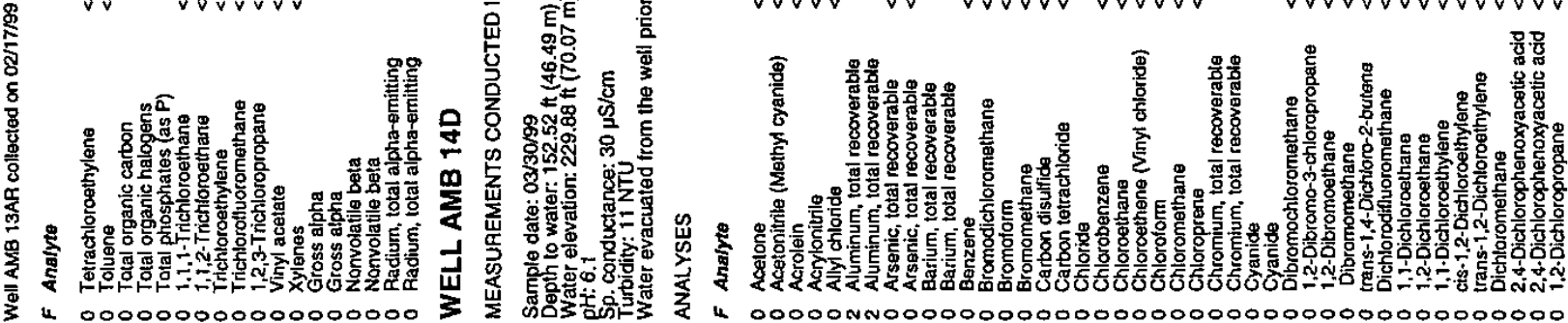

귬뮴

离

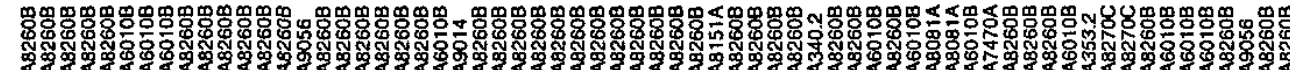

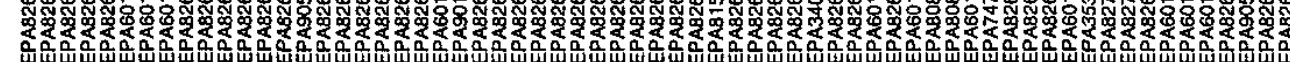

0

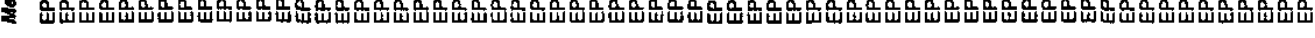

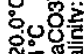

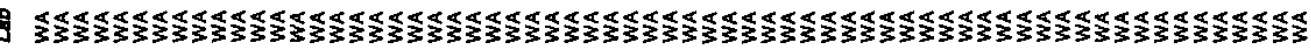

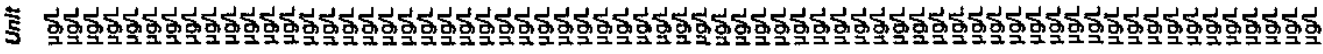

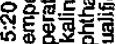

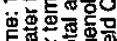

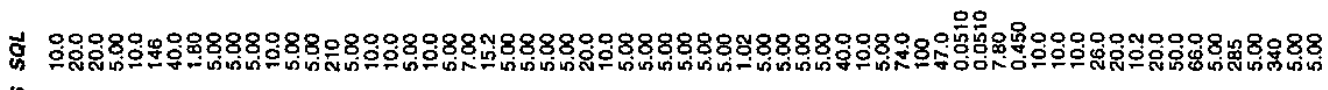
盖

os

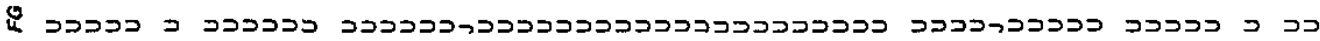

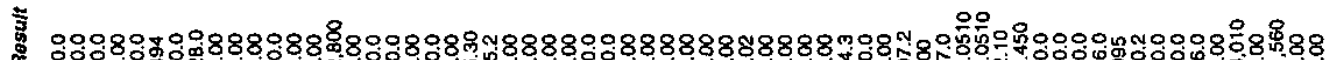

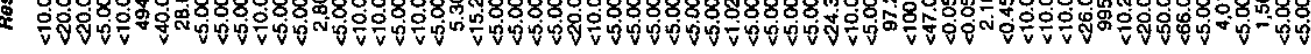

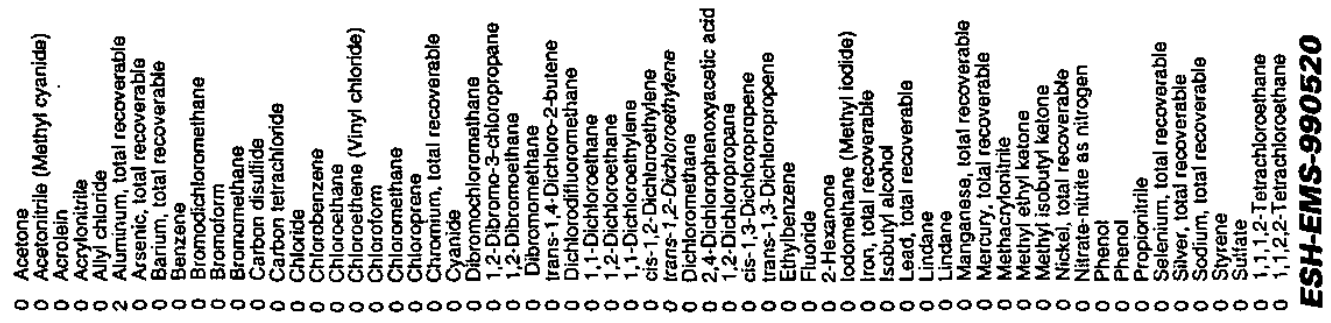



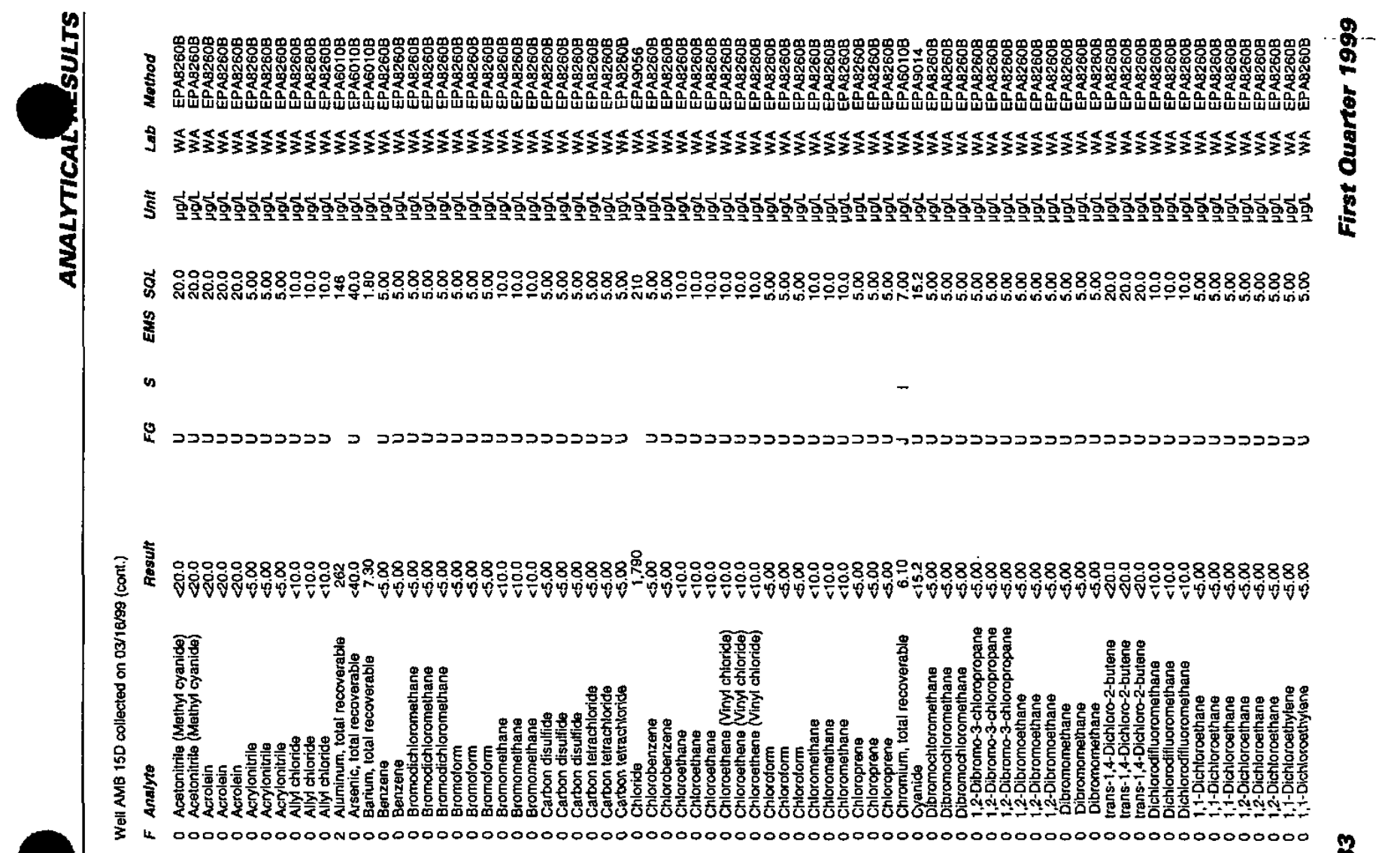

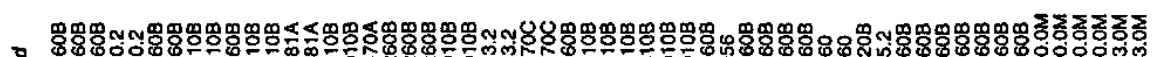 \\ 范

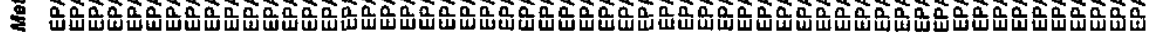

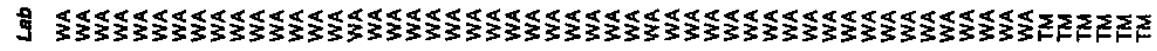

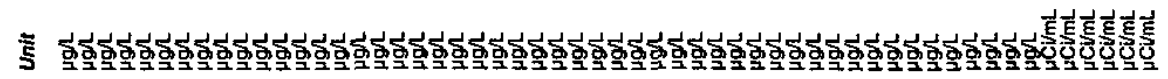

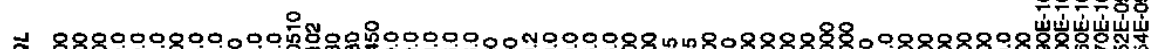

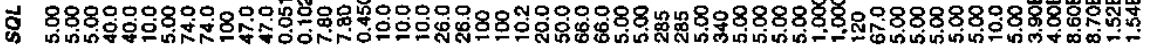
盗
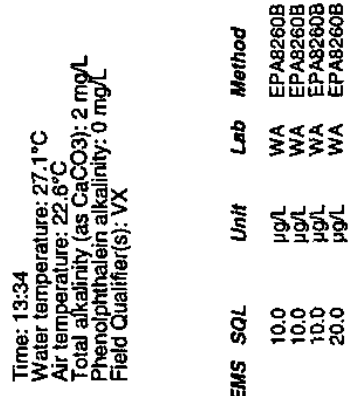

8

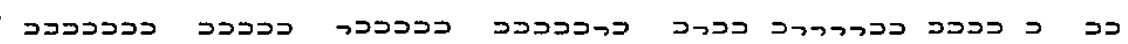

으옹ㅇㅇㅇㅡ

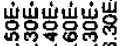

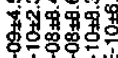

言 888 \%

$$
\text { . }
$$

.
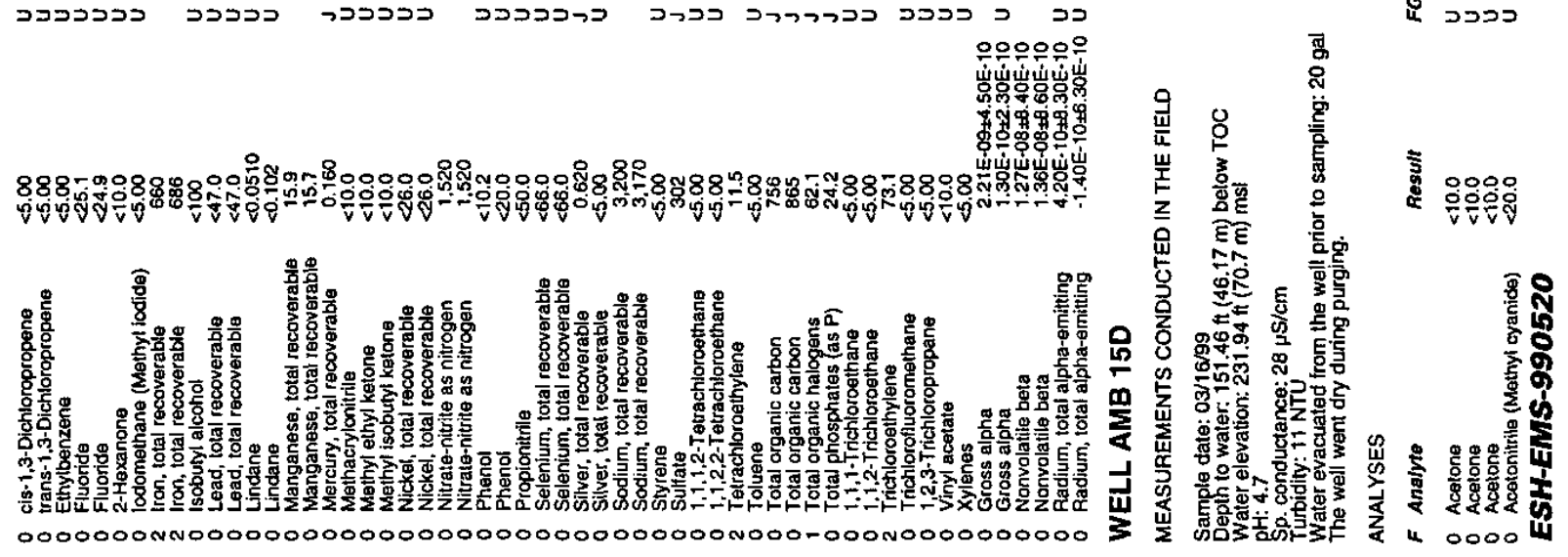


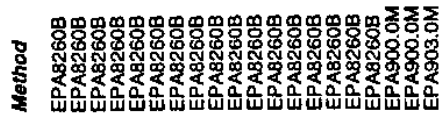

9

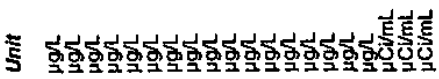

오응

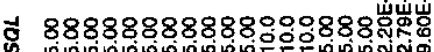

獣

es

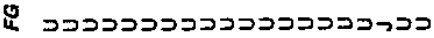

으융ㅇ

碗峞

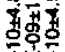

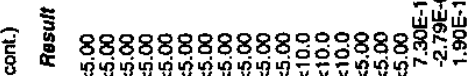
多 赵

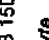

娄

क 400000000000000000000

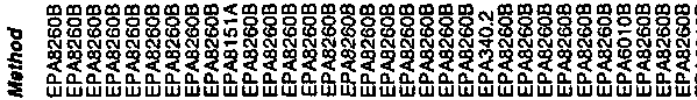

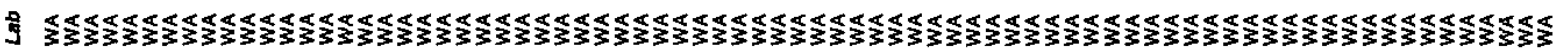

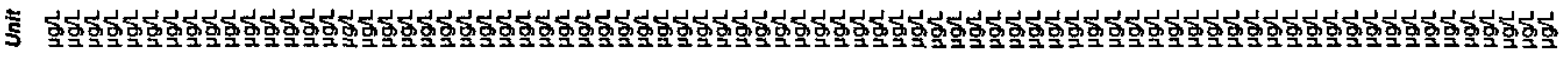

ఫ్ 誉

is
害

9

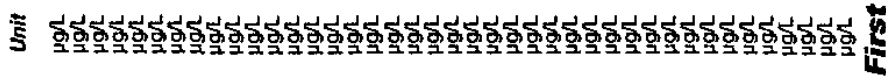

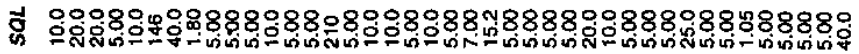
密

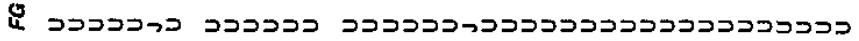

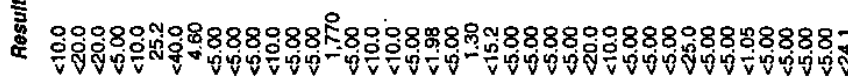

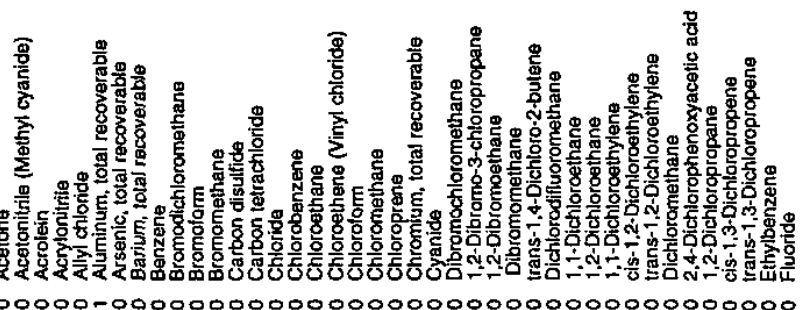

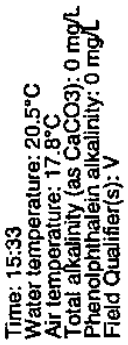

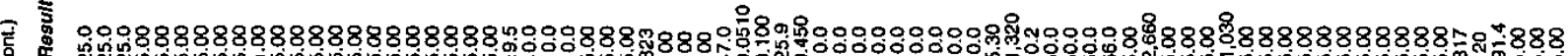

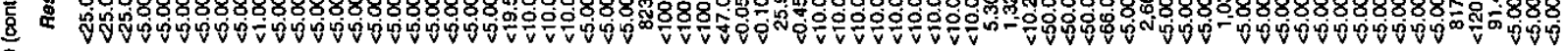

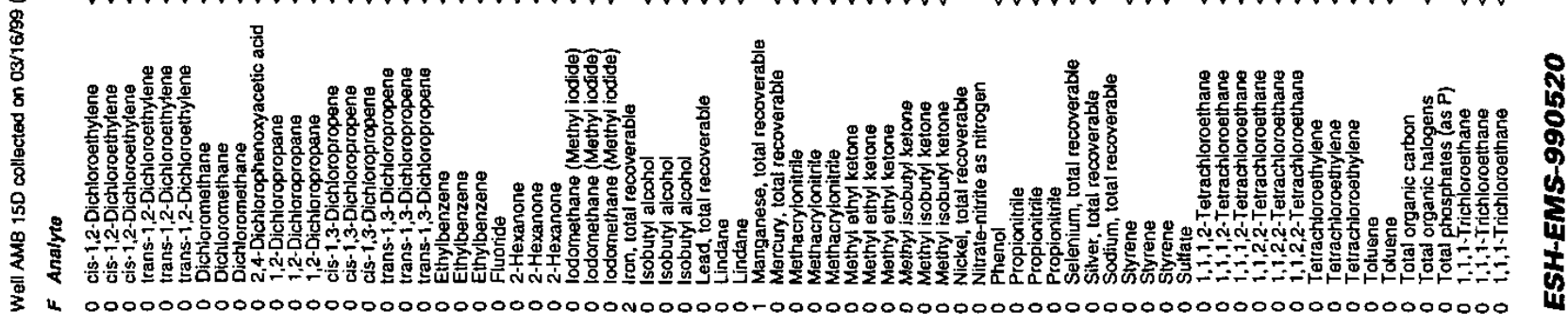



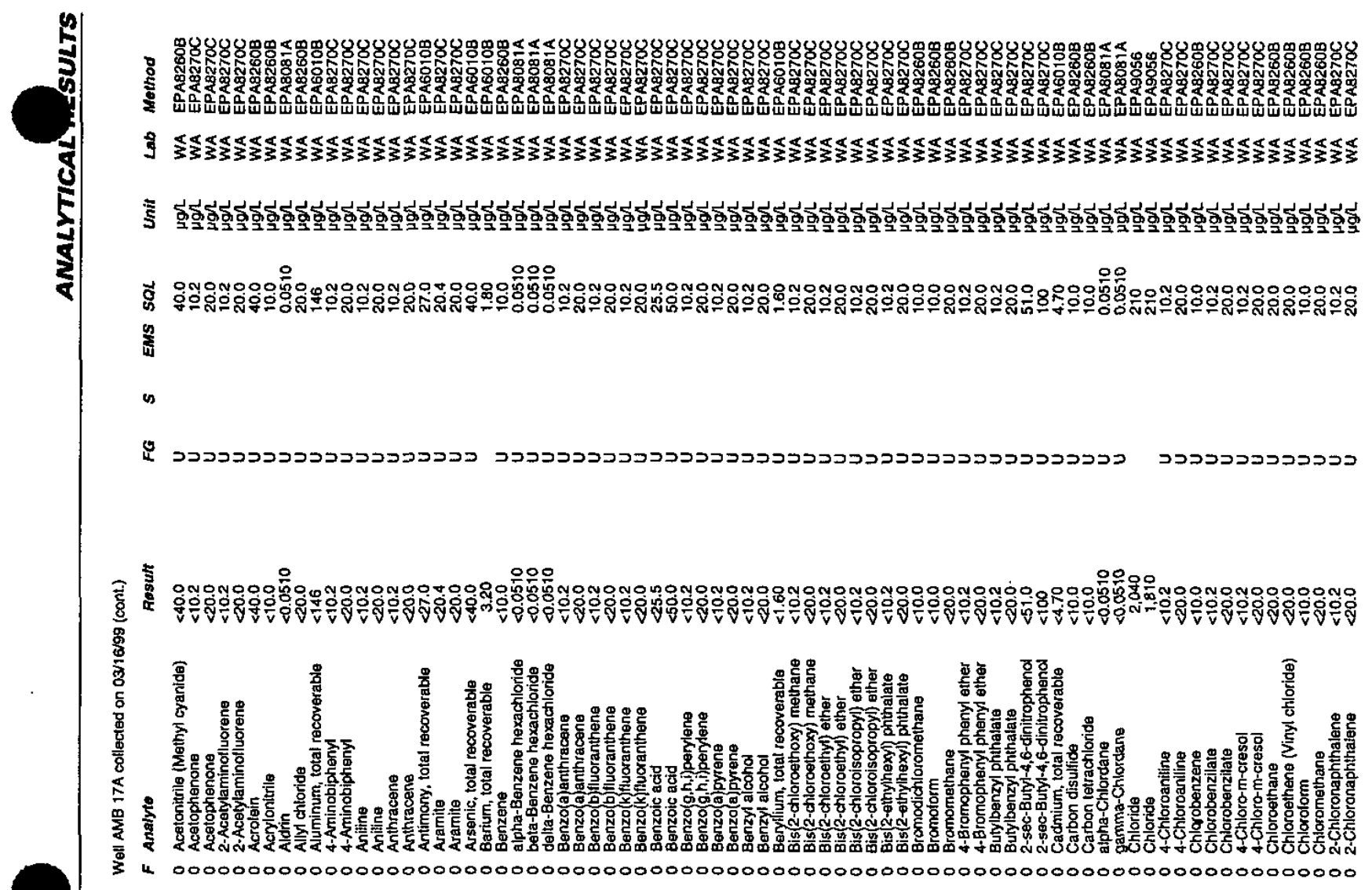

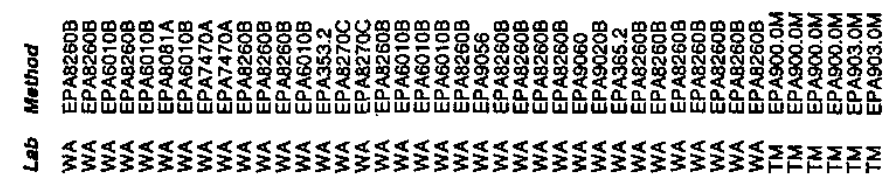

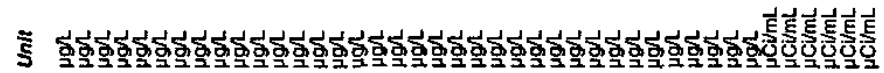

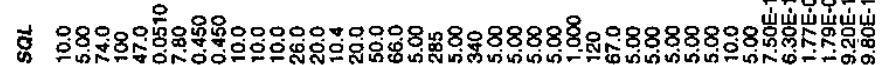
竞

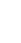

4

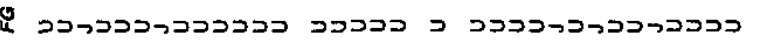

$$
8 \% 8888
$$

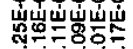

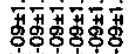

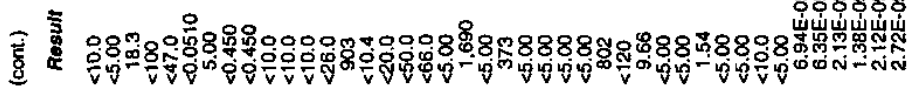

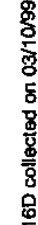

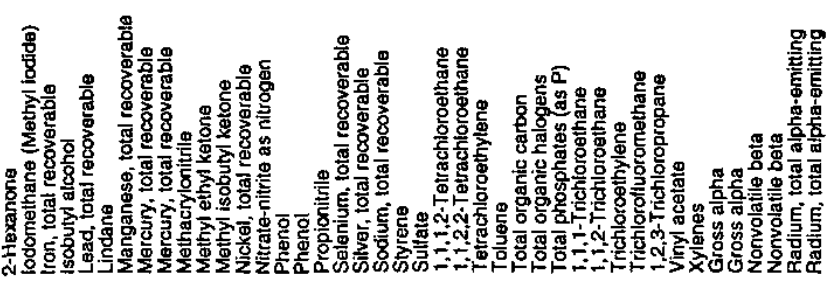

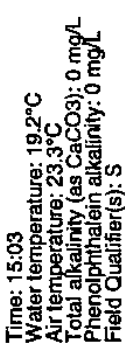

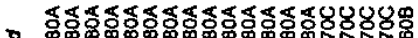

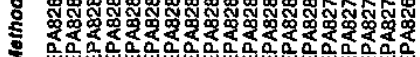

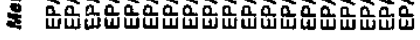

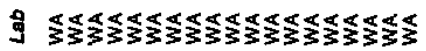

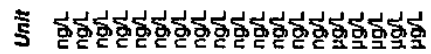

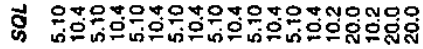
象

is

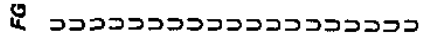

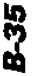




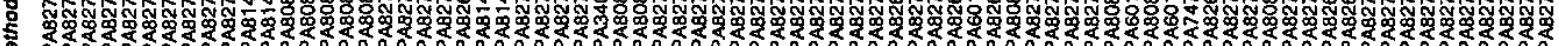

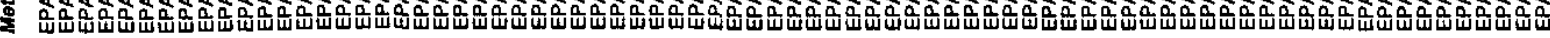

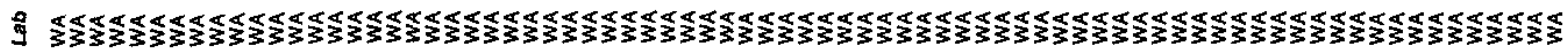

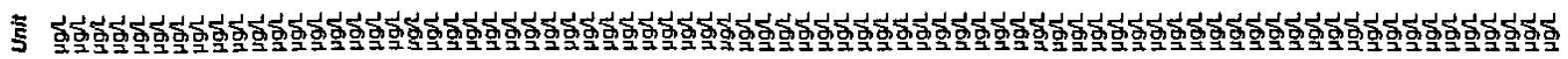

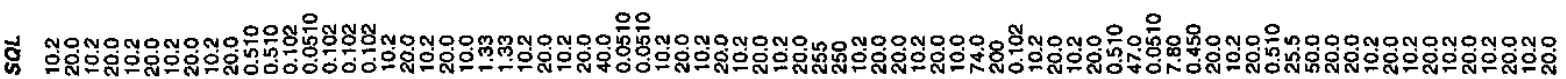
竓

is 00 oo $>$

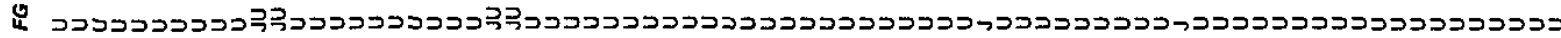

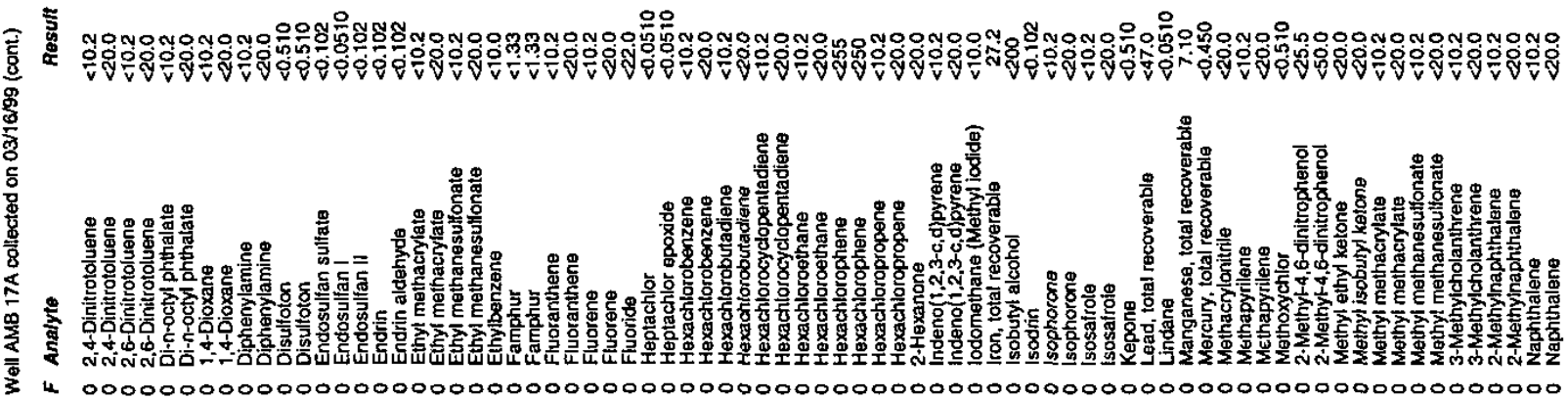

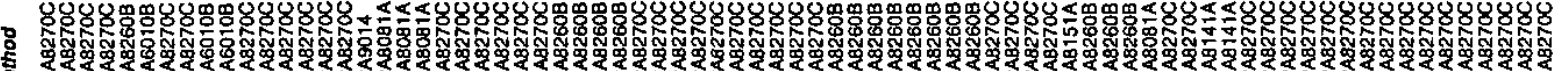

5

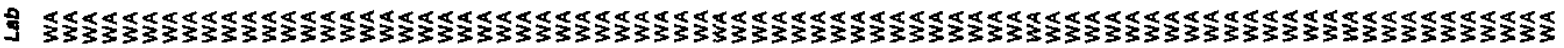

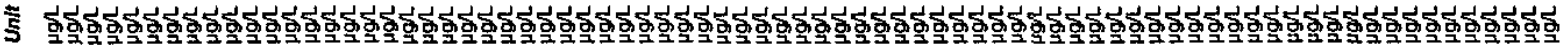

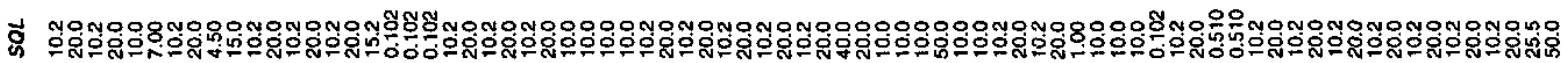
昜

00

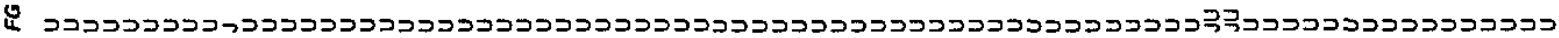

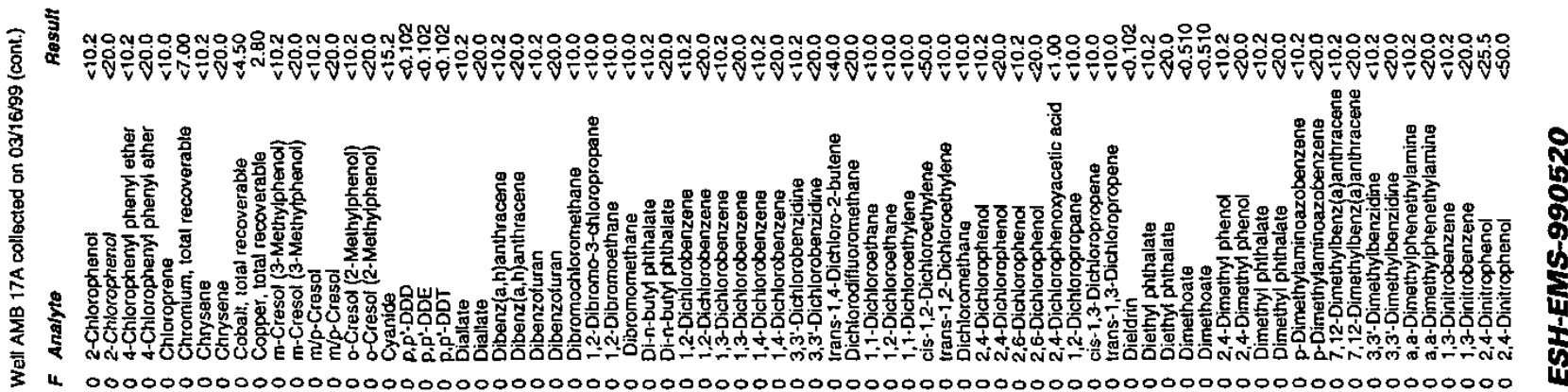

400000000000000000000000000000000000000000000000000000000000000000000000000000 

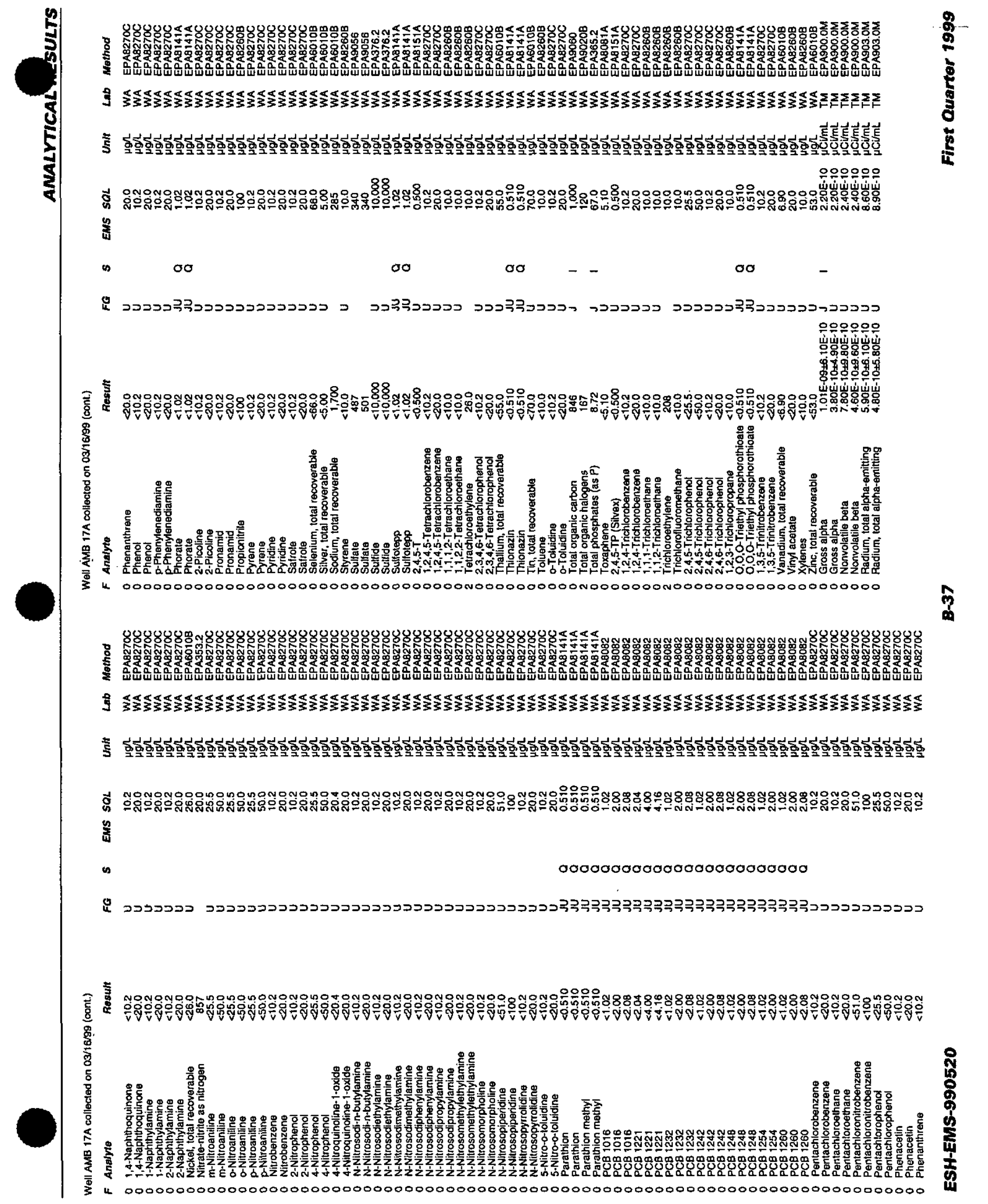


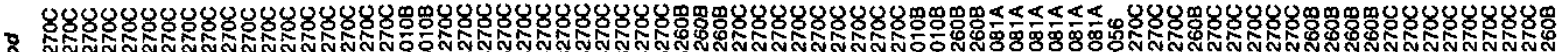

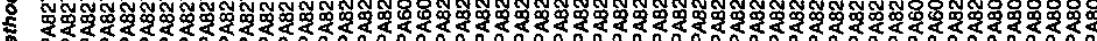

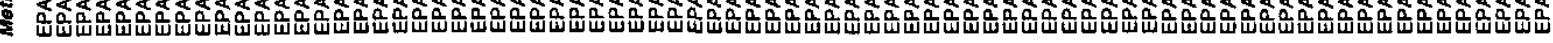

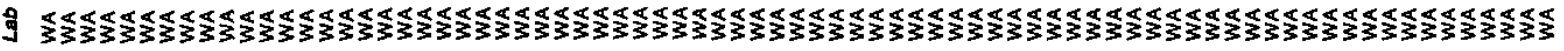

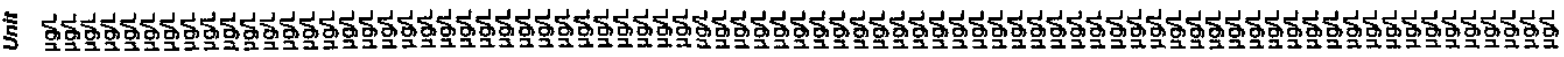

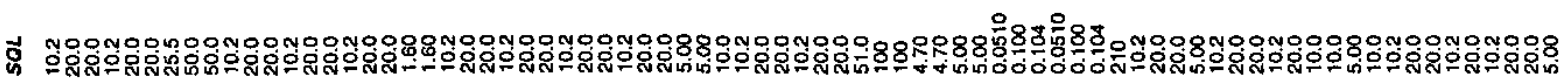
端

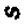

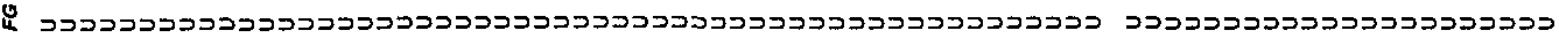

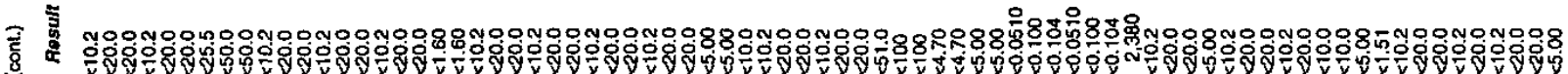

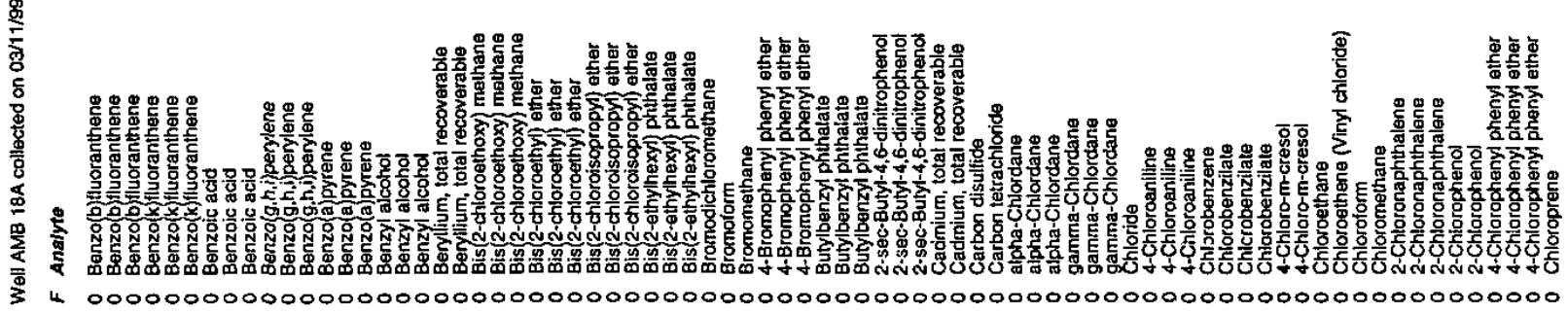

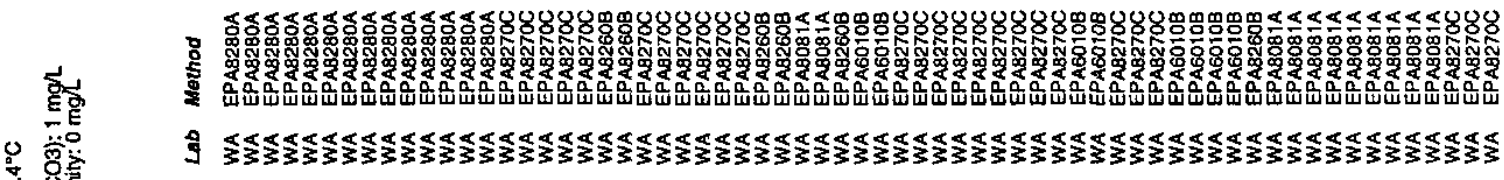

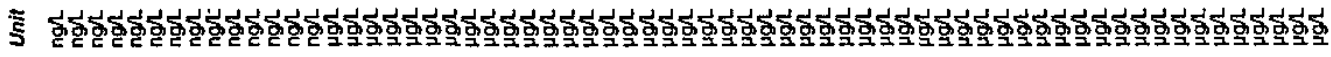

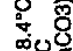

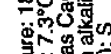

递密

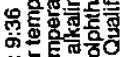

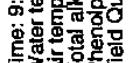

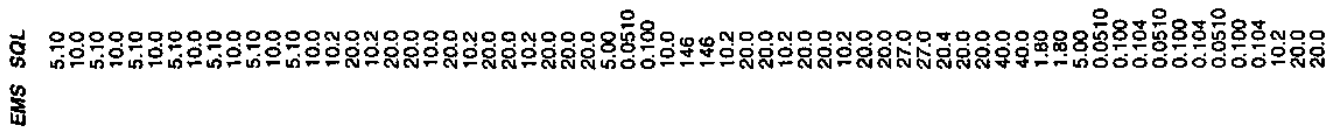

$-$

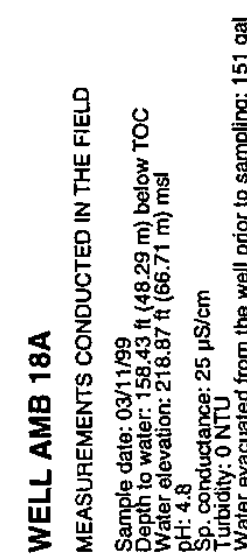
$\overline{\mathrm{s}}$

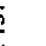

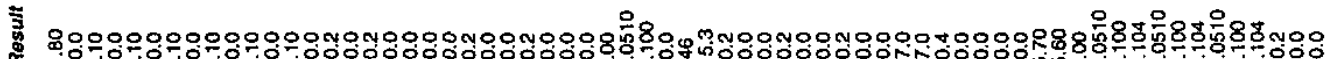

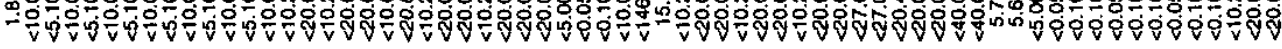

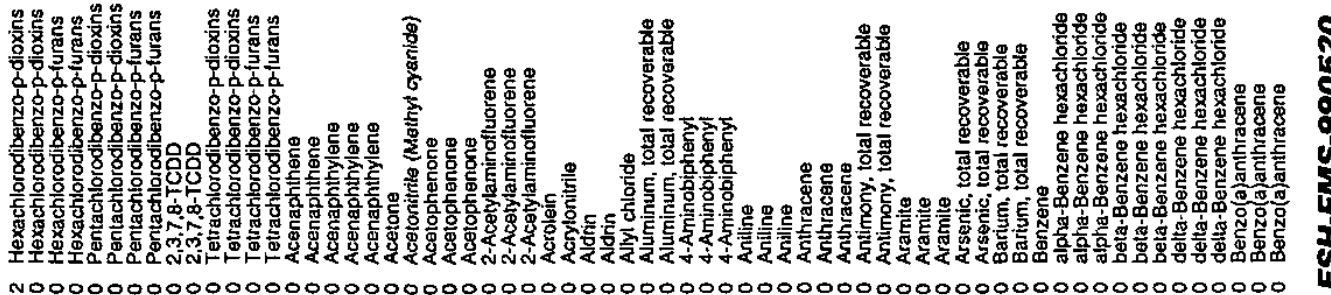



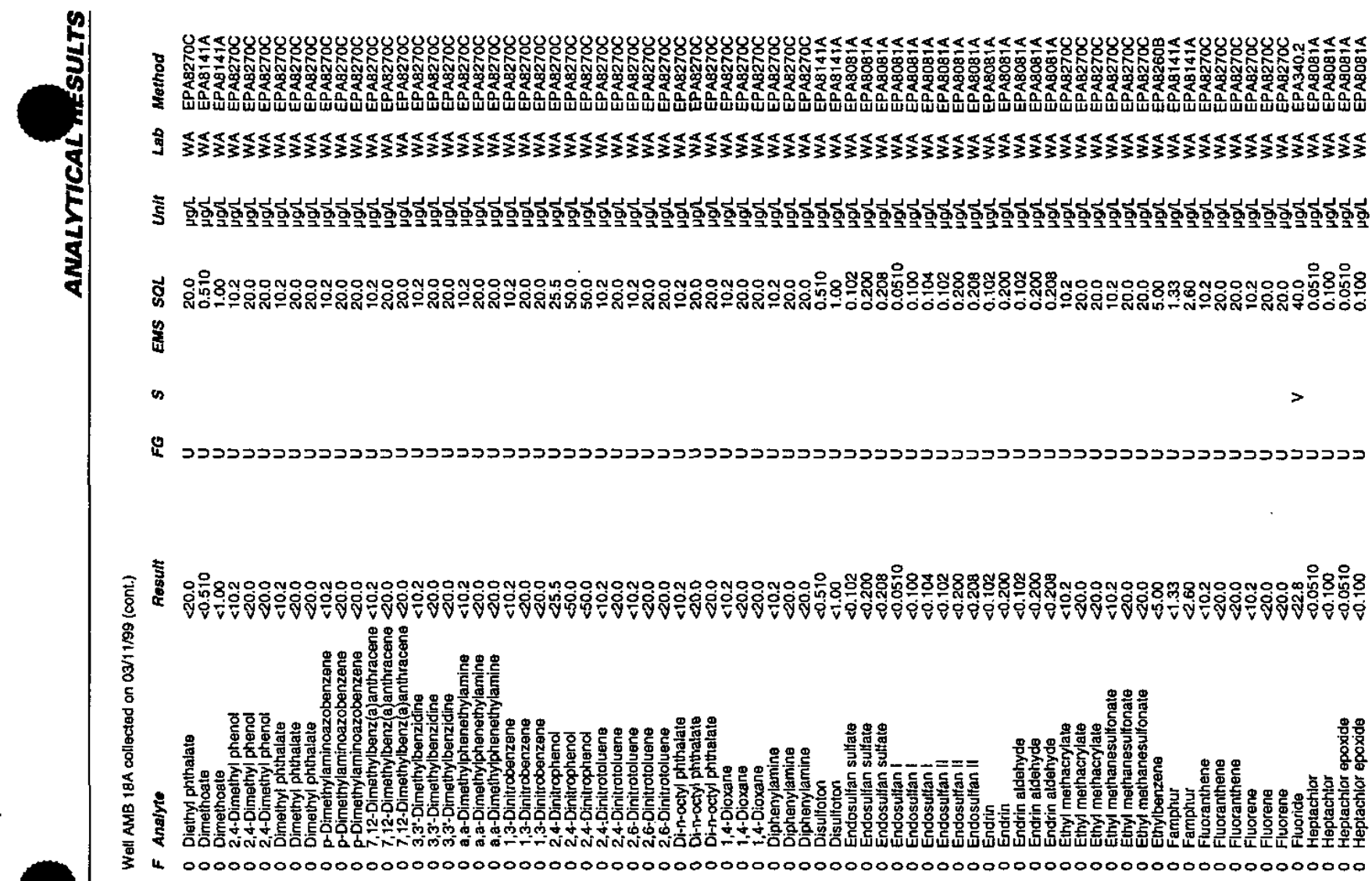

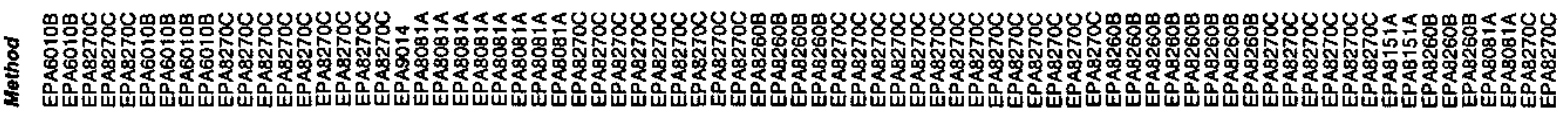

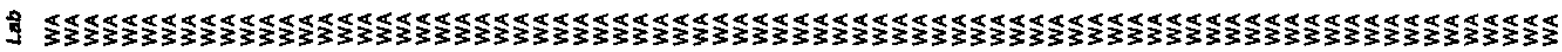

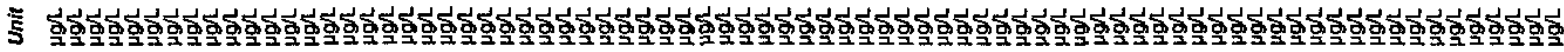

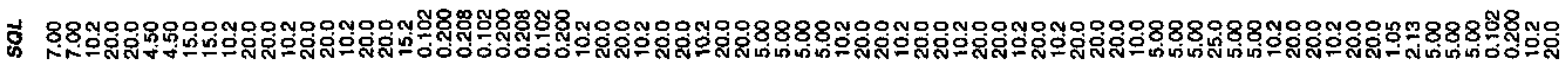
查

is

i

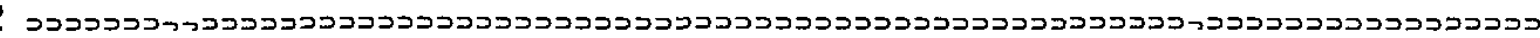

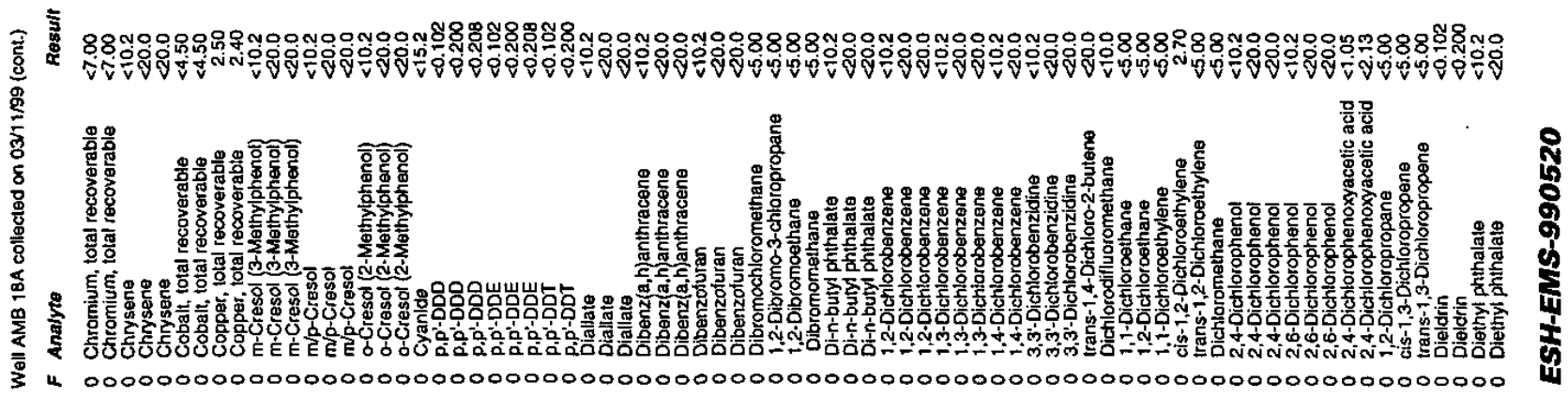




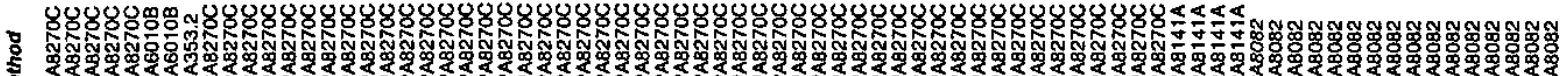

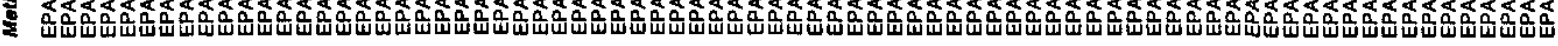

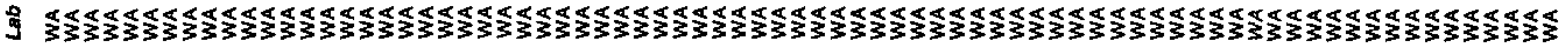

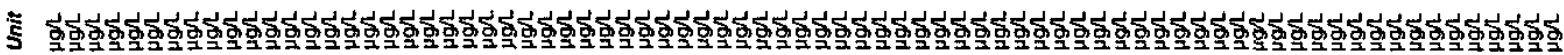

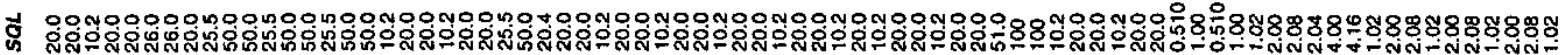
端

0000000000000000

[

害

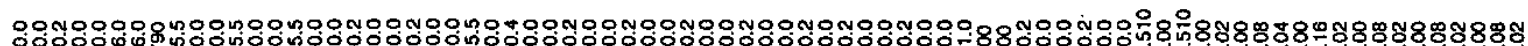
要

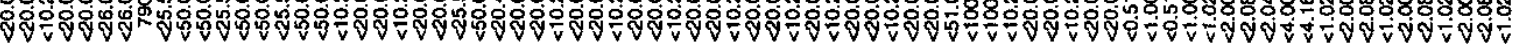

공

ᄃ

\begin{tabular}{l} 
要 \\
总 \\
\hline
\end{tabular}

S in 00000000000000000000000000000000000000000000000000000000000000000000000000000

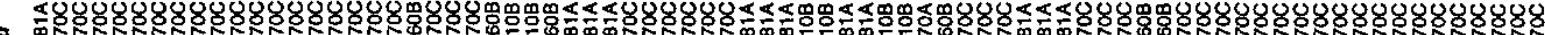

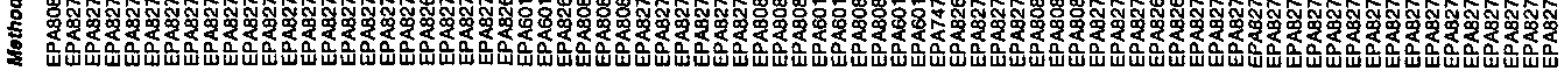

⿹

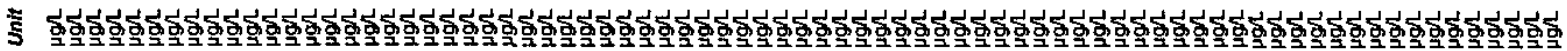

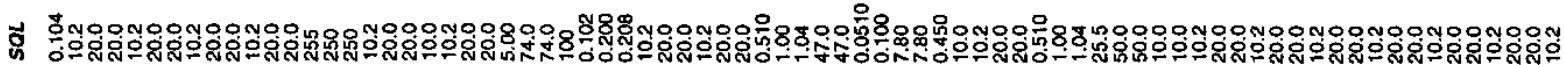
密

$\omega$

s.

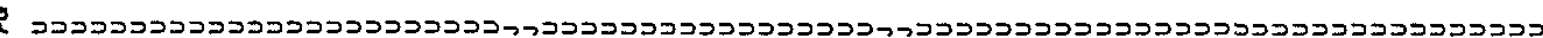

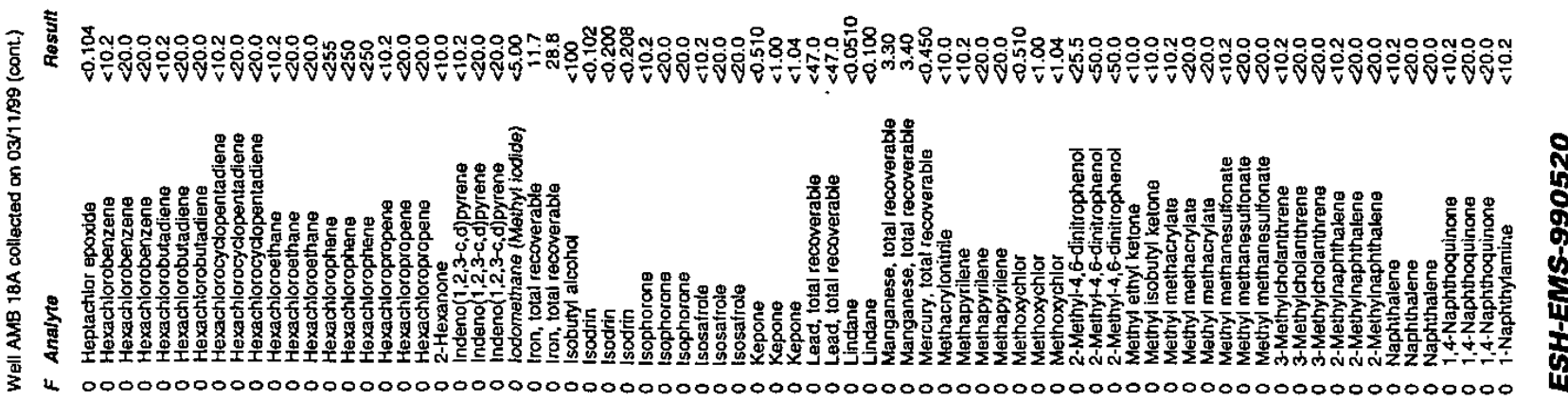



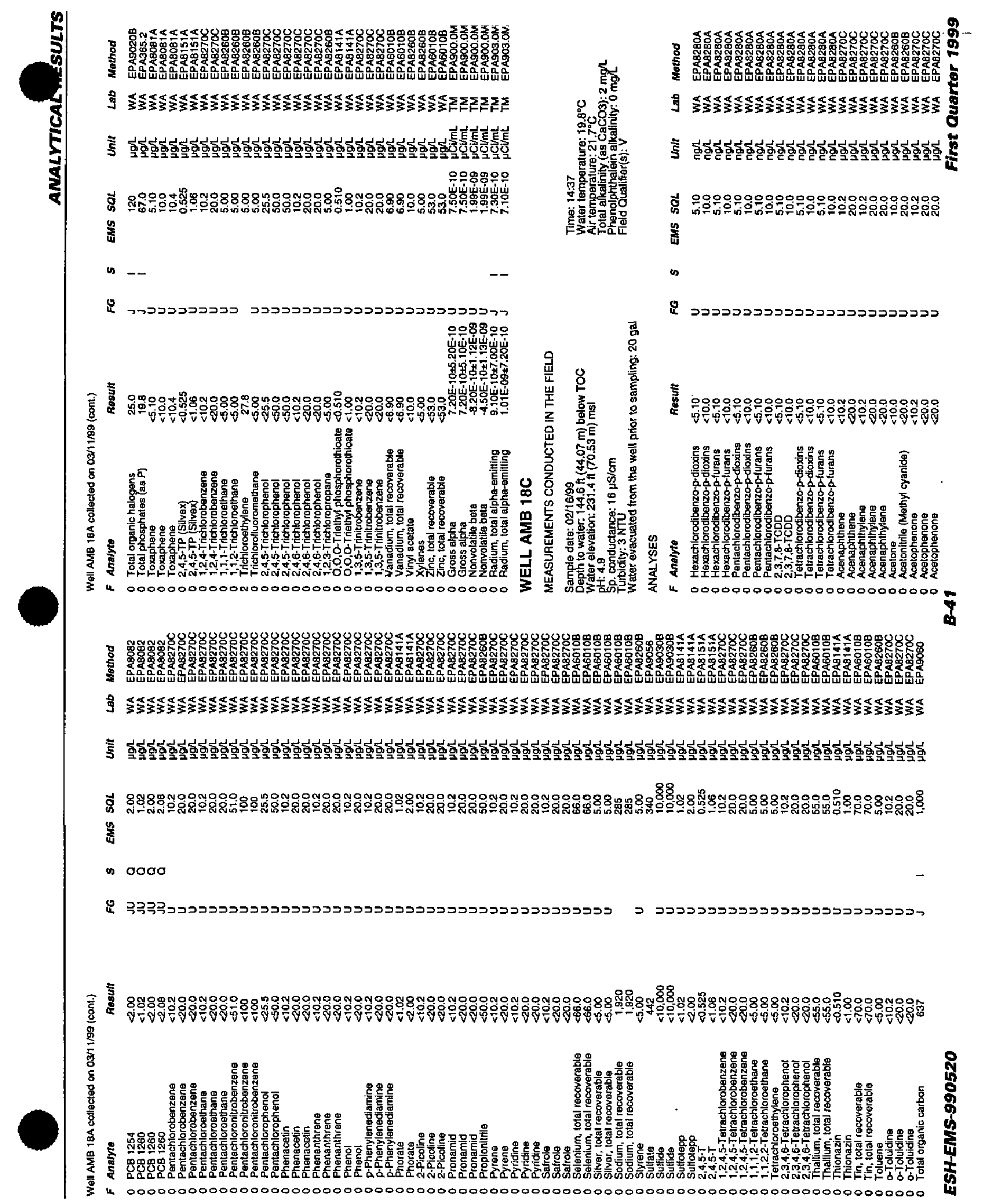
๔ $88 \%$ :

పึ

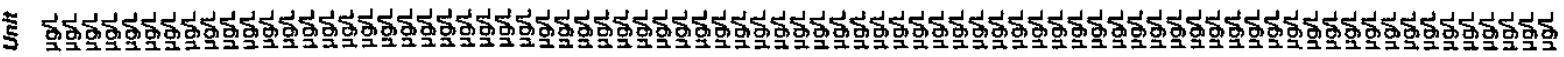

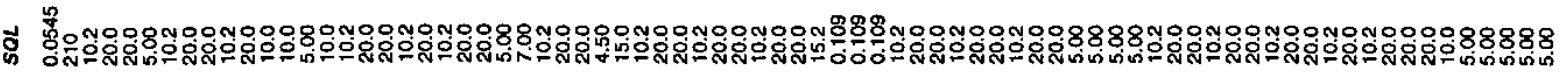
氧

us

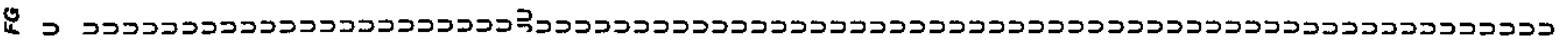

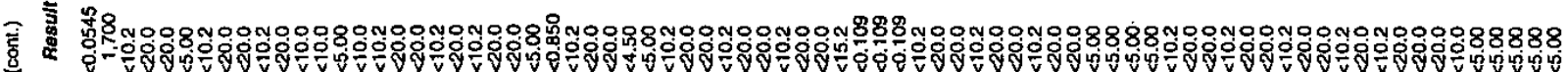

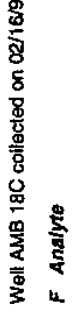
จงที่

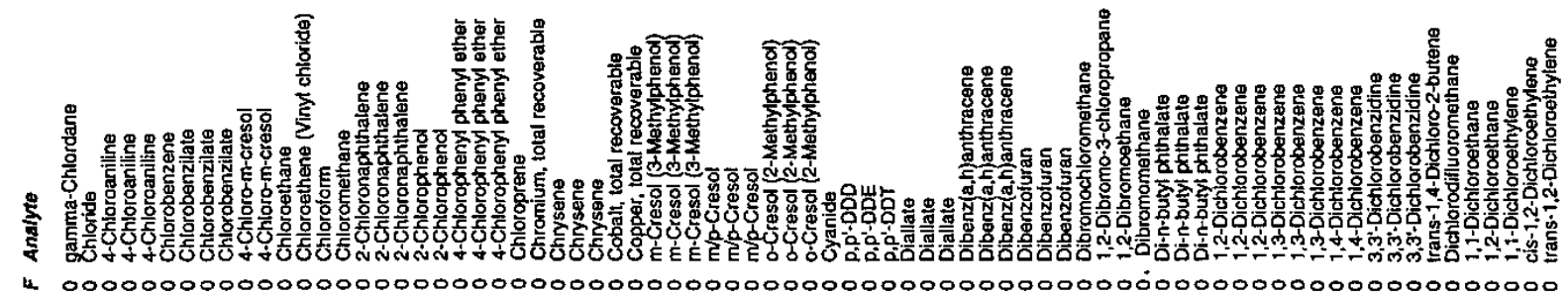

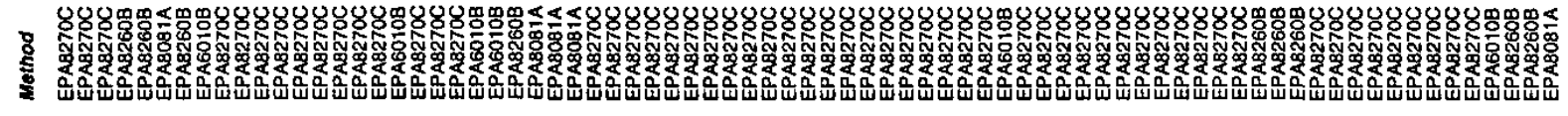

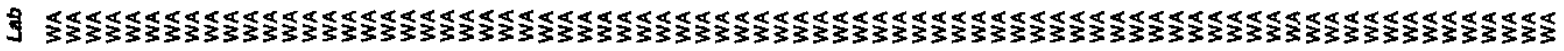

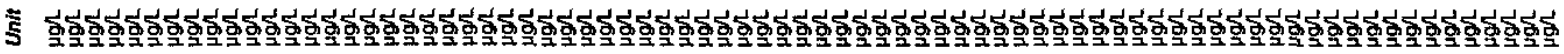

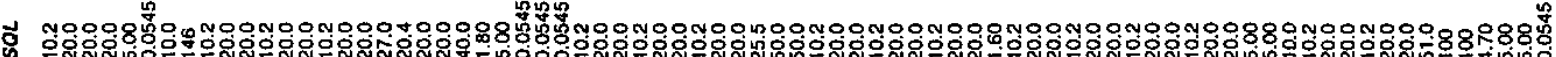
竞

4

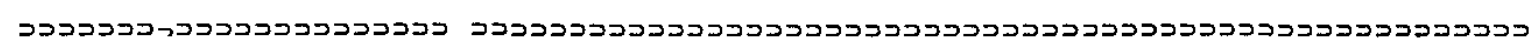

홍

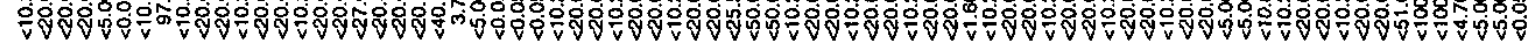

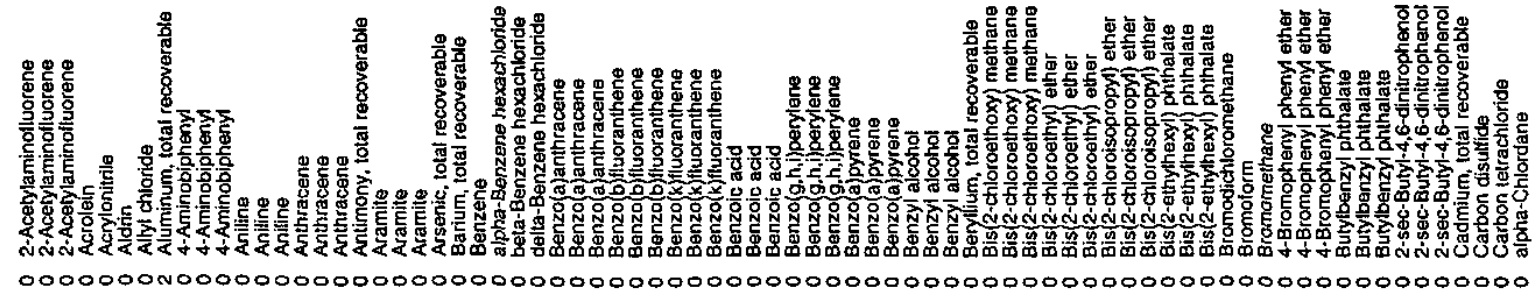


7 5

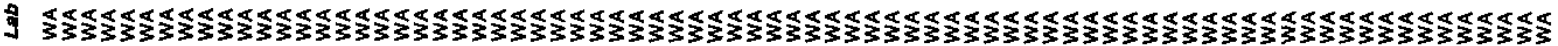

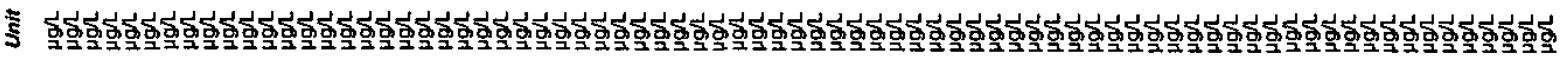

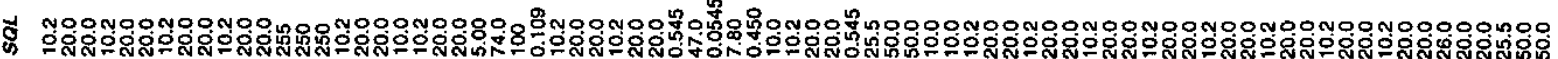
竞

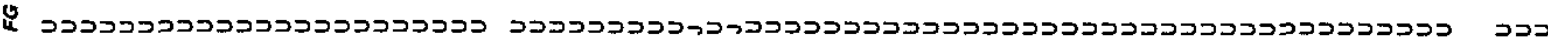

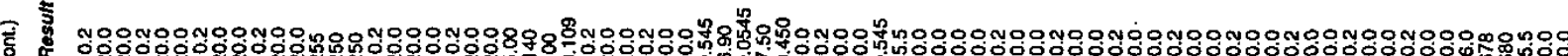
。

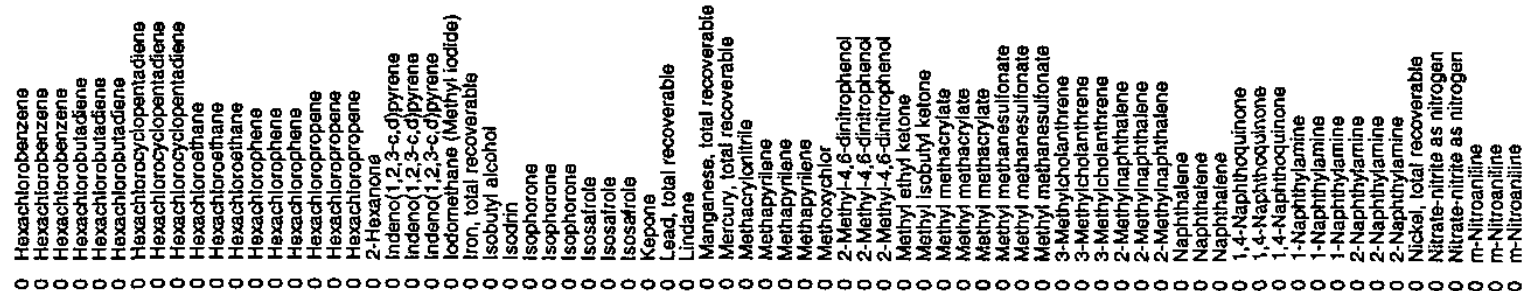

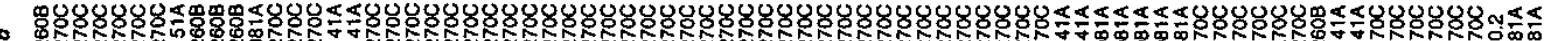

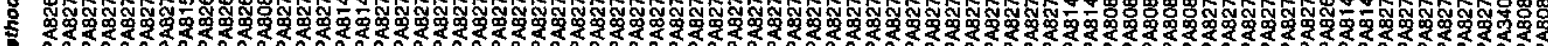

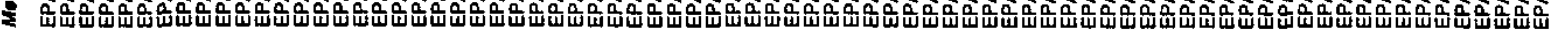

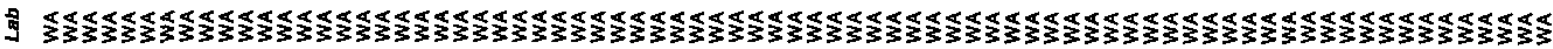

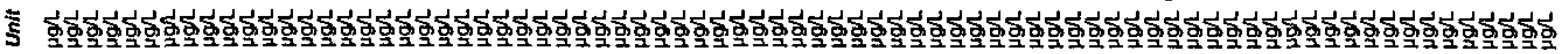

I 8ำoㄴ.

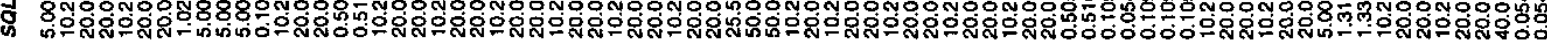
离 $\circ$

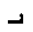

is

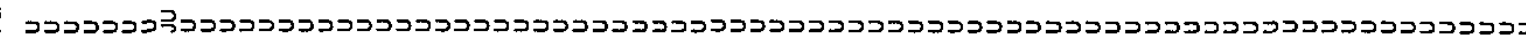

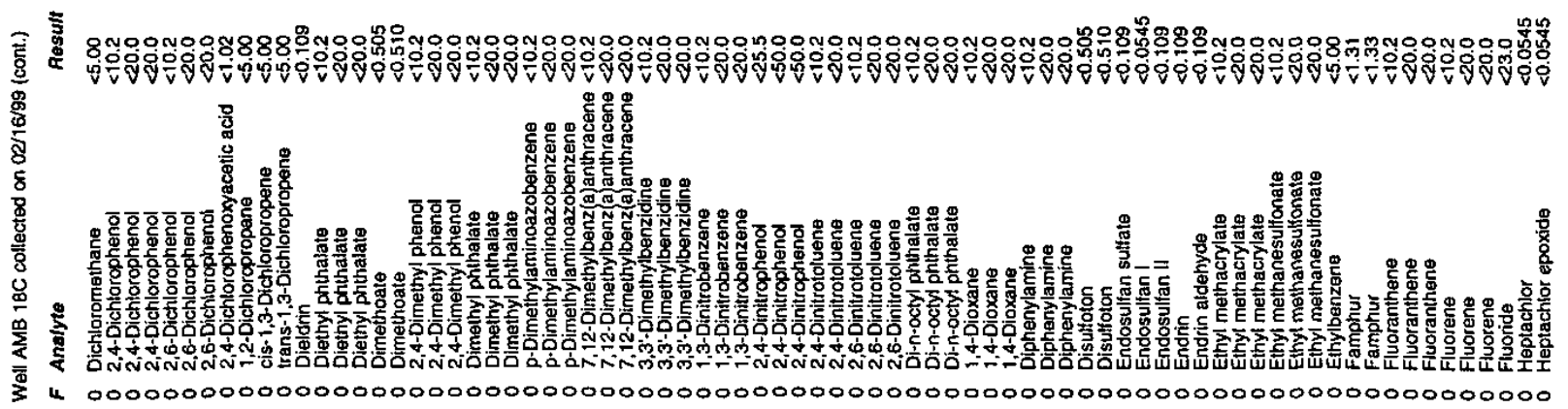


-

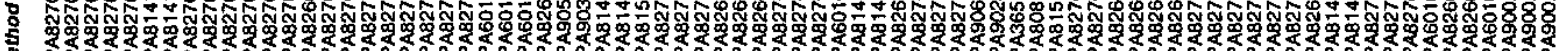

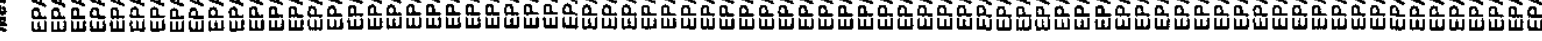

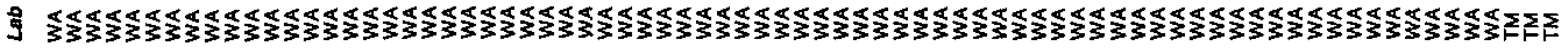

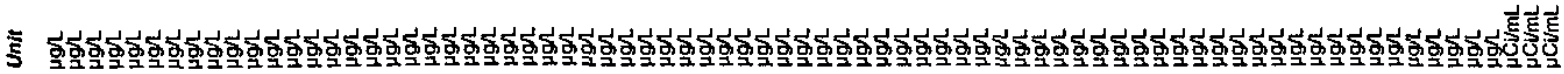

으융

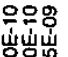

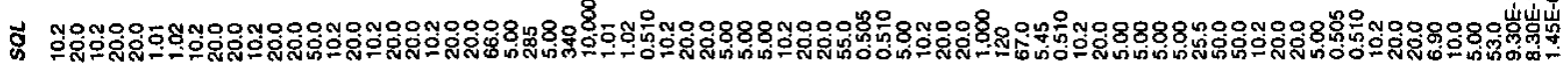
紊

$\circ$

\lrcorner $\circ$

$-\lrcorner$
0

$-\underline{x}$

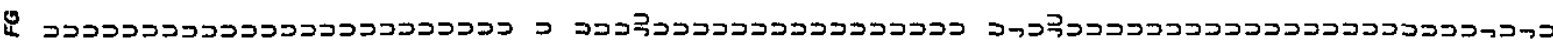
으유으

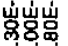
웅ํㅇ웅

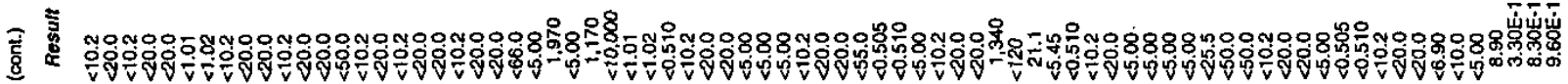

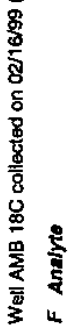

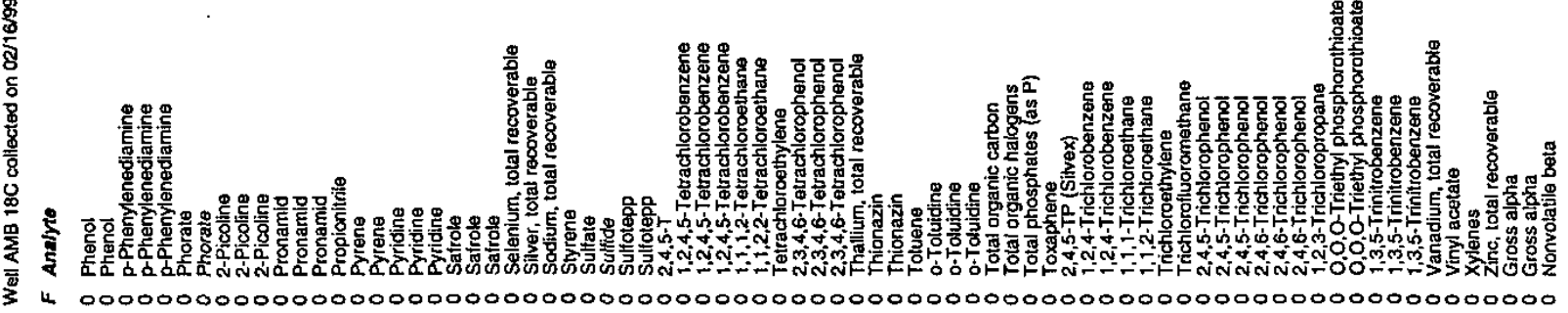

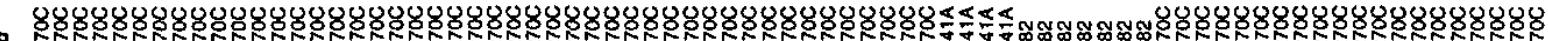 吉

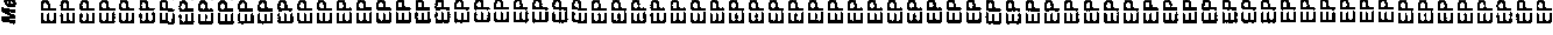

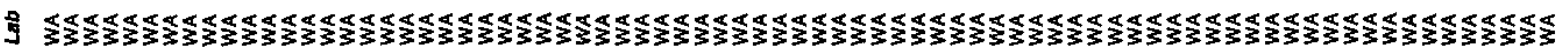

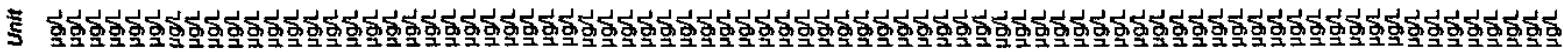

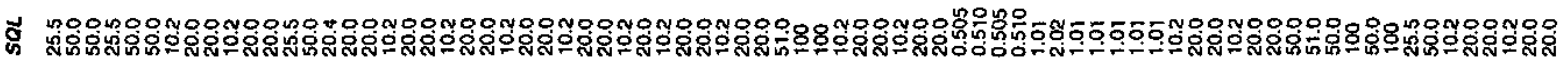
䆓

os

要

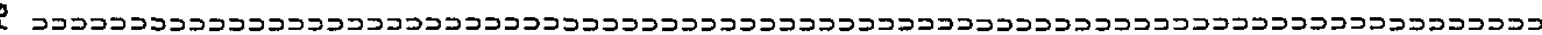

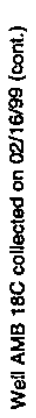

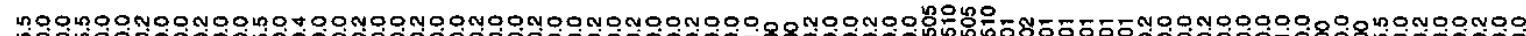
งึ.

(1)




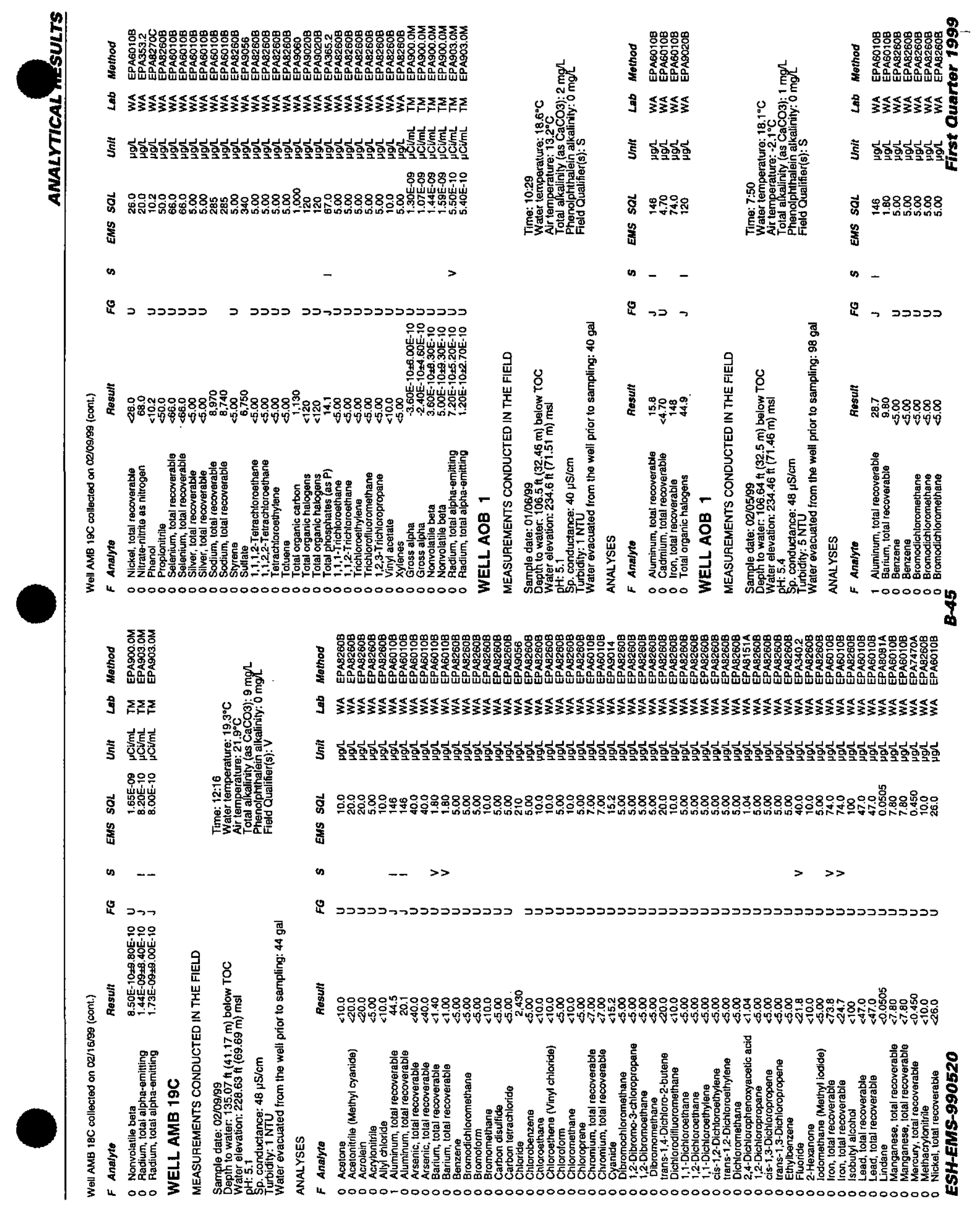




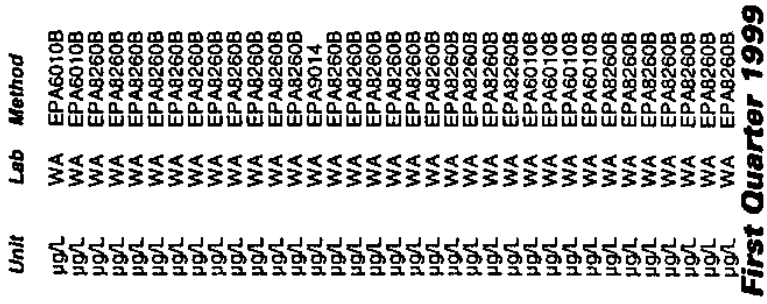

현 蛋

as - 000000000000000000000 - 0000000

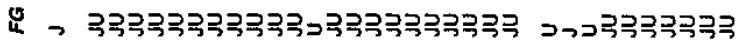

衰

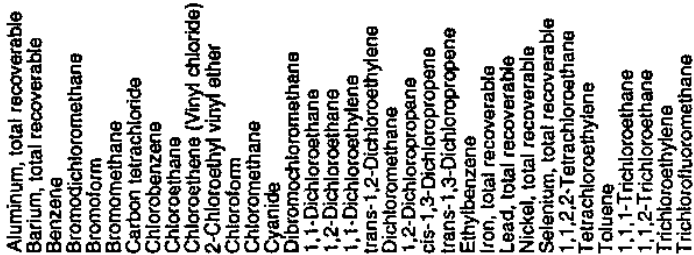

No0000000000000000000000N0000000000

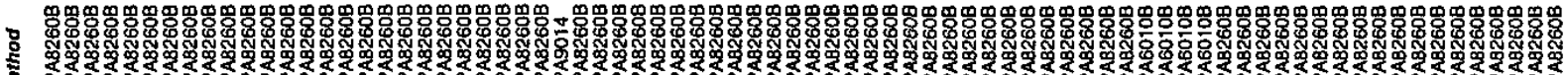
Wum

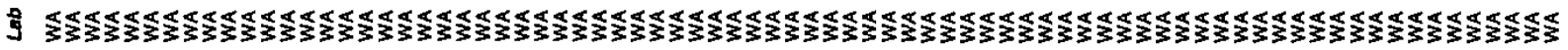

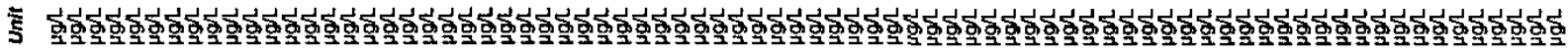

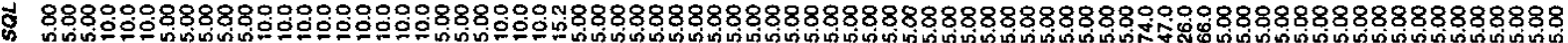
竞

a

8

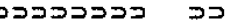

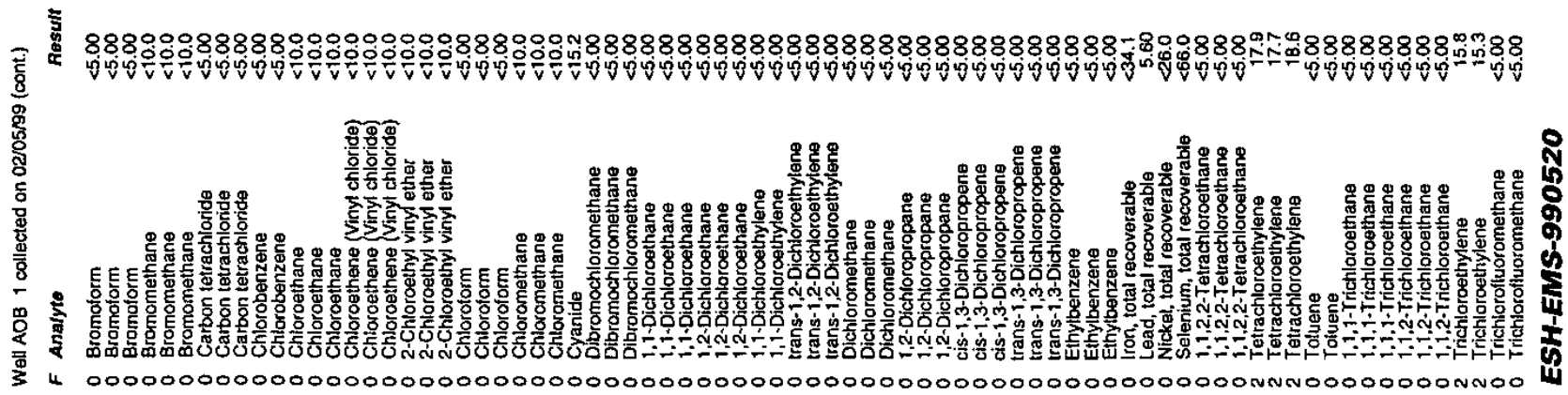




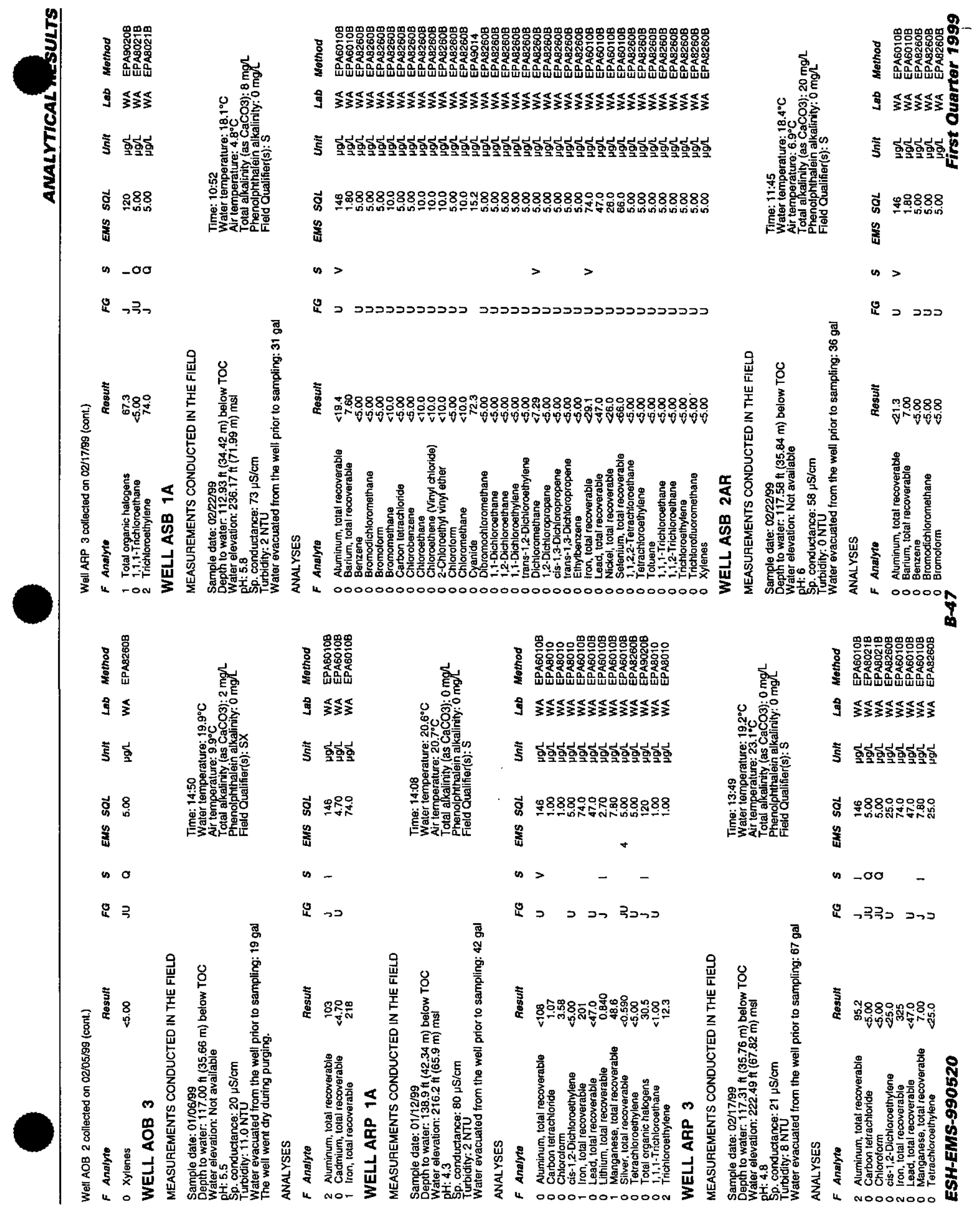



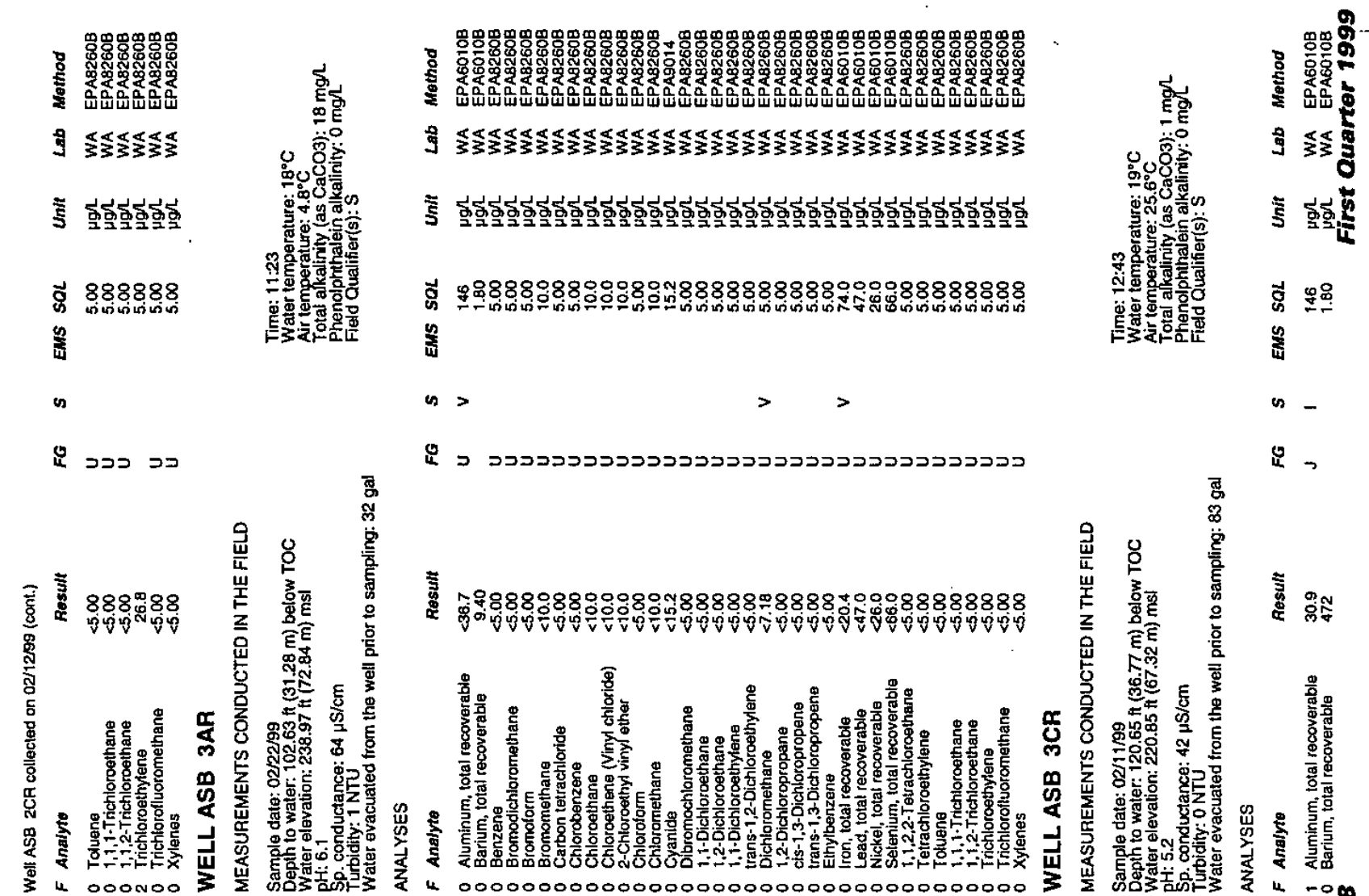

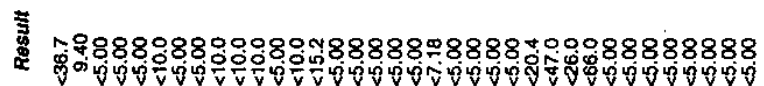
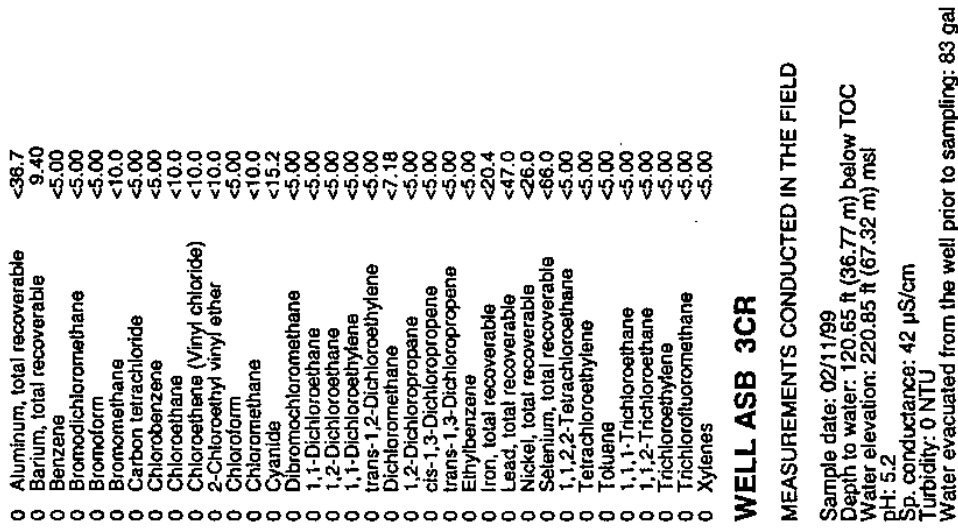

\& \& 000000000000000000000000000000000000

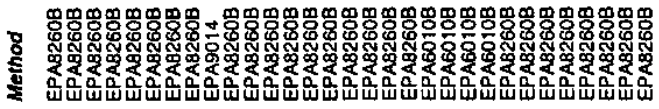

过

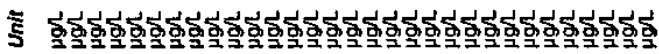

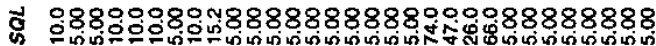
产

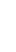

is

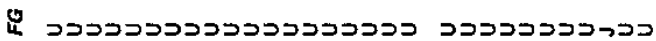

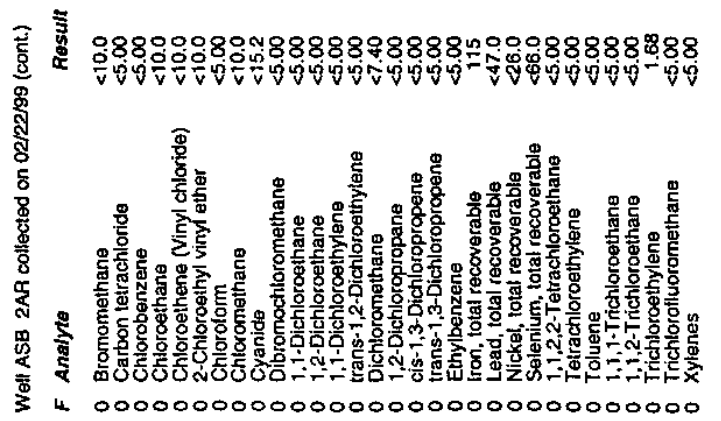

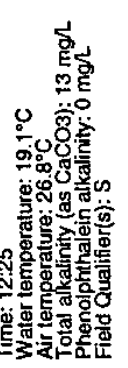

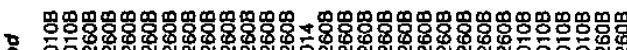
妾

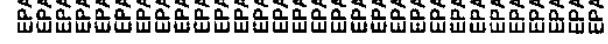

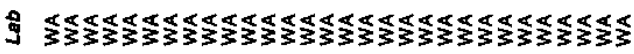

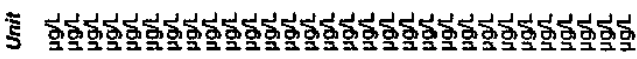

कै 善

is

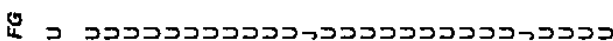

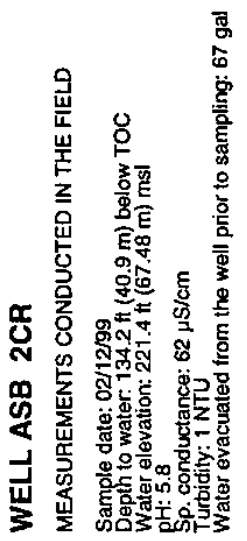

需

言

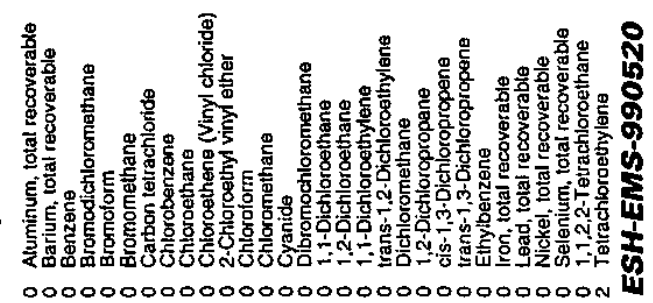



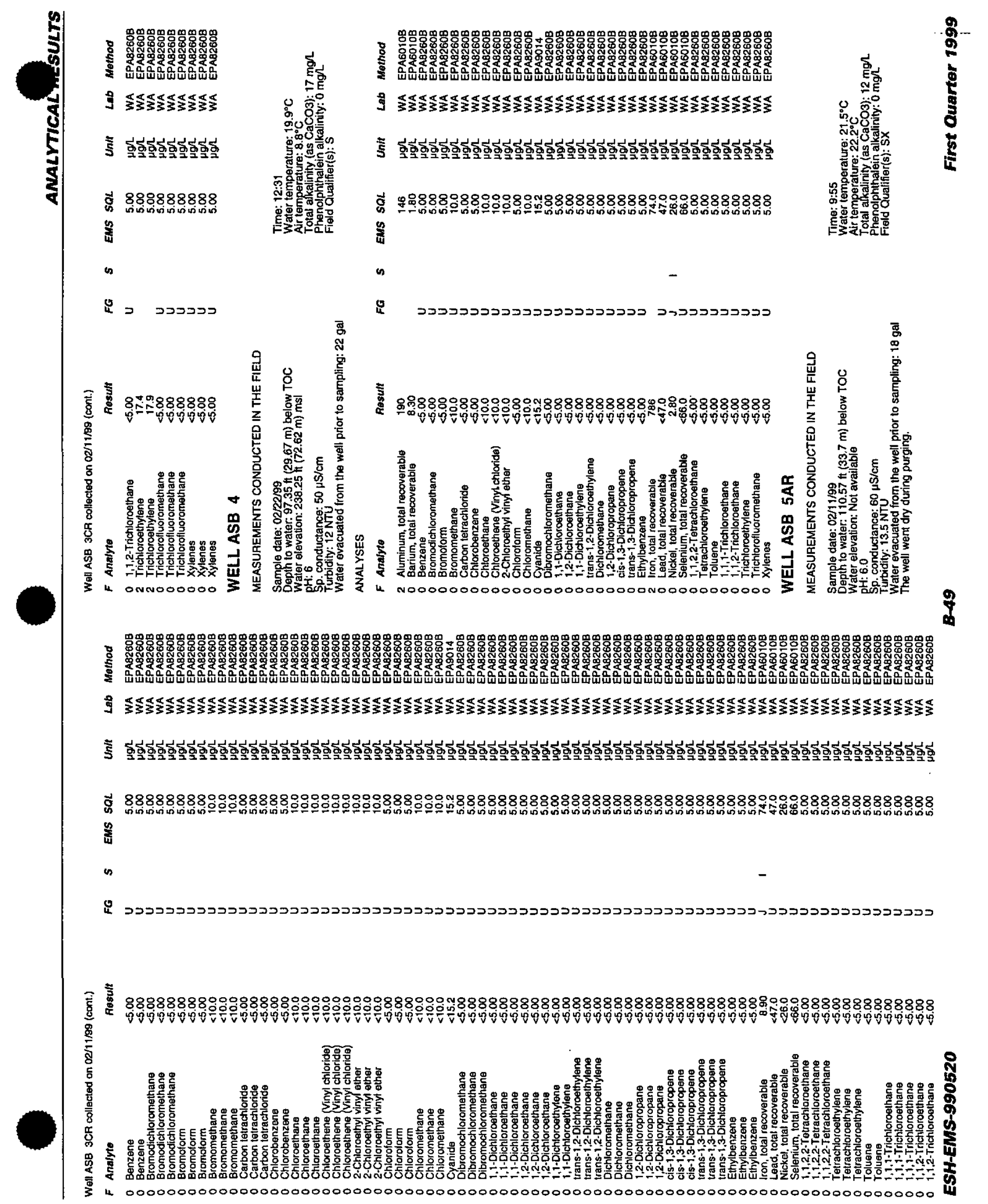


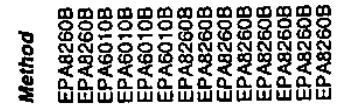

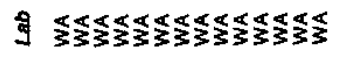

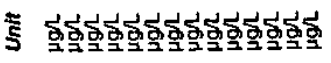

t) 880989888888888

蛋

s

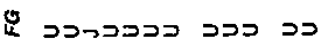

总总 落 فํำ

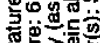
เ

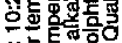

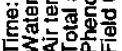

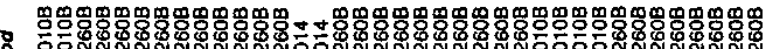
专

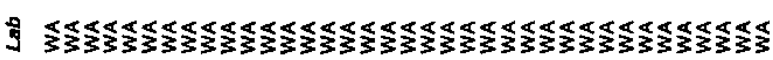

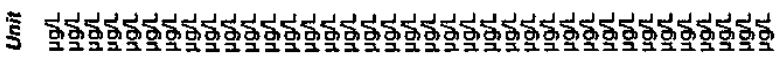

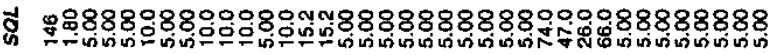
峦

$\infty>$

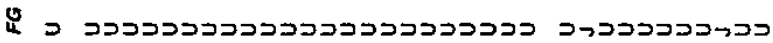

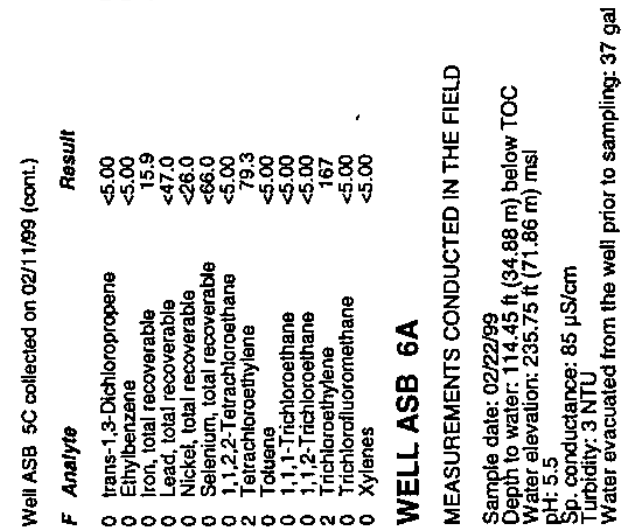
d

莺

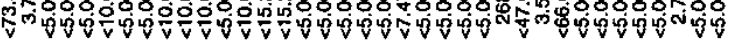

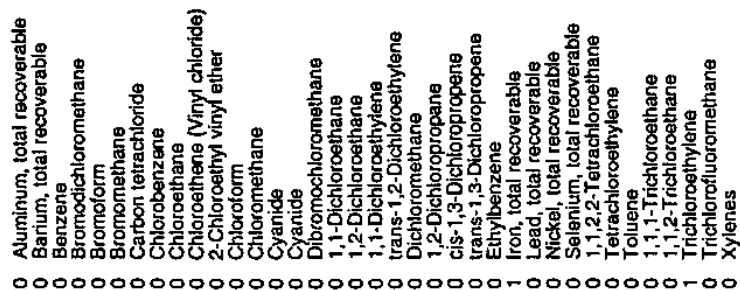

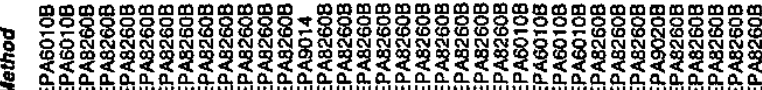

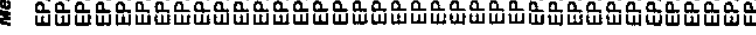

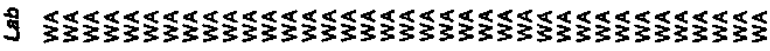

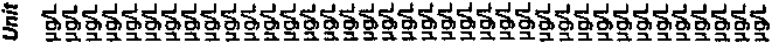

현

謇

n

品

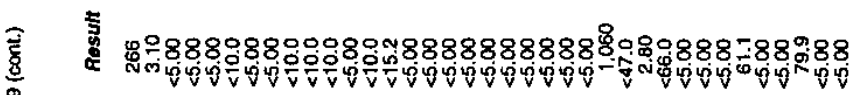

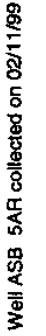

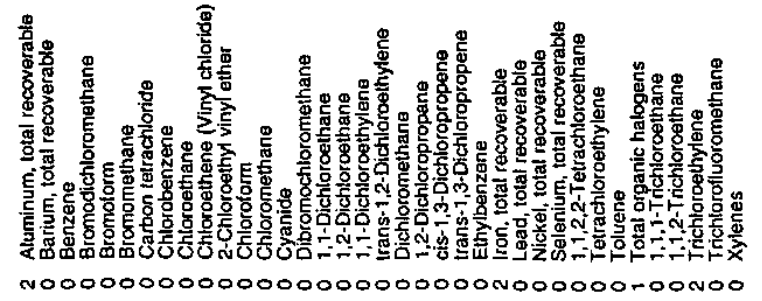

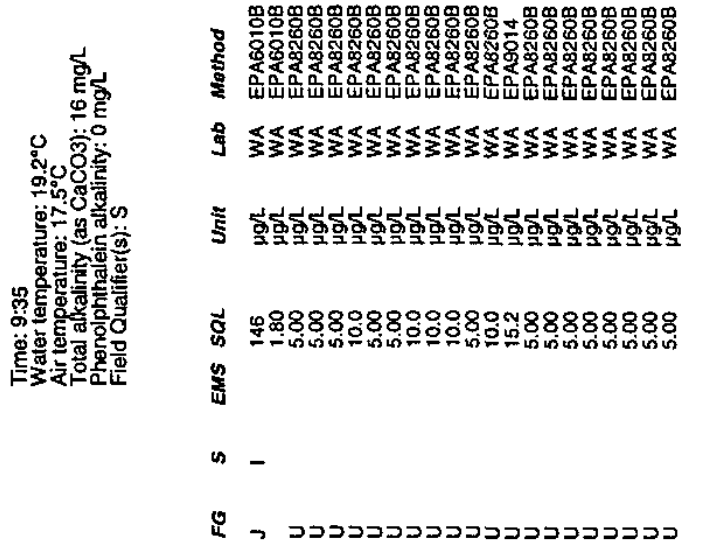

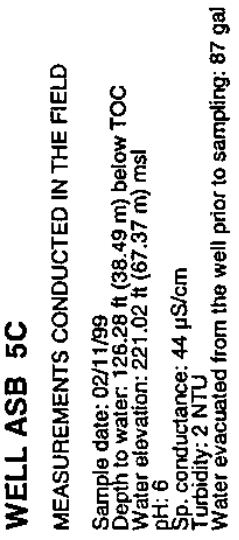

בכבככבכבכבכבכבכבכב

కั้

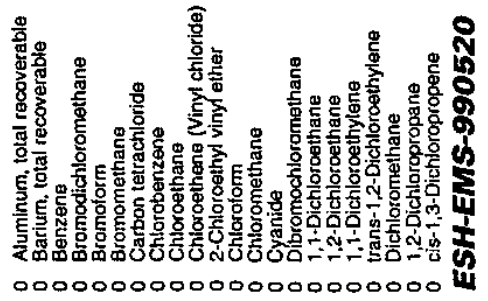









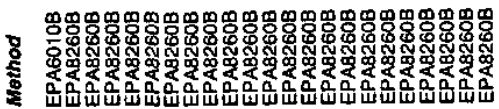

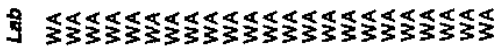

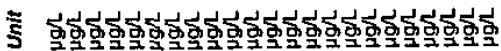

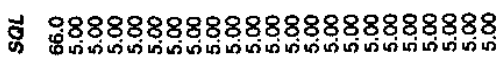
踤

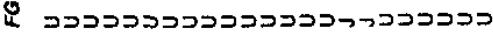

产

8

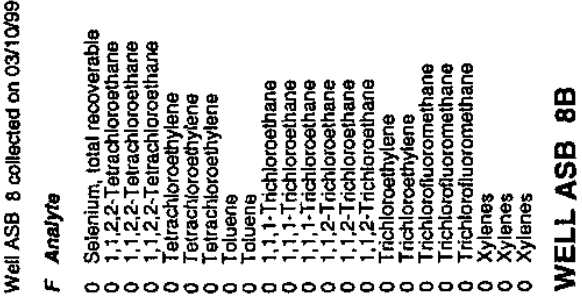

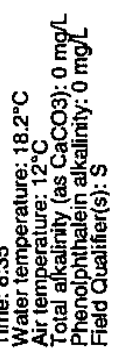

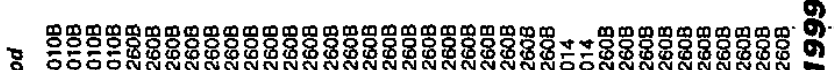
㐘

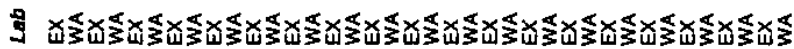

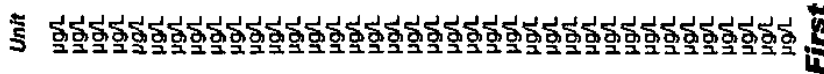

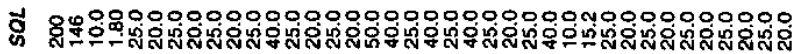
章

is

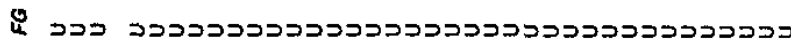

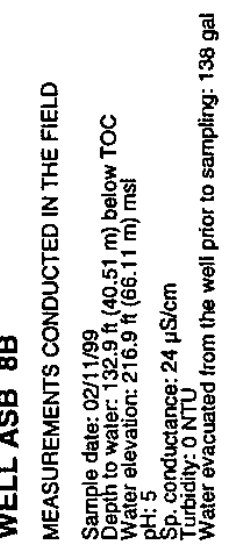

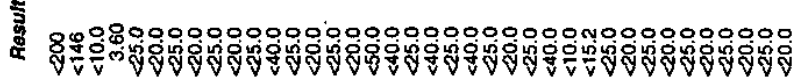

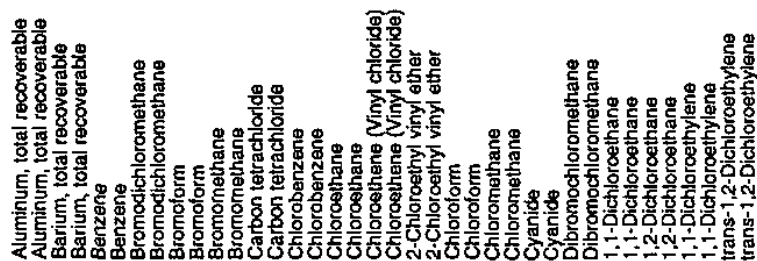

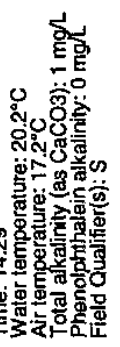

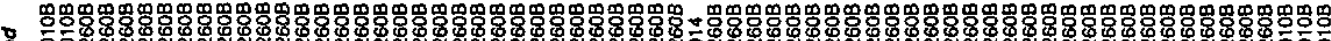
:

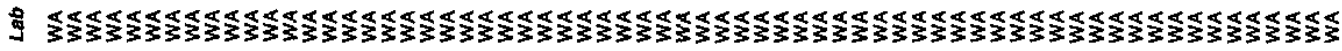

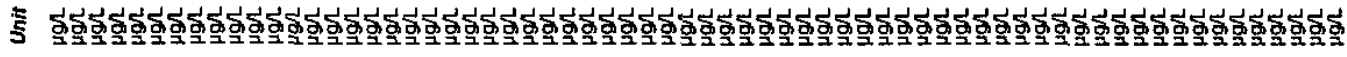

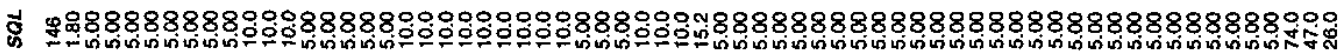
端

$\rightarrow \rightarrow$

$>>$

8

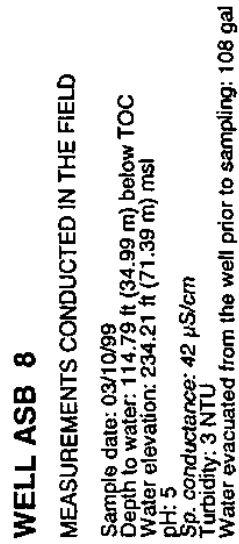

0 $\stackrel{\infty}{\circ}$

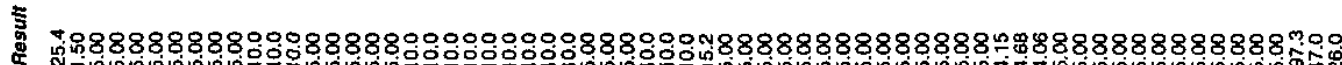

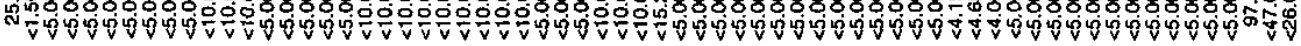
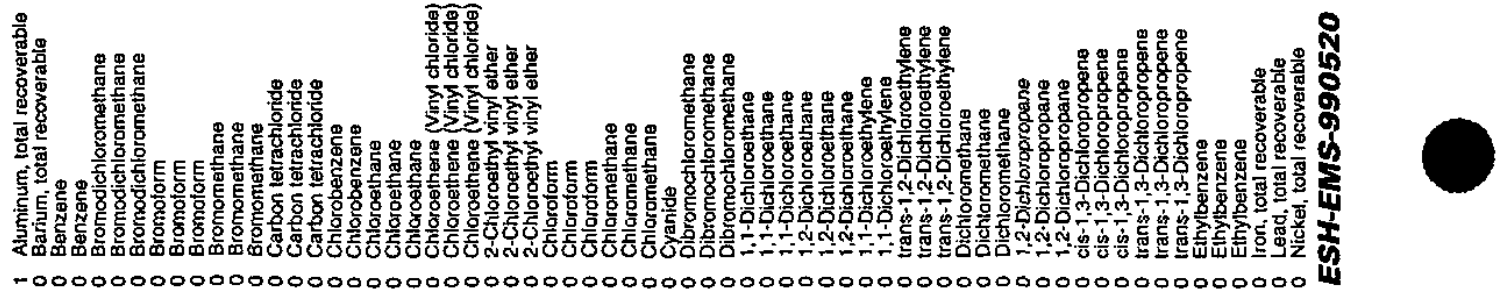


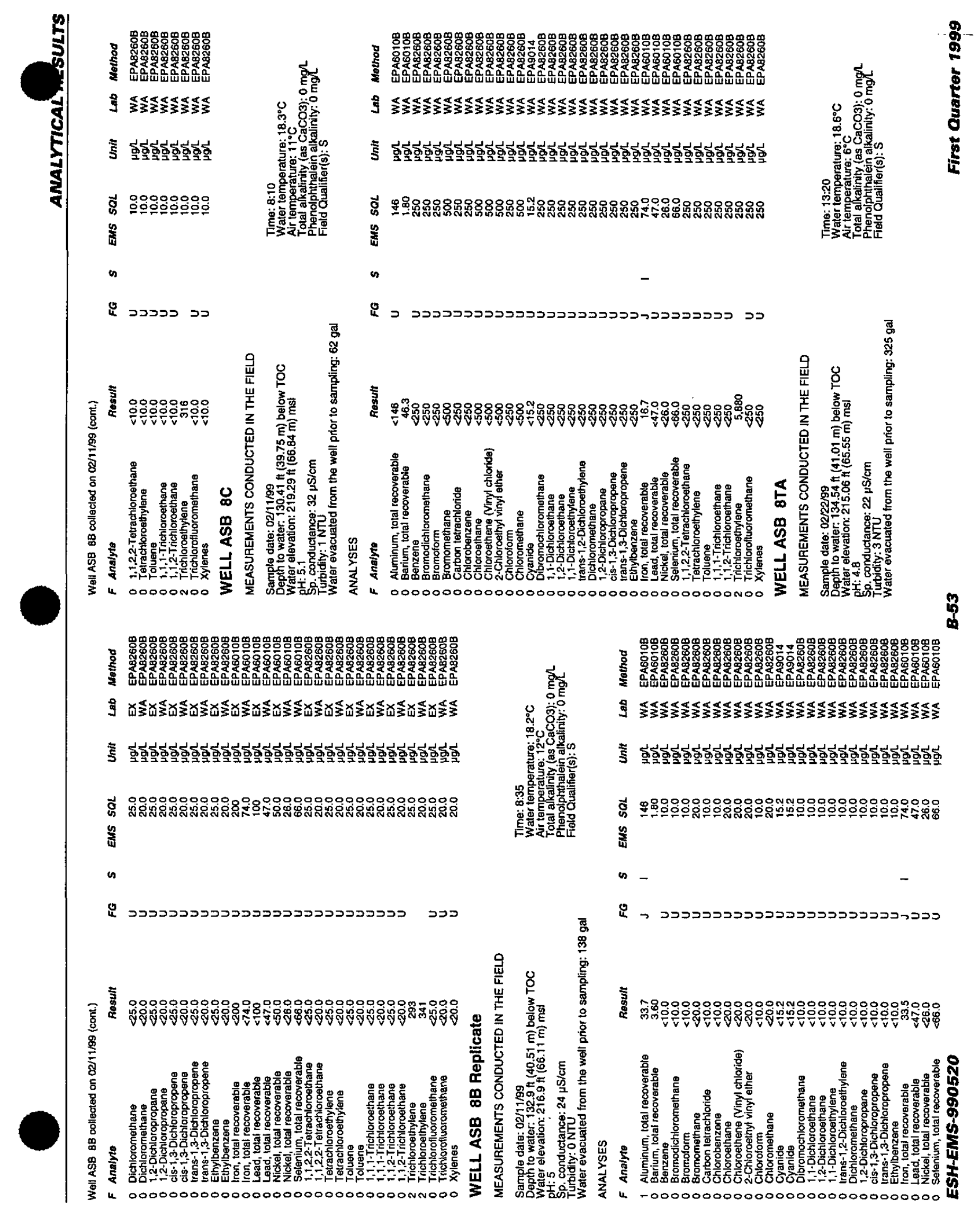




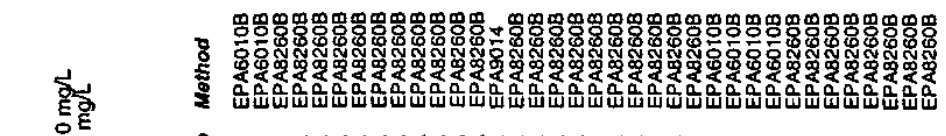

O 产.

$\therefore$ 记

象语心

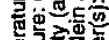

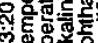

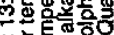

步娄

9

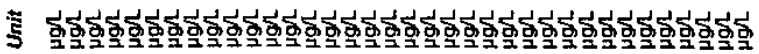

to 䓹

$n>$

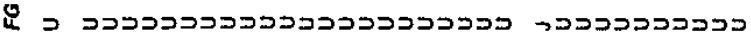

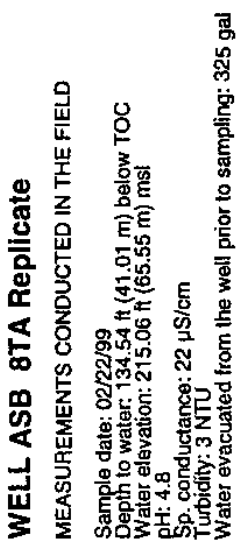

행

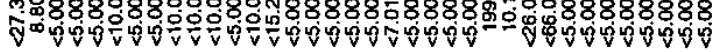

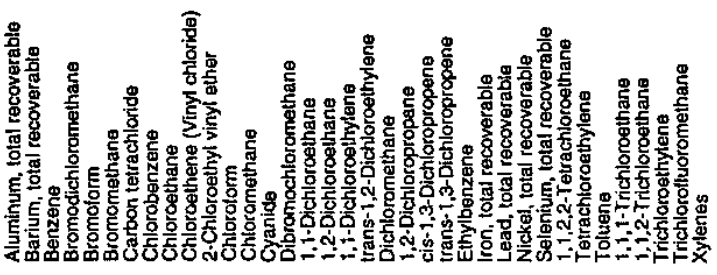

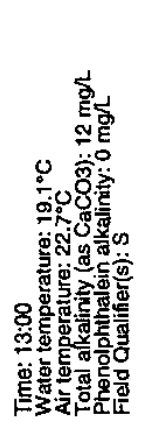

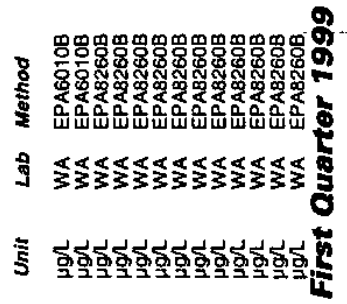

ㅎํ

总

s >

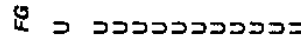

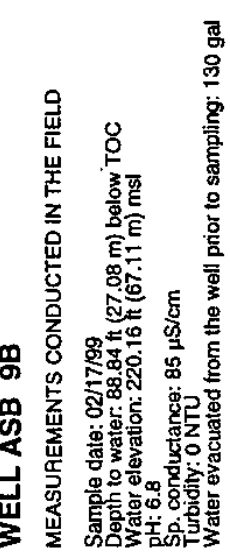

\section{芦}

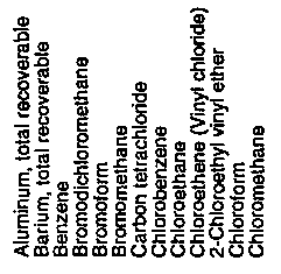

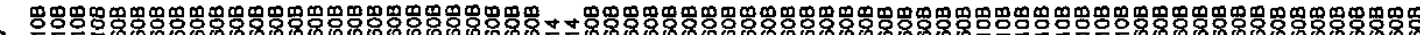

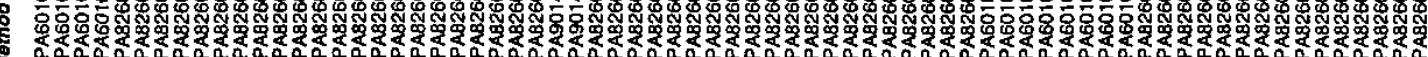

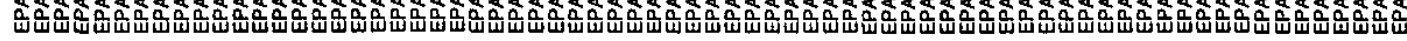

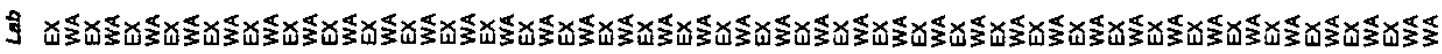

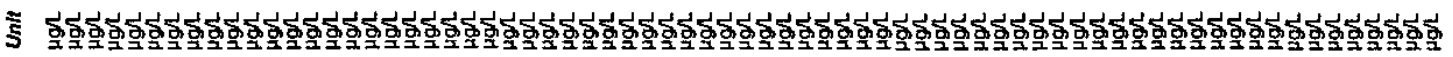

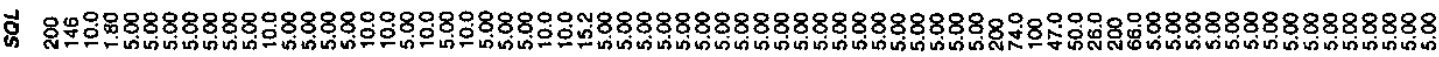

离

is >-

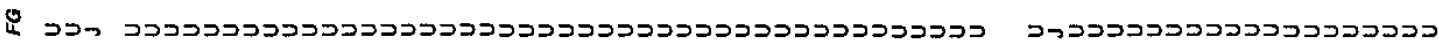

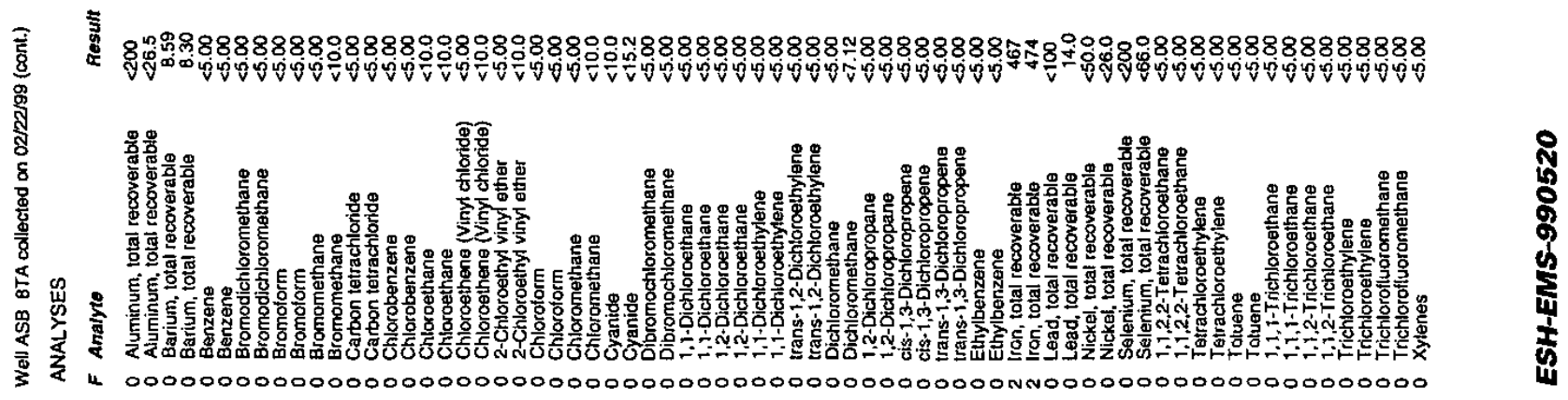




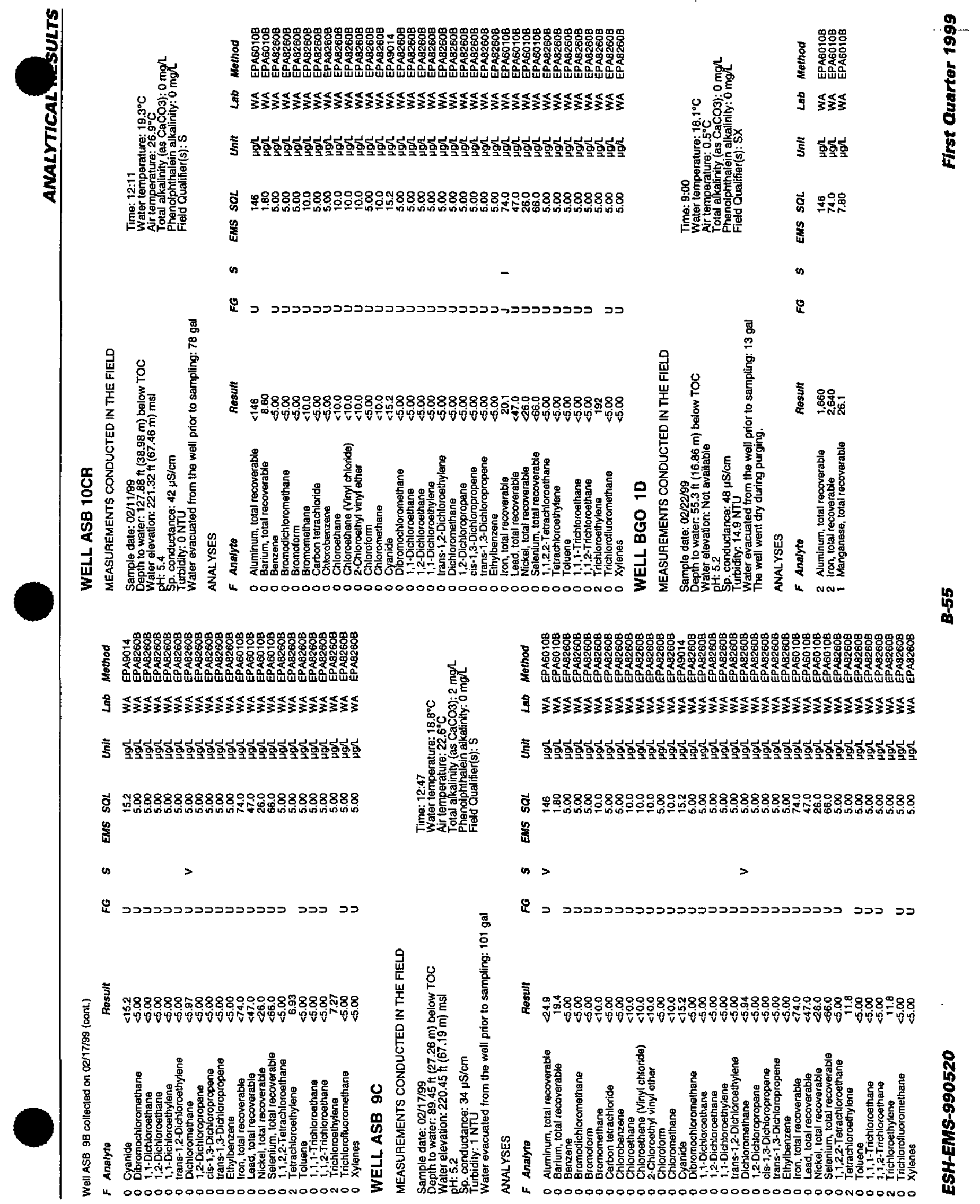


胥

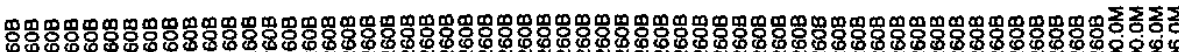

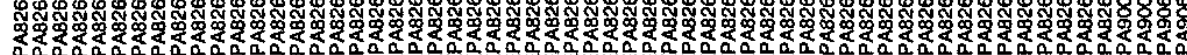

9

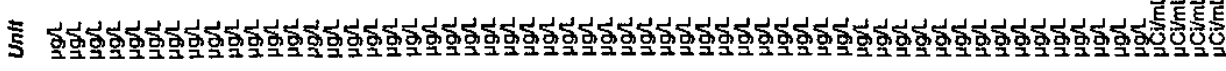

웅ํㅇํํ

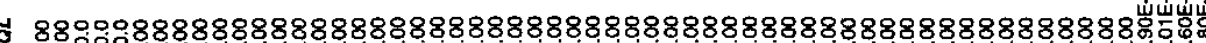

离

is

范

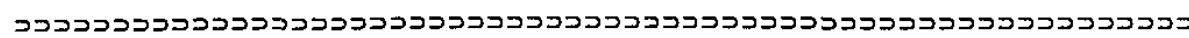

으옹후

岁岩楁崖

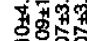

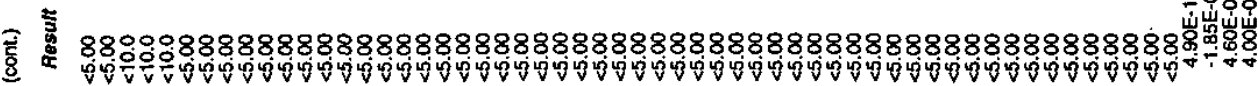

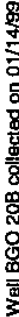
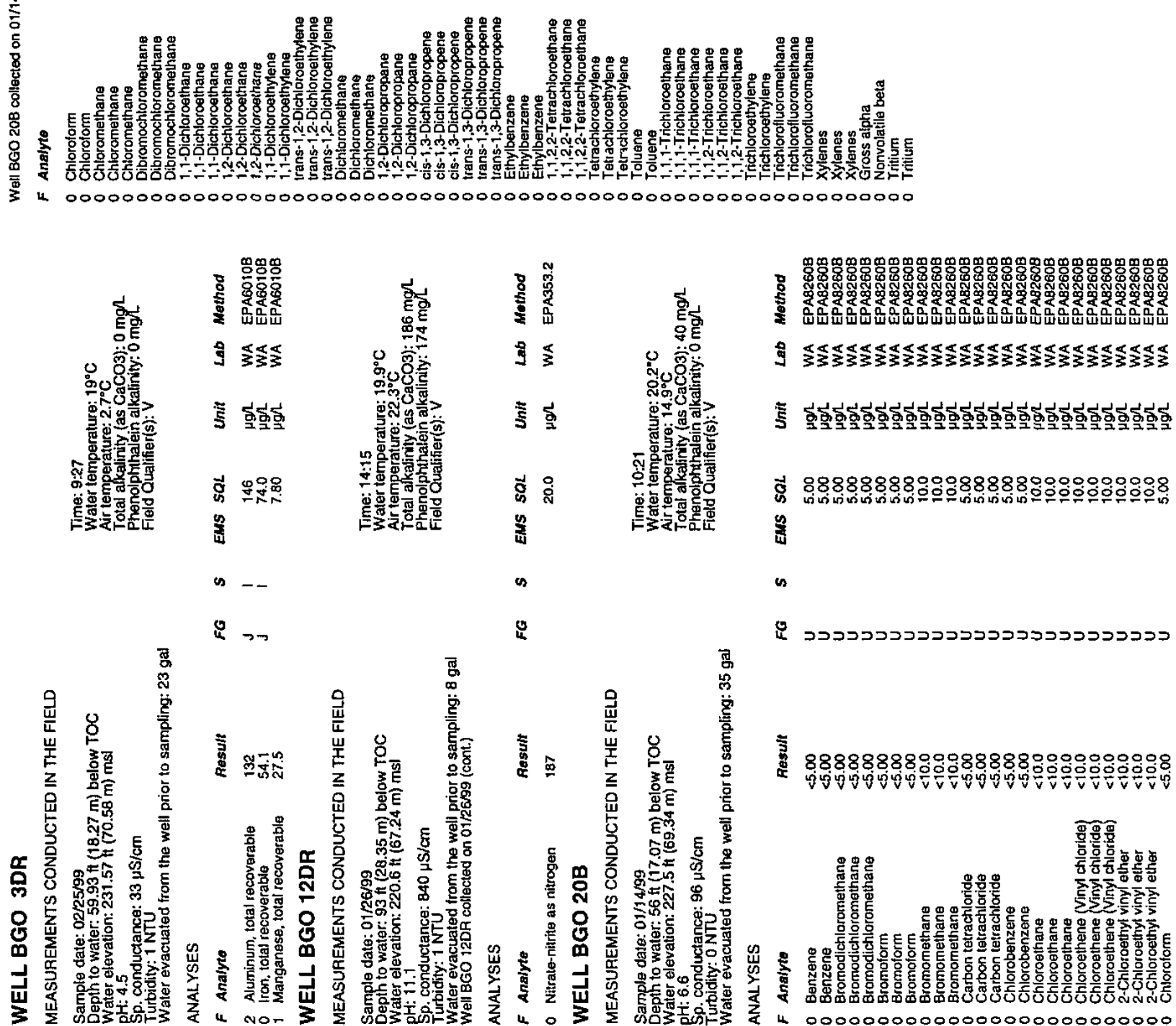
急
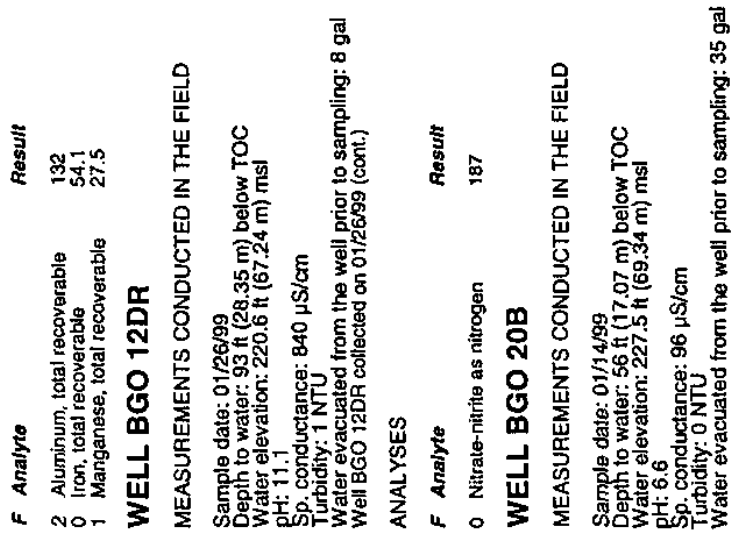

要

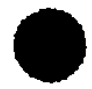

888888889000888880000000000008

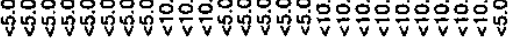

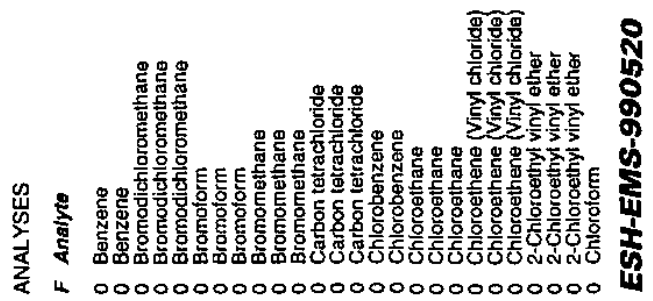




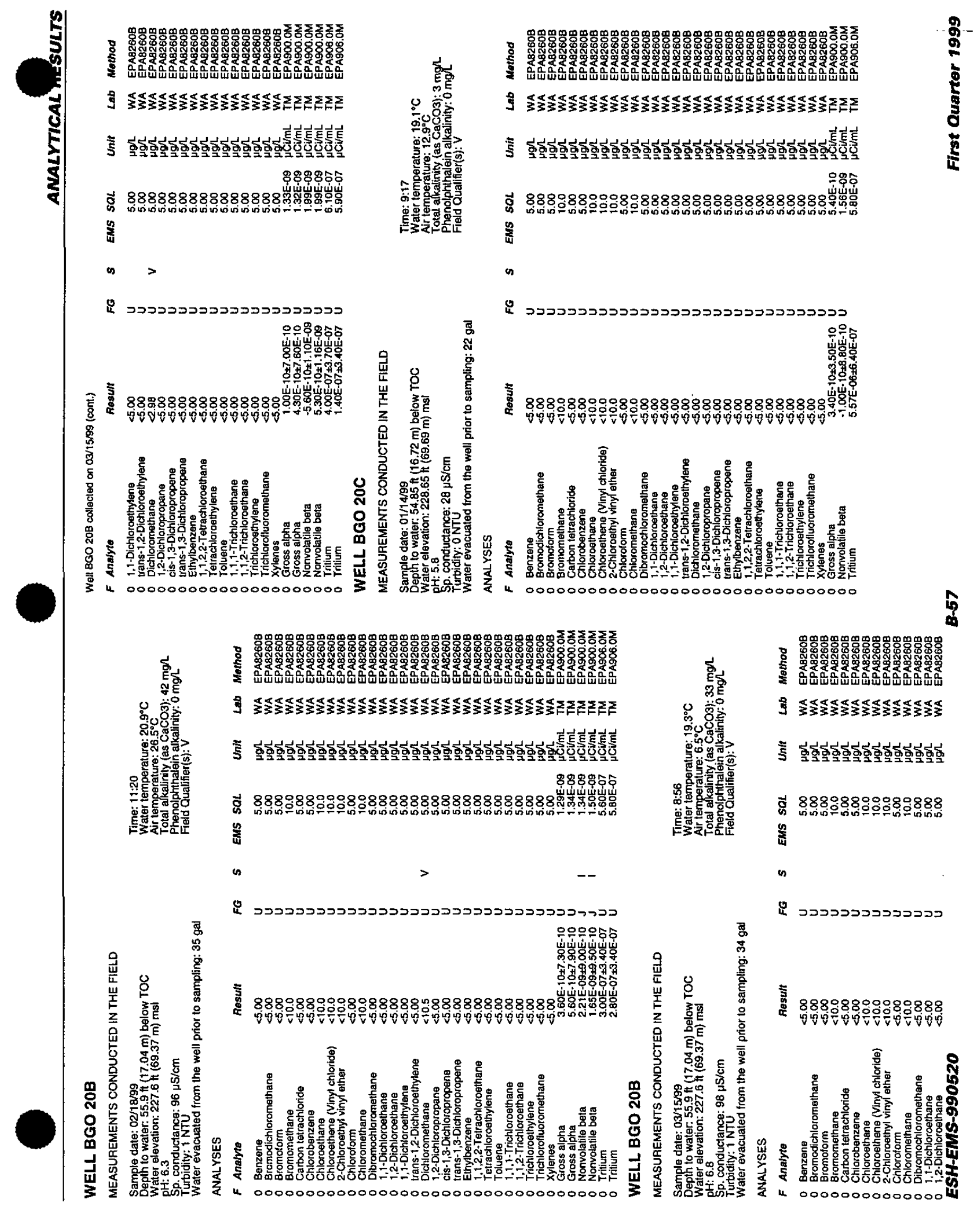




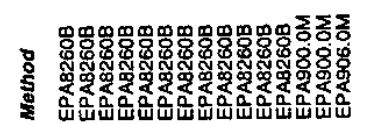

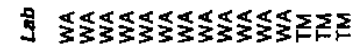

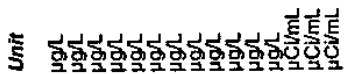
88 응

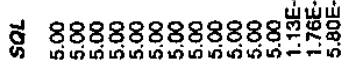

离

es

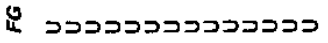

윴울웅

ำ की

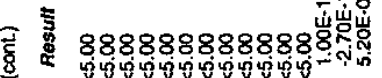

要

产

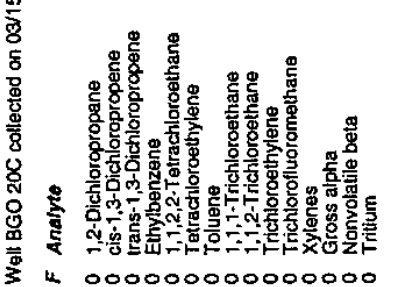

हु

:

กู่อ

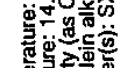

po

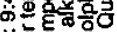

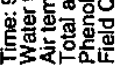

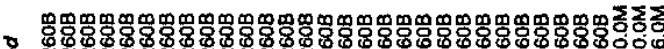

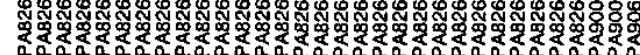

匀

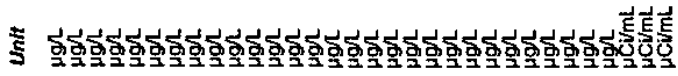

우웅후

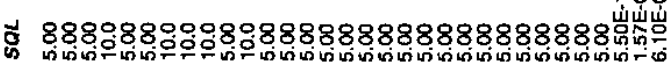

茜

n

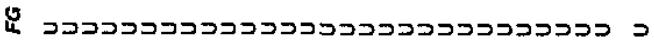

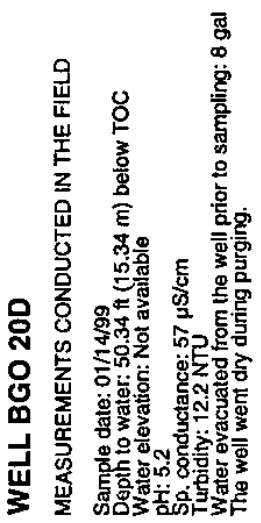

우요

刿㟧牢

今⿻ㅇㅇ융

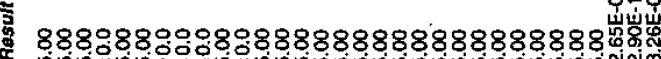

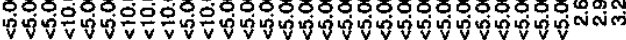

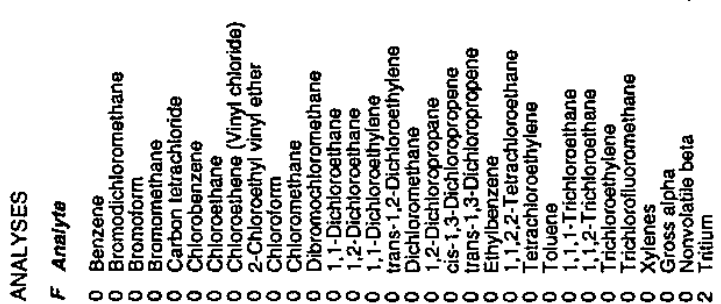

㟧

웅

o.jo

䎑

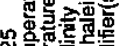

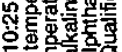

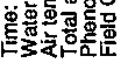

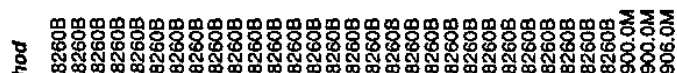

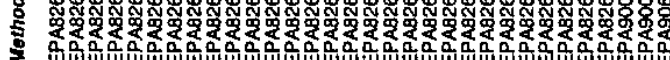

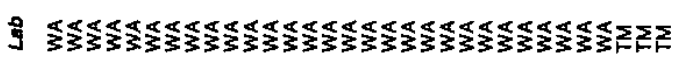

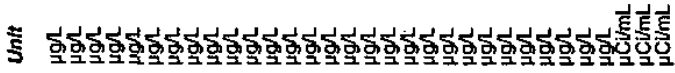

우옹

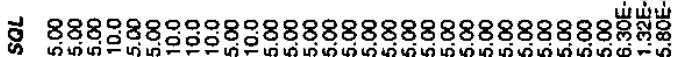

搴

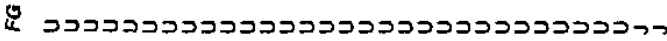

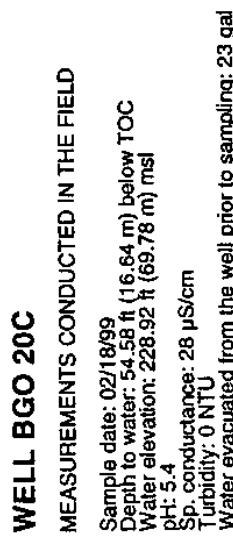

ฮั

(1)

$$
\text { 竎 } 88
$$

88808800080888880888888888880 楁

$$
\text { 으눙 }
$$

岁㟧岗

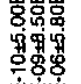

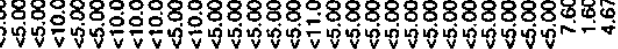

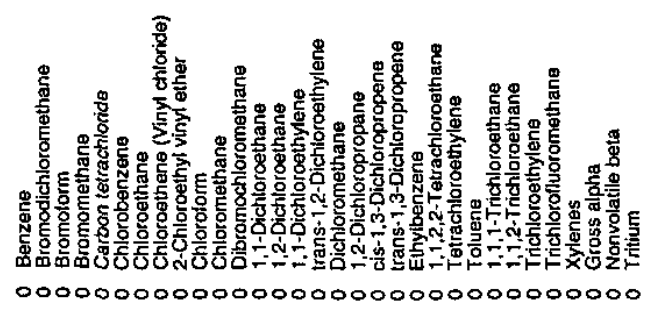

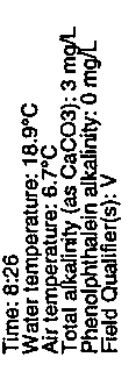

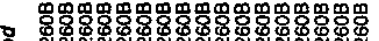

o

은

旬

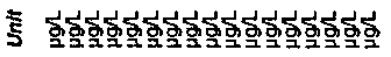

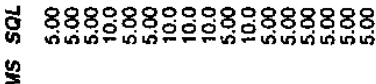

盖

$\infty$

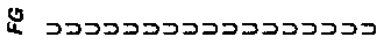

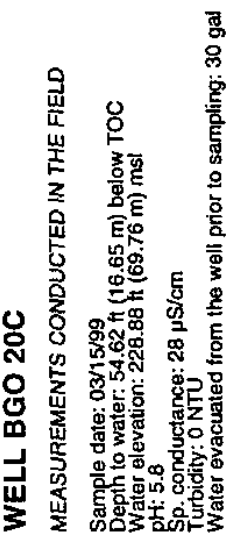

可

言 88898800080888890

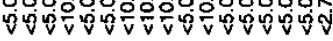

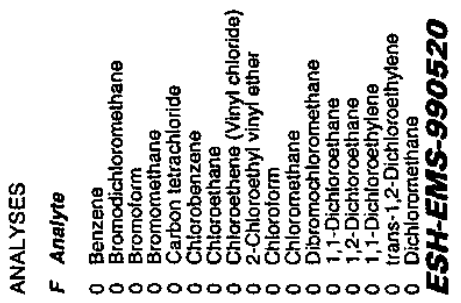


9

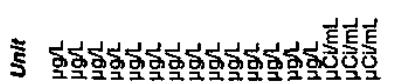

\section{웅훙}

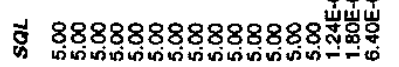

举

0

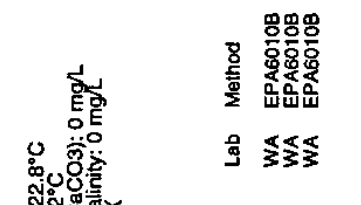

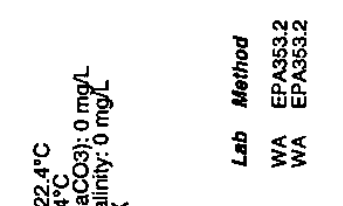

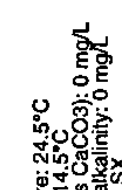

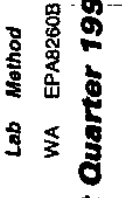

\section{홀 호호}

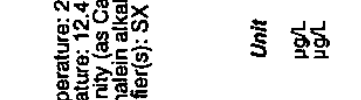

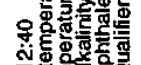

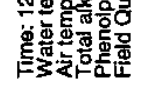

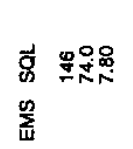

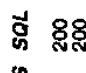

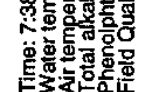

$\infty$

$\infty>$

$\underline{\underline{1}}$

i

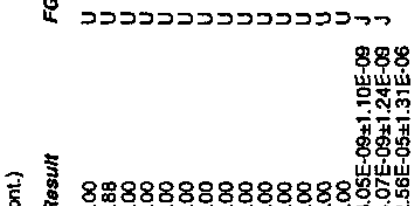

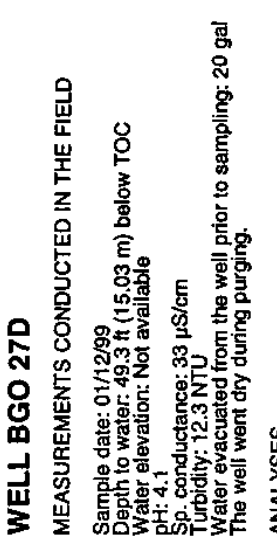

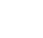

s.
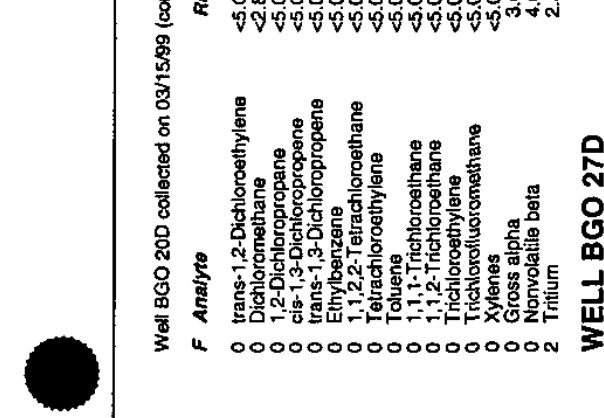

I L 00000000000000000

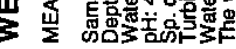
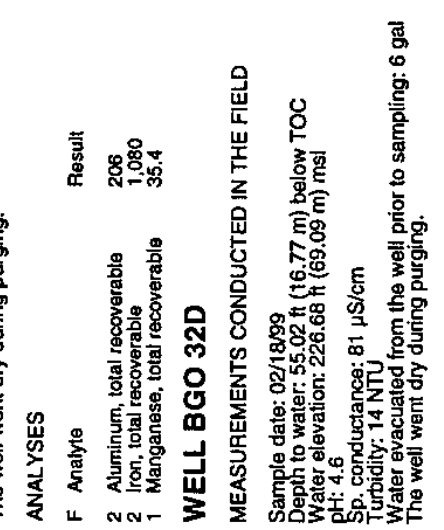

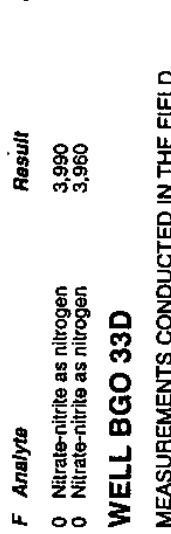

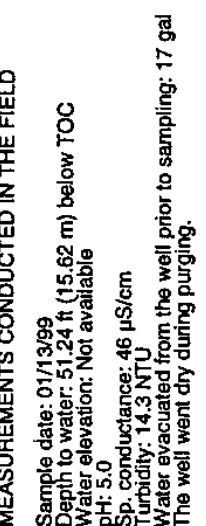

용 8

量

en

\&

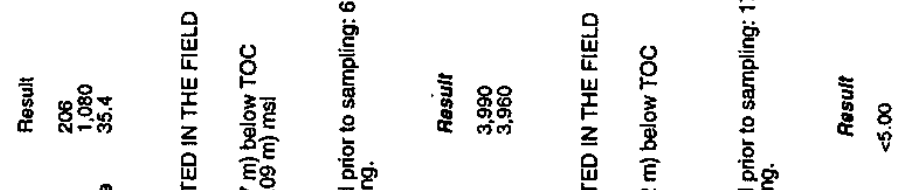

害点

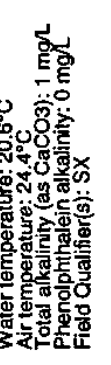

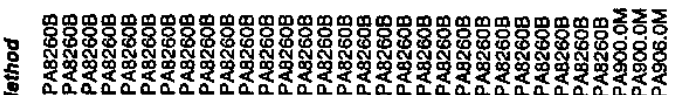

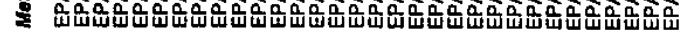

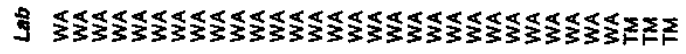

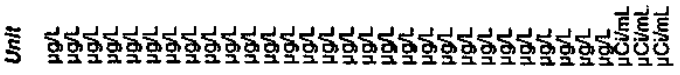

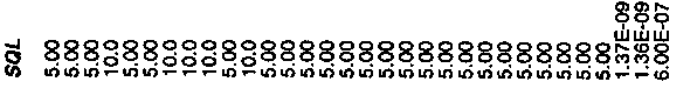

纁

0

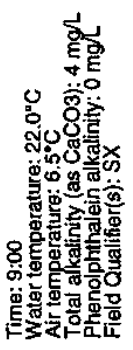

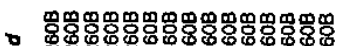

is

8

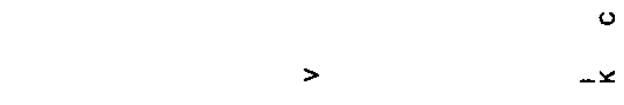

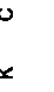

5.

ง

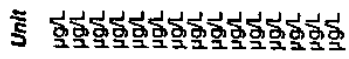

क् 888089800808898

$\infty$

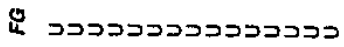

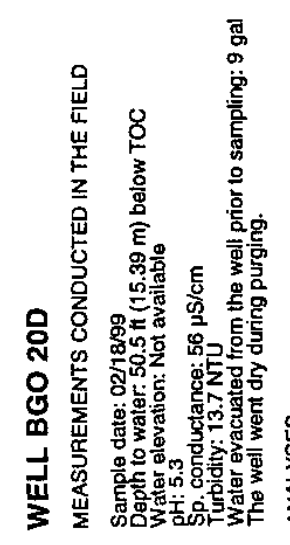

רים

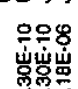

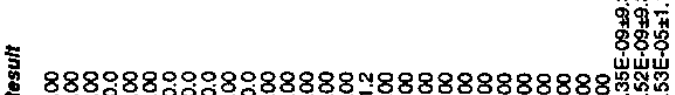

吪

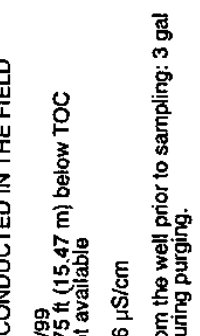

竎 8888880008088

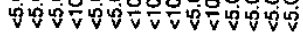




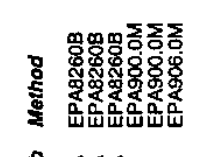

ง

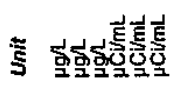

\%웅

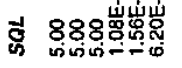

竞 0

is $-Y$

Q

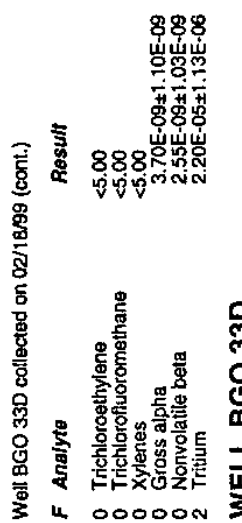

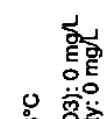

路
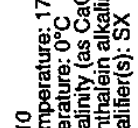

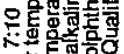

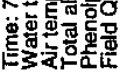

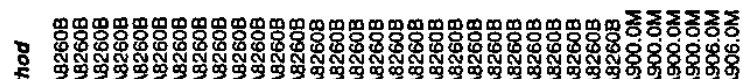

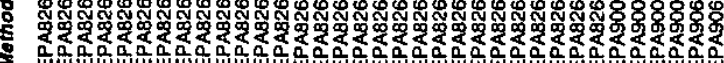

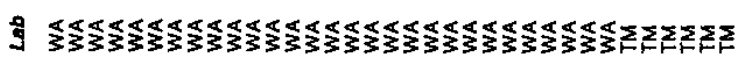

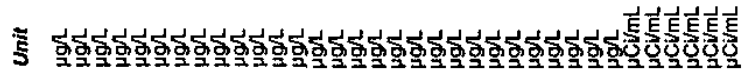

웅융ํㅇㅇ

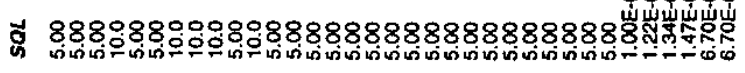

言

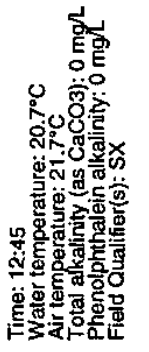

8

is

Q

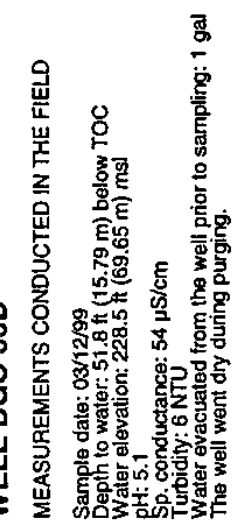

今.

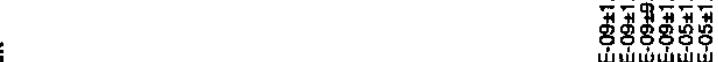
888인.
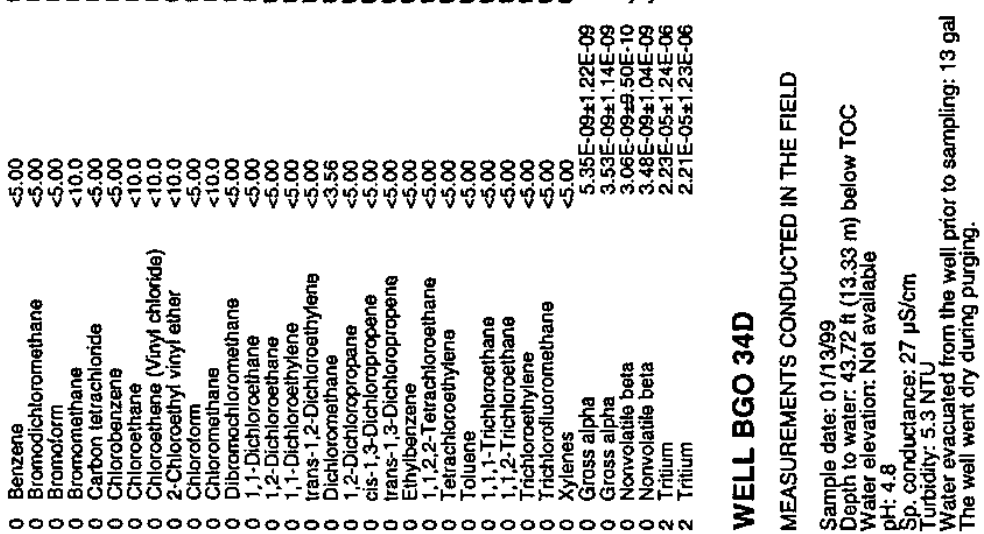

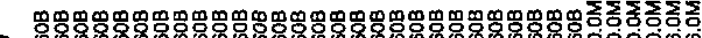

范

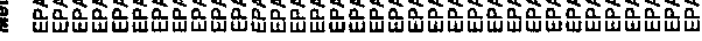

9

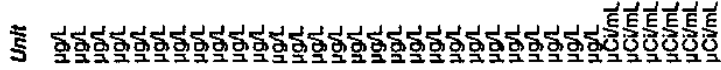

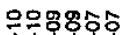

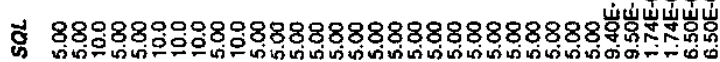

羍

in

S

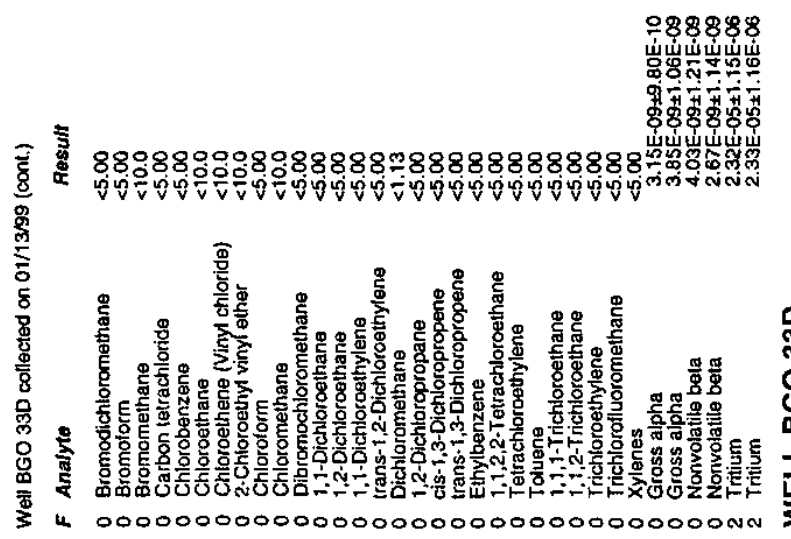

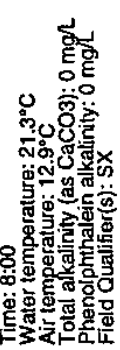

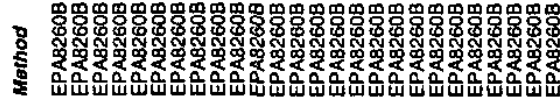

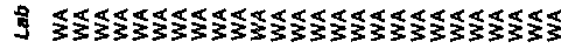

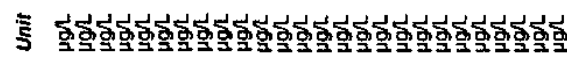

ปี 888888000808888888888888

急

os

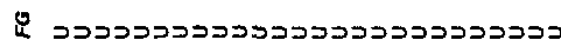

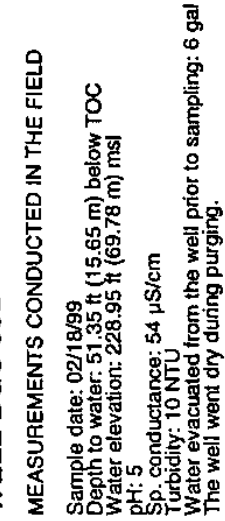

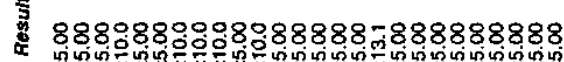

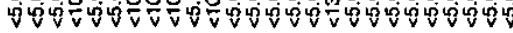

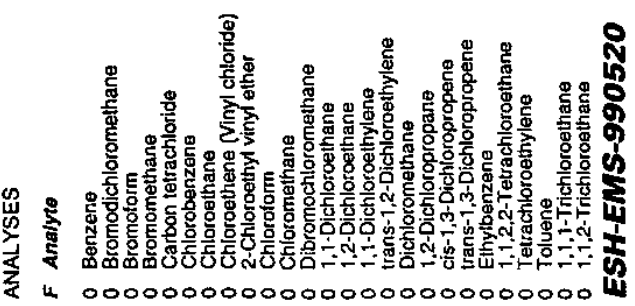




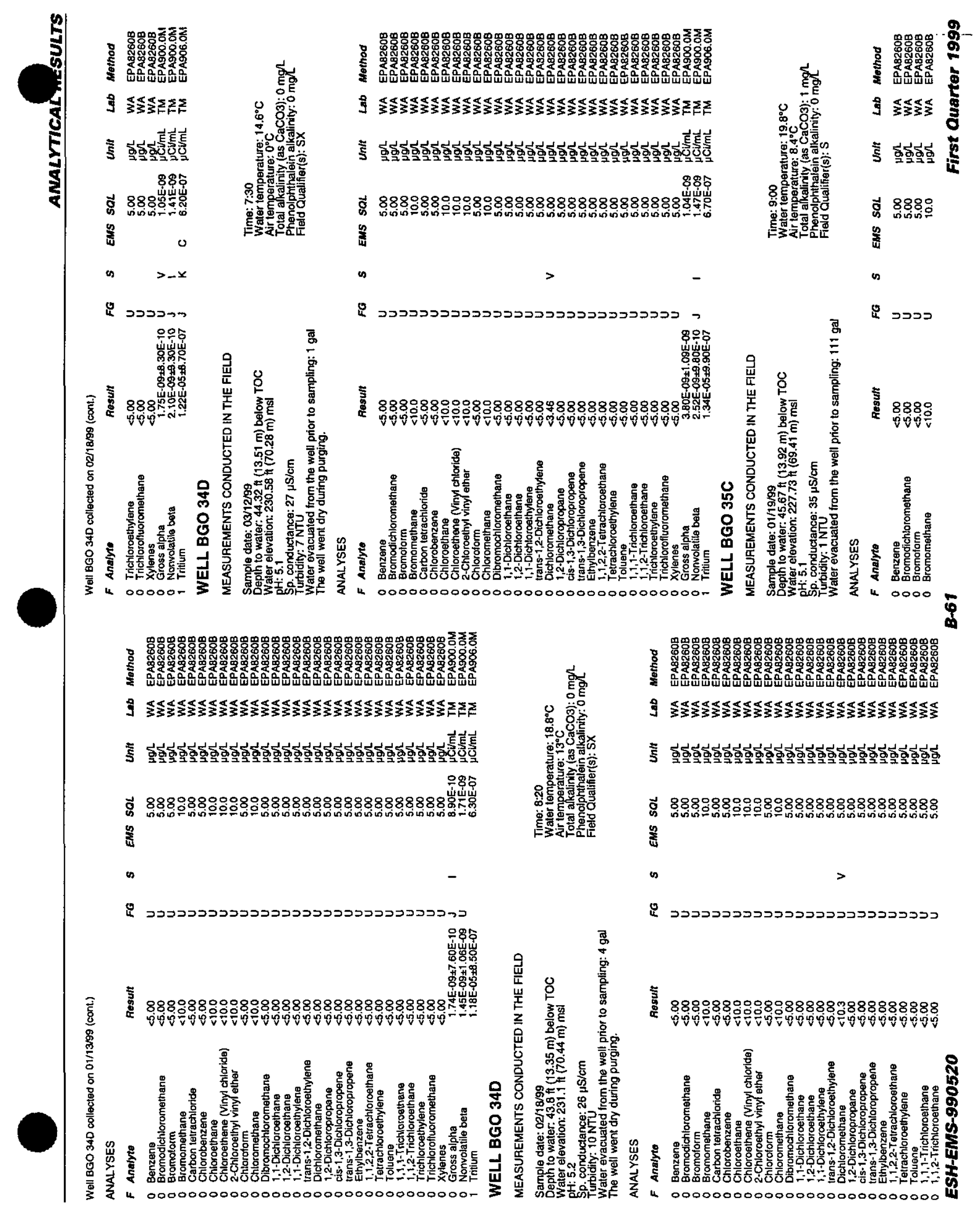



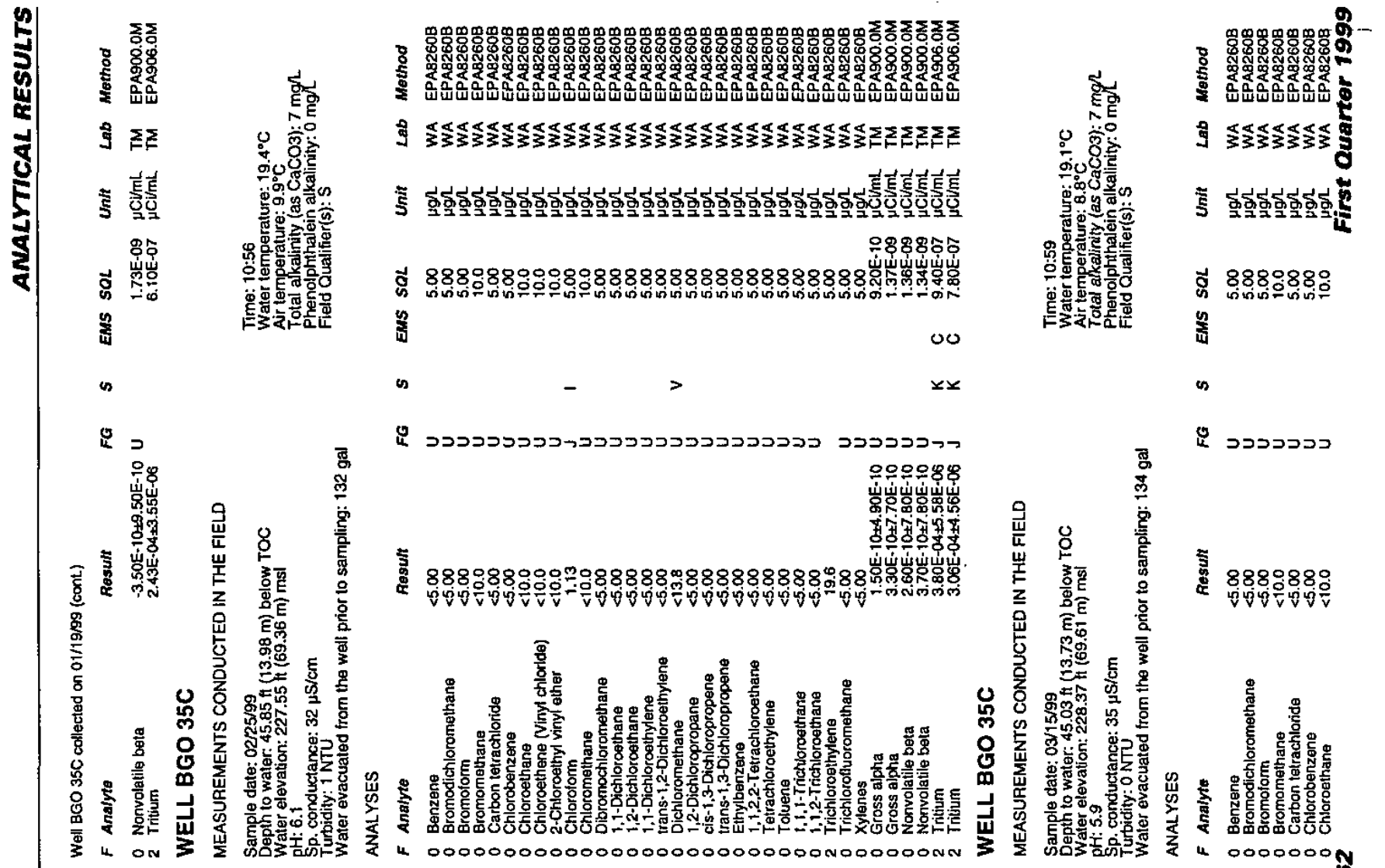

$$
\text { בכבכבכב }
$$

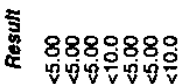

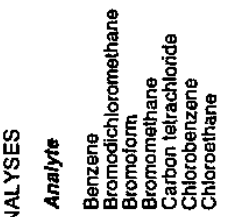

4 品

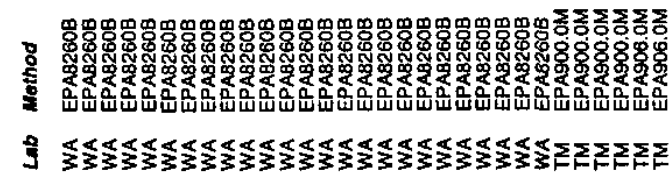

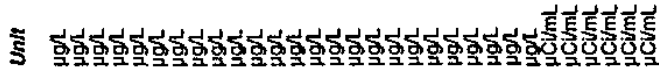

우우앵후우우

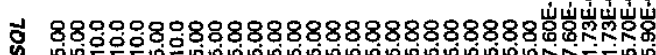

咨

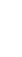

a

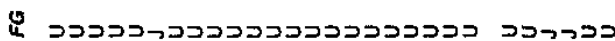

으응ㅇㅇㅜ 88

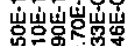

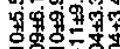

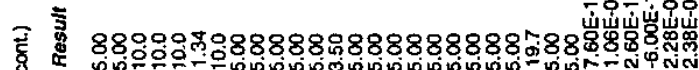

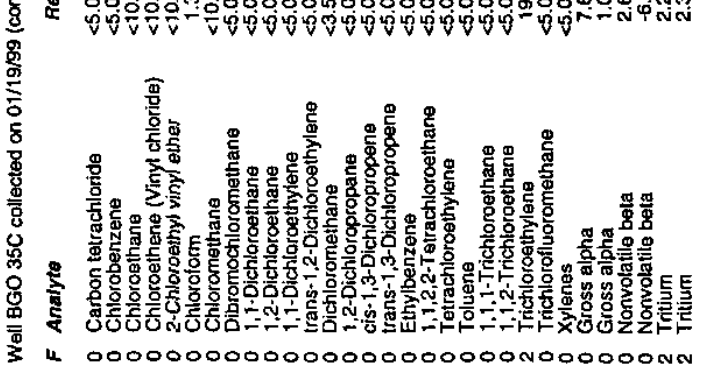

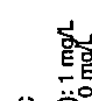

范品

\%ण

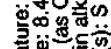

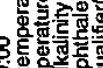

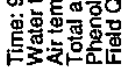
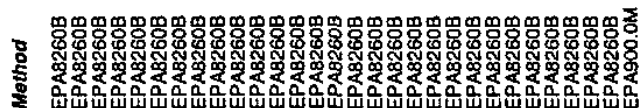

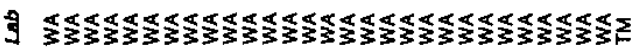

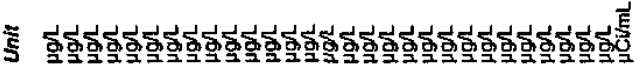

운

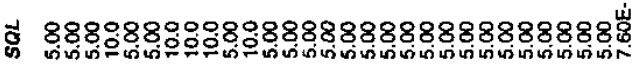

竞

is

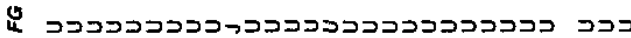

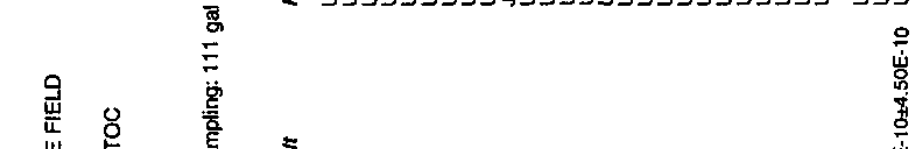

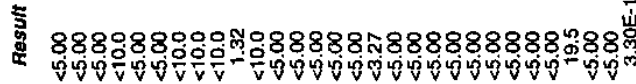

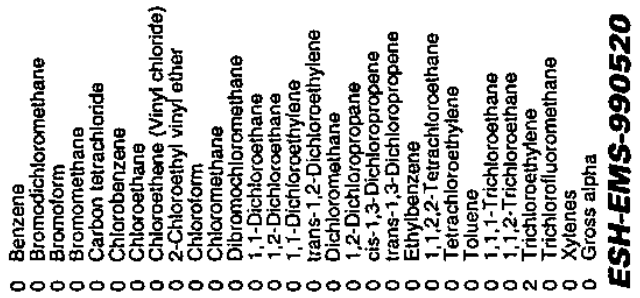




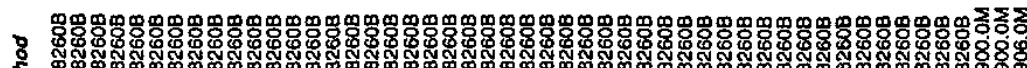

范

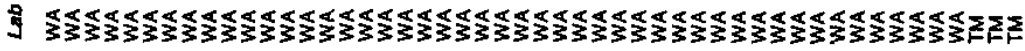

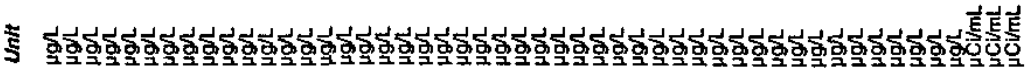

으웅

के 88888888888888988888888888888888888888888888888 \%

竞
要

幽范:

สำ

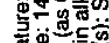

\%

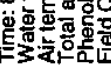

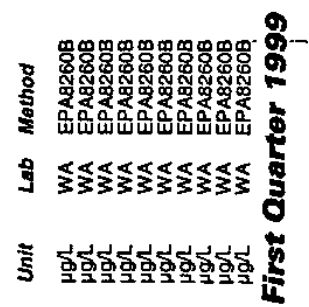

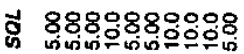

密

is

בכבכבכבכבי

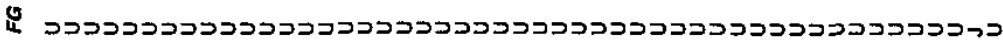

우용

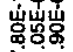

斗落

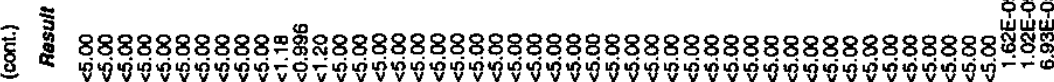

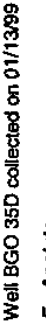

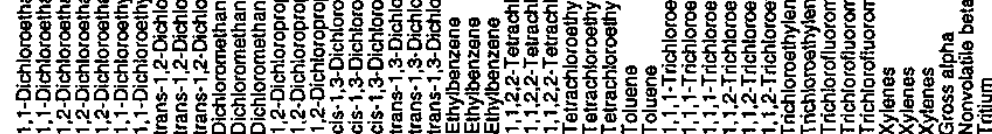

il $0000000000000000000000000000000000000000000000000 \mathrm{~N}$

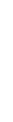

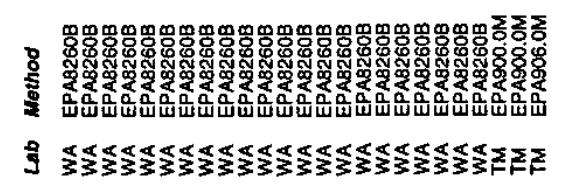

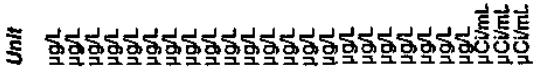

웅유

t) 8089888888888888888888

搴

is $\quad>$

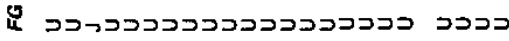

우웅

榢㸚

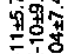

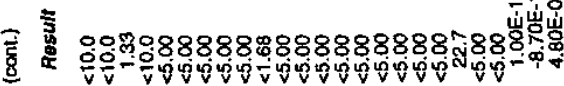

罗

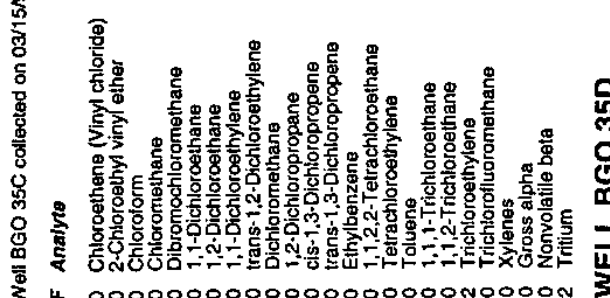

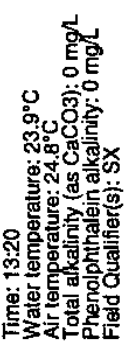

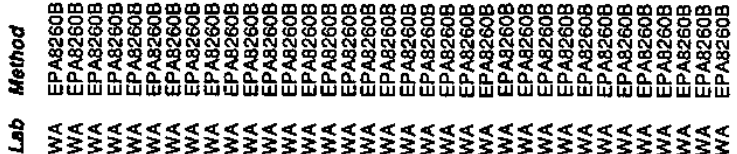

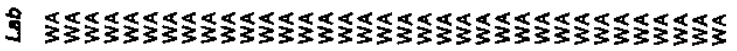

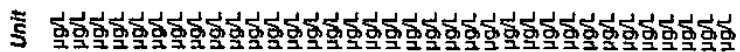

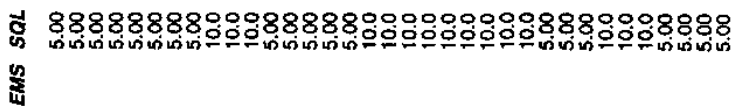

en

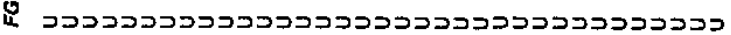

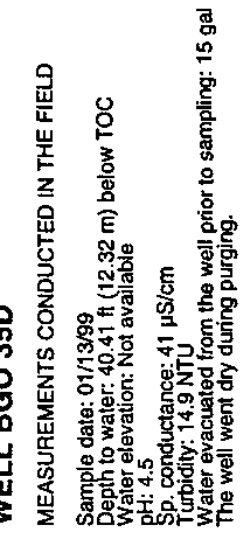

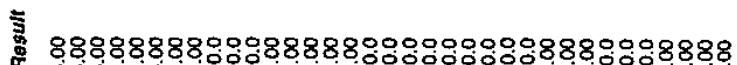

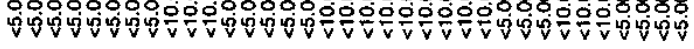

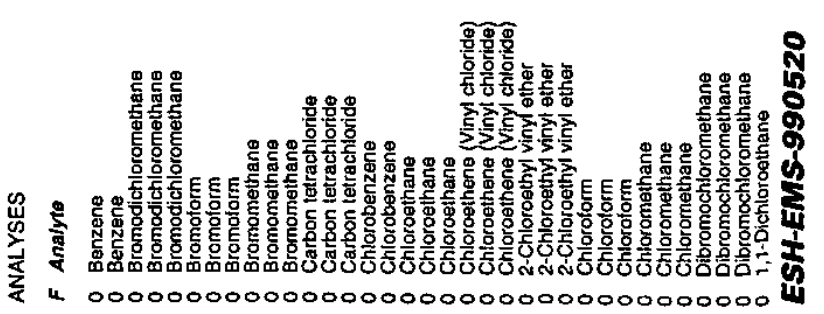




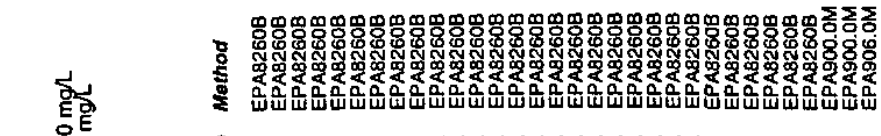

0

Si.

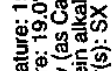

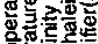

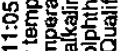

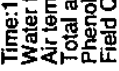

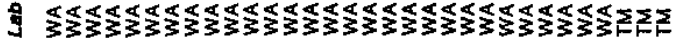

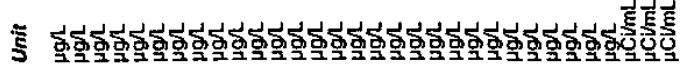

웅ㅎㅇ

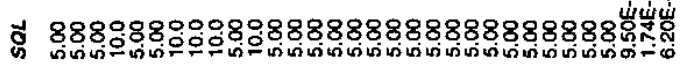

翣

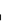

s.

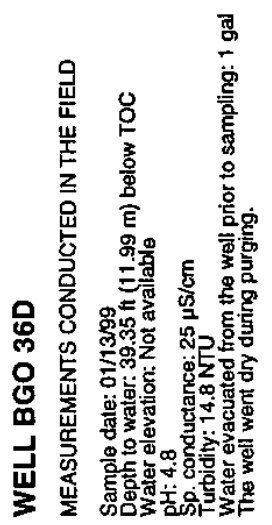

용ำ

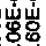

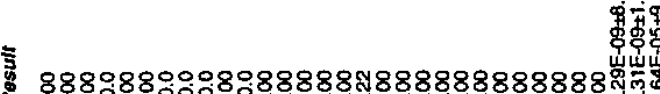

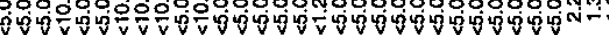

Бू.

范: Fig.

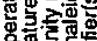

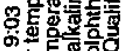

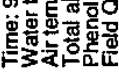

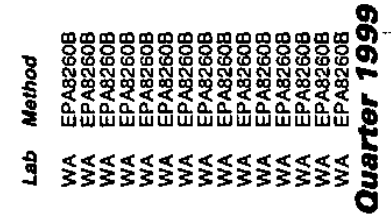

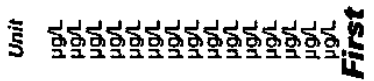

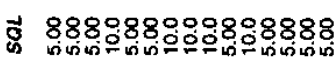

紊

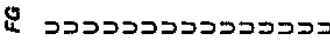

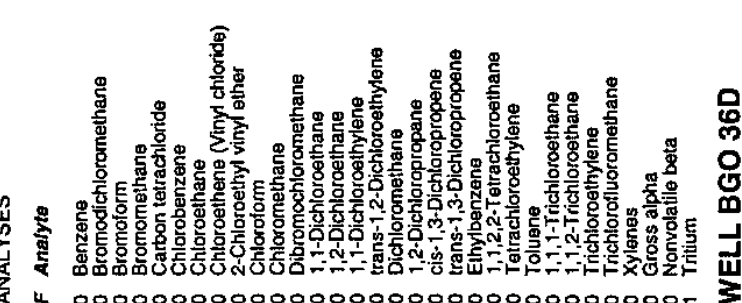

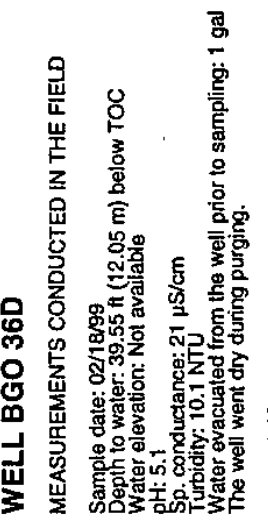

888988000808888

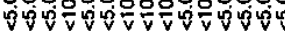

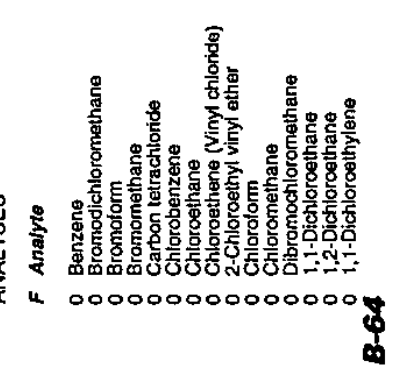

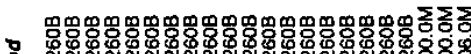

:

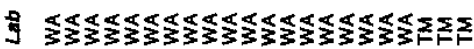

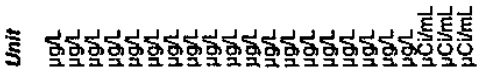

유은

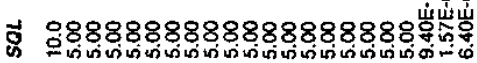

紊

n

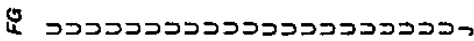

으으 8

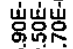

융음

氞

驾

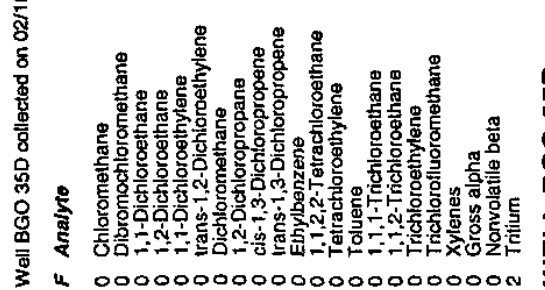

हृ่

品

Fuำ

这这通产

ᄂ

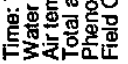

。

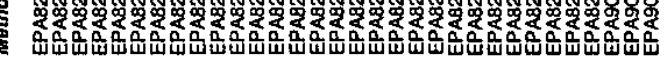

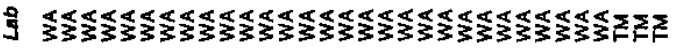

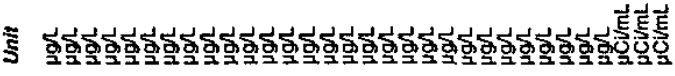

웅ㅎㅇ

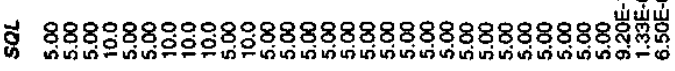

䱣

os

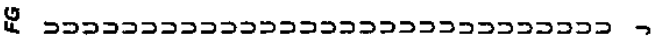

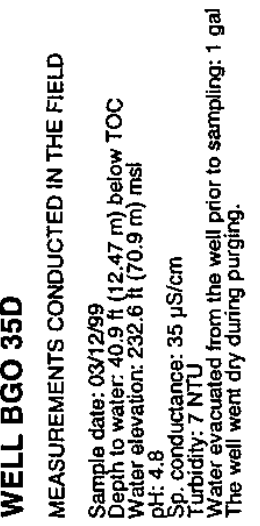

峈岩出

舟需落

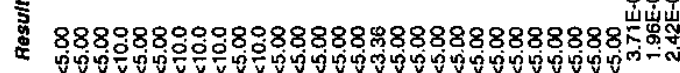

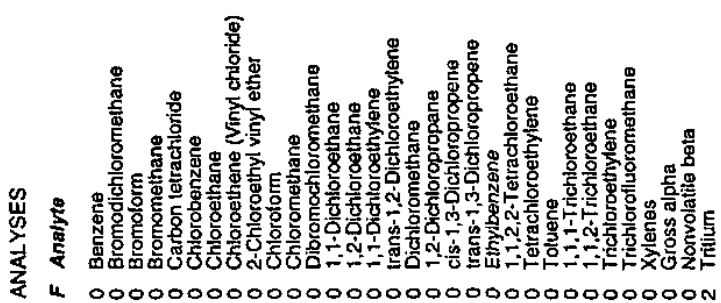




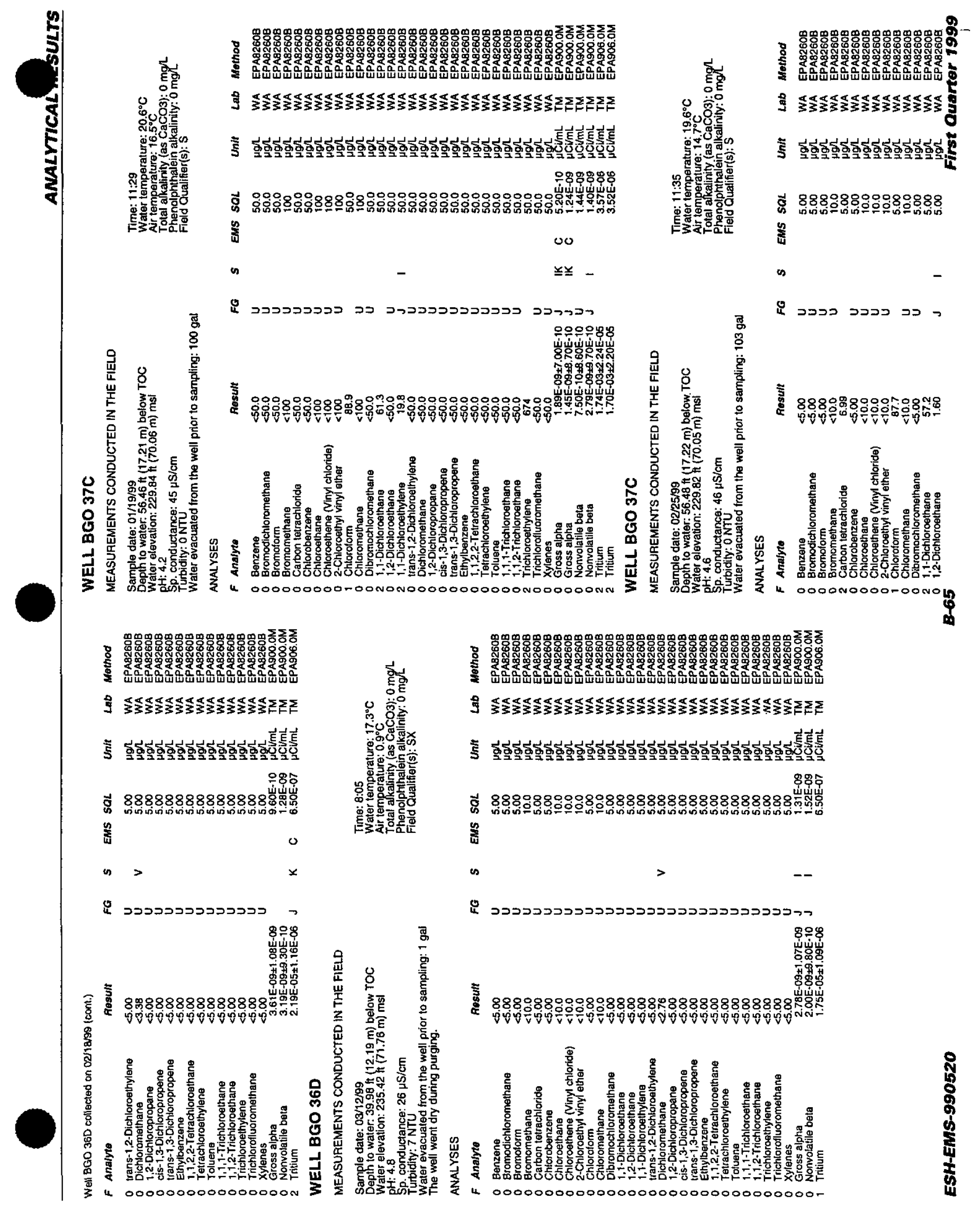



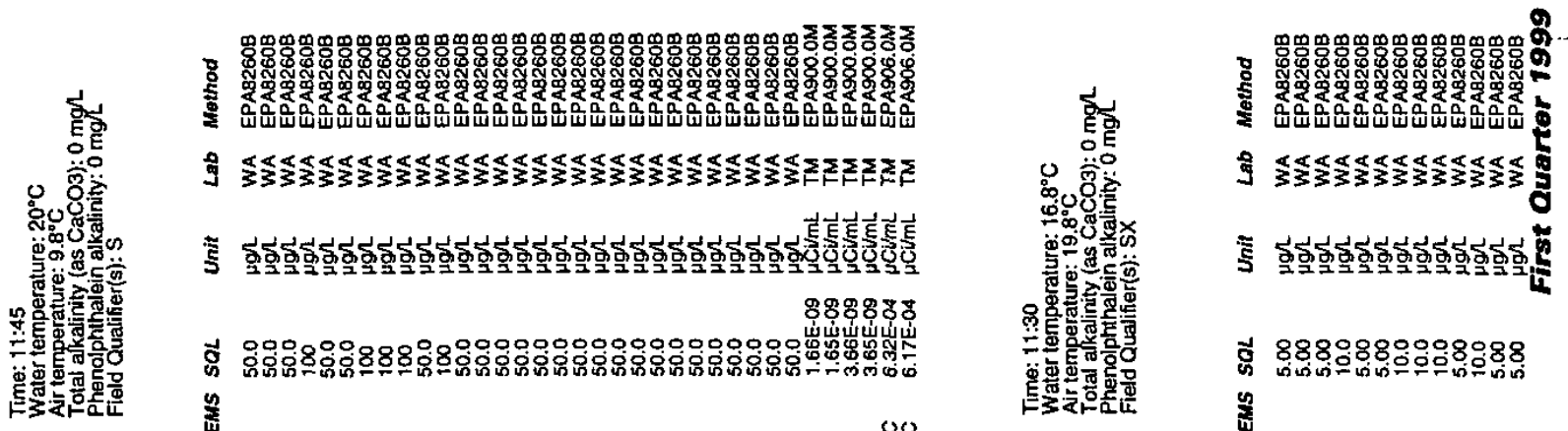

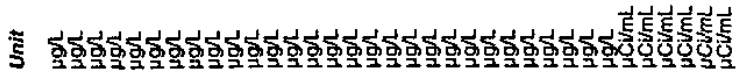

영영용ㅎㅇ

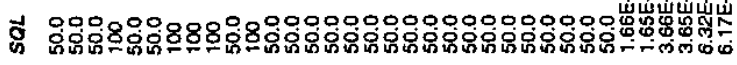
悉

oo

is 00000000000000900000000000000 - ــ

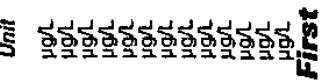

ธิ $8888 \% 80 \%$

密

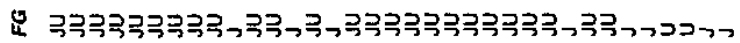

a

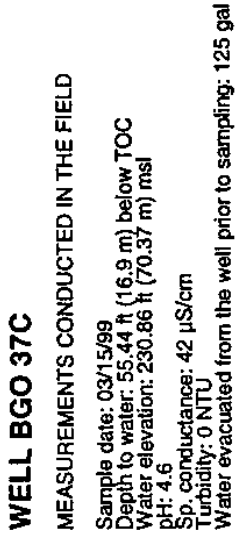
बुँ

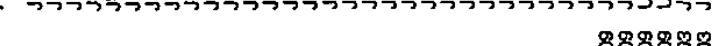

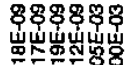

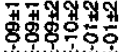

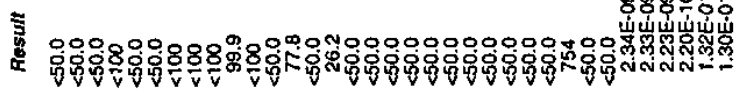

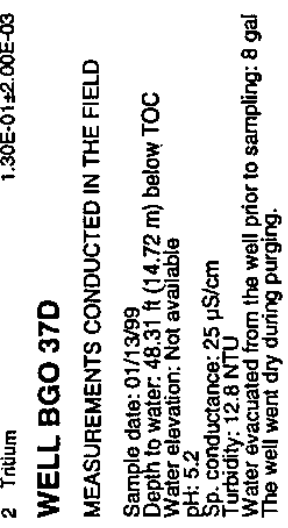

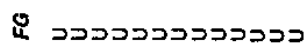

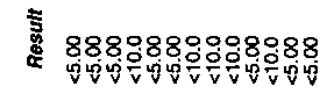
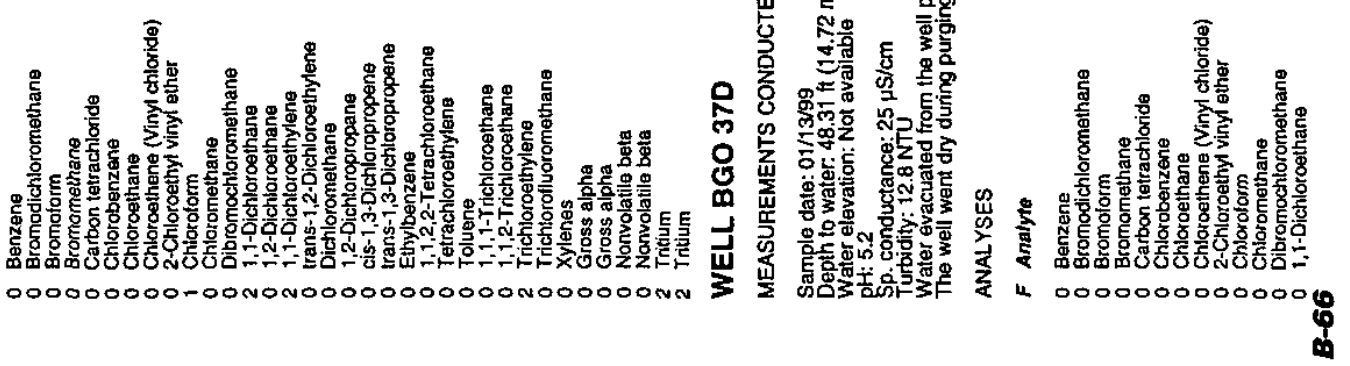

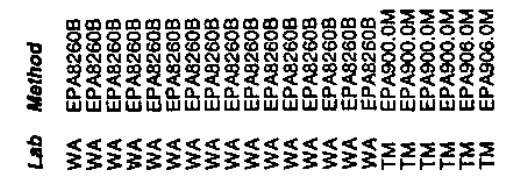

కั 호허 우요옹ㅇㅇ

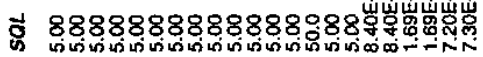
量 离 $-\infty$

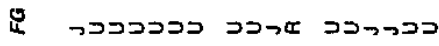

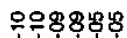

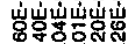
तित्ञ

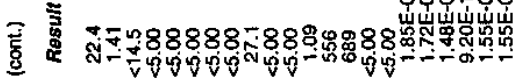

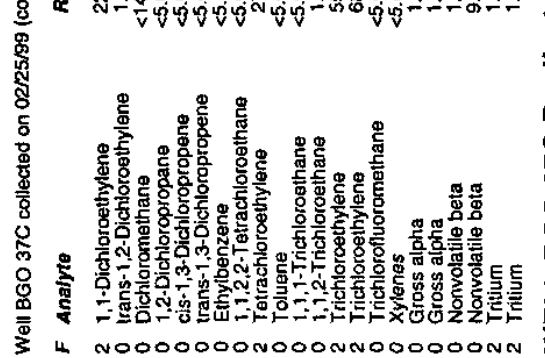

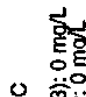

范茫 siर

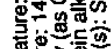
o

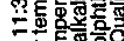

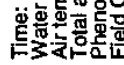

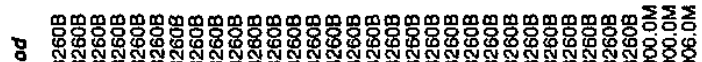
喜

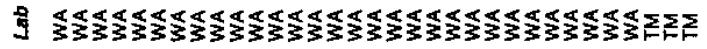

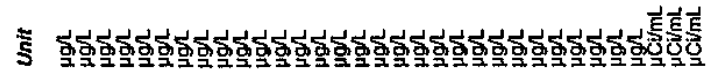
으용우

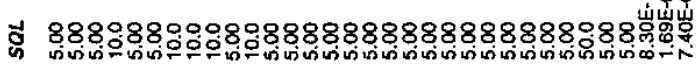
峦
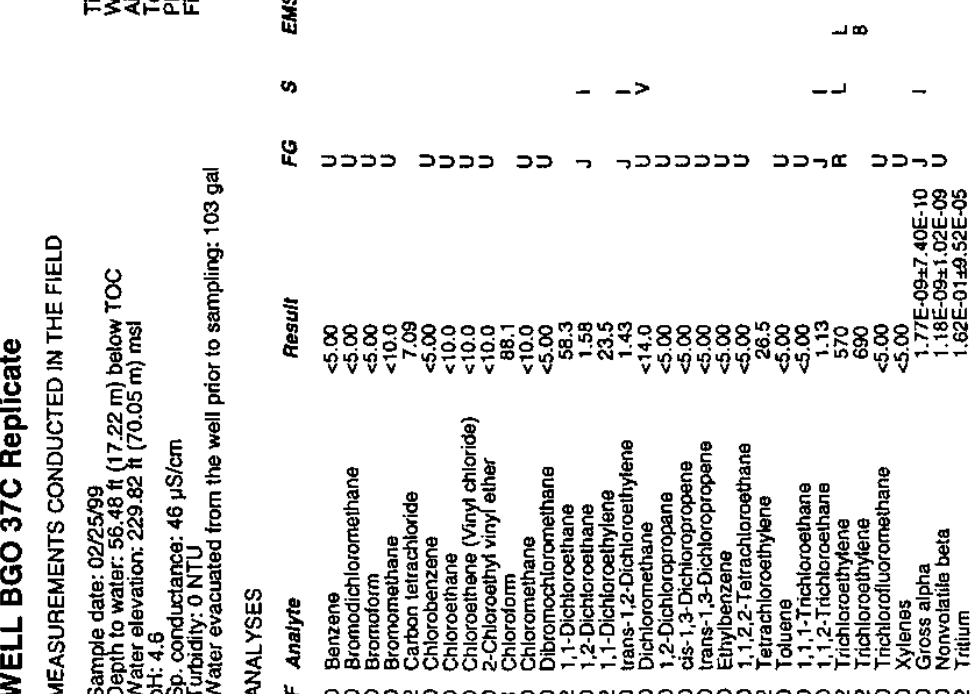

\begin{tabular}{l}
8 \\
\multirow{5}{5}{} \\
8 \\
8 \\
0 \\
$\frac{5}{5}$ \\
$\frac{1}{5}$ \\
$\frac{1}{4}$
\end{tabular} 


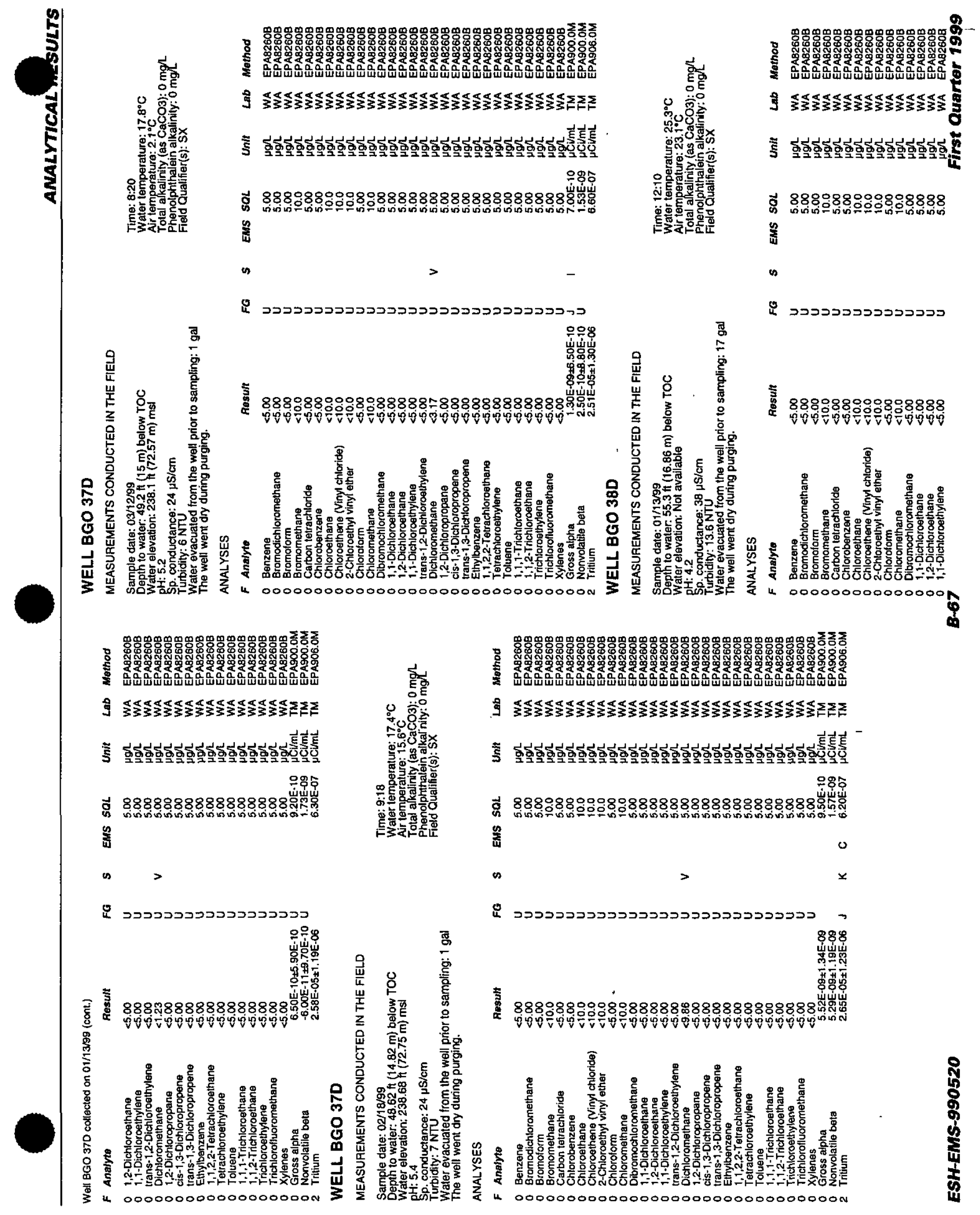




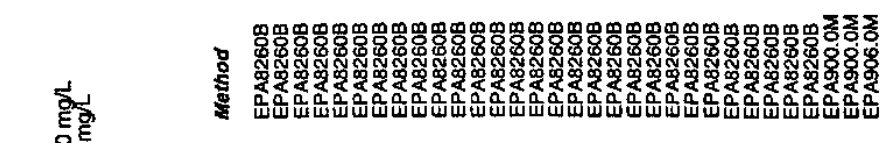

范

Note

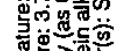

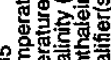

触

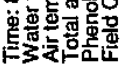

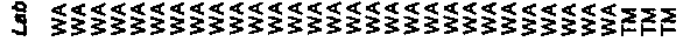

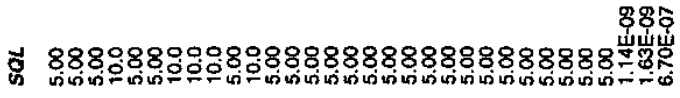

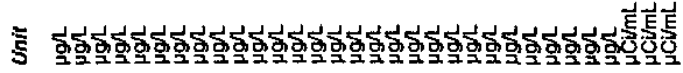

竞

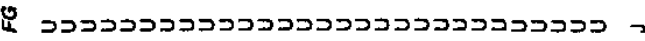

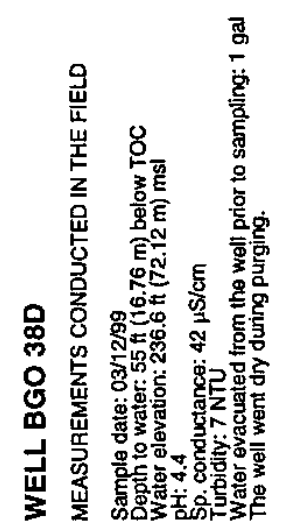

888

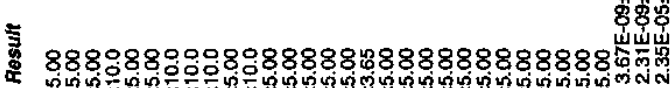

要

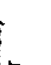

密

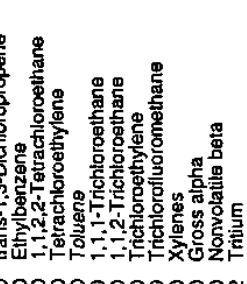

覀

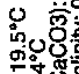

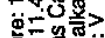

政

오에.

은.

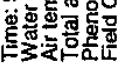

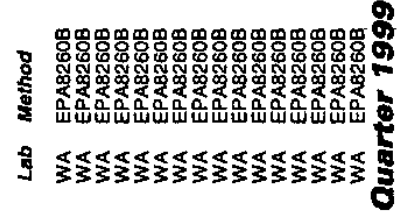

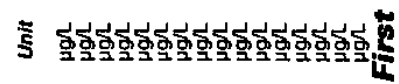

ปี 8880880008088888

竞

is

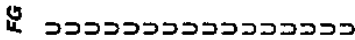

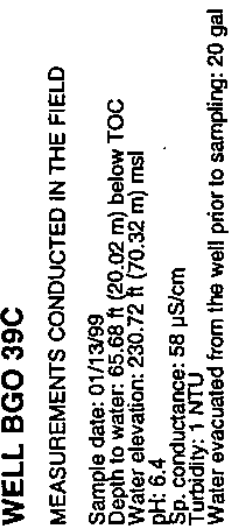
竞 8880880008088888

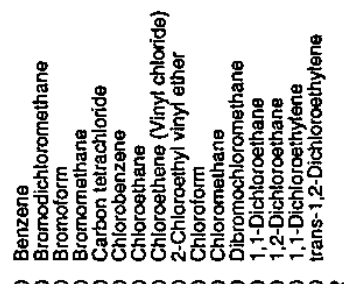

$0000000000000000 \%$

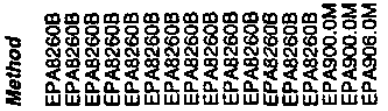

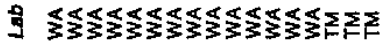

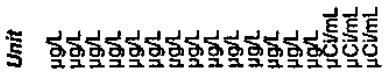

웅ํㅇ

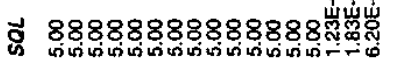

竞

जि

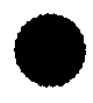

$\infty$

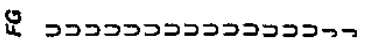

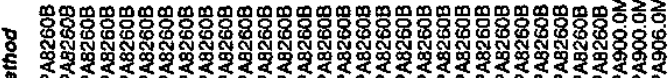

รู่

인

苑蓄

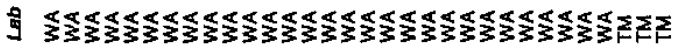

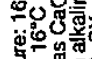

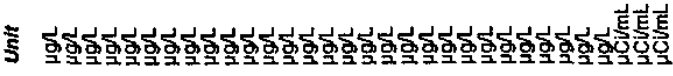

(웂

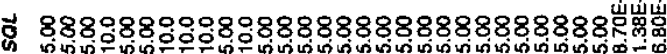

紊

0

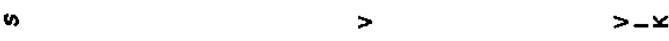

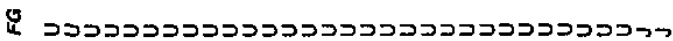

$88 \%$

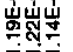

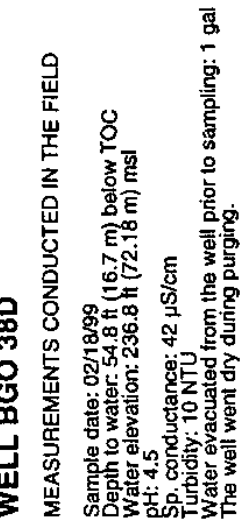

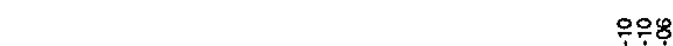

岁岸产

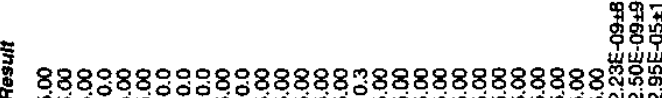

ชิ

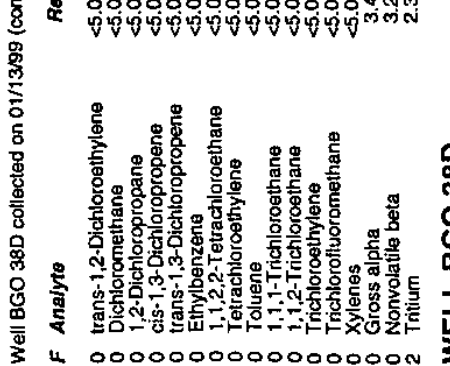

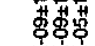

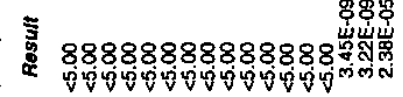

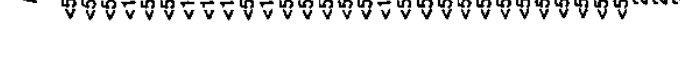

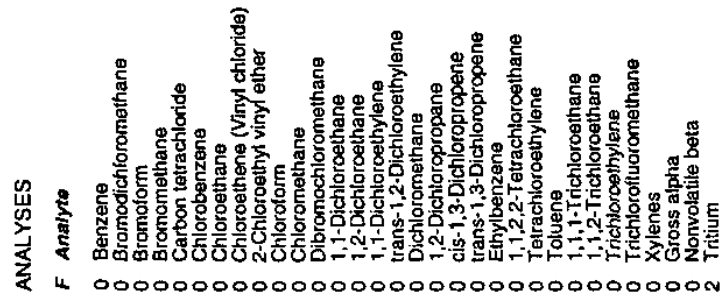




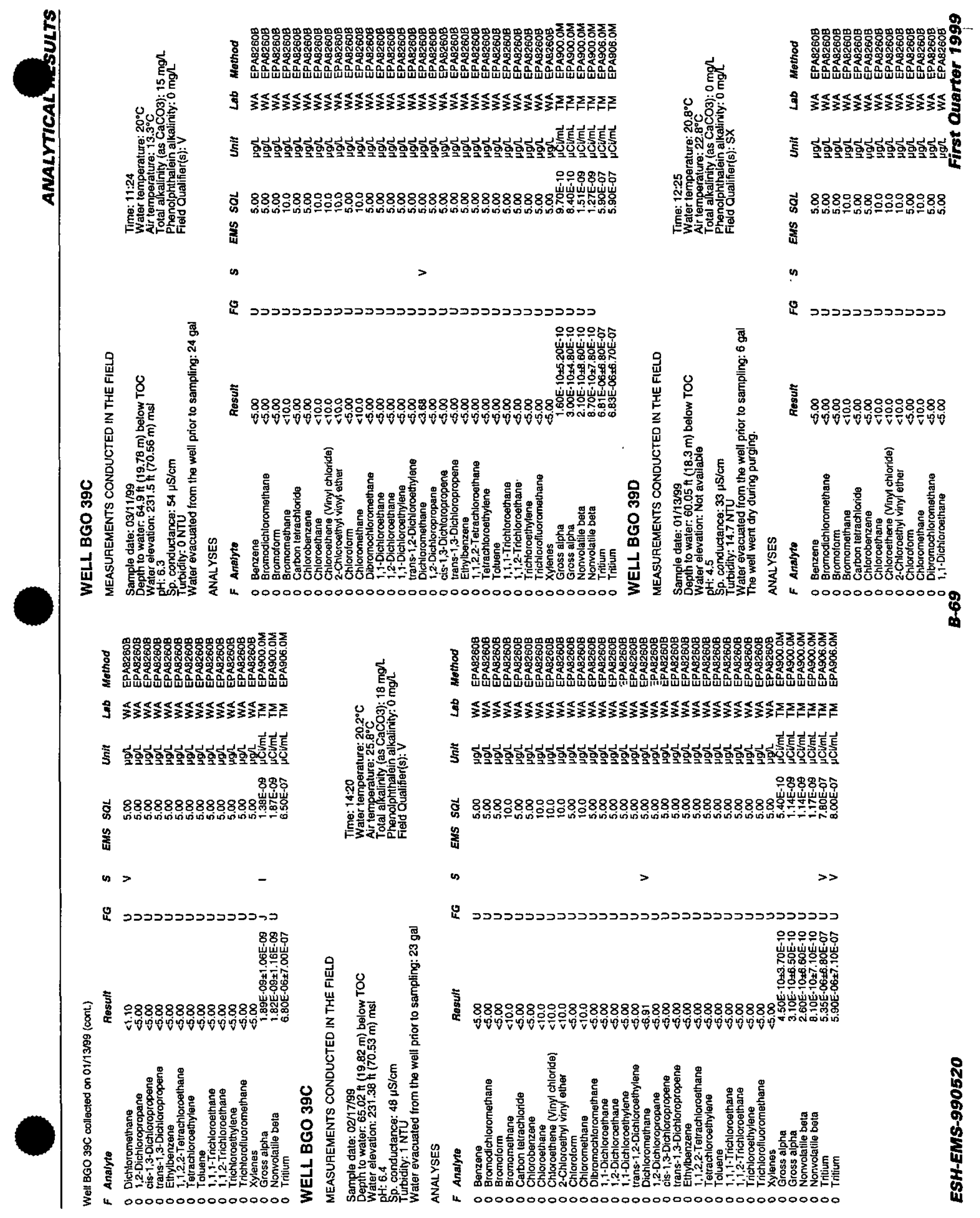




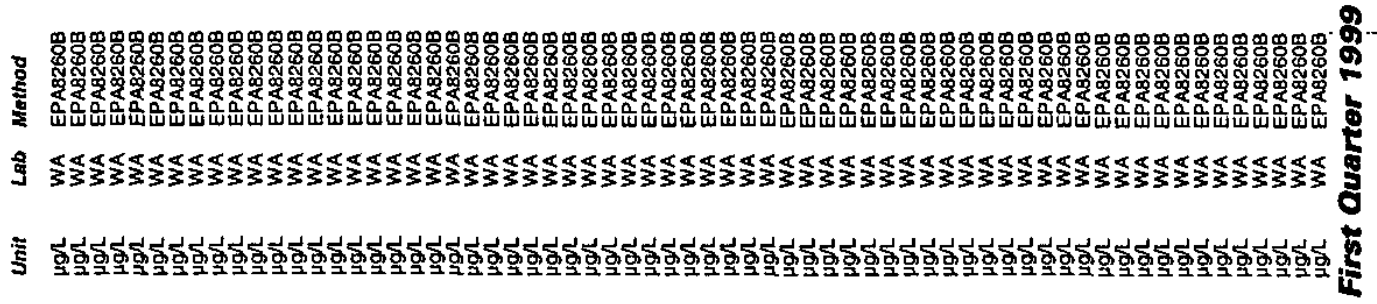

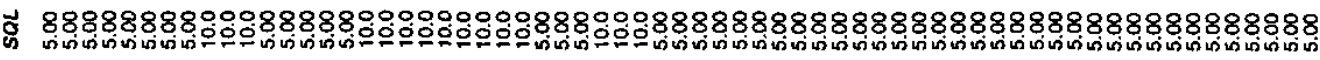
辛

n

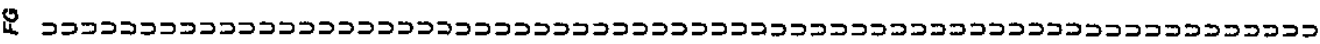

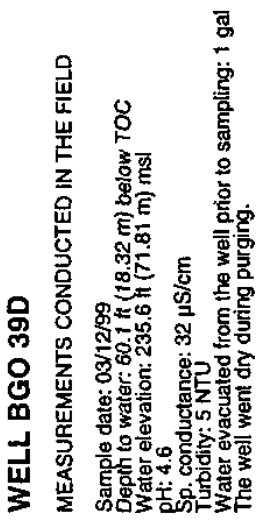

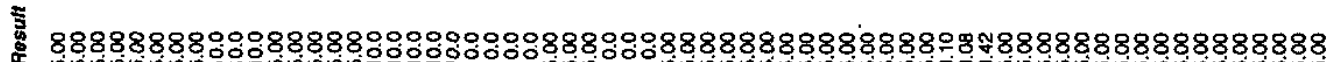

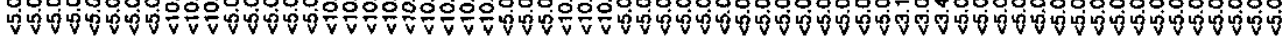

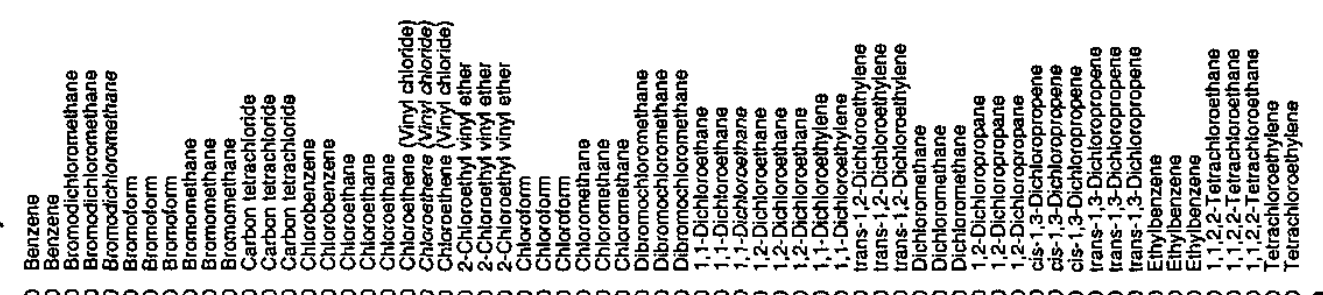

L. 000000000000000000000000000000000000000000000000000000000000000000

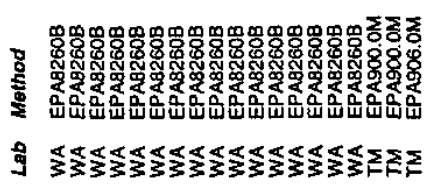

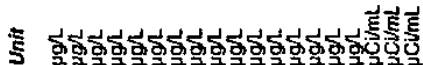

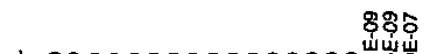

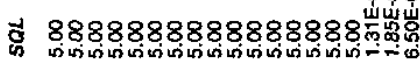

紊

as

Iㄴ

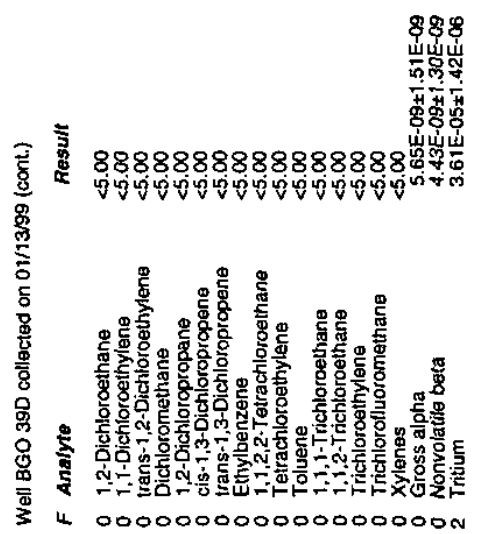

垔

㻤

กิ่0

हैं

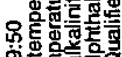

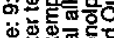

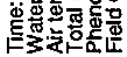

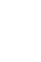

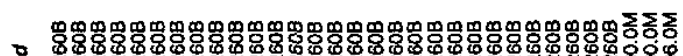

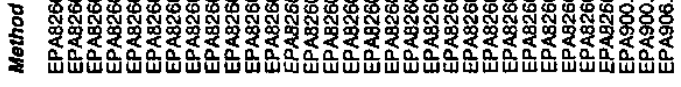

త

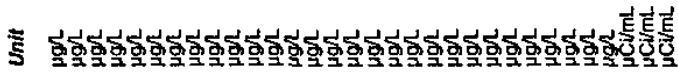

웅웅

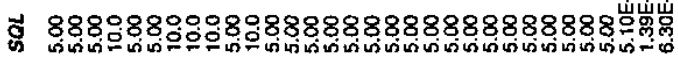

竞

0

m

$>$

$>\mathbf{x}$

幽

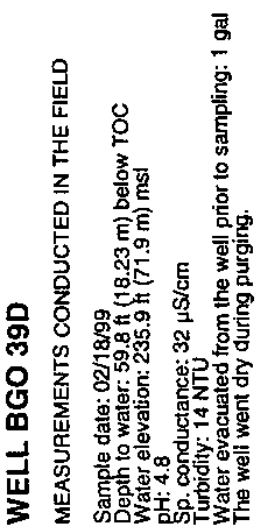

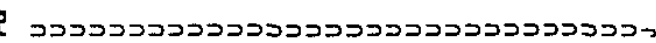
으요 8 岁演票 需贺

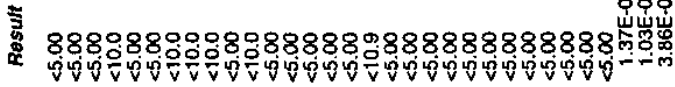

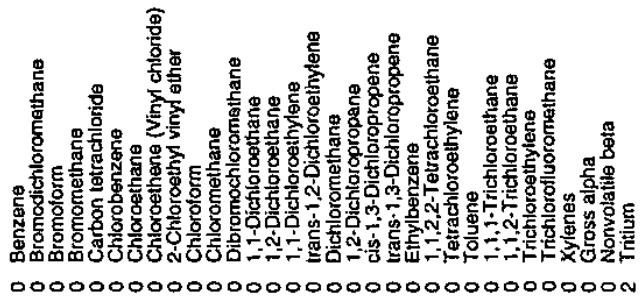




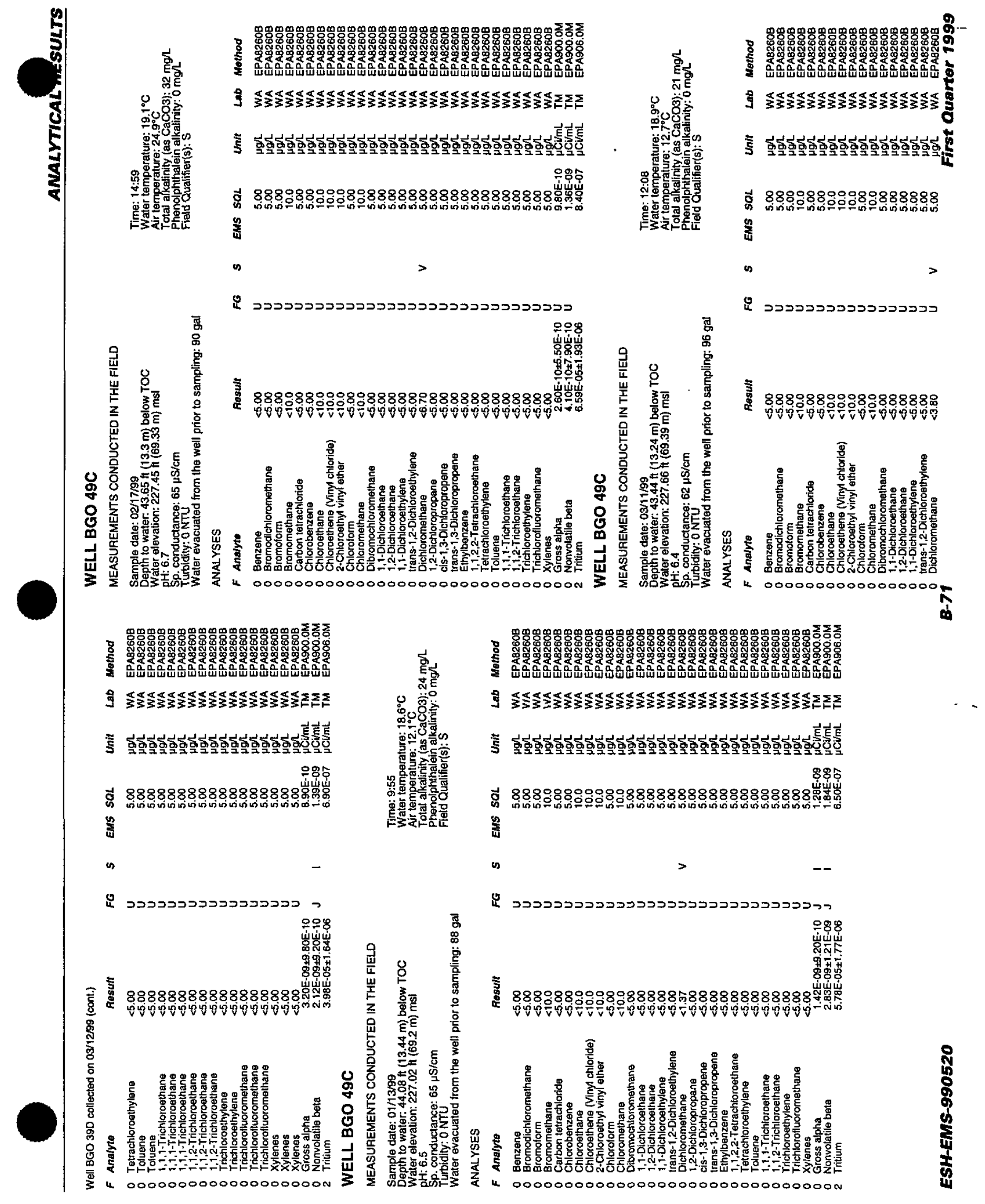



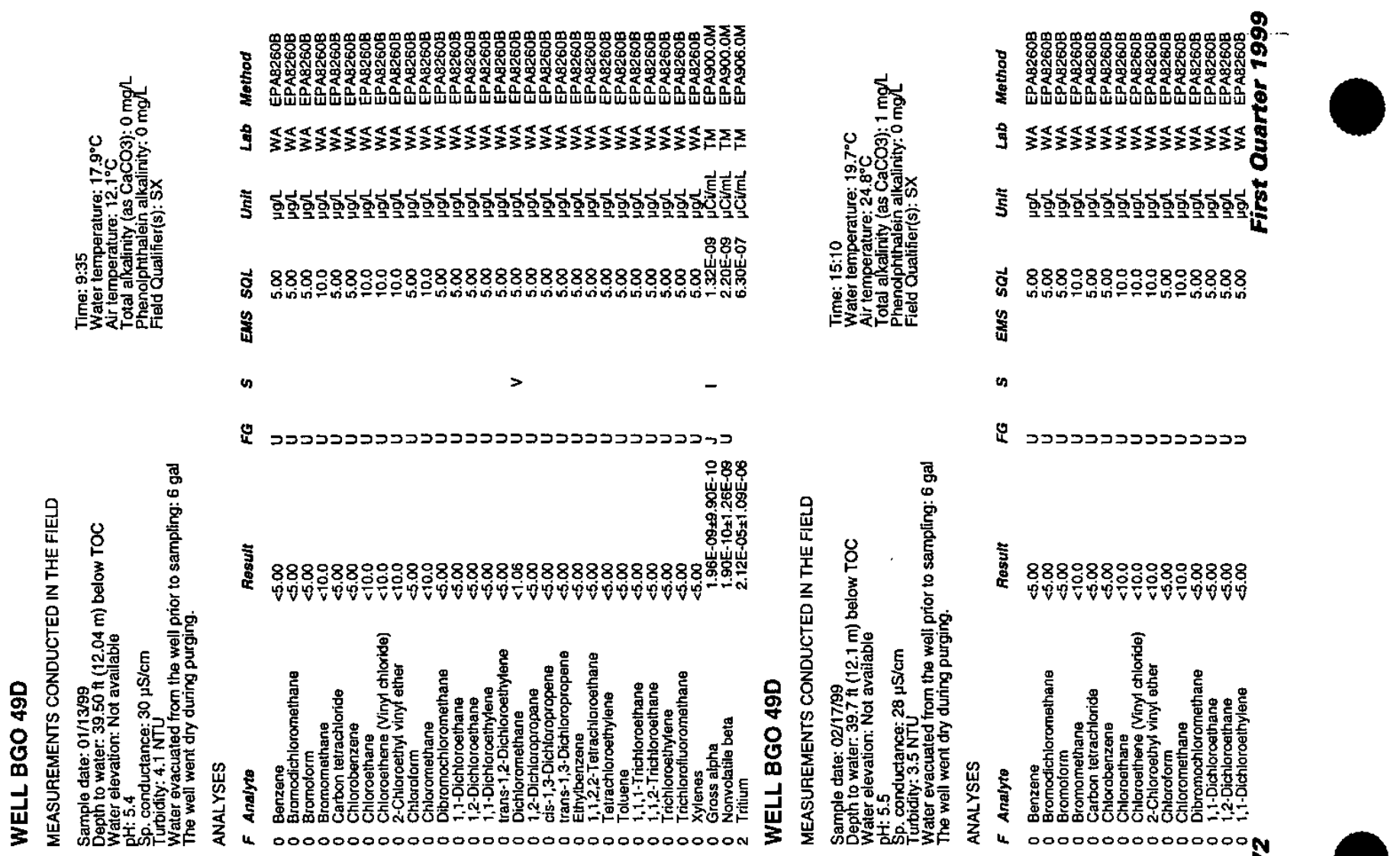

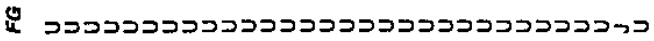

נבכבכבכבכבכבכב

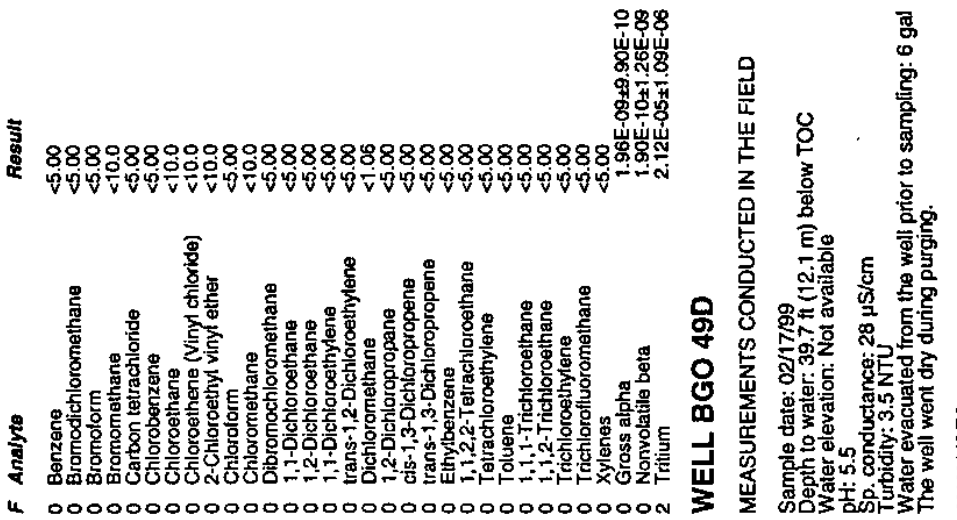
产 $888 \% 88000808888$

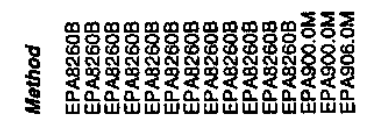

9

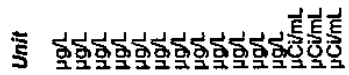

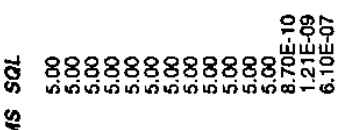
章

is

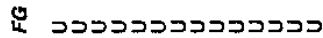

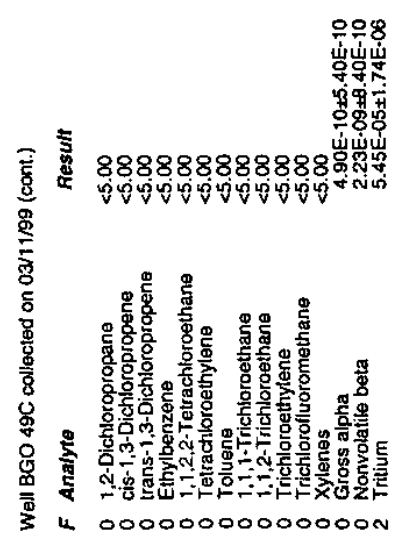

\section{.}

.
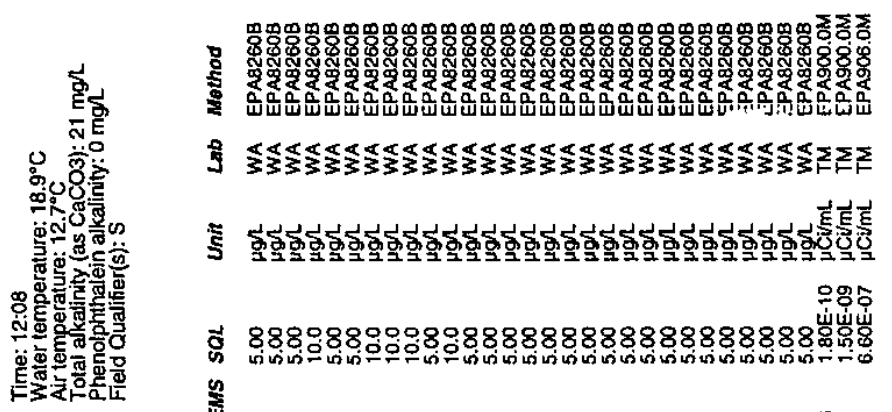

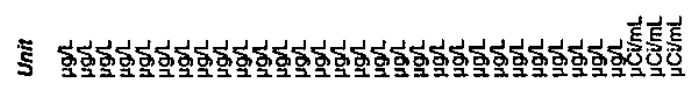

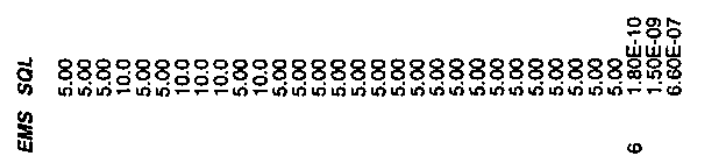

is

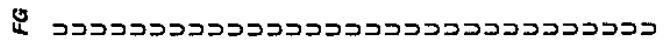

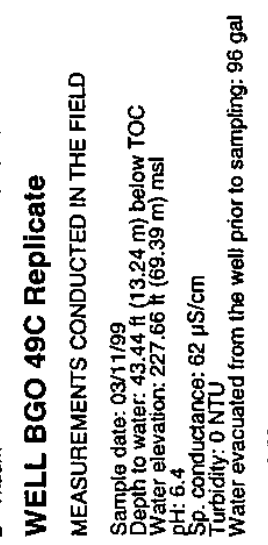

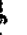

14.

等

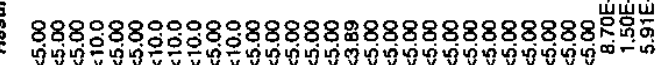

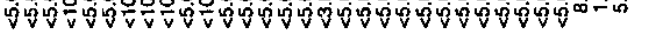

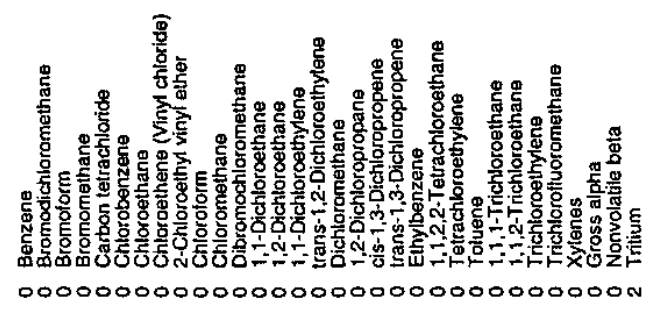

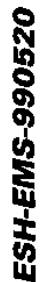



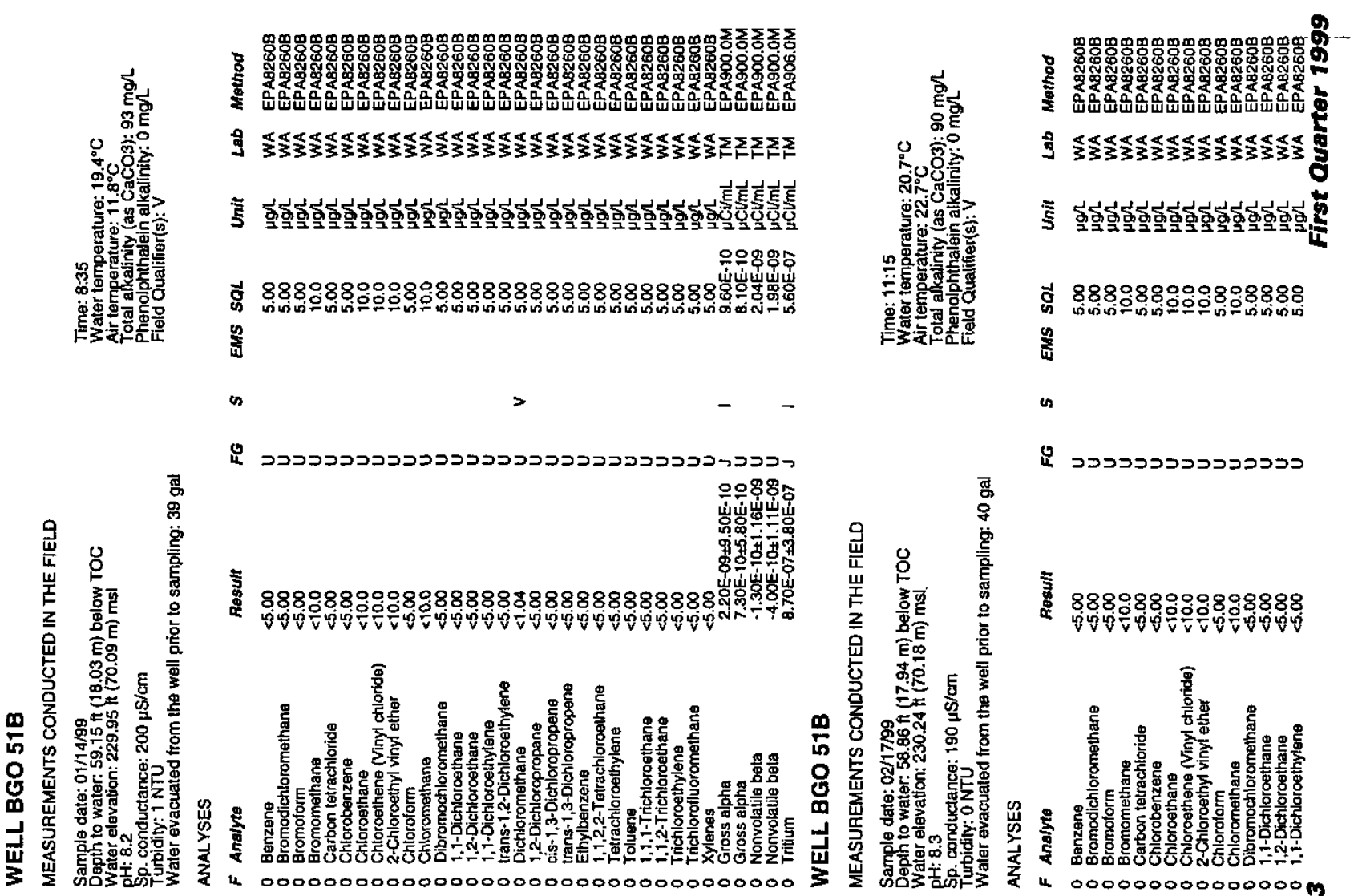

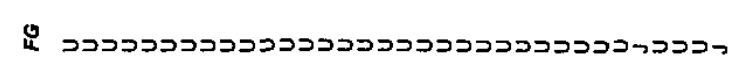

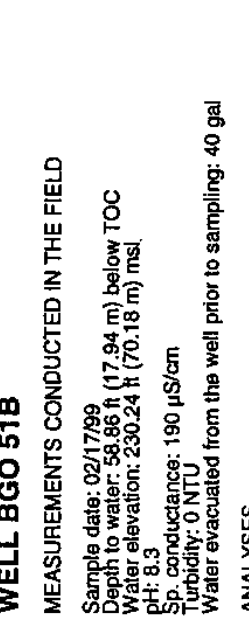

a

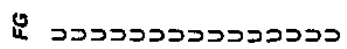

으유용

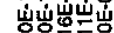

काजन

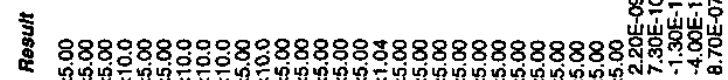

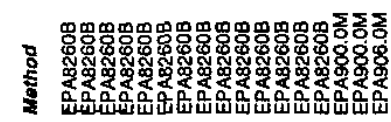

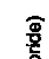

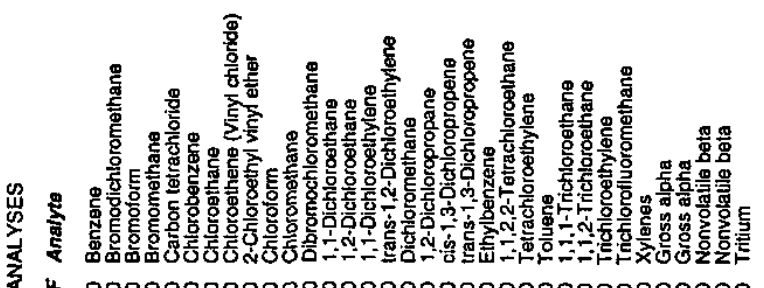

穿

言 888888800080888

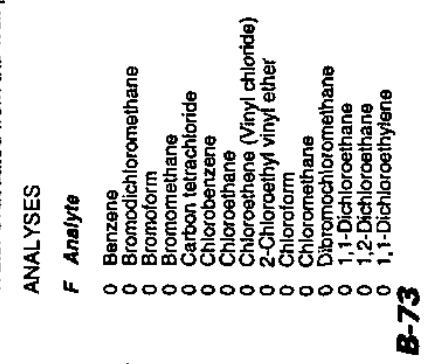

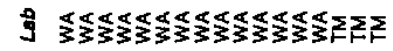

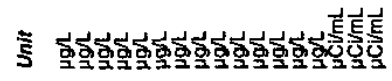

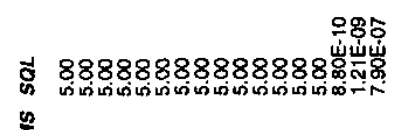
密

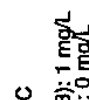

落:

\%ock

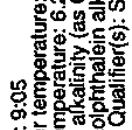

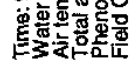

$\infty>$

בכבככככככבכככככנ

0.98

这岁岁

웡ㅎㅁ

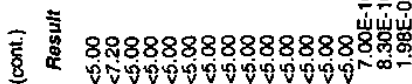

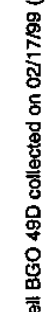

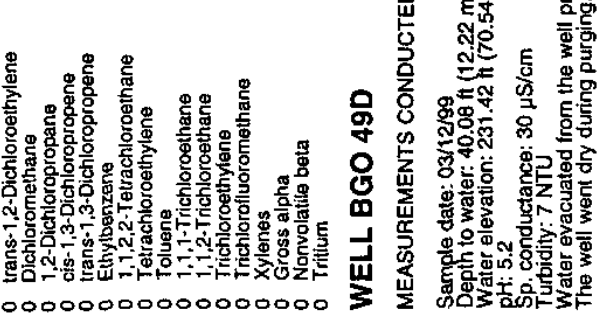

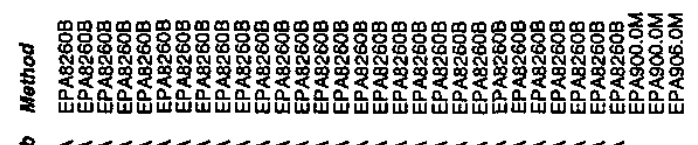

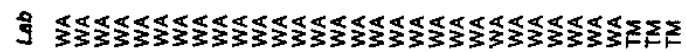

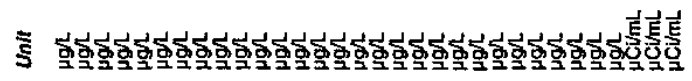

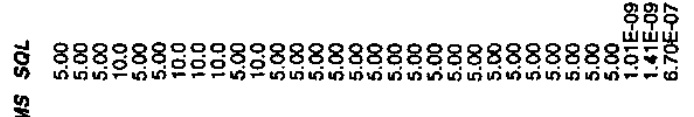
量

es

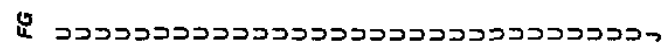
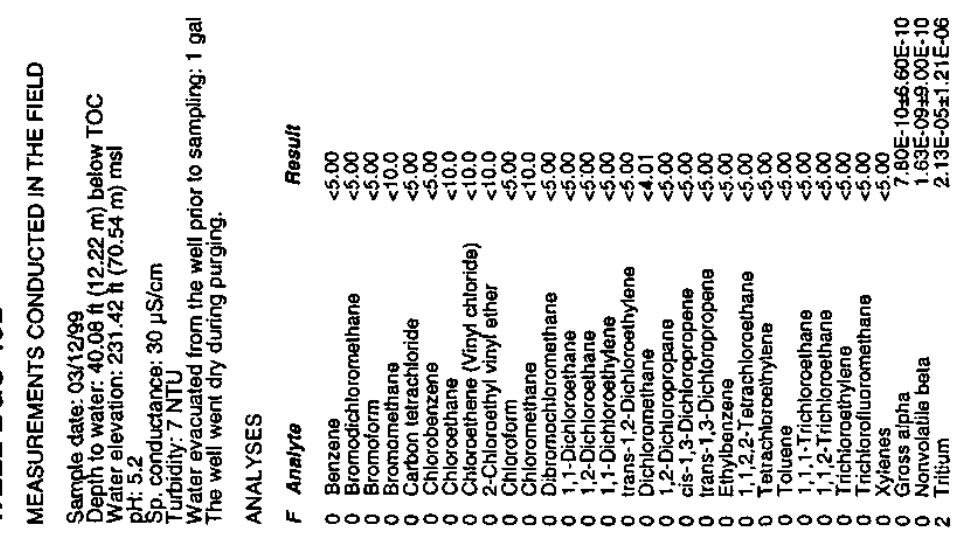


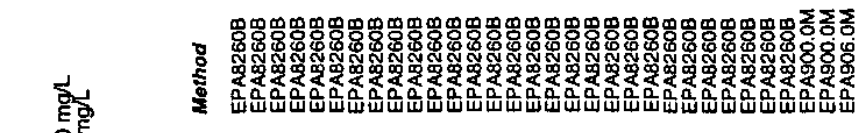



o.

政

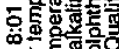

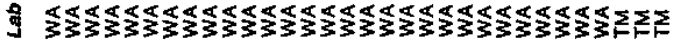

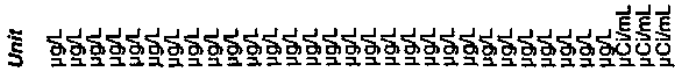

으양호

б.

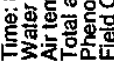
塞

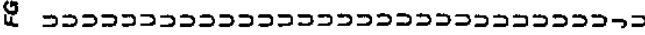

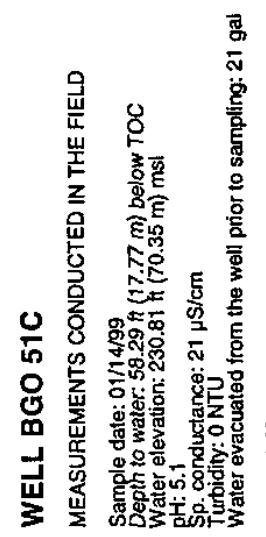

e.

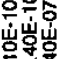

호을

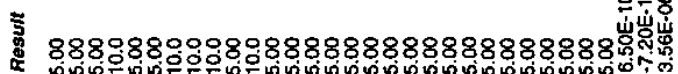

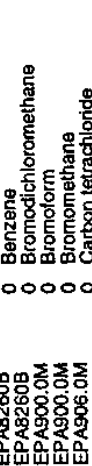

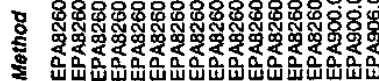

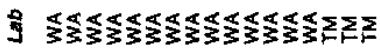

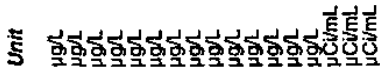

蛋

票<smiles>C1CCCC1</smiles>

\section{.}

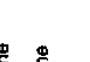

(1)

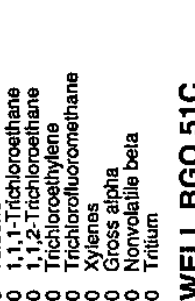

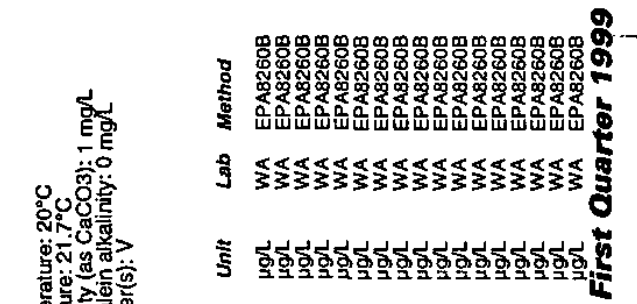

ธิ

管

ז

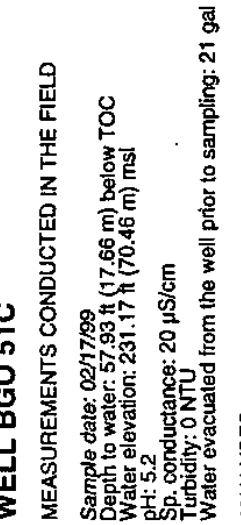

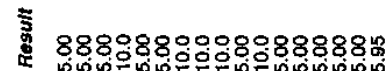

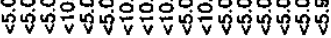

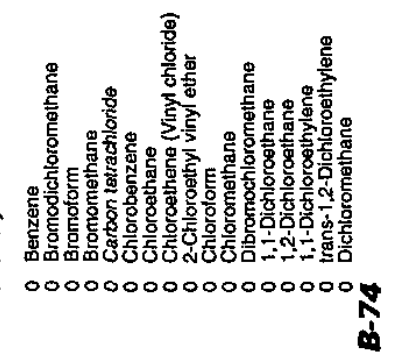

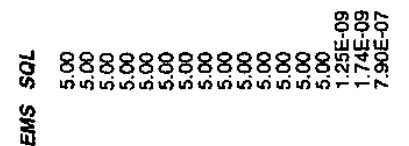

蔐

$\infty>$

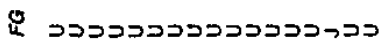

요웅

崩岁学

要势

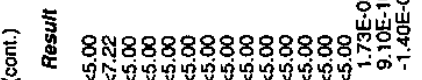

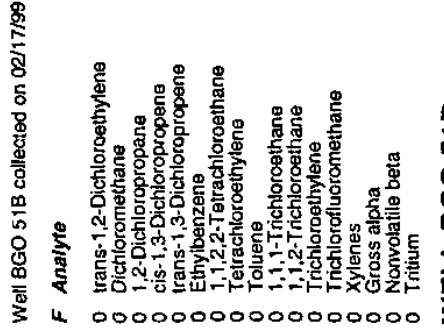

范.

o.

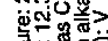

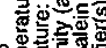

กั่

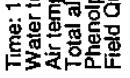

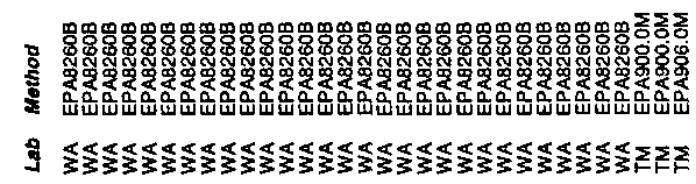

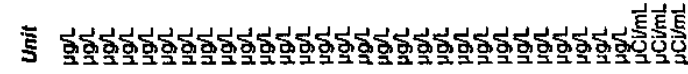

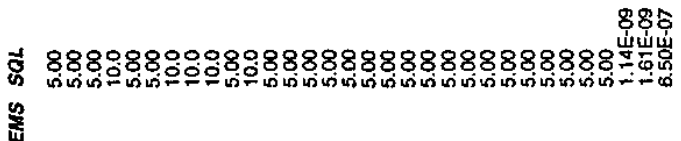

is

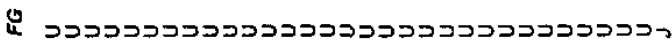

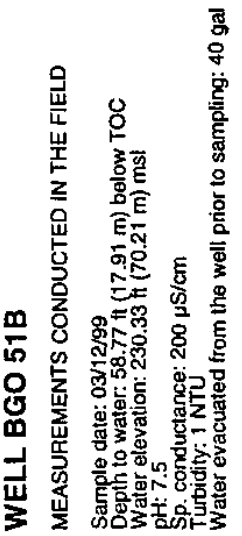

\section{要}

아요

कำ

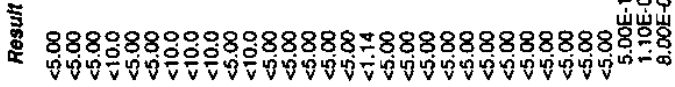

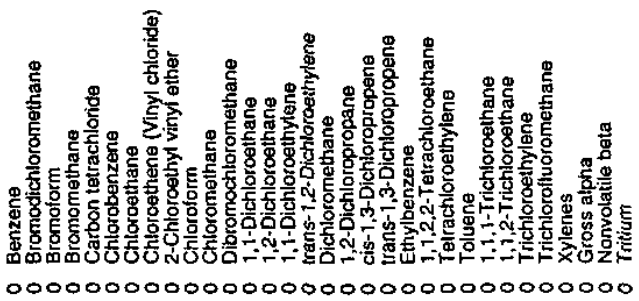




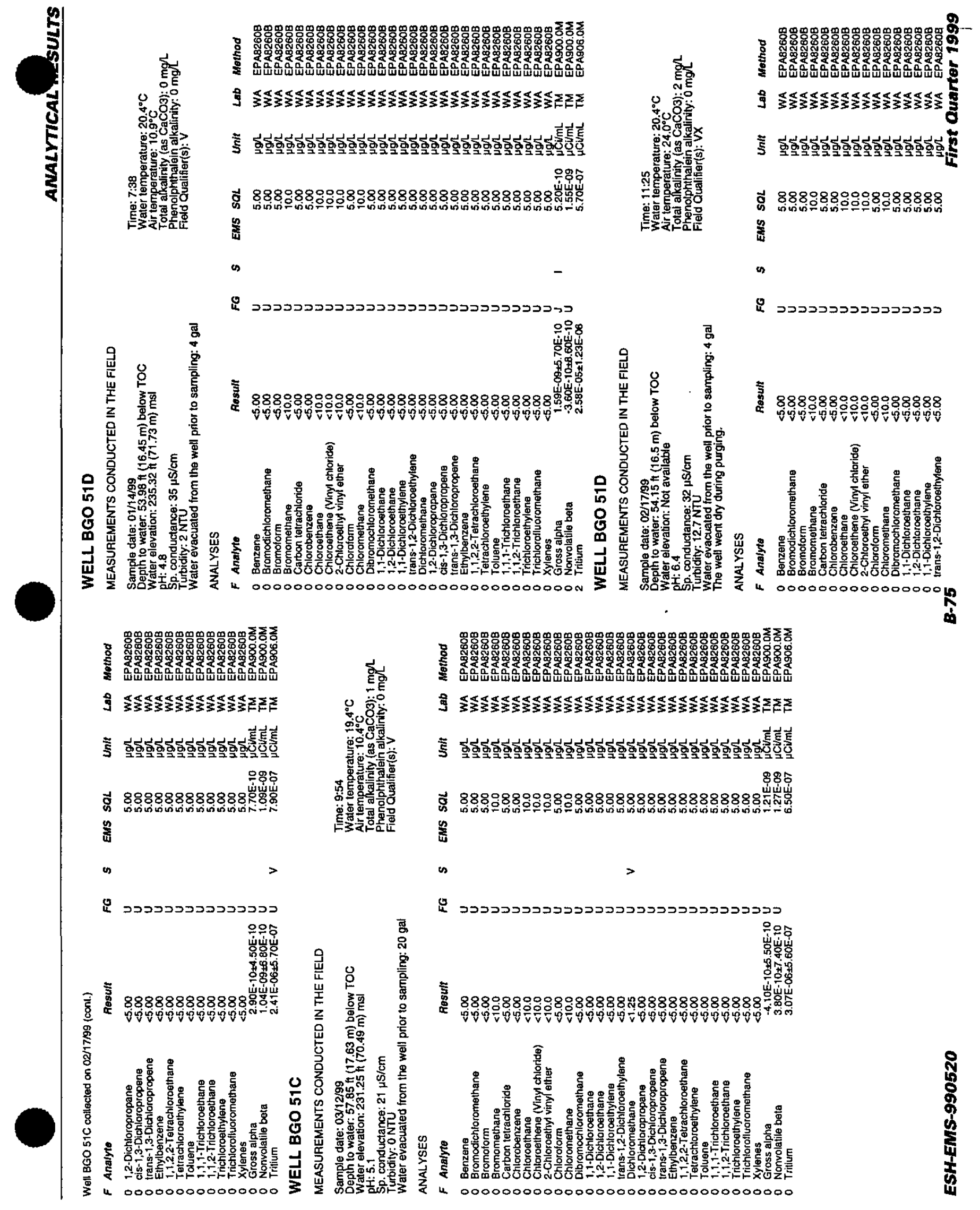



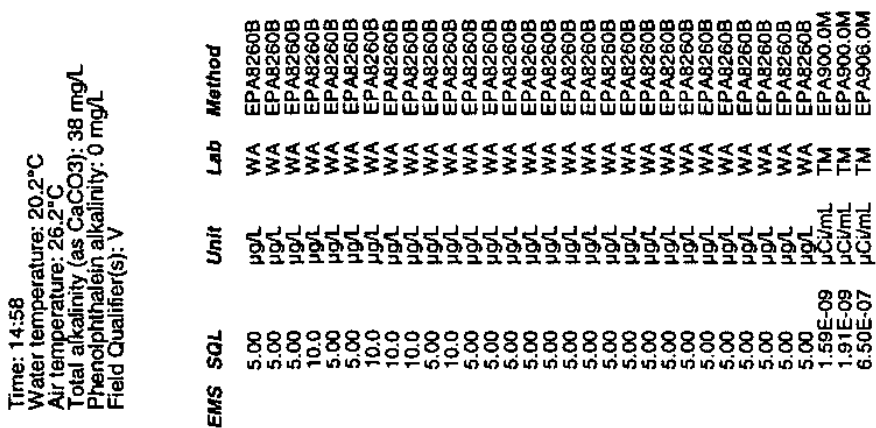

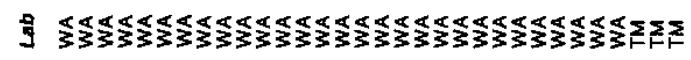

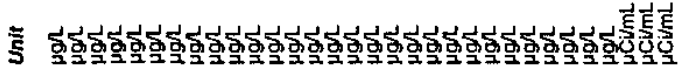

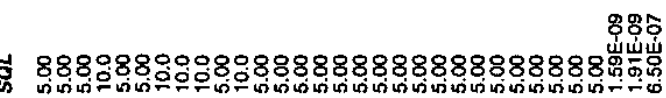

竞

(1)

is

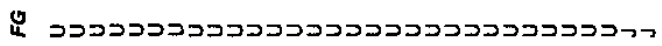

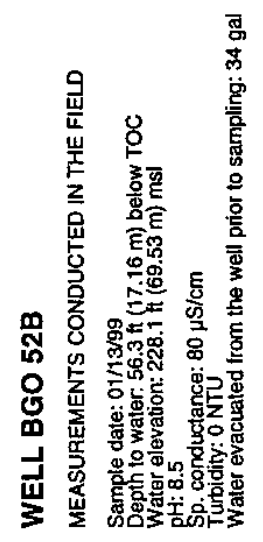

章

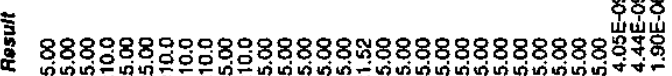

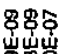

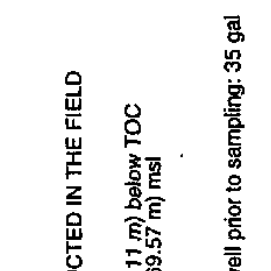

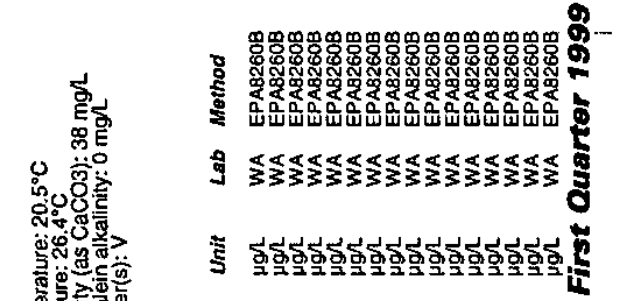

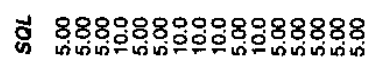

量

$\infty$

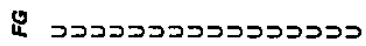

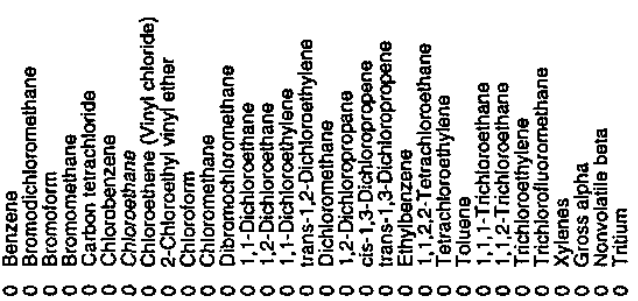

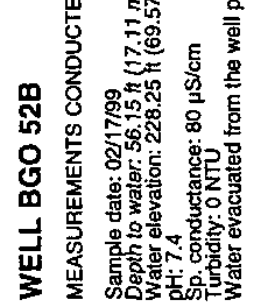

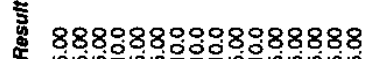

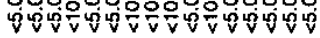

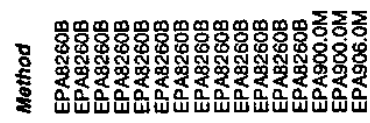

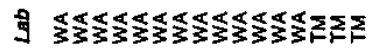

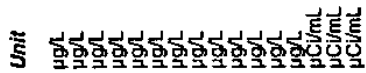

우웅

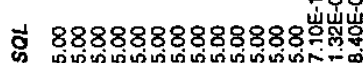

章

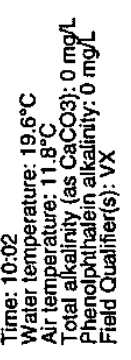

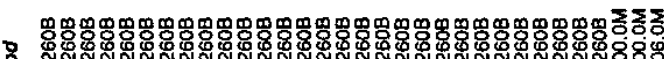

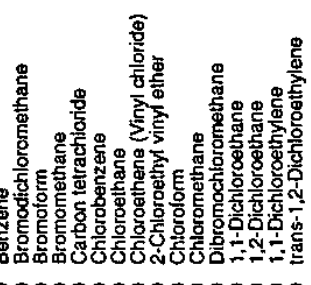

ร

\& in $0000000000000000 \%$

es

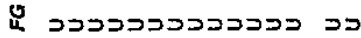

으웅

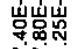

语突落

言

患

突
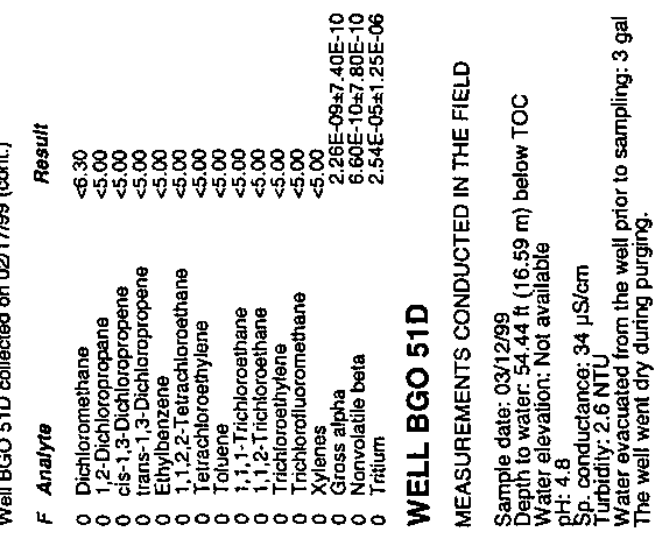

₹

9

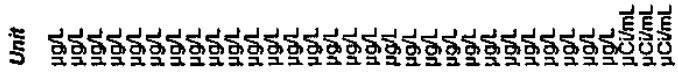

웅우

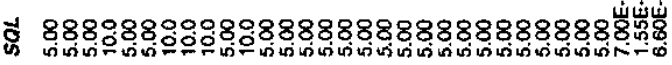

漓

s

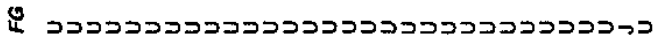

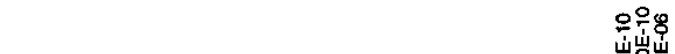

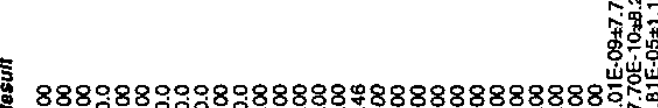

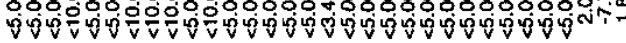

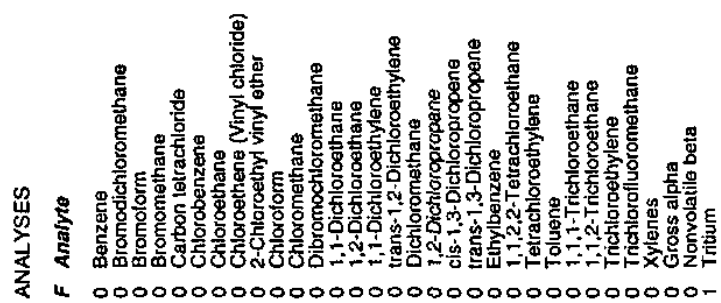




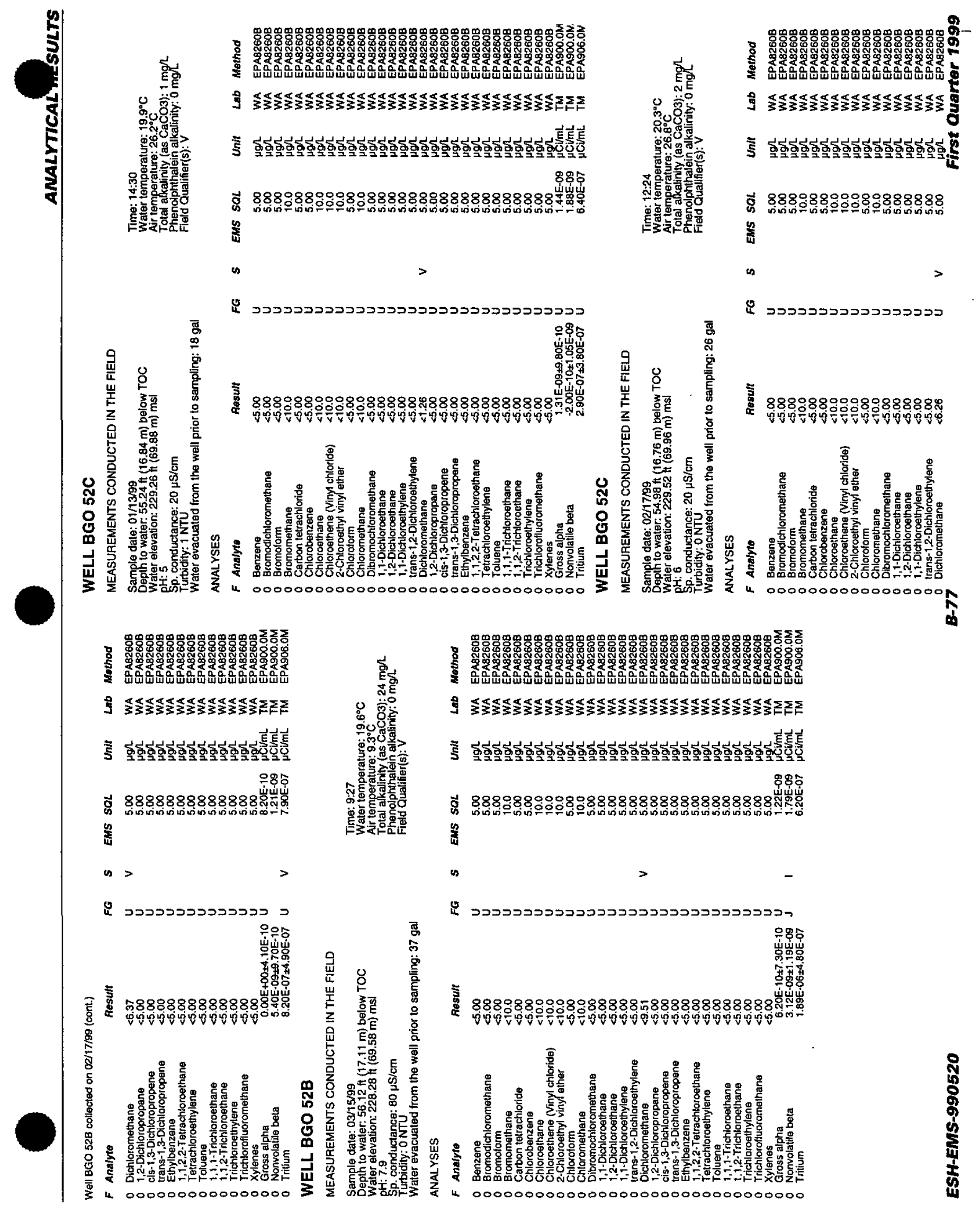



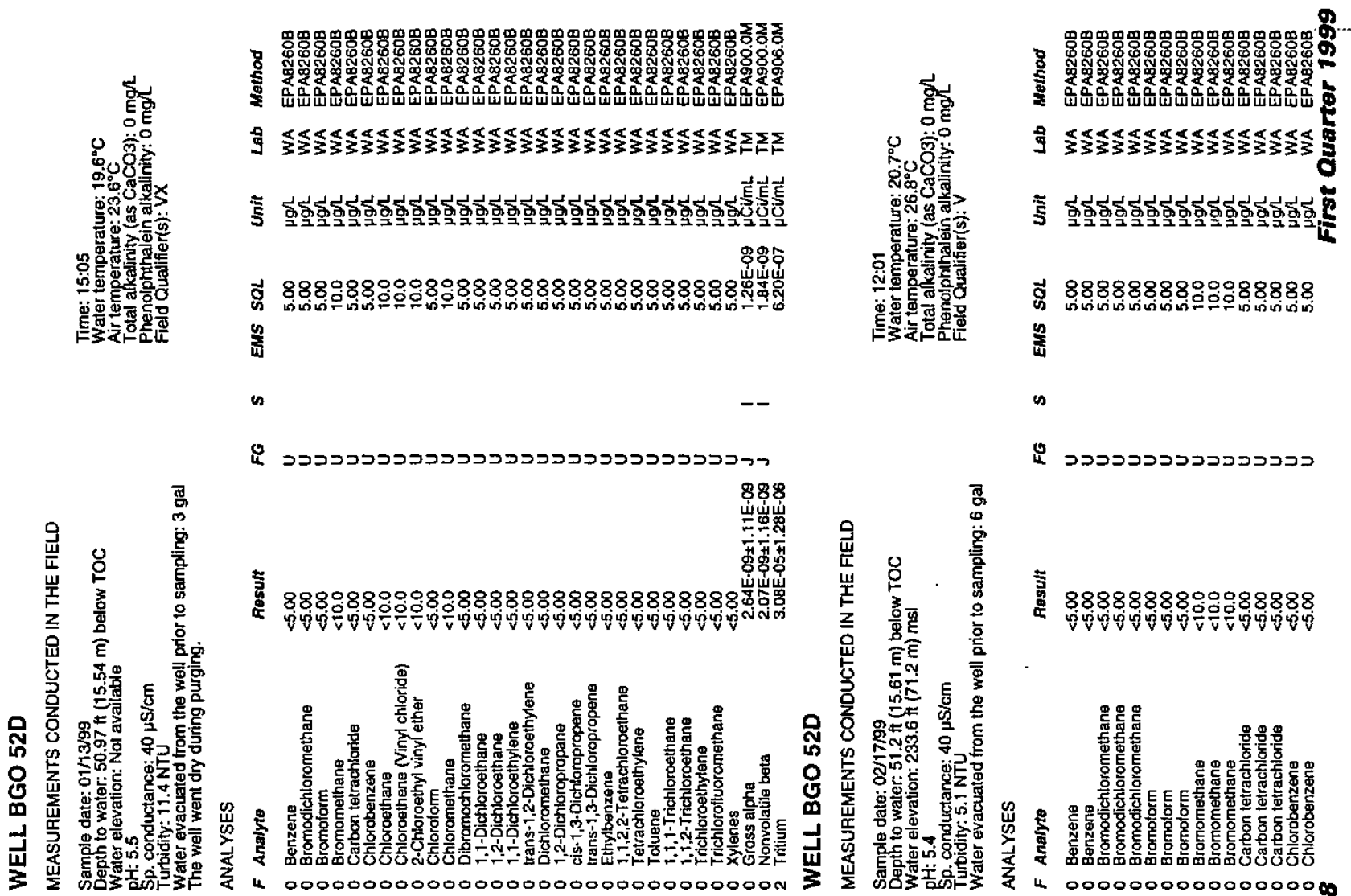
$\sqrt{10}$

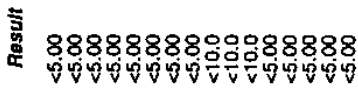

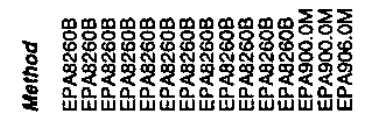

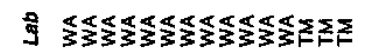

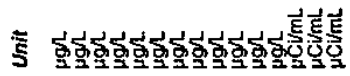

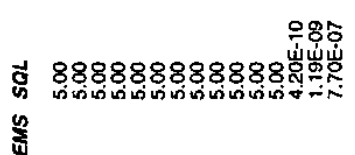

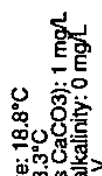

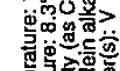

可

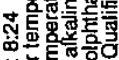

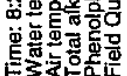

뜽

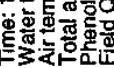
量

is

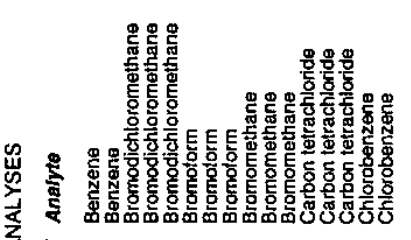

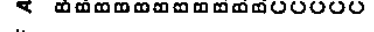

400000000000000000

as

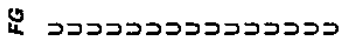

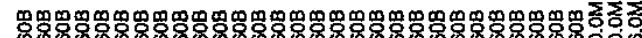

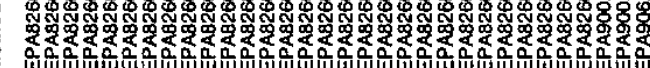

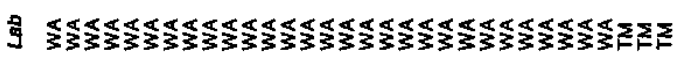

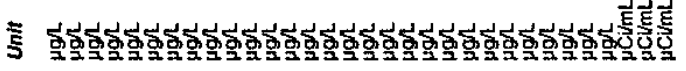

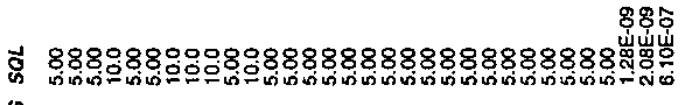
离

is

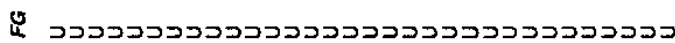

으옹

岗啱岩

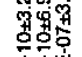

ह

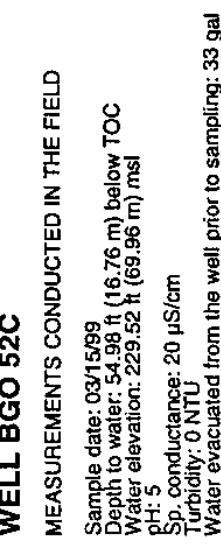

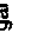

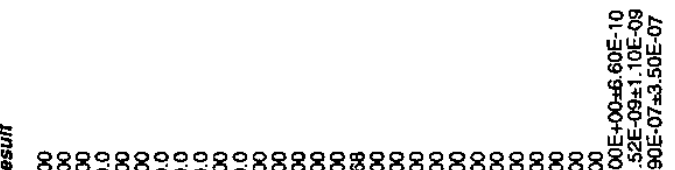

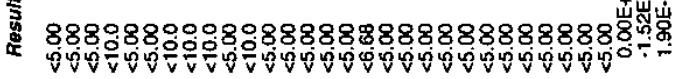

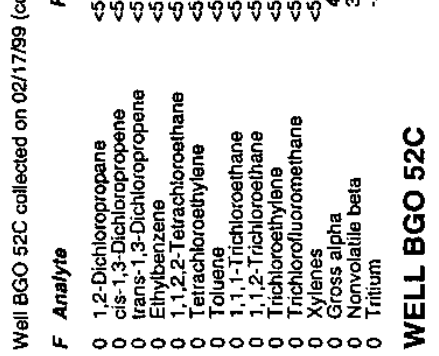



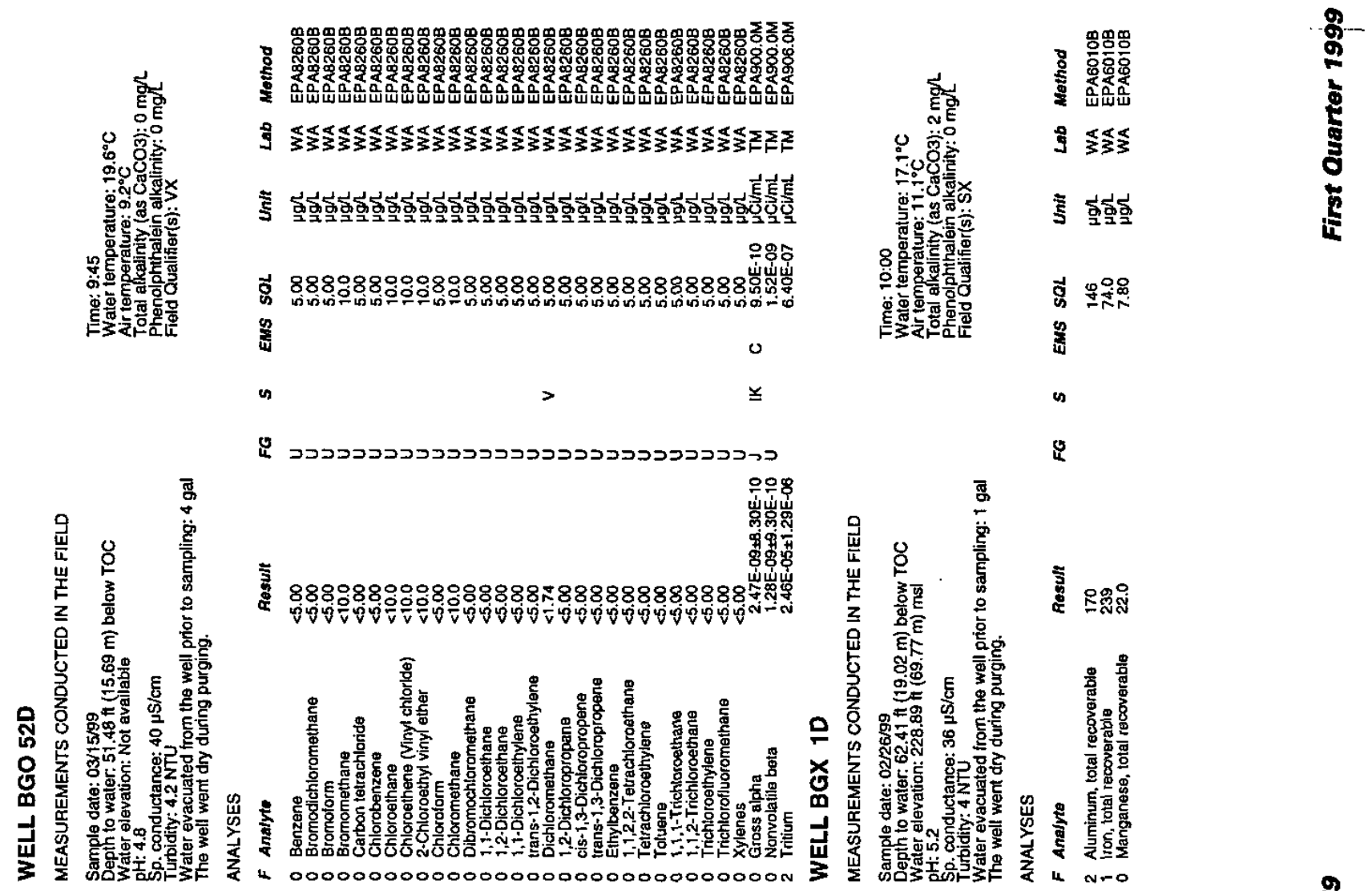

as

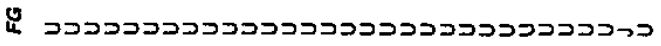

s.

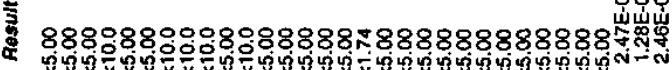

\section{음운 8}

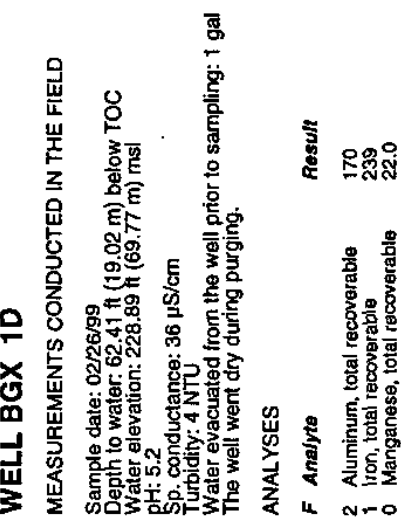

I

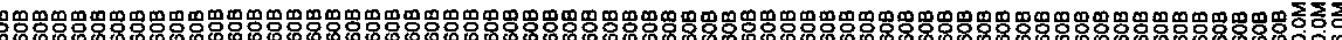

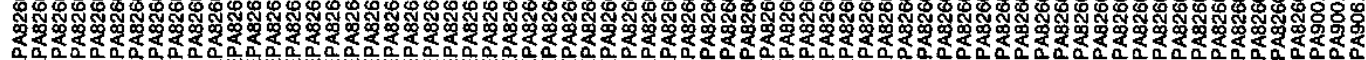

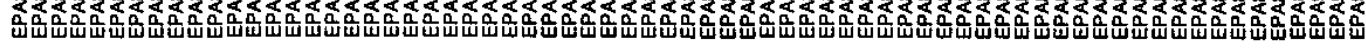

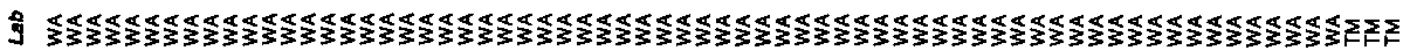

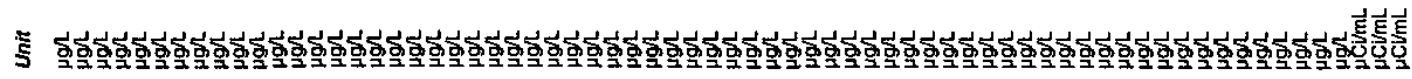

4.

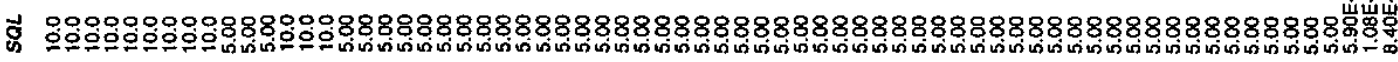
余

in

$>>$

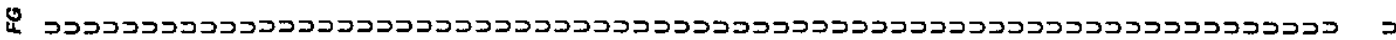
우요 ํํำํํำ

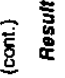
年

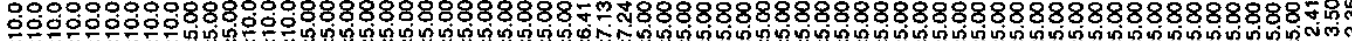$$
\text { g }
$$

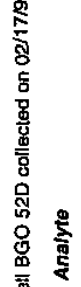<smiles></smiles>

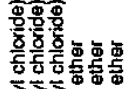

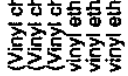

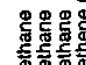

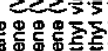




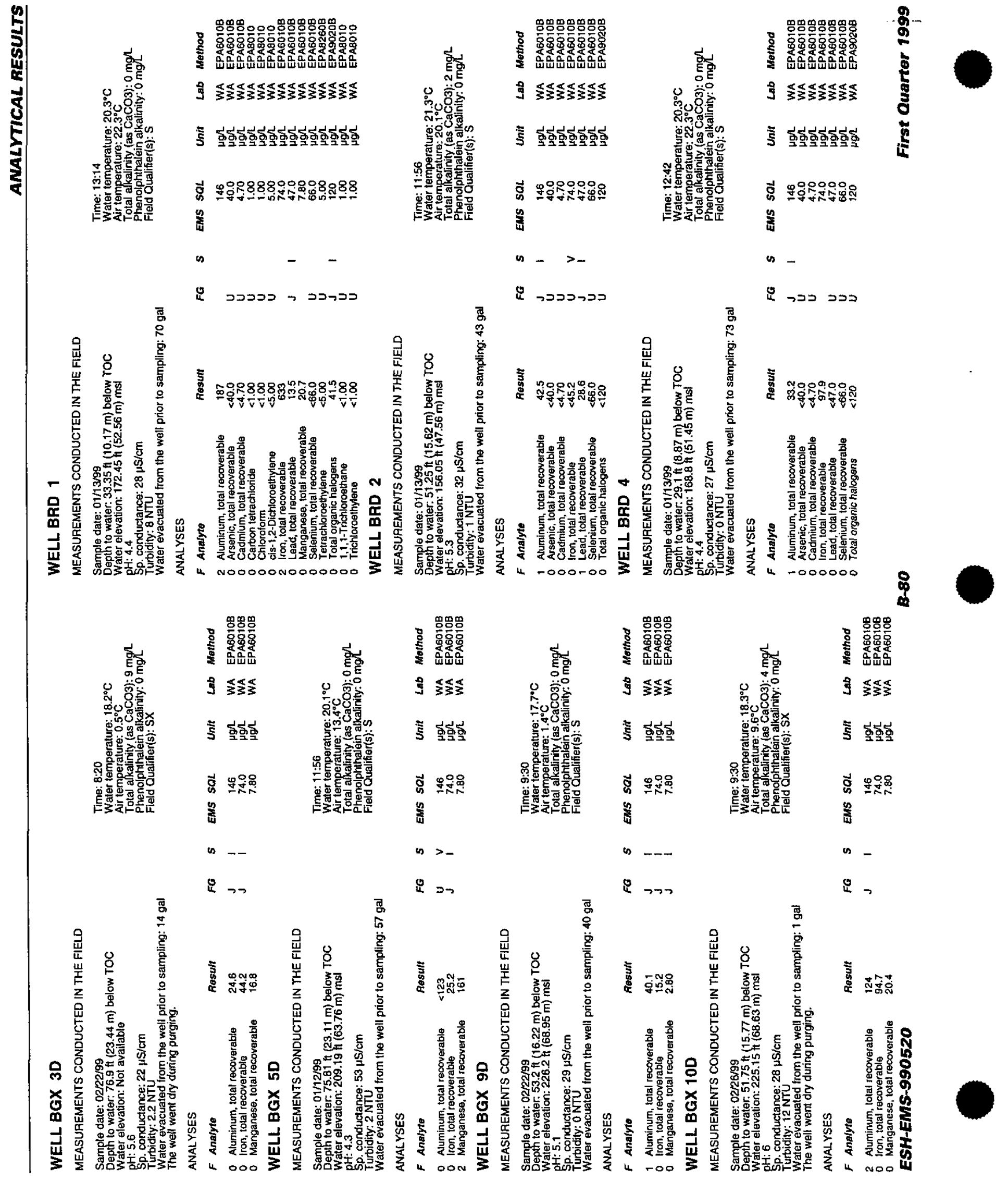




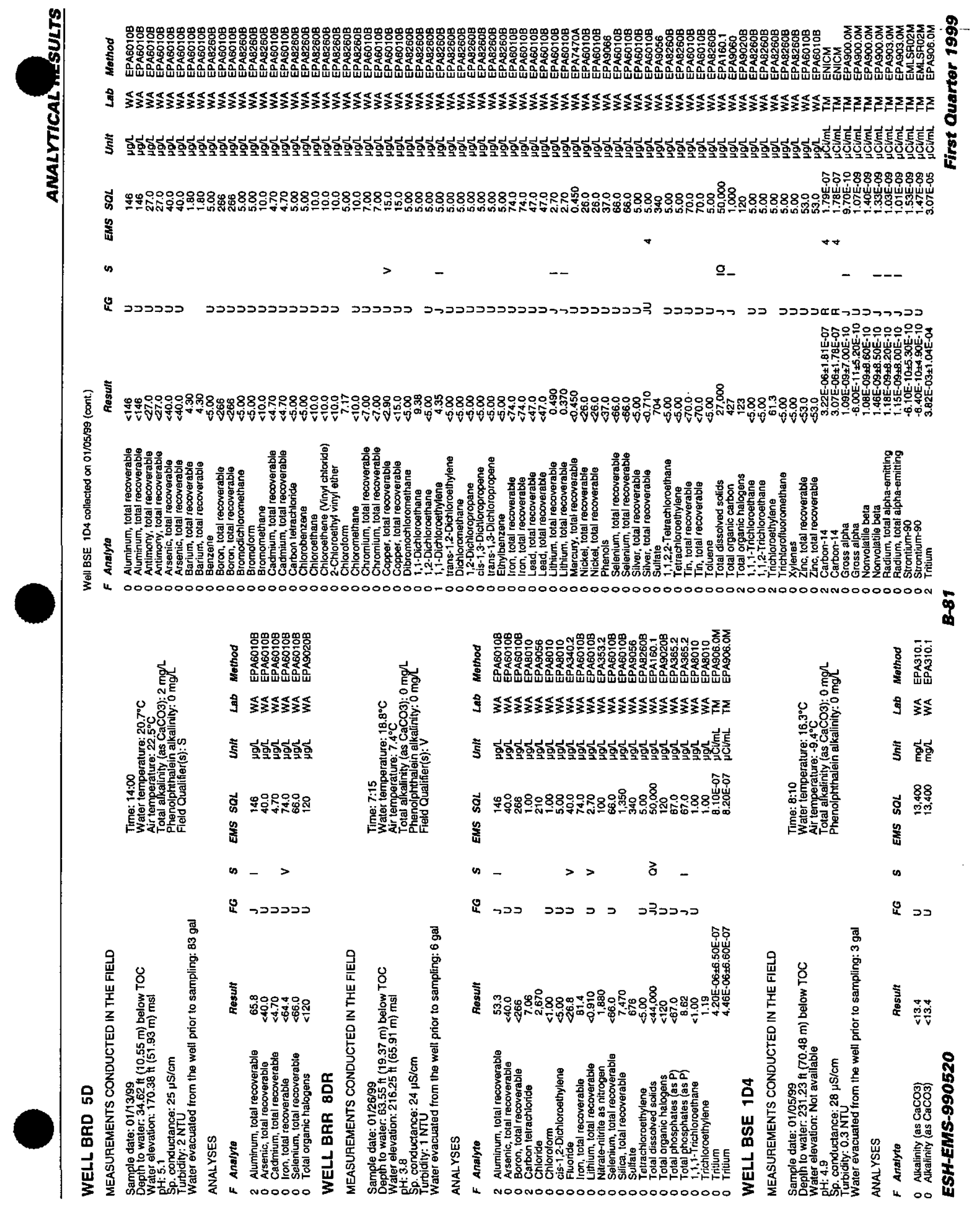




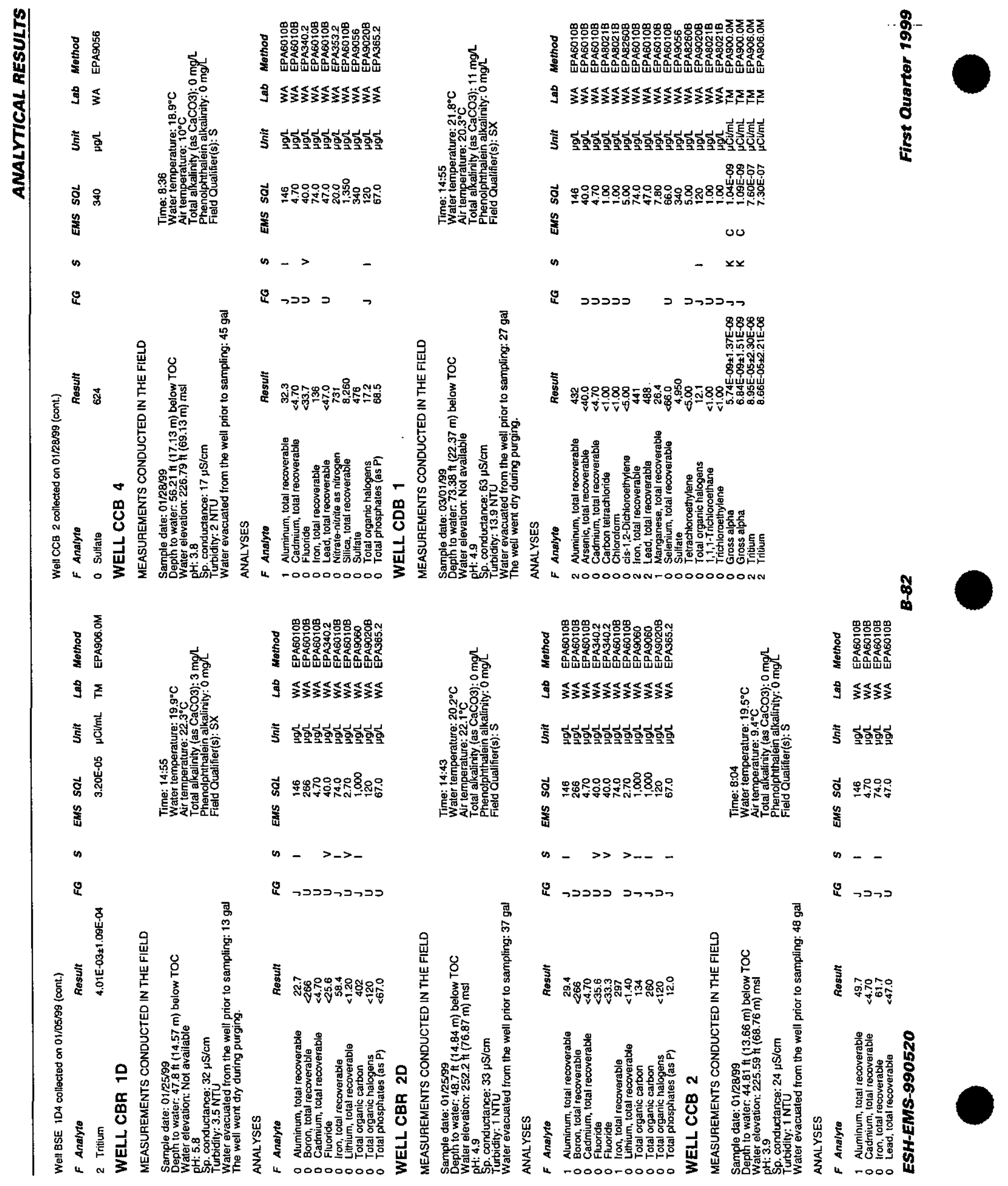









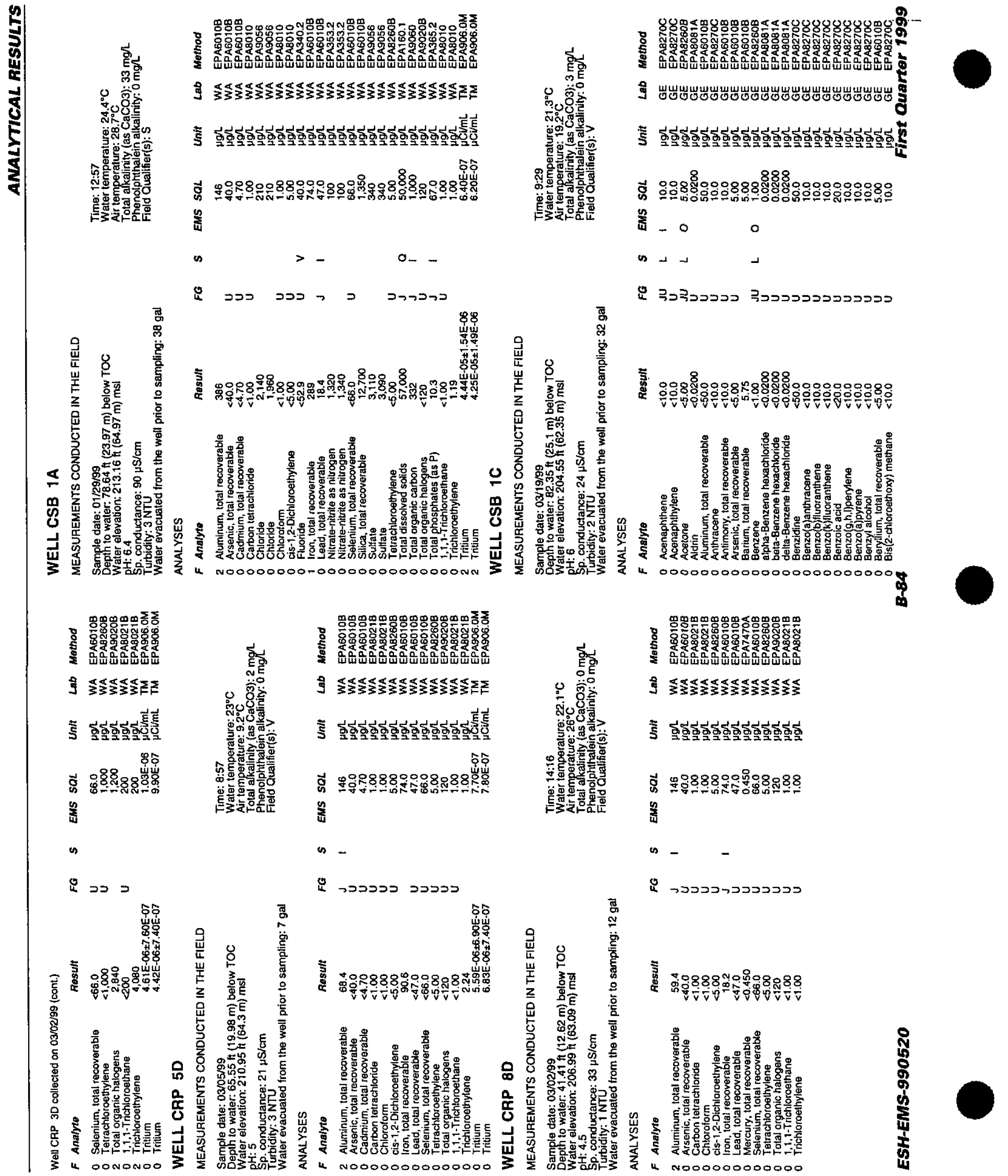




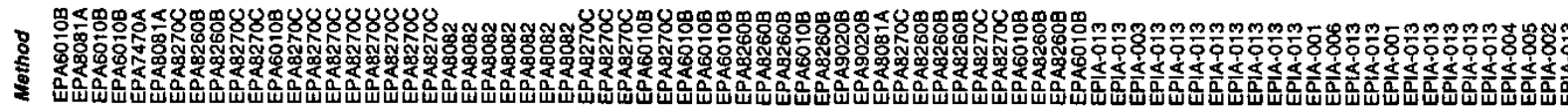

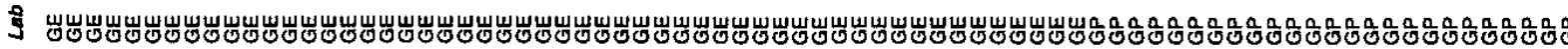

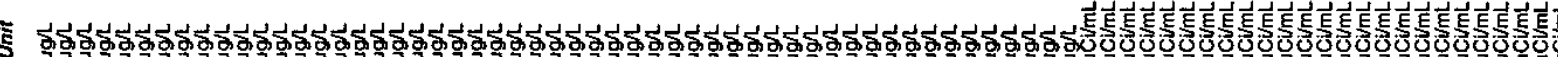

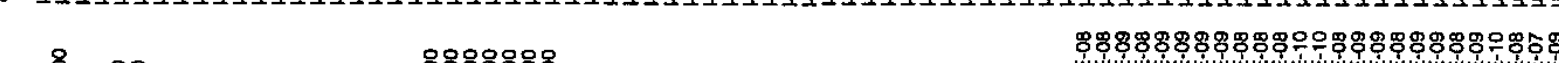

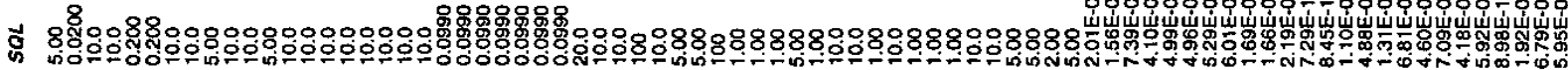

竞

00

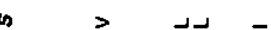

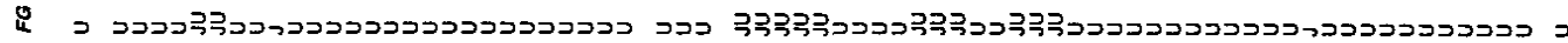
得8 8 \%

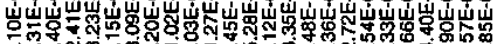

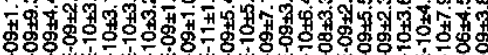

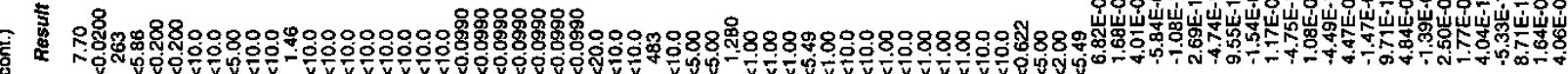

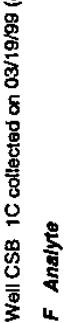

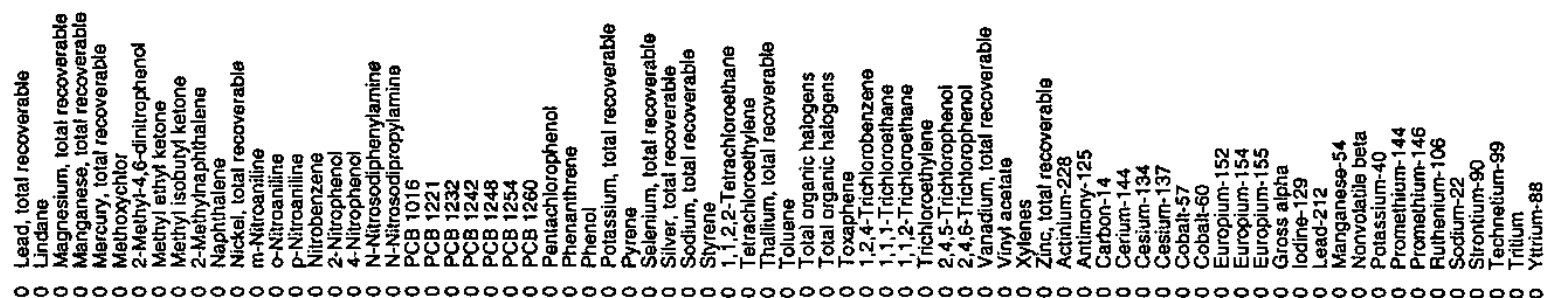

- 800

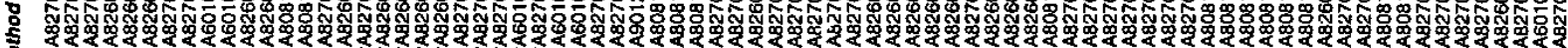

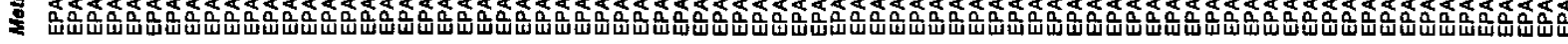

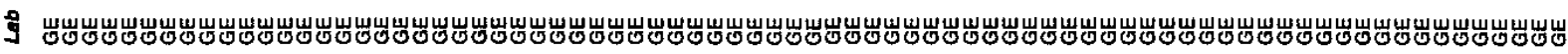

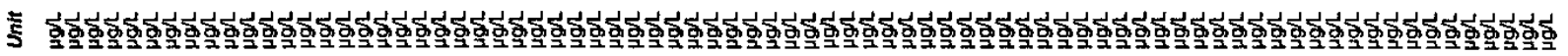

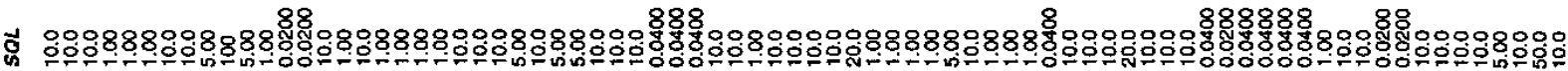
(n)

os

00000000

$\circ$

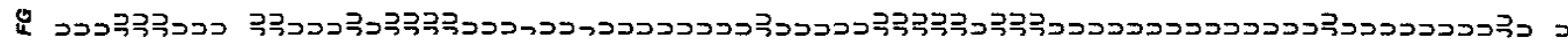

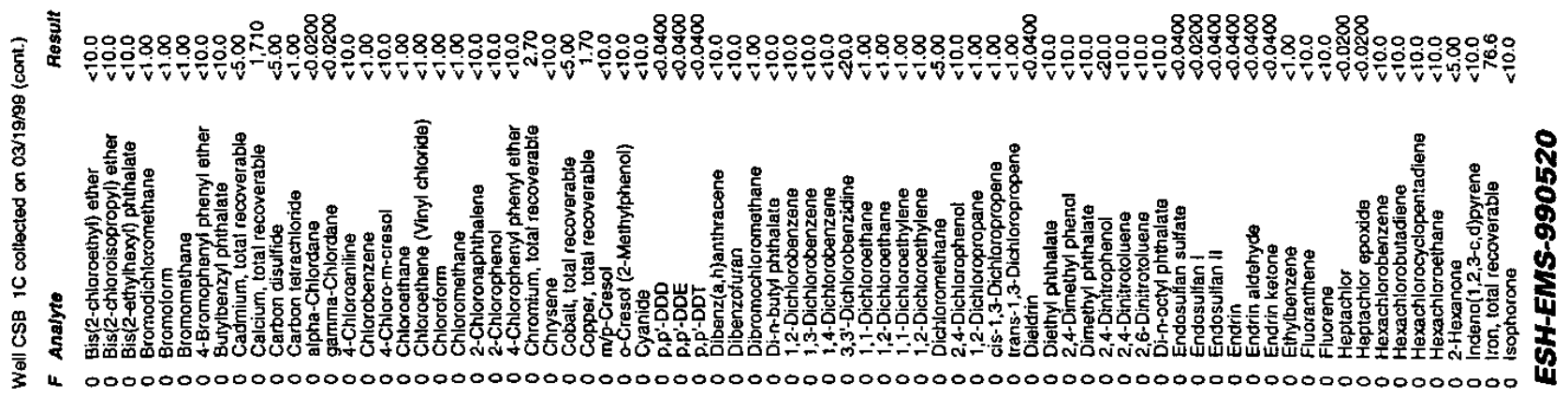




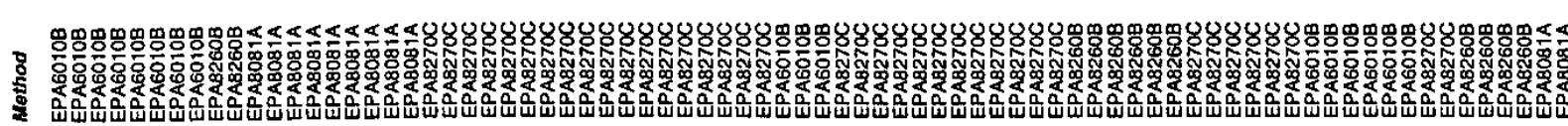

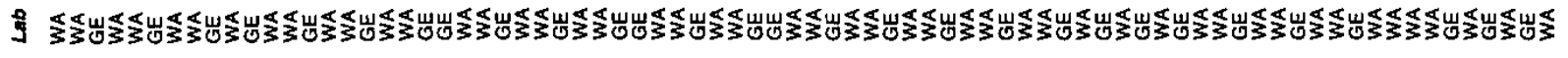

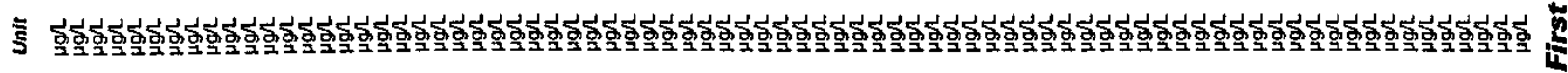

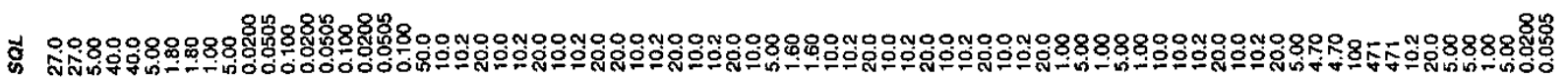

急

is

בסכנת
○

\lrcorner
$+$

000

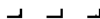

衰豆

\section{兽}

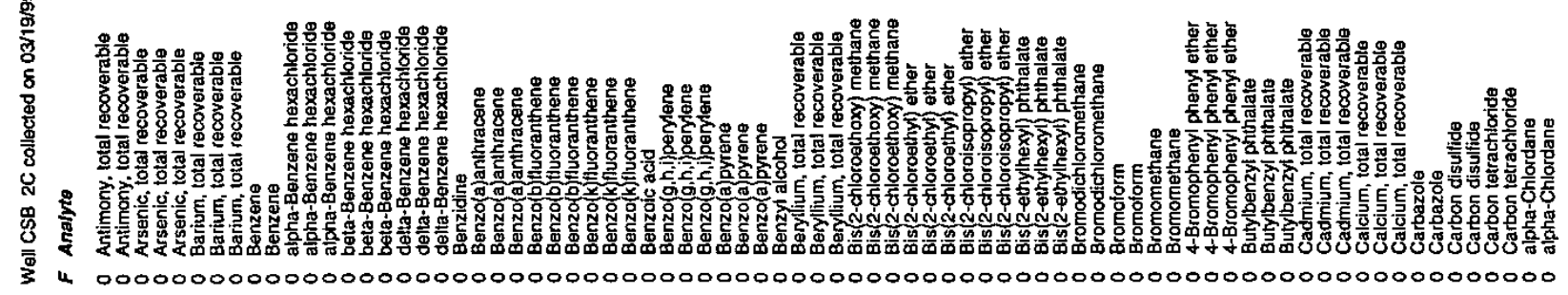

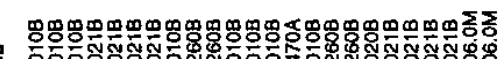
告

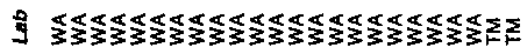

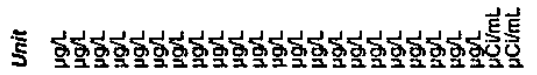

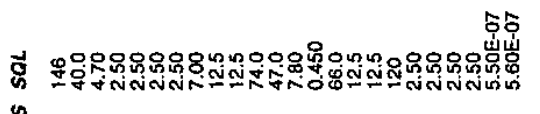
空

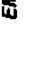

on

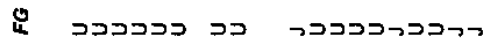

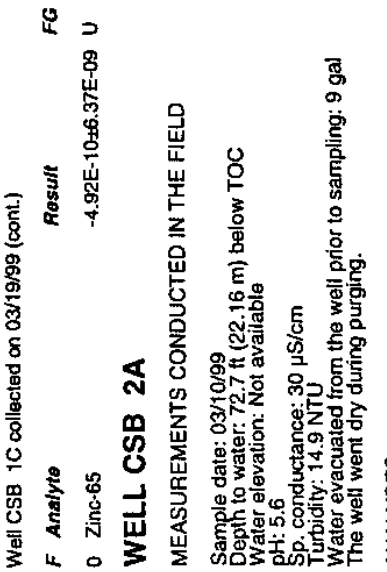

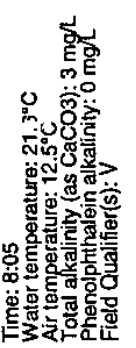

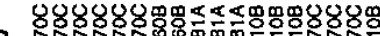

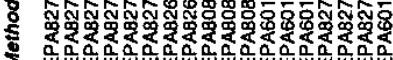
ब

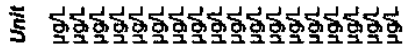

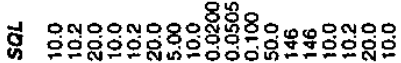
紊 -

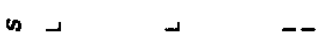

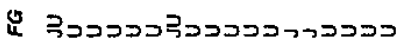

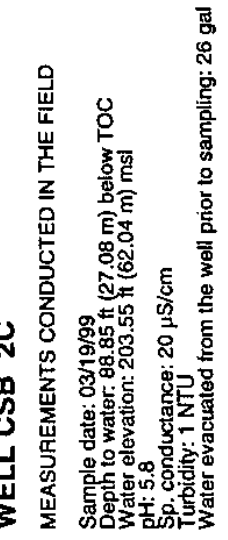

$\overrightarrow{8}$

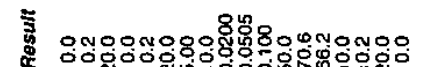

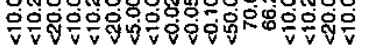

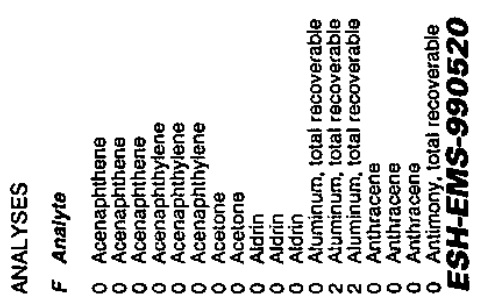




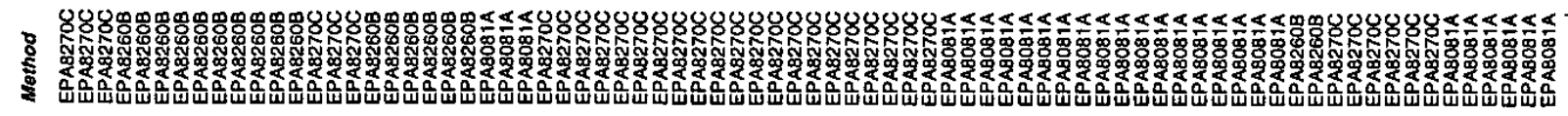

ง

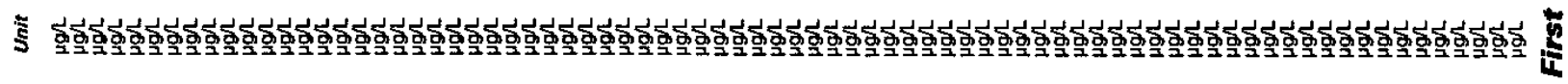

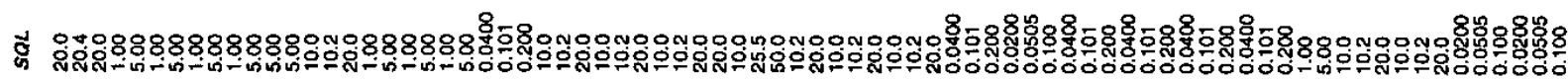

紊

00000000

a $\perp\lrcorner\lrcorner+\lrcorner$,

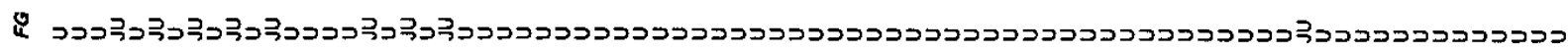

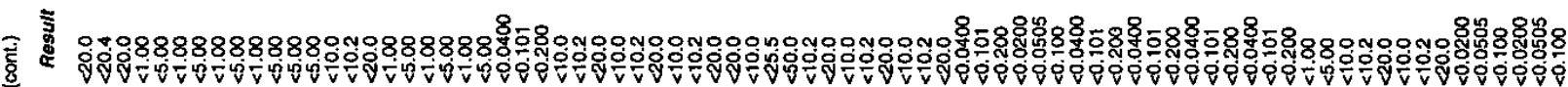

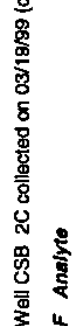

窵

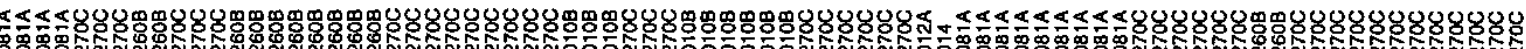

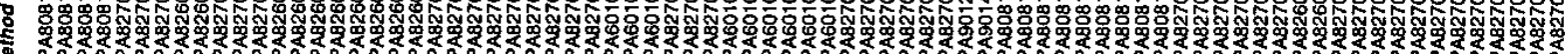

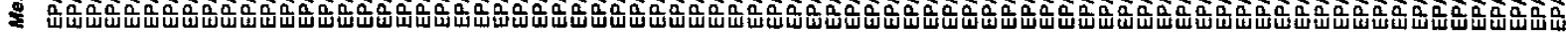

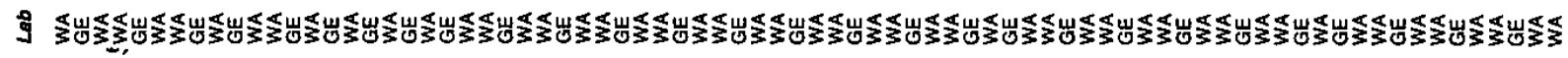

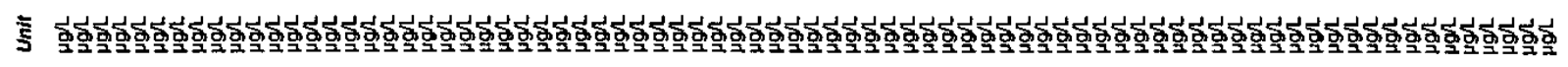

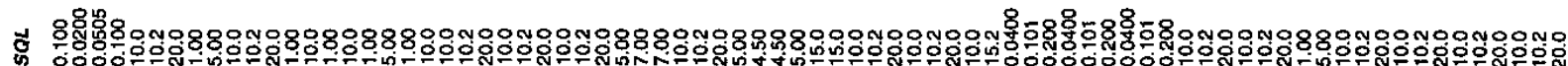
量

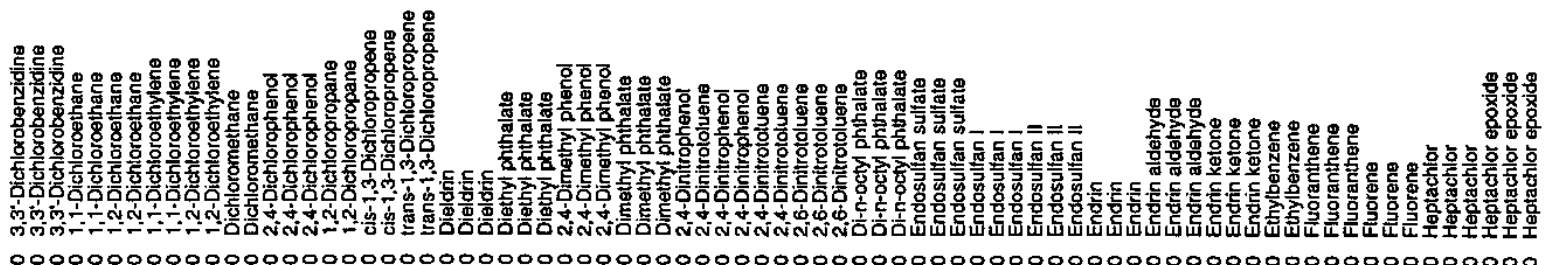

电 - 0000 $+\alpha$

\& 年

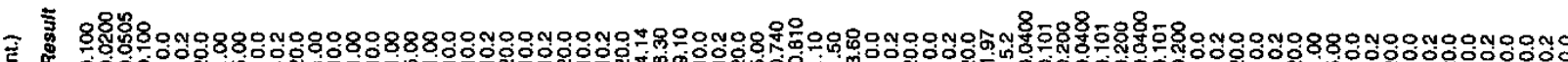
衰

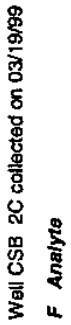

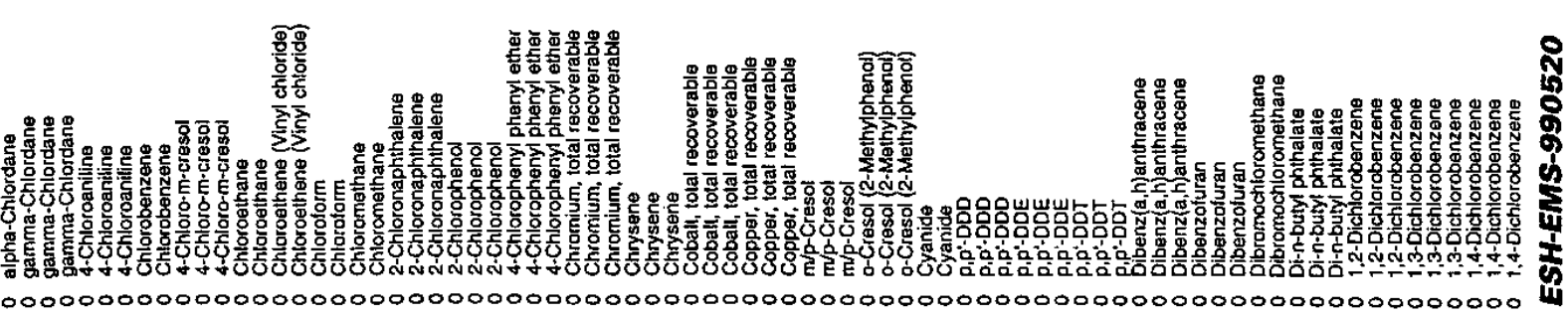


8.

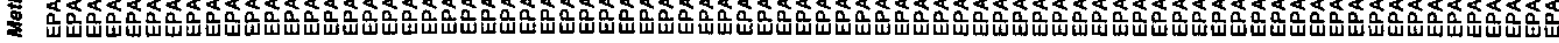

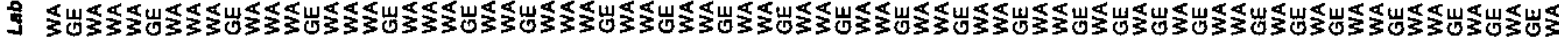

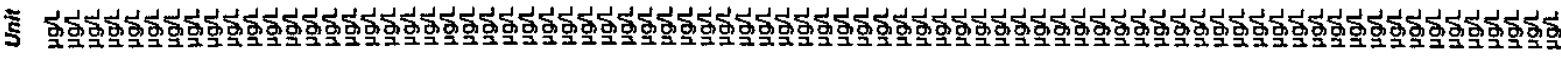

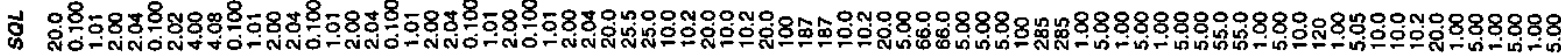

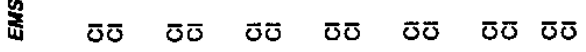

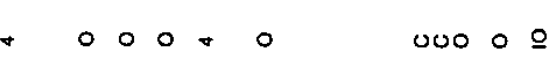

u 00 do 00 oo 00 do 00

$\rightarrow\lrcorner+\lrcorner\lrcorner\lrcorner$

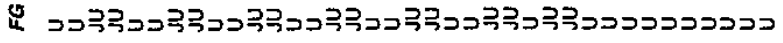

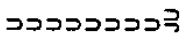

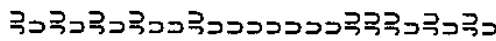

공

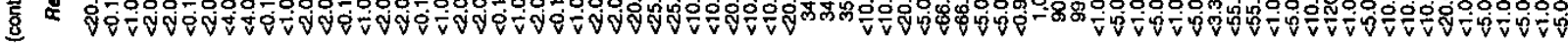

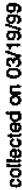

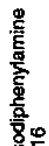<smiles>[Mg]=[W]=[W]</smiles>

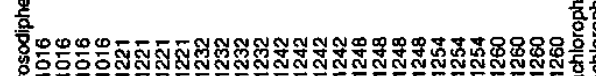<smiles>C1=C[As][As]1</smiles>

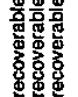

要累要

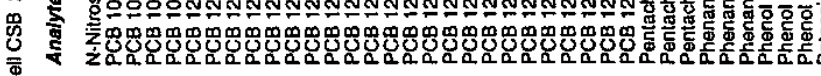

L 00000000000000000000000000000000000000000000000000000000000000000000000000000

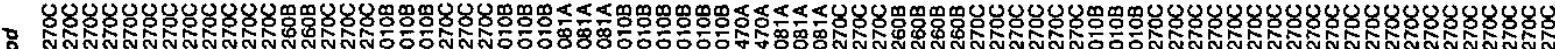

궁

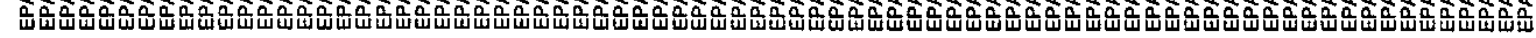

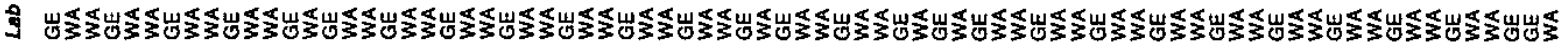

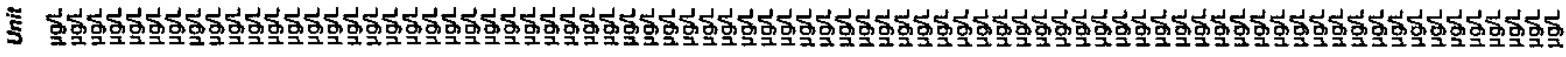

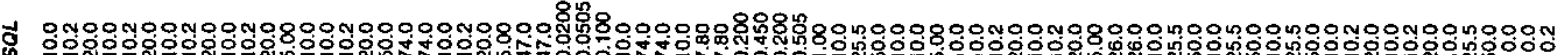
㝵

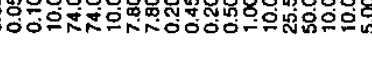

is

$\circ$

$\circ \circ$

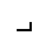

\lrcorner

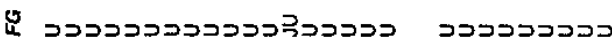

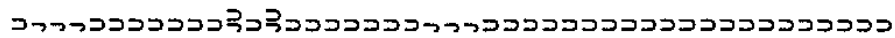

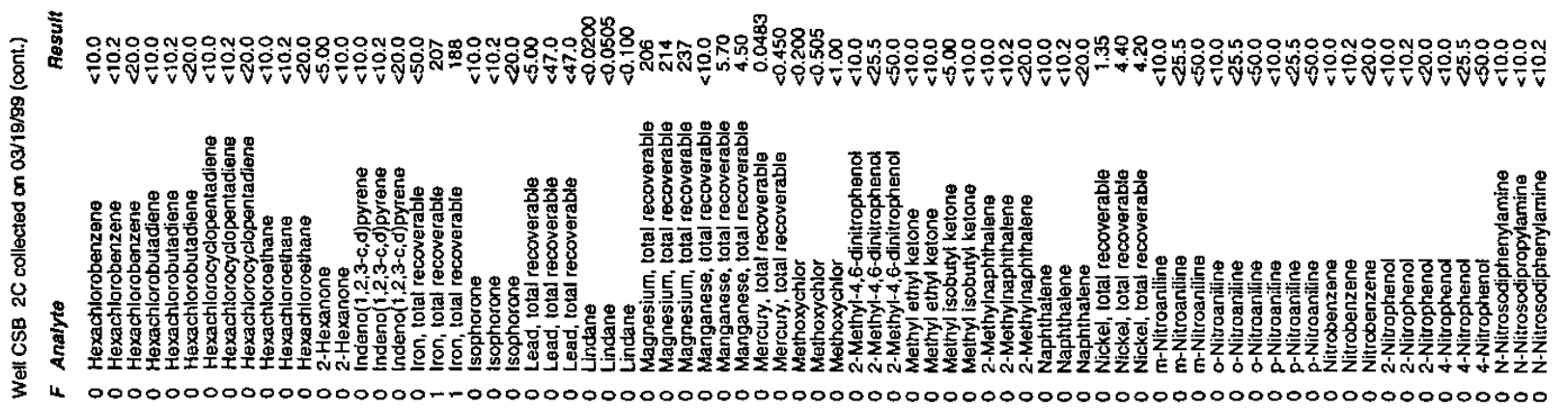




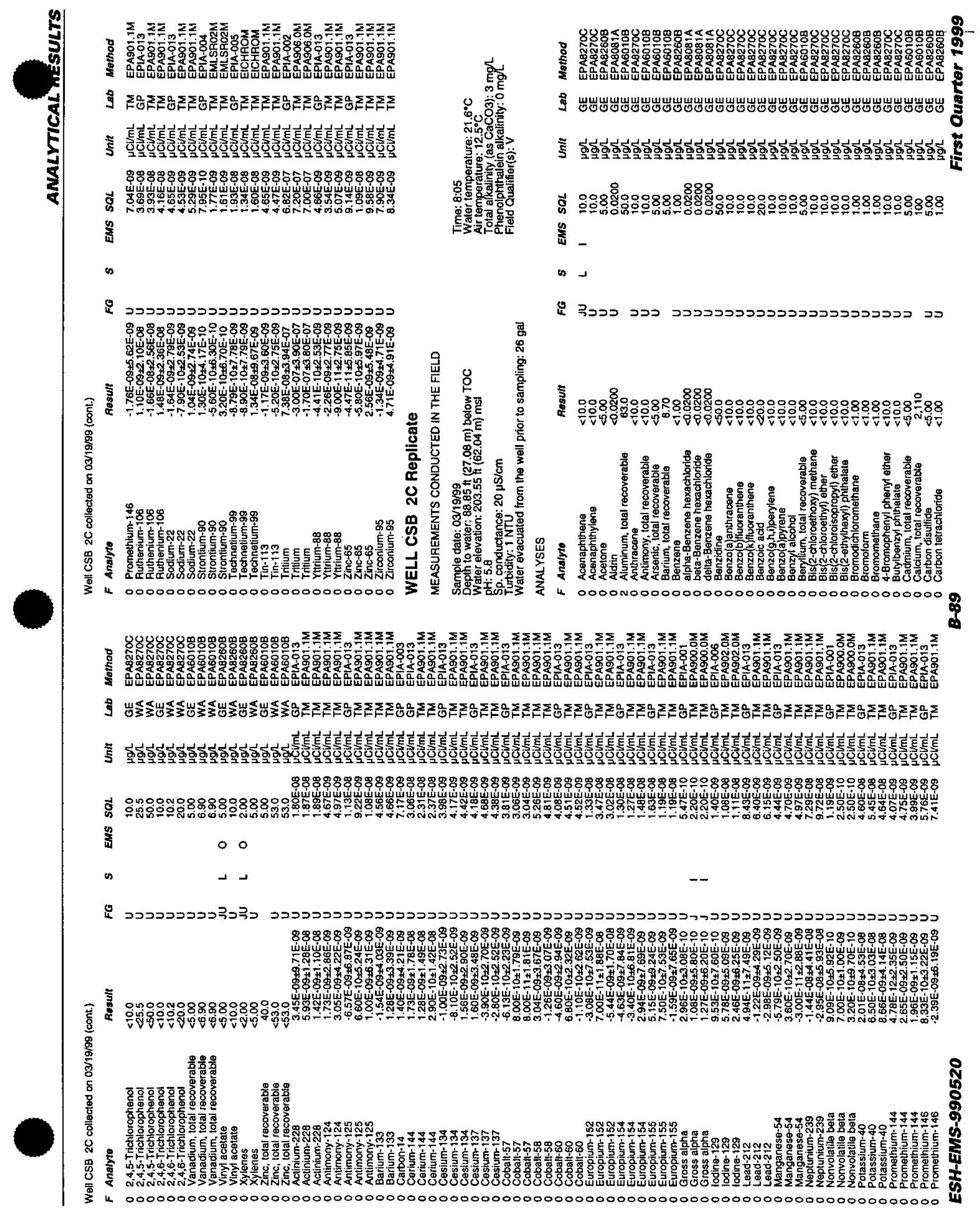


\%

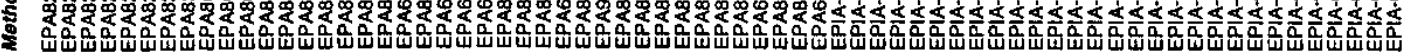

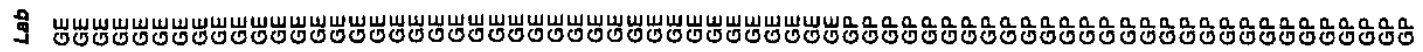

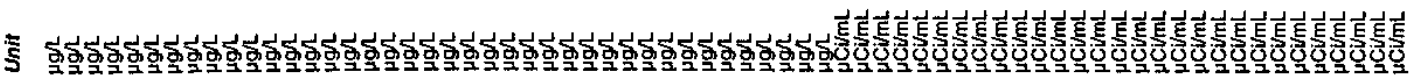

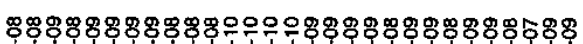

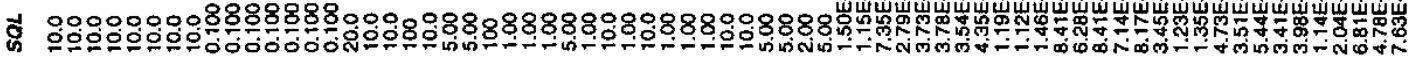

量

is

\&

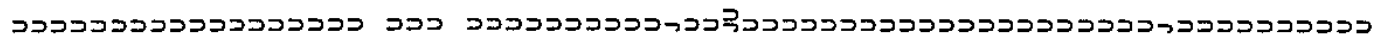

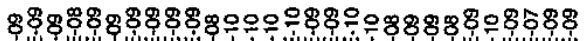

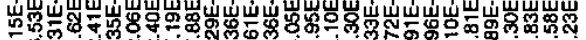

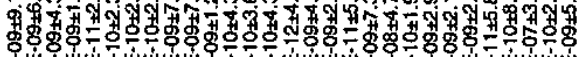

害

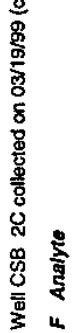

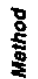

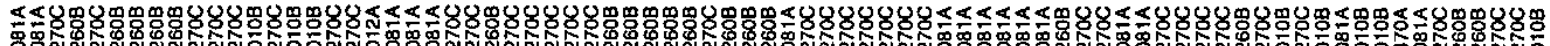
荡品 ₹

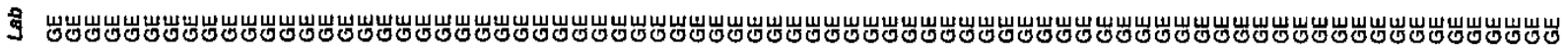

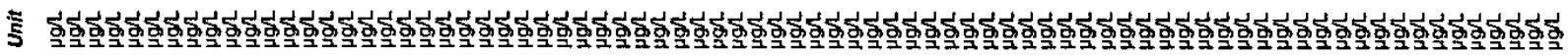

ఫ

竞

is

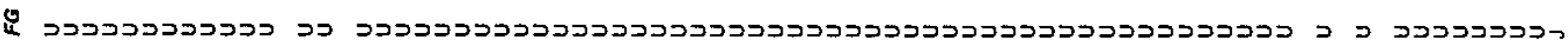

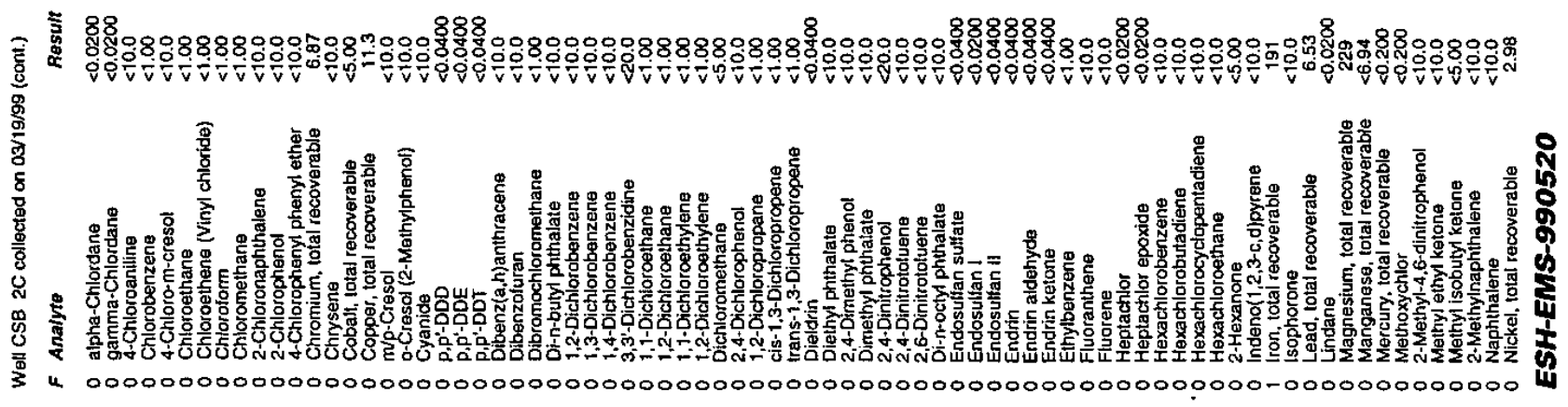

. 


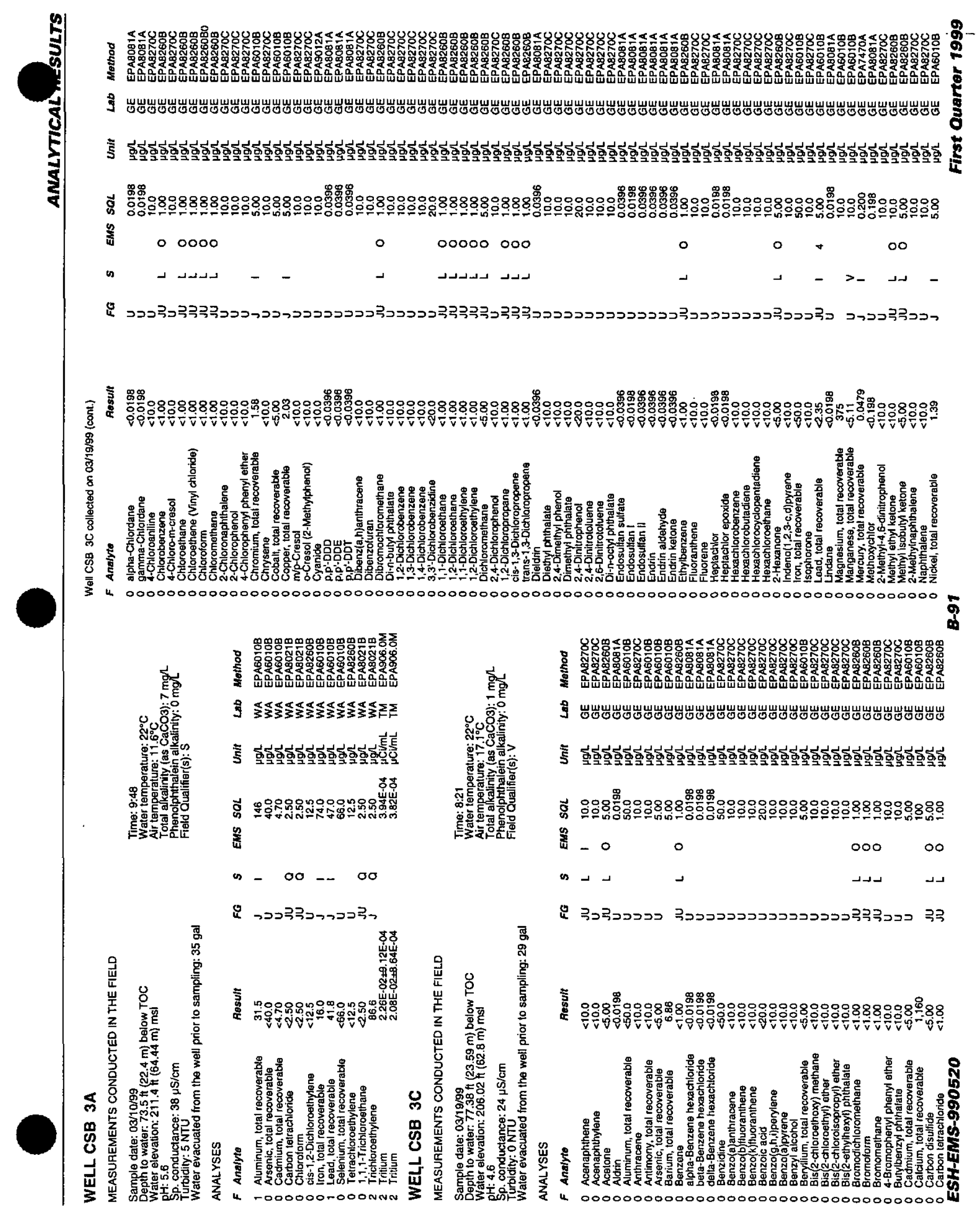




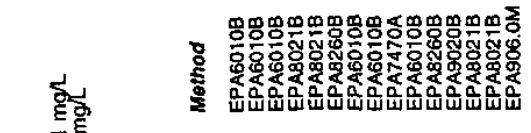

0 …ㅇ.

.

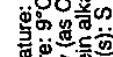

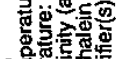

触

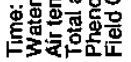

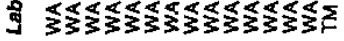

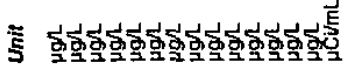

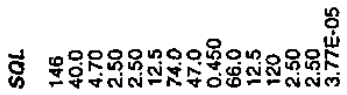

悉

s - 00 - $-0 \sigma$

צ

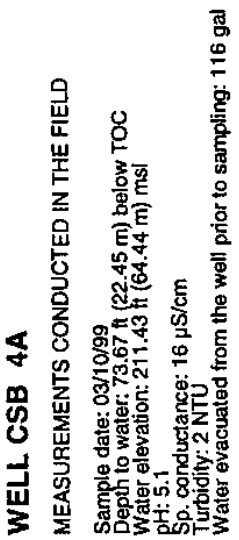

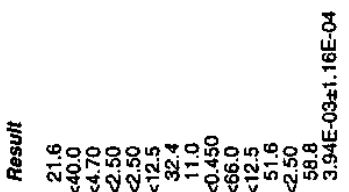
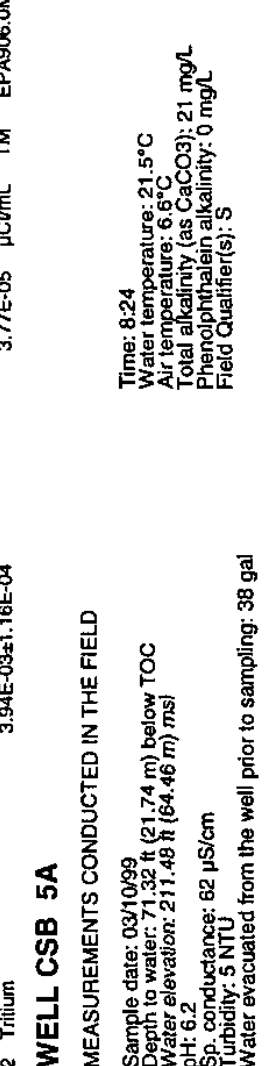

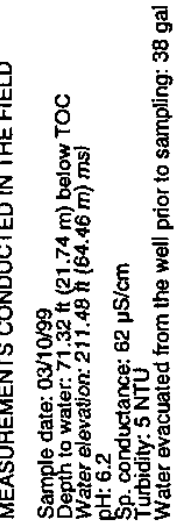

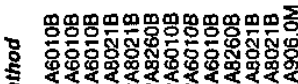

\%

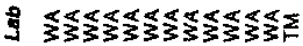

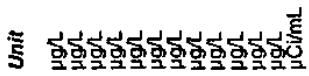

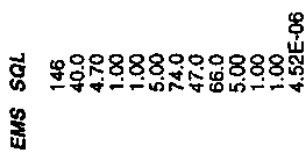

$\infty \quad 00-\infty \sigma$

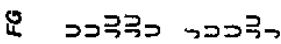
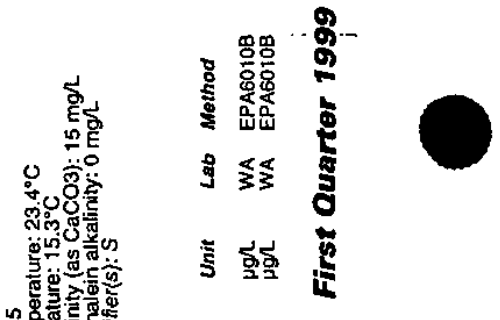

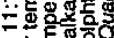

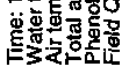

호ํ 果웅

釆

n-

כד

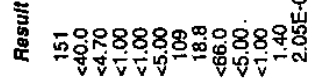

营
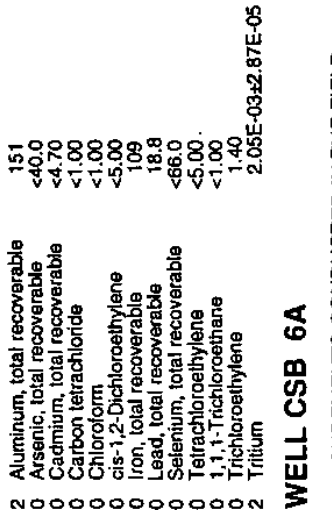

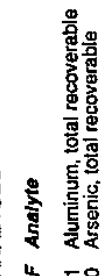

-

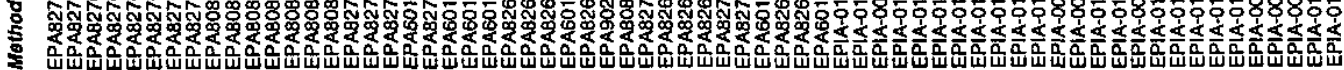

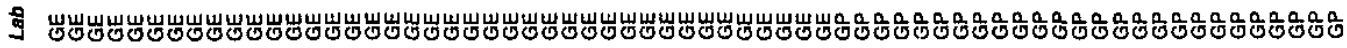

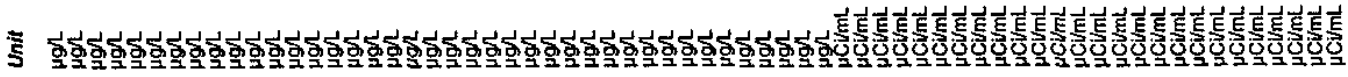

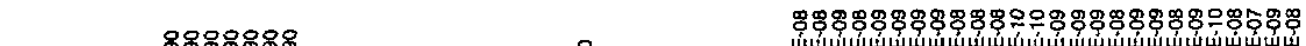

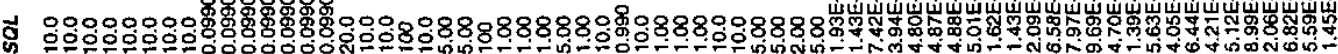
恙

$000 \circ 00000$

es

H

\$

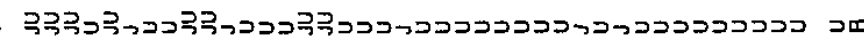

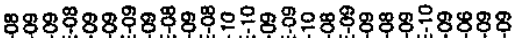

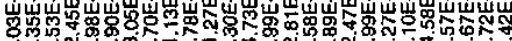

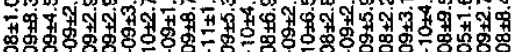

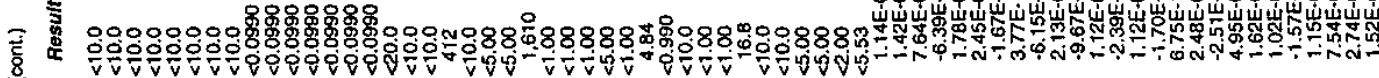

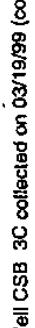

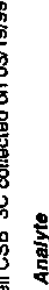

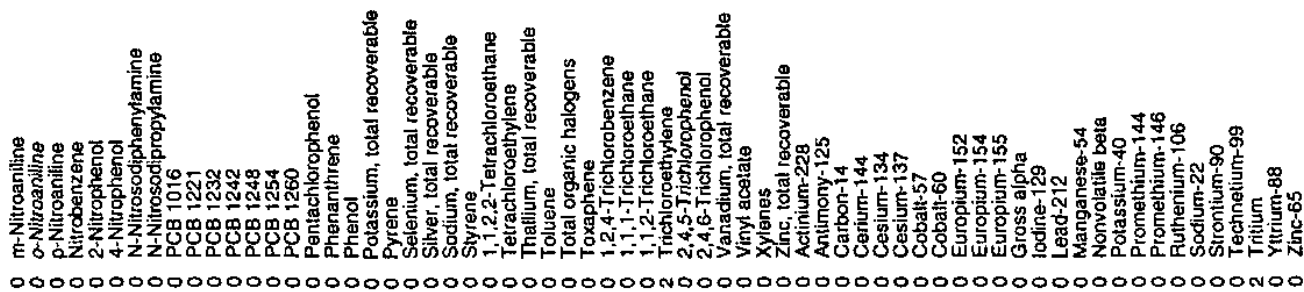




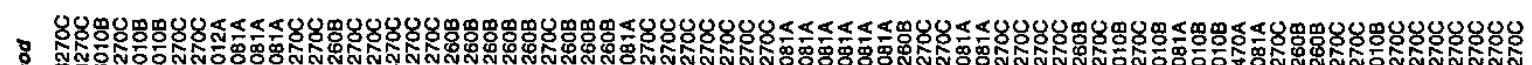
苾

1

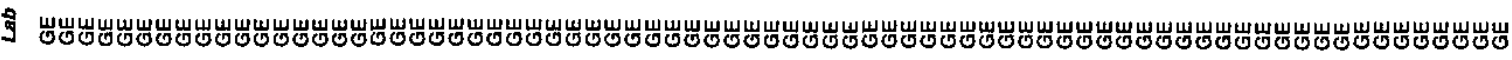

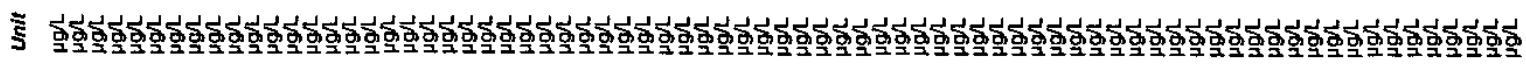

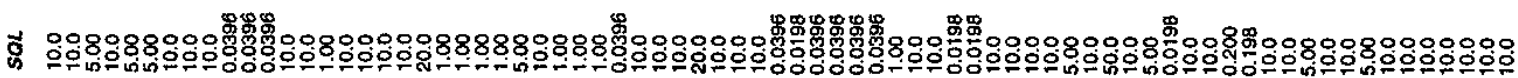

-

ניט

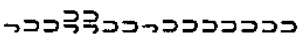

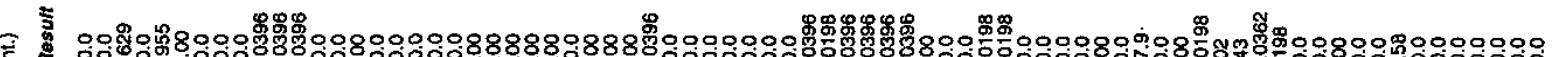

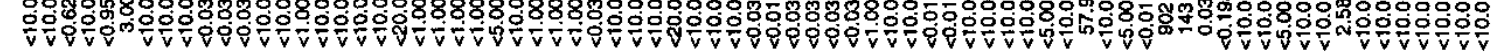

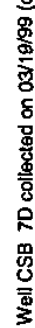

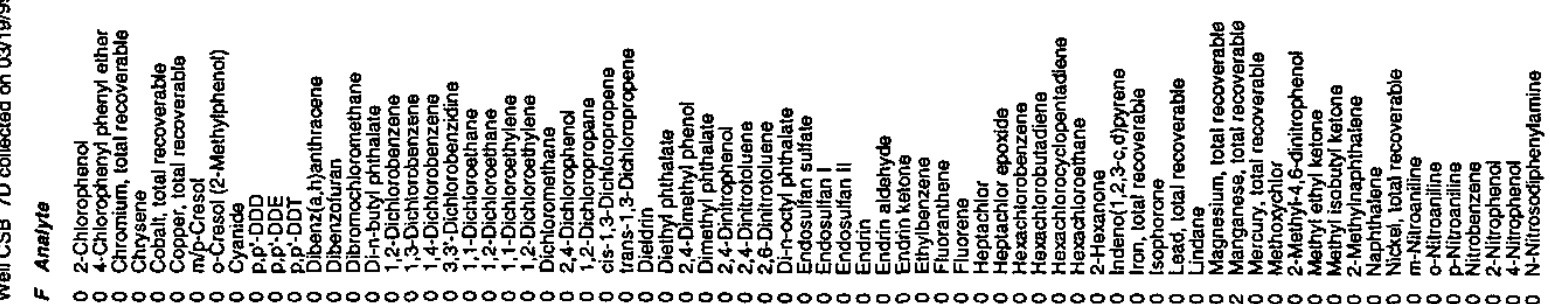

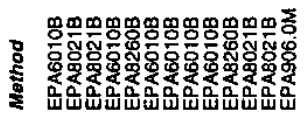

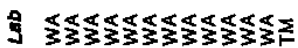

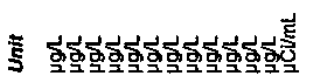
tᄒ
竞
a 00 - . 00

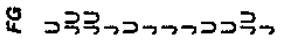
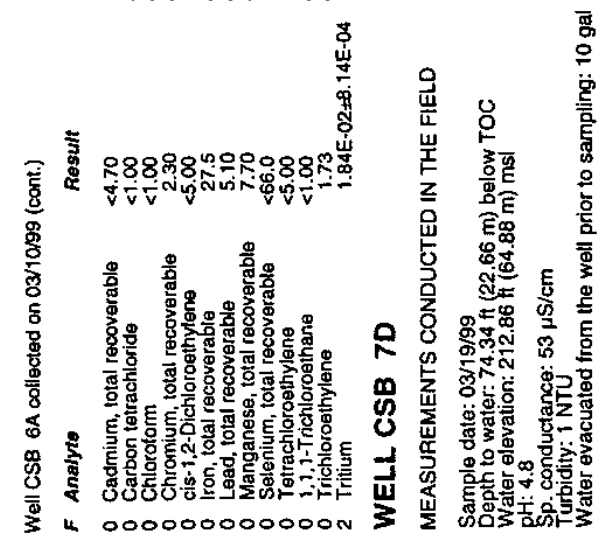

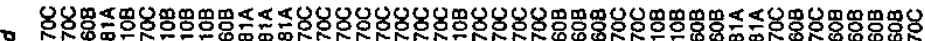

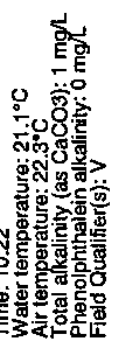

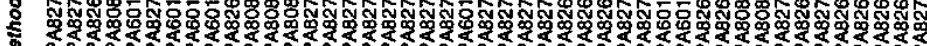

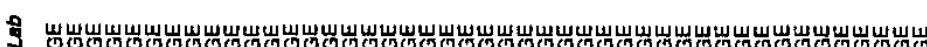

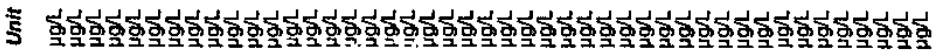

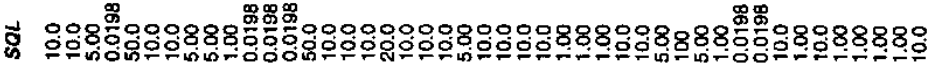
- 0 急

000

0000000

$\infty\lrcorner-+$

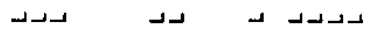

ב

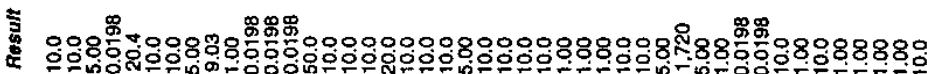

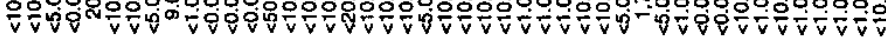

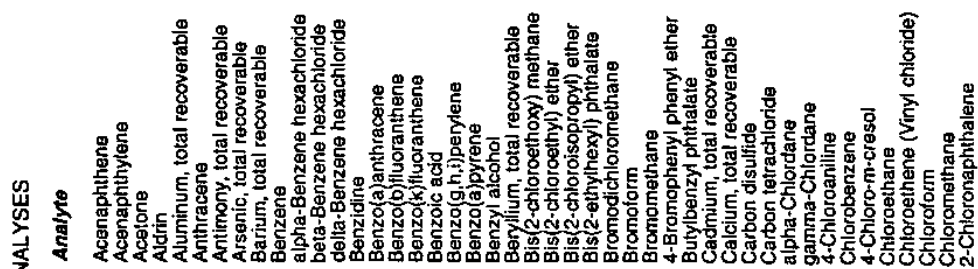


8 恶

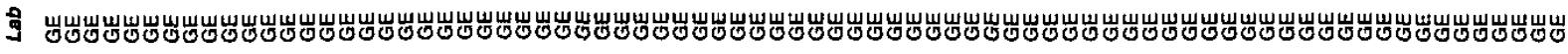

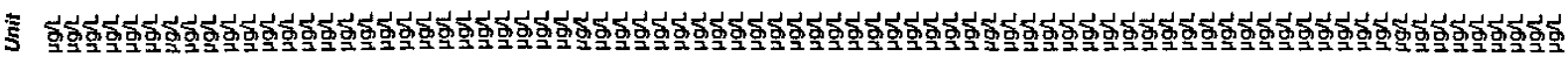

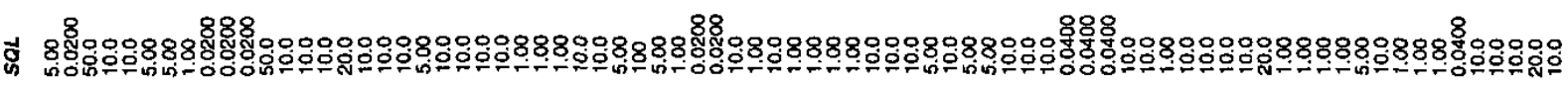

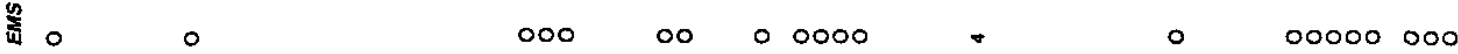

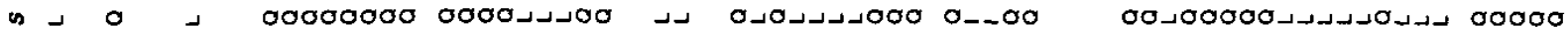

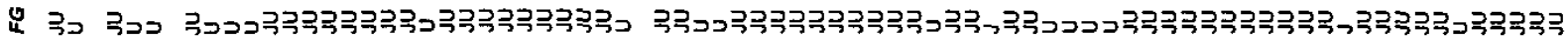

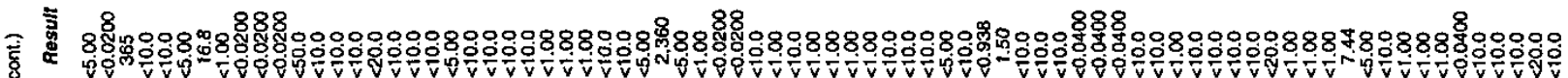

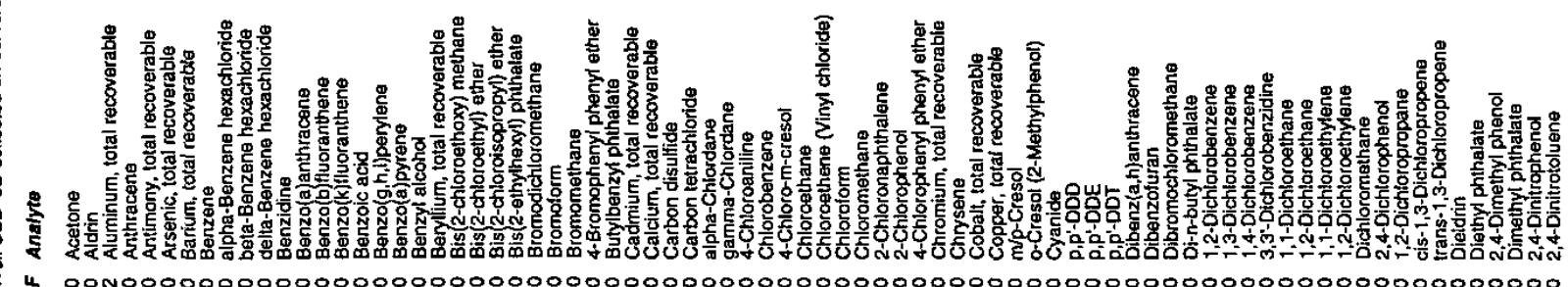

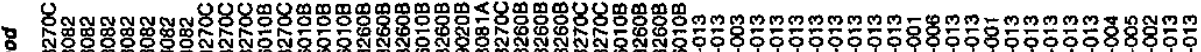

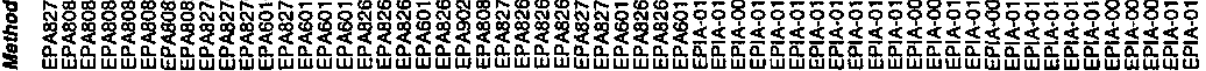

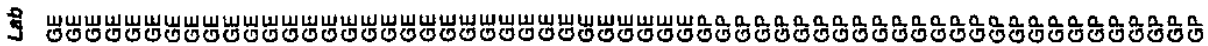

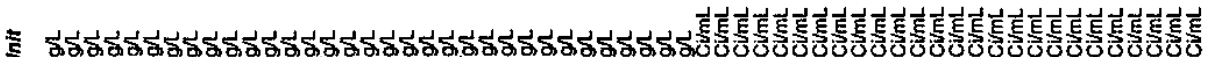

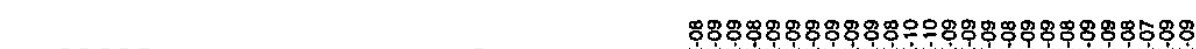
落 密

$000 \circ 00000$

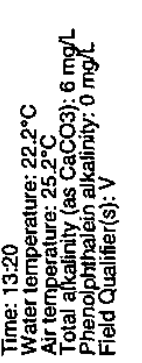

帘

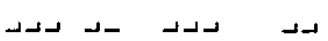

$-$

产

马 峞岕

e

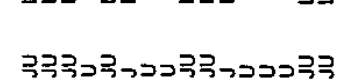

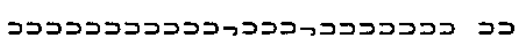

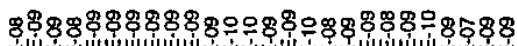

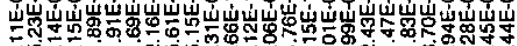

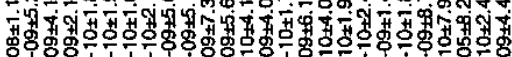

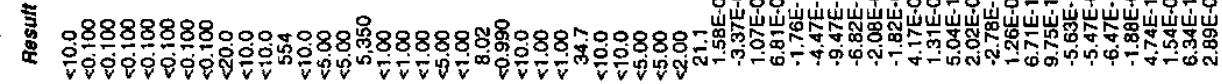

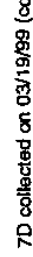
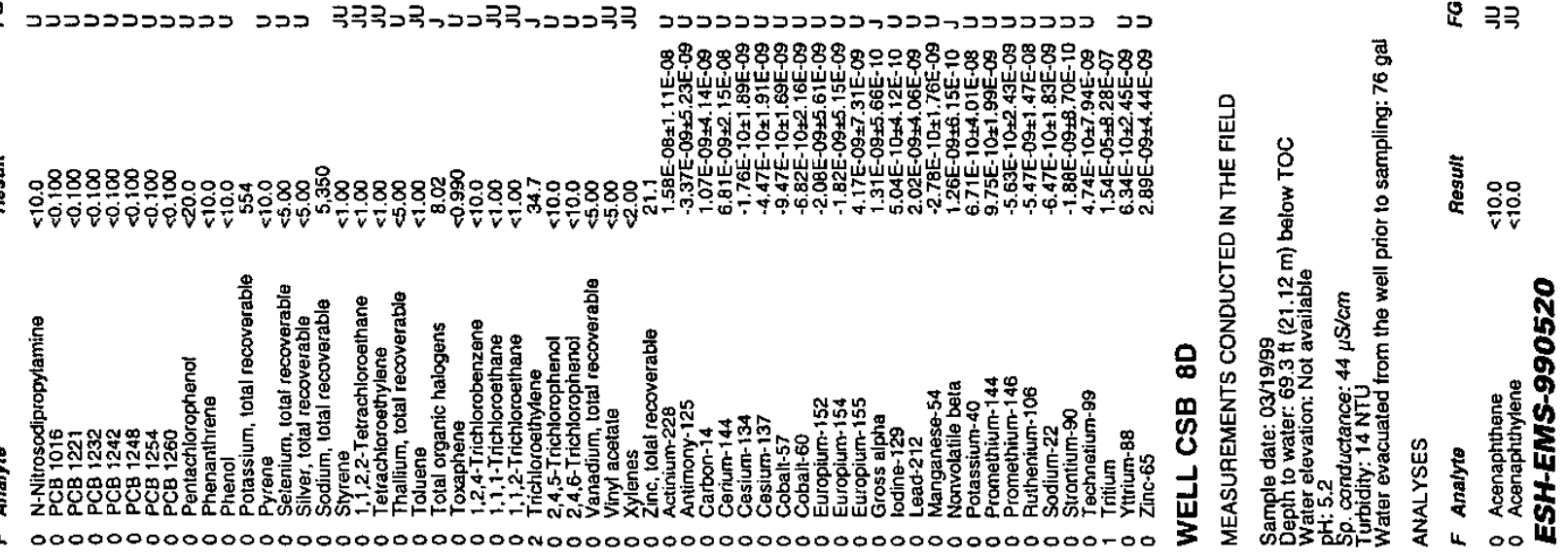

ริำ

ปี่

密

is So

注

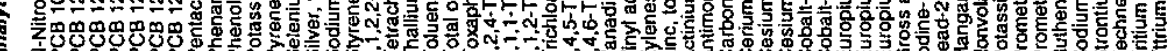

400000000000000000000000000 N00000000000000000000000000000-00 

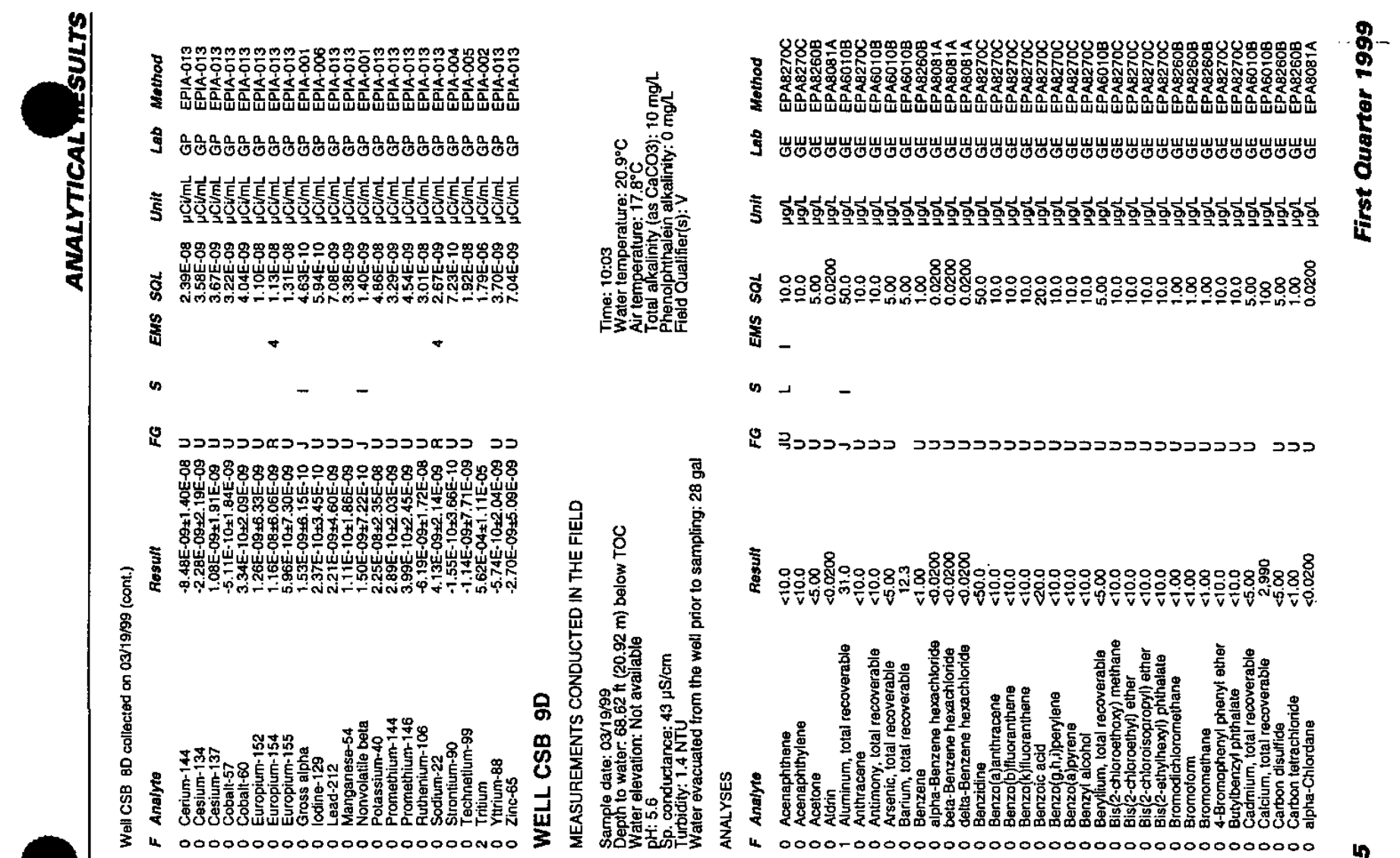

产

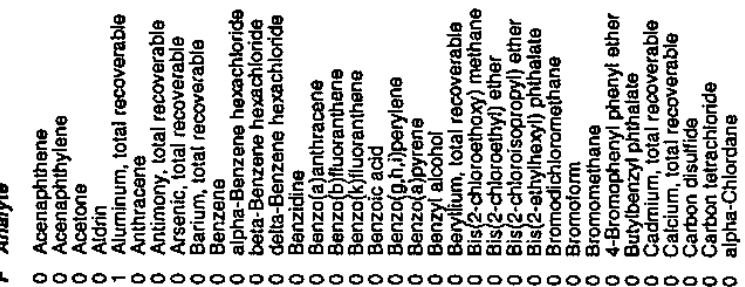

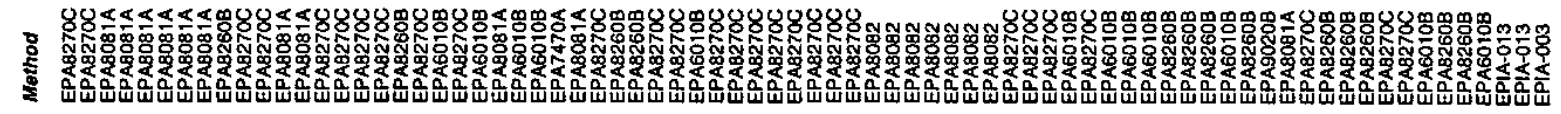

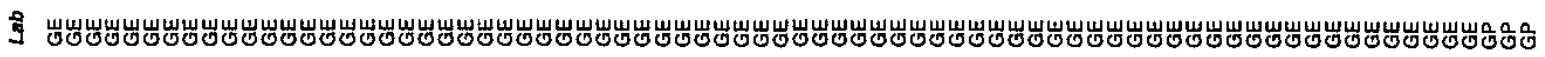

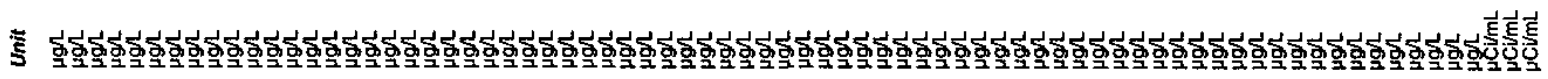

\%

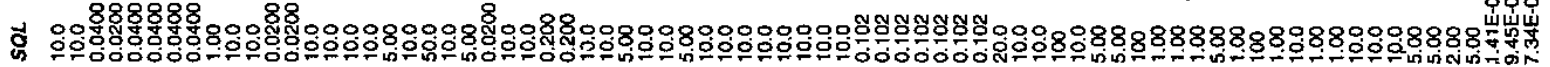
量
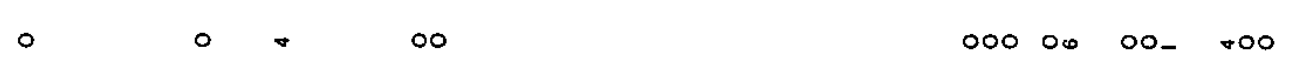

is 00

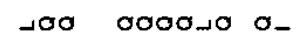

0-100_00000000

ooo 0

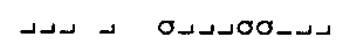

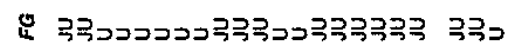

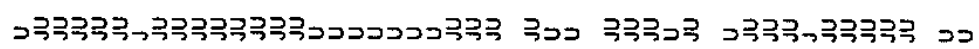

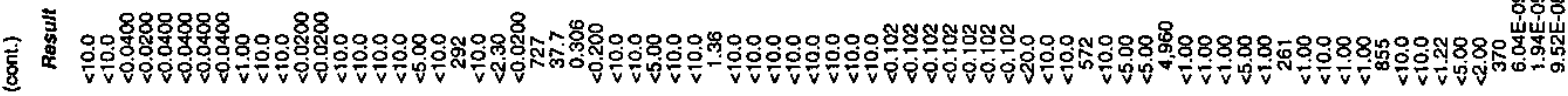

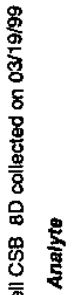

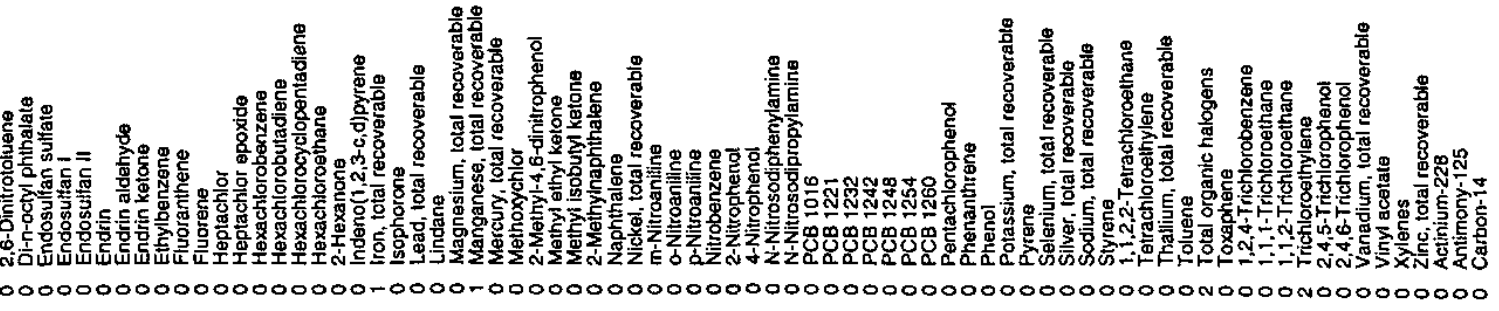


7

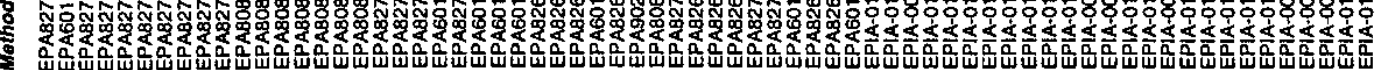

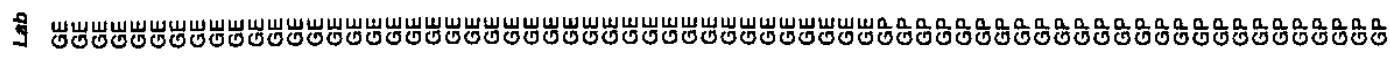

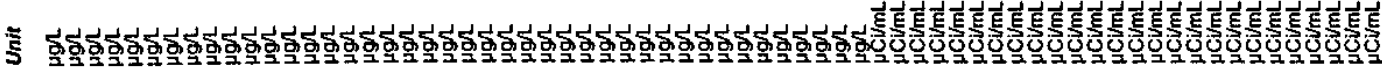

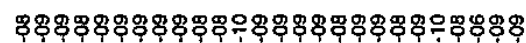

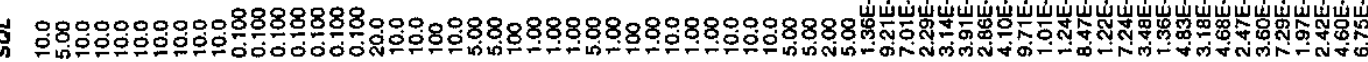
窝

is -

Q

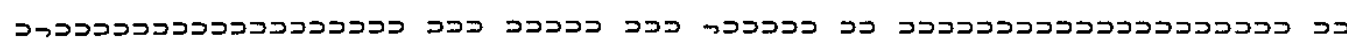
8 \% 8 8 8888 \$

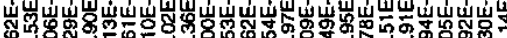

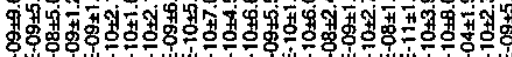

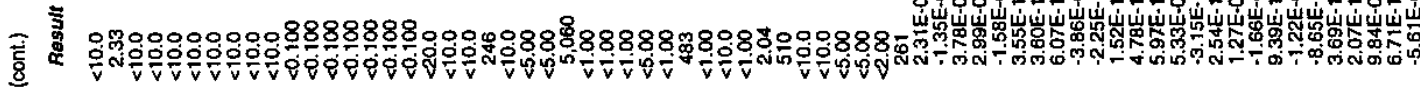

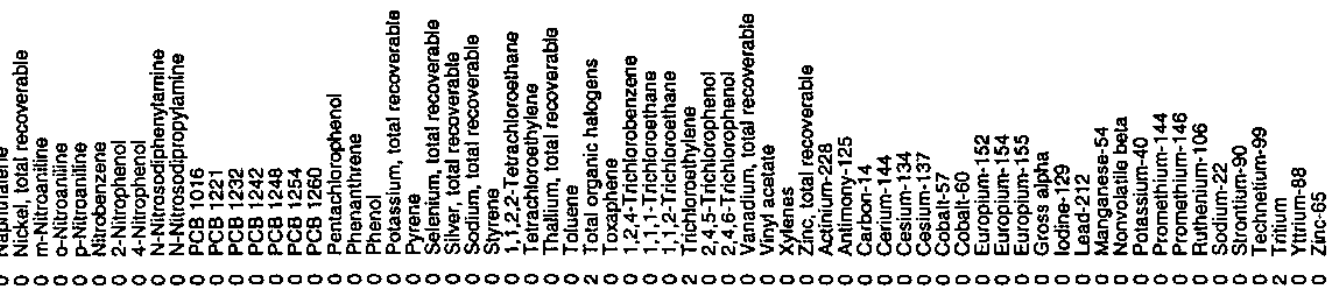

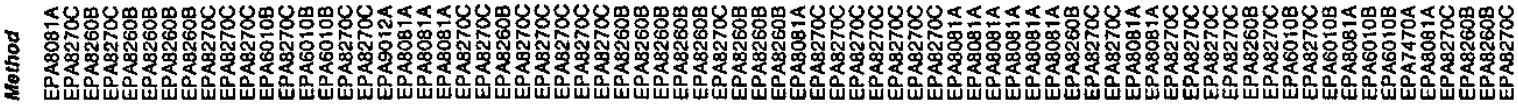

ఫ

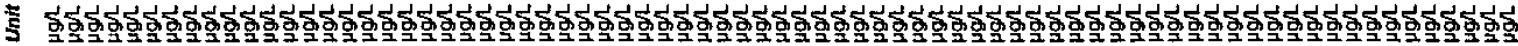

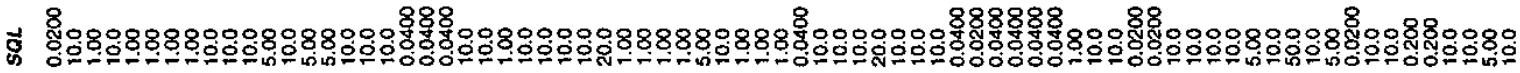
峦

佔

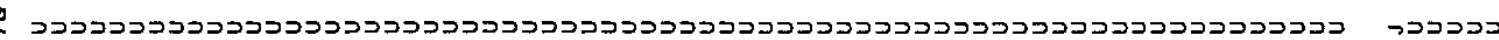

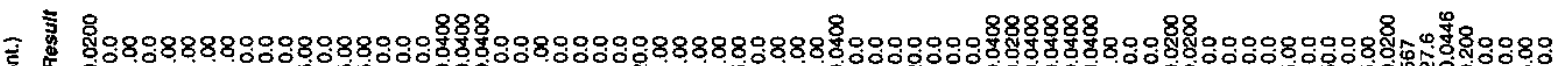
言 世

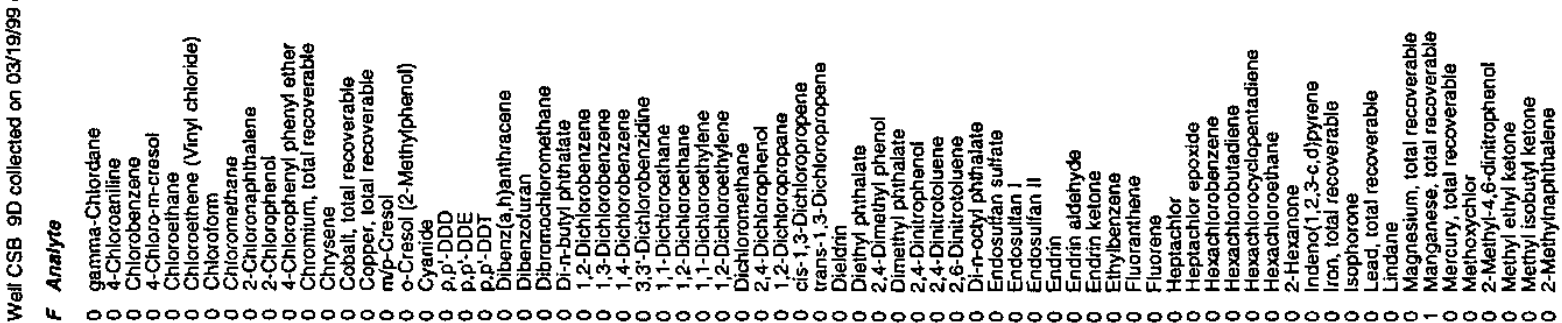




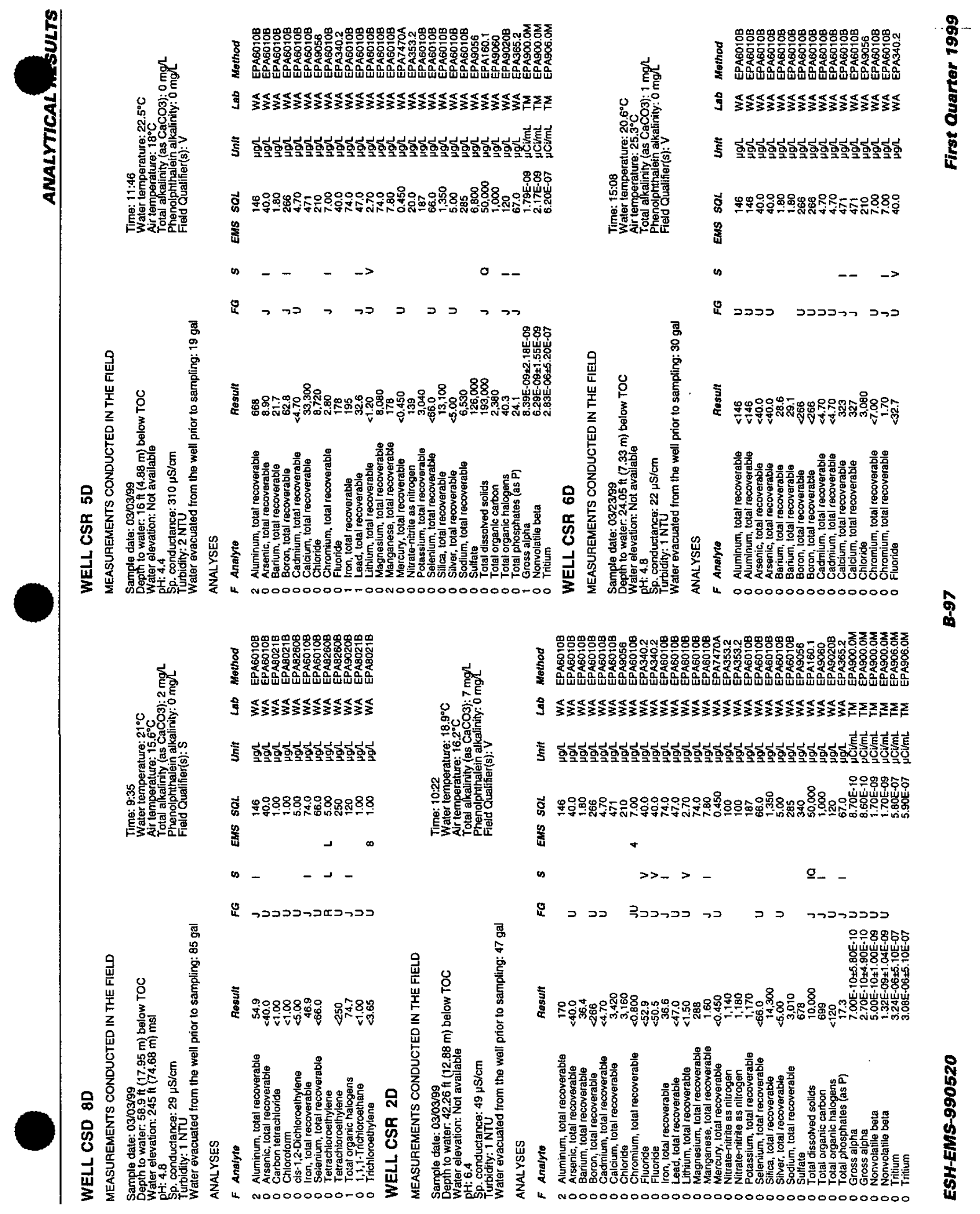




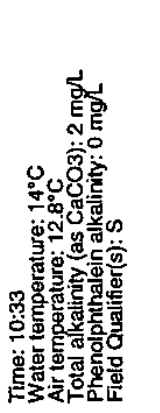

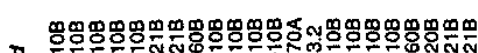
范

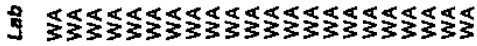

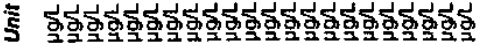

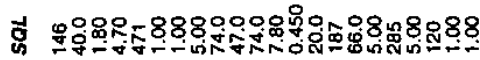
辤

s -

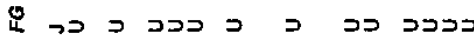

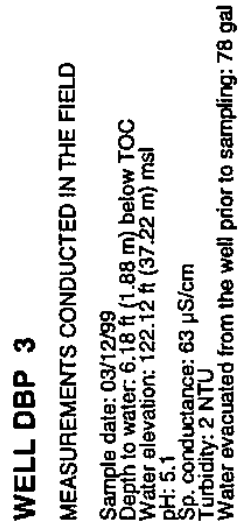

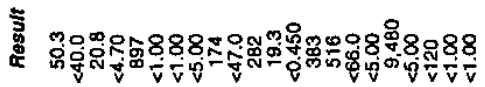

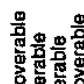

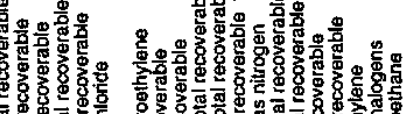

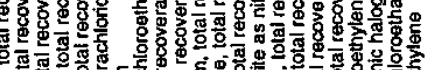

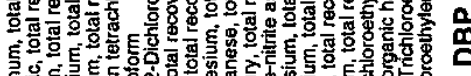

出

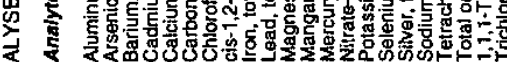

\& is N0000000-0000000000000

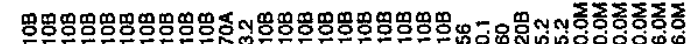

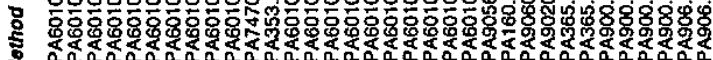

:

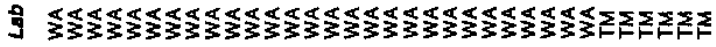

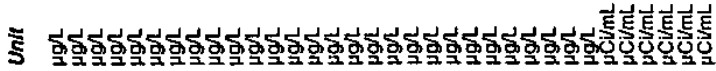

8 8워용후

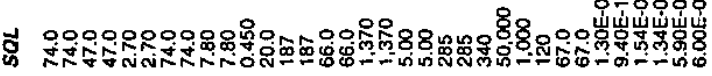

量

os -.

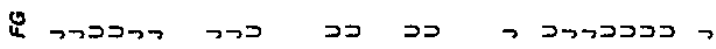

으으유유후우

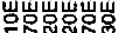

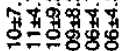

言 .

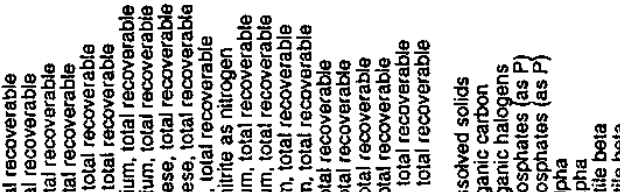
\% 虽 委 L 0000000000000000000000000000000000

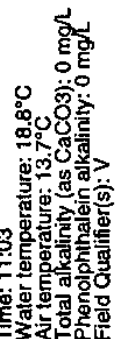

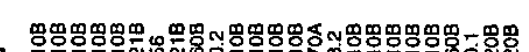
ऽ

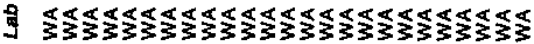

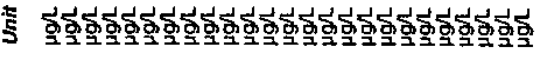

현 咅

n $\quad>--\quad$ g

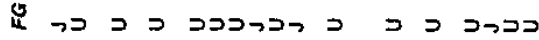

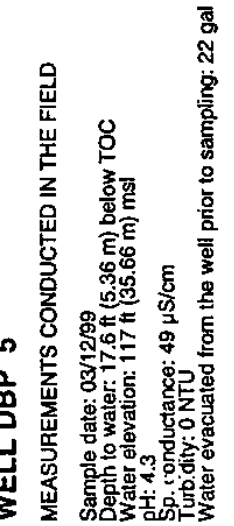

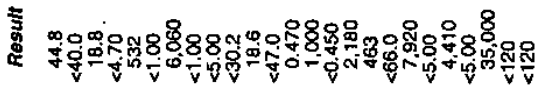

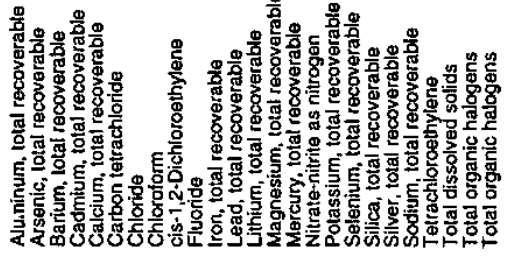

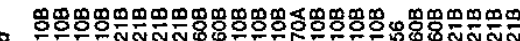
它

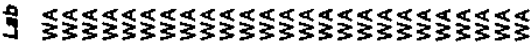

융.

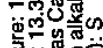

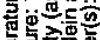

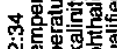

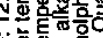

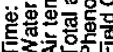

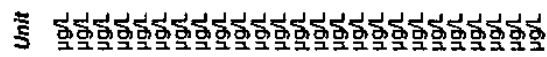

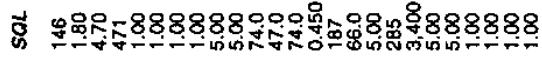
蛋

es

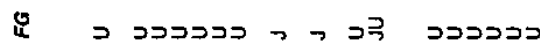
ब

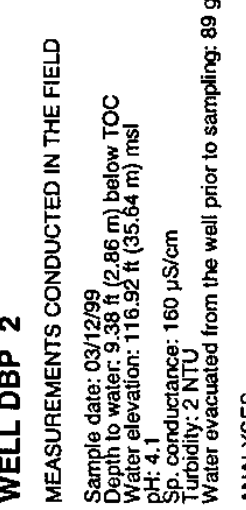

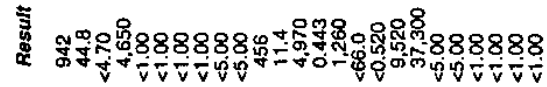

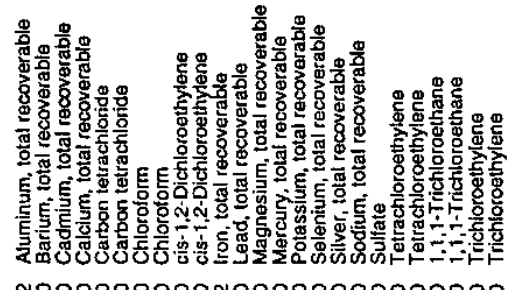




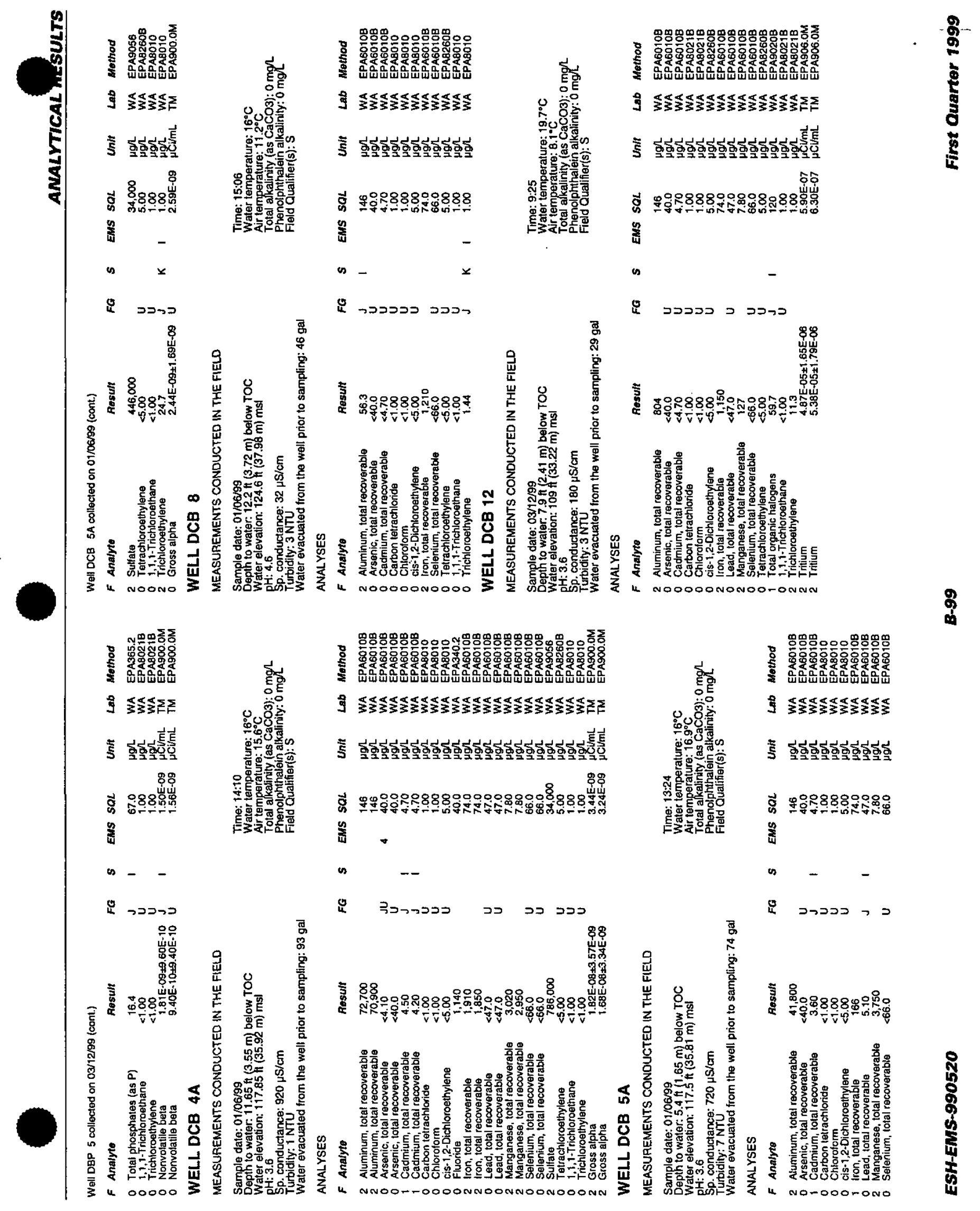



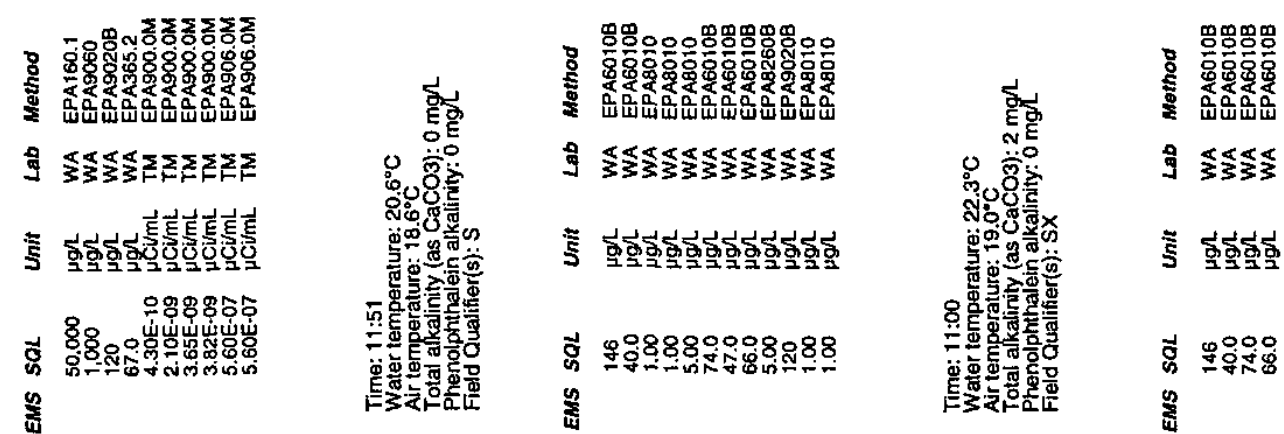

9
5
5
5
0
0
5

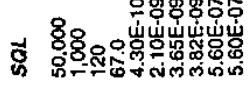

竞

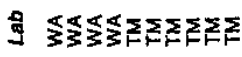

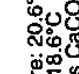

a 0 -

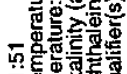

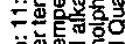

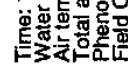

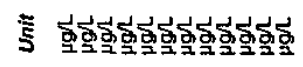

ㅎำ

密

粂

$\infty-$

n -

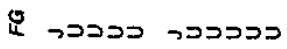

ב כ כ כ

Q 3

888888

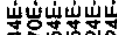
จู่

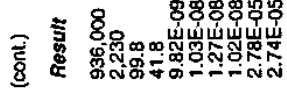

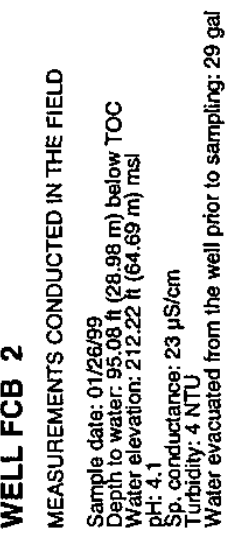

芦
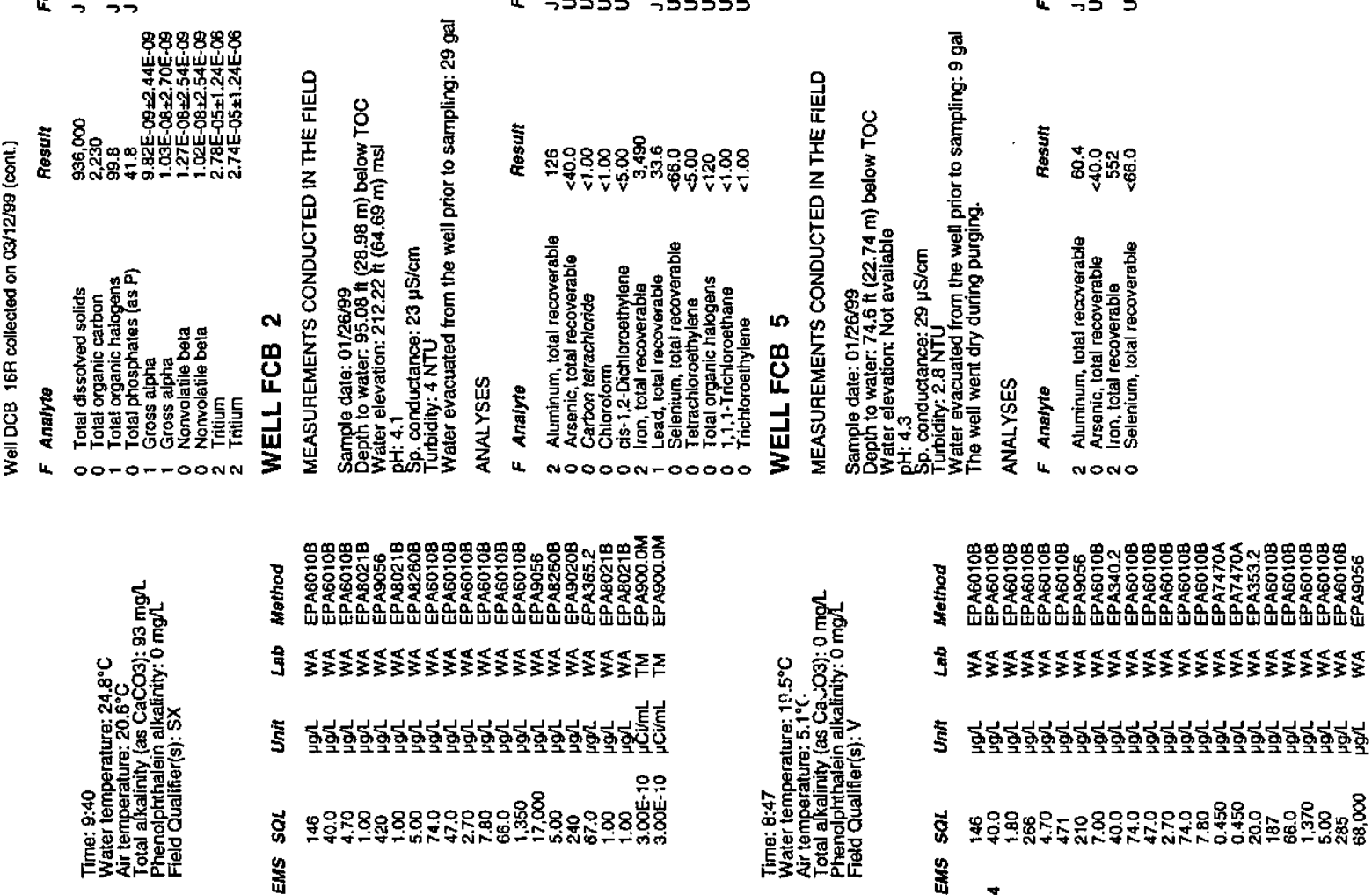

$\frac{8}{6}$

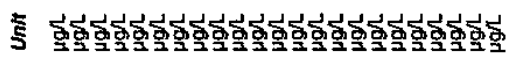

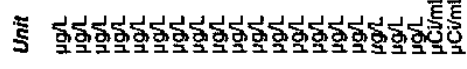

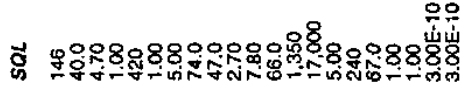
紊

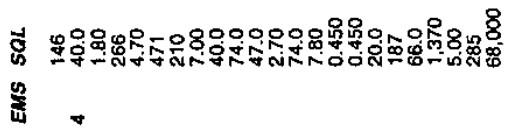

is --

a

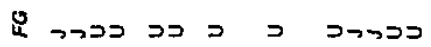
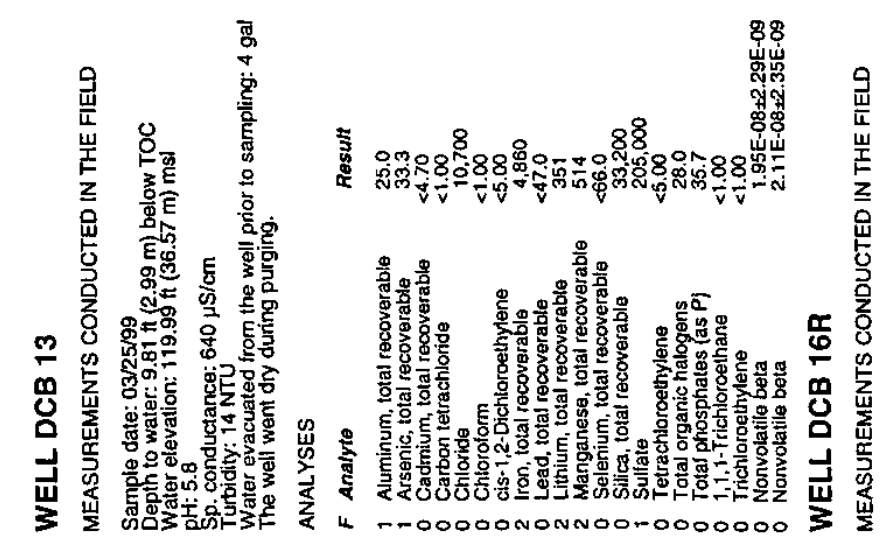

ल

\& $3 D \supset D \supset$

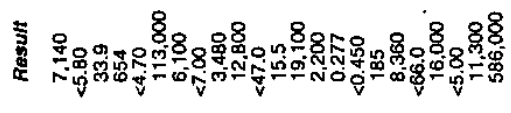

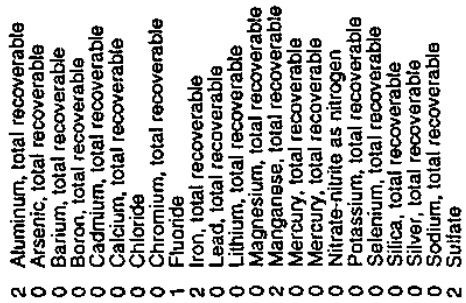

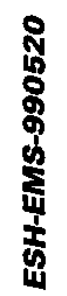




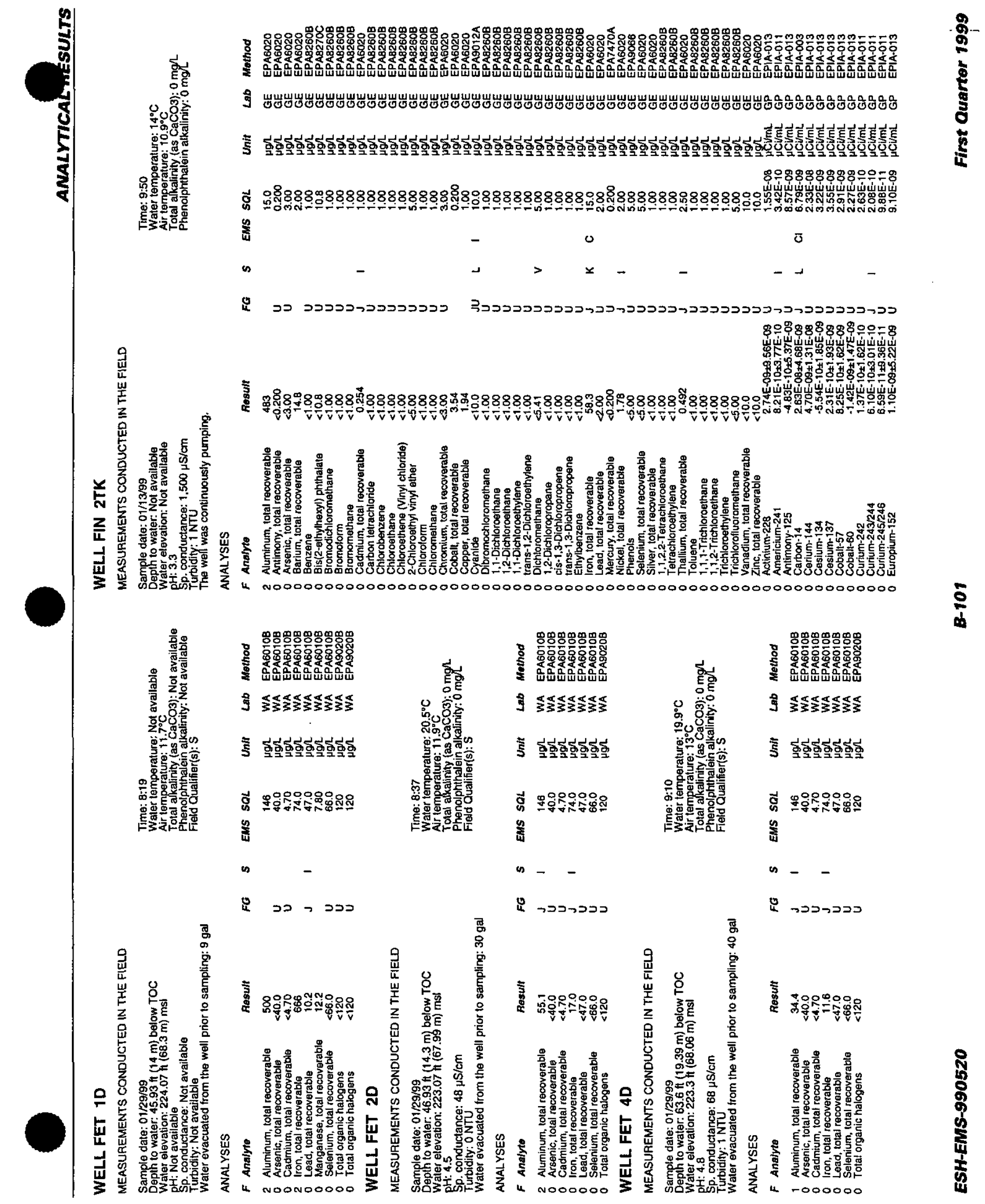


o 帝

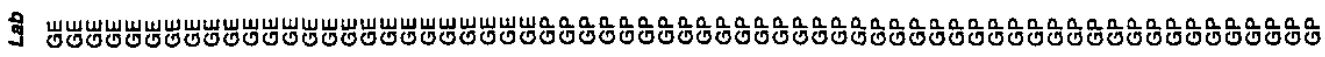

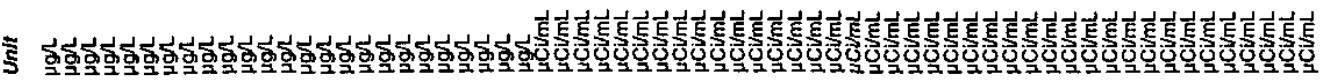

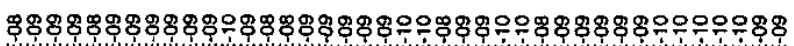

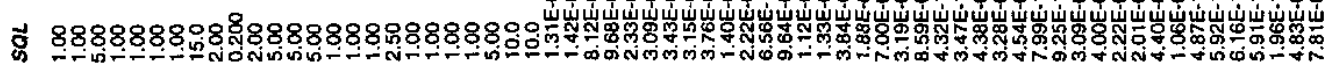
竞 $0000000 \quad 0000000$

is g979g9g9 - g9_9g9g9 0

000

ע

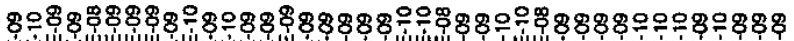

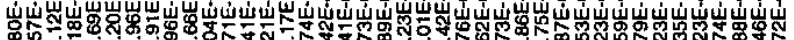

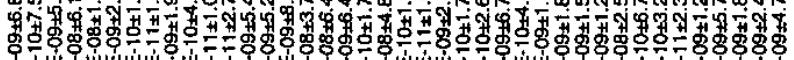

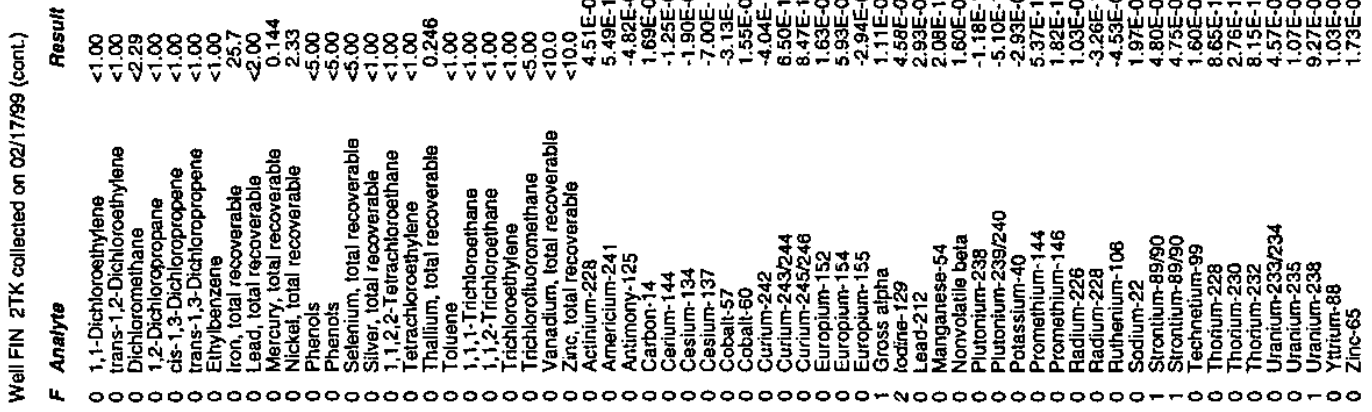

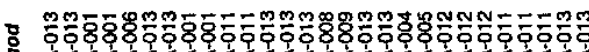

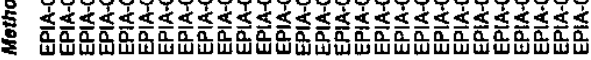

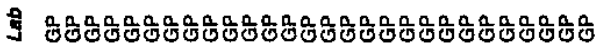

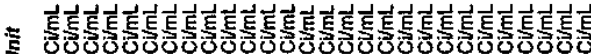

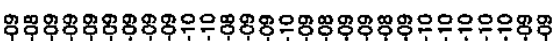

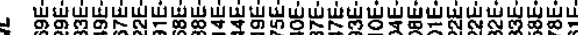

क्

章

$--\quad-\quad+$

is $\quad$ دي - - -

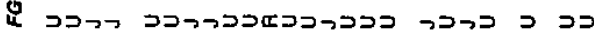

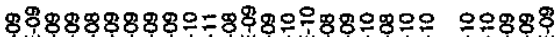

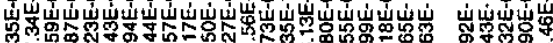

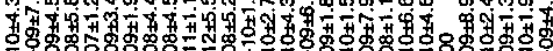

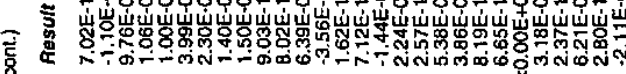

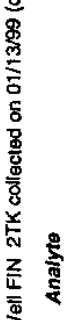

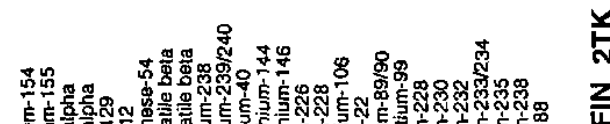

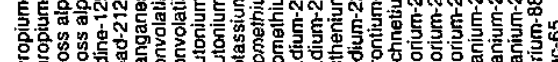
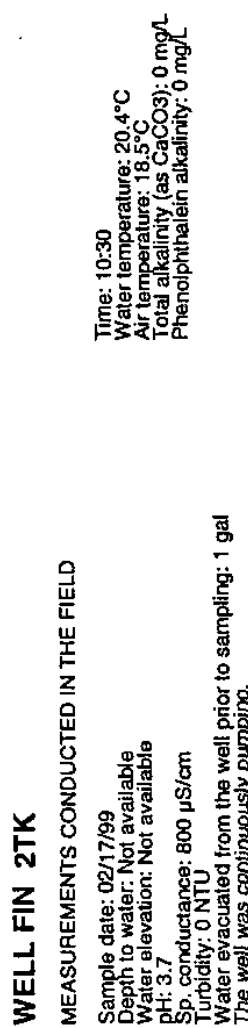
ब艿

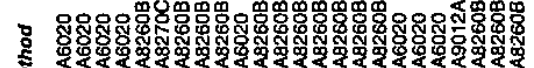

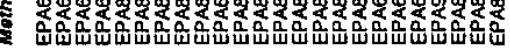

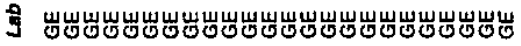

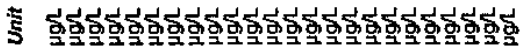

ธิ 0.

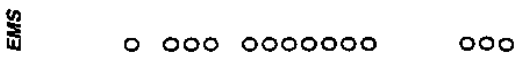

in 9 ggg gggggga ggg

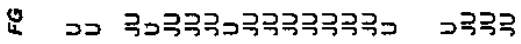

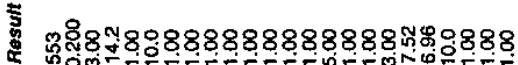

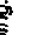

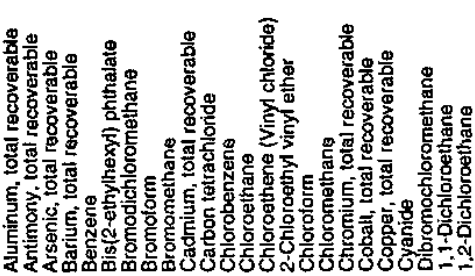

ำ 


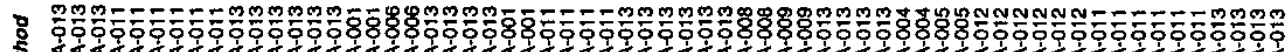

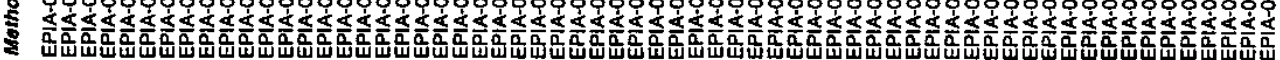

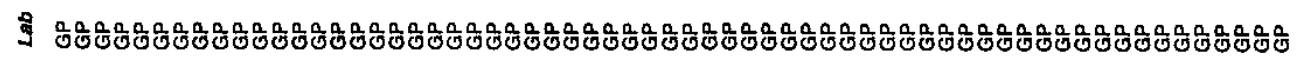

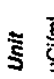

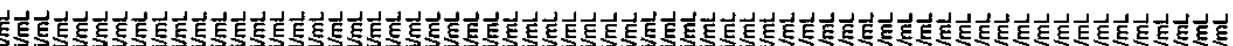

క

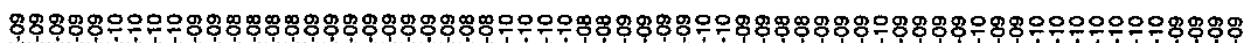

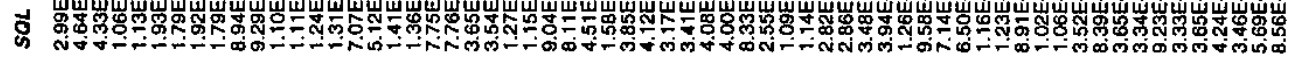

竞

()

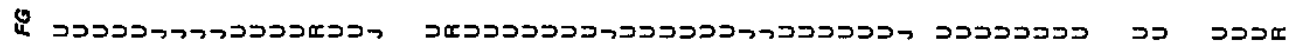

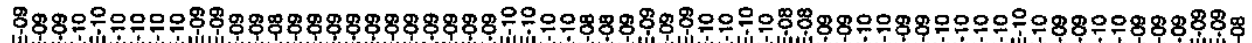

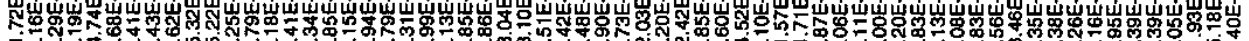

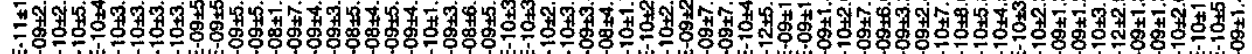

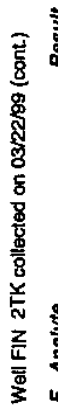

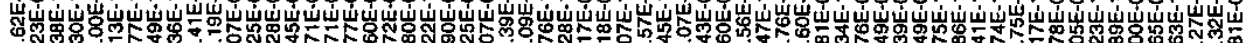

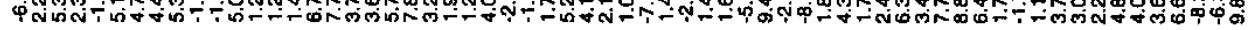

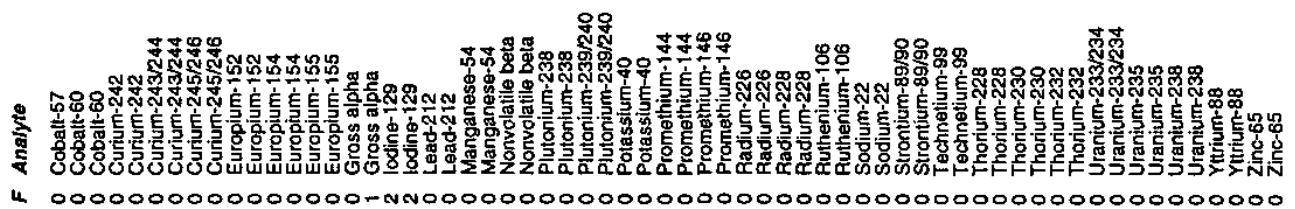

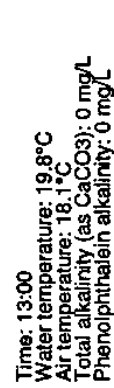

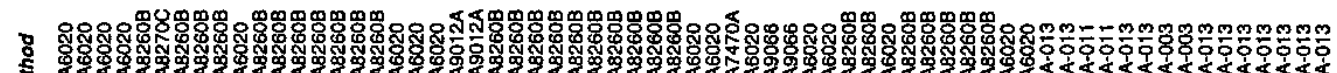

定

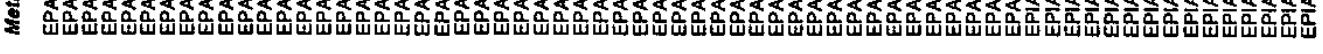

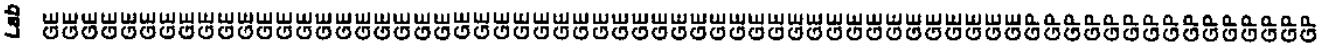

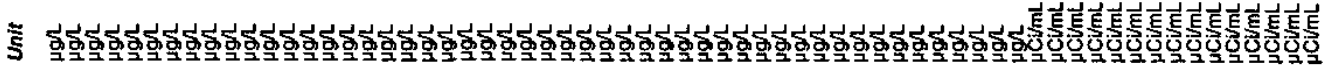

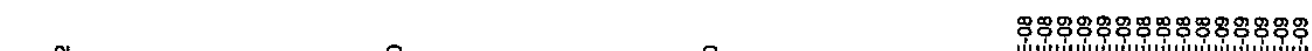

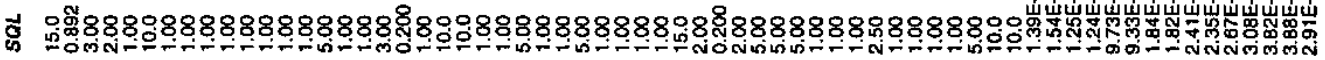
秀 $00000000000 \quad 0000000000 \quad 0000000$

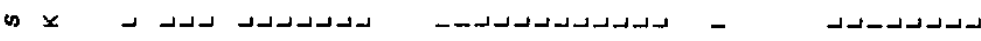

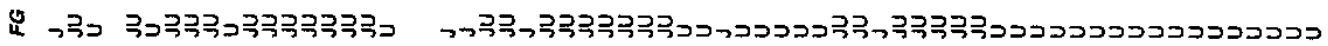

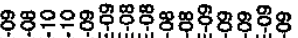

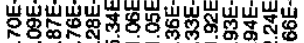

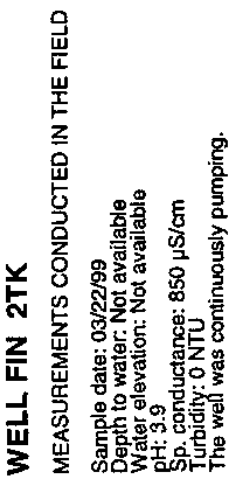

常 更

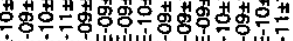



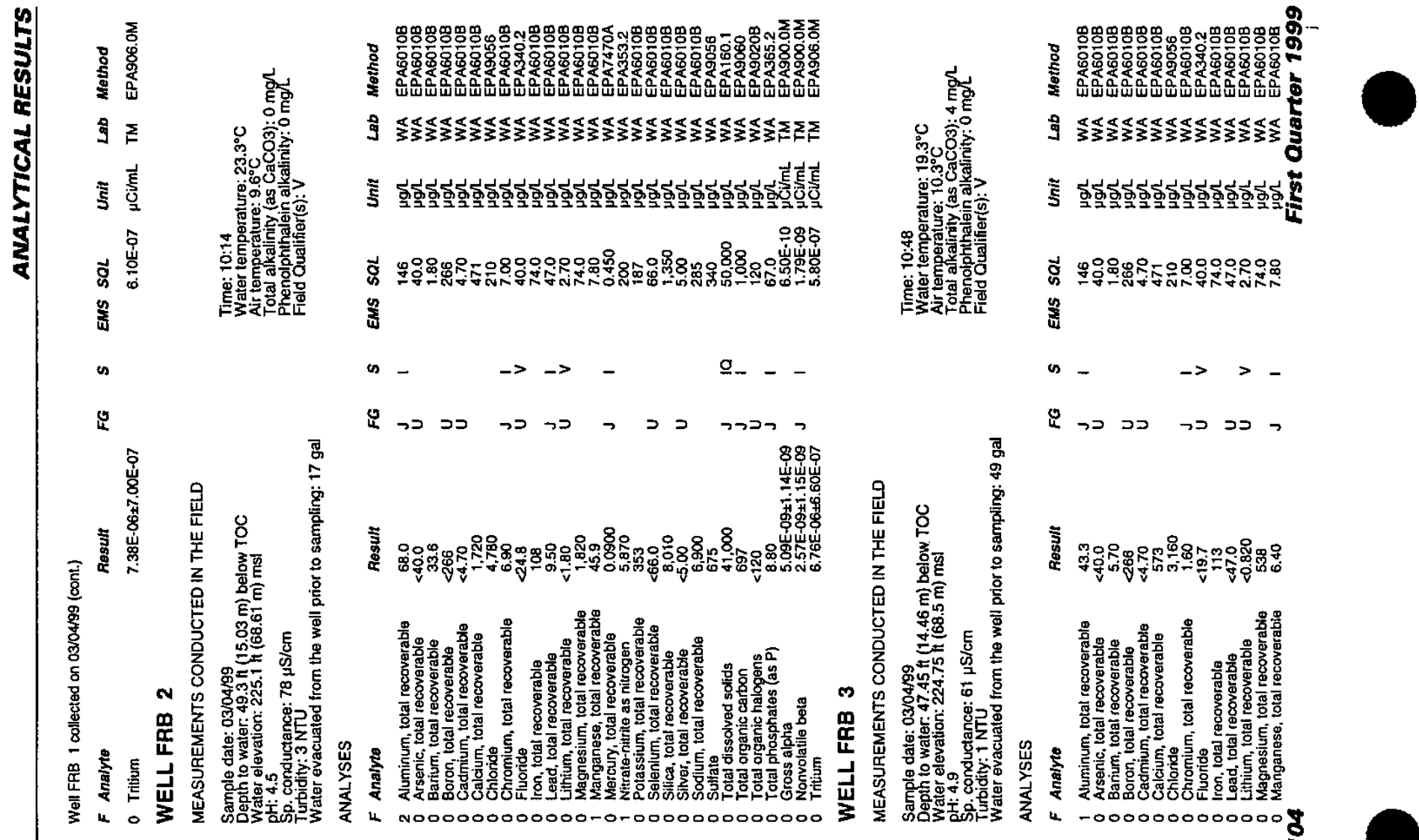

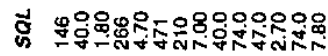

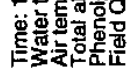
蛋

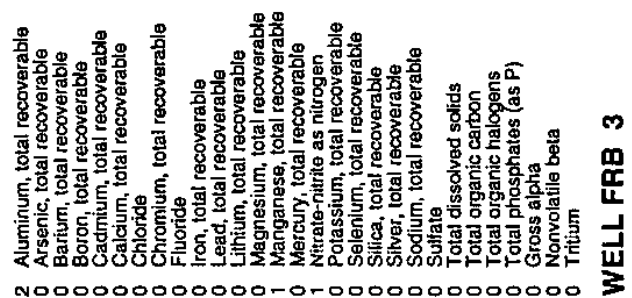

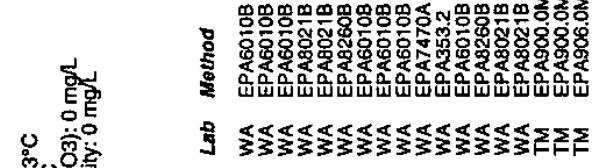

io.

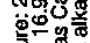

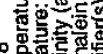

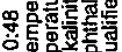

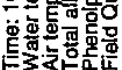

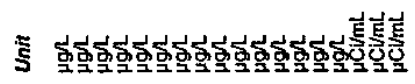

$88 \%$

혼
量

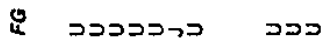

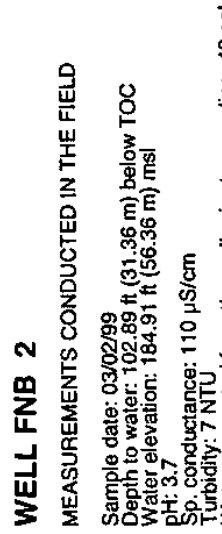

\section{बु,}

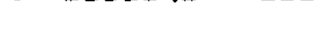

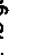

$$
\text { (1) }
$$

产

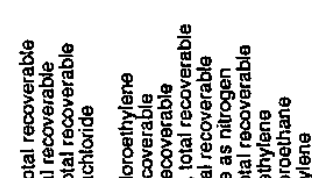

888

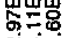

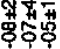

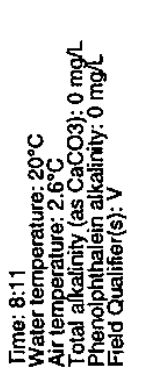

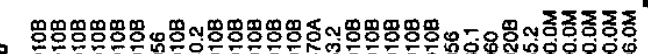

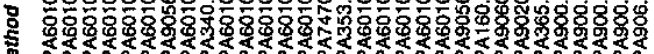

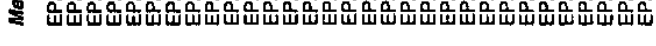

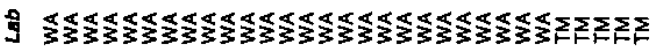

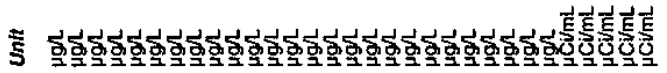

유유용무

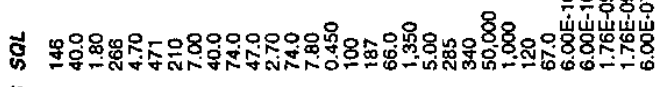
量

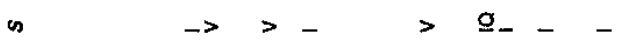

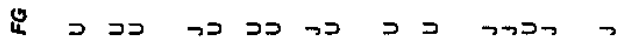

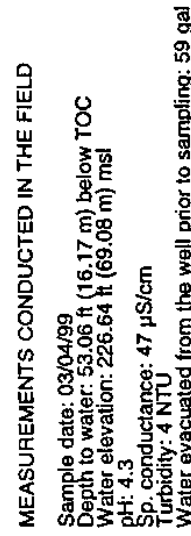

㟶

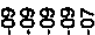

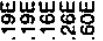
5

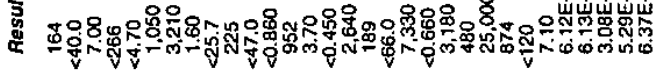

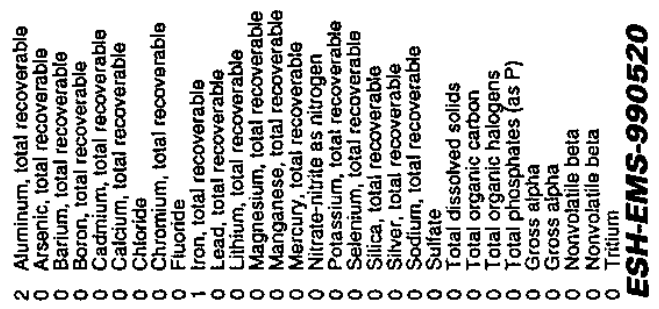

\section{g}

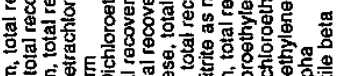




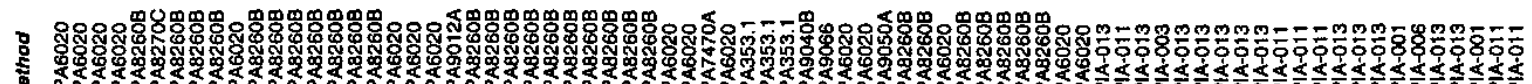

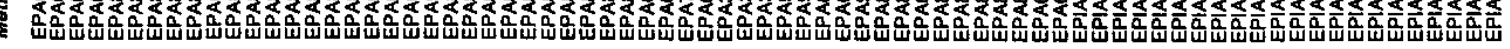

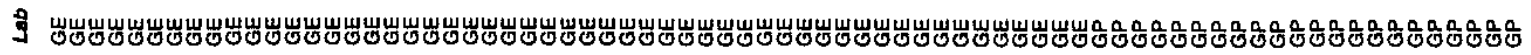

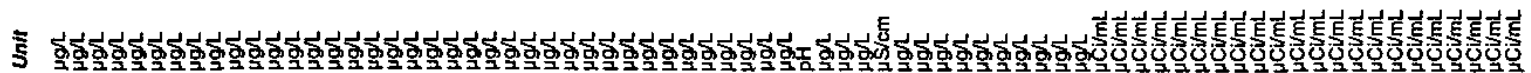

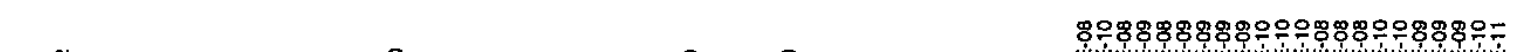
ธ్ .

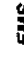
. 00000000000000000000009 es

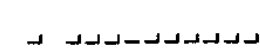

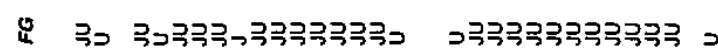
000000

00

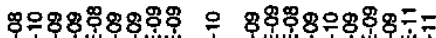

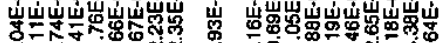

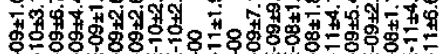

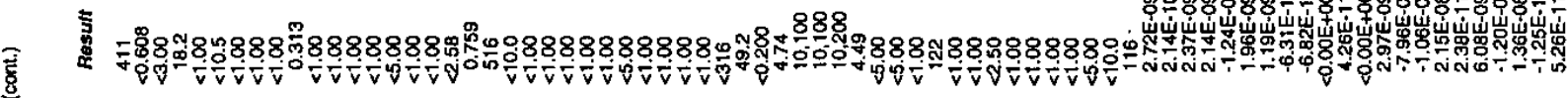
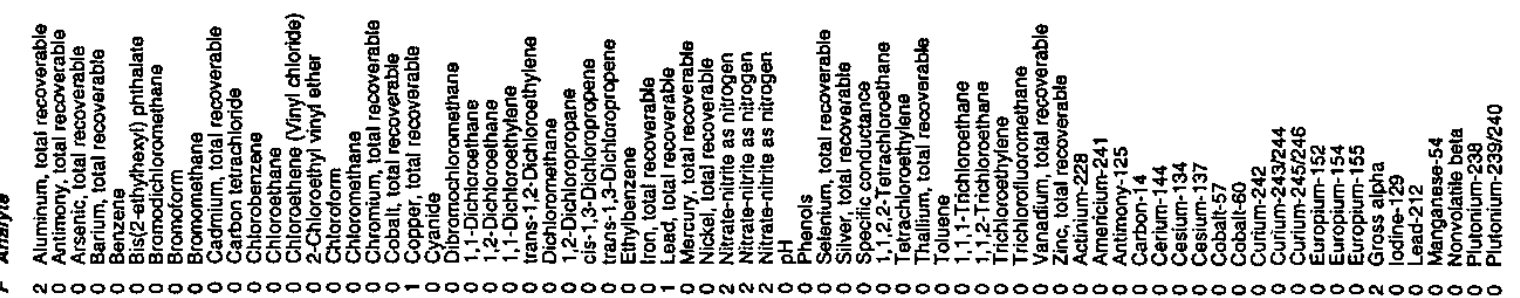

4 N000000000000000000-000000000000-00NNN000000000000000000000000000000N000000

\section{ธำ1900\% \\ 冬

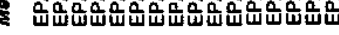

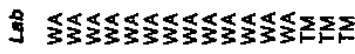

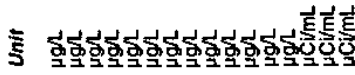

880

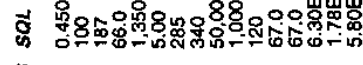

急

$\infty>$ O_ $_{-}$

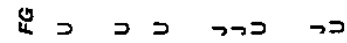

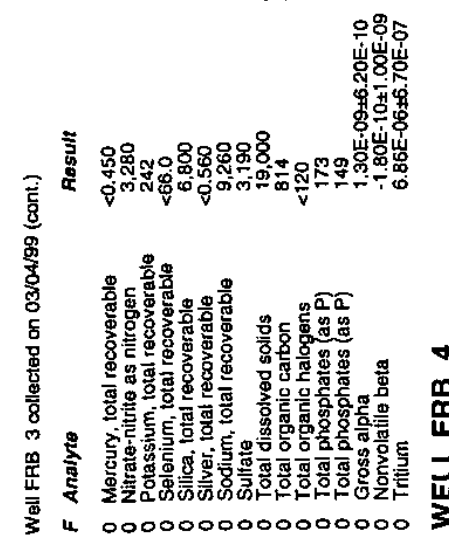

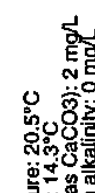

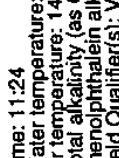

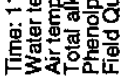

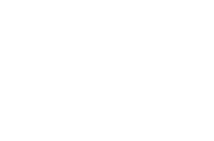

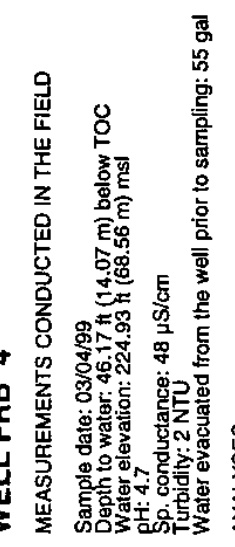

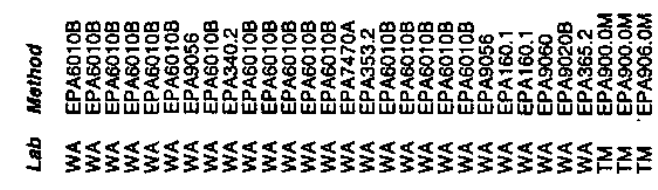

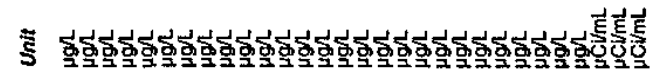

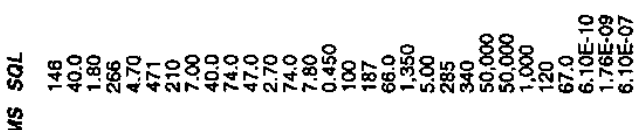

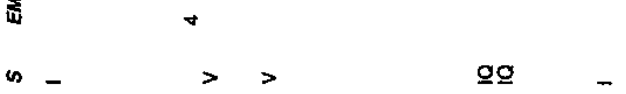

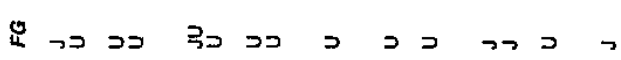

$\frac{\text { 象 }}{\dot{1}}$

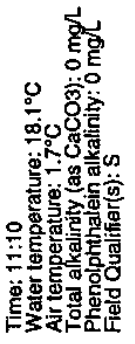

妾

으웅
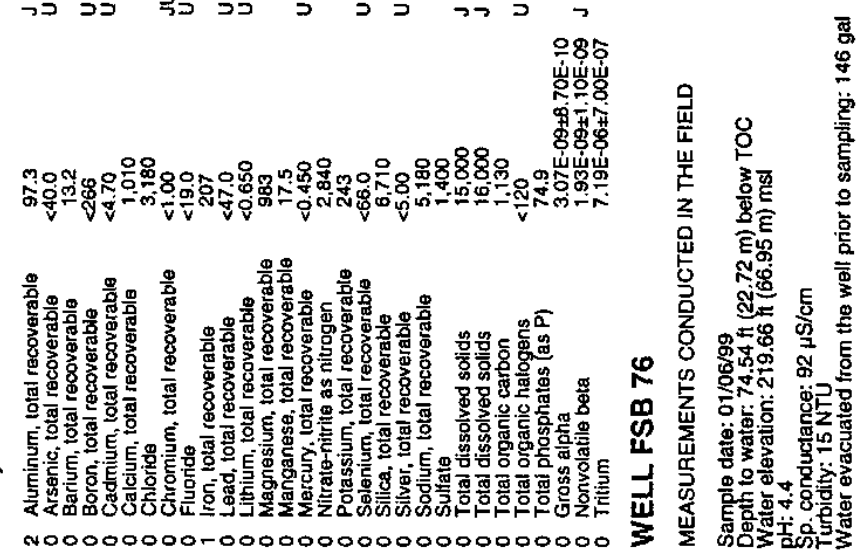


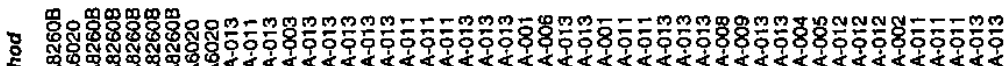
亮

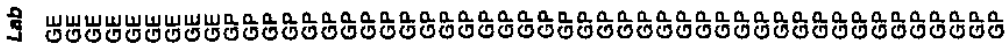

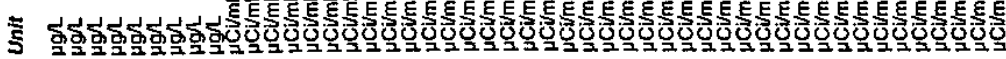

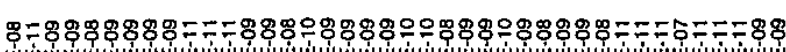

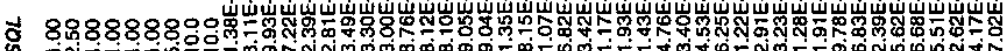
क -Nं 蛋 000000 oo - - $\checkmark$
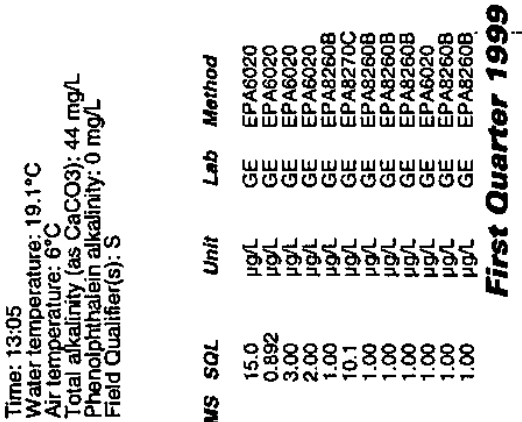

Q 1.<smiles>[Li][13CH]</smiles>

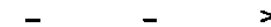

《

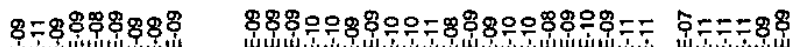

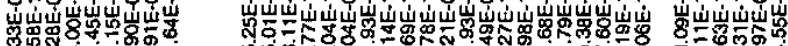

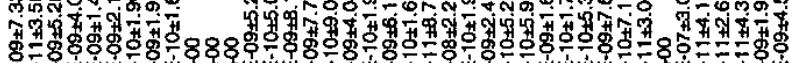

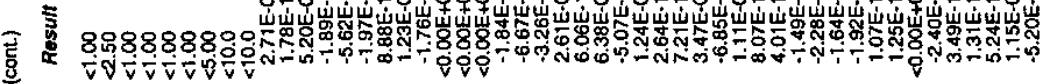

要
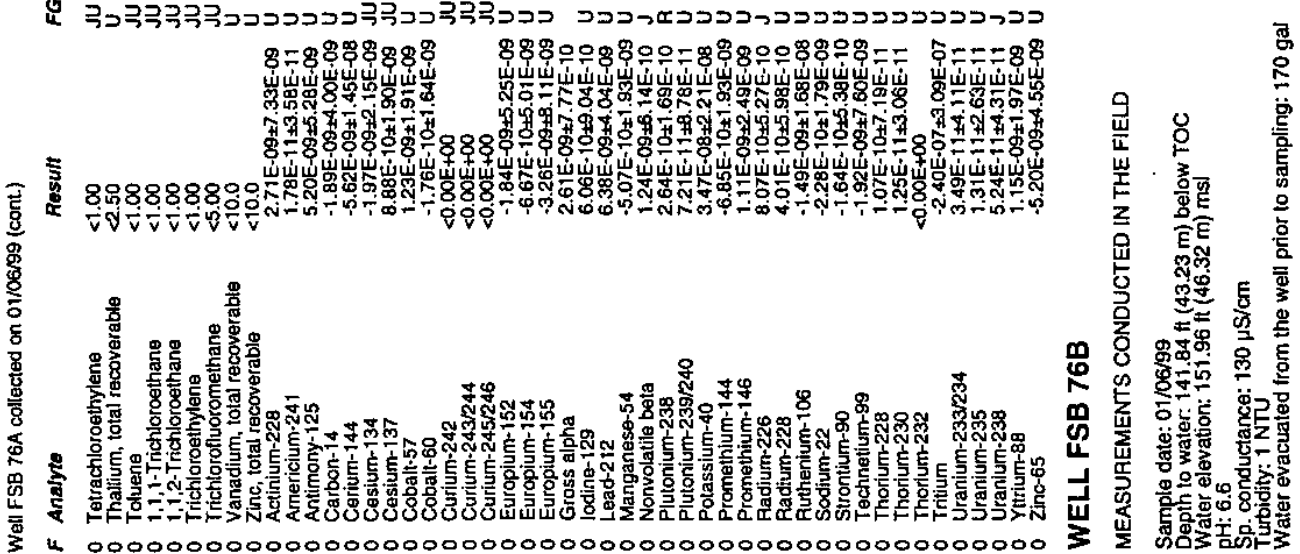

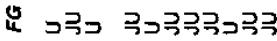

产

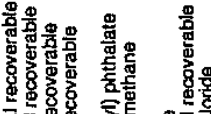

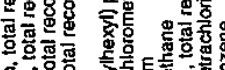

出

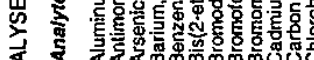

4h 000000000000

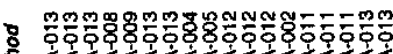

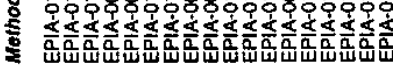

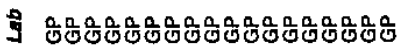

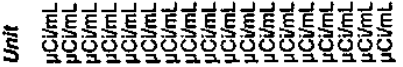

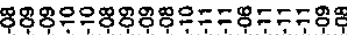

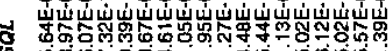

密

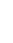

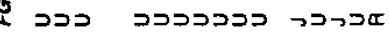

88880

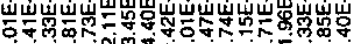

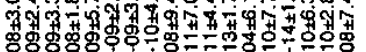

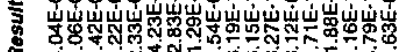

है व

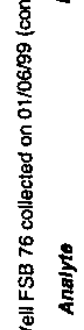

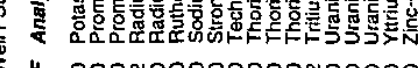

u 000 N00000000N00000

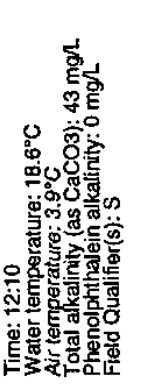

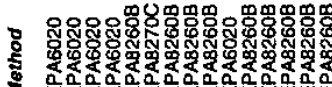

范

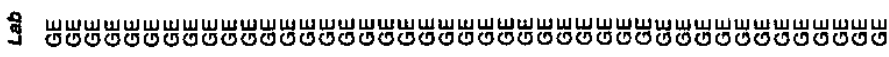

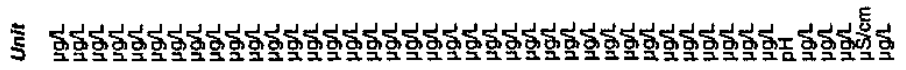

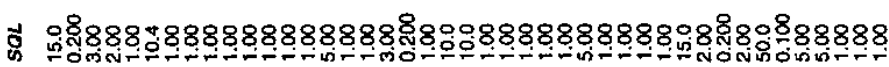

量

00000000000 \& 0000000000 0

o

is

ख్

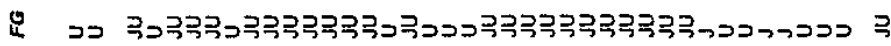

衰

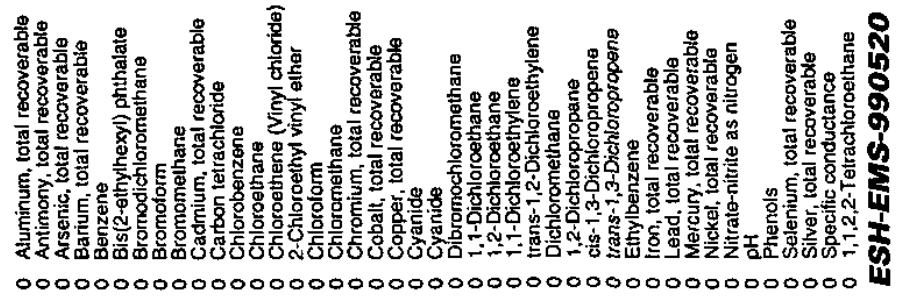

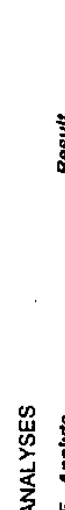

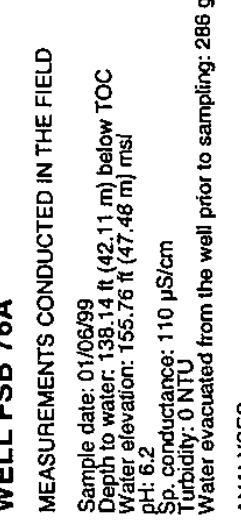




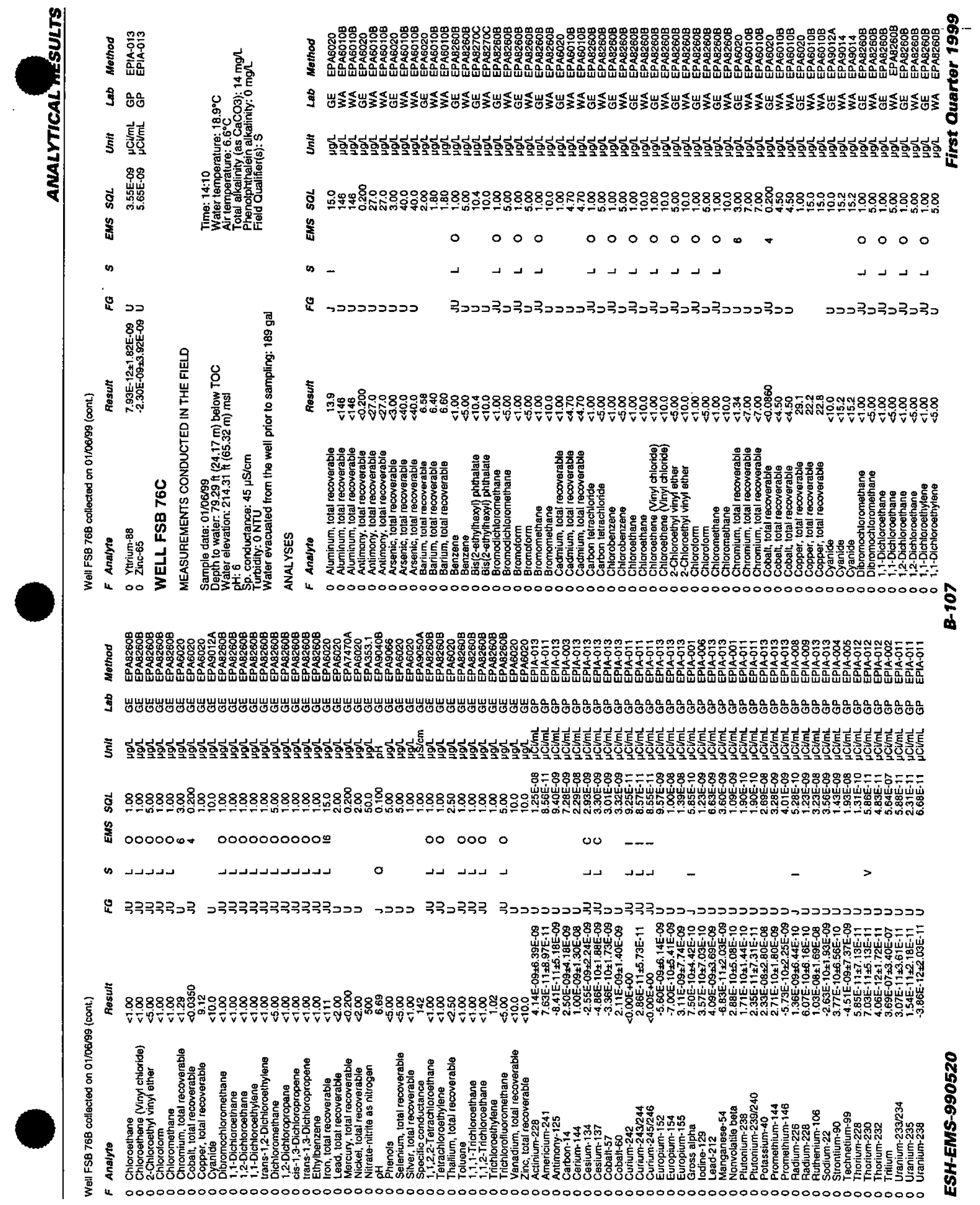




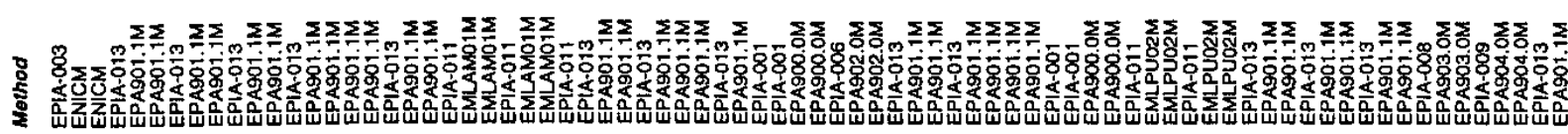

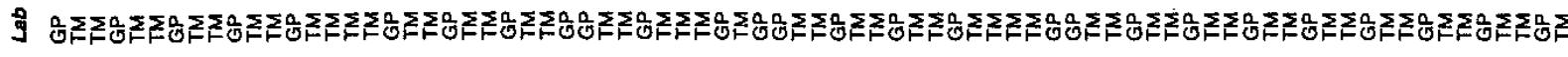

s

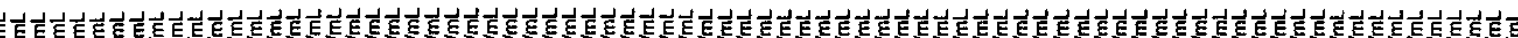

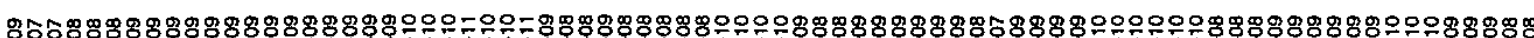

1 山्.

慈

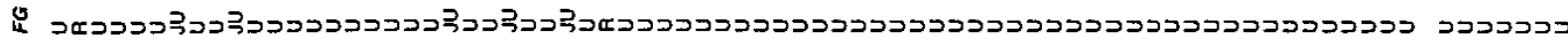

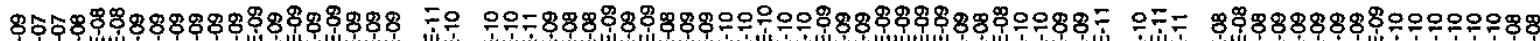

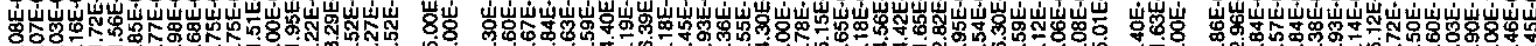

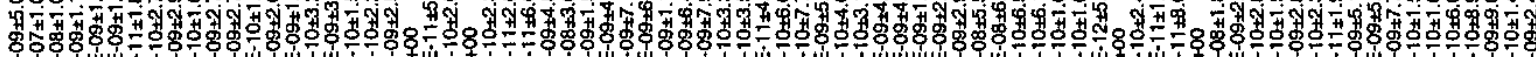
吾

軁

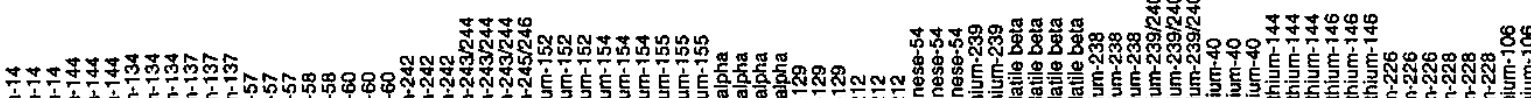

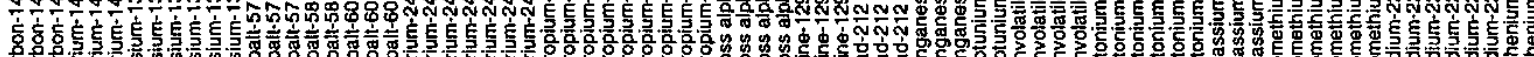
焉 \$ $40000000000000000000000000000-0000000000000000000000000000000000000000000000000$

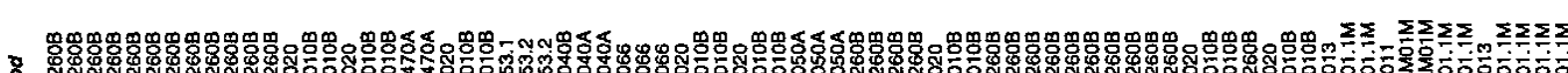

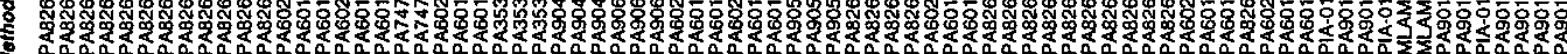

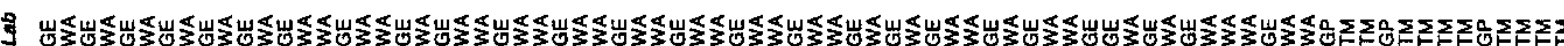

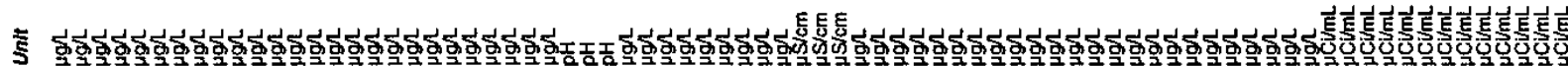

至

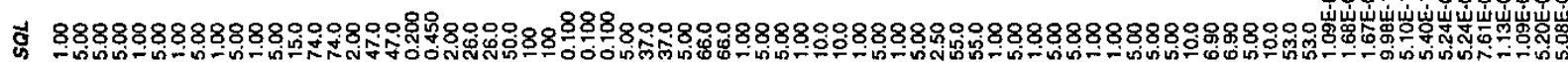
蛋 $000000 \varphi_{0}$

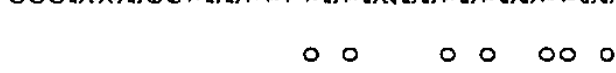

000

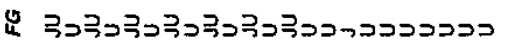

בכבכבכבכדתר

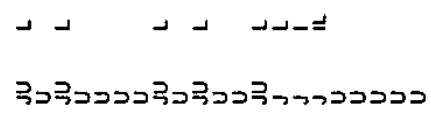

$--$

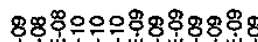

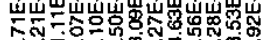

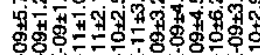

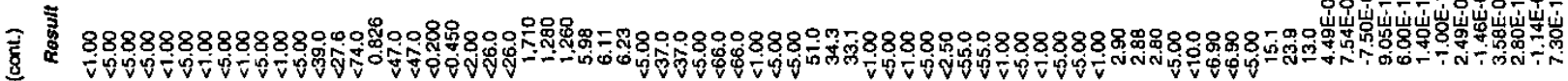

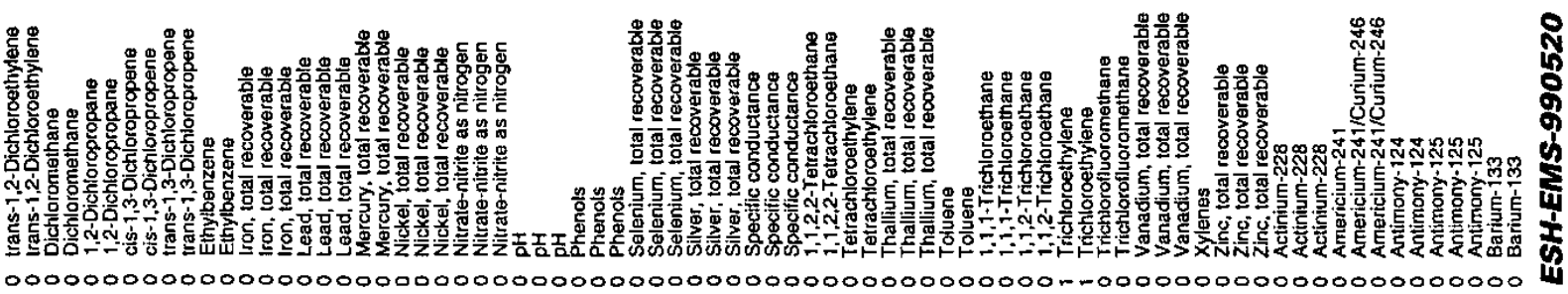




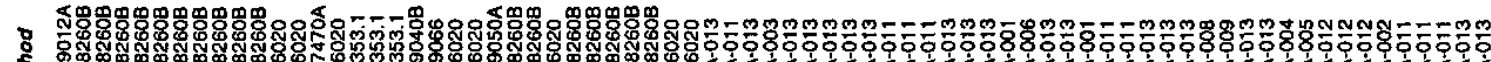

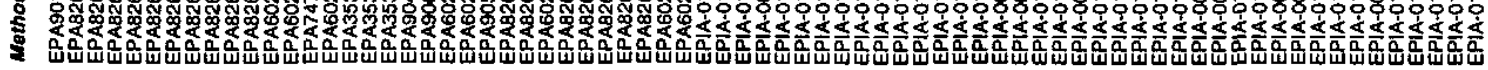

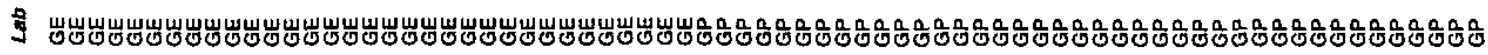

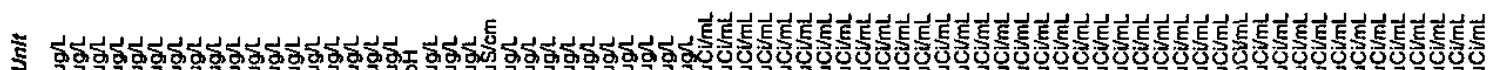
1

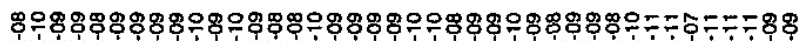

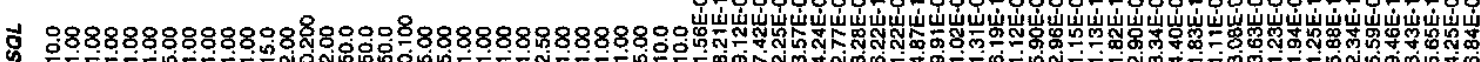
量

娄 00000000000

000000

00

Q $\lrcorner\lrcorner\lrcorner\lrcorner\lrcorner\lrcorner\lrcorner\lrcorner\lrcorner\lrcorner\lrcorner-$

o

لـد

$\rightarrow$

$+$

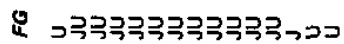

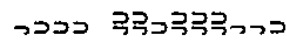

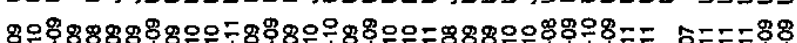

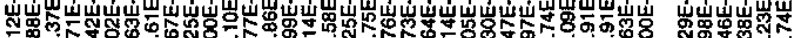

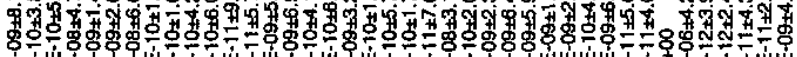

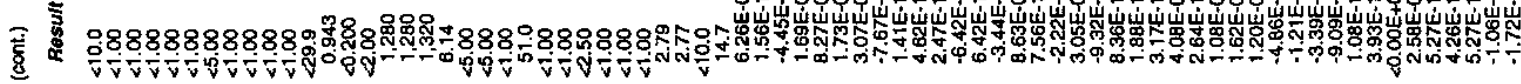

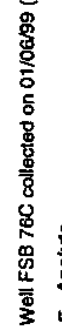
害 :

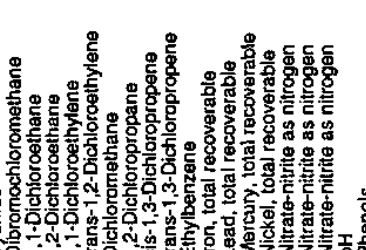

$400000000000000000000000000000-0000000000000000000000000000000000000000000$

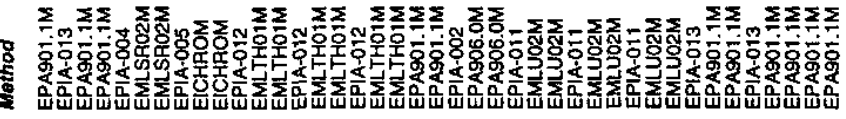

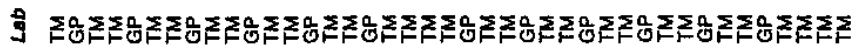

藏

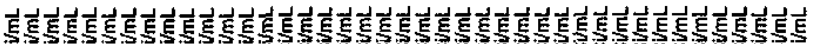

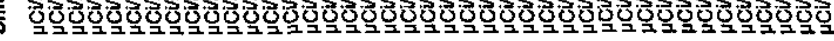

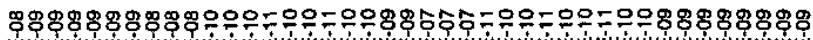

d

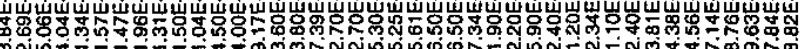
产

es

8

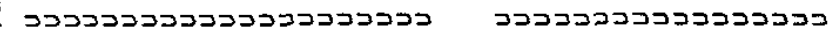

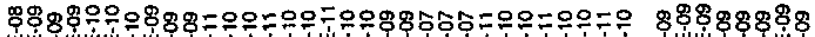

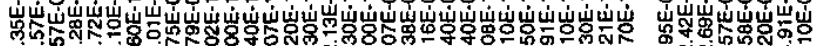

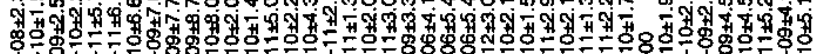

劳

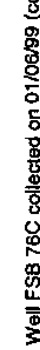

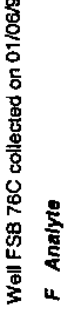

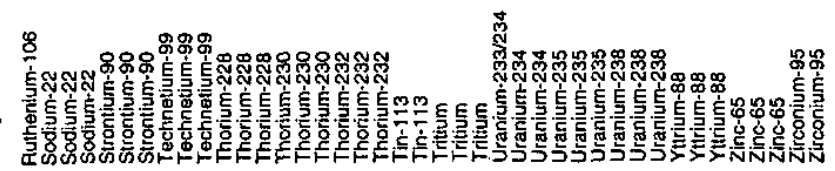

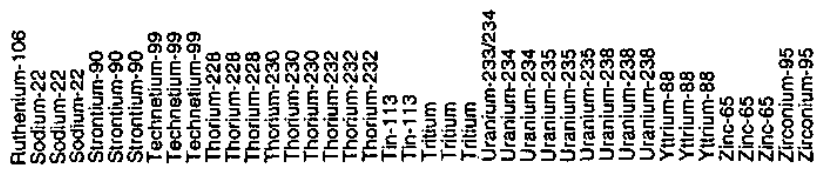

L 00000000000000000000000000000000000000000

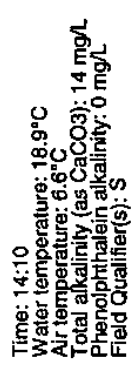

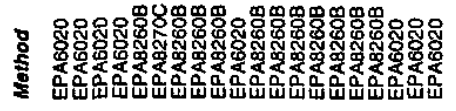

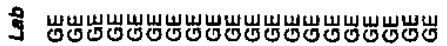

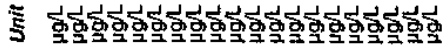

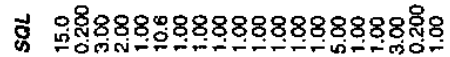

密

0000000000000

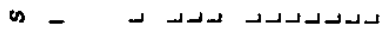

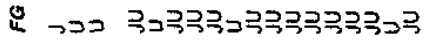

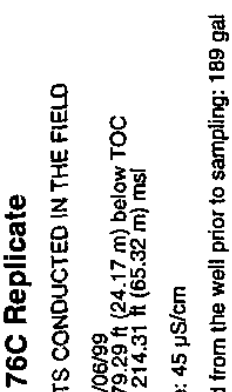

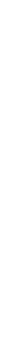

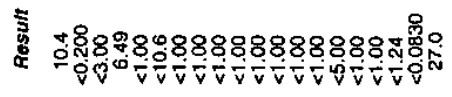

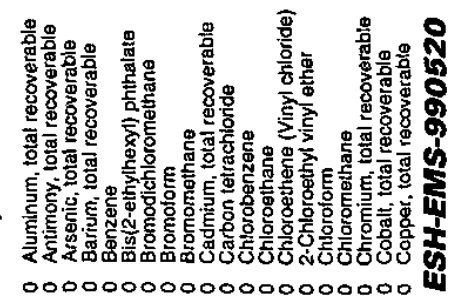


8 .

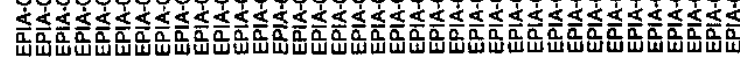

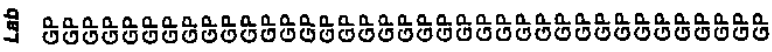

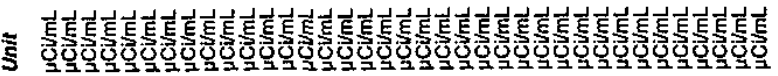

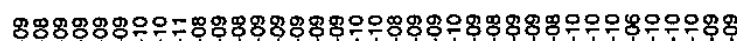

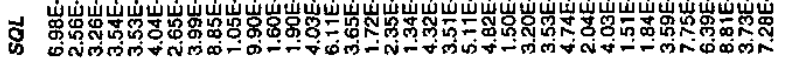

量的

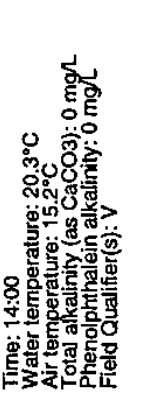

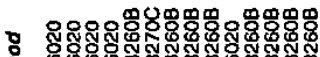

产

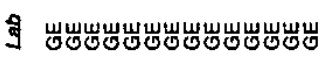

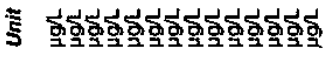

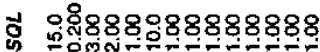

紊 00000000

a

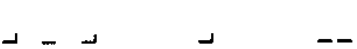

w

ـد - د.

נכ

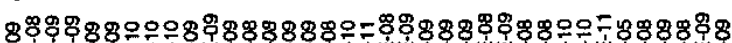

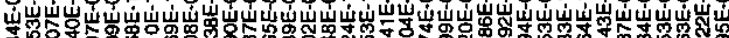

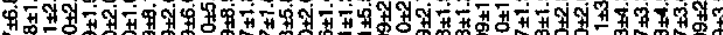

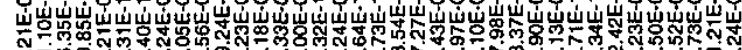

产

-

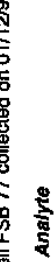

\%
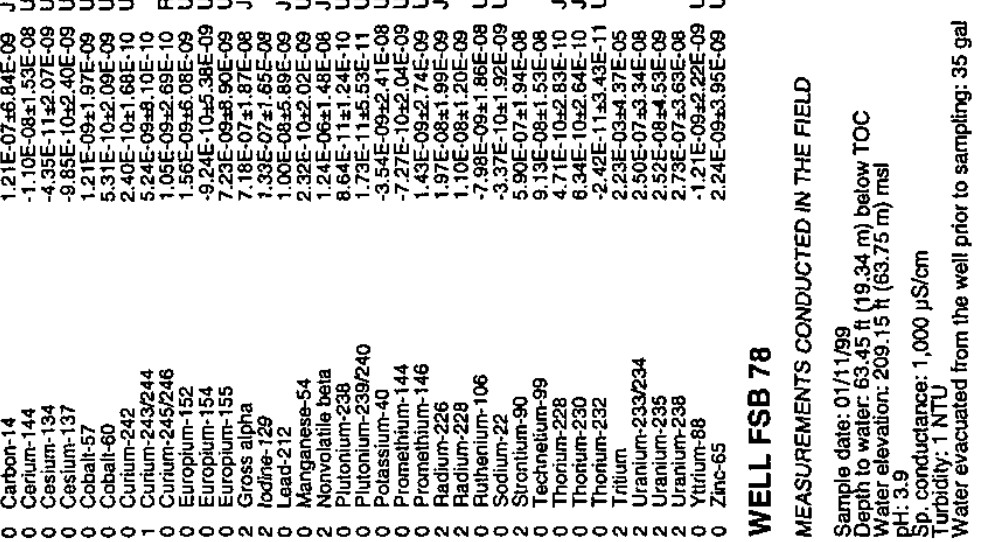

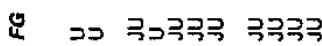

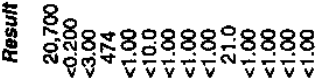

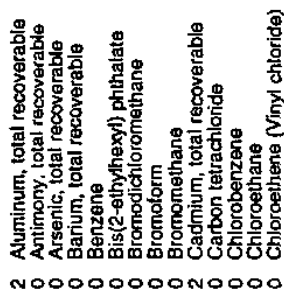

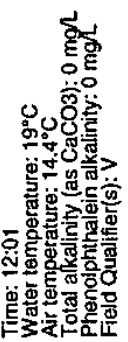

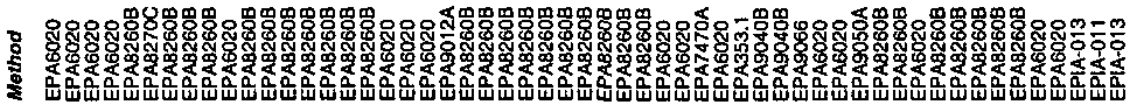

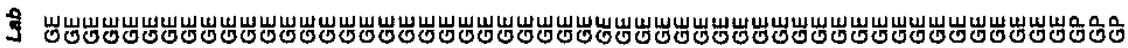

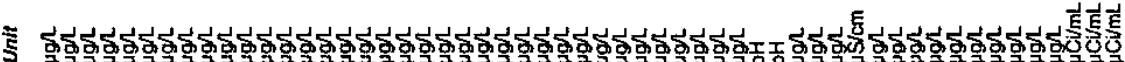

T)

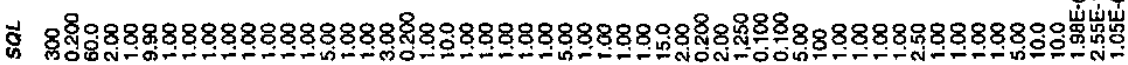

丞 00000000000

0000000000

0000000

क

ـ

-

נد

祎

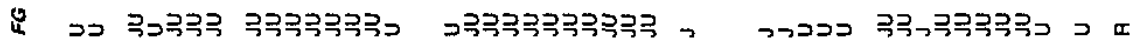

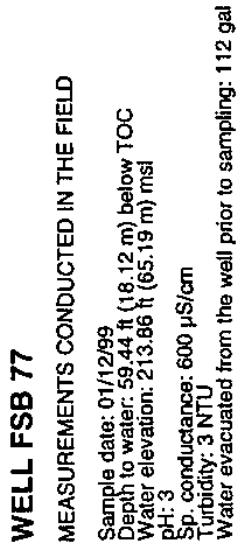

年

\% 8

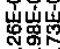

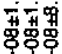

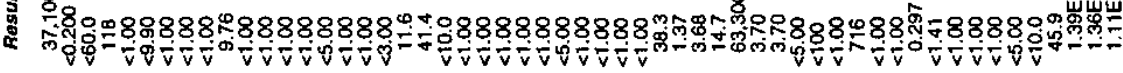

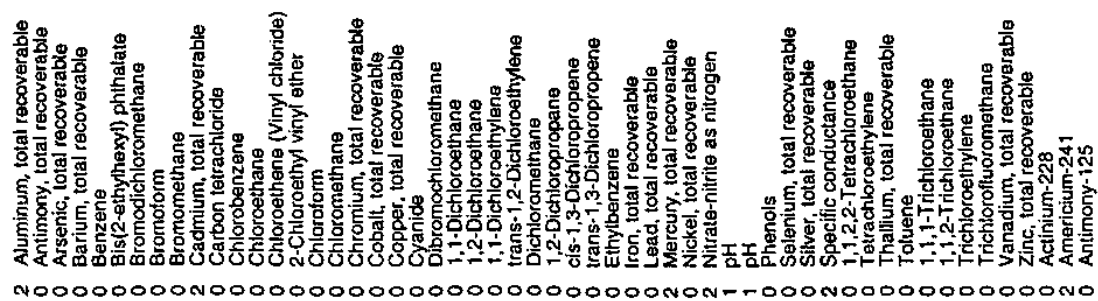




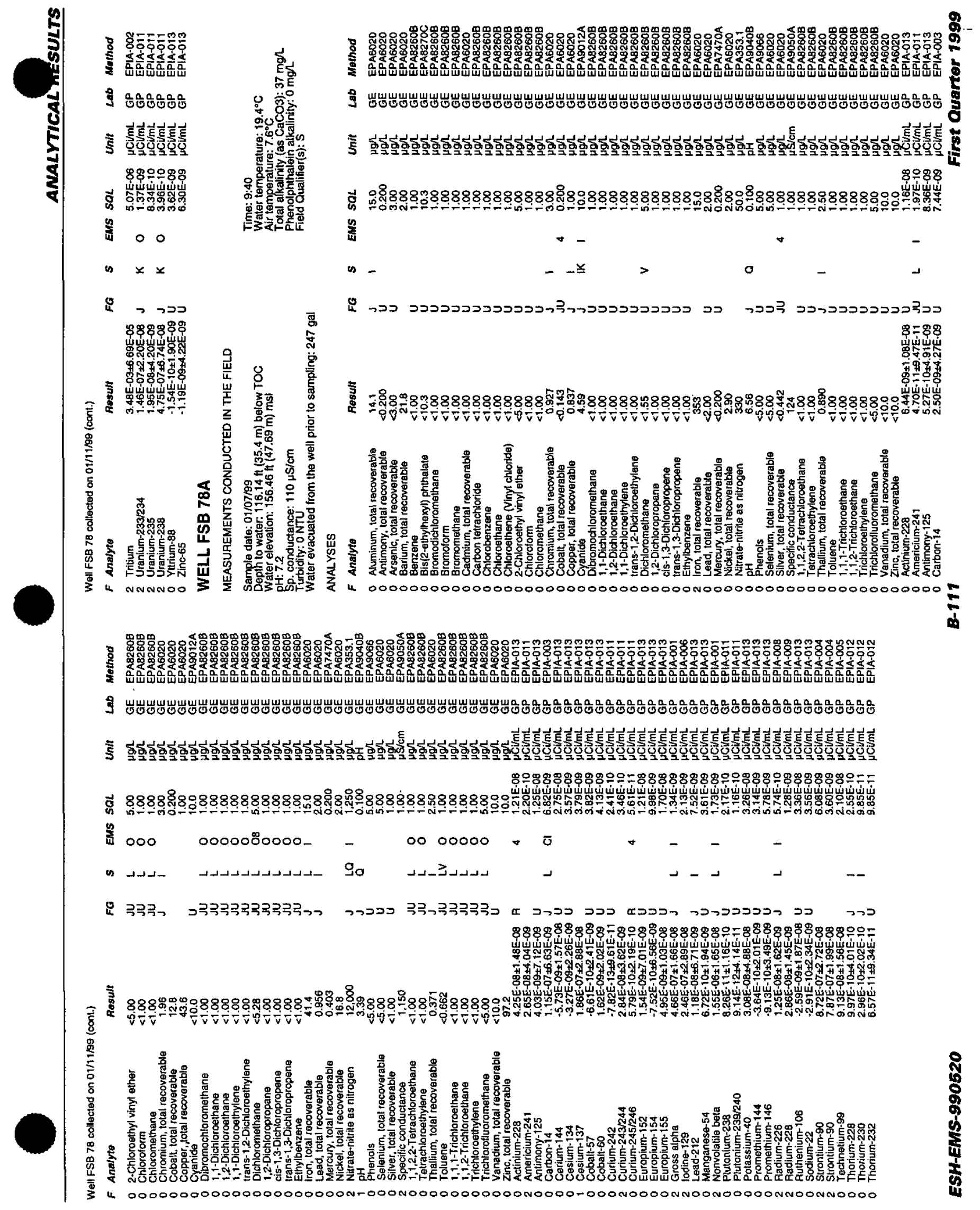




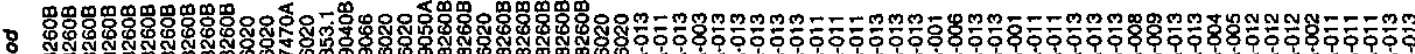
8.

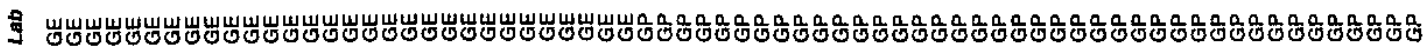

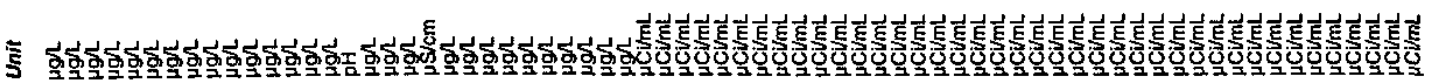

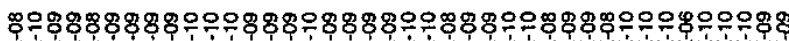

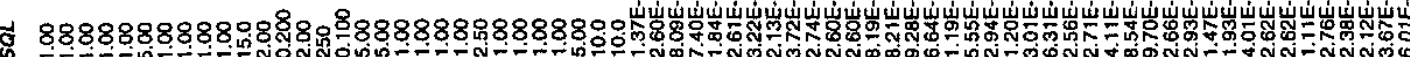
密

$-00$

$+000$

o

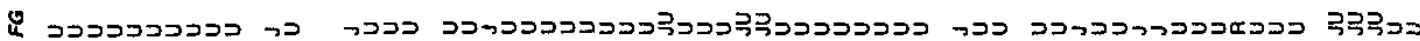

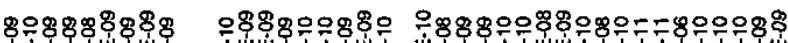

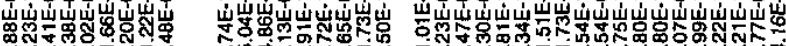

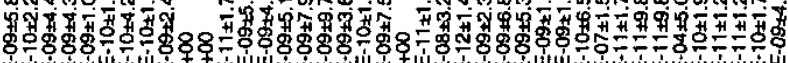

总 $8888888888 \%$

\%

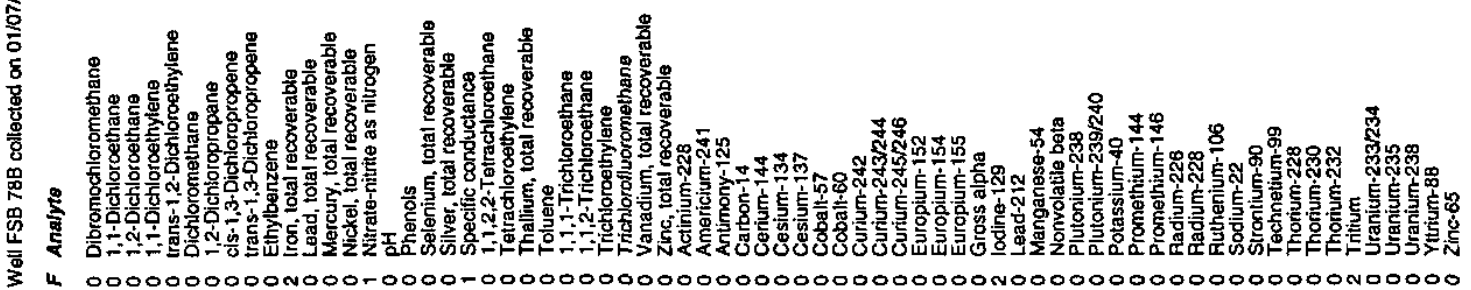

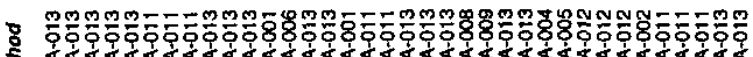
要

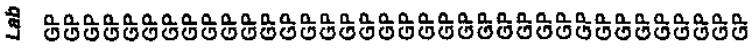

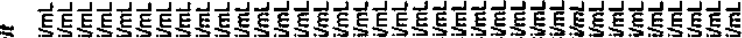

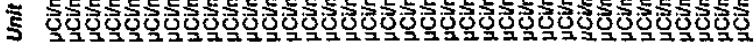

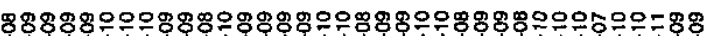

1

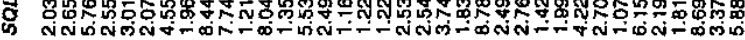

端

$\infty$

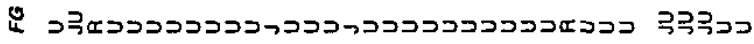

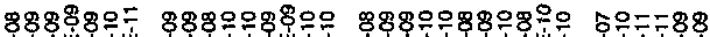

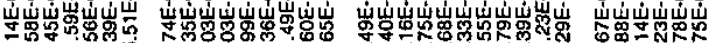

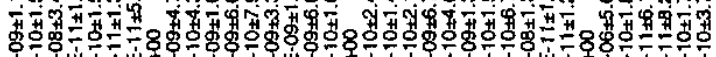

施

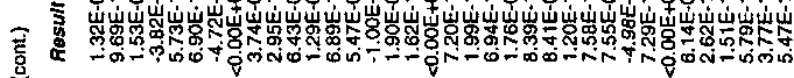

密

$\stackrel{\circ}{\circ}$

$\frac{8}{\overline{8}}$

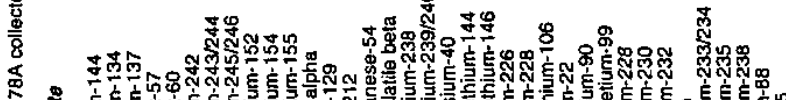

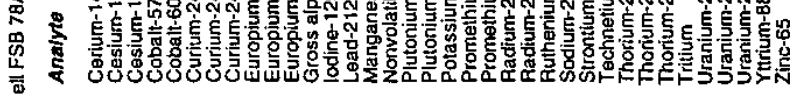

S 4000000000000000000000000000000000000

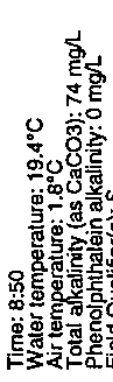

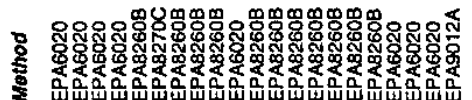

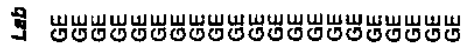

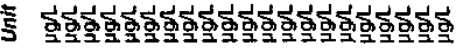

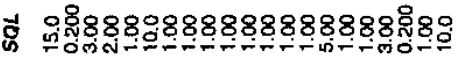

孳

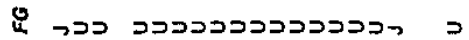

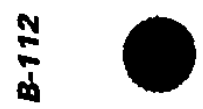

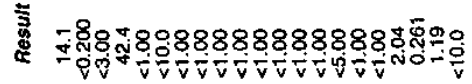

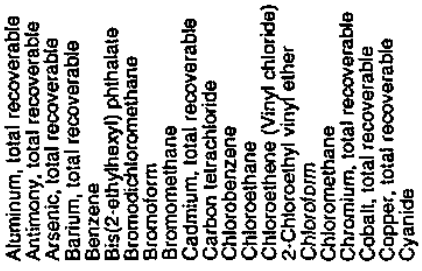

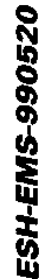









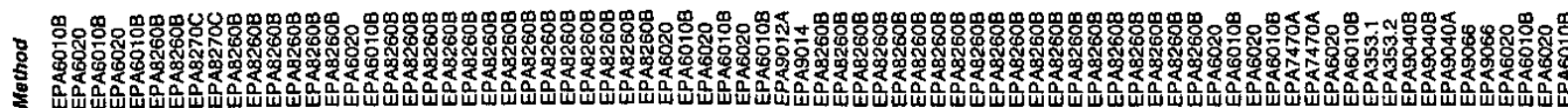

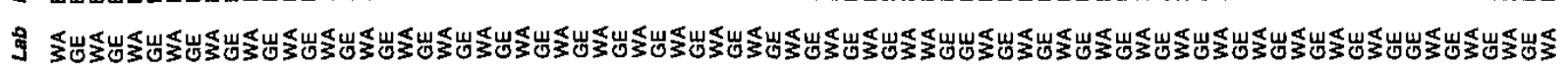

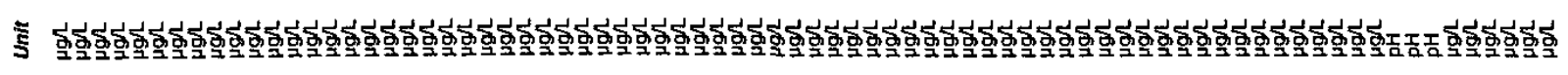

ఫ

盖 $00000000000+00000000000$

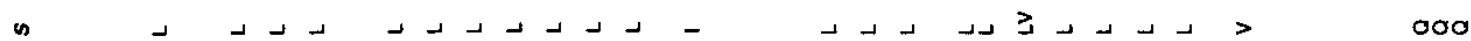

¿

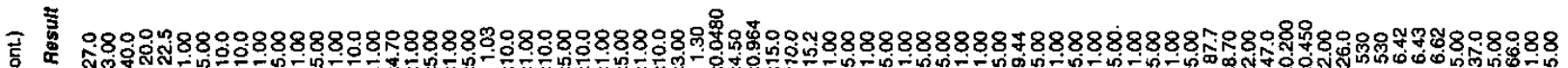

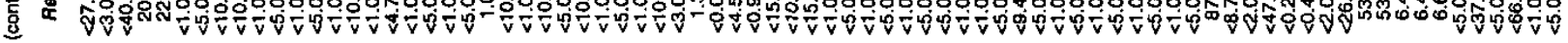

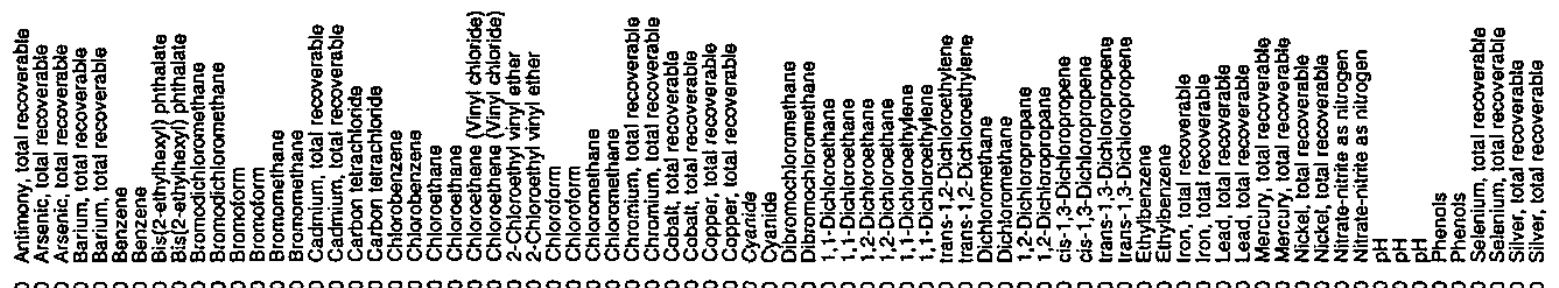

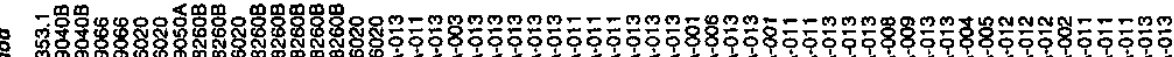
等

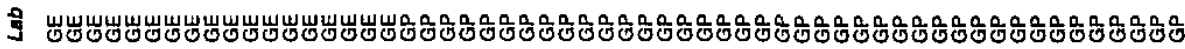

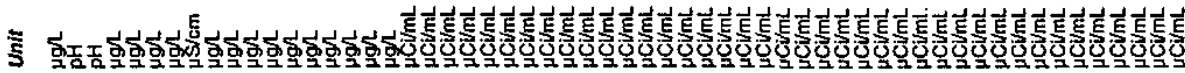

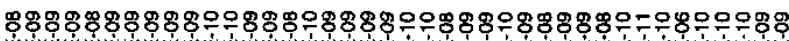

ఫ

竞
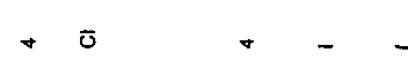

is $\infty 0$

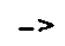

$+$

\&

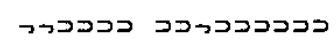

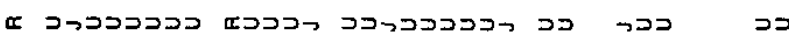

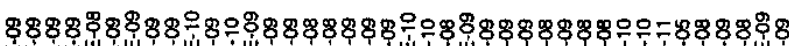

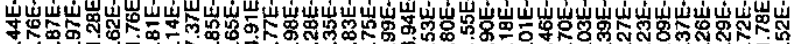

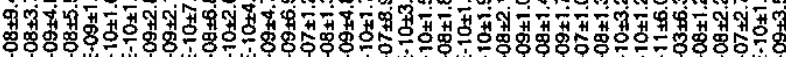

害

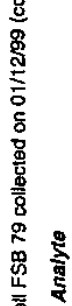<smiles>C=C[AsH2][AsH2]</smiles>

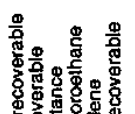

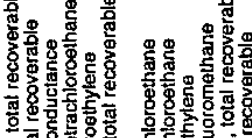

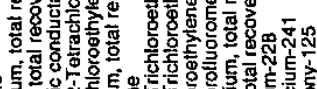

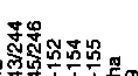

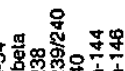
吅

等

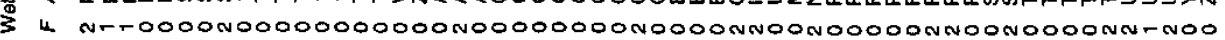
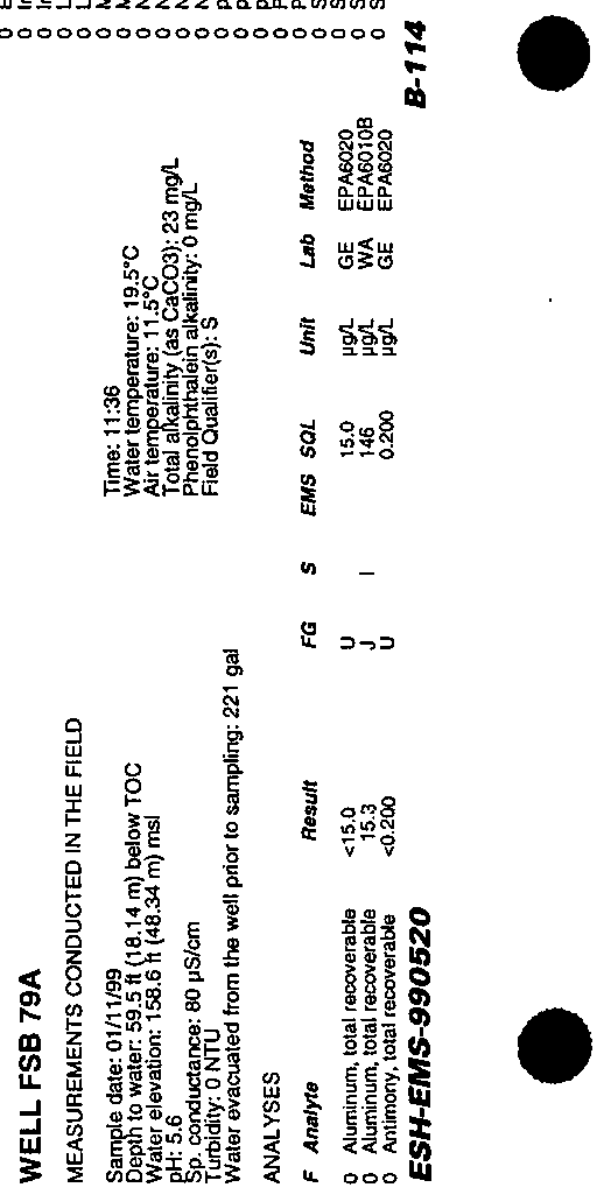

․․ำ

ํํㅇㅇํㅇ

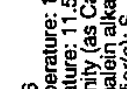

$$
\text { 禺 }
$$

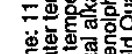

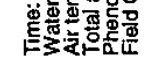

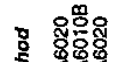

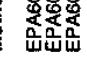

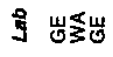

혹 햎허

호 윢융

竞

n -

S

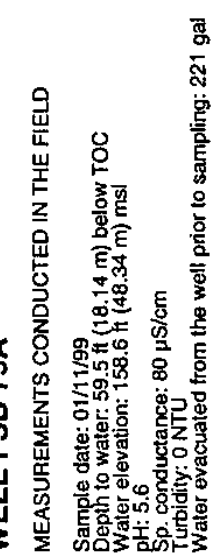

壱

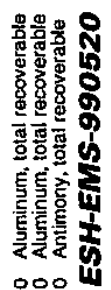




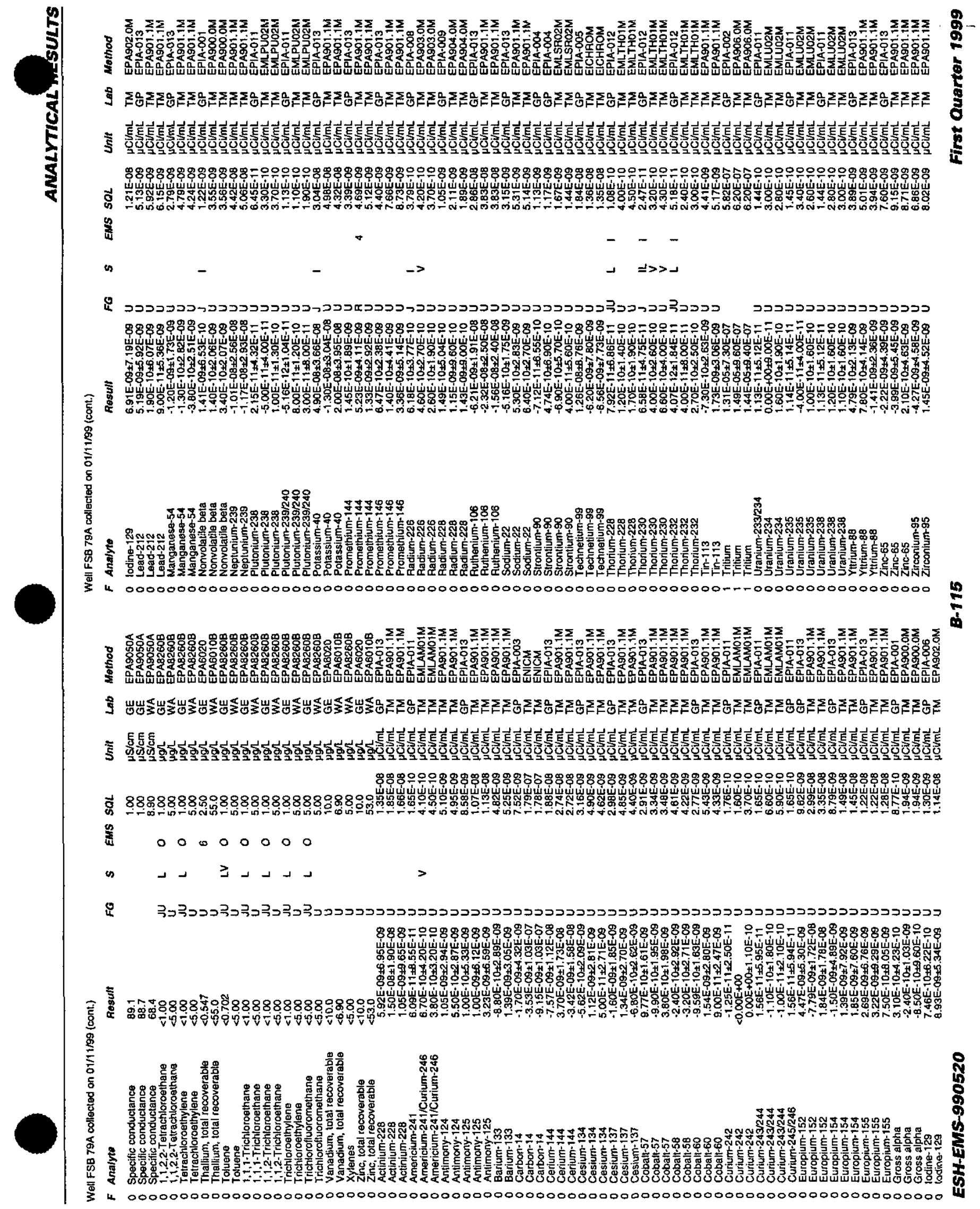




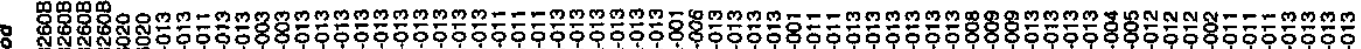

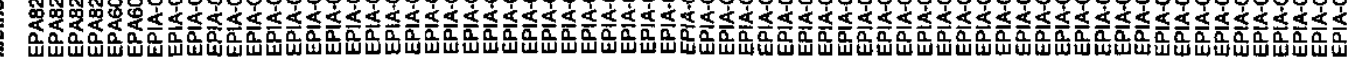

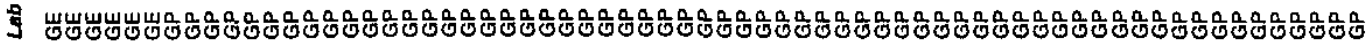
亏 ફ.

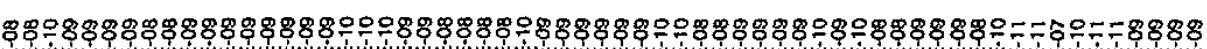

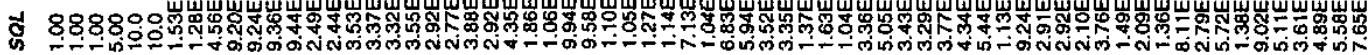
䆓 0000

גם

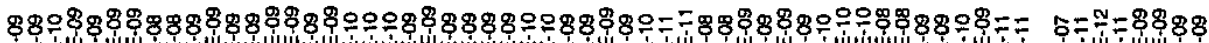

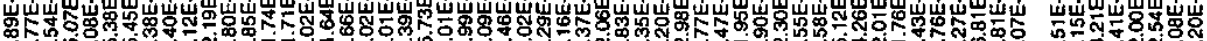

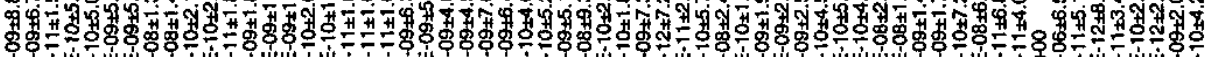

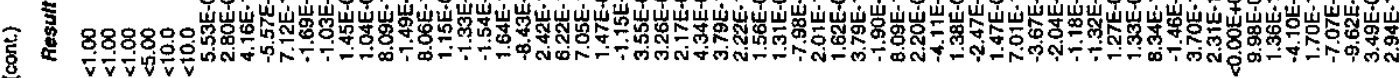

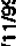

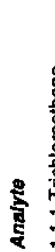

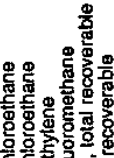

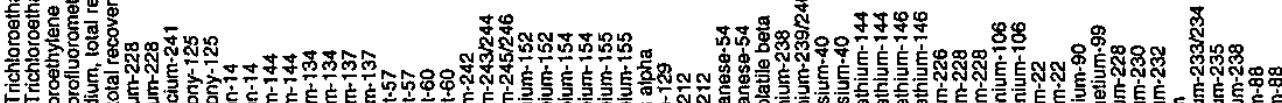

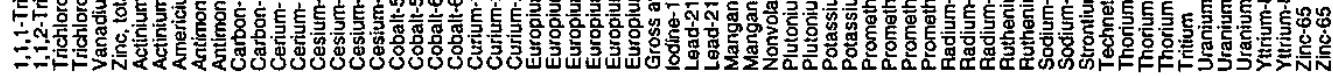

\$ 40000000000000000000000000000000000000000000000000000000000000000000

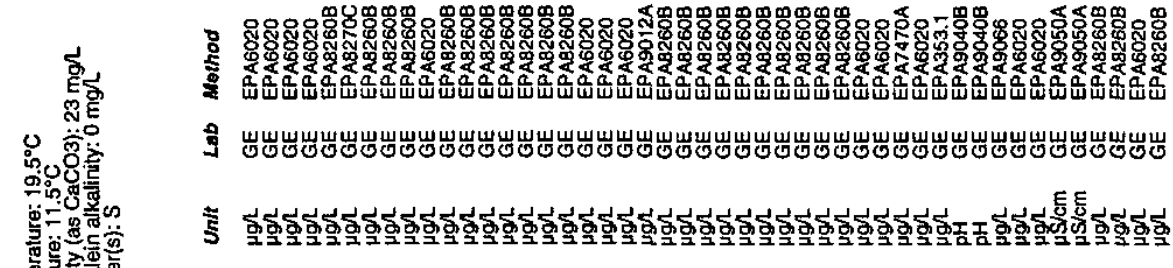

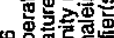

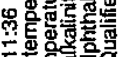

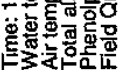

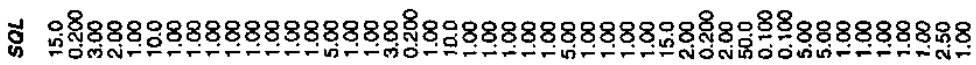
II $00000000000 \quad 0000000000 \quad 000$ is 马ु

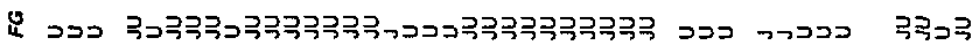

言 울 จ

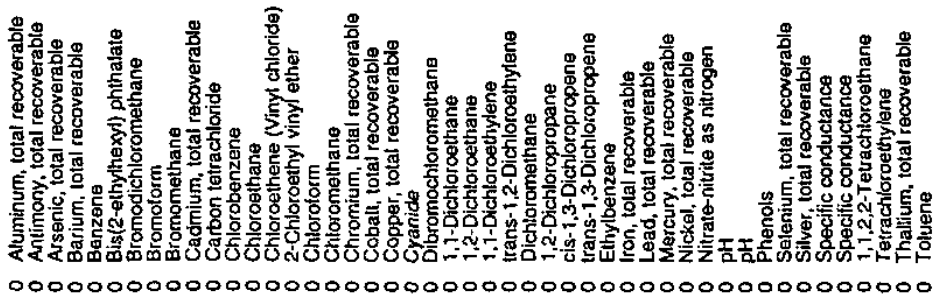




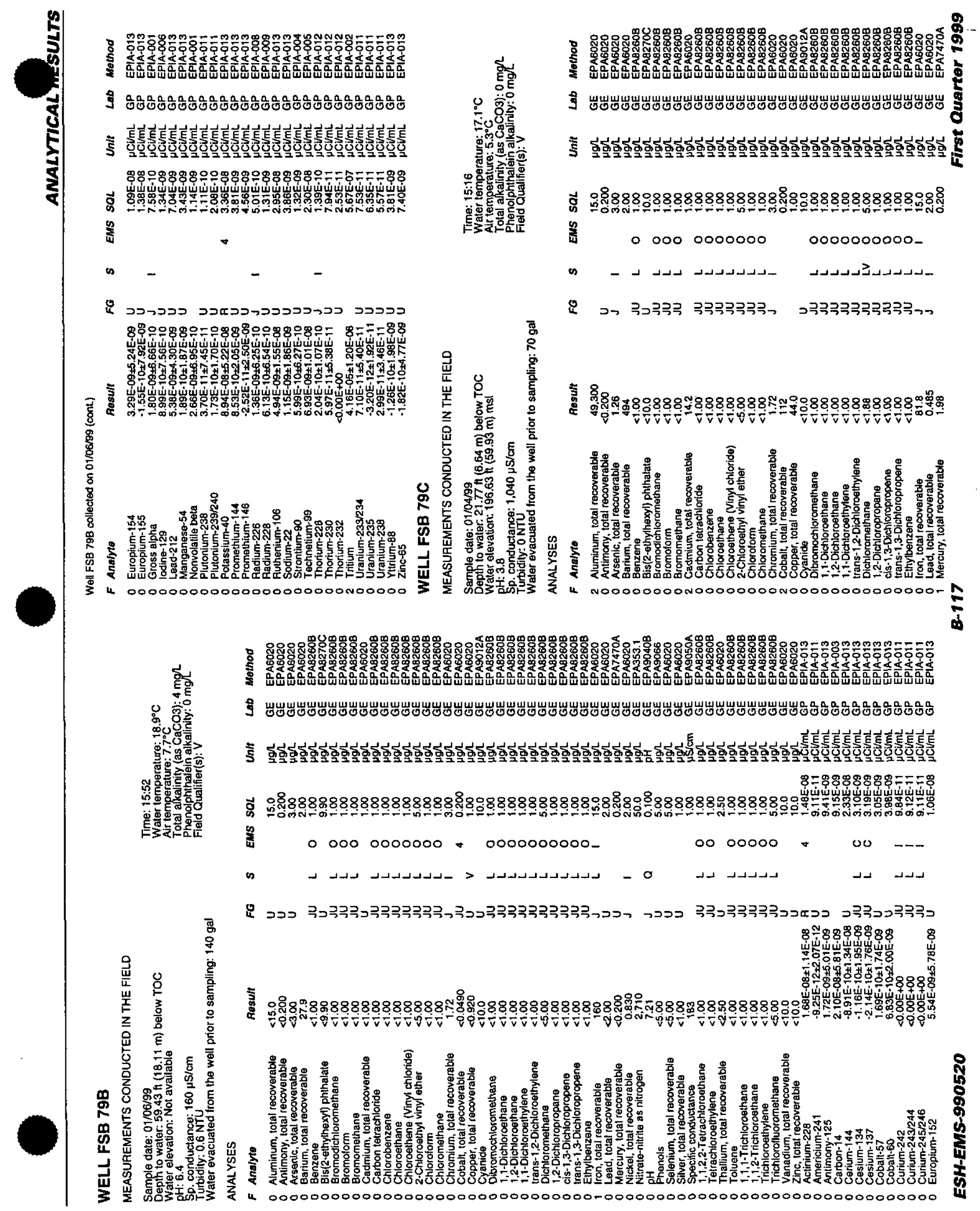




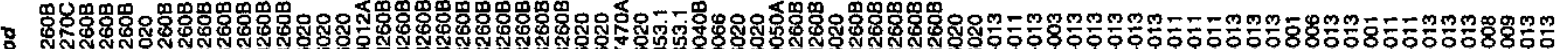
要

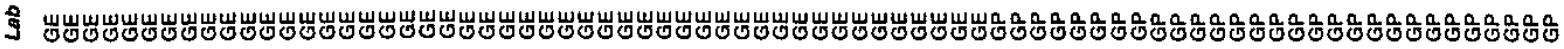

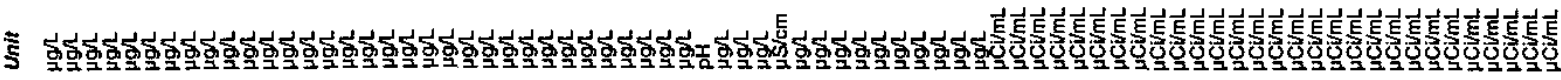

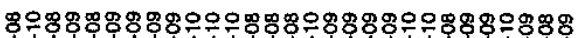

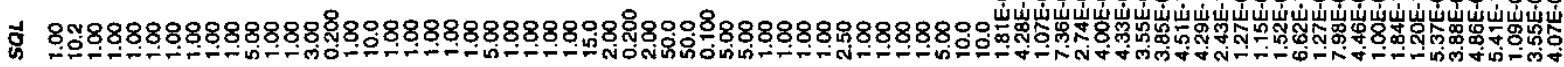

密

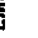

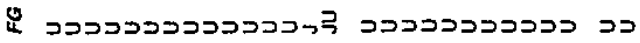

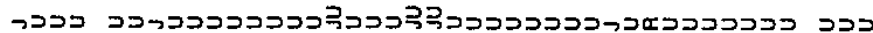

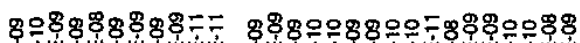

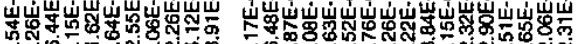

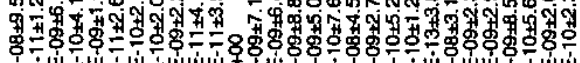

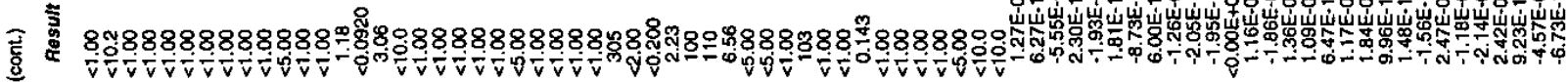

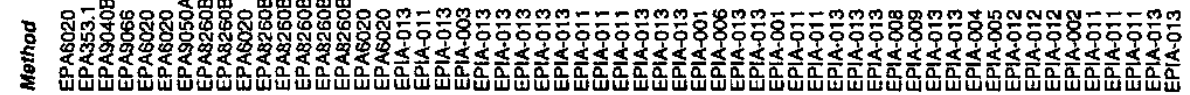

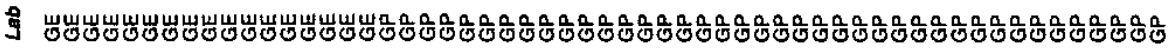

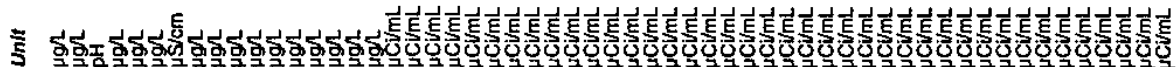

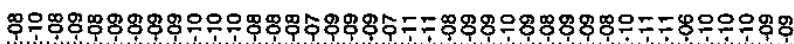

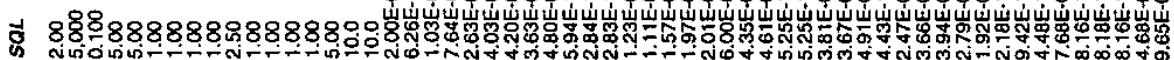
量

0000000

a 0

证

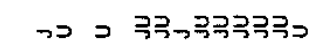

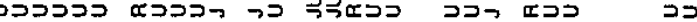

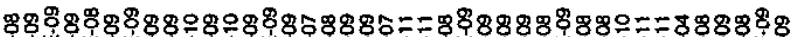

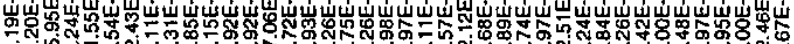

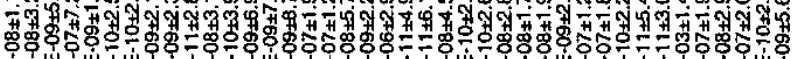

言

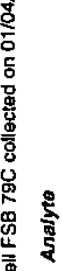

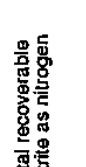

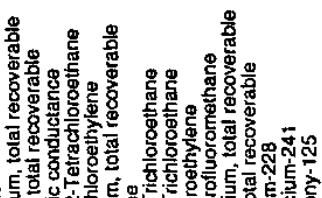

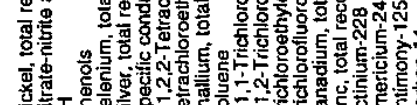

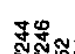

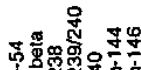

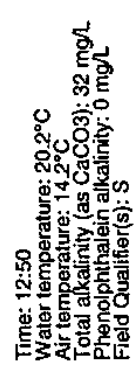

요요요

해

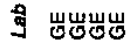

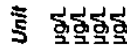

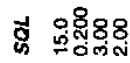

离

n-

怘

可

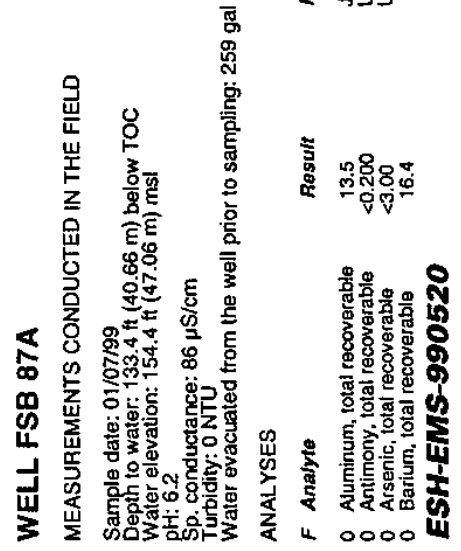

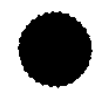

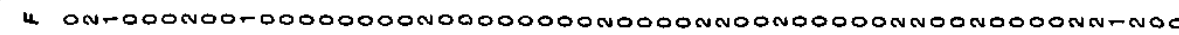




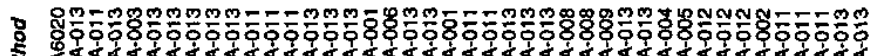
然

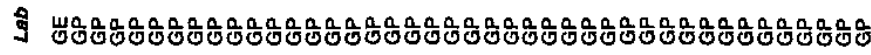

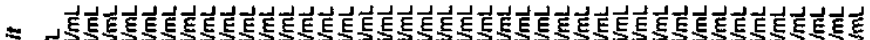

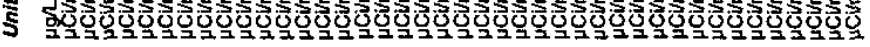

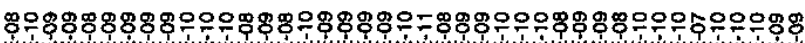

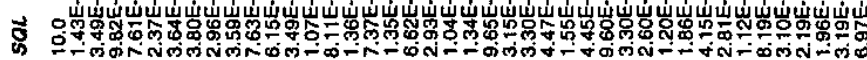

离 -

000

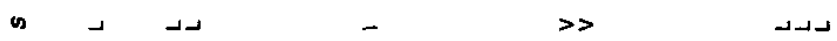

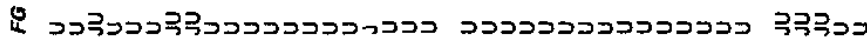

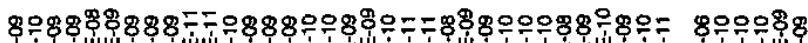

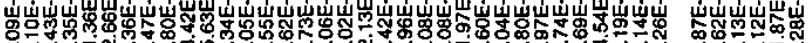

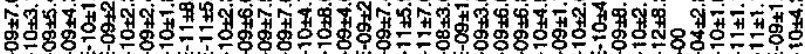

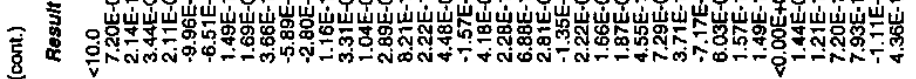

용

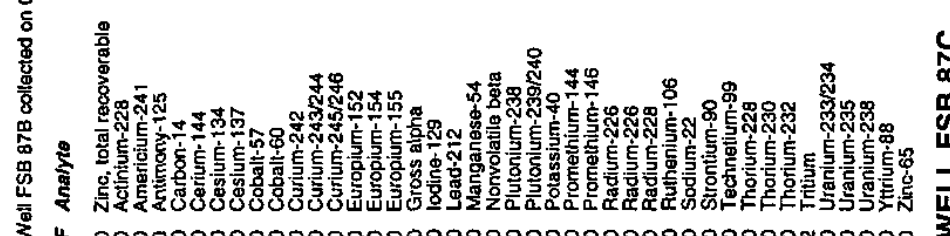

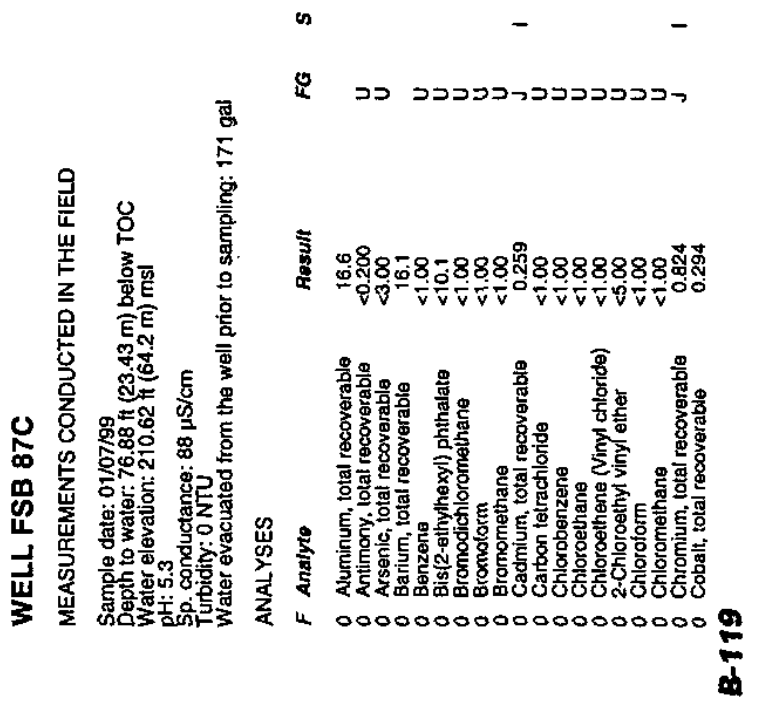

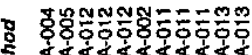

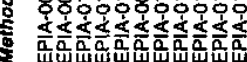

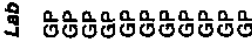

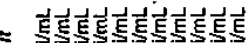

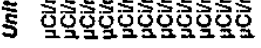

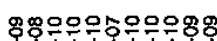

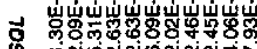

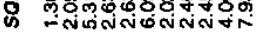

害

000

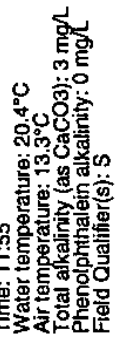

\%

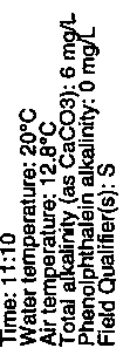

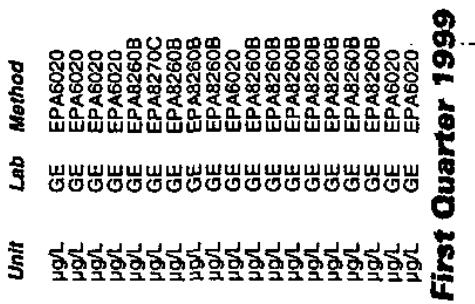

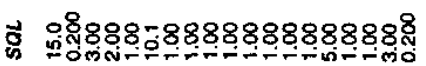

翣

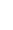

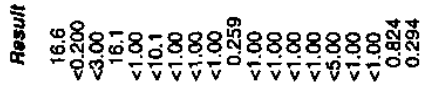

\& $\quad\lrcorner-\lrcorner$

טבני

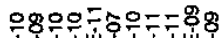

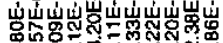

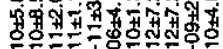

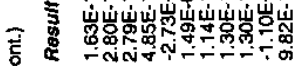

胥

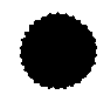

敢

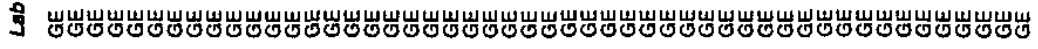

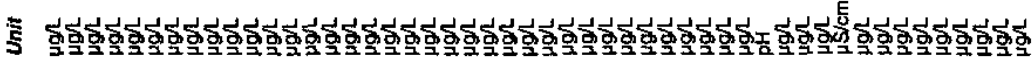

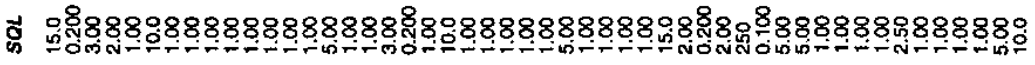

蛋

en 0

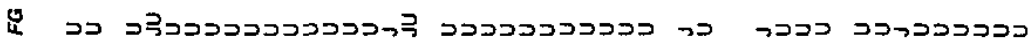

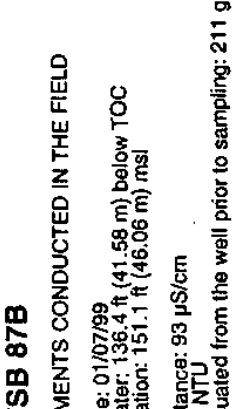

产

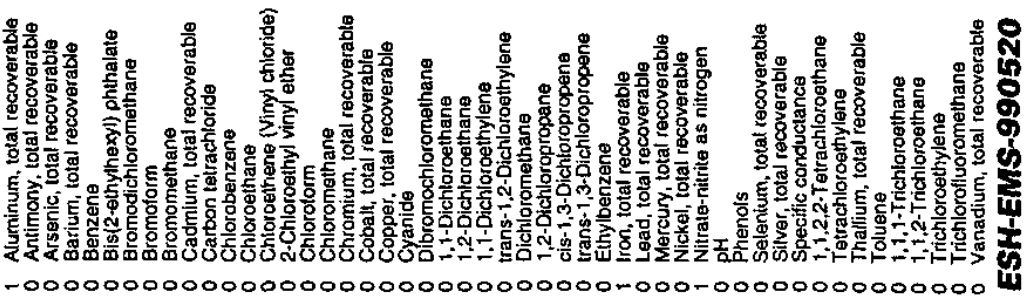




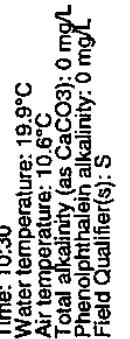

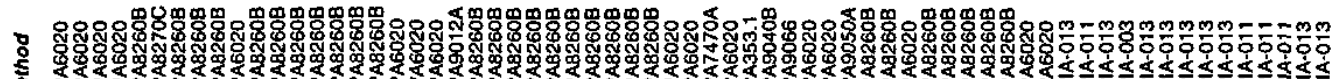

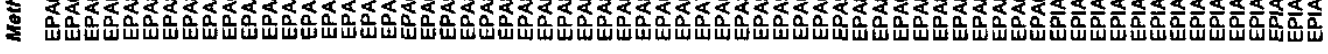

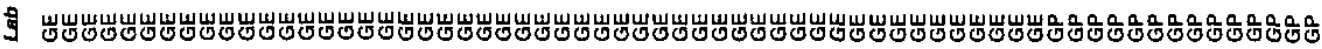

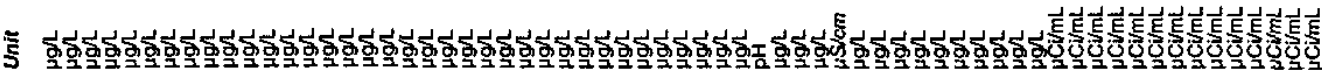

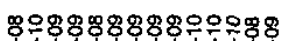

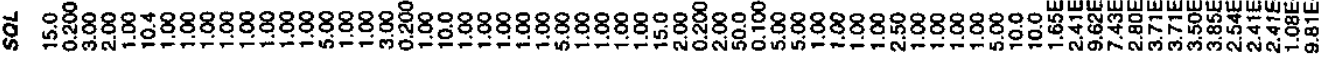
戀

$-00$

o

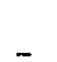

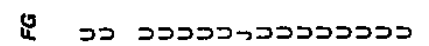

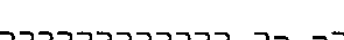

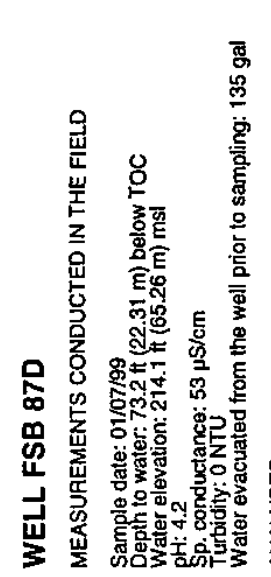
.

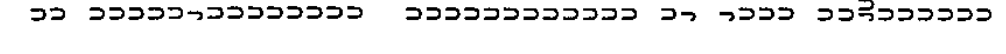

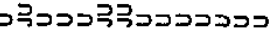
$808888888 \div 88$

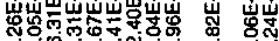

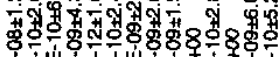
童

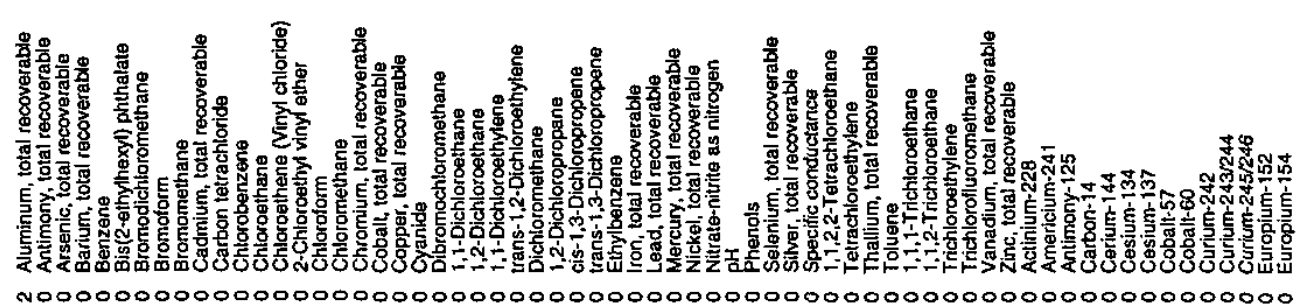

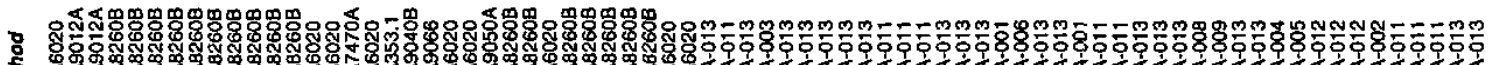
音

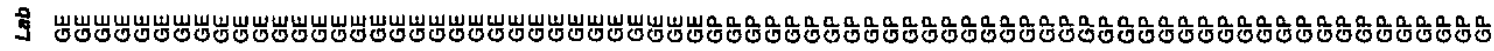

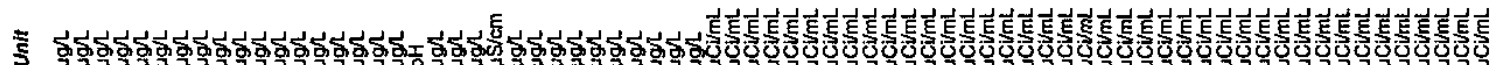

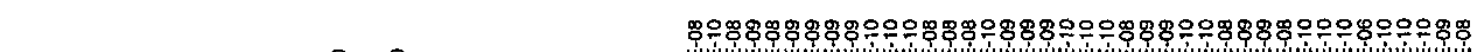

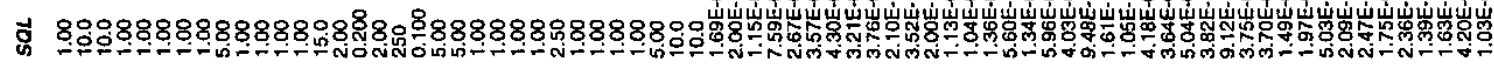
窝

is

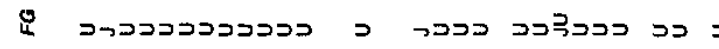

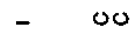

000

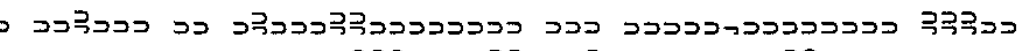

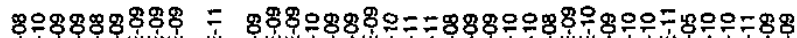

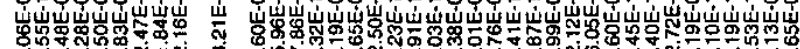

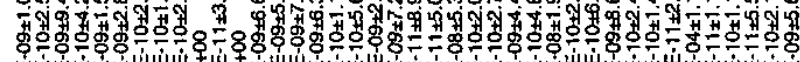

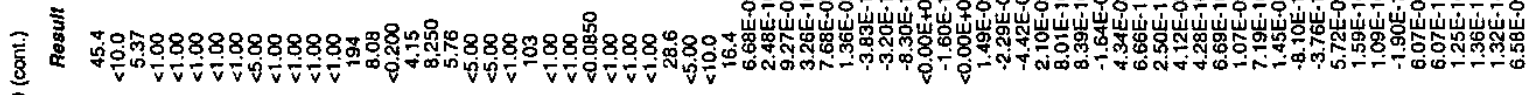


D

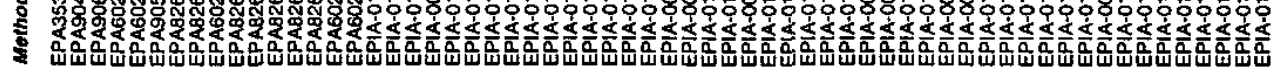

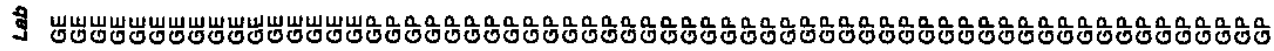

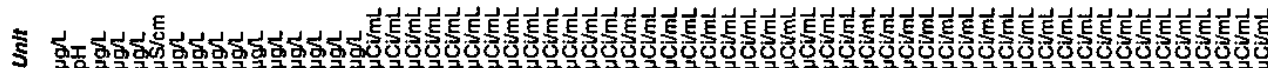

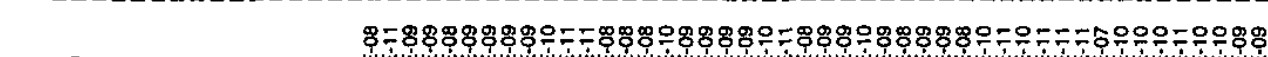

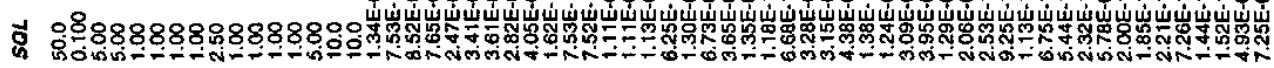
竞

采 $00+00000+\%$

is 0 נد

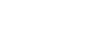

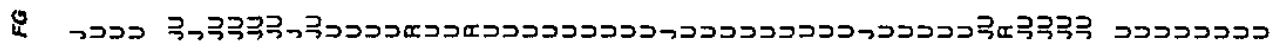

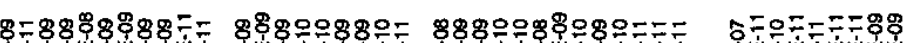

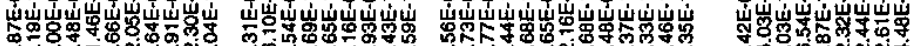

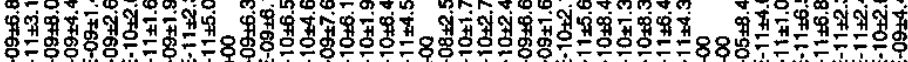

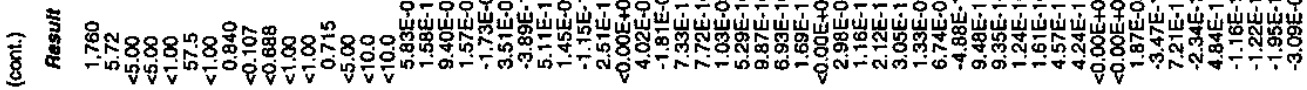
要
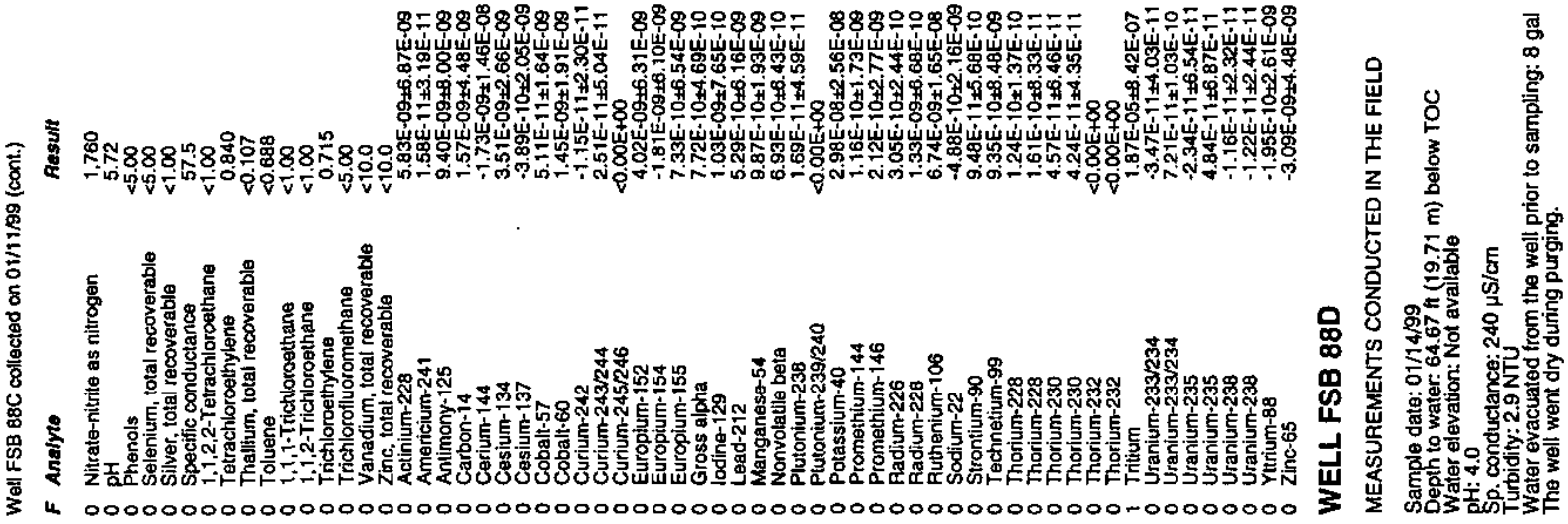

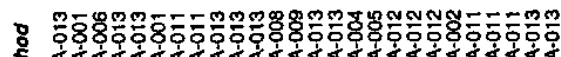

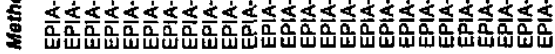

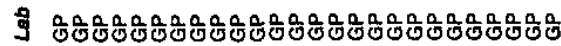

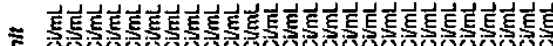

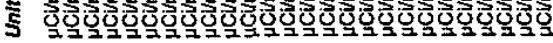

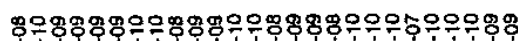

1

o 包

蛋

a - $\quad--$

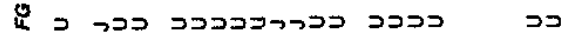
8 8

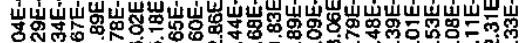

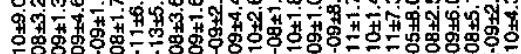

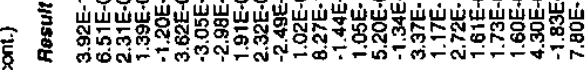$$
\text { . }
$$

옹
}
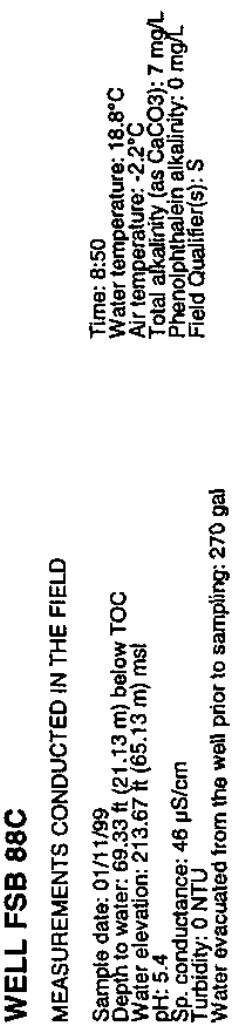

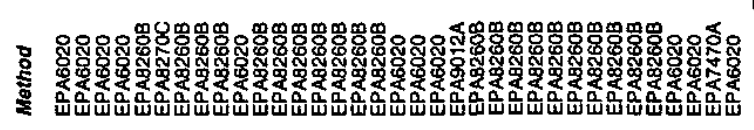

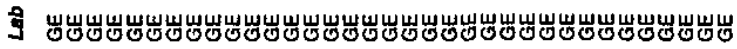

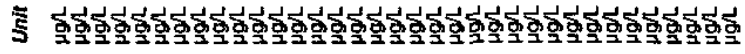

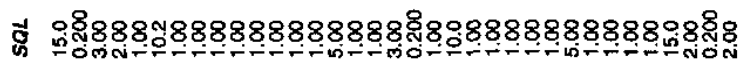

离 $00000000000 \quad 0000000000$

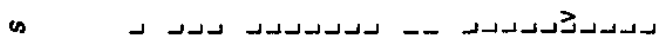

【

产

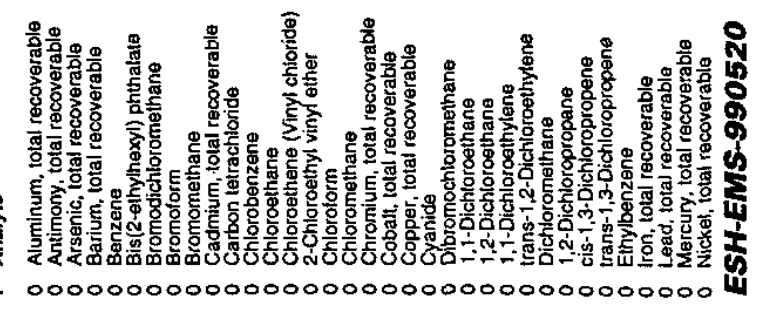




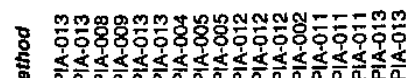
隻

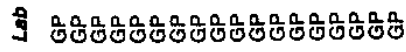

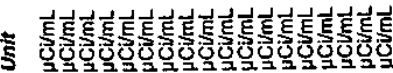

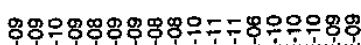

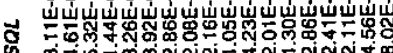

盖

n

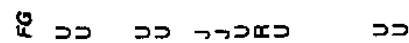

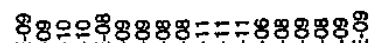

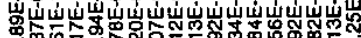

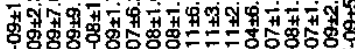

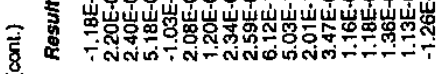

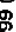

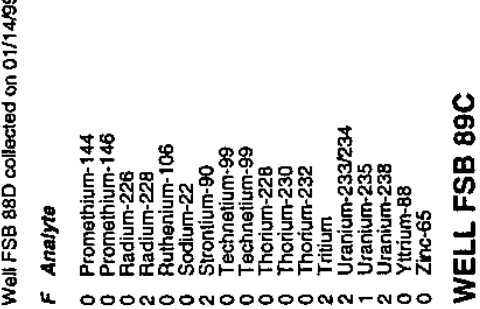

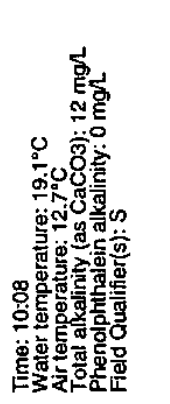

รั้

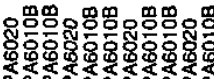

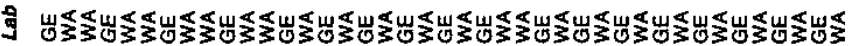

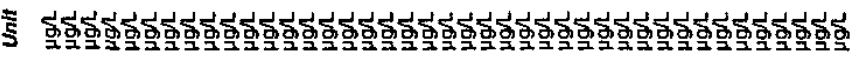

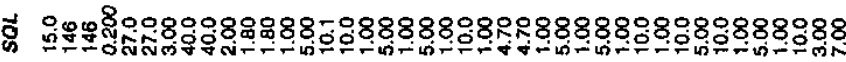
密

as

כברכנ כנב

可

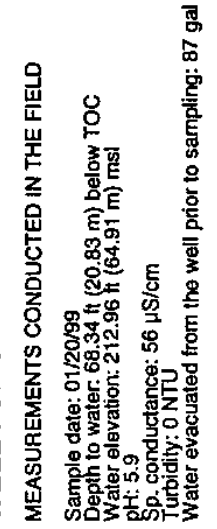

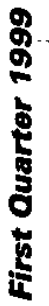

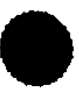

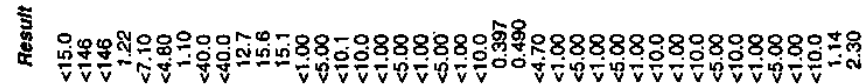

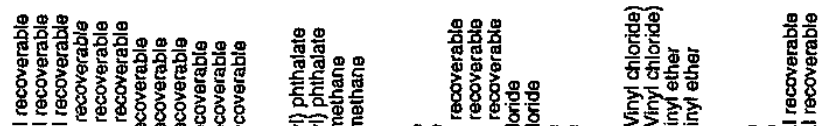

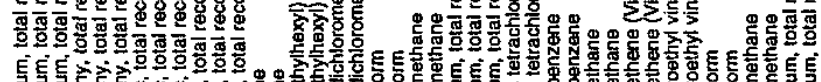
出 L. 00000000000000000000000000000000000000000

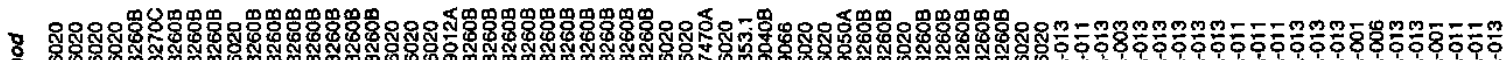
要

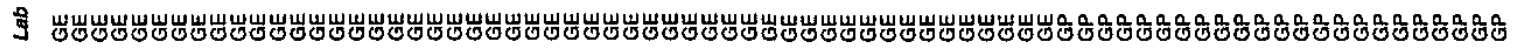

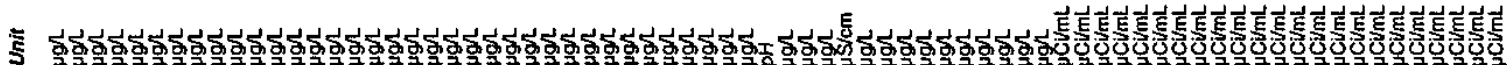

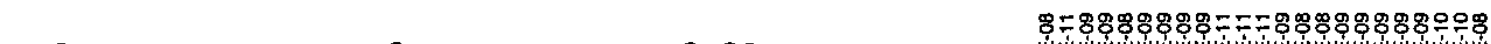

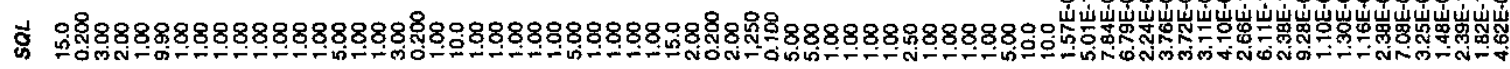

慈

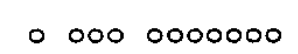

0000000000

0000000

is

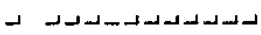

-

a

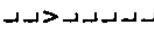

\& د クっว곡ㄱㄱㄱㄱㄱㄱ

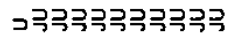

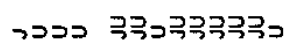
$88 \% 8 \% 8 \% 88=80 \% 8 \%$

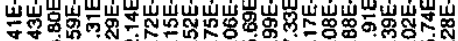

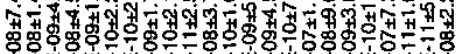

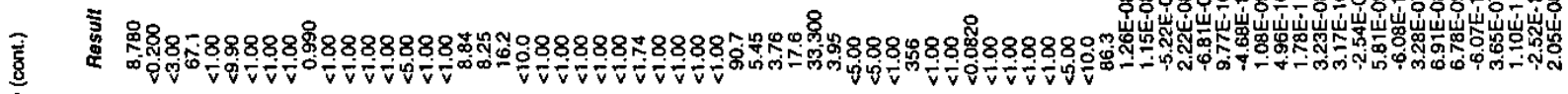
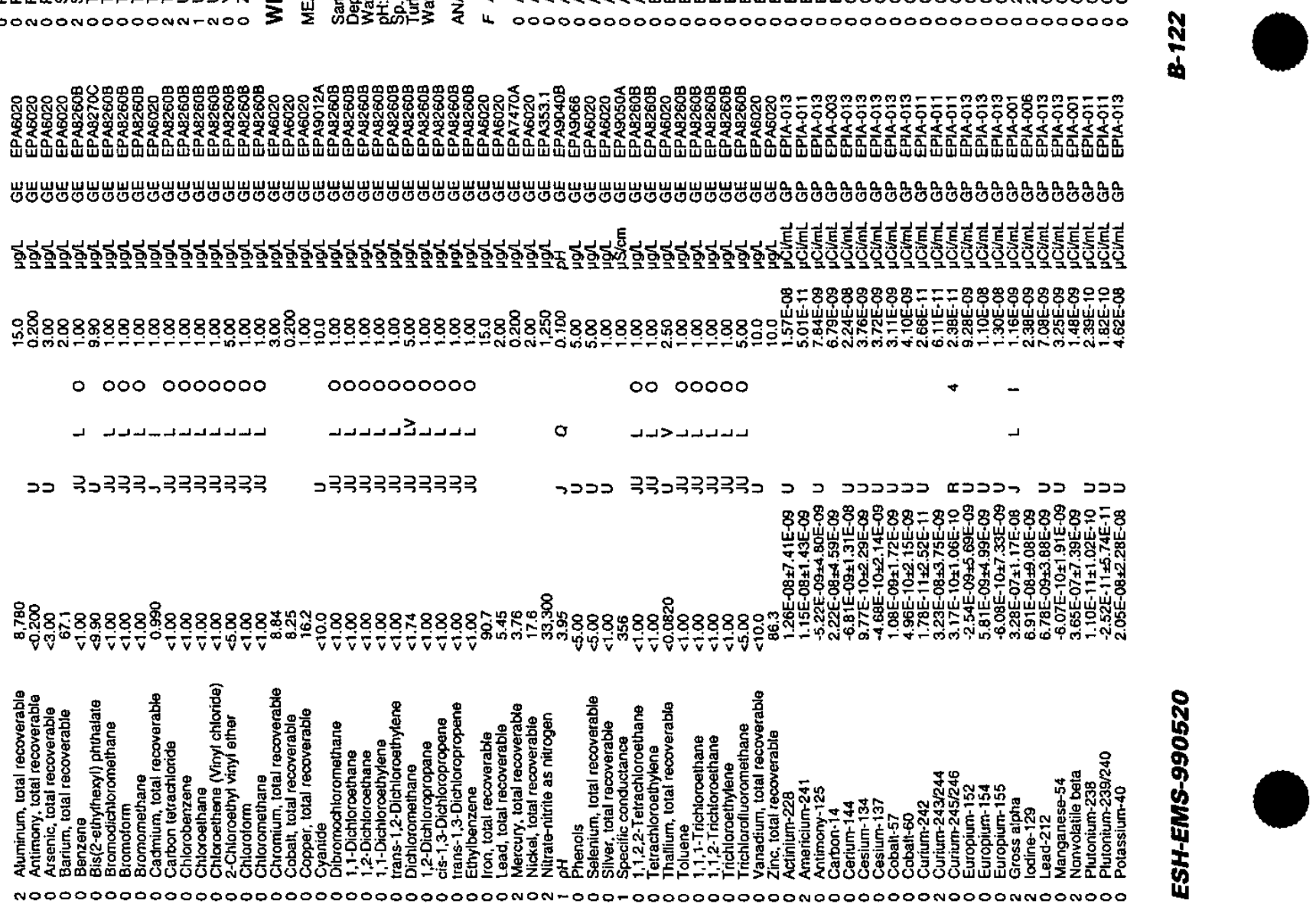


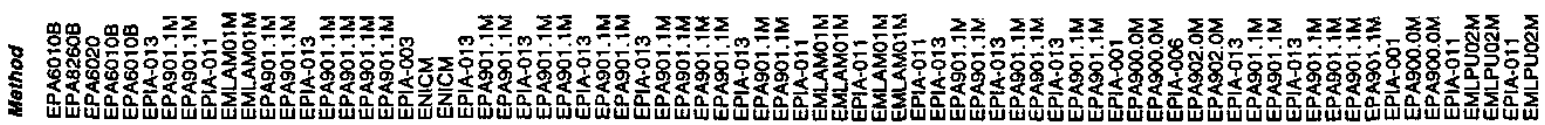

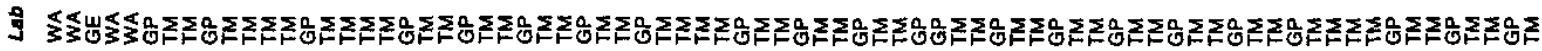

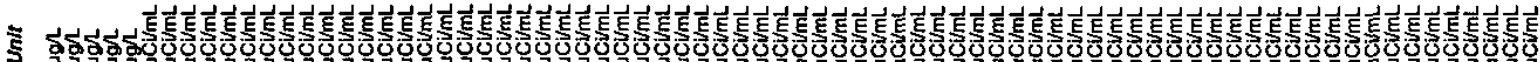

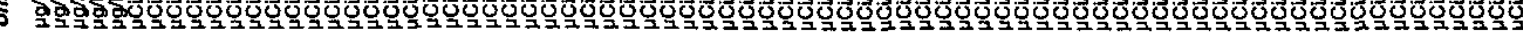

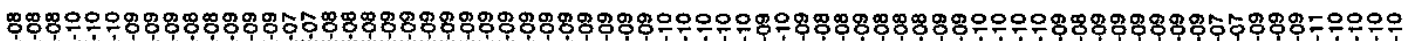

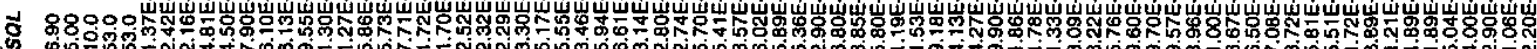

愛

a

$8>$ ב

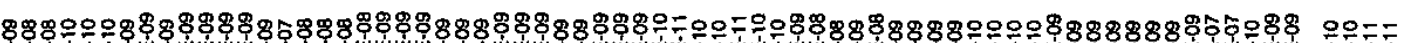

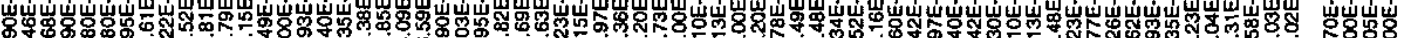

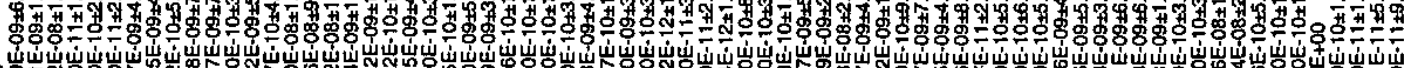

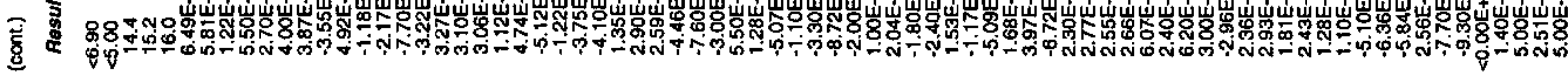

多

产 赵

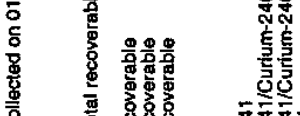

O

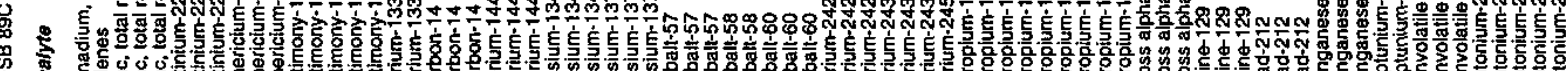
留

\$ 4 . 0000000000000000000000000000000000000000000000000000000000000000000000000000

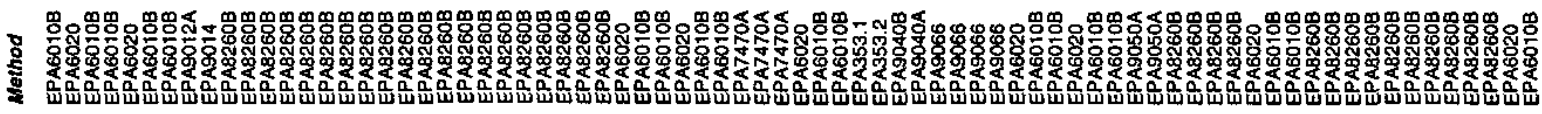

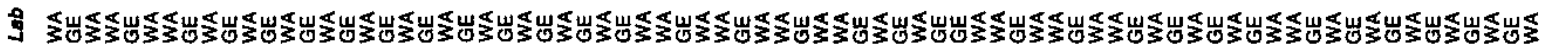

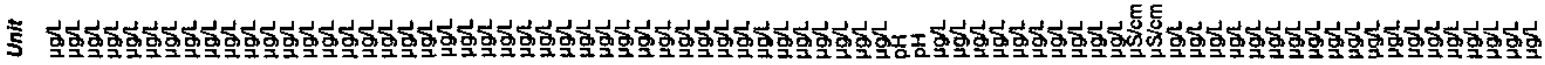

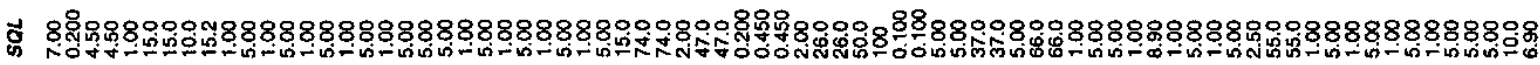

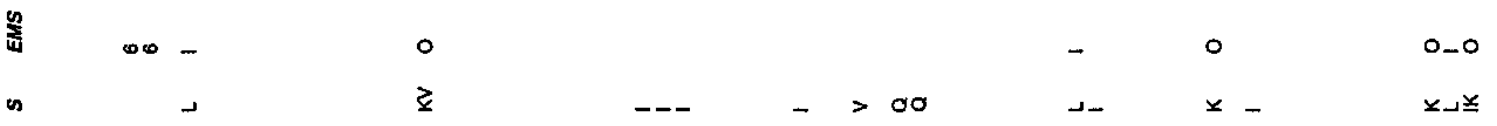

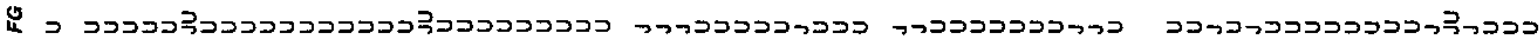

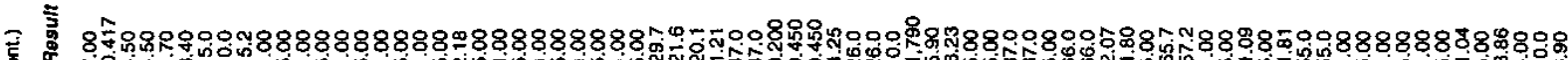

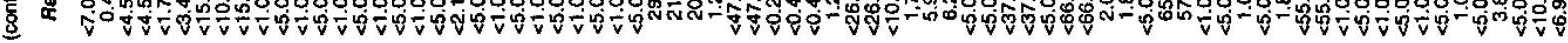

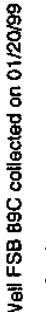

兴

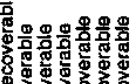

\section{놇ำ}

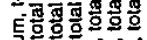

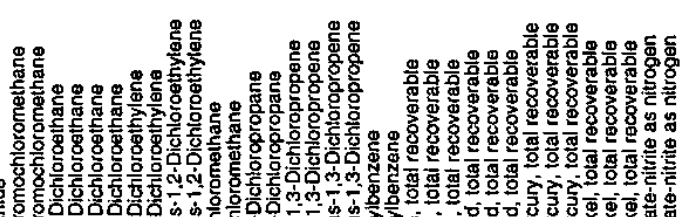

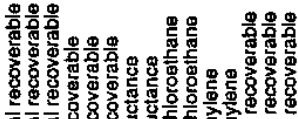

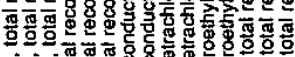

$\frac{m}{\infty}$

L. $0000000000000000000000000000000000000000000000000000000000000-00000000000000$ 


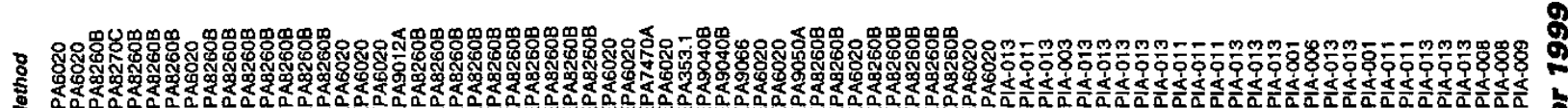

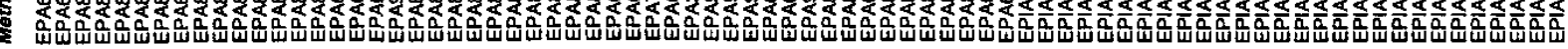

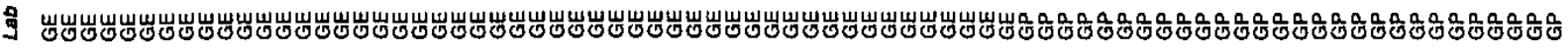

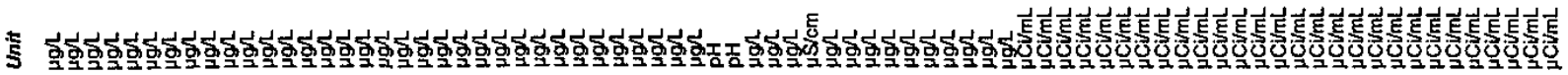

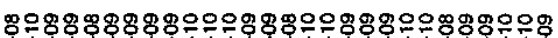

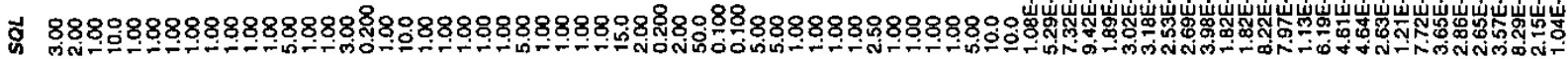
篦

is

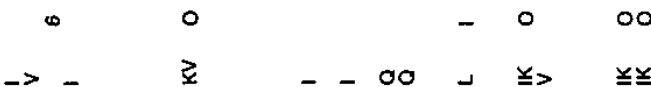

4

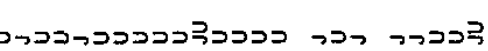

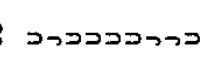

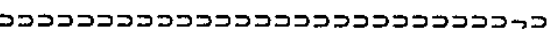

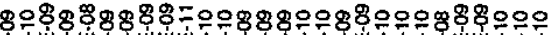

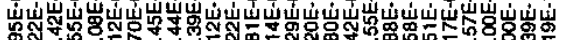

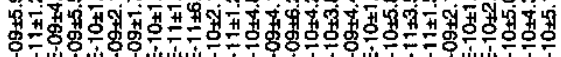

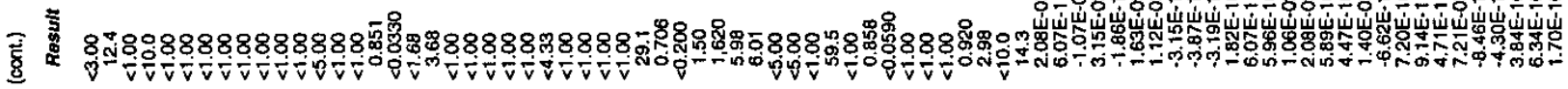

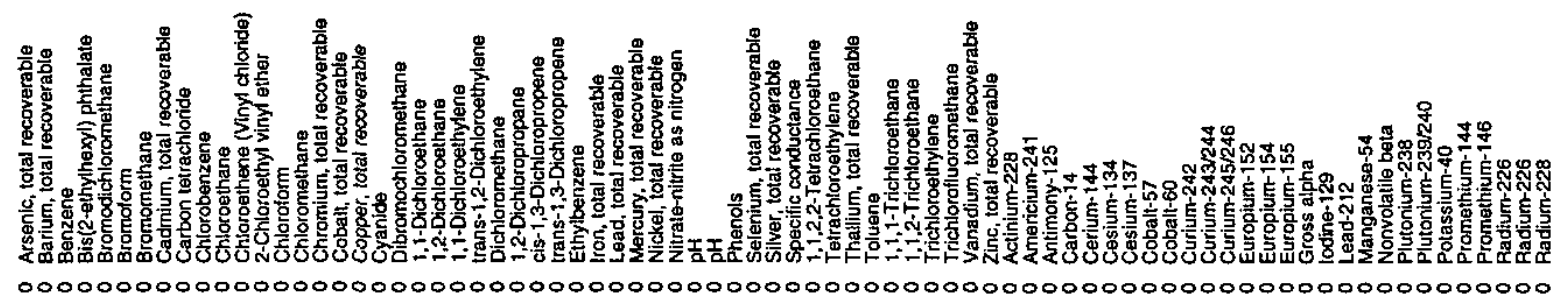

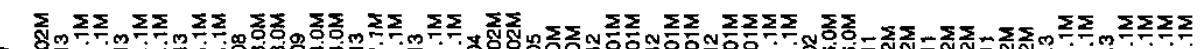

을

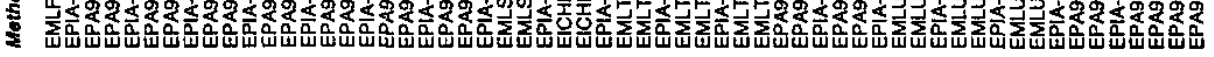

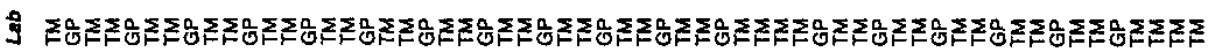

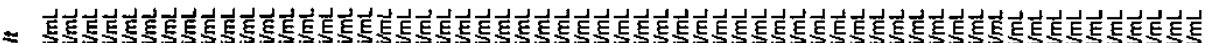

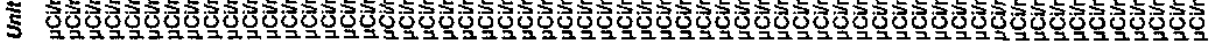

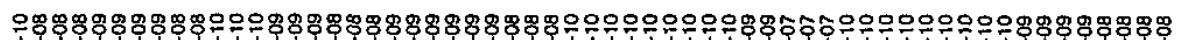

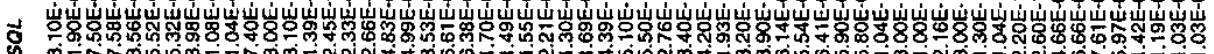

盖

is

o

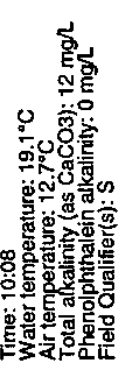

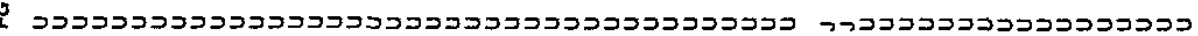

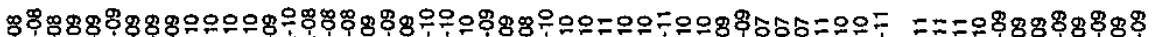

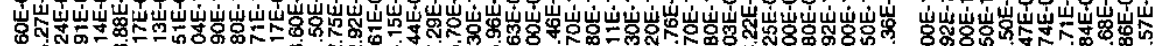

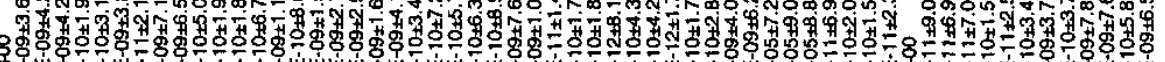

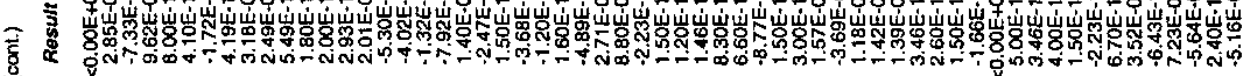

产

兽

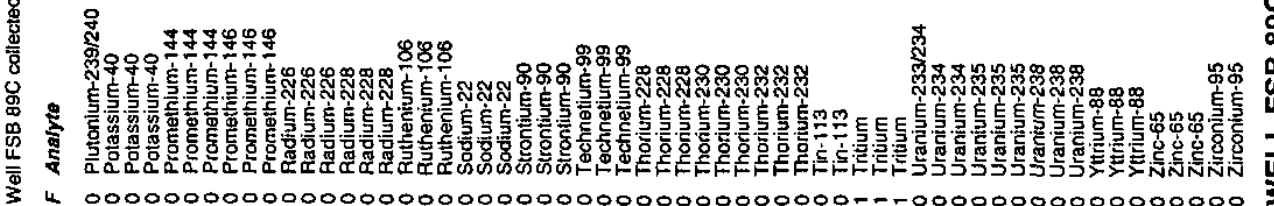

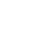




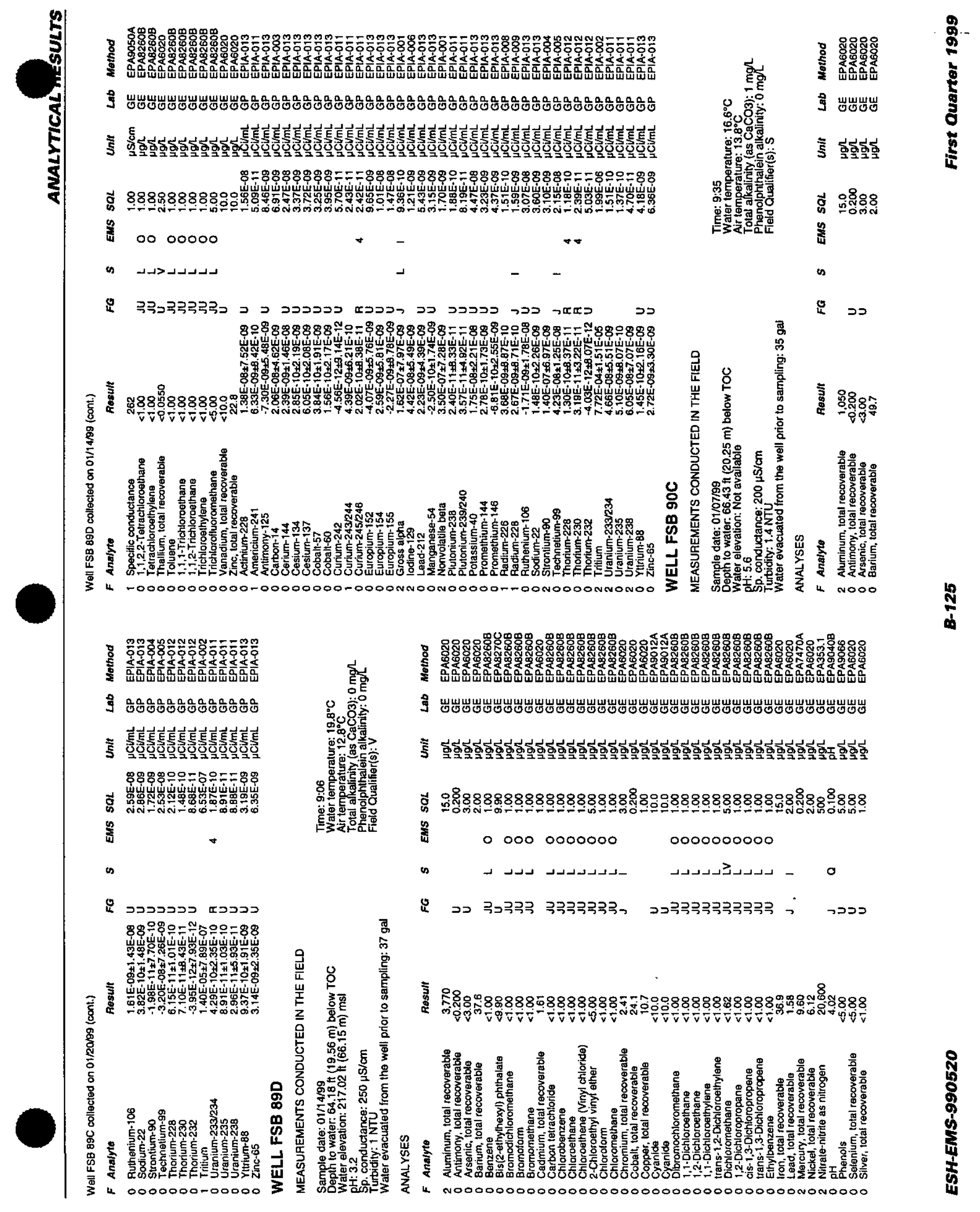




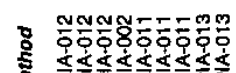

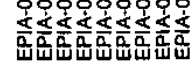

I 으요용요요용

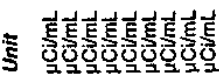
으유웅ㅇㅇㅇㅇㅇㅇㅇㅇㅇㅇㅇ

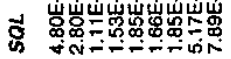

善

000

is

دכ 운=-8응ㄷㅇㅇㅇ

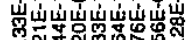

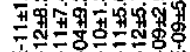

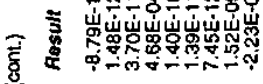

\%

홓

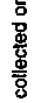

\&

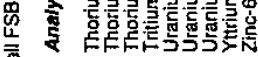

要 4000000000
Б્.

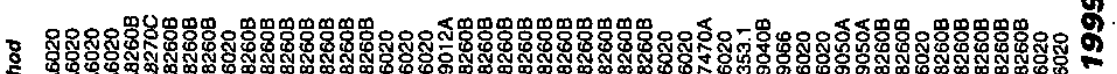
官

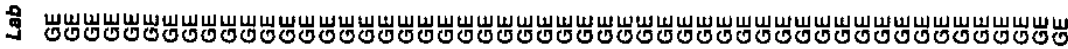

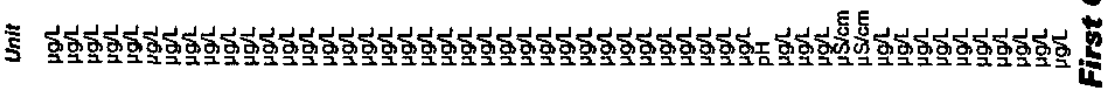

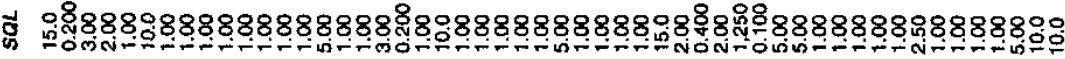

西

00000000000

0000000000

0000000

( )

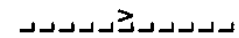

$\circ$

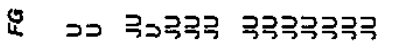

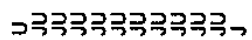

SOS

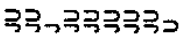

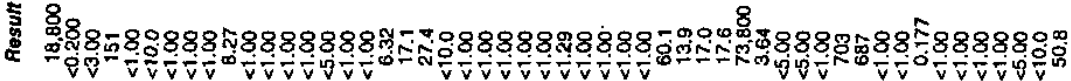

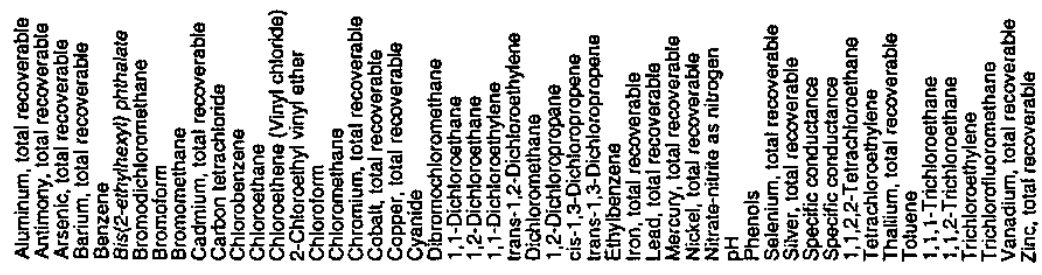

h No0000000N00000000000000000000000N0N-000NN0000000000

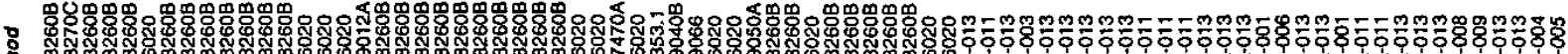

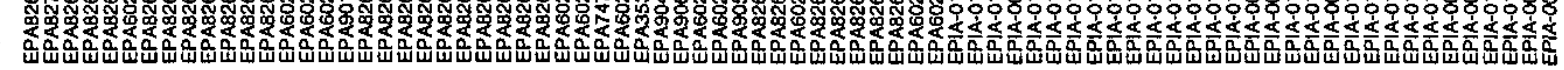

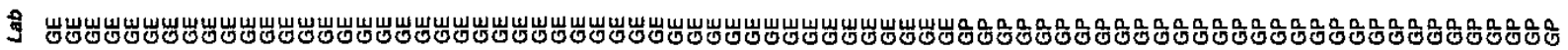

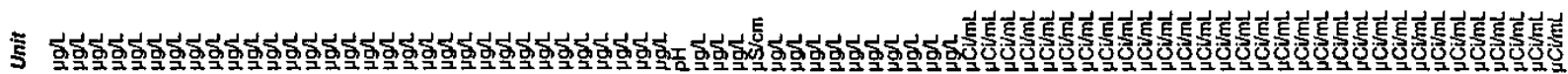

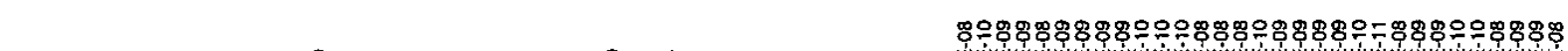

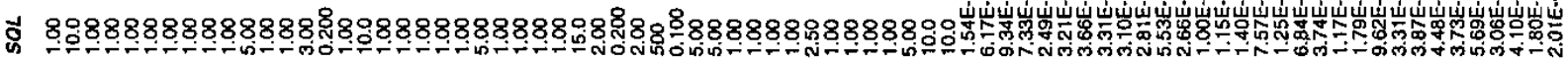
紊

(1)

is

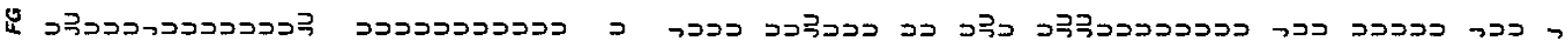

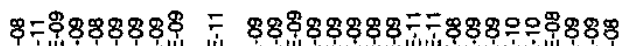

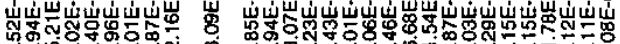

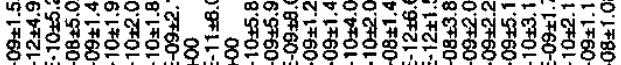

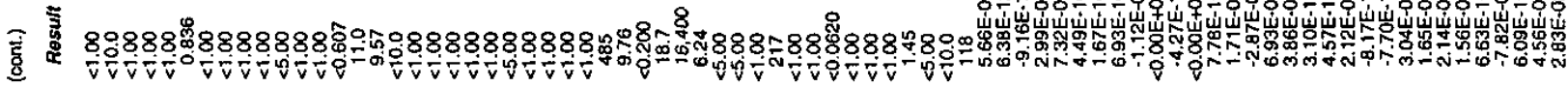

尊
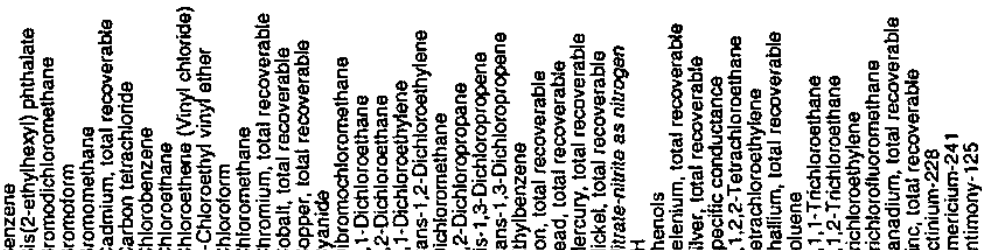






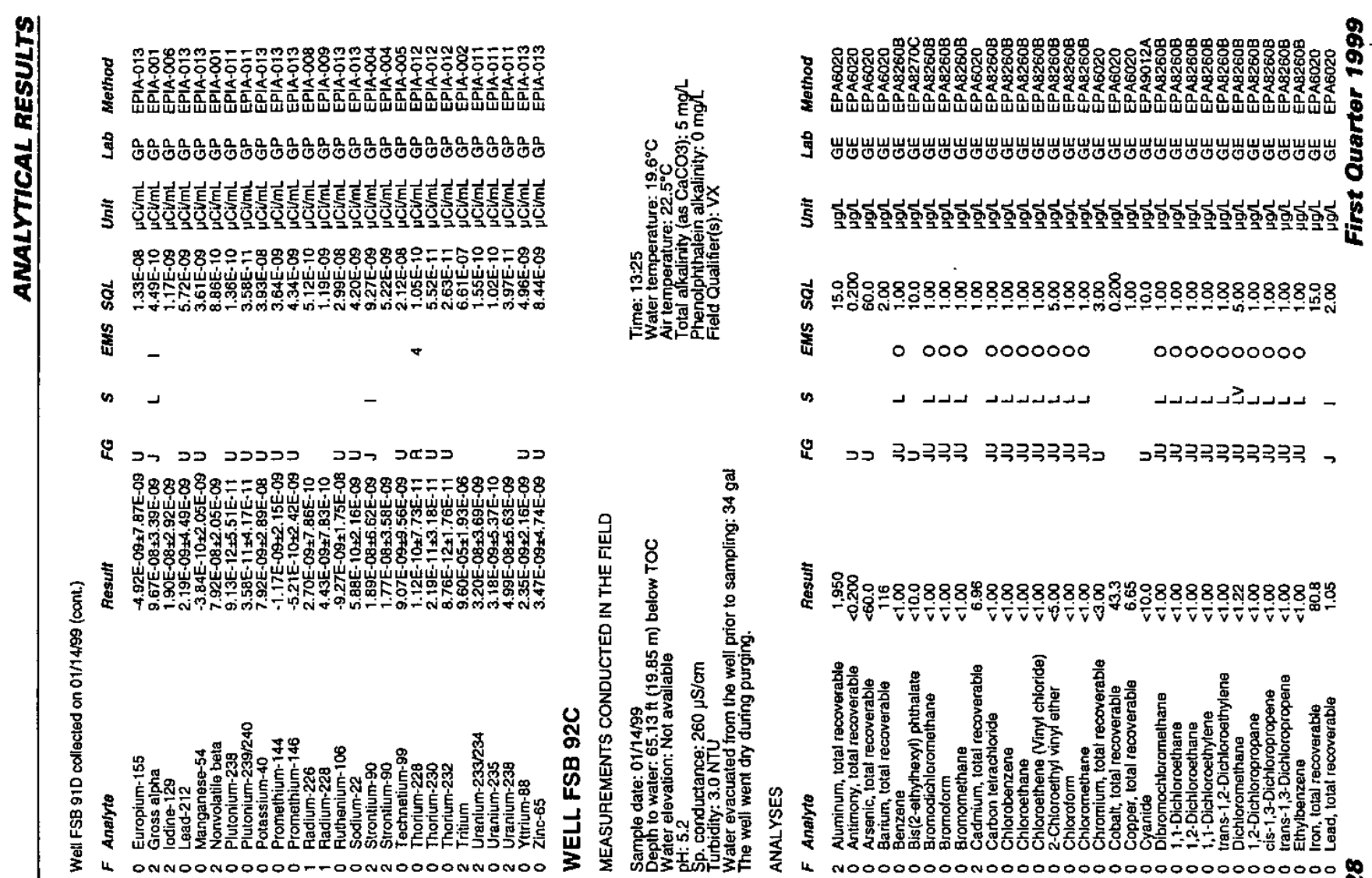
蛋

000000000000000000000

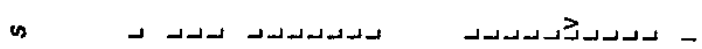

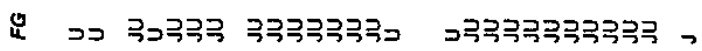

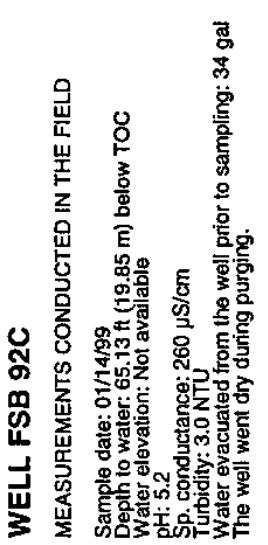

善

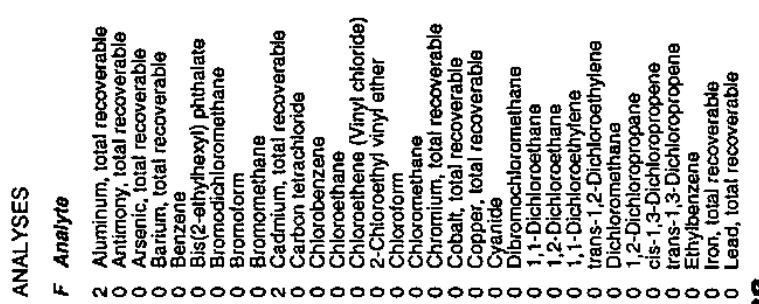

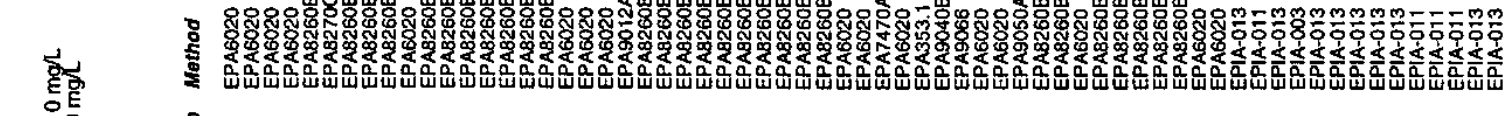

0 范

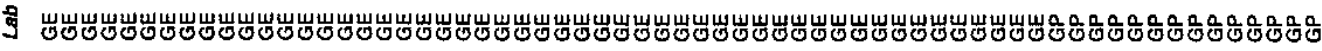

:o:

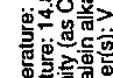

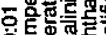

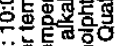

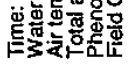

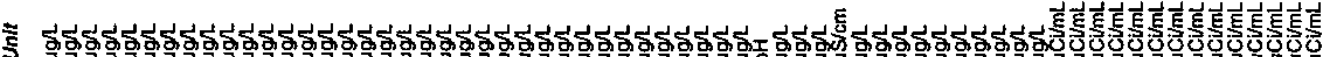
象:

ఫ

䍃 0000000000000000000000000000 -

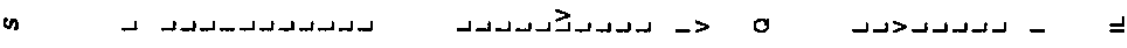

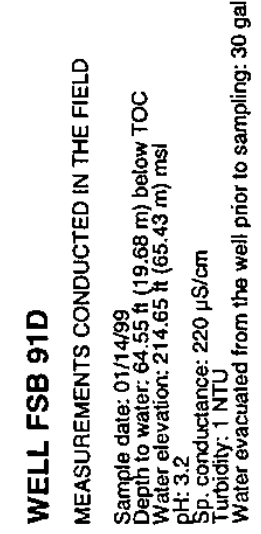

\& I

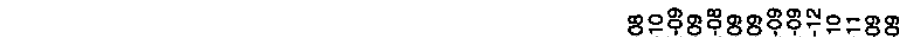

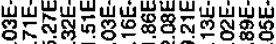

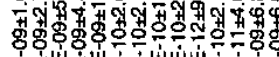

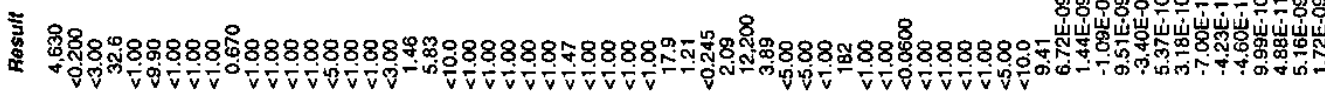

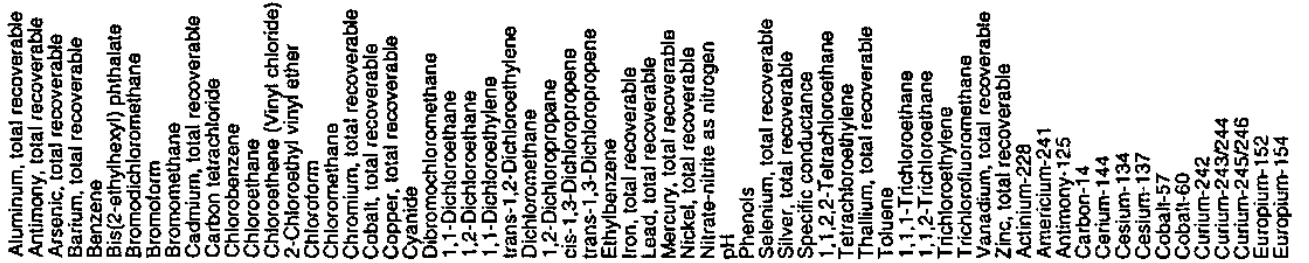


\& 年

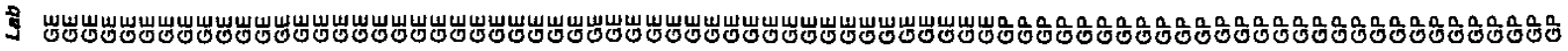

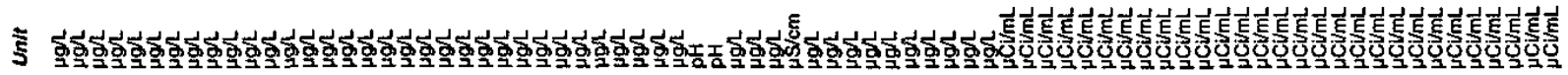

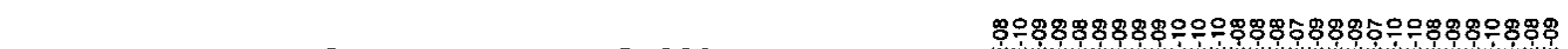

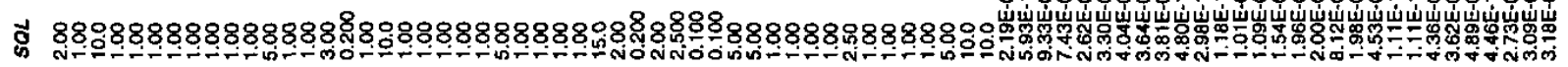

离 000000000000000000000 - 00000000 \& 00

(5) -

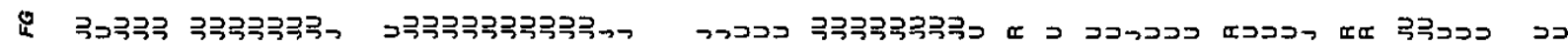

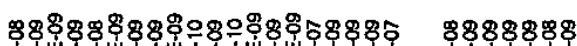

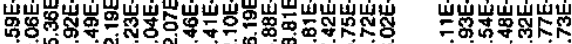

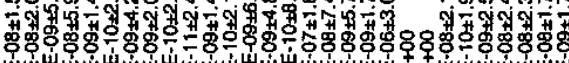

音

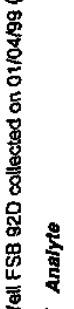

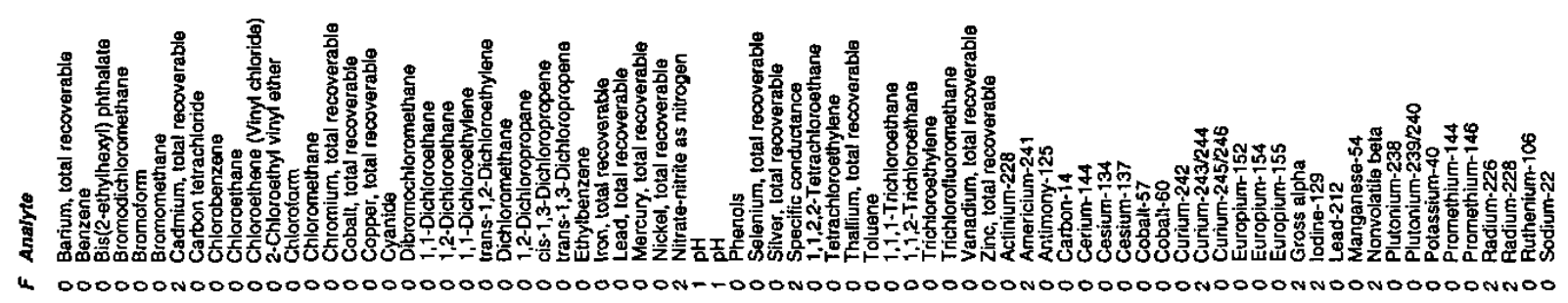

ఫ

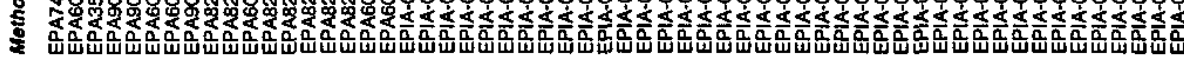

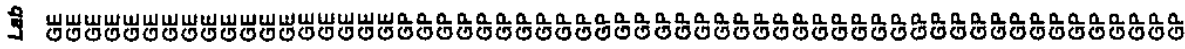

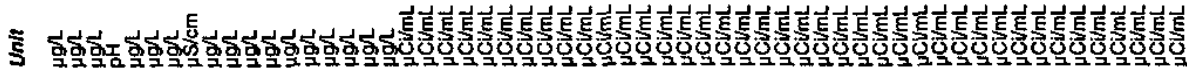

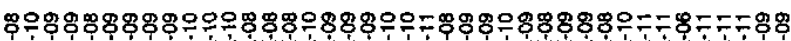
0

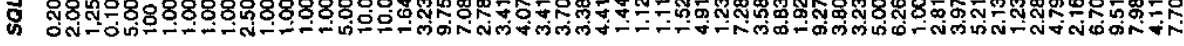
离 0000000
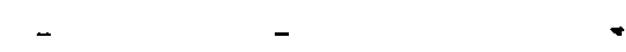

$\infty>0$

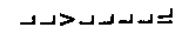

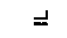

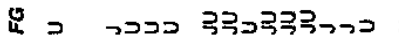

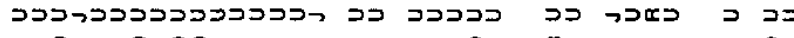

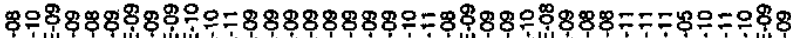

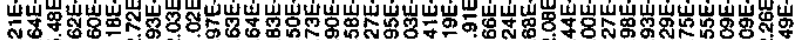

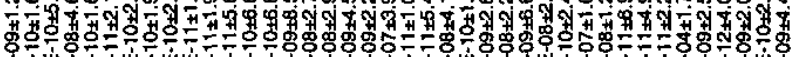

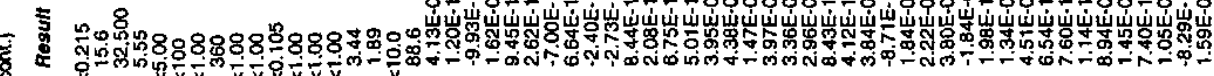

as

כ2

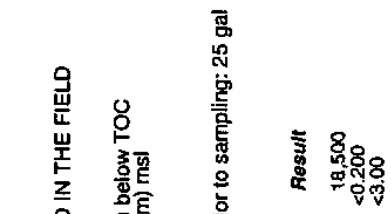




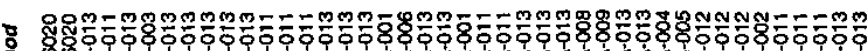

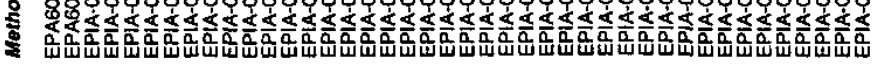

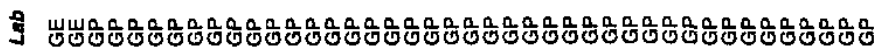

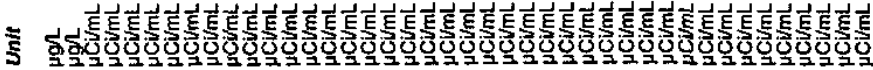

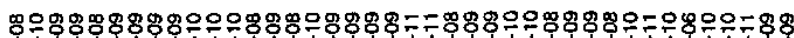

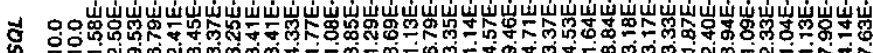

量

疍

- $\quad .000$

0

$-$

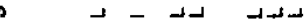

$x$

3

ניטי

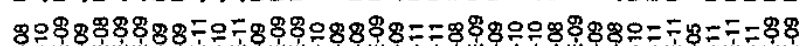

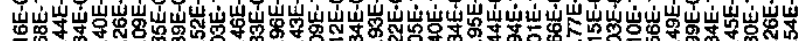

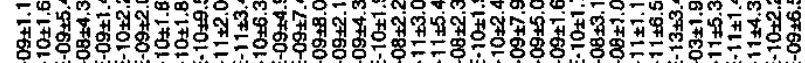

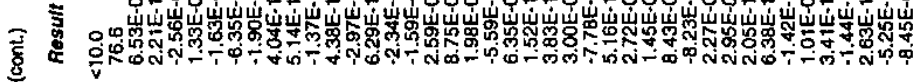

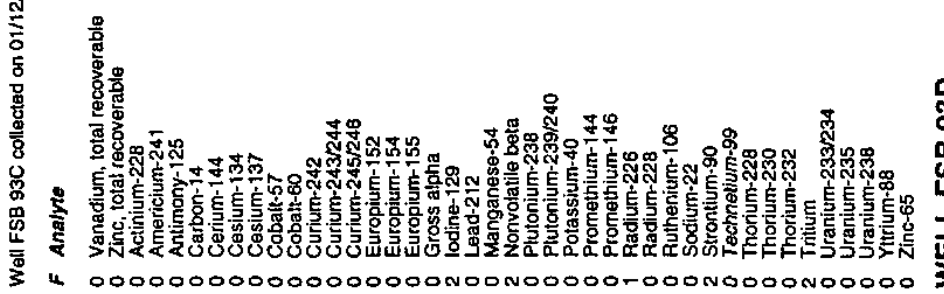

ל.

:

Nion

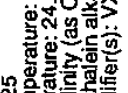

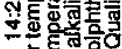

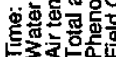

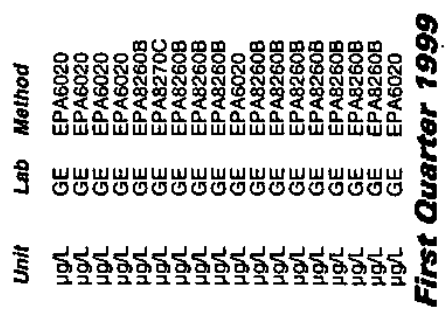

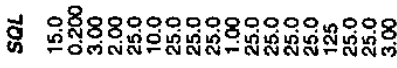

孳 $0 \quad 00000000000$

a

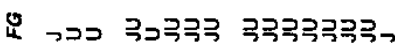

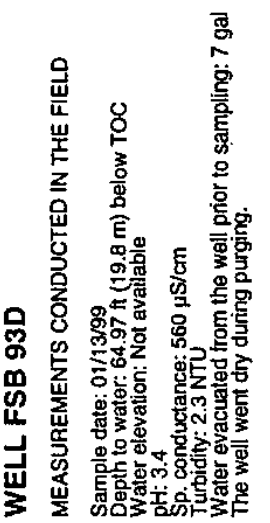

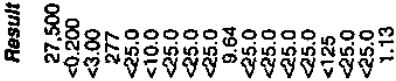

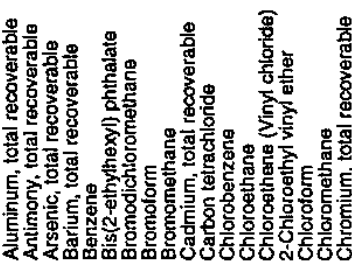

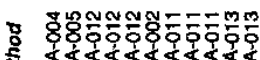

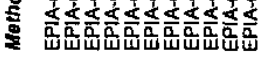

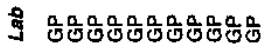

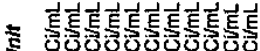

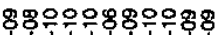

ปt

के

密 $0+$

$\infty x$

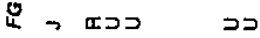
$880==8 \% 88 \%$

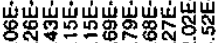

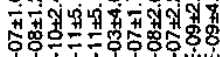

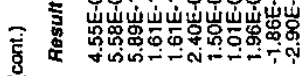

兽

¿

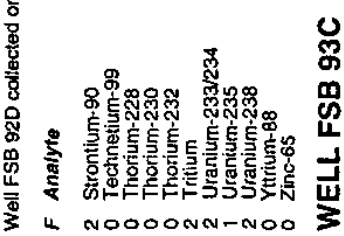

ร్.

ơ

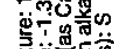

要

品

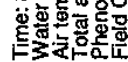

은.

बु

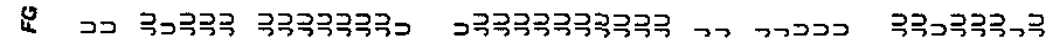

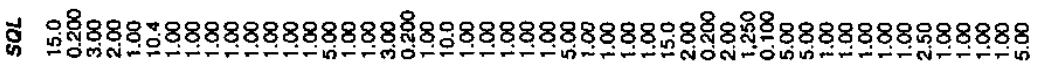

is $000000000000000000000 \quad$ - 0000000

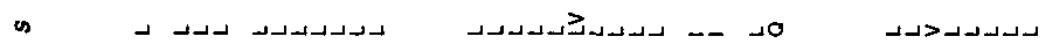

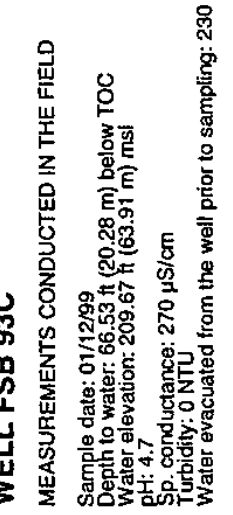

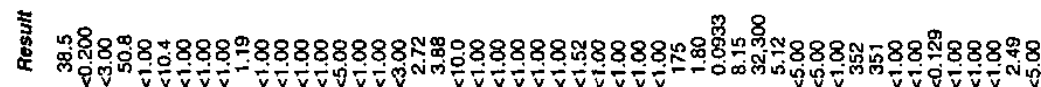

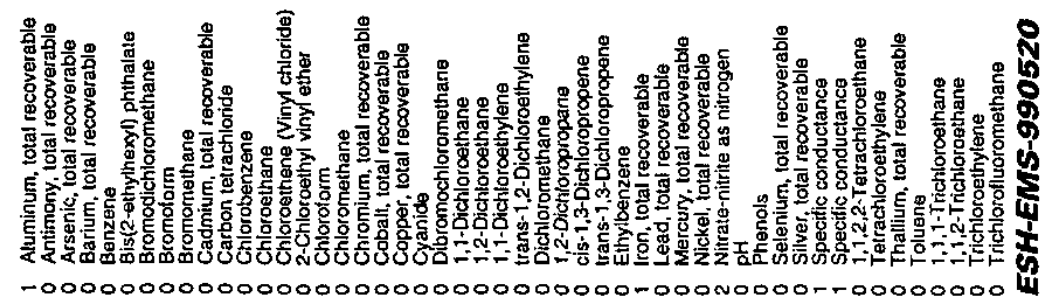




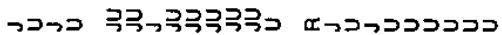

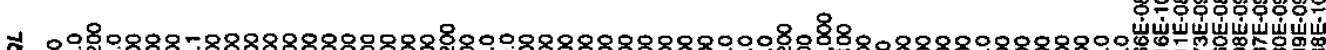
密 9. \%

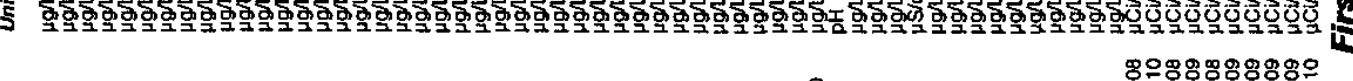

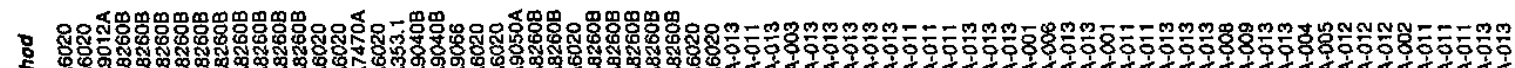

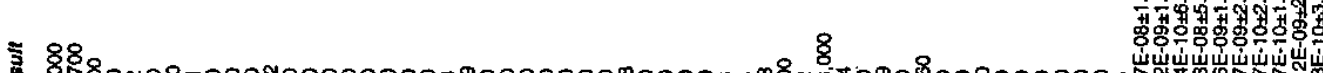

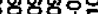

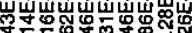

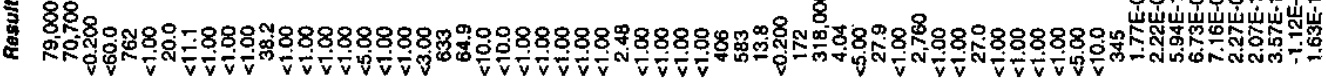
焉

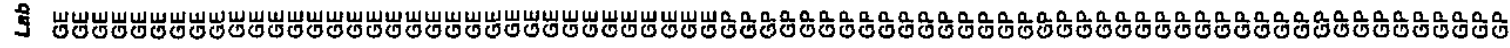

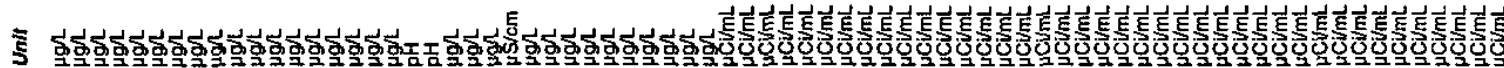

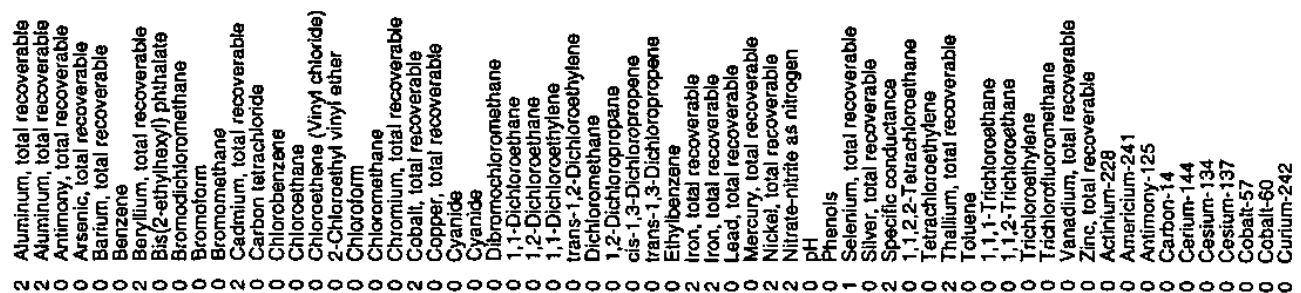

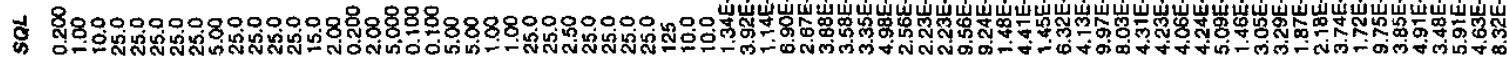

i

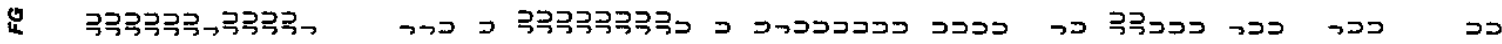

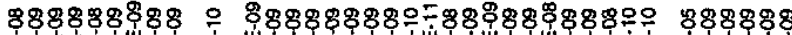

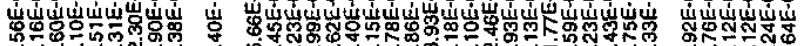

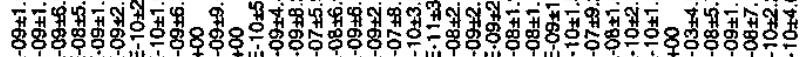

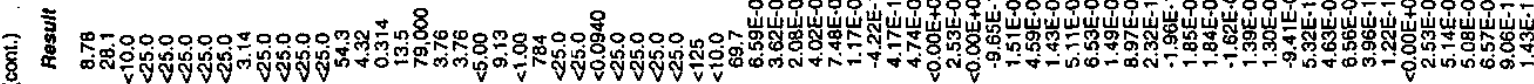




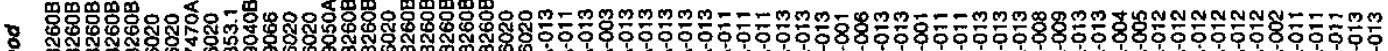
等

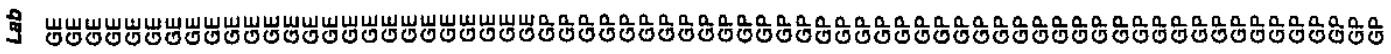

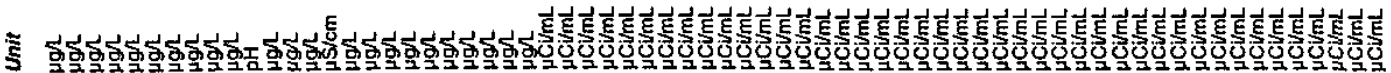

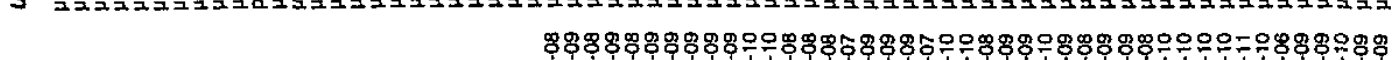

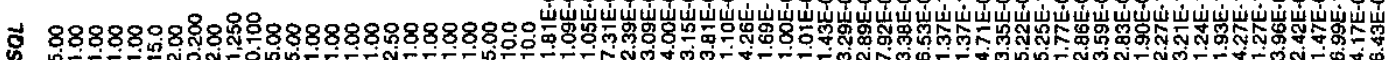

窝

00000

0000000

$\checkmark$

$+\bar{o}$

$0+\infty$

is

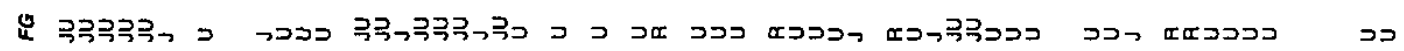
\%88 8

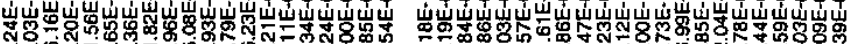

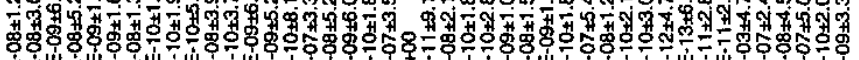

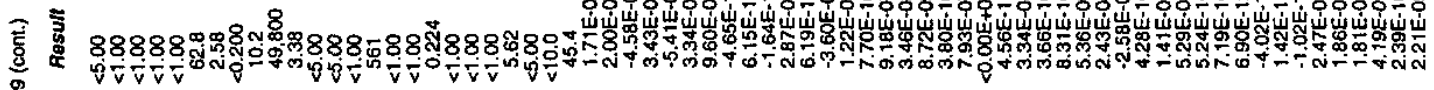

里产
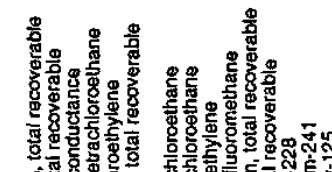

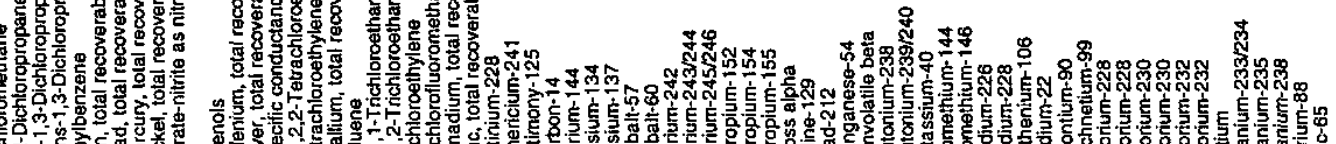

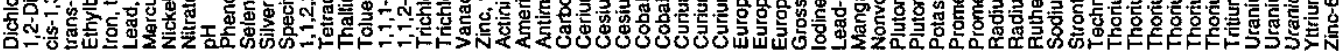

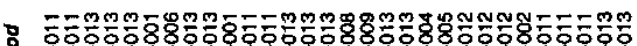

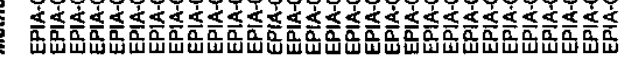

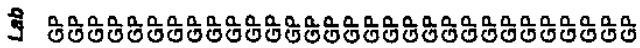

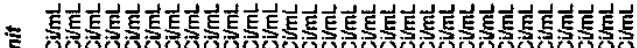

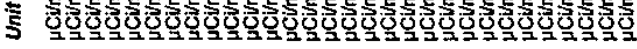

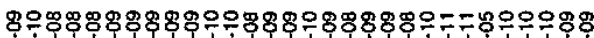

ป

善

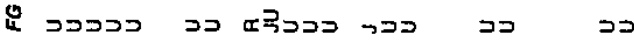

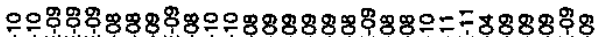

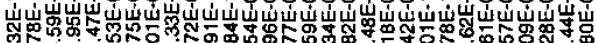

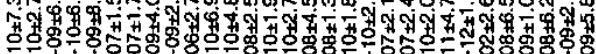

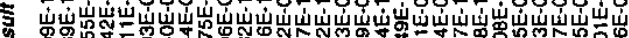

言

\%

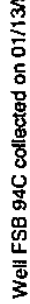

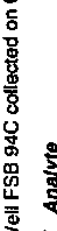

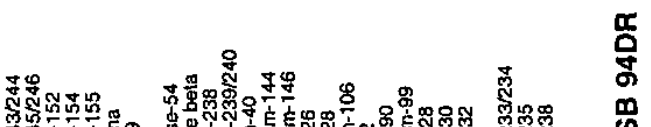

วิ

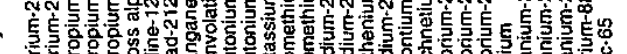

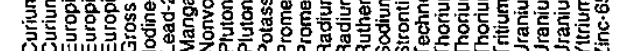

L OOOOONNOONOOOOONNOONOOOONNONOO

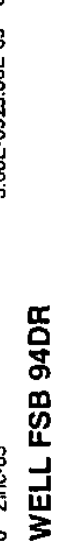

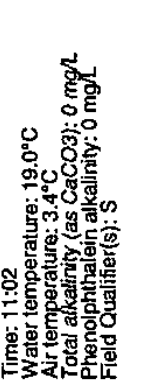

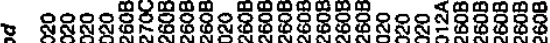

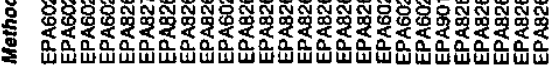

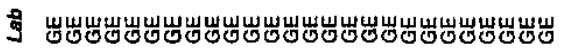

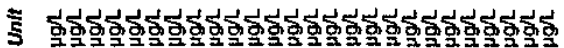

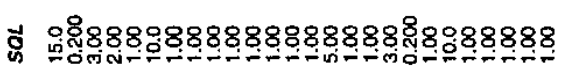

絭 $00000000000 \quad 00000$

i נגנגנ

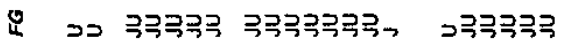

बु
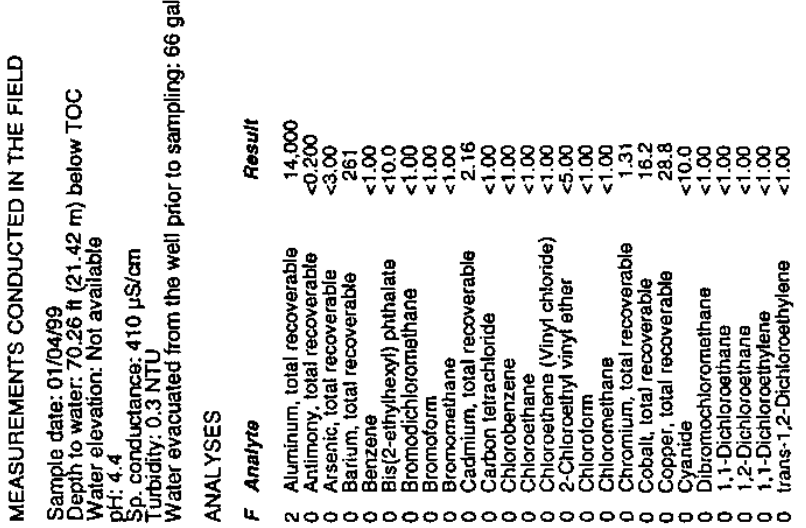


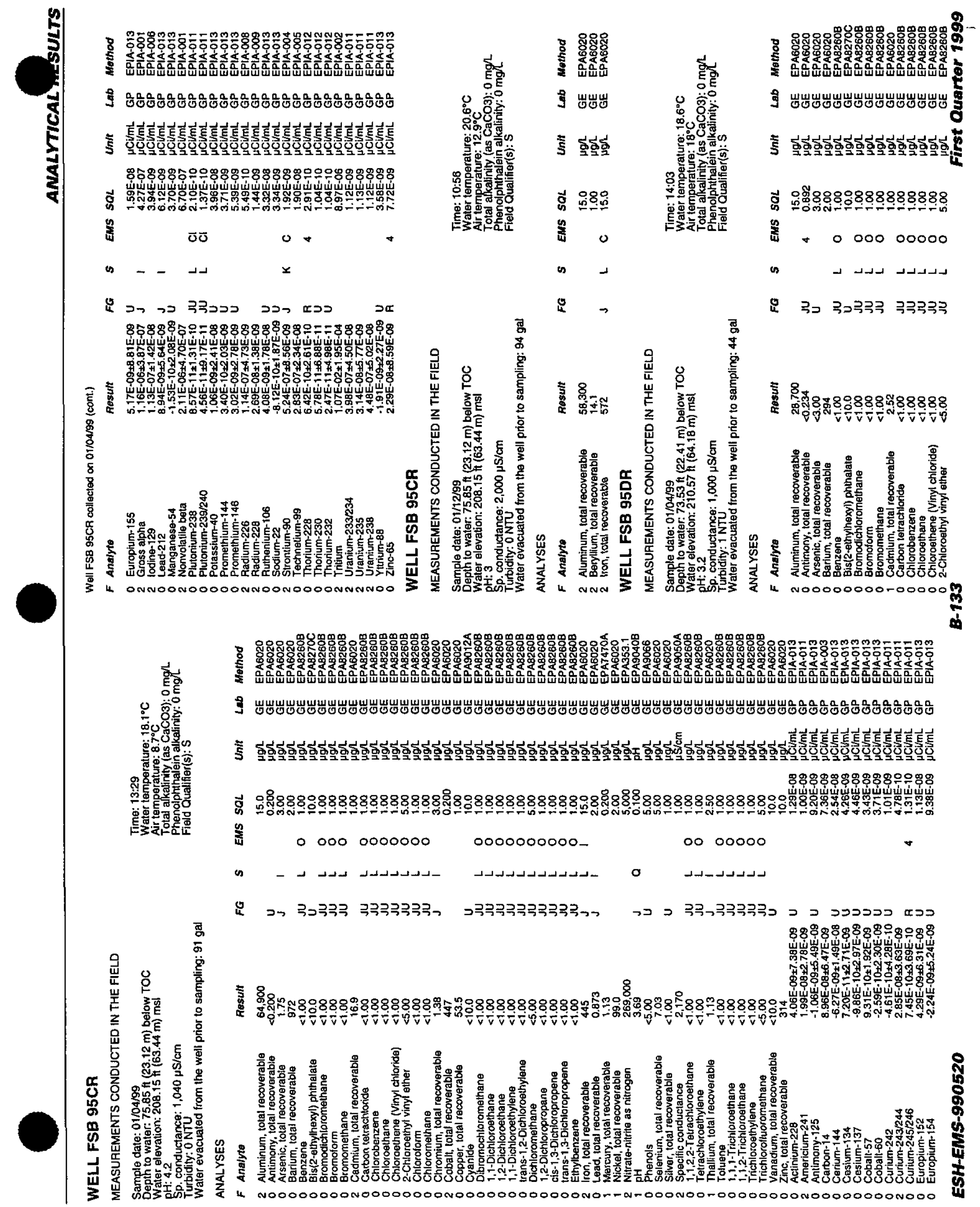




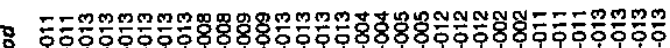

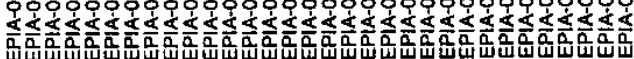

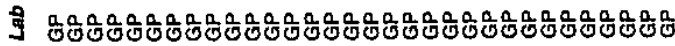

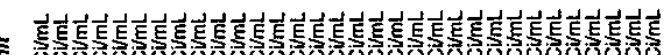

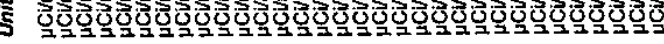

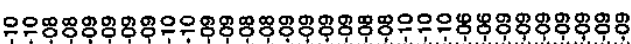

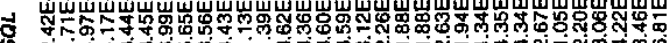

离 $\overline{\mathrm{W}}$

$00 \%$

es $\lrcorner$

$צ x$

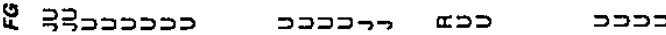

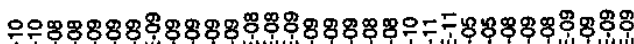

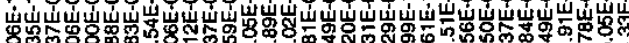

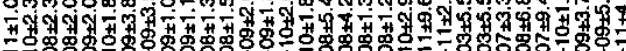

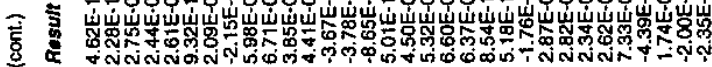

\%

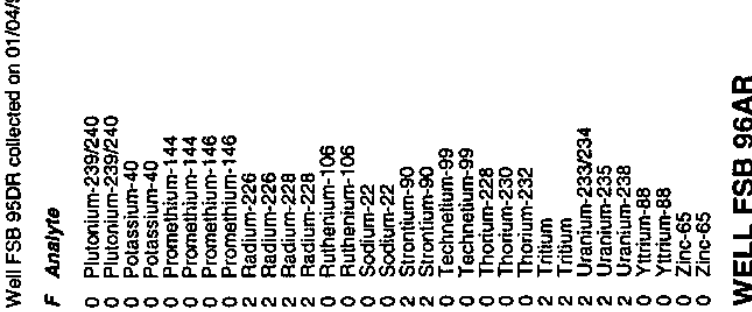

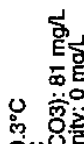

nind

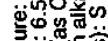

काँ 굴

吊

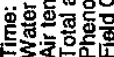

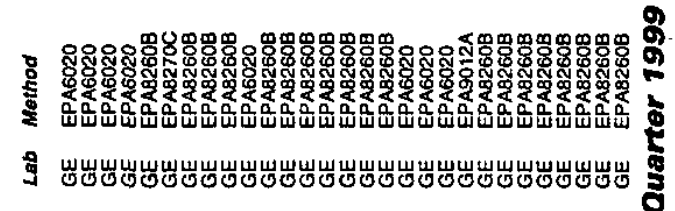

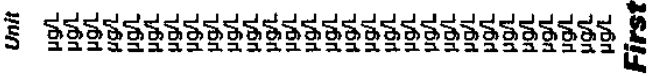

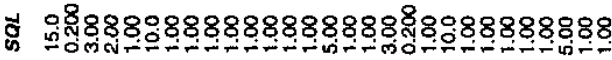

盗

is

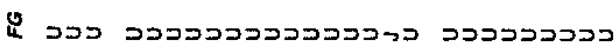

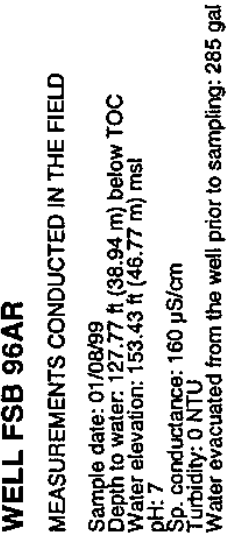

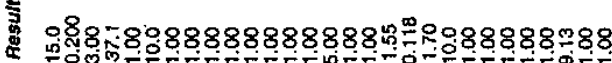
जั

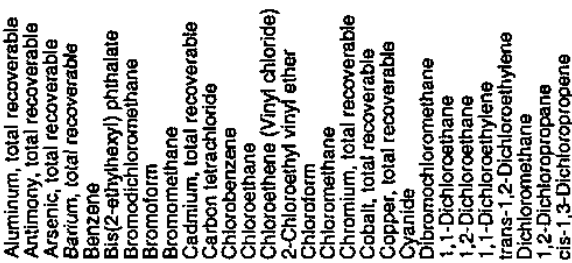

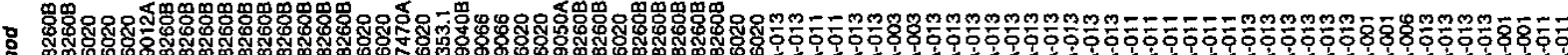
告

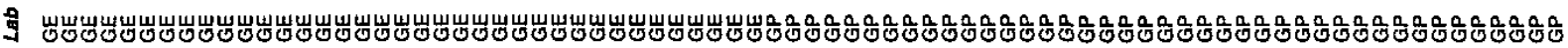

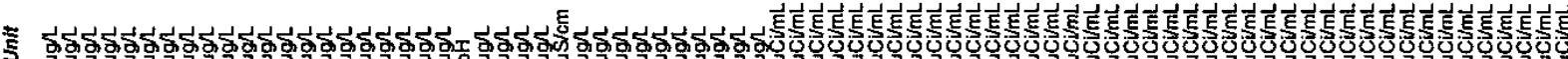

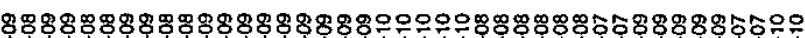
5 $8888 \%$ 㝕 000000000000 - 000000

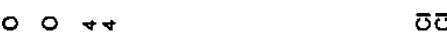

Q 1

\lrcorner$\lrcorner\lrcorner\lrcorner\lrcorner\lrcorner\lrcorner\lrcorner\lrcorner\lrcorner\lrcorner+0$

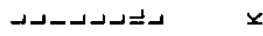

$\times \mathbf{x}$

$\overline{0}$

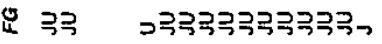

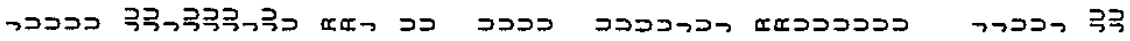
$8 \% 8888.8888 \%$

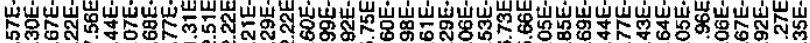

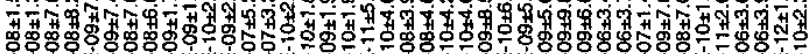

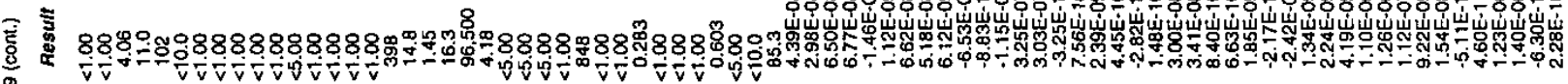

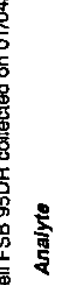
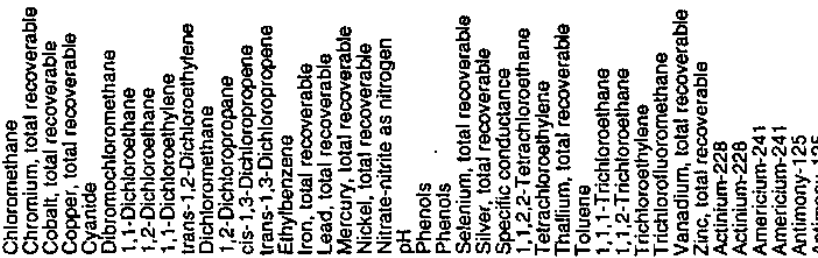

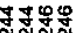

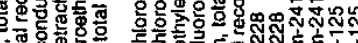

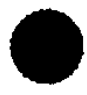




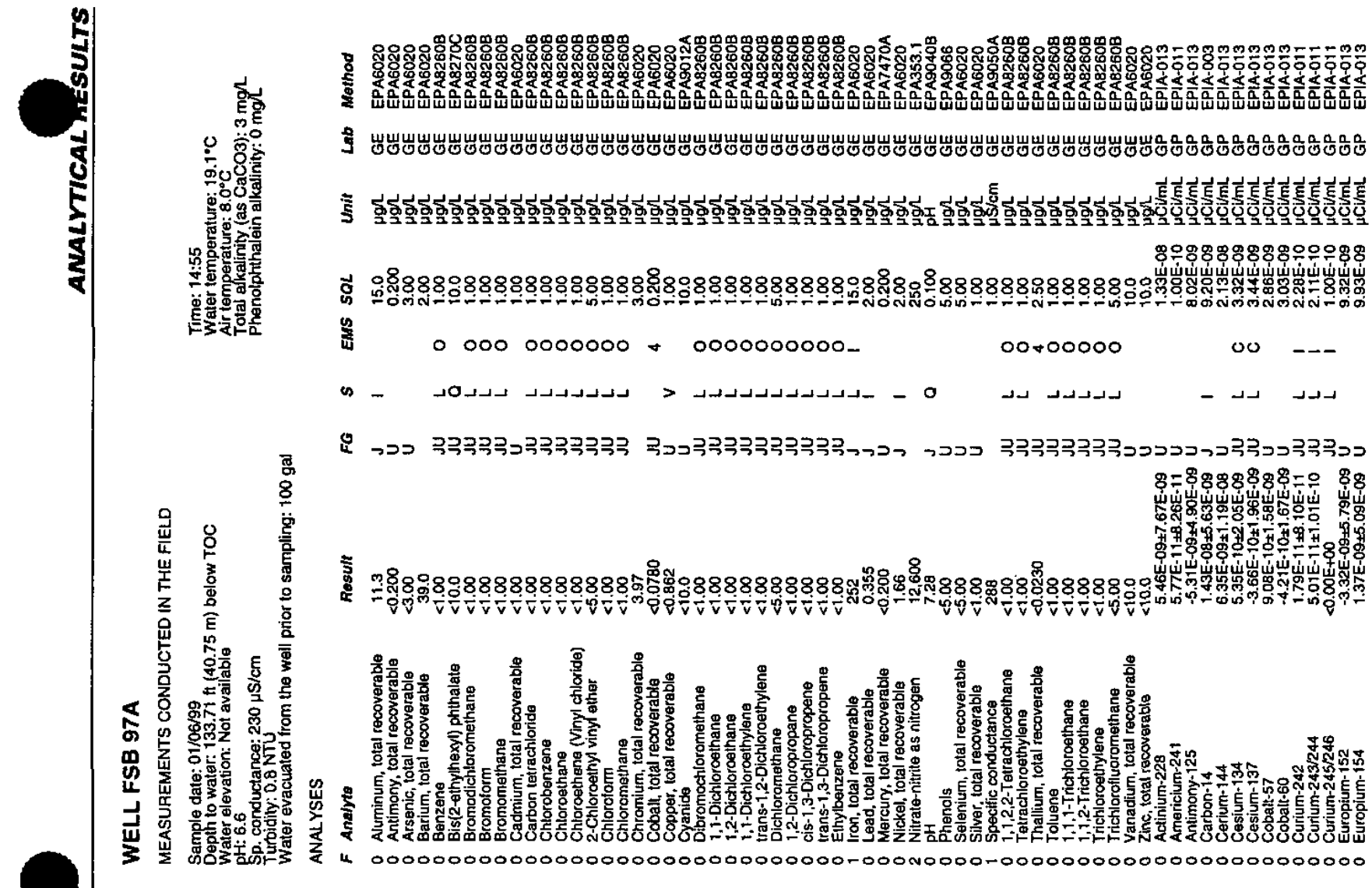

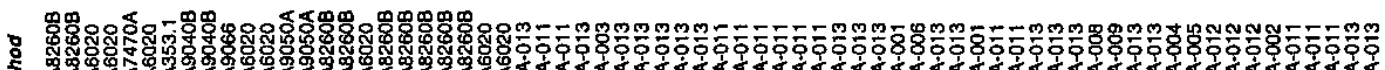 等}

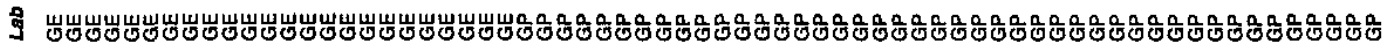

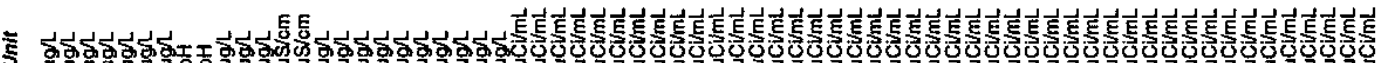

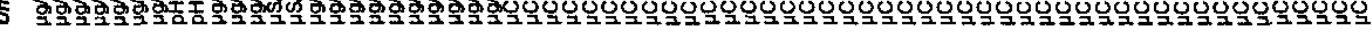

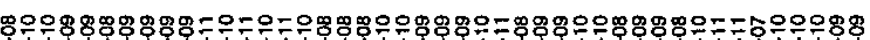

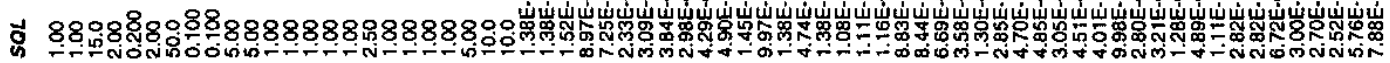

量

o $\lrcorner>$ oo oo $>>$

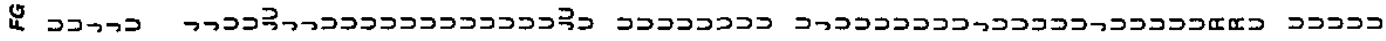

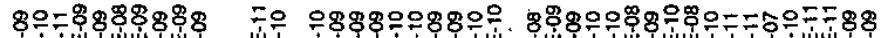

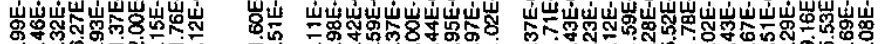

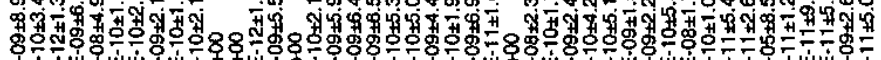

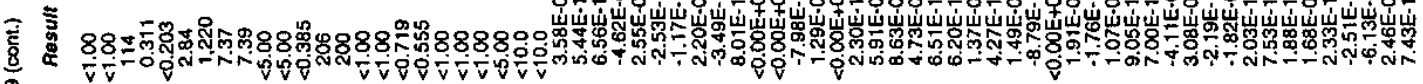

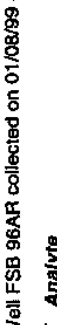
-
$->$
+

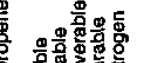

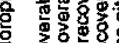

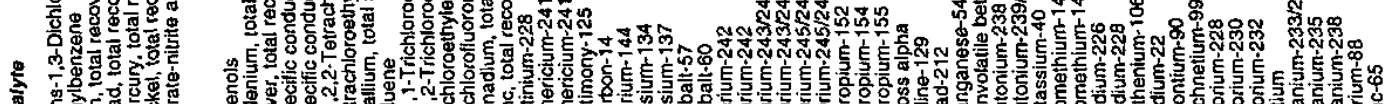

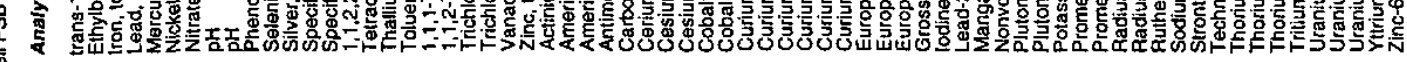
S L $00000000000000000000000000000000000000000000000000000000000000-00000$

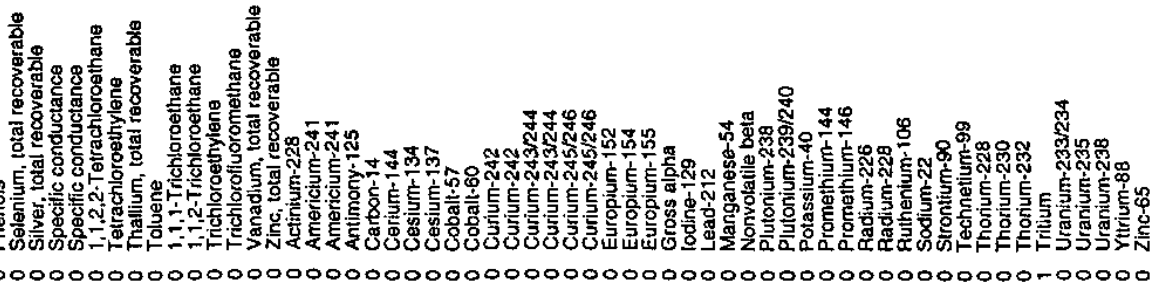




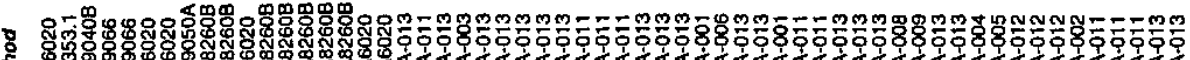

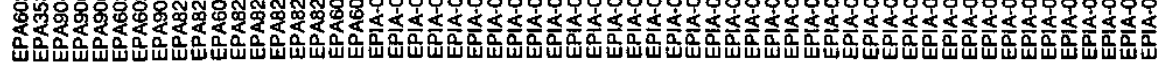

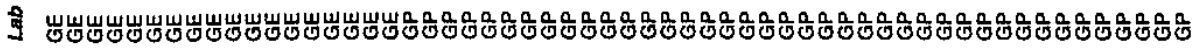

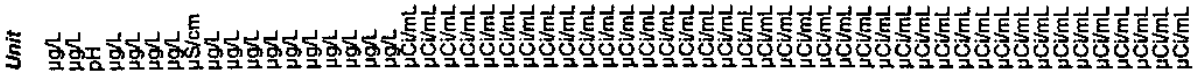

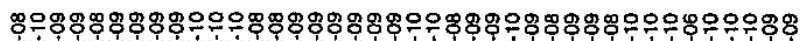

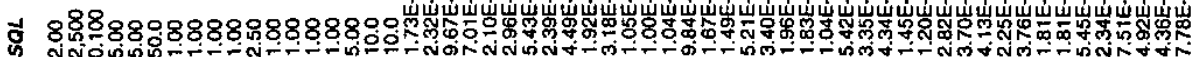

竞 (1)

$-$

0000000

$\overline{0} \checkmark$

$+$

$-$

$\circ$

a to

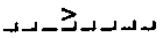

$+$

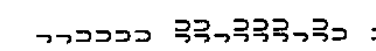
\$88 8 \%

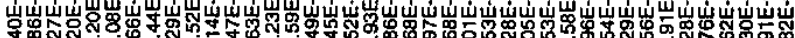

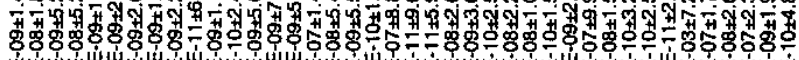
₹ 要

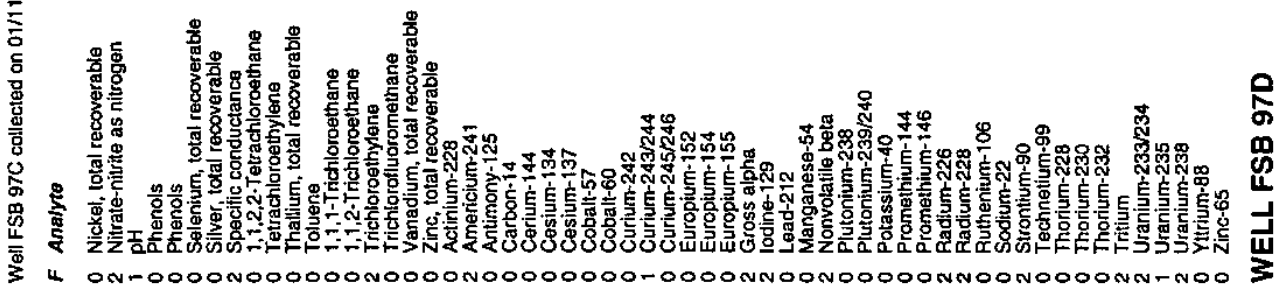

\&

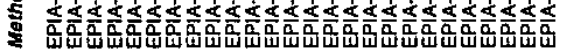

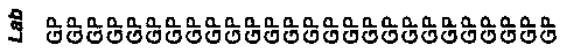

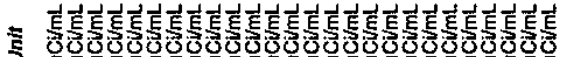

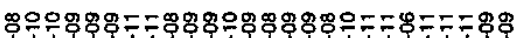

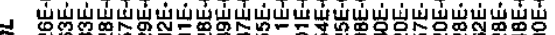

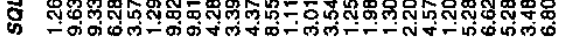

密

$\infty$

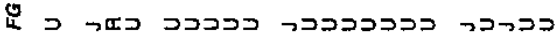

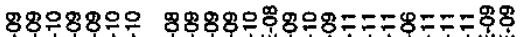

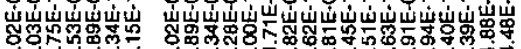

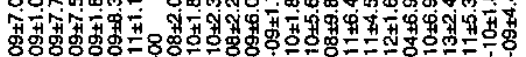

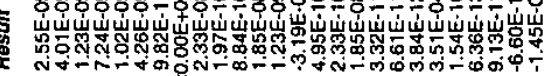

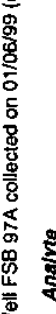

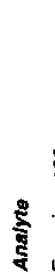

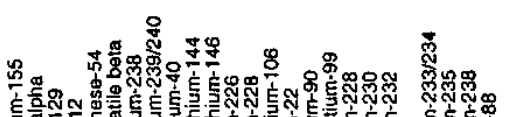

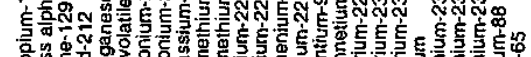

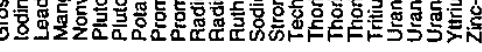

L 1400 N00000000N00000000N00000
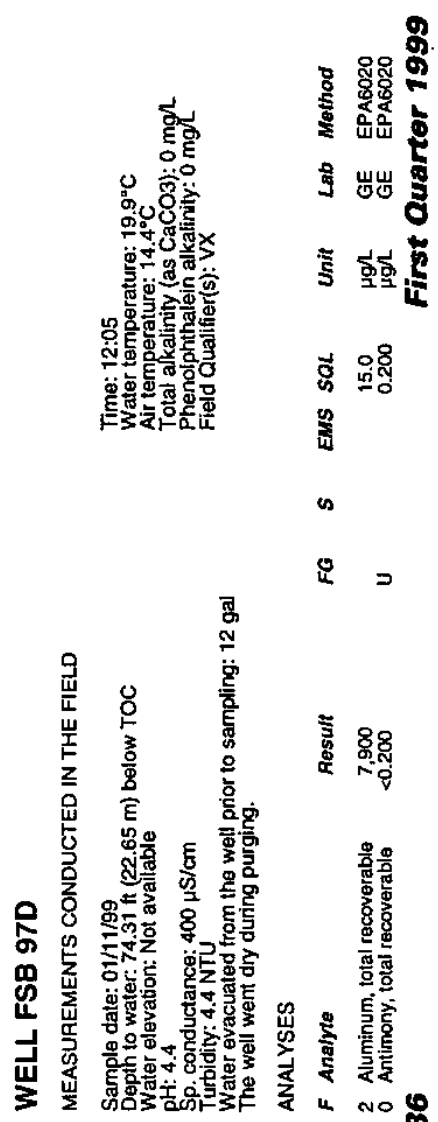

a

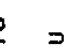

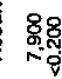

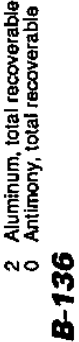

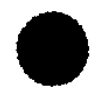

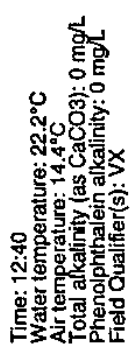

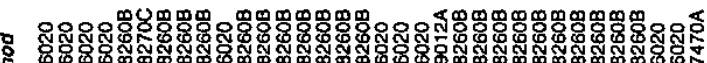

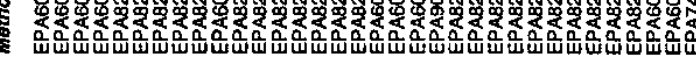

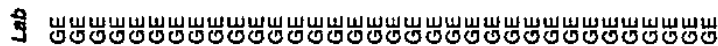

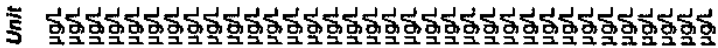

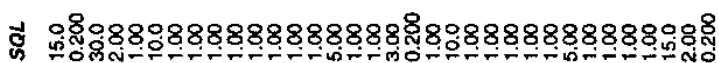

竞 $00000000000 \quad 0000000000$

a

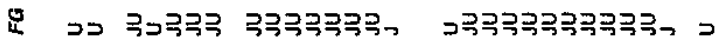

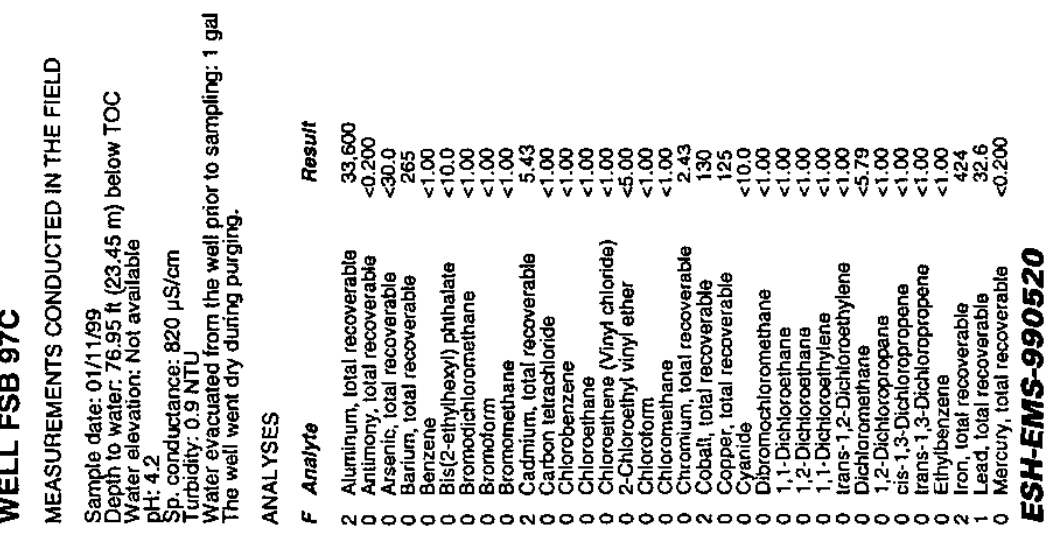




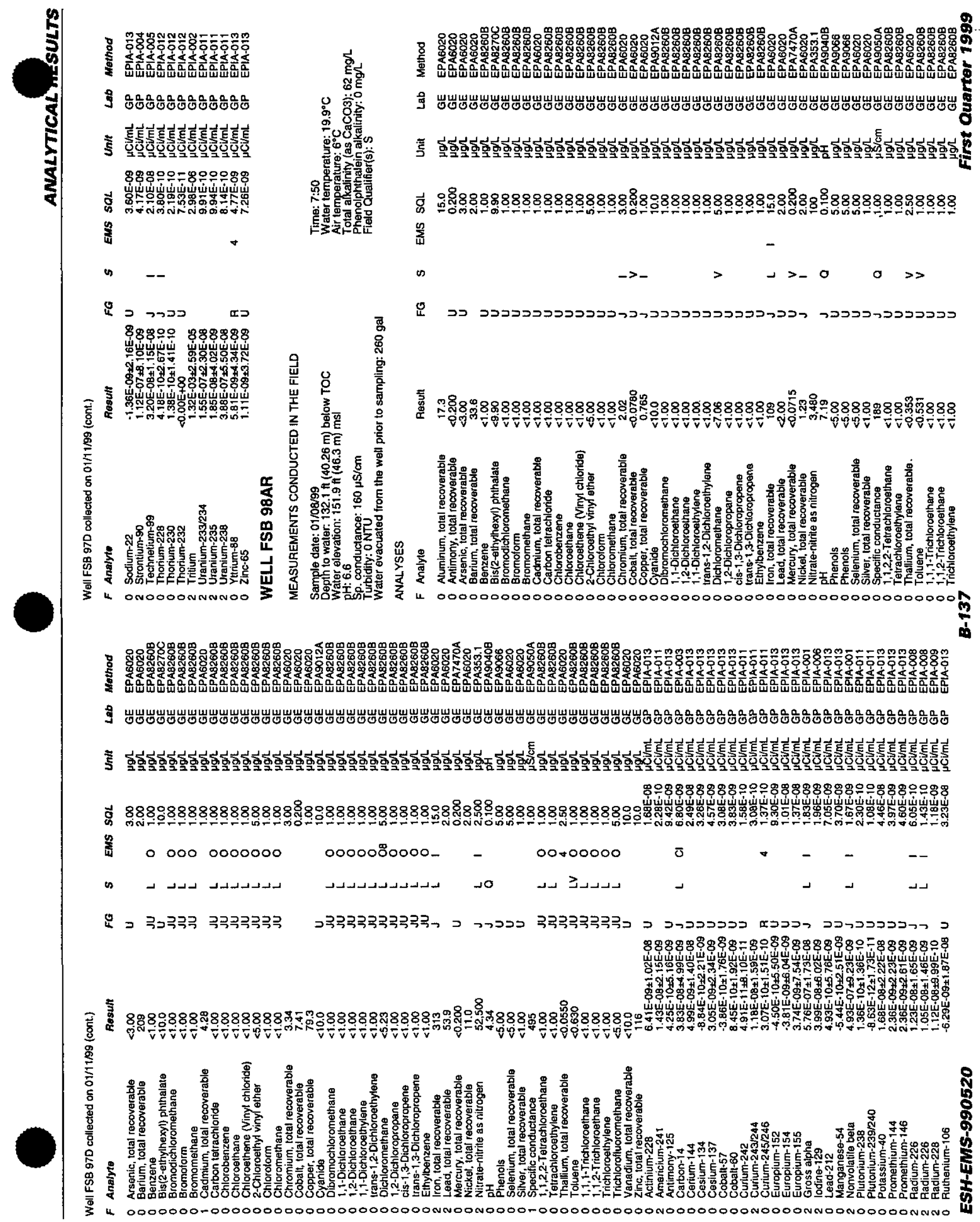




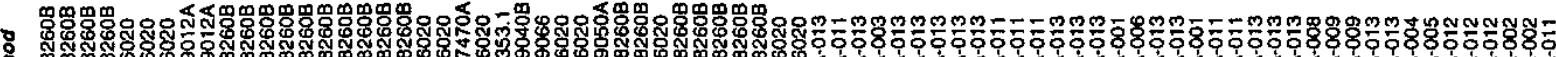

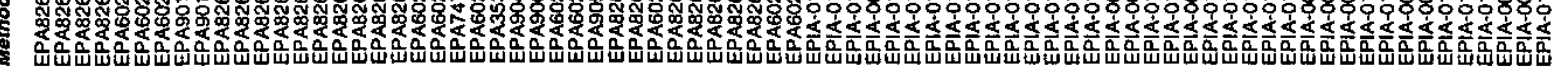

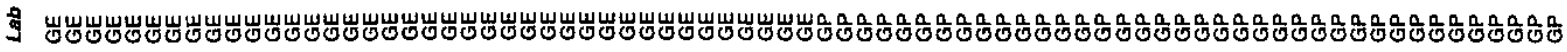

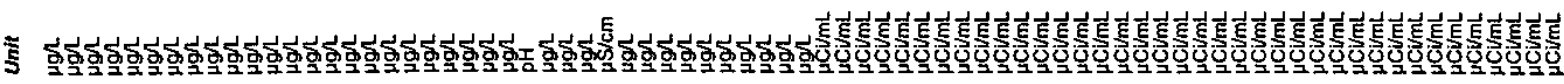

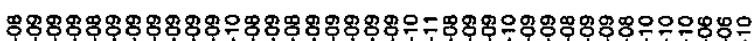

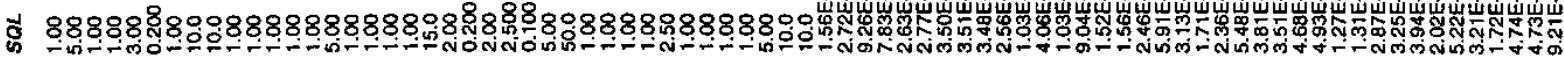

竞 0000

00000000000

(n) $\lrcorner\lrcorner\lrcorner\lrcorner$

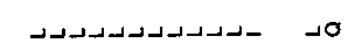

ــ

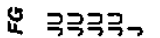

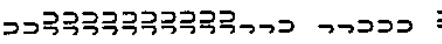

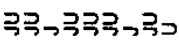

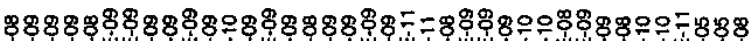

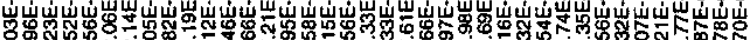

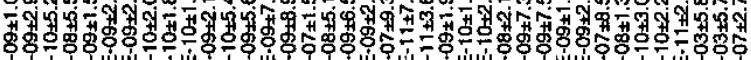

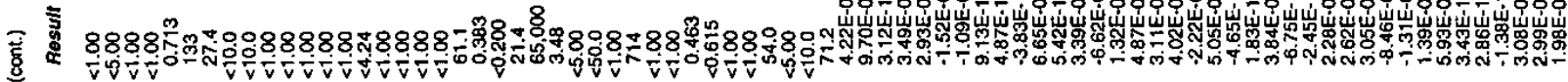

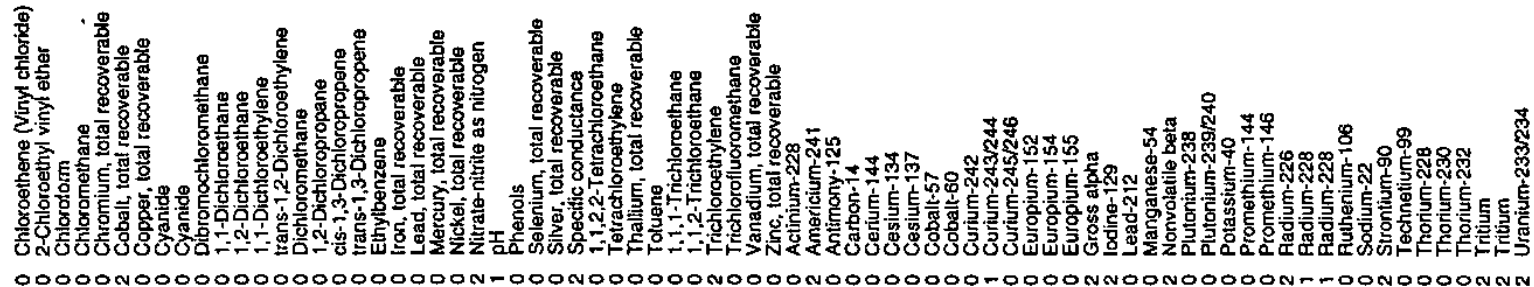

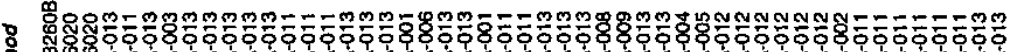
空

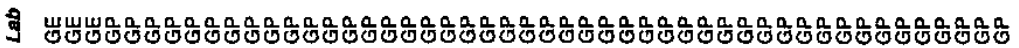

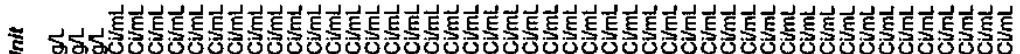

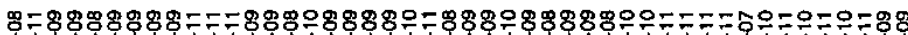

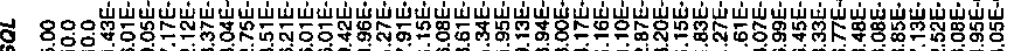

竞

-

$+$

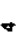

$\geq$

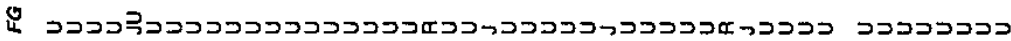

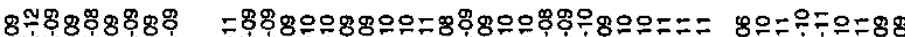

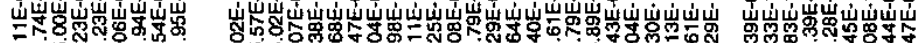

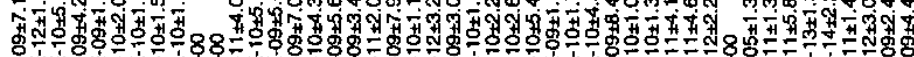

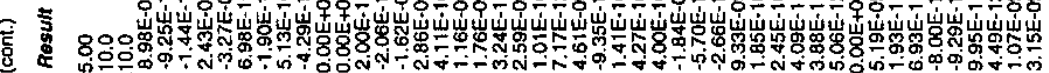

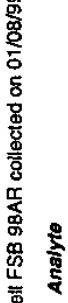

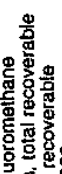

0 咅

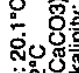

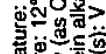

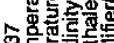

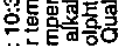

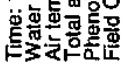

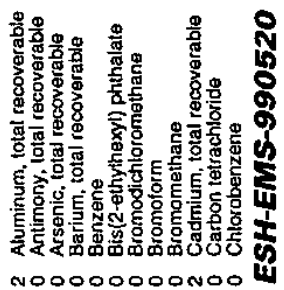

峦 00000

. 覀

芦

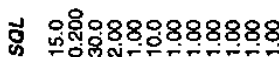

Q $\perp\lrcorner\lrcorner\lrcorner\lrcorner$

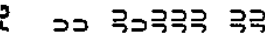
온

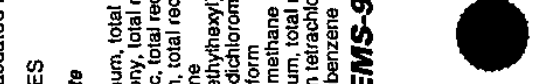




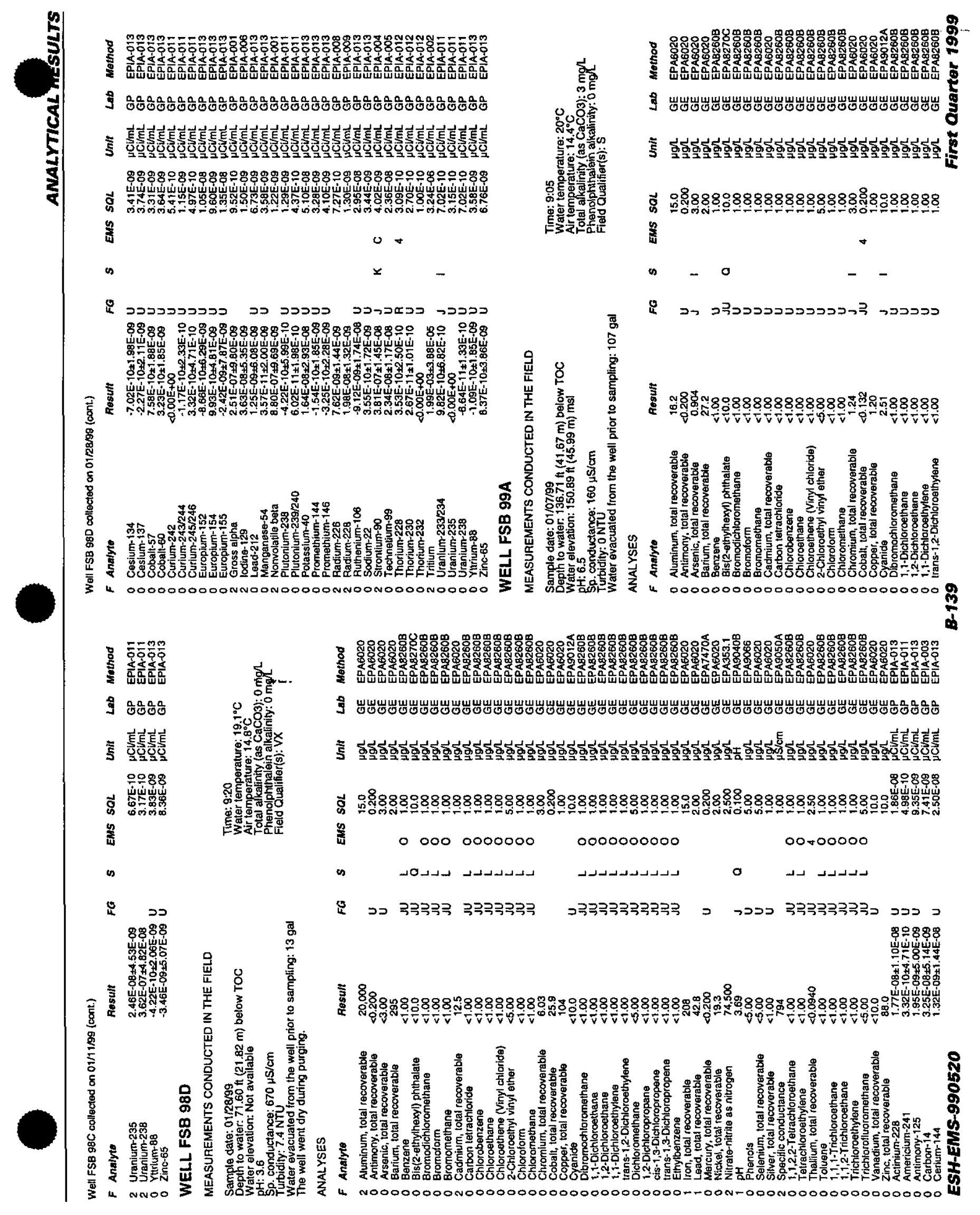




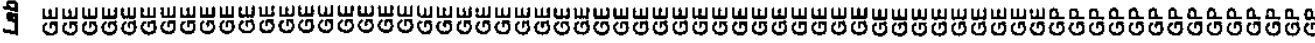

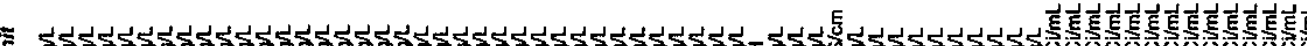

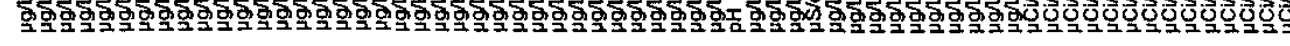

ప 盖

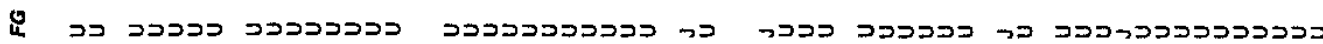
$8=888888 \%=-88$

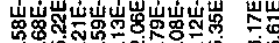

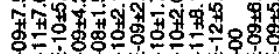

壱

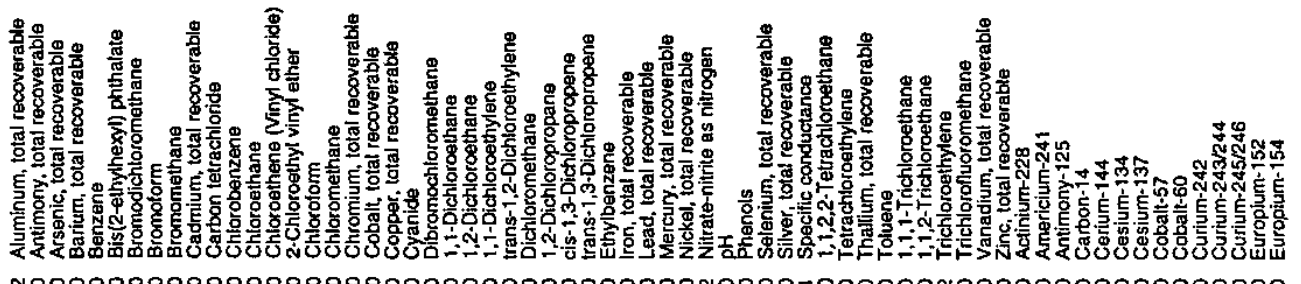

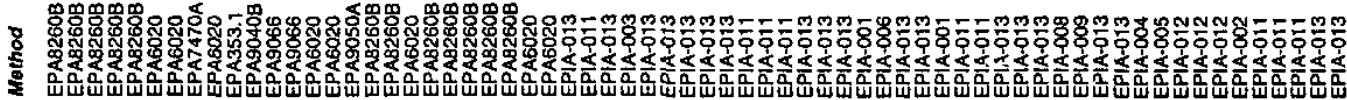

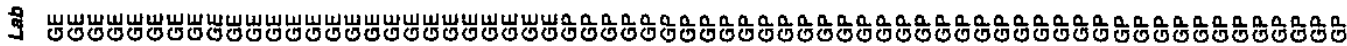

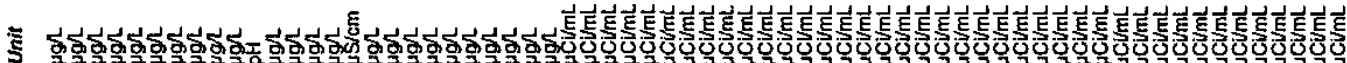

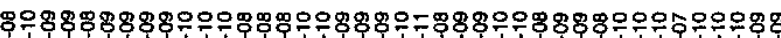

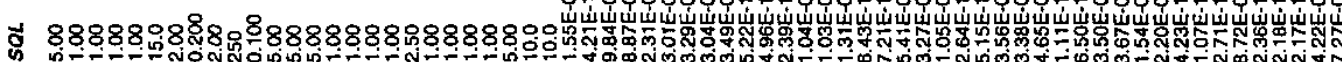

竞

$-00$

$+000$

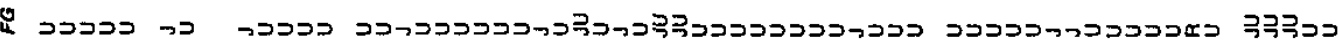

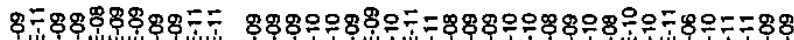

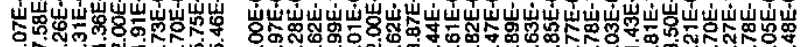

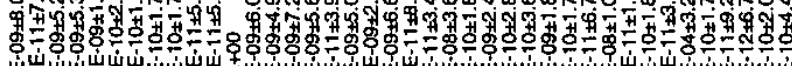

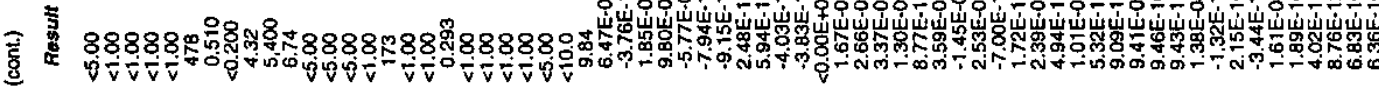

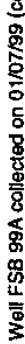

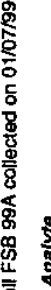

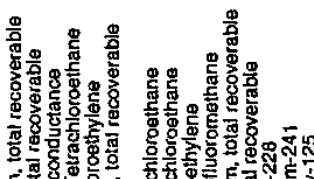

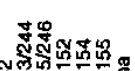

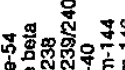
2.

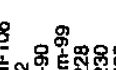

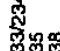

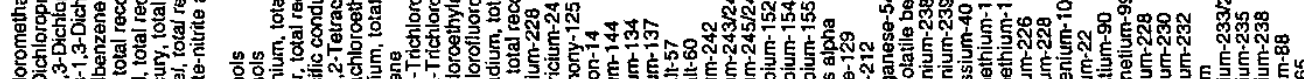

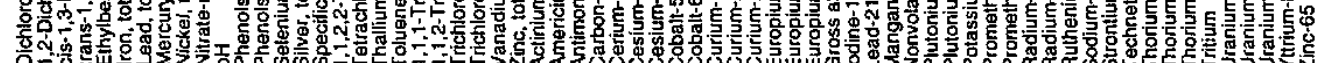
L 00000 N000 00000000000000000000000000000000000000000000000000 N00000 


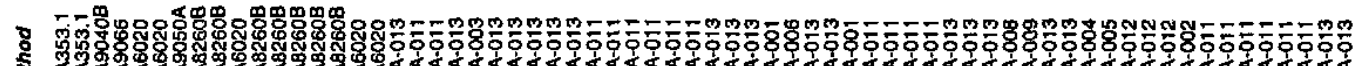

I

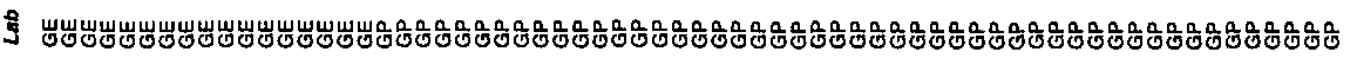

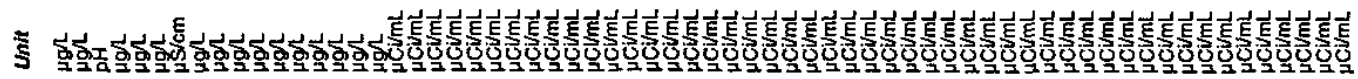

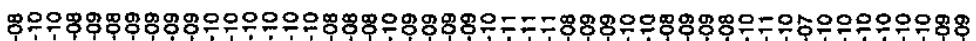

ఫ

蛋

s

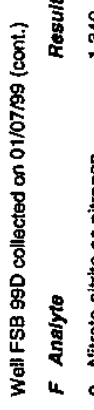$$
-00
$$$$
\text { - } \quad \text { - }
$$

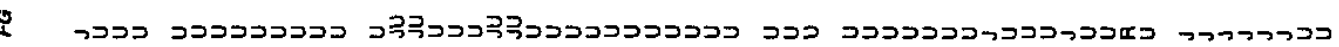

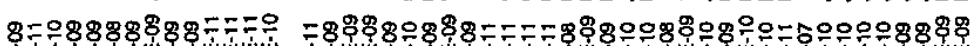

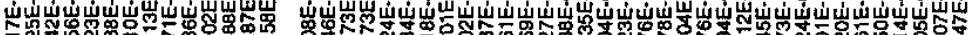

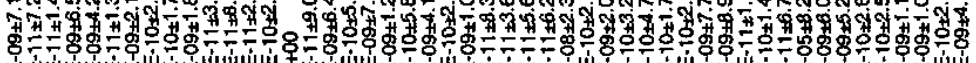

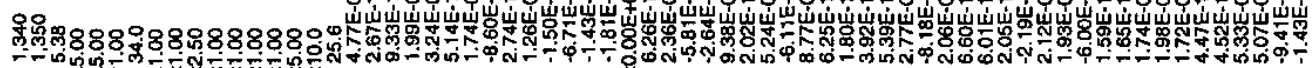

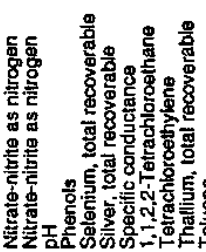

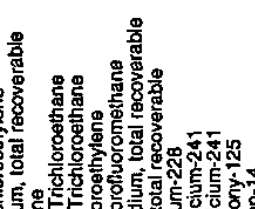

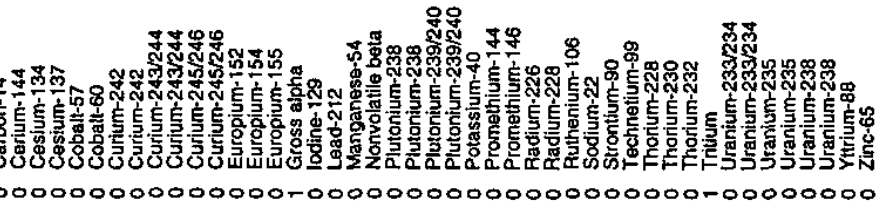

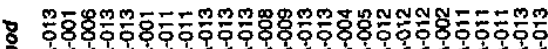
势

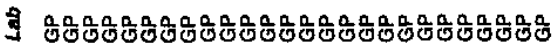

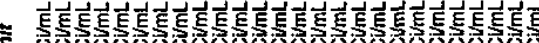

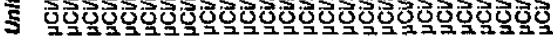

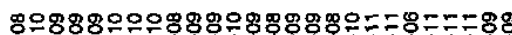

ᄀ

ธั

N

量

$-$

$\checkmark$

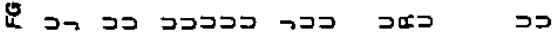

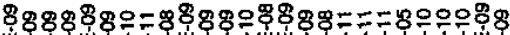

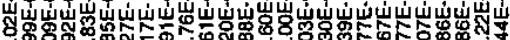

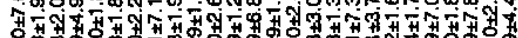

范

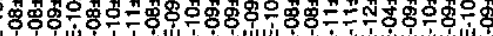

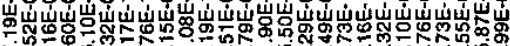
$\frac{8}{8}$ N

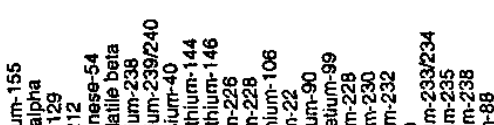
员

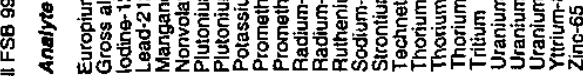
3 in ONNOONOOOOONOOONOOOONO0000

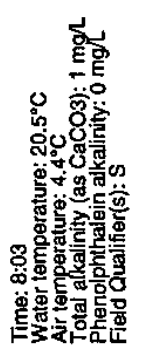

:

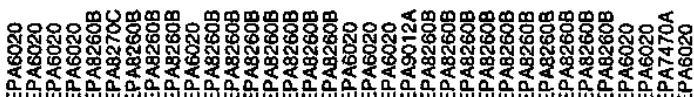

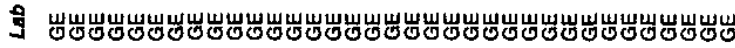

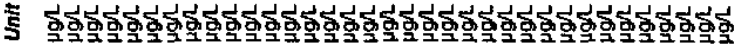

ธ్ .

竎

is

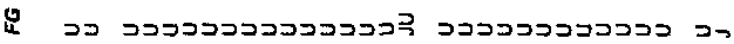

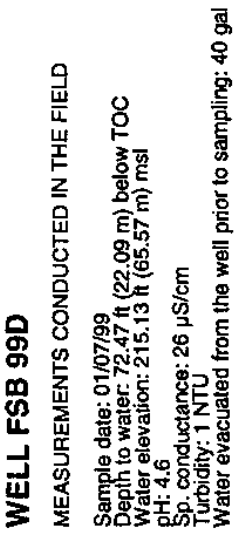

产 通8

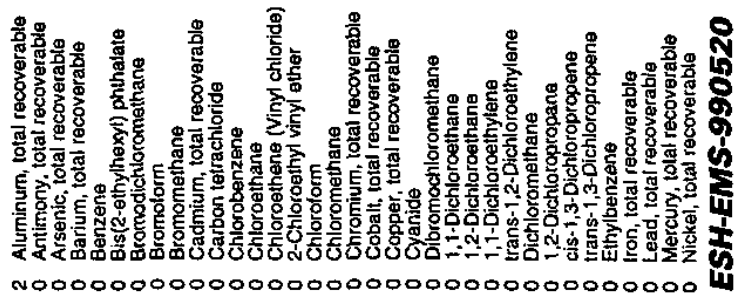




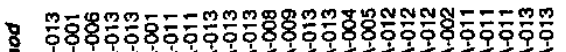

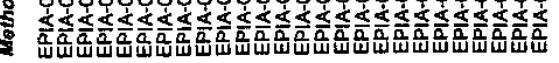

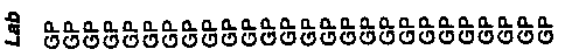

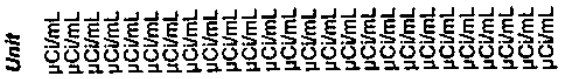

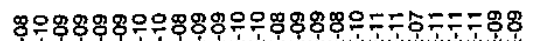

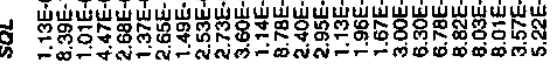

密

$\checkmark$

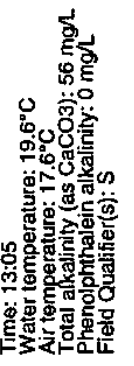

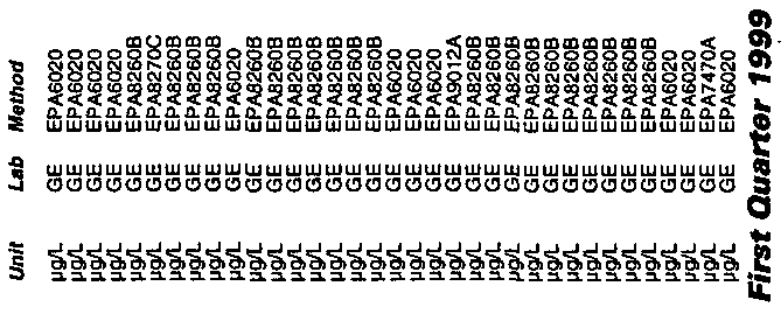

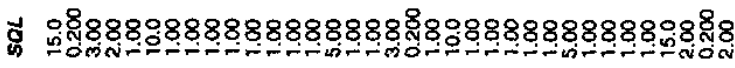

峦

is

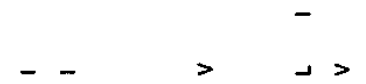

\&

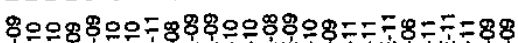

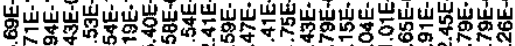

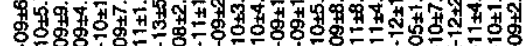

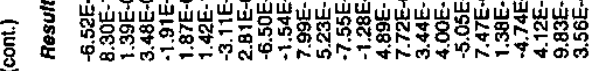

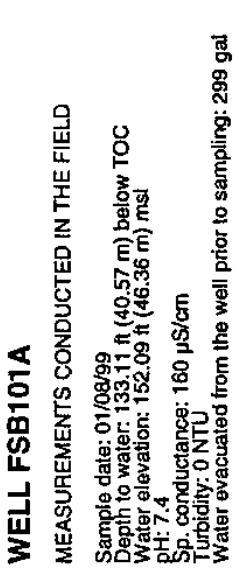

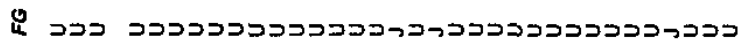

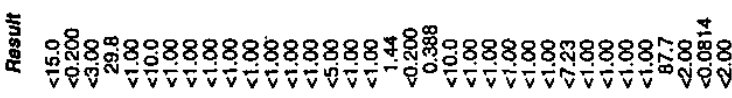

爱

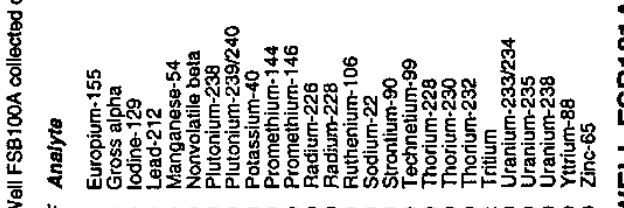

\ u 00000000000000000000 N00000

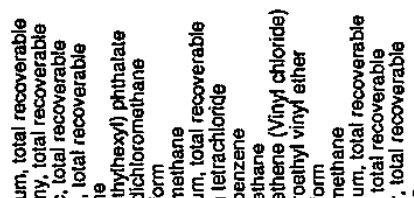

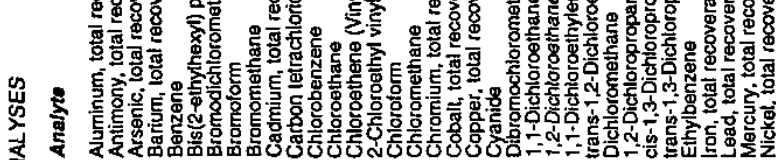

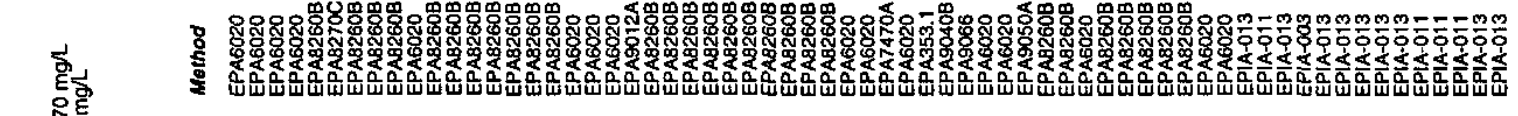

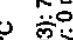

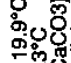

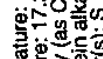

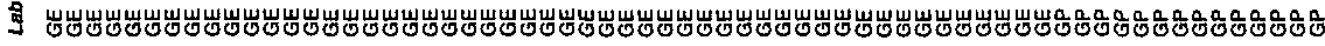

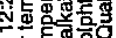

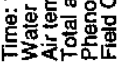

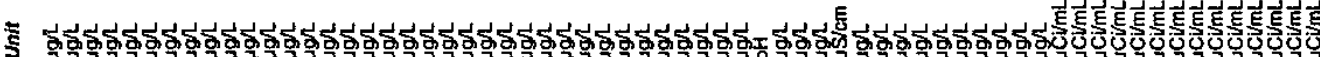

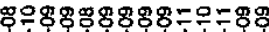
ఫ 誉

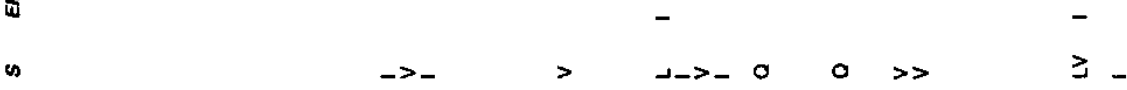

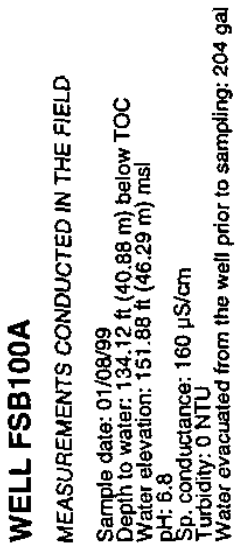

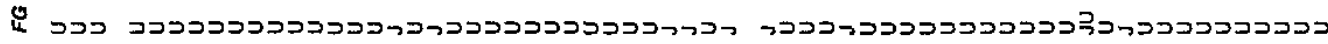

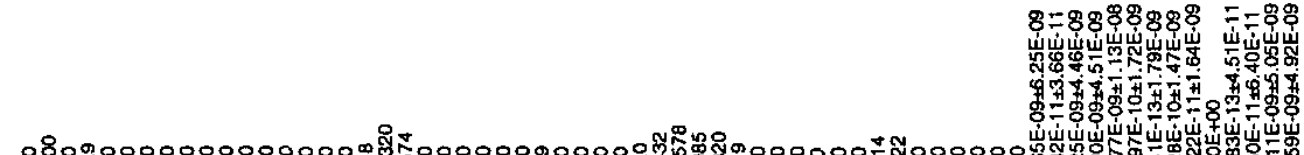
急

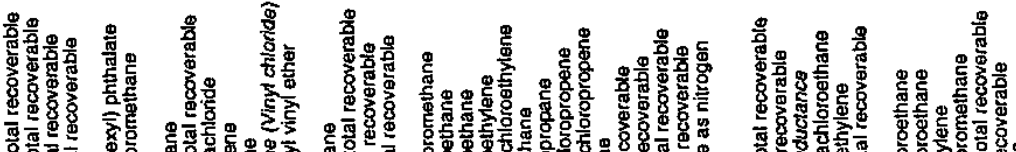

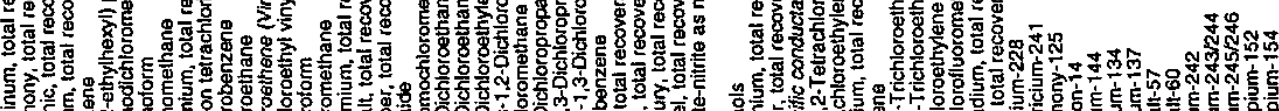
出 L 00000000000000000000000000000000000000000000000000000000000000000

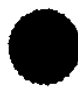




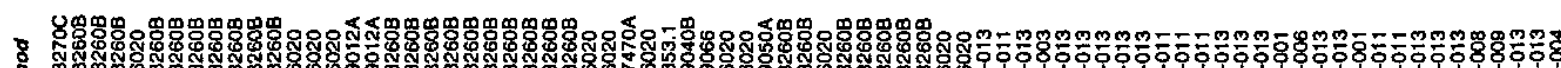

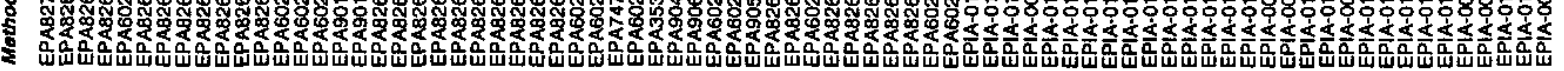

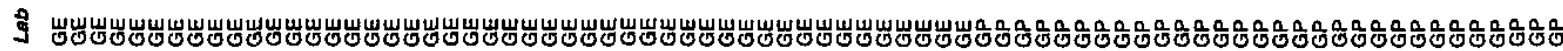

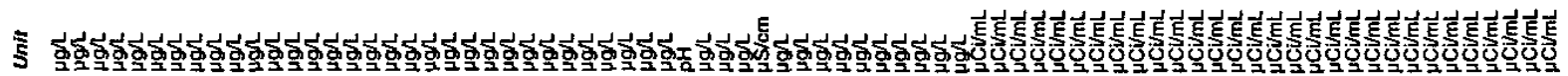

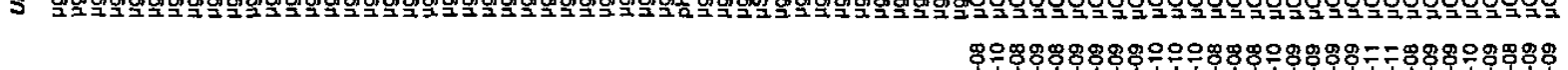

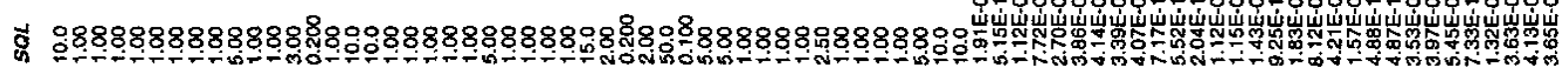
离

音

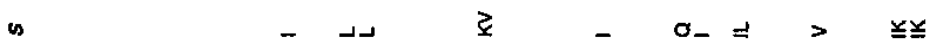

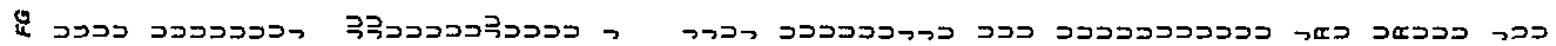

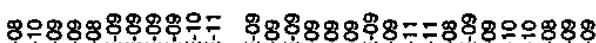

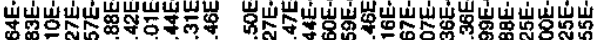

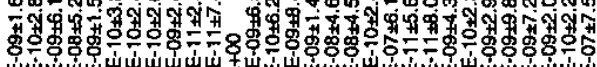

₹

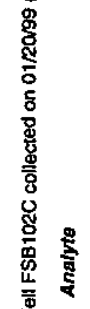

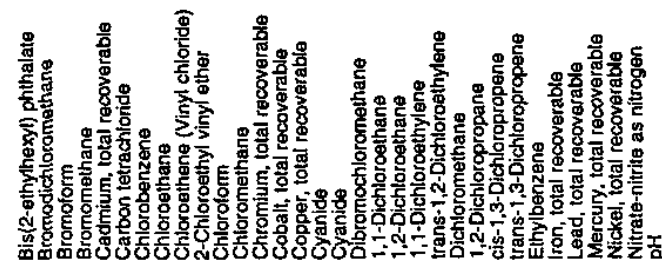

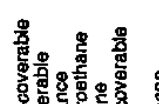

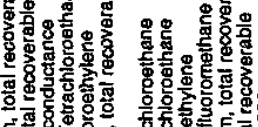

密

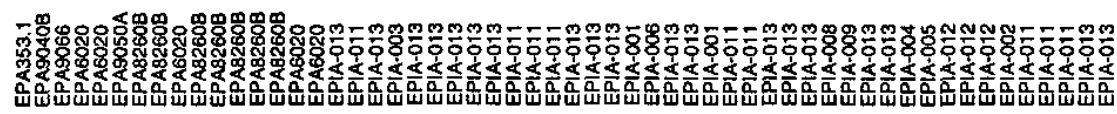

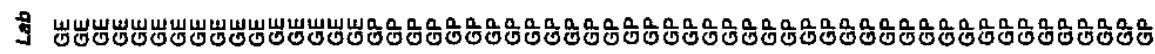

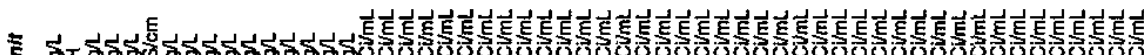

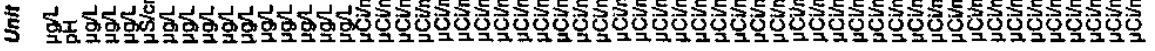

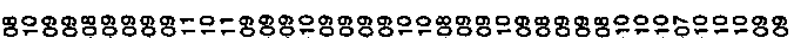

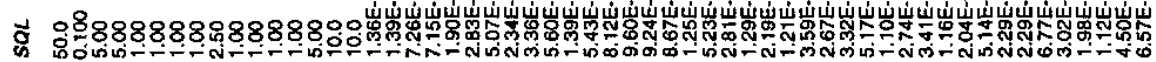

촌

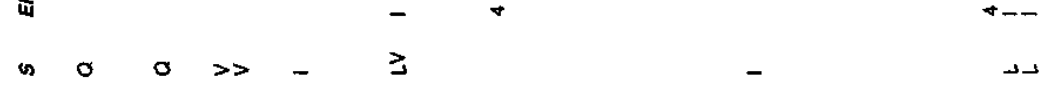

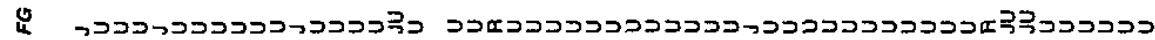

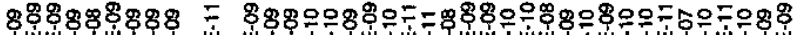

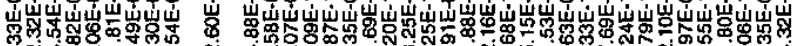

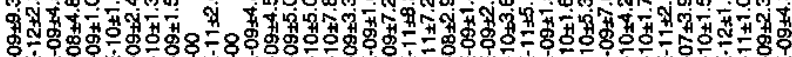
言

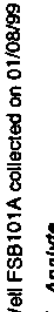
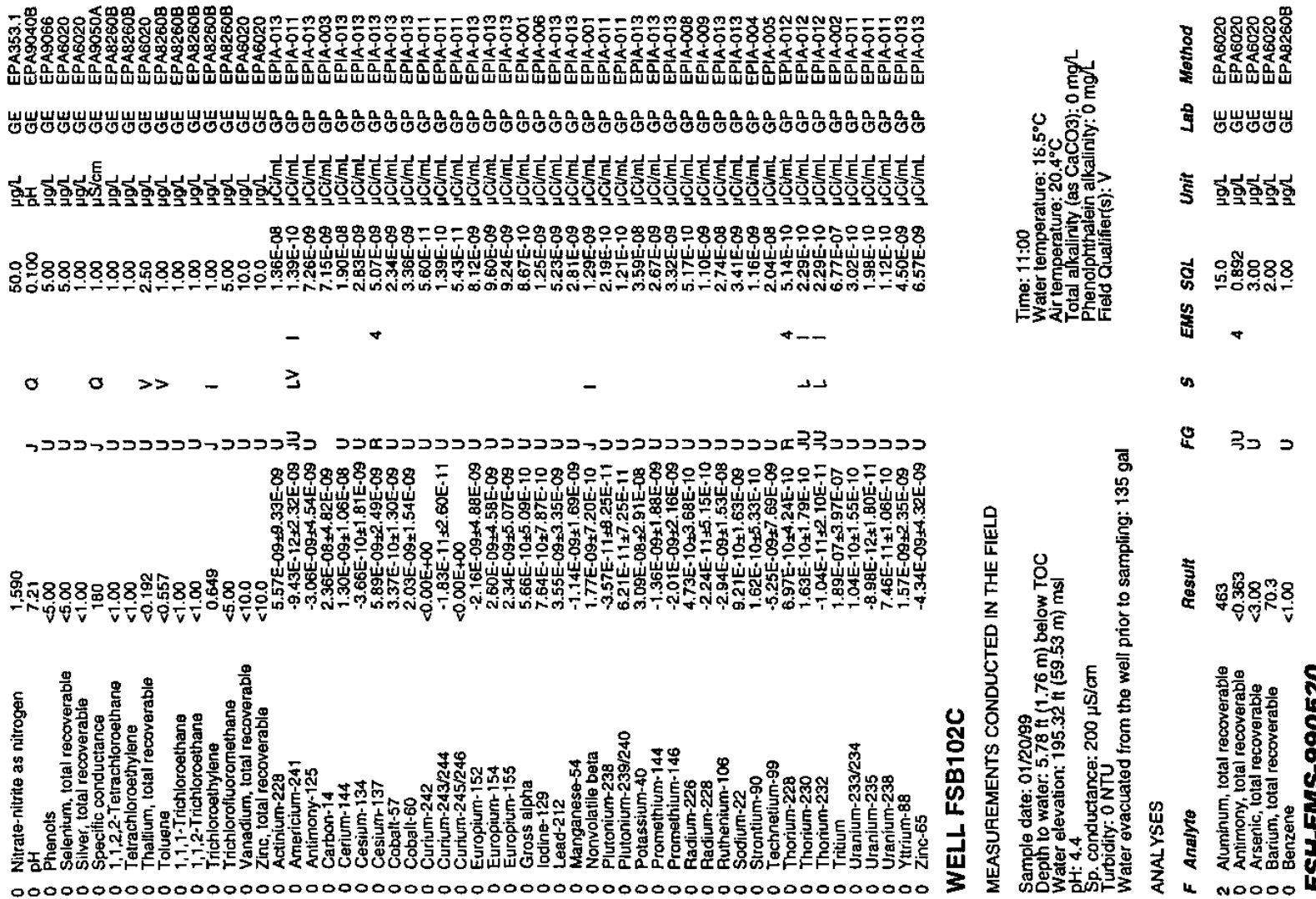

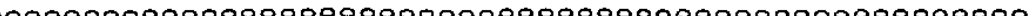




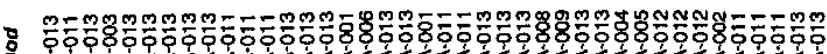
等

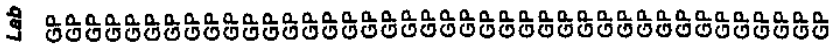

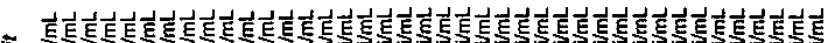

今

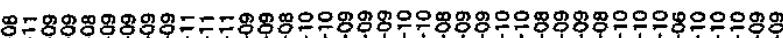

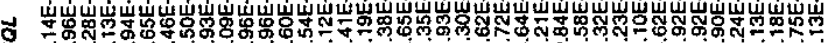

竞 $\checkmark+$

- +

a

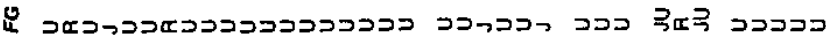

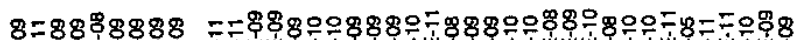

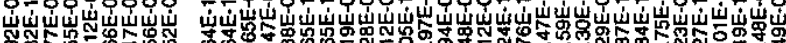

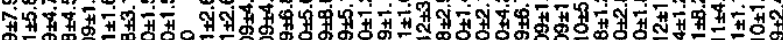

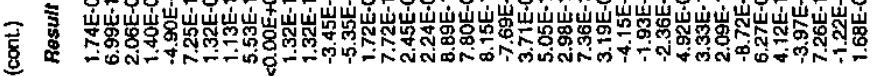
क

密

동

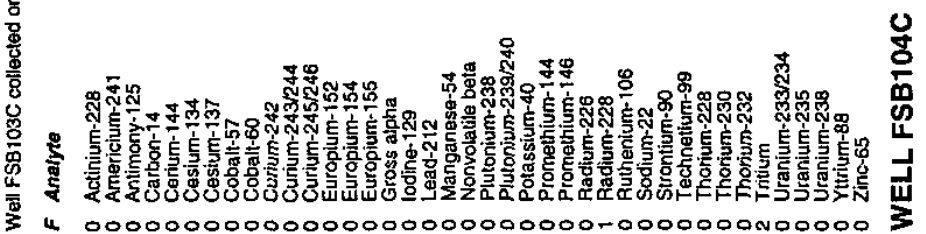

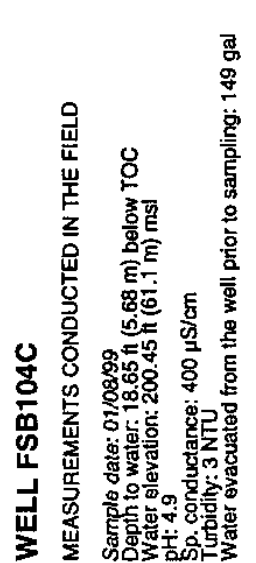

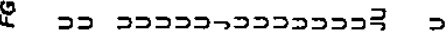

产

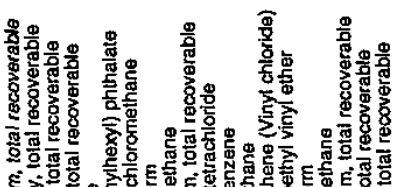

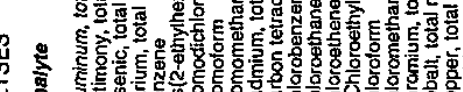

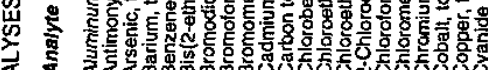

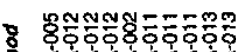
Iิ

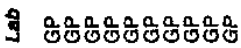

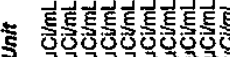

유유으우송으으으영요

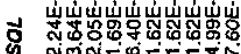

急

s

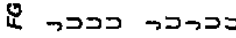

8 으 $=78$ 웅ㅇㅇㅇㅇㅛ

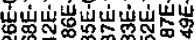

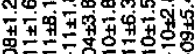

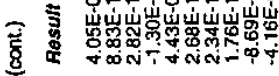

趂

.

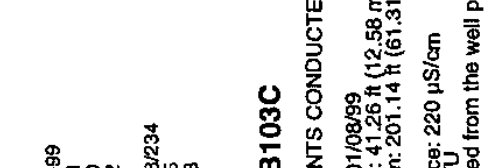

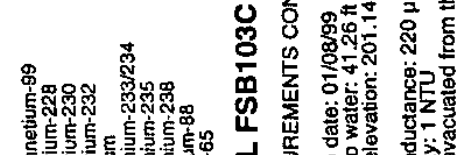
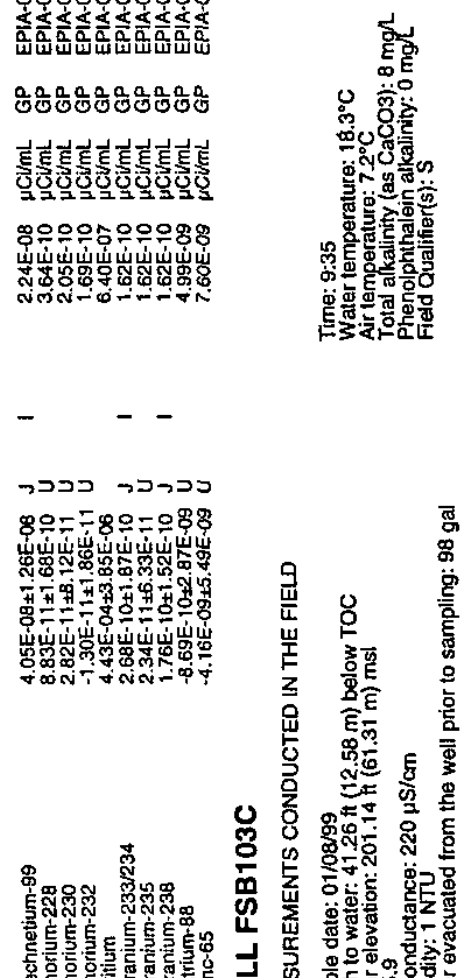

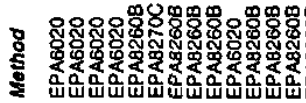

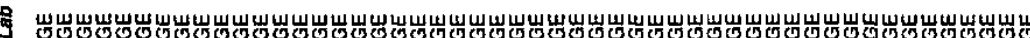

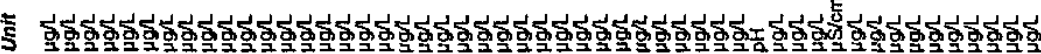

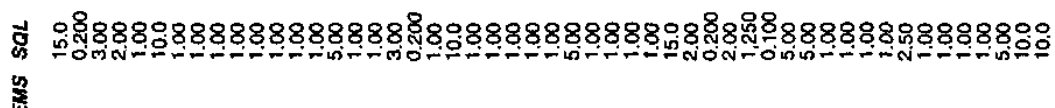

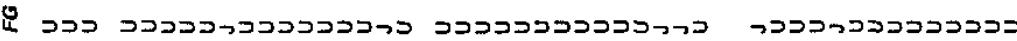

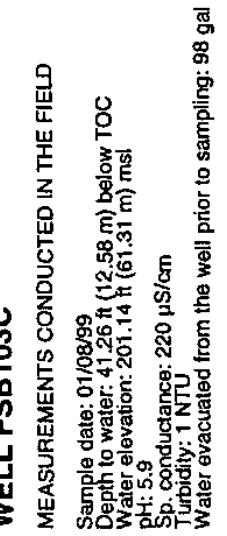

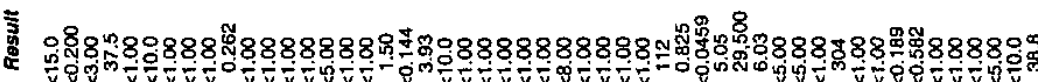

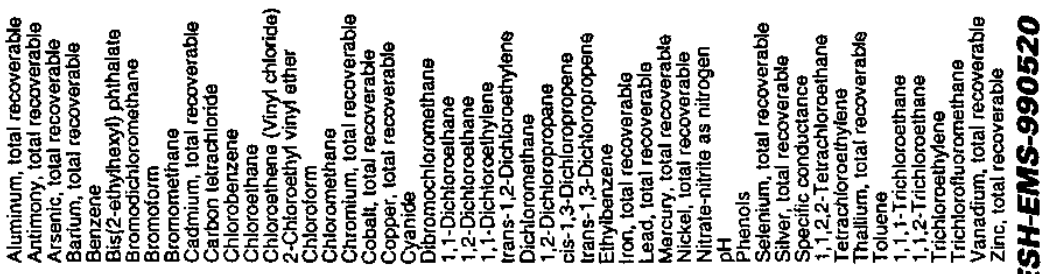

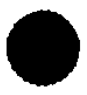

\& 


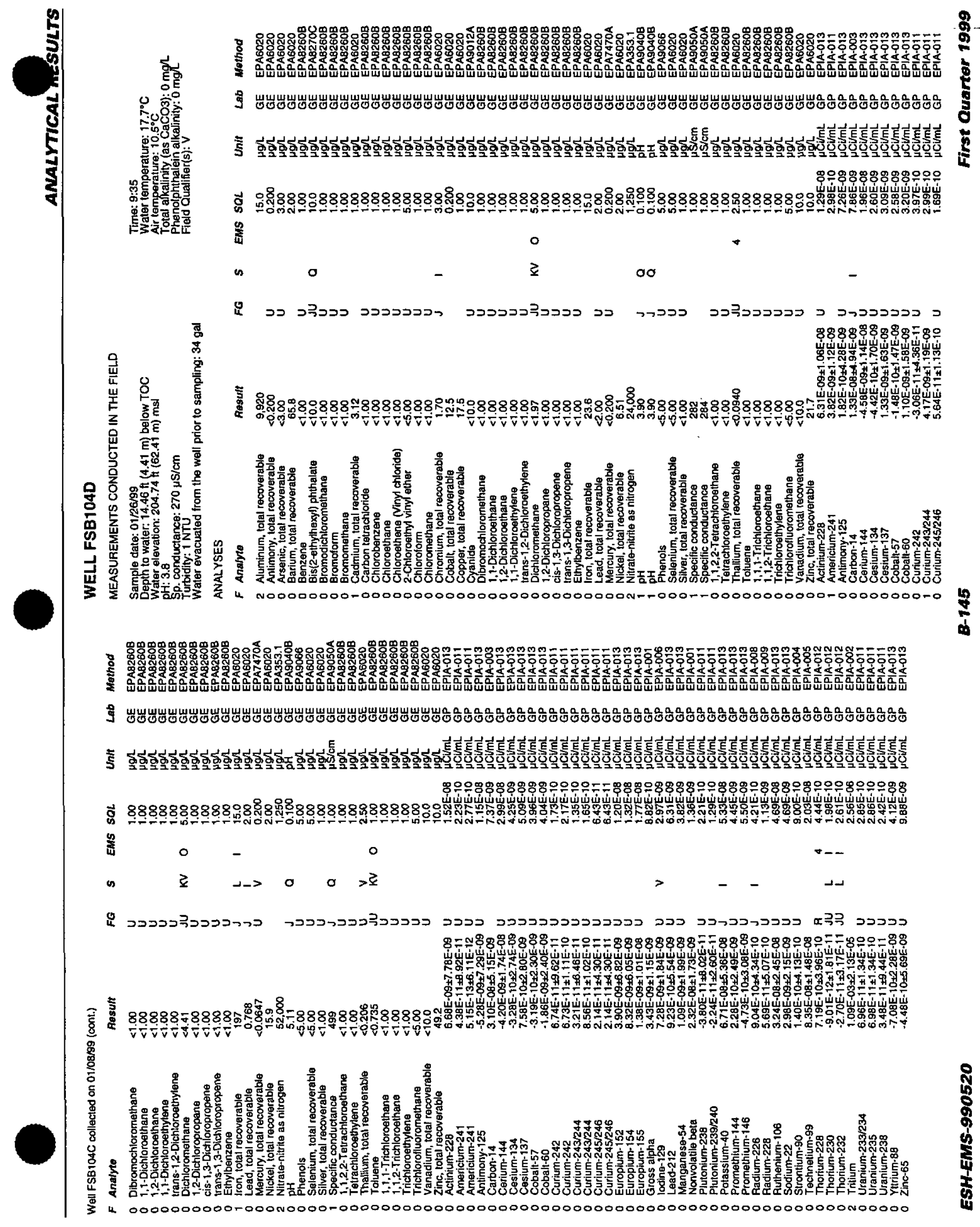




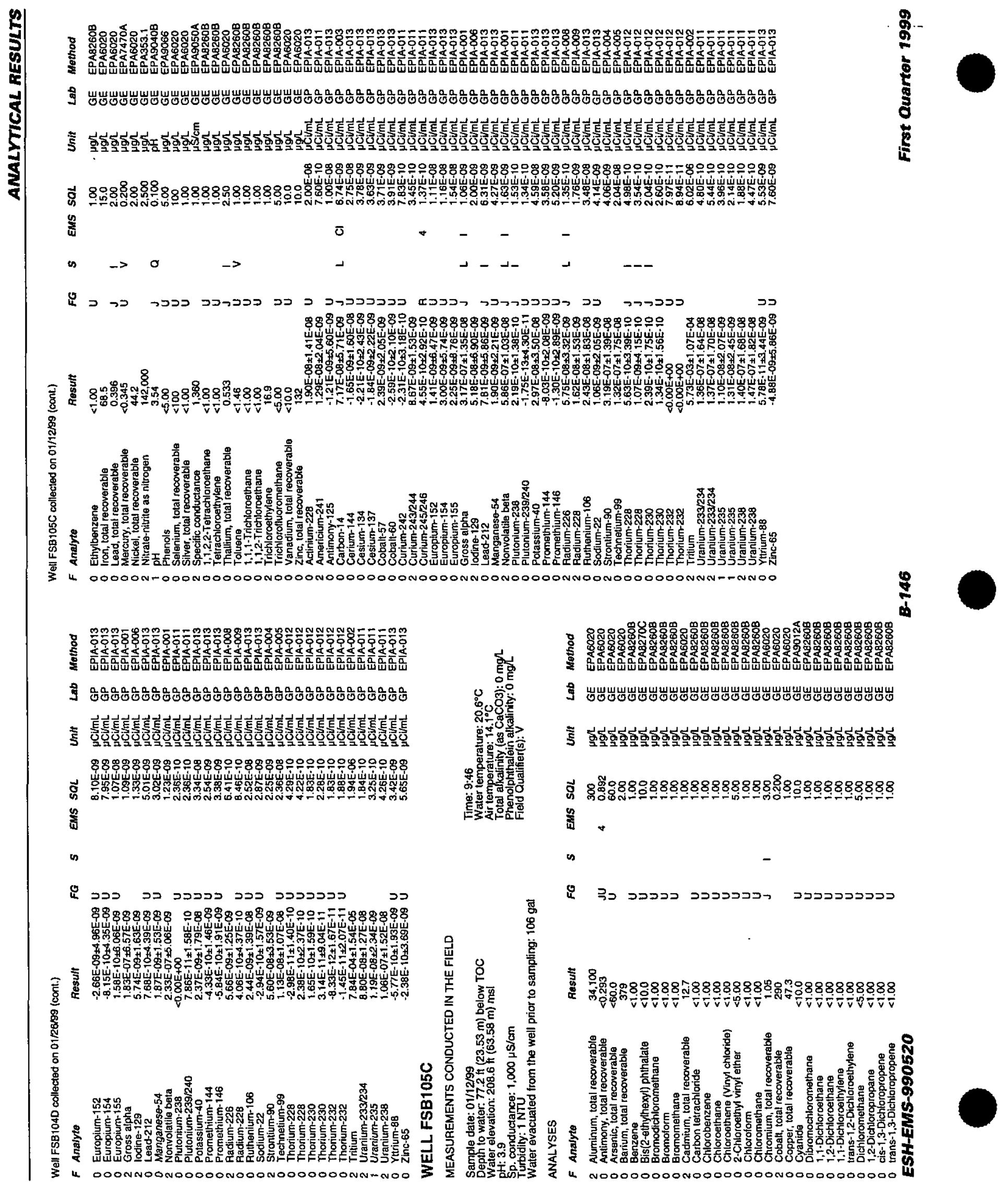




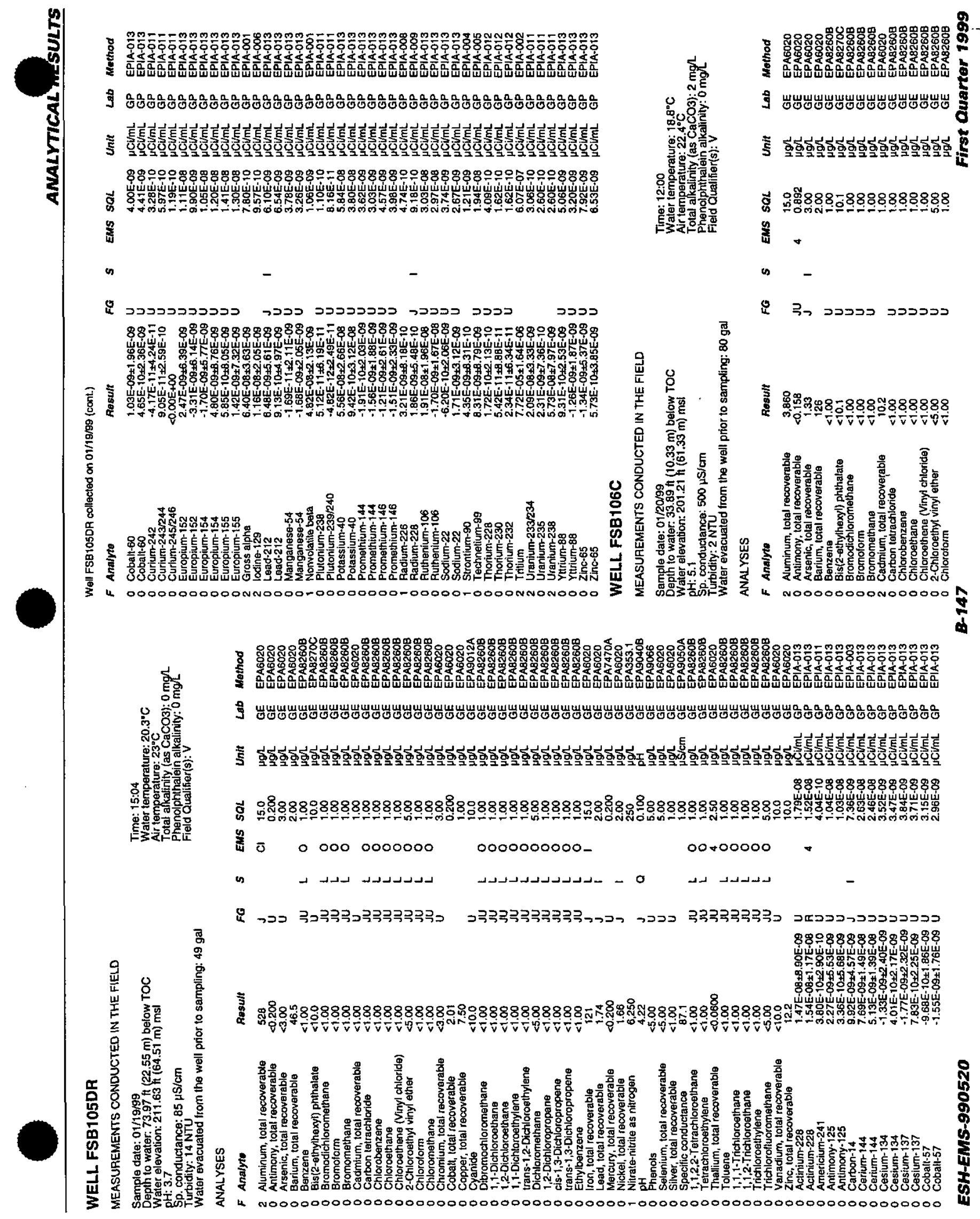


I

9

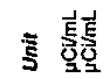

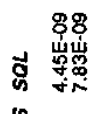

恙

$\infty$

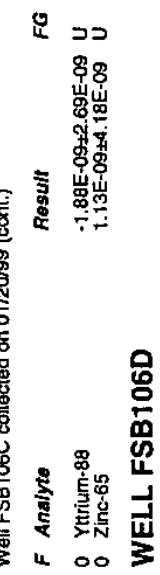

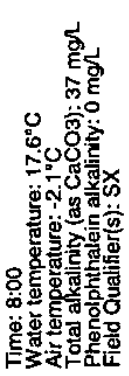

范

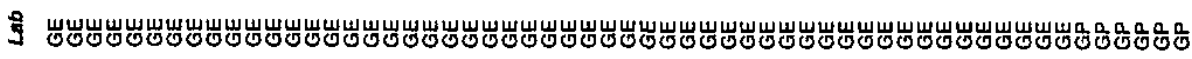

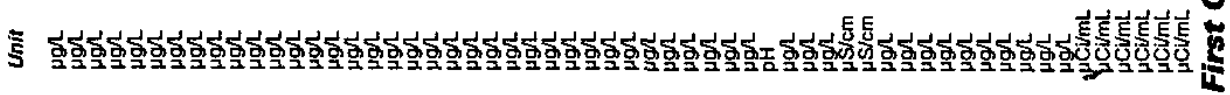

끙으영영용

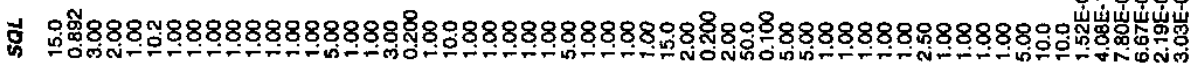

密

- 000000000000000000000

๘

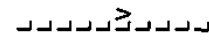

$-$

0000000

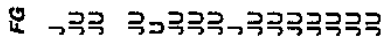

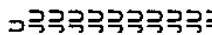

כ כמיכ

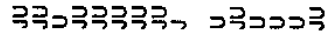

808898

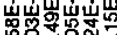

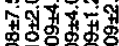

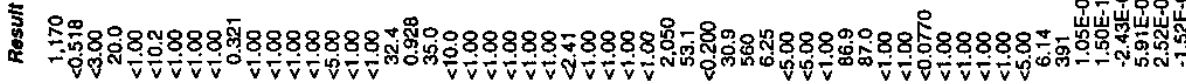

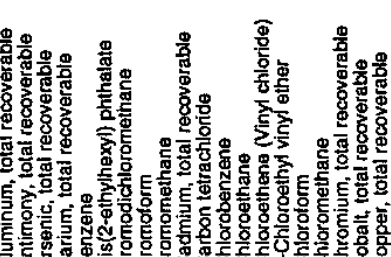

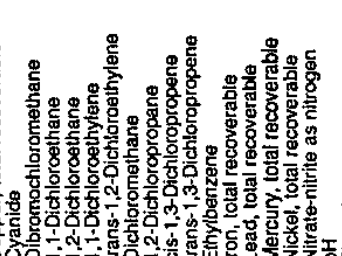
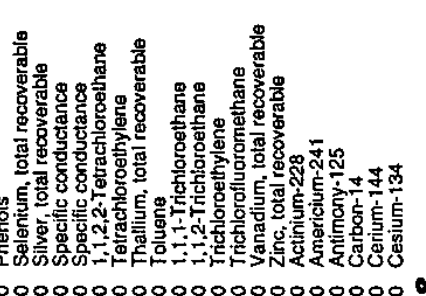

: 告

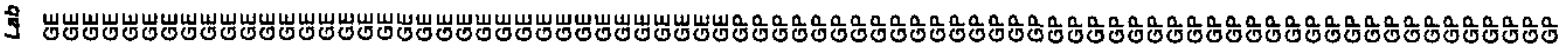

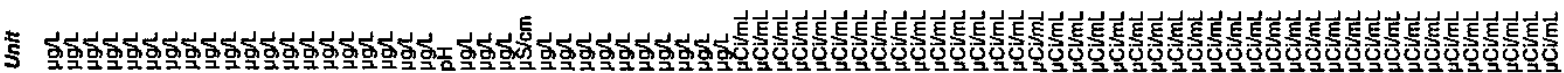

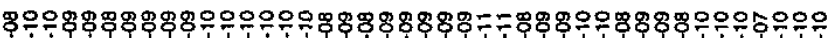

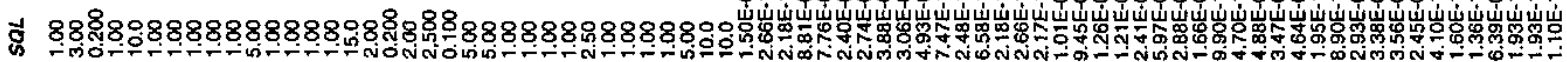
蛋

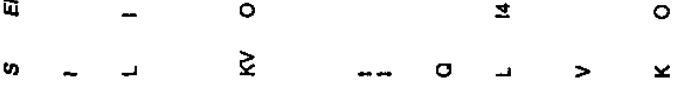

0

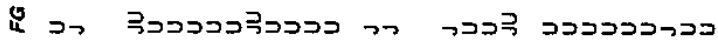

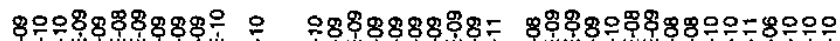

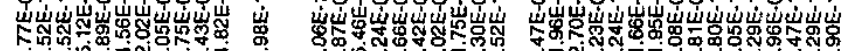

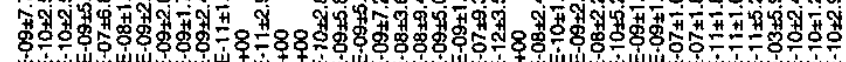

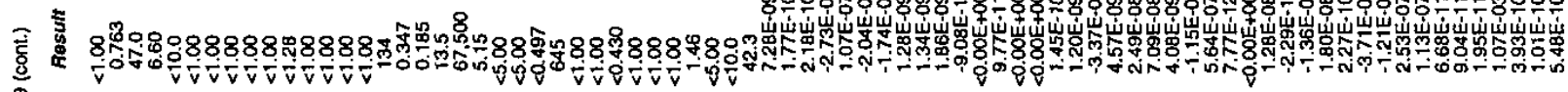

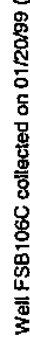
交

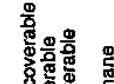
. 


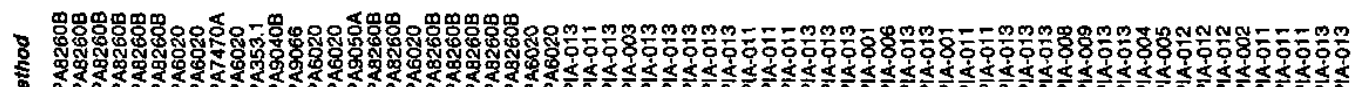

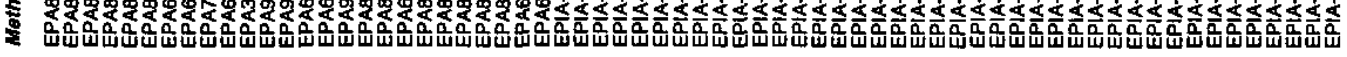

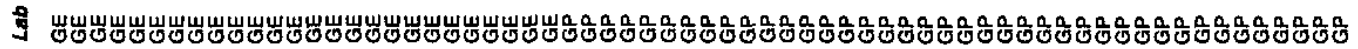

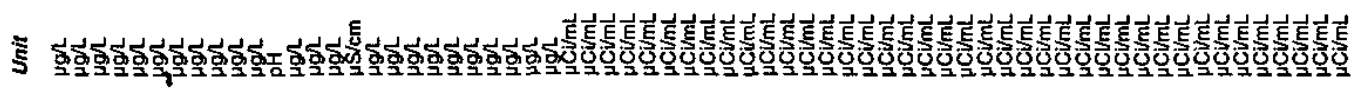

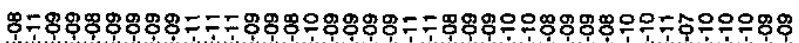

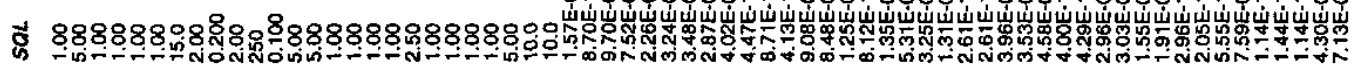

章

es $>\quad-\quad 0$

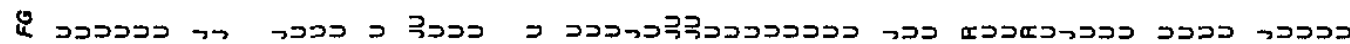

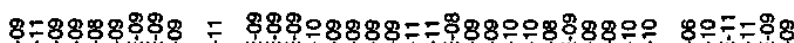

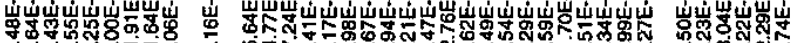

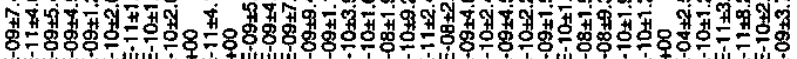

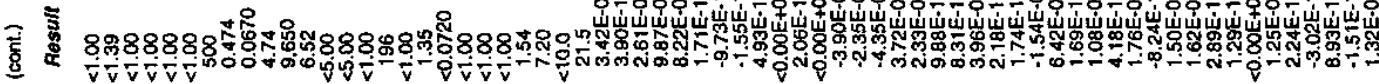

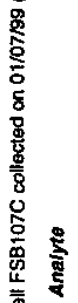
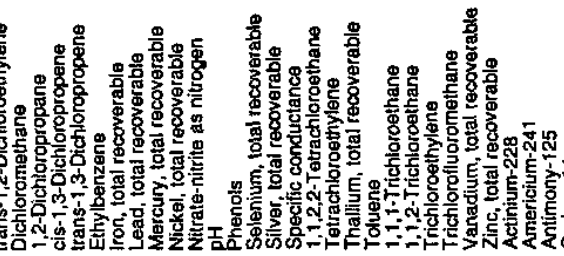

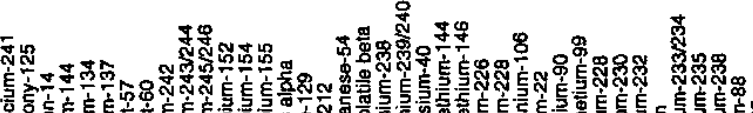

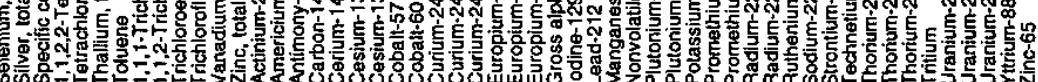

u 000000 N000-0000000000000000000000000000000N00-000000000N0000N00000

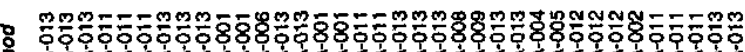

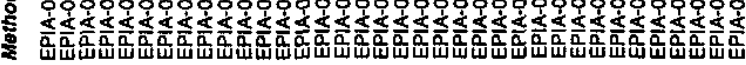

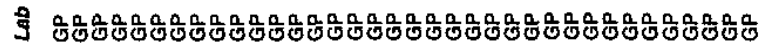

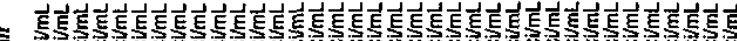

\$

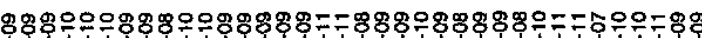

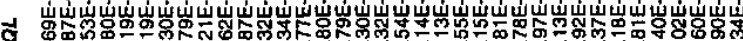

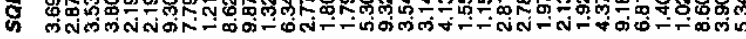

密

- 000 00 - 00 .

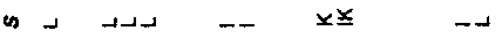

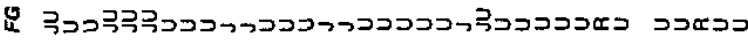

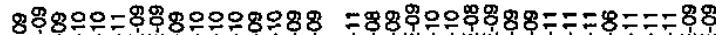

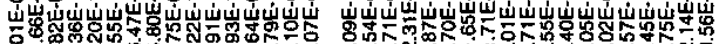

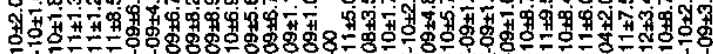

完芦

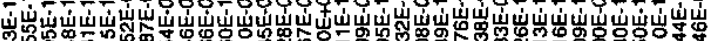

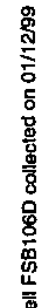

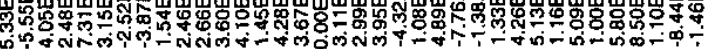

蓄

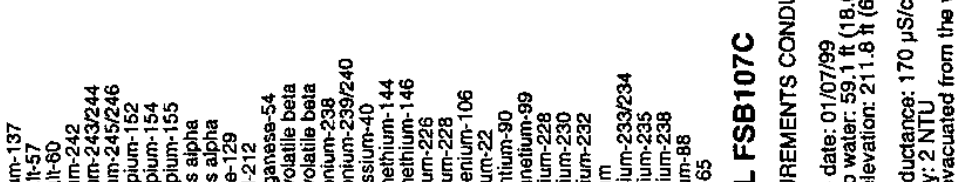

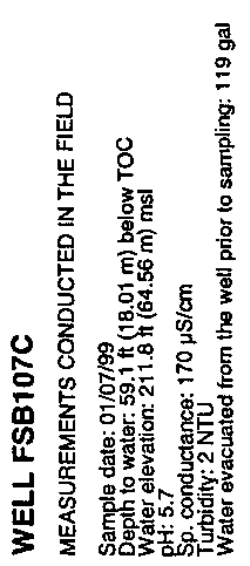

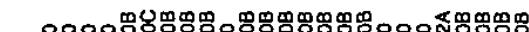
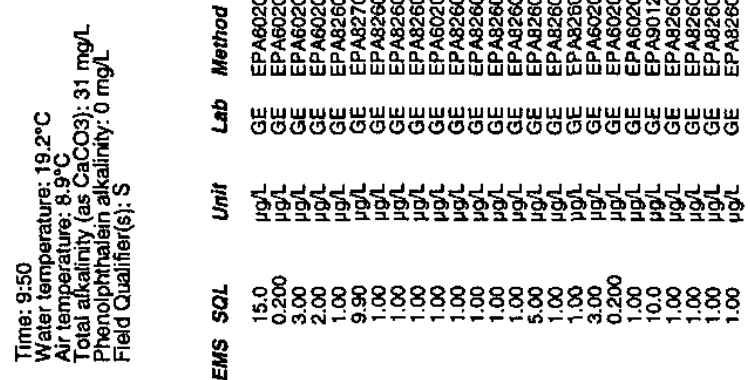

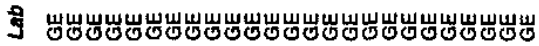

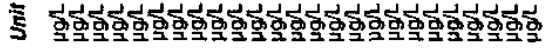

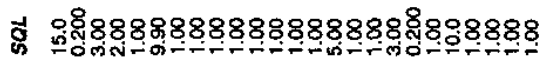

胥

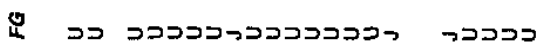

(1)

产

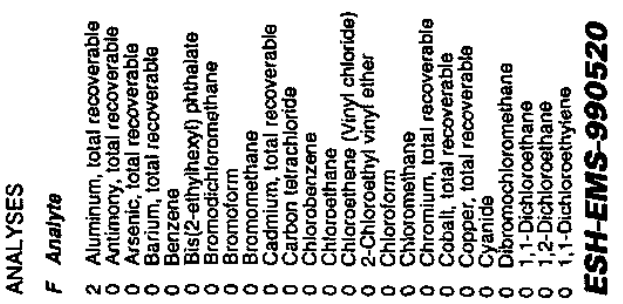




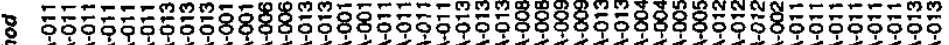

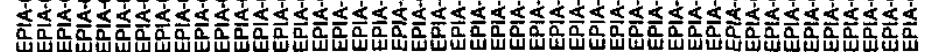

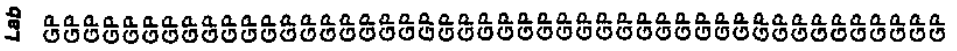

氜

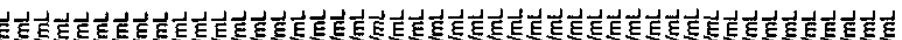

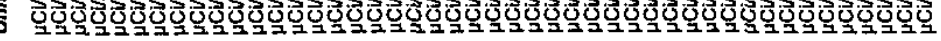

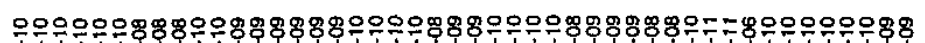

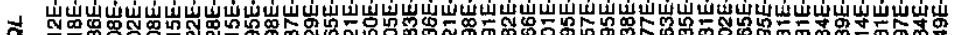

里

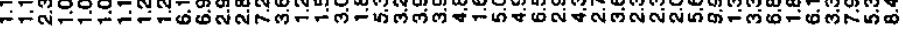

先

000

בט

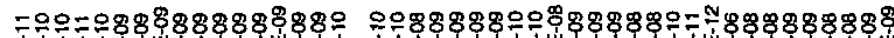

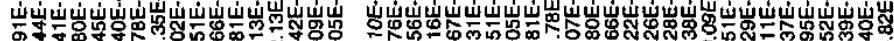

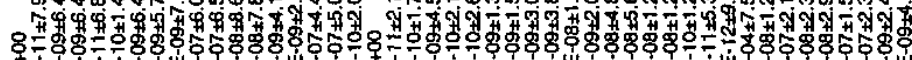

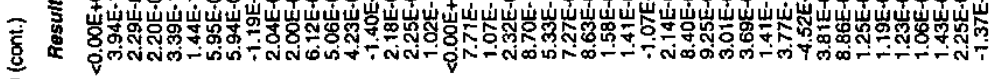
ชูํำ

密

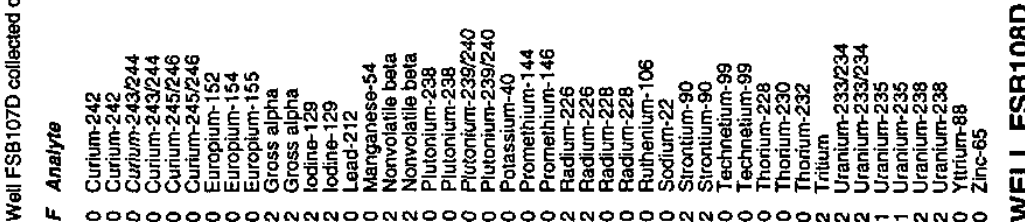

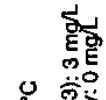

ज.

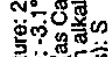

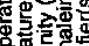

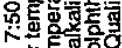

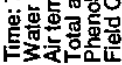

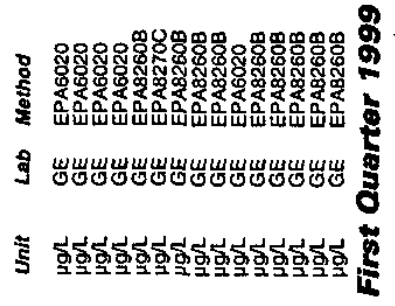

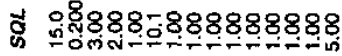

端 000000000

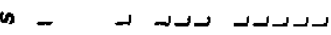

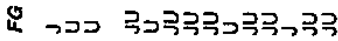

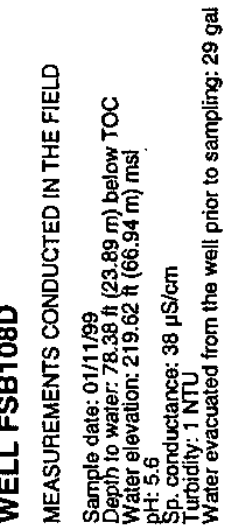

产 象8

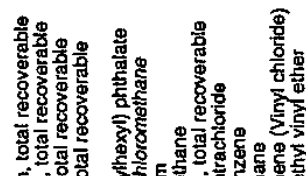

รू.

:

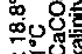

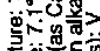

근

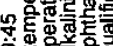
更

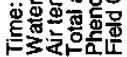

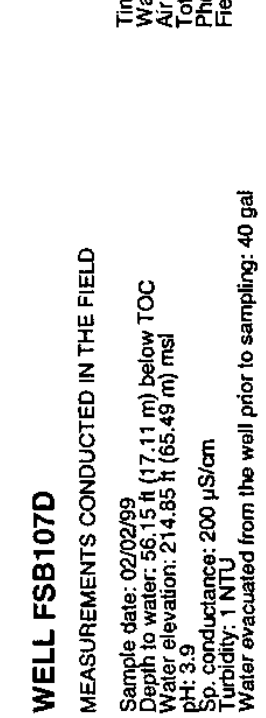

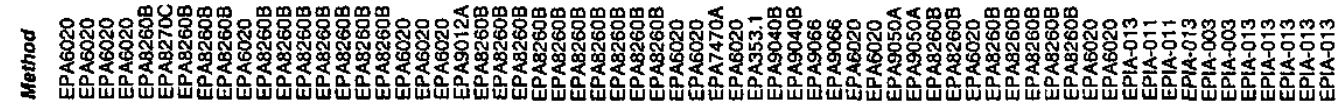

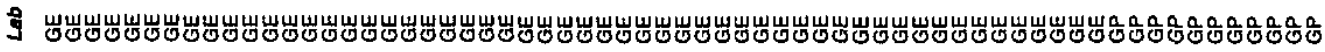

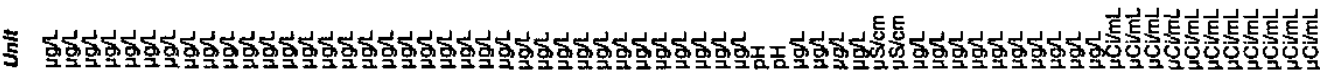

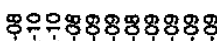

†

$00000000000 \quad 0000000000 \quad$ \% 0000000

is

ㄴ 尔

( $888888 \%$

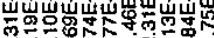

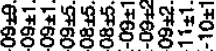

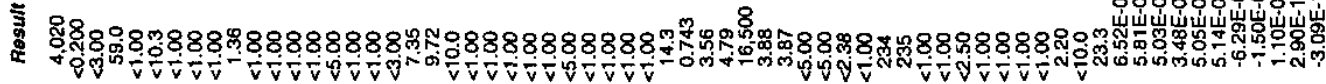
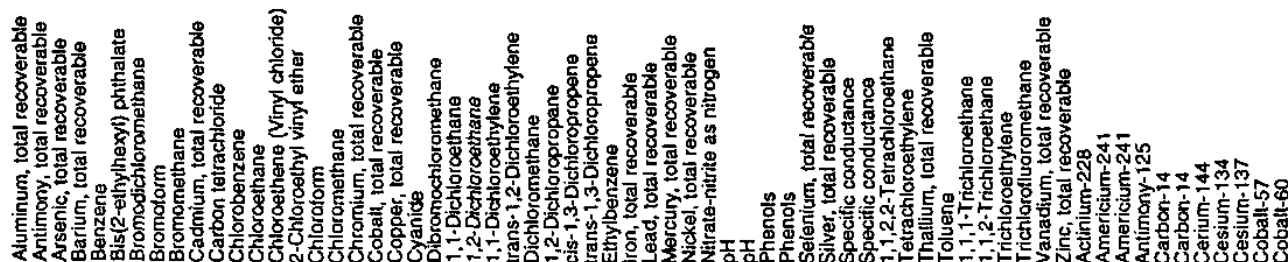


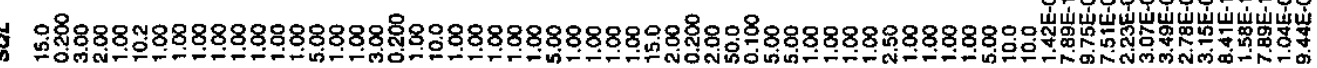
蛋

0 0000000000

0000000000

$00 \$ 00000$

$\infty$

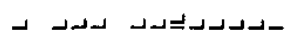

ג

o

دردנخ

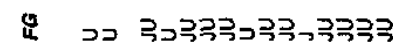

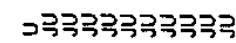

$\supset$

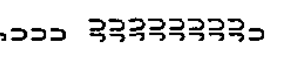

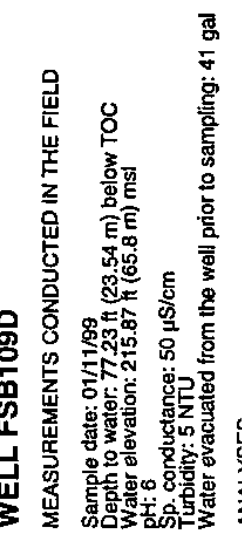

窟

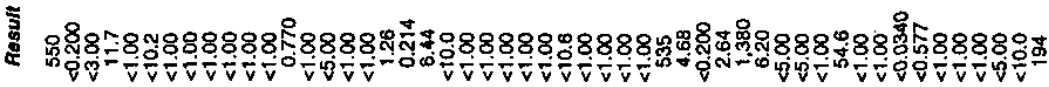

$8=8 \% 888 \%=\div=88$

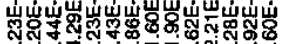

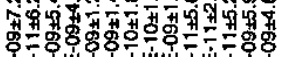

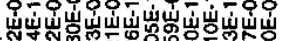

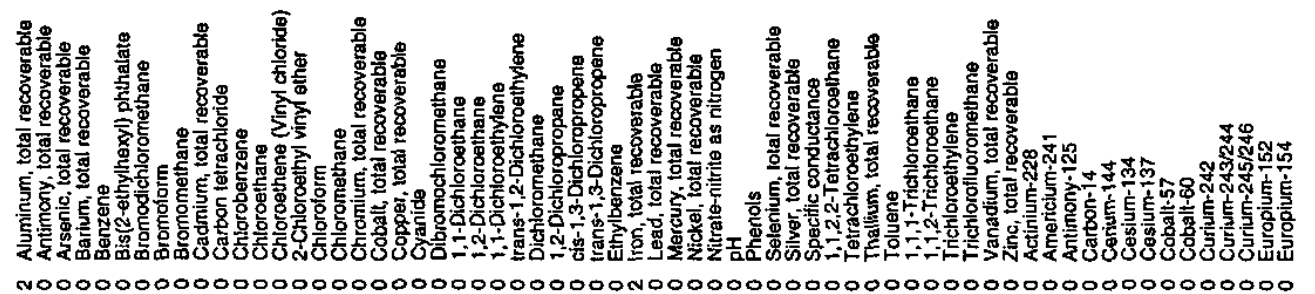

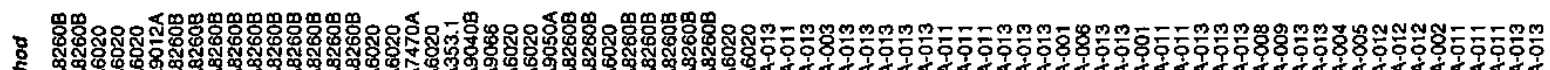
等

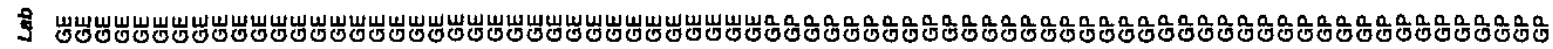

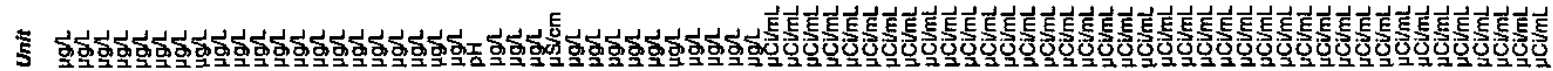

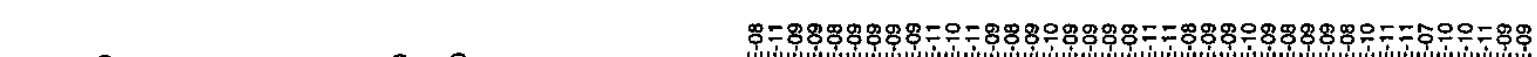

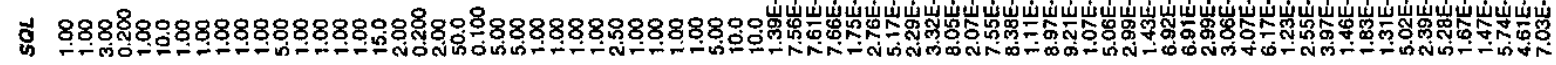

产

W $00000000000 \quad 00000000$

Q

$+$

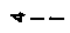

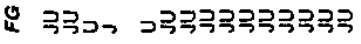

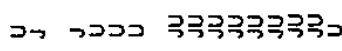

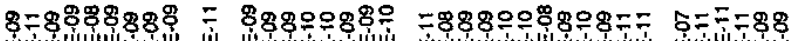

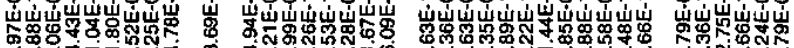

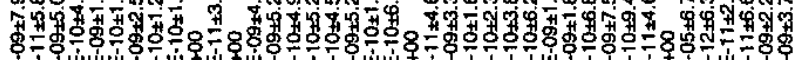

言 888 \%

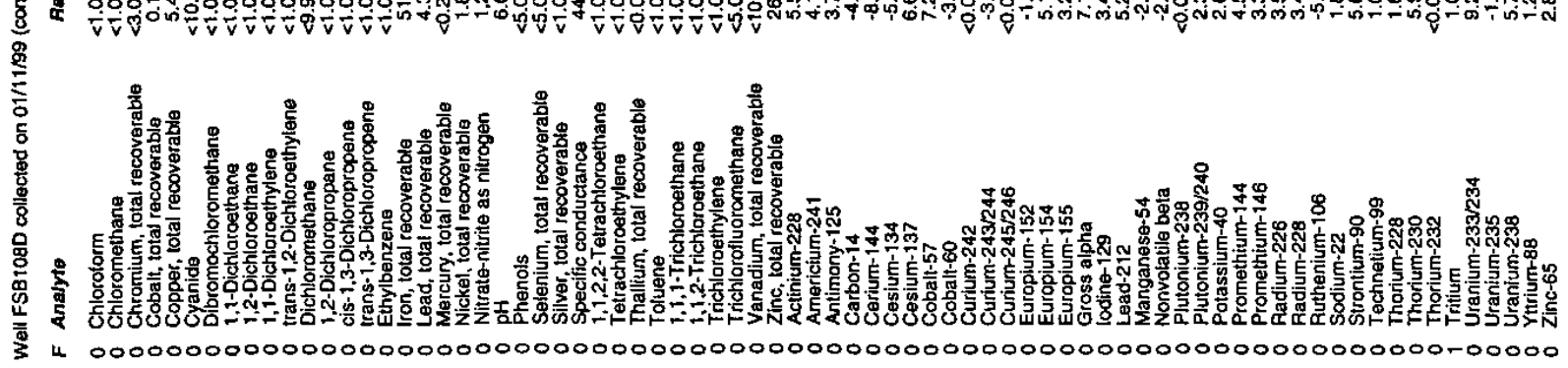




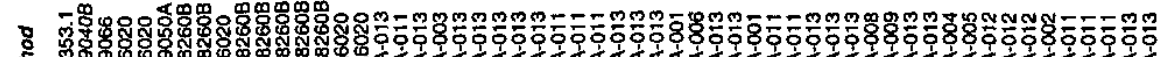
亳

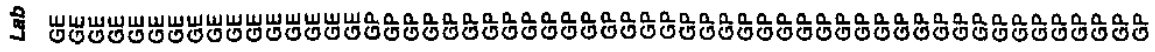

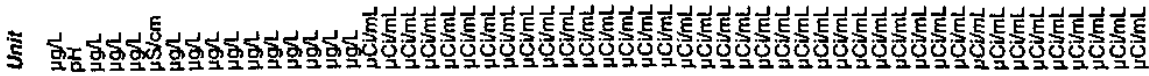

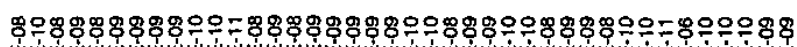

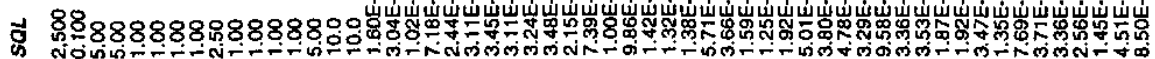

竞

a $00>$

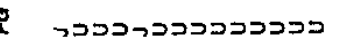

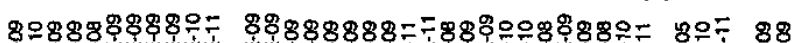

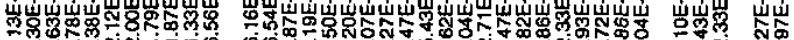
94

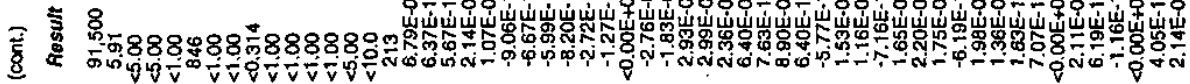
올

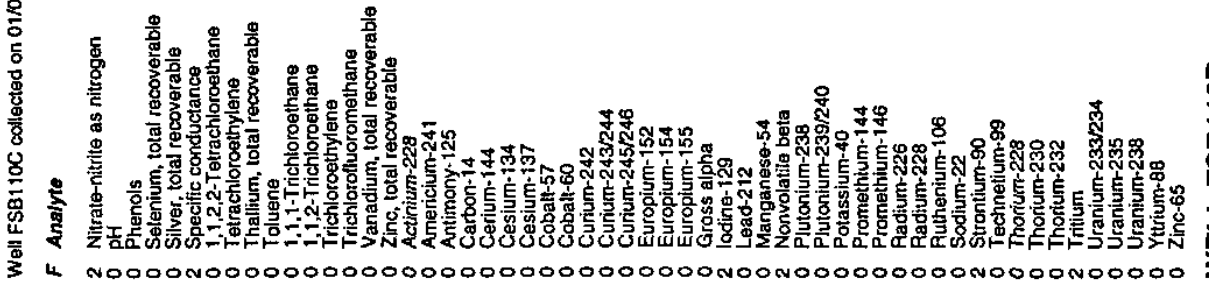

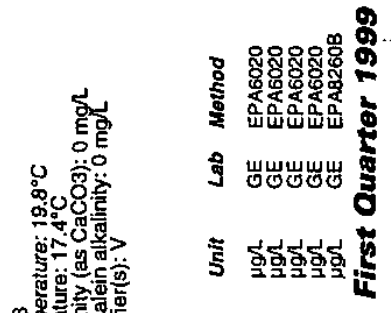

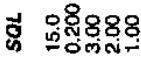

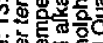

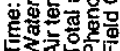

0

\& כ כ כ

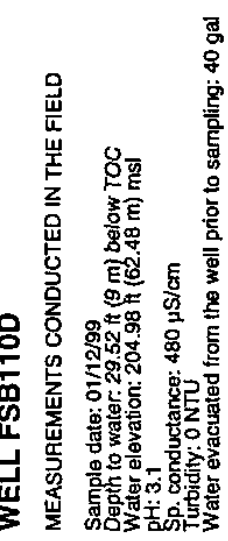

芦

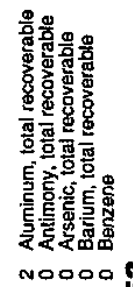

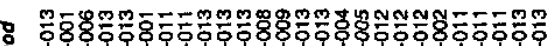

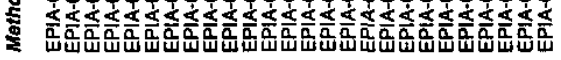

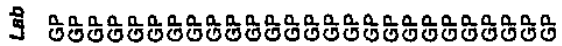

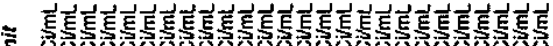

ริ

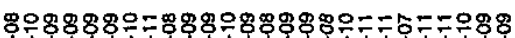

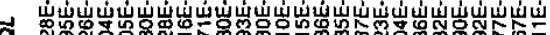

क्ष

㮍

$\nabla-$

8

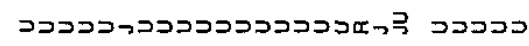

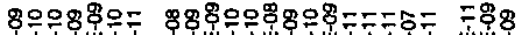

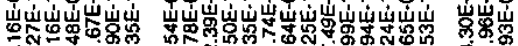

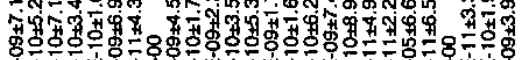

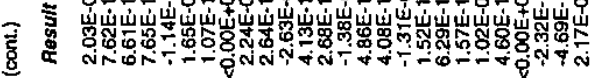

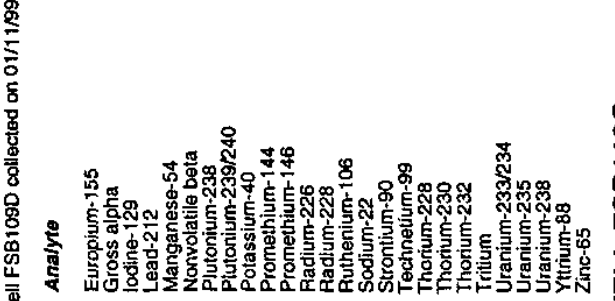

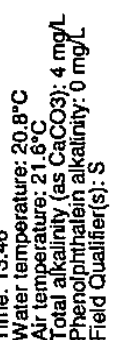

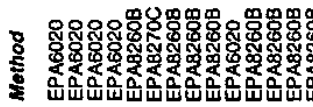

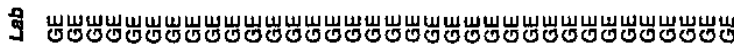

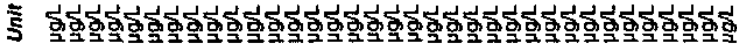

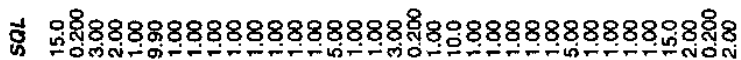

章

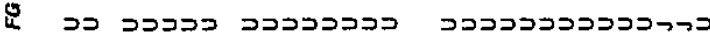

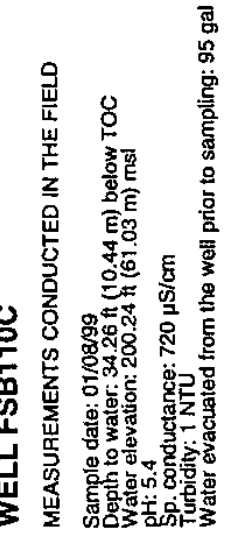

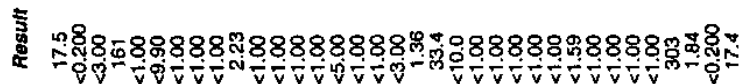

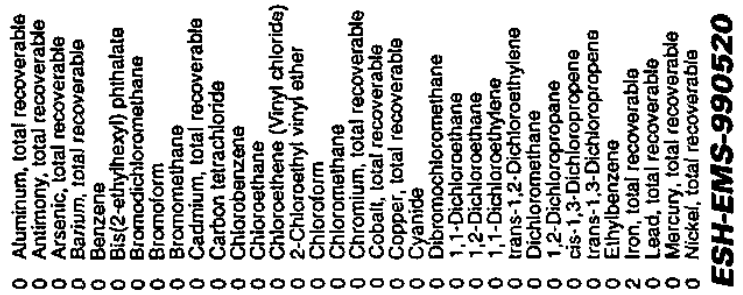




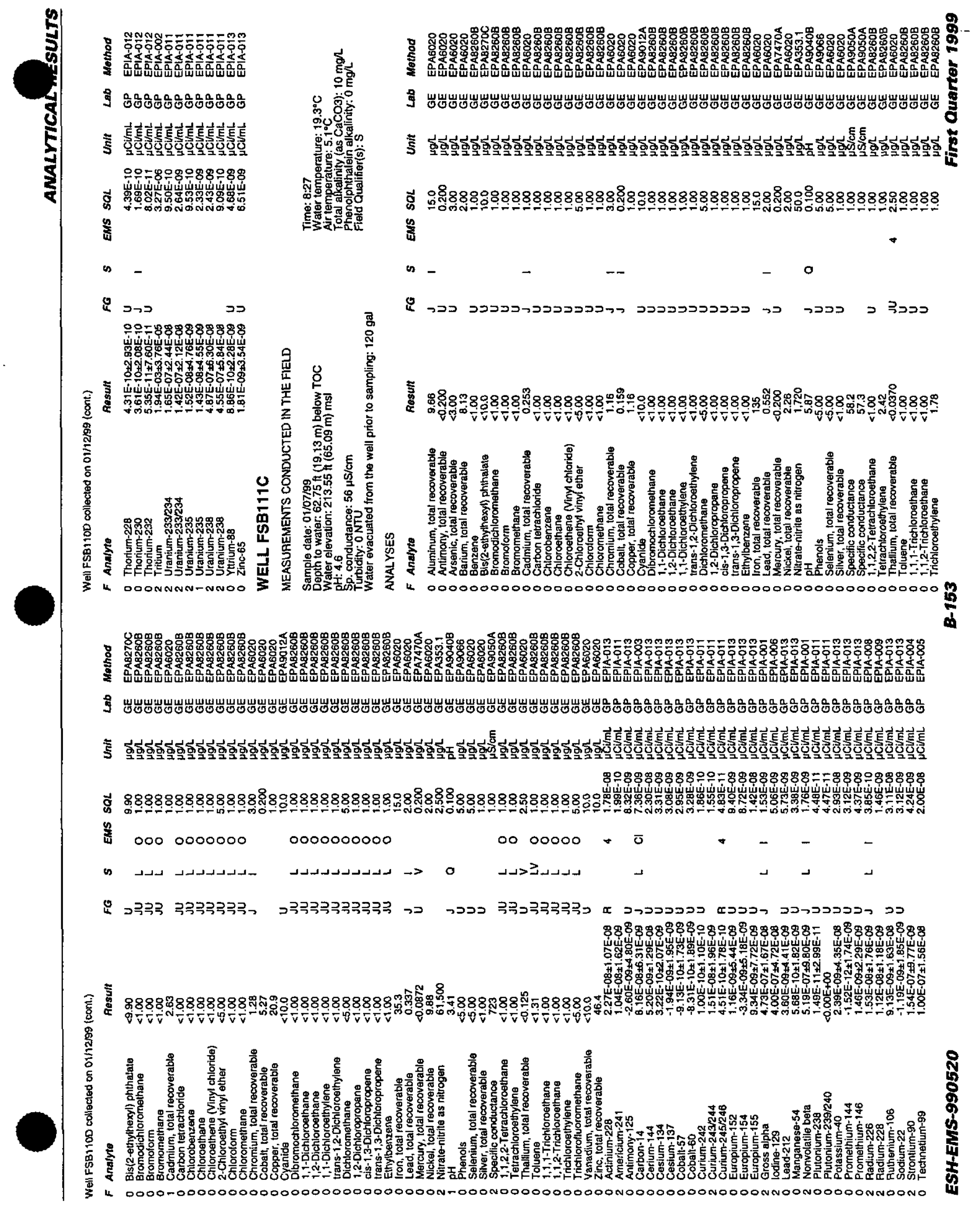


ริำ

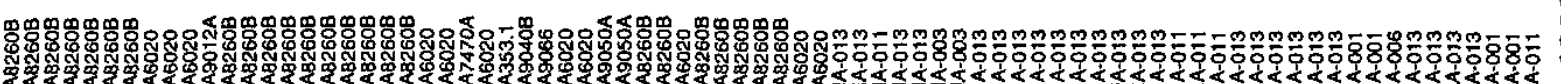
S.

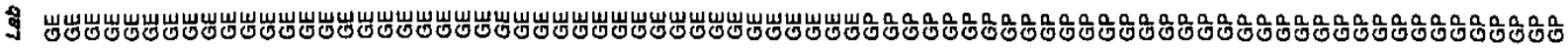

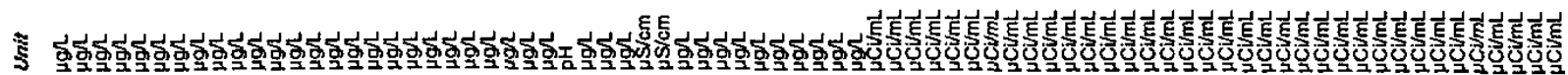

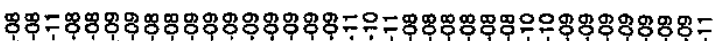

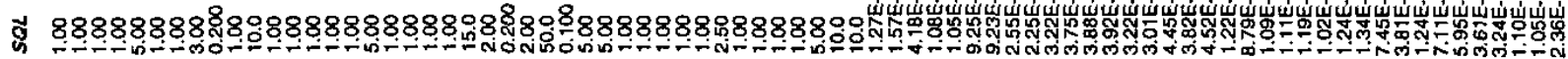

紊

(1)

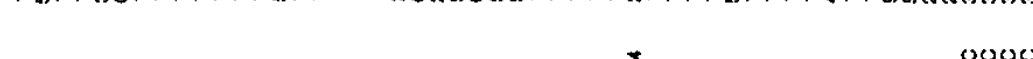

\&

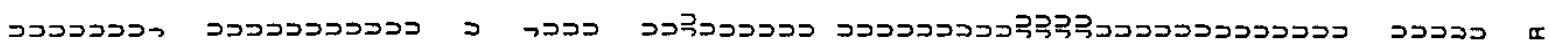

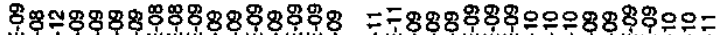

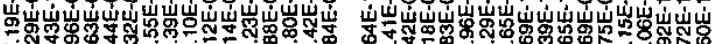

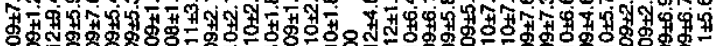

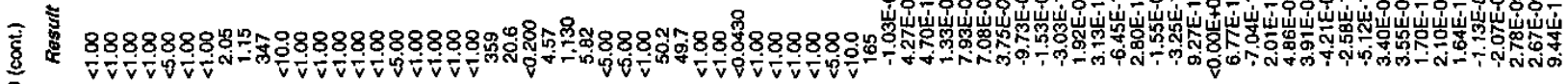

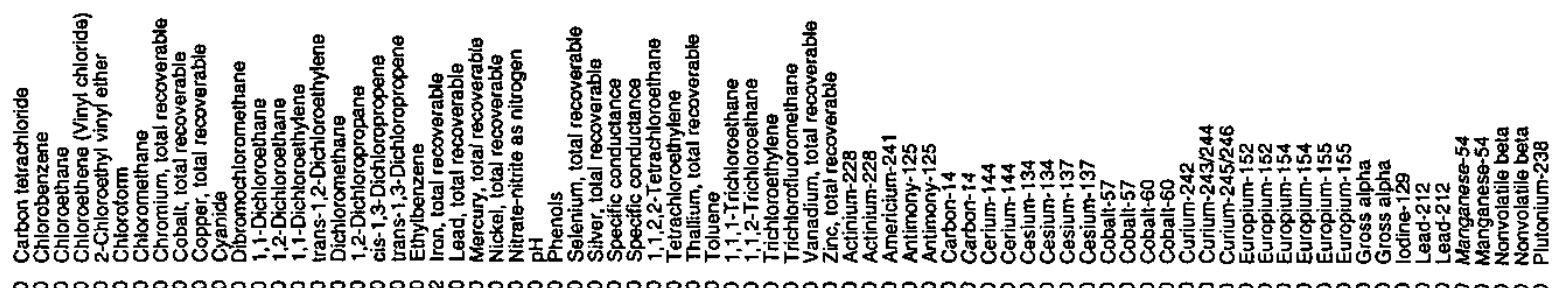

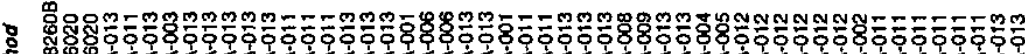
至

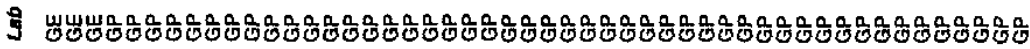

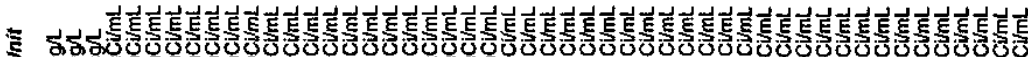

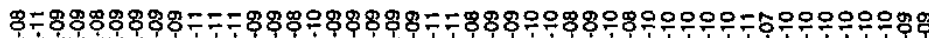

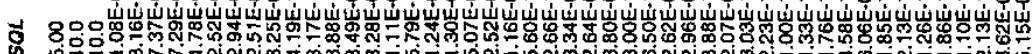

章

00

is

oo

$+$
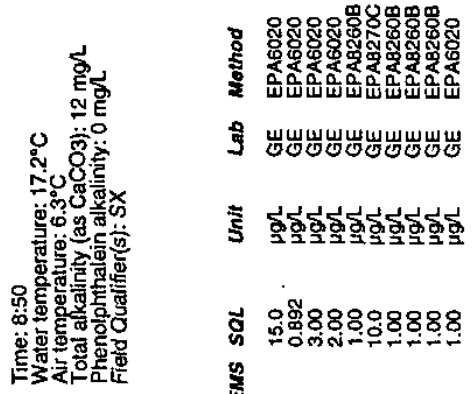

[ו

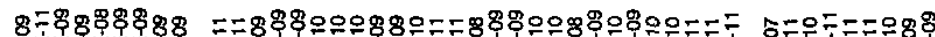

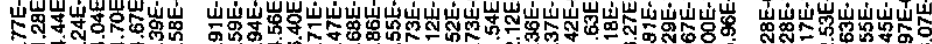

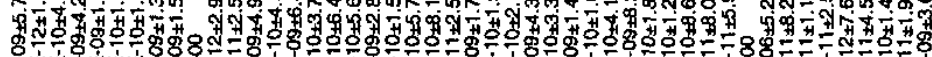

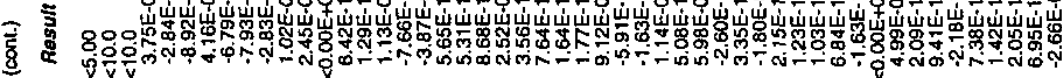

为

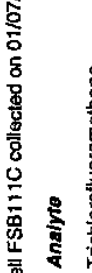

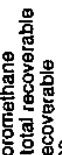

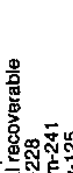

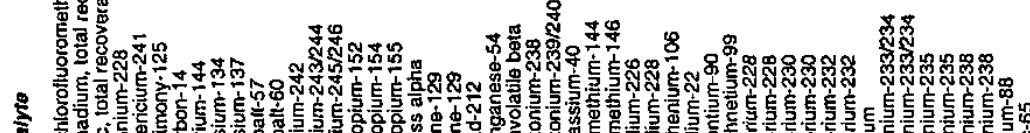

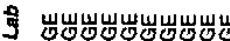

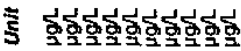

ตี 요유ำ

离.

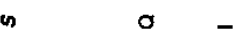

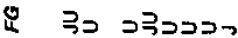

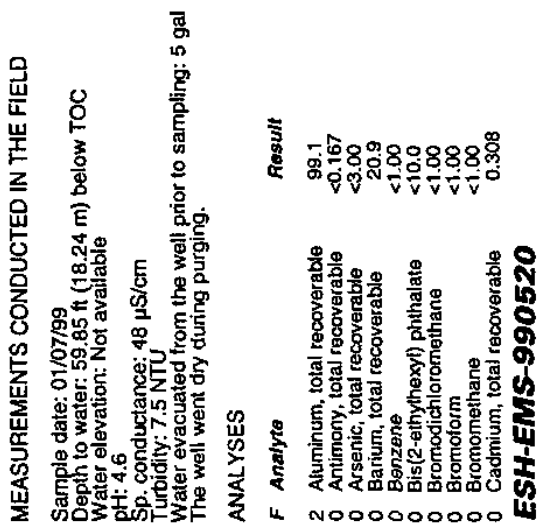




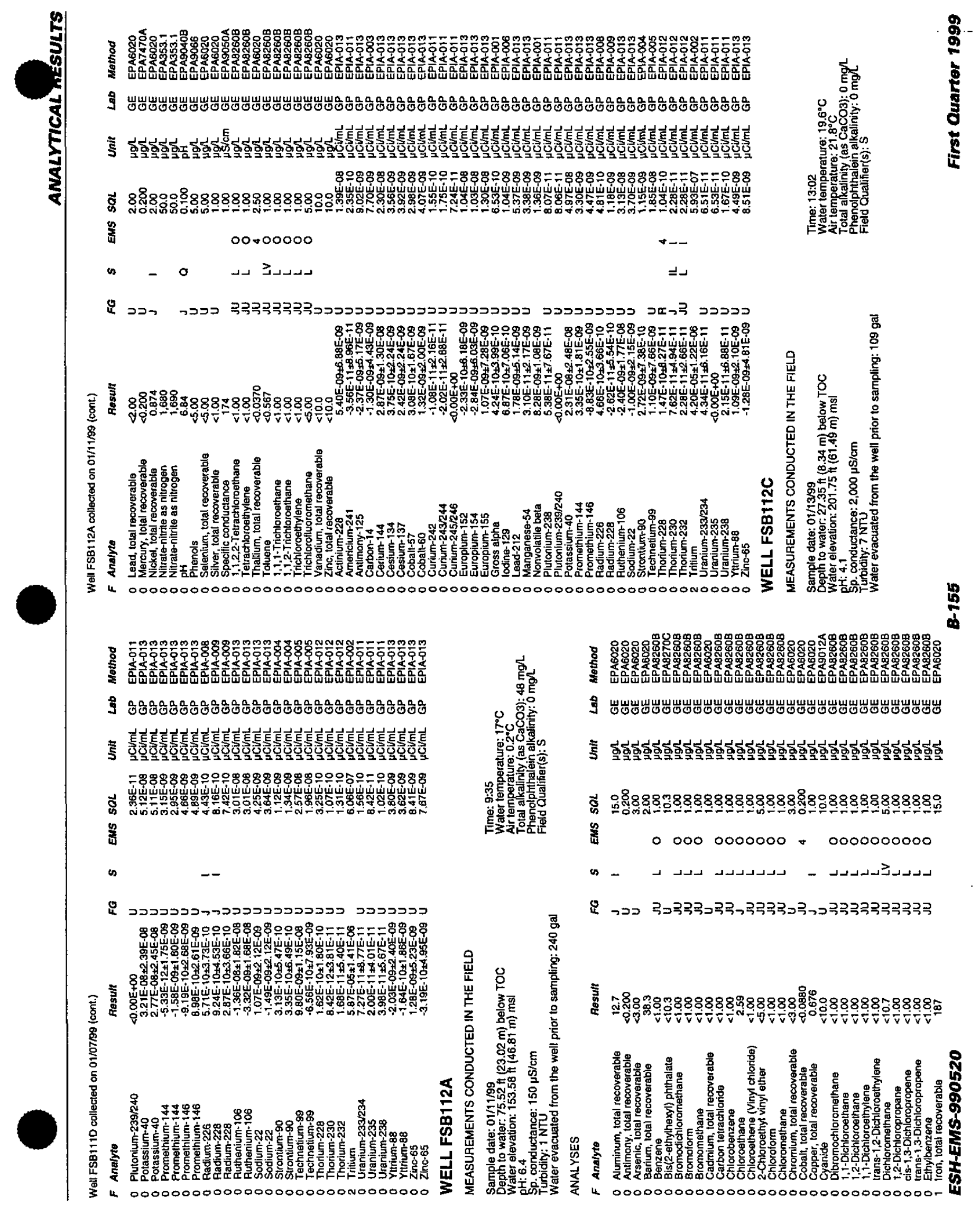




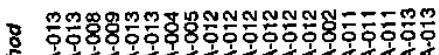

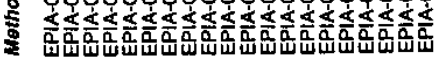

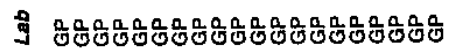

:

今ํ.

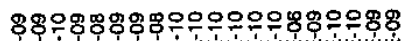

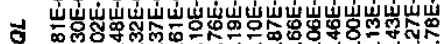

要

-

$n$

落

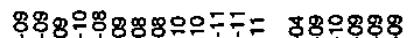

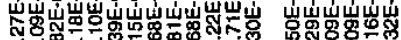

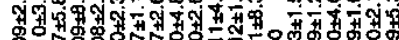

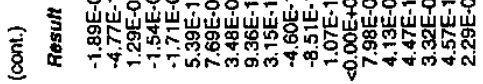

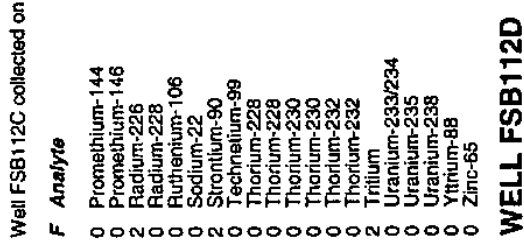

万ू్ 范

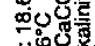

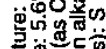

룬

为产

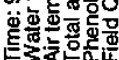

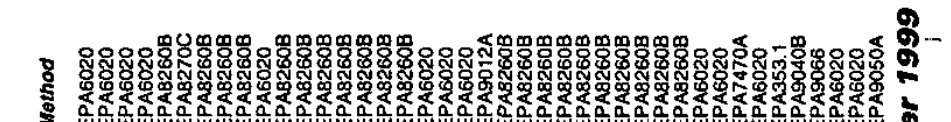

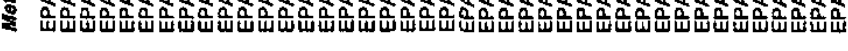

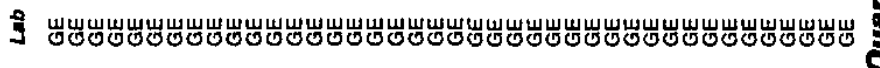

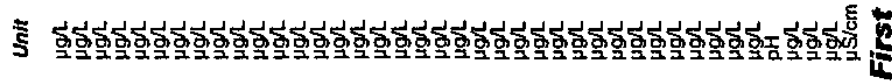

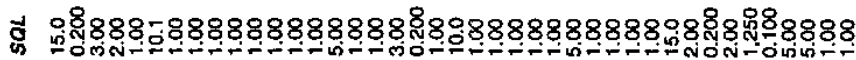
竞

$-120$

as

z

o -

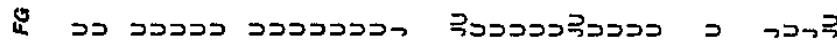

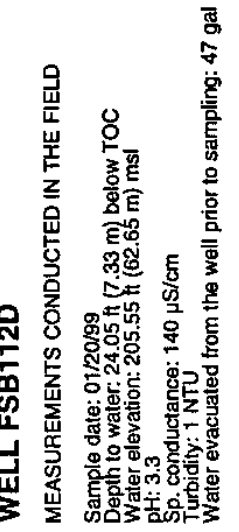

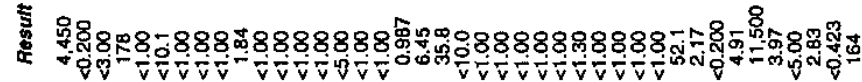

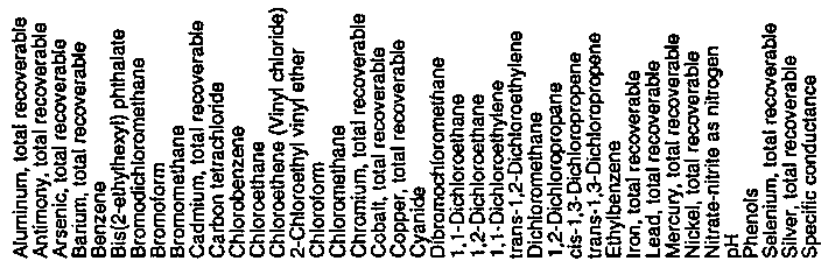

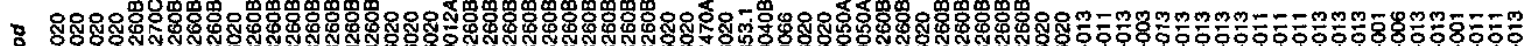
等

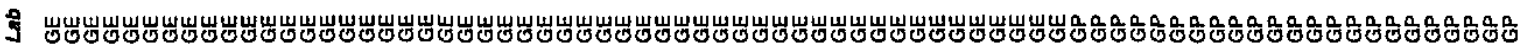

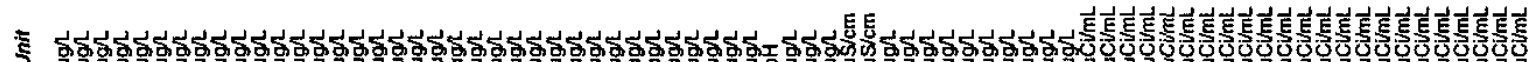

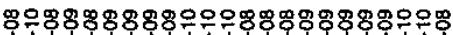

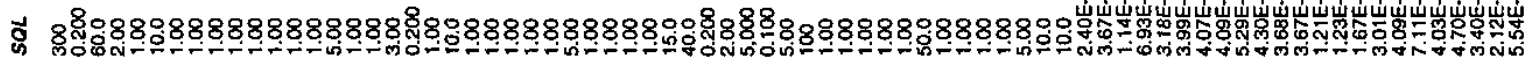

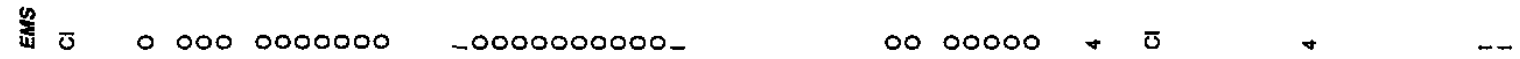
i د נ

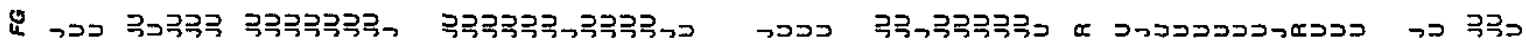

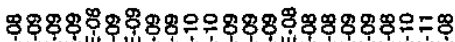

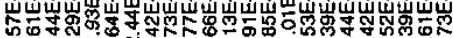

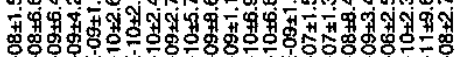

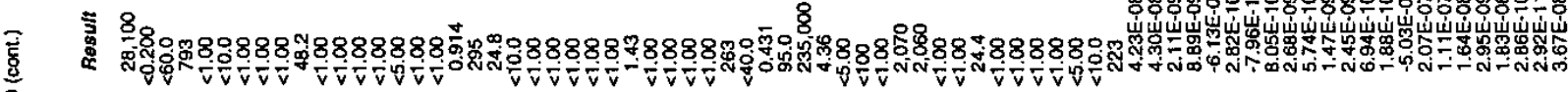




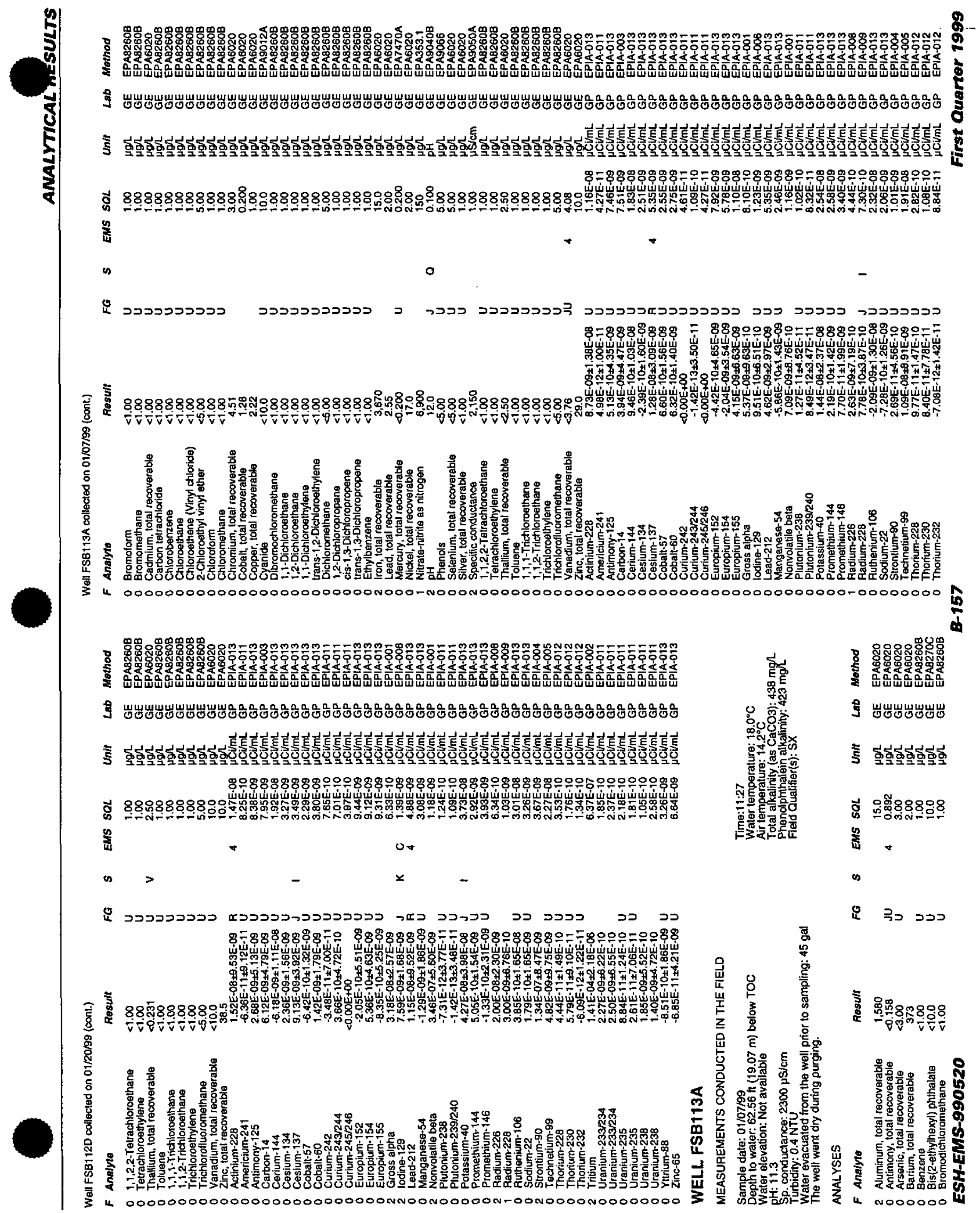


응 管

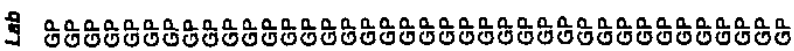

实

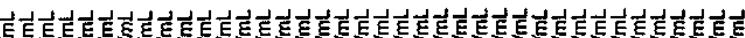

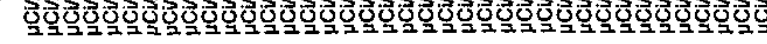

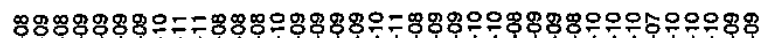

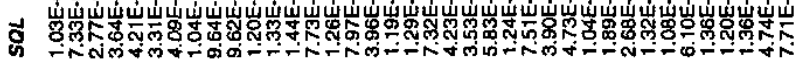
紊

$\infty$

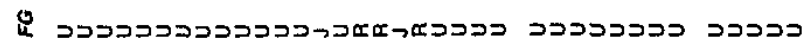

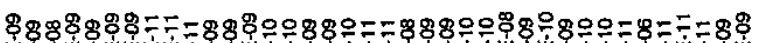

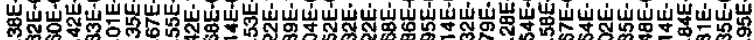

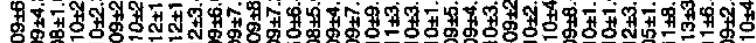

㝘

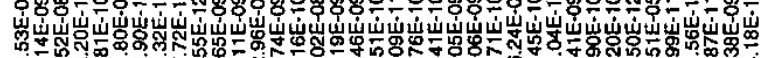
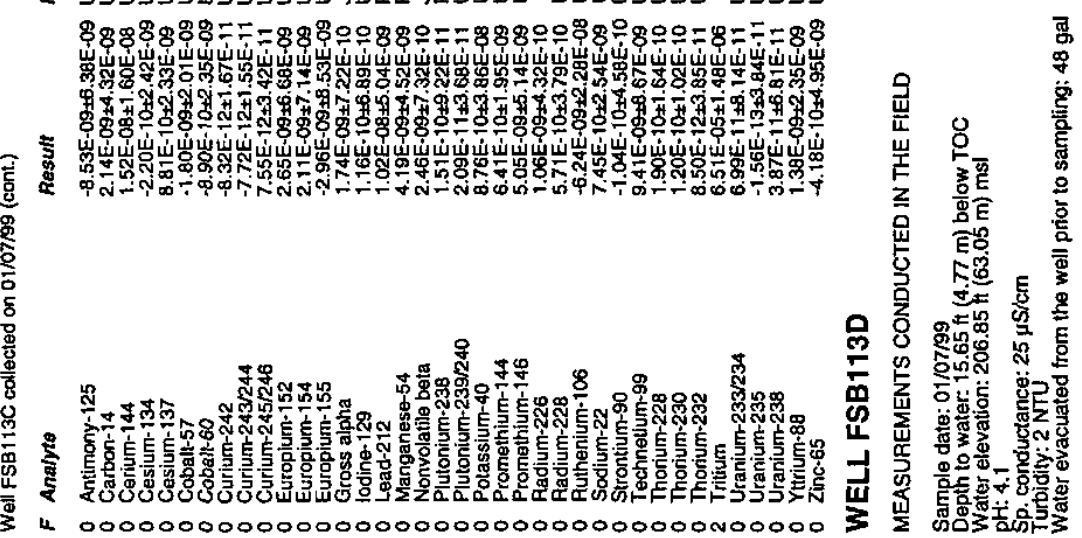

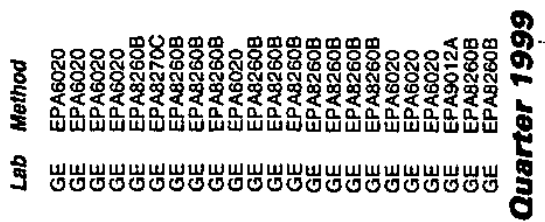

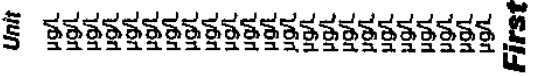

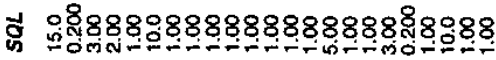

妥

is

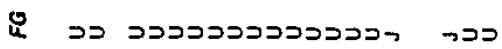

喜

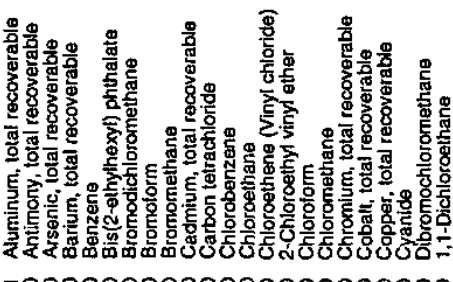

\&

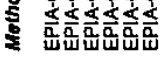

I 웅잉영용

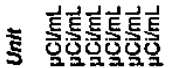

동ㅇ으으용요

ㅎำ

量

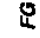

$8=-=88$

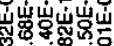

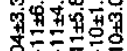

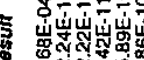

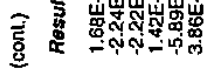

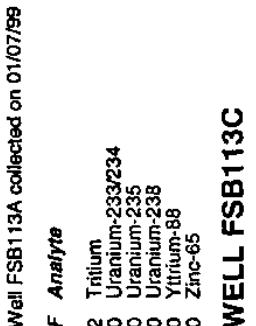

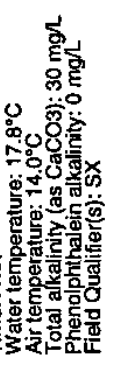

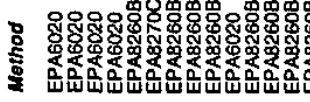

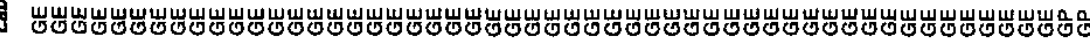

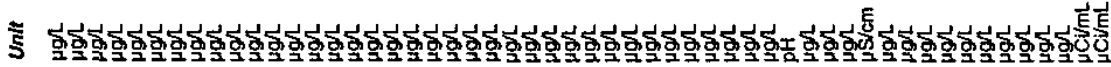

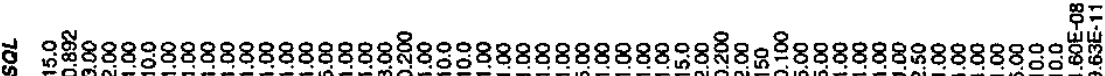

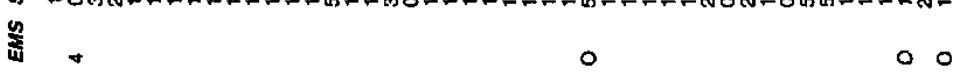

$\infty$

○
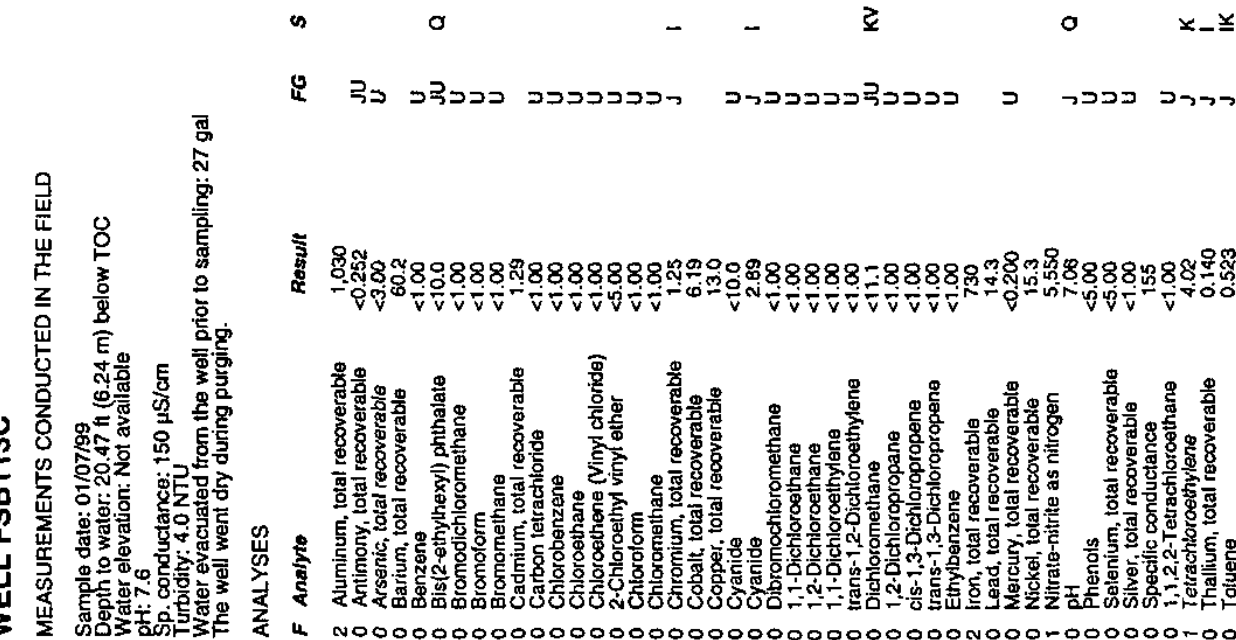

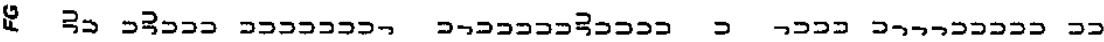

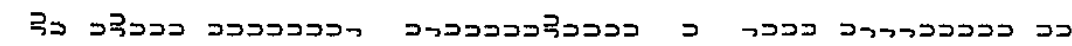

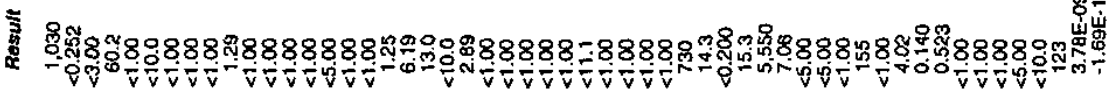

朔商

की

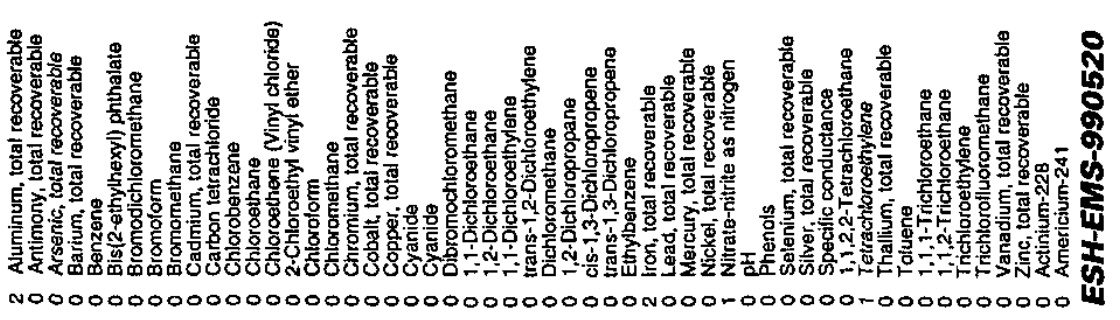

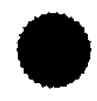




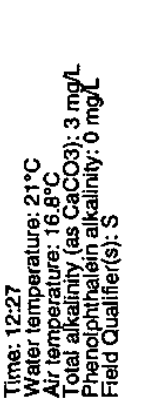

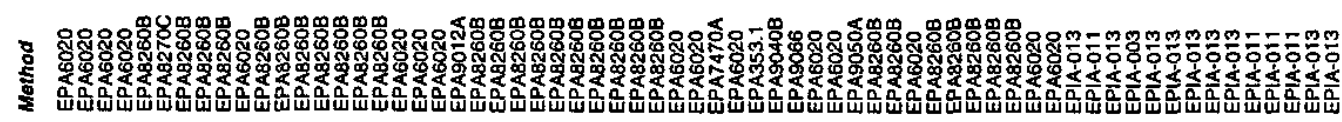

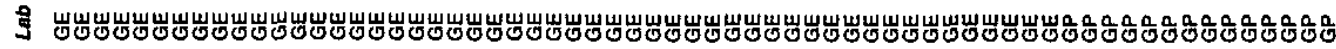

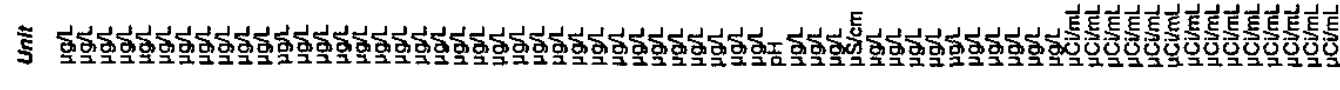

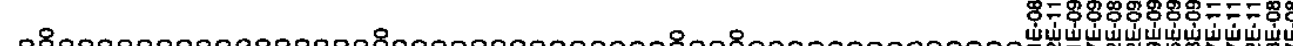

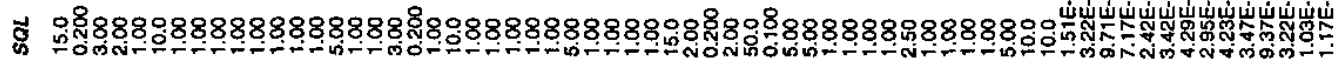
密

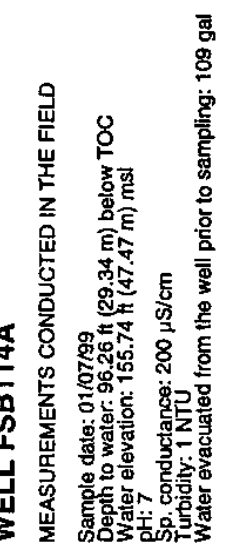

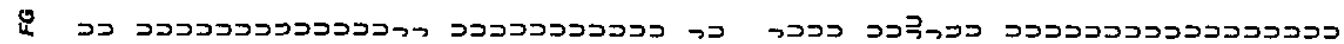
$8=8 \% 8 \% 888=88$

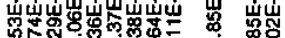

\% 衰

-

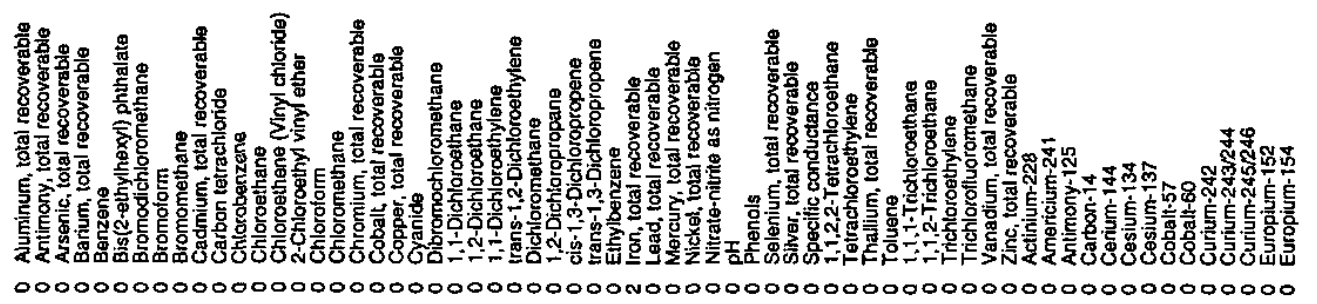

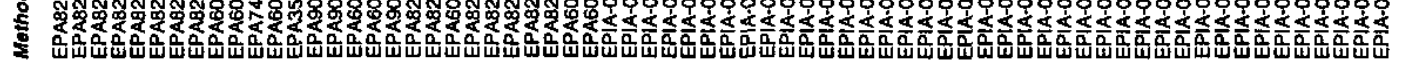

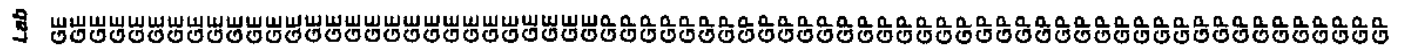

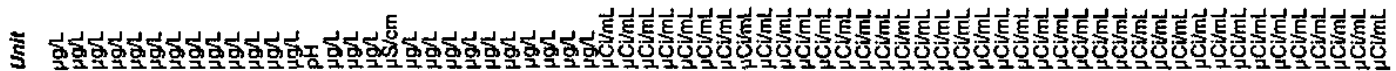

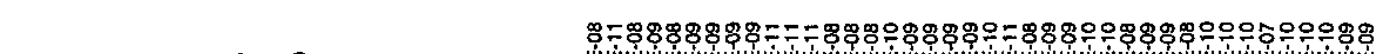

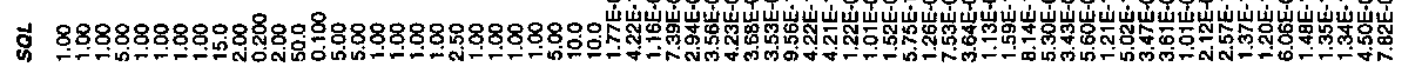

总

is $\geq--0 \quad \underline{0}$

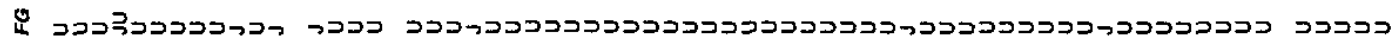
$8=8 \% 8 \%$

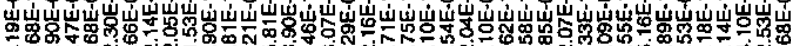

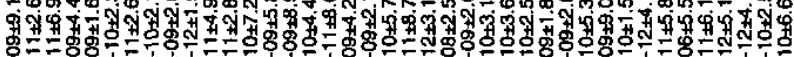

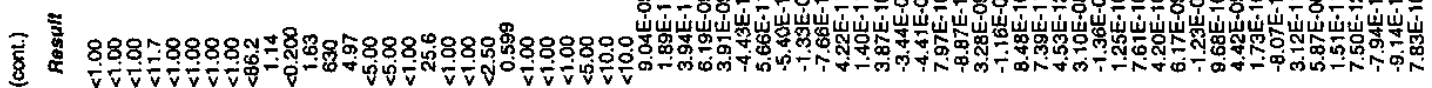

.

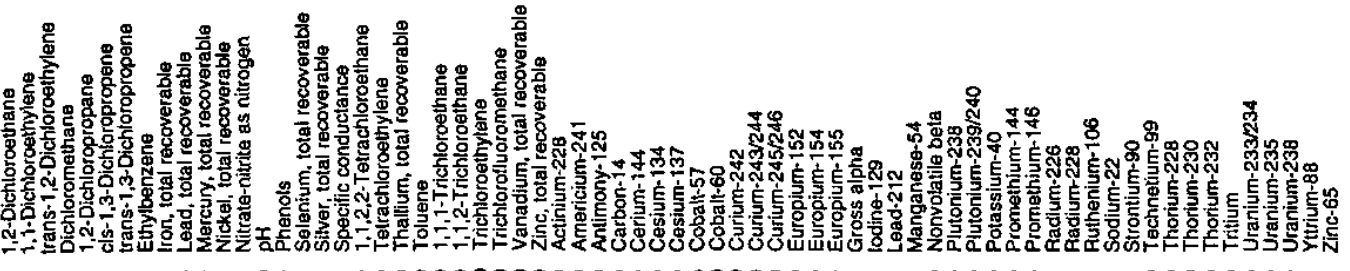




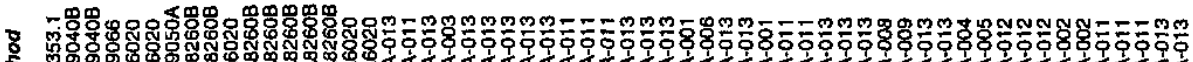
等

ปิ

₹

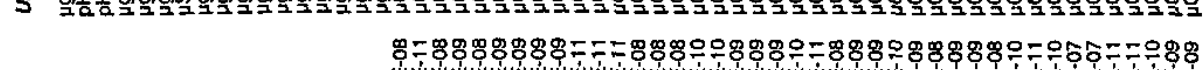

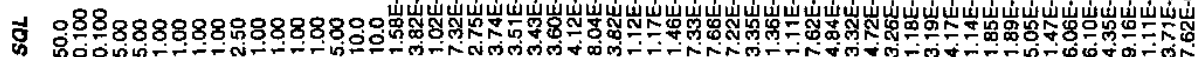

峦

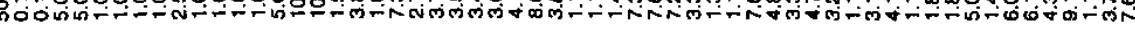

o

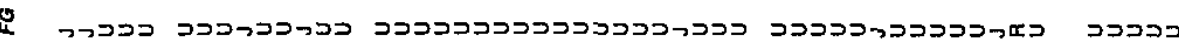
8: 8 \%

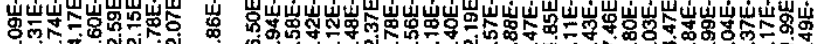

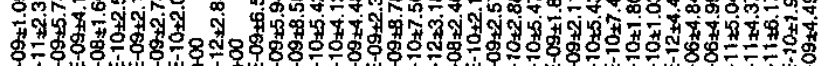
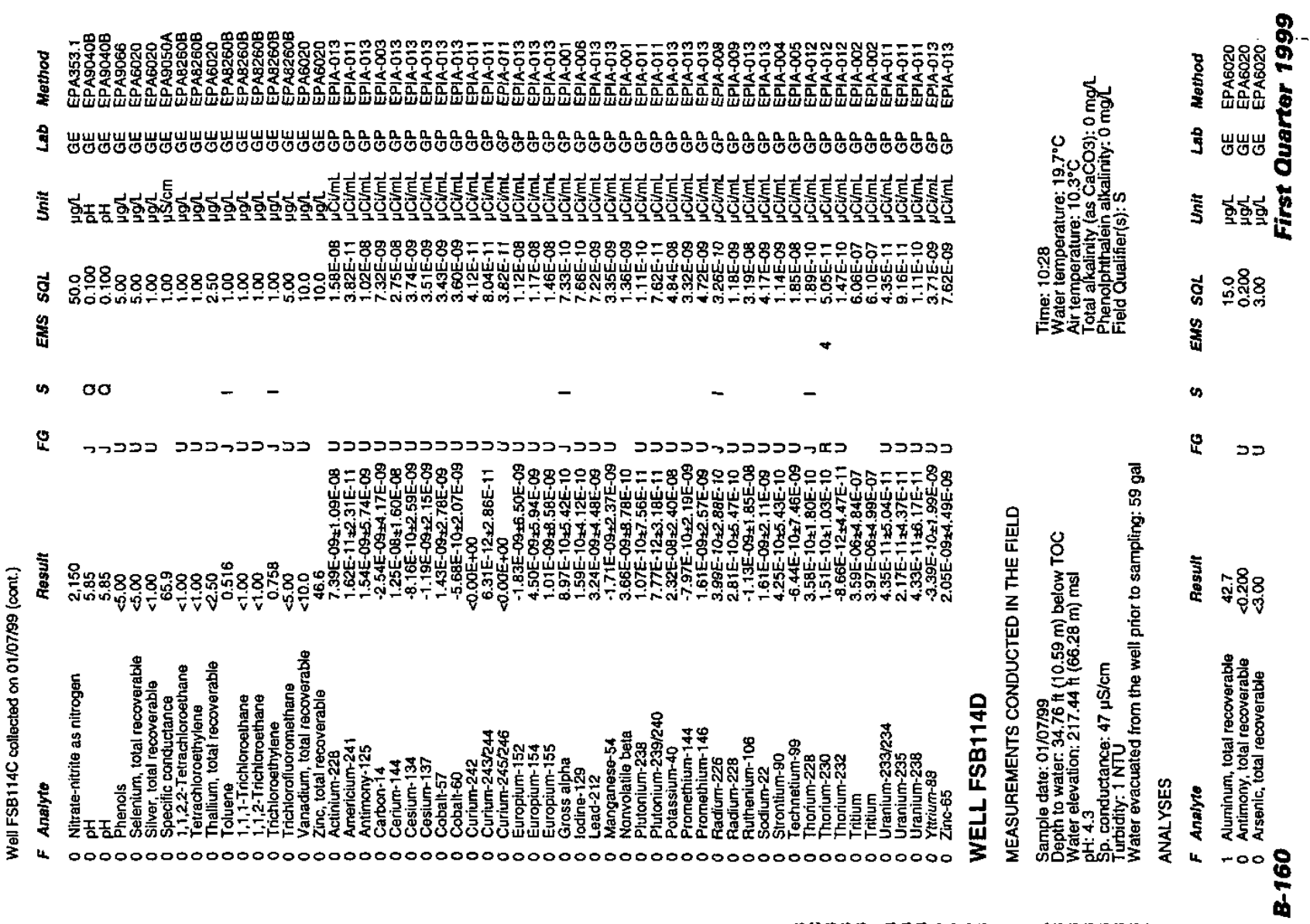

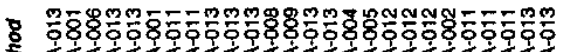
害

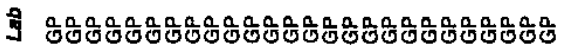

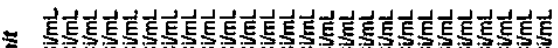

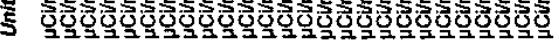

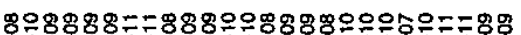

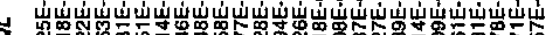

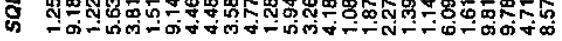

离

$\checkmark$

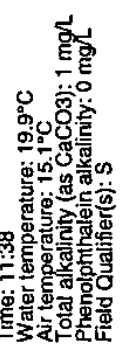

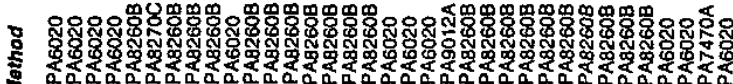

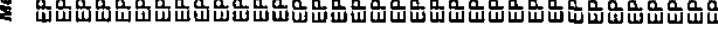

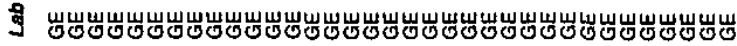

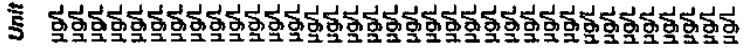

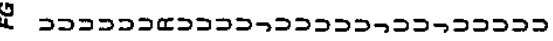

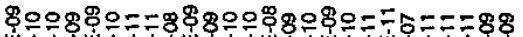

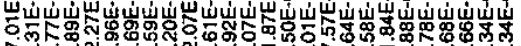

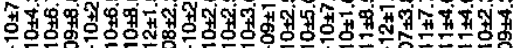

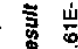

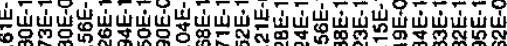

व 衷

:

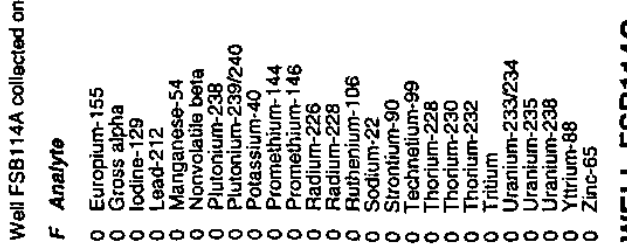

急

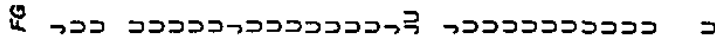

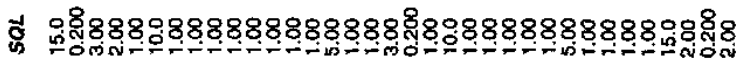
紊

$\infty$

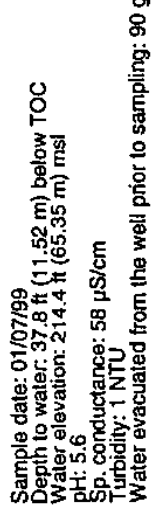

产

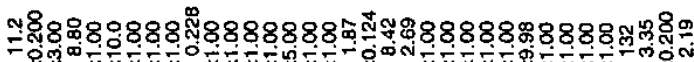

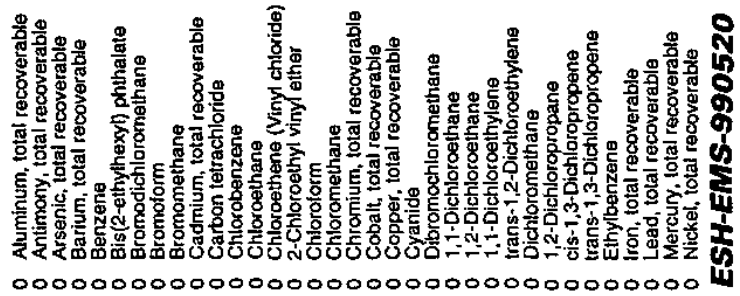

